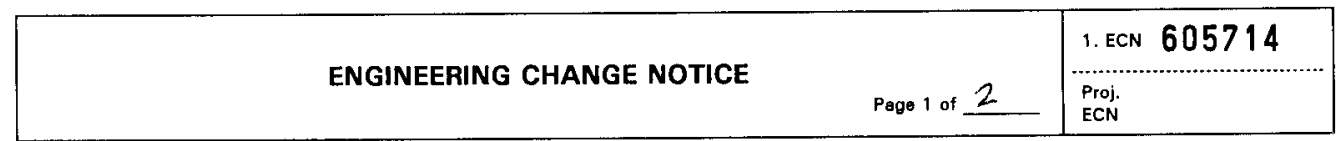

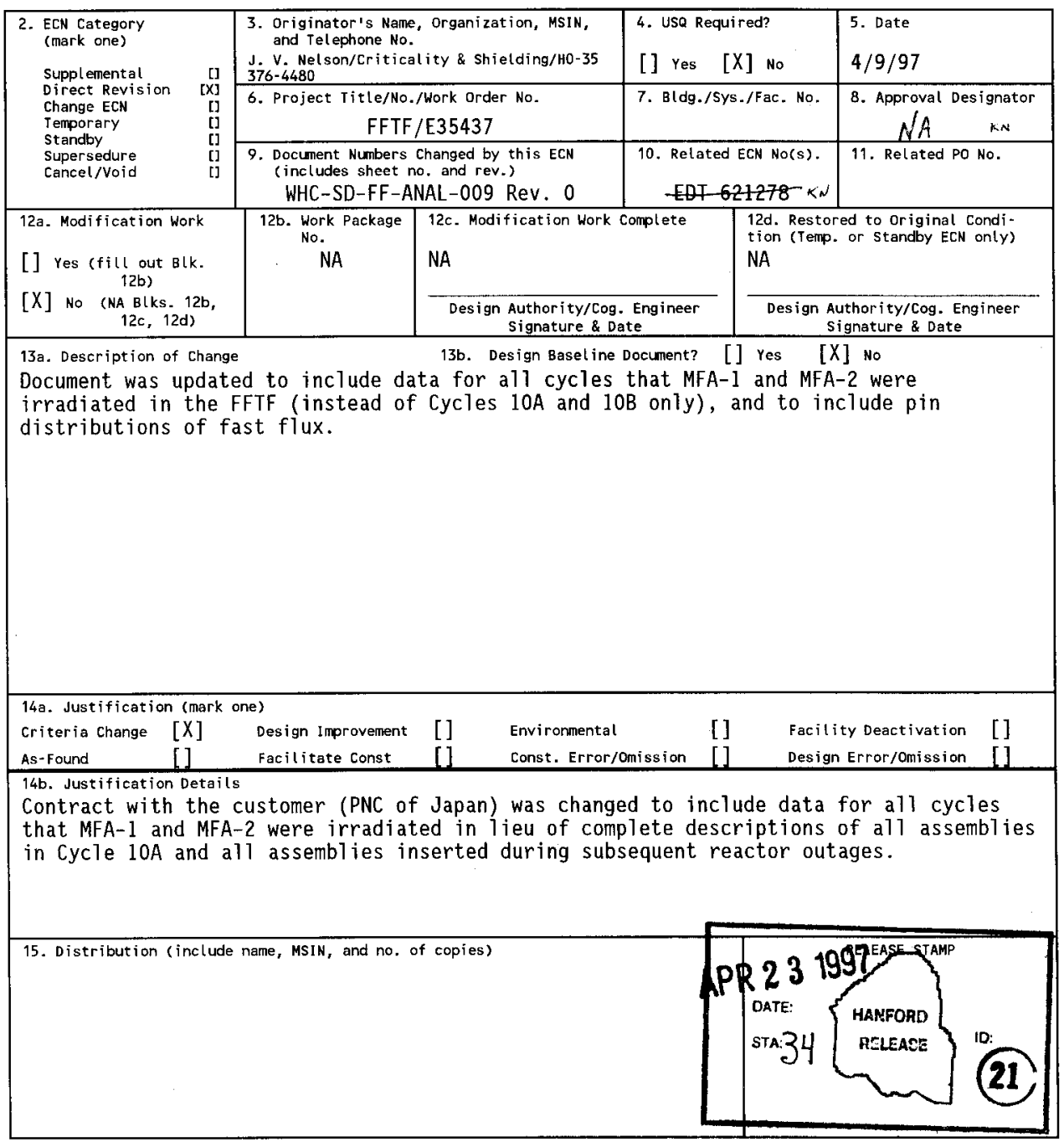

A-7900-013-2 (05/96) GEF095 


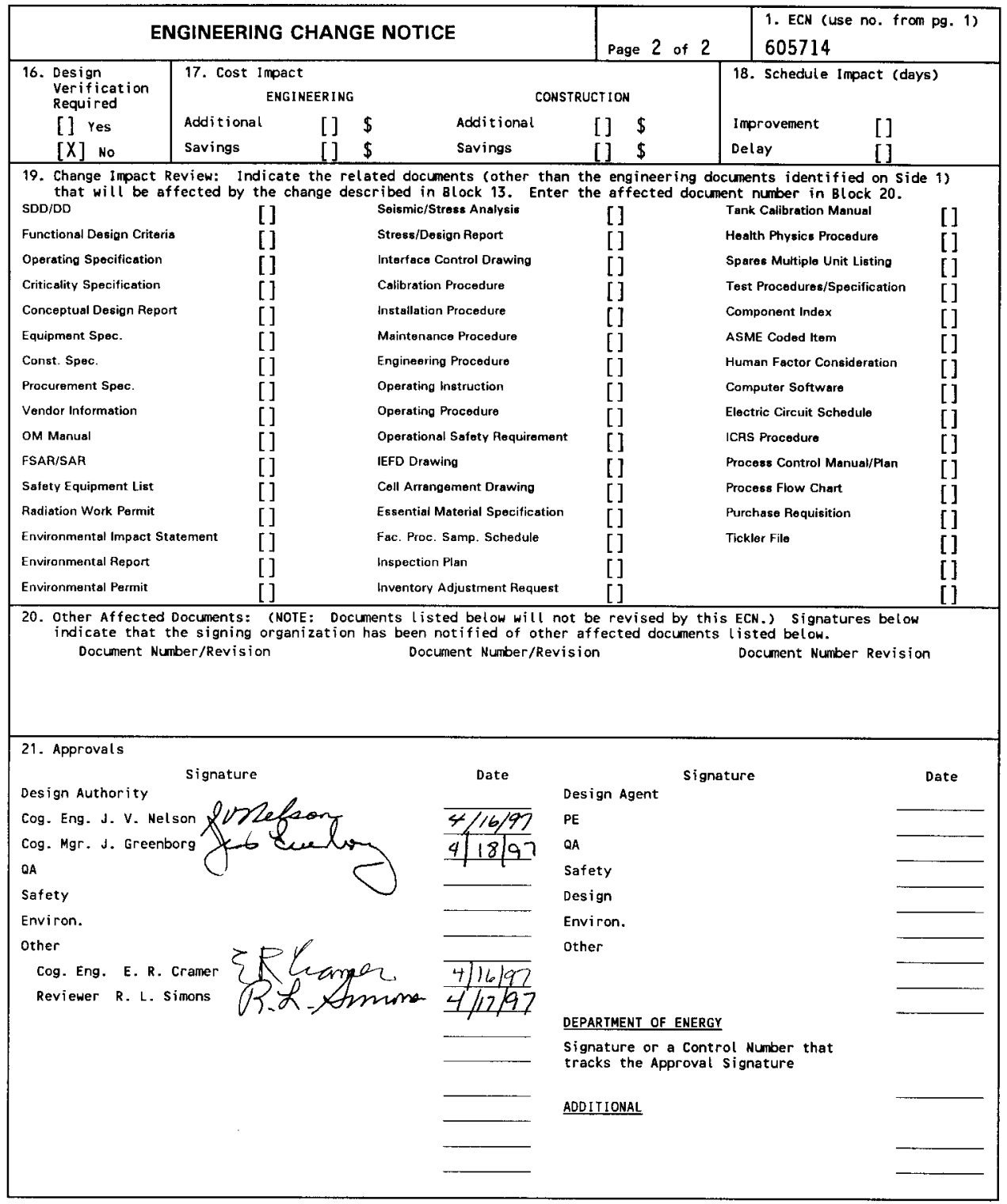




\section{Irradiation Data for the MFA-1 and MFA-2 Tests in the FFTF}

J. V. Nelson and E. R. Cramer

Fluor Daniel Northwest Inc./Numatec Hanford Company, Richland, WA 99352

U.S. Department of Energy Contract DE-AC06-96RL13200

\begin{tabular}{|c|c|c|c|}
\hline $\begin{array}{l}\text { EDT/ECN: } \\
\text { Org Code } \\
\text { B\&R Code }\end{array}$ & $\begin{array}{l}605714 \\
403 \\
\text { EX7050000 }\end{array}$ & $\begin{array}{l}\text { UC: } 532 \\
\text { Charge Code: } \\
\text { Total Pages: }\end{array}$ & $\begin{array}{l}E 35437 \\
434 \quad 435\end{array}$ \\
\hline
\end{tabular}

Key Words: FFTF, MONJU, Liquid Metal Reactor, fuel test, LMR, MFA

Abstract: This report provides key information on the irradiation environment of the MONJU fuel tests MFA-1 and MFA-2 in the Fast Flux Test Facility (FFTF). This information includes the fission powers, neutron fluxes, sodium temperatures and sodium flow rates in MFA-1, MFA2 and adjacent assemblies. It also includes MFA-1 and MFA-2 compositions as a function of exposure. The work was performed at the request of Power Reactor and Nuclear Fue]s Corporation (PNC) of Japan.

IRADEMARK DISCLAIMER - Reference herein to any specific commercial product, process, or service by trade name, trademark, manufacturer, or otherwise, does not necessarily constitute or imply its endorsement, recommendation, or favoring by the United States Government or any agency thereof or its contractors or subcontractors.

Printed in the United States of America. To obtain copies of this document, contact: Document Control Services, P.O. Box 950, Mailstop H6-08, Richland WA 99352, Phone (509) 372-2420; Fax (509) $376-4989$.
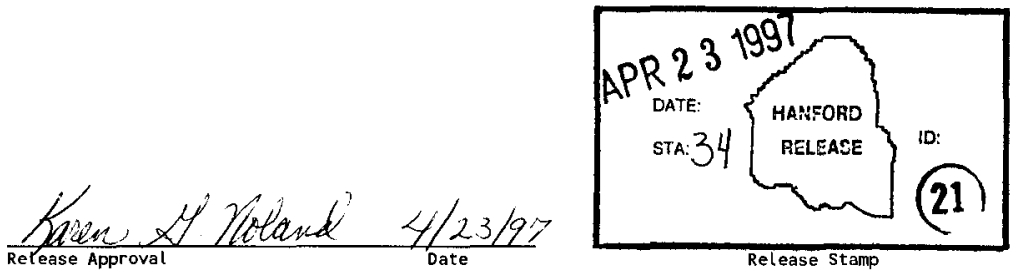

\section{Approved for Public Release}


(2) Title

Irradiation Data for the MFA-1 and MFA-2 Tests in the FFTF

CHANGE CONTROL RECORD

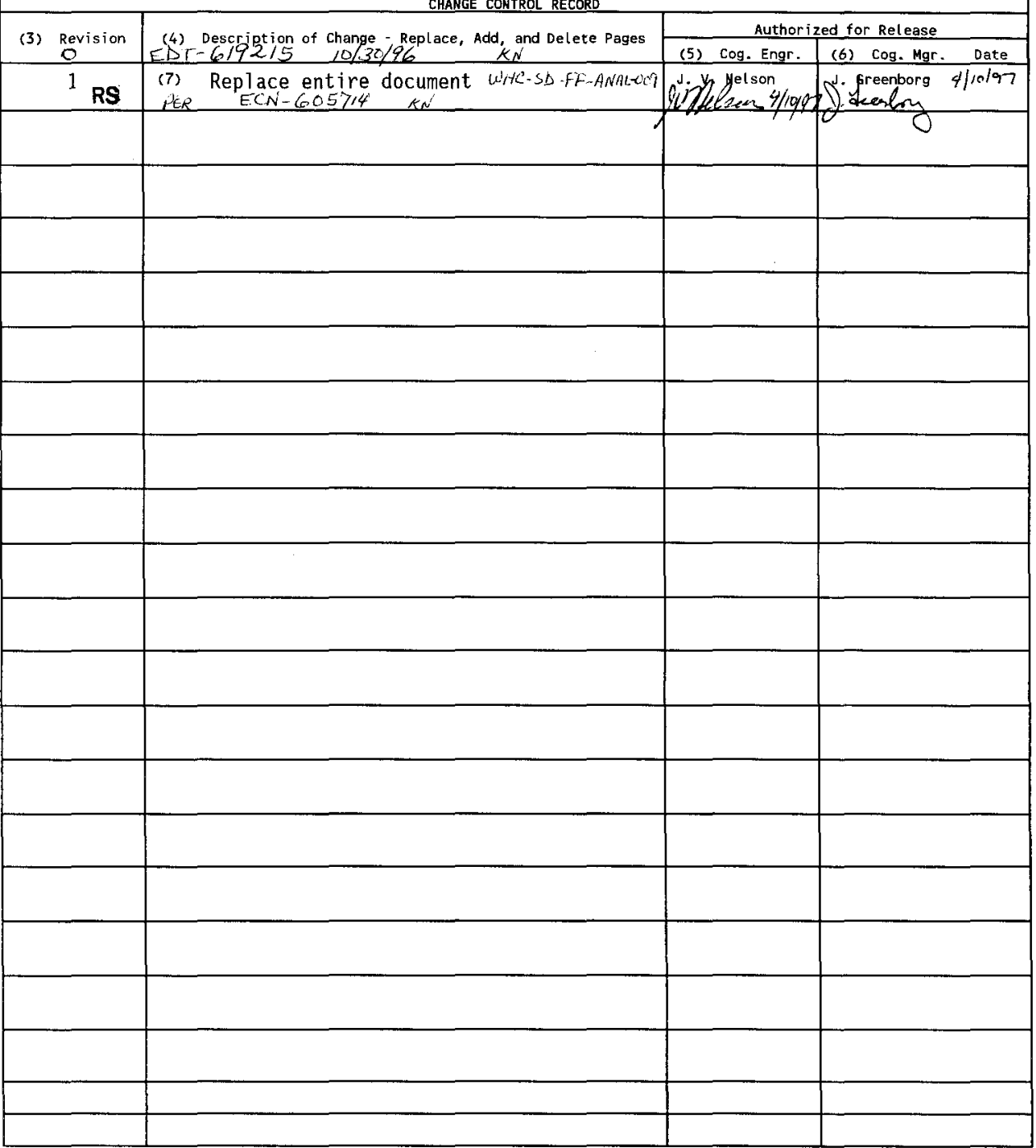


HNF-SD-FF-ANAL-009 Rev. 1

IRRADIATION DATA FOR THE MFA-1 AND MFA-2 TESTS IN THE FFTF

TABLE OF CONTENTS

1.0 Introduction . . . . . . . . . . . . . . . . . . 1

2.0 Description of Data Tables . . . . . . . . . . . . . 10

2.1 Assembly Fission Power ................ . . . . 10

2.2 Assembly Averaged Fast and Total Flux ............ 10

2.3 Axial Distributions of Total Flux, Fast Flux and Power : . 12

2.4 Fission Power Distribution by Pin ........... 12

2.5 Fast Flux Distribution by Pin . . . . . . . . . . . . . 12

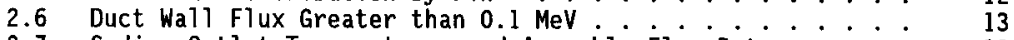

2.7 Sodium Outlet Temperatures and Assembly Flow Rates . . . . 13

2.8 Sodium Subchannel Temperatures . . . . . . . . . . . . . 13

2.9 Material Compositions . . . . . . . . . . . . . . . . . . 16

3.0 Contents of the Data Disk . . . . . . . . . . . . . . . 16

4.0 Data Tables for each Cycle . . . . . . . . . . . . . . 22

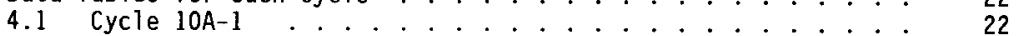

4.2 Cycle 10A-2

4.3 Cycle $10 A-3$. . . . . . . . . . . . . . . . . . . . 66

4.4 Cycle 10A-4 ........................ 78

4.5 Cycle $10 \mathrm{~B}$. . . . . . . . . . . . . . . . . . . . 98

4.6 Cycle $10 \mathrm{C}-1$. . . . . . . . . . . . . . . . . . . 130

4.7 Cycle $10 \mathrm{C}-3$. . . . . . . . . . . . . . . . . . 156

4.8 Cycle $11 \mathrm{~A}-1$. . . . . . . . . . . . . . . . . . . . . . . 181

4.9 Cycle $11 \mathrm{~A}-2$. . . . . . . . . . . . . . . . . . . . . . . 199

4.10 Cycle $11 A-3 \quad$. . . . . . . . . . . . . . . . . . . . . . 211

4.11 Cycle 11B-1 . . . . . . . . . . . . . . . . . . . . . . . 236

4.12 Cycle llB-2 ...................... 268

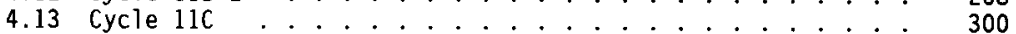

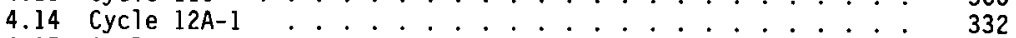

4.15 cycle $12 A-2$. . . . . . . . . . . . . . . . . . . 364

4.16 Cycle $12 \mathrm{~B}-1$. . . . . . . . . . . . . . . . . . 396

4.17 Cycle 12B-2 . . . . . . . . . . . . . . . . . . . . 414 
HNF-SD-FF-ANAL-009 Rev. 1

\section{IRRADIATION DATA FOR THE MFA-1 AND MFA-2 TESTS IN THE FFTF}

\subsection{Introduction}

This report provides data relevant to the irradiation of the MONJU fuel experiments MFA-1 and MFA-2 during their residence in the Fast Flux Test Facility (FFTF) core. MFA-1 was irradiated during operating Cycles 10A-1 through 12A-2, while MFA-2 was irradiated during Cycles 10A-1 through $12 \mathrm{~B}-2$. The operating history of these cycles is summarized in Table 1. The data are supplied in tabular form in this report, and most data are also available in electronic forms.

Data given for MFA-1 and MFA-2 include:

- assembly fission powers, fast neutron fluxes $(\phi>0.1 \mathrm{MeV})$, total fluxes, outlet temperatures and coolant flow rates,

- spatial distributions of fast flux and fission power by pin, and fast flux at the duct walls,

- subchannel sodium temperatures, and

- burnup dependant material compositions.

Also included are powers, outlet temperatures, and flow rates for the assemblies adjacent to MFA-1 and MFA-2. Composition data are given for the end of each cycle. Most of the other data are provided for the beginning and end of each of the 1 isted operating cycles, except for cycles less than 25 equivalent full power days (EFPD) in length. For these short cycles, only beginning of cycle data are included. However, no data are given for cycle $10 \mathrm{C}-2$ because its length was only 2.9 EFPD. Data for the end of Cycle $10 \mathrm{C}-1$ are very representative of the data for Cycle $10 \mathrm{C}-2$.

The irradiation history of MFA-1 and MFA-2 is 1isted in Table 2. Figure 1 shows the FFTF core loading for Cycle 10A-1. Loading changes made in core locations adjacent to MFA-1 and MFA-2 between Cycles 10A-1 and 10C-3 are identified in Table 3. As indicated in Table 2, MFA-1 and MFA-2 were both moved during the Cycle $11 \mathrm{~A}-1$ outage. Figure 2 shows the FFTF core loading for Cycle 11A-1. Loading changes made in core locations adjacent to MFA-1 and MFA-2 between Cycles $11 \mathrm{~A}-1$ and $11 \mathrm{~B}-1$ are identified in Table 4 .

MFA-1 was relocated to core location 1405 during the Cycle 11B-2 outage. Figure 3 shows the FFTF core loading for Cycle $11 \mathrm{~B}-2$. Loading changes made in core locations adjacent to MFA-1 and MFA-2 between Cycles $11 \mathrm{~B}-2$ and $12 \mathrm{~A}-2$ are identified in Table 5 . During the Cycle 12B-1 outage, MFA-1 was removed from the core and MFA-2 was moved to core location 2405. Figure 4 shows the FFTF core loading for Cycle $12 \mathrm{~B}-1$. There were no loading changes made to core locations adjacent to MFA-2 after Cycle 12B-1. 
HNF-SD-FF-ANAL-009 Rev. 1

Table 1. Summary of FFTF Cycles 10A-1 through 12B-2 Power Operation

\begin{tabular}{||c|c|c|c||}
\hline Cycle & Startup Date & Shutdown Date & $\begin{array}{c}\text { Cycle Length } \\
(\text { EFPO })\end{array}$ \\
\hline $10 A-1$ & $11 / 18 / 87$ & $1 / 26 / 88$ & 66.3 \\
\hline $10 A-2$ & $2 / 1 / 88$ & $2 / 23 / 88$ & 20.3 \\
\hline $10 A-3$ & $2 / 25 / 88$ & $3 / 4 / 88$ & 5.8 \\
\hline $10 A-4$ & $3 / 7 / 88$ & $5 / 7 / 88$ & 59.4 \\
\hline $10 B$ & $6 / 11 / 88$ & $10 / 17 / 88$ & 126.7 \\
\hline $10 C-1$ & $11 / 9 / 88$ & $1 / 8 / 89$ & 56.9 \\
\hline $10 C-2$ & $1 / 16 / 89$ & $1 / 20 / 89$ & 2.9 \\
\hline $10 C-3$ & $1 / 23 / 89$ & $3 / 13 / 89$ & 47.0 \\
\hline $11 \mathrm{~A}-1$ & $5 / 3 / 89$ & $5 / 16 / 89$ & 11.3 \\
\hline $11 \mathrm{~A}-2$ & $5 / 21 / 89$ & $6 / 2 / 89$ & 10.5 \\
\hline $11 \mathrm{~A}-3$ & $6 / 14 / 89$ & $9 / 19 / 89$ & 94.6 \\
\hline $11 \mathrm{~B}-1$ & $1 / 4 / 90$ & $4 / 8 / 90$ & 85.5 \\
\hline $11 \mathrm{~B}-2$ & $5 / 29 / 90$ & $10 / 27 / 90$ & 132.7 \\
\hline $11 \mathrm{C}$ & $12 / 15 / 90$ & $3 / 19 / 91$ & 81.5 \\
\hline $12 \mathrm{~A}-1$ & $5 / 27 / 91$ & $7 / 20 / 91$ & 52.8 \\
\hline $12 \mathrm{~A}-2$ & $8 / 1 / 91$ & $9 / 21 / 91$ & 48.6 \\
\hline $12 \mathrm{~B}-1$ & $11 / 22 / 91$ & $1 / 15 / 92$ & 50.3 \\
\hline $12 \mathrm{~B}-2$ & $1 / 26 / 92$ & $3 / 19 / 92$ & 51.6 \\
\hline
\end{tabular}

- EFPO = equivalent full power days. Full power $=291$ MH thermal. 
HNF-SD-FF-ANAL-009 Rev. I

Table 2. Irradiation History of MFA-1 and MFA-2

\begin{tabular}{|c|c|c|c|}
\hline \multirow[b]{2}{*}{ Cycle } & \multirow{2}{*}{$\begin{array}{c}\text { Cycle Length } \\
\text { (EFPD) }\end{array}$} & \multicolumn{2}{|c|}{ Assembly Location } \\
\hline & & MFA-1 & MFA-2 \\
\hline $10 \mathrm{~A}-1$ & 66.3 & \multirow{8}{*}{1506} & \multirow{8}{*}{2507} \\
\hline $10 \mathrm{~A}-2$ & 20.3 & & \\
\hline $10 \mathrm{~A}-3$ & 5.8 & & \\
\hline $10 A-4$ & 59.4 & & \\
\hline $10 \mathrm{~B}$ & 126.7 & & \\
\hline $10 C-1$ & 56.9 & & \\
\hline $10 C-2$ & 2.9 & & \\
\hline $10 C-3$ & 47.0 & & \\
\hline $11 \mathrm{~A}-1$ & 11.3 & \multirow[t]{4}{*}{1404} & \multirow[t]{8}{*}{2506} \\
\hline $11 \mathrm{~A}-2$ & 10.5 & & \\
\hline $11 \mathrm{~A}-3$ & 94.6 & & \\
\hline $11 \mathrm{~B}-1$ & 85.5 & & \\
\hline $11 \mathrm{~B}-2$ & 132.7 & \multirow[t]{4}{*}{1405} & \\
\hline $11 \mathrm{C}$ & 81.5 & & \\
\hline $12 \mathrm{~A}-1$ & 52.8 & & \\
\hline $12 \mathrm{~A}-2$ & 48.6 & & \\
\hline $12 \mathrm{~B}-1$ & 50.3 & & \multirow[t]{2}{*}{2405} \\
\hline $12 \mathrm{~B}-2$ & 51.6 & & \\
\hline
\end{tabular}




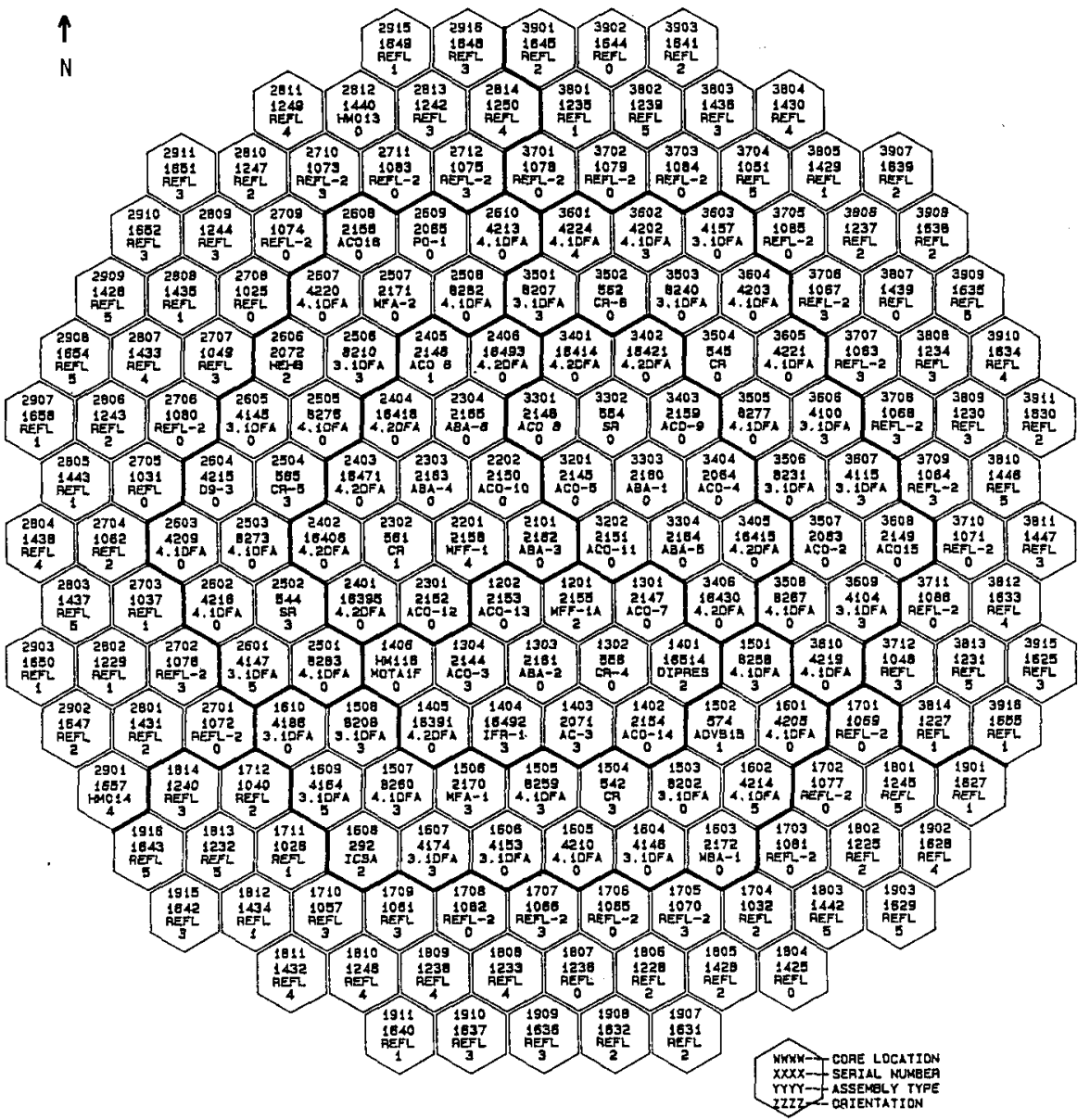

Figure 1. FFTF Cycle 10A-1 Core Loading 
Table 3. Loading Changes Made to Core Locations adjacent to MFA-1 and MFA-2 for Cycles 10A-2 through 10C-3

\begin{tabular}{|c|c|c|c|c|c|}
\hline \multirow{2}{*}{$\frac{\text { Cycle }}{10 \mathrm{~A}-2}$} & \multirow{2}{*}{$\begin{array}{c}\begin{array}{c}\text { Core } \\
\text { Position }\end{array} \\
2608 \\
\end{array}$} & \multicolumn{2}{|c|}{$\begin{array}{l}\text { Assembly Removed } \\
\text { (Serial No. \& Type) }\end{array}$} & \multicolumn{2}{|c|}{$\begin{array}{l}\text { Assembly Inserted } \\
\text { (serial No. \& Type) }\end{array}$} \\
\hline & & 2156 & $A C 0-16^{C}$ & 4114 & 3. $1 \mathrm{DFA}^{\mathrm{a}}$ \\
\hline $10 A-4$ & 2609 & 2065 & $P 0-1^{d}$ & 4140 & 3.1DFA \\
\hline \multirow[t]{5}{*}{$10 \mathrm{~B}$} & 1405 & 16391 & 4.2DFA & 2168 & $\mathrm{MFF}-2^{\mathrm{e}}$ \\
\hline & 1505 & 8259 & 4.1DFA & 8265 & 4. IDFA \\
\hline & 2405 & 2146 & $\mathrm{ACO}-6^{\mathrm{C}}$ & 16396 & 3.2DFA ${ }^{\mathrm{a}}$ \\
\hline & 2506 & 8210 & 3.1DFA & 8268 & 4.1DFA \\
\hline & 2609 & 4140 & 3.1DFA & 4152 & 4. 1DFA \\
\hline \multirow[t]{6}{*}{$10 C-1$} & 1404 & 16492 & IFR-1 $^{e}$ & 16391 & 4.2DFA \\
\hline & 1505 & 8265 & 4.1DFA & 8228 & 3.1DFA \\
\hline & 1507 & 8260 & 4. IDFA & 8209 & 3.1DFA \\
\hline & 1607 & 4174 & 3.1DFA & 4192 & 4. IDFA \\
\hline & 2405 & 16396 & 3.2DFA & 16390 & 3.2DFA \\
\hline & 2508 & 8282 & 4.1DFA & 8212 & 3.1DFA \\
\hline
\end{tabular}

\footnotetext{
3. IDFA and 3.2DFA are standard FFTF series I drivers

b 4.1DFA and 4.2DFA are standard FFTF series II drivers

c ACO-6 and ACO-16 are Core Demonstration Experiment (CDE) fuel assenblies

d $\mathrm{PO}-1$ is an advanced oxide fuel test

e MFF-2 and IFR-1 are metal fuel experiments
} 


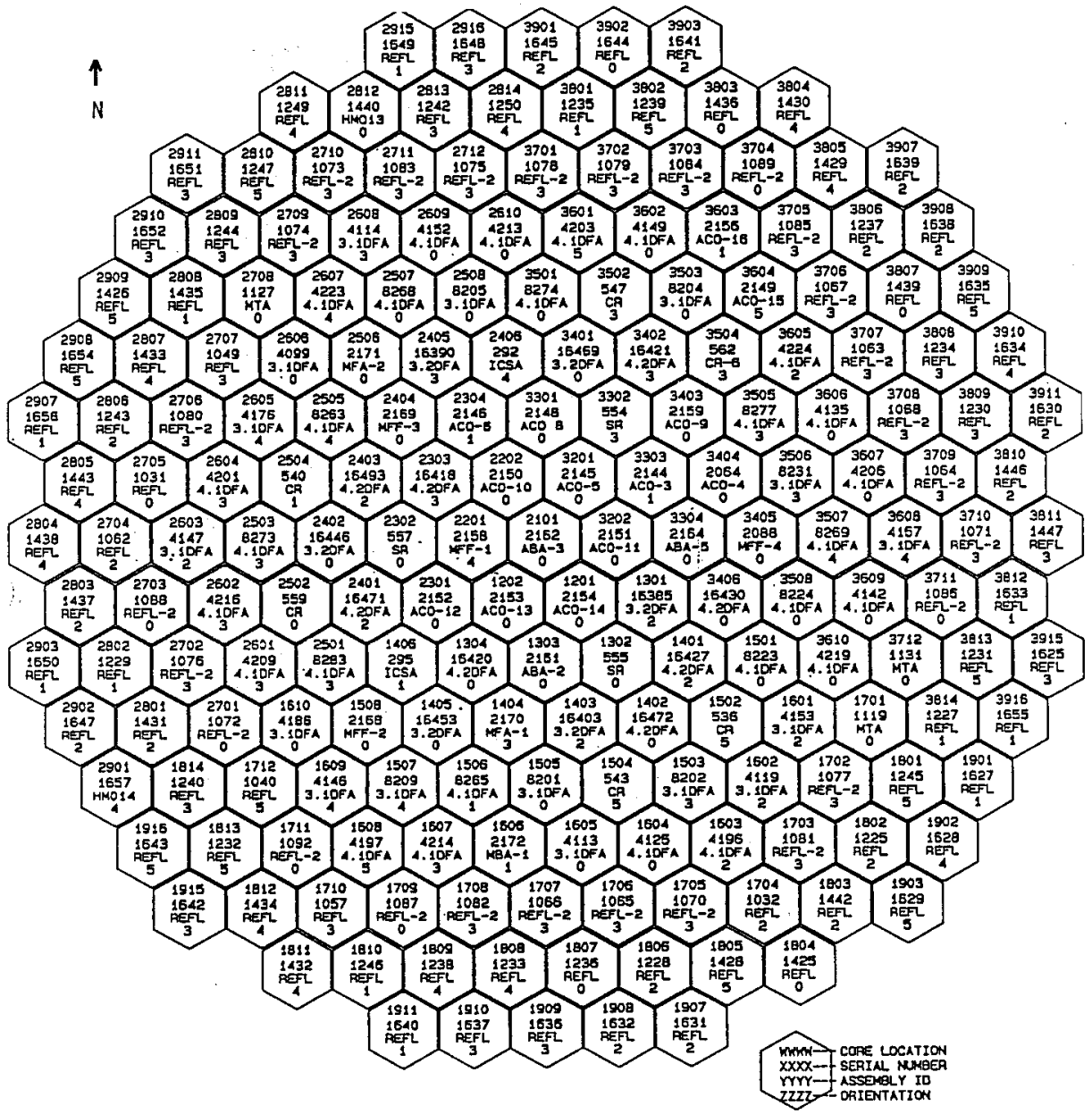

Figure 2. FFTF Cycle 11A-1 Core Loading 
Table 4. Loading Changes Made to Core Locations adjacent to MFA-1 and MFA-2 for Cycles 11A-2 through 11B-1

\begin{tabular}{|c|c|c|c|}
\hline Cycle & $\begin{array}{c}\text { Core } \\
\text { Position }\end{array}$ & $\begin{array}{c}\text { Assembly Removed } \\
\text { (Serial No. \& Type) }\end{array}$ & $\begin{array}{l}\text { Assembly Inserted } \\
\text { (serial No. \& Type) }\end{array}$ \\
\hline $11 \mathrm{~A}-3$ & 2607 & 4.1DFA ${ }^{\mathrm{b}}$ & 3.1DFA \\
\hline \multirow[t]{3}{*}{$11 \mathrm{~B}-1$} & 1304 & $16420 \quad 4.2 \mathrm{DFA}^{\mathrm{b}}$ & $M F F-5^{c}$ \\
\hline & 1403 & $16403 \quad 3.2$ DFA $^{\mathrm{a}}$ & $16420 \quad 4.2 D F A$ \\
\hline & 2405 & $16390 \quad 3.2 D F A$ & 2116 \\
\hline
\end{tabular}

a 3.1DFA and 3.20FA are standard FFTF series I drivers.

b 4 . 1DFA and 4.20FA are standard FFTF series II drivers.

c MFF-2 is a metal fuel experiment.

do- 1 is a fuel test containing 7 enriched $\mathrm{uO}_{2}$ pins and 210 mixed-oxide pins.

Table 5. Loading Changes Made to Core Locations adjacent to MFA-1 and MFA-2 for Cycles 11C through 12A-2

\begin{tabular}{|c|c|c|c|}
\hline Cycle & $\begin{array}{c}\text { Core } \\
\text { Position }\end{array}$ & $\begin{array}{l}\text { Assembly Removed } \\
\text { (Serial No. \& Type) }\end{array}$ & $\begin{array}{l}\text { Assembly Inserted } \\
\text { (serial No. \& Type) }\end{array}$ \\
\hline \multirow[t]{4}{*}{$11 \mathrm{C}$} & 2405 & UO- $1^{c}$ & $16388 \quad 3.2 D F A^{a}$ \\
\hline & 2505 & 4.1DFA & 4. 1DFA \\
\hline & 2507 & 4. IDFA & 4.1DFA \\
\hline & 2607 & 3. $1 \mathrm{DFA}^{3}$ & 4. 1DFA \\
\hline $12 \mathrm{~A}-1$ & 1406 & HM212 MOTA1G & $16498 \quad 4.2$ DFA \\
\hline $12 \mathrm{~A}-2$ & 1507 & 4.1DFA & 4.1DFA \\
\hline
\end{tabular}

a 3.1DFA and 3.2DFA are standard FFTF series I drivers

b 4.10FA and 4.2DFA are standard FFTF series II drivers

c vo- 1 is a fuel test containing 7 enriched $\mathrm{UO}_{2}$ pins and 210 mixed-oxide pins.

mOTAlg is a material open test assenbly 


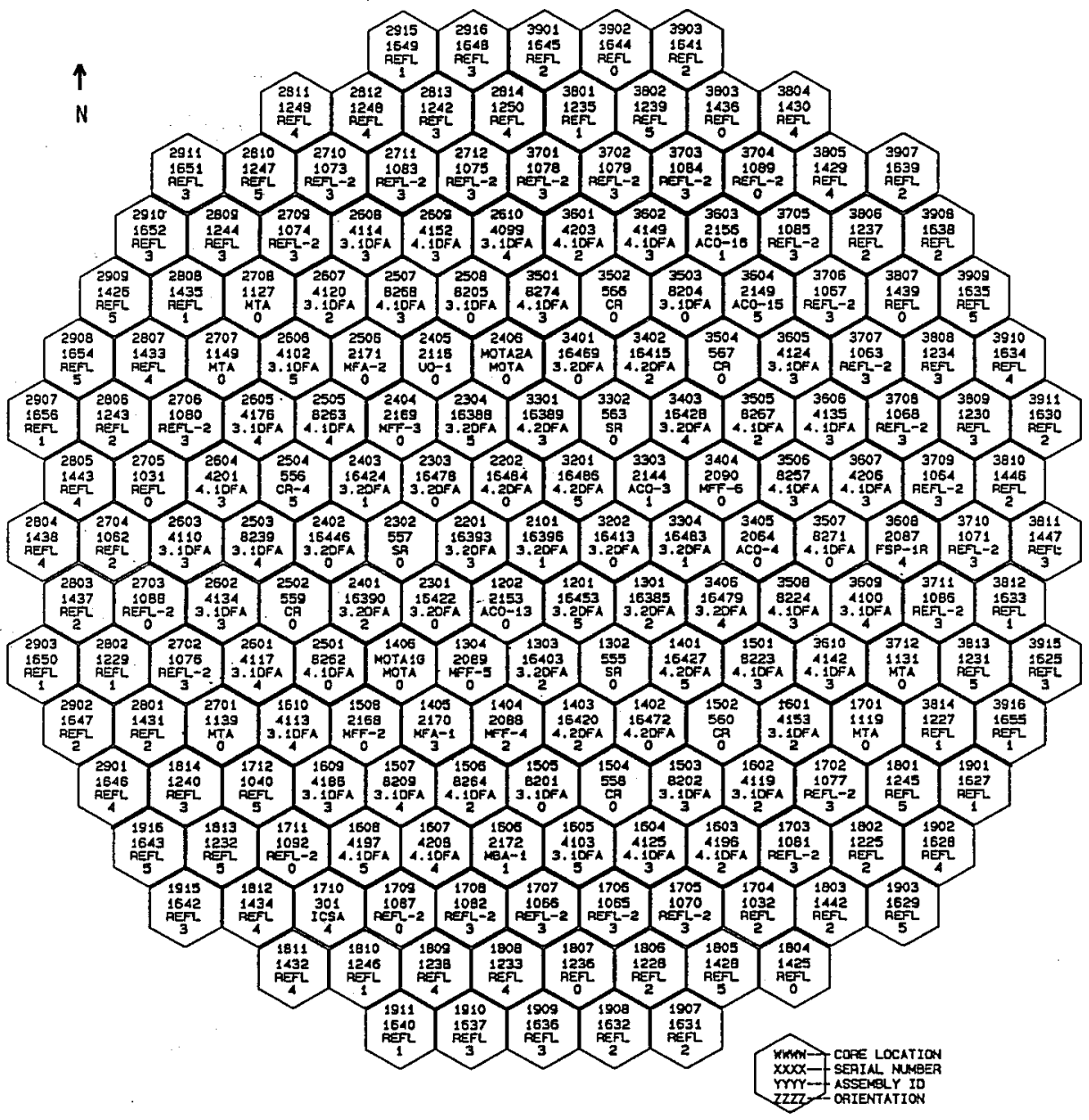

Figure 3. FFTF Cycle 11B-2 Core Loading 


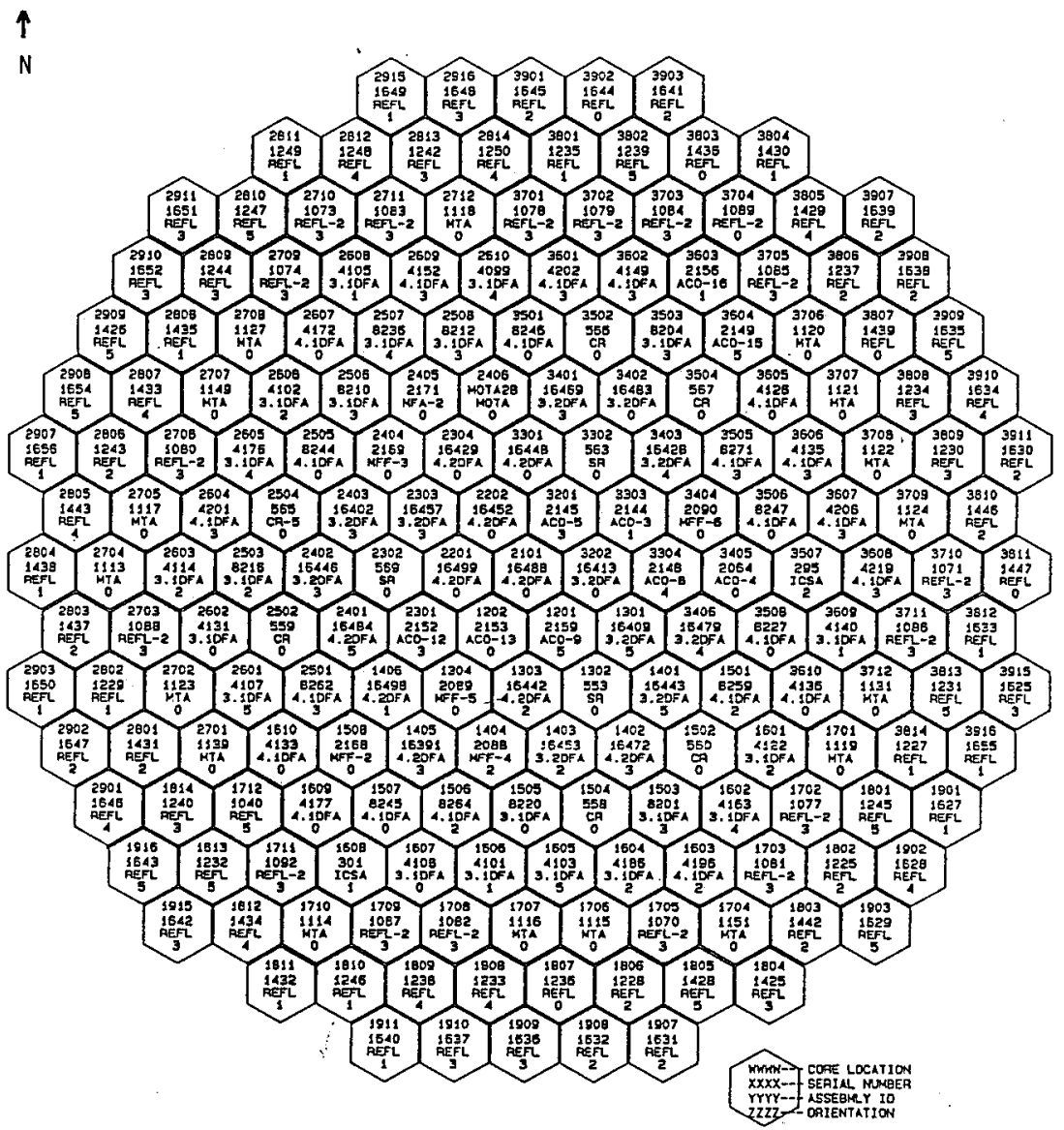

Figure 4. FFTF Cycle 12B-1 Core Loading 


\subsection{Description of Data Tables}

Included Sections 4.0 through 4.17 of this report are 18 different tables for each cycle. As indicated above, some of these tables were generated for beginning of cycle (BOC) conditions, some were generated for end of cycle (EOC) conditions, and many were generated for both $B O C$ and EOC conditions. Most tables are also available in electronic form on disk. However, others exist only in hard copy form from previous computer runs. These runs could not be repeated to obtain disk files because the computer programs needed have not been installed on any computer system that is currently available.

Table 6 summarizes the information contained in each of the 18 data tables, indicates whether this information is provided for $B O C$, EOC or both, and identifies the tables that are also available on disk. Data tables (both in printed form and on disk files) are identified using the following notation: TCCC.N, where $T$ is either the letter B (for beginning of cycle) or $E$ (for end of cycle), CCC is a cycle name (i.e., 10A-1, 10A-2,.., 12B-2), and $N$ is a table number $(1-18)$ identified in Table 6 . For example, Table BI0A-4.1 lists the fission power generated in MFA-1, MFA-2 and neighboring assemblies at the beginning of Cycle 10A-4 (BOC 10A-4).

The following sections explain the contents of each of the 18 types of tables.

\subsection{Assembly Fission Power}

The fission power generated in MFA-1 and each of its six adjacent assemblies, and in MFA-2 and each of its six adjacent assemblies are listed in Tables $\mathrm{B} 10 \mathrm{~A}-1.1, \mathrm{E} 10 \mathrm{~A}-1.1, \mathrm{~B} 10 \mathrm{~A}-2.1, \mathrm{E} 10 \mathrm{~A}-2.1$, etc. In these tables, the total fission power is given for each assembly. Also, a breakdown of the power by axial material region (fuel, axial blankets and $\mathrm{UO}_{2}$ insulator pellets) is included. Standard fuel assemblies that do not have axial blankets have insulator pellets between the fuel and the axial reflector regions.

Fission powers were computed using the three-dimensional diffusion theory and burnup program 3DB with nuclear cross section data in 12 energy groups. The computed flux and power data were adjusted by applying row-dependant bias factor that ranged in value from 0.98 to 1.02 . For rows 4 and 5 where MFA-1 and MFA-2 were located, the bias factors applied were 0.988 and 0.984 , respectively. The bias factors were determined by comparing the results of measurements made as part of the FFTF reactor characterization program, diffusion theory calculations and Monte Carlo (MCNP) calculations.

\subsection{Assembly Averaged Fast and Total Flux}

Assembly averaged fast flux $(\phi>0.1 \mathrm{MeV})$ and total flux data for MFA-1 and MFA-2 at the beginning and end of each cycle (except those noted in Table 6) are given in Tables $\mathrm{B} 10 \mathrm{~A}-1.2, \mathrm{E} 10 \mathrm{~A}-1.2$, B10A-2.2, etc. In computing the data in these tables, only the fuel region of each assembly was included in the averaging process. The group flux data were obtained from the same 3DB calculations used to produce the fission power data. Again, a bias factor $(0.988$ or 0.984$)$ was applied to the $30 \mathrm{~B}$ results. 
Table 6. Summary of Data Included for each Cycle

\begin{tabular}{|c|c|c|c|}
\hline $\begin{array}{l}\text { Table } \\
\text { No. }\end{array}$ & Description & $\begin{array}{l}\text { Created for } \\
\text { BOC or EOC? }\end{array}$ & $\begin{array}{l}\text { Included } \\
\text { on disk? }\end{array}$ \\
\hline 1 & Fission Power Generated in MFA-1, MFA-2 and Neighboring Assemblies & $B O C \& \operatorname{EOC}^{\circ}$ & yes \\
\hline 2 & Assembly Averaged Fast and Total Flux for MFA-1 and MFA-2 & BOC \& EOC & yes \\
\hline 3 & Axial Distribution of Total Flux, Fast Flux and Power in MFA-1 & $\mathrm{BOC} \& \mathrm{EOC}^{\mathrm{a}}$ & yes \\
\hline 4 & Axial Distribution of Total Flux, Fast Flux and Power in MFA-2 & $B O C \& \operatorname{EOC}^{8}$ & yes \\
\hline 5 & Fission Power Distribution by Pin in MFA-1 & $\operatorname{BOC} \& \mathrm{EOC}^{\mathrm{B}}$ & yes \\
\hline 6 & Fission Power Distribution by Pin in MFA-2 & $B O C \& \operatorname{EOC}^{\mathrm{A}}$ & yes \\
\hline 7 & Fast Flux Distribution by Pin in MFA-1 & $B O C \& \operatorname{EOC}^{\circ}$ & yes \\
\hline 8 & Fast Flux Distribution by Pin in MFA-2 & $\operatorname{SOC} \& \mathrm{EOC}^{\mathrm{a}}$ & yes \\
\hline 9 & MFA-1 and MFA-2 Duct Wall $F$ lux Greater than $0.1 \mathrm{MeV}$ & $\mathrm{BOC} \& \mathrm{EOC}^{\mathrm{\theta}}$ & yes \\
\hline 10 & $\begin{array}{l}\text { Sodium Outlet Temperatures and Flow Rates for MFA-1, MFA-2 and } \\
\text { Neighboring Assemblies }\end{array}$ & $B O C \& \operatorname{EOC}^{b}$ & yes \\
\hline 11 & MFA-1 Sodium Subchannel Temperatures at Top of Core Elevation & $\mathrm{BOC} \& \mathrm{EOC}^{\mathrm{C}}$ & no \\
\hline 12 & MFA-2 Sodium Subchannel Temperatures at Top of Core Elevation & $\mathrm{BOC} \& \mathrm{EOC}^{\mathrm{C}}$ & no \\
\hline 13 & $\begin{array}{l}\text { MFA-1 Sodium Subchannel Temperatures at Elevation of Upper Axial } \\
\text { Blanket }\end{array}$ & $B O C \& E^{C} C^{C}$ & no \\
\hline 14 & $\begin{array}{l}\text { MFA-2 Sodium Subchannet Temperatures at Elevation of Upper Axial } \\
\text { Blanket }\end{array}$ & $B O C \& E^{C} C^{C}$ & no \\
\hline 15 & MFA-1 Sodium Subchannel Temperatures at Top of Fuel Pin Bundle & $\operatorname{BOC} \& \mathrm{EOC}^{\mathrm{C}}$ & no \\
\hline 16 & MFA-2 Sodium Subchannel Temperatures at Top of Fuel Pin Bundle & $\mathrm{BOC} \& \mathrm{EOC}^{\mathrm{C}}$ & no \\
\hline 17 & Composition of MFA-1 & EOC & yes \\
\hline 18 & Composition of MFA- 2 & EOC & yes \\
\hline
\end{tabular}

a Table numbers $1-9$ are not include for the end of cycles 10A-2, 10A-3, 11A-1 and 11A-2 because of the short length of these cycles.

b rables of computed or measured sodium outlet temperatures and computed flow rates are given for the time periods tisted in Table 7 .

c sodium subchannel temperatures are available for only the times listed in rable 8. 


\subsection{Axial Distributions of Total Flux, Fast Flux and Power}

The axial distributions of total flux, fast flux $(\phi>0.1 \mathrm{MeV})$ and fission power at the beginning and end of each cycle (except those noted in Table 6) are given for MFA-1 in Tables B10A-1.3, E10A-1.3, B10A-2.3, etc. Likewise, the axial distributions of total flux, fast flux and fission power are given for MFA-2 in Tables B10A-1.4, E10A-1.4, B10A-2.4, etc.

The axial locations relative to the core midplane in units of centimeters $(\mathrm{cm})$ at hot, full power conditions are given in the first column of each table. Negative values indicate locations below the midplane and positive values indicate locations above the midplane. The flux and power data in the tables are normalized so that the axial average of each distribution over the active core region is 1.0 . The active core region extended from $-46.14 \mathrm{~cm}$ to 46.14 $\mathrm{cm}$ in the 3DB models of the FFTF at hot, full power conditions.

\subsection{Fission Power Distribution by Pin}

The distribution of fission power by pin at the beginning and end of each cycle is given for MFA-1 in Tables B1OA-1.5, E10A-1.5, B10A-2.5, etc. Likewise, the distribution of $f$ ission power by pin is given for MFA-2 in Tables B10A-1.6, E10A-1.6, B10A-2.6, etc. The direction north (N) is indicated in these tables.

The data in each table were based on a power distribution computed by 3DB. These distributions had six data points per assembiy at each axial elevation. Pin power data were generated by first axially averaging the 3DB computed powers, and then doing a least-squares quadratic fit to the available data. Finally, the pin powers obtained were normalized so that the linear power averaged over the fuel region of an assembly is 1.0 .

Using the given assembly powers, the axial power distributions and the pin power distributions, the linear power in any MFA-1 or MFA-2 pin at any axial elevation can be easily determined.

\subsection{Fast Flux Distribution by Pin}

The distribution of fast flux by pin is given at the beginning and end of each cycle for MFA-1 in Tables B10A-1.7, ElOA-1.7, B10A-2.7, etc. Likewise, the distribution of fast flux by $p$ in in MFA-2 is given in Tables B10A-1.8, E1OA-1.8, B10A-2.8, etc. These data were generated using the same method, described above, that was used to obtain the pin powers. Again, the flux data were normalized so that the average fast flux in the fuel region of MFA-1 or MFA-2 is 1.0 .

Using a given assembly averaged fast flux value, the corresponding axial fast flux distribution and pin distribution of fast flux, the fast flux at any axial level in any MFA-1 or MFA-2 pin can be easily determined.

Pin distributions of total flux are not given, but assembly-averaged total flux data and axial distributions of total flux are included in this report. Also, the pin distributions of total and fast flux are very similar. For example, the maximum difference for any pin in MFA-1 and MFA-2 during Cycles $10 A$ and $10 B$ was only about 2.6 percent. Thus, the total flux at any axial level in any MFA-1 or MFA-2 pin can be closely approximated. 
HNF-SD-FF-ANAL-009 Rev, 1

\subsection{Duct Wall Flux Greater than $0.1 \mathrm{MeV}$}

The fluxes greater than $0.1 \mathrm{MeV}$ at each of the six faces of the MFA-1 and MFA-2 ducts are given in Tables B10A-1.9, E10A-1.9, B10A-2.9, etc. The data given represent axial averages. To obtain the duct wall flux at a particular axial elevation, multiply by an axial factor from Tables B10A-1.3, B10A-1.4, ElOA-1.3, ElOA-1.4, etc.

Each of the six duct walls in each assembly is identified in the tables by the direction it faces - east (E), southeast (SE), southwest (SW), west (W), northwest (NW), or northeast (NE). Figures 1, 2, 3 and 4 show the orientation of MFA-1 and MFA-2. For example, in Figure 1, the east (E) duct wall of MFA-1 in position 1506, is adjacent to the assembly in lattice position 1505, and the east duct wall of MFA-2 in 2507 is adjacent to the assembly in lattice position 2508 .

Duct wall fluxes were generated by axially averaging the flux distributions computed by 3DB, integrating over energy to obtain the flux greater than 0.1 $\mathrm{MeV}$ at each space point, doing a linear interpolation between the two flux values on each side of a duct wall face, and finally applying the adjustment factor of 0.988 or 0.984 described earlier.

\subsection{Sodium Outlet Temperatures and Assembly Flow Rates}

Sodium outlet temperatures and flow rates for MFA-1, MFA-2 and the assemblies adjacent to them are given in Tables B10A-1.10, E10A-1.10, B10A-2.10, etc. In general, computed outlet temperatures are tabulated for the beginning and end of each cycle based on data extracted from FFTF core reload design reports and cycle reports. There are, however, a number of exceptions, which are identified in Table 7.

Assembly outlet temperature and flow rates for some time periods were not computed or reported because short cycle lengths and minor loading changes did not result in significant power or flow changes. Missing data can be estimated from data given for an earlier or later time period.

Assembly flow rate data were calculated for full power conditions. The calculated pressure drop and the total reactor flow rate upon which each assembly flow rate was based are given.

\subsection{Sodium Subchannel Temperatures}

Calculated sodium subchannel temperatures at three axial locations were available from previous analyses for the time periods listed in Table 8 . As with the assembly outlet temperatures, subchannel temperatures were not computed when short cycles and minor loading changes resulted in no significant temperature changes.

Subchannel temperatures at the top of core elevation are given for MFA-1 in Tables B1OA-1.11, B10A-2.11, etc., and for MFA-2 in Tables B10A-1.12, $B 10 A-2.12$, etc. Subchannel temperatures at the top of the upper axial blanket are given for MFA-1 in Tables BIOA-1.13, B1OA-2.13, etc, and for MFA-2 in 
Tables B10A-1.14, B10A-2.14, etc. SubchanneT temperatures at top of the fuel pin bundle are given for MFA-1 in Tables B10A-1.15, B10A-2.15, etc, and for MFA-2 in Tables $B 10 A-1.16$, B10A-2.16, etc.

All temperatures are in degrees Fahrenheit. The row of numbers just outside the asterisks on each map are calculated duct wall temperatures, and the outside row of temperatures on each map are the temperatures of the sodium between assemblies. The direction north is indicated on each map.

Table 7. Cycles for which Assembly outlet Temperatures and Flow Rates are Included

\begin{tabular}{||c|c|c|}
\hline \multirow{2}{*}{ Cycle } & \multicolumn{2}{|c|}{ Status $^{\mathrm{a}}$} \\
\cline { 2 - 3 } & $\mathrm{BOC}^{\mathrm{b}}$ & EOC $^{\mathrm{C}}$ \\
\hline $10 \mathrm{~A}-1$ & $\mathrm{C} \mathrm{M}$ & $\mathrm{C}$ \\
\hline $10 \mathrm{~A}-2$ & $\mathrm{C} \mathrm{M}$ & \\
\hline $10 \mathrm{~A}-3$ & $\mathrm{M}$ & \\
\hline $10 \mathrm{~A}-4$ & $\mathrm{M}$ & $\mathrm{C} \mathrm{M}$ \\
\hline $10 \mathrm{~B}$ & $\mathrm{C} \mathrm{M}$ & $\mathrm{C} \mathrm{M}$ \\
\hline $10 \mathrm{C}-1$ & $\mathrm{C}$ & \\
\hline $10 \mathrm{C}-2$ & & \\
\hline $10 \mathrm{C}-3$ & & $\mathrm{C}$ \\
\hline $11 \mathrm{~A}-1$ & $\mathrm{C}$ & \\
\hline $11 \mathrm{~A}-2$ & & \\
\hline $11 \mathrm{~A}-3$ & & $\mathrm{C}$ \\
\hline $11 \mathrm{~B}-1$ & $\mathrm{C}$ & \\
\hline $11 \mathrm{~B}-2$ & & $\mathrm{C}$ \\
\hline $11 \mathrm{C}$ & $\mathrm{C}$ & $\mathrm{C}$ \\
\hline $12 \mathrm{~A}-1$ & $\mathrm{C}$ & $\mathrm{C}$ \\
\hline $12 \mathrm{~A}-2$ & $\mathrm{C}$ & $\mathrm{C}$ \\
\hline $12 \mathrm{~B}-1$ & $\mathrm{C}$ & $\mathrm{C}$ \\
\hline $12 \mathrm{~B}-2$ & $\mathrm{C}$ & $\mathrm{C}$ \\
\hline
\end{tabular}
Status indication: A blank entry means no data are included. A non-blank entry means temperature and $f(0 w$ data are included. ' $C$ ' indicates that calculated temperatures are listed. ' $M$ ' indicates that measured temperatures are $t$ isted.

b $B O C=$ beginning of cycle

c EOC = end of cycle 
Table 8. Cycles for which Assembly Subchannel Temperatures Maps are Included

\begin{tabular}{||c|c|c||}
\hline \multirow{2}{*}{ Cycle } & \multicolumn{2}{|c|}{ Status $^{\mathrm{a}}$} \\
\hline & $\mathrm{BOC}^{\mathrm{b}}$ & EOC $^{\mathrm{C}}$ \\
\hline $10 \mathrm{~A}-1$ & $\mathrm{X}$ & \\
\hline $10 \mathrm{~A}-2$ & $\mathrm{X}$ & \\
\hline $10 \mathrm{~A}-3$ & & \\
\hline $10 \mathrm{~A}-4$ & & \\
\hline $10 \mathrm{~B}$ & $\mathrm{X}$ & $\mathrm{X}$ \\
\hline $10 \mathrm{C}-1$ & $\mathrm{X}$ & \\
\hline $10 \mathrm{C}-2$ & & \\
\hline $10 \mathrm{C}-3$ & & $X$ \\
\hline $11 \mathrm{~A}-1$ & $\mathrm{X}$ & \\
\hline $11 \mathrm{~A}-2$ & & \\
\hline $11 \mathrm{~A}-3$ & & $X$ \\
\hline $11 \mathrm{~B}-1$ & $\mathrm{X}$ & $\mathrm{X}$ \\
\hline $11 \mathrm{~B}-2$ & $\mathrm{X}$ & $\mathrm{X}$ \\
\hline $11 \mathrm{C}$ & $\mathrm{X}$ & $\mathrm{X}$ \\
\hline $12 \mathrm{~A}-1$ & $\mathrm{X}$ & $\mathrm{X}$ \\
\hline $12 \mathrm{~A}-2$ & $\mathrm{X}$ & $\mathrm{X}$ \\
\hline $12 \mathrm{~B}-1$ & $\mathrm{X}$ & $\mathrm{X}$ \\
\hline $12 \mathrm{~B}-2$ & $\mathrm{X}$ & $\mathrm{X}$ \\
\hline
\end{tabular}
Status indication: $' X$ ' indicates that subchannel temperature date are included. A blank entry means no data are included.

b $B O C=$ beginning of cycle

$c$ EOC $=$ end of cycle

The temperature calculations were performed using the SUPERENERGY code. The power of each pin, the assembly flow distribution and the reactor inlet temperature of $680^{\circ} \mathrm{F}$ were input to the code. SUPERENERGY calculates subchannel temperatures by using energy balances and correlations for subchannel mixing. SUPERENERGY has not been installed on any computer system that is currently available. For this reason, the calculations could not be repeated to obtain electronic copies of the temperature data, or missing data.

Fuel pin cladding temperatures are not available, but can be computed from the data provided. 


\subsection{Material Compositions}

Burnup-dependant material compositions for MFA-1 at the end of each cycle are given in Tables E1OA-1.17, E10A-2.17, etc, while the compositions for MFA-2 are given in Tables E1OA-1.18, E18A2.18, etc. Each table lists homogenized material compositions for 11 axial regions in MFA-1 or MFA-2. These 11 regions are identified in Table 9. The axial extent of each region, as modeled in $3 D B$, is listed in the material composition tables. The units are centimeters $(\mathrm{cm})$ relative to the core midplane at hot, full power conditions. The compositions are in units of atoms/b-cm at hot, full power conditions. Both the fresh (beginning of 1 ife) and EOC compositions are included in each table. Compositions of MFA-1 and MFA-2 at BOC 1OA-1 were the fresh values listed. Compositions at the beginning of any other cycle were essentially those listed for the end of the previous cycle. The only differences are due to decay of ${ }^{241} \mathrm{Pu}$ to ${ }^{241} \mathrm{Am}$.

The burnup-dependant compositions for the axial blankets (axial regions 3 and 10) and the fuel (axial regions 4 through 9) were computed using the threedimensional diffusion theory and burnup code, 3DB. The fuel was subdivided into six axial regions to properly account for the effect that the axial flux distribution has on the fuel depletion rate.

The constituents of the materials listed in the composition tables are individual isotopes with two exceptions. The first exception is that the constituent labelled "FP" represents an average pseudo fission product pair. An atom density for FP specifies the concentration of fission product pairs, not individual fission product nuclides. The microscopic cross section data used for fission products in the 3DB calculations were generated assuming all fissions were from ${ }^{239} \mathrm{Pu}$.

The second exception is that the constituent labelled "SS-316" represents type 316 stainless steel. An atom density given for SS-316 indicates the total number of atoms in stainless steel per unit volume. SS-316 was used in the calculational models for the ducts, cladding and wire wrap in both MFA-1 and MFA-2, even though these components in MFA-2 were fabricated from an advanced austenitic stainless steel. The composition used in generating nuclear cross section sets for SS-316 was based on the 1977 RDT \& ASTM standard, and is listed in Table 10 .

\subsection{Contents of the Data Disk}

The information identified in Table 6 was copied to a 3.5 inch floppy disk that is DOS formatted with 1.44 MB capacity. All information on the disk is contained in standard text (ASCII) files. There are 17 directories on the disk, one for each cycle analyzed. The directory names are 10A-1, 10A-2, $10 \mathrm{~A}-3,10 \mathrm{~A}-4,10-\mathrm{B}$, etc. As described earlier, the files on the disk are named the same as the data tables. For example, assembly fission powers for EOC 10A-4 are 1isted in Table ElOA-4.1 and on disk file ElOA-4.1, which is located in directory 10A-4. Table 6 indicates which of the data tables for each cycle are included on the disk. A table of contents of the disk is given in Table 11 . 
Table 9. Description of Axial Regions in Material Composition Tables

\begin{tabular}{|c|c|l|}
\hline $\begin{array}{c}\text { Axial } \\
\text { Level }\end{array}$ & $\begin{array}{c}\text { Axial Range } \\
\text { Relative to Core } \\
\text { Midplane (cm) }\end{array}$ & \multicolumn{1}{c|}{ Description } \\
\hline 1 & $-100.28^{\mathrm{b}}$ to -75.28 & Lower axial shield \\
\hline 2 & -75.28 to -62.78 & Pin attachment region \\
\hline 3 & -62.78 to -46.14 & Lower axial blanket \\
\hline $4-9$ & $\begin{array}{c}-46.14 \text { to } 46.14 \\
\text { (divided into } 6 \text { regions, } \\
\text { each } 15.38 \mathrm{~cm} \text { high) }\end{array}$ & Fuel region \\
\hline 10 & 46.14 to $62.78^{c}$ & Upper axial blanket \\
\hline 11 & 62.78 to $116.00^{\mathrm{b}}$ & Gas plenum \\
\hline
\end{tabular}

a Dimensions are representative of hot, full power conditions. Negative numbers indicate locations below the core midplane. Positive numbers indicate locations above the core midplane.

b The axial range in the $30 \mathrm{~B}$ models was $100.28 \mathrm{~cm}$ below the core midplane to $116.00 \mathrm{~cm}$ above the midplane.

c The upper axial blankets of MFA-1 and MFA-2 are actually $7.62 \mathrm{~cm}$ ( 3 in.) long. However, they were modeled as being $16.64 \mathrm{~cm}$ long (at hot, full power conditions) to match existing axial boundaries in the $30 \mathrm{DB}$ model.

Table 10. Composition of Type 316 Stainless Steel (SS-316)

\begin{tabular}{||c|c||c|c|}
\hline Constituent & $\begin{array}{c}\text { Weight } \\
\text { Percent }\end{array}$ & Constituent & $\begin{array}{c}\text { Weight } \\
\text { Percent }\end{array}$ \\
\hline $\mathrm{Fe}$ & 62.19 & $\mathrm{Mn}$ & 2.00 \\
\hline $\mathrm{Cr}$ & 18.00 & $\mathrm{Si}$ & 0.75 \\
\hline $\mathrm{Ni}$ & 14.00 & $\mathrm{C}$ & 0.06 \\
\hline $\mathrm{Mo}$ & 3.00 & & \\
\hline
\end{tabular}


HNF-SD-FF-ANAL-009 Rev. 1

Table 11. Disk Table of Contents (Sheet 1 of 4)

\begin{tabular}{|c|c|c|c|c|c|c|c|c|c|}
\hline \multicolumn{2}{|c|}{ File Name } & \multirow{2}{*}{$\begin{array}{c}\text { size } \\
\text { (bytes) }\end{array}$} & \multicolumn{2}{|c|}{ Greation } & \multicolumn{2}{|c|}{ File Name } & \multirow{2}{*}{$\begin{array}{c}\text { Size } \\
\text { (bytes) }\end{array}$} & \multicolumn{2}{|c|}{ Creation } \\
\hline Base & Ext. & & Date & Time & Base & Ext. & & Date & Time \\
\hline \multicolumn{5}{|c|}{ Directory of $10 \mathrm{~A}-1$} & \multicolumn{5}{|c|}{22 files, 40,971 bytes total } \\
\hline $\begin{array}{l}\text { B10A-1 } \\
\text { B10A-1 } \\
\text { B10A-1 } \\
\text { B10A-1 } \\
\text { B10A-1 } \\
\text { B10A-1 } \\
\text { B10A-1 } \\
\text { B10A-1 } \\
\text { B10A-1 } \\
\text { B10A-1 }\end{array}$ & $\begin{array}{l}1 \\
2 \\
3 \\
4 \\
5 \\
6 \\
7 \\
8 \\
9 \\
10\end{array}$ & $\begin{array}{r}989 \\
271 \\
2,767 \\
2,767 \\
1,615 \\
1,615 \\
1,611 \\
1,611 \\
350 \\
863\end{array}$ & $\begin{array}{l}12-30-96 \\
12-30-96 \\
12-30-96 \\
12-30-96 \\
12-30-96 \\
12-30-96 \\
12-30-96 \\
12-30-96 \\
01-06-97 \\
01-09-97\end{array}$ & $\begin{array}{l}2: 34 \mathrm{p} \\
2: 34 \mathrm{p} \\
2: 34 \mathrm{p} \\
2: 34 \mathrm{p} \\
2: 34 \mathrm{p} \\
2: 34 \mathrm{p} \\
2: 34 \mathrm{p} \\
2: 34 \mathrm{p} \\
4: 08 \mathrm{p} \\
9: 03 \mathrm{a}\end{array}$ & $\begin{array}{l}\text { E10A-1 } \\
\text { E10A-1 } \\
\text { E10A-1 } \\
\text { E1OA-1 } \\
\text { E10A-1 } \\
\text { E10A-1 } \\
\text { E1OA-1 } \\
\text { E1OA-1 } \\
\text { E1OA-1 } \\
\text { E1OA-1 } \\
\text { E1OA-1 } \\
\text { E1OA-1 }\end{array}$ & $\begin{array}{l}1 \\
2 \\
3 \\
4 \\
5 \\
6 \\
7 \\
8 \\
9 \\
10 \\
17 \\
18 \\
\end{array}$ & $\begin{array}{r}989 \\
271 \\
2,767 \\
2,767 \\
1,615 \\
1,615 \\
1,611 \\
1,611 \\
350 \\
708 \\
6,104 \\
6,104 \\
\end{array}$ & $\begin{array}{l}12-30-96 \\
12-30-96 \\
12-30-96 \\
12-30-96 \\
12-30-96 \\
12-30-96 \\
12-30-96 \\
12-30-96 \\
01-06-97 \\
01-09-97 \\
12-30-96 \\
12-30-96 \\
\end{array}$ & $\begin{array}{l}2: 35 p \\
2: 35 p \\
2: 35 p \\
2: 35 p \\
2: 35 p \\
2: 35 p \\
2: 35 p \\
2: 35 p \\
4: 10 p \\
9: 03 a \\
2: 35 p \\
2: 35 p\end{array}$ \\
\hline \multicolumn{5}{|c|}{ Directory of $10 \mathrm{~A}-2$} & \multicolumn{5}{|c|}{12 files, 26,667 bytes total } \\
\hline $\begin{array}{l}\text { B10A-2 } \\
\text { B10A-2 } \\
\text { B10A-2 } \\
\text { B10A-2 } \\
\text { B10A-2 } \\
\text { B10A-2 }\end{array}$ & $\begin{array}{l}1 \\
2 \\
3 \\
4 \\
5 \\
6 \\
\end{array}$ & $\begin{array}{r}989 \\
271 \\
2,767 \\
2,767 \\
1,615 \\
1,615 \\
\end{array}$ & $\begin{array}{l}12-30-96 \\
12-30-96 \\
12-30-96 \\
12-30-96 \\
12-30-96 \\
12-30-96 \\
\end{array}$ & $\begin{array}{l}2: 36 p \\
2: 36 p \\
2: 36 p \\
2: 36 p \\
2: 36 p \\
2: 36 p\end{array}$ & $\begin{array}{l}B 10 A-2 \\
B 10 A-2 \\
B 10 A-2 \\
B 10 A-2 \\
\text { E10A-2 } \\
\text { E10A-2 }\end{array}$ & $\begin{array}{l}7 \\
8 \\
9 \\
10 \\
17 \\
18 \\
\end{array}$ & $\begin{array}{r}1,611 \\
1,611 \\
350 \\
863 \\
6,104 \\
6,104 \\
\end{array}$ & $\begin{array}{l}12-30-96 \\
12-30-96 \\
01-06-97 \\
01-09-97 \\
12-30-96 \\
12-30-96 \\
\end{array}$ & $\begin{array}{l}2: 36 p \\
2: 36 p \\
4: 12 p \\
9: 04 a \\
2: 37 p \\
2: 37 p\end{array}$ \\
\hline \multicolumn{5}{|c|}{ Directory of 10A-3 } & \multicolumn{5}{|c|}{12 files, 26,494 bytes total } \\
\hline $\begin{array}{l}B 10 A-3 \\
B 10 A-3 \\
B 10 A-3 \\
B 10 A-3 \\
B 10 A-3 \\
B 10 A-3\end{array}$ & $\begin{array}{l}1 \\
2 \\
3 \\
4 \\
5 \\
6 \\
\end{array}$ & $\begin{array}{r}989 \\
271 \\
2,767 \\
2,767 \\
1,615 \\
1,615 \\
\end{array}$ & $\begin{array}{l}12-30-96 \\
12-30-96 \\
12-30-96 \\
12-30-96 \\
12-30-96 \\
12-30-96 \\
\end{array}$ & $\begin{array}{l}2: 38 p \\
2: 38 p \\
2: 38 p \\
2: 38 p \\
2: 38 p \\
2: 38 p \\
\end{array}$ & $\begin{array}{l}B 10 A-3 \\
B 10 A-3 \\
B 10 A-3 \\
B 10 A-3 \\
\text { E10A-3 } \\
\text { E10A-3 }\end{array}$ & $\begin{array}{l}7 \\
8 \\
9 \\
10 \\
17 \\
18 \\
\end{array}$ & $\begin{array}{r}1,611 \\
1,611 \\
350 \\
690 \\
6,104 \\
6,104 \\
\end{array}$ & $\begin{array}{l}12-30-96 \\
12-30-96 \\
01-06-97 \\
01-09-97 \\
12-30-96 \\
12-30-96 \\
\end{array}$ & $\begin{array}{l}2: 38 p \\
2: 38 p \\
4: 13 p \\
9: 04 a \\
2: 38 p \\
2: 38 p \\
\end{array}$ \\
\hline \multicolumn{5}{|c|}{ Directory of $10 \mathrm{~A}-4$} & \multicolumn{5}{|c|}{22 files, 40,952 bytes total } \\
\hline $\begin{array}{l}\text { B10A-4 } \\
\text { B10A-4 } \\
\text { B10A-4 } \\
\text { B10A-4 } \\
\text { B10A-4 } \\
\text { B10A-4 } \\
\text { B10A-4 } \\
\text { B10A-4 } \\
\text { B10A-4 } \\
\text { B 10A-4 }\end{array}$ & $\begin{array}{l}1 \\
2 \\
3 \\
4 \\
5 \\
6 \\
7 \\
8 \\
9 \\
10\end{array}$ & $\begin{array}{r}989 \\
271 \\
2,767 \\
2,767 \\
1,615 \\
1,615 \\
1,611 \\
1,611 \\
350 \\
689\end{array}$ & $\begin{array}{l}12-30-96 \\
12-30-96 \\
12-30-96 \\
12-30-96 \\
12-30-96 \\
12-30-96 \\
12-30-96 \\
12-30-96 \\
01-06-97 \\
01-09-97\end{array}$ & $\begin{array}{l}2: 39 p \\
2: 39 p \\
2: 39 p \\
2: 39 p \\
2: 39 p \\
2: 39 p \\
2: 39 p \\
2: 39 p \\
4: 14 p \\
9: 04 a\end{array}$ & $\begin{array}{l}\text { E10A-4 } \\
\text { E10A-4 } \\
\text { E1OA-4 } \\
\text { E10A-4 } \\
\text { E10A-4 } \\
\text { E10A-4 } \\
\text { E10A-4 } \\
\text { E10A-4 } \\
\text { E10A-4 } \\
\text { E10A-4 } \\
\text { E10A-4 } \\
\text { E10A-4 }\end{array}$ & $\begin{array}{l}1 \\
2 \\
3 \\
4 \\
5 \\
6 \\
7 \\
8 \\
9 \\
10 \\
17 \\
18 \\
\end{array}$ & $\begin{array}{r}989 \\
271 \\
2,767 \\
2,767 \\
1,615 \\
1,615 \\
1,611 \\
1,611 \\
350 \\
863 \\
6,104 \\
6,104 \\
\end{array}$ & $\begin{array}{l}12-30-96 \\
12-30-96 \\
12-30-96 \\
12-30-96 \\
12-30-96 \\
12-30-96 \\
12-30-96 \\
12-30-96 \\
01-06-97 \\
01-09-97 \\
12-30-96 \\
12-30-96 \\
\end{array}$ & $\begin{array}{l}2: 40 p \\
2: 40 p \\
2: 40 p \\
2: 40 p \\
2: 40 p \\
2: 40 p \\
2: 40 p \\
2: 40 p \\
4: 14 p \\
8: 58 a \\
2: 40 p \\
2: 40 p\end{array}$ \\
\hline \multicolumn{5}{|c|}{ Directory of 108} & \multicolumn{5}{|c|}{22 files, 41,050 bytes total } \\
\hline $\begin{array}{l}\text { B10B } \\
\text { B10B } \\
\text { B10B } \\
\text { B10B } \\
\text { B10B } \\
\text { B10B } \\
\text { B10B } \\
\text { B10B } \\
\text { B10B } \\
\text { B10B }\end{array}$ & $\begin{array}{l}1 \\
2 \\
3 \\
4 \\
5 \\
6 \\
7 \\
8 \\
9 \\
10\end{array}$ & $\begin{array}{r}987 \\
269 \\
2,763 \\
2,763 \\
1,611 \\
1,611 \\
1,607 \\
1,607 \\
346 \\
859\end{array}$ & $\begin{array}{l}12-30-96 \\
12-30-96 \\
12-30-96 \\
12-30-96 \\
12-30-96 \\
12-30-96 \\
12-30-96 \\
12-30-96 \\
01-06-97 \\
01-09-97\end{array}$ & $\begin{array}{l}2: 41 \mathrm{p} \\
2: 41 \mathrm{p} \\
2: 41 \mathrm{p} \\
2: 41 \mathrm{p} \\
2: 41 \mathrm{p} \\
2: 41 \mathrm{p} \\
2: 41 \mathrm{p} \\
2: 41 \mathrm{p} \\
4: 15 \mathrm{p} \\
9: 00 \mathrm{a}\end{array}$ & $\begin{array}{l}\text { E10B } \\
\text { E10B } \\
\text { E10B } \\
\text { E10B } \\
\text { E10B } \\
\text { E10B } \\
\text { E10B } \\
\text { E10B } \\
\text { E10B } \\
\text { E10B } \\
\text { E10B } \\
\text { E10B }\end{array}$ & $\begin{array}{l}1 \\
2 \\
3 \\
4 \\
5 \\
6 \\
7 \\
8 \\
9 \\
10 \\
17 \\
18\end{array}$ & $\begin{array}{r}987 \\
269 \\
2,763 \\
2,763 \\
1,611 \\
1,611 \\
1,607 \\
1,607 \\
346 \\
859 \\
6,102 \\
6,102 \\
\end{array}$ & $\begin{array}{l}12-30-96 \\
12-30-96 \\
12-30-96 \\
12-30-96 \\
12-30-96 \\
12-30-96 \\
12-30-96 \\
12-30-96 \\
01-06-97 \\
01-09-97 \\
12-30-96 \\
12-30-96\end{array}$ & $\begin{array}{l}2: 42 p \\
2: 42 p \\
2: 42 p \\
2: 42 p \\
2: 42 p \\
2: 42 p \\
2: 42 p \\
2: 42 p \\
4: 15 p \\
9: 01 p \\
2: 42 p \\
2: 42 p\end{array}$ \\
\hline
\end{tabular}


HNF-SD-FF-ANAL-009 Rev. 1

Table 11. Disk Table of Contents (Sheet 2 of 4)

\begin{tabular}{|c|c|c|c|c|c|c|c|c|c|}
\hline \multicolumn{2}{|c|}{ File Name } & \multirow{2}{*}{$\begin{array}{c}\text { size } \\
\text { (bytes) }\end{array}$} & \multicolumn{2}{|c|}{ Creation } & \multicolumn{2}{|c|}{ File Name } & \multirow{2}{*}{$\begin{array}{c}\text { size } \\
\text { (bytes) }\end{array}$} & \multicolumn{2}{|c|}{ Creation } \\
\hline Base E & Ext. & & Date & Time & Base & Ext. & & Date & Time \\
\hline \multicolumn{5}{|c|}{ Directory of $10 \mathrm{c}-1$} & \multicolumn{5}{|c|}{21 files, 40,109 bytes total } \\
\hline $\begin{array}{l}810 C-1 \\
\text { B10C-1 } \\
\text { B10C-1 } \\
\text { B10C-1 } \\
\text { B10C-1 } \\
\text { B10C-1 } \\
\text { B10C-1 } \\
\text { B10C-1 } \\
\text { B10C-1 } \\
\text { B10C-1 }\end{array}$ & $\begin{array}{l}1 \\
2 \\
3 \\
4 \\
5 \\
6 \\
7 \\
8 \\
9 \\
10\end{array}$ & $\begin{array}{r}989 \\
271 \\
2,767 \\
2,767 \\
1,615 \\
1,615 \\
1,611 \\
1,611 \\
350 \\
709\end{array}$ & $\begin{array}{l}12-30-96 \\
12-30-96 \\
12-30-96 \\
12-30-96 \\
12-30-96 \\
12-30-96 \\
12-30-96 \\
12-30-96 \\
01-06-97 \\
04-09-97\end{array}$ & $\begin{array}{l}2: 43 p \\
2: 43 p \\
2: 43 p \\
2: 43 p \\
2: 43 p \\
2: 43 p \\
2: 43 p \\
2: 43 p \\
4: 25 p \\
4: 55 p\end{array}$ & $\begin{array}{l}E 10 C-1 \\
E 10 C-1 \\
E 10 C-1 \\
E 10 C-1 \\
E 10 C-1 \\
E 10 C-1 \\
E 10 C-1 \\
E 10 C-1 \\
\text { E1OC-1 } \\
\text { E1OC-1 } \\
\text { E1OC-1 }\end{array}$ & $\begin{array}{l}1 \\
2 \\
3 \\
4 \\
5 \\
6 \\
7 \\
8 \\
9 \\
17 \\
18 \\
\end{array}$ & $\begin{array}{r}989 \\
271 \\
2,767 \\
2,767 \\
1,615 \\
1,615 \\
1,611 \\
1,611 \\
350 \\
6,104 \\
6,104 \\
\end{array}$ & $\begin{array}{l}12-30-96 \\
12-30-96 \\
12-30-96 \\
12-30-96 \\
12-30-96 \\
12-30-96 \\
12-30-96 \\
12-30-96 \\
01-06-97 \\
12-30-96 \\
12-30-96\end{array}$ & $\begin{array}{l}2: 43 p \\
2: 43 p \\
2: 43 p \\
2: 43 p \\
2: 43 p \\
2: 43 p \\
2: 43 p \\
2: 43 p \\
4: 26 p \\
2: 43 p \\
2: 43 p\end{array}$ \\
\hline \multicolumn{5}{|c|}{ Directory of $100-3$} & \multicolumn{5}{|c|}{$2\}$ files, 40,108 bytes total } \\
\hline $\begin{array}{l}\text { B10C-3 } \\
\text { B10C-3 } \\
\text { B10C-3 } \\
\text { B } 10 C-3 \\
\text { B } 10 C-3 \\
\text { B10C-3 } \\
\text { B } 10 C-3 \\
\text { B } 10 C-3 \\
\text { B } 10 C-3\end{array}$ & $\begin{array}{l}1 \\
2 \\
3 \\
4 \\
5 \\
6 \\
7 \\
8 \\
9\end{array}$ & $\begin{array}{r}989 \\
271 \\
2,767 \\
2,767 \\
1,615 \\
1,615 \\
1,611 \\
1,611 \\
350\end{array}$ & $\begin{array}{l}12-30-96 \\
12-30-96 \\
12-30-96 \\
12-30-96 \\
12-30-96 \\
12-30-96 \\
12-30-96 \\
12-30-96 \\
01-06-97\end{array}$ & $\begin{array}{l}2: 44 p \\
2: 44 p \\
2: 44 p \\
2: 44 p \\
2: 44 p \\
2: 44 p \\
2: 44 p \\
2: 44 p \\
4: 28 p\end{array}$ & $\begin{array}{l}E 10 C-3 \\
E 10 C-3 \\
E 10 C-3 \\
E 10 C-3 \\
E 10 C-3 \\
E 10 C-3 \\
E 10 C-3 \\
E 10 C-3 \\
E 10 C-3 \\
E 10 C-3 \\
E 10 C-3 \\
E 10 C-3\end{array}$ & $\begin{array}{l}1 \\
2 \\
3 \\
4 \\
5 \\
6 \\
7 \\
8 \\
9 \\
10 \\
17 \\
18 \\
\end{array}$ & $\begin{array}{r}989 \\
271 \\
2,767 \\
2,767 \\
1,615 \\
1,615 \\
1,611 \\
1,611 \\
350 \\
708 \\
6,104 \\
6,104 \\
\end{array}$ & $\begin{array}{l}12-30-96 \\
12-30-96 \\
12-30-96 \\
12-30-96 \\
12-30-96 \\
12-30-96 \\
12-30-96 \\
12-30-96 \\
01-06-97 \\
01-09-97 \\
12-30-96 \\
12-30-96 \\
\end{array}$ & $\begin{array}{l}2: 46 p \\
2: 46 p \\
2: 46 p \\
2: 46 p \\
2: 46 p \\
2: 46 p \\
2: 46 p \\
2: 46 p \\
4: 29 p \\
9: 02 a \\
2: 46 p \\
2: 46 p\end{array}$ \\
\hline \multicolumn{5}{|c|}{ Directory of 11A-1 } & \multicolumn{5}{|c|}{12 files, 26,514 bytes total } \\
\hline $\begin{array}{l}\text { B11A-1 } \\
\text { B11A-1 } \\
\text { B11A-1 } \\
\text { B11A-1 } \\
\text { B11A-1 } \\
\text { B11A-1 }\end{array}$ & $\begin{array}{l}1 \\
2 \\
3 \\
4 \\
5 \\
6 \\
\end{array}$ & $\begin{array}{r}989 \\
271 \\
2,767 \\
2,767 \\
1,615 \\
1,615 \\
\end{array}$ & $\begin{array}{l}12-30-96 \\
12-30-96 \\
12-30-96 \\
12-30-96 \\
12-30-96 \\
12-30-96\end{array}$ & $\begin{array}{l}2: 49 \mathrm{p} \\
2: 49 \mathrm{p} \\
2: 49 \mathrm{p} \\
2: 49 \mathrm{p} \\
2: 49 \mathrm{p} \\
2: 49 \mathrm{p}\end{array}$ & $\begin{array}{l}\text { B11A-1 } \\
\text { B11A-1 } \\
\text { B11A-1 } \\
\text { B11A-1 } \\
\text { E11A-1 } \\
\text { E11A-1 }\end{array}$ & $\begin{array}{l}7 \\
8 \\
9 \\
10 \\
17 \\
18 \\
\end{array}$ & $\begin{array}{r}1,611 \\
1,611 \\
350 \\
710 \\
6,104 \\
6,104 \\
\end{array}$ & $\begin{array}{l}12-30-96 \\
12-30-96 \\
01-06-97 \\
01-13-97 \\
12-30-96 \\
12-30-96 \\
\end{array}$ & $\begin{array}{r}2: 49 p \\
2: 49 p \\
4: 31 p \\
10: 35 p \\
2: 49 p \\
2: 49 p\end{array}$ \\
\hline \multicolumn{5}{|c|}{ Directory of 11A-2 } & \multicolumn{5}{|c|}{11 files, 25,804 bytes total } \\
\hline $\begin{array}{l}\text { B11A-2 } \\
\text { B11A-2 } \\
\text { B11A-2 } \\
\text { B11A-2 } \\
\text { B11A-2 } \\
\text { B11A-2 }\end{array}$ & $\begin{array}{l}1 \\
2 \\
3 \\
4 \\
5 \\
6 \\
\end{array}$ & $\begin{array}{r}989 \\
271 \\
2,767 \\
2,767 \\
1,615 \\
1,615 \\
\end{array}$ & $\begin{array}{l}12-30-96 \\
12-30-96 \\
12-30-96 \\
12-30-96 \\
12-30-96 \\
12-30-96\end{array}$ & $\begin{array}{l}2: 50 p \\
2: 50 p \\
2: 50 p \\
2: 50 p \\
2: 50 p \\
2: 50 p\end{array}$ & $\begin{array}{l}\text { B 11A-2 } \\
\text { B } 11 A-2 \\
\text { B } 11 A-2 \\
\text { E } 11 A-2 \\
\text { E } 11 A-2\end{array}$ & $\begin{array}{l}7 \\
8 \\
9 \\
17 \\
18\end{array}$ & $\begin{array}{l}1,611 \\
1,611 \\
350 \\
6,104 \\
6,104\end{array}$ & $\begin{array}{l}12-30-96 \\
12-30-96 \\
01-06-97 \\
12-30-96 \\
12-30-96\end{array}$ & $\begin{array}{l}2: 50 p \\
2: 50 p \\
4: 32 p \\
2: 51 p \\
2: 51 p\end{array}$ \\
\hline \multicolumn{5}{|c|}{ Directory of $11 \mathrm{~A}-3$} & \multicolumn{5}{|c|}{21 files, 40,110 bytes total } \\
\hline $\begin{array}{l}\text { B11A-3 } \\
\text { B11A-3 } \\
B 11 A-3 \\
\text { B11A-3 } \\
\text { B11A-3 } \\
\text { B11A-3 } \\
\text { B11A-3 } \\
\text { B11A-3 } \\
\text { B11A-3 }\end{array}$ & $\begin{array}{l}1 \\
2 \\
3 \\
4 \\
5 \\
6 \\
7 \\
8 \\
9\end{array}$ & $\begin{array}{r}989 \\
271 \\
2,767 \\
2,767 \\
1,615 \\
1,615 \\
1,611 \\
1,611 \\
350\end{array}$ & $\begin{array}{l}12-30-96 \\
12-30-96 \\
12-30-96 \\
12-30-96 \\
12-30-96 \\
12-30-96 \\
12-30-96 \\
12-30-96 \\
01-06-97\end{array}$ & $\begin{array}{l}2: 52 p \\
2: 52 p \\
2: 52 p \\
2: 52 p \\
2: 52 p \\
2: 52 p \\
2: 52 p \\
2: 52 p \\
4: 34 p\end{array}$ & $\begin{array}{l}E 11 A-3 \\
E 11 A-3 \\
E 11 A-3 \\
E 11 A-3 \\
E 11 A-3 \\
E 11 A-3 \\
E 11 A-3 \\
E 11 A-3 \\
\text { E11A-3 } \\
\text { E11A-3 } \\
\text { E11A-3 } \\
\text { E11A-3 }\end{array}$ & $\begin{array}{l}1 \\
2 \\
3 \\
4 \\
5 \\
6 \\
7 \\
8 \\
9 \\
10 \\
17 \\
18 \\
\end{array}$ & $\begin{array}{r}989 \\
271 \\
2,767 \\
2,767 \\
1,615 \\
1,615 \\
1,611 \\
1,611 \\
350 \\
710 \\
6,104 \\
6,104 \\
\end{array}$ & $\begin{array}{l}12-30-96 \\
12-30-96 \\
12-30-96 \\
12-30-96 \\
12-30-96 \\
12-30-96 \\
12-30-96 \\
12-30-96 \\
01-06-97 \\
01-13-97 \\
12-30-96 \\
12-30-96\end{array}$ & $\begin{array}{r}2: 52 p \\
2: 52 p \\
2: 52 p \\
2: 52 p \\
2: 52 p \\
2: 52 p \\
2: 52 p \\
2: 52 p \\
4: 34 p \\
10: 36 p \\
2: 52 p \\
2: 52 p \\
\end{array}$ \\
\hline
\end{tabular}


HNF-SD-FF-ANAL-009 Rev. 1

Table 11. Disk Table of Contents (Sheet 3 of 4)

\begin{tabular}{|c|c|c|c|c|c|c|c|c|c|}
\hline \multicolumn{2}{|c|}{ File Name } & \multirow{2}{*}{$\begin{array}{c}\text { Size } \\
\text { (bytes) }\end{array}$} & \multicolumn{2}{|c|}{ Creation } & \multicolumn{2}{|c|}{ File Name } & \multirow{2}{*}{$\begin{array}{c}\text { Size } \\
\text { (bytes) }\end{array}$} & \multicolumn{2}{|c|}{ Creation } \\
\hline Base & Ext. & & Date & Time & Base & Ext. & & Date & Time \\
\hline \multicolumn{5}{|c|}{ Directory of 118-1 } & \multicolumn{5}{|c|}{22 files, 40,820 bytes total } \\
\hline $\begin{array}{l}\text { B11B-1 } \\
\text { B11B-1 } \\
\text { B11B-1 } \\
\text { B11B-1 } \\
\text { B11B-1 } \\
\text { B11B-1 } \\
\text { B11B-1 } \\
\text { B11B-1 } \\
\text { B11B-1 } \\
\text { B11B-1 }\end{array}$ & $\begin{array}{l}1 \\
2 \\
3 \\
4 \\
5 \\
6 \\
7 \\
8 \\
9 \\
10\end{array}$ & $\begin{array}{r}989 \\
271 \\
2,767 \\
2,767 \\
1,615 \\
1,615 \\
1,611 \\
1,611 \\
350 \\
710\end{array}$ & $\begin{array}{l}12-30-96 \\
12-30-96 \\
12-30-96 \\
12-30-96 \\
12-30-96 \\
12-30-96 \\
12-30-96 \\
12-30-96 \\
01-06-97 \\
01-14-97\end{array}$ & $\begin{array}{l}2: 53 p \\
2: 53 p \\
2: 53 p \\
2: 53 p \\
2: 53 p \\
2: 53 p \\
2: 53 p \\
2: 53 p \\
4: 36 p \\
7: 35 p\end{array}$ & $\begin{array}{l}E 11 \mathrm{~B}-1 \\
\text { E11B-1 } \\
\text { E11B-1 } \\
\text { E11B-1 } \\
\text { E11B-1 } \\
\text { E11B-1 } \\
\text { E11B-1 } \\
\text { E11B-1 } \\
\text { E11B-1 } \\
\text { E11B-1 } \\
\text { E11B-1 } \\
\text { E11B-1 }\end{array}$ & $\begin{array}{l}1 \\
2 \\
3 \\
4 \\
5 \\
6 \\
7 \\
8 \\
9 \\
10 \\
17 \\
18 \\
\end{array}$ & $\begin{array}{r}989 \\
271 \\
2,767 \\
2,767 \\
1,615 \\
1,615 \\
1,611 \\
1,611 \\
350 \\
710 \\
6,104 \\
6,104 \\
\end{array}$ & $\begin{array}{l}12-30-96 \\
12-30-96 \\
12-30-96 \\
12-30-96 \\
12-30-96 \\
12-30-96 \\
12-30-96 \\
12-30-96 \\
01-06-97 \\
01-14-97 \\
12-30-96 \\
12-30-96 \\
\end{array}$ & $\begin{array}{l}2: 53 p \\
2: 53 p \\
2: 53 p \\
2: 53 p \\
2: 53 p \\
2: 53 p \\
2: 53 p \\
2: 53 p \\
4: 37 p \\
7: 40 p \\
2: 53 p \\
2: 53 p\end{array}$ \\
\hline \multicolumn{5}{|c|}{ Directory of $11 \mathrm{~B}-2$} & \multicolumn{5}{|c|}{22 files, 40,821 bytes total } \\
\hline $\begin{array}{l}\text { B } 11 B-2 \\
\text { B } 11 B-2 \\
\text { B } 11 B-2 \\
\text { B11B-2 } \\
\text { B11B-2 } \\
\text { B11B-2 } \\
\text { B11B-2 } \\
\text { B11B-2 } \\
\text { B11B-2 } \\
\text { B11B-2 }\end{array}$ & $\begin{array}{l}1 \\
2 \\
3 \\
4 \\
5 \\
6 \\
7 \\
8 \\
9 \\
10\end{array}$ & $\begin{array}{r}989 \\
271 \\
2,767 \\
2,767 \\
1,615 \\
1,615 \\
1,611 \\
1,611 \\
350 \\
711\end{array}$ & $\begin{array}{l}12-30-96 \\
12-30-96 \\
12-30-96 \\
12-30-96 \\
12-30-96 \\
12-30-96 \\
12-30-96 \\
12-30-96 \\
01-06-97 \\
01-16-97\end{array}$ & $\begin{array}{l}2: 54 p \\
2: 54 p \\
2: 54 p \\
2: 54 p \\
2: 54 p \\
2: 54 p \\
2: 54 p \\
2: 54 p \\
4: 38 p \\
1: 26 p\end{array}$ & $\begin{array}{l}E 11 B-2 \\
\text { E11B-2 } \\
E 11 B-2 \\
\text { E19B-2 } \\
\text { E19B-2 } \\
\text { E11B-2 } \\
\text { E11B-2 } \\
\text { E11B-2 } \\
\text { E11B-2 } \\
\text { E11B-2 } \\
\text { E11B-2 } \\
\text { E11B-2 }\end{array}$ & $\begin{array}{l}1 \\
2 \\
3 \\
4 \\
5 \\
6 \\
7 \\
8 \\
9 \\
10 \\
17 \\
18 \\
\end{array}$ & $\begin{array}{r}989 \\
271 \\
2,767 \\
2,767 \\
1,615 \\
1,615 \\
1,611 \\
1,611 \\
350 \\
710 \\
6,104 \\
6,104 \\
\end{array}$ & $\begin{array}{l}12-30-96 \\
12-30-96 \\
12-30-96 \\
12-30-96 \\
12-30-96 \\
12-30-96 \\
12-30-96 \\
12-30-96 \\
01-06-97 \\
01-14-97 \\
12-30-96 \\
12-30-96 \\
\end{array}$ & $\begin{array}{l}2: 56 p \\
2: 56 p \\
2: 56 p \\
2: 56 p \\
2: 56 p \\
2: 56 p \\
2: 56 p \\
2: 56 p \\
4: 39 p \\
8: 04 p \\
2: 56 p \\
2: 56 p \\
\end{array}$ \\
\hline \multicolumn{5}{|c|}{ Directory of $11 \mathrm{C}$} & \multicolumn{5}{|c|}{22 files, 40,744 bytes total } \\
\hline $\begin{array}{l}B 11 C \\
B 11 C \\
B 11 C \\
B 11 C \\
B 11 C \\
B 11 C \\
B 11 C \\
B 11 C \\
B 11 C \\
B 11 C\end{array}$ & $\begin{array}{l}1 \\
2 \\
3 \\
4 \\
5 \\
6 \\
7 \\
8 \\
9 \\
10\end{array}$ & $\begin{array}{r}987 \\
269 \\
2,763 \\
2,763 \\
1,611 \\
1,611 \\
1,607 \\
1,607 \\
346 \\
706\end{array}$ & $\begin{array}{l}12-30-96 \\
12-30-96 \\
12-30-96 \\
12-30-96 \\
12-30-96 \\
12-30-96 \\
12-30-96 \\
12-30-96 \\
01-06-97 \\
01-15-97\end{array}$ & $\begin{array}{l}2: 57 p \\
2: 57 p \\
2: 57 p \\
2: 57 p \\
2: 57 p \\
2: 57 p \\
2: 57 p \\
2: 57 p \\
4: 40 p \\
7: 23 p\end{array}$ & $\begin{array}{l}\text { E11C } \\
\text { E11C } \\
\text { E11C } \\
\text { E11C } \\
\text { E11C } \\
\text { E11C } \\
\text { E11C } \\
\text { E11C } \\
\text { E11C } \\
\text { E11C } \\
\text { E11C } \\
\text { E11C }\end{array}$ & $\begin{array}{l}1 \\
2 \\
3 \\
4 \\
5 \\
6 \\
7 \\
8 \\
9 \\
10 \\
17 \\
18 \\
\end{array}$ & $\begin{array}{r}987 \\
269 \\
2,763 \\
2,763 \\
1,611 \\
1,611 \\
1,607 \\
1,607 \\
346 \\
706 \\
6,102 \\
6,102 \\
\end{array}$ & $\begin{array}{l}12-30-96 \\
12-30-96 \\
12-30-96 \\
12-30-96 \\
12-30-96 \\
12-30-96 \\
12-30-96 \\
12-30-96 \\
01-06-97 \\
01-15-97 \\
12-30-96 \\
12-30-96 \\
\end{array}$ & $\begin{array}{l}2: 57 p \\
2: 57 p \\
2: 57 p \\
2: 57 p \\
2: 57 p \\
2: 57 p \\
2: 57 p \\
2: 57 p \\
4: 41 p \\
7: 28 p \\
2: 57 p \\
2: 57 p \\
\end{array}$ \\
\hline \multicolumn{5}{|c|}{ Directory of $12 \mathrm{~A}-1$} & \multicolumn{5}{|c|}{22 files, 40,820 bytes total } \\
\hline $\begin{array}{l}B 12 A-1 \\
B 12 A-1 \\
B 12 A-1 \\
B 12 A-1 \\
B 12 A-1 \\
B 12 A-1 \\
B 12 A-1 \\
B 12 A-1 \\
B 12 A-1 \\
B 12 A-1\end{array}$ & $\begin{array}{l}1 \\
2 \\
3 \\
4 \\
5 \\
6 \\
7 \\
8 \\
9 \\
10\end{array}$ & $\begin{array}{r}989 \\
271 \\
2,767 \\
2,767 \\
1,615 \\
1,615 \\
1,611 \\
1,611 \\
350 \\
710\end{array}$ & $\begin{array}{l}12-30-96 \\
12-30-96 \\
12-30-96 \\
12-30-96 \\
12-30-96 \\
12-30-96 \\
12-30-96 \\
12-30-96 \\
01-06-97 \\
01-16-97\end{array}$ & $\begin{array}{l}2: 57 \mathrm{p} \\
2: 57 \mathrm{p} \\
2: 57 \mathrm{p} \\
2: 57 \mathrm{p} \\
2: 57 \mathrm{p} \\
2: 57 \mathrm{p} \\
2: 57 \mathrm{p} \\
2: 57 \mathrm{p} \\
4: 42 \mathrm{p} \\
1: 34 \mathrm{p}\end{array}$ & $\begin{array}{l}\text { E12A-1 } \\
\text { E12A-1 } \\
\text { E12A-1 } \\
\text { E12A-1 } \\
\text { E12A-1 } \\
\text { E12A-1 } \\
\text { E12A-1 } \\
\text { E12A-1 } \\
\text { E12A-1 } \\
\text { E12A-1 } \\
\text { E12A-1 } \\
\text { E12A-1 }\end{array}$ & $\begin{array}{l}1 \\
2 \\
3 \\
4 \\
5 \\
6 \\
7 \\
8 \\
9 \\
10 \\
17 \\
18 \\
\end{array}$ & $\begin{array}{r}989 \\
271 \\
2,767 \\
2,767 \\
1,615 \\
1,615 \\
1,611 \\
1,611 \\
350 \\
710 \\
6,104 \\
6,104 \\
\end{array}$ & $\begin{array}{l}12-30-96 \\
12-30-96 \\
12-30-96 \\
12-30-96 \\
12-30-96 \\
12-30-96 \\
12-30-96 \\
12-30-96 \\
01-06-97 \\
01-16-97 \\
12-30-96 \\
12-30-96 \\
\end{array}$ & $\begin{array}{l}2: 58 p \\
2: 58 p \\
2: 58 p \\
2: 58 p \\
2: 58 p \\
2: 58 p \\
2: 58 p \\
2: 58 p \\
4: 42 p \\
1: 35 p \\
2: 58 p \\
2: 58 p\end{array}$ \\
\hline
\end{tabular}


Table 11. Disk Table of Contents (Sheet 4 of 4 )

\begin{tabular}{|c|c|c|c|c|c|c|c|c|c|}
\hline \multicolumn{2}{|c|}{ File Name } & \multirow{2}{*}{$\begin{array}{c}\text { Size } \\
\text { (bytes) }\end{array}$} & \multicolumn{2}{|c|}{ Creation } & \multicolumn{2}{|c|}{ File Nane } & \multirow{2}{*}{$\begin{array}{l}\text { size } \\
\text { (bytes) }\end{array}$} & \multicolumn{2}{|c|}{ Crestion } \\
\hline Base & Ext. & & 1 Date & Time & Base & Ext. & & Date & Time \\
\hline \multicolumn{5}{|c|}{ Directory of $12 \mathrm{~A}-2$} & \multicolumn{5}{|c|}{22 files, 40,820 bytes total } \\
\hline $\begin{array}{l}\text { B12A-2 } \\
\text { B12A-2 } \\
\text { B12A-2 } \\
\text { B12A-2 } \\
\text { B12A-2 } \\
\text { B12A-2 } \\
\text { B12A-2 } \\
\text { B12A-2 } \\
\text { B12A-2 } \\
\text { B12A-2 }\end{array}$ & $\begin{array}{l}1 \\
2 \\
3 \\
4 \\
5 \\
6 \\
7 \\
8 \\
9 \\
10\end{array}$ & $\begin{array}{r}989 \\
271 \\
2,767 \\
2,767 \\
1,615 \\
1,615 \\
1,611 \\
1,611 \\
350 \\
710\end{array}$ & $\begin{array}{l}12-30-96 \\
12-30-96 \\
12-30-96 \\
12-30-96 \\
12-30-96 \\
12-30-96 \\
12-30-96 \\
12-30-96 \\
01-06-97 \\
01-16-97\end{array}$ & $\begin{array}{l}2: 58 p \\
2: 58 p \\
2: 58 p \\
2: 58 p \\
2: 58 p \\
2: 58 p \\
2: 58 p \\
2: 58 p \\
4: 43 p \\
1: 36 p\end{array}$ & $\begin{array}{l}E 12 A-2 \\
E 12 A-2 \\
E 12 A-2 \\
E 12 A-2 \\
\text { E12A-2 } \\
\text { E12A-2 } \\
\text { E12A-2 } \\
\text { E12A-2 } \\
\text { E12A-2 } \\
\text { E12A-2 } \\
\text { E12A-2 } \\
\text { E12A-2 }\end{array}$ & $\begin{array}{l}1 \\
2 \\
3 \\
4 \\
5 \\
6 \\
7 \\
8 \\
9 \\
10 \\
17 \\
18 \\
\end{array}$ & $\begin{array}{r}989 \\
271 \\
2,767 \\
2,767 \\
1,615 \\
1,615 \\
1,611 \\
1,611 \\
350 \\
710 \\
6,104 \\
6,104 \\
\end{array}$ & $\begin{array}{l}12-30-96 \\
12-30-96 \\
12-30-96 \\
12-30-96 \\
12-30-96 \\
12-30-96 \\
12-30-96 \\
12-30-96 \\
01-06-97 \\
01-16-97 \\
12-30-96 \\
12-30-96 \\
\end{array}$ & $\begin{array}{l}2: 59 p \\
2: 59 p \\
2: 59 p \\
2: 59 p \\
2: 59 p \\
2: 59 p \\
2: 59 p \\
2: 59 p \\
4: 44 p \\
1: 37 p \\
2: 59 p \\
2: 59 p \\
\end{array}$ \\
\hline \multicolumn{5}{|c|}{ Directory of $12 \mathrm{~B}-1$} & \multicolumn{5}{|c|}{22 files, 40,402 bytes total } \\
\hline $\begin{array}{l}\text { В } 12 B-1 \\
\text { В12B-1 } \\
\text { B } 12 B-1 \\
\text { B } 12 B-1 \\
\text { B } 12 B-1 \\
\text { B } 12 B-1 \\
\text { B } 12 B-1 \\
\text { B } 12 B-1 \\
\text { B } 12 B-1 \\
\text { B } 12 B-1\end{array}$ & $\begin{array}{l}1 \\
2 \\
3 \\
4 \\
5 \\
6 \\
7 \\
8 \\
9 \\
10\end{array}$ & $\begin{array}{r}989 \\
271 \\
2,767 \\
2,767 \\
1,615 \\
1,615 \\
1,611 \\
1,611 \\
350 \\
501\end{array}$ & $\begin{array}{l}12-30-96 \\
12-30-96 \\
12-30-96 \\
12-30-96 \\
12-30-96 \\
12-30-96 \\
12-30-96 \\
12-30-96 \\
01-06-97 \\
01-16-97\end{array}$ & $\begin{array}{l}3: 00 p \\
3: 00 p \\
3: 00 p \\
3: 00 p \\
3: 00 p \\
3: 00 p \\
3: 00 p \\
3: 00 p \\
4: 46 p \\
1: 37 p\end{array}$ & $\begin{array}{l}E 12 B-1 \\
E 12 B-1 \\
E 12 B-1 \\
E 12 B-1 \\
E 12 B-1 \\
E 12 B-1 \\
E 12 B-1 \\
E 12 B-1 \\
\text { E12B-1 } \\
\text { E12B-1 } \\
\text { E12B-1 } \\
\text { E12B-1 }\end{array}$ & $\begin{array}{l}1 \\
2 \\
3 \\
4 \\
5 \\
6 \\
7 \\
8 \\
9 \\
10 \\
17 \\
18 \\
\end{array}$ & $\begin{array}{r}989 \\
271 \\
2,767 \\
2,767 \\
1,615 \\
1,615 \\
1.611 \\
1,611 \\
350 \\
501 \\
6,104 \\
6,104 \\
\end{array}$ & $\begin{array}{l}12-30-96 \\
12-30-96 \\
12-30-96 \\
12-30-96 \\
12-30-96 \\
12-30-96 \\
12-30-96 \\
12-30-96 \\
01-06-97 \\
01-16-97 \\
12-30-96 \\
12-30-96 \\
\end{array}$ & $\begin{array}{l}3: 00 p \\
3: 00 p \\
3: 00 p \\
3: 00 p \\
3: 00 p \\
3: 00 p \\
3: 00 p \\
3: 00 p \\
4: 46 p \\
1: 38 p \\
3: 00 p \\
3: 00 p\end{array}$ \\
\hline \multicolumn{5}{|c|}{ Directory of $12 \mathrm{~B}-2$} & \multicolumn{5}{|c|}{22 files, 40,402 bytes total } \\
\hline $\begin{array}{l}\text { B12B-2 } \\
\text { B12B-2 } \\
\text { B12B-2 } \\
\text { B12B-2 } \\
\text { B12B-2 } \\
\text { B12B-2 } \\
\text { B12B-2 } \\
\text { B12B-2 } \\
\text { B12B-2 } \\
\text { B12B-2 }\end{array}$ & $\begin{array}{l}1 \\
2 \\
3 \\
4 \\
5 \\
6 \\
7 \\
8 \\
9 \\
10\end{array}$ & $\begin{array}{r}989 \\
271 \\
2,767 \\
2,767 \\
1,615 \\
1,615 \\
1,611 \\
1,611 \\
350 \\
501\end{array}$ & $\begin{array}{l}12-30-96 \\
12-30-96 \\
12-30-96 \\
12-30-96 \\
12-30-96 \\
12-30-96 \\
12-30-96 \\
12-30-96 \\
01-06-97 \\
01-16-97\end{array}$ & $\begin{array}{l}3: 00 p \\
3: 00 p \\
3: 00 p \\
3: 00 p \\
3: 00 p \\
3: 00 p \\
3: 00 p \\
3: 00 p \\
4: 47 p \\
1: 39 p\end{array}$ & $\begin{array}{l}E 12 B-2 \\
E 12 B-2 \\
E 12 B-2 \\
E 12 B-2 \\
E 12 B-2 \\
E 12 B-2 \\
E 12 B-2 \\
E 12 B-2 \\
E 12 B-2 \\
E 12 B-2 \\
E 12 B-2 \\
E 12 B-2 \\
\end{array}$ & $\begin{array}{l}1 \\
2 \\
3 \\
4 \\
5 \\
6 \\
7 \\
8 \\
9 \\
10 \\
17 \\
18 \\
\end{array}$ & $\begin{array}{r}989 \\
271 \\
2,767 \\
2,767 \\
1,615 \\
1,615 \\
1,611 \\
1,611 \\
350 \\
501 \\
6,104 \\
6,104 \\
\end{array}$ & $\begin{array}{l}12-30-96 \\
12-30-96 \\
12-30-96 \\
12-30-96 \\
12-30-96 \\
12-30-96 \\
12-30-96 \\
12-30-96 \\
01-06-97 \\
01-16-97 \\
12-30-96 \\
12-30-96 \\
\end{array}$ & $\begin{array}{l}3: 01 p \\
3: 01 p \\
3: 01 p \\
3: 01 p \\
3: 01 p \\
3: 01 p \\
3: 01 p \\
3: 01 p \\
4: 47 p \\
2: 00 p \\
3: 01 p \\
3: 01 p\end{array}$ \\
\hline
\end{tabular}




\subsection{Data Tables for each Cycle}

\subsection{Cycle 10A-1}

Table B10A-1.1. Fission Power Generated in MFA-1, MFA-2 and Neighboring Assemblies at BOC 10A-1

\begin{tabular}{|c|c|c|c|c|}
\hline $\begin{array}{l}\text { CORE } \\
\text { POS. } \\
1506 \\
1404 \\
1405 \\
1505 \\
1507 \\
1606 \\
1607 \\
2507 \\
2405 \\
2506 \\
2508 \\
2607 \\
2608 \\
2509\end{array}$ & $\begin{array}{l}\star \star \star \star \star \star \star \\
\text { BELOW CORE } \\
2.246 \mathrm{E}-02 \\
7.618 \mathrm{E}-02 \\
8.659 \mathrm{E}-03 \\
1.088 \mathrm{E}-02 \\
9.496 \mathrm{E}-03 \\
5.669 \mathrm{E}-03 \\
8.989 \mathrm{E}-03 \\
2.105 \mathrm{E}-02 \\
5.745 \mathrm{E}-02 \\
9.759 \mathrm{E}-03 \\
9.327 \mathrm{E}-03 \\
6.612 \mathrm{E}-03 \\
1.328 \mathrm{E}-02 \\
8.462 \mathrm{E}-02\end{array}$ & $\begin{array}{l}\text { POWER IN } \\
\text { IN CORE } \\
4.196 E+00 \\
4.933 E+00 \\
4.127 E+00 \\
3.800 E+00 \\
3.586 E+00 \\
3.720 E+00 \\
3.110 E+00 \\
3.963 E+00 \\
4.173 E+00 \\
3.659 E+00 \\
4.376 E+00 \\
3.376 E+00 \\
3.186 E+00 \\
2.998 E+00\end{array}$ & $\begin{array}{c}\text { MEGAWATTS } \\
\text { ABOVE CORE } \\
1.709 \mathrm{E}-02 \\
4.233 \mathrm{E}-02 \\
6.616 \mathrm{E}-03 \\
6.491 \mathrm{E}-03 \\
7.131 \mathrm{E}-03 \\
4.286 \mathrm{E}-03 \\
6.475 \mathrm{E}-03 \\
1.649 \mathrm{E}-02 \\
3.298 \mathrm{E}-02 \\
6.848 \mathrm{E}-03 \\
6.847 \mathrm{E}-03 \\
4.948 \mathrm{E}-03 \\
1.062 \mathrm{E}-02 \\
4.569 \mathrm{E}-02\end{array}$ & $\begin{array}{l}\star \star \star \star \star \star \\
\text { TOTAL PWR } \\
4.235 \mathrm{E}+00 \\
5.052 \mathrm{E}+00 \\
4.143 \mathrm{E}+00 \\
3.818 \mathrm{E}+00 \\
3.602 \mathrm{E}+00 \\
3.730 \mathrm{E}+00 \\
3.126 \mathrm{E}+00 \\
4.001 \mathrm{E}+00 \\
4.264 \mathrm{E}+00 \\
3.676 \mathrm{E}+00 \\
4.392 \mathrm{E}+00 \\
3.388 \mathrm{E}+00 \\
3.210 \mathrm{E}+00 \\
3.128 \mathrm{E}+00\end{array}$ \\
\hline
\end{tabular}

Table B10A-1.2. Assembly Averaged Total and Fast Flux in MFA-1 and MFA-2 at BOC $10 \mathrm{~A}-1$

\begin{tabular}{cccc} 
& Core & \multicolumn{2}{c}{ Flux $\left(\mathrm{n} / \mathrm{cm}^{2}-\mathrm{sec}\right)$} \\
Assembly & Pos. & Total & $>0.1 \mathrm{MeV}$ \\
MFA-1 & 1506 & $3.006 \mathrm{E}+15$ & $1.951 \mathrm{E}+15$ \\
MFA-2 & 2507 & $2.836 \mathrm{E}+15$ & $1.821 \mathrm{E}+15$
\end{tabular}


Table Bl0A-1.3. Axial Distribution of Total Flux, Fast Flux and Power in MFA-1 at BOC 10A-1

$\begin{array}{rccc}\text { z }(\mathrm{cm}) & \text { Total Flux } & \text { Flux }>0.1 \text { MeV } & \text { Power } \\ -97.78 & 2.6526 \mathrm{E}-02 & 1.3341 \mathrm{E}-02 & 0.0000 \mathrm{E}+00 \\ -92.78 & 5.7045 \mathrm{E}-02 & 2.5782 \mathrm{E}-02 & 0.0000 \mathrm{E}+00 \\ -87.78 & 9.1548 \mathrm{E}-02 & 4.1301 \mathrm{E}-02 & 0.0000 \mathrm{E}+00 \\ -82.78 & 1.3267 \mathrm{E}-01 & 6.1694 \mathrm{E}-02 & 0.0000 \mathrm{E}+00 \\ -77.78 & 1.8310 \mathrm{E}-01 & 8.9179 \mathrm{E}-02 & 0.0000 \mathrm{E}+00 \\ -72.15 & 2.4031 \mathrm{E}-01 & 1.2480 \mathrm{E}-01 & 0.0000 \mathrm{E}+00 \\ -65.90 & 2.9764 \mathrm{E}-01 & 1.6976 \mathrm{E}-01 & 0.0000 \mathrm{E}+00 \\ -60.35 & 3.5878 \mathrm{E}-01 & 2.3041 \mathrm{E}-01 & 1.6617 \mathrm{E}-02 \\ -55.49 & 4.4025 \mathrm{E}-01 & 3.1379 \mathrm{E}-01 & 2.4379 \mathrm{E}-02 \\ -50.62 & 5.3615 \mathrm{E}-01 & 4.2430 \mathrm{E}-01 & 3.7703 \mathrm{E}-02 \\ -47.17 & 6.1441 \mathrm{E}-01 & 5.3037 \mathrm{E}-01 & 5.4264 \mathrm{E}-02 \\ -43.58 & 6.9713 \mathrm{E}-01 & 6.5148 \mathrm{E}-01 & 7.3365 \mathrm{E}-01 \\ -38.45 & 8.1621 \mathrm{E}-01 & 7.9755 \mathrm{E}-01 & 8.2583 \mathrm{E}-01 \\ -33.32 & 9.2677 \mathrm{E}-01 & 9.2234 \mathrm{E}-01 & 9.2756 \mathrm{E}-01 \\ -28.20 & 1.0236 \mathrm{E}+00 & 1.0268 \mathrm{E}+00 & 1.0207 \mathrm{E}+00 \\ -23.07 & 1.1043 \mathrm{E}+00 & 1.1116 \mathrm{E}+00 & 1.0995 \mathrm{E}+00 \\ -17.94 & 1.1673 \mathrm{E}+00 & 1.1768 \mathrm{E}+00 & 1.1613 \mathrm{E}+00 \\ -12.82 & 1.2115 \mathrm{E}+00 & 1.2220 \mathrm{E}+00 & 1.2047 \mathrm{E}+00 \\ -7.69 & 1.2365 \mathrm{E}+00 & 1.2476 \mathrm{E}+00 & 1.2293 \mathrm{E}+00 \\ -2.56 & 1.2421 \mathrm{E}+00 & 1.2534 \mathrm{E}+00 & 1.2347 \mathrm{E}+00 \\ 2.56 & 1.2284 \mathrm{E}+00 & 1.2397 \mathrm{E}+00 & 1.2209 \mathrm{E}+00 \\ 7.69 & 1.1955 \mathrm{E}+00 & 1.2068 \mathrm{E}+00 & 1.1882 \mathrm{E}+00 \\ 12.82 & 1.1445 \mathrm{E}+00 & 1.1555 \mathrm{E}+00 & 1.1376 \mathrm{E}+00 \\ 17.94 & 1.0769 \mathrm{E}+00 & 1.0873 \mathrm{E}+00 & 1.0706 \mathrm{E}+00 \\ 23.07 & 9.9430 \mathrm{E}-01 & 1.0030 \mathrm{E}+00 & 9.8896 \mathrm{E}-01 \\ 28.20 & 8.9899 \mathrm{E}-01 & 9.0449 \mathrm{E}-01 & 8.9515 \mathrm{E}-01 \\ 33.32 & 7.9329 \mathrm{E}-01 & 7.9301 \mathrm{E}-01 & 7.9209 \mathrm{E}-01 \\ 38.45 & 6.7981 \mathrm{E}-01 & 6.6873 \mathrm{E}-01 & 6.8447 \mathrm{E}-01 \\ 43.58 & 5.6299 \mathrm{E}-01 & 5.3189 \mathrm{E}-01 & 5.8471 \mathrm{E}-01 \\ 47.17 & 4.8378 \mathrm{E}-01 & 4.2522 \mathrm{E}-01 & 4.3606 \mathrm{E}-02 \\ 50.62 & 4.0918 \mathrm{E}-01 & 3.3194 \mathrm{E}-01 & 2.9525 \mathrm{E}-02 \\ 55.49 & 3.1815 \mathrm{E}-01 & 2.3509 \mathrm{E}-01 & 1.8093 \mathrm{E}-02 \\ 60.35 & 2.3997 \mathrm{E}-01 & 1.6184 \mathrm{E}-01 & 1.1296 \mathrm{E}-02 \\ 64.98 & 1.9222 \mathrm{E}-01 & 1.2052 \mathrm{E}-01 & 0.0000 \mathrm{E}+00 \\ 69.39 & 1.6460 \mathrm{E}-01 & 9.8965 \mathrm{E}-02 & 0.0000 \mathrm{E}+00 \\ 76.46 & 1.2535 \mathrm{E}-01 & 7.0195 \mathrm{E}-02 & 0.0000 \mathrm{E}+00 \\ 86.80 & 8.1928 \mathrm{E}-02 & 4.2306 \mathrm{E}-02 & 0.0000 \mathrm{E}+00 \\ 98.14 & 4.7337 \mathrm{E}-02 & 2.3110 \mathrm{E}-02 & 0.0000 \mathrm{E}+00 \\ 110.00 & 2.0307 \mathrm{E}-02 & 1.0362 \mathrm{E}-02 & 0.0000 \mathrm{E}+00\end{array}$


Table B10A-1.4. Axial Distribution of Total Flux, Fast Flux and Power in MFA-2 at BOC 10A-1

$\begin{array}{rccc}\text { z }(\mathrm{cm}) & \text { Total F1ux } & \text { F1ux }>0.1 \mathrm{MeV} & \text { Power } \\ -97.78 & 2.5594 \mathrm{E}-02 & 1.2927 \mathrm{E}-02 & 0.0000 \mathrm{E}+00 \\ -92.78 & 5.5013 \mathrm{E}-02 & 2.4935 \mathrm{E}-02 & 0.0000 \mathrm{E}+00 \\ -87.78 & 8.8199 \mathrm{E}-02 & 3.9859 \mathrm{E}-02 & 0.0000 \mathrm{E}+00 \\ -82.78 & 1.2771 \mathrm{E}-01 & 5.9417 \mathrm{E}-02 & 0.0000 \mathrm{E}+00 \\ -77.78 & 1.7617 \mathrm{E}-01 & 8.5771 \mathrm{E}-02 & 0.0000 \mathrm{E}+00 \\ -72.15 & 2.3120 \mathrm{E}-01 & 1.2002 \mathrm{E}-01 & 0.0000 \mathrm{E}+00 \\ -65.90 & 2.8707 \mathrm{E}-01 & 1.6387 \mathrm{E}-01 & 0.0000 \mathrm{E}+00 \\ -60.35 & 3.4727 \mathrm{E}-01 & 2.2388 \mathrm{E}-01 & 1.6431 \mathrm{E}-02 \\ -55.49 & 4.2863 \mathrm{E}-01 & 3.0663 \mathrm{E}-01 & 2.4217 \mathrm{E}-02 \\ -50.62 & 5.2581 \mathrm{E}-01 & 4.1680 \mathrm{E}-01 & 3.7476 \mathrm{E}-02 \\ -47.17 & 6.0568 \mathrm{E}-01 & 5.2258 \mathrm{E}-01 & 5.3907 \mathrm{E}-02 \\ -43.58 & 6.8985 \mathrm{E}-01 & 6.4366 \mathrm{E}-01 & 7.2306 \mathrm{E}-01 \\ -38.45 & 8.0993 \mathrm{E}-01 & 7.9061 \mathrm{E}-01 & 8.1912 \mathrm{E}-01 \\ -33.32 & 9.2109 \mathrm{E}-01 & 9.1643 \mathrm{E}-01 & 9.2189 \mathrm{E}-01 \\ -28.20 & 1.0184 \mathrm{E}+00 & 1.0219 \mathrm{E}+00 & 1.0154 \mathrm{E}+00 \\ -23.07 & 1.0999 \mathrm{E}+00 & 1.1079 \mathrm{E}+00 & 1.0949 \mathrm{E}+00 \\ -17.94 & 1.1639 \mathrm{E}+00 & 1.1743 \mathrm{E}+00 & 1.1578 \mathrm{E}+00 \\ -12.82 & 1.2092 \mathrm{E}+00 & 1.2207 \mathrm{E}+00 & 1.2025 \mathrm{E}+00 \\ -7.69 & 1.2352 \mathrm{E}+00 & 1.2471 \mathrm{E}+00 & 1.2281 \mathrm{E}+00 \\ -2.56 & 1.2415 \mathrm{E}+00 & 1.2537 \mathrm{E}+00 & 1.2342 \mathrm{E}+00 \\ 2.56 & 1.2283 \mathrm{E}+00 & 1.2406 \mathrm{E}+00 & 1.2210 \mathrm{E}+00 \\ 7.69 & 1.1963 \mathrm{E}+00 & 1.2084 \mathrm{E}+00 & 1.1892 \mathrm{E}+00 \\ 12.82 & 1.1466 \mathrm{E}+00 & 1.1583 \mathrm{E}+00 & 1.1400 \mathrm{E}+00 \\ 17.94 & 1.0806 \mathrm{E}+00 & 1.0913 \mathrm{E}+00 & 1.0748 \mathrm{E}+00 \\ 23.07 & 9.9947 \mathrm{E}-01 & 1.0081 \mathrm{E}+00 & 9.9473 \mathrm{E}-01 \\ 28.20 & 9.0506 \mathrm{E}-01 & 9.1006 \mathrm{E}-01 & 9.0196 \mathrm{E}-01 \\ 33.32 & 7.9976 \mathrm{E}-01 & 7.9844 \mathrm{E}-01 & 7.9937 \mathrm{E}-01 \\ 38.45 & 6.8613 \mathrm{E}-01 & 6.7337 \mathrm{E}-01 & 6.9155 \mathrm{E}-01 \\ 43.58 & 5.6869 \mathrm{E}-01 & 5.3526 \mathrm{E}-01 & 5.9037 \mathrm{E}-01 \\ 47.17 & 4.8861 \mathrm{E}-01 & 4.2753 \mathrm{E}-01 & 4.4266 \mathrm{E}-02 \\ 50.62 & 4.1312 \mathrm{E}-01 & 3.3359 \mathrm{E}-01 & 3.0062 \mathrm{E}-02 \\ 55.49 & 3.2164 \mathrm{E}-01 & 2.3629 \mathrm{E}-01 & 1.8544 \mathrm{E}-02 \\ 60.35 & 2.4413 \mathrm{E}-01 & 1.6316 \mathrm{E}-01 & 1.1680 \mathrm{E}-02 \\ 64.98 & 1.9720 \mathrm{E}-01 & 1.2185 \mathrm{E}-01 & 0.0000 \mathrm{E}+00 \\ 69.39 & 1.6933 \mathrm{E}-01 & 1.0004 \mathrm{E}-01 & 0.0000 \mathrm{E}+00 \\ 76.46 & 1.2922 \mathrm{E}-01 & 7.0908 \mathrm{E}-02 & 0.0000 \mathrm{E}+00 \\ 86.80 & 8.4577 \mathrm{E}-02 & 4.2724 \mathrm{E}-02 & 0.0000 \mathrm{E}+00 \\ 98.14 & 4.8841 \mathrm{E}-02 & 2.3310 \mathrm{E}-02 & 0.0000 \mathrm{E}+00 \\ 110.00 & 2.0742 \mathrm{E}-02 & 1.0361 \mathrm{E}-02 & 0.0000 \mathrm{E}+00\end{array}$


HNF-SD-FF-ANAL-009 Rev. 1

Table B10A-1.5. Fission Power Distribution by Pin in MFA-1 at BOC 10A-1 CORE POSITION 1506

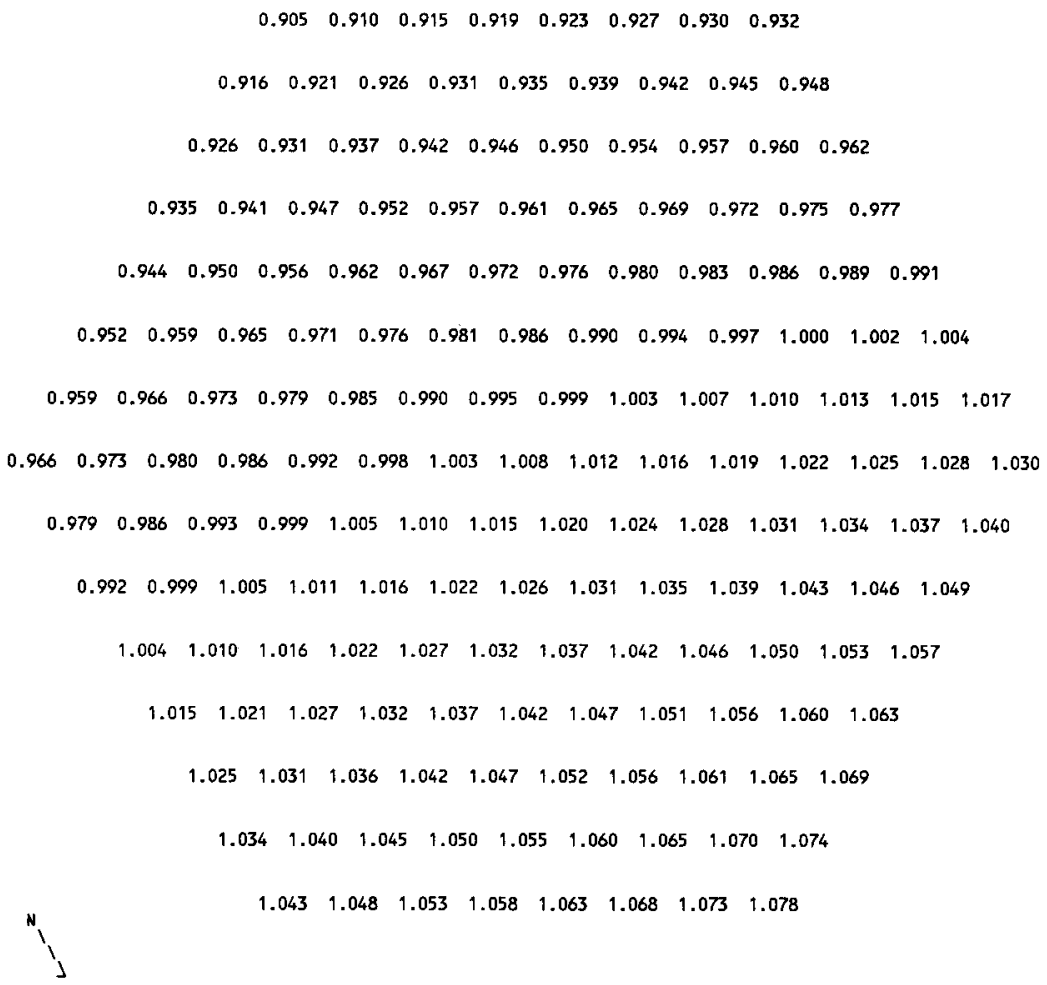


HNF-SD-FF-ANAL-009 Rev. 1

Table B10A-1.6. Fission Power Distribution by Pin in MFA-2 at BOC 10A-1 CORE POSITION 2507

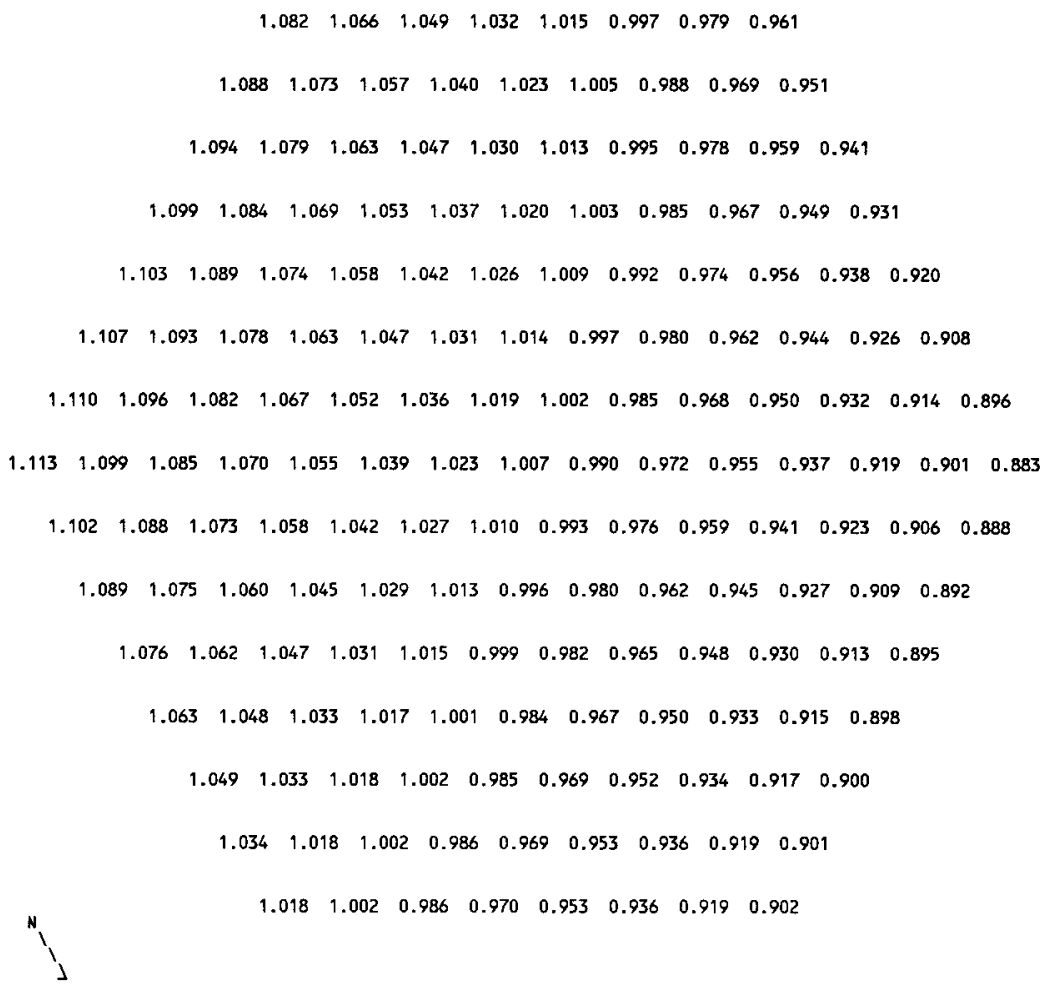


HNF-SD-FF-ANAL-009 Rev. 1

Table B10A-1.7. Fast Flux Distribution by Pin in MFA-1 at BOC 10A-1 CORE POSITION 1506

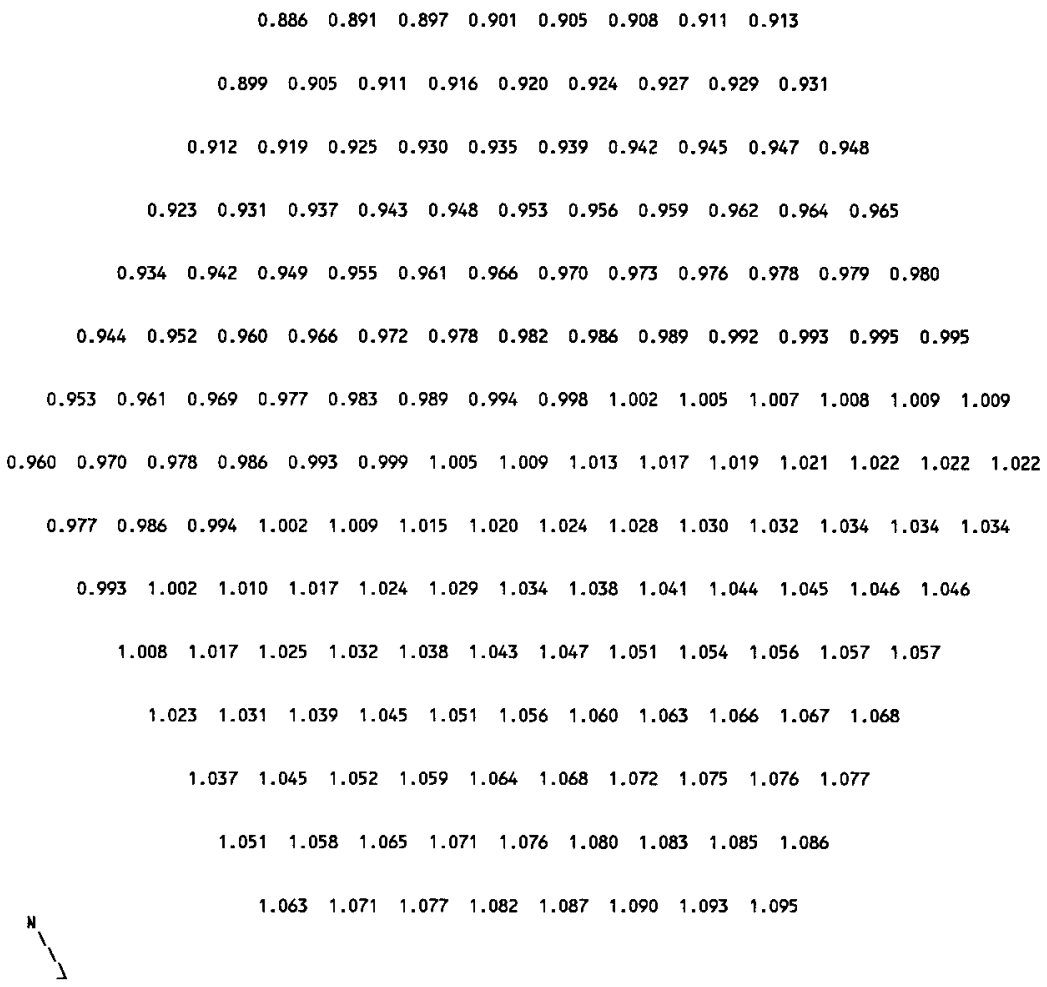


HNF-SD-FF-ANAL-009 Rev. 1

Table BlOA-1.8. Fast Flux Distribution by Pin in MFA-2 at BOC 10A-1 CORE POSITION 2507

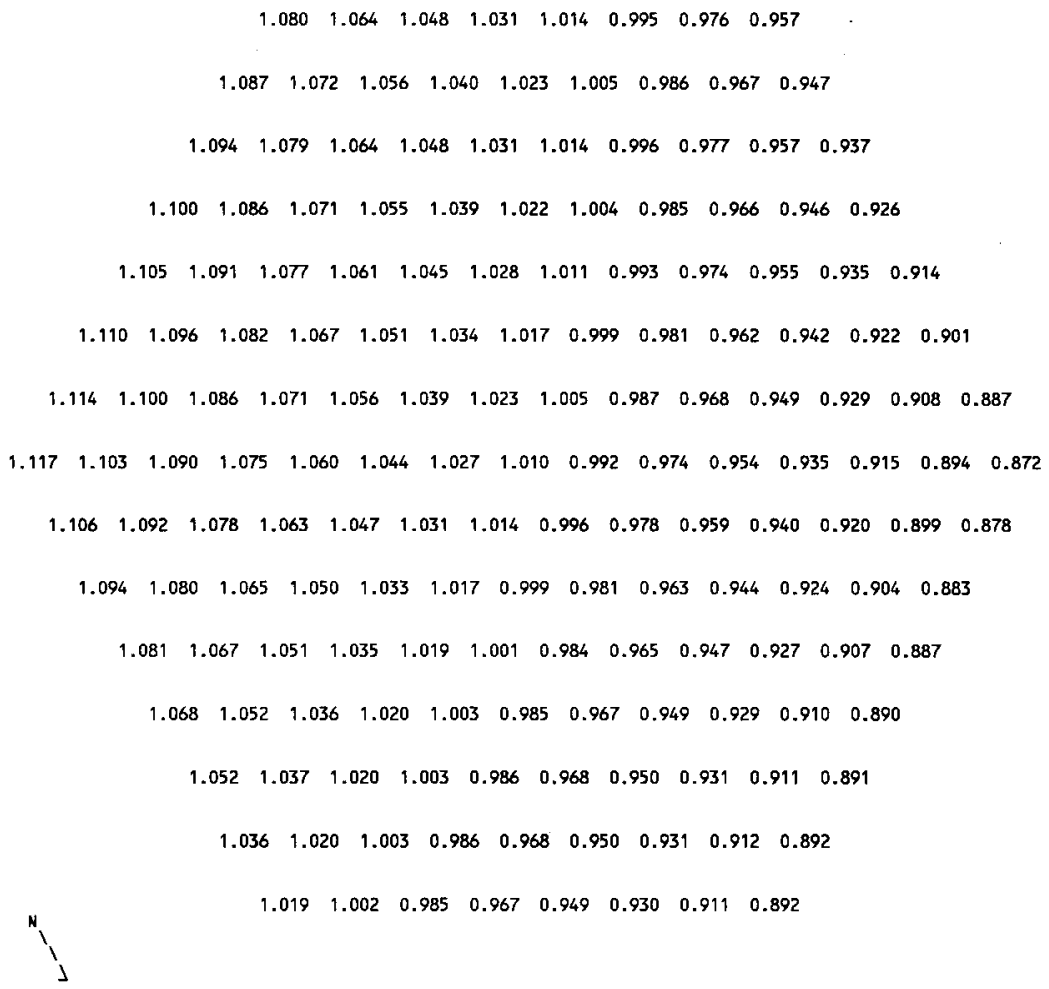


Table B10A-1.9. MFA-1 and MFA-2 Duct Wall Fast Flux Data at BOC 10A-1

$\begin{array}{ccc}\text { Side } & \begin{array}{c}\text { Flux }>0.1 \\ \text { MFA-1 }\end{array} & \begin{array}{c}\mathrm{MeV}\left(\mathrm{n} / \mathrm{cm}^{2}-\mathrm{sec}\right) \\ \text { MFA-2 }\end{array} \\ \text { E } & 1.9719 E+15 & 1.9751 \mathrm{E}+15 \\ \text { SE } & 1.7724 \mathrm{E}+15 & 2.0325 \mathrm{E}+15 \\ \text { SW } & 1.7082 E+15 & 1.8566 \mathrm{E}+15 \\ \text { W } & 1.8752 E+15 & 1.6328 \mathrm{E}+15 \\ \text { NW } & 2.0845 E+15 & 1.5643 \mathrm{E}+15 \\ \text { NE } & 2.1367 \mathrm{E}+15 & 1.7117 \mathrm{E}+15\end{array}$

Table B10A-1.10. Assembly Outlet Temperatures and Flow Rates at BOC 10A-1

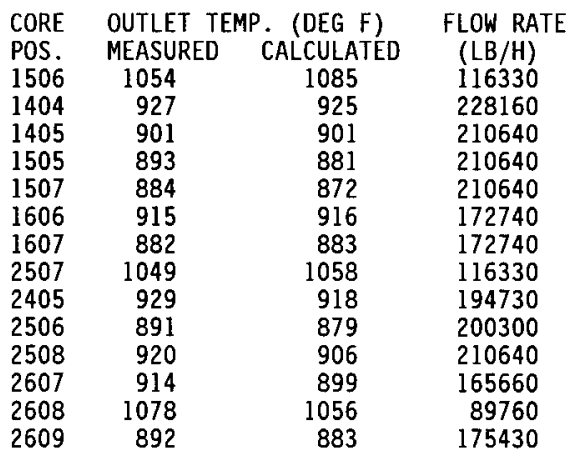

Assembly flows are based on a calculated core pressure drop of $113.3 \mathrm{psi}$ at a total reactor flow rate of $16.74 \mathrm{E}+06 \mathrm{lb} / \mathrm{hr}$. 
Table B10A-1.11. MFA-1 Sodium Subchannel Temperatures at Top of Core Elevation for BOC 1OA-1

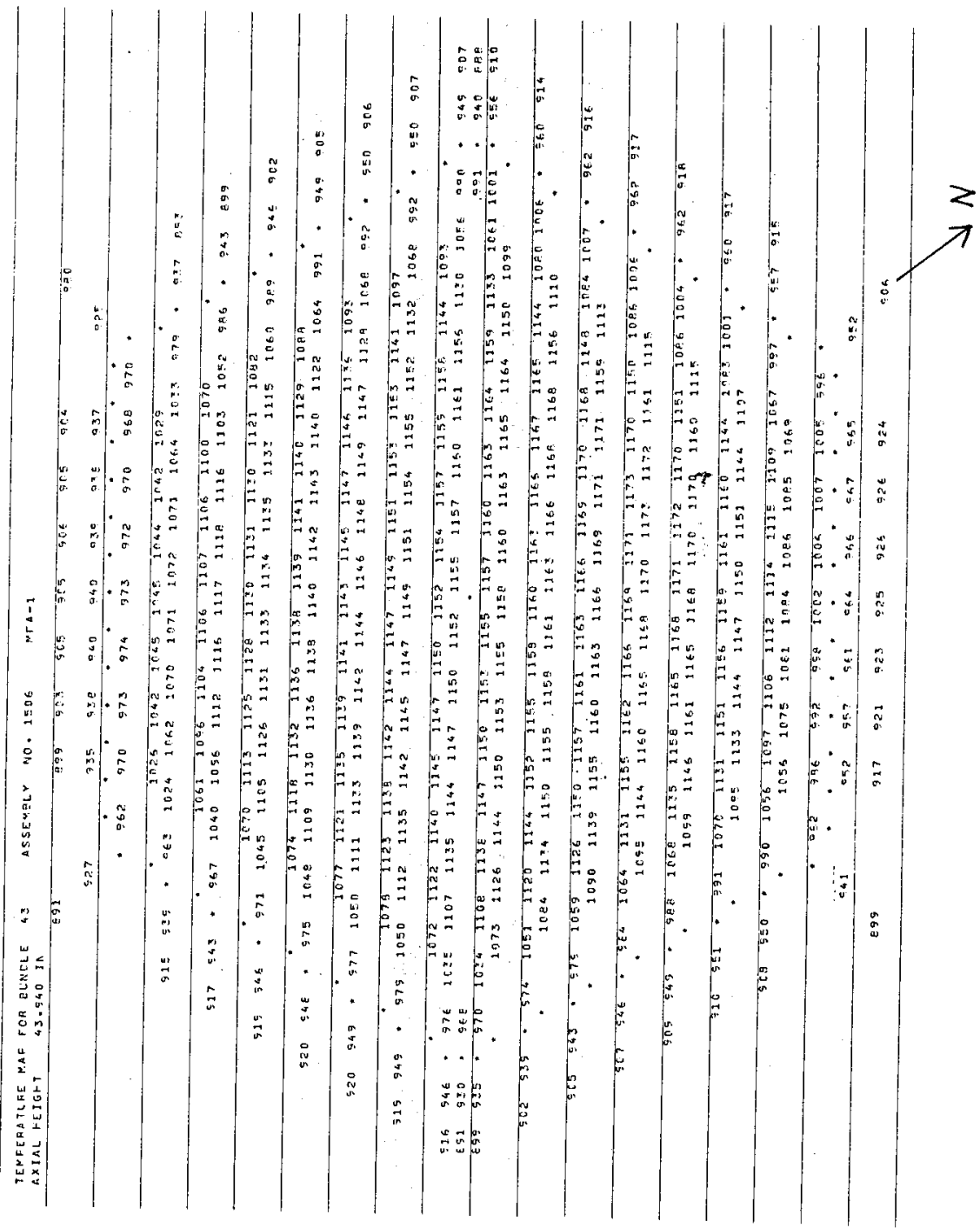




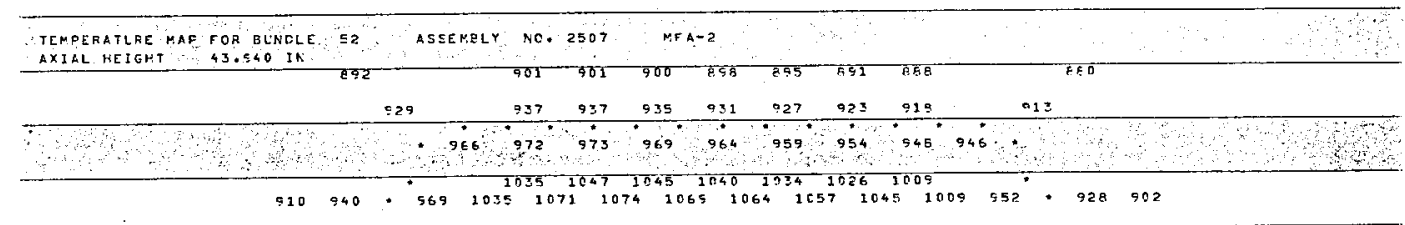

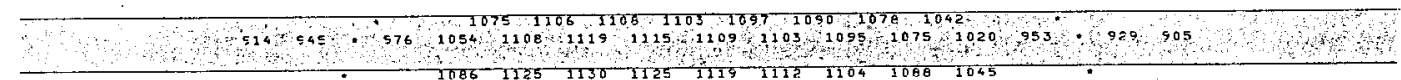

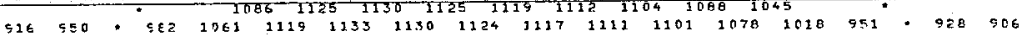

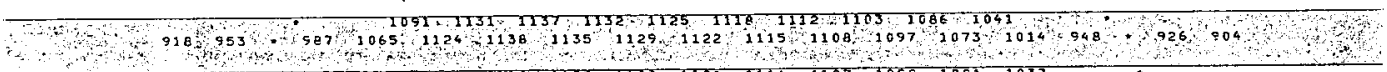

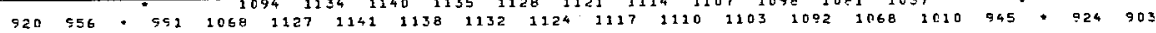

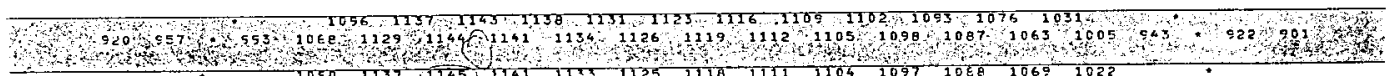

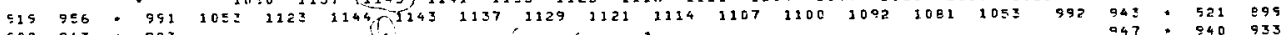

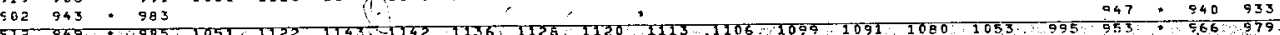

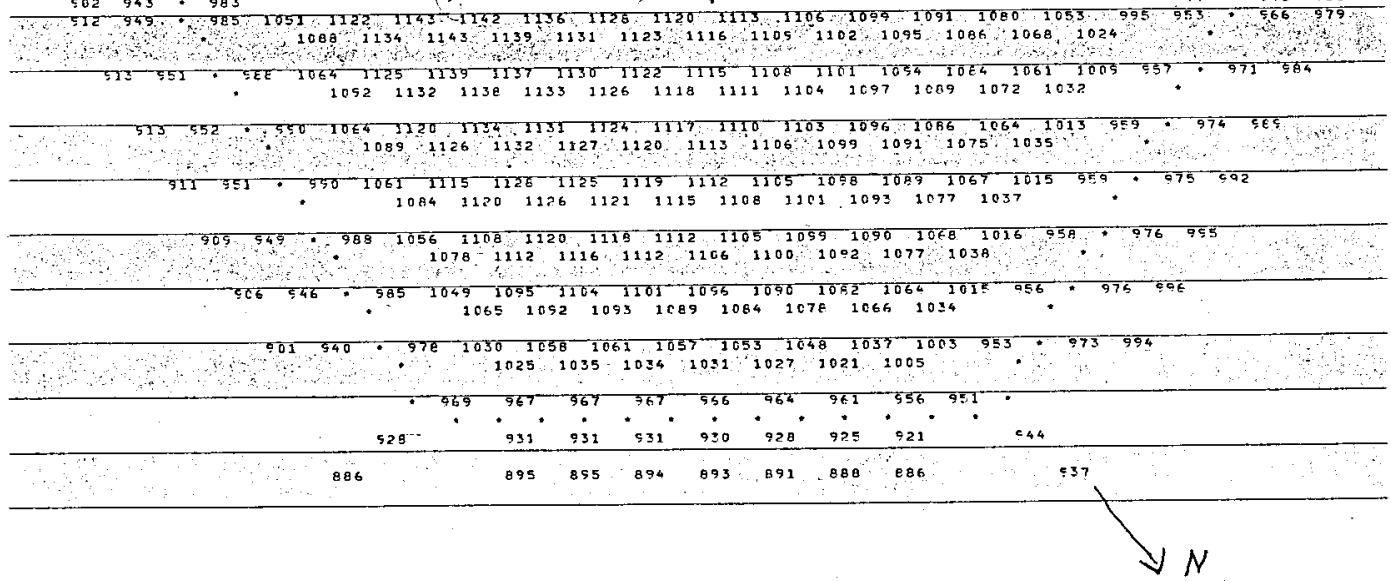


Table BlOA-1.13. MFA-1 Sodium Subchannel Temperatures at Elevation of Upper Axial Blanket for BOC $10 \mathrm{~A}-1$

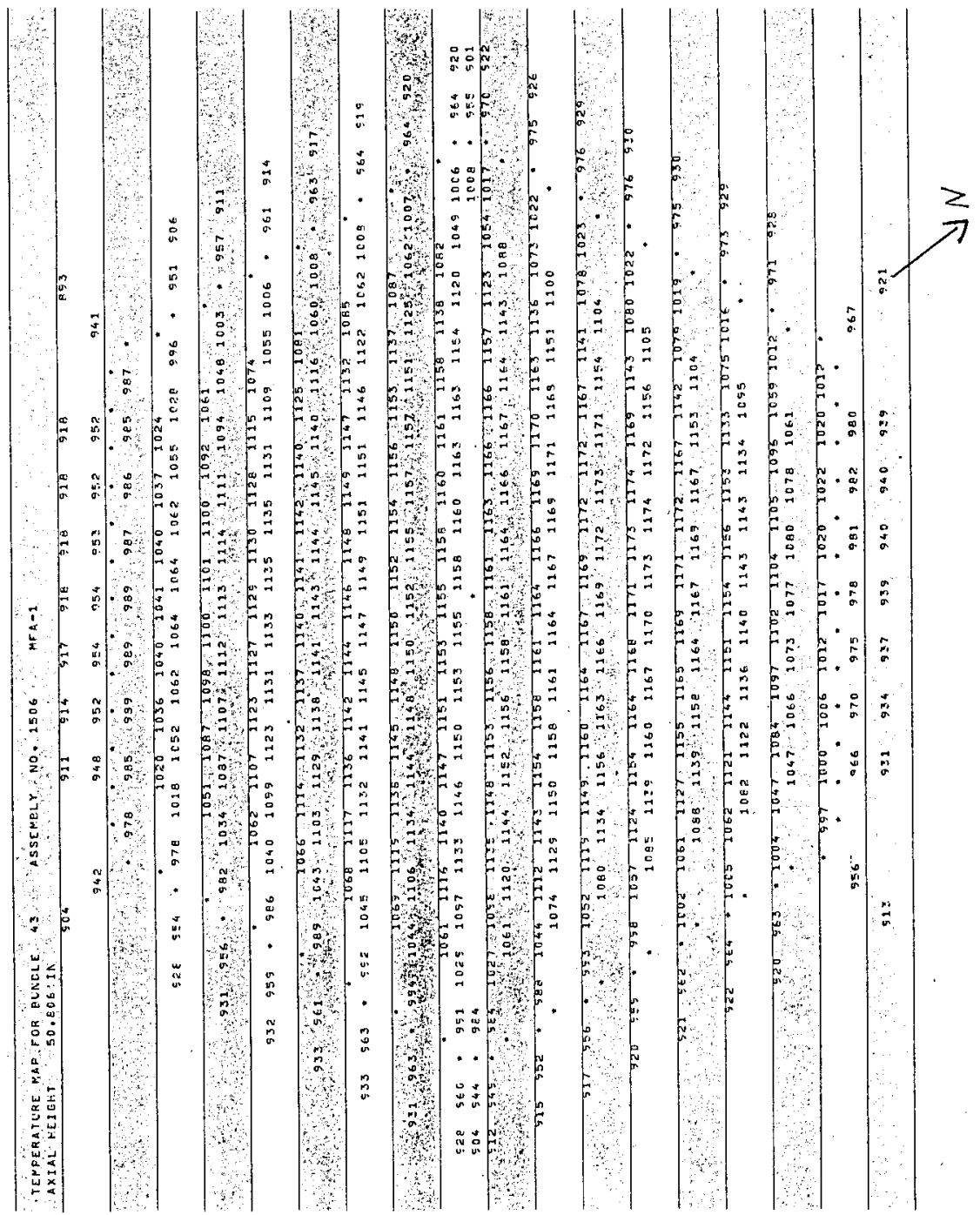


Table BlOA-1.14. MFA-2 Sodium Subchannel Temperatures at Elevation of Upper Axial Blanket for BOC 1OA-1

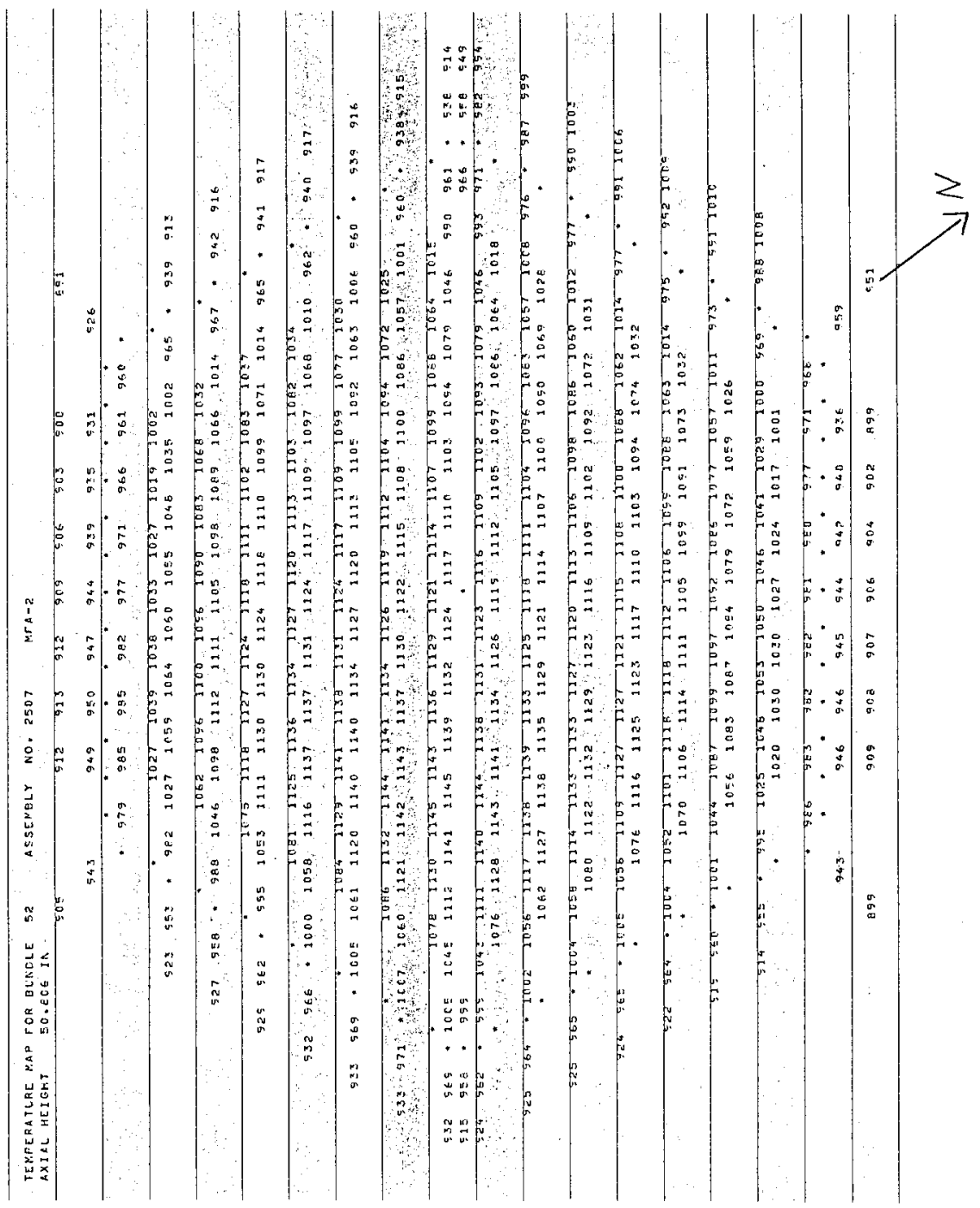


Table B10A-1.15. MFA-1 Sodium Subchannel Temperatures at Top of Fuel Pin Bundle for BOC 1OA-1

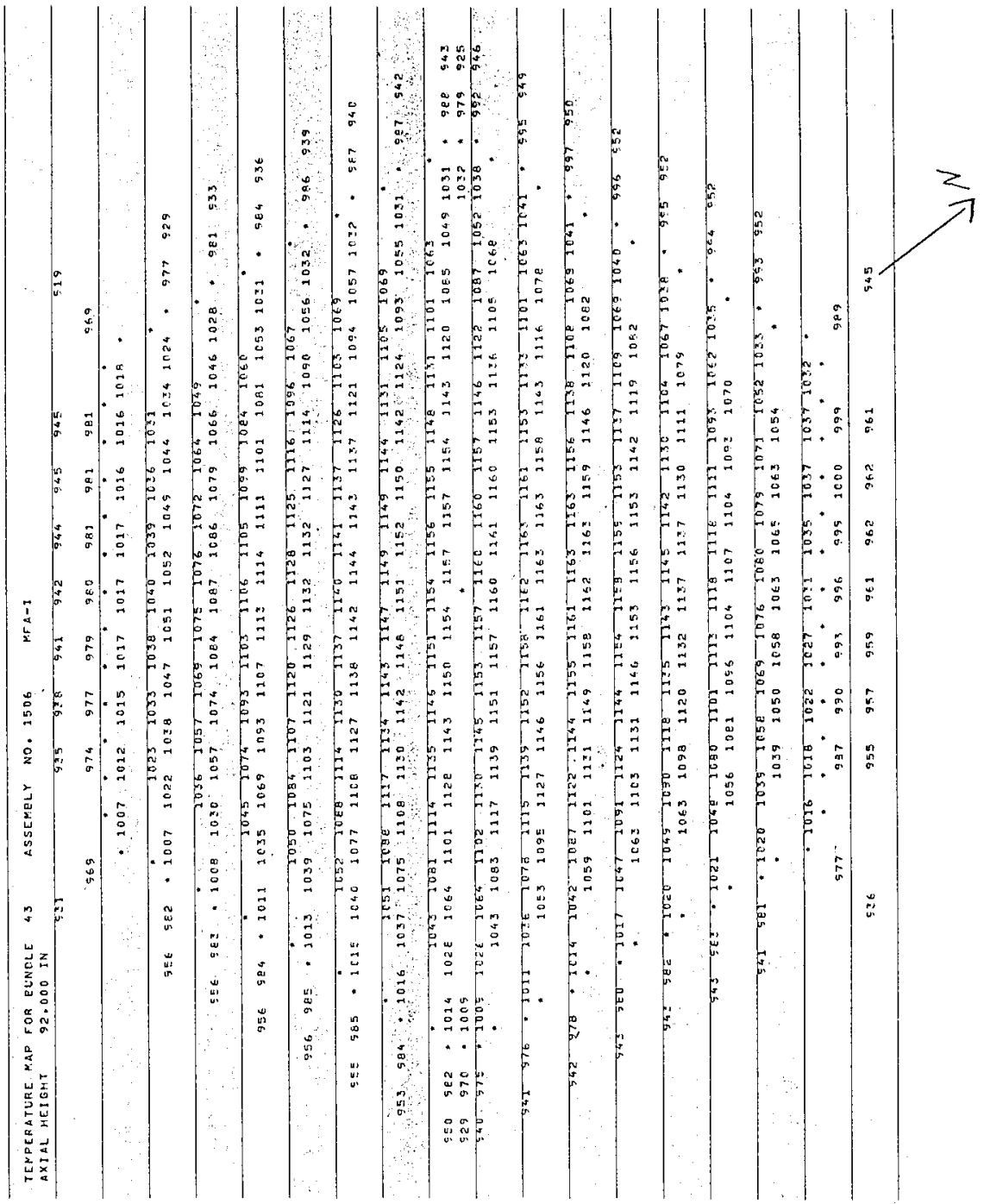


Table B10A-1.16. MFA-2 Sodium Subchannel Temperatures at Top of Fuel Pin Bundle for BOC 10A-1

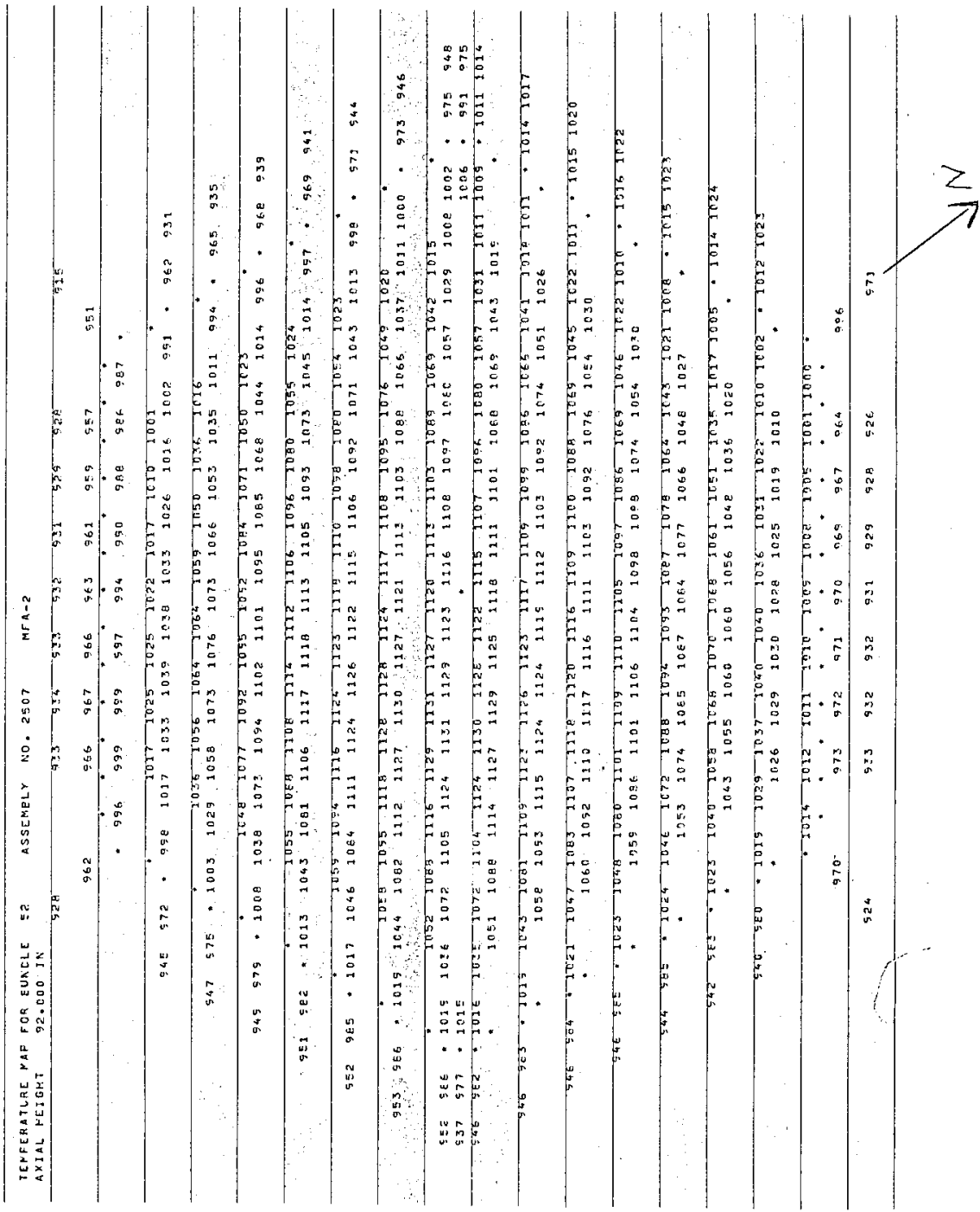


Table E10A-1.1. Fission Power Generated in MFA-1, MFA-2 and Neighboring Assemblies at EOC 10A-1

\begin{tabular}{lrrrr} 
CORE & $* * * * * *$ & \multicolumn{2}{c}{ POWER IN MEGAWATTS } & $* * * * * *$ \\
POS. & BELOW CORE & IN CORE & ABOVE CORE & TOTAL PWR \\
1506 & $2.758 \mathrm{E}-02$ & $4.106 \mathrm{E}+00$ & $2.092 \mathrm{E}-02$ & $4.154 \mathrm{E}+00$ \\
1404 & $8.078 \mathrm{E}-02$ & $4.846 \mathrm{E}+00$ & $4.798 \mathrm{E}-02$ & $4.975 \mathrm{E}+00$ \\
1405 & $9.261 \mathrm{E}-03$ & $4.037 \mathrm{E}+00$ & $7.469 \mathrm{E}-03$ & $4.054 \mathrm{E}+00$ \\
1505 & $1.121 \mathrm{E}-02$ & $3.795 \mathrm{E}+00$ & $7.296 \mathrm{E}-03$ & $3.814 \mathrm{E}+00$ \\
1507 & $9.750 \mathrm{E}-03$ & $3.510 \mathrm{E}+00$ & $7.821 \mathrm{E}-03$ & $3.528 \mathrm{E}+00$ \\
1606 & $6.117 \mathrm{E}-03$ & $3.692 \mathrm{E}+00$ & $4.921 \mathrm{E}-03$ & $3.703 \mathrm{E}+00$ \\
1607 & $9.165 \mathrm{E}-03$ & $3.063 \mathrm{E}+00$ & $7.099 \mathrm{E}-03$ & $3.079 \mathrm{E}+00$ \\
2507 & $2.522 \mathrm{E}-02$ & $3.880 \mathrm{E}+00$ & $1.995 \mathrm{E}-02$ & $3.925 \mathrm{E}+00$ \\
2405 & $6.120 \mathrm{E}-02$ & $4.089 \mathrm{E}+00$ & $3.771 \mathrm{E}-02$ & $4.188 \mathrm{E}+00$ \\
2506 & $1.013 \mathrm{E}-02$ & $3.614 \mathrm{E}+00$ & $7.616 \mathrm{E}-03$ & $3.631 \mathrm{E}+00$ \\
2508 & $9.791 \mathrm{E}-03$ & $4.299 \mathrm{E}+00$ & $7.682 \mathrm{E}-03$ & $4.317 \mathrm{E}+00$ \\
2607 & $6.923 \mathrm{E}-03$ & $3.335 \mathrm{E}+00$ & $5.509 \mathrm{E}-03$ & $3.348 \mathrm{E}+00$ \\
2608 & $1.574 \mathrm{E}-02$ & $3.135 \mathrm{E}+00$ & $1.288 \mathrm{E}-02$ & $3.163 \mathrm{E}+00$ \\
2609 & $8.413 \mathrm{E}-02$ & $2.972 \mathrm{E}+00$ & $4.964 \mathrm{E}-02$ & $3.106 \mathrm{E}+00$
\end{tabular}

Table E1OA-1.2. Assembly Averaged Total and Fast Flux in MFA-1 and MFA-2 at EOC $10 \mathrm{~A}-1$

\begin{tabular}{cccc} 
& Core & \multicolumn{2}{c}{ Flux $\left(\mathrm{n} / \mathrm{cm}^{2}-\mathrm{sec}\right)$} \\
Assembly & Pos. & Total & $>0.1 \mathrm{MeV}$ \\
MFA-1 & 1506 & $3.013 \mathrm{E}+15$ & $1.945 \mathrm{E}+15$ \\
MFA-2 & 2507 & $2.840 \mathrm{E}+15$ & $1.814 \mathrm{E}+15$
\end{tabular}


Table E10A-1.3. Axial Distribution of Total Flux, Fast Flux and Power in MFA-1 at EOC 1OA-1

$\begin{array}{rccc}\text { Z }(\mathrm{cm}) & \text { Total Flux } & \text { Flux }>0.1 \text { MeV } & \text { Power } \\ -97.78 & 2.5837 \mathrm{E}-02 & 1.3075 \mathrm{E}-02 & 0.0000 \mathrm{E}+00 \\ -92.78 & 5.5558 \mathrm{E}-02 & 2.5263 \mathrm{E}-02 & 0.0000 \mathrm{E}+00 \\ -87.78 & 8.9146 \mathrm{E}-02 & 4.0461 \mathrm{E}-02 & 0.0000 \mathrm{E}+00 \\ -82.78 & 1.2917 \mathrm{E}-01 & 6.0424 \mathrm{E}-02 & 0.0000 \mathrm{E}+00 \\ -77.78 & 1.7822 \mathrm{E}-01 & 8.7325 \mathrm{E}-02 & 0.0000 \mathrm{E}+00 \\ -72.15 & 2.3384 \mathrm{E}-01 & 1.2218 \mathrm{E}-01 & 0.0000 \mathrm{E}+00 \\ -65.90 & 2.8951 \mathrm{E}-01 & 1.6617 \mathrm{E}-01 & 0.0000 \mathrm{E}+00 \\ -60.35 & 3.4874 \mathrm{E}-01 & 2.2560 \mathrm{E}-01 & 2.3875 \mathrm{E}-02 \\ -55.49 & 4.2772 \mathrm{E}-01 & 3.0667 \mathrm{E}-01 & 3.2031 \mathrm{E}-02 \\ -50.62 & 5.2060 \mathrm{E}-01 & 4.1369 \mathrm{E}-01 & 4.5485 \mathrm{E}-02 \\ -47.17 & 5.9628 \mathrm{E}-01 & 5.1612 \mathrm{E}-01 & 6.1785 \mathrm{E}-02 \\ -43.58 & 6.7629 \mathrm{E}-01 & 6.3292 \mathrm{E}-01 & 7.1450 \mathrm{E}-01 \\ -38.45 & 7.9201 \mathrm{E}-01 & 7.7461 \mathrm{E}-01 & 8.0485 \mathrm{E}-01 \\ -33.32 & 9.0008 \mathrm{E}-01 & 8.9622 \mathrm{E}-01 & 9.0491 \mathrm{E}-01 \\ -28.20 & 9.9561 \mathrm{E}-01 & 9.9876 \mathrm{E}-01 & 9.9138 \mathrm{E}-01 \\ -23.07 & 1.0766 \mathrm{E}+00 & 1.0835 \mathrm{E}+00 & 1.0704 \mathrm{E}+00 \\ -17.94 & 1.1414 \mathrm{E}+00 & 1.1503 \mathrm{E}+00 & 1.1339 \mathrm{E}+00 \\ -12.82 & 1.1891 \mathrm{E}+00 & 1.1987 \mathrm{E}+00 & 1.1773 \mathrm{E}+00 \\ -7.69 & 1.2194 \mathrm{E}+00 & 1.2295 \mathrm{E}+00 & 1.2070 \mathrm{E}+00 \\ -2.56 & 1.2319 \mathrm{E}+00 & 1.2423 \mathrm{E}+00 & 1.2193 \mathrm{E}+00 \\ 2.56 & 1.2264 \mathrm{E}+00 & 1.2370 \mathrm{E}+00 & 1.2148 \mathrm{E}+00 \\ 7.69 & 1.2026 \mathrm{E}+00 & 1.2134 \mathrm{E}+00 & 1.1913 \mathrm{E}+00 \\ 12.82 & 1.1608 \mathrm{E}+00 & 1.1717 \mathrm{E}+00 & 1.1501 \mathrm{E}+00 \\ 17.94 & 1.1015 \mathrm{E}+00 & 1.1121 \mathrm{E}+00 & 1.0966 \mathrm{E}+00 \\ 23.07 & 1.0249 \mathrm{E}+00 & 1.0341 \mathrm{E}+00 & 1.0209 \mathrm{E}+00 \\ 28.20 & 9.3261 \mathrm{E}-01 & 9.3866 \mathrm{E}-01 & 9.2994 \mathrm{E}-01 \\ 33.32 & 8.2702 \mathrm{E}-01 & 8.2732 \mathrm{E}-01 & 8.3248 \mathrm{E}-01 \\ 38.45 & 7.1124 \mathrm{E}-01 & 7.0030 \mathrm{E}-01 & 7.2195 \mathrm{E}-01 \\ 43.58 & 5.9066 \mathrm{E}-01 & 5.5866 \mathrm{E}-01 & 6.1844 \mathrm{E}-01 \\ 47.17 & 5.0845 \mathrm{E}-01 & 4.4762 \mathrm{E}-01 & 5.1239 \mathrm{E}-02 \\ 50.62 & 4.3082 \mathrm{E}-01 & 3.5033 \mathrm{E}-01 & 3.6074 \mathrm{E}-02 \\ 55.49 & 3.3565 \mathrm{E}-01 & 2.4883 \mathrm{E}-01 & 2.3419 \mathrm{E}-02 \\ 60.35 & 2.5367 \mathrm{E}-01 & 1.7175 \mathrm{E}-01 & 1.5577 \mathrm{E}-02 \\ 64.98 & 2.0357 \mathrm{E}-01 & 1.2806 \mathrm{E}-01 & 0.0000 \mathrm{E}+00 \\ 69.39 & 1.7450 \mathrm{E}-01 & 1.0524 \mathrm{E}-01 & 0.0000 \mathrm{E}+00 \\ 76.46 & 1.3306 \mathrm{E}-01 & 7.4740 \mathrm{E}-02 & 0.0000 \mathrm{E}+00 \\ 86.80 & 8.7042 \mathrm{E}-02 & 4.5095 \mathrm{E}-02 & 0.0000 \mathrm{E}+00 \\ 98.14 & 5.0290 \mathrm{E}-02 & 2.4643 \mathrm{E}-02 & 0.0000 \mathrm{E}+00 \\ 110.00 & 2.1534 \mathrm{E}-02 & 1.1037 \mathrm{E}-02 & 0.0000 \mathrm{E}+00\end{array}$


Table ElOA-1.4. Axial Distribution of Total Flux, Fast Flux and Power in MFA-2 at EOC 10A-1

$\begin{array}{rccc}\text { z }(\mathrm{cm}) & \text { Total Flux } & \text { Flux }>0.1 \text { MeV } & \text { Power } \\ -97.78 & 2.5014 \mathrm{E}-02 & 1.2714 \mathrm{E}-02 & 0.0000 \mathrm{E}+00 \\ -92.78 & 5.3755 \mathrm{E}-02 & 2.4520 \mathrm{E}-02 & 0.0000 \mathrm{E}+00 \\ -87.78 & 8.6170 \mathrm{E}-02 & 3.9187 \mathrm{E}-02 & 0.0000 \mathrm{E}+00 \\ -82.78 & 1.2475 \mathrm{E}-01 & 5.8402 \mathrm{E}-02 & 0.0000 \mathrm{E}+00 \\ -77.78 & 1.7203 \mathrm{E}-01 & 8.4284 \mathrm{E}-02 & 0.0000 \mathrm{E}+00 \\ -72.15 & 2.2570 \mathrm{E}-01 & 1.1791 \mathrm{E}-01 & 0.0000 \mathrm{E}+00 \\ -65.90 & 2.8013 \mathrm{E}-01 & 1.6097 \mathrm{E}-01 & 0.0000 \mathrm{E}+00 \\ -60.35 & 3.3871 \mathrm{E}-01 & 2.1998 \mathrm{E}-01 & 2.2643 \mathrm{E}-02 \\ -55.49 & 4.1782 \mathrm{E}-01 & 3.0072 \mathrm{E}-01 & 3.0807 \mathrm{E}-02 \\ -50.62 & 5.1230 \mathrm{E}-01 & 4.0778 \mathrm{E}-01 & 4.4321 \mathrm{E}-02 \\ -47.17 & 5.8983 \mathrm{E}-01 & 5.1029 \mathrm{E}-01 & 6.0658 \mathrm{E}-02 \\ -43.58 & 6.7155 \mathrm{E}-01 & 6.2756 \mathrm{E}-01 & 7.0665 \mathrm{E}-01 \\ -38.45 & 7.8864 \mathrm{E}-01 & 7.7056 \mathrm{E}-01 & 8.0111 \mathrm{E}-01 \\ -33.32 & 8.9759 \mathrm{E}-01 & 8.9354 \mathrm{E}-01 & 9.0244 \mathrm{E}-01 \\ -28.20 & 9.9383 \mathrm{E}-01 & 9.9728 \mathrm{E}-01 & 9.8976 \mathrm{E}-01 \\ -23.07 & 1.0756 \mathrm{E}+00 & 1.0832 \mathrm{E}+00 & 1.0694 \mathrm{E}+00 \\ -17.94 & 1.1414 \mathrm{E}+00 & 1.1511 \mathrm{E}+00 & 1.1340 \mathrm{E}+00 \\ -12.82 & 1.1900 \mathrm{E}+00 & 1.2005 \mathrm{E}+00 & 1.1786 \mathrm{E}+00 \\ -7.69 & 1.2208 \mathrm{E}+00 & 1.2317 \mathrm{E}+00 & 1.2088 \mathrm{E}+00 \\ -2.56 & 1.2332 \mathrm{E}+00 & 1.2444 \mathrm{E}+00 & 1.2210 \mathrm{E}+00 \\ 2.56 & 1.2271 \mathrm{E}+00 & 1.2386 \mathrm{E}+00 & 1.2158 \mathrm{E}+00 \\ 7.69 & 1.2027 \mathrm{E}+00 & 1.2143 \mathrm{E}+00 & 1.1916 \mathrm{E}+00 \\ 12.82 & 1.1604 \mathrm{E}+00 & 1.1718 \mathrm{E}+00 & 1.1500 \mathrm{E}+00 \\ 17.94 & 1.1010 \mathrm{E}+00 & 1.1119 \mathrm{E}+00 & 1.0962 \mathrm{E}+00 \\ 23.07 & 1.0249 \mathrm{E}+00 & 1.0339 \mathrm{E}+00 & 1.0211 \mathrm{E}+00 \\ 28.20 & 9.3348 \mathrm{E}-01 & 9.3904 \mathrm{E}-01 & 9.3126 \mathrm{E}-01 \\ 33.32 & 8.2917 \mathrm{E}-01 & 8.2842 \mathrm{E}-01 & 8.3497 \mathrm{E}-01 \\ 38.45 & 7.1444 \mathrm{E}-01 & 7.0185 \mathrm{E}-01 & 7.2553 \mathrm{E}-01 \\ 43.58 & 5.9443 \mathrm{E}-01 & 5.6016 \mathrm{E}-01 & 6.2175 \mathrm{E}-01 \\ 47.17 & 5.1195 \mathrm{E}-01 & 4.4868 \mathrm{E}-01 & 5.1566 \mathrm{E}-02 \\ 50.62 & 4.3397 \mathrm{E}-01 & 3.5131 \mathrm{E}-01 & 3.6332 \mathrm{E}-02 \\ 55.49 & 3.3891 \mathrm{E}-01 & 2.4986 \mathrm{E}-01 & 2.3653 \mathrm{E}-02 \\ 60.35 & 2.5801 \mathrm{E}-01 & 1.7319 \mathrm{E}-01 & 1.5841 \mathrm{E}-02 \\ 64.98 & 2.0899 \mathrm{E}-01 & 1.2963 \mathrm{E}-01 & 0.0000 \mathrm{E}+00 \\ 69.39 & 1.7972 \mathrm{E}-01 & 1.0657 \mathrm{E}-01 & 0.0000 \mathrm{E}+00 \\ 76.46 & 1.3742 \mathrm{E}-01 & 7.5673 \mathrm{E}-02 & 0.0000 \mathrm{E}+00 \\ 86.80 & 9.0081 \mathrm{E}-02 & 4.5673 \mathrm{E}-02 & 0.0000 \mathrm{E}+00 \\ 98.14 & 5.2051 \mathrm{E}-02 & 2.4941 \mathrm{E}-02 & 0.0000 \mathrm{E}+00 \\ 110.00 & 2.2096 \mathrm{E}-02 & 1.1084 \mathrm{E}-02 & 0.0000 \mathrm{E}+00\end{array}$


HNF-SD-FF-ANAL-009 Rev. 1

Table E10A-1.5. Fission Power Distribution by Pin in MFA-1 at EOC 10A-1 CORE POSITION 1506

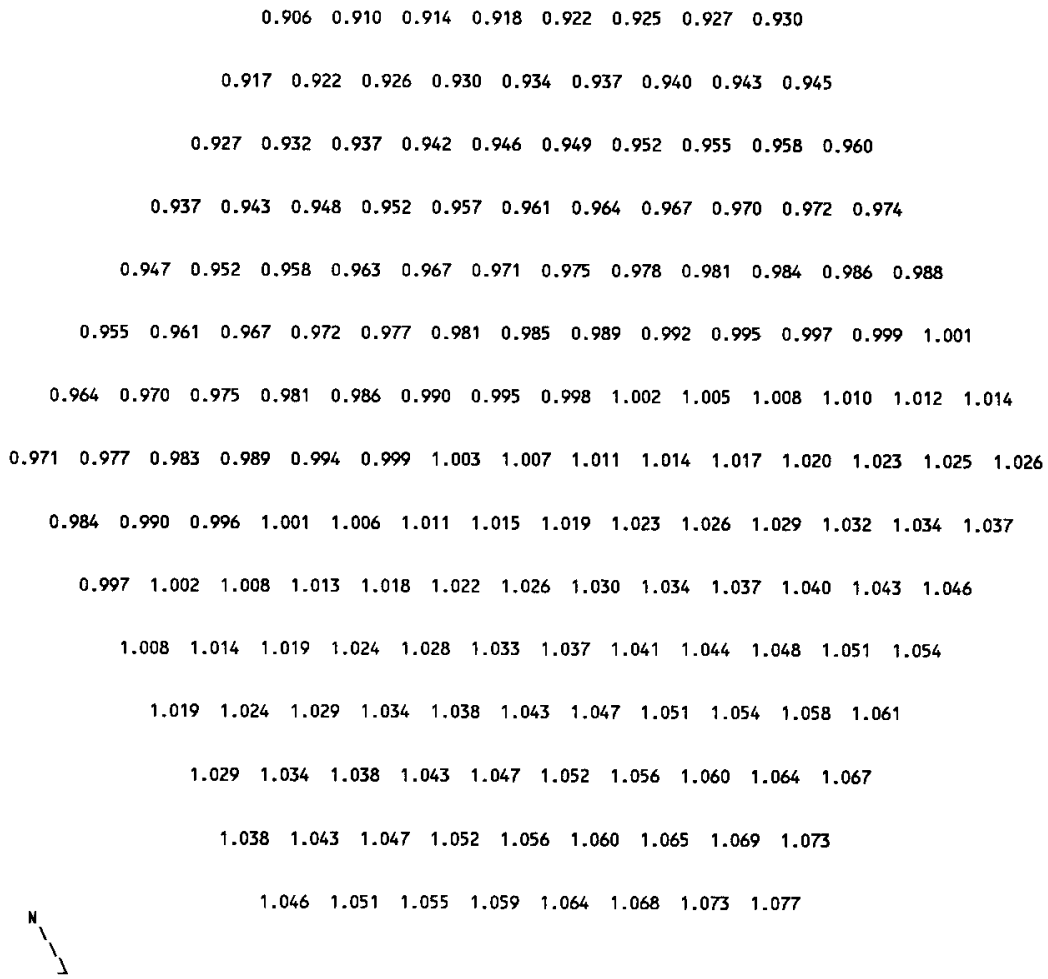


HNF-SD-FF-ANAL-009 Rev, I

Table ElOA-1.6. Fission Power Distribution by Pin in MFA-2 at EOC 10A-1 CORE POSITION 2507

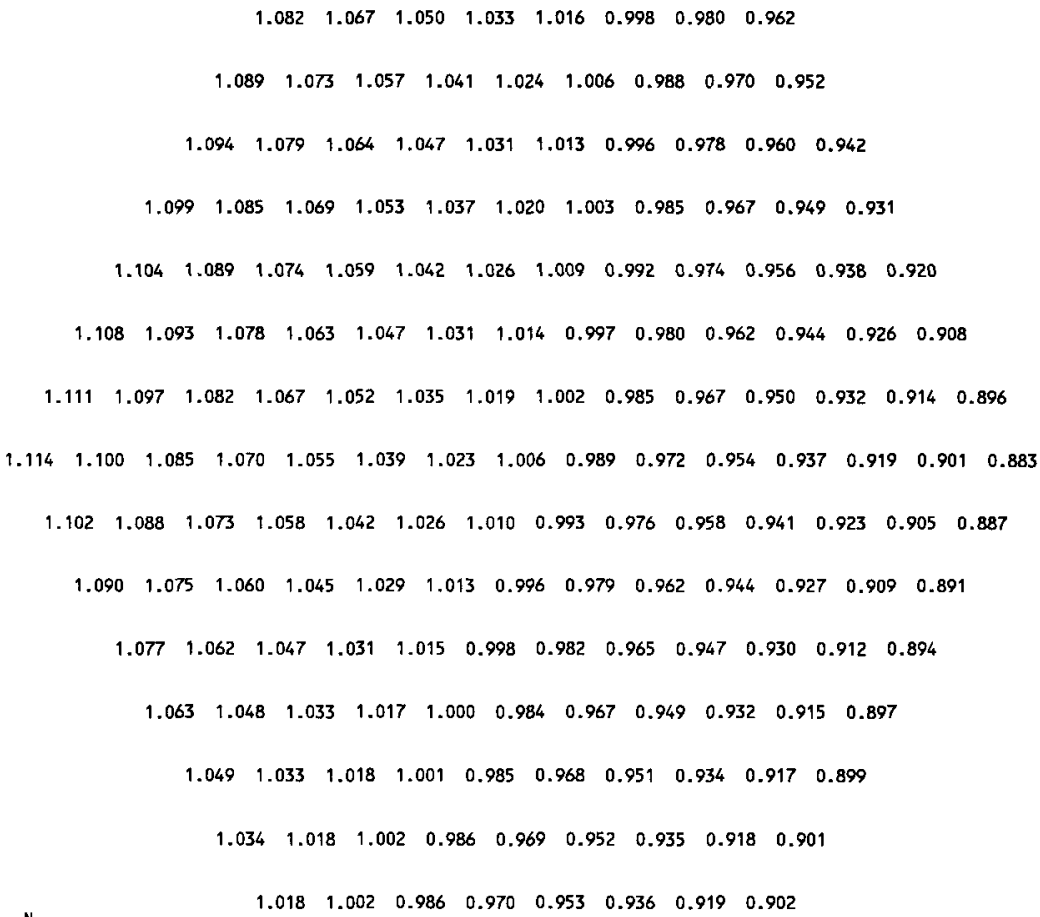


HNF-SD-FF-ANAL-009 Rev, 1

Table E1OA-1.7. Fast Flux Distribution by Pin in MFA-1 at EOC 10A-1

CORE POSITION 1506

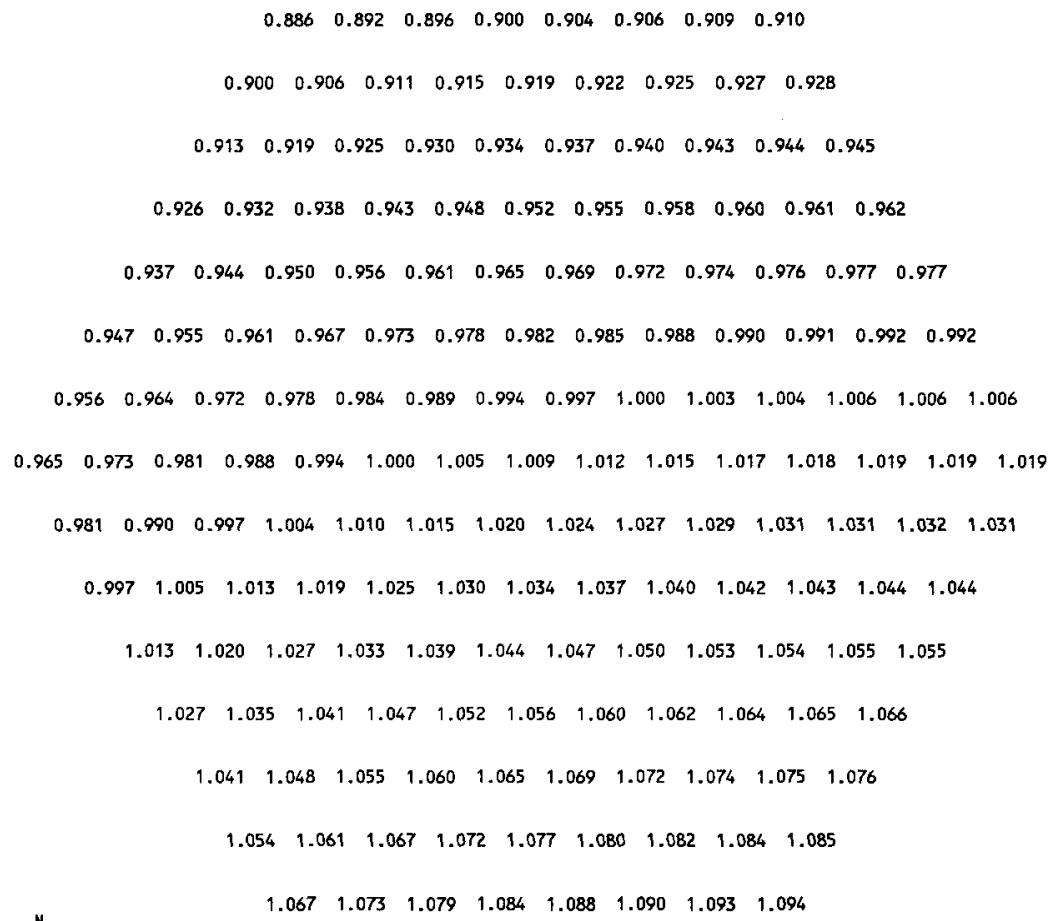




\section{HNF-SD-FF-ANAL-009 Rev. 1}

Table E10A-1.8. Fast Flux Distribution by Pin in MFA-2 at EOC 10A-1 CORE POSITION 2507

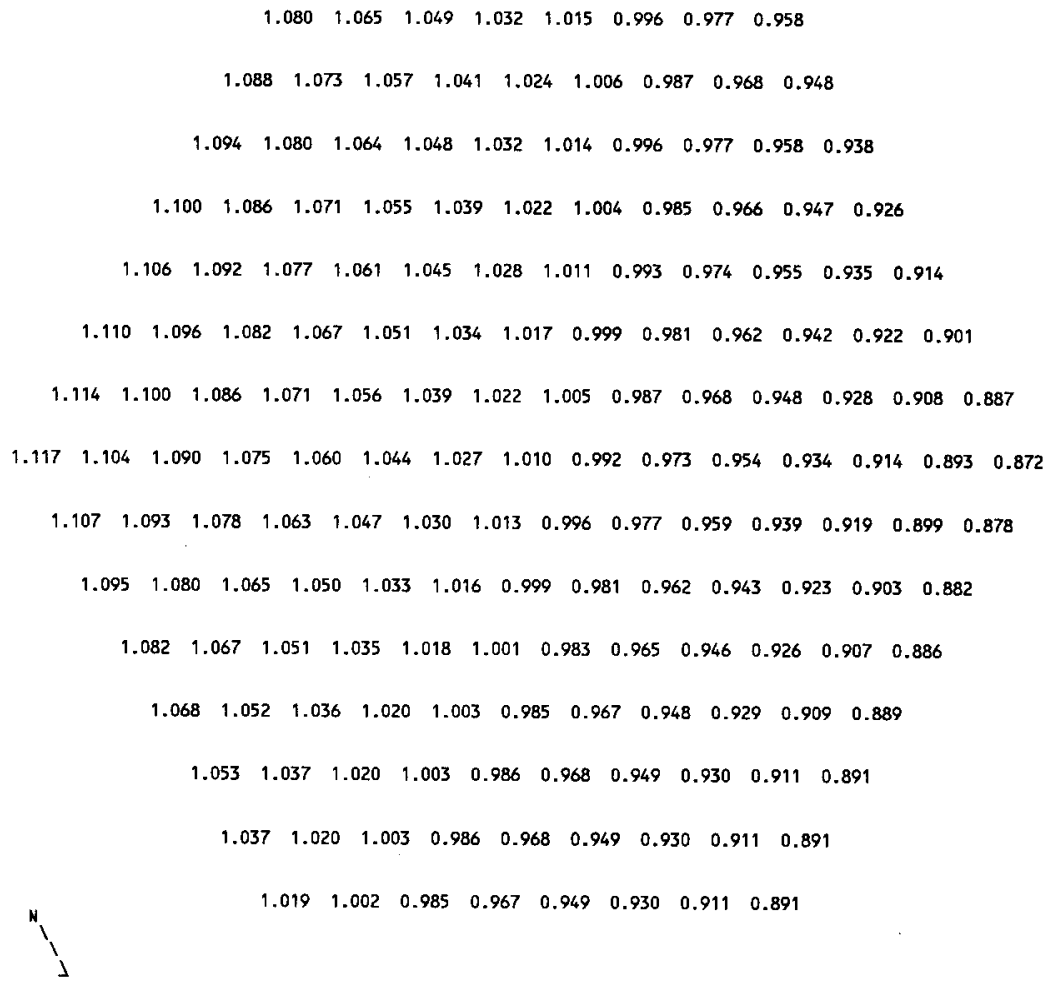


Table E10A-1.9. MFA-1 and MFA-2 Duct Wall

Fast Flux Data at EOC $10 \mathrm{~A}-1$

$\begin{array}{ccc}\text { Side } & \begin{array}{c}\text { Flux }>0.1 \\ \text { MFA-1 }\end{array} & \begin{array}{c}\text { MeV } \\ \left.\text { (n/ } / \mathrm{cm}^{2}-\mathrm{sec}\right) \\ \text { EFA-2 }\end{array} \\ \text { SE } & 1.9763 \mathrm{E}+15 & 1.9693 \mathrm{E}+15 \\ \text { SW } & 1.7725 \mathrm{E}+15 & 2.0264 \mathrm{E}+15 \\ \text { W } & 1.7004 \mathrm{E}+15 & 1.8524 \mathrm{E}+15 \\ \text { NW } & 1.8632 \mathrm{E}+15 & 1.6275 \mathrm{E}+15 \\ \text { NE } & 2.0730 \mathrm{E}+15 & 1.5572 \mathrm{E}+15 \\ & 2.1323 \mathrm{E}+15 & 1.7056 \mathrm{E}+15\end{array}$

Table E10A-1.10. Assembly Outlet Temperatures and Flow Rates at EOC 10A-1

$\begin{array}{lcl} & \text { CALCULATED } \\ \text { CORE } & \text { OUTLET TEMP. } & \text { FLOW RATE } \\ \text { POS. } & \text { (DEG F) } & \text { (LB/H) } \\ 1506 & 1077 & 116330 \\ 1404 & 922 & 228160 \\ 1405 & 894 & 210640 \\ 1505 & 884 & 210640 \\ 1507 & 868 & 210640 \\ 1606 & 918 & 172740 \\ 1607 & 880 & 172740 \\ 2507 & 1038 & 116330 \\ 2405 & 907 & 194730 \\ 2506 & 872 & 200300 \\ 2508 & 895 & 210640 \\ 2607 & 890 & 165660 \\ 2608 & 1038 & 89760 \\ 2609 & 875 & 175430\end{array}$

Assembly flows are based on a calculated core pressure drop of 113.3 psi at a total reactor flow rate of 16. $74 \mathrm{E}+06 \mathrm{lb} / \mathrm{hr}$. 
Table E10A-1.17. Composition of MFA-1 at EOC 10A-1

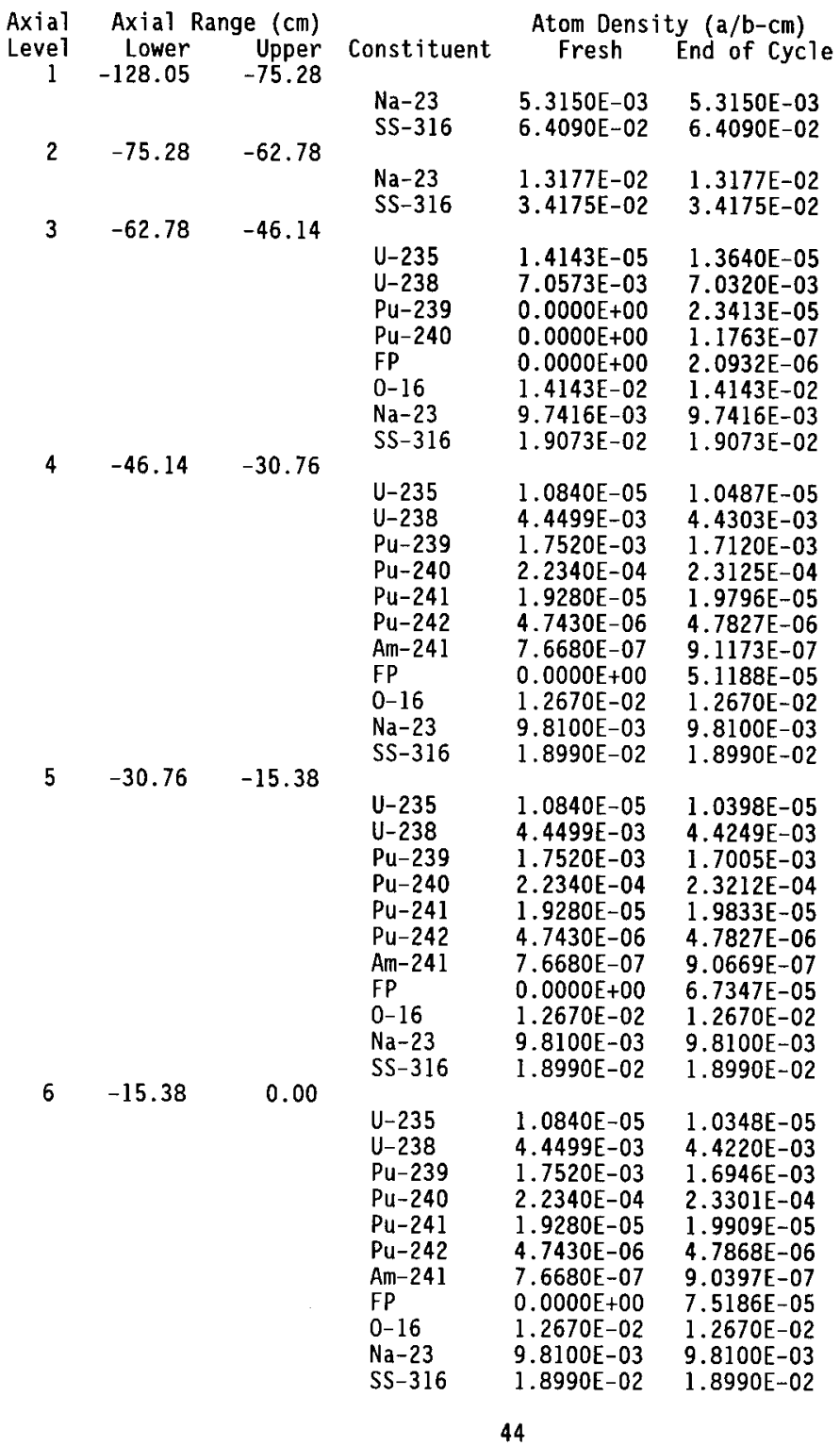


HNF-SD-FF-ANAL-009 Rev. 1

\begin{tabular}{|c|c|c|c|c|c|}
\hline \multirow[t]{2}{*}{7} & 0.00 & 15.38 & & & \\
\hline & & & $\begin{array}{l}\mathrm{U}-235 \\
\mathrm{U}-238 \\
\mathrm{Pu}-239 \\
\mathrm{Pu}-240 \\
\mathrm{Pu}-241 \\
\mathrm{Pu}-242 \\
\mathrm{Am}-241 \\
\mathrm{FP} \\
0-16 \\
\mathrm{Na}-23 \\
\mathrm{SS}-316\end{array}$ & $\begin{array}{l}1.0840 \mathrm{E}-05 \\
4.4499 \mathrm{E}-03 \\
1.7520 \mathrm{E}-03 \\
2.2340 \mathrm{E}-04 \\
1.9280 \mathrm{E}-05 \\
4.7430 \mathrm{E}-06 \\
7.6680 \mathrm{E}-07 \\
0.0000 \mathrm{E}+00 \\
1.2670 \mathrm{E}-02 \\
9.8100 \mathrm{E}-03 \\
1.8990 \mathrm{E}-02\end{array}$ & $\begin{array}{l}1.0364 \mathrm{E}-05 \\
4.4230 \mathrm{E}-03 \\
1.6964 \mathrm{E}-03 \\
2.3268 \mathrm{E}-04 \\
1.9880 \mathrm{E}-05 \\
4.7852 \mathrm{E}-06 \\
9.0486 \mathrm{E}-07 \\
7.2720 \mathrm{E}-05 \\
1.2670 \mathrm{E}-02 \\
9.8100 \mathrm{E}-03 \\
1.8990 \mathrm{E}-02\end{array}$ \\
\hline \multirow[t]{2}{*}{8} & 15.38 & 30.76 & & & \\
\hline & & & $\begin{array}{l}\mathrm{U}-235 \\
\mathrm{U}-238 \\
\mathrm{Pu}-239 \\
\mathrm{Pu}-240 \\
\mathrm{Pu}-241 \\
\mathrm{Pu}-242 \\
\mathrm{Am}-241 \\
\mathrm{FP} \\
0-16 \\
\mathrm{Na}-23 \\
\mathrm{SS}-316\end{array}$ & $\begin{array}{l}1.0840 \mathrm{E}-05 \\
4.4499 \mathrm{E}-03 \\
1.7520 \mathrm{E}-03 \\
2.2340 \mathrm{E}-04 \\
1.9280 \mathrm{E}-05 \\
4.7430 \mathrm{E}-06 \\
7.6680 \mathrm{E}-07 \\
0.0000 \mathrm{E}+00 \\
1.2670 \mathrm{E}-02 \\
9.8100 \mathrm{E}-03 \\
1.8990 \mathrm{E}-02\end{array}$ & $\begin{array}{l}1.0442 \mathrm{E}-05 \\
4.4274 \mathrm{E}-03 \\
1.7056 \mathrm{E}-03 \\
2.3120 \mathrm{E}-04 \\
1.9753 \mathrm{E}-05 \\
4.7780 \mathrm{E}-06 \\
9.0909 \mathrm{E}-07 \\
6.0716 \mathrm{E}-05 \\
1.2670 \mathrm{E}-02 \\
9.8100 \mathrm{E}-03 \\
1.8990 \mathrm{E}-02\end{array}$ \\
\hline \multirow[t]{2}{*}{9} & 30.76 & 46.14 & & & \\
\hline & & & $\begin{array}{l}\mathrm{U}-235 \\
\mathrm{U}-238 \\
\mathrm{Pu}-239 \\
\mathrm{Pu}-240 \\
\mathrm{Pu}-241 \\
\mathrm{Pu}-242 \\
\mathrm{Am}-241 \\
\mathrm{FP} \\
0-16 \\
\mathrm{Na}-23 \\
\mathrm{SS}-316\end{array}$ & $\begin{array}{l}1.0840 \mathrm{E}-05 \\
4.4499 \mathrm{E}-03 \\
1.7520 \mathrm{E}-03 \\
2.2340 \mathrm{E}-04 \\
1.9280 \mathrm{E}-05 \\
4.7430 \mathrm{E}-06 \\
7.6680 \mathrm{E}-07 \\
0.0000 \mathrm{E}+00 \\
1.2670 \mathrm{E}-02 \\
9.8100 \mathrm{E}-03 \\
1.8990 \mathrm{E}-02\end{array}$ & $\begin{array}{l}1.0549 \mathrm{E}-05 \\
4.4338 \mathrm{E}-03 \\
1.7189 \mathrm{E}-03 \\
2.2970 \mathrm{E}-04 \\
1.9650 \mathrm{E}-05 \\
4.7738 \mathrm{E}-06 \\
9.1509 \mathrm{E}-07 \\
4.2496 \mathrm{E}-05 \\
1.2670 \mathrm{E}-02 \\
9.8100 \mathrm{E}-03 \\
1.8990 \mathrm{E}-02\end{array}$ \\
\hline \multirow[t]{2}{*}{10} & 46.14 & 62.78 & & & \\
\hline & & & $\begin{array}{l}U-235 \\
U-238 \\
P u-239 \\
P u-240 \\
F P \\
0-16 \\
\mathrm{Na}-23 \\
S S-316\end{array}$ & $\begin{array}{l}1.4143 E-05 \\
7.0573 E-03 \\
0.0000 E+00 \\
0.0000 E+00 \\
0.0000 E+00 \\
1.4143 E-02 \\
9.7416 E-03 \\
1.9073 E-02\end{array}$ & $\begin{array}{l}1.3807 \mathrm{E}-05 \\
7.0399 \mathrm{E}-03 \\
1.6111 \mathrm{E}-05 \\
5.1836 \mathrm{E}-08 \\
1.5227 \mathrm{E}-06 \\
1.4143 \mathrm{E}-02 \\
9.7416 \mathrm{E}-03 \\
1.9073 \mathrm{E}-02\end{array}$ \\
\hline 11 & 62.78 & 172. & $\begin{array}{l}\mathrm{Na}-23 \\
\mathrm{SS}-316\end{array}$ & $\begin{array}{l}8.2400 \mathrm{E}-03 \\
2.5562 \mathrm{E}-02\end{array}$ & $\begin{array}{l}8.2400 E-03 \\
2.5562 E-02\end{array}$ \\
\hline
\end{tabular}


Table E10A-1.18. Composition of MFA-2 at EOC 10A-1

\begin{tabular}{|c|c|c|c|c|c|}
\hline ial & Axial & ge $(\mathrm{cm})$ & & Atom Densi & $a / b-c m)$ \\
\hline $\begin{array}{c}\text { Leve } 1 \\
\quad 1\end{array}$ & $\begin{array}{r}\text { Lower } \\
-128.05\end{array}$ & $\begin{array}{r}\text { Upper } \\
-75.28\end{array}$ & Constituent & Fresh & End of Cycle \\
\hline & & & $\begin{array}{l}\mathrm{Na}-23 \\
\mathrm{SS}-316\end{array}$ & $\begin{array}{l}5.3150 \mathrm{E}-03 \\
6.4090 \mathrm{E}-02\end{array}$ & $\begin{array}{l}5.3150 \mathrm{E}-03 \\
6.4090 \mathrm{E}-02\end{array}$ \\
\hline 2 & -75.28 & -62.78 & & & \\
\hline & & & $\begin{array}{l}\mathrm{Na}-23 \\
\mathrm{SS}-316\end{array}$ & $\begin{array}{l}1.3177 \mathrm{E}-02 \\
3.4175 \mathrm{E}-02\end{array}$ & $\begin{array}{l}1.3177 \mathrm{E}-02 \\
3.4175 \mathrm{E}-02\end{array}$ \\
\hline 3 & -62.78 & -46.14 & $\begin{array}{l}\text { U-235 } \\
U-238 \\
\mathrm{Pu}-239 \\
\mathrm{Pu}-240 \\
\mathrm{FP} \\
\mathrm{O}-16 \\
\mathrm{Na}-23 \\
\mathrm{SS}-316\end{array}$ & $\begin{array}{l}1.4143 \mathrm{E}-05 \\
7.0573 \mathrm{E}-03 \\
0.0000 \mathrm{E}+00 \\
0.0000 \mathrm{E}+00 \\
0.0000 \mathrm{E}+00 \\
1.4143 \mathrm{E}-02 \\
9.7416 \mathrm{E}-03 \\
1.9073 \mathrm{E}-02\end{array}$ & $\begin{array}{l}1.3696 \mathrm{E}-05 \\
7.0344 \mathrm{E}-03 \\
2.1249 \mathrm{E}-05 \\
9.3514 \mathrm{E}-08 \\
1.9325 \mathrm{E}-06 \\
1.4143 \mathrm{E}-02 \\
9.7416 \mathrm{E}-03 \\
1.9073 \mathrm{E}-02\end{array}$ \\
\hline 4 & -46.14 & -30.76 & $\begin{array}{l}U-235 \\
U-238 \\
P u-239 \\
P u-240 \\
P u-241 \\
P u-242 \\
\text { Am-241 } \\
F P \\
0-16 \\
\mathrm{Na}-23 \\
\text { SS }-316\end{array}$ & $\begin{array}{l}1.0160 \mathrm{E}-05 \\
4.4270 \mathrm{E}-03 \\
1.7483 \mathrm{E}-03 \\
2.2290 \mathrm{E}-04 \\
1.9040 \mathrm{E}-05 \\
4.7330 \mathrm{E}-06 \\
7.5700 \mathrm{E}-07 \\
0.0000 \mathrm{E}+00 \\
1.2760 \mathrm{E}-02 \\
9.8100 \mathrm{E}-03 \\
1.8990 \mathrm{E}-02\end{array}$ & $\begin{array}{l}9.8470 \mathrm{E}-06 \\
4.4086 \mathrm{E}-03 \\
1.7108 \mathrm{E}-03 \\
2.3041 \mathrm{E}-04 \\
1.9532 \mathrm{E}-05 \\
4.7699 \mathrm{E}-06 \\
9.0125 \mathrm{E}-07 \\
4.7929 \mathrm{E}-05 \\
1.2760 \mathrm{E}-02 \\
9.8100 \mathrm{E}-03 \\
1.8990 \mathrm{E}-02\end{array}$ \\
\hline 5 & -30.76 & -15.38 & $\begin{array}{l}U-235 \\
U-238 \\
P u-239 \\
P u-240 \\
P u-241 \\
P u-242 \\
\text { Am-241 } \\
\text { FP } \\
0-16 \\
\mathrm{Na}-23 \\
S S-316\end{array}$ & $\begin{array}{l}1.0160 \mathrm{E}-05 \\
4.4270 \mathrm{E}-03 \\
1.7483 \mathrm{E}-03 \\
2.2290 \mathrm{E}-04 \\
1.9040 \mathrm{E}-05 \\
4.7330 \mathrm{E}-06 \\
7.5700 \mathrm{E}-07 \\
0.0000 \mathrm{E}+00 \\
1.2760 \mathrm{E}-02 \\
9.8100 \mathrm{E}-03 \\
1.8990 \mathrm{E}-02\end{array}$ & $\begin{array}{l}9.7661 \mathrm{E}-06 \\
4.4033 \mathrm{E}-03 \\
1.6997 \mathrm{E}-03 \\
2.3132 \mathrm{E}-04 \\
1.9581 \mathrm{E}-05 \\
4.7704 \mathrm{E}-06 \\
8.9642 \mathrm{E}-07 \\
6.3395 \mathrm{E}-05 \\
1.2760 \mathrm{E}-02 \\
9.8100 \mathrm{E}-03 \\
1.8990 \mathrm{E}-02\end{array}$ \\
\hline 6 & -15.38 & 0.00 & $\begin{array}{l}\text { U-235 } \\
\mathrm{U}-238 \\
\mathrm{Pu}-239 \\
\mathrm{Pu}-240 \\
\mathrm{Pu}-241 \\
\mathrm{Pu}-242 \\
\mathrm{Am}-241 \\
\mathrm{FP} \\
0-16 \\
\mathrm{Na}-23 \\
\mathrm{SS}-316\end{array}$ & $\begin{array}{l}1.0160 \mathrm{E}-05 \\
4.4270 \mathrm{E}-03 \\
1.7483 \mathrm{E}-03 \\
2.2290 \mathrm{E}-04 \\
1.9040 \mathrm{E}-05 \\
4.7330 \mathrm{E}-06 \\
7.5700 \mathrm{E}-07 \\
0.0000 \mathrm{E}+00 \\
1.2760 \mathrm{E}-02 \\
9.8100 \mathrm{E}-03 \\
1.8990 \mathrm{E}-02\end{array}$ & $\begin{array}{l}9.7206 \mathrm{E}-06 \\
4.4005 \mathrm{E}-03 \\
1.6940 \mathrm{E}-03 \\
2.3220 \mathrm{E}-04 \\
1.9659 \mathrm{E}-05 \\
4.7745 \mathrm{E}-06 \\
8.9380 \mathrm{E}-07 \\
7.0989 \mathrm{E}-05 \\
1.2760 \mathrm{E}-02 \\
9.8100 \mathrm{E}-03 \\
1.8990 \mathrm{E}-02\end{array}$ \\
\hline
\end{tabular}




\begin{tabular}{|c|c|c|c|c|c|}
\hline \multirow{2}{*}{7} & 0.00 & 15.38 & & & \\
\hline & & & $\begin{array}{l}U-235 \\
U-238 \\
P u-239 \\
P u-240 \\
P u-241 \\
P u-242 \\
A m-241 \\
F P \\
0-16 \\
N a-23 \\
S S-316\end{array}$ & $\begin{array}{l}1.0160 \mathrm{E}-05 \\
4.4270 \mathrm{E}-03 \\
1.7483 \mathrm{E}-03 \\
2.2290 \mathrm{E}-04 \\
1.9040 \mathrm{E}-05 \\
4.7330 \mathrm{E}-06 \\
7.5700 \mathrm{E}-07 \\
0.0000 \mathrm{E}+00 \\
1.2760 \mathrm{E}-02 \\
9.8100 \mathrm{E}-03 \\
1.8990 \mathrm{E}-02\end{array}$ & $\begin{array}{l}9.7343 \mathrm{E}-06 \\
4.4014 \mathrm{E}-03 \\
1.6956 \mathrm{E}-03 \\
2.3190 \mathrm{E}-04 \\
1.9632 \mathrm{E}-05 \\
4.7730 \mathrm{E}-06 \\
8.9458 \mathrm{E}-07 \\
6.8798 \mathrm{E}-05 \\
1.2760 \mathrm{E}-02 \\
9.8100 \mathrm{E}-03 \\
1.8990 \mathrm{E}-02\end{array}$ \\
\hline \multirow[t]{2}{*}{8} & 15.38 & 30.76 & & & \\
\hline & & & $\begin{array}{l}U-235 \\
U-238 \\
\mathrm{Pu}-239 \\
\mathrm{Pu}-240 \\
\mathrm{Pu}-241 \\
\mathrm{Pu}-242 \\
\mathrm{Am}-241 \\
\mathrm{FP} \\
\mathrm{O}-16 \\
\mathrm{Na}-23 \\
\mathrm{SS}-316\end{array}$ & $\begin{array}{l}1.0160 \mathrm{E}-05 \\
4.4270 \mathrm{E}-03 \\
1.7483 \mathrm{E}-03 \\
2.2290 \mathrm{E}-04 \\
1.9040 \mathrm{E}-05 \\
4.7330 \mathrm{E}-06 \\
7.5700 \mathrm{E}-07 \\
0.0000 \mathrm{E}+00 \\
1.2760 \mathrm{E}-02 \\
9.8100 \mathrm{E}-03 \\
1.8990 \mathrm{E}-02\end{array}$ & $\begin{array}{l}9.8018 \mathrm{E}-06 \\
4.4055 \mathrm{E}-03 \\
1.7041 \mathrm{E}-03 \\
2.3053 \mathrm{E}-04 \\
1.9513 \mathrm{E}-05 \\
4.7666 \mathrm{E}-06 \\
8.9845 \mathrm{E}-07 \\
5.7703 \mathrm{E}-05 \\
1.2760 \mathrm{E}-02 \\
9.8100 \mathrm{E}-03 \\
1.8990 \mathrm{E}-02\end{array}$ \\
\hline \multirow[t]{2}{*}{9} & 30.76 & 46.14 & & & \\
\hline & & & $\begin{array}{l}U-235 \\
U-238 \\
P u-239 \\
P u-240 \\
P u-241 \\
P u-242 \\
A m-241 \\
F P \\
0-16 \\
\mathrm{Na}-23 \\
S S-316\end{array}$ & $\begin{array}{l}1.0160 \mathrm{E}-05 \\
4.4270 \mathrm{E}-03 \\
1.7483 \mathrm{E}-03 \\
2.2290 \mathrm{E}-04 \\
1.9040 \mathrm{E}-05 \\
4.7330 \mathrm{E}-06 \\
7.5700 \mathrm{E}-07 \\
0.0000 \mathrm{E}+00 \\
1.2760 \mathrm{E}-02 \\
9.8100 \mathrm{E}-03 \\
1.8990 \mathrm{E}-02\end{array}$ & $\begin{array}{l}9.8972 \mathrm{E}-06 \\
4.4115 \mathrm{E}-03 \\
1.7167 \mathrm{E}-03 \\
2.2909 \mathrm{E}-04 \\
1.9409 \mathrm{E}-05 \\
4.7626 \mathrm{E}-06 \\
9.0406 \mathrm{E}-07 \\
4.0541 \mathrm{E}-05 \\
1.2760 \mathrm{E}-02 \\
9.8100 \mathrm{E}-03 \\
1.8990 \mathrm{E}-02\end{array}$ \\
\hline \multirow[t]{2}{*}{10} & 46.14 & 62.78 & & & \\
\hline & & & $\begin{array}{l}U-235 \\
U-238 \\
\mathrm{Pu}-239 \\
\mathrm{Pu}-240 \\
\mathrm{FP} \\
0-16 \\
\mathrm{Na}-23 \\
\mathrm{SS}-316\end{array}$ & $\begin{array}{l}1.4143 \mathrm{E}-05 \\
7.0573 \mathrm{E}-03 \\
0.0000 \mathrm{E}+00 \\
0.0000 \mathrm{E}+00 \\
0.0000 \mathrm{E}+00 \\
1.4143 \mathrm{E}-02 \\
9.7416 \mathrm{E}-03 \\
1.9073 \mathrm{E}-02\end{array}$ & $\begin{array}{l}1.3827 \mathrm{E}-05 \\
7.0406 \mathrm{E}-03 \\
1.5406 \mathrm{E}-05 \\
4.6476 \mathrm{E}-08 \\
1.4617 \mathrm{E}-06 \\
1.4143 \mathrm{E}-02 \\
9.7416 \mathrm{E}-03 \\
1.9073 \mathrm{E}-02\end{array}$ \\
\hline \multirow[t]{2}{*}{11} & 62.78 & 172.84 & & & \\
\hline & & & $\begin{array}{l}\mathrm{Na}-23 \\
\mathrm{SS}-316\end{array}$ & $\begin{array}{l}8.2400 \mathrm{E}-03 \\
2.5562 \mathrm{E}-02\end{array}$ & $\begin{array}{l}8.2400 E-03 \\
2.5562 E-02\end{array}$ \\
\hline
\end{tabular}




\subsection{Cycle 10A-2}

Table B1OA-2.1. Fission Power Generated in MFA-1, MFA-2 and Neighboring Assemblies at BOC 10A-2

\begin{tabular}{lrrrrr} 
CORE & $* * * * * *$ & \multicolumn{2}{c}{ POWER IN } & MEGAWATTS & $* * * * * *$ \\
POS. & BELOW CORE & IN CORE & ABOVE CORE & TOTAL PWR \\
1506 & $2.734 \mathrm{E}-02$ & $3.888 \mathrm{E}+00$ & $1.919 \mathrm{E}-02$ & $3.935 \mathrm{E}+00$ \\
1404 & $8.085 \mathrm{E}-02$ & $4.652 \mathrm{E}+00$ & $4.446 \mathrm{E}-02$ & $4.778 \mathrm{E}+00$ \\
1405 & $9.264 \mathrm{E}-03$ & $3.890 \mathrm{E}+00$ & $6.952 \mathrm{E}-03$ & $3.906 \mathrm{E}+00$ \\
1505 & $1.106 \mathrm{E}-02$ & $3.550 \mathrm{E}+00$ & $6.539 \mathrm{E}-03$ & $3.567 \mathrm{E}+00$ \\
1507 & $9.617 \mathrm{E}-03$ & $3.291 \mathrm{E}+00$ & $7.200 \mathrm{E}-03$ & $3.308 \mathrm{E}+00$ \\
1606 & $6.006 \mathrm{E}-03$ & $3.453 \mathrm{E}+00$ & $4.422 \mathrm{E}-03$ & $3.464 \mathrm{E}+00$ \\
1607 & $8.993 \mathrm{E}-03$ & $2.851 \mathrm{E}+00$ & $6.503 \mathrm{E}-03$ & $2.867 \mathrm{E}+00$ \\
2507 & $2.827 \mathrm{E}-02$ & $4.180 \mathrm{E}+00$ & $2.107 \mathrm{E}-02$ & $4.229 \mathrm{E}+00$ \\
2405 & $7.001 \mathrm{E}-02$ & $4.460 \mathrm{E}+00$ & $4.071 \mathrm{E}-02$ & $4.571 \mathrm{E}+00$ \\
2506 & $1.122 \mathrm{E}-02$ & $3.876 \mathrm{E}+00$ & $7.938 \mathrm{E}-03$ & $3.895 \mathrm{E}+00$ \\
2508 & $1.083 \mathrm{E}-02$ & $4.596 \mathrm{E}+00$ & $7.977 \mathrm{E}-03$ & $4.615 \mathrm{E}+00$ \\
2607 & $7.671 \mathrm{E}-03$ & $3.560 \mathrm{E}+00$ & $5.748 \mathrm{E}-03$ & $3.574 \mathrm{E}+00$ \\
2608 & $4.839 \mathrm{E}-03$ & $3.421 \mathrm{E}+00$ & $4.041 \mathrm{E}-03$ & $3.430 \mathrm{E}+00$ \\
2609 & $9.386 \mathrm{E}-02$ & $3.167 \mathrm{E}+00$ & $5.186 \mathrm{E}-02$ & $3.313 \mathrm{E}+00$
\end{tabular}

Table B1OA-2.2. Assembly Averaged Total and Fast Flux in MFA-1 and MFA-2 at BOC 1OA-2

\begin{tabular}{|c|c|c|}
\hline & Core & Flux \\
\hline $\begin{array}{l}\text { sssembly } \\
\text { MFA-1 } \\
\text { MFA-2 }\end{array}$ & $\begin{array}{l}\text { Pos. } \\
1506 \\
2507\end{array}$ & $\begin{array}{c}\text { Total } \\
2.855 \mathrm{E}+15 \\
3.063 \mathrm{E}+15\end{array}$ \\
\hline
\end{tabular}


Table BlOA-2.3. Axial Distribution of Total Flux, Fast Flux and Power in MFA-1 at BOC 10A-2

$\begin{array}{rccc}\text { z }(\mathrm{cm}) & \text { Total Flux } & \text { Flux }>0.1 \text { MeV } & \text { Power } \\ -97.78 & 2.7490 \mathrm{E}-02 & 1.3904 \mathrm{E}-02 & 0.0000 \mathrm{E}+00 \\ -92.78 & 5.9094 \mathrm{E}-02 & 2.6852 \mathrm{E}-02 & 0.0000 \mathrm{E}+00 \\ -87.78 & 9.4762 \mathrm{E}-02 & 4.2972 \mathrm{E}-02 & 0.0000 \mathrm{E}+00 \\ -82.78 & 1.3719 \mathrm{E}-01 & 6.4106 \mathrm{E}-02 & 0.0000 \mathrm{E}+00 \\ -77.78 & 1.8909 \mathrm{E}-01 & 9.2523 \mathrm{E}-02 & 0.0000 \mathrm{E}+00 \\ -72.15 & 2.4780 \mathrm{E}-01 & 1.2927 \mathrm{E}-01 & 0.0000 \mathrm{E}+00 \\ -65.90 & 3.0612 \mathrm{E}-01 & 1.7541 \mathrm{E}-01 & 0.0000 \mathrm{E}+00 \\ -60.35 & 3.6758 \mathrm{E}-01 & 2.3738 \mathrm{E}-01 & 2.5138 \mathrm{E}-02 \\ -55.49 & 4.4930 \mathrm{E}-01 & 3.2169 \mathrm{E}-01 & 3.3599 \mathrm{E}-02 \\ -50.62 & 5.4496 \mathrm{E}-01 & 4.3254 \mathrm{E}-01 & 4.7534 \mathrm{E}-02 \\ -47.17 & 6.2244 \mathrm{E}-01 & 5.3821 \mathrm{E}-01 & 6.4395 \mathrm{E}-02 \\ -43.58 & 7.0413 \mathrm{E}-01 & 6.5858 \mathrm{E}-01 & 7.4425 \mathrm{E}-01 \\ -38.45 & 8.2126 \mathrm{E}-01 & 8.0299 \mathrm{E}-01 & 8.3479 \mathrm{E}-01 \\ -33.32 & 9.2956 \mathrm{E}-01 & 9.2555 \mathrm{E}-01 & 9.3464 \mathrm{E}-01 \\ -28.20 & 1.0240 \mathrm{E}+00 & 1.0273 \mathrm{E}+00 & 1.0197 \mathrm{E}+00 \\ -23.07 & 1.1025 \mathrm{E}+00 & 1.1098 \mathrm{E}+00 & 1.0962 \mathrm{E}+00 \\ -17.94 & 1.1636 \mathrm{E}+00 & 1.1729 \mathrm{E}+00 & 1.1560 \mathrm{E}+00 \\ -12.82 & 1.2063 \mathrm{E}+00 & 1.2163 \mathrm{E}+00 & 1.1944 \mathrm{E}+00 \\ -7.69 & 1.2306 \mathrm{E}+00 & 1.2409 \mathrm{E}+00 & 1.2181 \mathrm{E}+00 \\ -2.56 & 1.2360 \mathrm{E}+00 & 1.2466 \mathrm{E}+00 & 1.2233 \mathrm{E}+00 \\ 2.56 & 1.2227 \mathrm{E}+00 & 1.2335 \mathrm{E}+00 & 1.2111 \mathrm{E}+00 \\ 7.69 & 1.1909 \mathrm{E}+00 & 1.2018 \mathrm{E}+00 & 1.1796 \mathrm{E}+00 \\ 12.82 & 1.1415 \mathrm{E}+00 & 1.1523 \mathrm{E}+00 & 1.1307 \mathrm{E}+00 \\ 17.94 & 1.0758 \mathrm{E}+00 & 1.0864 \mathrm{E}+00 & 1.0707 \mathrm{E}+00 \\ 23.07 & 9.9519 \mathrm{E}-01 & 1.0044 \mathrm{E}+00 & 9.9089 \mathrm{E}-01 \\ 28.20 & 9.0174 \mathrm{E}-01 & 9.0781 \mathrm{E}-01 & 8.9891 \mathrm{E}-01 \\ 33.32 & 7.9785 \mathrm{E}-01 & 7.9824 \mathrm{E}-01 & 8.0306 \mathrm{E}-01 \\ 38.45 & 6.8590 \mathrm{E}-01 & 6.7530 \mathrm{E}-01 & 6.9632 \mathrm{E}-01 \\ 43.58 & 5.7029 \mathrm{E}-01 & 5.3923 \mathrm{E}-01 & 5.9727 \mathrm{E}-01 \\ 47.17 & 4.9156 \mathrm{E}-01 & 4.3247 \mathrm{E}-01 & 4.9498 \mathrm{E}-02 \\ 50.62 & 4.1725 \mathrm{E}-01 & 3.3897 \mathrm{E}-01 & 3.4897 \mathrm{E}-02 \\ 55.49 & 3.2587 \mathrm{E}-01 & 2.4130 \mathrm{E}-01 & 2.2711 \mathrm{E}-02 \\ 60.35 & 2.4701 \mathrm{E}-01 & 1.6701 \mathrm{E}-01 & 1.5153 \mathrm{E}-02 \\ 64.98 & 1.9879 \mathrm{E}-01 & 1.2488 \mathrm{E}-01 & 0.0000 \mathrm{E}+00 \\ 69.39 & 1.7069 \mathrm{E}-01 & 1.0281 \mathrm{E}-01 & 0.0000 \mathrm{E}+00 \\ 76.46 & 1.3050 \mathrm{E}-01 & 7.3220 \mathrm{E}-02 & 0.0000 \mathrm{E}+00 \\ 86.80 & 8.5638 \mathrm{E}-02 & 4.4335 \mathrm{E}-02 & 0.0000 \mathrm{E}+00 \\ 98.14 & 4.9635 \mathrm{E}-02 & 2.4314 \mathrm{E}-02 & 0.0000 \mathrm{E}+00 \\ 110.00 & 2.1333 \mathrm{E}-02 & 1.0931 \mathrm{E}-02 & 0.0000 \mathrm{E}+00\end{array}$


Table B10A-2.4. Axial Distribution of Total Flux, Fast Flux and Power in MFA-2 at BOC 10A-2

$\begin{array}{rccc}\text { Z }(\mathrm{cm}) & \text { Total Flux } & \text { Flux }>0.1 \text { MeV } & \text { Power } \\ -97.78 & 2.5287 \mathrm{E}-02 & 1.2895 \mathrm{E}-02 & 0.0000 \mathrm{E}+00 \\ -92.78 & 5.4304 \mathrm{E}-02 & 2.4889 \mathrm{E}-02 & 0.0000 \mathrm{E}+00 \\ -87.78 & 8.7078 \mathrm{E}-02 & 3.9832 \mathrm{E}-02 & 0.0000 \mathrm{E}+00 \\ -82.78 & 1.2618 \mathrm{E}-01 & 5.9474 \mathrm{E}-02 & 0.0000 \mathrm{E}+00 \\ -77.78 & 1.7434 \mathrm{E}-01 & 8.6027 \mathrm{E}-02 & 0.0000 \mathrm{E}+00 \\ -72.15 & 2.2951 \mathrm{E}-01 & 1.2067 \mathrm{E}-01 & 0.0000 \mathrm{E}+00 \\ -65.90 & 2.8617 \mathrm{E}-01 & 1.6524 \mathrm{E}-01 & 0.0000 \mathrm{E}+00 \\ -60.35 & 3.4757 \mathrm{E}-01 & 2.2611 \mathrm{E}-01 & 2.3606 \mathrm{E}-02 \\ -55.49 & 4.2917 \mathrm{E}-01 & 3.0902 \mathrm{E}-01 & 3.2126 \mathrm{E}-02 \\ -50.62 & 5.2569 \mathrm{E}-01 & 4.1864 \mathrm{E}-01 & 4.6080 \mathrm{E}-02 \\ -47.17 & 6.0460 \mathrm{E}-01 & 5.2362 \mathrm{E}-01 & 6.2868 \mathrm{E}-02 \\ -43.58 & 6.8800 \mathrm{E}-01 & 6.4360 \mathrm{E}-01 & 7.2608 \mathrm{E}-01 \\ -38.45 & 8.0749 \mathrm{E}-01 & 7.8929 \mathrm{E}-01 & 8.2053 \mathrm{E}-01 \\ -33.32 & 9.1807 \mathrm{E}-01 & 9.1397 \mathrm{E}-01 & 9.2286 \mathrm{E}-01 \\ -28.20 & 1.0148 \mathrm{E}+00 & 1.0182 \mathrm{E}+00 & 1.0104 \mathrm{E}+00 \\ -23.07 & 1.0957 \mathrm{E}+00 & 1.1033 \mathrm{E}+00 & 1.0893 \mathrm{E}+00 \\ -17.94 & 1.1595 \mathrm{E}+00 & 1.1691 \mathrm{E}+00 & 1.1518 \mathrm{E}+00 \\ -12.82 & 1.2048 \mathrm{E}+00 & 1.2151 \mathrm{E}+00 & 1.1930 \mathrm{E}+00 \\ -7.69 & 1.2311 \mathrm{E}+00 & 1.2418 \mathrm{E}+00 & 1.2188 \mathrm{E}+00 \\ -2.56 & 1.2382 \mathrm{E}+00 & 1.2491 \mathrm{E}+00 & 1.2257 \mathrm{E}+00 \\ 2.56 & 1.2263 \mathrm{E}+00 & 1.2374 \mathrm{E}+00 & 1.2147 \mathrm{E}+00 \\ 7.69 & 1.1958 \mathrm{E}+00 & 1.2070 \mathrm{E}+00 & 1.1846 \mathrm{E}+00 \\ 12.82 & 1.1478 \mathrm{E}+00 & 1.1588 \mathrm{E}+00 & 1.1373 \mathrm{E}+00 \\ 17.94 & 1.0836 \mathrm{E}+00 & 1.0941 \mathrm{E}+00 & 1.0786 \mathrm{E}+00 \\ 23.07 & 1.0040 \mathrm{E}+00 & 1.0128 \mathrm{E}+00 & 1.0000 \mathrm{E}+00 \\ 28.20 & 9.1078 \mathrm{E}-01 & 9.1632 \mathrm{E}-01 & 9.0834 \mathrm{E}-01 \\ 33.32 & 8.0627 \mathrm{E}-01 & 8.0591 \mathrm{E}-01 & 8.1171 \mathrm{E}-01 \\ 38.45 & 6.9279 \mathrm{E}-01 & 6.8129 \mathrm{E}-01 & 7.0360 \mathrm{E}-01 \\ 43.58 & 5.7507 \mathrm{E}-01 & 5.4293 \mathrm{E}-01 & 6.0278 \mathrm{E}-01 \\ 47.17 & 4.9475 \mathrm{E}-01 & 4.3465 \mathrm{E}-01 & 5.0486 \mathrm{E}-02 \\ 50.62 & 4.1915 \mathrm{E}-01 & 3.4011 \mathrm{E}-01 & 3.5631 \mathrm{E}-02 \\ 55.49 & 3.2700 \mathrm{E}-01 & 2.4186 \mathrm{E}-01 & 2.3215 \mathrm{E}-02 \\ 60.35 & 2.4813 \mathrm{E}-01 & 1.6747 \mathrm{E}-01 & 1.5505 \mathrm{E}-02 \\ 64.98 & 1.9982 \mathrm{E}-01 & 1.2503 \mathrm{E}-01 & 0.0000 \mathrm{E}+00 \\ 69.39 & 1.7116 \mathrm{E}-01 & 1.0252 \mathrm{E}-01 & 0.0000 \mathrm{E}+00 \\ 76.46 & 1.3009 \mathrm{E}-01 & 7.2401 \mathrm{E}-02 & 0.0000 \mathrm{E}+00 \\ 86.80 & 8.4762 \mathrm{E}-02 & 4.3366 \mathrm{E}-02 & 0.0000 \mathrm{E}+00 \\ 98.14 & 4.8762 \mathrm{E}-02 & 2.3512 \mathrm{E}-02 & 0.0000 \mathrm{E}+00 \\ 110.00 & 2.0658 \mathrm{E}-02 & 1.0394 \mathrm{E}-02 & 0.0000 \mathrm{E}+00\end{array}$


HNF-SD-FF-ANAL-009 Rev. 1

Table B1OA-2.5. Fission Power Distribution by Pin in MFA-1 at BOC $10 A-2$ CORE POSITION 1506

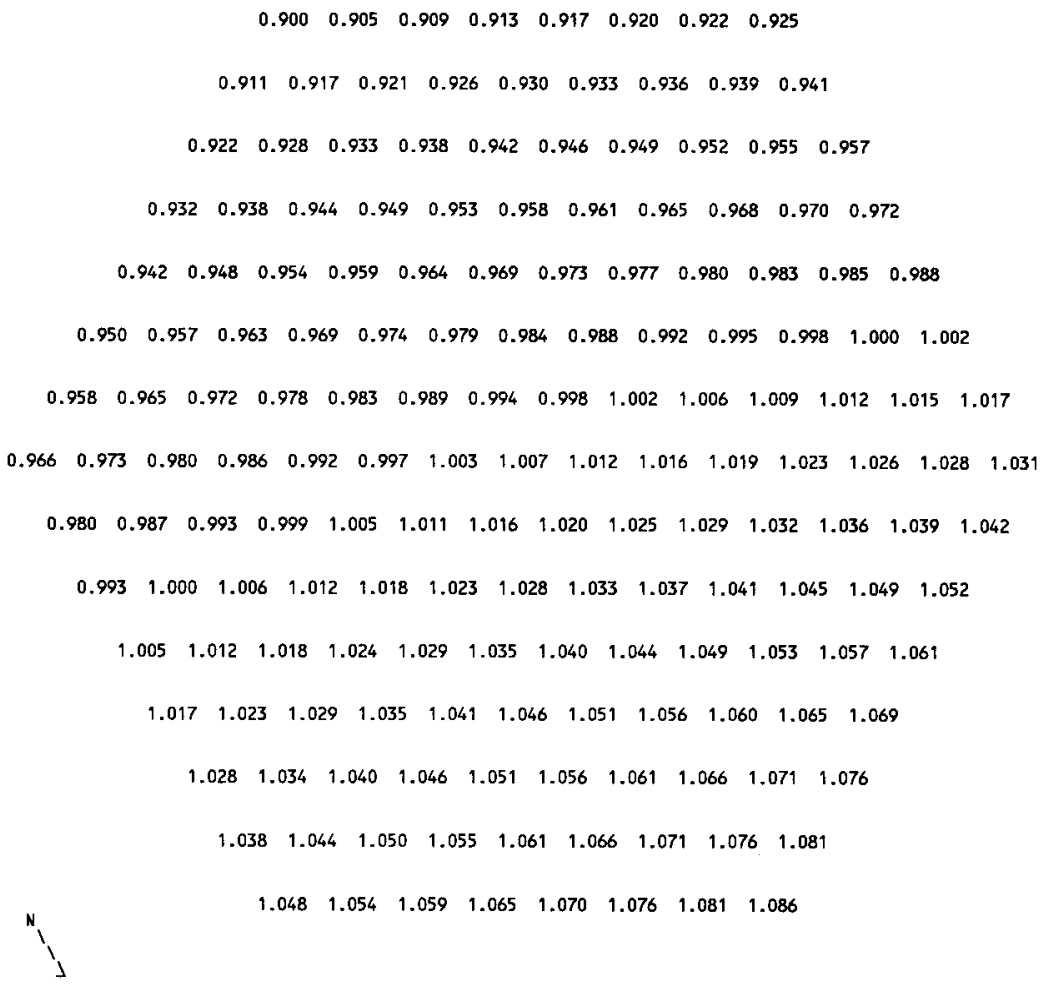


HNF-SD-FF-ANAL-009 Rev. 1

Table BlOA-2.6. Fission Power Distribution by Pin in MFA-2 at BOC 10A-2 CORE POSITION 2507

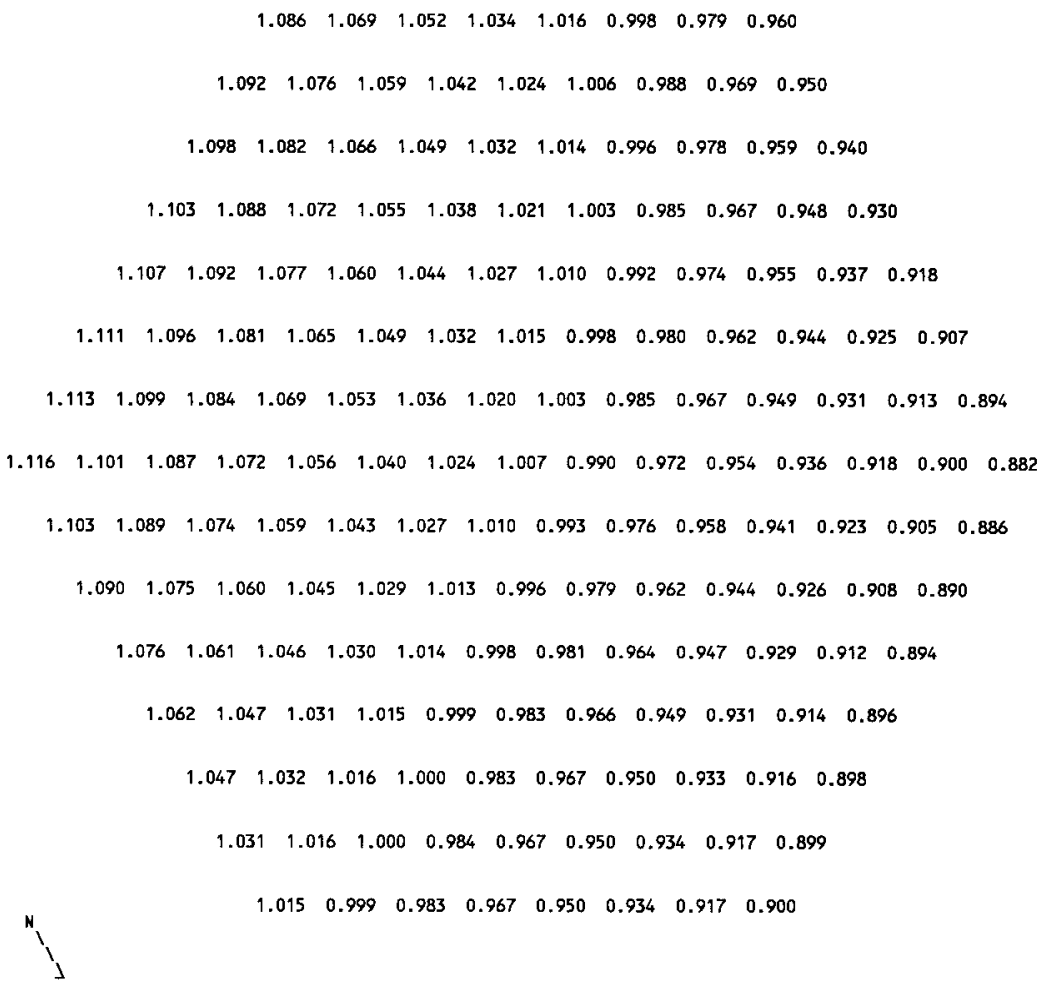


HNF-SD-FF-ANAL-009 Rev. 1

Table BIOA-2.7. Fast Flux Distribution by Pin in MFA-1 at BOC $10 \mathrm{~A}-2$ CORE POSITION 1506

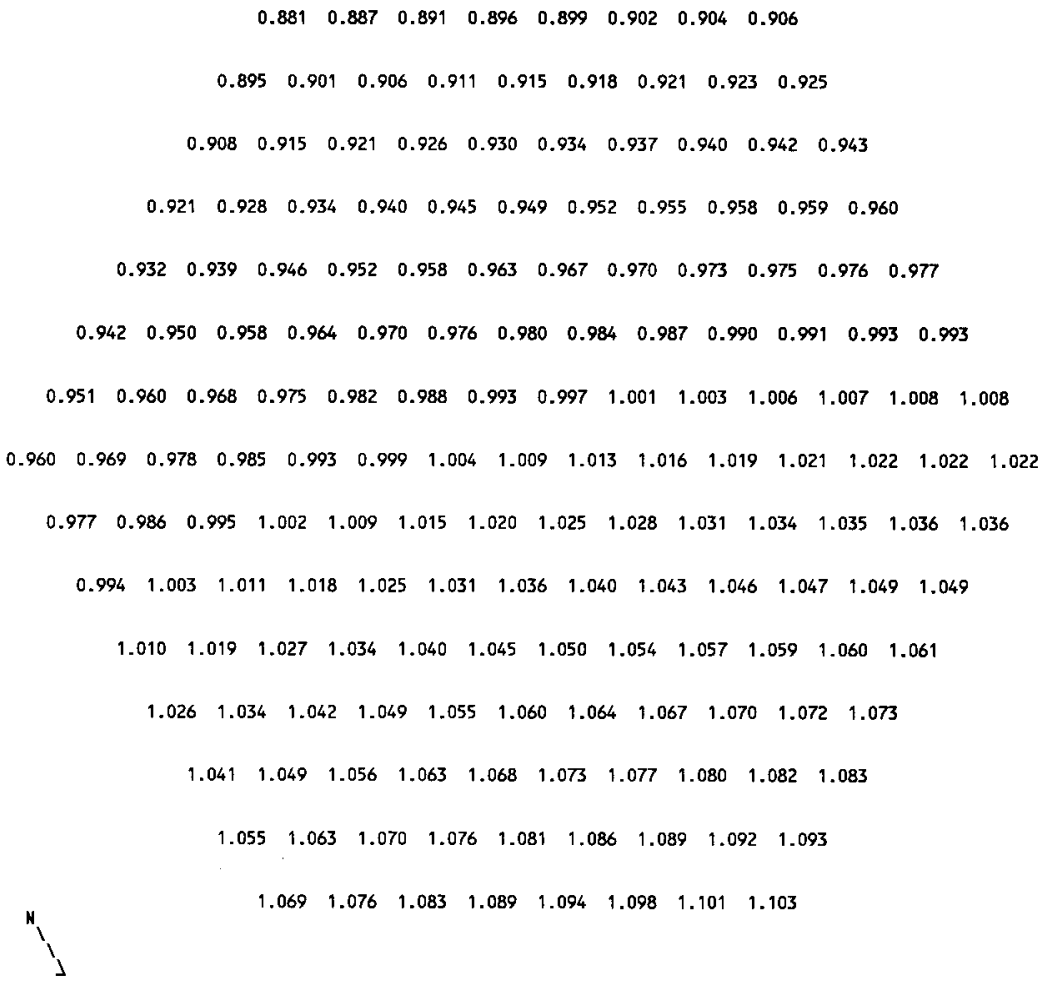


Table BIOA-2.8. Fast Flux Distribution by Pin in MFA-2 at BOC $10 \mathrm{~A}-2$ CORE POSITION 2507

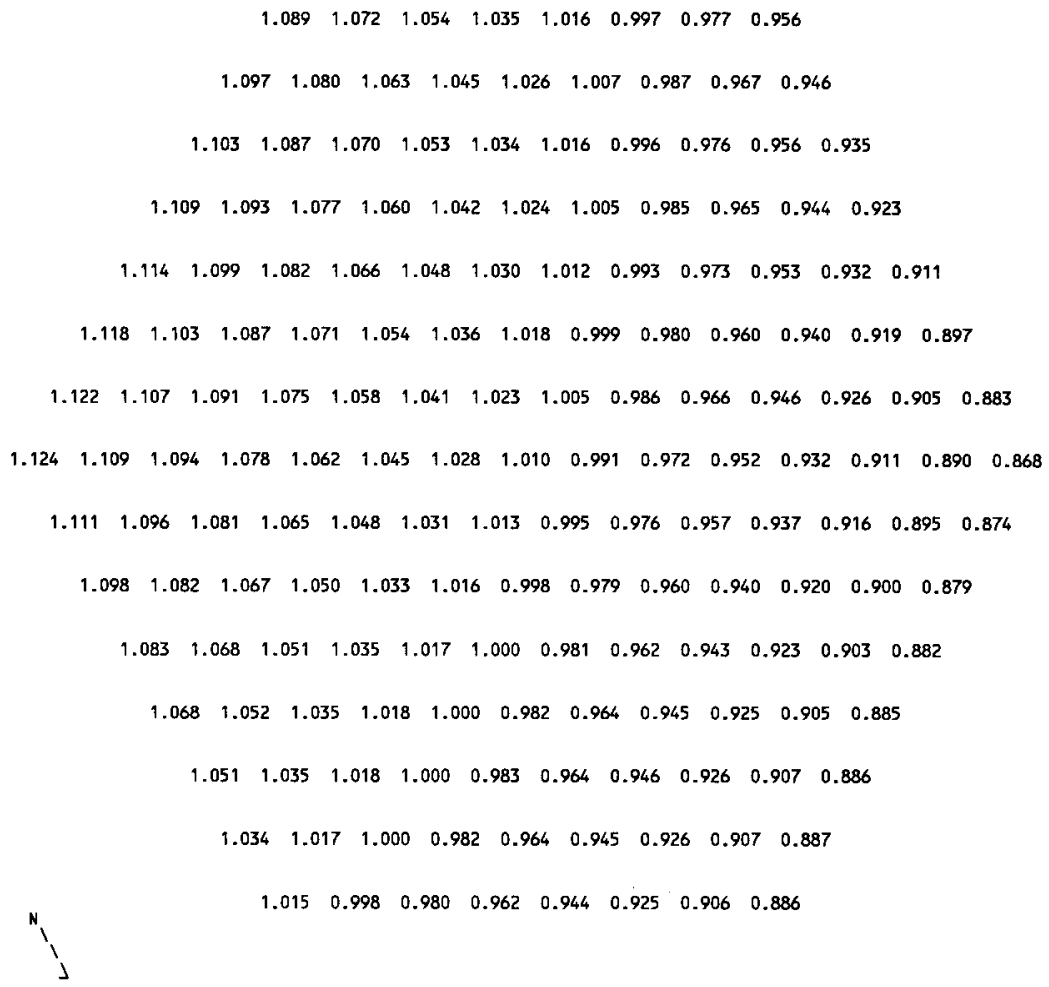


Table BlOA-2.9. MFA-1 and MFA-2 Duct Wall

Fast Flux Data at BOC 10A-2

$\begin{array}{lcc}\text { Side } & \begin{array}{c}\text { Flux }>0.1 \\ \text { MFA-1 }\end{array} & \begin{array}{c}\text { MeV } \\ \left(\mathrm{n} / \mathrm{cm}^{2}-\mathrm{sec}\right) \\ \text { MFA-2 }\end{array} \\ \text { E } & 1.8656 \mathrm{E}+15 & 2.1390 \mathrm{E}+15 \\ \text { SE } & 1.6677 \mathrm{E}+15 & 2.2249 \mathrm{E}+15 \\ \text { SW } & 1.6006 \mathrm{E}+15 & 2.0170 \mathrm{E}+15 \\ \text { W } & 1.7613 \mathrm{E}+15 & 1.7603 \mathrm{E}+15 \\ \text { NW } & 1.9781 \mathrm{E}+15 & 1.6834 \mathrm{E}+15 \\ \text { NE } & 2.0318 \mathrm{E}+15 & 1.8419 \mathrm{E}+15\end{array}$

Table B10A-2.10. Assembly Outlet Temperatures and Flow Rates at $\mathrm{BOC} 10 \mathrm{~A}-2$

$\begin{array}{lccc}\text { CORE } & \text { OUTLET TEMP. } & \text { (DEG F) } & \text { FLOW RATE } \\ \text { POS. } & \text { MEASURED } & \text { CALCULATED } & (\text { LB/H) } \\ 1506 & 1034 & 1039 & 114940 \\ 1404 & 917 & 911 & 225440 \\ 1405 & 891 & 887 & 208130 \\ 1505 & 883 & 867 & 208130 \\ 1507 & 873 & 855 & 208130 \\ 1606 & 902 & 898 & 170680 \\ 1607 & 871 & 864 & 170680 \\ 2507 & 1063 & 1065 & 114940 \\ 2405 & 949 & 938 & 192410 \\ 2506 & 902 & 894 & 197920 \\ 2508 & 932 & 922 & 208130 \\ 2607 & 926 & 911 & 163690 \\ 2608 & 903 & 900 & 170680 \\ 2609 & 898 & 895 & 173340\end{array}$

Assembly flows are based on a calculated core pressure drop of $110.6 \mathrm{psi}$ at a total reactor flow rate of $16.74 \mathrm{E}+06 \mathrm{lb} / \mathrm{hr}$. 
Table B10A-2.11. MFA-1 Sodium Subchannel Temperatures at Top of Core Elevation for BOC 10A-2

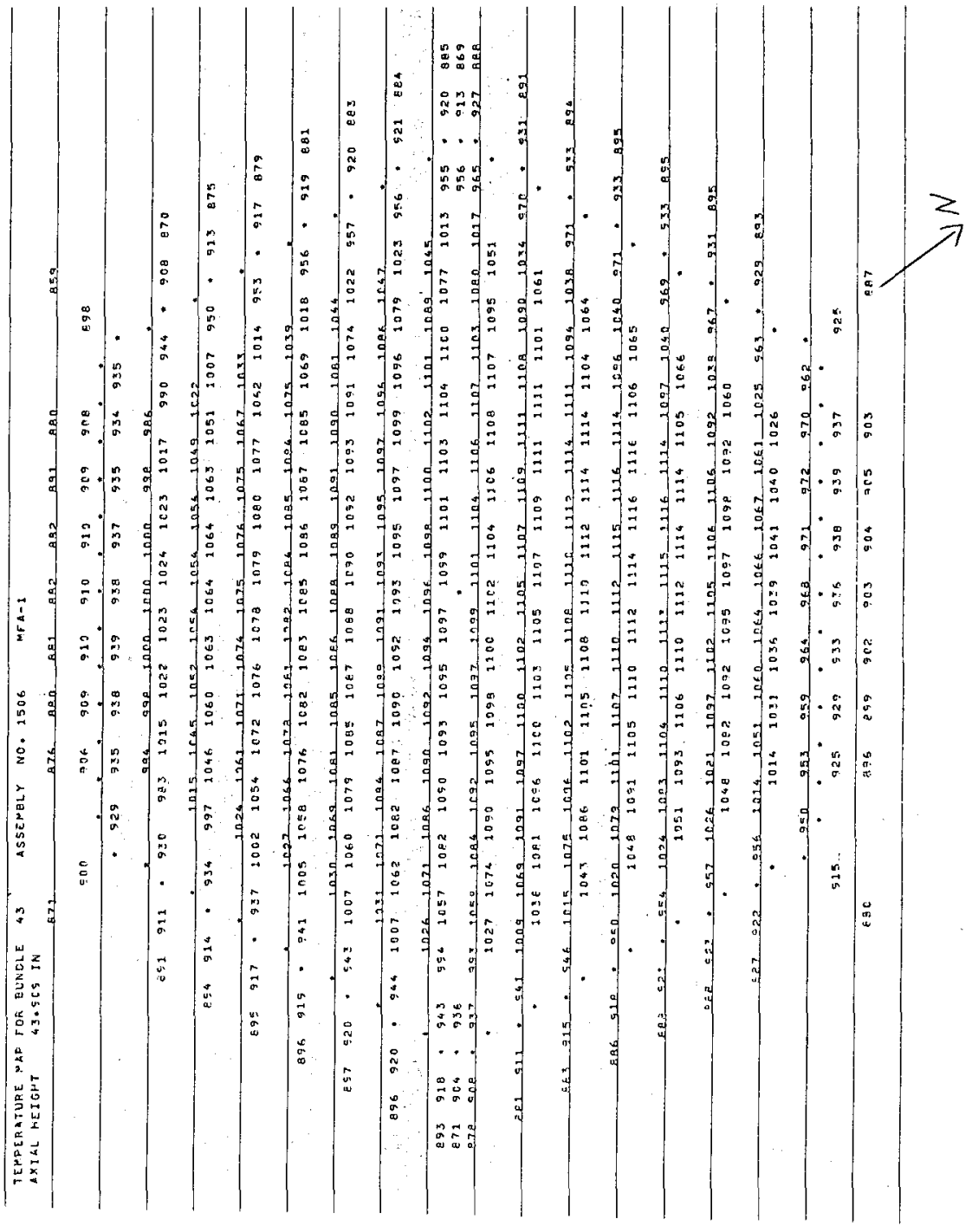


Table B1OA-2.12. MFA-2 Sodium Subchannel Temperatures at Top of Core Elevation for BOC $10 \mathrm{~A}-2$

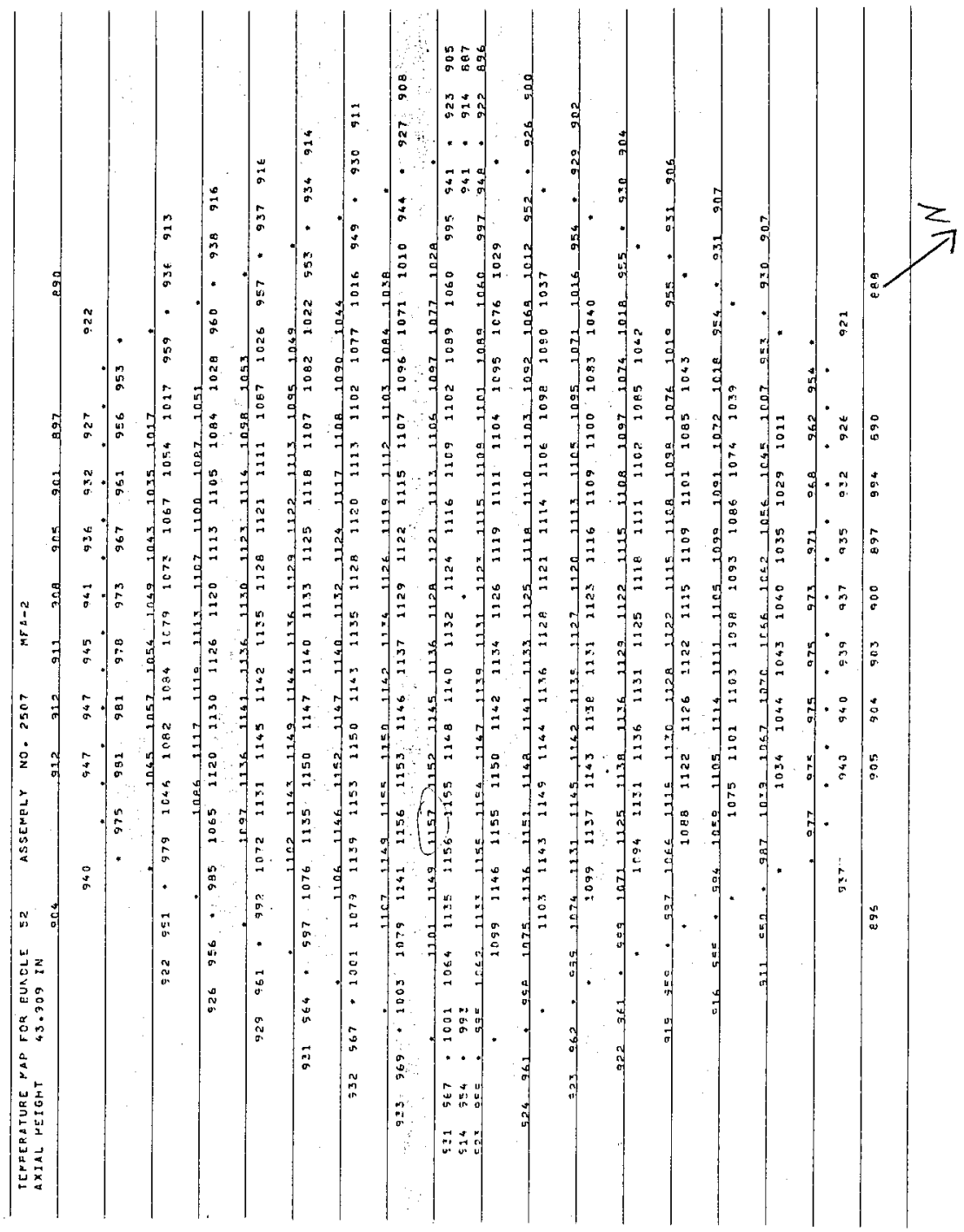


Table B10A-2.13. MFA-1 Sodium Subchannel Temperatures at Elevation of Upper Axial Blanket for BOC $10 \mathrm{~A}-2$

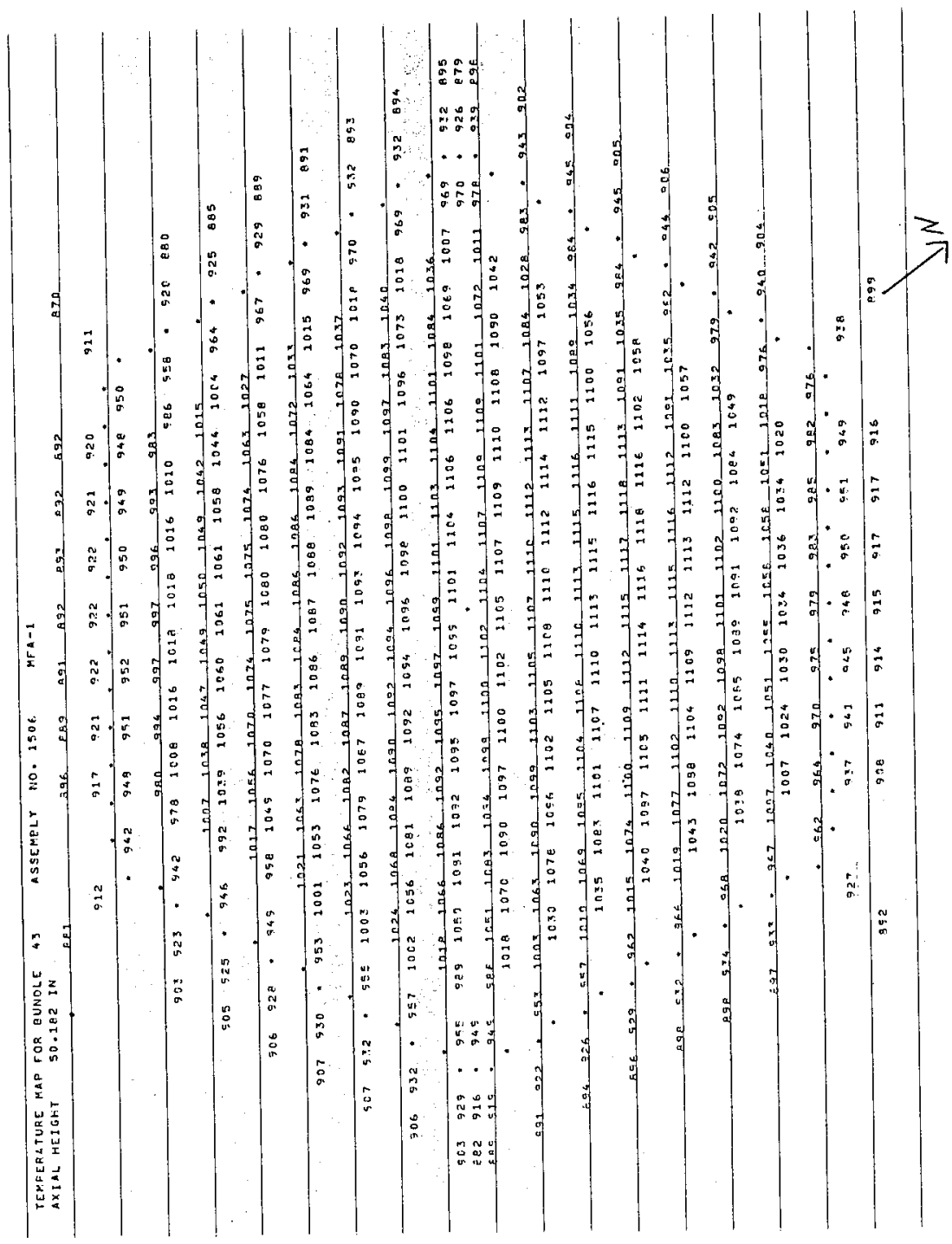




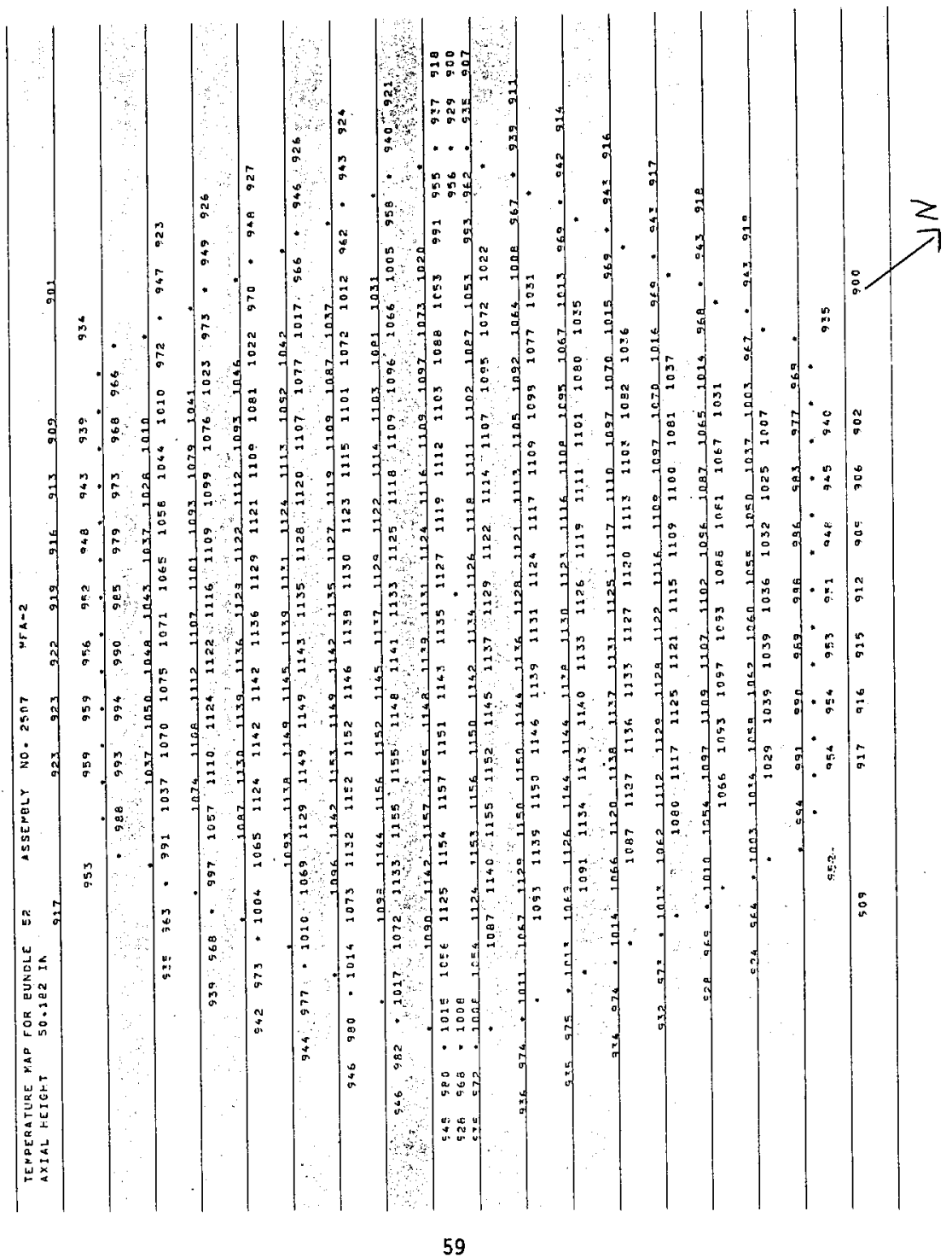




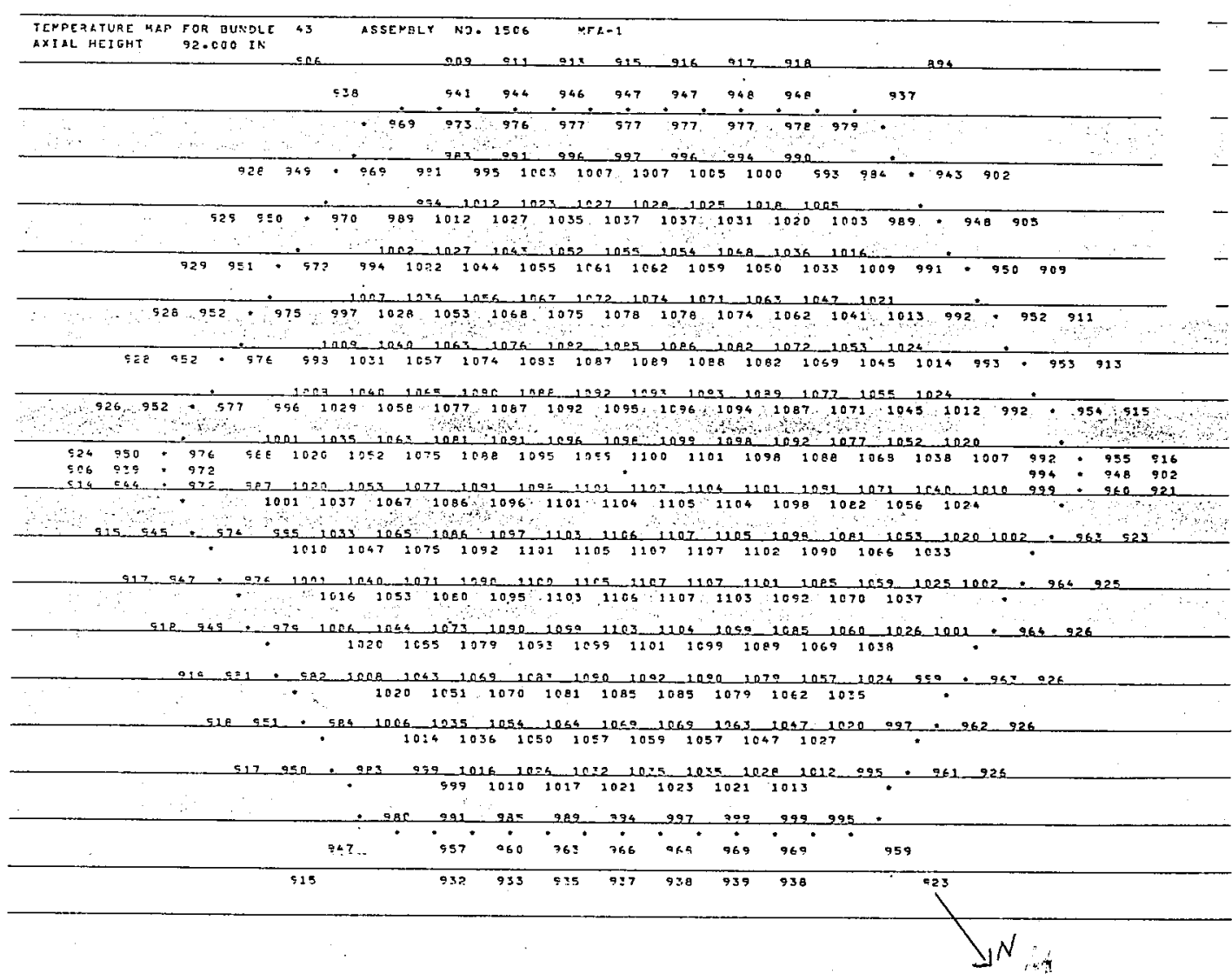


Table B10A-2.16. MFA-2 Sodium Subchannel Temperatures at Top of Fuel Pin Bundle for BOC 10A-2

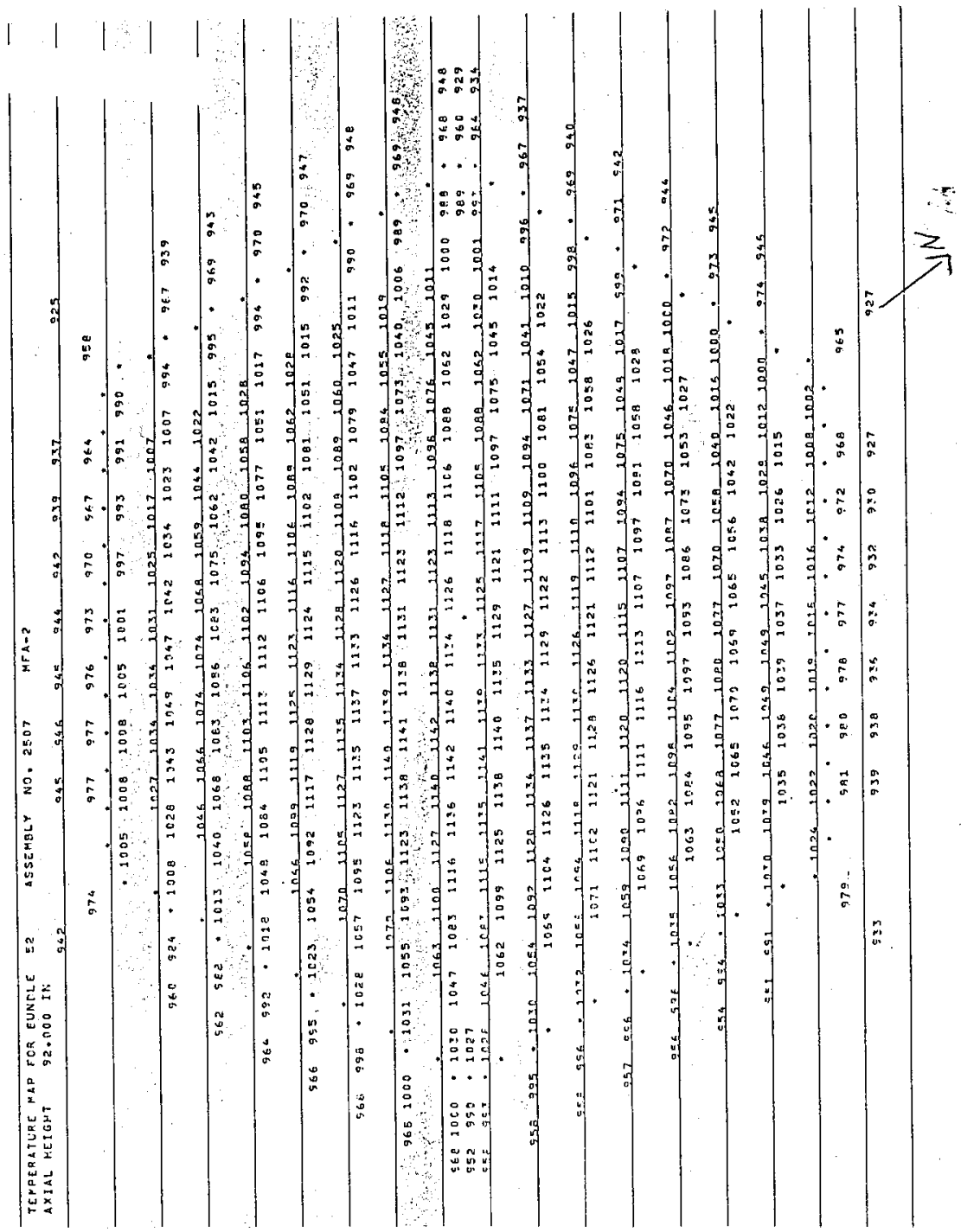


HNF-SD-FF-ANAL-009 Rev. I

Table E10A-2.17. Composition of MFA-1 at EOC 10A-2

\begin{tabular}{|c|c|c|c|c|c|}
\hline \multirow{3}{*}{$\begin{array}{c}\text { Axial } \\
\text { Level } \\
1\end{array}$} & \multicolumn{2}{|c|}{ Axial Range $(\mathrm{cm})$} & \multirow[b]{2}{*}{ Constituent } & \multicolumn{2}{|c|}{ Atom Density (a/b-cm) } \\
\hline & $\begin{array}{r}\text { Lower } \\
-128.05\end{array}$ & $\begin{array}{r}\text { Upper } \\
-75,28\end{array}$ & & Fresh & End of Cycle \\
\hline & & & $\begin{array}{l}\mathrm{Na}-23 \\
\mathrm{SS}-316\end{array}$ & $\begin{array}{l}5.3150 \mathrm{E}-03 \\
6.4090 \mathrm{E}-02\end{array}$ & $\begin{array}{l}5.3150 \mathrm{E}-03 \\
6.4090 \mathrm{E}-02\end{array}$ \\
\hline 2 & -75.28 & -62.78 & & & \\
\hline & & & $\begin{array}{l}\mathrm{Na}-23 \\
\mathrm{SS}-316\end{array}$ & $\begin{array}{l}1.3177 \mathrm{E}-02 \\
3.4175 \mathrm{E}-02\end{array}$ & $\begin{array}{l}1.3177 \mathrm{E}-02 \\
3.4175 \mathrm{E}-02\end{array}$ \\
\hline 3 & -62.78 & -46.14 & & & \\
\hline & & & $\begin{array}{l}\mathrm{U}-235 \\
\mathrm{U}-238 \\
\mathrm{Pu}-239 \\
\mathrm{Pu}-240 \\
\mathrm{FP} \\
0-16 \\
\mathrm{Na}-23 \\
\mathrm{SS}-316\end{array}$ & $\begin{array}{l}1.4143 \mathrm{E}-05 \\
7.0573 \mathrm{E}-03 \\
0.0000 \mathrm{E}+00 \\
0.0000 \mathrm{E}+00 \\
0.0000 \mathrm{E}+00 \\
1.4143 \mathrm{E}-02 \\
9.7416 \mathrm{E}-03 \\
1.9073 \mathrm{E}-02\end{array}$ & $\begin{array}{l}1.3496 \mathrm{E}-05 \\
7.0246 \mathrm{E}-03 \\
3.0158 \mathrm{E}-05 \\
1.9609 \mathrm{E}-07 \\
2.7995 \mathrm{E}-06 \\
1.4143 \mathrm{E}-02 \\
9.7416 \mathrm{E}-03 \\
1.9073 \mathrm{E}-02\end{array}$ \\
\hline 4 & -46.14 & -30.76 & & & \\
\hline & & & $\begin{array}{l}\mathrm{U}-235 \\
\mathrm{U}-238 \\
\mathrm{Pu}-239 \\
\mathrm{Pu}-240 \\
\mathrm{Pu}-241 \\
\mathrm{Pu}-242 \\
\mathrm{Am}-241 \\
\mathrm{FP} \\
\mathrm{O}-16 \\
\mathrm{Na}-23 \\
\mathrm{SS}-316\end{array}$ & $\begin{array}{l}1.0840 \mathrm{E}-05 \\
4.4499 \mathrm{E}-03 \\
1.7520 \mathrm{E}-03 \\
2.2340 \mathrm{E}-04 \\
1.9280 \mathrm{E}-05 \\
4.7430 \mathrm{E}-06 \\
7.6680 \mathrm{E}-07 \\
0.0000 \mathrm{E}+00 \\
1.2670 \mathrm{E}-02 \\
9.8100 \mathrm{E}-03 \\
1.8990 \mathrm{E}-02\end{array}$ & $\begin{array}{l}1.0386 \mathrm{E}-05 \\
4.4246 \mathrm{E}-03 \\
1.7006 \mathrm{E}-03 \\
2.3350 \mathrm{E}-04 \\
1.9937 \mathrm{E}-05 \\
4.7949 \mathrm{E}-06 \\
9.7032 \mathrm{E}-07 \\
6.5884 \mathrm{E}-05 \\
1.2670 \mathrm{E}-02 \\
9.8100 \mathrm{E}-03 \\
1.8990 \mathrm{E}-02\end{array}$ \\
\hline 5 & -30.76 & -15.38 & & & \\
\hline & & & $\begin{array}{l}\mathrm{U}-235 \\
\mathrm{U}-238 \\
\mathrm{Pu}-239 \\
\mathrm{Pu}-240 \\
\mathrm{Pu}-241 \\
\mathrm{Pu}-242 \\
\mathrm{Am}-241 \\
\mathrm{FP} \\
\mathrm{O}-16 \\
\mathrm{Na}-23 \\
\mathrm{SS}-316\end{array}$ & $\begin{array}{l}1.0840 \mathrm{E}-05 \\
4.4499 \mathrm{E}-03 \\
1.7520 \mathrm{E}-03 \\
2.2340 \mathrm{E}-04 \\
1.9280 \mathrm{E}-05 \\
4.7430 \mathrm{E}-06 \\
7.6680 \mathrm{E}-07 \\
0.0000 \mathrm{E}+00 \\
1.2670 \mathrm{E}-02 \\
9.8100 \mathrm{E}-03 \\
1.8990 \mathrm{E}-02\end{array}$ & $\begin{array}{l}1.0273 \mathrm{E}-05 \\
4.4177 \mathrm{E}-03 \\
1.6860 \mathrm{E}-03 \\
2.3458 \mathrm{E}-04 \\
1.9985 \mathrm{E}-05 \\
4.7950 \mathrm{E}-06 \\
9.6380 \mathrm{E}-07 \\
8.6461 \mathrm{E}-05 \\
1.2670 \mathrm{E}-02 \\
9.8100 \mathrm{E}-03 \\
1.8990 \mathrm{E}-02\end{array}$ \\
\hline 6 & -15.38 & 0.00 & & & \\
\hline & & & $\begin{array}{l}\mathrm{U}-235 \\
\mathrm{U}-238 \\
\mathrm{Pu}-239 \\
\mathrm{Pu}-240 \\
\mathrm{Pu}-241 \\
\mathrm{Pu}-242 \\
\mathrm{Am}-241 \\
\mathrm{FP} \\
\mathrm{O}-16 \\
\mathrm{Na}-23 \\
\mathrm{SS}-316\end{array}$ & $\begin{array}{l}1.0840 \mathrm{E}-05 \\
4.4499 \mathrm{E}-03 \\
1.7520 \mathrm{E}-03 \\
2.2340 \mathrm{E}-04 \\
1.9280 \mathrm{E}-05 \\
4.7430 \mathrm{E}-06 \\
7.6680 \mathrm{E}-07 \\
0.0000 \mathrm{E}+00 \\
1.2670 \mathrm{E}-02 \\
9.8100 \mathrm{E}-03 \\
1.8990 \mathrm{E}-02\end{array}$ & $\begin{array}{l}1.0210 \mathrm{E}-05 \\
4.4140 \mathrm{E}-03 \\
1.6785 \mathrm{E}-03 \\
2.3571 \mathrm{E}-04 \\
2.0084 \mathrm{E}-05 \\
4.8005 \mathrm{E}-06 \\
9.6042 \mathrm{E}-07 \\
9.6417 \mathrm{E}-05 \\
1.2670 \mathrm{E}-02 \\
9.8100 \mathrm{E}-03 \\
1.8990 \mathrm{E}-02\end{array}$ \\
\hline
\end{tabular}


HNF-SD-FF-ANAL-009 Rev. 1

$\begin{array}{lll}7 & 0.00 & 15.38\end{array}$

$\begin{array}{lll}\mathrm{U}-235 & 1.0840 \mathrm{E}-05 & 1.0230 \mathrm{E}-05 \\ \mathrm{U}-238 & 4.4499 \mathrm{E}-03 & 4.4152 \mathrm{E}-03 \\ \mathrm{Pu}-239 & 1.7520 \mathrm{E}-03 & 1.6808 \mathrm{E}-03 \\ \mathrm{Pu}-240 & 2.2340 \mathrm{E}-04 & 2.3529 \mathrm{E}-04 \\ \mathrm{Pu}-241 & 1.9280 \mathrm{E}-05 & 2.0046 \mathrm{E}-05 \\ \mathrm{Pu}-242 & 4.7430 \mathrm{E}-06 & 4.7983 \mathrm{E}-06 \\ \mathrm{Am}-241 & 7.6680 \mathrm{E}-07 & 9.6151 \mathrm{E}-07 \\ \mathrm{FP} & 0.0000 \mathrm{E}+00 & 9.3286 \mathrm{E}-05 \\ 0-16 & 1.2670 \mathrm{E}-02 & 1.2670 \mathrm{E}-02 \\ \mathrm{Na}-23 & 9.8100 \mathrm{E}-03 & 9.8100 \mathrm{E}-03 \\ \mathrm{SS}-316 & 1.8990 \mathrm{E}-02 & 1.8990 \mathrm{E}-02\end{array}$

$\begin{array}{lll}8 & 15.38 & 30.76\end{array}$

$\begin{array}{lll}\mathrm{U}-235 & 1.0840 \mathrm{E}-05 & 1.0329 \mathrm{E}-05 \\ \mathrm{U}-238 & 4.4499 \mathrm{E}-03 & 4.4209 \mathrm{E}-03 \\ \mathrm{Pu}-239 & 1.7520 \mathrm{E}-03 & 1.6925 \mathrm{E}-03 \\ \mathrm{Pu}-240 & 2.2340 \mathrm{E}-04 & 2.3341 \mathrm{E}-04 \\ \mathrm{Pu}-241 & 1.9280 \mathrm{E}-05 & 1.9881 \mathrm{E}-05 \\ \mathrm{Pu}-242 & 4.7430 \mathrm{E}-06 & 4.7888 \mathrm{E}-06 \\ \mathrm{Am}-241 & 7.6680 \mathrm{E}-07 & 9.6677 \mathrm{E}-07 \\ \mathrm{FP} & 0.0000 \mathrm{E}+00 & 7.8016 \mathrm{E}-05 \\ 0-16 & 1.2670 \mathrm{E}-02 & 1.2670 \mathrm{E}-02 \\ \mathrm{Na}-23 & 9.8100 \mathrm{E}-03 & 9.8100 \mathrm{E}-03 \\ \mathrm{SS}-316 & 1.8990 \mathrm{E}-02 & 1.8990 \mathrm{E}-02\end{array}$

$\begin{array}{lll}9 & 30.76 & 46.14\end{array}$

$\begin{array}{lll}\text { U-235 } & 1.0840 \mathrm{E}-05 & 1.0465 \mathrm{E}-05 \\ \mathrm{U}-238 & 4.4499 \mathrm{E}-03 & 4.4291 \mathrm{E}-03 \\ \mathrm{Pu}-239 & 1.7520 \mathrm{E}-03 & 1.7094 \mathrm{E}-03 \\ \mathrm{Pu}-240 & 2.2340 \mathrm{E}-04 & 2.3152 \mathrm{E}-04 \\ \mathrm{Pu}-241 & 1.9280 \mathrm{E}-05 & 1.9747 \mathrm{E}-05 \\ \mathrm{Pu}-242 & 4.7430 \mathrm{E}-06 & 4.7832 \mathrm{E}-06 \\ \mathrm{Am}-241 & 7.6680 \mathrm{E}-07 & 9.7442 \mathrm{E}-07 \\ \text { FP } & 0.0000 \mathrm{E}+00 & 5.4760 \mathrm{E}-05 \\ 0-16 & 1.2670 \mathrm{E}-02 & 1.2670 \mathrm{E}-02 \\ \mathrm{Na}-23 & 9.8100 \mathrm{E}-03 & 9.8100 \mathrm{E}-03 \\ \text { SS-316 } & 1.8990 \mathrm{E}-02 & 1.8990 \mathrm{E}-02\end{array}$

$\begin{array}{lll}10 & 46.14 & 62.78\end{array}$

$\begin{array}{lll}\text { U-235 } & 1.4143 \mathrm{E}-05 & 1.3709 \mathrm{E}-05 \\ \mathrm{U}-238 & 7.0573 \mathrm{E}-03 & 7.0347 \mathrm{E}-03 \\ \mathrm{Pu}-239 & 0.0000 \mathrm{E}+00 & 2.0819 \mathrm{E}-05 \\ \mathrm{Pu}-240 & 0.0000 \mathrm{E}+00 & 8.6890 \mathrm{E}-08 \\ \mathrm{FP} & 0.0000 \mathrm{E}+00 & 2.0138 \mathrm{E}-06 \\ \mathrm{O}-16 & 1.4143 \mathrm{E}-02 & 1.4143 \mathrm{E}-02 \\ \mathrm{Na}-23 & 9.7416 \mathrm{E}-03 & 9.7416 \mathrm{E}-03 \\ \mathrm{SS}-316 & 1.9073 \mathrm{E}-02 & 1.9073 \mathrm{E}-02\end{array}$

$11 \quad 62.78 \quad 172.84$

$\begin{array}{lll}\mathrm{Na}-23 & 8.2400 \mathrm{E}-03 & 8.2400 \mathrm{E}-03 \\ \mathrm{SS}-316 & 2.5562 \mathrm{E}-02 & 2.5562 \mathrm{E}-02\end{array}$


Table E10A-2.18. Composition of MFA-2 at EOC 10A-2

\begin{tabular}{|c|c|c|c|c|c|}
\hline & Axial $R$ & $(\mathrm{~cm})$ & & Atom Den & n) \\
\hline & $\begin{array}{l}\text { Lower } \\
-128\end{array}$ & $\begin{array}{l}\text { Upper } \\
\text { Un }\end{array}$ & Constituent & & \\
\hline & & & $\begin{array}{l}\mathrm{Na}-23 \\
\mathrm{SS}-316\end{array}$ & $\begin{array}{l}5.3150 \mathrm{E}-03 \\
6.4090 \mathrm{E}-02\end{array}$ & $\begin{array}{l}5.3150 \mathrm{E}-03 \\
6.4090 \mathrm{E}-02\end{array}$ \\
\hline 2 & -75.28 & -62.78 & & & \\
\hline & & & $\begin{array}{l}\mathrm{Na}-23 \\
\mathrm{SS}-316\end{array}$ & $\begin{array}{l}1.3177 \mathrm{E}-02 \\
3.4175 \mathrm{E}-02\end{array}$ & $\begin{array}{l}1.3177 \mathrm{E}-02 \\
3.4175 \mathrm{E}-02\end{array}$ \\
\hline 3 & -62.78 & -46.14 & $\begin{array}{l}U-235 \\
U-238 \\
\text { Pu-239 } \\
\text { Pu-240 } \\
\text { FP } \\
0-16 \\
\text { Na-23 } \\
\text { SS-316 }\end{array}$ & $\begin{array}{l}1.4143 \mathrm{E}-05 \\
7.0573 \mathrm{E}-03 \\
0.0000 \mathrm{E}+00 \\
0.0000 \mathrm{E}+00 \\
0.0000 \mathrm{E}+00 \\
1.4143 \mathrm{E}-02 \\
9.7416 \mathrm{E}-03 \\
1.9073 \mathrm{E}-02\end{array}$ & $\begin{array}{l}1.3549 \mathrm{E}-05 \\
7.0268 \mathrm{E}-03 \\
2.8137 \mathrm{E}-05 \\
1.6635 \mathrm{E}-07 \\
2.6628 \mathrm{E}-06 \\
1.4143 \mathrm{E}-02 \\
9.7416 \mathrm{E}-03 \\
1.9073 \mathrm{E}-02\end{array}$ \\
\hline 4 & -46.14 & -30.76 & $\begin{array}{l}U-235 \\
U-238 \\
P u-239 \\
P u-240 \\
P u-241 \\
P u-242 \\
\text { Am-241 } \\
\text { FP } \\
0-16 \\
\text { Na-23 } \\
\text { SS }-316\end{array}$ & $\begin{array}{l}1.0160 \mathrm{E}-05 \\
4.4270 \mathrm{E}-03 \\
1.7483 \mathrm{E}-03 \\
2.2290 \mathrm{E}-04 \\
1.9040 \mathrm{E}-05 \\
4.7330 \mathrm{E}-06 \\
7.5700 \mathrm{E}-07 \\
0.0000 \mathrm{E}+00 \\
1.2760 \mathrm{E}-02 \\
9.8100 \mathrm{E}-03 \\
1.8990 \mathrm{E}-02\end{array}$ & $\begin{array}{l}9.7467 \mathrm{E}-06 \\
4.4026 \mathrm{E}-03 \\
1.6987 \mathrm{E}-03 \\
2.3279 \mathrm{E}-04 \\
1.9688 \mathrm{E}-05 \\
4.7824 \mathrm{E}-06 \\
9.5864 \mathrm{E}-07 \\
6.3447 \mathrm{E}-05 \\
1.2760 \mathrm{E}-02 \\
9.8100 \mathrm{E}-03 \\
1.8990 \mathrm{E}-02\end{array}$ \\
\hline 5 & -30.76 & -15.38 & $\begin{array}{l}U-235 \\
U-238 \\
P u-239 \\
P u-240 \\
P u-241 \\
P u-242 \\
\text { Am-241 } \\
\mathrm{FP} \\
0-16 \\
\mathrm{Na}-23 \\
\mathrm{SS}-316\end{array}$ & $\begin{array}{l}1.0160 \mathrm{E}-05 \\
4.4270 \mathrm{E}-03 \\
1.7483 \mathrm{E}-03 \\
2.2290 \mathrm{E}-04 \\
1.9040 \mathrm{E}-05 \\
4.7330 \mathrm{E}-06 \\
7.5700 \mathrm{E}-07 \\
0.0000 \mathrm{E}+00 \\
1.2760 \mathrm{E}-02 \\
9.8100 \mathrm{E}-03 \\
1.8990 \mathrm{E}-02\end{array}$ & $\begin{array}{l}9.6407 \mathrm{E}-06 \\
4.3956 \mathrm{E}-03 \\
1.6842 \mathrm{E}-03 \\
2.3396 \mathrm{E}-04 \\
1.9753 \mathrm{E}-05 \\
4.7831 \mathrm{E}-06 \\
9.5219 \mathrm{E}-07 \\
8.3808 \mathrm{E}-05 \\
1.2760 \mathrm{E}-02 \\
9.8100 \mathrm{E}-03 \\
1.8990 \mathrm{E}-02\end{array}$ \\
\hline 6 & -15.38 & 0.00 & $\begin{array}{l}U-235 \\
U-238 \\
P u-239 \\
P u-240 \\
P u-241 \\
P u-242 \\
\text { Am-241 } \\
\text { FP } \\
\text { O-16 } \\
\mathrm{Na}-23 \\
\text { SS }-316\end{array}$ & $\begin{array}{l}1.0160 \mathrm{E}-05 \\
4.4270 \mathrm{E}-03 \\
1.7483 \mathrm{E}-03 \\
2.2290 \mathrm{E}-04 \\
1.9040 \mathrm{E}-05 \\
4.7330 \mathrm{E}-06 \\
7.5700 \mathrm{E}-07 \\
0.0000 \mathrm{E}+00 \\
1.2760 \mathrm{E}-02 \\
9.8100 \mathrm{E}-03 \\
1.8990 \mathrm{E}-02\end{array}$ & $\begin{array}{l}9.5810 \mathrm{E}-06 \\
4.3919 \mathrm{E}-03 \\
1.6767 \mathrm{E}-03 \\
2.3511 \mathrm{E}-04 \\
1.9858 \mathrm{E}-05 \\
4.7887 \mathrm{E}-06 \\
9.4879 \mathrm{E}-07 \\
9.3816 \mathrm{E}-05 \\
1.2760 \mathrm{E}-02 \\
9.8100 \mathrm{E}-03 \\
1.8990 \mathrm{E}-02\end{array}$ \\
\hline
\end{tabular}


HNF-SD-FF-ANAL-009 Rev. 1

7

0.00

15.38

8

9

30.76

10

46.14

11

15.38

$\begin{array}{ll}\mathrm{U}-235 & 1.0160 \mathrm{E}-05 \\ \mathrm{U}-238 & 4.4270 \mathrm{E}-03 \\ \mathrm{Pu}-239 & 1.7483 \mathrm{E}-03 \\ \mathrm{Pu}-240 & 2.2290 \mathrm{E}-04 \\ \mathrm{Pu}-241 & 1.9040 \mathrm{E}-05 \\ \mathrm{Pu}-242 & 4.7330 \mathrm{E}-06 \\ \mathrm{Am}-241 & 7.5700 \mathrm{E}-07 \\ \mathrm{FP} & 0.0000 \mathrm{E}+00 \\ \mathrm{O}-16 & 1.2760 \mathrm{E}-02 \\ \mathrm{Na}-23 & 9.8100 \mathrm{E}-03 \\ \mathrm{SS}-316 & 1.8990 \mathrm{E}-02\end{array}$

9.5986E-06

4. $3930 \mathrm{E}-03$

$1.6788 \mathrm{E}-03$

2. $3473 \mathrm{E}-04$

1. $9822 \mathrm{E}-05$

4.7867E-06

$9.4976 \mathrm{E}-07$

9.0994E-05

$1.2760 \mathrm{E}-02$

9.8100E-03

$1.8990 \mathrm{E}-02$

30.76

$\begin{array}{ll}\mathrm{U}-235 & 1.0160 \mathrm{E}-05 \\ \mathrm{U}-238 & 4.4270 \mathrm{E}-03 \\ \mathrm{Pu}-239 & 1.7483 \mathrm{E}-03 \\ \mathrm{Pu}-240 & 2.2290 \mathrm{E}-04 \\ \mathrm{Pu}-241 & 1.9040 \mathrm{E}-05 \\ \mathrm{Pu}-242 & 4.7330 \mathrm{E}-06 \\ \mathrm{Am}-241 & 7.5700 \mathrm{E}-07 \\ \mathrm{FP} & 0.0000 \mathrm{E}+00 \\ \mathrm{O}-16 & 1.2760 \mathrm{E}-02 \\ \mathrm{Na}-23 & 9.8100 \mathrm{E}-03 \\ \mathrm{SS}-316 & 1.8990 \mathrm{E}-02\end{array}$

$9.6866 \mathrm{E}-06$

4.3984E-03

$1.6898 \mathrm{E}-03$

$2.3295 \mathrm{E}-04$

$1.9663 \mathrm{E}-05$

$4.7780 \mathrm{E}-06$

9.5474E-07

$7.6462 \mathrm{E}-05$

$1.2760 \mathrm{E}-02$

9.8100E-03

$1.8990 \mathrm{E}-02$

46.14

$\begin{array}{ll}\mathrm{U}-235 & 1.0160 \mathrm{E}-05 \\ \mathrm{U}-238 & 4.4270 \mathrm{E}-03 \\ \mathrm{Pu}-239 & 1.7483 \mathrm{E}-03 \\ \mathrm{Pu}-240 & 2.2290 \mathrm{E}-04 \\ \mathrm{Pu}-241 & 1.9040 \mathrm{E}-05 \\ \mathrm{Pu}-242 & 4.7330 \mathrm{E}-06 \\ \mathrm{Am}-241 & 7.5700 \mathrm{E}-07 \\ \mathrm{FP} & 0.0000 \mathrm{E}+00 \\ 0-16 & 1.2760 \mathrm{E}-02 \\ \mathrm{Na}-23 & 9.8100 \mathrm{E}-03 \\ \mathrm{SS}-316 & 1.8990 \mathrm{E}-02\end{array}$

9.8117E-06

4.4064E-03

$1.7064 \mathrm{E}-03$

2.3109E-04

$1.9525 \mathrm{E}-05$

4.7726E-06

9.6214E-07

5.3857E-05

$1.2760 \mathrm{E}-02$

$9.8100 \mathrm{E}-03$

$1.8990 \mathrm{E}-02$

62.78

$\begin{array}{lll}\text { U-235 } & 1.4143 \mathrm{E}-05 & 1.3721 \mathrm{E}-05 \\ \mathrm{U}-238 & 7.0573 \mathrm{E}-03 & 7.0350 \mathrm{E}-03 \\ \mathrm{Pu}-239 & 0.0000 \mathrm{E}+00 & 2.0506 \mathrm{E}-05 \\ \mathrm{Pu}-240 & 0.0000 \mathrm{E}+00 & 8.3485 \mathrm{E}-08 \\ \mathrm{FP} & 0.0000 \mathrm{E}+00 & 2.0017 \mathrm{E}-06 \\ 0-16 & 1.4143 \mathrm{E}-02 & 1.4143 \mathrm{E}-02 \\ \mathrm{Na}-23 & 9.7416 \mathrm{E}-03 & 9.7416 \mathrm{E}-03 \\ \text { SS-316 } & 1.9073 \mathrm{E}-02 & 1.9073 \mathrm{E}-02\end{array}$

$\mathrm{Na}-23 \quad 8.2400 \mathrm{E}-03$

8. $2400 \mathrm{E}-03$

SS -316

2. 5562E-02

2.5562E-02 


\subsection{Cycle 10A-3}

Table B10A-3.1. Fission Power Generated in MFA-1, MFA-2 and Neighboring Assemblies at BOC 10A-3

\begin{tabular}{lrrrrr} 
CORE & $* * * * * *$ & \multicolumn{2}{c}{ POWER IN } & MEGAWATTS & $* * * * * *$ \\
POS. & BELOW CORE & IN CORE & ABOVE CORE & TOTAL PWR \\
1506 & $2.923 \mathrm{E}-02$ & $3.888 \mathrm{E}+00$ & $2.016 \mathrm{E}-02$ & $3.937 \mathrm{E}+00$ \\
1404 & $8.347 \mathrm{E}-02$ & $4.655 \mathrm{E}+00$ & $4.578 \mathrm{E}-02$ & $4.785 \mathrm{E}+00$ \\
1405 & $9.573 \mathrm{E}-03$ & $3.886 \mathrm{E}+00$ & $7.157 \mathrm{E}-03$ & $3.903 \mathrm{E}+00$ \\
1505 & $1.134 \mathrm{E}-02$ & $3.560 \mathrm{E}+00$ & $6.692 \mathrm{E}-03$ & $3.578 \mathrm{E}+00$ \\
1507 & $9.828 \mathrm{E}-03$ & $3.290 \mathrm{E}+00$ & $7.354 \mathrm{E}-03$ & $3.307 \mathrm{E}+00$ \\
1606 & $6.233 \mathrm{E}-03$ & $3.462 \mathrm{E}+00$ & $4.559 \mathrm{E}-03$ & $3.473 \mathrm{E}+00$ \\
1607 & $9.179 \mathrm{E}-03$ & $2.857 \mathrm{E}+00$ & $6.639 \mathrm{E}-03$ & $2.872 \mathrm{E}+00$ \\
2507 & $3.000 \mathrm{E}-02$ & $4.145 \mathrm{E}+00$ & $2.203 \mathrm{E}-02$ & $4.197 \mathrm{E}+00$ \\
2405 & $7.196 \mathrm{E}-02$ & $4.423 \mathrm{E}+00$ & $4.182 \mathrm{E}-02$ & $4.536 \mathrm{E}+00$ \\
2506 & $1.144 \mathrm{E}-02$ & $3.850 \mathrm{E}+00$ & $8.082 \mathrm{E}-03$ & $3.870 \mathrm{E}+00$ \\
2508 & $1.108 \mathrm{E}-02$ & $4.557 \mathrm{E}+00$ & $8.140 \mathrm{E}-03$ & $4.576 \mathrm{E}+00$ \\
2607 & $7.842 \mathrm{E}-03$ & $3.538 \mathrm{E}+00$ & $5.861 \mathrm{E}-03$ & $3.551 \mathrm{E}+00$ \\
2608 & $4.991 \mathrm{E}-03$ & $3.398 \mathrm{E}+00$ & $4.147 \mathrm{E}-03$ & $3.408 \mathrm{E}+00$ \\
2609 & $9.444 \mathrm{E}-02$ & $3.150 \mathrm{E}+00$ & $5.240 \mathrm{E}-02$ & $3.297 \mathrm{E}+00$
\end{tabular}

Table B10A-3.2. Assembly Averaged Total and Fast Flux in MFA-1 and MFA-2 at BOC 10A-3

\begin{tabular}{|c|c|c|}
\hline & Core & Flux $\left(\mathrm{n} / \mathrm{cm}^{2}-\mathrm{sec}\right)$ \\
\hline $\begin{array}{c}\text { As sembly } \\
\text { MFA-1 } \\
\text { MFA-2 }\end{array}$ & $\begin{array}{l}\text { Pos. } \\
1506 \\
2507\end{array}$ & $\begin{array}{l}>0.1 \mathrm{MeV} \\
1.853 \mathrm{E}+15 \\
1.967 \mathrm{E}+15\end{array}$ \\
\hline
\end{tabular}


Table BlOA-3.3. Axial Distribution of Total Flux, Fast Flux and Power in MFA-1 at BOC 10A-3

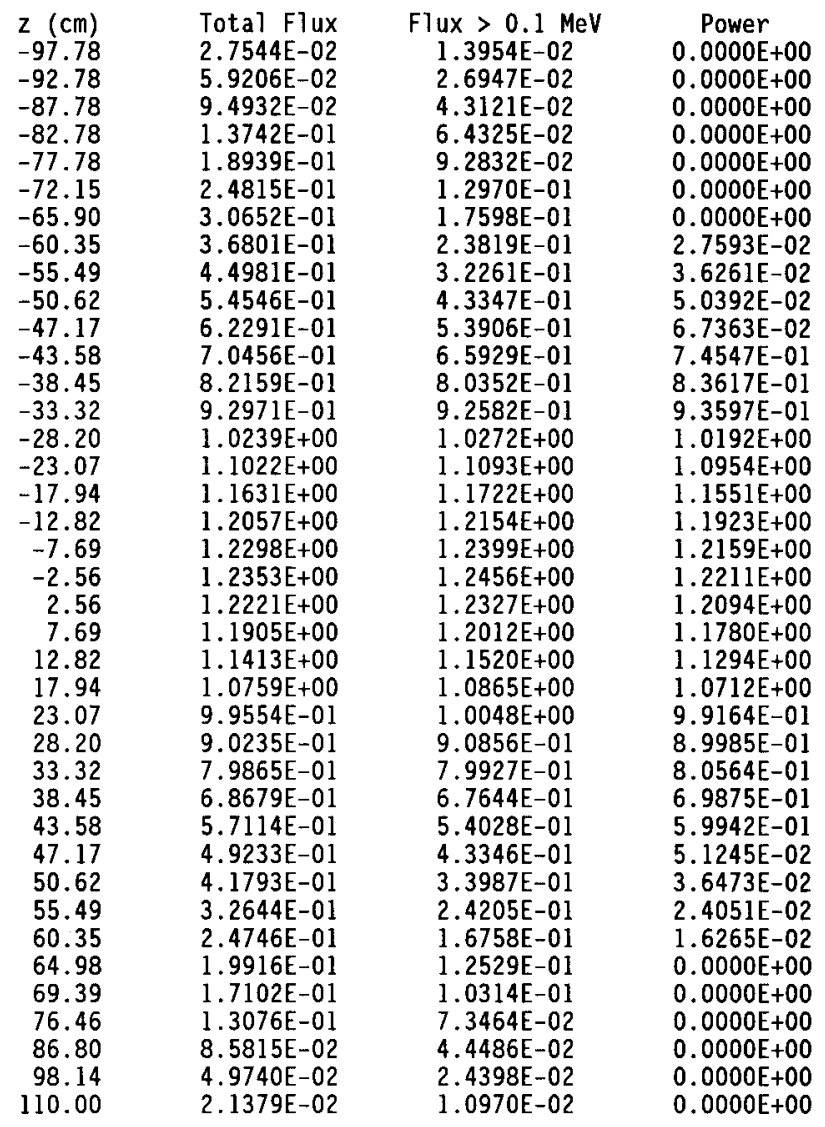


Table BlOA-3.4. Axial Distribution of Total Flux, Fast Flux and Power in MFA-2 at BOC $10 A-3$

\begin{tabular}{|c|c|c|c|}
\hline $\begin{array}{r}z(\mathrm{~cm}) \\
-97.78 \\
-92.78 \\
-87.78 \\
-82.78 \\
-77.78 \\
-72.15 \\
-65.90 \\
-60.35 \\
-55.49 \\
-50.62 \\
-47.17 \\
-43.58 \\
-38.45 \\
-33.32 \\
-28.20 \\
-23.07 \\
-17.94 \\
-12.82 \\
-7.69 \\
-2.56 \\
2.56 \\
7.69 \\
12.82 \\
17.94 \\
23.07 \\
28.20 \\
33.32 \\
38.45 \\
43.58 \\
47.17 \\
50.62 \\
55.49 \\
60.35 \\
64.98 \\
69.39 \\
76.46 \\
86.80 \\
98.14 \\
110.00\end{array}$ & $\begin{array}{l}\text { Total Flux } \\
2.5372 \mathrm{E}-02 \\
5.4484 \mathrm{E}-02 \\
8.7356 \mathrm{E}-02 \\
1.2656 \mathrm{E}-01 \\
1.7484 \mathrm{E}-01 \\
2.3013 \mathrm{E}-01 \\
2.8687 \mathrm{E}-01 \\
3.4830 \mathrm{E}-01 \\
4.2994 \mathrm{E}-01 \\
5.2644 \mathrm{E}-01 \\
6.0532 \mathrm{E}-01 \\
6.8870 \mathrm{E}-01 \\
8.0800 \mathrm{E}-01 \\
9.1834 \mathrm{E}-01 \\
1.0147 \mathrm{E}+00 \\
1.0954 \mathrm{E}+00 \\
1.1588 \mathrm{E}+00 \\
1.2039 \mathrm{E}+00 \\
1.2301 \mathrm{E}+00 \\
1.2372 \mathrm{E}+00 \\
1.2254 \mathrm{E}+00 \\
1.1951 \mathrm{E}+00 \\
1.1475 \mathrm{E}+00 \\
1.0836 \mathrm{E}+00 \\
1.0043 \mathrm{E}+00 \\
9.1152 \mathrm{E}-01 \\
8.0726 \mathrm{E}-01 \\
6.9394 \mathrm{E}-01 \\
5.7621 \mathrm{E}-01 \\
4.9585 \mathrm{E}-01 \\
4.2020 \mathrm{E}-01 \\
3.2791 \mathrm{E}-01 \\
2.4890 \mathrm{E}-01 \\
2.0052 \mathrm{E}-01 \\
1.7179 \mathrm{E}-01 \\
1.3062 \mathrm{E}-01 \\
8.5140 \mathrm{E}-02 \\
4.8997 \mathrm{E}-02 \\
2.0763 \mathrm{E}-02\end{array}$ & $\begin{array}{c}\text { Flux > } 0.1 \mathrm{MeV} \\
1.2956 \mathrm{E}-02 \\
2.5004 \mathrm{E}-02 \\
4.0013 \mathrm{E}-02 \\
5.9739 \mathrm{E}-02 \\
8.6392 \mathrm{E}-02 \\
1.2117 \mathrm{E}-01 \\
1.6591 \mathrm{E}-01 \\
2.2703 \mathrm{E}-01 \\
3.1008 \mathrm{E}-01 \\
4.1975 \mathrm{E}-01 \\
5.2466 \mathrm{E}-01 \\
6.4452 \mathrm{E}-01 \\
7.9004 \mathrm{E}-01 \\
9.1440 \mathrm{E}-01 \\
1.0181 \mathrm{E}+00 \\
1.1029 \mathrm{E}+00 \\
1.1683 \mathrm{E}+00 \\
1.2139 \mathrm{E}+00 \\
1.2405 \mathrm{E}+00 \\
1.2478 \mathrm{E}+00 \\
1.2363 \mathrm{E}+00 \\
1.2061 \mathrm{E}+00 \\
1.1584 \mathrm{E}+00 \\
1.0941 \mathrm{E}+00 \\
1.0133 \mathrm{E}+00 \\
9.1720 \mathrm{E}-01 \\
8.0719 \mathrm{E}-01 \\
6.8268 \mathrm{E}-01 \\
5.4432 \mathrm{E}-01 \\
4.3595 \mathrm{E}-01 \\
3.4131 \mathrm{E}-01 \\
2.4286 \mathrm{E}-01 \\
1.6825 \mathrm{E}-01 \\
1.2563 \mathrm{E}-01 \\
1.0303 \mathrm{E}-01 \\
7.2781 \mathrm{E}-02 \\
4.3610 \mathrm{E}-02 \\
2.3654 \mathrm{E}-02 \\
1.0461 \mathrm{E}-02 \\
\end{array}$ & $\begin{array}{c}\text { Power } \\
0.0000 \mathrm{E}+00 \\
0.0000 \mathrm{E}+00 \\
0.0000 \mathrm{E}+00 \\
0.0000 \mathrm{E}+00 \\
0.0000 \mathrm{E}+00 \\
0.0000 \mathrm{E}+00 \\
0.0000 \mathrm{E}+00 \\
2.5974 \mathrm{E}-02 \\
3.4715 \mathrm{E}-02 \\
4.8897 \mathrm{E}-02 \\
6.5829 \mathrm{E}-02 \\
7.2770 \mathrm{E}-01 \\
8.2223 \mathrm{E}-01 \\
9.2450 \mathrm{E}-01 \\
1.0100 \mathrm{E}+00 \\
1.0885 \mathrm{E}+00 \\
1.1507 \mathrm{E}+00 \\
1.1905 \mathrm{E}+00 \\
1.2163 \mathrm{E}+00 \\
1.2232 \mathrm{E}+00 \\
1.2126 \mathrm{E}+00 \\
1.1827 \mathrm{E}+00 \\
1.1357 \mathrm{E}+00 \\
1.0789 \mathrm{E}+00 \\
1.0007 \mathrm{E}+00 \\
9.0934 \mathrm{E}-01 \\
8.1464 \mathrm{E}-01 \\
7.0643 \mathrm{E}-01 \\
6.0535 \mathrm{E}-01 \\
5.2402 \mathrm{E}-02 \\
3.7358 \mathrm{E}-02 \\
2.4688 \mathrm{E}-02 \\
1.6738 \mathrm{E}-02 \\
0.0000 \mathrm{E}+00 \\
0.0000 \mathrm{E}+00 \\
0.0000 \mathrm{E}+00 \\
0.0000 \mathrm{E}+00 \\
0.0000 \mathrm{E}+00 \\
0.0000 \mathrm{E}+00\end{array}$ \\
\hline
\end{tabular}


HNF-SD-FF-ANAL-009 Rev. 1

Table B10A-3.5. Fission Power Distribution by Pin in MFA-1 at BOC $10 A-3$ CORE POSITION 1506

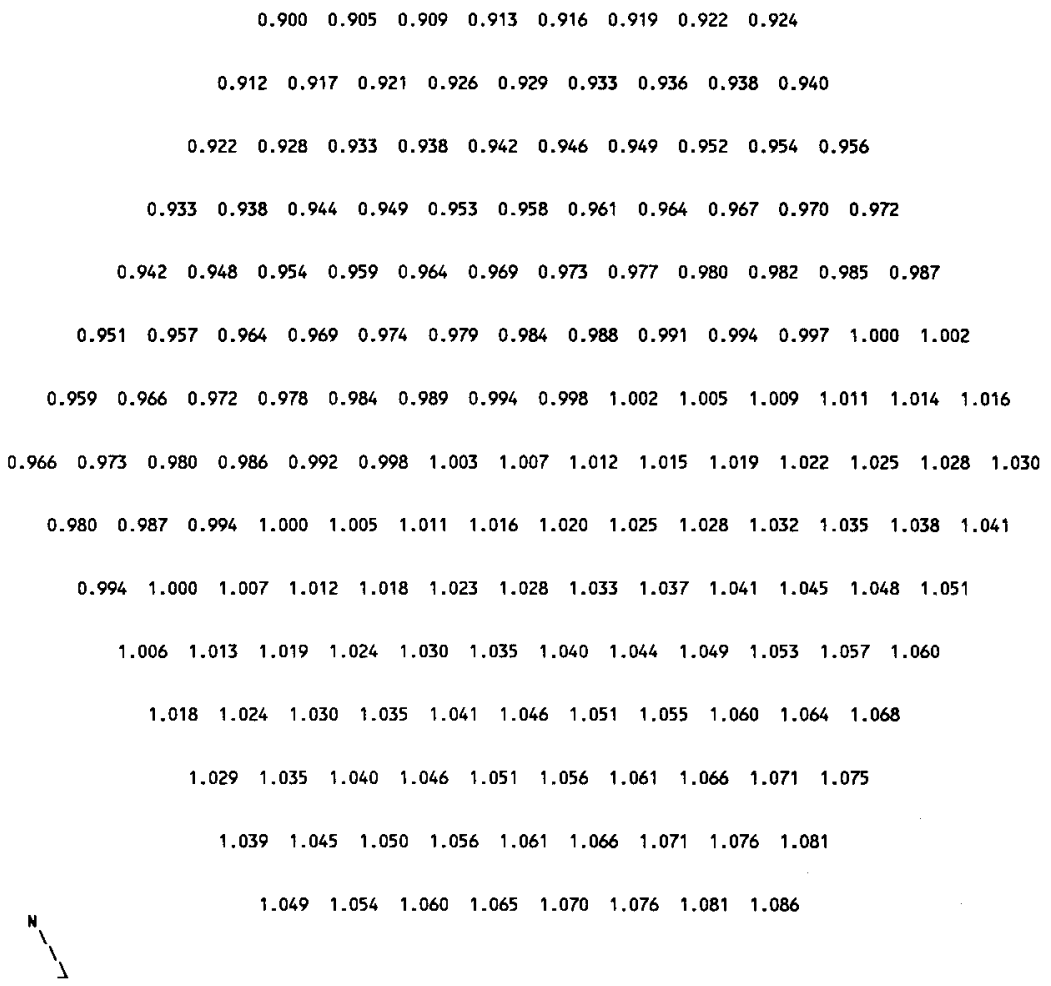


HNF-SD-FF-ANAL-009 Rev. 1

Table B10A-3.6. Fission Power Distribution by Pin in MFA-2 at BOC 10A-3 CORE POSITION 2507

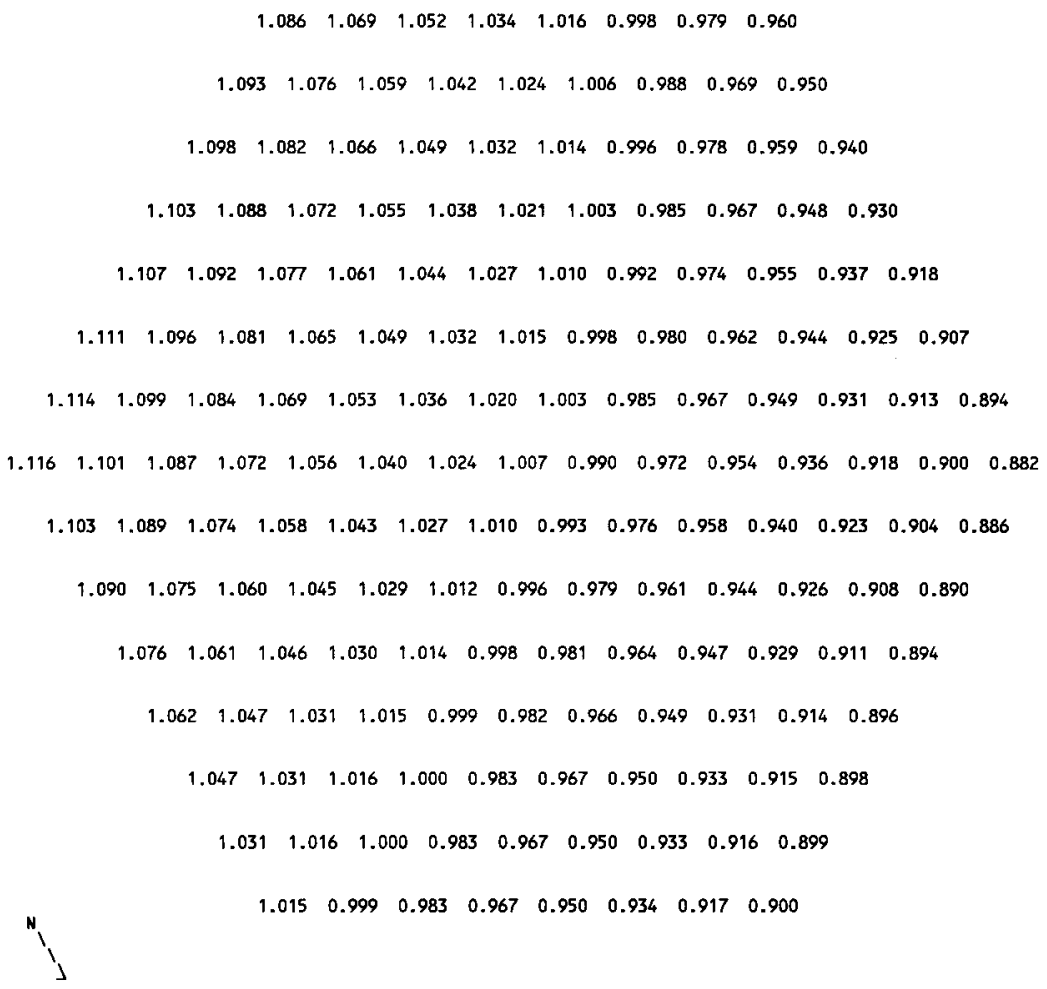


HNF-SD-FF-ANAL-009 Rev. 1

Table B10A-3.7. Fast Flux Distribution by Pin in MFA-1 at BOC 10A-3 CORE POSITION 1506

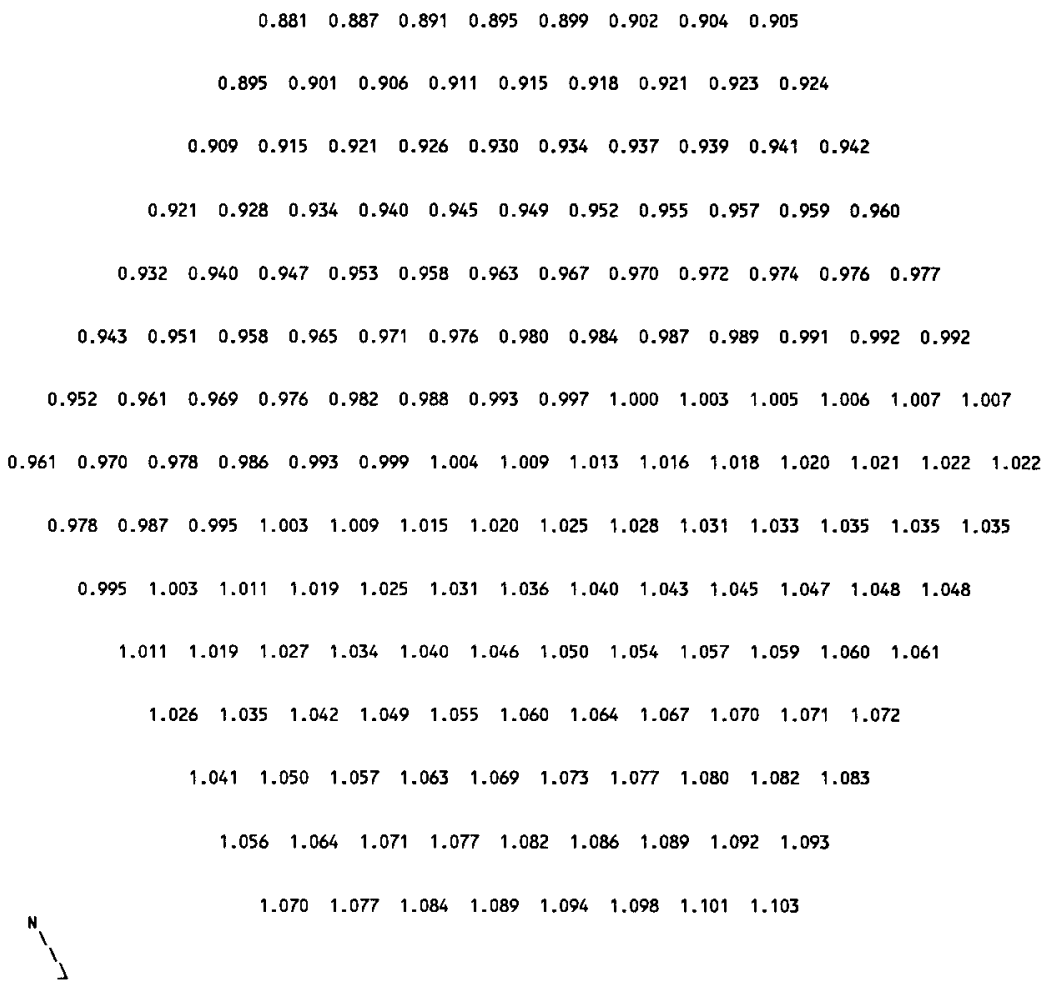




\section{HNF-SD-FF-ANAL-009 Rev. I}

Table B10A-3.8. Fast Flux Distribution by Pin in MFA-2 at BOC 10A-3 CORE POSITION 2507

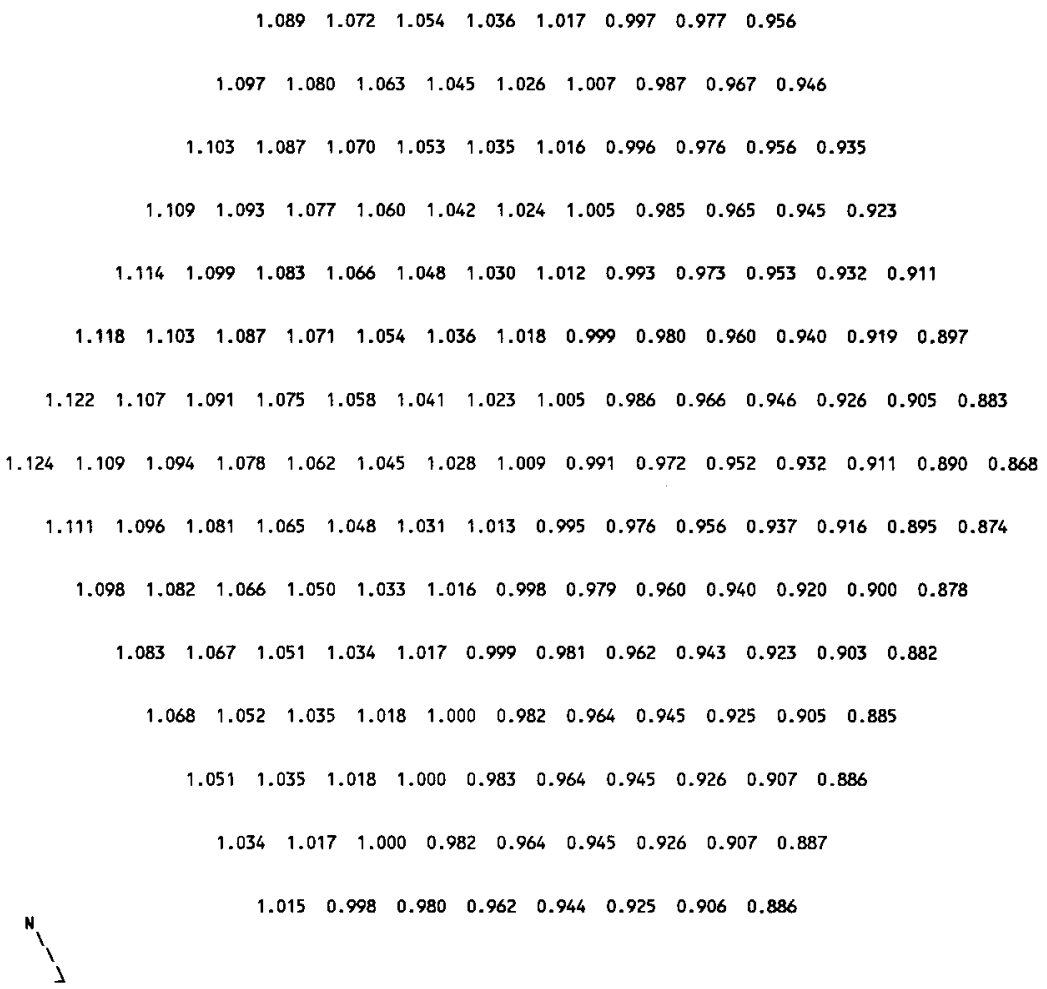


Table B1OA-3.9. MFA-1 and MFA-2 Duct Wall

Fast Flux Data at BOC 10A-3

$\begin{array}{ccc}\text { Side } & \begin{array}{c}\text { Flux }>0.1 \\ \text { MFA-1 }\end{array} & \begin{array}{c}\text { MeV } \\ \left(\mathrm{n} / \mathrm{cm}^{2}-\mathrm{sec}\right) \\ \text { MFA-2 }\end{array} \\ \text { E } & 1.8787 \mathrm{E}+15 & 2.1340 \mathrm{E}+15 \\ \text { SE } & 1.6787 \mathrm{E}+15 & 2.2202 \mathrm{E}+15 \\ \text { SW } & 1.6099 \mathrm{E}+15 & 2.0130 \mathrm{E}+15 \\ \text { W } & 1.7705 \mathrm{E}+15 & 1.7564 \mathrm{E}+15 \\ \text { NW } & 1.9888 \mathrm{E}+15 & 1.6795 \mathrm{E}+15 \\ \text { NE } & 2.0449 \mathrm{E}+15 & 1.8375 \mathrm{E}+15\end{array}$

Table B10A-3.10. Assembly Outlet Temperatures and Flow Rates at $\mathrm{BOC}$ 10A-3

\begin{tabular}{lcl}
\multicolumn{3}{c}{$\begin{array}{c}\text { MEASURED } \\
\text { CORE } \\
\text { OUTLET TEMP. }\end{array}$} \\
POS. & $\begin{array}{l}\text { FLOW RATE } \\
\text { (DEG F) }\end{array}$ \\
1506 & 1033 & 114940 \\
1404 & 916 & 225440 \\
1405 & 890 & 208130 \\
1505 & 884 & 208130 \\
1507 & 873 & 208130 \\
1606 & 902 & 170680 \\
1607 & 871 & 170680 \\
2507 & 1058 & 114940 \\
2405 & 945 & 192410 \\
2506 & 900 & 197920 \\
2508 & 927 & 208130 \\
2607 & 922 & 163690 \\
2608 & 899 & 170680 \\
2609 & 897 & 173340
\end{tabular}

Assembly flows are based on a calculated core pressure drop of $110.6 \mathrm{psi}$ at a total reactor flow rate of $16.74 \mathrm{E}+06 \mathrm{lb} / \mathrm{hr}$. 
Table E1OA-3.17. Composition of MFA-1 at EOC 1OA-3

\begin{tabular}{|c|c|c|c|c|c|}
\hline \multirow{3}{*}{$\begin{array}{c}\text { Axial } \\
\text { Level } \\
1\end{array}$} & \multicolumn{2}{|c|}{ Axial Range $(\mathrm{cm})$} & \multirow[b]{2}{*}{ Constituent } & \multicolumn{2}{|c|}{ Atom Density $(a / b-c m)$} \\
\hline & Lower & Upper & & & End of Cycle \\
\hline & & & $\begin{array}{l}\mathrm{Na}-23 \\
\text { SS-316 }\end{array}$ & $\begin{array}{l}5.3150 \mathrm{E}-03 \\
6.4090 \mathrm{E}-02\end{array}$ & $\begin{array}{l}5.3150 \mathrm{E}-03 \\
6.4090 \mathrm{E}-02\end{array}$ \\
\hline 2 & -75.28 & -62.78 & & & \\
\hline & & & $\begin{array}{l}\mathrm{Na}-23 \\
\text { SS-316 }\end{array}$ & $\begin{array}{l}1.3177 \mathrm{E}-02 \\
3.4175 \mathrm{E}-02\end{array}$ & $\begin{array}{l}1.3177 \mathrm{E}-02 \\
3.4175 \mathrm{E}-02\end{array}$ \\
\hline 3 & -62.78 & -46.14 & $\begin{array}{l}U-235 \\
U-238 \\
P u-239 \\
P U-240 \\
F P \\
O-16 \\
\mathrm{Na}-23 \\
\text { SS }-316\end{array}$ & $\begin{array}{l}1.4143 E-05 \\
7.0573 E-03 \\
0.0000 E+00 \\
0.0000 E+00 \\
0.0000 E+00 \\
1.4143 E-02 \\
9.7416 E-03 \\
1.9073 E-02\end{array}$ & $\begin{array}{l}1.3455 \mathrm{E}-05 \\
7.0225 \mathrm{E}-03 \\
3.2081 \mathrm{E}-05 \\
2.2219 \mathrm{E}-07 \\
3.0103 \mathrm{E}-06 \\
1.4143 \mathrm{E}-02 \\
9.7416 \mathrm{E}-03 \\
1.9073 \mathrm{E}-02\end{array}$ \\
\hline 4 & -46.14 & -30.76 & $\begin{array}{l}U-235 \\
U-238 \\
P u-239 \\
P u-240 \\
P u-241 \\
P u-242 \\
A m-241 \\
F P \\
O-16 \\
\mathrm{Na}-23 \\
\text { SS }-316\end{array}$ & $\begin{array}{l}1.0840 \mathrm{E}-05 \\
4.4499 \mathrm{E}-03 \\
1.7520 \mathrm{E}-03 \\
2.2340 \mathrm{E}-04 \\
1.9280 \mathrm{E}-05 \\
4.7430 \mathrm{E}-06 \\
7.6680 \mathrm{E}-07 \\
0.0000 \mathrm{E}+00 \\
1.2670 \mathrm{E}-02 \\
9.8100 \mathrm{E}-03 \\
1.8990 \mathrm{E}-02\end{array}$ & $\begin{array}{l}1.0357 \mathrm{E}-05 \\
4.4230 \mathrm{E}-03 \\
1.6973 \mathrm{E}-03 \\
2.3414 \mathrm{E}-04 \\
1.9977 \mathrm{E}-05 \\
4.7984 \mathrm{E}-06 \\
9.8823 \mathrm{E}-07 \\
7.0090 \mathrm{E}-05 \\
1.2670 \mathrm{E}-02 \\
9.8100 \mathrm{E}-03 \\
1.8990 \mathrm{E}-02\end{array}$ \\
\hline 5 & -30.76 & -15.38 & $\begin{array}{l}U-235 \\
U-238 \\
P u-239 \\
P u-240 \\
P u-241 \\
P u-242 \\
\text { Am-241 } \\
\mathrm{FP} \\
0-16 \\
\mathrm{Na}-23 \\
\text { SS }-316\end{array}$ & $\begin{array}{l}1.0840 \mathrm{E}-05 \\
4.4499 \mathrm{E}-03 \\
1.7520 \mathrm{E}-03 \\
2.2340 \mathrm{E}-04 \\
1.9280 \mathrm{E}-05 \\
4.7430 \mathrm{E}-06 \\
7.6680 \mathrm{E}-07 \\
0.0000 \mathrm{E}+00 \\
1.2670 \mathrm{E}-02 \\
9.8100 \mathrm{E}-03 \\
1.8990 \mathrm{E}-02\end{array}$ & $\begin{array}{l}1.0237 \mathrm{E}-05 \\
4.4156 \mathrm{E}-03 \\
1.6819 \mathrm{E}-03 \\
2.3528 \mathrm{E}-04 \\
2.0028 \mathrm{E}-05 \\
4.7986 \mathrm{E}-06 \\
9.8128 \mathrm{E}-07 \\
9.1922 \mathrm{E}-05 \\
1.2670 \mathrm{E}-02 \\
9.8100 \mathrm{E}-03 \\
1.8990 \mathrm{E}-02\end{array}$ \\
\hline 6 & -15.38 & 0.00 & $\begin{array}{l}U-235 \\
U-238 \\
P u-239 \\
P u-240 \\
P u-241 \\
P u-242 \\
A m-241 \\
F P \\
O-16 \\
\mathrm{Na}-23 \\
\text { SS }-316\end{array}$ & $\begin{array}{l}1.0840 \mathrm{E}-05 \\
4.4499 \mathrm{E}-03 \\
1.7520 \mathrm{E}-03 \\
2.2340 \mathrm{E}-04 \\
1.9280 \mathrm{E}-05 \\
4.7430 \mathrm{E}-06 \\
7.6680 \mathrm{E}-07 \\
0.0000 \mathrm{E}+00 \\
1.2670 \mathrm{E}-02 \\
9.8100 \mathrm{E}-03 \\
1.8990 \mathrm{E}-02\end{array}$ & $\begin{array}{l}1.0171 \mathrm{E}-05 \\
4.4117 \mathrm{E}-03 \\
1.6739 \mathrm{E}-03 \\
2.3648 \mathrm{E}-04 \\
2.0134 \mathrm{E}-05 \\
4.8045 \mathrm{E}-06 \\
9.7771 \mathrm{E}-07 \\
1.0248 \mathrm{E}-04 \\
1.2670 \mathrm{E}-02 \\
9.8100 \mathrm{E}-03 \\
1.8990 \mathrm{E}-02\end{array}$ \\
\hline
\end{tabular}


HNF-SD-FF-ANAL-009 Rev. 1

7

0.00

15.38

8

15.38

30.76

$\begin{array}{ll}\text { U-235 } & 1.0840 \mathrm{E}-05 \\ \mathrm{U}-238 & 4.4499 \mathrm{E}-03 \\ \text { Pu-239 } & 1.7520 \mathrm{E}-03 \\ \mathrm{Pu}-240 & 2.2340 \mathrm{E}-04 \\ \mathrm{Pu}-241 & 1.9280 \mathrm{E}-05 \\ \mathrm{Pu}-242 & 4.7430 \mathrm{E}-06 \\ \mathrm{Am}-241 & 7.6680 \mathrm{E}-07 \\ \text { FP } & 0.0000 \mathrm{E}+00 \\ \text { O-16 } & 1.2670 \mathrm{E}-02 \\ \mathrm{Na}-23 & 9.8100 \mathrm{E}-03 \\ \text { SS-316 } & 1.8990 \mathrm{E}-02\end{array}$

1.0192E-05

4.4130E-03

$1.6764 \mathrm{E}-03$

2. 3603E-04

2.0094E-05

4.8021E-06

$9.7885 \mathrm{E}-07$

$9.9159 \mathrm{E}-05$

$1.2670 \mathrm{E}-02$

$9.8100 \mathrm{E}-03$

1.8990E-02

$\begin{array}{ll}\text { U-235 } & 1.0840 E-05 \\ \text { U-238 } & 4.4499 E-03 \\ \text { Pu-239 } & 1.7520 E-03 \\ \text { Pu-240 } & 2.2340 E-04 \\ \text { Pu-241 } & 1.9280 E-05 \\ \text { Pu-242 } & 4.7430 E-06 \\ \text { Am-241 } & 7.6680 E-07 \\ \text { FP } & 0.0000 E+00 \\ \text { O-16 } & 1.2670 E-02 \\ \mathrm{Na}-23 & 9.8100 E-03 \\ \text { SS-316 } & 1.8990 E-02\end{array}$

$1.0297 \mathrm{E}-05$

4. 4190E-03

$1.6888 \mathrm{E}-03$

2.3404E-04

$1.9917 \mathrm{E}-05$

4.7919E-06

9.8439E-07

8.2964E-05

1.2670E-02

$9.8100 \mathrm{E}-03$

1. $8990 \mathrm{E}-02$

$9 \quad 30.76$

46.14

U-235 $\quad 1.0840 E-05$

1.0441E-05

$\mathrm{U}-238$

4. 4499E-03

$4.4277 \mathrm{E}-03$

$\mathrm{Pu}-239$

1. $7520 \mathrm{E}-03$

$1.7067 \mathrm{E}-03$

$\mathrm{Pu}-240 \quad 2.2340 \mathrm{E}-04$

2. 3204E-04

$\mathrm{Pu}-241$

1. $9280 \mathrm{E}-05$

$1.9774 \mathrm{E}-05$

$\mathrm{Pu}-242$

4. $7430 \mathrm{E}-06$

Am-241

$7.6680 \mathrm{E}-07$

FP

$0.0000 \mathrm{E}+00$

4.7859E-06

$0-16$

1.2670E-02

9.9251E-07

$\mathrm{Na}-23$

$9.8100 \mathrm{E}-03$

$5.8275 \mathrm{E}-05$

$1.2670 \mathrm{E}-02$

SS -316

1. $8990 \mathrm{E}-02$

$9.8100 \mathrm{E}-03$

$1.8990 \mathrm{E}-02$

10

46.14

62.78

U-235 $\quad 1.4143 \mathrm{E}-05$

1. 3681E-05

$\mathrm{U}-238$

$7.0573 \mathrm{E}-03$

$7.0332 \mathrm{E}-03$

$\mathrm{Pu}-239$

$0.0000 \mathrm{E}+00$

$2.2166 \mathrm{E}-05$

$\mathrm{Pu}-240$

$0.0000 \mathrm{E}+00$

9.8597E-08

FP

$0-16$

$0.0000 \mathrm{E}+00$

$2.1588 \mathrm{E}-06$

$\mathrm{Na}-23$

$1.4143 \mathrm{E}-02$

$1.4143 \mathrm{E}-02$

SS-316

$9.7416 \mathrm{E}-03$

9.7416E-03

1. $9073 \mathrm{E}-02$

$11 \quad 62.78 \quad 172.84$

$\begin{array}{lll}\mathrm{Na}-23 & 8.2400 \mathrm{E}-03 & 8.2400 \mathrm{E}-03 \\ \mathrm{SS}-316 & 2.5562 \mathrm{E}-02 & 2.5562 \mathrm{E}-02\end{array}$


Table E1OA-3.18. Composition of MFA-2 at EOC 10A-3

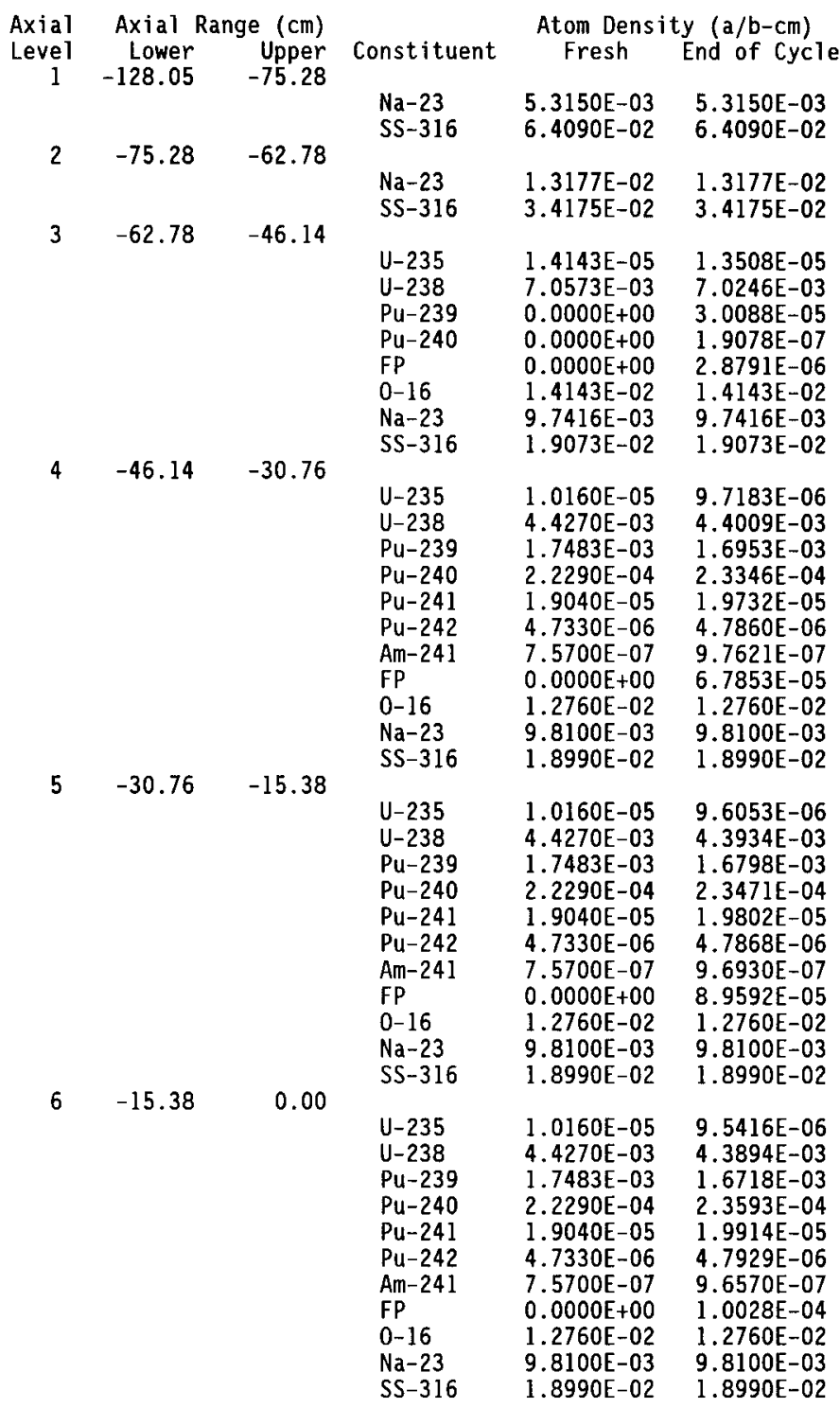


HNF-SD-FF-ANAL-009 Rev. 1

7

0.00

15.38

$\begin{array}{ll}\text { U-235 } & 1.0160 \mathrm{E}-05 \\ \mathrm{U}-238 & 4.4270 \mathrm{E}-03 \\ \text { Pu-239 } & 1.7483 \mathrm{E}-03 \\ \text { Pu-240 } & 2.2290 \mathrm{E}-04 \\ \text { Pu-241 } & 1.9040 \mathrm{E}-05 \\ \text { Pu-242 } & 4.7330 \mathrm{E}-06 \\ \text { Am-241 } & 7.5700 \mathrm{E}-07 \\ \text { FP } & 0.0000 \mathrm{E}+00 \\ 0-16 & 1.2760 \mathrm{E}-02 \\ \mathrm{Na}-23 & 9.8100 \mathrm{E}-03 \\ \text { SS-316 } & 1.8990 \mathrm{E}-02\end{array}$

30.76

8

15.38

30.76

46.14

$\begin{array}{ll}\text { U-235 } & 1.0160 \mathrm{E}-05 \\ \mathrm{U}-238 & 4.4270 \mathrm{E}-03 \\ \mathrm{Pu}-239 & 1.7483 \mathrm{E}-03 \\ \mathrm{Pu}-240 & 2.2290 \mathrm{E}-04 \\ \mathrm{Pu}-241 & 1.9040 \mathrm{E}-05 \\ \mathrm{Pu}-242 & 4.7330 \mathrm{E}-06 \\ \mathrm{Am}-241 & 7.5700 \mathrm{E}-07 \\ \mathrm{FP} & 0.0000 \mathrm{E}+00 \\ \mathrm{O}-16 & 1.2760 \mathrm{E}-02 \\ \mathrm{Na}-23 & 9.8100 \mathrm{E}-03 \\ \mathrm{SS}-316 & 1.8990 \mathrm{E}-02\end{array}$

$-316$

$\begin{array}{ll}\text { U-235 } & 1.0160 \mathrm{E}-05 \\ \mathrm{U}-238 & 4.4270 \mathrm{E}-03 \\ \text { Pu-239 } & 1.7483 \mathrm{E}-03 \\ \mathrm{Pu}-240 & 2.2290 \mathrm{E}-04 \\ \mathrm{Pu}-241 & 1.9040 \mathrm{E}-05 \\ \mathrm{Pu}-242 & 4.7330 \mathrm{E}-06 \\ \mathrm{Am}-241 & 7.5700 \mathrm{E}-07 \\ \mathrm{FP} & 0.0000 \mathrm{E}+00 \\ 0-16 & 1.2760 \mathrm{E}-02 \\ \mathrm{Na}-23 & 9.8100 \mathrm{E}-03 \\ \mathrm{SS}-316 & 1.8990 \mathrm{E}-02\end{array}$

62.78

10

46.14

11

$\begin{array}{ll}\text { U-235 } & 1.4143 \mathrm{E}-05 \\ \mathrm{U}-238 & 7.0573 \mathrm{E}-03 \\ \mathrm{PU}-239 & 0.0000 \mathrm{E}+00 \\ \mathrm{PU}-240 & 0.0000 \mathrm{E}+00 \\ \mathrm{FP} & 0.0000 \mathrm{E}+00 \\ 0-16 & 1.4143 \mathrm{E}-02 \\ \mathrm{Na}-23 & 9.7416 \mathrm{E}-03 \\ \mathrm{SS}-316 & 1.9073 \mathrm{E}-02\end{array}$

$\mathrm{Na}-23$

SS-316
8. 2400E-03

2.5562E-02
9.5603E-06

$4.3906 \mathrm{E}-03$

$1.6740 \mathrm{E}-03$

2.3553E-04

$1.9876 \mathrm{E}-05$

$4.7907 \mathrm{E}-06$

9.6671E-07

9.7279E-05

$1.2760 \mathrm{E}-02$

$9.8100 \mathrm{E}-03$

$1.8990 \mathrm{E}-02$

9. $6540 \mathrm{E}-06$

4. 3964E-03

$1.6858 \mathrm{E}-03$

2.3363E-04

$1.9705 \mathrm{E}-05$

4.7813E-06

9.7198E-07

8.1783E-05

1. $2760 \mathrm{E}-02$

$9.8100 \mathrm{E}-03$

$1.8990 \mathrm{E}-02$

$9.7874 \mathrm{E}-06$

4. $4049 \mathrm{E}-03$

$1.7035 \mathrm{E}-03$

2.3166E-04

$1.9557 \mathrm{E}-05$

$4.7755 \mathrm{E}-06$

$9.7988 \mathrm{E}-07$

5.7644E-05

1. $2760 \mathrm{E}-02$

$9.8100 \mathrm{E}-03$

1.8990E-02

1.3691E-05

$7.0334 \mathrm{E}-03$

2. $1955 \mathrm{E}-05$

$9.5959 \mathrm{E}-08$

2. $1603 \mathrm{E}-06$

$1.4143 \mathrm{E}-02$

$9.7416 \mathrm{E}-03$

1.9073E-02

8. $2400 \mathrm{E}-03$

2.5562E-02 


\subsection{Cycle 10A-4}

Table B10A-4.1. Fission Power Generated in MFA-1, MFA-2 and Neighboring Assemblies at BOC 10A-4

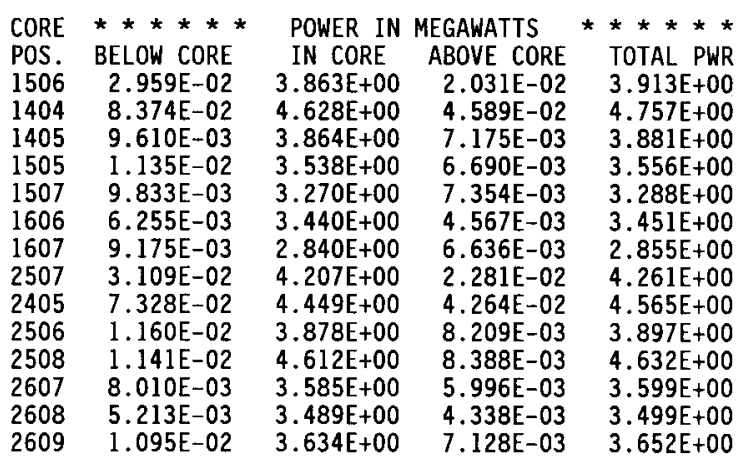

Table B10A-4.2. Assembly Averaged Total and Fast Flux in MFA-1 and MFA-2 at BOC 10A-4

\begin{tabular}{|c|c|c|}
\hline & Core & Flux $\left(\mathrm{n} / \mathrm{cm}^{2}-\mathrm{sec}\right)$ \\
\hline $\begin{array}{l}\text { Assembly } \\
\text { MFA-1 } \\
\text { MFA-2 }\end{array}$ & $\begin{array}{l}\text { Pos. } \\
1506 \\
2507\end{array}$ & $\begin{array}{l}>0.1 \mathrm{MeV} \\
1.844 \mathrm{E}+15 \\
2.004 \mathrm{E}+15\end{array}$ \\
\hline
\end{tabular}


Table BlOA-4.3. Axial Distribution of Total Flux, Fast Flux and Power in MFA-1 at BOC 10A-4

$\begin{array}{rccc}\text { z }(\mathrm{cm}) & \text { Total Flux } & \text { Flux }>0.1 \text { MeV } & \text { Power } \\ -97.78 & 2.7595 \mathrm{E}-02 & 1.3987 \mathrm{E}-02 & 0.0000 \mathrm{E}+00 \\ -92.78 & 5.9310 \mathrm{E}-02 & 2.7007 \mathrm{E}-02 & 0.0000 \mathrm{E}+00 \\ -87.78 & 9.5095 \mathrm{E}-02 & 4.3215 \mathrm{E}-02 & 0.0000 \mathrm{E}+00 \\ -82.78 & 1.3765 \mathrm{E}-01 & 6.4455 \mathrm{E}-02 & 0.0000 \mathrm{E}+00 \\ -77.78 & 1.8968 \mathrm{E}-01 & 9.3017 \mathrm{E}-02 & 0.0000 \mathrm{E}+00 \\ -72.15 & 2.4851 \mathrm{E}-01 & 1.2993 \mathrm{E}-01 & 0.0000 \mathrm{E}+00 \\ -65.90 & 3.0691 \mathrm{E}-01 & 1.7628 \mathrm{E}-01 & 0.0000 \mathrm{E}+00 \\ -60.35 & 3.6841 \mathrm{E}-01 & 2.3856 \mathrm{E}-01 & 2.8315 \mathrm{E}-02 \\ -55.49 & 4.5020 \mathrm{E}-01 & 3.2302 \mathrm{E}-01 & 3.7039 \mathrm{E}-02 \\ -50.62 & 5.4584 \mathrm{E}-01 & 4.3389 \mathrm{E}-01 & 5.1220 \mathrm{E}-02 \\ -47.17 & 6.2322 \mathrm{E}-01 & 5.3942 \mathrm{E}-01 & 6.8216 \mathrm{E}-02 \\ -43.58 & 7.0484 \mathrm{E}-01 & 6.5960 \mathrm{E}-01 & 7.4599 \mathrm{E}-01 \\ -38.45 & 8.2181 \mathrm{E}-01 & 8.0374 \mathrm{E}-01 & 8.3666 \mathrm{E}-01 \\ -33.32 & 9.2983 \mathrm{E}-01 & 9.2595 \mathrm{E}-01 & 9.3643 \mathrm{E}-01 \\ -28.20 & 1.0239 \mathrm{E}+00 & 1.0272 \mathrm{E}+00 & 1.0192 \mathrm{E}+00 \\ -23.07 & 1.1021 \mathrm{E}+00 & 1.1093 \mathrm{E}+00 & 1.0953 \mathrm{E}+00 \\ -17.94 & 1.1629 \mathrm{E}+00 & 1.1720 \mathrm{E}+00 & 1.1548 \mathrm{E}+00 \\ -12.82 & 1.2054 \mathrm{E}+00 & 1.2151 \mathrm{E}+00 & 1.1917 \mathrm{E}+00 \\ -7.69 & 1.2295 \mathrm{E}+00 & 1.2396 \mathrm{E}+00 & 1.2152 \mathrm{E}+00 \\ -2.56 & 1.2350 \mathrm{E}+00 & 1.2452 \mathrm{E}+00 & 1.2204 \mathrm{E}+00 \\ 2.56 & 1.2218 \mathrm{E}+00 & 1.2323 \mathrm{E}+00 & 1.2088 \mathrm{E}+00 \\ 7.69 & 1.1903 \mathrm{E}+00 & 1.2009 \mathrm{E}+00 & 1.1776 \mathrm{E}+00 \\ 12.82 & 1.1412 \mathrm{E}+00 & 1.1519 \mathrm{E}+00 & 1.1290 \mathrm{E}+00 \\ 17.94 & 1.0759 \mathrm{E}+00 & 1.0865 \mathrm{E}+00 & 1.0712 \mathrm{E}+00 \\ 23.07 & 9.9562 \mathrm{E}-01 & 1.0049 \mathrm{E}+00 & 9.9181 \mathrm{E}-01 \\ 28.20 & 9.0249 \mathrm{E}-01 & 9.0877 \mathrm{E}-01 & 9.0007 \mathrm{E}-01 \\ 33.32 & 7.9887 \mathrm{E}-01 & 7.9953 \mathrm{E}-01 & 8.0638 \mathrm{E}-01 \\ 38.45 & 6.8706 \mathrm{E}-01 & 6.7678 \mathrm{E}-01 & 6.9947 \mathrm{E}-01 \\ 43.58 & 5.7143 \mathrm{E}-01 & 5.4065 \mathrm{E}-01 & 6.0009 \mathrm{E}-01 \\ 47.17 & 4.9264 \mathrm{E}-01 & 4.3380 \mathrm{E}-01 & 5.1742 \mathrm{E}-02 \\ 50.62 & 4.1825 \mathrm{E}-01 & 3.4020 \mathrm{E}-01 & 3.6926 \mathrm{E}-02 \\ 55.49 & 3.2674 \mathrm{E}-01 & 2.4234 \mathrm{E}-01 & 2.4441 \mathrm{E}-02 \\ 60.35 & 2.4774 \mathrm{E}-01 & 1.6782 \mathrm{E}-01 & 1.6592 \mathrm{E}-02 \\ 64.98 & 1.9943 \mathrm{E}-01 & 1.2549 \mathrm{E}-01 & 0.0000 \mathrm{E}+00 \\ 69.39 & 1.7128 \mathrm{E}-01 & 1.0332 \mathrm{E}-01 & 0.0000 \mathrm{E}+00 \\ 76.46 & 1.3099 \mathrm{E}-01 & 7.3607 \mathrm{E}-02 & 0.0000 \mathrm{E}+00 \\ 86.80 & 8.5984 \mathrm{E}-02 & 4.4584 \mathrm{E}-02 & 0.0000 \mathrm{E}+00 \\ 98.14 & 4.9848 \mathrm{E}-02 & 2.4457 \mathrm{E}-02 & 0.0000 \mathrm{E}+00 \\ 110.00 & 2.1429 \mathrm{E}-02 & 1.0999 \mathrm{E}-02 & 0.0000 \mathrm{E}+00\end{array}$


Table B10A-4.4. Axial Distribution of Total Flux, Fast Flux and Power in MFA-2 at BOC 10A-4

$\begin{array}{rccc}z(\mathrm{~cm}) & \text { Total Flux } & \text { Flux }>0.1 \text { MeV } & \text { Power } \\ -97.78 & 2.5060 \mathrm{E}-02 & 1.2741 \mathrm{E}-02 & 0.0000 \mathrm{E}+00 \\ -92.78 & 5.3832 \mathrm{E}-02 & 2.4595 \mathrm{E}-02 & 0.0000 \mathrm{E}+00 \\ -87.78 & 8.6354 \mathrm{E}-02 & 3.9368 \mathrm{E}-02 & 0.0000 \mathrm{E}+00 \\ -82.78 & 1.2523 \mathrm{E}-01 & 5.8792 \mathrm{E}-02 & 0.0000 \mathrm{E}+00 \\ -77.78 & 1.7333 \mathrm{E}-01 & 8.5065 \mathrm{E}-02 & 0.0000 \mathrm{E}+00 \\ -72.15 & 2.2887 \mathrm{E}-01 & 1.1941 \mathrm{E}-01 & 0.0000 \mathrm{E}+00 \\ -65.90 & 2.8629 \mathrm{E}-01 & 1.6365 \mathrm{E}-01 & 0.0000 \mathrm{E}+00 \\ -60.35 & 3.4853 \mathrm{E}-01 & 2.2408 \mathrm{E}-01 & 2.6713 \mathrm{E}-02 \\ -55.49 & 4.3044 \mathrm{E}-01 & 3.0680 \mathrm{E}-01 & 3.5515 \mathrm{E}-02 \\ -50.62 & 5.2667 \mathrm{E}-01 & 4.1681 \mathrm{E}-01 & 4.9790 \mathrm{E}-02 \\ -47.17 & 6.0506 \mathrm{E}-01 & 5.2251 \mathrm{E}-01 & 6.6856 \mathrm{E}-02 \\ -43.58 & 6.8785 \mathrm{E}-01 & 6.4307 \mathrm{E}-01 & 7.3100 \mathrm{E}-01 \\ -38.45 & 8.0698 \mathrm{E}-01 & 7.8882 \mathrm{E}-01 & 8.2217 \mathrm{E}-01 \\ -33.32 & 9.1736 \mathrm{E}-01 & 9.1336 \mathrm{E}-01 & 9.2367 \mathrm{E}-01 \\ -28.20 & 1.0139 \mathrm{E}+00 & 1.0174 \mathrm{E}+00 & 1.0086 \mathrm{E}+00 \\ -23.07 & 1.0948 \mathrm{E}+00 & 1.1024 \mathrm{E}+00 & 1.0873 \mathrm{E}+00 \\ -17.94 & 1.1585 \mathrm{E}+00 & 1.1681 \mathrm{E}+00 & 1.1497 \mathrm{E}+00 \\ -12.82 & 1.2038 \mathrm{E}+00 & 1.2140 \mathrm{E}+00 & 1.1895 \mathrm{E}+00 \\ -7.69 & 1.2303 \mathrm{E}+00 & 1.2408 \mathrm{E}+00 & 1.2154 \mathrm{E}+00 \\ -2.56 & 1.2375 \mathrm{E}+00 & 1.2483 \mathrm{E}+00 & 1.2225 \mathrm{E}+00 \\ 2.56 & 1.2258 \mathrm{E}+00 & 1.2369 \mathrm{E}+00 & 1.2121 \mathrm{E}+00 \\ 7.69 & 1.1956 \mathrm{E}+00 & 1.2068 \mathrm{E}+00 & 1.1822 \mathrm{E}+00 \\ 12.82 & 1.1479 \mathrm{E}+00 & 1.1589 \mathrm{E}+00 & 1.1353 \mathrm{E}+00 \\ 17.94 & 1.0840 \mathrm{E}+00 & 1.0946 \mathrm{E}+00 & 1.0788 \mathrm{E}+00 \\ 23.07 & 1.0047 \mathrm{E}+00 & 1.0137 \mathrm{E}+00 & 1.0006 \mathrm{E}+00 \\ 28.20 & 9.1185 \mathrm{E}-01 & 9.1753 \mathrm{E}-01 & 9.0926 \mathrm{E}-01 \\ 33.32 & 8.0764 \mathrm{E}-01 & 8.0748 \mathrm{E}-01 & 8.1520 \mathrm{E}-01 \\ 38.45 & 6.9441 \mathrm{E}-01 & 6.8304 \mathrm{E}-01 & 7.0766 \mathrm{E}-01 \\ 43.58 & 5.7696 \mathrm{E}-01 & 5.4466 \mathrm{E}-01 & 6.0898 \mathrm{E}-01 \\ 47.17 & 4.9702 \mathrm{E}-01 & 4.3591 \mathrm{E}-01 & 5.3237 \mathrm{E}-02 \\ 50.62 & 4.2160 \mathrm{E}-01 & 3.4074 \mathrm{E}-01 & 3.8040 \mathrm{E}-02 \\ 55.49 & 3.2928 \mathrm{E}-01 & 2.4201 \mathrm{E}-01 & 2.5221 \mathrm{E}-02 \\ 60.35 & 2.4974 \mathrm{E}-01 & 1.6744 \mathrm{E}-01 & 1.7153 \mathrm{E}-02 \\ 64.98 & 2.0073 \mathrm{E}-01 & 1.2495 \mathrm{E}-01 & 0.0000 \mathrm{E}+00 \\ 69.39 & 1.7166 \mathrm{E}-01 & 1.0240 \mathrm{E}-01 & 0.0000 \mathrm{E}+00 \\ 76.46 & 1.3019 \mathrm{E}-01 & 7.2258 \mathrm{E}-02 & 0.0000 \mathrm{E}+00 \\ 86.80 & 8.4695 \mathrm{E}-02 & 4.3239 \mathrm{E}-02 & 0.0000 \mathrm{E}+00 \\ 98.14 & 4.8685 \mathrm{E}-02 & 2.3423 \mathrm{E}-02 & 0.0000 \mathrm{E}+00 \\ 110.00 & 2.0618 \mathrm{E}-02 & 1.0349 \mathrm{E}-02 & 0.0000 \mathrm{E}+00\end{array}$


HNF-SD-FF-ANAL-009 Rev. 1

Table B10A-4.5. Fission Power Distribution by Pin in MFA-1 at BOC 10A-4 CORE POSITION 1506

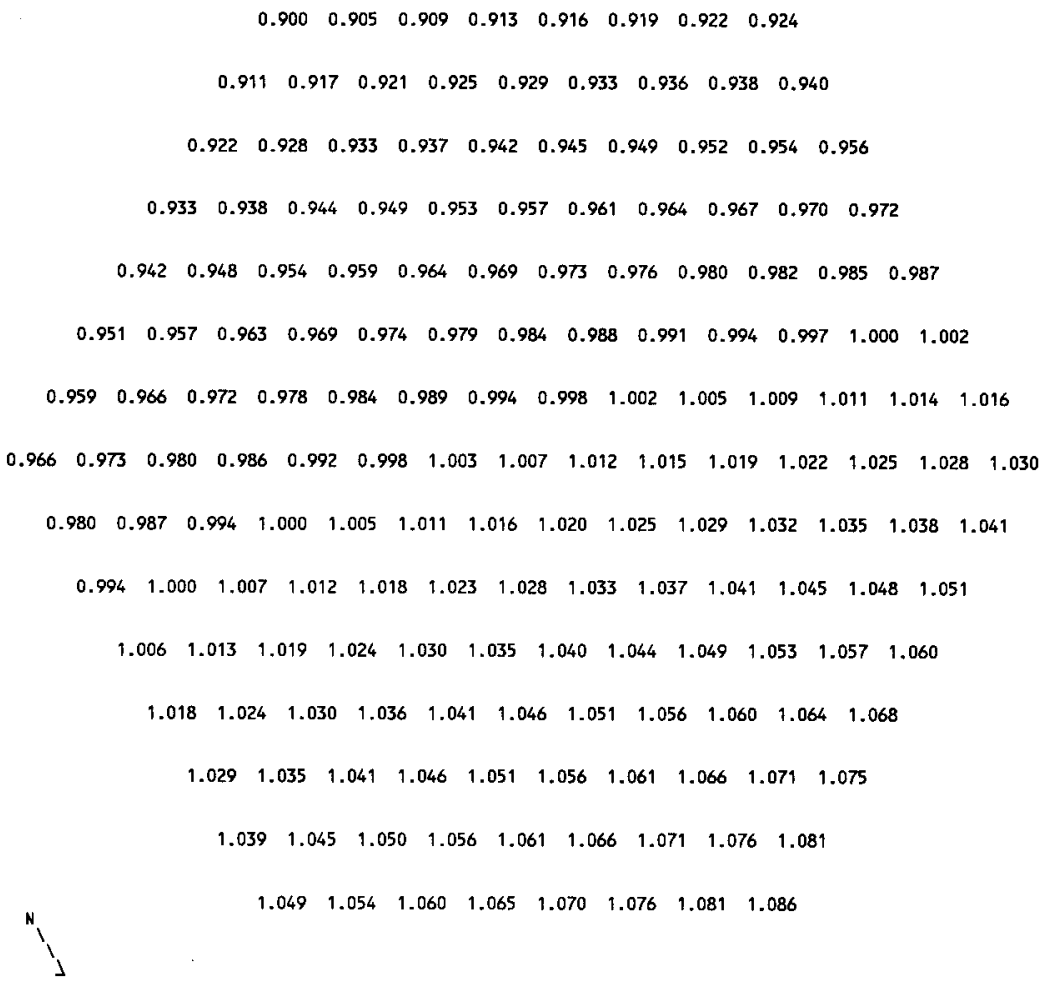


Table B10A-4.6. Fission Power Distribution by Pin in MFA-2 at BOC 10A-4 CORE POSITION 2507

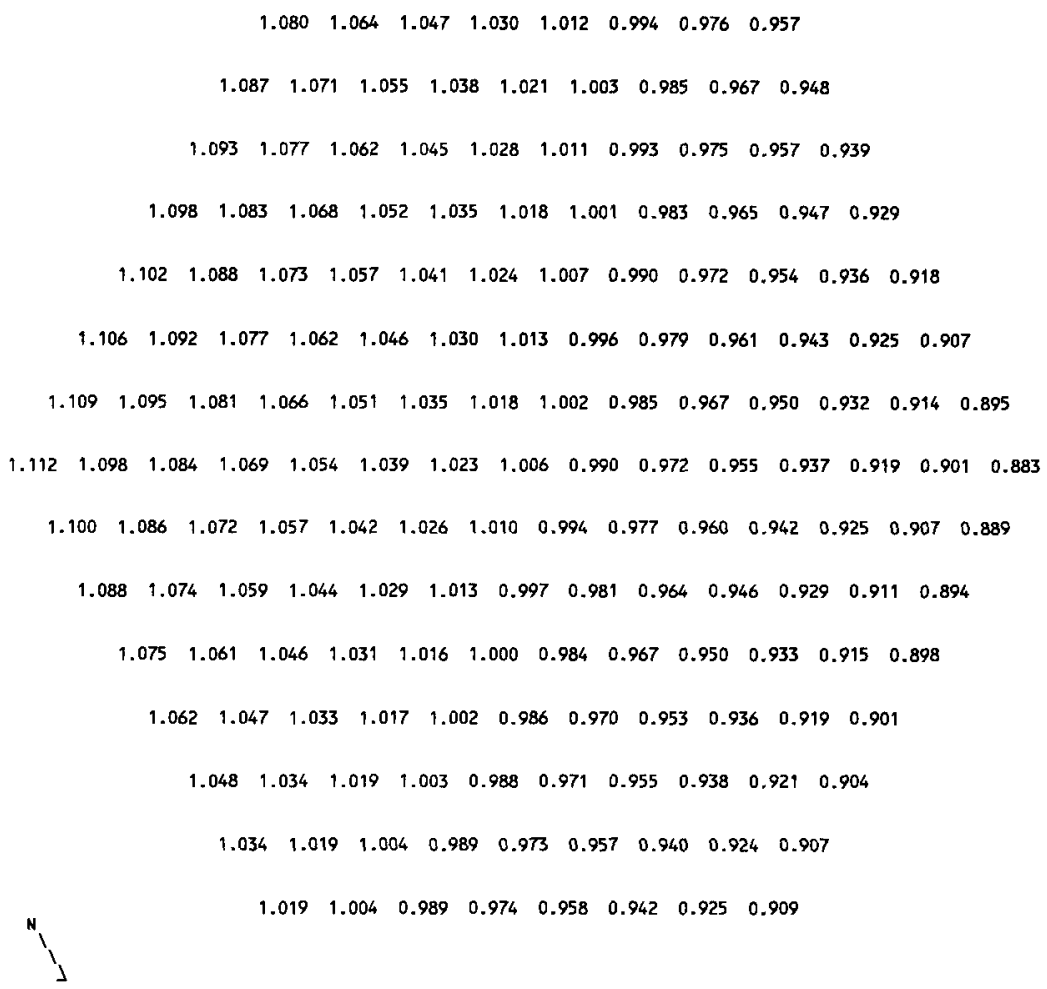


HNF-SD-FF-ANAL-009 Rev. 1

Table B10A-4.7. Fast Flux Distribution by Pin in MFA-1 at BOC 10A-4 CORE POSITION 1506

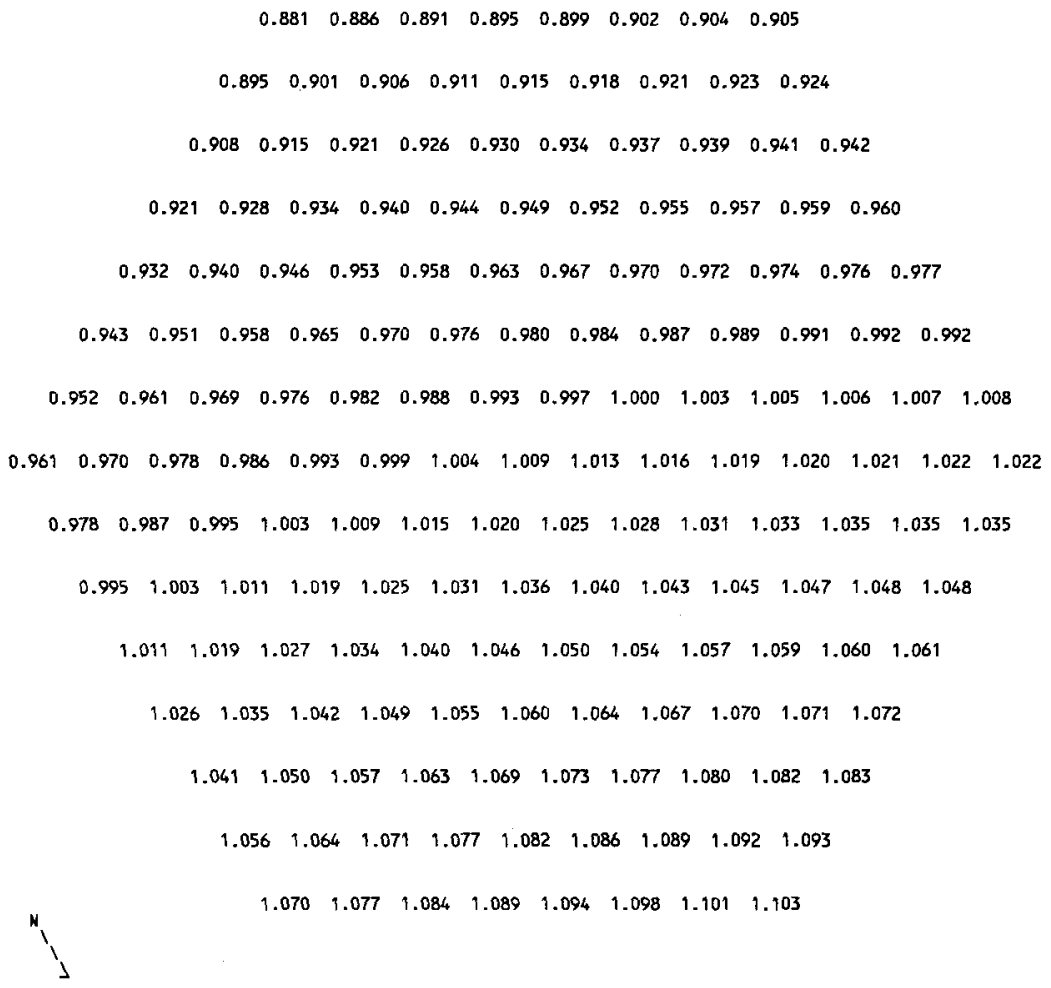


HNF-SD-FF-ANAL-009 Rev. 1

Table B10A-4.8. Fast Flux Distribution by Pin in MFA-2 at BOC 10A-4 CORE POSITION 2507

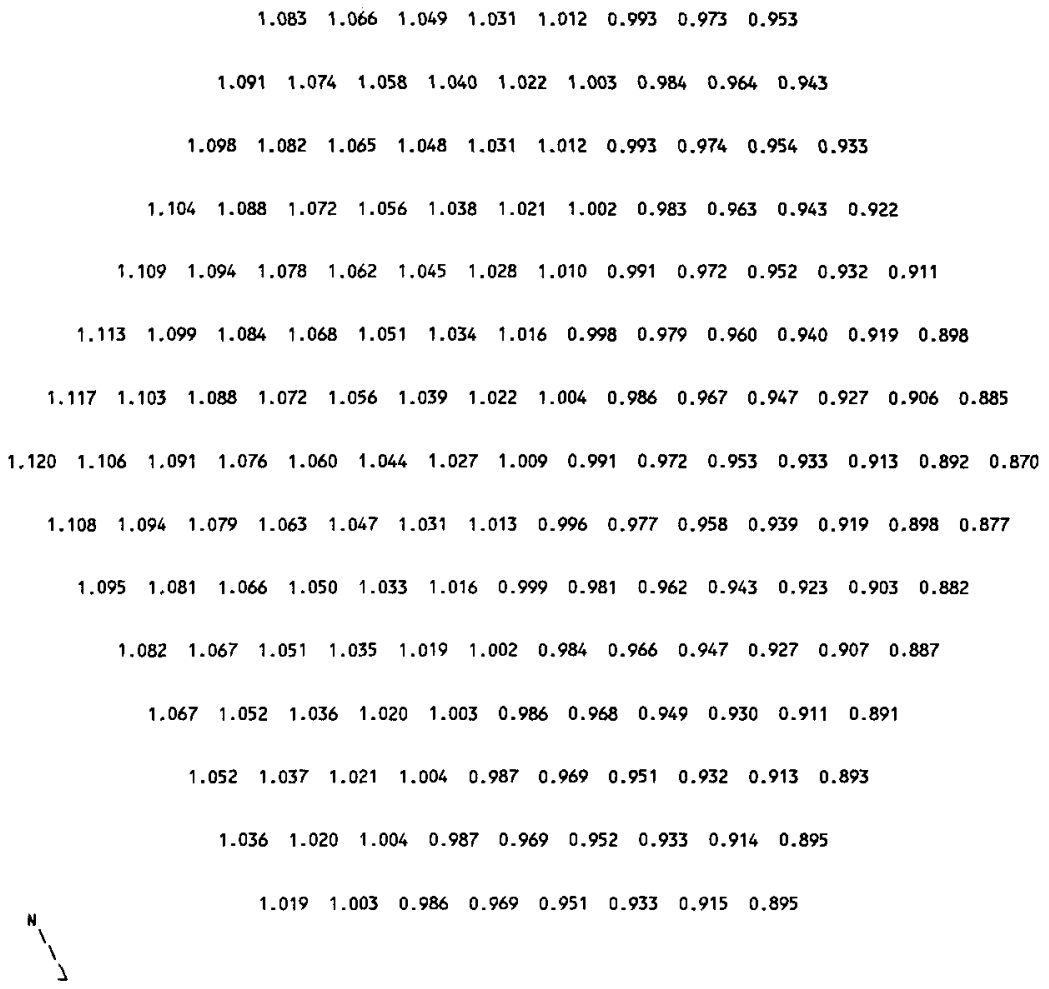


Table B1OA-4.9. MFA-1 and MFA-2 Duct Wall

Fast Flux Data at BOC 10A-4

$\begin{array}{ccc}\text { Side } & \begin{array}{c}\text { Flux }>0.1 \\ \text { MFA-1 }\end{array} & \begin{array}{c}\text { MeV } \\ \left(\mathrm{n} / \mathrm{cm}^{2}-\mathrm{sec}\right) \\ \text { MFA-2 }\end{array} \\ \text { E } & 1.8694 \mathrm{E}+15 & 2.1715 \mathrm{E}+15 \\ \text { SE } & 1.6702 \mathrm{E}+15 & 2.2491 \mathrm{E}+15 \\ \text { SW } & 1.6019 \mathrm{E}+15 & 2.0399 \mathrm{E}+15 \\ \text { W } & 1.7619 \mathrm{E}+15 & 1.7879 \mathrm{E}+15 \\ \text { NW } & 1.9794 \mathrm{E}+15 & 1.7228 \mathrm{E}+15 \\ \text { NE } & 2.0353 \mathrm{E}+15 & 1.8899 \mathrm{E}+15\end{array}$

Table BIOA-4.10. Assembly Outlet Temperatures and Flow Rates at BOC 10A-4

MEASURED

CORE OUTLET TEMP. FLOW RATE

POS. (DEG F) (LB/H)

$\begin{array}{lll}1506 & 1029 & 114940\end{array}$

$1404 \quad 914 \quad 225440$

$\begin{array}{lll}1405 & 889 & 208130\end{array}$

$\begin{array}{lll}1505 & 882 & 208130\end{array}$

$\begin{array}{lll}1507 & 872 & 208130\end{array}$

$1606 \quad 900 \quad 170680$

$\begin{array}{lll}1607 & 869 & 170680\end{array}$

$2507 \quad 1063 \quad 114940$

$2405 \quad 946 \quad 192410$

$2506 \quad 901 \quad 197920$

$2508 \quad 931 \quad 208130$

$2607 \quad 926 \quad 163690$

$2608 \quad 905 \quad 170680$

$\begin{array}{lll}2609 & 914 & 170680\end{array}$

Assembly flows are based on a calculated core pressure drop of $110.6 \mathrm{psi}$ at a total reactor flow rate of 16. $74 \mathrm{E}+06 \mathrm{lb} / \mathrm{hr}$. 
Table E10A-4.1. Fission Power Generated in MFA-1, MFA-2 and Neighboring Assemblies at EOC 10A-4

\begin{tabular}{lcccc} 
CORE & $* * * * * *$ & \multicolumn{2}{c}{ POWER IN MEGAWATTS } & $* * * * * *$ \\
POS. & BELOW CORE & IN CORE & ABOVE CORE & TOTAL PWR \\
1506 & $3.241 \mathrm{E}-02$ & $3.799 \mathrm{E}+00$ & $2.518 \mathrm{E}-02$ & $3.857 \mathrm{E}+00$ \\
1404 & $8.461 \mathrm{E}-02$ & $4.559 \mathrm{E}+00$ & $5.420 \mathrm{E}-02$ & $4.698 \mathrm{E}+00$ \\
1405 & $9.766 \mathrm{E}-03$ & $3.786 \mathrm{E}+00$ & $8.299 \mathrm{E}-03$ & $3.804 \mathrm{E}+00$ \\
1505 & $1.127 \mathrm{E}-02$ & $3.577 \mathrm{E}+00$ & $8.230 \mathrm{E}-03$ & $3.597 \mathrm{E}+00$ \\
1507 & $9.733 \mathrm{E}-03$ & $3.213 \mathrm{E}+00$ & $8.314 \mathrm{E}-03$ & $3.231 \mathrm{E}+00$ \\
1606 & $6.404 \mathrm{E}-03$ & $3.447 \mathrm{E}+00$ & $5.535 \mathrm{E}-03$ & $3.459 \mathrm{E}+00$ \\
1607 & $9.048 \mathrm{E}-03$ & $2.810 \mathrm{E}+00$ & $7.536 \mathrm{E}-03$ & $2.826 \mathrm{E}+00$ \\
2507 & $3.402 \mathrm{E}-02$ & $4.044 \mathrm{E}+00$ & $2.767 \mathrm{E}-02$ & $4.106 \mathrm{E}+00$ \\
2405 & $7.395 \mathrm{E}-02$ & $4.282 \mathrm{E}+00$ & $4.958 \mathrm{E}-02$ & $4.406 \mathrm{E}+00$ \\
2506 & $1.148 \mathrm{E}-02$ & $3.770 \mathrm{E}+00$ & $9.332 \mathrm{E}-03$ & $3.791 \mathrm{E}+00$ \\
2508 & $1.138 \mathrm{E}-02$ & $4.459 \mathrm{E}+00$ & $9.609 \mathrm{E}-03$ & $4.480 \mathrm{E}+00$ \\
2607 & $7.988 \mathrm{E}-03$ & $3.483 \mathrm{E}+00$ & $6.782 \mathrm{E}-03$ & $3.498 \mathrm{E}+00$ \\
2608 & $5.315 \mathrm{E}-03$ & $3.370 \mathrm{E}+00$ & $4.923 \mathrm{E}-03$ & $3.381 \mathrm{E}+00$ \\
2609 & $1.072 \mathrm{E}-02$ & $3.522 \mathrm{E}+00$ & $8.034 \mathrm{E}-03$ & $3.541 \mathrm{E}+00$
\end{tabular}

Table E10A-4.2. Assembly Averaged Total and Fast Flux in MFA-1 and MFA-2 at EOC $10 \mathrm{~A}-4$

\begin{tabular}{|c|c|c|}
\hline & Core & Flux $\left(\mathrm{n} / \mathrm{cm}^{2}-\mathrm{sec}\right)$ \\
\hline $\begin{array}{l}\text { Assembly } \\
\text { MFA-1 } \\
\text { MFA-2 }\end{array}$ & $\begin{array}{l}\text { Pos } \\
1506 \\
2507\end{array}$ & $\begin{array}{c}\text { Total } \\
2.869 \mathrm{E}+15 \\
3.051 \mathrm{E}+15\end{array}$ \\
\hline
\end{tabular}


Table E10A-4.3. Axial Distribution of Total Flux, Fast Flux and Power in MFA-1 at EOC 10A-4

$\begin{array}{rccc}\text { Z }(\mathrm{cm}) & \text { Tota1 Flux } & \text { Flux }>0.1 \mathrm{MeV} & \text { Power } \\ -97.78 & 2.6179 \mathrm{E}-02 & 1.3345 \mathrm{E}-02 & 0.0000 \mathrm{E}+00 \\ -92.78 & 5.6273 \mathrm{E}-02 & 2.5769 \mathrm{E}-02 & 0.0000 \mathrm{E}+00 \\ -87.78 & 9.0229 \mathrm{E}-02 & 4.1233 \mathrm{E}-02 & 0.0000 \mathrm{E}+00 \\ -82.78 & 1.3062 \mathrm{E}-01 & 6.1502 \mathrm{E}-02 & 0.0000 \mathrm{E}+00 \\ -77.78 & 1.8001 \mathrm{E}-01 & 8.8751 \mathrm{E}-02 & 0.0000 \mathrm{E}+00 \\ -72.15 & 2.3585 \mathrm{E}-01 & 1.2398 \mathrm{E}-01 & 0.0000 \mathrm{E}+00 \\ -65.90 & 2.9127 \mathrm{E}-01 & 1.6822 \mathrm{E}-01 & 0.0000 \mathrm{E}+00 \\ -60.35 & 3.4958 \mathrm{E}-01 & 2.2778 \mathrm{E}-01 & 3.3357 \mathrm{E}-02 \\ -55.49 & 4.2719 \mathrm{E}-01 & 3.0808 \mathrm{E}-01 & 4.2119 \mathrm{E}-02 \\ -50.62 & 5.1790 \mathrm{E}-01 & 4.1317 \mathrm{E}-01 & 5.5966 \mathrm{E}-02 \\ -47.17 & 5.9130 \mathrm{E}-01 & 5.1298 \mathrm{E}-01 & 7.2236 \mathrm{E}-02 \\ -43.58 & 6.6870 \mathrm{E}-01 & 6.2663 \mathrm{E}-01 & 7.1007 \mathrm{E}-01 \\ -38.45 & 7.8058 \mathrm{E}-01 & 7.6406 \mathrm{E}-01 & 7.9754 \mathrm{E}-01 \\ -33.32 & 8.8508 \mathrm{E}-01 & 8.8184 \mathrm{E}-01 & 8.9462 \mathrm{E}-01 \\ -28.20 & 9.7757 \mathrm{E}-01 & 9.8093 \mathrm{E}-01 & 9.7199 \mathrm{E}-01 \\ -23.07 & 1.0565 \mathrm{E}+00 & 1.0633 \mathrm{E}+00 & 1.0489 \mathrm{E}+00 \\ -17.94 & 1.1205 \mathrm{E}+00 & 1.1290 \mathrm{E}+00 & 1.1115 \mathrm{E}+00 \\ -12.82 & 1.1685 \mathrm{E}+00 & 1.1775 \mathrm{E}+00 & 1.1510 \mathrm{E}+00 \\ -7.69 & 1.2006 \mathrm{E}+00 & 1.2099 \mathrm{E}+00 & 1.1823 \mathrm{E}+00 \\ -2.56 & 1.2164 \mathrm{E}+00 & 1.2261 \mathrm{E}+00 & 1.1978 \mathrm{E}+00 \\ 2.56 & 1.2157 \mathrm{E}+00 & 1.2257 \mathrm{E}+00 & 1.1989 \mathrm{E}+00 \\ 7.69 & 1.1980 \mathrm{E}+00 & 1.2085 \mathrm{E}+00 & 1.1815 \mathrm{E}+00 \\ 12.82 & 1.1635 \mathrm{E}+00 & 1.1743 \mathrm{E}+00 & 1.1476 \mathrm{E}+00 \\ 17.94 & 1.1122 \mathrm{E}+00 & 1.1232 \mathrm{E}+00 & 1.1077 \mathrm{E}+00 \\ 23.07 & 1.0437 \mathrm{E}+00 & 1.0536 \mathrm{E}+00 & 1.0401 \mathrm{E}+00 \\ 28.20 & 9.5881 \mathrm{E}-01 & 9.6573 \mathrm{E}-01 & 9.5681 \mathrm{E}-01 \\ 33.32 & 8.5936 \mathrm{E}-01 & 8.6032 \mathrm{E}-01 & 8.7280 \mathrm{E}-01 \\ 38.45 & 7.4714 \mathrm{E}-01 & 7.3606 \mathrm{E}-01 & 7.6554 \mathrm{E}-01 \\ 43.58 & 6.2724 \mathrm{E}-01 & 5.9339 \mathrm{E}-01 & 6.6331 \mathrm{E}-01 \\ 47.17 & 5.4366 \mathrm{E}-01 & 4.7864 \mathrm{E}-01 & 6.2155 \mathrm{E}-02 \\ 50.62 & 4.6413 \mathrm{E}-01 & 3.7764 \mathrm{E}-01 & 4.5746 \mathrm{E}-02 \\ 55.49 & 3.6477 \mathrm{E}-01 & 2.7079 \mathrm{E}-01 & 3.1534 \mathrm{E}-02 \\ 60.35 & 2.7810 \mathrm{E}-01 & 1.8863 \mathrm{E}-01 & 2.2269 \mathrm{E}-02 \\ 64.98 & 2.2492 \mathrm{E}-01 & 1.4162 \mathrm{E}-01 & 0.0000 \mathrm{E}+00 \\ 69.39 & 1.9361 \mathrm{E}-01 & 1.1684 \mathrm{E}-01 & 0.0000 \mathrm{E}+00 \\ 76.46 & 1.4850 \mathrm{E}-01 & 8.3473 \mathrm{E}-02 & 0.0000 \mathrm{E}+00 \\ 86.80 & 9.7675 \mathrm{E}-02 & 5.0667 \mathrm{E}-02 & 0.0000 \mathrm{E}+00 \\ 98.14 & 5.6647 \mathrm{E}-02 & 2.7816 \mathrm{E}-02 & 0.0000 \mathrm{E}+00 \\ 110.00 & 2.4303 \mathrm{E}-02 & 1.2495 \mathrm{E}-02 & 0.0000 \mathrm{E}+00 \\ & & & \end{array}$


Table El0A-4.4. Axial Distribution of Total Flux, Fast Flux and Power in MFA-2 at EOC 10A-4

$\begin{array}{rccc}z(\mathrm{~cm}) & \text { Total Flux } & \text { Flux }>0.1 \text { MeV } & \text { Power } \\ -97.78 & 2.4099 \mathrm{E}-02 & 1.2324 \mathrm{E}-02 & 0.0000 \mathrm{E}+00 \\ -92.78 & 5.1768 \mathrm{E}-02 & 2.3786 \mathrm{E}-02 & 0.0000 \mathrm{E}+00 \\ -87.78 & 8.3038 \mathrm{E}-02 & 3.8064 \mathrm{E}-02 & 0.0000 \mathrm{E}+00 \\ -82.78 & 1.2041 \mathrm{E}-01 & 5.6826 \mathrm{E}-02 & 0.0000 \mathrm{E}+00 \\ -77.78 & 1.6662 \mathrm{E}-01 & 8.2190 \mathrm{E}-02 & 0.0000 \mathrm{E}+00 \\ -72.15 & 2.1997 \mathrm{E}-01 & 1.1533 \mathrm{E}-01 & 0.0000 \mathrm{E}+00 \\ -65.90 & 2.7501 \mathrm{E}-01 & 1.5801 \mathrm{E}-01 & 0.0000 \mathrm{E}+00 \\ -60.35 & 3.3448 \mathrm{E}-01 & 2.1636 \mathrm{E}-01 & 3.2407 \mathrm{E}-02 \\ -55.49 & 4.1279 \mathrm{E}-01 & 2.9572 \mathrm{E}-01 & 4.1384 \mathrm{E}-02 \\ -50.62 & 5.0478 \mathrm{E}-01 & 4.0091 \mathrm{E}-01 & 5.5481 \mathrm{E}-02 \\ -47.17 & 5.7957 \mathrm{E}-01 & 5.0173 \mathrm{E}-01 & 7.1958 \mathrm{E}-02 \\ -43.58 & 6.5863 \mathrm{E}-01 & 6.1665 \mathrm{E}-01 & 7.0285 \mathrm{E}-01 \\ -38.45 & 7.7316 \mathrm{E}-01 & 7.5659 \mathrm{E}-01 & 7.9120 \mathrm{E}-01 \\ -33.32 & 8.8034 \mathrm{E}-01 & 8.7714 \mathrm{E}-01 & 8.9029 \mathrm{E}-01 \\ -28.20 & 9.7536 \mathrm{E}-01 & 9.7901 \mathrm{E}-01 & 9.6944 \mathrm{E}-01 \\ -23.07 & 1.0568 \mathrm{E}+00 & 1.0642 \mathrm{E}+00 & 1.0486 \mathrm{E}+00 \\ -17.94 & 1.1232 \mathrm{E}+00 & 1.1322 \mathrm{E}+00 & 1.1136 \mathrm{E}+00 \\ -12.82 & 1.1732 \mathrm{E}+00 & 1.1828 \mathrm{E}+00 & 1.1549 \mathrm{E}+00 \\ -7.69 & 1.2067 \mathrm{E}+00 & 1.2164 \mathrm{E}+00 & 1.1876 \mathrm{E}+00 \\ -2.56 & 1.2228 \mathrm{E}+00 & 1.2329 \mathrm{E}+00 & 1.2033 \mathrm{E}+00 \\ 2.56 & 1.2215 \mathrm{E}+00 & 1.2320 \mathrm{E}+00 & 1.2037 \mathrm{E}+00 \\ 7.69 & 1.2027 \mathrm{E}+00 & 1.2136 \mathrm{E}+00 & 1.1852 \mathrm{E}+00 \\ 12.82 & 1.1667 \mathrm{E}+00 & 1.1777 \mathrm{E}+00 & 1.1499 \mathrm{E}+00 \\ 17.94 & 1.1137 \mathrm{E}+00 & 1.1246 \mathrm{E}+00 & 1.1085 \mathrm{E}+00 \\ 23.07 & 1.0436 \mathrm{E}+00 & 1.0531 \mathrm{E}+00 & 1.0395 \mathrm{E}+00 \\ 28.20 & 9.5740 \mathrm{E}-01 & 9.6365 \mathrm{E}-01 & 9.5492 \mathrm{E}-01 \\ 33.32 & 8.5680 \mathrm{E}-01 & 8.5695 \mathrm{E}-01 & 8.7025 \mathrm{E}-01 \\ 38.45 & 7.4381 \mathrm{E}-01 & 7.3177 \mathrm{E}-01 & 7.6302 \mathrm{E}-01 \\ 43.58 & 6.2360 \mathrm{E}-01 & 5.8861 \mathrm{E}-01 & 6.6320 \mathrm{E}-01 \\ 47.17 & 5.4045 \mathrm{E}-01 & 4.7374 \mathrm{E}-01 & 6.3544 \mathrm{E}-02 \\ 50.62 & 4.6149 \mathrm{E}-01 & 3.7284 \mathrm{E}-01 & 4.7019 \mathrm{E}-02 \\ 55.49 & 3.6344 \mathrm{E}-01 & 2.6709 \mathrm{E}-01 & 3.2682 \mathrm{E}-02 \\ 60.35 & 2.7806 \mathrm{E}-01 & 1.8643 \mathrm{E}-01 & 2.3303 \mathrm{E}-02 \\ 64.98 & 2.2528 \mathrm{E}-01 & 1.4009 \mathrm{E}-01 & 0.0000 \mathrm{E}+00 \\ 69.39 & 1.9351 \mathrm{E}-01 & 1.1529 \mathrm{E}-01 & 0.0000 \mathrm{E}+00 \\ 76.46 & 1.4766 \mathrm{E}-01 & 8.1858 \mathrm{E}-02 & 0.0000 \mathrm{E}+00 \\ 86.80 & 9.6566 \mathrm{E}-02 & 4.9259 \mathrm{E}-02 & 0.0000 \mathrm{E}+00 \\ 98.14 & 5.5677 \mathrm{E}-02 & 2.6783 \mathrm{E}-02 & 0.0000 \mathrm{E}+00 \\ 110.00 & 2.3593 \mathrm{E}-02 & 1.1851 \mathrm{E}-02 & 0.0000 \mathrm{E}+00\end{array}$


HNF-SD-FF-ANAL-009 Rev. 1

Table E1OA-4.5. Fission Power Distribution by Pin in MFA-1 at EOC 10A-4 CORE POSITION 1506

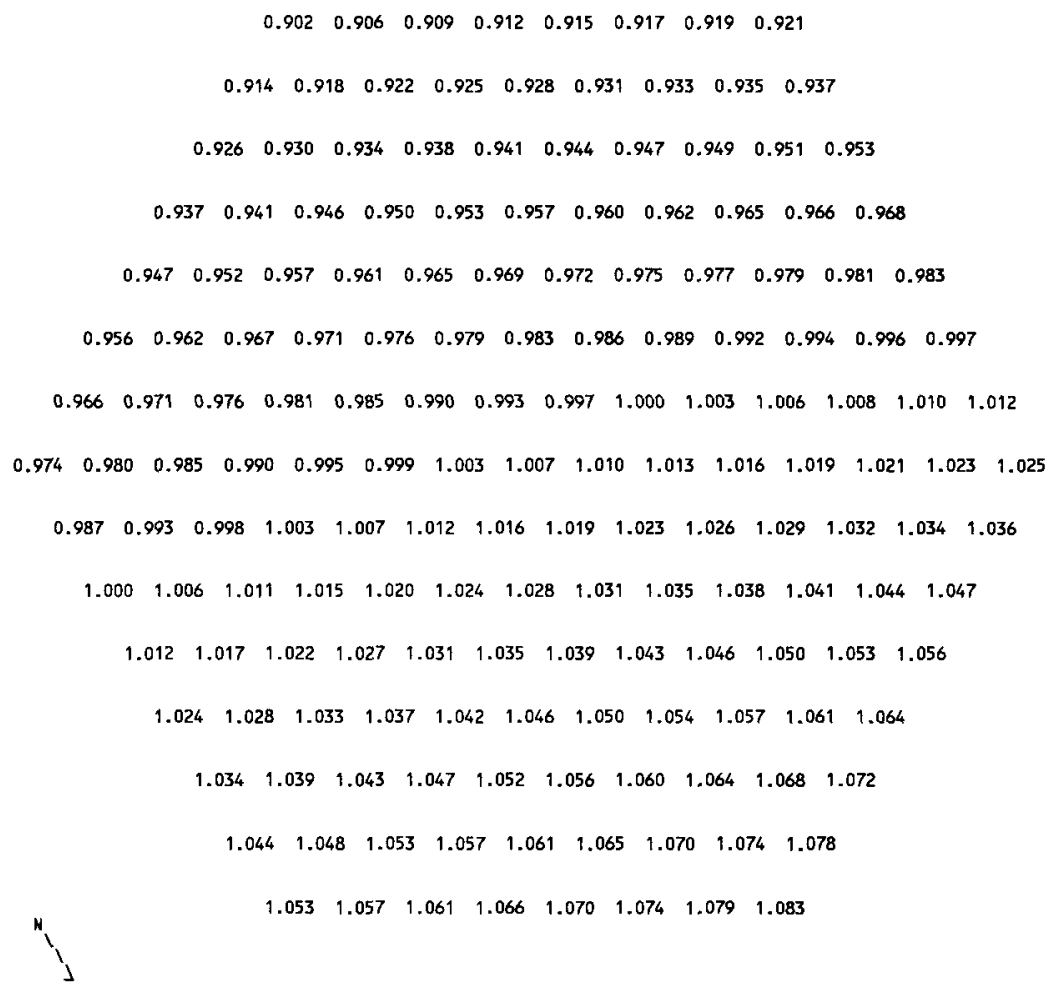


Table E1OA-4.6. Fission Power Distribution by Pin in MFA-2 at EOC 10A-4 CORE POSIIION 2507

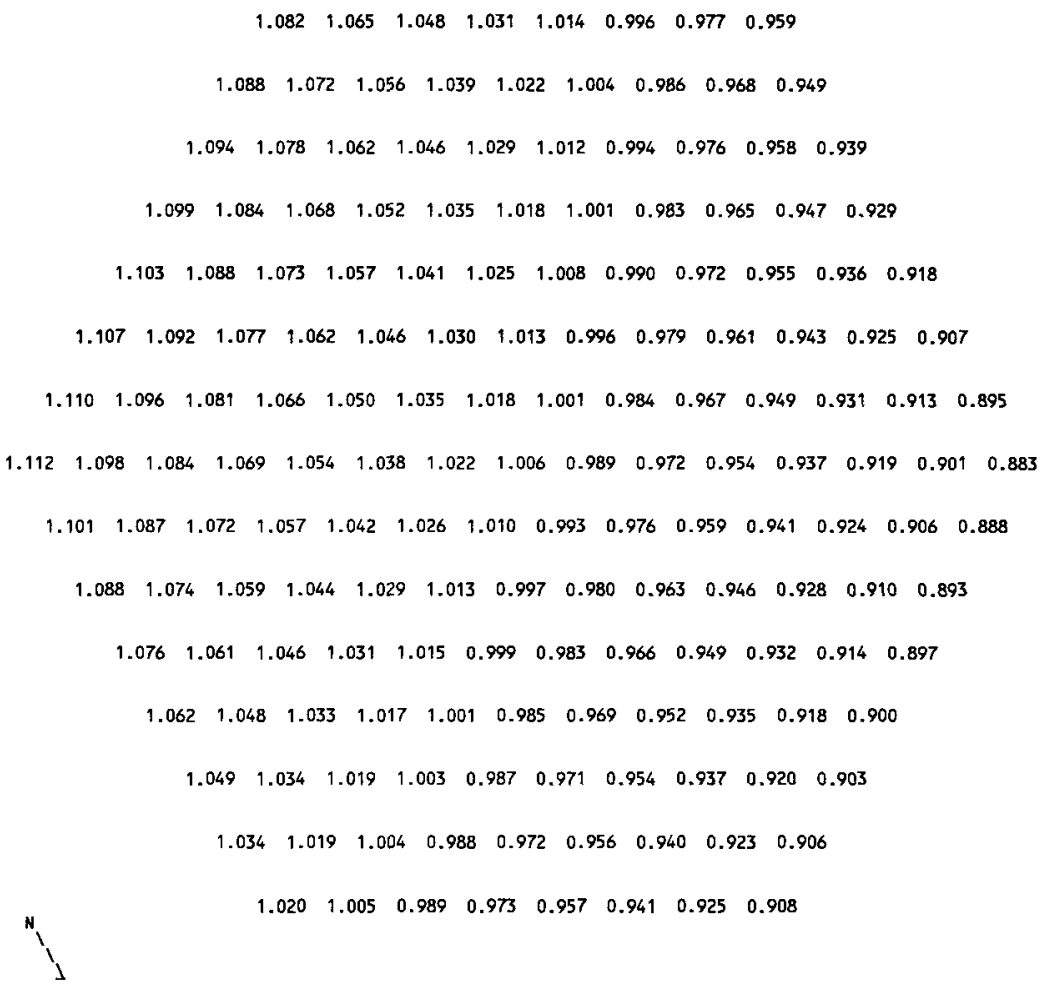


HNF-SD-FF-ANAL-009 Rev. 1

Table ElOA-4.7. Fast Flux Distribution by Pin in MFA-1 at EOC 10A-4 CORE POSITION 1506

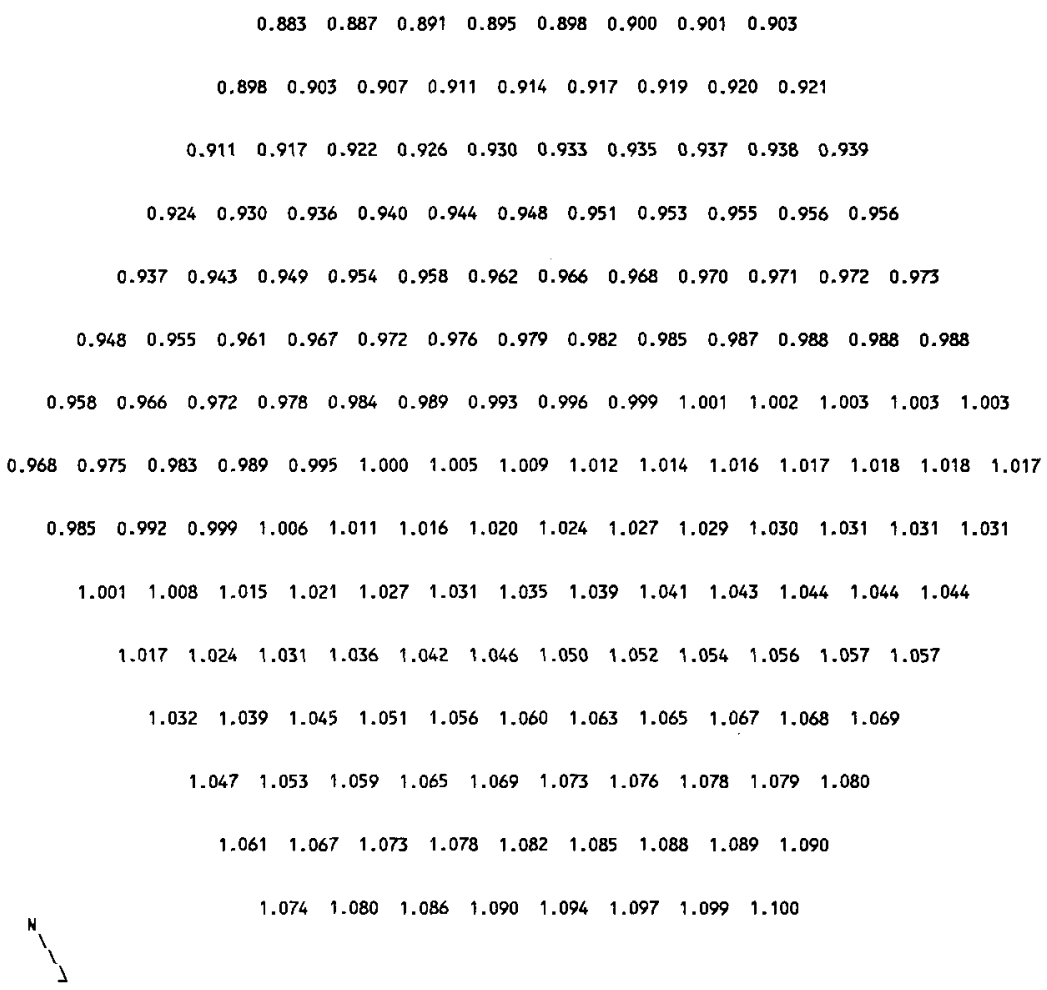


HNF-SD-FF-ANAL-009 Rev. 1

Table ElOA-4.8. Fast Flux Distribution by Pin in MFA-2 at EOC 10A-4 CORE POSITION 2507

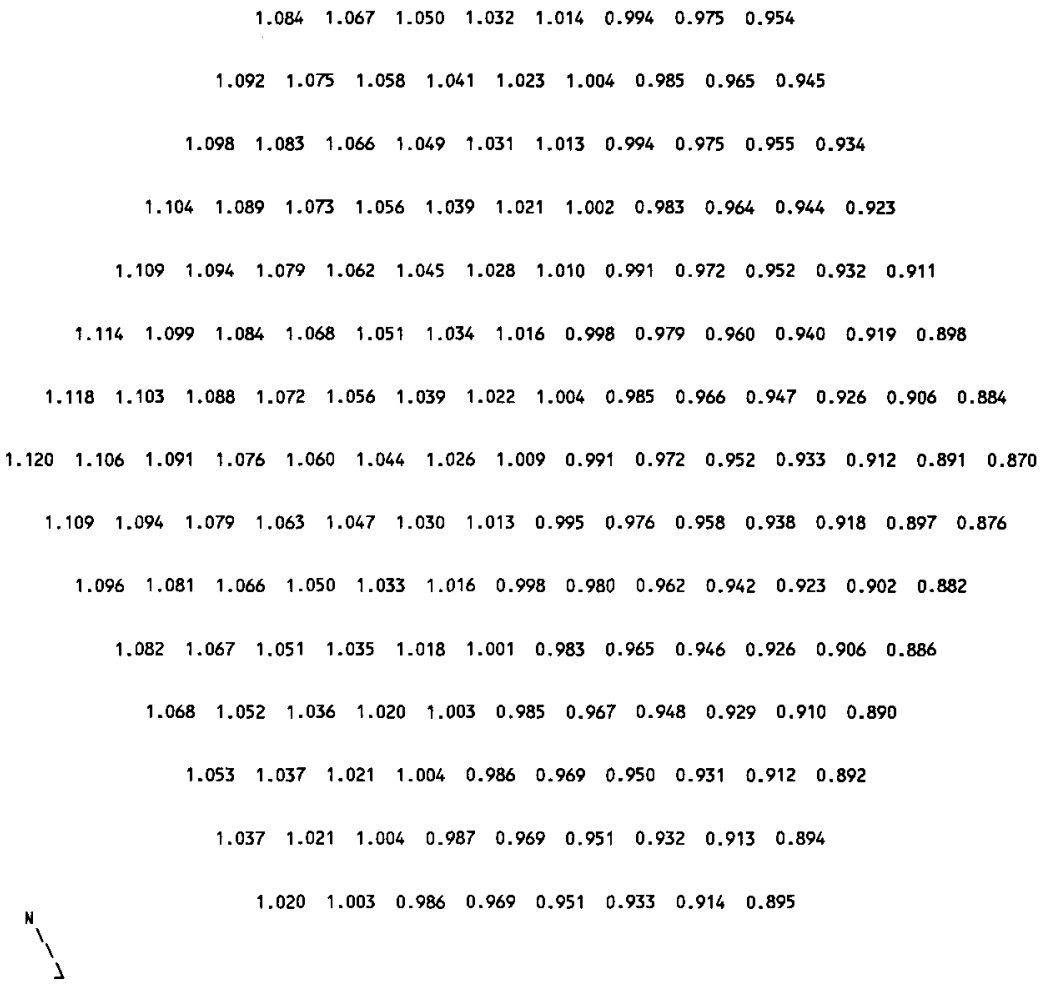


Table E10A-4.9. MFA-1 and MFA-2 Duct Wall

Fast Flux Data at EOC $10 \mathrm{~A}-4$

$\begin{array}{ccc}\text { Side } & \begin{array}{c}\text { Flux }>0.1 \\ \text { MFA-1 }\end{array} & \begin{array}{c}\text { MeV } \\ \left(\mathrm{n} / \mathrm{cm}^{2}-\mathrm{sec}\right) \\ \text { MFA-2 }\end{array} \\ \text { E } & 1.8777 \mathrm{E}+15 & 2.1200 \mathrm{E}+15 \\ \text { SE } & 1.6743 \mathrm{E}+15 & 2.1949 \mathrm{E}+15 \\ \text { SW } & 1.5952 \mathrm{E}+15 & 1.9935 \mathrm{E}+15 \\ \text { W } & 1.7491 \mathrm{E}+15 & 1.7451 \mathrm{E}+15 \\ \text { NW } & 1.9651 \mathrm{E}+15 & 1.6780 \mathrm{E}+15 \\ \text { NE } & 2.0293 \mathrm{E}+15 & 1.8430 \mathrm{E}+15\end{array}$

Table E10A-4.10. Assembly Outlet Temperatures and Flow Rates at EOC 10A-4

\begin{tabular}{|c|c|c|c|}
\hline $\begin{array}{l}\text { CORE } \\
\text { POS. } \\
1506 \\
1404 \\
1405 \\
1505 \\
1507 \\
1606 \\
1607 \\
2507 \\
2405 \\
2506 \\
2508 \\
2607 \\
2608 \\
2609\end{array}$ & $\begin{array}{c}\text { OUTLET T } \\
\text { MEASURED } \\
1027 \\
911 \\
885 \\
884 \\
866 \\
899 \\
868 \\
1050 \\
938 \\
895 \\
920 \\
918 \\
899 \\
909\end{array}$ & $\begin{array}{c}\text { P. (DEG F) } \\
\text { CALCULATED } \\
1032 \\
908 \\
883 \\
870 \\
852 \\
898 \\
862 \\
1053 \\
930 \\
887 \\
915 \\
906 \\
894 \\
892\end{array}$ & $\begin{array}{c}\text { FLOW RATE } \\
(\text { LB } / H) \\
114940 \\
225440 \\
208130 \\
208130 \\
208130 \\
170680 \\
170680 \\
114940 \\
192410 \\
197920 \\
208130 \\
163690 \\
170680 \\
170680\end{array}$ \\
\hline
\end{tabular}

Assembly flows are based on a calculated core pressure drop of $110.6 \mathrm{psi}$ at a total reactor flow rate of $16.74 \mathrm{E}+06 \mathrm{lb} / \mathrm{hr}$. 
HNF-SD-FF-ANAL-009 Rev. 1

Table E1OA-4.17. Composition of MFA-1 at EOC 10A-4

\begin{tabular}{|c|c|c|c|c|c|}
\hline Axial & Axial R & e $(\mathrm{cm})$ & & Atom Dens & $y(a / b-c m)$ \\
\hline Level & $\begin{array}{r}\text { Lower } \\
-128.05\end{array}$ & $\begin{array}{r}\text { Upper } \\
-75,28\end{array}$ & Constituent & Fresh & End of Cycle \\
\hline & & & $\begin{array}{l}\mathrm{Na}-23 \\
\mathrm{SS}-316\end{array}$ & $\begin{array}{l}5.3150 \mathrm{E}-03 \\
6.4090 \mathrm{E}-02\end{array}$ & $\begin{array}{l}5.3150 \mathrm{E}-03 \\
6.4090 \mathrm{E}-02\end{array}$ \\
\hline 2 & -75.28 & -62.78 & & & \\
\hline & & & $\begin{array}{l}\mathrm{Na}-23 \\
\mathrm{SS}-316\end{array}$ & $\begin{array}{l}1.3177 \mathrm{E}-02 \\
3.4175 \mathrm{E}-02\end{array}$ & $\begin{array}{l}1.3177 \mathrm{E}-02 \\
3.4175 \mathrm{E}-02\end{array}$ \\
\hline 3 & -62.78 & -46.14 & & & \\
\hline & & & $\begin{array}{l}\mathrm{U}-235 \\
\mathrm{U}-238 \\
\mathrm{Pu}-239 \\
\mathrm{Pu}-240 \\
\mathrm{FP} \\
0-16 \\
\mathrm{Na}-23 \\
\mathrm{SS}-316\end{array}$ & $\begin{array}{l}1.4143 \mathrm{E}-05 \\
7.0573 \mathrm{E}-03 \\
0.0000 \mathrm{E}+00 \\
0.0000 \mathrm{E}+00 \\
0.0000 \mathrm{E}+00 \\
1.4143 \mathrm{E}-02 \\
9.7416 \mathrm{E}-03 \\
1.9073 \mathrm{E}-02\end{array}$ & $\begin{array}{l}1.3056 \mathrm{E}-05 \\
7.0015 \mathrm{E}-03 \\
5.0743 \mathrm{E}-05 \\
5.6431 \mathrm{E}-07 \\
5.2535 \mathrm{E}-06 \\
1.4143 \mathrm{E}-02 \\
9.7416 \mathrm{E}-03 \\
1.9073 \mathrm{E}-02\end{array}$ \\
\hline 4 & -46.14 & -30.76 & & & \\
\hline & & & $\begin{array}{l}\mathrm{U}-235 \\
\mathrm{U}-238 \\
\mathrm{Pu}-239 \\
\mathrm{Pu}-240 \\
\mathrm{Pu}-241 \\
\mathrm{Pu}-242 \\
\mathrm{Am}-241 \\
\mathrm{FP} \\
0-16 \\
\mathrm{Na}-23 \\
\mathrm{SS}-316\end{array}$ & $\begin{array}{l}1.0840 \mathrm{E}-05 \\
4.4499 \mathrm{E}-03 \\
1.7520 \mathrm{E}-03 \\
2.2340 \mathrm{E}-04 \\
1.9280 \mathrm{E}-05 \\
4.7430 \mathrm{E}-06 \\
7.6680 \mathrm{E}-07 \\
0.0000 \mathrm{E}+00 \\
1.2670 \mathrm{E}-02 \\
9.8100 \mathrm{E}-03 \\
1.8990 \mathrm{E}-02\end{array}$ & $\begin{array}{l}1.0076 \mathrm{E}-05 \\
4.4069 \mathrm{E}-03 \\
1.6657 \mathrm{E}-03 \\
2.4037 \mathrm{E}-04 \\
2.0419 \mathrm{E}-05 \\
4.8344 \mathrm{E}-06 \\
1.1275 \mathrm{E}-06 \\
1.1114 \mathrm{E}-04 \\
1.2670 \mathrm{E}-02 \\
9.8100 \mathrm{E}-03 \\
1.8990 \mathrm{E}-02\end{array}$ \\
\hline 5 & -30.76 & -15.38 & & & \\
\hline & & & $\begin{array}{l}\mathrm{U}-235 \\
\mathrm{U}-238 \\
\mathrm{Pu}-239 \\
\mathrm{Pu}-240 \\
\mathrm{Pu}-241 \\
\mathrm{Pu}-242 \\
\mathrm{Am}-241 \\
\mathrm{FP} \\
\mathrm{O}-16 \\
\mathrm{Na}-23 \\
\mathrm{SS}-316\end{array}$ & $\begin{array}{l}1.0840 \mathrm{E}-05 \\
4.4499 \mathrm{E}-03 \\
1.7520 \mathrm{E}-03 \\
2.2340 \mathrm{E}-04 \\
1.9280 \mathrm{E}-05 \\
4.7430 \mathrm{E}-06 \\
7.6680 \mathrm{E}-07 \\
0.0000 \mathrm{E}+00 \\
1.2670 \mathrm{E}-02 \\
9.8100 \mathrm{E}-03 \\
1.8990 \mathrm{E}-02\end{array}$ & $\begin{array}{l}9.8896 \mathrm{E}-06 \\
4.3951 \mathrm{E}-03 \\
1.6416 \mathrm{E}-03 \\
2.4211 \mathrm{E}-04 \\
2.0512 \mathrm{E}-05 \\
4.8357 \mathrm{E}-06 \\
1.1158 \mathrm{E}-06 \\
1.4547 \mathrm{E}-04 \\
1.2670 \mathrm{E}-02 \\
9.8100 \mathrm{E}-03 \\
1.8990 \mathrm{E}-02\end{array}$ \\
\hline 6 & -15.38 & 0.00 & & & \\
\hline & & & $\begin{array}{l}\mathrm{U}-235 \\
\mathrm{U}-238 \\
\mathrm{Pu}-239 \\
\mathrm{Pu}-240 \\
\mathrm{Pu}-241 \\
\mathrm{Pu}-242 \\
\mathrm{Am}-241 \\
\mathrm{FP} \\
0-16 \\
\mathrm{Na}-23 \\
\mathrm{SS}-316\end{array}$ & $\begin{array}{l}1.0840 \mathrm{E}-05 \\
4.4499 \mathrm{E}-03 \\
1.7520 \mathrm{E}-03 \\
2.2340 \mathrm{E}-04 \\
1.9280 \mathrm{E}-05 \\
4.7430 \mathrm{E}-06 \\
7.6680 \mathrm{E}-07 \\
0.0000 \mathrm{E}+00 \\
1.2670 \mathrm{E}-02 \\
9.8100 \mathrm{E}-03 \\
1.8990 \mathrm{E}-02\end{array}$ & $\begin{array}{l}9.7832 \mathrm{E}-06 \\
4.3886 \mathrm{E}-03 \\
1.6288 \mathrm{E}-03 \\
2.4402 \mathrm{E}-04 \\
2.0697 \mathrm{E}-05 \\
4.8466 \mathrm{E}-06 \\
1.1100 \mathrm{E}-06 \\
1.6258 \mathrm{E}-04 \\
1.2670 \mathrm{E}-02 \\
9.8100 \mathrm{E}-03 \\
1.8990 \mathrm{E}-02\end{array}$ \\
\hline & & & & & \\
\hline
\end{tabular}


HNF-SD-FF-ANAL-009 Rev. 1

$\begin{array}{lll}7 & 0.00 & 15.38\end{array}$

$\begin{array}{lll}8 & 15.38 & 30.76\end{array}$

$\begin{array}{lll}\text { U-235 } & 1.0840 \mathrm{E}-05 & 9.8089 \mathrm{E}-06 \\ \mathrm{U}-238 & 4.4499 \mathrm{E}-03 & 4.3902 \mathrm{E}-03 \\ \mathrm{Pu}-239 & 1.7520 \mathrm{E}-03 & 1.6319 \mathrm{E}-03 \\ \text { Pu-240 } & 2.2340 \mathrm{E}-04 & 2.4347 \mathrm{E}-04 \\ \mathrm{Pu}-241 & 1.9280 \mathrm{E}-05 & 2.0644 \mathrm{E}-05 \\ \mathrm{Pu}-242 & 4.7430 \mathrm{E}-06 & 4.8432 \mathrm{E}-06 \\ \mathrm{Am}-241 & 7.6680 \mathrm{E}-07 & 1.1112 \mathrm{E}-06 \\ \mathrm{FP} & 0.0000 \mathrm{E}+00 & 1.5852 \mathrm{E}-04 \\ 0-16 & 1.2670 \mathrm{E}-02 & 1.2670 \mathrm{E}-02 \\ \mathrm{Na}-23 & 9.8100 \mathrm{E}-03 & 9.8100 \mathrm{E}-03 \\ \mathrm{SS}-316 & 1.8990 \mathrm{E}-02 & 1.8990 \mathrm{E}-02\end{array}$

$9 \quad 30.76 \quad 46.14$

$\begin{array}{lll}\mathrm{U}-235 & 1.0840 \mathrm{E}-05 & 9.9651 \mathrm{E}-06 \\ \mathrm{U}-238 & 4.4499 \mathrm{E}-03 & 4.3995 \mathrm{E}-03 \\ \mathrm{Pu}-239 & 1.7520 \mathrm{E}-03 & 1.6502 \mathrm{E}-03 \\ \mathrm{Pu}-240 & 2.2340 \mathrm{E}-04 & 2.4053 \mathrm{E}-04 \\ \mathrm{Pu}-241 & 1.9280 \mathrm{E}-05 & 2.0361 \mathrm{E}-05 \\ \mathrm{Pu}-242 & 4.7430 \mathrm{E}-06 & 4.8262 \mathrm{E}-06 \\ \mathrm{Am}-241 & 7.6680 \mathrm{E}-07 & 1.1193 \mathrm{E}-06 \\ \mathrm{FP} & 0.0000 \mathrm{E}+00 & 1.3413 \mathrm{E}-04 \\ 0-16 & 1.2670 \mathrm{E}-02 & 1.2670 \mathrm{E}-02 \\ \mathrm{Na}-23 & 9.8100 \mathrm{E}-03 & 9.8100 \mathrm{E}-03 \\ \mathrm{SS}-316 & 1.8990 \mathrm{E}-02 & 1.8990 \mathrm{E}-02\end{array}$

$\begin{array}{lll}\mathrm{U}-235 & 1.0840 \mathrm{E}-05 & 1.0190 \mathrm{E}-05 \\ \mathrm{U}-238 & 4.4499 \mathrm{E}-03 & 4.4134 \mathrm{E}-03 \\ \mathrm{Pu}-239 & 1.7520 \mathrm{E}-03 & 1.6783 \mathrm{E}-03 \\ \mathrm{Pu}-240 & 2.2340 \mathrm{E}-04 & 2.3749 \mathrm{E}-04 \\ \mathrm{Pu}-241 & 1.9280 \mathrm{E}-05 & 2.0125 \mathrm{E}-05 \\ \mathrm{Pu}-242 & 4.7430 \mathrm{E}-06 & 4.8156 \mathrm{E}-06 \\ \mathrm{Am}-241 & 7.6680 \mathrm{E}-07 & 1.1324 \mathrm{E}-06 \\ \mathrm{FP} & 0.0000 \mathrm{E}+00 & 9.5157 \mathrm{E}-05 \\ 0-16 & 1.2670 \mathrm{E}-02 & 1.2670 \mathrm{E}-02 \\ \mathrm{Na}-23 & 9.8100 \mathrm{E}-03 & 9.8100 \mathrm{E}-03 \\ \mathrm{SS}-316 & 1.8990 \mathrm{E}-02 & 1.8990 \mathrm{E}-02\end{array}$

$\begin{array}{lll}10 & 46.14 & 62.78\end{array}$

$\begin{array}{lll}\text { U-235 } & 1.4143 \mathrm{E}-05 & 1.3385 \mathrm{E}-05 \\ \mathrm{U}-238 & 7.0573 \mathrm{E}-03 & 7.0174 \mathrm{E}-03 \\ \mathrm{Pu}-239 & 0.0000 \mathrm{E}+00 & 3.6387 \mathrm{E}-05 \\ \mathrm{Pu}-240 & 0.0000 \mathrm{E}+00 & 2.6913 \mathrm{E}-07 \\ \mathrm{FP} & 0.0000 \mathrm{E}+00 & 3.7929 \mathrm{E}-06 \\ \mathrm{O}-16 & 1.4143 \mathrm{E}-02 & 1.4143 \mathrm{E}-02 \\ \mathrm{Na}-23 & 9.7416 \mathrm{E}-03 & 9.7416 \mathrm{E}-03 \\ \mathrm{SS}-316 & 1.9073 \mathrm{E}-02 & 1.9073 \mathrm{E}-02\end{array}$

$\begin{array}{lll}11 & 62.78 & 172.84\end{array}$

$\begin{array}{lll}\mathrm{Na}-23 & 8.2400 \mathrm{E}-03 & 8.2400 \mathrm{E}-03 \\ \mathrm{SS}-316 & 2.5562 \mathrm{E}-02 & 2.5562 \mathrm{E}-02\end{array}$


Table ElOA-4.18. Composition of MFA-2 at EOC 10A-4

\begin{tabular}{|c|c|c|c|c|c|}
\hline \multirow{3}{*}{$\begin{array}{c}\text { Axial } \\
\text { Level } \\
1\end{array}$} & \multicolumn{2}{|c|}{ Axial Range $(\mathrm{cm})$} & & \multicolumn{2}{|c|}{ Atom Density $(\mathrm{a} / \mathrm{b}-\mathrm{cm})$} \\
\hline & $\begin{array}{r}\text { Lower } \\
-128.05\end{array}$ & Upper & Constituent & Fresh & End of Cycle \\
\hline & & & $\begin{array}{l}\mathrm{Na}-23 \\
\mathrm{SS}-316\end{array}$ & $\begin{array}{l}5.3150 \mathrm{E}-03 \\
6.4090 \mathrm{E}-02\end{array}$ & $\begin{array}{l}5.3150 \mathrm{E}-03 \\
6.4090 \mathrm{E}-02\end{array}$ \\
\hline 2 & -75.28 & -62.78 & & & \\
\hline & & & $\begin{array}{l}\mathrm{Na}-23 \\
\text { SS-316 }\end{array}$ & $\begin{array}{l}1.3177 \mathrm{E}-02 \\
3.4175 \mathrm{E}-02\end{array}$ & $\begin{array}{l}1.3177 \mathrm{E}-02 \\
3.4175 \mathrm{E}-02\end{array}$ \\
\hline 3 & -62.78 & -46.14 & $\begin{array}{l}U-235 \\
U-238 \\
P u-239 \\
P U-240 \\
F P \\
\text { O-16 } \\
\mathrm{Na}-23 \\
\text { SS-316 }\end{array}$ & $\begin{array}{l}1.4143 \mathrm{E}-05 \\
7.0573 \mathrm{E}-03 \\
0.0000 \mathrm{E}+00 \\
0.0000 \mathrm{E}+00 \\
0.0000 \mathrm{E}+00 \\
1.4143 \mathrm{E}-02 \\
9.7416 \mathrm{E}-03 \\
1.9073 \mathrm{E}-02\end{array}$ & $\begin{array}{l}1.3078 \mathrm{E}-05 \\
7.0024 \mathrm{E}-03 \\
4.9911 \mathrm{E}-05 \\
5.4997 \mathrm{E}-07 \\
5.2479 \mathrm{E}-06 \\
1.4143 \mathrm{E}-02 \\
9.7416 \mathrm{E}-03 \\
1.9073 \mathrm{E}-02\end{array}$ \\
\hline 4 & -46.14 & -30.76 & & & \\
\hline & & & $\begin{array}{l}\text { U-235 } \\
U-238 \\
\mathrm{Pu}-239 \\
\mathrm{Pu}-240 \\
\mathrm{Pu}-241 \\
\mathrm{Pu}-242 \\
\mathrm{Am}-241 \\
\mathrm{FP} \\
\mathrm{O}-16 \\
\mathrm{Na}-23 \\
\mathrm{SS}-316\end{array}$ & $\begin{array}{l}1.0160 \mathrm{E}-05 \\
4.4270 \mathrm{E}-03 \\
1.7483 \mathrm{E}-03 \\
2.2290 \mathrm{E}-04 \\
1.9040 \mathrm{E}-05 \\
4.7330 \mathrm{E}-06 \\
7.5700 \mathrm{E}-07 \\
0.0000 \mathrm{E}+00 \\
1.2760 \mathrm{E}-02 \\
9.8100 \mathrm{E}-03 \\
1.8990 \mathrm{E}-02\end{array}$ & $\begin{array}{l}9.4363 \mathrm{E}-06 \\
4.3837 \mathrm{E}-03 \\
1.6614 \mathrm{E}-03 \\
2.4016 \mathrm{E}-04 \\
2.0234 \mathrm{E}-05 \\
4.8239 \mathrm{E}-06 \\
1.1123 \mathrm{E}-06 \\
1.1174 \mathrm{E}-04 \\
1.2760 \mathrm{E}-02 \\
9.8100 \mathrm{E}-03 \\
1.8990 \mathrm{E}-02\end{array}$ \\
\hline 5 & -30.76 & -15.38 & & & \\
\hline & & & $\begin{array}{l}U-235 \\
U-238 \\
P u-239 \\
P u-240 \\
P u-241 \\
P u-242 \\
\text { Am-241 } \\
\text { FP } \\
0-16 \\
\mathrm{Na}-23 \\
\text { SS-316 }\end{array}$ & $\begin{array}{l}1.0160 \mathrm{E}-05 \\
4.4270 \mathrm{E}-03 \\
1.7483 \mathrm{E}-03 \\
2.2290 \mathrm{E}-04 \\
1.9040 \mathrm{E}-05 \\
4.7330 \mathrm{E}-06 \\
7.5700 \mathrm{E}-07 \\
0.0000 \mathrm{E}+00 \\
1.2760 \mathrm{E}-02 \\
9.8100 \mathrm{E}-03 \\
1.8990 \mathrm{E}-02\end{array}$ & $\begin{array}{l}9.2542 \mathrm{E}-06 \\
4.3713 \mathrm{E}-03 \\
1.6363 \mathrm{E}-03 \\
2.4205 \mathrm{E}-04 \\
2.0349 \mathrm{E}-05 \\
4.8256 \mathrm{E}-06 \\
1.1004 \mathrm{E}-06 \\
1.4732 \mathrm{E}-04 \\
1.2760 \mathrm{E}-02 \\
9.8100 \mathrm{E}-03 \\
1.8990 \mathrm{E}-02\end{array}$ \\
\hline 6 & -15.38 & 0.00 & $\begin{array}{l}U-235 \\
U-238 \\
P u-239 \\
P u-240 \\
P u-241 \\
P u-242 \\
\text { Am-241 } \\
\text { FP } \\
0-16 \\
\mathrm{Na}-23 \\
\text { SS }-316\end{array}$ & $\begin{array}{l}1.0160 \mathrm{E}-05 \\
4.4270 \mathrm{E}-03 \\
1.7483 \mathrm{E}-03 \\
2.2290 \mathrm{E}-04 \\
1.9040 \mathrm{E}-05 \\
4.7330 \mathrm{E}-06 \\
7.5700 \mathrm{E}-07 \\
0.0000 \mathrm{E}+00 \\
1.2760 \mathrm{E}-02 \\
9.8100 \mathrm{E}-03 \\
1.8990 \mathrm{E}-02\end{array}$ & $\begin{array}{l}9.1478 \mathrm{E}-06 \\
4.3644 \mathrm{E}-03 \\
1.6229 \mathrm{E}-03 \\
2.4407 \mathrm{E}-04 \\
2.0551 \mathrm{E}-05 \\
4.8372 \mathrm{E}-06 \\
1.0942 \mathrm{E}-06 \\
1.6542 \mathrm{E}-04 \\
1.2760 \mathrm{E}-02 \\
9.8100 \mathrm{E}-03 \\
1.8990 \mathrm{E}-02\end{array}$ \\
\hline
\end{tabular}


HNF-SD-FF-ANAL-009 Rev. 1

$\begin{array}{lll}7 & 0.00 & 15.38\end{array}$

$\begin{array}{lll}8 & 15.38 & 30.76\end{array}$

$\begin{array}{lll}\mathrm{U}-235 & 1.0160 \mathrm{E}-05 & 9.1713 \mathrm{E}-06 \\ \mathrm{U}-238 & 4.4270 \mathrm{E}-03 & 4.3660 \mathrm{E}-03 \\ \mathrm{Pu}-239 & 1.7483 \mathrm{E}-03 & 1.6257 \mathrm{E}-03 \\ \mathrm{Pu}-240 & 2.2290 \mathrm{E}-04 & 2.4356 \mathrm{E}-04 \\ \mathrm{Pu}-241 & 1.9040 \mathrm{E}-05 & 2.0499 \mathrm{E}-05 \\ \mathrm{Pu}-242 & 4.7330 \mathrm{E}-06 & 4.8340 \mathrm{E}-06 \\ \mathrm{Am}-241 & 7.5700 \mathrm{E}-07 & 1.0953 \mathrm{E}-06 \\ \mathrm{FP} & 0.0000 \mathrm{E}+00 & 1.6162 \mathrm{E}-04 \\ 0-16 & 1.2760 \mathrm{E}-02 & 1.2760 \mathrm{E}-02 \\ \mathrm{Na}-23 & 9.8100 \mathrm{E}-03 & 9.8100 \mathrm{E}-03 \\ \mathrm{SS}-316 & 1.8990 \mathrm{E}-02 & 1.8990 \mathrm{E}-02 \\ & & \\ \mathrm{U}-235 & 1.0160 \mathrm{E}-05 & 9.3168 \mathrm{E}-06 \\ \mathrm{U}-238 & 4.4270 \mathrm{E}-03 & 4.3753 \mathrm{E}-03 \\ \mathrm{Pu}-239 & 1.7483 \mathrm{E}-03 & 1.6440 \mathrm{E}-03 \\ \mathrm{Pu}-240 & 2.2290 \mathrm{E}-04 & 2.4066 \mathrm{E}-04 \\ \mathrm{Pu}-241 & 1.9040 \mathrm{E}-05 & 2.0214 \mathrm{E}-05 \\ \mathrm{Pu}-242 & 4.7330 \mathrm{E}-06 & 4.8175 \mathrm{E}-06 \\ \mathrm{Am}-241 & 7.5700 \mathrm{E}-07 & 1.1034 \mathrm{E}-06 \\ \mathrm{FP} & 0.0000 \mathrm{E}+00 & 1.3721 \mathrm{E}-04 \\ 0-16 & 1.2760 \mathrm{E}-02 & 1.2760 \mathrm{E}-02 \\ \mathrm{Na}-23 & 9.8100 \mathrm{E}-03 & 9.8100 \mathrm{E}-03 \\ \mathrm{SS}-316 & 1.8990 \mathrm{E}-02 & 1.8990 \mathrm{E}-02\end{array}$

$\begin{array}{lll}9 & 30.76 & 46.14\end{array}$

$\begin{array}{lll}\mathrm{U}-235 & 1.0160 \mathrm{E}-05 & 9.5310 \mathrm{E}-06 \\ \mathrm{U}-238 & 4.4270 \mathrm{E}-03 & 4.3893 \mathrm{E}-03 \\ \mathrm{Pu}-239 & 1.7483 \mathrm{E}-03 & 1.6725 \mathrm{E}-03 \\ \mathrm{Pu}-240 & 2.2290 \mathrm{E}-04 & 2.3765 \mathrm{E}-04 \\ \mathrm{Pu}-241 & 1.9040 \mathrm{E}-05 & 1.9974 \mathrm{E}-05 \\ \mathrm{Pu}-242 & 4.7330 \mathrm{E}-06 & 4.8077 \mathrm{E}-06 \\ \mathrm{Am}-241 & 7.5700 \mathrm{E}-07 & 1.1166 \mathrm{E}-06 \\ \mathrm{FP} & 0.0000 \mathrm{E}+00 & 9.7751 \mathrm{E}-05 \\ 0-16 & 1.2760 \mathrm{E}-02 & 1.2760 \mathrm{E}-02 \\ \mathrm{Na}-23 & 9.8100 \mathrm{E}-03 & 9.8100 \mathrm{E}-03 \\ \mathrm{SS}-316 & 1.8990 \mathrm{E}-02 & 1.8990 \mathrm{E}-02\end{array}$

$\begin{array}{lll}10 & 46.14 & 62.78\end{array}$

$\begin{array}{lll}\mathrm{U}-235 & 1.4143 \mathrm{E}-05 & 1.3355 \mathrm{E}-05 \\ \mathrm{U}-238 & 7.0573 \mathrm{E}-03 & 7.0158 \mathrm{E}-03 \\ \mathrm{Pu}-239 & 0.0000 \mathrm{E}+00 & 3.7793 \mathrm{E}-05 \\ \mathrm{Pu}-240 & 0.0000 \mathrm{E}+00 & 2.9713 \mathrm{E}-07 \\ \mathrm{FP} & 0.0000 \mathrm{E}+00 & 3.9889 \mathrm{E}-06 \\ \mathrm{O}-16 & 1.4143 \mathrm{E}-02 & 1.4143 \mathrm{E}-02 \\ \mathrm{Na}-23 & 9.7416 \mathrm{E}-03 & 9.7416 \mathrm{E}-03 \\ \mathrm{SS}-316 & 1.9073 \mathrm{E}-02 & 1.9073 \mathrm{E}-02\end{array}$

$\begin{array}{lll}11 & 62.78 & 172.84\end{array}$

$\begin{array}{lll}\mathrm{Na}-23 & 8.2400 \mathrm{E}-03 & 8.2400 \mathrm{E}-03 \\ \mathrm{SS}-316 & 2.5562 \mathrm{E}-02 & 2.5562 \mathrm{E}-02\end{array}$




\subsection{Cycle 1OB}

Table BlOB.1. Fission Power Generated in MFA-1, MFA-2 and Neighboring Assemblies at BOC 10B

\begin{tabular}{lcccc} 
CORE & $* * * * * \star$ & \multicolumn{2}{c}{ POWER IN MEGAWATTS } & $* * * * * *$ \\
POS. & BELOW CORE & IN CORE & ABOVE CORE & TOTAL PWR \\
1506 & $3.473 \mathrm{E}-02$ & $3.674 \mathrm{E}+00$ & $2.243 \mathrm{E}-02$ & $3.731 \mathrm{E}+00$ \\
1404 & $8.999 \mathrm{E}-02$ & $4.349 \mathrm{E}+00$ & $4.727 \mathrm{E}-02$ & $4.486 \mathrm{E}+00$ \\
1405 & $0.000 \mathrm{E}+00$ & $6.471 \mathrm{E}+00$ & $0.000 \mathrm{E}+00$ & $6.471 \mathrm{E}+00$ \\
1505 & $6.642 \mathrm{E}-03$ & $4.040 \mathrm{E}+00$ & $4.411 \mathrm{E}-03$ & $4.051 \mathrm{E}+00$ \\
1507 & $1.025 \mathrm{E}-02$ & $3.107 \mathrm{E}+00$ & $7.320 \mathrm{E}-03$ & $3.124 \mathrm{E}+00$ \\
1606 & $6.841 \mathrm{E}-03$ & $3.304 \mathrm{E}+00$ & $4.704 \mathrm{E}-03$ & $3.316 \mathrm{E}+00$ \\
1607 & $9.531 \mathrm{E}-03$ & $2.732 \mathrm{E}+00$ & $6.635 \mathrm{E}-03$ & $2.748 \mathrm{E}+00$ \\
2507 & $3.646 \mathrm{E}-02$ & $4.003 \mathrm{E}+00$ & $2.503 \mathrm{E}-02$ & $4.065 \mathrm{E}+00$ \\
2405 & $1.008 \mathrm{E}-02$ & $4.134 \mathrm{E}+00$ & $7.467 \mathrm{E}-03$ & $4.151 \mathrm{E}+00$ \\
2506 & $6.915 \mathrm{E}-03$ & $4.386 \mathrm{E}+00$ & $5.308 \mathrm{E}-03$ & $4.398 \mathrm{E}+00$ \\
2508 & $1.223 \mathrm{E}-02$ & $4.393 \mathrm{E}+00$ & $8.597 \mathrm{E}-03$ & $4.414 \mathrm{E}+00$ \\
2607 & $8.402 \mathrm{E}-03$ & $3.401 \mathrm{E}+00$ & $6.054 \mathrm{E}-03$ & $3.415 \mathrm{E}+00$ \\
2608 & $5.672 \mathrm{E}-03$ & $3.352 \mathrm{E}+00$ & $4.515 \mathrm{E}-03$ & $3.362 \mathrm{E}+00$ \\
2609 & $6.230 \mathrm{E}-03$ & $4.224 \mathrm{E}+00$ & $4.945 \mathrm{E}-03$ & $4.235 \mathrm{E}+00$
\end{tabular}

Table B10B.2. Assembly Averaged Total and Fast Flux in MFA-1 and MFA-2 at BOC 10B

\begin{tabular}{|c|c|c|}
\hline & Core & Flux $\left(\mathrm{n} / \mathrm{cm}^{2}-\right.$ \\
\hline $\begin{array}{l}\text { Assembly } \\
\text { MFA-1 } \\
\text { MFA-2 }\end{array}$ & $\begin{array}{l}\text { Pos. } \\
1506 \\
2507\end{array}$ & $\begin{array}{c}\text { Total } \\
2.791 \mathrm{E}+15 \\
3.016 \mathrm{E}+15\end{array}$ \\
\hline
\end{tabular}


Table B10B.3. Axial Distribution of Total Flux, Fast Flux and Power in MFA-1 at BOC $10 \mathrm{~B}$

\begin{tabular}{|c|c|c|c|}
\hline $\begin{array}{r}Z(\mathrm{~cm}) \\
-97.78 \\
-92.78 \\
-87.78 \\
-82.78 \\
-77.78 \\
-72.15 \\
-65.90 \\
-60.35 \\
-55.49 \\
-50.62 \\
-47.17 \\
-43.58 \\
-38.45 \\
-33.32 \\
-28.20 \\
-23.07 \\
-17.94 \\
-12.82 \\
-7.69 \\
-2.56 \\
2.56 \\
7.69 \\
12.82 \\
17.94 \\
23.07 \\
28.20 \\
33.32 \\
38.45 \\
43.58 \\
47.17 \\
50.62 \\
55.49 \\
60.35 \\
64.98 \\
69.39 \\
76.46 \\
86.80 \\
98.14 \\
110.00\end{array}$ & $\begin{array}{l}\text { Total Flux } \\
2.8660 \mathrm{E}-02 \\
6.1544 \mathrm{E}-02 \\
9.8613 \mathrm{E}-02 \\
1.4263 \mathrm{E}-01 \\
1.9637 \mathrm{E}-01 \\
2.5706 \mathrm{E}-01 \\
3.1736 \mathrm{E}-01 \\
3.8094 \mathrm{E}-01 \\
4.6530 \mathrm{E}-01 \\
5.6352 \mathrm{E}-01 \\
6.4245 \mathrm{E}-01 \\
7.2536 \mathrm{E}-01 \\
8.4374 \mathrm{E}-01 \\
9.5221 \mathrm{E}-01 \\
1.0454 \mathrm{E}+00 \\
1.1212 \mathrm{E}+00 \\
1.1782 \mathrm{E}+00 \\
1.2154 \mathrm{E}+00 \\
1.2331 \mathrm{E}+00 \\
1.2317 \mathrm{E}+00 \\
1.2121 \mathrm{E}+00 \\
1.1757 \mathrm{E}+00 \\
1.1239 \mathrm{E}+00 \\
1.0580 \mathrm{E}+00 \\
9.7848 \mathrm{E}-01 \\
8.8675 \mathrm{E}-01 \\
7.8459 \mathrm{E}-01 \\
6.7410 \mathrm{E}-01 \\
5.6003 \mathrm{E}-01 \\
4.8402 \mathrm{E}-01 \\
4.1383 \mathrm{E}-01 \\
3.2800 \mathrm{E}-01 \\
2.5277 \mathrm{E}-01 \\
2.0457 \mathrm{E}-01 \\
1.7565 \mathrm{E}-01 \\
1.3414 \mathrm{E}-01 \\
8.8291 \mathrm{E}-02 \\
5.1709 \mathrm{E}-02 \\
2.2429 \mathrm{E}-02\end{array}$ & $\begin{array}{c}\text { Flux > } 0.1 \mathrm{MeV} \\
1.4202 \mathrm{E}-02 \\
2.7429 \mathrm{E}-02 \\
4.3911 \mathrm{E}-02 \\
6.5541 \mathrm{E}-02 \\
9.4660 \mathrm{E}-02 \\
1.3238 \mathrm{E}-01 \\
1.8002 \mathrm{E}-01 \\
2.4458 \mathrm{E}-01 \\
3.3192 \mathrm{E}-01 \\
4.4652 \mathrm{E}-01 \\
5.5513 \mathrm{E}-01 \\
6.7808 \mathrm{E}-01 \\
8.2492 \mathrm{E}-01 \\
9.4819 \mathrm{E}-01 \\
1.0490 \mathrm{E}+00 \\
1.1289 \mathrm{E}+00 \\
1.1879 \mathrm{E}+00 \\
1.2259 \mathrm{E}+00 \\
1.2439 \mathrm{E}+00 \\
1.2427 \mathrm{E}+00 \\
1.2232 \mathrm{E}+00 \\
1.1869 \mathrm{E}+00 \\
1.1350 \mathrm{E}+00 \\
1.0687 \mathrm{E}+00 \\
9.8763 \mathrm{E}-01 \\
8.9271 \mathrm{E}-01 \\
7.8479 \mathrm{E}-01 \\
6.6317 \mathrm{E}-01 \\
5.2841 \mathrm{E}-01 \\
4.2513 \mathrm{E}-01 \\
3.3603 \mathrm{E}-01 \\
2.4309 \mathrm{E}-01 \\
1.7129 \mathrm{E}-01 \\
1.2864 \mathrm{E}-01 \\
1.0560 \mathrm{E}-01 \\
7.4736 \mathrm{E}-02 \\
4.5086 \mathrm{E}-02 \\
2.4785 \mathrm{E}-02 \\
1.1198 \mathrm{E}-02\end{array}$ & $\begin{array}{l}\text { Power } \\
0.0000 E+00 \\
0.0000 E+00 \\
0.0000 E+00 \\
0.0000 E+00 \\
0.0000 E+00 \\
0.0000 E+00 \\
0.0000 E+00 \\
3.6638 E-02 \\
4.6500 E-02 \\
6.2213 E-02 \\
8.0727 E-02 \\
7.6944 E-01 \\
8.6192 E-01 \\
9.6281 E-01 \\
1.0401 E+00 \\
1.1140 E+00 \\
1.1697 E+00 \\
1.1982 E+00 \\
1.2153 E+00 \\
1.2136 E+00 \\
1.1960 E+00 \\
1.1600 E+00 \\
1.1090 E+00 \\
1.0540 E+00 \\
9.7527 E-01 \\
8.8479 E-01 \\
7.9642 E-01 \\
6.8965 E-01 \\
5.8982 E-01 \\
5.7224 E-02 \\
4.2069 E-02 \\
2.9073 E-02 \\
2.0592 E-02 \\
0.0000 E+00 \\
0.0000 E+00 \\
0.0000 E+00 \\
0.0000 E+00 \\
0.0000 E+00 \\
0.0000 E+00\end{array}$ \\
\hline
\end{tabular}


Table B10B.4. Axial Distribution of Total Flux, Fast Flux and Power in MFA-2 at BOC 10B

$\begin{array}{rccc}\text { z }(\mathrm{cm}) & \text { Total Flux } & \text { Flux }>0.1 \text { MeV } & \text { Power } \\ -97.78 & 2.5586 \mathrm{E}-02 & 1.2970 \mathrm{E}-02 & 0.0000 \mathrm{E}+00 \\ -92.78 & 5.4933 \mathrm{E}-02 & 2.5033 \mathrm{E}-02 & 0.0000 \mathrm{E}+00 \\ -87.78 & 8.8076 \mathrm{E}-02 & 4.0058 \mathrm{E}-02 & 0.0000 \mathrm{E}+00 \\ -82.78 & 1.2768 \mathrm{E}-01 & 5.9806 \mathrm{E}-02 & 0.0000 \mathrm{E}+00 \\ -77.78 & 1.7671 \mathrm{E}-01 & 8.6514 \mathrm{E}-02 & 0.0000 \mathrm{E}+00 \\ -72.15 & 2.3360 \mathrm{E}-01 & 1.2149 \mathrm{E}-01 & 0.0000 \mathrm{E}+00 \\ -65.90 & 2.9288 \mathrm{E}-01 & 1.6681 \mathrm{E}-01 & 0.0000 \mathrm{E}+00 \\ -60.35 & 3.5697 \mathrm{E}-01 & 2.2888 \mathrm{E}-01 & 3.5079 \mathrm{E}-02 \\ -55.49 & 4.4044 \mathrm{E}-01 & 3.1310 \mathrm{E}-01 & 4.4821 \mathrm{E}-02 \\ -50.62 & 5.3782 \mathrm{E}-01 & 4.2488 \mathrm{E}-01 & 6.0040 \mathrm{E}-02 \\ -47.17 & 6.1702 \mathrm{E}-01 & 5.3230 \mathrm{E}-01 & 7.7936 \mathrm{E}-02 \\ -43.58 & 7.0086 \mathrm{E}-01 & 6.5493 \mathrm{E}-01 & 7.5091 \mathrm{E}-01 \\ -38.45 & 8.2168 \mathrm{E}-01 & 8.0305 \mathrm{E}-01 & 8.4138 \mathrm{E}-01 \\ -33.32 & 9.3305 \mathrm{E}-01 & 9.2889 \mathrm{E}-01 & 9.4359 \mathrm{E}-01 \\ -28.20 & 1.0295 \mathrm{E}+00 & 1.0331 \mathrm{E}+00 & 1.0232 \mathrm{E}+00 \\ -23.07 & 1.1091 \mathrm{E}+00 & 1.1170 \mathrm{E}+00 & 1.1004 \mathrm{E}+00 \\ -17.94 & 1.1702 \mathrm{E}+00 & 1.1803 \mathrm{E}+00 & 1.1603 \mathrm{E}+00 \\ -12.82 & 1.2119 \mathrm{E}+00 & 1.2229 \mathrm{E}+00 & 1.1930 \mathrm{E}+00 \\ -7.69 & 1.2340 \mathrm{E}+00 & 1.2453 \mathrm{E}+00 & 1.2145 \mathrm{E}+00 \\ -2.56 & 1.2367 \mathrm{E}+00 & 1.2482 \mathrm{E}+00 & 1.2170 \mathrm{E}+00 \\ 2.56 & 1.2205 \mathrm{E}+00 & 1.2322 \mathrm{E}+00 & 1.2027 \mathrm{E}+00 \\ 7.69 & 1.1867 \mathrm{E}+00 & 1.1982 \mathrm{E}+00 & 1.1694 \mathrm{E}+00 \\ 12.82 & 1.1365 \mathrm{E}+00 & 1.1476 \mathrm{E}+00 & 1.1200 \mathrm{E}+00 \\ 17.94 & 1.0711 \mathrm{E}+00 & 1.0815 \mathrm{E}+00 & 1.0659 \mathrm{E}+00 \\ 23.07 & 9.9147 \mathrm{E}-01 & 9.9994 \mathrm{E}-01 & 9.8731 \mathrm{E}-01 \\ 28.20 & 8.9903 \mathrm{E}-01 & 9.0411 \mathrm{E}-01 & 8.9641 \mathrm{E}-01 \\ 33.32 & 7.9575 \mathrm{E}-01 & 7.9495 \mathrm{E}-01 & 8.0787 \mathrm{E}-01 \\ 38.45 & 6.8386 \mathrm{E}-01 & 6.7202 \mathrm{E}-01 & 7.0127 \mathrm{E}-01 \\ 43.58 & 5.6799 \mathrm{E}-01 & 5.3567 \mathrm{E}-01 & 6.0483 \mathrm{E}-01 \\ 47.17 & 4.8951 \mathrm{E}-01 & 4.2874 \mathrm{E}-01 & 5.8736 \mathrm{E}-02 \\ 50.62 & 4.1562 \mathrm{E}-01 & 3.3536 \mathrm{E}-01 & 4.3165 \mathrm{E}-02 \\ 55.49 & 3.2514 \mathrm{E}-01 & 2.3852 \mathrm{E}-01 & 2.9747 \mathrm{E}-02 \\ 60.35 & 2.4675 \mathrm{E}-01 & 1.6520 \mathrm{E}-01 & 2.0985 \mathrm{E}-02 \\ 64.98 & 1.9826 \mathrm{E}-01 & 1.2316 \mathrm{E}-01 & 0.0000 \mathrm{E}+00 \\ 69.39 & 1.6952 \mathrm{E}-01 & 1.0089 \mathrm{E}-01 & 0.0000 \mathrm{E}+00 \\ 76.46 & 1.2865 \mathrm{E}-01 & 7.1169 \mathrm{E}-02 & 0.0000 \mathrm{E}+00 \\ 86.80 & 8.4008 \mathrm{E}-02 & 4.2658 \mathrm{E}-02 & 0.0000 \mathrm{E}+00 \\ 98.14 & 4.8631 \mathrm{E}-02 & 2.3205 \mathrm{E}-02 & 0.0000 \mathrm{E}+00 \\ 110.00 & 2.0726 \mathrm{E}-02 & 1.0302 \mathrm{E}-02 & 0.0000 \mathrm{E}+00\end{array}$


HNF-SD-FF-ANAL-009 Rev. 1

Table B1OB.5. Fission Power Distribution by Pin in MFA-1 at BOC 10B CORE POSITION 1506

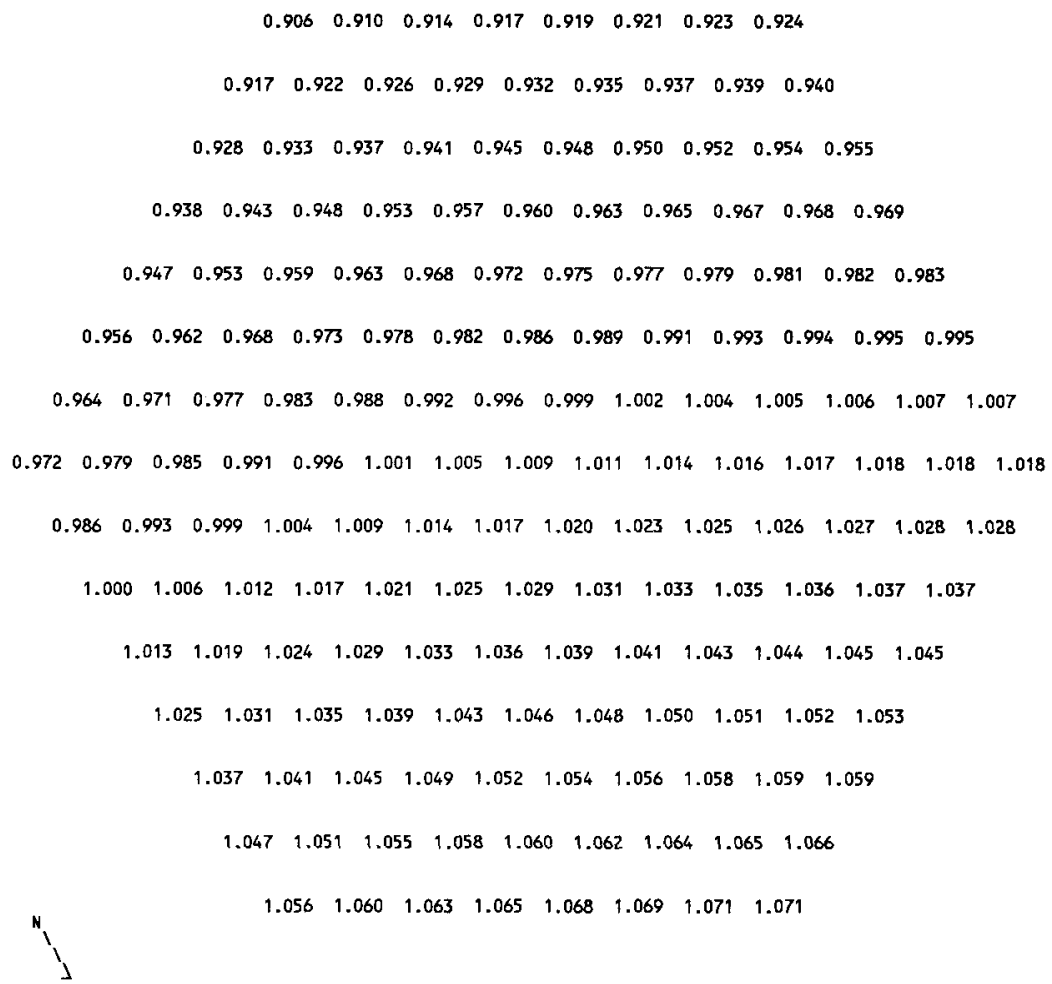


HNF-SD-FF-ANAL-009 Rev. 1

Table B10B.6. Fission Power Distribution by Pin in MFA-2 at BOC $10 \mathrm{~B}$ CORE POSITION 2507

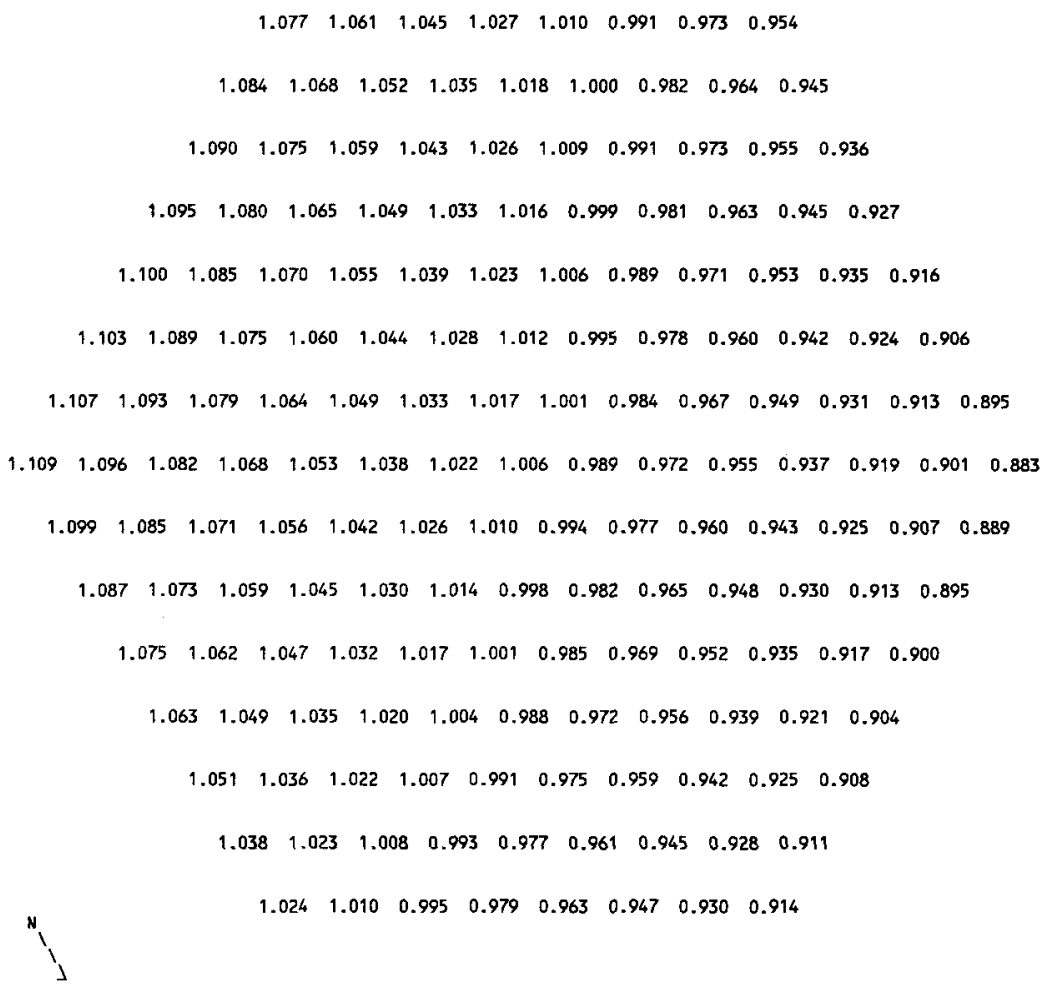


HNF-SD-FF-ANAL-009 Rev. 1

Table B10B.7. Fast Flux Distribution by Pin in MFA-1 at BOC 10B CORE POSITION 1506

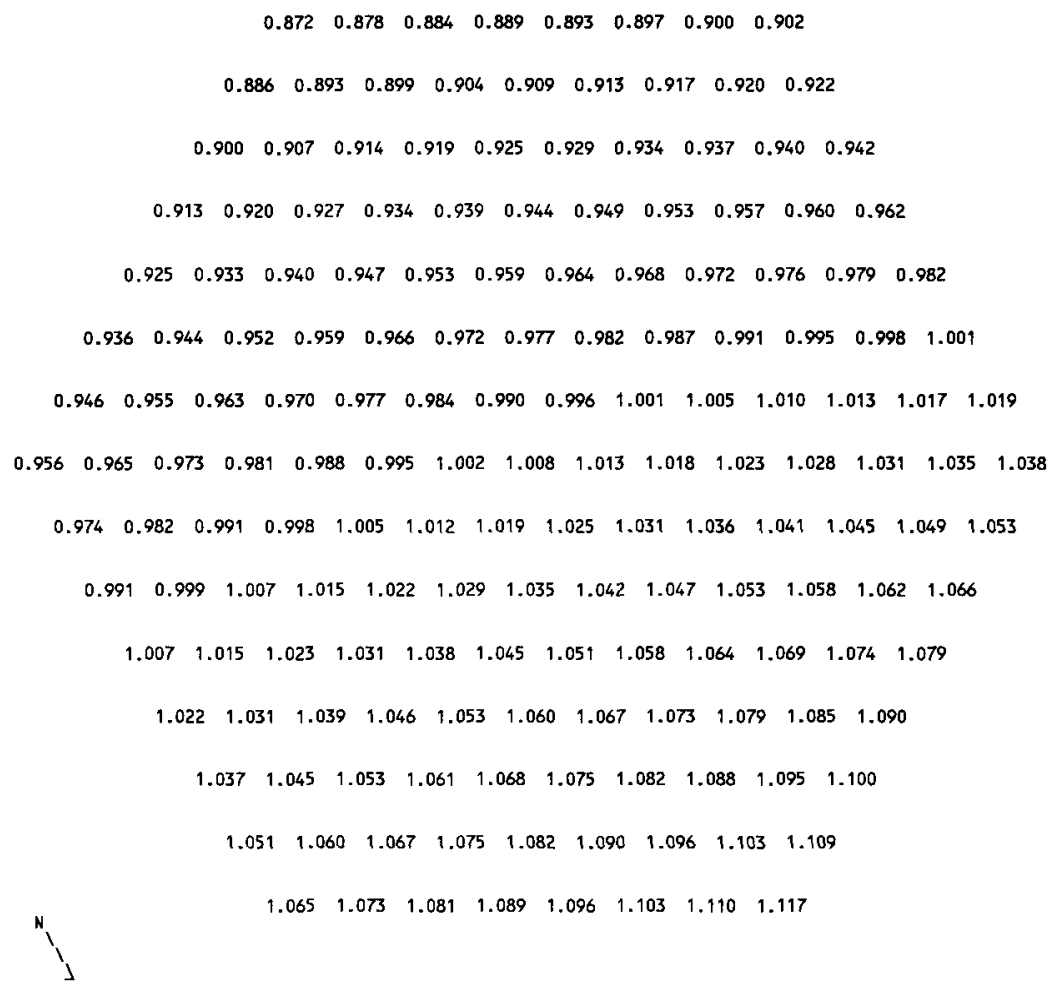


HNF-SD-FF-ANAL-009 Rev, 1

Table B10B.8. Fast Flux Distribution by Pin in MFA-2 at BOC $10 \mathrm{~B}$ CORE POSITION 2507

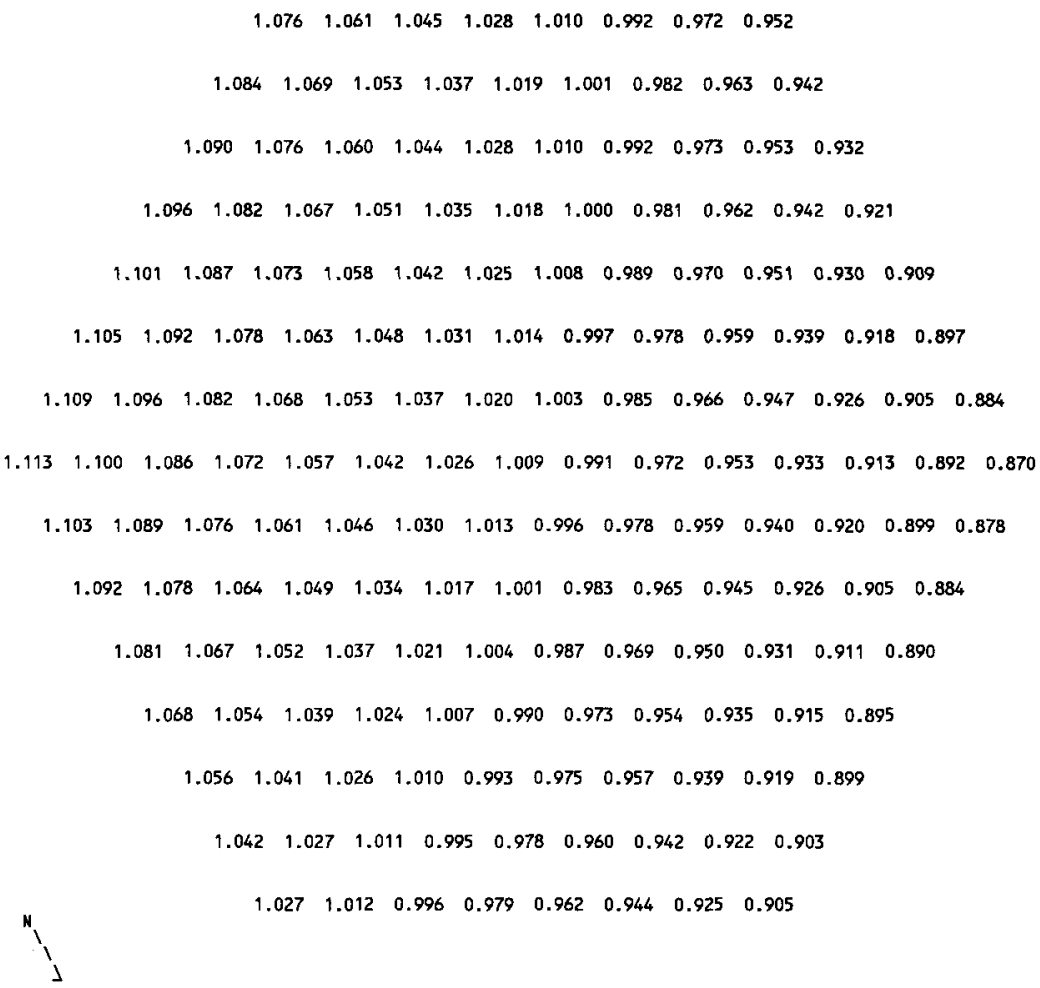


Table BlOB.9. MFA-1 and MFA-2 Duct Wall

Fast Flux Data at BOC 10B

$\begin{array}{ccc}\text { Side } & \begin{array}{c}\text { Flux }>0.1 \\ \text { MFA-1 }\end{array} & \begin{array}{c}\text { MeV }\left(\mathrm{n} / \mathrm{cm}^{2}-\mathrm{sec}\right) \\ \text { MFA-2 }\end{array} \\ \text { E } & 1.8738 \mathrm{E}+15 & 2.1211 \mathrm{E}+15 \\ \text { SE } & 1.6634 \mathrm{E}+15 & 2.1766 \mathrm{E}+15 \\ \text { SW } & 1.5985 \mathrm{E}+15 & 1.9890 \mathrm{E}+15 \\ \text { W } & 1.7757 \mathrm{E}+15 & 1.7425 \mathrm{E}+15 \\ \text { NW } & 2.0314 \mathrm{E}+15 & 1.6907 \mathrm{E}+15 \\ \text { NE } & 2.0435 \mathrm{E}+15 & 1.8708 \mathrm{E}+15\end{array}$

Table Bl0B.10. Assembly 0utlet Temperatures and Flow Rates at BOC $10 \mathrm{~B}$

$\begin{array}{lccc}\text { CORE } & \text { OUTLET TEMP. } & \text { (DEG F) } & \text { FLOW RATE } \\ \text { POS. } & \text { MEASURED } & \text { CALCULATED } & \text { (LB } / H \text { ) } \\ 1506 & 1022 & 1023 & 115440 \\ 1404 & 906 & 899 & 228160 \\ 1405 & 1042 & 1037 & 188170 \\ 1505 & 898 & 890 & 209040 \\ 1507 & 864 & 847 & 209040 \\ 1606 & 893 & 890 & 171430 \\ 1607 & 867 & 856 & 171430 \\ 2507 & 1043 & 1051 & 115440 \\ 2405 & 897 & 894 & 209060 \\ 2506 & 913 & 905 & 209040 \\ 2508 & 917 & 912 & 209040 \\ 2607 & 910 & 901 & 164410 \\ 2608 & 894 & 896 & 171430 \\ 2609 & 950 & 959 & 164410\end{array}$

Assembly flows are based on a calculated core pressure drop of $111.5 \mathrm{psi}$ at a total reactor flow rate of $16.74 \mathrm{E}+06 \mathrm{lb} / \mathrm{hr}$. 


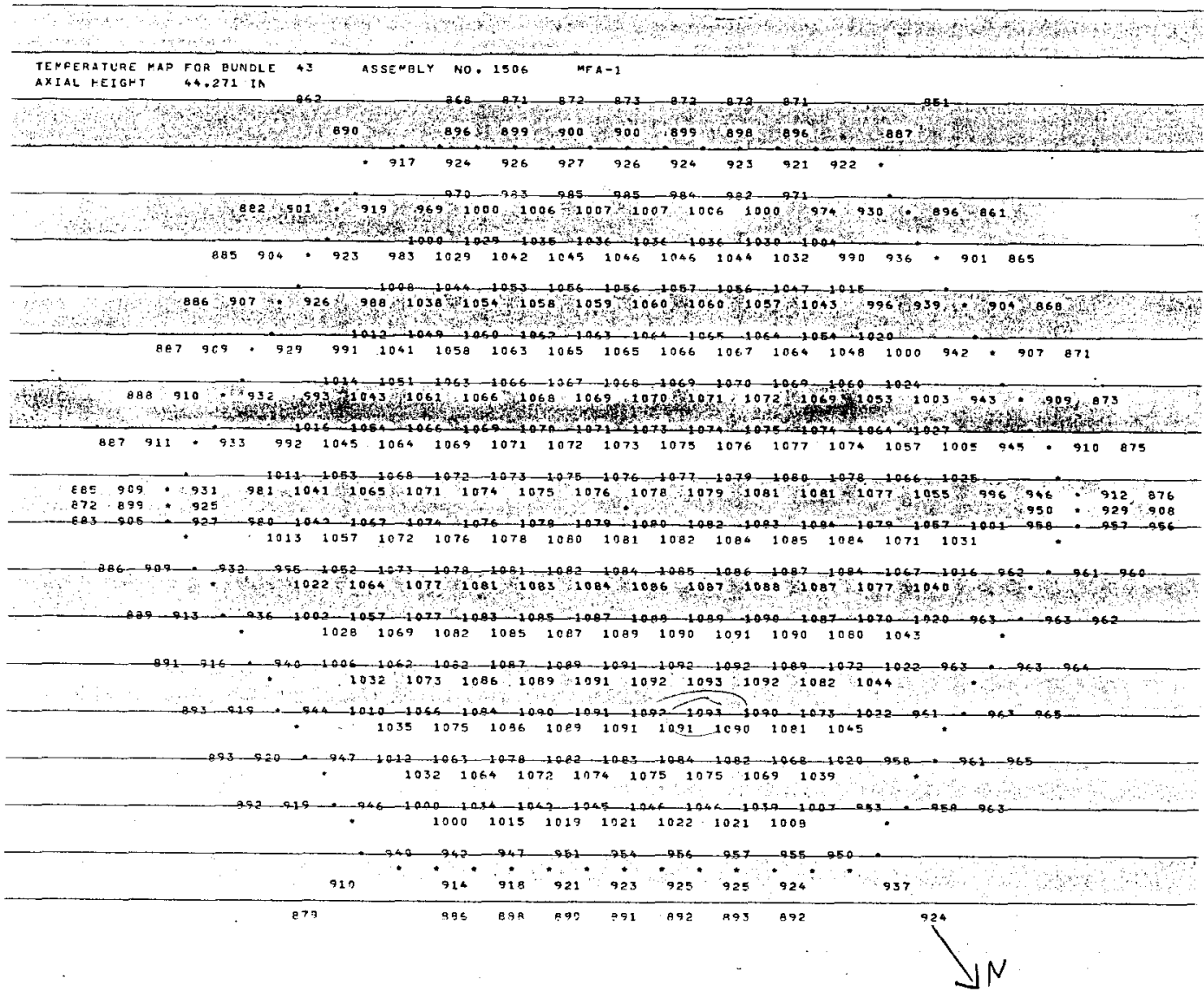


Table B10B.12. MFA-2 Sodium Subchannel Temperatures at Top of Core Elevation for BOC $10 \mathrm{~B}$

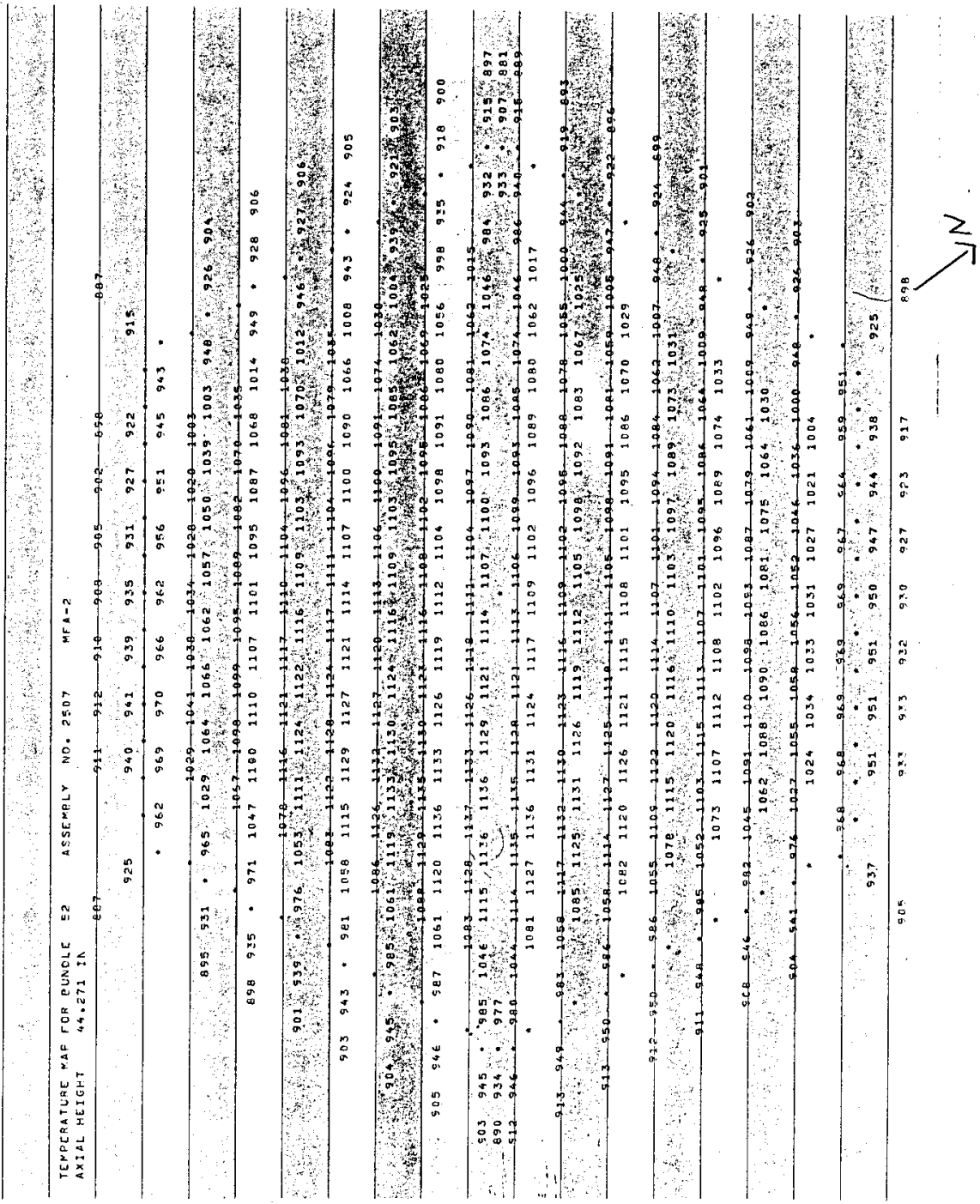


Table B10B.13. MFA-1 Sodium Subchannel Temperatures at Elevation of Upper Axial Blanket for BOC $10 B$

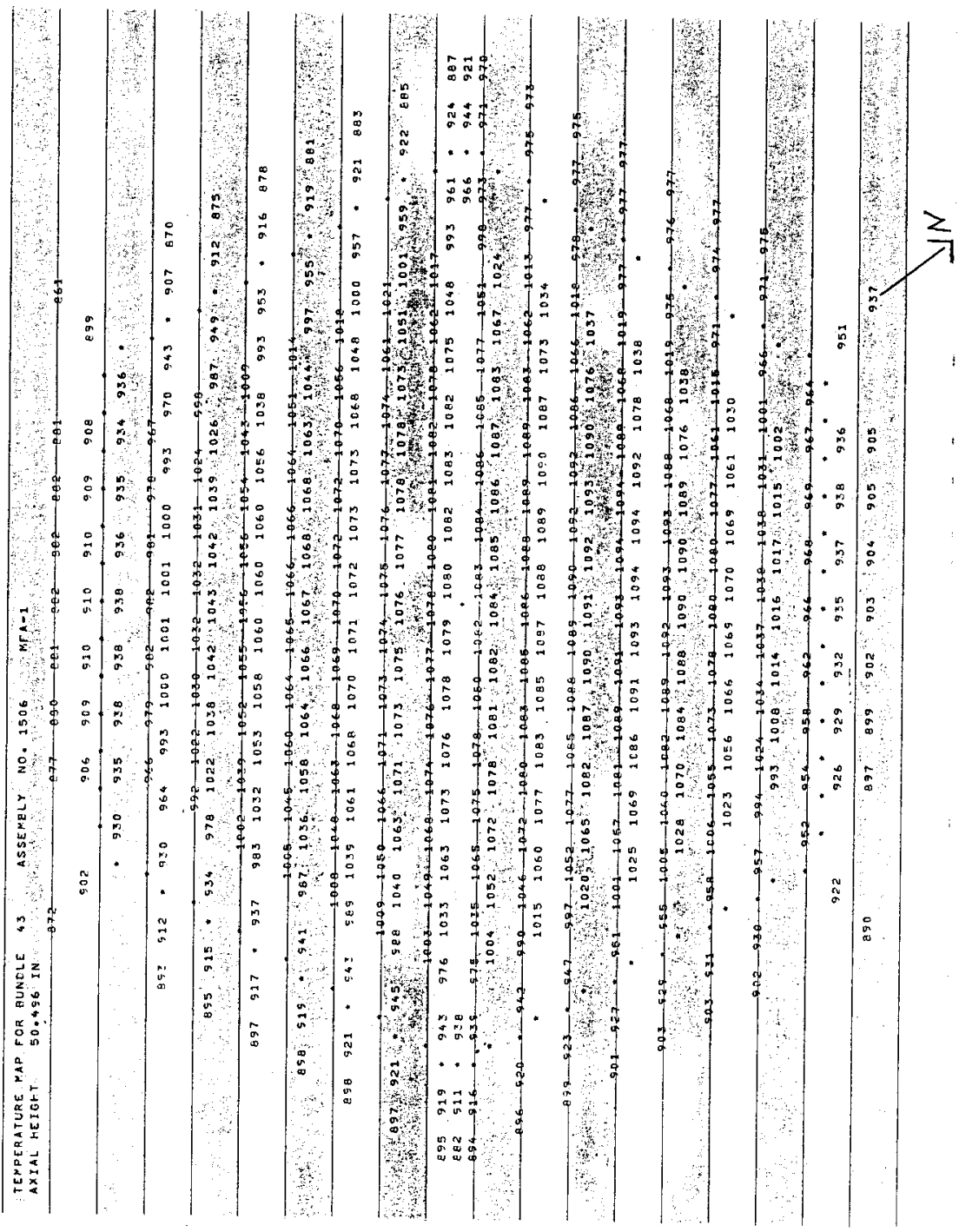




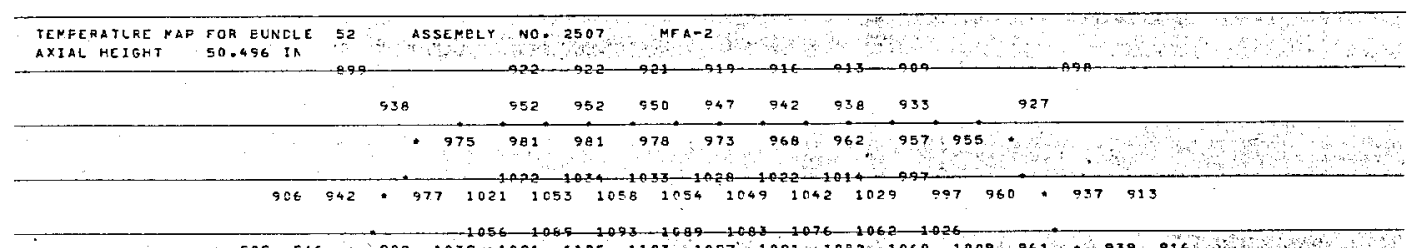

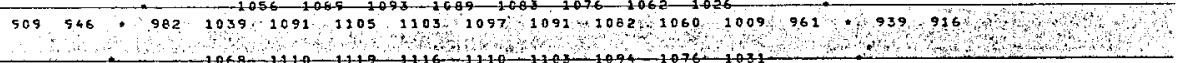

912550 * $988 \quad 1047 \quad 1104 \quad 1122 \quad 1122 \quad 1116 \quad 1110 \quad 1103 \quad 1092 \quad 10651008 \quad 959 \quad 938917$

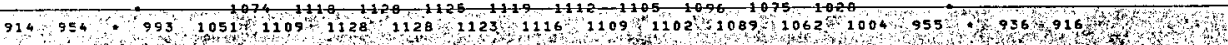

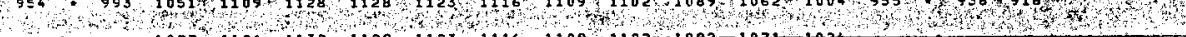

$915557.997 \quad 20541112$ 1132 1132 1126 1119111211051097100510571000952.993994

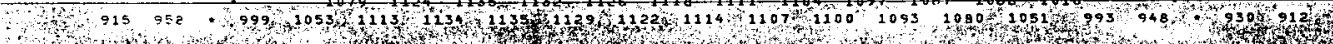

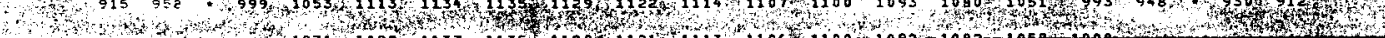

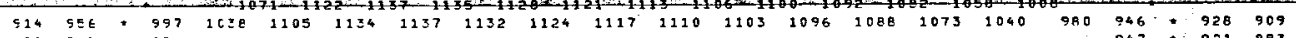

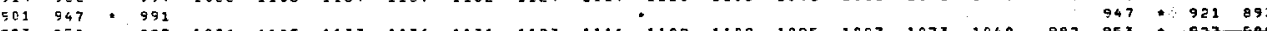

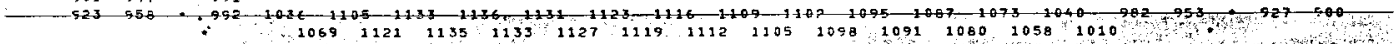

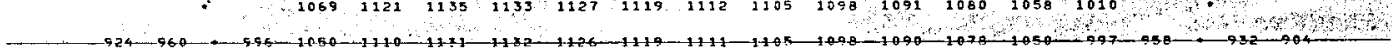
$1076 \quad 112011311128 \quad 112211151108 \quad 1101 \quad 1094 \quad 1084 \quad 1064 \quad 1020$

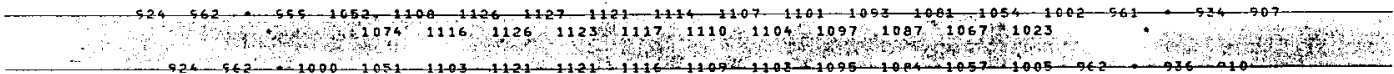
1071111111201117111211051099108910702026

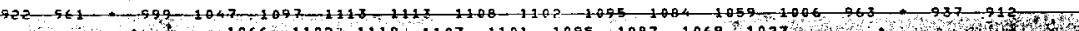

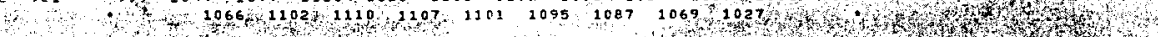

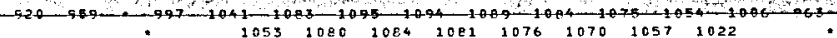

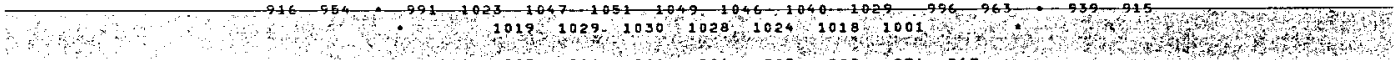

939

$55 ?$

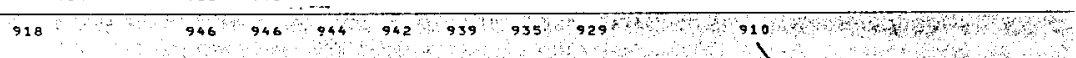


Table B10B.15. MFA-1 Sodium Subchannel Temperatures at Top of Fuel Pin Bundle for $B O C$ 1OB

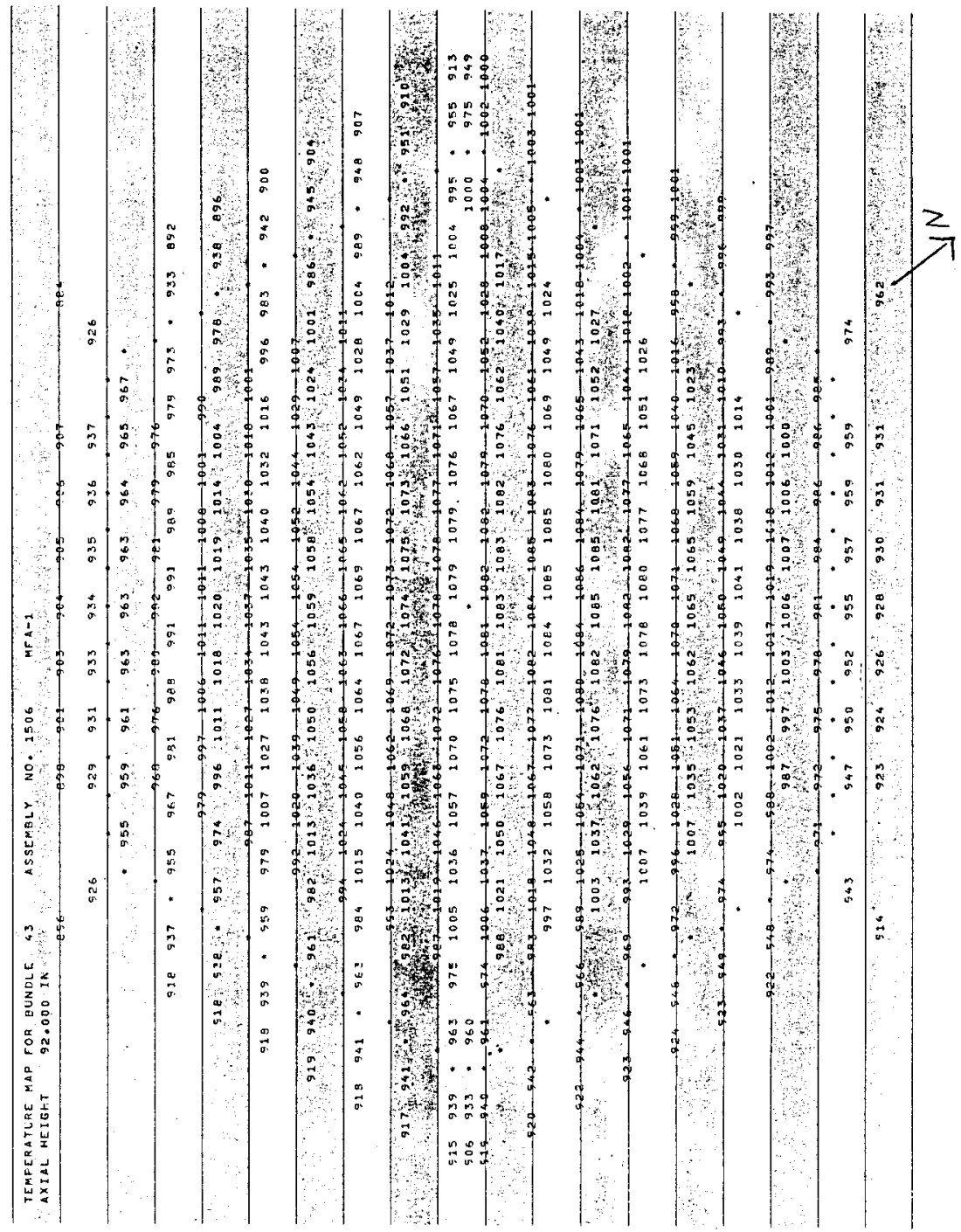




\begin{tabular}{l} 
TENFERATURE MAP FOR EUNELE S2 ASSEMELY NO. 2507 \\
AXIAL HEIGTT M2.0OOIN \\
\hline
\end{tabular}

$959 \quad 970 \quad 970969 \quad 967 \quad 964 \quad 961 \quad 959 \quad 951$

- 993 997 $997994990 \quad 9869839909996$

$531963 \cdot 9951013102910341033102810211010995902.957930$

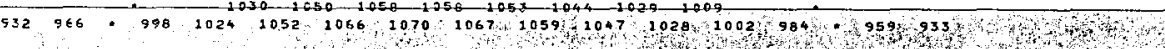

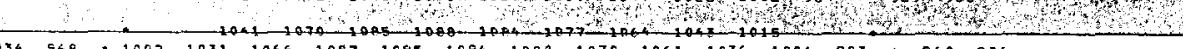

$934968 \quad 1002 \quad 1031 \quad 1066 \quad 1087 \quad 1095 \quad 1094 \quad 108 B \quad 1078 \quad 1061 \quad 1036 \quad 1004983 \quad 960936$

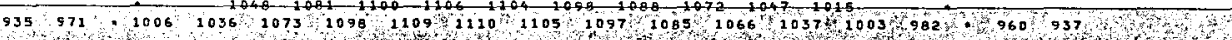

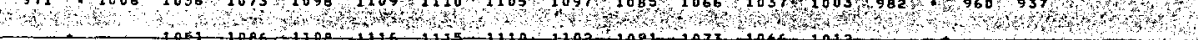

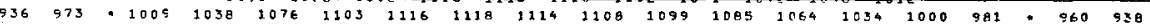

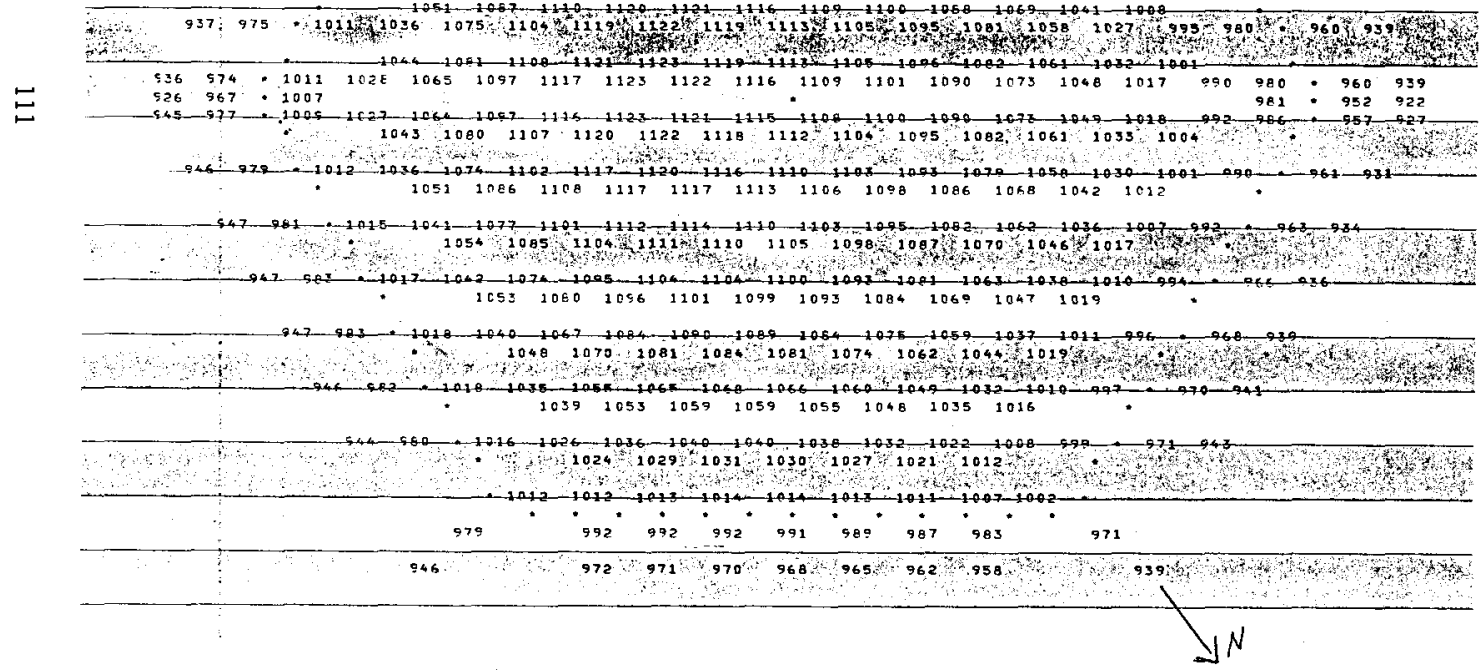


Table ElOB.1. Fission Power Generated in MFA-1, MFA-2 and Neighboring Assemblies at EOC 10B

\begin{tabular}{lccccc} 
CORE & $* \star * \star * *$ & \multicolumn{2}{c}{ POWER IN MEGAWATTS } & $* * * * \star *$ \\
POS. & BELOW CORE & IN CORE & ABOVE CORE & TOTAL PWR \\
1506 & $4.147 \mathrm{E}-02$ & $3.600 \mathrm{E}+00$ & $3.064 \mathrm{E}-02$ & $3.672 \mathrm{E}+00$ \\
1404 & $9.507 \mathrm{E}-02$ & $4.277 \mathrm{E}+00$ & $6.009 \mathrm{E}-02$ & $4.432 \mathrm{E}+00$ \\
1405 & $0.000 \mathrm{E}+00$ & $6.248 \mathrm{E}+00$ & $0.000 \mathrm{E}+00$ & $6.248 \mathrm{E}+00$ \\
1505 & $7.608 \mathrm{E}-03$ & $4.130 \mathrm{E}+00$ & $6.111 \mathrm{E}-03$ & $4.143 \mathrm{E}+00$ \\
1507 & $1.043 \mathrm{E}-02$ & $3.040 \mathrm{E}+00$ & $8.763 \mathrm{E}-03$ & $3.060 \mathrm{E}+00$ \\
1606 & $7.433 \mathrm{E}-03$ & $3.351 \mathrm{E}+00$ & $6.214 \mathrm{E}-03$ & $3.365 \mathrm{E}+00$ \\
1607 & $9.673 \mathrm{E}-03$ & $2.711 \mathrm{E}+00$ & $8.021 \mathrm{E}-03$ & $2.729 \mathrm{E}+00$ \\
2507 & $4.353 \mathrm{E}-02$ & $3.746 \mathrm{E}+00$ & $3.353 \mathrm{E}-02$ & $3.823 \mathrm{E}+00$ \\
2405 & $1.103 \mathrm{E}-02$ & $3.904 \mathrm{E}+00$ & $9.356 \mathrm{E}-03$ & $3.925 \mathrm{E}+00$ \\
2506 & $7.814 \mathrm{E}-03$ & $4.175 \mathrm{E}+00$ & $6.790 \mathrm{E}-03$ & $4.190 \mathrm{E}+00$ \\
2508 & $1.258 \mathrm{E}-02$ & $4.150 \mathrm{E}+00$ & $1.046 \mathrm{E}-02$ & $4.173 \mathrm{E}+00$ \\
2607 & $8.646 \mathrm{E}-03$ & $3.251 \mathrm{E}+00$ & $7.244 \mathrm{E}-03$ & $3.267 \mathrm{E}+00$ \\
2608 & $6.043 \mathrm{E}-03$ & $3.165 \mathrm{E}+00$ & $5.465 \mathrm{E}-03$ & $3.176 \mathrm{E}+00$ \\
2609 & $6.872 \mathrm{E}-03$ & $3.982 \mathrm{E}+00$ & $6.211 \mathrm{E}-03$ & $3.995 \mathrm{E}+00$
\end{tabular}

Table E10B.2. Assembly Averaged Total and Fast Flux in MFA-1 and MFA-2 at EOC 10B

\begin{tabular}{|c|c|c|c|}
\hline & Core & Flux & $m^{2}-\mathrm{sec}$ ) \\
\hline $\begin{array}{c}\text { Assembly } \\
\text { MFA-1 } \\
\text { MFA-2 }\end{array}$ & $\begin{array}{l}\text { Pos. } \\
1506 \\
2507\end{array}$ & $\begin{array}{c}\text { Total } \\
2.848 \mathrm{E}+15 \\
2.948 \mathrm{E}+15\end{array}$ & $\begin{array}{l}>0.1 \mathrm{MeV} \\
1.870 \mathrm{E}+15 \\
1.888 \mathrm{E}+15\end{array}$ \\
\hline
\end{tabular}


Table ElOB.3. Axial Distribution of Total Flux, Fast Flux and Power in MFA-1 at EOC 10B

$\begin{array}{rccc}\text { z }(\mathrm{cm}) & \text { Total Flux } & \text { Flux }>0.1 \text { MeV } & \text { Power } \\ -97.78 & 2.6556 \mathrm{E}-02 & 1.3311 \mathrm{E}-02 & 0.0000 \mathrm{E}+00 \\ -92.78 & 5.7032 \mathrm{E}-02 & 2.5707 \mathrm{E}-02 & 0.0000 \mathrm{E}+00 \\ -87.78 & 9.1393 \mathrm{E}-02 & 4.1152 \mathrm{E}-02 & 0.0000 \mathrm{E}+00 \\ -82.78 & 1.3221 \mathrm{E}-01 & 6.1421 \mathrm{E}-02 & 0.0000 \mathrm{E}+00 \\ -77.78 & 1.8205 \mathrm{E}-01 & 8.8716 \mathrm{E}-02 & 0.0000 \mathrm{E}+00 \\ -72.15 & 2.3834 \mathrm{E}-01 & 1.2408 \mathrm{E}-01 & 0.0000 \mathrm{E}+00 \\ -65.90 & 2.9425 \mathrm{E}-01 & 1.6878 \mathrm{E}-01 & 0.0000 \mathrm{E}+00 \\ -60.35 & 3.5314 \mathrm{E}-01 & 2.2955 \mathrm{E}-01 & 4.8073 \mathrm{E}-02 \\ -55.49 & 4.3135 \mathrm{E}-01 & 3.1083 \mathrm{E}-01 & 5.8282 \mathrm{E}-02 \\ -50.62 & 5.2237 \mathrm{E}-01 & 4.1682 \mathrm{E}-01 & 7.3765 \mathrm{E}-02 \\ -47.17 & 5.9540 \mathrm{E}-01 & 5.1685 \mathrm{E}-01 & 9.1281 \mathrm{E}-02 \\ -43.58 & 6.7219 \mathrm{E}-01 & 6.3005 \mathrm{E}-01 & 7.1728 \mathrm{E}-01 \\ -38.45 & 7.8345 \mathrm{E}-01 & 7.6728 \mathrm{E}-01 & 8.0557 \mathrm{E}-01 \\ -33.32 & 8.8740 \mathrm{E}-01 & 8.8458 \mathrm{E}-01 & 9.0324 \mathrm{E}-01 \\ -28.20 & 9.7932 \mathrm{E}-01 & 9.8288 \mathrm{E}-01 & 9.7144 \mathrm{E}-01 \\ -23.07 & 1.0577 \mathrm{E}+00 & 1.0647 \mathrm{E}+00 & 1.0477 \mathrm{E}+00 \\ -17.94 & 1.1211 \mathrm{E}+00 & 1.1297 \mathrm{E}+00 & 1.1097 \mathrm{E}+00 \\ -12.82 & 1.1687 \mathrm{E}+00 & 1.1777 \mathrm{E}+00 & 1.1434 \mathrm{E}+00 \\ -7.69 & 1.2005 \mathrm{E}+00 & 1.2098 \mathrm{E}+00 & 1.1741 \mathrm{E}+00 \\ -2.56 & 1.2160 \mathrm{E}+00 & 1.2257 \mathrm{E}+00 & 1.1892 \mathrm{E}+00 \\ 2.56 & 1.2150 \mathrm{E}+00 & 1.2251 \mathrm{E}+00 & 1.1920 \mathrm{E}+00 \\ 7.69 & 1.1973 \mathrm{E}+00 & 1.2078 \mathrm{E}+00 & 1.1747 \mathrm{E}+00 \\ 12.82 & 1.1627 \mathrm{E}+00 & 1.1735 \mathrm{E}+00 & 1.1409 \mathrm{E}+00 \\ 17.94 & 1.1114 \mathrm{E}+00 & 1.1225 \mathrm{E}+00 & 1.1093 \mathrm{E}+00 \\ 23.07 & 1.0427 \mathrm{E}+00 & 1.0526 \mathrm{E}+00 & 1.0414 \mathrm{E}+00 \\ 28.20 & 9.5759 \mathrm{E}-01 & 9.6441 \mathrm{E}-01 & 9.5755 \mathrm{E}-01 \\ 33.32 & 8.5765 \mathrm{E}-01 & 8.5853 \mathrm{E}-01 & 8.8248 \mathrm{E}-01 \\ 38.45 & 7.4477 \mathrm{E}-01 & 7.3329 \mathrm{E}-01 & 7.7260 \mathrm{E}-01 \\ 43.58 & 6.2448 \mathrm{E}-01 & 5.8979 \mathrm{E}-01 & 6.6739 \mathrm{E}-01 \\ 47.17 & 5.4275 \mathrm{E}-01 & 4.7724 \mathrm{E}-01 & 7.4707 \mathrm{E}-02 \\ 50.62 & 4.6655 \mathrm{E}-01 & 3.7967 \mathrm{E}-01 & 5.7303 \mathrm{E}-02 \\ 55.49 & 3.7180 \mathrm{E}-01 & 2.7652 \mathrm{E}-01 & 4.1724 \mathrm{E}-02 \\ 60.35 & 2.8787 \mathrm{E}-01 & 1.9594 \mathrm{E}-01 & 3.0995 \mathrm{E}-02 \\ 64.98 & 2.3396 \mathrm{E}-01 & 1.4764 \mathrm{E}-01 & 0.0000 \mathrm{E}+00 \\ 69.39 & 2.0126 \mathrm{E}-01 & 1.2142 \mathrm{E}-01 & 0.0000 \mathrm{E}+00 \\ 76.46 & 1.5393 \mathrm{E}-01 & 8.6116 \mathrm{E}-02 & 0.0000 \mathrm{E}+00 \\ 86.80 & 1.0102 \mathrm{E}-01 & 5.1935 \mathrm{E}-02 & 0.0000 \mathrm{E}+00 \\ 98.14 & 5.8524 \mathrm{E}-02 & 2.8396 \mathrm{E}-02 & 0.0000 \mathrm{E}+00 \\ 110.00 & 2.5111 \mathrm{E}-02 & 1.2728 \mathrm{E}-02 & 0.0000 \mathrm{E}+00\end{array}$


Table E10B.4. Axial Distribution of Total Flux, Fast Flux and Power in MFA-2 at EOC $10 \mathrm{~B}$

$\begin{array}{rccc}\text { z }(\mathrm{cm}) & \text { Total Flux } & \text { Flux }>0.1 \text { MeV } & \text { Power } \\ -97.78 & 2.4343 \mathrm{E}-02 & 1.2478 \mathrm{E}-02 & 0.0000 \mathrm{E}+00 \\ -92.78 & 5.2264 \mathrm{E}-02 & 2.4073 \mathrm{E}-02 & 0.0000 \mathrm{E}+00 \\ -87.78 & 8.3781 \mathrm{E}-02 & 3.8498 \mathrm{E}-02 & 0.0000 \mathrm{E}+00 \\ -82.78 & 1.2141 \mathrm{E}-01 & 5.7431 \mathrm{E}-02 & 0.0000 \mathrm{E}+00 \\ -77.78 & 1.6797 \mathrm{E}-01 & 8.3004 \mathrm{E}-02 & 0.0000 \mathrm{E}+00 \\ -72.15 & 2.2192 \mathrm{E}-01 & 1.1645 \mathrm{E}-01 & 0.0000 \mathrm{E}+00 \\ -65.90 & 2.7786 \mathrm{E}-01 & 1.5972 \mathrm{E}-01 & 0.0000 \mathrm{E}+00 \\ -60.35 & 3.3789 \mathrm{E}-01 & 2.1907 \mathrm{E}-01 & 4.8702 \mathrm{E}-02 \\ -55.49 & 4.1625 \mathrm{E}-01 & 2.9863 \mathrm{E}-01 & 5.9101 \mathrm{E}-02 \\ -50.62 & 5.0746 \mathrm{E}-01 & 4.0350 \mathrm{E}-01 & 7.4249 \mathrm{E}-02 \\ -47.17 & 5.8144 \mathrm{E}-01 & 5.0374 \mathrm{E}-01 & 9.1260 \mathrm{E}-02 \\ -43.58 & 6.5967 \mathrm{E}-01 & 6.1810 \mathrm{E}-01 & 7.1220 \mathrm{E}-01 \\ -38.45 & 7.7387 \mathrm{E}-01 & 7.5769 \mathrm{E}-01 & 7.9880 \mathrm{E}-01 \\ -33.32 & 8.8079 \mathrm{E}-01 & 8.7784 \mathrm{E}-01 & 8.9793 \mathrm{E}-01 \\ -28.20 & 9.7550 \mathrm{E}-01 & 9.7917 \mathrm{E}-01 & 9.6699 \mathrm{E}-01 \\ -23.07 & 1.0566 \mathrm{E}+00 & 1.0639 \mathrm{E}+00 & 1.0456 \mathrm{E}+00 \\ -17.94 & 1.1226 \mathrm{E}+00 & 1.1316 \mathrm{E}+00 & 1.1101 \mathrm{E}+00 \\ -12.82 & 1.1723 \mathrm{E}+00 & 1.1817 \mathrm{E}+00 & 1.1448 \mathrm{E}+00 \\ -7.69 & 1.2055 \mathrm{E}+00 & 1.2151 \mathrm{E}+00 & 1.1770 \mathrm{E}+00 \\ -2.56 & 1.2216 \mathrm{E}+00 & 1.2316 \mathrm{E}+00 & 1.1926 \mathrm{E}+00 \\ 2.56 & 1.2204 \mathrm{E}+00 & 1.2307 \mathrm{E}+00 & 1.1948 \mathrm{E}+00 \\ 7.69 & 1.2018 \mathrm{E}+00 & 1.2125 \mathrm{E}+00 & 1.1766 \mathrm{E}+00 \\ 12.82 & 1.1661 \mathrm{E}+00 & 1.1769 \mathrm{E}+00 & 1.1419 \mathrm{E}+00 \\ 17.94 & 1.1136 \mathrm{E}+00 & 1.1243 \mathrm{E}+00 & 1.1097 \mathrm{E}+00 \\ 23.07 & 1.0439 \mathrm{E}+00 & 1.0532 \mathrm{E}+00 & 1.0409 \mathrm{E}+00 \\ 28.20 & 9.5798 \mathrm{E}-01 & 9.6416 \mathrm{E}-01 & 9.5666 \mathrm{E}-01 \\ 33.32 & 8.5773 \mathrm{E}-01 & 8.5803 \mathrm{E}-01 & 8.8274 \mathrm{E}-01 \\ 38.45 & 7.4497 \mathrm{E}-01 & 7.3319 \mathrm{E}-01 & 7.7482 \mathrm{E}-01 \\ 43.58 & 6.2503 \mathrm{E}-01 & 5.9026 \mathrm{E}-01 & 6.7578 \mathrm{E}-01 \\ 47.17 & 5.4250 \mathrm{E}-01 & 4.7582 \mathrm{E}-01 & 7.7442 \mathrm{E}-02 \\ 50.62 & 4.6424 \mathrm{E}-01 & 3.7539 \mathrm{E}-01 & 5.9987 \mathrm{E}-02 \\ 55.49 & 3.6663 \mathrm{E}-01 & 2.6980 \mathrm{E}-01 & 4.4168 \mathrm{E}-02 \\ 60.35 & 2.8101 \mathrm{E}-01 & 1.8885 \mathrm{E}-01 & 3.3072 \mathrm{E}-02 \\ 64.98 & 2.2784 \mathrm{E}-01 & 1.4187 \mathrm{E}-01 & 0.0000 \mathrm{E}+00 \\ 69.39 & 1.9574 \mathrm{E}-01 & 1.1675 \mathrm{E}-01 & 0.0000 \mathrm{E}+00 \\ 76.46 & 1.4946 \mathrm{E}-01 & 8.2926 \mathrm{E}-02 & 0.0000 \mathrm{E}+00 \\ 86.80 & 9.7887 \mathrm{E}-02 & 4.9962 \mathrm{E}-02 & 0.0000 \mathrm{E}+00 \\ 98.14 & 5.6532 \mathrm{E}-02 & 2.7207 \mathrm{E}-02 & 0.0000 \mathrm{E}+00 \\ 110.00 & 2.3989 \mathrm{E}-02 & 1.2054 \mathrm{E}-02 & 0.0000 \mathrm{E}+00\end{array}$


HNF-SD-FF-ANAL-009 Rev. 1

Table E10B.5. Fission Power Distribution by Pin in MFA-1 at EOC 10B CORE POSITION 1506

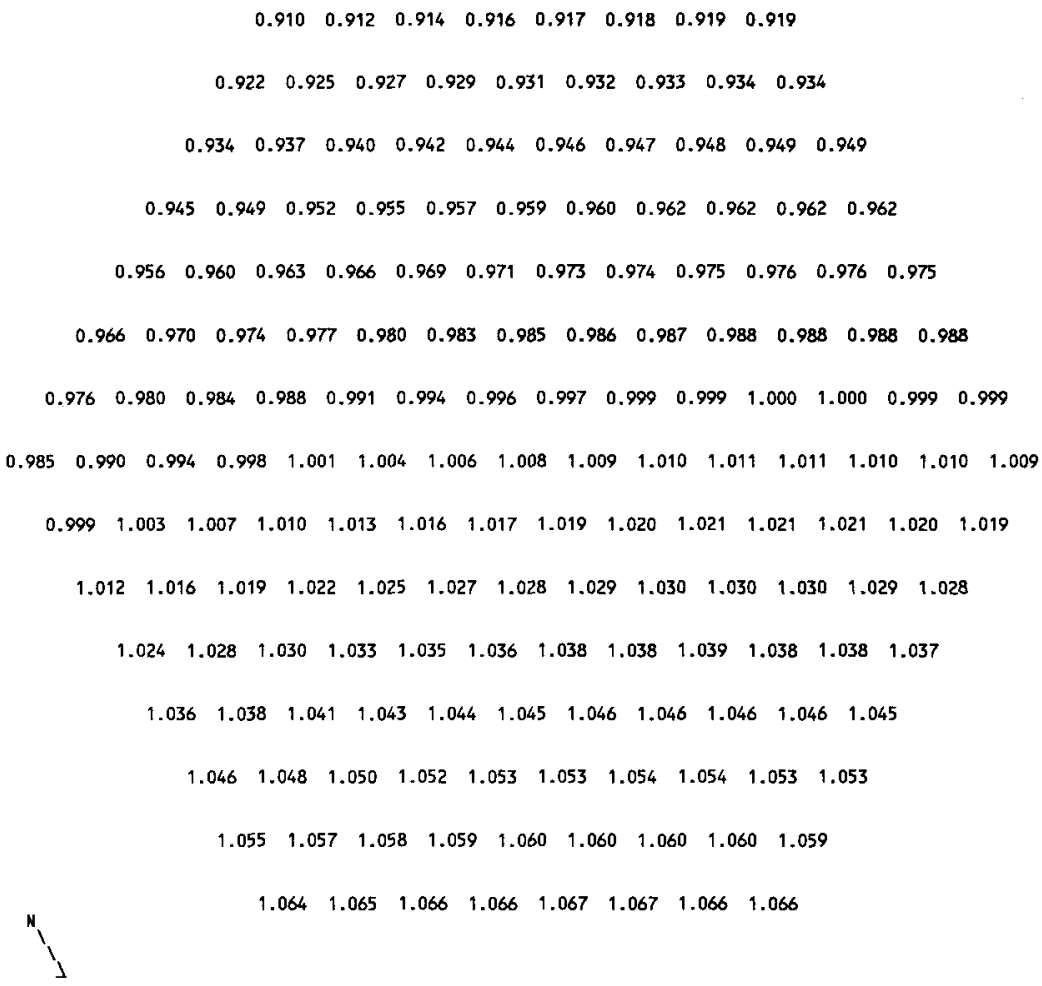


HNF-SD-FF-ANAL-009 Rev. 1

Table E10B.6. Fission Power Distribution by Pin in MFA-2 at EOC 10B CORE POSITION 2507

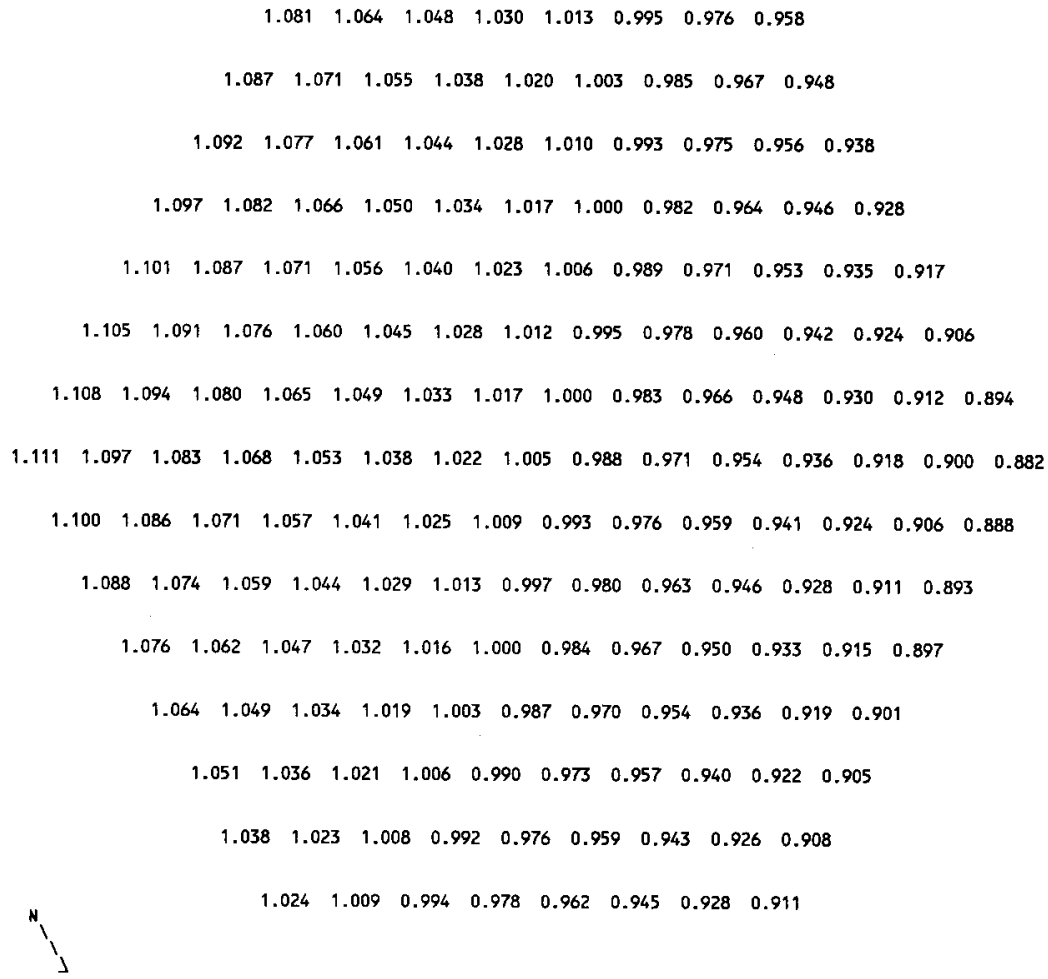


HNF-SD-FF-ANAL-009 Rev, 1

Table E1OB.7. Fast Flux Distribution by Pin in MFA-1 at EOC $10 B$ CORE POSITION 1506

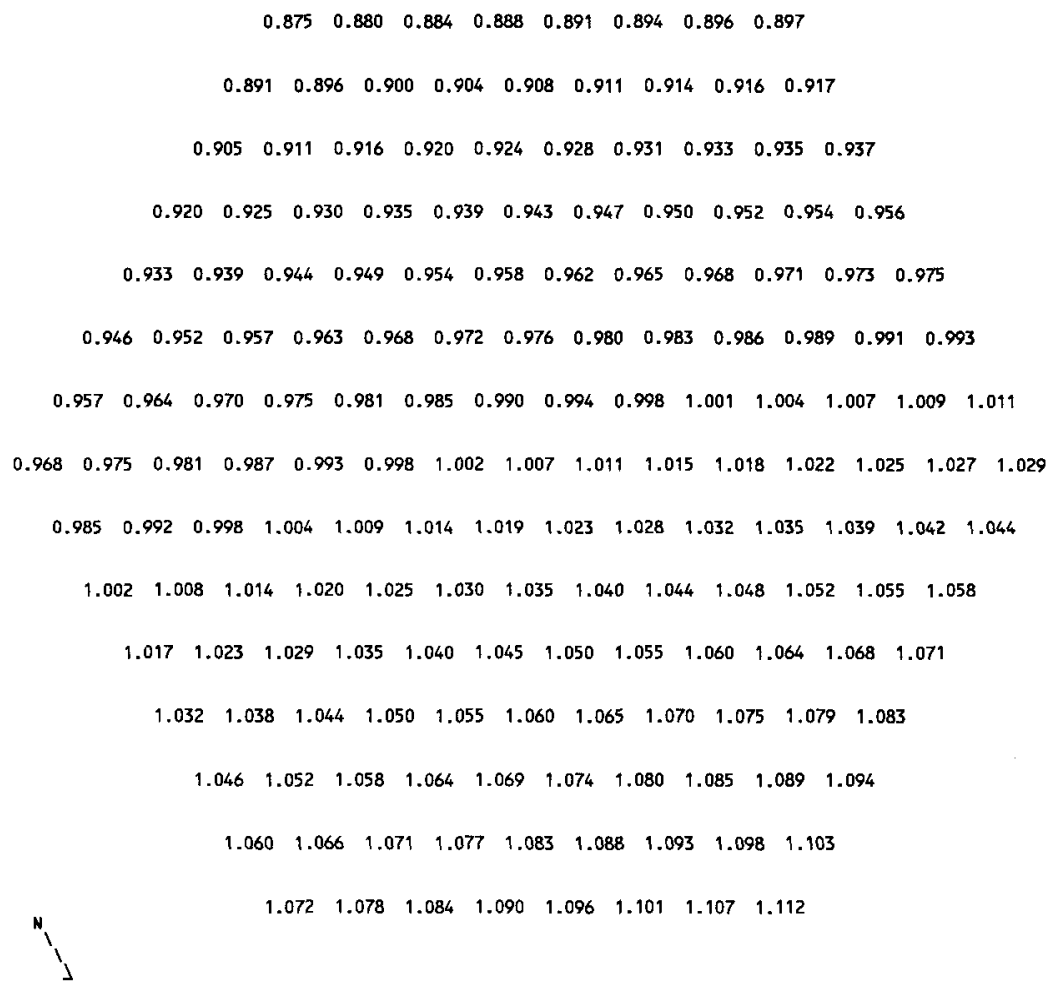


HNF-SD-FF-ANAL-009 Rev. 1

Table E10B.8. Fast Flux Distribution by Pin in MFA-2 at EOC 10B CORE POSITION 2507

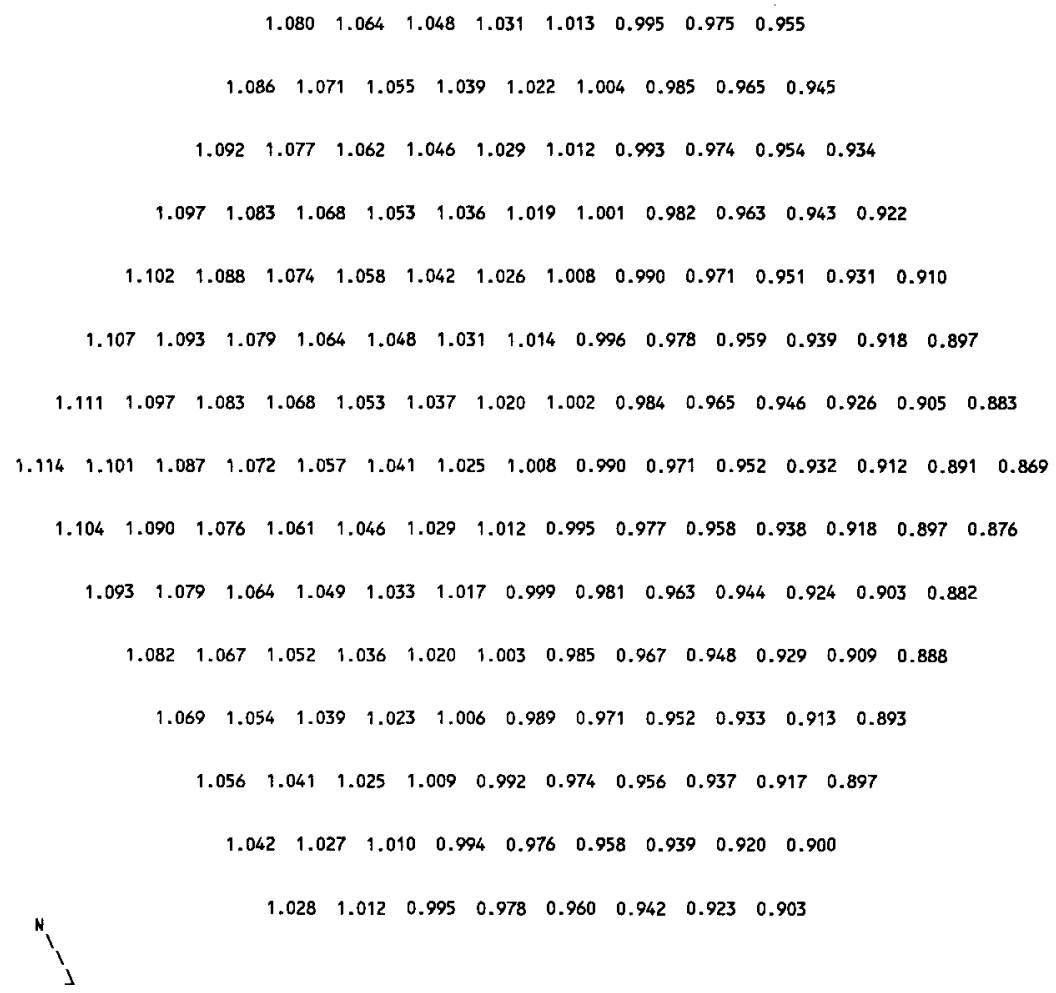


Table E10B.9. MFA-1 and MFA-2 Duct Wall

Fast Flux Data at EOC 10B

$\begin{array}{ccc}\text { Side } & \begin{array}{c}\text { Flux }>0.1 \\ \text { MFA-1 }\end{array} & \begin{array}{c}\text { MeV } \\ \left(\mathrm{n} / \mathrm{cm}^{2}-\mathrm{sec}\right) \\ \text { MFA-2 }\end{array} \\ \text { E } & 1.9156 \mathrm{E}+15 & 2.0498 \mathrm{E}+15 \\ \text { SE } & 1.6949 \mathrm{E}+15 & 2.1040 \mathrm{E}+15 \\ \text { SW } & 1.6100 \mathrm{E}+15 & 1.9269 \mathrm{E}+15 \\ \text { W } & 1.7782 \mathrm{E}+15 & 1.6837 \mathrm{E}+15 \\ \text { NW } & 2.0340 \mathrm{E}+15 & 1.6263 \mathrm{E}+15 \\ \text { NE } & 2.0627 \mathrm{E}+15 & 1.8024 \mathrm{E}+15\end{array}$

Table ElOB.10. Assembly outlet Temperatures and Flow Rates at EOC 10B

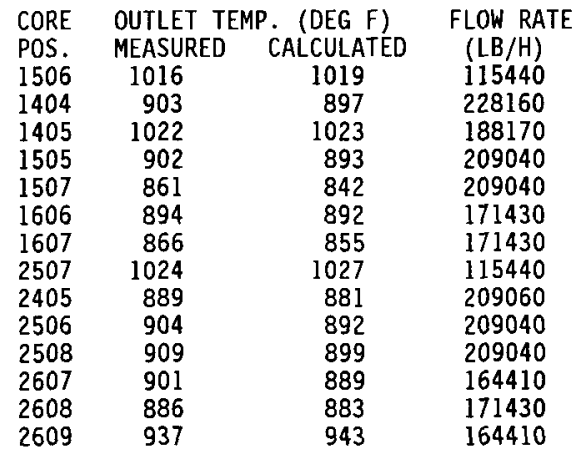

Assembly flows are based on a calculated core pressure drop of $111.5 \mathrm{psi}$ at a total reactor flow rate of 16.74E+06 $\mathrm{lb} / \mathrm{hr}$. 


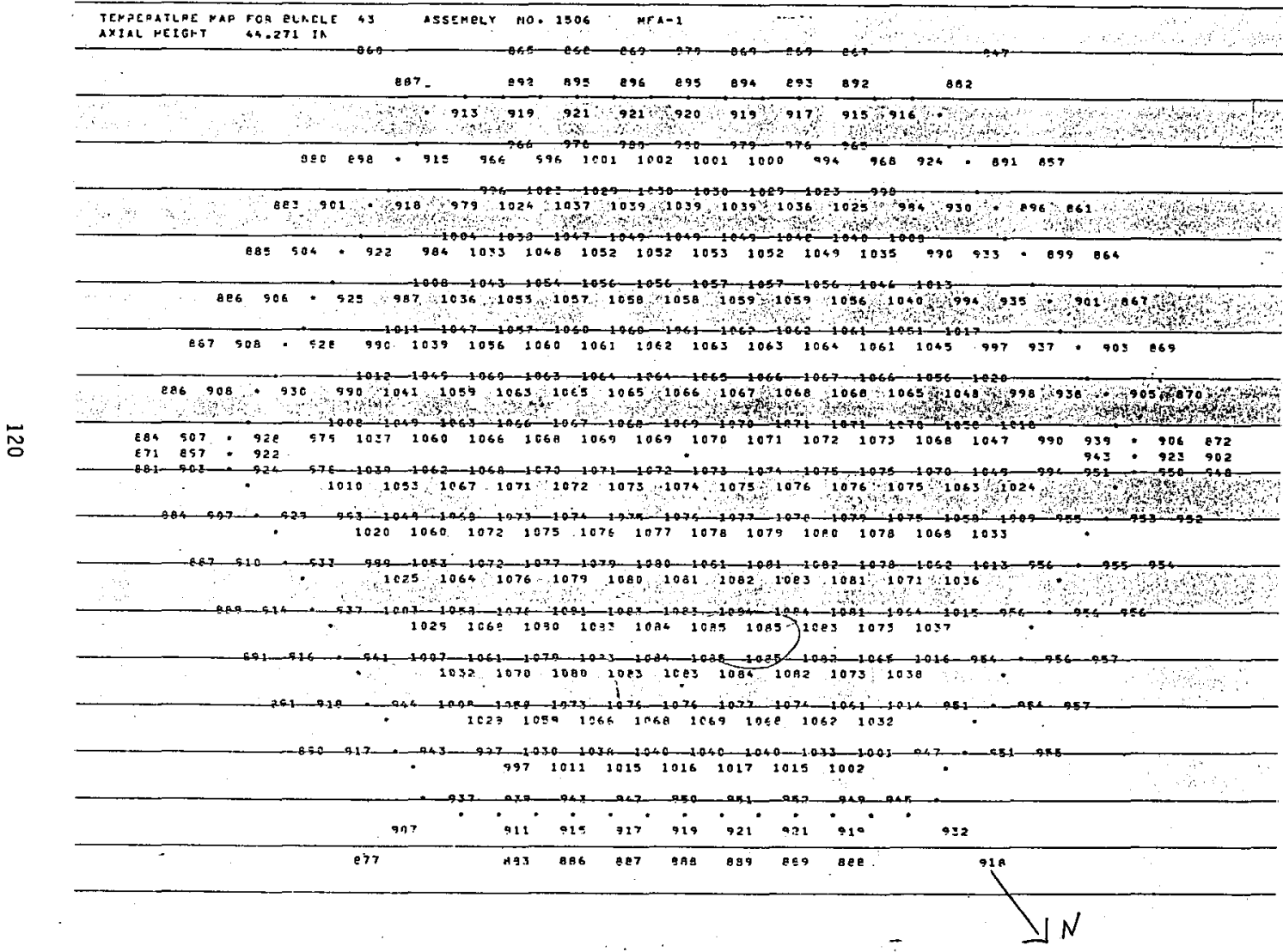




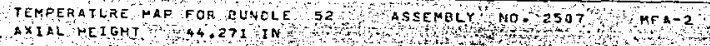

913

-

$885918 * 9511013104010481044103910331022988935.914894$

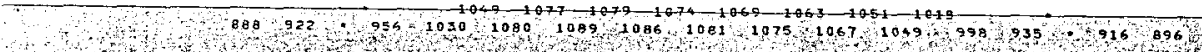

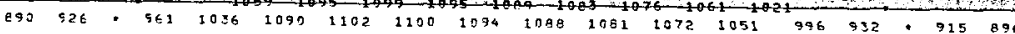

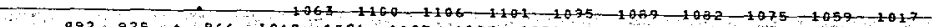

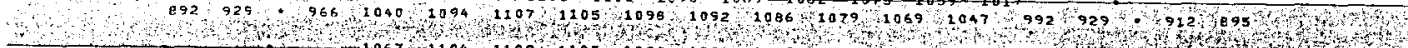

993932 969 10421097111011071101109410871091107410651042989925.909092

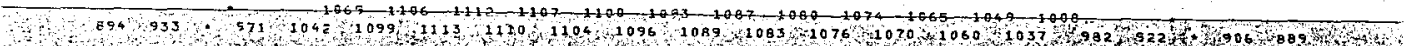
-

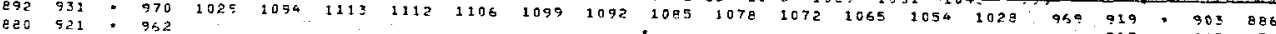

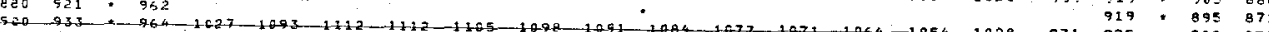

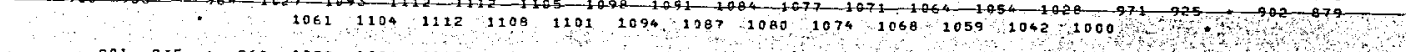

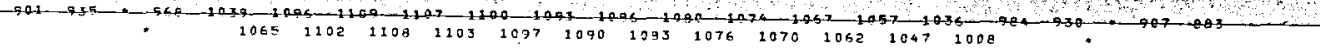

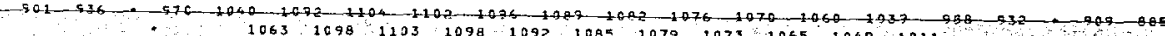

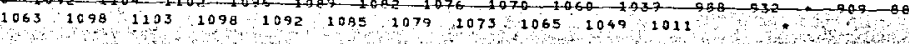

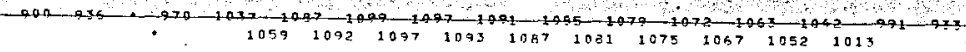

$\rightarrow+\infty+80$

and

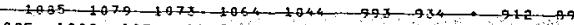

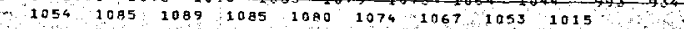

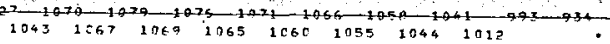

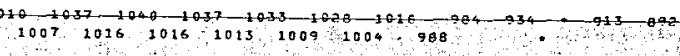

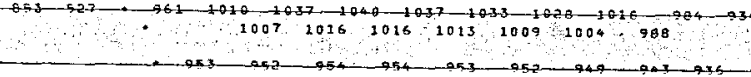

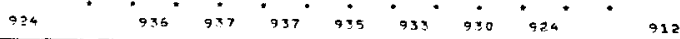

$094 \quad 920 \quad 920 \quad 919 \quad 917.914 \quad 910 \quad 905$

$\int_{\searrow N}^{A 87}$ 


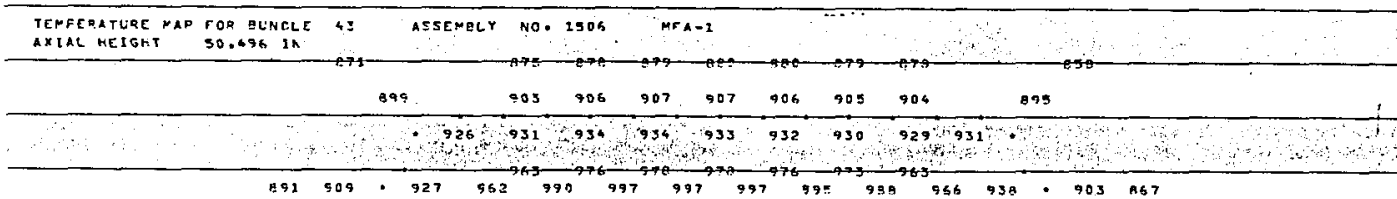

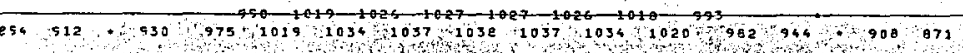

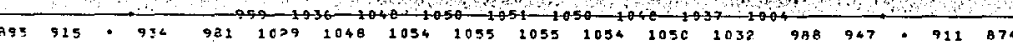

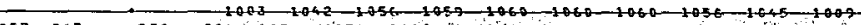

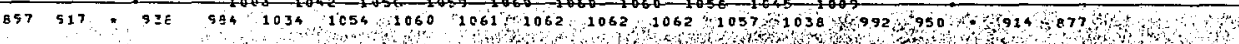

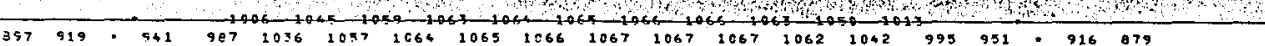

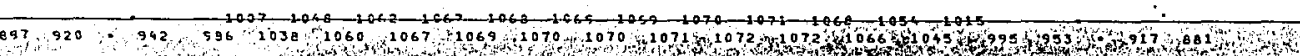

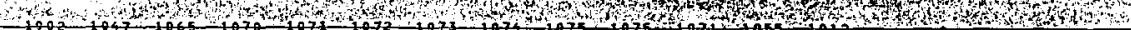

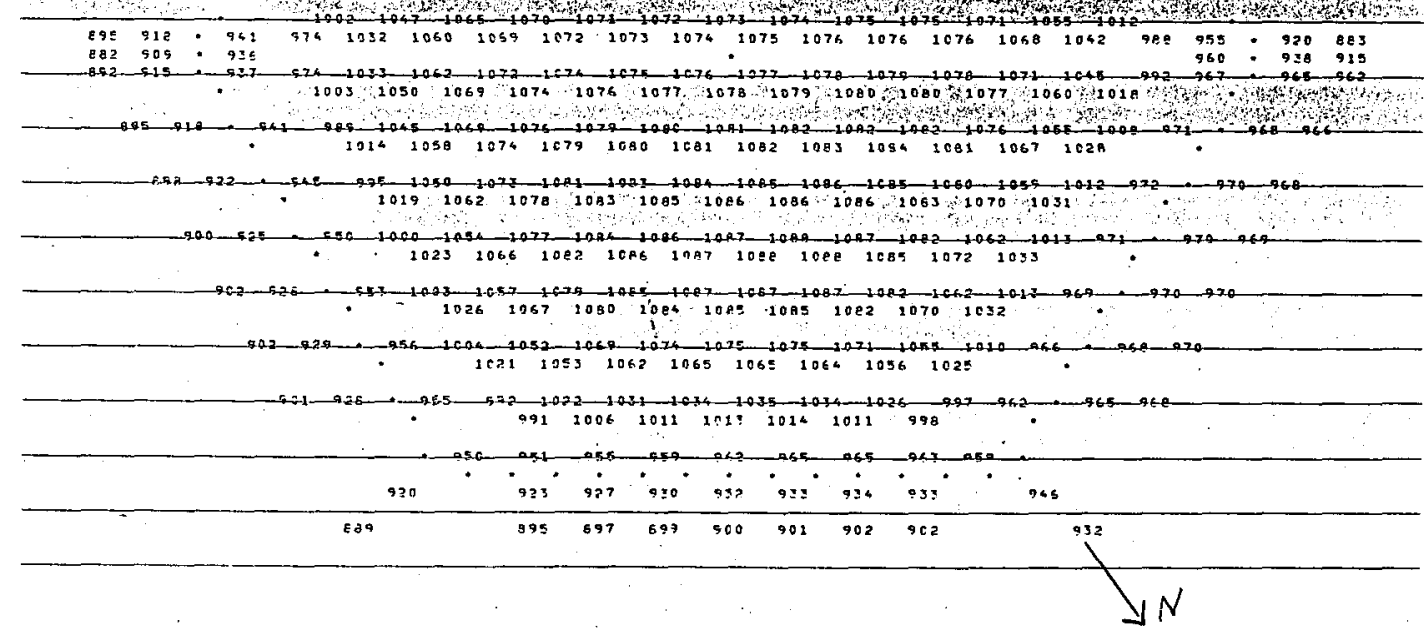

$\stackrel{\vec{a}}{\sim}$

紧

요

$<\infty$

$\Xi$ w

.

옥즉

돈

苗的

r吕

文.

四

চ

畟

帘岂

$\rightarrow$

홍

m言

응

品

윰ำ 


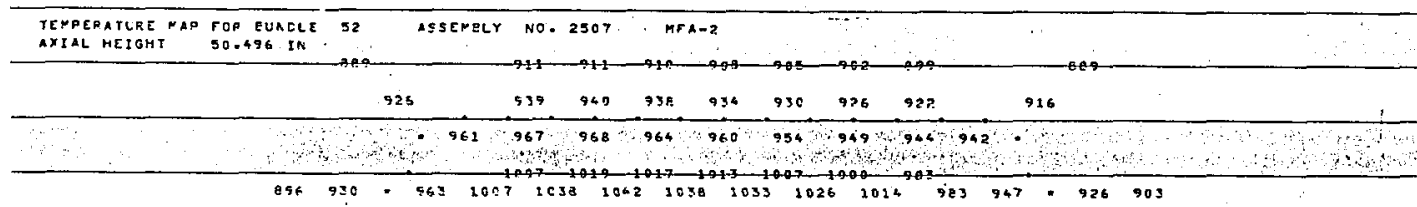

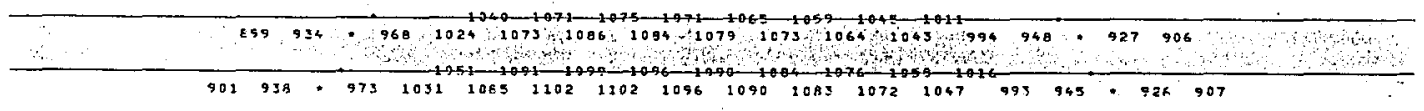

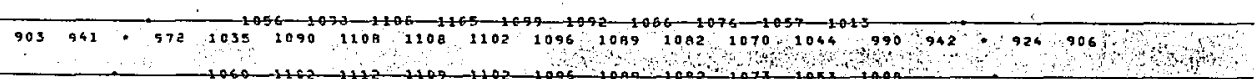

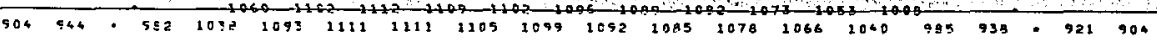

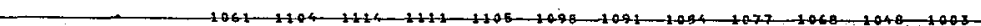

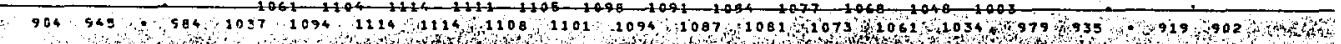

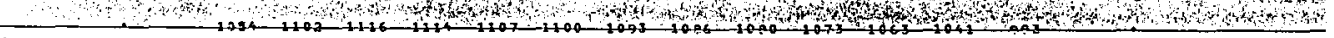

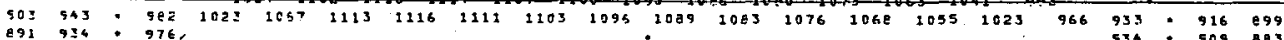

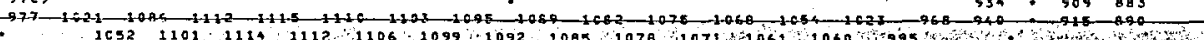

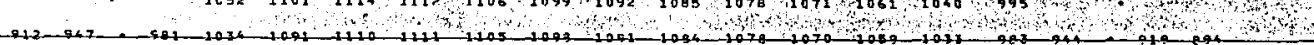

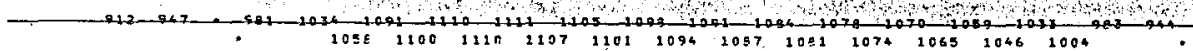

5.13 548 .

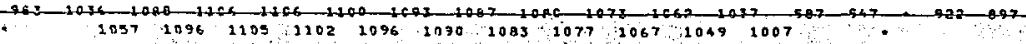

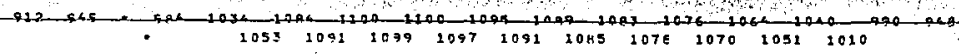

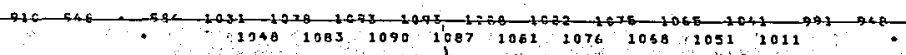

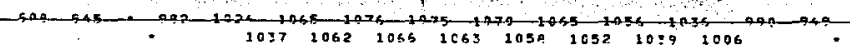

$-26-902$

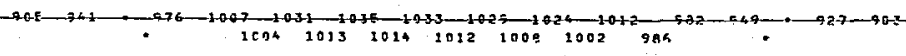

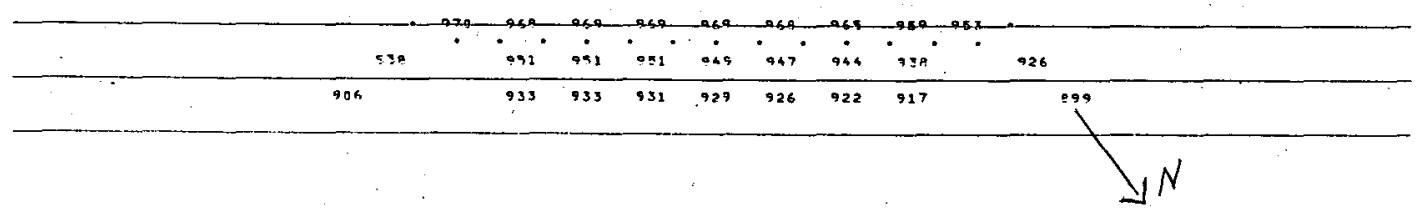

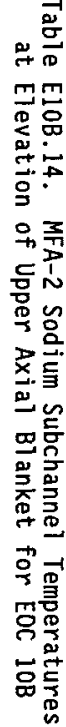


Table E10B.15. MFA-1 Sodium Subchannel Temperatures at Top of Fuel Pin Bundle for EOC $10 B$

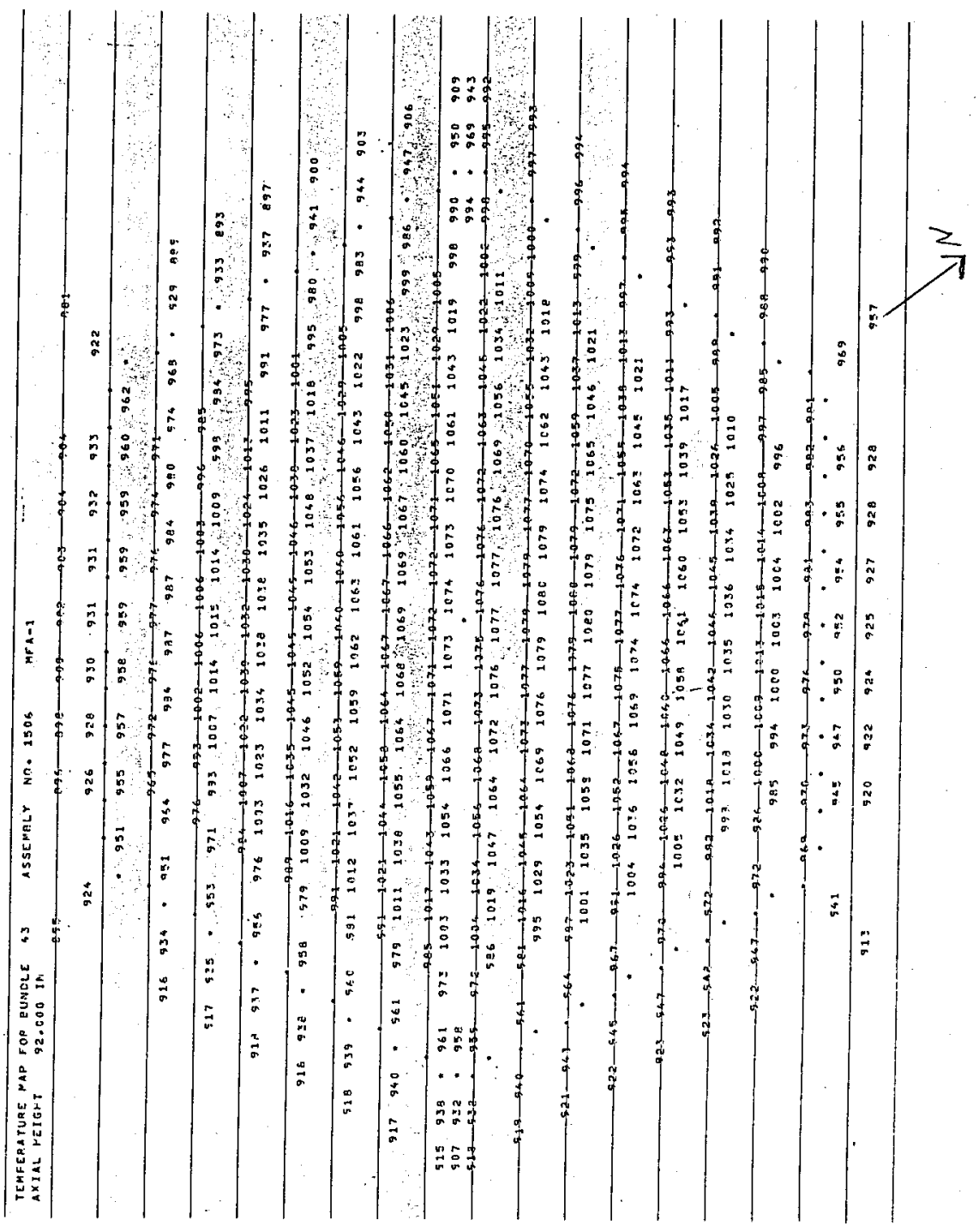


Table E10B.16. MFA-2 Sodium Subchannel Temperatures at Top of Fuel Pin Bundle for EOC $10 B$

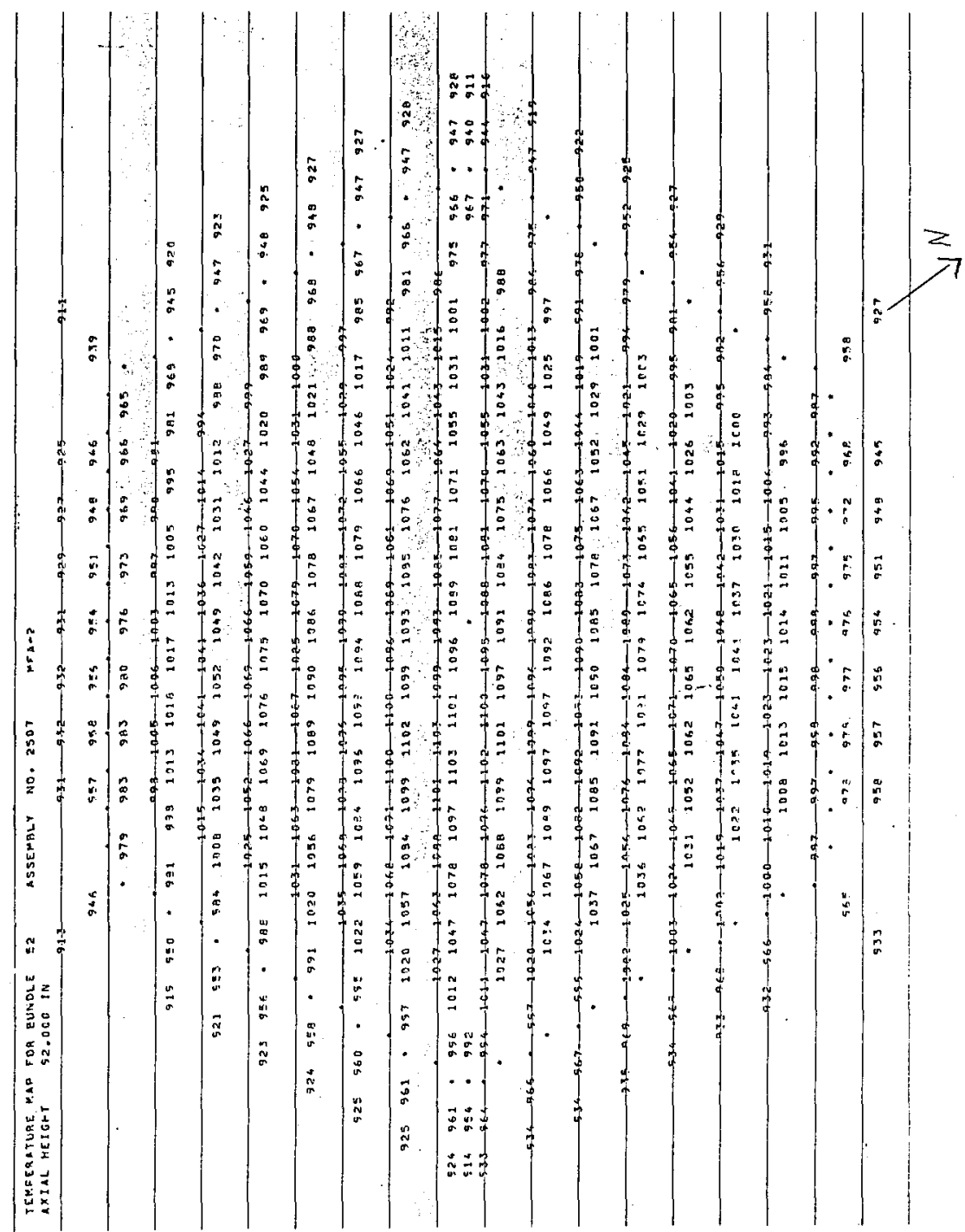


HNF-SD-FF-ANAL-009 Rev. 1

Table ElOB.17. Composition of MFA-1 at EOC 1OB

\begin{tabular}{|c|c|c|c|c|c|}
\hline & Axial & ge $(\mathrm{cm})$ & & Atom De & m) \\
\hline & $\begin{array}{r}\text { Lower } \\
-12805\end{array}$ & $\begin{array}{l}\text { Upper } \\
-75\end{array}$ & Constituent & & \\
\hline & & & $\begin{array}{l}\mathrm{Na}-23 \\
\text { SS-316 }\end{array}$ & $\begin{array}{l}5.3150 \mathrm{E}-03 \\
6.4090 \mathrm{E}-02\end{array}$ & $\begin{array}{l}5.3150 \mathrm{E}-03 \\
6.4090 \mathrm{E}-02\end{array}$ \\
\hline 2 & -75.28 & -62.78 & & & \\
\hline 3 & -62.78 & -46.14 & $\begin{array}{l}\text { Na-23 } \\
\text { SS-316 }\end{array}$ & $\begin{array}{l}1.3177 \mathrm{E}-02 \\
3.4175 \mathrm{E}-02\end{array}$ & $\begin{array}{l}1.3177 \mathrm{E}-02 \\
3.4175 \mathrm{E}-02\end{array}$ \\
\hline & & & $\begin{array}{l}U-235 \\
U-238 \\
\mathrm{Pu}-239 \\
\mathrm{Pu}-240 \\
\mathrm{FP} \\
\mathrm{O}-16 \\
\mathrm{Na}-23 \\
\mathrm{SS}-316\end{array}$ & $\begin{array}{l}1.4143 \mathrm{E}-05 \\
7.0573 \mathrm{E}-03 \\
0.0000 \mathrm{E}+00 \\
0.0000 \mathrm{E}+00 \\
0.0000 \mathrm{E}+00 \\
1.4143 \mathrm{E}-02 \\
9.7416 \mathrm{E}-03 \\
1.9073 \mathrm{E}-02\end{array}$ & $\begin{array}{l}1.2238 \mathrm{E}-05 \\
6.9566 \mathrm{E}-03 \\
8.8941 \mathrm{E}-05 \\
1.7886 \mathrm{E}-06 \\
1.1291 \mathrm{E}-05 \\
1.4143 \mathrm{E}-02 \\
9.7416 \mathrm{E}-03 \\
1.9073 \mathrm{E}-02\end{array}$ \\
\hline 4 & -46.14 & -30.76 & $\begin{array}{l}U-235 \\
U-238 \\
P u-239 \\
P u-240 \\
P u-241 \\
P u-242 \\
\mathrm{Am}-241 \\
\mathrm{FP} \\
0-16 \\
\mathrm{Na}-23 \\
\text { SS }-316\end{array}$ & $\begin{array}{l}1.0840 \mathrm{E}-05 \\
4.4499 \mathrm{E}-03 \\
1.7520 \mathrm{E}-03 \\
2.2340 \mathrm{E}-04 \\
1.9280 \mathrm{E}-05 \\
4.7430 \mathrm{E}-06 \\
7.6680 \mathrm{E}-07 \\
0.0000 \mathrm{E}+00 \\
1.2670 \mathrm{E}-02 \\
9.8100 \mathrm{E}-03 \\
1.8990 \mathrm{E}-02\end{array}$ & $\begin{array}{l}9.5078 \mathrm{E}-06 \\
4.3731 \mathrm{E}-03 \\
1.6001 \mathrm{E}-03 \\
2.5209 \mathrm{E}-04 \\
2.1258 \mathrm{E}-05 \\
4.9086 \mathrm{E}-06 \\
1.4957 \mathrm{E}-06 \\
1.9784 \mathrm{E}-04 \\
1.2670 \mathrm{E}-02 \\
9.8100 \mathrm{E}-03 \\
1.8990 \mathrm{E}-02\end{array}$ \\
\hline 3 & -30.10 & -15.38 & $\begin{array}{l}U-235 \\
U-238 \\
P u-239 \\
P u-240 \\
P u-241 \\
P u-242 \\
\text { Am-241 } \\
F P \\
0-16 \\
\mathrm{Na}-23 \\
\text { SS }-316\end{array}$ & $\begin{array}{l}1.0840 \mathrm{E}-05 \\
4.4499 \mathrm{E}-03 \\
1.7520 \mathrm{E}-03 \\
2.2340 \mathrm{E}-04 \\
1.9280 \mathrm{E}-05 \\
4.7430 \mathrm{E}-05 \\
7.6680 \mathrm{E}-07 \\
0.0000 \mathrm{E}+00 \\
1.2670 \mathrm{E}-02 \\
9.8100 \mathrm{E}-03 \\
1.8990 \mathrm{E}-02\end{array}$ & $\begin{array}{l}9.2007 \mathrm{E}-06 \\
4.3525 \mathrm{E}-03 \\
1.5596 \mathrm{E}-03 \\
2.5435 \mathrm{E}-04 \\
2.1405 \mathrm{E}-05 \\
4.9098 \mathrm{E}-06 \\
1.4732 \mathrm{E}-06 \\
2.5693 \mathrm{E}-04 \\
1.2670 \mathrm{E}-02 \\
9.8100 \mathrm{E}-03 \\
1.8990 \mathrm{E}-02\end{array}$ \\
\hline 0 & -15.38 & 0.00 & $\begin{array}{l}U-235 \\
U-238 \\
P u-239 \\
P u-240 \\
P u-241 \\
P u-242 \\
\text { Am-241 } \\
\mathrm{FP} \\
0-16 \\
\mathrm{Na}-23 \\
S S-316\end{array}$ & $\begin{array}{l}1.0840 \mathrm{E}-05 \\
4.4499 \mathrm{E}-03 \\
1.7520 \mathrm{E}-03 \\
2.2340 \mathrm{E}-04 \\
1.9280 \mathrm{E}-05 \\
4.7430 \mathrm{E}-06 \\
7.6680 \mathrm{E}-07 \\
0.0000 \mathrm{E}+00 \\
1.2670 \mathrm{E}-02 \\
9.8100 \mathrm{E}-03 \\
1.8990 \mathrm{E}-02\end{array}$ & $\begin{array}{l}9.0293 \mathrm{E}-05 \\
4.3414 \mathrm{E}-03 \\
1.5388 \mathrm{E}-03 \\
2.5720 \mathrm{E}-04 \\
2.1727 \mathrm{E}-05 \\
4.9304 \mathrm{E}-06 \\
1.4646 \mathrm{E}-06 \\
2.8557 \mathrm{E}-04 \\
1.2670 \mathrm{E}-02 \\
9.8100 \mathrm{E}-03 \\
1.8990 \mathrm{E}-02\end{array}$ \\
\hline
\end{tabular}


HNF-SD-FF-ANAL-009 Rev. 1

\begin{tabular}{|c|c|c|c|c|c|}
\hline \multirow{2}{*}{7} & 0.00 & 15.38 & & & \\
\hline & & & $\begin{array}{l}U-235 \\
U-238 \\
P u-239 \\
P u-240 \\
P u-241 \\
P u-242 \\
A m-241 \\
F P \\
0-16 \\
N a-23 \\
S S-316\end{array}$ & $\begin{array}{l}1.0840 \mathrm{E}-05 \\
4.4499 \mathrm{E}-03 \\
1.7520 \mathrm{E}-03 \\
2.2340 \mathrm{E}-04 \\
1.9280 \mathrm{E}-05 \\
4.7430 \mathrm{E}-06 \\
7.6680 \mathrm{E}-07 \\
0.0000 \mathrm{E}+00 \\
1.2670 \mathrm{E}-02 \\
9.8100 \mathrm{E}-03 \\
1.8990 \mathrm{E}-02\end{array}$ & $\begin{array}{l}9.0781 \mathrm{E}-06 \\
4.3446 \mathrm{E}-03 \\
1.5446 \mathrm{E}-03 \\
2.5624 \mathrm{E}-04 \\
2.1618 \mathrm{E}-05 \\
4.9231 \mathrm{E}-06 \\
1.4670 \mathrm{E}-06 \\
2.7762 \mathrm{E}-04 \\
1.2670 \mathrm{E}-02 \\
9.8100 \mathrm{E}-03 \\
1.8990 \mathrm{E}-02\end{array}$ \\
\hline \multirow[t]{2}{*}{8} & 15.38 & 30.76 & & & \\
\hline & & & $\begin{array}{l}U-235 \\
U-238 \\
\mathrm{Pu}-239 \\
\mathrm{Pu}-240 \\
\mathrm{Pu}-241 \\
\mathrm{Pu}-242 \\
\mathrm{Am}-241 \\
\mathrm{FP} \\
\mathrm{O}-16 \\
\mathrm{Na}-23 \\
\mathrm{SS}-316\end{array}$ & $\begin{array}{l}1.0840 \mathrm{E}-05 \\
4.4499 \mathrm{E}-03 \\
1.7520 \mathrm{E}-03 \\
2.2340 \mathrm{E}-04 \\
1.9280 \mathrm{E}-05 \\
4.7430 \mathrm{E}-06 \\
7.6680 \mathrm{E}-07 \\
0.0000 \mathrm{E}+00 \\
1.2670 \mathrm{E}-02 \\
9.8100 \mathrm{E}-03 \\
1.8990 \mathrm{E}-02\end{array}$ & $\begin{array}{l}9.3414 \mathrm{E}-06 \\
4.3613 \mathrm{E}-03 \\
1.5758 \mathrm{E}-03 \\
2.5158 \mathrm{E}-04 \\
2.1101 \mathrm{E}-05 \\
4.8900 \mathrm{E}-06 \\
1.4800 \mathrm{E}-06 \\
2.3484 \mathrm{E}-04 \\
1.2670 \mathrm{E}-02 \\
9.8100 \mathrm{E}-03 \\
1.8990 \mathrm{E}-02\end{array}$ \\
\hline \multirow[t]{2}{*}{9} & 30.76 & 46.14 & & & \\
\hline & & & $\begin{array}{l}\mathrm{U}-235 \\
\mathrm{U}-238 \\
\mathrm{Pu}-239 \\
\mathrm{Pu}-240 \\
\mathrm{Pu}-241 \\
\mathrm{Pu}-242 \\
\mathrm{Am}-241 \\
\mathrm{FP} \\
0-16 \\
\mathrm{Na}-23 \\
\mathrm{SS}-316\end{array}$ & $\begin{array}{l}1.0840 \mathrm{E}-05 \\
4.4499 \mathrm{E}-03 \\
1.7520 \mathrm{E}-03 \\
2.2340 \mathrm{E}-04 \\
1.9280 \mathrm{E}-05 \\
4.7430 \mathrm{E}-06 \\
7.6680 \mathrm{E}-07 \\
0.0000 \mathrm{E}+00 \\
1.2670 \mathrm{E}-02 \\
9.8100 \mathrm{E}-03 \\
1.8990 \mathrm{E}-02\end{array}$ & $\begin{array}{l}9.7206 \mathrm{E}-06 \\
4.3858 \mathrm{E}-03 \\
1.6238 \mathrm{E}-03 \\
2.4697 \mathrm{E}-04 \\
2.0681 \mathrm{E}-05 \\
4.8705 \mathrm{E}-06 \\
1.5038 \mathrm{E}-06 \\
1.6702 \mathrm{E}-04 \\
1.2670 \mathrm{E}-02 \\
9.8100 \mathrm{E}-03 \\
1.8990 \mathrm{E}-02\end{array}$ \\
\hline \multirow[t]{2}{*}{10} & 46.14 & 62.78 & & & \\
\hline & & & $\begin{array}{l}\mathrm{U}-235 \\
\mathrm{U}-238 \\
\mathrm{Pu}-239 \\
\mathrm{Pu}-240 \\
\mathrm{FP} \\
\mathrm{O}-16 \\
\mathrm{Na}-23 \\
\mathrm{SS}-316\end{array}$ & $\begin{array}{l}1.4143 \mathrm{E}-05 \\
7.0573 \mathrm{E}-03 \\
0.0000 \mathrm{E}+00 \\
0.0000 \mathrm{E}+00 \\
0.0000 \mathrm{E}+00 \\
1.4143 \mathrm{E}-02 \\
9.7416 \mathrm{E}-03 \\
1.9073 \mathrm{E}-02\end{array}$ & $\begin{array}{l}1.2827 \mathrm{E}-05 \\
6.9864 \mathrm{E}-03 \\
6.3300 \mathrm{E}-05 \\
8.2206 \mathrm{E}-07 \\
7.6817 \mathrm{E}-06 \\
1.4143 \mathrm{E}-02 \\
9.7416 \mathrm{E}-03 \\
1.9073 \mathrm{E}-02\end{array}$ \\
\hline 11 & 62.78 & 172.84 & $\begin{array}{l}\mathrm{Na}-23 \\
\mathrm{SS}-316\end{array}$ & $\begin{array}{l}8.2400 E-03 \\
2.5562 E-02\end{array}$ & $\begin{array}{l}8.2400 E-03 \\
2.5562 E-02\end{array}$ \\
\hline
\end{tabular}


HNF-SD-FF-ANAL-009 Rev. 1

Table E10B.18. Composition of MFA-2 at EOC 1OB

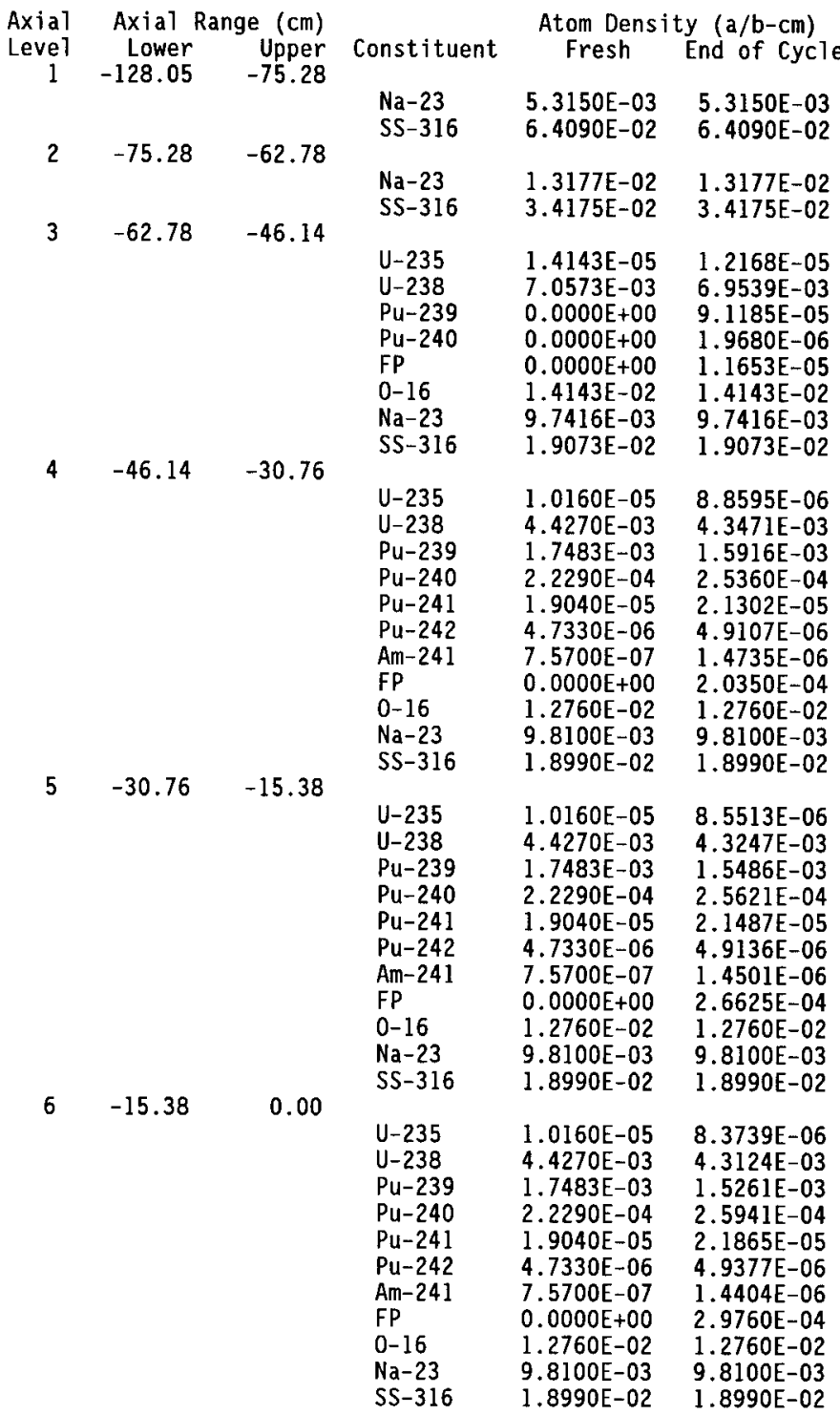


HNF-SD-FF-ANAL-009 Rev. 1

$\begin{array}{lll}7 & 0.00 & 15.38\end{array}$

$\begin{array}{lll}\text { U-235 } & 1.0160 \mathrm{E}-05 & 8.4177 \mathrm{E}-06 \\ \mathrm{U}-238 & 4.4270 \mathrm{E}-03 & 4.3155 \mathrm{E}-03 \\ \mathrm{Pu}-239 & 1.7483 \mathrm{E}-03 & 1.5314 \mathrm{E}-03 \\ \mathrm{Pu}-240 & 2.2290 \mathrm{E}-04 & 2.5850 \mathrm{E}-04 \\ \mathrm{Pu}-241 & 1.9040 \mathrm{E}-05 & 2.1756 \mathrm{E}-05 \\ \mathrm{Pu}-242 & 4.7330 \mathrm{E}-06 & 4.9304 \mathrm{E}-06 \\ \mathrm{Am}-241 & 7.5700 \mathrm{E}-07 & 1.4426 \mathrm{E}-06 \\ \mathrm{FP} & 0.0000 \mathrm{E}+00 & 2.9017 \mathrm{E}-04 \\ 0-16 & 1.2760 \mathrm{E}-02 & 1.2760 \mathrm{E}-02 \\ \mathrm{Na}-23 & 9.8100 \mathrm{E}-03 & 9.8100 \mathrm{E}-03 \\ \mathrm{SS}-316 & 1.8990 \mathrm{E}-02 & 1.8990 \mathrm{E}-02\end{array}$

$\begin{array}{lll}8 & 15.38 & 30.76\end{array}$

$\begin{array}{lll}\mathrm{U}-235 & 1.0160 \mathrm{E}-05 & 8.6688 \mathrm{E}-06 \\ \mathrm{U}-238 & 4.4270 \mathrm{E}-03 & 4.3327 \mathrm{E}-03 \\ \mathrm{Pu}-239 & 1.7483 \mathrm{E}-03 & 1.5631 \mathrm{E}-03 \\ \mathrm{Pu}-240 & 2.2290 \mathrm{E}-04 & 2.5373 \mathrm{E}-04 \\ \mathrm{Pu}-241 & 1.9040 \mathrm{E}-05 & 2.1205 \mathrm{E}-05 \\ \mathrm{Pu}-242 & 4.7330 \mathrm{E}-06 & 4.8955 \mathrm{E}-06 \\ \mathrm{Am}-241 & 7.5700 \mathrm{E}-07 & 1.4559 \mathrm{E}-06 \\ \mathrm{FP} & 0.0000 \mathrm{E}+00 & 2.4655 \mathrm{E}-04 \\ 0-16 & 1.2760 \mathrm{E}-02 & 1.2760 \mathrm{E}-02 \\ \mathrm{Na}-23 & 9.8100 \mathrm{E}-03 & 9.8100 \mathrm{E}-03 \\ \mathrm{SS}-316 & 1.8990 \mathrm{E}-02 & 1.8990 \mathrm{E}-02\end{array}$

$\begin{array}{lll}9 & 30.76 & 46.14\end{array}$

$\begin{array}{lll}\mathrm{U}-235 & 1.0160 \mathrm{E}-05 & 9.0368 \mathrm{E}-06 \\ \mathrm{U}-238 & 4.4270 \mathrm{E}-03 & 4.3583 \mathrm{E}-03 \\ \mathrm{Pu}-239 & 1.7483 \mathrm{E}-03 & 1.6125 \mathrm{E}-03 \\ \mathrm{Pu}-240 & 2.2290 \mathrm{E}-04 & 2.4899 \mathrm{E}-04 \\ \mathrm{Pu}-241 & 1.9040 \mathrm{E}-05 & 2.0764 \mathrm{E}-05 \\ \mathrm{Pu}-242 & 4.7330 \mathrm{E}-06 & 4.8756 \mathrm{E}-06 \\ \mathrm{Am}-241 & 7.5700 \mathrm{E}-07 & 1.4806 \mathrm{E}-06 \\ \mathrm{FP} & 0.0000 \mathrm{E}+00 & 1.7654 \mathrm{E}-04 \\ 0-16 & 1.2760 \mathrm{E}-02 & 1.2760 \mathrm{E}-02 \\ \mathrm{Na}-23 & 9.8100 \mathrm{E}-03 & 9.8100 \mathrm{E}-03 \\ \mathrm{SS}-316 & 1.8990 \mathrm{E}-02 & 1.8990 \mathrm{E}-02\end{array}$

$\begin{array}{lll}10 & 46.14 & 62.78\end{array}$

$\begin{array}{lll}\text { U-235 } & 1.4143 \mathrm{E}-05 & 1.2691 \mathrm{E}-05 \\ \mathrm{U}-238 & 7.0573 \mathrm{E}-03 & 6.9803 \mathrm{E}-03 \\ \mathrm{Pu}-239 & 0.0000 \mathrm{E}+00 & 6.8652 \mathrm{E}-05 \\ \mathrm{Pu}-240 & 0.0000 \mathrm{E}+00 & 1.0361 \mathrm{E}-06 \\ \mathrm{FP} & 0.0000 \mathrm{E}+00 & 8.3704 \mathrm{E}-06 \\ 0-16 & 1.4143 \mathrm{E}-02 & 1.4143 \mathrm{E}-02 \\ \mathrm{Na}-23 & 9.7416 \mathrm{E}-03 & 9.7416 \mathrm{E}-03 \\ \mathrm{SS}-316 & 1.9073 \mathrm{E}-02 & 1.9073 \mathrm{E}-02\end{array}$

$\begin{array}{lll}11 & 62.78 & 172.84\end{array}$

$\begin{array}{lll}\mathrm{Na}-23 & 8.2400 \mathrm{E}-03 & 8.2400 \mathrm{E}-03 \\ \mathrm{SS}-316 & 2.5562 \mathrm{E}-02 & 2.5562 \mathrm{E}-02\end{array}$


HNF-SD-FF-ANAL-009 Rev. I

\subsection{Cycle 10C-1}

Table B10C-1,1. Fission Power Generated in MFA-1, MFA-2 and Neighboring Assemblies at BOC $10 \mathrm{C}-1$

\begin{tabular}{lrrrrr} 
CORE & $* * * * * *$ & \multicolumn{2}{c}{ POWER IN MEGAWATTS } & $* * * * * *$ \\
POS. & BELOW CORE & IN CORE & ABOVE CORE & TOTAL PWR \\
1506 & $4.498 \mathrm{E}-02$ & $3.489 \mathrm{E}+00$ & $2.737 \mathrm{E}-02$ & $3.562 \mathrm{E}+00$ \\
1404 & $1.255 \mathrm{E}-02$ & $3.635 \mathrm{E}+00$ & $8.021 \mathrm{E}-03$ & $3.655 \mathrm{E}+00$ \\
1405 & $0.000 \mathrm{E}+00$ & $6.311 \mathrm{E}+00$ & $0.000 \mathrm{E}+00$ & $6.311 \mathrm{E}+00$ \\
1505 & $1.120 \mathrm{E}-02$ & $3.173 \mathrm{E}+00$ & $6.603 \mathrm{E}-03$ & $3.191 \mathrm{E}+00$ \\
1507 & $8.006 \mathrm{E}-03$ & $3.287 \mathrm{E}+00$ & $5.434 \mathrm{E}-03$ & $3.301 \mathrm{E}+00$ \\
1606 & $7.571 \mathrm{E}-03$ & $3.055 \mathrm{E}+00$ & $5.076 \mathrm{E}-03$ & $3.067 \mathrm{E}+00$ \\
1607 & $9.894 \mathrm{E}-03$ & $2.860 \mathrm{E}+00$ & $6.403 \mathrm{E}-03$ & $2.877 \mathrm{E}+00$ \\
2507 & $4.934 \mathrm{E}-02$ & $4.039 \mathrm{E}+00$ & $3.267 \mathrm{E}-02$ & $4.121 \mathrm{E}+00$ \\
2405 & $1.373 \mathrm{E}-02$ & $4.030 \mathrm{E}+00$ & $9.583 \mathrm{E}-03$ & $4.053 \mathrm{E}+00$ \\
2506 & $9.444 \mathrm{E}-03$ & $4.689 \mathrm{E}+00$ & $6.984 \mathrm{E}-03$ & $4.705 \mathrm{E}+00$ \\
2508 & $1.408 \mathrm{E}-02$ & $4.214 \mathrm{E}+00$ & $8.945 \mathrm{E}-03$ & $4.237 \mathrm{E}+00$ \\
2607 & $1.025 \mathrm{E}-02$ & $3.719 \mathrm{E}+00$ & $7.530 \mathrm{E}-03$ & $3.737 \mathrm{E}+00$ \\
2608 & $6.908 \mathrm{E}-03$ & $3.439 \mathrm{E}+00$ & $5.455 \mathrm{E}-03$ & $3.452 \mathrm{E}+00$ \\
2609 & $7.564 \mathrm{E}-03$ & $4.111 \mathrm{E}+00$ & $5.864 \mathrm{E}-03$ & $4.125 \mathrm{E}+00$
\end{tabular}

Table $B 10 C-1.2$. Assembly Averaged Total and Fast Flux in MFA-1 and MFA-2 at $B O C$ 10C-1

\begin{tabular}{|c|c|c|c|}
\hline & Core & Flux & $\left(m^{2}-s e c\right)$ \\
\hline $\begin{array}{l}\text { Assembly } \\
\text { MFA-1 } \\
\text { MFA-2 }\end{array}$ & $\begin{array}{l}\text { Pos. } \\
1506 \\
2507\end{array}$ & $\begin{array}{c}\text { Tota } 1 \\
2.744 E+15 \\
3.191 E+15\end{array}$ & $\begin{array}{l}>0.1 \mathrm{MeV} \\
1.784 \mathrm{E}+15 \\
2.069 \mathrm{E}+15\end{array}$ \\
\hline
\end{tabular}


Table BlOC-1.3. Axial Distribution of Total Flux, Fast Flux and Power in MFA-1 at BOC $10 \mathrm{C}-1$

$\begin{array}{rccc}\text { z }(\mathrm{cm}) & \text { Total Flux } & \text { Flux }>0.1 \text { MeV } & \text { Power } \\ -97.78 & 2.8606 \mathrm{E}-02 & 1.4407 \mathrm{E}-02 & 0.0000 \mathrm{E}+00 \\ -92.78 & 6.1416 \mathrm{E}-02 & 2.7786 \mathrm{E}-02 & 0.0000 \mathrm{E}+00 \\ -87.78 & 9.8382 \mathrm{E}-02 & 4.4390 \mathrm{E}-02 & 0.0000 \mathrm{E}+00 \\ -82.78 & 1.4234 \mathrm{E}-01 & 6.6089 \mathrm{E}-02 & 0.0000 \mathrm{E}+00 \\ -77.78 & 1.9634 \mathrm{E}-01 & 9.5217 \mathrm{E}-02 & 0.0000 \mathrm{E}+00 \\ -72.15 & 2.5833 \mathrm{E}-01 & 1.3304 \mathrm{E}-01 & 0.0000 \mathrm{E}+00 \\ -65.90 & 3.2123 \mathrm{E}-01 & 1.8130 \mathrm{E}-01 & 0.0000 \mathrm{E}+00 \\ -60.35 & 3.8673 \mathrm{E}-01 & 2.4660 \mathrm{E}-01 & 5.4673 \mathrm{E}-02 \\ -55.49 & 4.7156 \mathrm{E}-01 & 3.3369 \mathrm{E}-01 & 6.5758 \mathrm{E}-02 \\ -50.62 & 5.6869 \mathrm{E}-01 & 4.4784 \mathrm{E}-01 & 8.1938 \mathrm{E}-02 \\ -47.17 & 6.4592 \mathrm{E}-01 & 5.5618 \mathrm{E}-01 & 1.0018 \mathrm{E}-01 \\ -43.58 & 7.2696 \mathrm{E}-01 & 6.7898 \mathrm{E}-01 & 7.8500 \mathrm{E}-01 \\ -38.45 & 8.4406 \mathrm{E}-01 & 8.2512 \mathrm{E}-01 & 8.7046 \mathrm{E}-01 \\ -33.32 & 9.5155 \mathrm{E}-01 & 9.4742 \mathrm{E}-01 & 9.6907 \mathrm{E}-01 \\ -28.20 & 1.0438 \mathrm{E}+00 & 1.0469 \mathrm{E}+00 & 1.0352 \mathrm{E}+00 \\ -23.07 & 1.1190 \mathrm{E}+00 & 1.1261 \mathrm{E}+00 & 1.1079 \mathrm{E}+00 \\ -17.94 & 1.1754 \mathrm{E}+00 & 1.1845 \mathrm{E}+00 & 1.1628 \mathrm{E}+00 \\ -12.82 & 1.2123 \mathrm{E}+00 & 1.2220 \mathrm{E}+00 & 1.1853 \mathrm{E}+00 \\ -7.69 & 1.2300 \mathrm{E}+00 & 1.2401 \mathrm{E}+00 & 1.2021 \mathrm{E}+00 \\ -2.56 & 1.2288 \mathrm{E}+00 & 1.2393 \mathrm{E}+00 & 1.2006 \mathrm{E}+00 \\ 2.56 & 1.2098 \mathrm{E}+00 & 1.2207 \mathrm{E}+00 & 1.1855 \mathrm{E}+00 \\ 7.69 & 1.1741 \mathrm{E}+00 & 1.1853 \mathrm{E}+00 & 1.1505 \mathrm{E}+00 \\ 12.82 & 1.1233 \mathrm{E}+00 & 1.1347 \mathrm{E}+00 & 1.1008 \mathrm{E}+00 \\ 17.94 & 1.0586 \mathrm{E}+00 & 1.0700 \mathrm{E}+00 & 1.0552 \mathrm{E}+00 \\ 23.07 & 9.8023 \mathrm{E}-01 & 9.9023 \mathrm{E}-01 & 9.7760 \mathrm{E}-01 \\ 28.20 & 8.8952 \mathrm{E}-01 & 8.9645 \mathrm{E}-01 & 8.8830 \mathrm{E}-01 \\ 33.32 & 7.8841 \mathrm{E}-01 & 7.8965 \mathrm{E}-01 & 8.1046 \mathrm{E}-01 \\ 38.45 & 6.7870 \mathrm{E}-01 & 6.6861 \mathrm{E}-01 & 7.0456 \mathrm{E}-01 \\ 43.58 & 5.6547 \mathrm{E}-01 & 5.3391 \mathrm{E}-01 & 6.0864 \mathrm{E}-01 \\ 47.17 & 4.9071 \mathrm{E}-01 & 4.3019 \mathrm{E}-01 & 6.8281 \mathrm{E}-02 \\ 50.62 & 4.2151 \mathrm{E}-01 & 3.4092 \mathrm{E}-01 & 5.2626 \mathrm{E}-02 \\ 55.49 & 3.3610 \mathrm{E}-01 & 2.4788 \mathrm{E}-01 & 3.8612 \mathrm{E}-02 \\ 60.35 & 2.6025 \mathrm{E}-01 & 1.7585 \mathrm{E}-01 & 2.8812 \mathrm{E}-02 \\ 64.98 & 2.1074 \mathrm{E}-01 & 1.3242 \mathrm{E}-01 & 0.0000 \mathrm{E}+00 \\ 69.39 & 1.8030 \mathrm{E}-01 & 1.0852 \mathrm{E}-01 & 0.0000 \mathrm{E}+00 \\ 76.46 & 1.3673 \mathrm{E}-01 & 7.6438 \mathrm{E}-02 & 0.0000 \mathrm{E}+00 \\ 86.80 & 8.9382 \mathrm{E}-02 & 4.5838 \mathrm{E}-02 & 0.0000 \mathrm{E}+00 \\ 98.14 & 5.2058 \mathrm{E}-02 & 2.5037 \mathrm{E}-02 & 0.0000 \mathrm{E}+00 \\ 110.00 & 2.2338 \mathrm{E}-02 & 1.1174 \mathrm{E}-02 & 0.0000 \mathrm{E}+00\end{array}$


Table BlOC-1.4. Axial Distribution of Total Flux, Fast Flux and Power in MFA-2 at BOC $10 \mathrm{C}-1$

$\begin{array}{rccc}\text { Z }(\mathrm{cm}) & \text { Total Flux } & \text { Flux }>0.1 \mathrm{MeV} & \text { Power } \\ -97.78 & 2.5080 \mathrm{E}-02 & 1.2786 \mathrm{E}-02 & 0.0000 \mathrm{E}+00 \\ -92.78 & 5.3820 \mathrm{E}-02 & 2.4682 \mathrm{E}-02 & 0.0000 \mathrm{E}+00 \\ -87.78 & 8.6275 \mathrm{E}-02 & 3.9513 \mathrm{E}-02 & 0.0000 \mathrm{E}+00 \\ -82.78 & 1.2506 \mathrm{E}-01 & 5.9032 \mathrm{E}-02 & 0.0000 \mathrm{E}+00 \\ -77.78 & 1.7307 \mathrm{E}-01 & 8.5490 \mathrm{E}-02 & 0.0000 \mathrm{E}+00 \\ -72.15 & 2.2882 \mathrm{E}-01 & 1.2024 \mathrm{E}-01 & 0.0000 \mathrm{E}+00 \\ -65.90 & 2.8749 \mathrm{E}-01 & 1.6568 \mathrm{E}-01 & 0.0000 \mathrm{E}+00 \\ -60.35 & 3.5187 \mathrm{E}-01 & 2.2878 \mathrm{E}-01 & 5.0602 \mathrm{E}-02 \\ -55.49 & 4.3585 \mathrm{E}-01 & 3.1370 \mathrm{E}-01 & 6.1927 \mathrm{E}-02 \\ -50.62 & 5.3370 \mathrm{E}-01 & 4.2560 \mathrm{E}-01 & 7.8435 \mathrm{E}-02 \\ -47.17 & 6.1299 \mathrm{E}-01 & 5.3206 \mathrm{E}-01 & 9.6890 \mathrm{E}-02 \\ -43.58 & 6.9687 \mathrm{E}-01 & 6.5308 \mathrm{E}-01 & 7.5043 \mathrm{E}-01 \\ -38.45 & 8.1807 \mathrm{E}-01 & 8.0069 \mathrm{E}-01 & 8.4398 \mathrm{E}-01 \\ -33.32 & 9.2992 \mathrm{E}-01 & 9.2632 \mathrm{E}-01 & 9.4824 \mathrm{E}-01 \\ -28.20 & 1.0266 \mathrm{E}+00 & 1.0299 \mathrm{E}+00 & 1.0184 \mathrm{E}+00 \\ -23.07 & 1.1062 \mathrm{E}+00 & 1.1132 \mathrm{E}+00 & 1.0956 \mathrm{E}+00 \\ -17.94 & 1.1670 \mathrm{E}+00 & 1.1759 \mathrm{E}+00 & 1.1550 \mathrm{E}+00 \\ -12.82 & 1.2084 \mathrm{E}+00 & 1.2179 \mathrm{E}+00 & 1.1810 \mathrm{E}+00 \\ -7.69 & 1.2307 \mathrm{E}+00 & 1.2405 \mathrm{E}+00 & 1.2025 \mathrm{E}+00 \\ -2.56 & 1.2342 \mathrm{E}+00 & 1.2442 \mathrm{E}+00 & 1.2058 \mathrm{E}+00 \\ 2.56 & 1.2195 \mathrm{E}+00 & 1.2298 \mathrm{E}+00 & 1.1948 \mathrm{E}+00 \\ 7.69 & 1.1873 \mathrm{E}+00 & 1.1977 \mathrm{E}+00 & 1.1634 \mathrm{E}+00 \\ 12.82 & 1.1388 \mathrm{E}+00 & 1.1492 \mathrm{E}+00 & 1.1160 \mathrm{E}+00 \\ 17.94 & 1.0749 \mathrm{E}+00 & 1.0853 \mathrm{E}+00 & 1.0719 \mathrm{E}+00 \\ 23.07 & 9.9622 \mathrm{E}-01 & 1.0053 \mathrm{E}+00 & 9.9407 \mathrm{E}-01 \\ 28.20 & 9.0423 \mathrm{E}-01 & 9.1030 \mathrm{E}-01 & 9.0343 \mathrm{E}-01 \\ 33.32 & 8.0098 \mathrm{E}-01 & 8.0159 \mathrm{E}-01 & 8.2442 \mathrm{E}-01 \\ 38.45 & 6.8842 \mathrm{E}-01 & 6.7810 \mathrm{E}-01 & 7.1545 \mathrm{E}-01 \\ 43.58 & 5.7169 \mathrm{E}-01 & 5.4091 \mathrm{E}-01 & 6.1572 \mathrm{E}-01 \\ 47.17 & 4.9281 \mathrm{E}-01 & 4.3411 \mathrm{E}-01 & 7.1157 \mathrm{E}-02 \\ 50.62 & 4.1870 \mathrm{E}-01 & 3.4063 \mathrm{E}-01 & 5.4589 \mathrm{E}-02 \\ 55.49 & 3.2779 \mathrm{E}-01 & 2.4310 \mathrm{E}-01 & 3.9643 \mathrm{E}-02 \\ 60.35 & 2.4887 \mathrm{E}-01 & 1.6878 \mathrm{E}-01 & 2.9256 \mathrm{E}-02 \\ 64.98 & 1.9982 \mathrm{E}-01 & 1.2575 \mathrm{E}-01 & 0.0000 \mathrm{E}+00 \\ 69.39 & 1.7076 \mathrm{E}-01 & 1.0296 \mathrm{E}-01 & 0.0000 \mathrm{E}+00 \\ 76.46 & 1.2944 \mathrm{E}-01 & 7.2517 \mathrm{E}-02 & 0.0000 \mathrm{E}+00 \\ 86.80 & 8.4409 \mathrm{E}-02 & 4.3373 \mathrm{E}-02 & 0.0000 \mathrm{E}+00 \\ 98.14 & 4.8815 \mathrm{E}-02 & 2.3550 \mathrm{E}-02 & 0.0000 \mathrm{E}+00 \\ 110.00 & 2.0809 \mathrm{E}-02 & 1.0444 \mathrm{E}-02 & 0.0000 \mathrm{E}+00\end{array}$


HNF-SD-FF-ANAL-009 Rev. 1

Table BlOC-1.5. Fission Power Distribution by Pin in MFA-1 at BOC $10 \mathrm{C}-1$ CORE POSITION 1506

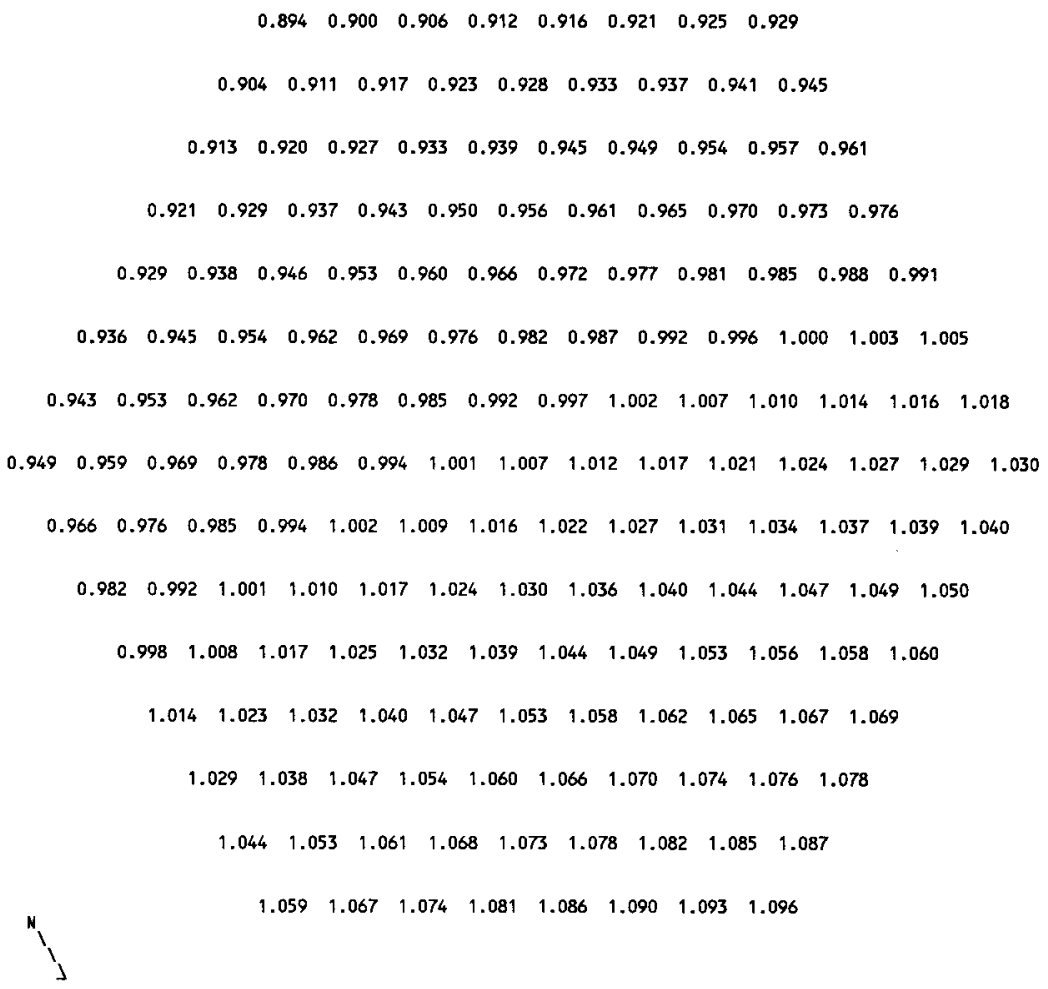


HNF-SD-FF-ANAL-009 Rev. 1

Table B10C-1.6. Fission Power Distribution by Pin in MFA-2 at BOC $10 \mathrm{C}-1$ CORE POSITION 2507

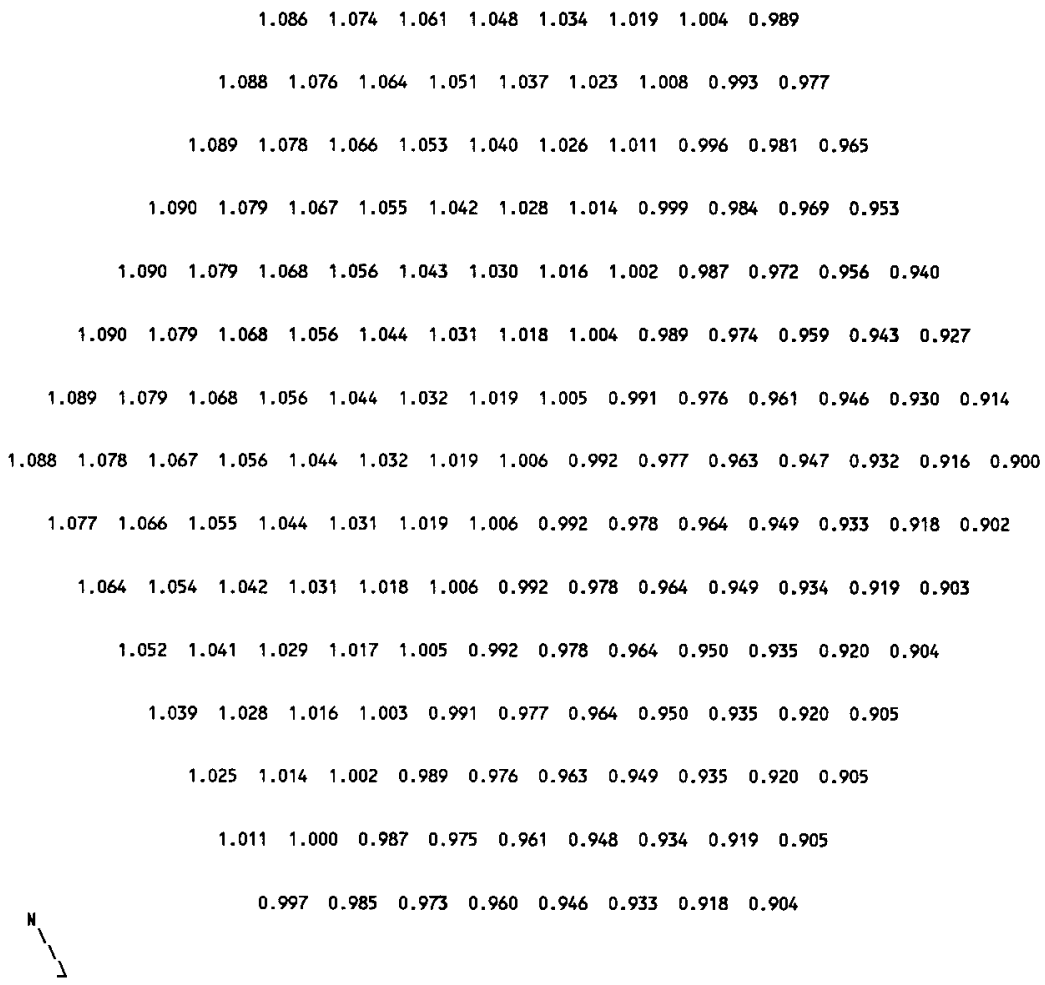


HNF-SD-FF-ANAL-009 Rev. 1

Table B10C-1.7. Fast Flux Distribution by Pin in MFA-1 at BOC $10 \mathrm{C}-1$ CORE POSITION 1506

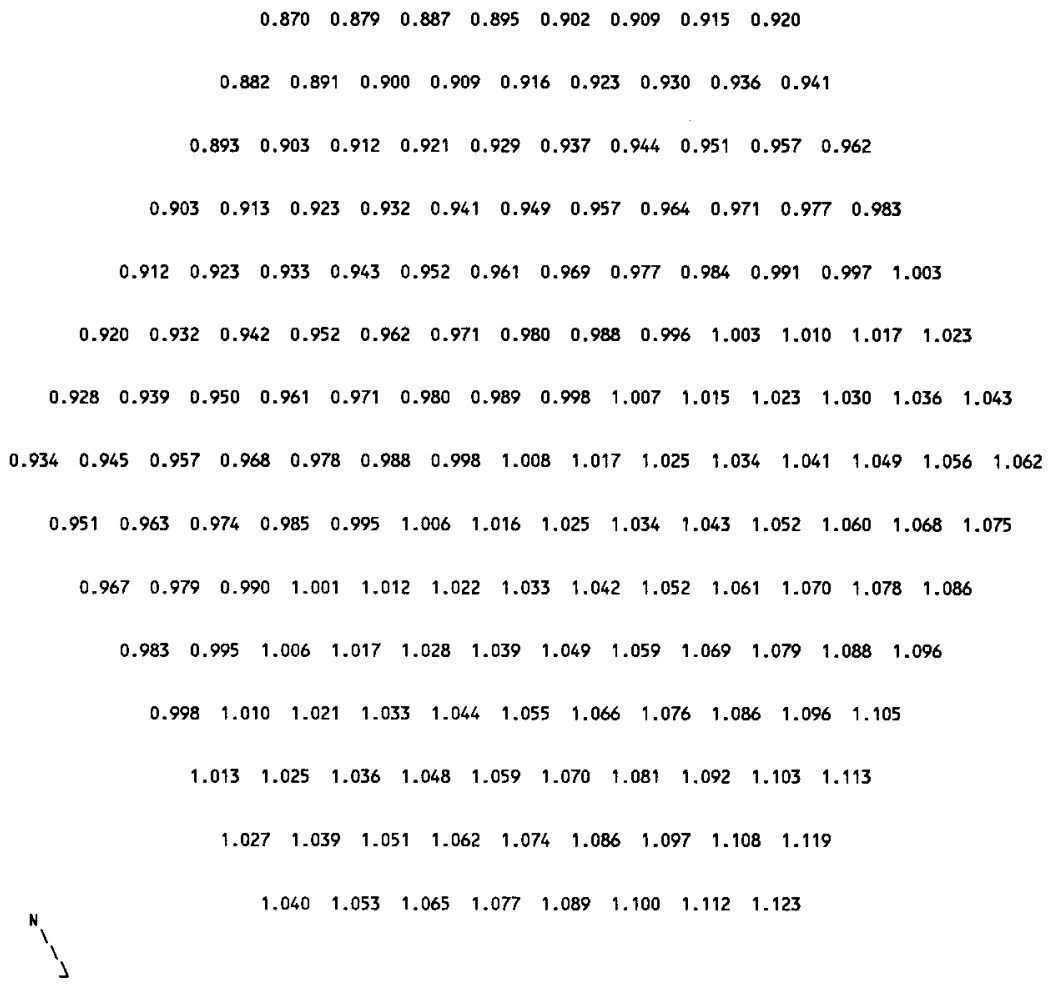


HNF-SD-FF-ANAL-009 Rev. 1

Table B1OC-1.8. Fast Flux Distribution by Pin in MFA-2 at BOC $10 \mathrm{C}-1$ CORE POSITION 2507

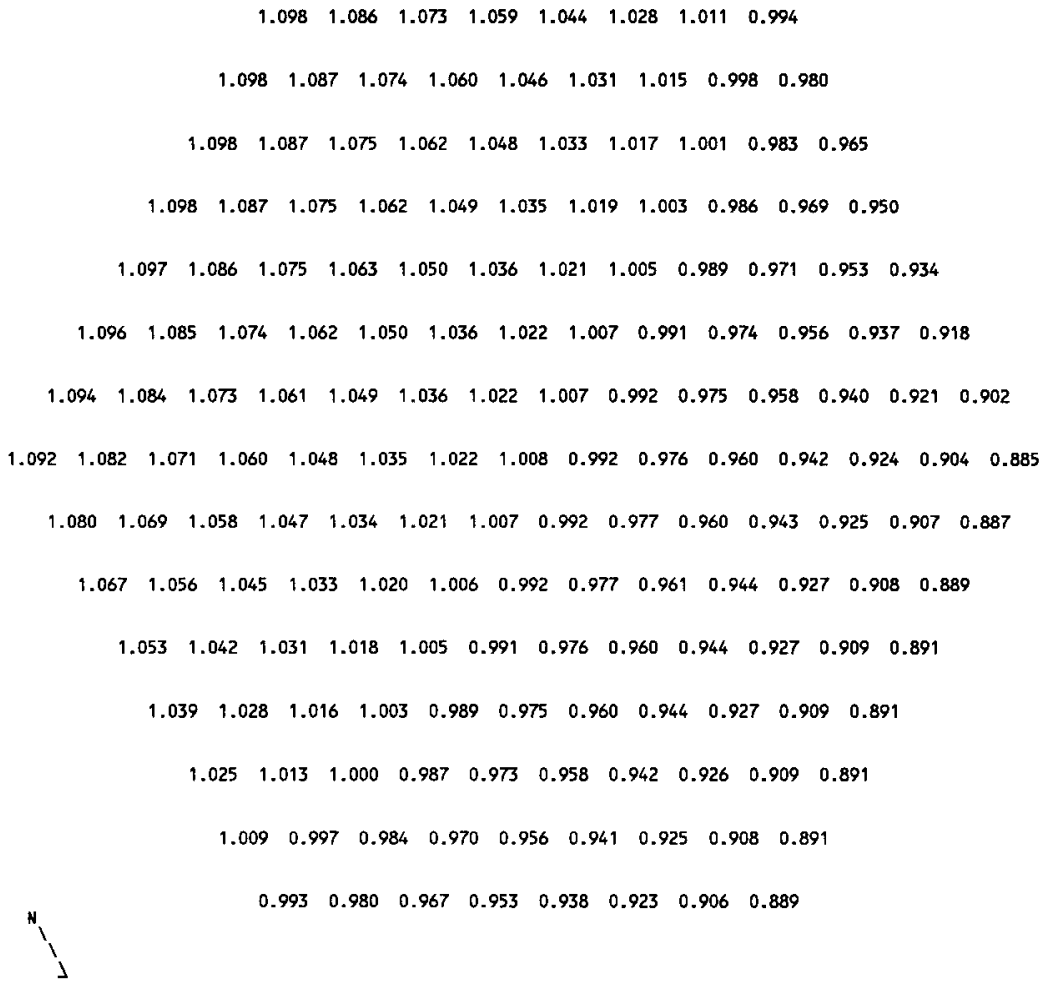


Table B10C-1.9. MFA-1 and MFA-2 Duct Wa11

Fast Flux Data at BOC 10C-1

$\begin{array}{ccc}\text { Side } & \begin{array}{c}\text { Flux }>0.1 \mathrm{MeV}\left(\mathrm{M} / \mathrm{cm}^{2}-\mathrm{sec}\right) \\ \text { MFA-1 }\end{array} & \begin{array}{c}\text { MFA-2 } \\ \text { E }\end{array} \\ 1.7529 E+15 & 2.1736 \mathrm{E}+15 \\ \text { SE } & 1.5775 \mathrm{E}+15 & 2.2992 \mathrm{E}+15 \\ \text { SW } & 1.5547 \mathrm{E}+15 & 2.1863 \mathrm{E}+15 \\ \text { W } & 1.7513 \mathrm{E}+15 & 1.9090 \mathrm{E}+15 \\ \text { NW } & 1.9921 \mathrm{E}+15 & 1.7845 \mathrm{E}+15 \\ \text { NE } & 1.9481 \mathrm{E}+15 & 1.9208 \mathrm{E}+15\end{array}$

Table B10C-1.10. Assembly Outlet Temperatures and Flow Rates at BOC $10 \mathrm{C}-1$

$\begin{array}{lcl} & \text { CALCULATED } \\ \text { CORE } & \text { OUTLET TEMP. } & \text { FLOW RATE } \\ \text { POS. } & \text { (DEG F) } & \text { (LB/H) } \\ 1506 & 1004 & 114180 \\ 1404 & 874 & 206750 \\ 1405 & 1026 & 186110 \\ 1505 & 855 & 196600 \\ 1507 & 863 & 196600 \\ 1606 & 873 & 169550 \\ 1607 & 870 & 162600 \\ 2507 & 1064 & 114180 \\ 2405 & 900 & 206770 \\ 2506 & 930 & 206750 \\ 2508 & 907 & 196500 \\ 2607 & 931 & 162600 \\ 2608 & 906 & 169550 \\ 2609 & 957 & 162600\end{array}$

Assembly flows are based on a calculated core pressure drop of $113.3 \mathrm{psi}$ at a total reactor flow rate of $16.67 \mathrm{E}+06 \mathrm{lb} / \mathrm{hr}$. 
Table BloC-1.11. MFA-1 Sodium Subchannel Temperatures at Top of Core Elevation for BOC $10 C-1$

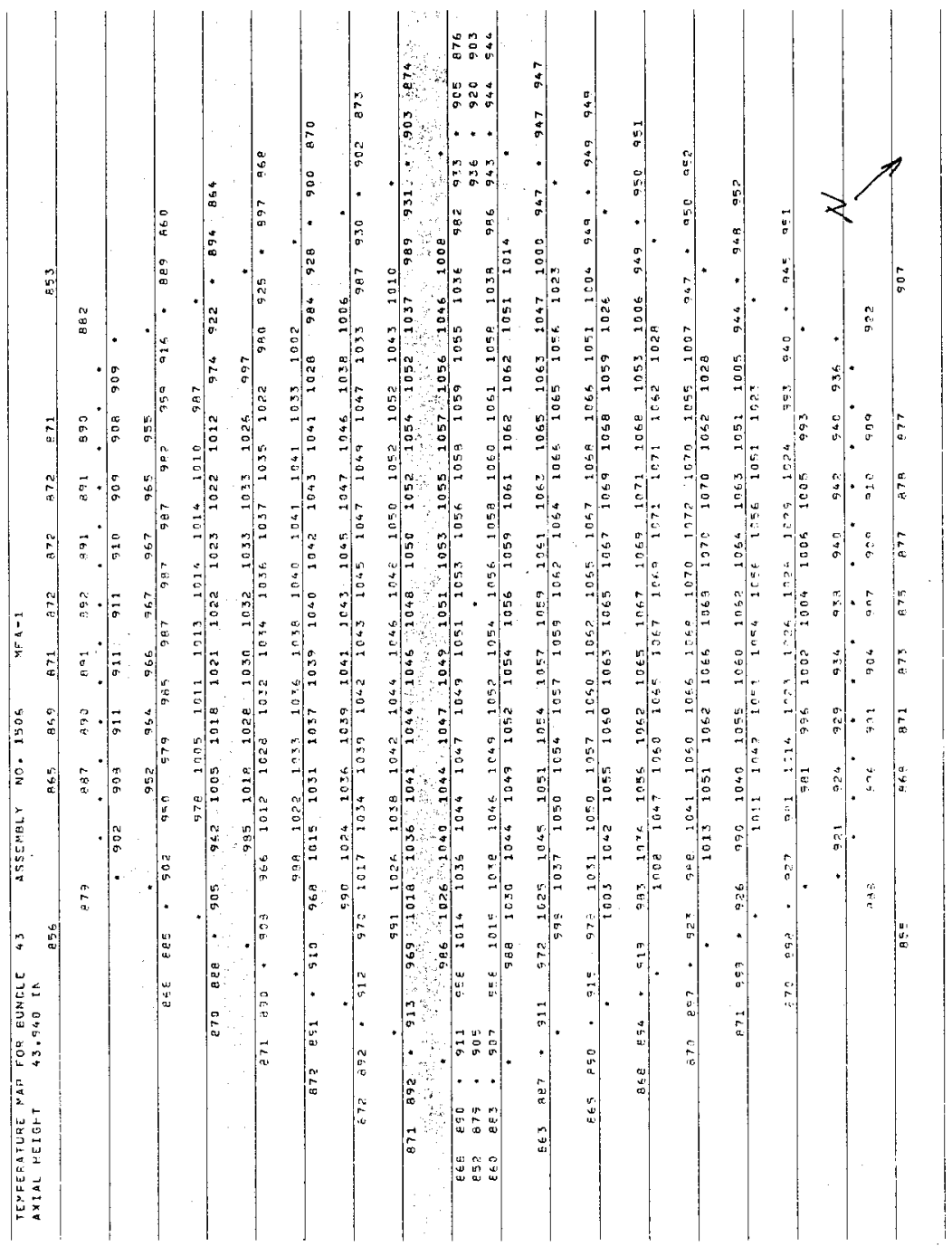


Table B10C-1.12. MFA-2 Sodium Subchannel Temperatures at Top of Core Elevation for $B O C$ 1OC-1

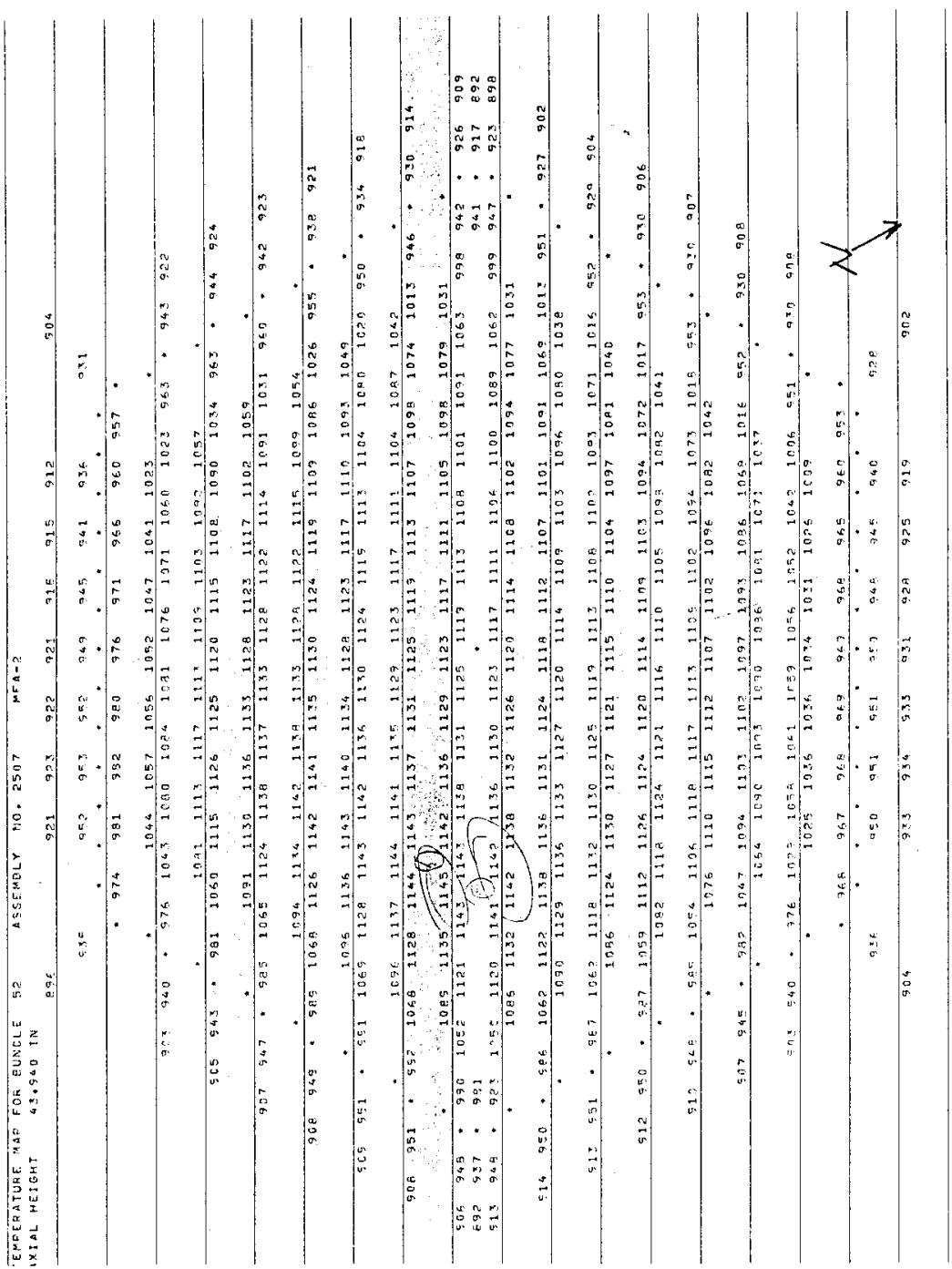


Table B10C-1.13. MFA-1 Sodium Subchanne1 Temperatures at Elevation of Upper Axial Blanket for BOC $10 \mathrm{C}-1$

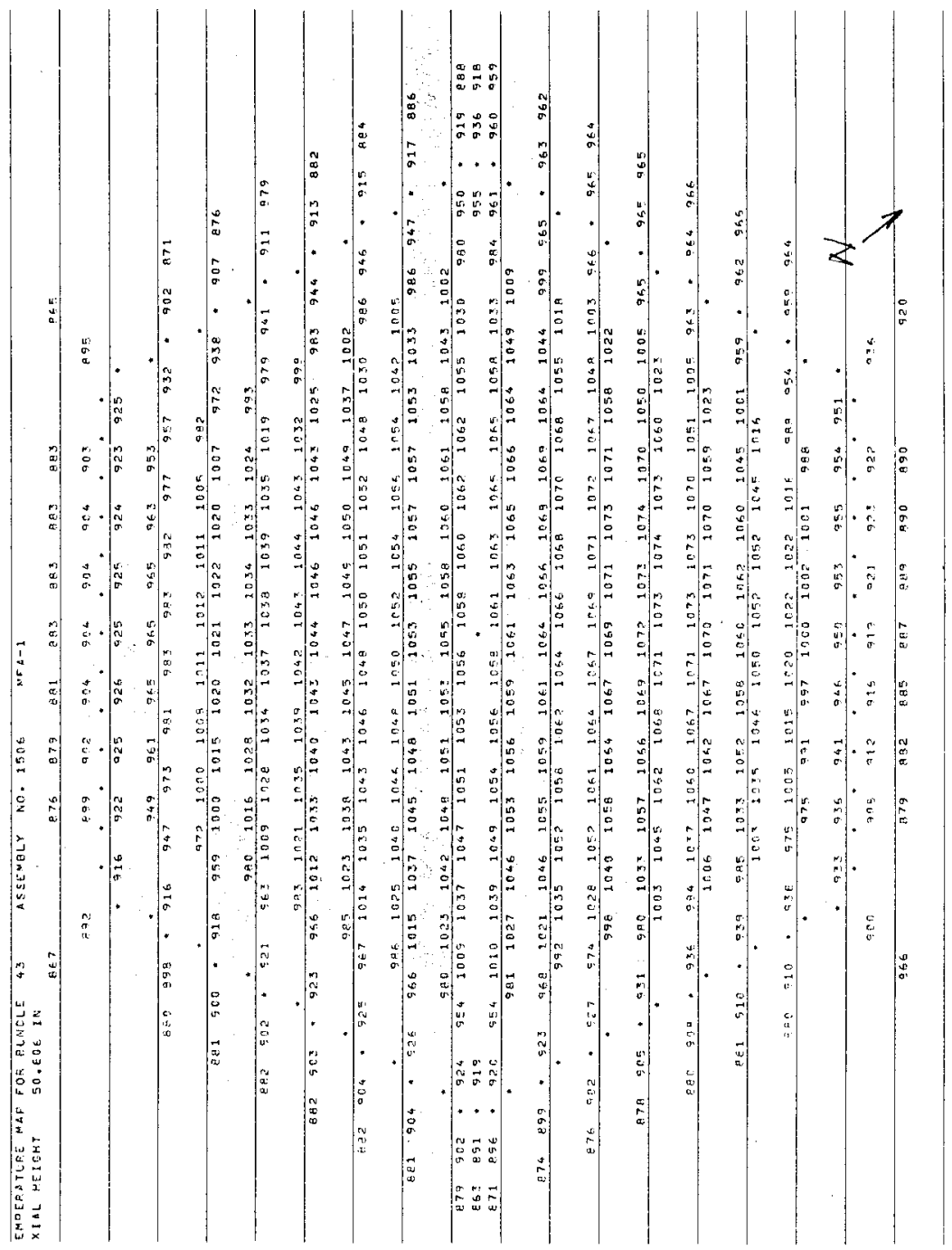


Table B10C-1.14. MFA-2 Sodium Subchannel Temperatures at Elevation of Upper Axial Blanket for BOC 10C-1

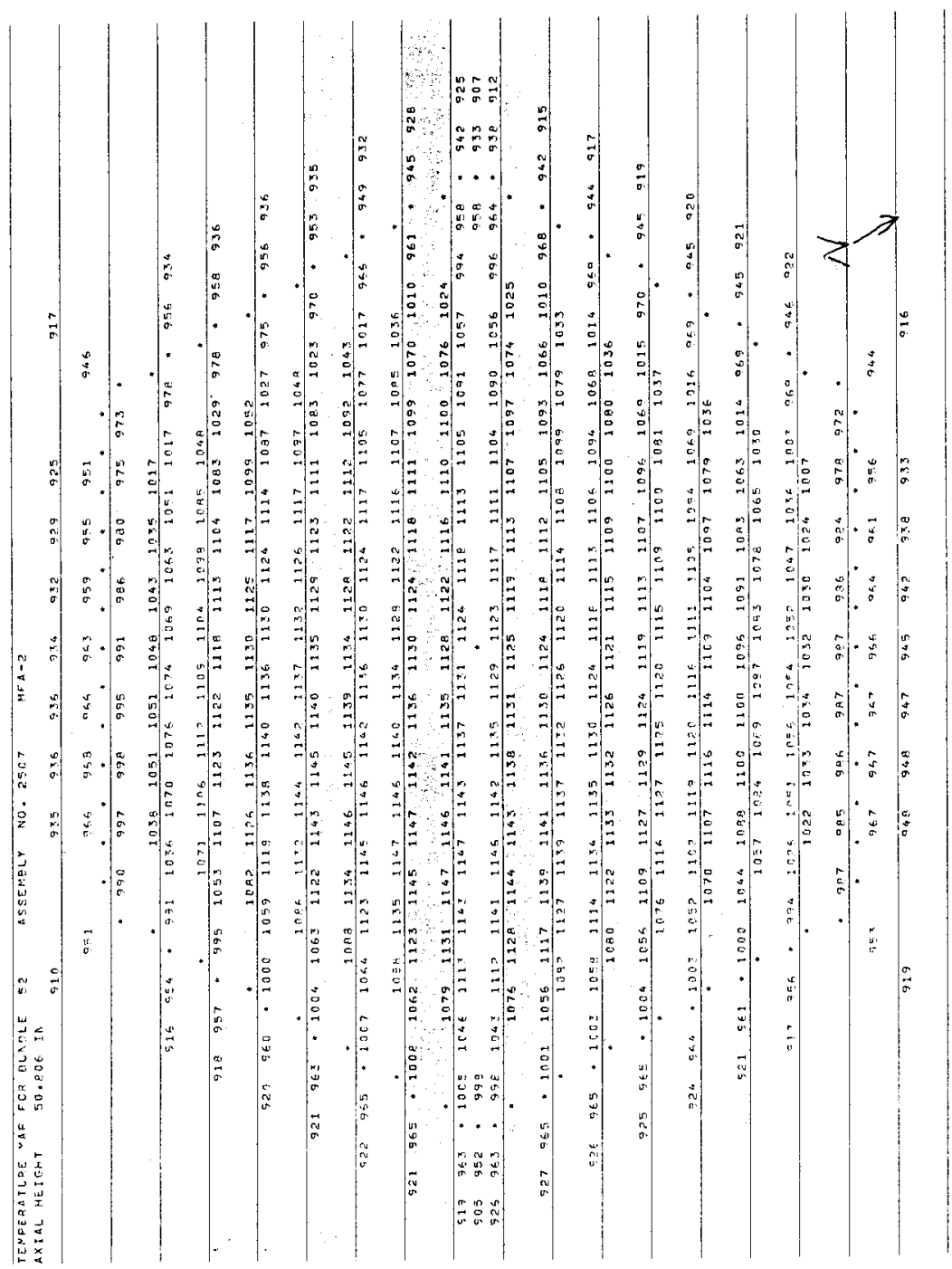


Table B10C-1.15. MFA-1 Sodium Subchannel Temperatures at Top of Fuel Pin Bundle for $B O C$ $10 C-1$

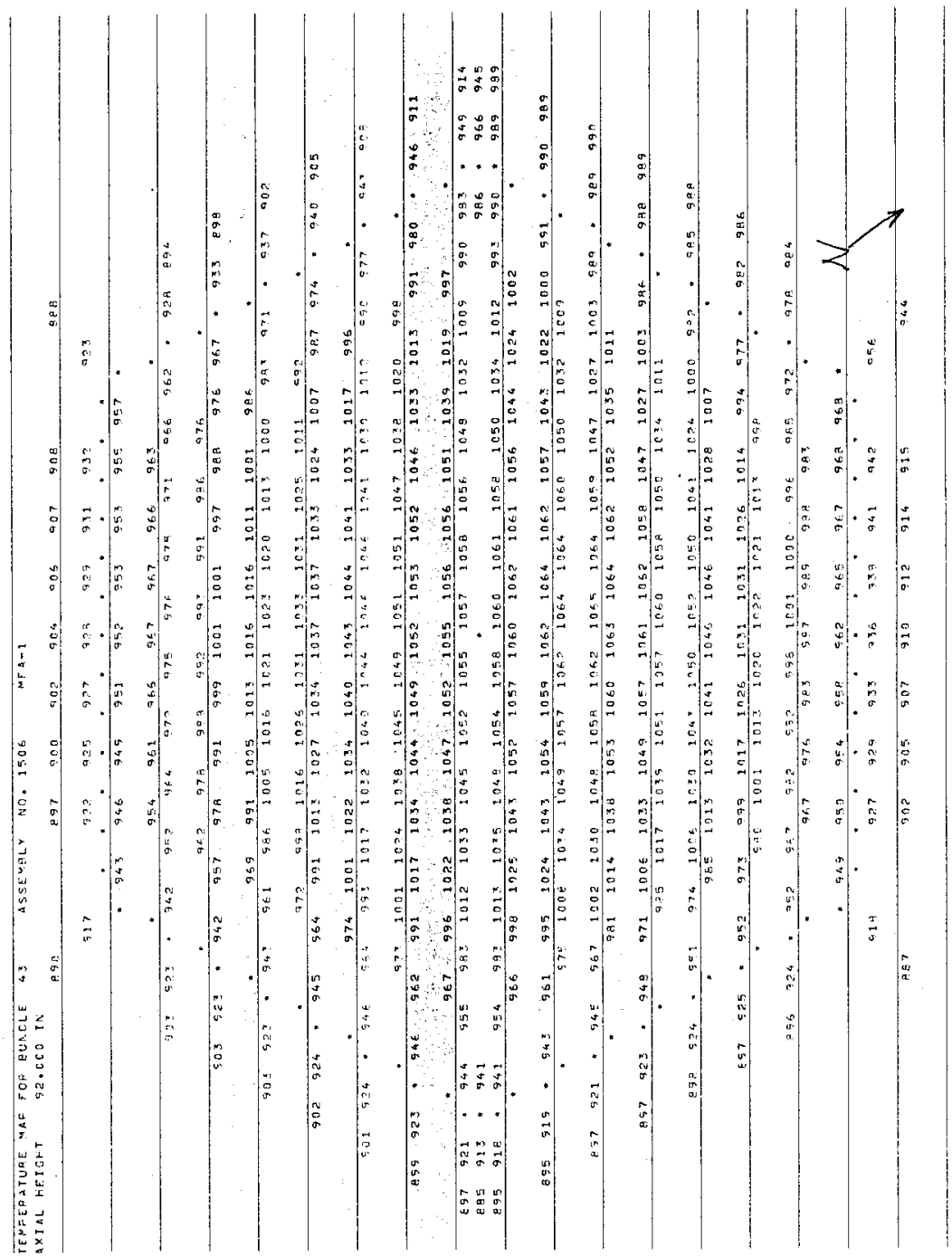


Table B10C-1.16. MFA-2 Sodium Subchannel Temperatures

at Top of Fuel Pin Bundle for BOC 10C-1

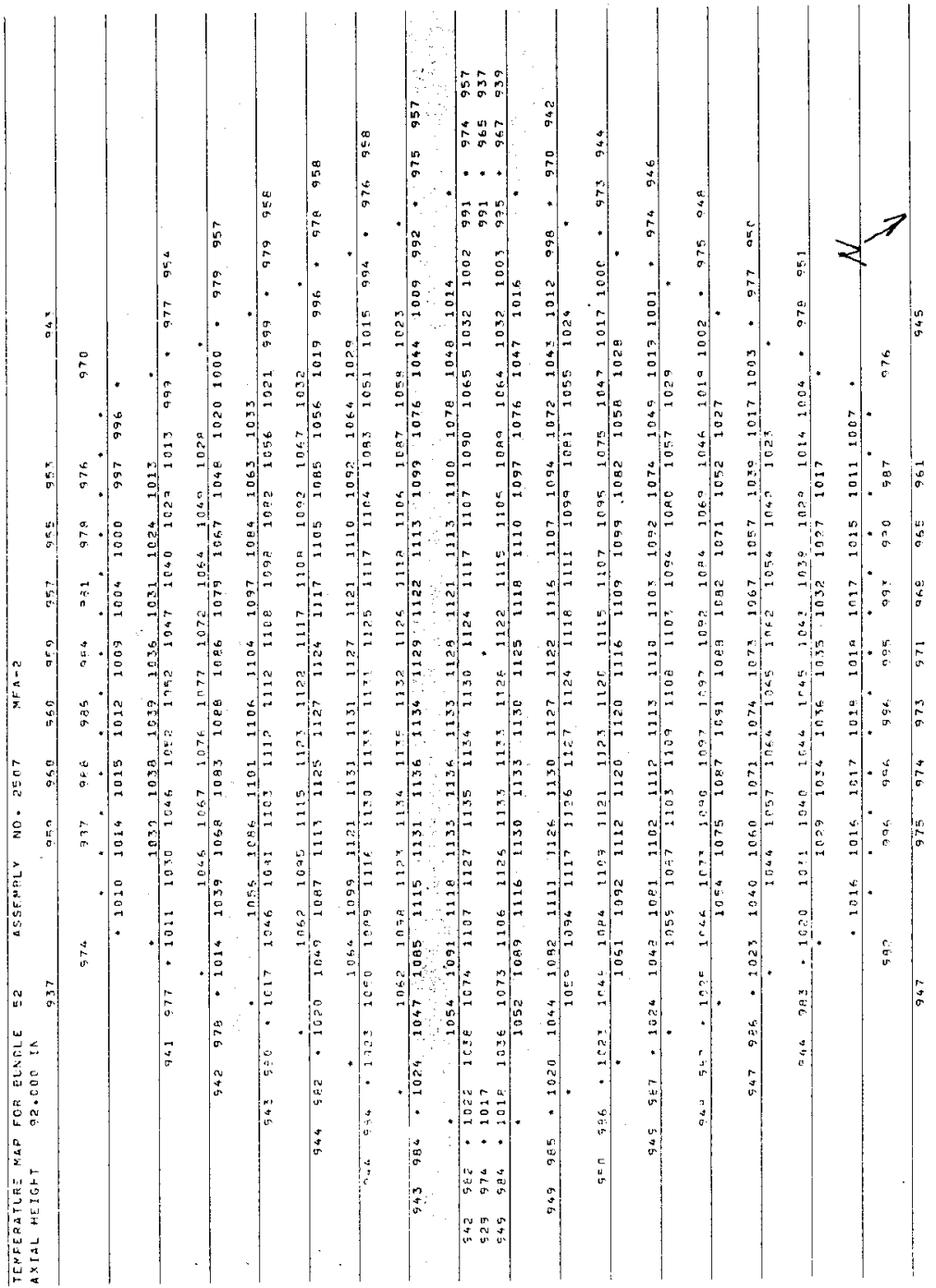


Table ElOC-1.1. Fission Power Generated in MFA-1, MFA-2 and Neighboring Assemblies at EOC $10 \mathrm{C}-1$

\begin{tabular}{lcccc} 
CORE & $* * * * * *$ & \multicolumn{2}{c}{ POWER IN MEGAWATTS } & $* * * * * * *$ \\
POS. & BELOW CORE & IN CORE & ABOVE CORE & TOTAL PWR \\
1506 & $4.883 \mathrm{E}-02$ & $3.467 \mathrm{E}+00$ & $3.014 \mathrm{E}-02$ & $3.546 \mathrm{E}+00$ \\
1404 & $1.306 \mathrm{E}-02$ & $3.615 \mathrm{E}+00$ & $8.578 \mathrm{E}-03$ & $3.637 \mathrm{E}+00$ \\
1405 & $0.000 \mathrm{E}+00$ & $6.220 \mathrm{E}+00$ & $0.000 \mathrm{E}+00$ & $6.220 \mathrm{E}+00$ \\
1505 & $1.162 \mathrm{E}-02$ & $3.230 \mathrm{E}+00$ & $7.041 \mathrm{E}-03$ & $3.248 \mathrm{E}+00$ \\
1507 & $8.342 \mathrm{E}-03$ & $3.259 \mathrm{E}+00$ & $5.837 \mathrm{E}-03$ & $3.273 \mathrm{E}+00$ \\
1606 & $7.922 \mathrm{E}-03$ & $3.089 \mathrm{E}+00$ & $5.459 \mathrm{E}-03$ & $3.102 \mathrm{E}+00$ \\
1607 & $1.008 \mathrm{E}-02$ & $2.854 \mathrm{E}+00$ & $6.772 \mathrm{E}-03$ & $2.871 \mathrm{E}+00$ \\
2507 & $5.286 \mathrm{E}-02$ & $3.906 \mathrm{E}+00$ & $3.553 \mathrm{E}-02$ & $3.995 \mathrm{E}+00$ \\
2405 & $1.414 \mathrm{E}-02$ & $3.920 \mathrm{E}+00$ & $1.012 \mathrm{E}-02$ & $3.945 \mathrm{E}+00$ \\
2506 & $9.911 \mathrm{E}-03$ & $4.558 \mathrm{E}+00$ & $7.449 \mathrm{E}-03$ & $4.575 \mathrm{E}+00$ \\
2508 & $1.432 \mathrm{E}-02$ & $4.107 \mathrm{E}+00$ & $9.413 \mathrm{E}-03$ & $4.131 \mathrm{E}+00$ \\
2607 & $1.042 \mathrm{E}-02$ & $3.620 \mathrm{E}+00$ & $7.871 \mathrm{E}-03$ & $3.638 \mathrm{E}+00$ \\
2608 & $7.131 \mathrm{E}-03$ & $3.339 \mathrm{E}+00$ & $5.753 \mathrm{E}-03$ & $3.352 \mathrm{E}+00$ \\
2609 & $7.904 \mathrm{E}-03$ & $3.997 \mathrm{E}+00$ & $6.244 \mathrm{E}-03$ & $4.011 \mathrm{E}+00$
\end{tabular}

Table E1OC-1.2. Assembly Averaged Total and Fast Flux in MFA-1 and MFA-2 at EOC $10 \mathrm{C}-1$

\begin{tabular}{|c|c|c|}
\hline & Core & Flux \\
\hline $\begin{array}{l}\text { issembly } \\
\text { MFA-1 } \\
\text { MFA-2 }\end{array}$ & $\begin{array}{l}\text { Pos. } \\
1506 \\
2507\end{array}$ & $\begin{array}{c}\text { Total } \\
2.776 \mathrm{E}+15 \\
3.151 \mathrm{E}+15\end{array}$ \\
\hline
\end{tabular}


Table El0C-1.3. Axial Distribution of Total Flux, Fast Flux and Power in MFA-1 at EOC $10 \mathrm{C}-1$

$\begin{array}{rccc}z(\mathrm{~cm}) & \text { Total Flux } & \text { F1ux }>0.1 \mathrm{MeV} & \text { Power } \\ -97.78 & 2.7935 \mathrm{E}-02 & 1.4139 \mathrm{E}-02 & 0.0000 \mathrm{E}+00 \\ -92.78 & 5.9980 \mathrm{E}-02 & 2.7269 \mathrm{E}-02 & 0.0000 \mathrm{E}+00 \\ -87.78 & 9.6092 \mathrm{E}-02 & 4.3564 \mathrm{E}-02 & 0.0000 \mathrm{E}+00 \\ -82.78 & 1.3904 \mathrm{E}-01 & 6.4861 \mathrm{E}-02 & 0.0000 \mathrm{E}+00 \\ -77.78 & 1.9182 \mathrm{E}-01 & 9.3445 \mathrm{E}-02 & 0.0000 \mathrm{E}+00 \\ -72.15 & 2.5242 \mathrm{E}-01 & 1.3057 \mathrm{E}-01 & 0.0000 \mathrm{E}+00 \\ -65.90 & 3.1388 \mathrm{E}-01 & 1.7795 \mathrm{E}-01 & 0.0000 \mathrm{E}+00 \\ -60.35 & 3.7785 \mathrm{E}-01 & 2.4219 \mathrm{E}-01 & 6.1127 \mathrm{E}-02 \\ -55.49 & 4.6072 \mathrm{E}-01 & 3.2744 \mathrm{E}-01 & 7.2510 \mathrm{E}-02 \\ -50.62 & 5.5564 \mathrm{E}-01 & 4.3890 \mathrm{E}-01 & 8.8669 \mathrm{E}-02 \\ -47.17 & 6.3107 \mathrm{E}-01 & 5.4445 \mathrm{E}-01 & 1.0652 \mathrm{E}-01 \\ -43.58 & 7.1021 \mathrm{E}-01 & 6.6413 \mathrm{E}-01 & 7.6871 \mathrm{E}-01 \\ -38.45 & 8.2534 \mathrm{E}-01 & 8.0746 \mathrm{E}-01 & 8.5338 \mathrm{E}-01 \\ -33.32 & 9.3196 \mathrm{E}-01 & 9.2831 \mathrm{E}-01 & 9.5163 \mathrm{E}-01 \\ -28.20 & 1.0246 \mathrm{E}+00 & 1.0278 \mathrm{E}+00 & 1.0149 \mathrm{E}+00 \\ -23.07 & 1.1017 \mathrm{E}+00 & 1.1086 \mathrm{E}+00 & 1.0894 \mathrm{E}+00 \\ -17.94 & 1.1616 \mathrm{E}+00 & 1.1703 \mathrm{E}+00 & 1.1476 \mathrm{E}+00 \\ -12.82 & 1.2034 \mathrm{E}+00 & 1.2125 \mathrm{E}+00 & 1.1730 \mathrm{E}+00 \\ -7.69 & 1.2271 \mathrm{E}+00 & 1.2365 \mathrm{E}+00 & 1.1958 \mathrm{E}+00 \\ -2.56 & 1.2325 \mathrm{E}+00 & 1.2422 \mathrm{E}+00 & 1.2009 \mathrm{E}+00 \\ 2.56 & 1.2196 \mathrm{E}+00 & 1.2297 \mathrm{E}+00 & 1.1929 \mathrm{E}+00 \\ 7.69 & 1.1884 \mathrm{E}+00 & 1.1989 \mathrm{E}+00 & 1.1624 \mathrm{E}+00 \\ 12.82 & 1.1398 \mathrm{E}+00 & 1.1506 \mathrm{E}+00 & 1.1149 \mathrm{E}+00 \\ 17.94 & 1.0752 \mathrm{E}+00 & 1.0863 \mathrm{E}+00 & 1.0729 \mathrm{E}+00 \\ 23.07 & 9.9551 \mathrm{E}-01 & 1.0055 \mathrm{E}+00 & 9.9392 \mathrm{E}-01 \\ 28.20 & 9.0279 \mathrm{E}-01 & 9.0994 \mathrm{E}-01 & 9.0251 \mathrm{E}-01 \\ 33.32 & 7.9961 \mathrm{E}-01 & 8.0137 \mathrm{E}-01 & 8.2668 \mathrm{E}-01 \\ 38.45 & 6.8792 \mathrm{E}-01 & 6.7827 \mathrm{E}-01 & 7.1823 \mathrm{E}-01 \\ 43.58 & 5.7294 \mathrm{E}-01 & 5.4164 \mathrm{E}-01 & 6.2028 \mathrm{E}-01 \\ 47.17 & 4.9730 \mathrm{E}-01 & 4.3668 \mathrm{E}-01 & 7.3979 \mathrm{E}-02 \\ 50.62 & 4.2726 \mathrm{E}-01 & 3.4633 \mathrm{E}-01 & 5.7875 \mathrm{E}-02 \\ 55.49 & 3.4068 \mathrm{E}-01 & 2.5200 \mathrm{E}-01 & 4.3191 \mathrm{E}-02 \\ 60.35 & 2.6373 \mathrm{E}-01 & 1.7885 \mathrm{E}-01 & 3.2682 \mathrm{E}-02 \\ 64.98 & 2.1350 \mathrm{E}-01 & 1.3462 \mathrm{E}-01 & 0.0000 \mathrm{E}+00 \\ 69.39 & 1.8256 \mathrm{E}-01 & 1.1030 \mathrm{E}-01 & 0.0000 \mathrm{E}+00 \\ 76.46 & 1.3823 \mathrm{E}-01 & 7.7637 \mathrm{E}-02 & 0.0000 \mathrm{E}+00 \\ 85.80 & 8.9917 \mathrm{E}-02 & 4.6440 \mathrm{E}-02 & 0.0000 \mathrm{E}+00 \\ 98.14 & 5.1775 \mathrm{E}-02 & 2.5205 \mathrm{E}-02 & 0.0000 \mathrm{E}+00 \\ 110.00 & 2.2018 \mathrm{E}-02 & 1.1170 \mathrm{E}-02 & 0.0000 \mathrm{E}+00\end{array}$


Table E10C-1.4. Axial Distribution of Total Flux, Fast Flux and Power in MFA-2 at EOC $10 \mathrm{C}-1$

$\begin{array}{rccc}\text { z }(\mathrm{cm}) & \text { Total Flux } & \text { Flux }>0.1 \text { MeV } & \text { Power } \\ -97.78 & 2.4837 \mathrm{E}-02 & 1.2725 \mathrm{E}-02 & 0.0000 \mathrm{E}+00 \\ -92.78 & 5.3297 \mathrm{E}-02 & 2.4557 \mathrm{E}-02 & 0.0000 \mathrm{E}+00 \\ -87.78 & 8.5430 \mathrm{E}-02 & 3.9299 \mathrm{E}-02 & 0.0000 \mathrm{E}+00 \\ -82.78 & 1.2380 \mathrm{E}-01 & 5.8688 \mathrm{E}-02 & 0.0000 \mathrm{E}+00 \\ -77.78 & 1.7128 \mathrm{E}-01 & 8.4939 \mathrm{E}-02 & 0.0000 \mathrm{E}+00 \\ -72.15 & 2.2638 \mathrm{E}-01 & 1.1940 \mathrm{E}-01 & 0.0000 \mathrm{E}+00 \\ -65.90 & 2.8420 \mathrm{E}-01 & 1.6440 \mathrm{E}-01 & 0.0000 \mathrm{E}+00 \\ -60.35 & 3.4737 \mathrm{E}-01 & 2.2690 \mathrm{E}-01 & 5.7530 \mathrm{E}-02 \\ -55.49 & 4.2985 \mathrm{E}-01 & 3.1056 \mathrm{E}-01 & 6.9298 \mathrm{E}-02 \\ -50.62 & 5.2582 \mathrm{E}-01 & 4.2043 \mathrm{E}-01 & 8.5980 \mathrm{E}-02 \\ -47.17 & 6.0343 \mathrm{E}-01 & 5.2467 \mathrm{E}-01 & 1.0424 \mathrm{E}-01 \\ -43.58 & 6.8553 \mathrm{E}-01 & 6.4314 \mathrm{E}-01 & 7.4092 \mathrm{E}-01 \\ -38.45 & 8.0480 \mathrm{E}-01 & 7.8820 \mathrm{E}-01 & 8.3340 \mathrm{E}-01 \\ -33.32 & 9.1548 \mathrm{E}-01 & 9.1228 \mathrm{E}-01 & 9.3705 \mathrm{E}-01 \\ -28.20 & 1.0120 \mathrm{E}+00 & 1.0153 \mathrm{E}+00 & 1.0027 \mathrm{E}+00 \\ -23.07 & 1.0926 \mathrm{E}+00 & 1.0994 \mathrm{E}+00 & 1.0809 \mathrm{E}+00 \\ -17.94 & 1.1557 \mathrm{E}+00 & 1.1641 \mathrm{E}+00 & 1.1424 \mathrm{E}+00 \\ -12.82 & 1.2003 \mathrm{E}+00 & 1.2090 \mathrm{E}+00 & 1.1689 \mathrm{E}+00 \\ -7.69 & 1.2266 \mathrm{E}+00 & 1.2356 \mathrm{E}+00 & 1.1942 \mathrm{E}+00 \\ -2.56 & 1.2345 \mathrm{E}+00 & 1.2437 \mathrm{E}+00 & 1.2018 \mathrm{E}+00 \\ 2.56 & 1.2240 \mathrm{E}+00 & 1.2336 \mathrm{E}+00 & 1.1959 \mathrm{E}+00 \\ 7.69 & 1.1953 \mathrm{E}+00 & 1.2052 \mathrm{E}+00 & 1.1680 \mathrm{E}+00 \\ 12.82 & 1.1493 \mathrm{E}+00 & 1.1594 \mathrm{E}+00 & 1.1232 \mathrm{E}+00 \\ 17.94 & 1.0869 \mathrm{E}+00 & 1.0973 \mathrm{E}+00 & 1.0848 \mathrm{E}+00 \\ 23.07 & 1.0087 \mathrm{E}+00 & 1.0180 \mathrm{E}+00 & 1.0074 \mathrm{E}+00 \\ 28.20 & 9.1636 \mathrm{E}-01 & 9.2289 \mathrm{E}-01 & 9.1636 \mathrm{E}-01 \\ 33.32 & 8.1236 \mathrm{E}-01 & 8.1363 \mathrm{E}-01 & 8.4158 \mathrm{E}-01 \\ 38.45 & 6.9874 \mathrm{E}-01 & 6.8897 \mathrm{E}-01 & 7.3090 \mathrm{E}-01 \\ 43.58 & 5.8079 \mathrm{E}-01 & 5.5021 \mathrm{E}-01 & 6.2969 \mathrm{E}-01 \\ 47.17 & 5.0121 \mathrm{E}-01 & 4.4222 \mathrm{E}-01 & 7.7943 \mathrm{E}-02 \\ 50.62 & 4.2632 \mathrm{E}-01 & 3.4762 \mathrm{E}-01 & 6.0830 \mathrm{E}-02 \\ 55.49 & 3.3423 \mathrm{E}-01 & 2.4860 \mathrm{E}-01 & 4.5068 \mathrm{E}-02 \\ 60.35 & 2.5407 \mathrm{E}-01 & 1.7292 \mathrm{E}-01 & 3.3821 \mathrm{E}-02 \\ 64.98 & 2.0425 \mathrm{E}-01 & 1.2895 \mathrm{E}-01 & 0.0000 \mathrm{E}+00 \\ 69.39 & 1.7462 \mathrm{E}-01 & 1.0563 \mathrm{E}-01 & 0.0000 \mathrm{E}+00 \\ 76.46 & 1.3239 \mathrm{E}-01 & 7.4464 \mathrm{E}-02 & 0.0000 \mathrm{E}+00 \\ 86.80 & 8.6170 \mathrm{E}-02 & 4.4526 \mathrm{E}-02 & 0.0000 \mathrm{E}+00 \\ 98.14 & 4.9587 \mathrm{E}-02 & 2.4120 \mathrm{E}-02 & 0.0000 \mathrm{E}+00 \\ 110.00 & 2.1040 \mathrm{E}-02 & 1.0663 \mathrm{E}-02 & 0.0000 \mathrm{E}+00\end{array}$


HNF-SD-FF-ANAL-009 Rev. 1

Table ElOC-1.5. Fission Power Distribution by Pin in MFA-1 at EOC 1OC-1 CORE POSIIIION 1506

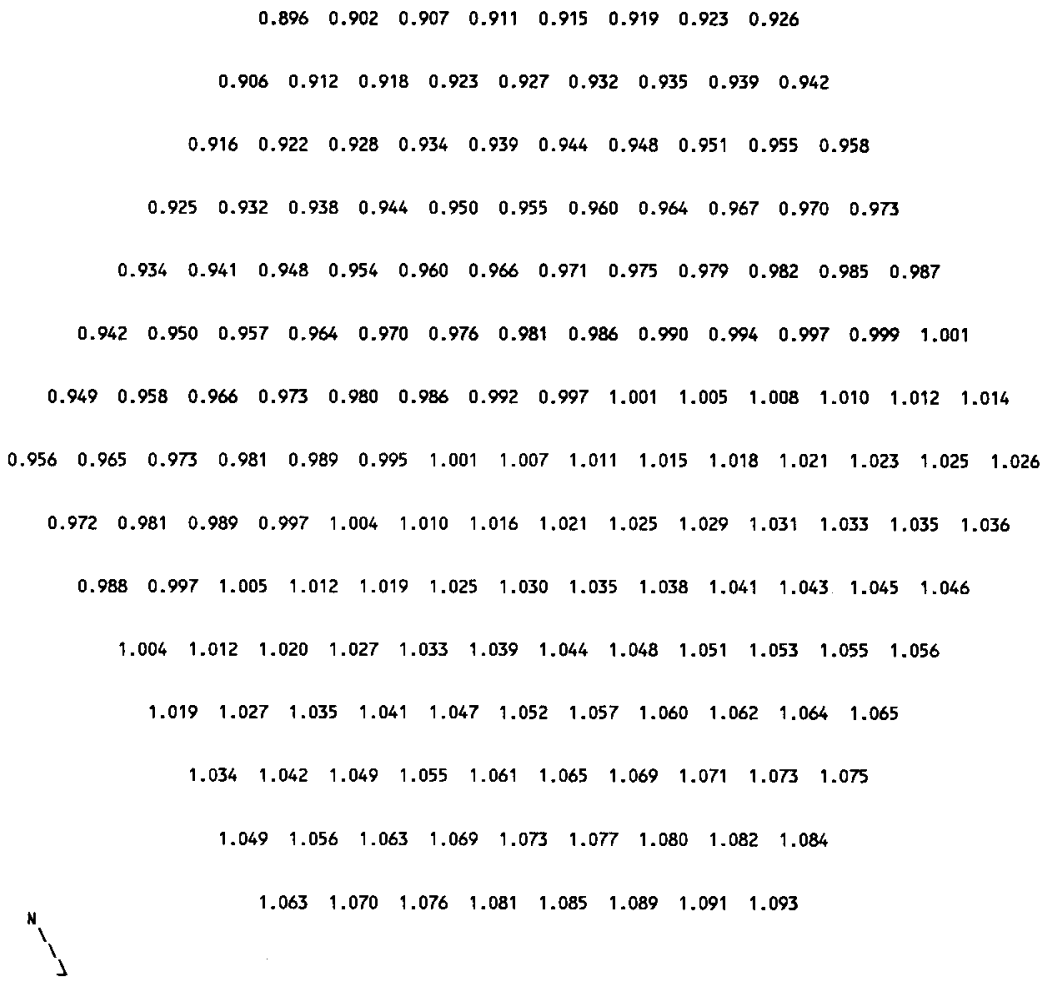


HNF-SD-FF-ANAL-009 Rev. 1

Table E10C-1.6. Fission Power Distribution by Pin in MFA-2 at EOC 1OC-1 CORE POSITION 2507

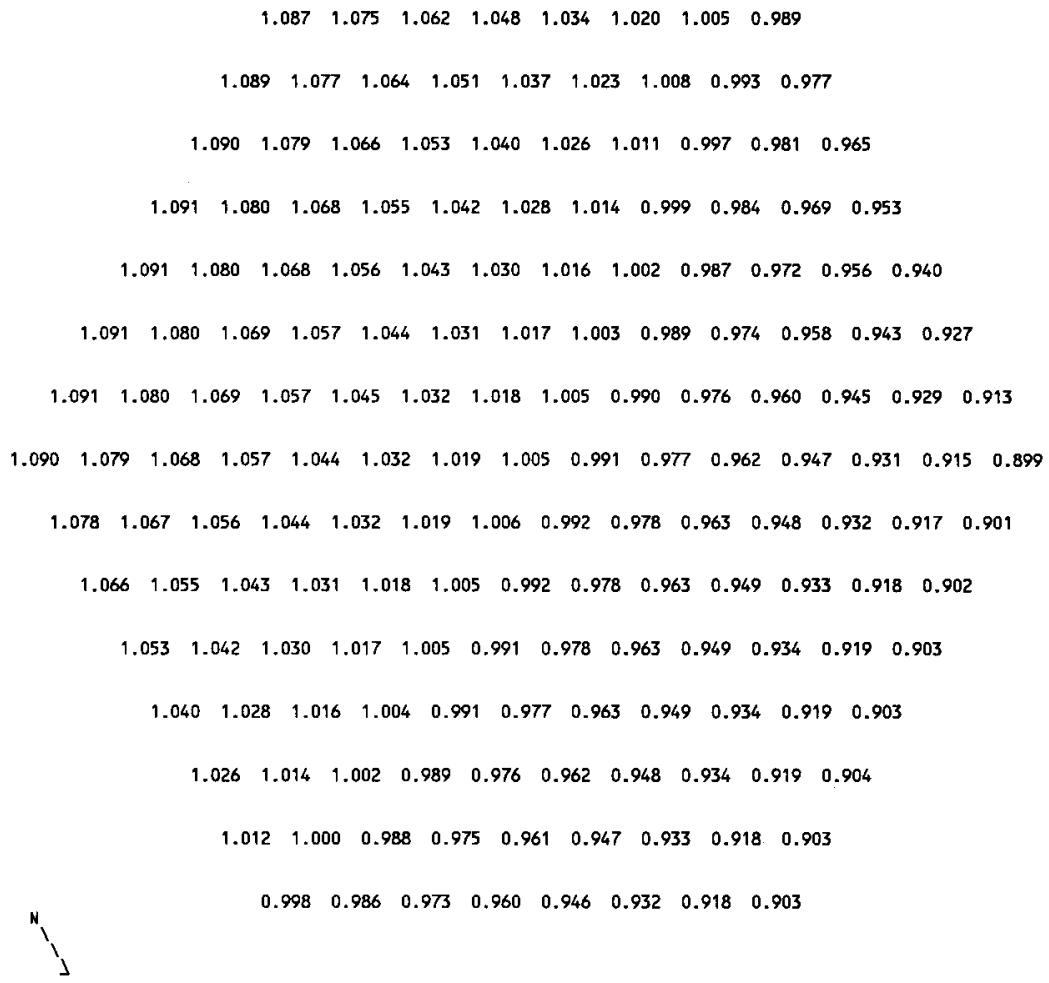


HNF-SD-FF-ANAL-009 Rev. 1

Table E10C-1.7. Fast Flux Distribution by Pin in MFA-1 at EOC 10C-1 CORE POSIIION 1506

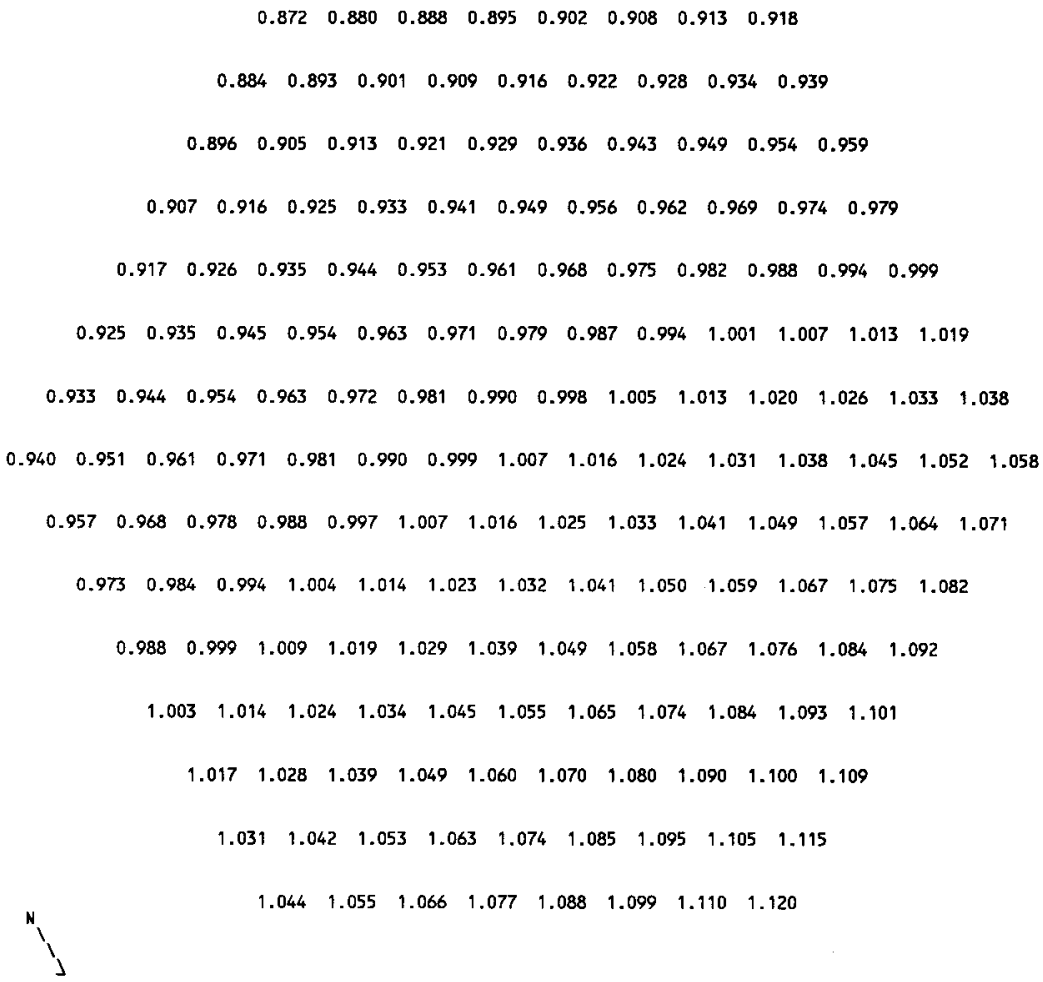


Table E1OC-1.8. Fast Flux Distribution by Pin in MFA-2 at EOC $10 \mathrm{C}-1$ CORE POSITION 2507

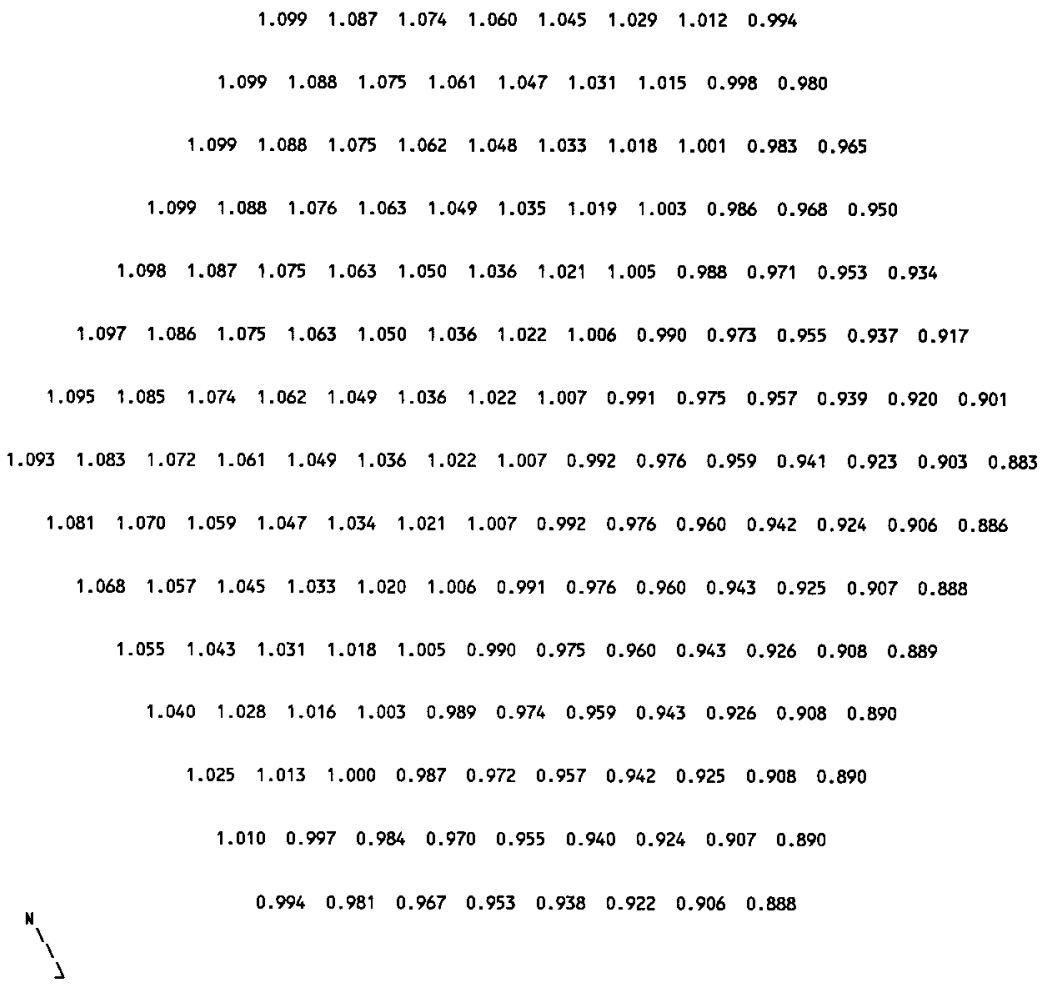




\section{HNF-SD-FF-ANAL-009 Rev. 1}

Table E10C-1.9. MFA-1 and MFA-2 Duct Wall

Fast Flux Data at EOC $10 \mathrm{C}-1$

$\begin{array}{ccc}\text { Side } & \begin{array}{c}\text { Flux }>0.1 \\ \text { MFA-1 }\end{array} & \begin{array}{c}\text { MeV }\left(\mathrm{n} / \mathrm{cm}^{2}-\mathrm{sec}\right) \\ \text { MFA-2 }\end{array} \\ \text { E } & 1.7757 \mathrm{E}+15 & 2.1369 \mathrm{E}+15 \\ \text { SE } & 1.5955 \mathrm{E}+15 & 2.2597 \mathrm{E}+15 \\ \text { SW } & 1.5631 \mathrm{E}+15 & 2.1487 \mathrm{E}+15 \\ \text { W } & 1.7554 \mathrm{E}+15 & 1.8732 \mathrm{E}+15 \\ \text { NW } & 1.9962 \mathrm{E}+15 & 1.7488 \mathrm{E}+15 \\ \text { NE } & 1.9598 \mathrm{E}+15 & 1.8850 \mathrm{E}+15\end{array}$


Table ElOC-1.17. Composition of MFA-1 at EOC 1OC-1

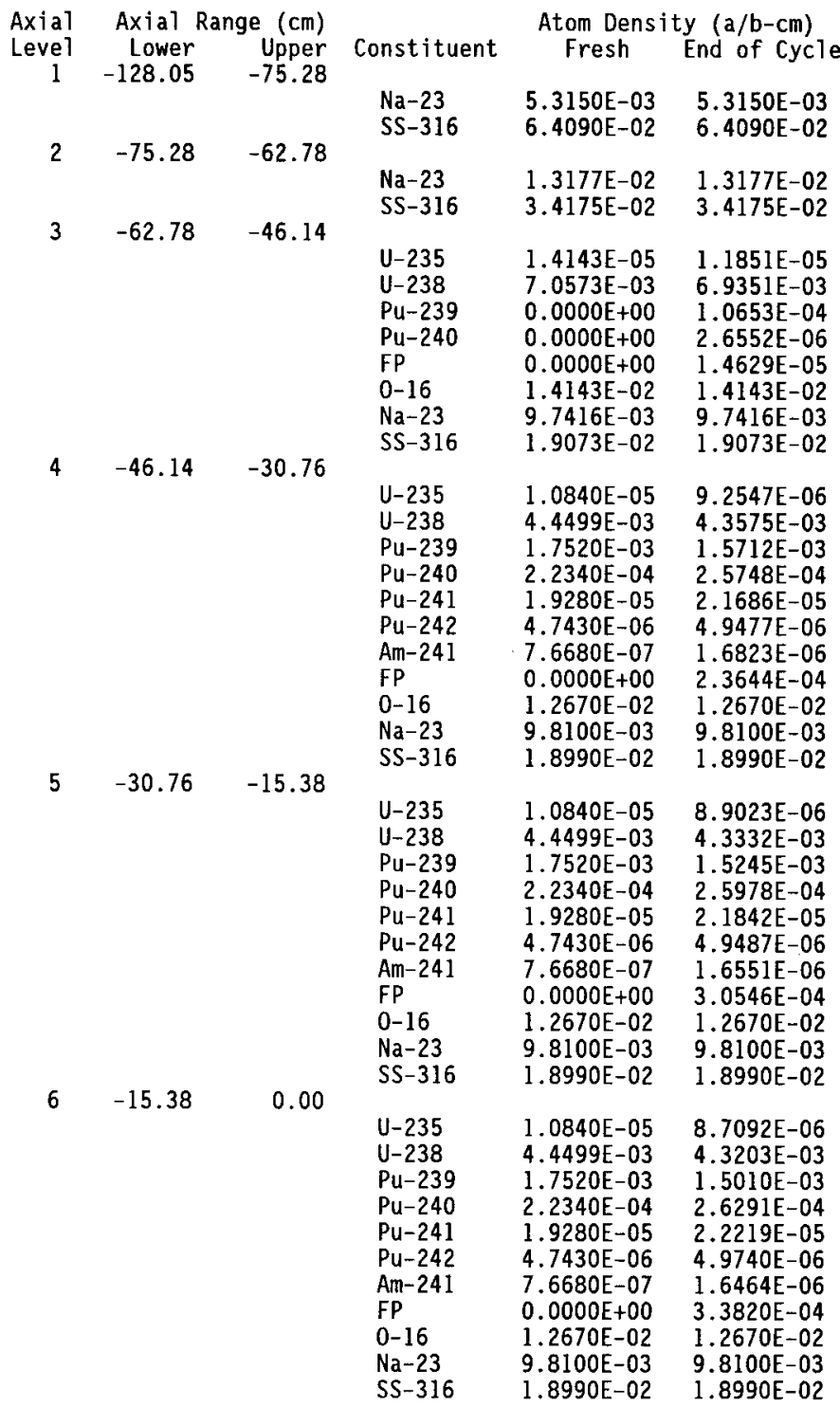


HNF-SD-FF-ANAL-009 Rev. 1

$\begin{array}{lll}7 & 0.00 \quad 15.38\end{array}$

$\begin{array}{lll}8 & 15.38 & 30.76\end{array}$

$\begin{array}{lll}\mathrm{U}-235 & 1.0840 \mathrm{E}-05 & 8.7711 \mathrm{E}-06 \\ \mathrm{U}-238 & 4.4499 \mathrm{E}-03 & 4.3245 \mathrm{E}-03 \\ \mathrm{Pu}-239 & 1.7520 \mathrm{E}-03 & 1.5083 \mathrm{E}-03 \\ \mathrm{Pu}-240 & 2.2340 \mathrm{E}-04 & 2.6170 \mathrm{E}-04 \\ \mathrm{Pu}-241 & 1.9280 \mathrm{E}-05 & 2.2074 \mathrm{E}-05 \\ \mathrm{Pu}-242 & 4.7430 \mathrm{E}-06 & 4.9638 \mathrm{E}-06 \\ \mathrm{Am}-241 & 7.6680 \mathrm{E}-07 & 1.6495 \mathrm{E}-06 \\ \mathrm{FP} & 0.0000 \mathrm{E}+00 & 3.2806 \mathrm{E}-04 \\ \mathrm{O}-16 & 1.2670 \mathrm{E}-02 & 1.2670 \mathrm{E}-02 \\ \mathrm{Na}-23 & 9.8100 \mathrm{E}-03 & 9.8100 \mathrm{E}-03 \\ \mathrm{SS}-316 & 1.8990 \mathrm{E}-02 & 1.8990 \mathrm{E}-02\end{array}$

U-235 $\quad 1.0840 E-05 \quad 9.0767 E-06$

U-238 4.4499E-03 4.3444E-03

$\mathrm{Pu}-239 \quad 1.7520 \mathrm{E}-03 \quad 1.5446 \mathrm{E}-03$

$\mathrm{Pu}-240 \quad 2.2340 \mathrm{E}-04 \quad 2.5637 \mathrm{E}-04$

$\mathrm{Pu}-241 \quad 1.9280 \mathrm{E}-05 \quad 2.1445 \mathrm{E}-05$

$\mathrm{Pu}-242 \quad 4.7430 \mathrm{E}-06 \quad 4.9220 \mathrm{E}-06$

$\begin{array}{lll}A m-241 & 7.6680 \mathrm{E}-07 & 1.6634 \mathrm{E}-06\end{array}$

FP $\quad 0.0000 E+00 \quad 2.7777 \mathrm{E}-04$

0-16 $\quad 1.2670 \mathrm{E}-02 \quad 1.2670 \mathrm{E}-02$

$\mathrm{Na}-23 \quad 9.8100 \mathrm{E}-03 \quad 9.8100 \mathrm{E}-03$

SS-316 $1.8990 \mathrm{E}-02 \quad 1.8990 \mathrm{E}-02$

$\begin{array}{lll}9 & 30.76 & 46.14\end{array}$

$\begin{array}{lll}\text { U-235 } & 1.0840 \mathrm{E}-05 & 9.5158 \mathrm{E}-06 \\ \mathrm{U}-238 & 4.4499 \mathrm{E}-03 & 4.3734 \mathrm{E}-03 \\ \mathrm{Pu}-23.9 & 1.7520 \mathrm{E}-03 & 1.6003 \mathrm{E}-03 \\ \mathrm{Pu}-240 & 2.2340 \mathrm{E}-04 & 2.5123 \mathrm{E}-04 \\ \mathrm{Pu}-241 & 1.9280 \mathrm{E}-05 & 2.0949 \mathrm{E}-05 \\ \mathrm{Pu}-242 & 4.7430 \mathrm{E}-06 & 4.8980 \mathrm{E}-06 \\ \mathrm{Am}-241 & 7.6680 \mathrm{E}-07 & 1.6912 \mathrm{E}-06 \\ \mathrm{FP} & 0.0000 \mathrm{E}+00 & 1.9830 \mathrm{E}-04 \\ 0-16 & 1.2670 \mathrm{E}-02 & 1.2670 \mathrm{E}-02 \\ \mathrm{Na}-23 & 9.8100 \mathrm{E}-03 & 9.8100 \mathrm{E}-03 \\ \text { SS-316 } & 1.8990 \mathrm{E}-02 & 1.8990 \mathrm{E}-02\end{array}$

$\begin{array}{lll}10 & 46.14 & 62.78\end{array}$

$\begin{array}{lll}\text { U-235 } & 1.4143 \mathrm{E}-05 & 1.2566 \mathrm{E}-05 \\ \mathrm{U}-238 & 7.0573 \mathrm{E}-03 & 6.9719 \mathrm{E}-03 \\ \mathrm{Pu}-239 & 0.0000 \mathrm{E}+00 & 7.5587 \mathrm{E}-05 \\ \mathrm{Pu}-240 & 0.0000 \mathrm{E}+00 & 1.2034 \mathrm{E}-06 \\ \mathrm{FP} & 0.0000 \mathrm{E}+00 & 9.6893 \mathrm{E}-06 \\ \mathrm{O}-16 & 1.4143 \mathrm{E}-02 & 1.4143 \mathrm{E}-02 \\ \mathrm{Na}-23 & 9.7416 \mathrm{E}-03 & 9.7416 \mathrm{E}-03 \\ \mathrm{SS}-316 & 1.9073 \mathrm{E}-02 & 1.9073 \mathrm{E}-02\end{array}$

$\begin{array}{lll}11 & 62.78 & 172.84\end{array}$

$\begin{array}{lll}\mathrm{Na}-23 & 8.2400 \mathrm{E}-03 & 8.2400 \mathrm{E}-03 \\ \mathrm{SS}-316 & 2.5562 \mathrm{E}-02 & 2.5562 \mathrm{E}-02\end{array}$


Table ElOC-1.18. Composition of MFA-2 at EOC 1OC-1

Axial Axial Range (cm) Leve1 Lower Upper $1-128.05 \quad-75.28$

$2 \quad-75.28 \quad-62.78$

$3 \quad-62.78-46.14$

$4 \quad-46.14 \quad-30.76$

$5 \quad-30.76 \quad-15.38$

$6 \quad-15.38$

0.00
Atom Density $(\mathrm{a} / \mathrm{b}-\mathrm{cm})$

Fresh End of Cycle

$\begin{array}{lll}\mathrm{Na}-23 & 5.3150 \mathrm{E}-03 & 5.3150 \mathrm{E}-03 \\ \mathrm{SS}-316 & 6.4090 \mathrm{E}-02 & 6.4090 \mathrm{E}-02\end{array}$

$\begin{array}{lll}\mathrm{Na}-23 & 5.3150 \mathrm{E}-03 & 5.3150 \mathrm{E}-03 \\ \mathrm{SS}-316 & 6.4090 \mathrm{E}-02 & 6.4090 \mathrm{E}-02\end{array}$

Na-23 $\quad 1.3177 \mathrm{E}-02 \quad 1.3177 \mathrm{E}-02$

SS-316 3.4175E-02 3.4175E-02

U-235 $1.4143 \mathrm{E}-05 \quad 1.1762 \mathrm{E}-05$

U-238 $7.0573 \mathrm{E}-03 \quad 6.9310 \mathrm{E}-03$

$\mathrm{Pu}-239 \quad 0.0000 \mathrm{E}+00 \quad 1.0974 \mathrm{E}-04$

Pu-240 $\quad 0.0000 E+00 \quad 2.9000 E-06$

$\begin{array}{lll}\text { FP } & 0.0000 E+00 & 1.5319 E-05\end{array}$

0-16 $1.4143 \mathrm{E}-02 \quad 1.4143 \mathrm{E}-02$

$\mathrm{Na}-23 \quad 9.7416 \mathrm{E}-03 \quad 9.7416 \mathrm{E}-03$

SS-316 $1.9073 \mathrm{E}-02 \quad 1.9073 \mathrm{E}-02$

U-235 $\quad 1.0160 \mathrm{E}-05 \quad 8.5944 \mathrm{E}-06$

U-238 $4.4270 \mathrm{E}-03 \quad 4.3296 \mathrm{E}-03$

Pu-239 $1.7483 E-03 \quad 1.5593 E-03$

Pu-240 2.2290E-04 2.5958E-04

Pu-241 $\quad 1.9040 \mathrm{E}-05 \quad 2.1816 \mathrm{E}-05$

Pu-242 4.7330E-06 4.9549E-06

Am-241 $\quad 7.5700 \mathrm{E}-07 \quad 1.6571 \mathrm{E}-06$

$\begin{array}{lll}\mathrm{FP} & 0.0000 \mathrm{E}+00 \quad 2.4675 \mathrm{E}-04\end{array}$

$0-16 \quad 1.2760 \mathrm{E}-02 \quad 1.2760 \mathrm{E}-02$

$\begin{array}{lll}\mathrm{Na}-23 & 9.8100 \mathrm{E}-03 \quad 9.8100 \mathrm{E}-03\end{array}$

SS-316 $1.8990 \mathrm{E}-02 \quad 1.8990 \mathrm{E}-02$

U-235 $\quad 1.0160 \mathrm{E}-05 \quad 8.2320 \mathrm{E}-06$

U-238 $4.4270 \mathrm{E}-03 \quad 4.3025 \mathrm{E}-03$

$\begin{array}{lll}\mathrm{Pu}-239 & 1.7483 \mathrm{E}-03 & 1.5085 \mathrm{E}-03\end{array}$

Pu-240 2.2290E-04 2.6240E-04

Pu-24l $1.9040 \mathrm{E}-05 \quad 2.2041 \mathrm{E}-05$

Pu-242 4.7330E-06 4.9595E-06

Am-241 7.5700E-07 $1.6276 \mathrm{E}-06$

$\begin{array}{lll}\text { FP } & 0.0000 \mathrm{E}+00 \quad 3.2173 \mathrm{E}-04\end{array}$

$\begin{array}{lll}0-16 & 1.2760 \mathrm{E}-02 & 1.2760 \mathrm{E}-02\end{array}$

Na-23 $\quad 9.8100 \mathrm{E}-03 \quad 9.8100 \mathrm{E}-03$

SS-316 $1.8990 \mathrm{E}-02 \quad 1.8990 \mathrm{E}-02$

$\begin{array}{lll}\mathrm{U}-235 & 1.0160 \mathrm{E}-05 & 8.0279 \mathrm{E}-06\end{array}$

U-238 $4.4270 \mathrm{E}-03 \quad 4.2879 \mathrm{E}-03$

Pu-239 $\quad 1.7483 \mathrm{E}-03 \quad 1.4825 \mathrm{E}-03$

Pu-240 2.2290E-04 2.6598E-04

Pu-241 $\quad 1.9040 \mathrm{E}-05 \quad 2.2497 \mathrm{E}-05$

Pu-242 4.7330E-06 4.9903E-06

Am-241 7.5700E-07 1.6173E-06

FP $\quad 0.0000 \mathrm{E}+00 \quad 3.5844 \mathrm{E}-04$

$\begin{array}{lll}0-16 & 1.2760 \mathrm{E}-02 & 1.2760 \mathrm{E}-02\end{array}$

$\begin{array}{lll}\mathrm{Na}-23 & 9.8100 \mathrm{E}-03 & 9.8100 \mathrm{E}-03\end{array}$

SS-316 $1.8990 \mathrm{E}-02 \quad 1.8990 \mathrm{E}-02$ 


\begin{tabular}{|c|c|c|c|c|c|}
\hline \multirow{2}{*}{7} & 0.00 & 15.38 & & & \\
\hline & & & $\begin{array}{l}\mathrm{U}-235 \\
\mathrm{U}-238 \\
\mathrm{Pu}-239 \\
\mathrm{Pu}-240 \\
\mathrm{Pu}-241 \\
\mathrm{Pu}-242 \\
\mathrm{Am}-241 \\
\mathrm{FP} \\
0-16 \\
\mathrm{Na}-23 \\
\mathrm{SS}-316\end{array}$ & $\begin{array}{l}1.0160 \mathrm{E}-05 \\
4.4270 \mathrm{E}-03 \\
1.7483 \mathrm{E}-03 \\
2.2290 \mathrm{E}-04 \\
1.9040 \mathrm{E}-05 \\
4.7330 \mathrm{E}-06 \\
7.5700 \mathrm{E}-07 \\
0.0000 \mathrm{E}+00 \\
1.2760 \mathrm{E}-02 \\
9.8100 \mathrm{E}-03 \\
1.8990 \mathrm{E}-02\end{array}$ & $\begin{array}{l}8.0820 \mathrm{E}-06 \\
4.2918 \mathrm{E}-03 \\
1.4891 \mathrm{E}-03 \\
2.6488 \mathrm{E}-04 \\
2.2355 \mathrm{E}-05 \\
4.9803 \mathrm{E}-06 \\
1.6201 \mathrm{E}-06 \\
3.4909 \mathrm{E}-04 \\
1.2760 \mathrm{E}-02 \\
9.8100 \mathrm{E}-03 \\
1.8990 \mathrm{E}-02\end{array}$ \\
\hline \multirow[t]{2}{*}{8} & 15.38 & 30.76 & & & \\
\hline & & & $\begin{array}{l}\mathrm{U}-235 \\
\mathrm{U}-238 \\
\mathrm{Pu}-239 \\
\mathrm{Pu}-240 \\
\mathrm{Pu}-241 \\
\mathrm{Pu}-242 \\
\mathrm{Am}-241 \\
\mathrm{FP} \\
0-16 \\
\mathrm{Na}-23 \\
\mathrm{SS}-316\end{array}$ & $\begin{array}{l}1.0160 \mathrm{E}-05 \\
4.4270 \mathrm{E}-03 \\
1.7483 \mathrm{E}-03 \\
2.2290 \mathrm{E}-04 \\
1.9040 \mathrm{E}-05 \\
4.7330 \mathrm{E}-06 \\
7.5700 \mathrm{E}-07 \\
0.0000 \mathrm{E}+00 \\
1.2760 \mathrm{E}-02 \\
9.8100 \mathrm{E}-03 \\
1.8990 \mathrm{E}-02\end{array}$ & $\begin{array}{l}8.3773 \mathrm{E}-06 \\
4.3127 \mathrm{E}-03 \\
1.5265 \mathrm{E}-03 \\
2.5938 \mathrm{E}-04 \\
2.1675 \mathrm{E}-05 \\
4.9350 \mathrm{E}-06 \\
1.6348 \mathrm{E}-06 \\
2.9699 \mathrm{E}-04 \\
1.2760 \mathrm{E}-02 \\
9.8100 \mathrm{E}-03 \\
1.8990 \mathrm{E}-02\end{array}$ \\
\hline \multirow[t]{2}{*}{9} & 30.76 & 46.14 & & & \\
\hline & & & $\begin{array}{l}\mathrm{U}-235 \\
\mathrm{U}-238 \\
\mathrm{Pu}-239 \\
\mathrm{Pu}-240 \\
\mathrm{Pu}-241 \\
\mathrm{Pu}-242 \\
\mathrm{Am}-241 \\
\mathrm{FP} \\
0-16 \\
\mathrm{Na}-23 \\
\mathrm{SS}-316\end{array}$ & $\begin{array}{l}1.0160 \mathrm{E}-05 \\
4.4270 \mathrm{E}-03 \\
1.7483 \mathrm{E}-03 \\
2.2290 \mathrm{E}-04 \\
1.9040 \mathrm{E}-05 \\
4.7330 \mathrm{E}-06 \\
7.5700 \mathrm{E}-07 \\
0.0000 \mathrm{E}+00 \\
1.2760 \mathrm{E}-02 \\
9.8100 \mathrm{E}-03 \\
1.8990 \mathrm{E}-02\end{array}$ & $\begin{array}{l}8.8116 \mathrm{E}-06 \\
4.3437 \mathrm{E}-03 \\
1.5849 \mathrm{E}-03 \\
2.5400 \mathrm{E}-04 \\
2.1133 \mathrm{E}-05 \\
4.9089 \mathrm{E}-06 \\
1.6646 \mathrm{E}-06 \\
2.1326 \mathrm{E}-04 \\
1.2760 \mathrm{E}-02 \\
9.8100 \mathrm{E}-03 \\
1.8990 \mathrm{E}-02\end{array}$ \\
\hline \multirow[t]{2}{*}{10} & 46.14 & 62.78 & & & \\
\hline & & & $\begin{array}{l}\mathrm{U}-235 \\
\mathrm{U}-238 \\
\mathrm{Pu}-239 \\
\mathrm{Pu}-240 \\
\mathrm{FP} \\
0-16 \\
\mathrm{Na}-23 \\
\mathrm{SS}-316\end{array}$ & $\begin{array}{l}1.4143 \mathrm{E}-05 \\
7.0573 \mathrm{E}-03 \\
0.0000 \mathrm{E}+00 \\
0.0000 \mathrm{E}+00 \\
0.0000 \mathrm{E}+00 \\
1.4143 \mathrm{E}-02 \\
9.7416 \mathrm{E}-03 \\
1.9073 \mathrm{E}-02\end{array}$ & $\begin{array}{l}1.2393 \mathrm{E}-05 \\
6.9637 \mathrm{E}-03 \\
8.2580 \mathrm{E}-05 \\
1.5164 \mathrm{E}-06 \\
1.0775 \mathrm{E}-05 \\
1.4143 \mathrm{E}-02 \\
9.7416 \mathrm{E}-03 \\
1.9073 \mathrm{E}-02\end{array}$ \\
\hline \multirow[t]{2}{*}{11} & 62.78 & 172.84 & & & \\
\hline & & & $\begin{array}{l}\mathrm{Na}-23 \\
\mathrm{SS}-316\end{array}$ & $\begin{array}{l}8.2400 \mathrm{E}-03 \\
2.5562 \mathrm{E}-02\end{array}$ & $\begin{array}{l}8.2400 \mathrm{E}-03 \\
2.5562 \mathrm{E}-02\end{array}$ \\
\hline
\end{tabular}




\subsection{Cycle 10C-3}

Table BlOC-3.1. Fission Power Generated in MFA-1, MFA-2 and Neighboring Assemblies at $\mathrm{BOC}$ 1OC-3

\begin{tabular}{lcccc} 
CORE & $* * * * * *$ & \multicolumn{2}{c}{ POWER IN MEGAWATTS } & $* * * * * *$ \\
POS. & BELOW CORE & IN CORE & ABOVE CORE & \multicolumn{1}{c}{ TOTAL PWR } \\
1506 & $4.898 \mathrm{E}-02$ & $3.503 \mathrm{E}+00$ & $3.016 \mathrm{E}-02$ & $3.582 \mathrm{E}+00$ \\
1404 & $1.309 \mathrm{E}-02$ & $3.633 \mathrm{E}+00$ & $8.594 \mathrm{E}-03$ & $3.655 \mathrm{E}+00$ \\
1405 & $0.000 \mathrm{E}+00$ & $6.256 \mathrm{E}+00$ & $0.000 \mathrm{E}+00$ & $6.256 \mathrm{E}+00$ \\
1505 & $1.169 \mathrm{E}-02$ & $3.250 \mathrm{E}+00$ & $7.073 \mathrm{E}-03$ & $3.269 \mathrm{E}+00$ \\
1507 & $8.393 \mathrm{E}-03$ & $3.342 \mathrm{E}+00$ & $5.854 \mathrm{E}-03$ & $3.356 \mathrm{E}+00$ \\
1606 & $7.970 \mathrm{E}-03$ & $3.117 \mathrm{E}+00$ & $5.487 \mathrm{E}-03$ & $3.130 \mathrm{E}+00$ \\
1607 & $1.017 \mathrm{E}-02$ & $2.924 \mathrm{E}+00$ & $6.791 \mathrm{E}-03$ & $2.941 \mathrm{E}+00$ \\
2507 & $5.305 \mathrm{E}-02$ & $3.895 \mathrm{E}+00$ & $3.560 \mathrm{E}-02$ & $3.984 \mathrm{E}+00$ \\
2405 & $1.416 \mathrm{E}-02$ & $3.910 \mathrm{E}+00$ & $1.012 \mathrm{E}-02$ & $3.934 \mathrm{E}+00$ \\
2506 & $9.925 \mathrm{E}-03$ & $4.542 \mathrm{E}+00$ & $7.450 \mathrm{E}-03$ & $4.560 \mathrm{E}+00$ \\
2508 & $1.439 \mathrm{E}-02$ & $4.099 \mathrm{E}+00$ & $9.436 \mathrm{E}-03$ & $4.123 \mathrm{E}+00$ \\
2607 & $1.042 \mathrm{E}-02$ & $3.608 \mathrm{E}+00$ & $7.866 \mathrm{E}-03$ & $3.626 \mathrm{E}+00$ \\
2608 & $7.143 \mathrm{E}-03$ & $3.331 \mathrm{E}+00$ & $5.757 \mathrm{E}-03$ & $3.344 \mathrm{E}+00$ \\
2609 & $7.942 \mathrm{E}-03$ & $3.991 \mathrm{E}+00$ & $6.260 \mathrm{E}-03$ & $4.005 \mathrm{E}+00$
\end{tabular}

Table B10C-3.2. Assembly Averaged Total and Fast Flux in MFA- 1 and MFA-2 at BOC $10 \mathrm{C}-3$

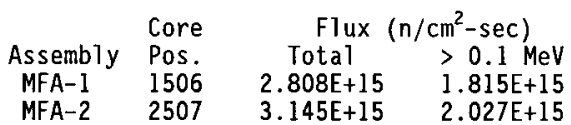


Table BloC-3.3. Axial Distribution of Total Flux, Fast Flux and Power in MFA-1 at BOC $10 \mathrm{C}-3$

$\begin{array}{rccc}\text { z }(\mathrm{cm}) & \text { Total Flux } & \text { Flux }>0.1 \text { MeV } & \text { Power } \\ -97.78 & 2.7391 \mathrm{E}-02 & 1.3831 \mathrm{E}-02 & 0.0000 \mathrm{E}+00 \\ -92.78 & 5.8830 \mathrm{E}-02 & 2.6681 \mathrm{E}-02 & 0.0000 \mathrm{E}+00 \\ -87.78 & 9.4269 \mathrm{E}-02 & 4.2637 \mathrm{E}-02 & 0.0000 \mathrm{E}+00 \\ -82.78 & 1.3644 \mathrm{E}-01 & 6.3506 \mathrm{E}-02 & 0.0000 \mathrm{E}+00 \\ -77.78 & 1.8832 \mathrm{E}-01 & 9.1546 \mathrm{E}-02 & 0.0000 \mathrm{E}+00 \\ -72.15 & 2.4796 \mathrm{E}-01 & 1.2803 \mathrm{E}-01 & 0.0000 \mathrm{E}+00 \\ -65.90 & 3.0866 \mathrm{E}-01 & 1.7475 \mathrm{E}-01 & 0.0000 \mathrm{E}+00 \\ -60.35 & 3.7219 \mathrm{E}-01 & 2.3843 \mathrm{E}-01 & 6.0577 \mathrm{E}-02 \\ -55.49 & 4.5482 \mathrm{E}-01 & 3.2321 \mathrm{E}-01 & 7.1944 \mathrm{E}-02 \\ -50.62 & 5.4982 \mathrm{E}-01 & 4.3440 \mathrm{E}-01 & 8.8103 \mathrm{E}-02 \\ -47.17 & 6.2569 \mathrm{E}-01 & 5.3999 \mathrm{E}-01 & 1.0595 \mathrm{E}-01 \\ -43.58 & 7.0538 \mathrm{E}-01 & 6.5977 \mathrm{E}-01 & 7.6367 \mathrm{E}-01 \\ -38.45 & 8.2172 \mathrm{E}-01 & 8.0401 \mathrm{E}-01 & 8.4986 \mathrm{E}-01 \\ -33.32 & 9.2976 \mathrm{E}-01 & 9.2619 \mathrm{E}-01 & 9.4964 \mathrm{E}-01 \\ -28.20 & 1.0238 \mathrm{E}+00 & 1.0270 \mathrm{E}+00 & 1.0141 \mathrm{E}+00 \\ -23.07 & 1.1022 \mathrm{E}+00 & 1.1090 \mathrm{E}+00 & 1.0899 \mathrm{E}+00 \\ -17.94 & 1.1632 \mathrm{E}+00 & 1.1718 \mathrm{E}+00 & 1.1493 \mathrm{E}+00 \\ -12.82 & 1.2058 \mathrm{E}+00 & 1.2148 \mathrm{E}+00 & 1.1753 \mathrm{E}+00 \\ -7.69 & 1.2301 \mathrm{E}+00 & 1.2394 \mathrm{E}+00 & 1.1986 \mathrm{E}+00 \\ -2.56 & 1.2358 \mathrm{E}+00 & 1.2453 \mathrm{E}+00 & 1.2039 \mathrm{E}+00 \\ 2.56 & 1.2227 \mathrm{E}+00 & 1.2327 \mathrm{E}+00 & 1.1959 \mathrm{E}+00 \\ 7.69 & 1.1911 \mathrm{E}+00 & 1.2014 \mathrm{E}+00 & 1.1650 \mathrm{E}+00 \\ 12.82 & 1.1419 \mathrm{E}+00 & 1.1525 \mathrm{E}+00 & 1.1169 \mathrm{E}+00 \\ 17.94 & 1.0764 \mathrm{E}+00 & 1.0874 \mathrm{E}+00 & 1.0744 \mathrm{E}+00 \\ 23.07 & 9.9581 \mathrm{E}-01 & 1.0057 \mathrm{E}+00 & 9.9450 \mathrm{E}-01 \\ 28.20 & 9.0218 \mathrm{E}-01 & 9.0928 \mathrm{E}-01 & 9.0211 \mathrm{E}-01 \\ 33.32 & 7.9793 \mathrm{E}-01 & 7.9978 \mathrm{E}-01 & 8.2523 \mathrm{E}-01 \\ 38.45 & 6.8509 \mathrm{E}-01 & 6.7571 \mathrm{E}-01 & 7.1542 \mathrm{E}-01 \\ 43.58 & 5.6906 \mathrm{E}-01 & 5.3826 \mathrm{E}-01 & 6.1613 \mathrm{E}-01 \\ 47.17 & 4.9291 \mathrm{E}-01 & 4.3315 \mathrm{E}-01 & 7.3489 \mathrm{E}-02 \\ 50.62 & 4.2255 \mathrm{E}-01 & 3.4274 \mathrm{E}-01 & 5.7388 \mathrm{E}-02 \\ 55.49 & 3.3604 \mathrm{E}-01 & 2.4865 \mathrm{E}-01 & 4.2735 \mathrm{E}-02 \\ 60.35 & 2.5947 \mathrm{E}-01 & 1.7593 \mathrm{E}-01 & 3.2270 \mathrm{E}-02 \\ 64.98 & 2.0964 \mathrm{E}-01 & 1.3209 \mathrm{E}-01 & 0.0000 \mathrm{E}+00 \\ 69.39 & 1.7905 \mathrm{E}-01 & 1.0807 \mathrm{E}-01 & 0.0000 \mathrm{E}+00 \\ 76.46 & 1.3533 \mathrm{E}-01 & 7.5891 \mathrm{E}-02 & 0.0000 \mathrm{E}+00 \\ 86.80 & 8.7881 \mathrm{E}-02 & 4.5290 \mathrm{E}-02 & 0.0000 \mathrm{E}+00 \\ 98.14 & 5.0528 \mathrm{E}-02 & 2.4533 \mathrm{E}-02 & 0.0000 \mathrm{E}+00 \\ 110.00 & 2.1463 \mathrm{E}-02 & 1.0855 \mathrm{E}-02 & 0.0000 \mathrm{E}+00\end{array}$


Table BlOC-3.4. Axial Distribution of Total Flux, Fast Flux and Power in MFA-2 at BOC $10 \mathrm{C}-3$

$\begin{array}{rccc}\text { Z }(\mathrm{cm}) & \text { Total Flux } & \text { Flux }>0.1 \text { MeV } & \text { Power } \\ -97.78 & 2.4791 \mathrm{E}-02 & 1.2691 \mathrm{E}-02 & 0.0000 \mathrm{E}+00 \\ -92.78 & 5.3209 \mathrm{E}-02 & 2.4498 \mathrm{E}-02 & 0.0000 \mathrm{E}+00 \\ -87.78 & 8.5306 \mathrm{E}-02 & 3.9214 \mathrm{E}-02 & 0.0000 \mathrm{E}+00 \\ -82.78 & 1.2366 \mathrm{E}-01 & 5.8578 \mathrm{E}-02 & 0.0000 \mathrm{E}+00 \\ -77.78 & 1.7116 \mathrm{E}-01 & 8.4808 \mathrm{E}-02 & 0.0000 \mathrm{E}+00 \\ -72.15 & 2.2631 \mathrm{E}-01 & 1.1926 \mathrm{E}-01 & 0.0000 \mathrm{E}+00 \\ -65.90 & 2.8418 \mathrm{E}-01 & 1.6428 \mathrm{E}-01 & 0.0000 \mathrm{E}+00 \\ -60.35 & 3.4741 \mathrm{E}-01 & 2.2687 \mathrm{E}-01 & 5.7998 \mathrm{E}-02 \\ -55.49 & 4.2994 \mathrm{E}-01 & 3.1062 \mathrm{E}-01 & 6.9790 \mathrm{E}-02 \\ -50.62 & 5.2599 \mathrm{E}-01 & 4.2061 \mathrm{E}-01 & 8.6494 \mathrm{E}-02 \\ -47.17 & 6.0367 \mathrm{E}-01 & 5.2495 \mathrm{E}-01 & 1.0476 \mathrm{E}-01 \\ -43.58 & 6.8580 \mathrm{E}-01 & 6.4345 \mathrm{E}-01 & 7.4151 \mathrm{E}-01 \\ -38.45 & 8.0513 \mathrm{E}-01 & 7.8861 \mathrm{E}-01 & 8.3394 \mathrm{E}-01 \\ -33.32 & 9.1588 \mathrm{E}-01 & 9.1275 \mathrm{E}-01 & 9.3761 \mathrm{E}-01 \\ -28.20 & 1.0124 \mathrm{E}+00 & 1.0157 \mathrm{E}+00 & 1.0030 \mathrm{E}+00 \\ -23.07 & 1.0930 \mathrm{E}+00 & 1.0998 \mathrm{E}+00 & 1.0812 \mathrm{E}+00 \\ -17.94 & 1.1560 \mathrm{E}+00 & 1.1644 \mathrm{E}+00 & 1.1426 \mathrm{E}+00 \\ -12.82 & 1.2005 \mathrm{E}+00 & 1.2092 \mathrm{E}+00 & 1.1689 \mathrm{E}+00 \\ -7.69 & 1.2267 \mathrm{E}+00 & 1.2356 \mathrm{E}+00 & 1.1941 \mathrm{E}+00 \\ -2.56 & 1.2345 \mathrm{E}+00 & 1.2436 \mathrm{E}+00 & 1.2015 \mathrm{E}+00 \\ 2.56 & 1.2239 \mathrm{E}+00 & 1.2334 \mathrm{E}+00 & 1.1956 \mathrm{E}+00 \\ 7.69 & 1.1951 \mathrm{E}+00 & 1.2050 \mathrm{E}+00 & 1.1676 \mathrm{E}+00 \\ 12.82 & 1.1490 \mathrm{E}+00 & 1.1591 \mathrm{E}+00 & 1.1227 \mathrm{E}+00 \\ 17.94 & 1.0866 \mathrm{E}+00 & 1.0969 \mathrm{E}+00 & 1.0845 \mathrm{E}+00 \\ 23.07 & 1.0083 \mathrm{E}+00 & 1.0176 \mathrm{E}+00 & 1.0070 \mathrm{E}+00 \\ 28.20 & 9.1604 \mathrm{E}-01 & 9.2255 \mathrm{E}-01 & 9.1607 \mathrm{E}-01 \\ 33.32 & 8.1207 \mathrm{E}-01 & 8.1332 \mathrm{E}-01 & 8.4149 \mathrm{E}-01 \\ 38.45 & 6.9850 \mathrm{E}-01 & 6.8880 \mathrm{E}-01 & 7.3088 \mathrm{E}-01 \\ 43.58 & 5.8061 \mathrm{E}-01 & 5.5009 \mathrm{E}-01 & 6.2976 \mathrm{E}-01 \\ 47.17 & 5.0108 \mathrm{E}-01 & 4.4215 \mathrm{E}-01 & 7.8219 \mathrm{E}-02 \\ 50.62 & 4.2625 \mathrm{E}-01 & 3.4758 \mathrm{E}-01 & 6.1101 \mathrm{E}-02 \\ 55.49 & 3.3414 \mathrm{E}-01 & 2.4859 \mathrm{E}-01 & 4.5320 \mathrm{E}-02 \\ 60.35 & 2.5402 \mathrm{E}-01 & 1.7291 \mathrm{E}-01 & 3.4042 \mathrm{E}-02 \\ 64.98 & 2.0421 \mathrm{E}-01 & 1.2893 \mathrm{E}-01 & 0.0000 \mathrm{E}+00 \\ 69.39 & 1.7458 \mathrm{E}-01 & 1.0561 \mathrm{E}-01 & 0.0000 \mathrm{E}+00 \\ 76.46 & 1.3234 \mathrm{E}-01 & 7.4433 \mathrm{E}-02 & 0.0000 \mathrm{E}+00 \\ 86.80 & 8.6110 \mathrm{E}-02 & 4.4495 \mathrm{E}-02 & 0.0000 \mathrm{E}+00 \\ 98.14 & 4.9536 \mathrm{E}-02 & 2.4093 \mathrm{E}-02 & 0.0000 \mathrm{E}+00 \\ 110.00 & 2.1013 \mathrm{E}-02 & 1.0647 \mathrm{E}-02 & 0.0000 \mathrm{E}+00 \\ & & & \end{array}$




\section{HNF-SD-FF-ANAL-009 Rev. 1}

Table B10C-3.5. Fission Power Distribution by Pin in MFA-1 at BOC $10 \mathrm{C}-3$ CORE POSITION 1506

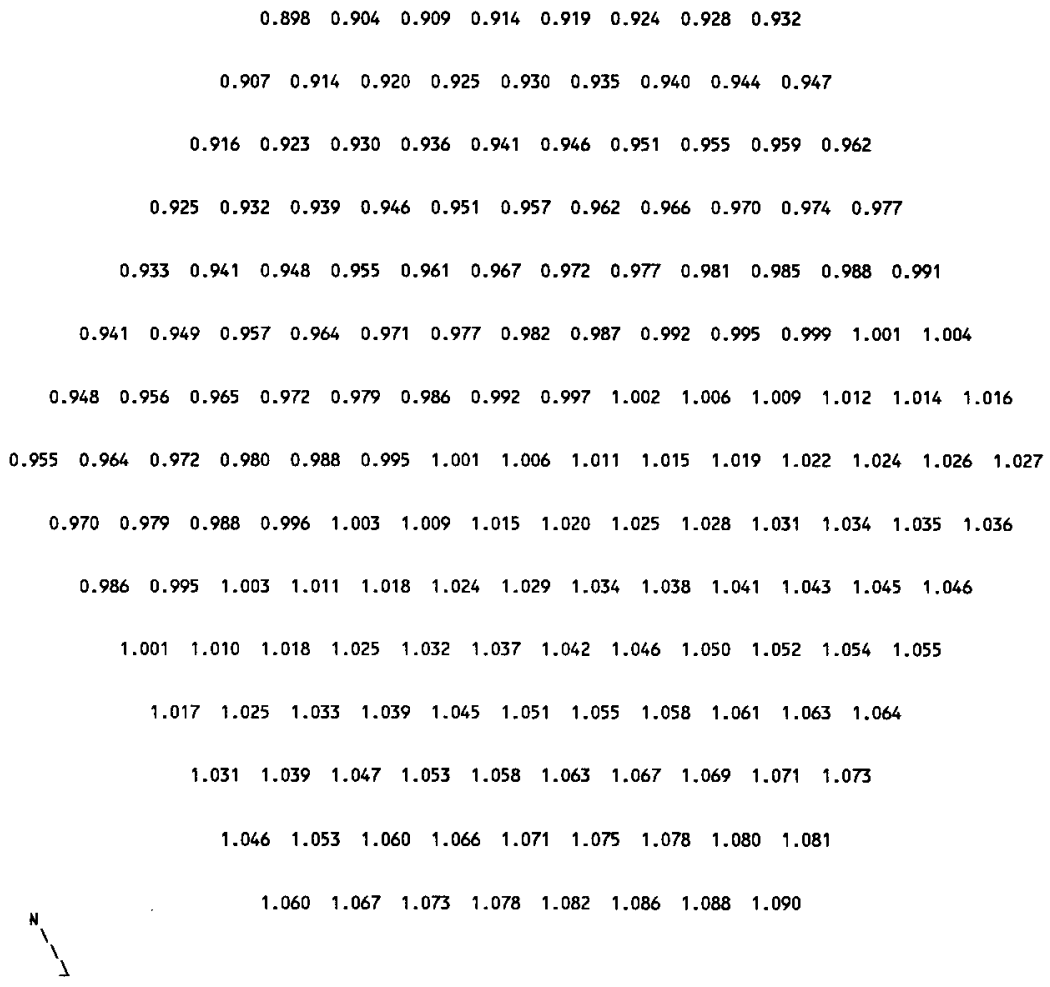


HNF-SD-FF-ANAL-009 Rev. 1

Table B10C-3.6. Fission Power Distribution by Pin in MFA-2 at BOC 10C-3 CORE POSITION 2507

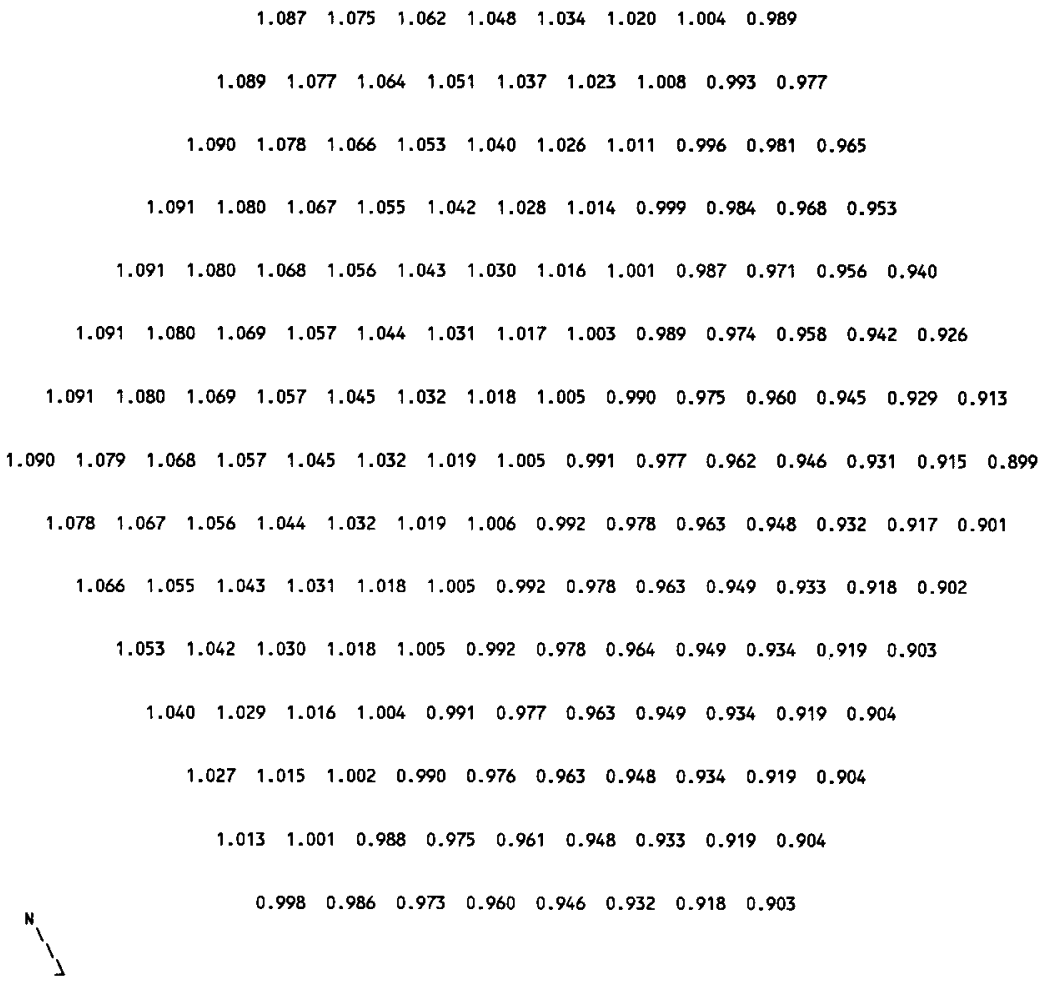




\section{HNF-SD-FF-ANAL-009 Rev. 1}

Table B10C-3.7. Fast Flux Distribution by $P$ in in MFA-1 at BOC $10 C-3$ CORE POSITION 1506

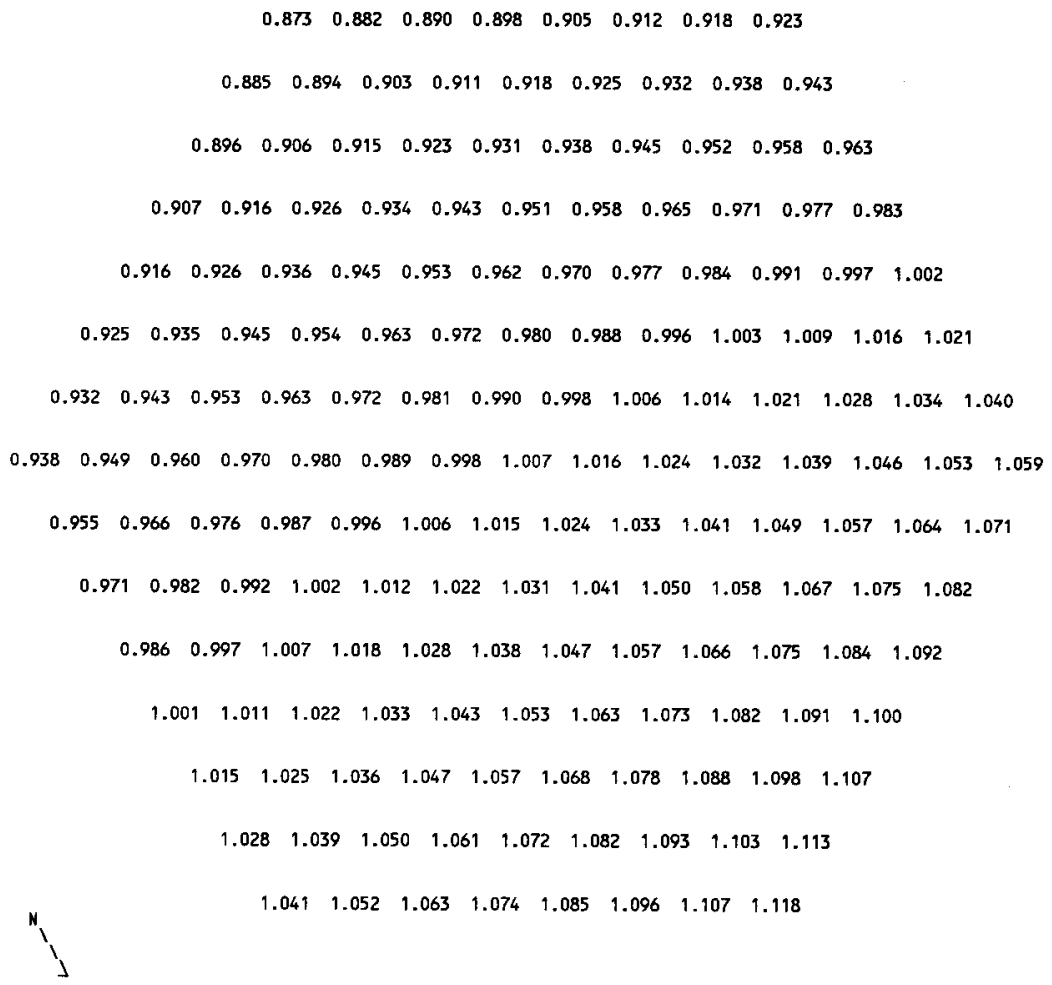


HNF-SD-FF-ANAL-009 Rev. 1

Table B10C-3.8. Fast Flux Distribution by Pin in MFA-2 at BOC $10 \mathrm{C}-3$

CORE POSITION 2507

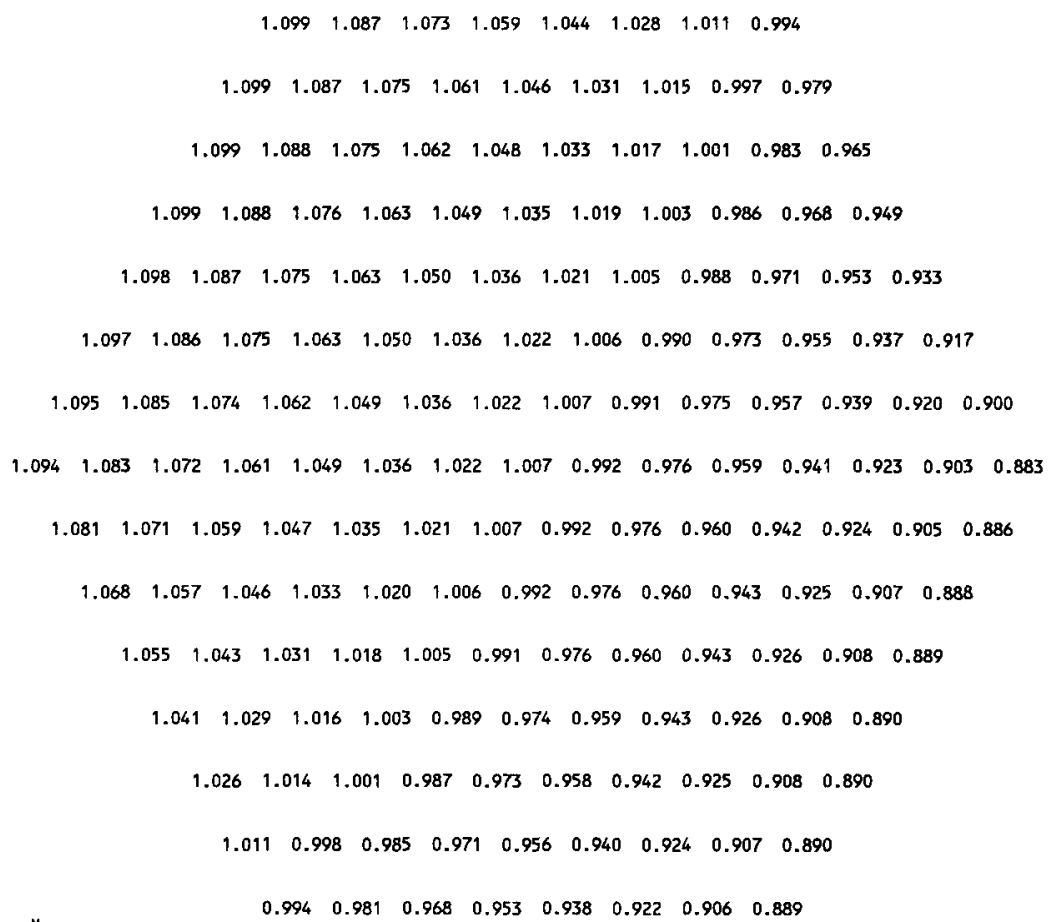


Table B10C-3.9. MFA-1 and MFA-2 Duct Wall

Fast Flux Data at BOC $10 \mathrm{C}-3$

$\begin{array}{ccc}\text { Side } & \begin{array}{c}\text { Flux }>0.1 \\ \text { MFA-1 }\end{array} & \begin{array}{c}\text { MeV } \\ \left(\mathrm{n} / \mathrm{cm}^{2}-\mathrm{sec}\right) \\ \text { MFA-2 }\end{array} \\ \text { E } & 1.7917 \mathrm{E}+15 & 2.1335 \mathrm{E}+15 \\ \text { SE } & 1.6133 \mathrm{E}+15 & 2.2550 \mathrm{E}+15 \\ \text { SW } & 1.5874 \mathrm{E}+15 & 2.1435 \mathrm{E}+15 \\ \text { W } & 1.7839 \mathrm{E}+15 & 1.8688 \mathrm{E}+15 \\ \text { NW } & 2.0169 \mathrm{E}+15 & 1.7453 \mathrm{E}+15 \\ \text { NE } & 1.9766 \mathrm{E}+15 & 1.8824 \mathrm{E}+15\end{array}$

Table E10C-3.1. Fission Power Generated in MFA-1, MFA-2 and Neighboring Assemblies at EOC $10 C-3$

\begin{tabular}{lcccc} 
CORE & $* * * * * *$ & \multicolumn{2}{c}{ POWER IN MEGAWATTS } & $* * * * * *$ \\
POS. & BELOW CORE & IN CORE & ABOVE CORE & TOTAL PWR \\
1506 & $5.119 \mathrm{E}-02$ & $3.480 \mathrm{E}+00$ & $3.371 \mathrm{E}-02$ & $3.565 \mathrm{E}+00$ \\
1404 & $1.329 \mathrm{E}-02$ & $3.613 \mathrm{E}+00$ & $9.387 \mathrm{E}-03$ & $3.635 \mathrm{E}+00$ \\
1405 & $0.000 \mathrm{E}+00$ & $6.171 \mathrm{E}+00$ & $0.000 \mathrm{E}+00$ & $6.171 \mathrm{E}+00$ \\
1505 & $1.183 \mathrm{E}-02$ & $3.291 \mathrm{E}+00$ & $7.814 \mathrm{E}-03$ & $3.311 \mathrm{E}+00$ \\
1507 & $8.538 \mathrm{E}-03$ & $3.313 \mathrm{E}+00$ & $6.384 \mathrm{E}-03$ & $3.328 \mathrm{E}+00$ \\
1606 & $8.118 \mathrm{E}-03$ & $3.138 \mathrm{E}+00$ & $6.068 \mathrm{E}-03$ & $3.152 \mathrm{E}+00$ \\
1607 & $1.017 \mathrm{E}-02$ & $2.914 \mathrm{E}+00$ & $7.331 \mathrm{E}-03$ & $2.931 \mathrm{E}+00$ \\
2507 & $5.497 \mathrm{E}-02$ & $3.800 \mathrm{E}+00$ & $3.920 \mathrm{E}-02$ & $3.894 \mathrm{E}+00$ \\
2405 & $1.429 \mathrm{E}-02$ & $3.831 \mathrm{E}+00$ & $1.090 \mathrm{E}-02$ & $3.856 \mathrm{E}+00$ \\
2506 & $1.015 \mathrm{E}-02$ & $4.447 \mathrm{E}+00$ & $8.101 \mathrm{E}-03$ & $4.465 \mathrm{E}+00$ \\
2508 & $1.438 \mathrm{E}-02$ & $4.023 \mathrm{E}+00$ & $1.017 \mathrm{E}-02$ & $4.048 \mathrm{E}+00$ \\
2607 & $1.042 \mathrm{E}-02$ & $3.537 \mathrm{E}+00$ & $8.402 \mathrm{E}-03$ & $3.556 \mathrm{E}+00$ \\
2608 & $7.225 \mathrm{E}-03$ & $3.260 \mathrm{E}+00$ & $6.171 \mathrm{E}-03$ & $3.273 \mathrm{E}+00$ \\
2609 & $8.099 \mathrm{E}-03$ & $3.910 \mathrm{E}+00$ & $6.786 \mathrm{E}-03$ & $3.925 \mathrm{E}+00$
\end{tabular}

Table E10C-3.2. Assembly Averaged Total and Fast Flux in MFA-1 and MFA-2 at EOC $10 \mathrm{C}-3$

\begin{tabular}{|c|c|c|c|}
\hline & Core & Flux & $\mathrm{cm}^{2}-\mathrm{sec}$ ) \\
\hline $\begin{array}{l}\text { Assembly } \\
\text { MFA-1 } \\
\text { MFA-2 }\end{array}$ & $\begin{array}{l}\text { Pos. } \\
1506 \\
2507\end{array}$ & $\begin{array}{c}\text { Total } \\
2.830 E+15 \\
3.119 E+15\end{array}$ & $\begin{array}{l}>0.1 \mathrm{MeV} \\
1.822 \mathrm{E}+15 \\
2.001 \mathrm{E}+15\end{array}$ \\
\hline
\end{tabular}


Table E10C-3.3. Axial Distribution of Total Flux, Fast Flux and Power in MFA-1 at EOC $10 \mathrm{C}-3$

\begin{tabular}{|c|c|c|c|}
\hline $\begin{array}{r}z(\mathrm{~cm}) \\
-97.78 \\
-92.78 \\
-87.78 \\
-82.78 \\
-77.78 \\
-72.15 \\
-65.90 \\
-60.35 \\
-55.49 \\
-50.62 \\
-47.17 \\
-43.58 \\
-38.45 \\
-33.32 \\
-28.20 \\
-23.07 \\
-17.94 \\
-12.82 \\
-7.69 \\
-2.56 \\
2.56 \\
7.69 \\
12.82 \\
17.94 \\
23.07 \\
28.20 \\
33.32 \\
38.45 \\
43.58 \\
47.17 \\
50.62 \\
55.49 \\
60.35 \\
64.98 \\
69.39 \\
76.46 \\
86.80 \\
98.14 \\
110.00\end{array}$ & $\begin{array}{l}\text { Total Flux } \\
2.6519 \mathrm{E}-02 \\
5.6962 \mathrm{E}-02 \\
9.1282 \mathrm{E}-02 \\
1.3213 \mathrm{E}-01 \\
1.8239 \mathrm{E}-01 \\
2.4017 \mathrm{E}-01 \\
2.9897 \mathrm{E}-01 \\
3.6048 \mathrm{E}-01 \\
4.4056 \mathrm{E}-01 \\
5.3266 \mathrm{E}-01 \\
6.0613 \mathrm{E}-01 \\
6.8338 \mathrm{E}-01 \\
7.9680 \mathrm{E}-01 \\
9.0281 \mathrm{E}-01 \\
9.9613 \mathrm{E}-01 \\
1.0752 \mathrm{E}+00 \\
1.1385 \mathrm{E}+00 \\
1.1849 \mathrm{E}+00 \\
1.2145 \mathrm{E}+00 \\
1.2269 \mathrm{E}+00 \\
1.2219 \mathrm{E}+00 \\
1.1991 \mathrm{E}+00 \\
1.1586 \mathrm{E}+00 \\
1.1009 \mathrm{E}+00 \\
1.0258 \mathrm{E}+00 \\
9.3466 \mathrm{E}-01 \\
8.3014 \mathrm{E}-01 \\
7.1481 \mathrm{E}-01 \\
5.9500 \mathrm{E}-01 \\
5.1600 \mathrm{E}-01 \\
4.4289 \mathrm{E}-01 \\
3.5263 \mathrm{E}-01 \\
2.7256 \mathrm{E}-01 \\
2.2047 \mathrm{E}-01 \\
1.8840 \mathrm{E}-01 \\
1.4250 \mathrm{E}-01 \\
9.2544 \mathrm{E}-02 \\
5.3175 \mathrm{E}-02 \\
2.2531 \mathrm{E}-02\end{array}$ & $\begin{array}{c}\text { Flux }>0.1 \mathrm{MeV} \\
1.3443 \mathrm{E}-02 \\
2.5933 \mathrm{E}-02 \\
4.1441 \mathrm{E}-02 \\
6.1721 \mathrm{E}-02 \\
8.8979 \mathrm{E}-02 \\
1.2444 \mathrm{E}-01 \\
1.6987 \mathrm{E}-01 \\
2.3190 \mathrm{E}-01 \\
3.1416 \mathrm{E}-01 \\
4.2186 \mathrm{E}-01 \\
5.2400 \mathrm{E}-01 \\
6.3984 \mathrm{E}-01 \\
7.8015 \mathrm{E}-01 \\
8.9972 \mathrm{E}-01 \\
9.9937 \mathrm{E}-01 \\
1.0819 \mathrm{E}+00 \\
1.1467 \mathrm{E}+00 \\
1.1934 \mathrm{E}+00 \\
1.2233 \mathrm{E}+00 \\
1.2360 \mathrm{E}+00 \\
1.2315 \mathrm{E}+00 \\
1.2091 \mathrm{E}+00 \\
1.1691 \mathrm{E}+00 \\
1.1119 \mathrm{E}+00 \\
1.0358 \mathrm{E}+00 \\
9.4191 \mathrm{E}-01 \\
8.3216 \mathrm{E}-01 \\
7.0519 \mathrm{E}-01 \\
5.6298 \mathrm{E}-01 \\
4.5370 \mathrm{E}-01 \\
3.5957 \mathrm{E}-01 \\
2.6128 \mathrm{E}-01 \\
1.8511 \mathrm{E}-01 \\
1.3908 \mathrm{E}-01 \\
1.1384 \mathrm{E}-01 \\
7.9997 \mathrm{E}-02 \\
4.7759 \mathrm{E}-02 \\
2.5861 \mathrm{E}-02 \\
1.1422 \mathrm{E}-02\end{array}$ & $\begin{array}{c}\text { Power } \\
0.0000 \mathrm{E}+00 \\
0.0000 \mathrm{E}+00 \\
0.0000 \mathrm{E}+00 \\
0.0000 \mathrm{E}+00 \\
0.0000 \mathrm{E}+00 \\
0.0000 \mathrm{E}+00 \\
0.0000 \mathrm{E}+00 \\
6.4695 \mathrm{E}-02 \\
7.6158 \mathrm{E}-02 \\
9.2128 \mathrm{E}-02 \\
1.0948 \mathrm{E}-01 \\
7.4132 \mathrm{E}-01 \\
8.2585 \mathrm{E}-01 \\
9.2417 \mathrm{E}-01 \\
9.8552 \mathrm{E}-01 \\
1.0620 \mathrm{E}+00 \\
1.1235 \mathrm{E}+00 \\
1.1517 \mathrm{E}+00 \\
1.1801 \mathrm{E}+00 \\
1.1921 \mathrm{E}+00 \\
1.1925 \mathrm{E}+00 \\
1.1703 \mathrm{E}+00 \\
1.1311 \mathrm{E}+00 \\
1.0997 \mathrm{E}+00 \\
1.0252 \mathrm{E}+00 \\
9.3526 \mathrm{E}-01 \\
8.6256 \mathrm{E}-01 \\
7.4994 \mathrm{E}-01 \\
6.4728 \mathrm{E}-01 \\
8.1158 \mathrm{E}-02 \\
6.4155 \mathrm{E}-02 \\
4.8439 \mathrm{E}-02 \\
3.6995 \mathrm{E}-02 \\
0.0000 \mathrm{E}+00 \\
0.0000 \mathrm{E}+00 \\
0.0000 \mathrm{E}+00 \\
0.0000 \mathrm{E}+00 \\
0.0000 \mathrm{E}+00 \\
0.0000 \mathrm{E}+00\end{array}$ \\
\hline
\end{tabular}


Table E10C-3.4. Axial Distribution of Total Flux, Fast Flux and Power in MFA-2 at EOC $10 \mathrm{C}-3$

$\begin{array}{rccc}z(c m) & \text { Total Flux } & \text { Flux }>\text { O.1 MeV } & \text { Power } \\ -97.78 & 2.4241 \mathrm{E}-02 & 1.2457 \mathrm{E}-02 & 0.0000 \mathrm{E}+00 \\ -92.78 & 5.2030 \mathrm{E}-02 & 2.4043 \mathrm{E}-02 & 0.0000 \mathrm{E}+00 \\ -87.78 & 8.3411 \mathrm{E}-02 & 3.8477 \mathrm{E}-02 & 0.0000 \mathrm{E}+00 \\ -82.78 & 1.2090 \mathrm{E}-01 & 5.7460 \mathrm{E}-02 & 0.0000 \mathrm{E}+00 \\ -77.78 & 1.6732 \mathrm{E}-01 & 8.3172 \mathrm{E}-02 & 0.0000 \mathrm{E}+00 \\ -72.15 & 2.2120 \mathrm{E}-01 & 1.1692 \mathrm{E}-01 & 0.0000 \mathrm{E}+00 \\ -65.90 & 2.7764 \mathrm{E}-01 & 1.6100 \mathrm{E}-01 & 0.0000 \mathrm{E}+00 \\ -60.35 & 3.3915 \mathrm{E}-01 & 2.2233 \mathrm{E}-01 & 6.2613 \mathrm{E}-02 \\ -55.49 & 4.1953 \mathrm{E}-01 & 3.0407 \mathrm{E}-01 & 7.4612 \mathrm{E}-02 \\ -50.62 & 5.1304 \mathrm{E}-01 & 4.1119 \mathrm{E}-01 & 9.1268 \mathrm{E}-02 \\ -47.17 & 5.8860 \mathrm{E}-01 & 5.1263 \mathrm{E}-01 & 1.0920 \mathrm{E}-01 \\ -43.58 & 6.6848 \mathrm{E}-01 & 6.2781 \mathrm{E}-01 & 7.2482 \mathrm{E}-01 \\ -38.45 & 7.8512 \mathrm{E}-01 & 7.6946 \mathrm{E}-01 & 8.1562 \mathrm{E}-01 \\ -33.32 & 8.9396 \mathrm{E}-01 & 8.9123 \mathrm{E}-01 & 9.1789 \mathrm{E}-01 \\ -28.20 & 9.8965 \mathrm{E}-01 & 9.9299 \mathrm{E}-01 & 9.7947 \mathrm{E}-01 \\ -23.07 & 1.0708 \mathrm{E}+00 & 1.0772 \mathrm{E}+00 & 1.0581 \mathrm{E}+00 \\ -17.94 & 1.1356 \mathrm{E}+00 & 1.1435 \mathrm{E}+00 & 1.1212 \mathrm{E}+00 \\ -12.82 & 1.1833 \mathrm{E}+00 & 1.1913 \mathrm{E}+00 & 1.1487 \mathrm{E}+00 \\ -7.69 & 1.2139 \mathrm{E}+00 & 1.2222 \mathrm{E}+00 & 1.1782 \mathrm{E}+00 \\ -2.56 & 1.2273 \mathrm{E}+00 & 1.2359 \mathrm{E}+00 & 1.1911 \mathrm{E}+00 \\ 2.56 & 1.2232 \mathrm{E}+00 & 1.2322 \mathrm{E}+00 & 1.1919 \mathrm{E}+00 \\ 7.69 & 1.2012 \mathrm{E}+00 & 1.2108 \mathrm{E}+00 & 1.1707 \mathrm{E}+00 \\ 12.82 & 1.1618 \mathrm{E}+00 & 1.1718 \mathrm{E}+00 & 1.1325 \mathrm{E}+00 \\ 17.94 & 1.1053 \mathrm{E}+00 & 1.1159 \mathrm{E}+00 & 1.1035 \mathrm{E}+00 \\ 23.07 & 1.0315 \mathrm{E}+00 & 1.0412 \mathrm{E}+00 & 1.0306 \mathrm{E}+00 \\ 28.20 & 9.4191 \mathrm{E}-01 & 9.4887 \mathrm{E}-01 & 9.4229 \mathrm{E}-01 \\ 33.32 & 8.3878 \mathrm{E}-01 & 8.4048 \mathrm{E}-01 & 8.7337 \mathrm{E}-01 \\ 38.45 & 7.2420 \mathrm{E}-01 & 7.1452 \mathrm{E}-01 & 7.6154 \mathrm{E}-01 \\ 43.58 & 6.0400 \mathrm{E}-01 & 5.7258 \mathrm{E}-01 & 6.5854 \mathrm{E}-01 \\ 47.17 & 5.2241 \mathrm{E}-01 & 4.6127 \mathrm{E}-01 & 8.6415 \mathrm{E}-02 \\ 50.62 & 4.4543 \mathrm{E}-01 & 3.6360 \mathrm{E}-01 & 6.8451 \mathrm{E}-02 \\ 55.49 & 3.5019 \mathrm{E}-01 & 2.6088 \mathrm{E}-01 & 5.1599 \mathrm{E}-02 \\ 60.35 & 2.6699 \mathrm{E}-01 & 1.8202 \mathrm{E}-01 & 3.9296 \mathrm{E}-02 \\ 64.98 & 2.1523 \mathrm{E}-01 & 1.3601 \mathrm{E}-01 & 0.0000 \mathrm{E}+00 \\ 69.39 & 1.8428 \mathrm{E}-01 & 1.1156 \mathrm{E}-01 & 0.0000 \mathrm{E}+00 \\ 76.46 & 1.3998 \mathrm{E}-01 & 7.8790 \mathrm{E}-02 & 0.0000 \mathrm{E}+00 \\ 86.80 & 9.1232 \mathrm{E}-02 & 4.7187 \mathrm{E}-02 & 0.0000 \mathrm{E}+00 \\ 98.14 & 5.2522 \mathrm{E}-02 & 2.5579 \mathrm{E}-02 & 0.0000 \mathrm{E}+00 \\ 110.00 & 2.2270 \mathrm{E}-02 & 1.1304 \mathrm{E}-02 & 0.0000 \mathrm{E}+00\end{array}$




\section{HNF-SD-FF-ANAL-009 Rev, I}

Table E1OC-3.5. Fission Power Distribution by Pin in MFA-1 at EOC $10 \mathrm{C}-3$ CORE POSIIION 1506

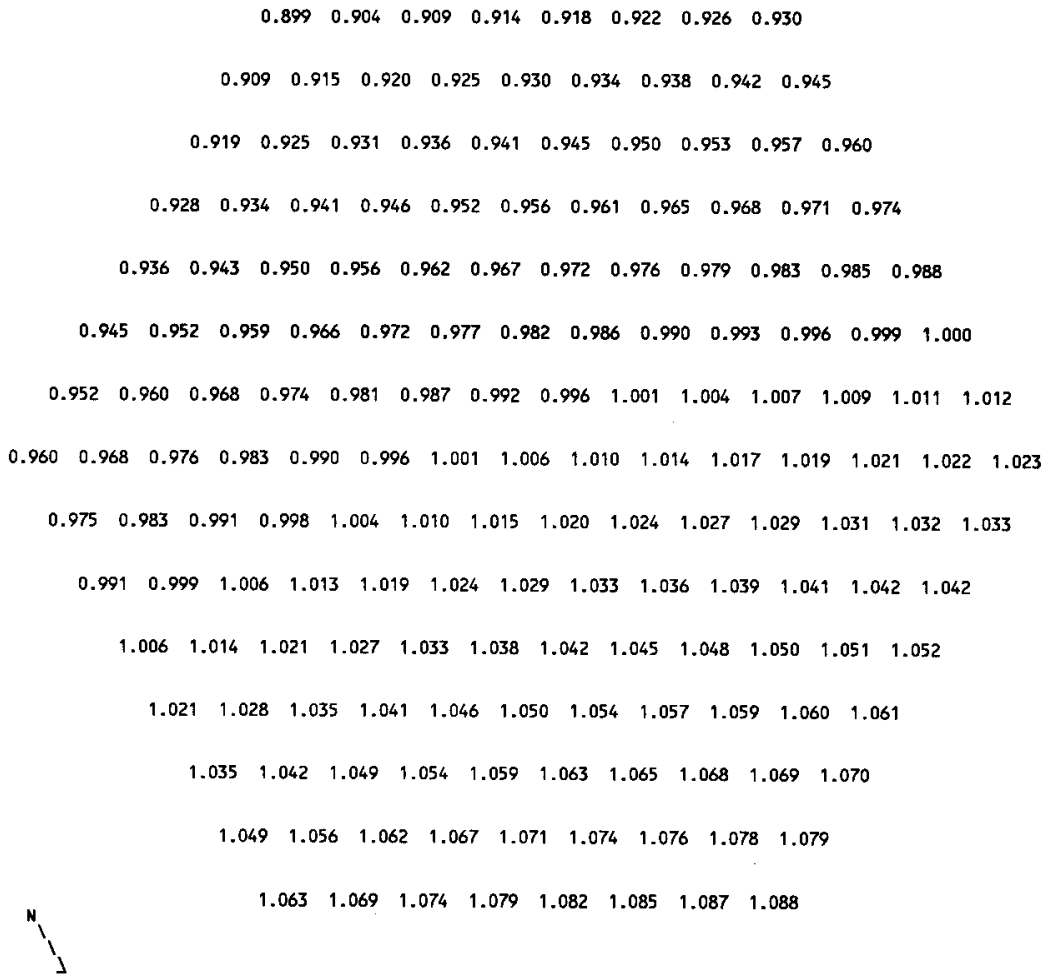


HNF-SD-FF-ANAL-009 Rev. 1

Table E1OC-3.6. Fission Power Distribution by Pin in MFA-2 at EOC 10C-3 CORE POSITION 2507

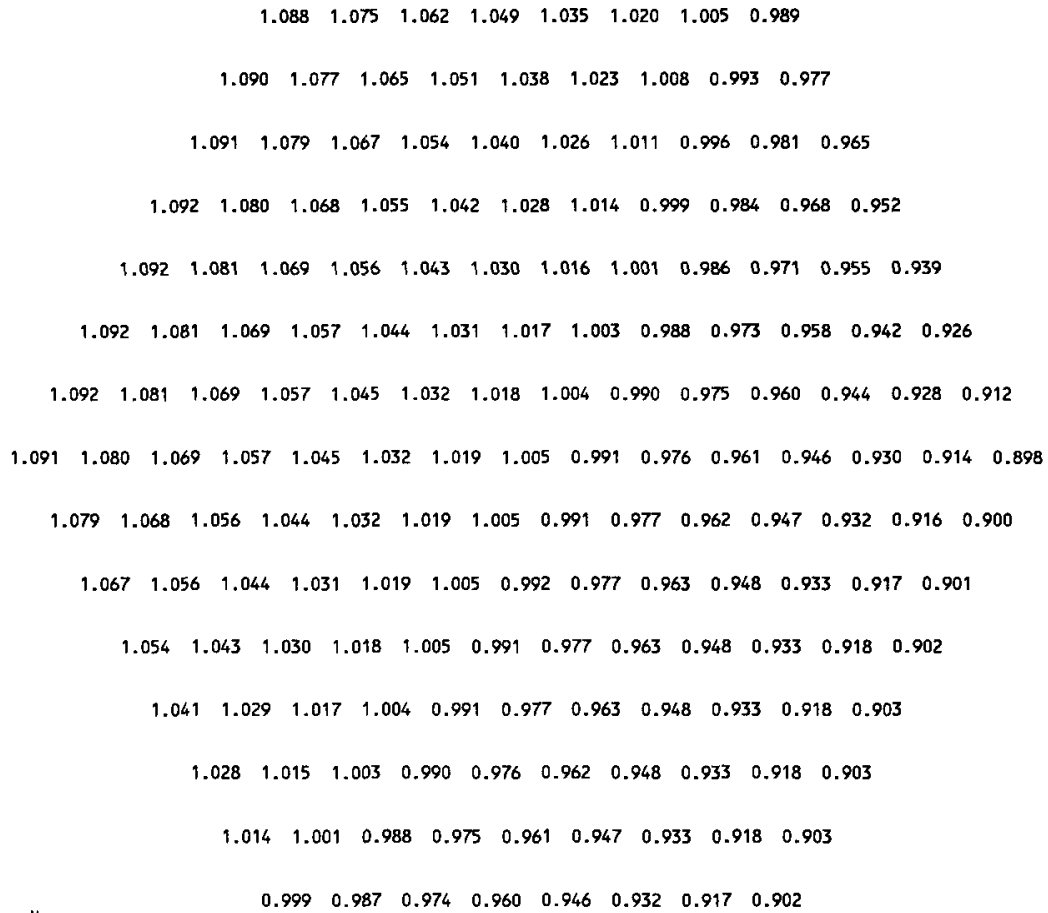


HNF-SD-FF-ANAL-009 Rev. 1

Table E1OC-3.7. Fast Flux Distribution by Pin in MFA-1 at EOC 10C-3

CORE POSIIION 1506

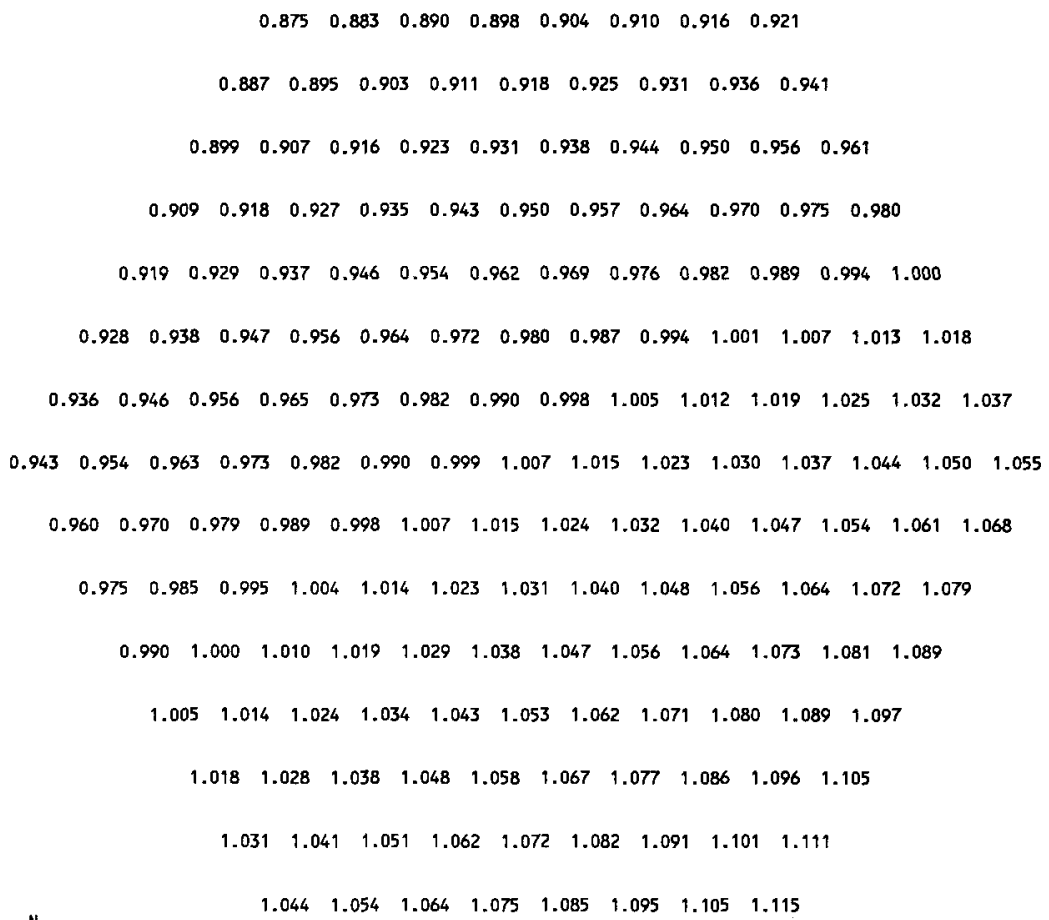


HNF-SD-FF-ANAL-009 Rev. 1

Table ElOC-3.8. Fast Flux Distribution by Pin in MFA-2 at EOC $10 \mathrm{C}-3$

CORE POSITION 2507

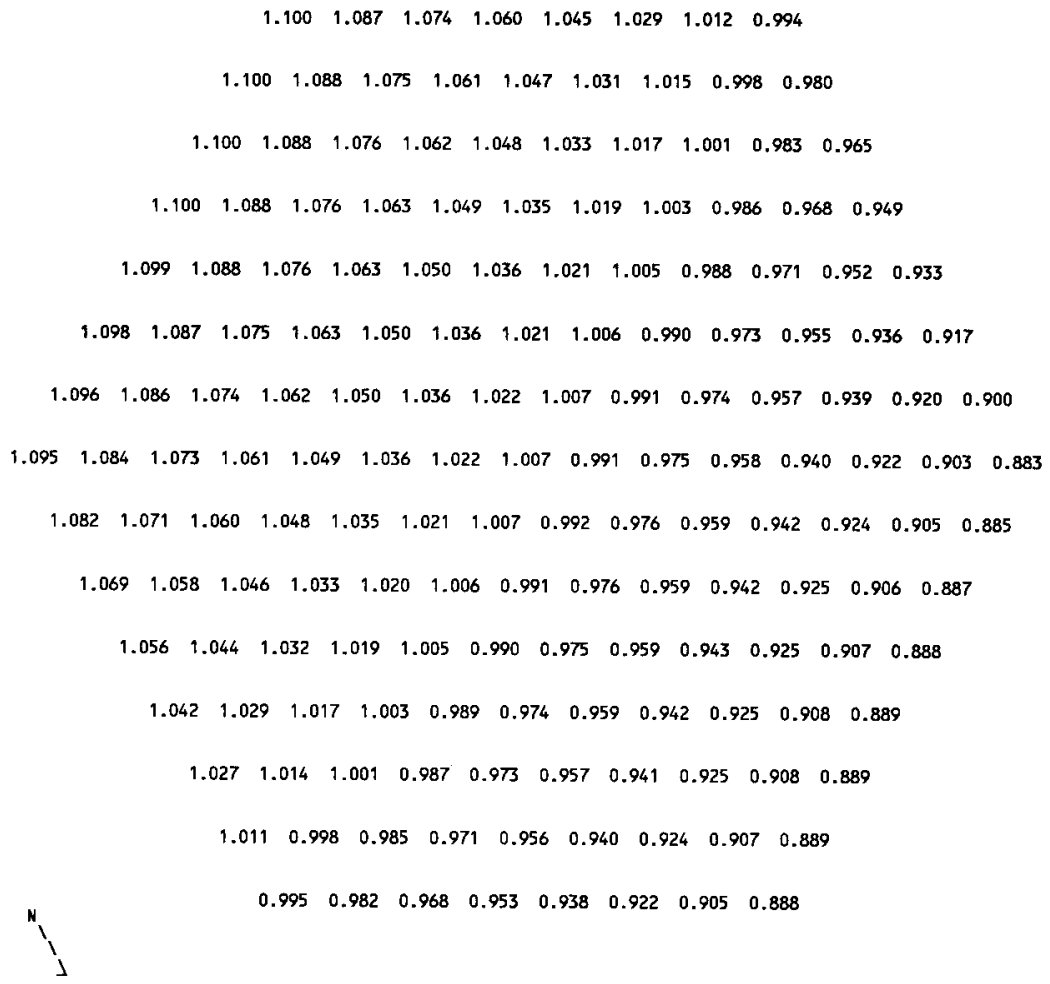


Table E10C-3.9. MFA-1 and MFA-2 Duct Wal1

Fast Flux Data at EOC $10 \mathrm{C}-3$

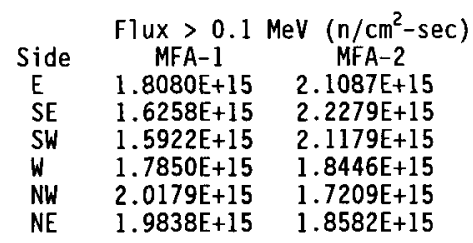

Table ElOC-3.10. Assembly Outlet Temperatures and Flow Rates at EOC 10C-3

$\begin{array}{lcl}\text { CALCULATED } & \\ \text { CORE } & \text { OUTLET TEMP. } & \begin{array}{l}\text { FLOW RATE } \\ \text { (LB } / H)\end{array} \\ \text { POS. } & \text { (DEG F) } & \begin{array}{l}\text { (DE) } \\ 1506\end{array} \\ 1001 & 114180 \\ 1404 & 873 & 206750 \\ 1405 & 1018 & 186110 \\ 1505 & 861 & 196600 \\ 1507 & 860 & 196600 \\ 1606 & 877 & 169550 \\ 1607 & 870 & 162600 \\ 2507 & 1045 & 114180 \\ 2405 & 891 & 206770 \\ 2506 & 920 & 206750 \\ 2508 & 898 & 196600 \\ 2607 & 921 & 162600 \\ 2608 & 897 & 169550 \\ 2609 & 945 & 162600\end{array}$

Assembly flows are based on a calculated core pressure drop of 113.3 psi at a total reactor flow rate of 16. $67 \mathrm{E}+06 \mathrm{lb} / \mathrm{hr}$. 
Table ElOC-3.11. MFA-1 Sodium Subchannel Temperatures at Top of Core Elevation for EOC $10 \mathrm{C}-3$
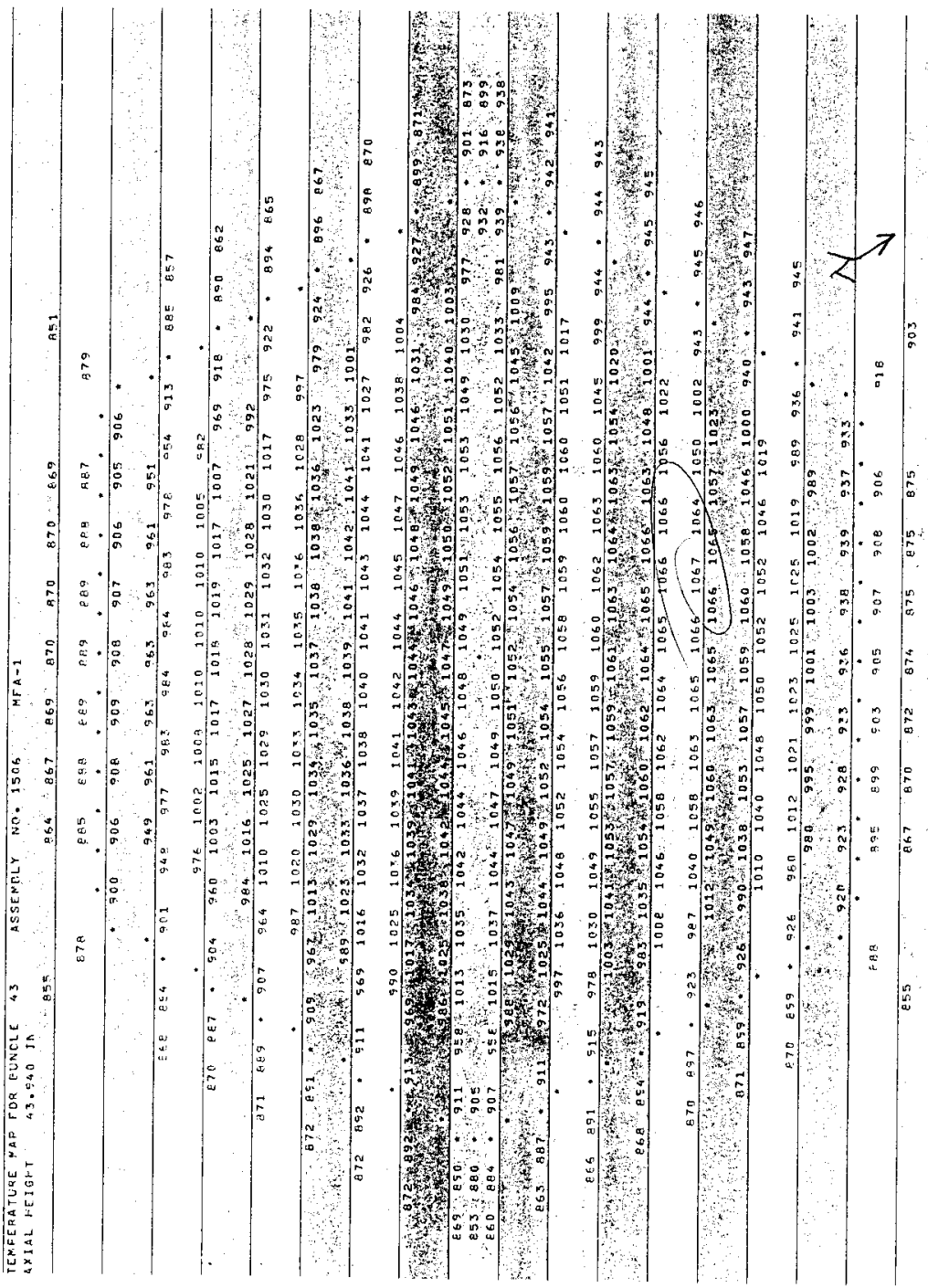
Table E1OC-3.12. MFA-2 Sodium Subchannel Temperatures at Top of Core Elevation for EOC $10 \mathrm{C}-3$
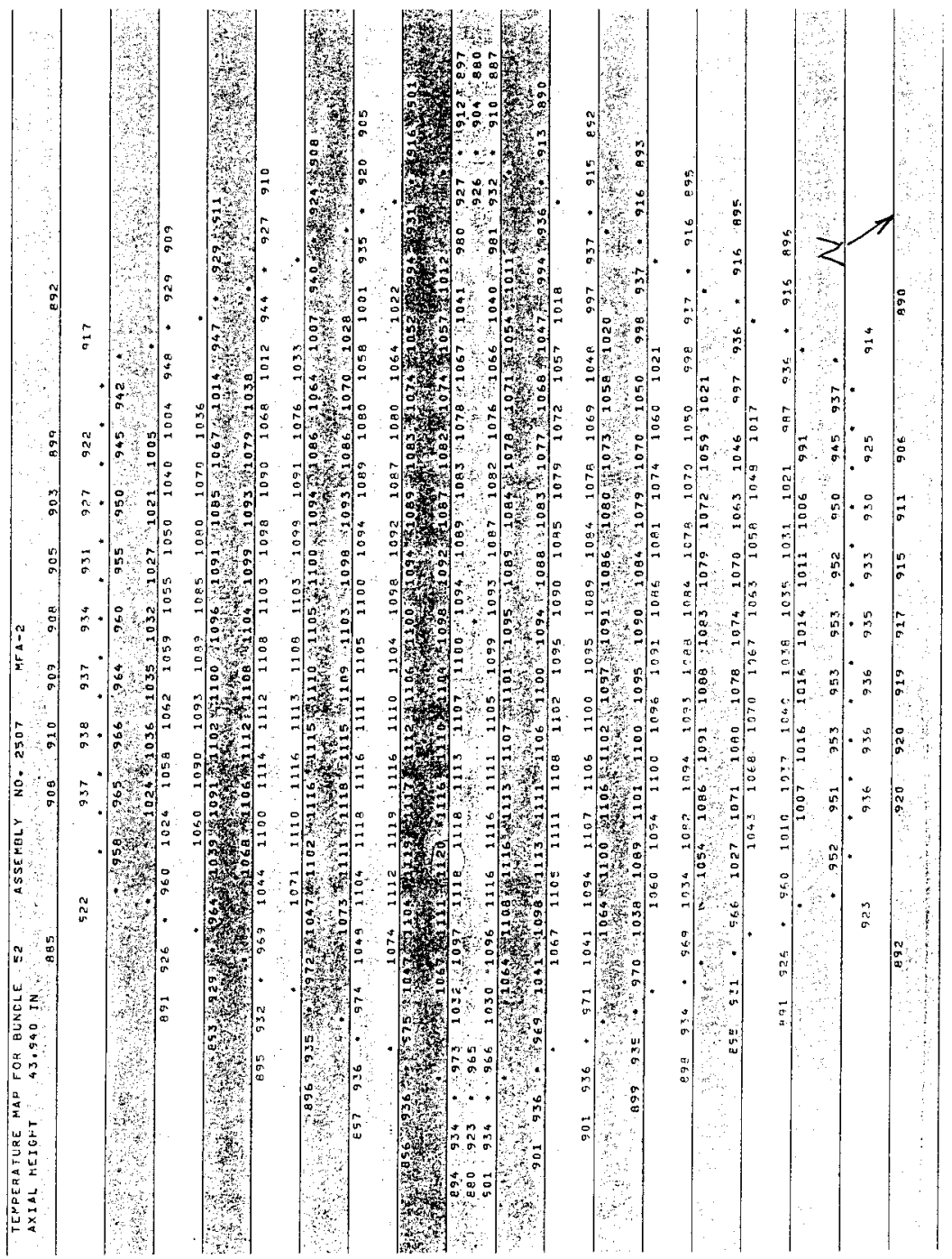
Table E1OC-3.13. MFA-1 Sodium Subchannel Temperatures at Elevation of Upper Axial Blanket for EOC $10 \mathrm{C}-3$

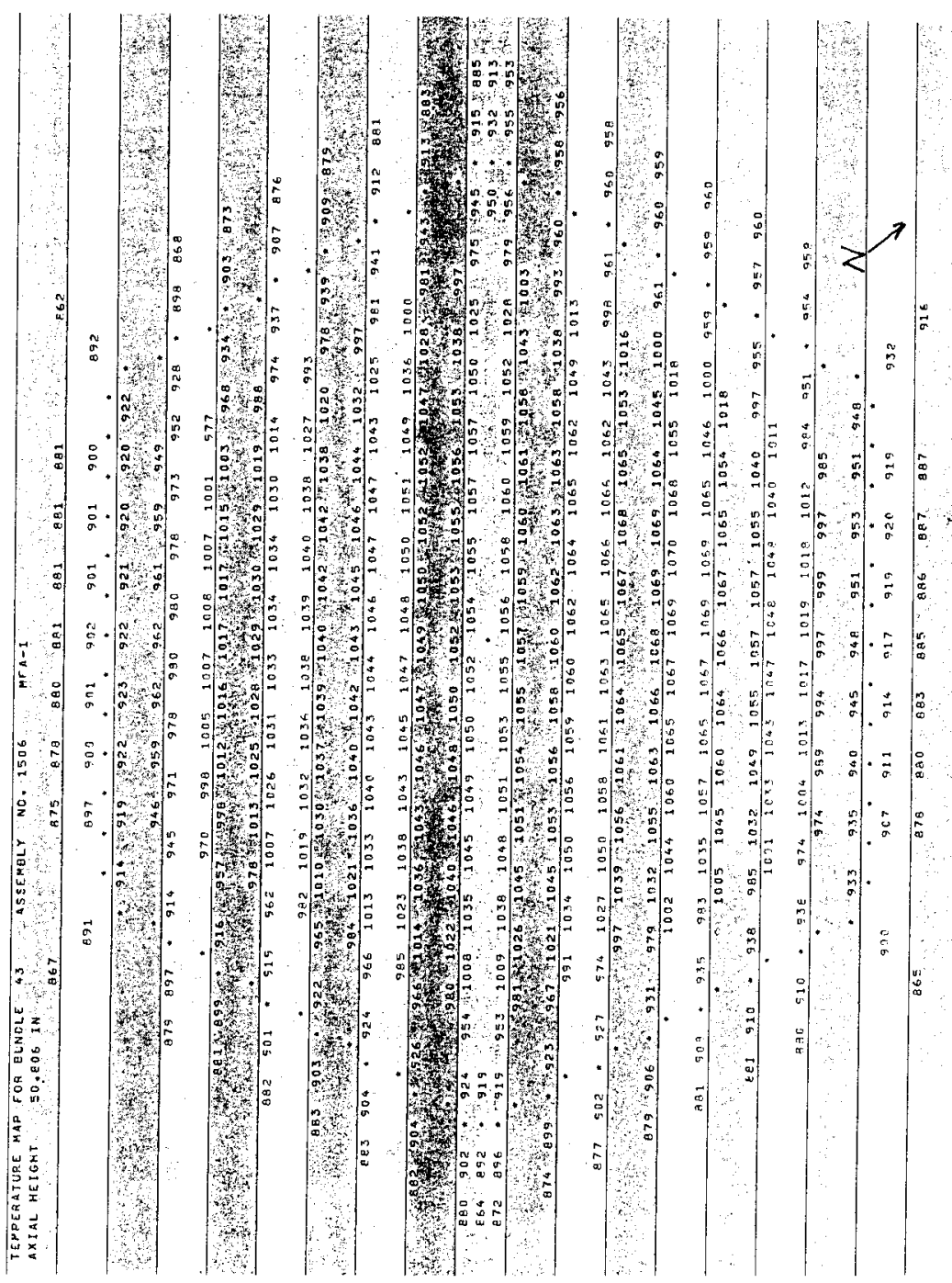


Table E10C-3.14. MFA-2 Sodium Subchannel Temperatures at Elevation of Upper Axial Blanket for EOC $10 \mathrm{C}-3$

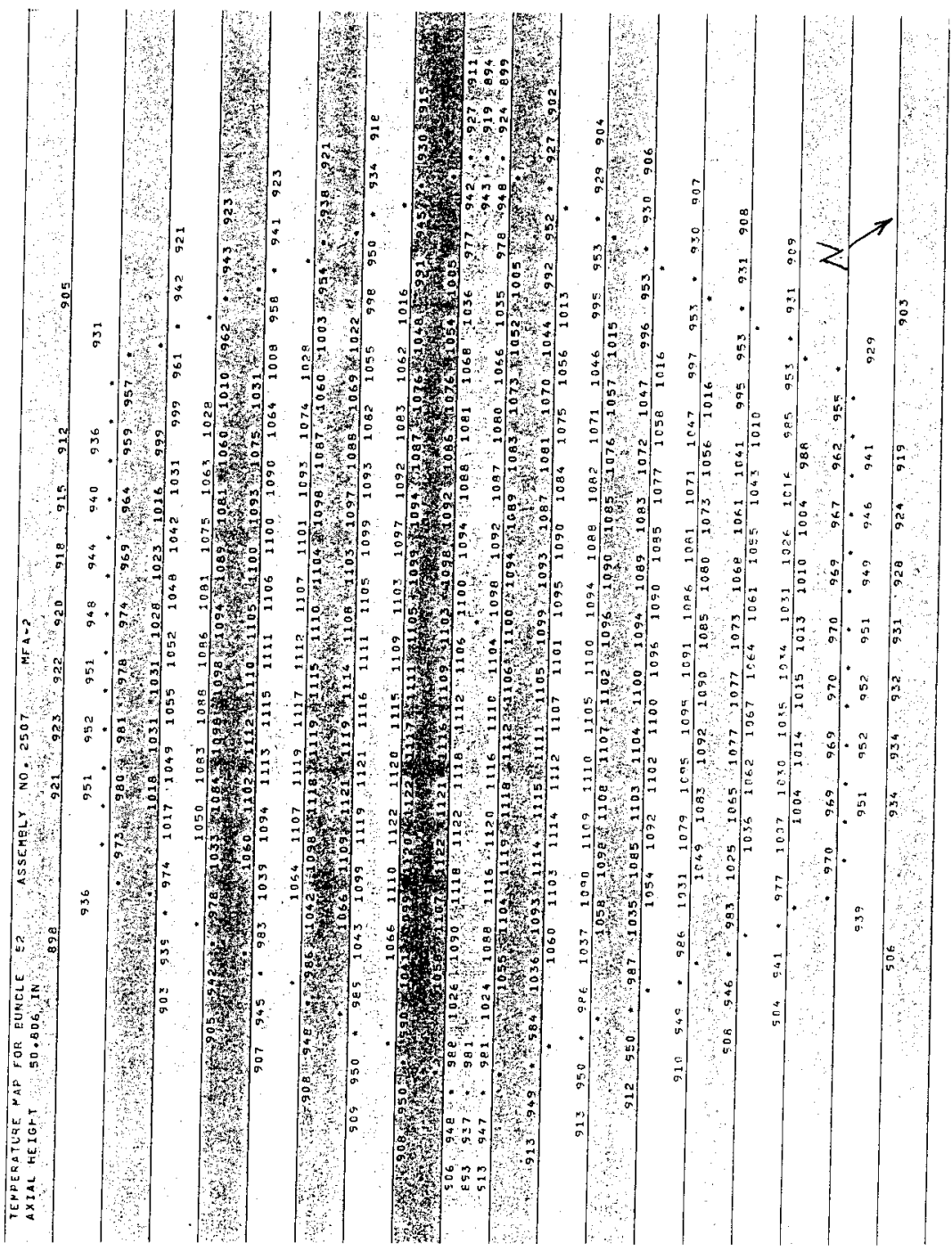


Table E10C-3.15. MFA-1 Sodium Subchannel Temperatures at Top of Fuel Pin Bundle for EOC 10C-3
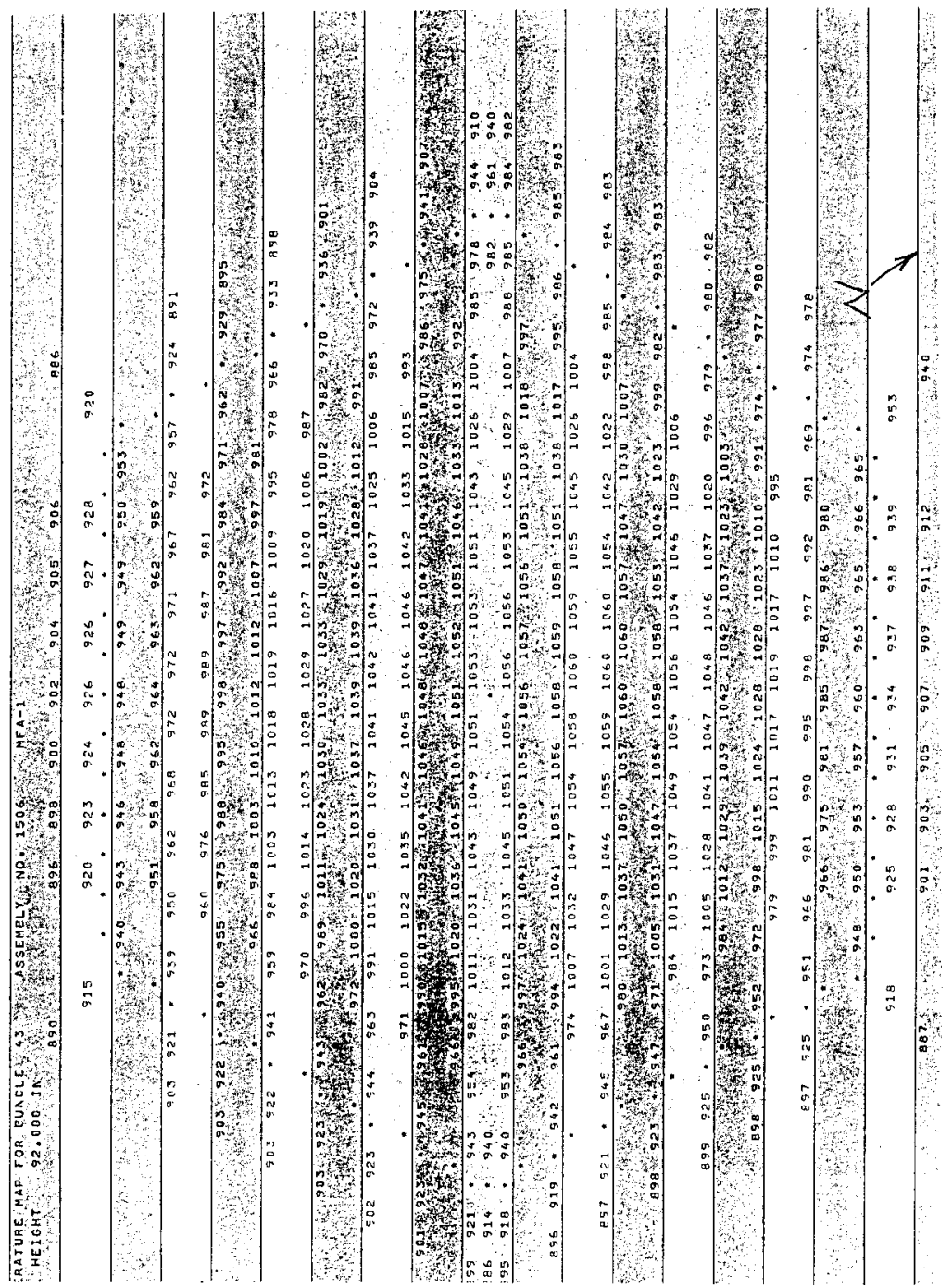
Table E10C-3.16. MFA-2 Sodium Subchannel Temperatures at Top of Fuel Pin Bundle for EOC $10 C-3$

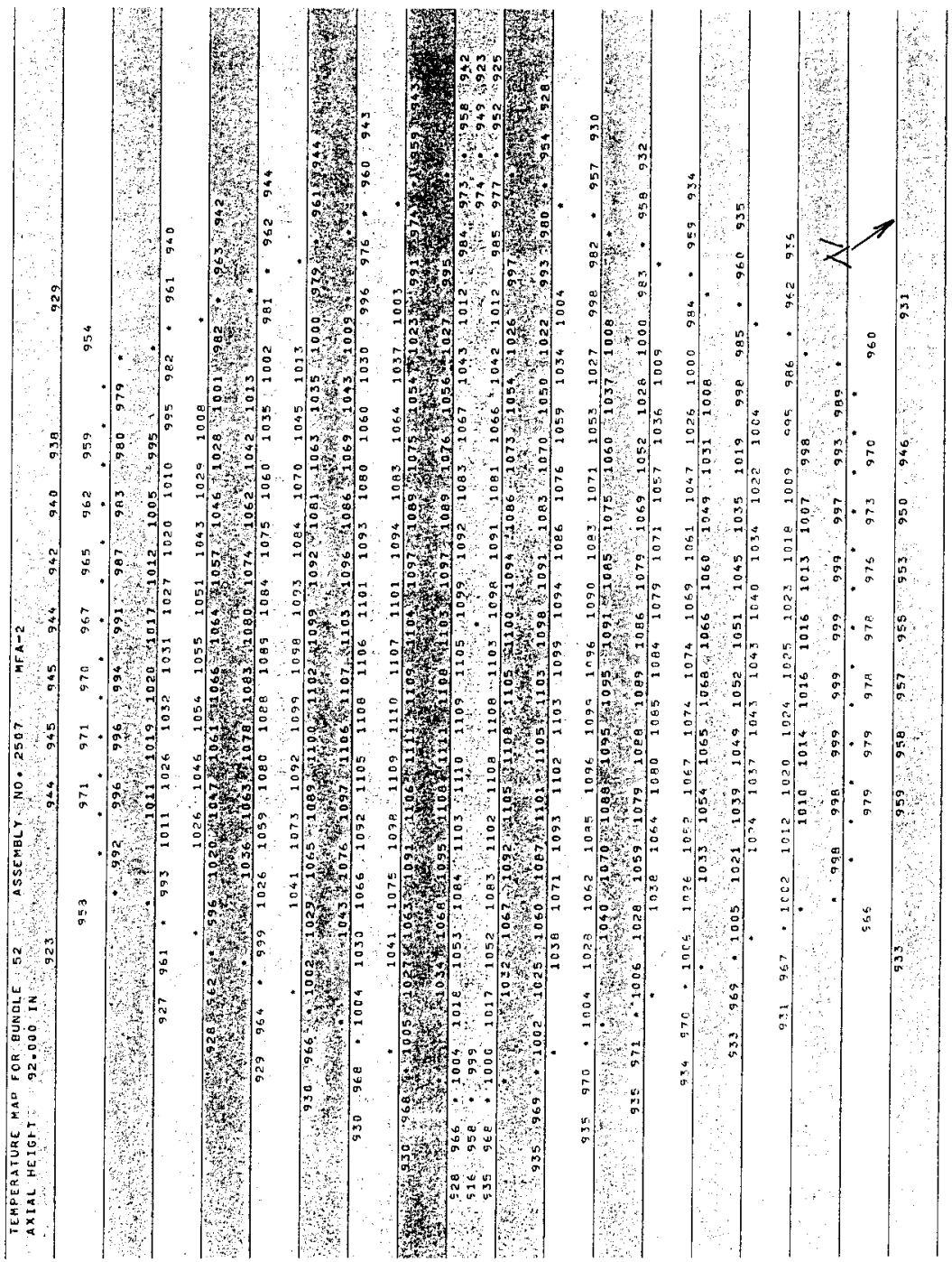


Table E1OC-3.17. Composition of MFA-1 at EOC 1OC-3

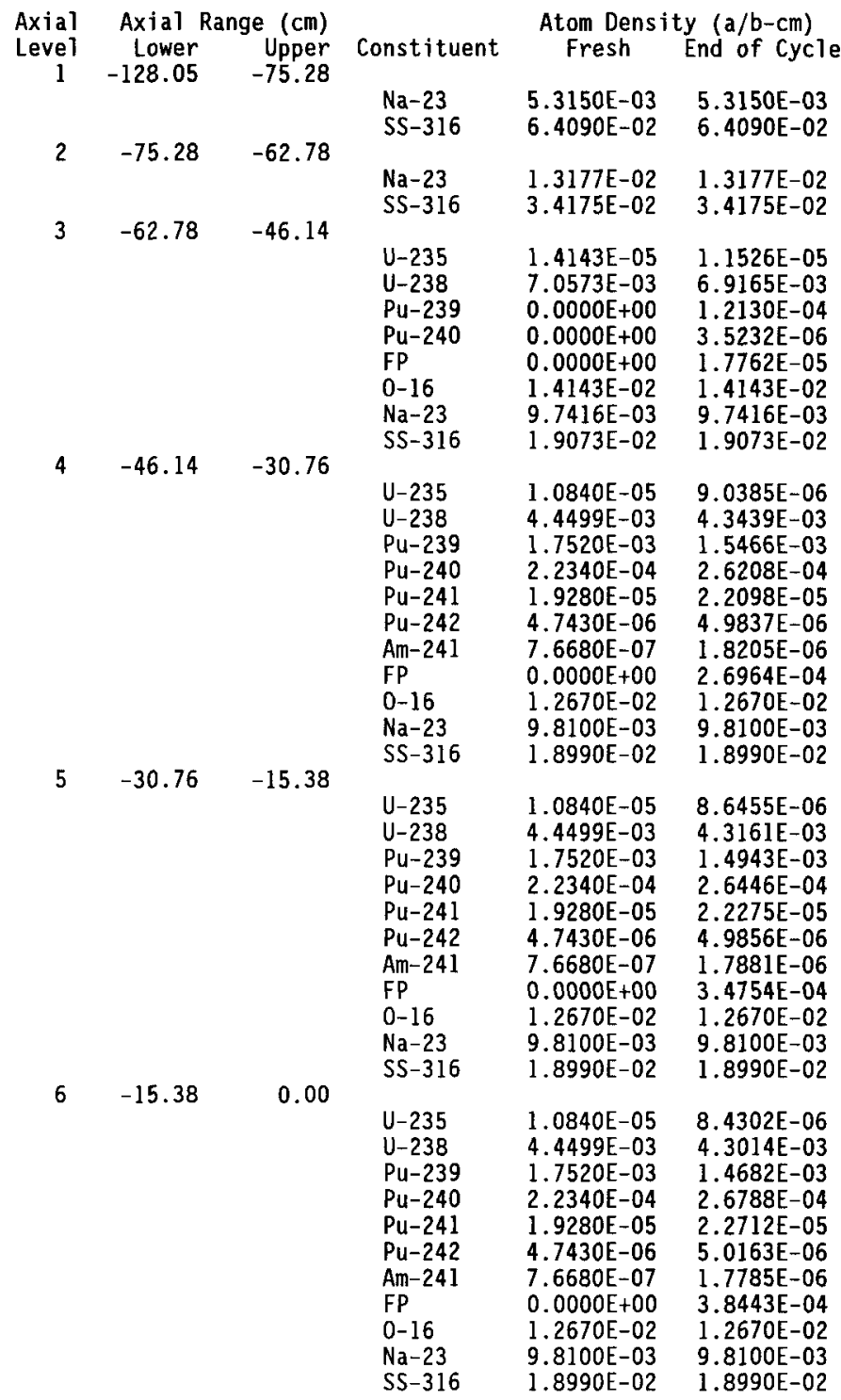


HNF-SD-FF-ANAL-009 Rev. 1

$0.00 \quad 15.38$

8

$15.38 \quad 30.76$

$\begin{array}{ll}\mathrm{U}-235 & 1.0840 \mathrm{E}-05 \\ \mathrm{U}-238 & 4.4499 \mathrm{E}-03 \\ \mathrm{Pu}-239 & 1.7520 \mathrm{E}-03 \\ \mathrm{Pu}-240 & 2.2340 \mathrm{E}-04 \\ \mathrm{Pu}-241 & 1.9280 \mathrm{E}-05 \\ \mathrm{Pu}-242 & 4.7430 \mathrm{E}-06 \\ \mathrm{Am}-241 & 7.6680 \mathrm{E}-07 \\ \mathrm{FP} & 0.0000 \mathrm{E}+00 \\ 0-16 & 1.2670 \mathrm{E}-02 \\ \mathrm{Na}-23 & 9.8100 \mathrm{E}-03 \\ \mathrm{SS}-316 & 1.8990 \mathrm{E}-02\end{array}$

8. 4993E-06

4.3061E-03

$1.4763 \mathrm{E}-03$

2. 6655E-04

2. 2543E-05

5. $0039 \mathrm{E}-06$

1.7817E-06

3. $7302 \mathrm{E}-04$

1.2670E-02

$9.8100 \mathrm{E}-03$

1.8990E-02

$\begin{array}{lll}\mathrm{U}-235 & 1.0840 \mathrm{E}-05 & 8.8408 \mathrm{E}-06 \\ \mathrm{U}-238 & 4.4499 \mathrm{E}-03 & 4.3290 \mathrm{E}-03 \\ \mathrm{Pu}-239 & 1.7520 \mathrm{E}-03 & 1.5169 \mathrm{E}-03 \\ \mathrm{Pu}-240 & 2.2340 \mathrm{E}-04 & 2.6065 \mathrm{E}-04 \\ \mathrm{Pu}-241 & 1.9280 \mathrm{E}-05 & 2.1811 \mathrm{E}-05 \\ \mathrm{Pu}-242 & 4.7430 \mathrm{E}-06 & 4.9533 \mathrm{E}-06 \\ \mathrm{Am}-241 & 7.6680 \mathrm{E}-07 & 1.7969 \mathrm{E}-06 \\ \mathrm{FP} & 0.0000 \mathrm{E}+00 & 3.1622 \mathrm{E}-04 \\ 0-16 & 1.2670 \mathrm{E}-02 & 1.2670 \mathrm{E}-02 \\ \mathrm{Na}-23 & 9.8100 \mathrm{E}-03 & 9.8100 \mathrm{E}-03 \\ \mathrm{SS}-316 & 1.8990 \mathrm{E}-02 & 1.8990 \mathrm{E}-02\end{array}$

9

$30.76 \quad 46.14$

$\begin{array}{ll}\mathrm{U}-235 & 1.0840 \mathrm{E}-05 \\ \mathrm{U}-238 & 4.4499 \mathrm{E}-03 \\ \mathrm{Pu}-239 & 1.7520 \mathrm{E}-03 \\ \mathrm{Pu}-240 & 2.2340 \mathrm{E}-04 \\ \mathrm{Pu}-241 & 1.9280 \mathrm{E}-05 \\ \mathrm{Pu}-242 & 4.7430 \mathrm{E}-06 \\ \mathrm{Am}-241 & 7.6680 \mathrm{E}-07 \\ \mathrm{FP} & 0.0000 \mathrm{E}+00 \\ \mathrm{O}-16 & 1.2670 \mathrm{E}-02 \\ \mathrm{Na}-23 & 9.8100 \mathrm{E}-03 \\ \mathrm{SS}-316 & 1.8990 \mathrm{E}-02\end{array}$

9.3336E-06

4. $3622 \mathrm{E}-03$

$1.5794 \mathrm{E}-03$

2. 5502E-04

2. $1233 \mathrm{E}-05$

4. $9240 \mathrm{E}-06$

1.8292E-06

2. 2626E-04

$1.2670 \mathrm{E}-02$

9.8100E-03

$\begin{array}{lll}10 & 46.14 & 62.78\end{array}$

$\begin{array}{lll}\mathrm{U}-235 & 1.4143 \mathrm{E}-05 & 1.2337 \mathrm{E}-05 \\ \mathrm{U}-238 & 7.0573 \mathrm{E}-03 & 6.9590 \mathrm{E}-03 \\ \mathrm{Pu}-239 & 0.0000 \mathrm{E}+00 & 8.6411 \mathrm{E}-05 \\ \mathrm{Pu}-240 & 0.0000 \mathrm{E}+00 & 1.6016 \mathrm{E}-06 \\ \mathrm{FP} & 0.0000 \mathrm{E}+00 & 1.1607 \mathrm{E}-05 \\ 0-16 & 1.4143 \mathrm{E}-02 & 1.4143 \mathrm{E}-02 \\ \mathrm{Na}-23 & 9.7416 \mathrm{E}-03 & 9.7416 \mathrm{E}-03 \\ \mathrm{SS}-316 & 1.9073 \mathrm{E}-02 & 1.9073 \mathrm{E}-02\end{array}$

$11 \quad 62.78 \quad 172.84$

$\mathrm{Na}-23 \quad 8.2400 \mathrm{E}-03 \quad 8.2400 \mathrm{E}-03$

SS-316 2.5562E-02 2.5562E-02 
HNF-SD-FF-ANAL-009 Rev. 1

Table E1OC-3.18. Composition of MFA-2 at EOC $10 \mathrm{C}-3$

\begin{tabular}{|c|c|c|c|c|c|}
\hline Axial & Axial & e $(\mathrm{cm})$ & & Atom Dens & y $(a / b-c m)$ \\
\hline Level & $\begin{array}{r}\text { Lower } \\
-128.05\end{array}$ & $\begin{array}{r}\text { Upper } \\
-75.28\end{array}$ & Constituent & Fresh & End of Cycle \\
\hline & & & $\begin{array}{l}\mathrm{Na}-23 \\
\mathrm{SS}-316\end{array}$ & $\begin{array}{l}5.3150 \mathrm{E}-03 \\
6.4090 \mathrm{E}-02\end{array}$ & $\begin{array}{l}5.3150 \mathrm{E}-03 \\
6.4090 \mathrm{E}-02\end{array}$ \\
\hline 2 & -75.28 & -62.78 & & & \\
\hline & & & $\begin{array}{l}\mathrm{Na}-23 \\
\mathrm{SS}-316\end{array}$ & $\begin{array}{l}1.3177 \mathrm{E}-02 \\
3.4175 \mathrm{E}-02\end{array}$ & $\begin{array}{l}1.3177 \mathrm{E}-02 \\
3.4175 \mathrm{E}-02\end{array}$ \\
\hline 3 & -62.78 & -46.14 & & & \\
\hline & & & $\begin{array}{l}U-235 \\
U-238 \\
P u-239 \\
P u-240 \\
\text { FP } \\
0-16 \\
\mathrm{Na}-23 \\
\mathrm{SS}-316\end{array}$ & $\begin{array}{l}1.4143 \mathrm{E}-05 \\
7.0573 \mathrm{E}-03 \\
0.0000 \mathrm{E}+00 \\
0.0000 \mathrm{E}+00 \\
0.0000 \mathrm{E}+00 \\
1.4143 \mathrm{E}-02 \\
9.7416 \mathrm{E}-03 \\
1.9073 \mathrm{E}-02\end{array}$ & $\begin{array}{l}1.1426 \mathrm{E}-05 \\
6.9116 \mathrm{E}-03 \\
1.2509 \mathrm{E}-04 \\
3.8246 \mathrm{E}-06 \\
1.8713 \mathrm{E}-05 \\
1.4143 \mathrm{E}-02 \\
9.7416 \mathrm{E}-03 \\
1.9073 \mathrm{E}-02\end{array}$ \\
\hline 4 & -46.14 & -30.76 & & & \\
\hline & & & $\begin{array}{l}U-235 \\
U-238 \\
P u-239 \\
P u-240 \\
P u-241 \\
P u-242 \\
\mathrm{Am}-241 \\
\mathrm{FP} \\
0-16 \\
\mathrm{Na}-23 \\
\mathrm{SS}-316\end{array}$ & $\begin{array}{l}1.0160 \mathrm{E}-05 \\
4.4270 \mathrm{E}-03 \\
1.7483 \mathrm{E}-03 \\
2.2290 \mathrm{E}-04 \\
1.9040 \mathrm{E}-05 \\
4.7330 \mathrm{E}-06 \\
7.5700 \mathrm{E}-07 \\
0.0000 \mathrm{E}+00 \\
1.2760 \mathrm{E}-02 \\
9.8100 \mathrm{E}-03 \\
1.8990 \mathrm{E}-02\end{array}$ & $\begin{array}{l}8.3743 \mathrm{E}-06 \\
4.3147 \mathrm{E}-03 \\
1.5325 \mathrm{E}-03 \\
2.6454 \mathrm{E}-04 \\
2.2289 \mathrm{E}-05 \\
4.9949 \mathrm{E}-06 \\
1.7938 \mathrm{E}-06 \\
2.8292 \mathrm{E}-04 \\
1.2760 \mathrm{E}-02 \\
9.8100 \mathrm{E}-03 \\
1.8990 \mathrm{E}-02\end{array}$ \\
\hline 5 & -30.76 & -15.38 & & & \\
\hline & & & $\begin{array}{l}\mathrm{U}-235 \\
\mathrm{U}-238 \\
\mathrm{Pu}-239 \\
\mathrm{Pu}-240 \\
\mathrm{Pu}-241 \\
\mathrm{Pu}-242 \\
\mathrm{Am}-241 \\
\mathrm{FP} \\
0-16 \\
\mathrm{Na}-23 \\
\mathrm{SS}-316\end{array}$ & $\begin{array}{l}1.0160 \mathrm{E}-05 \\
4.4270 \mathrm{E}-03 \\
1.7483 \mathrm{E}-03 \\
2.2290 \mathrm{E}-04 \\
1.9040 \mathrm{E}-05 \\
4.7330 \mathrm{E}-06 \\
7.5700 \mathrm{E}-07 \\
0.0000 \mathrm{E}+00 \\
1.2760 \mathrm{E}-02 \\
9.8100 \mathrm{E}-03 \\
1.8990 \mathrm{E}-02\end{array}$ & $\begin{array}{l}7.9675 \mathrm{E}-06 \\
4.2836 \mathrm{E}-03 \\
1.4754 \mathrm{E}-03 \\
2.6751 \mathrm{E}-04 \\
2.2554 \mathrm{E}-05 \\
5.0019 \mathrm{E}-06 \\
1.7585 \mathrm{E}-06 \\
3.6809 \mathrm{E}-04 \\
1.2760 \mathrm{E}-02 \\
9.8100 \mathrm{E}-03 \\
1.8990 \mathrm{E}-02\end{array}$ \\
\hline 6 & -15.38 & 0.00 & & & \\
\hline & & & $\begin{array}{l}\mathrm{U}-235 \\
\mathrm{U}-238 \\
\mathrm{Pu}-239 \\
\mathrm{Pu}-240 \\
\mathrm{Pu}-241 \\
\mathrm{Pu}-242 \\
\mathrm{Am}-241 \\
\mathrm{FP} \\
0-16 \\
\mathrm{Na}-23 \\
\mathrm{SS}-316\end{array}$ & $\begin{array}{l}1.0160 \mathrm{E}-05 \\
4.4270 \mathrm{E}-03 \\
1.7483 \mathrm{E}-03 \\
2.2290 \mathrm{E}-04 \\
1.9040 \mathrm{E}-05 \\
4.7330 \mathrm{E}-06 \\
7.5700 \mathrm{E}-07 \\
0.0000 \mathrm{E}+00 \\
1.2760 \mathrm{E}-02 \\
9.8100 \mathrm{E}-03 \\
1.8990 \mathrm{E}-02\end{array}$ & $\begin{array}{l}7.7398 \mathrm{E}-06 \\
4.2668 \mathrm{E}-03 \\
1.4464 \mathrm{E}-03 \\
2.7143 \mathrm{E}-04 \\
2.3086 \mathrm{E}-05 \\
5.0397 \mathrm{E}-06 \\
1.7473 \mathrm{E}-06 \\
4.0961 \mathrm{E}-04 \\
1.2760 \mathrm{E}-02 \\
9.8100 \mathrm{E}-03 \\
1.8990 \mathrm{E}-02\end{array}$ \\
\hline & & & & & \\
\hline
\end{tabular}


HNF-SD-FF-ANAL-009 Rev. 1

$\begin{array}{lll}7 & 0.00 \quad 15.38\end{array}$

$\begin{array}{lll}\text { U-235 } & 1.0160 \mathrm{E}-05 & 7.7995 \mathrm{E}-06 \\ \mathrm{U}-238 & 4.4270 \mathrm{E}-03 & 4.2712 \mathrm{E}-03 \\ \mathrm{Pu}-239 & 1.7483 \mathrm{E}-03 & 1.4536 \mathrm{E}-03 \\ \mathrm{Pu}-240 & 2.2290 \mathrm{E}-04 & 2.7024 \mathrm{E}-04 \\ \mathrm{Pu}-241 & 1.9040 \mathrm{E}-05 & 2.2922 \mathrm{E}-05 \\ \mathrm{Pu}-242 & 4.7330 \mathrm{E}-06 & 5.0275 \mathrm{E}-06 \\ \mathrm{Am}-241 & 7.5700 \mathrm{E}-07 & 1.7502 \mathrm{E}-06 \\ \mathrm{FP} & 0.0000 \mathrm{E}+00 & 3.9914 \mathrm{E}-04 \\ 0-16 & 1.2760 \mathrm{E}-02 & 1.2760 \mathrm{E}-02 \\ \mathrm{Na}-23 & 9.8100 \mathrm{E}-03 & 9.8100 \mathrm{E}-03 \\ \mathrm{SS}-316 & 1.8990 \mathrm{E}-02 & 1.8990 \mathrm{E}-02\end{array}$

$\begin{array}{lll}8 & 15.38 & 30.76\end{array}$

U-235 $\quad 1.0160 E-05 \quad 8.1294 E-06$

$\mathrm{U}-238 \quad 4.4270 \mathrm{E}-03 \quad 4.2952 \mathrm{E}-03$

$\mathrm{Pu}-239 \quad 1.7483 \mathrm{E}-03 \quad 1.4955 \mathrm{E}-03$

Pu-240 2.2290E-04 2.6418E-04

Pu-241 1.9040E-05 2.2130E-05

Pu-242 4.7330E-06 4.9722E-06

Am-241 7.5700E-07 $1.7660 \mathrm{E}-06$

FP $\quad 0.0000 E+00 \quad 3.4023 \mathrm{E}-04$

$0-16 \quad 1.2760 \mathrm{E}-02 \quad 1.2760 \mathrm{E}-02$

$\mathrm{Na}-23 \quad 9.8100 \mathrm{E}-03 \quad 9.8100 \mathrm{E}-03$

SS-316 $1.8990 \mathrm{E}-02 \quad 1.8990 \mathrm{E}-02$

$9 \quad 30.76 \quad 46.14$

U-235 $\quad 1.0160 \mathrm{E}-05 \quad 8.6180 \mathrm{E}-06$

U-238 4.4270E-03 4.3309E-03

$\mathrm{Pu}-239 \quad 1.7483 \mathrm{E}-03 \quad 1.5612 \mathrm{E}-03$

Pu-240 2.2290E-04 2.5831E-04

Pu-241 1.9040E-05 2.1495E-05

$\mathrm{Pu}-242 \quad 4.7330 \mathrm{E}-06 \quad 4.9398 \mathrm{E}-06$

Am-241 7.5700E-07 1.8008E-06

$\mathrm{FP} \quad 0.0000 \mathrm{E}+00 \quad 2.4498 \mathrm{E}-04$

$0-161.2760 \mathrm{E}-02 \quad 1.2760 \mathrm{E}-02$

$\mathrm{Na}-23 \quad 9.8100 \mathrm{E}-03 \quad 9.8100 \mathrm{E}-03$

SS-316 $1.8990 \mathrm{E}-02 \quad 1.8990 \mathrm{E}-02$

$\begin{array}{lll}10 & 46.14 & 62.78\end{array}$

U-235 $\quad 1.4143 \mathrm{E}-05 \quad 1.2136 \mathrm{E}-05$

$\mathrm{U}-238 \quad 7.0573 \mathrm{E}-03 \quad 6.9491 \mathrm{E}-03$

Pu-239 $\quad 0.0000 \mathrm{E}+00 \quad 9.4601 \mathrm{E}-05$

Pu-240 $\quad 0.0000 E+00 \quad 2.0115 E-06$

FP $\quad 0.0000 E+00 \quad 1.3042 \mathrm{E}-05$

$0-161.4143 \mathrm{E}-02 \quad 1.4143 \mathrm{E}-02$

$\mathrm{Na}-23 \quad 9.7416 \mathrm{E}-03 \quad 9.7416 \mathrm{E}-03$

SS-316 $\quad 1.9073 \mathrm{E}-02 \quad 1.9073 \mathrm{E}-02$

$11 \quad 62.78 \quad 172.84$

$\begin{array}{lll}\mathrm{Na}-23 & 8.2400 \mathrm{E}-03 & 8.2400 \mathrm{E}-03 \\ \mathrm{SS}-3 \mathrm{I} 6 & 2.5562 \mathrm{E}-02 & 2.5562 \mathrm{E}-02\end{array}$




\subsection{Cycle 11A-1}

Table B11A-1.1. Fission Power Generated in MFA-1, MFA-2 and Neighboring Assemblies at BOC 11A-1

\begin{tabular}{lccccc} 
CORE & $* * * * * *$ & \multicolumn{2}{c}{ POWER IN } & MEGAWATTS & $* * * * * * *$ \\
POS. & BELOW CORE & IN CORE & ABOVE CORE & TOTAL PWR \\
1404 & $6.085 \mathrm{E}-02$ & $4.110 \mathrm{E}+00$ & $3.409 \mathrm{E}-02$ & $4.205 \mathrm{E}+00$ \\
1303 & $1.404 \mathrm{E}-01$ & $1.753 \mathrm{E}+00$ & $6.482 \mathrm{E}-02$ & $1.958 \mathrm{E}+00$ \\
1304 & $9.466 \mathrm{E}-03$ & $4.457 \mathrm{E}+00$ & $6.901 \mathrm{E}-03$ & $4.474 \mathrm{E}+00$ \\
1403 & $1.384 \mathrm{E}-02$ & $3.372 \mathrm{E}+00$ & $7.286 \mathrm{E}-03$ & $3.393 \mathrm{E}+00$ \\
1405 & $7.053 \mathrm{E}-03$ & $3.819 \mathrm{E}+00$ & $5.489 \mathrm{E}-03$ & $3.831 \mathrm{E}+00$ \\
1505 & $6.327 \mathrm{E}-03$ & $3.917 \mathrm{E}+00$ & $4.270 \mathrm{E}-03$ & $3.928 \mathrm{E}+00$ \\
1506 & $8.765 \mathrm{E}-03$ & $3.894 \mathrm{E}+00$ & $6.190 \mathrm{E}-03$ & $3.909 \mathrm{E}+00$ \\
2506 & $5.978 \mathrm{E}-02$ & $3.888 \mathrm{E}+00$ & $3.772 \mathrm{E}-02$ & $3.986 \mathrm{E}+00$ \\
2404 & $0.000 \mathrm{E}+00$ & $7.201 \mathrm{E}+00$ & $0.000 \mathrm{E}+00$ & $7.201 \mathrm{E}+00$ \\
2505 & $1.284 \mathrm{E}-02$ & $4.088 \mathrm{E}+00$ & $6.851 \mathrm{E}-03$ & $4.108 \mathrm{E}+00$ \\
2405 & $1.409 \mathrm{E}-02$ & $3.658 \mathrm{E}+00$ & $9.874 \mathrm{E}-03$ & $3.682 \mathrm{E}+00$ \\
2606 & $6.053 \mathrm{E}-03$ & $3.953 \mathrm{E}+00$ & $4.677 \mathrm{E}-03$ & $3.963 \mathrm{E}+00$ \\
2607 & $1.330 \mathrm{E}-02$ & $3.421 \mathrm{E}+00$ & $7.627 \mathrm{E}-03$ & $3.442 \mathrm{E}+00$ \\
2507 & $9.345 \mathrm{E}-03$ & $4.037 \mathrm{E}+00$ & $7.145 \mathrm{E}-03$ & $4.054 \mathrm{E}+00$
\end{tabular}

Table B11A-1.2. Assembly Averaged Total and Fast Flux in MFA-1 and MFA-2 at BOC 11A-1

\begin{tabular}{cccc} 
& Core & \multicolumn{2}{c}{ Flux $\left(\mathrm{n} / \mathrm{cm}^{2}-\mathrm{sec}\right)$} \\
Assembly & Pos. & Total & $>0.1 \mathrm{MeV}$ \\
MFA-1 & 1404 & $3.341 \mathrm{E}+15$ & $2.061 \mathrm{E}+15$ \\
MFA-2 & 2506 & $3.210 \mathrm{E}+15$ & $2.098 \mathrm{E}+15$
\end{tabular}


Table BllA-1.3. Axial Distribution of Total Flux, Fast Flux and Power in MFA-1 at BOC 11A-1

$\begin{array}{rccc}\text { z }(\mathrm{cm}) & \text { Total Flux } & \text { Flux }>0.1 \mathrm{MeV} & \text { Power } \\ -97.78 & 2.8900 \mathrm{E}-02 & 1.5414 \mathrm{E}-02 & 0.0000 \mathrm{E}+00 \\ -92.78 & 6.2037 \mathrm{E}-02 & 2.9706 \mathrm{E}-02 & 0.0000 \mathrm{E}+00 \\ -87.78 & 9.9360 \mathrm{E}-02 & 4.7415 \mathrm{E}-02 & 0.0000 \mathrm{E}+00 \\ -82.78 & 1.4364 \mathrm{E}-01 & 7.0505 \mathrm{E}-02 & 0.0000 \mathrm{E}+00 \\ -77.78 & 1.9764 \mathrm{E}-01 & 1.0136 \mathrm{E}-01 & 0.0000 \mathrm{E}+00 \\ -72.15 & 2.5852 \mathrm{E}-01 & 1.4101 \mathrm{E}-01 & 0.0000 \mathrm{E}+00 \\ -65.90 & 3.1917 \mathrm{E}-01 & 1.9031 \mathrm{E}-01 & 0.0000 \mathrm{E}+00 \\ -60.35 & 3.8225 \mathrm{E}-01 & 2.5570 \mathrm{E}-01 & 6.5143 \mathrm{E}-02 \\ -55.49 & 4.6476 \mathrm{E}-01 & 3.4106 \mathrm{E}-01 & 7.6558 \mathrm{E}-02 \\ -50.62 & 5.5963 \mathrm{E}-01 & 4.5024 \mathrm{E}-01 & 9.2787 \mathrm{E}-02 \\ -47.17 & 6.3498 \mathrm{E}-01 & 5.5224 \mathrm{E}-01 & 1.1023 \mathrm{E}-01 \\ -43.58 & 7.1408 \mathrm{E}-01 & 6.6838 \mathrm{E}-01 & 7.6972 \mathrm{E}-01 \\ -38.45 & 8.2850 \mathrm{E}-01 & 8.0945 \mathrm{E}-01 & 8.6005 \mathrm{E}-01 \\ -33.32 & 9.3490 \mathrm{E}-01 & 9.3033 \mathrm{E}-01 & 9.5930 \mathrm{E}-01 \\ -28.20 & 1.0281 \mathrm{E}+00 & 1.0312 \mathrm{E}+00 & 1.0191 \mathrm{E}+00 \\ -23.07 & 1.1060 \mathrm{E}+00 & 1.1138 \mathrm{E}+00 & 1.0942 \mathrm{E}+00 \\ -17.94 & 1.1669 \mathrm{E}+00 & 1.1771 \mathrm{E}+00 & 1.1532 \mathrm{E}+00 \\ -12.82 & 1.2095 \mathrm{E}+00 & 1.2207 \mathrm{E}+00 & 1.1771 \mathrm{E}+00 \\ -7.69 & 1.2336 \mathrm{E}+00 & 1.2453 \mathrm{E}+00 & 1.2001 \mathrm{E}+00 \\ -2.56 & 1.2387 \mathrm{E}+00 & 1.2505 \mathrm{E}+00 & 1.2048 \mathrm{E}+00 \\ 2.56 & 1.2247 \mathrm{E}+00 & 1.2365 \mathrm{E}+00 & 1.1965 \mathrm{E}+00 \\ 7.69 & 1.1916 \mathrm{E}+00 & 1.2030 \mathrm{E}+00 & 1.1641 \mathrm{E}+00 \\ 12.82 & 1.1403 \mathrm{E}+00 & 1.1511 \mathrm{E}+00 & 1.1140 \mathrm{E}+00 \\ 17.94 & 1.0726 \mathrm{E}+00 & 1.0826 \mathrm{E}+00 & 1.0719 \mathrm{E}+00 \\ 23.07 & 9.8984 \mathrm{E}-01 & 9.9787 \mathrm{E}-01 & 9.8964 \mathrm{E}-01 \\ 28.20 & 8.9465 \mathrm{E}-01 & 8.9913 \mathrm{E}-01 & 8.9546 \mathrm{E}-01 \\ 33.32 & 7.8940 \mathrm{E}-01 & 7.8826 \mathrm{E}-01 & 8.2031 \mathrm{E}-01 \\ 38.45 & 6.7635 \mathrm{E}-01 & 6.6474 \mathrm{E}-01 & 7.0820 \mathrm{E}-01 \\ 43.58 & 5.6025 \mathrm{E}-01 & 5.2995 \mathrm{E}-01 & 6.0221 \mathrm{E}-01 \\ 47.17 & 4.8182 \mathrm{E}-01 & 4.2649 \mathrm{E}-01 & 7.1930 \mathrm{E}-02 \\ 50.62 & 4.0783 \mathrm{E}-01 & 3.3600 \mathrm{E}-01 & 5.5945 \mathrm{E}-02 \\ 55.49 & 3.1648 \mathrm{E}-01 & 2.4061 \mathrm{E}-01 & 4.0944 \mathrm{E}-02 \\ 60.35 & 2.3769 \mathrm{E}-01 & 1.6740 \mathrm{E}-01 & 3.0189 \mathrm{E}-02 \\ 64.98 & 1.8895 \mathrm{E}-01 & 1.2522 \mathrm{E}-01 & 0.0000 \mathrm{E}+00 \\ 69.39 & 1.6085 \mathrm{E}-01 & 1.0302 \mathrm{E}-01 & 0.0000 \mathrm{E}+00 \\ 76.46 & 1.2144 \mathrm{E}-01 & 7.3261 \mathrm{E}-02 & 0.0000 \mathrm{E}+00 \\ 86.80 & 7.8816 \mathrm{E}-02 & 4.4267 \mathrm{E}-02 & 0.0000 \mathrm{E}+00 \\ 98.14 & 4.5361 \mathrm{E}-02 & 2.4214 \mathrm{E}-02 & 0.0000 \mathrm{E}+00 \\ 110.00 & 1.9445 \mathrm{E}-02 & 1.0830 \mathrm{E}-02 & 0.0000 \mathrm{E}+00\end{array}$


HNF-SD-FF-ANAL-009 Rev. 1

Table B11A-1.4. Axial Distribution of Total Flux, Fast Flux and Power in MFA-2 at BOC 11A-1

\begin{tabular}{|c|c|c|c|}
\hline $\begin{array}{l}Z(\mathrm{~cm}) \\
-97.78 \\
-92.78 \\
-87.78 \\
-82.78 \\
-77.78 \\
-72.15 \\
-65.90 \\
-60.35 \\
-55.49 \\
-50.62 \\
-47.17 \\
-43.58 \\
-38.45 \\
-33.32 \\
-28.20 \\
-23.07 \\
-17.94 \\
-12.82 \\
-7.69 \\
-2.56 \\
2.56 \\
7.69 \\
12.82 \\
17.94 \\
23.07 \\
28.20 \\
33.32 \\
38.45 \\
43.58 \\
47.17 \\
50.62 \\
55.49 \\
60.35 \\
64.98 \\
69.39 \\
76.46 \\
86.80 \\
98.14 \\
110.00\end{array}$ & $\begin{array}{l}\text { Total Flux } \\
2.6257 \mathrm{E}-02 \\
5.6350 \mathrm{E}-02 \\
9.0358 \mathrm{E}-02 \\
1.3096 \mathrm{E}-01 \\
1.8109 \mathrm{E}-01 \\
2.3897 \mathrm{E}-01 \\
2.9893 \mathrm{E}-01 \\
3.6304 \mathrm{E}-01 \\
4.4615 \mathrm{E}-01 \\
5.4192 \mathrm{E}-01 \\
6.1844 \mathrm{E}-01 \\
6.9898 \mathrm{E}-01 \\
8.1646 \mathrm{E}-01 \\
9.2559 \mathrm{E}-01 \\
1.0207 \mathrm{E}+00 \\
1.1001 \mathrm{E}+00 \\
1.1621 \mathrm{E}+00 \\
1.2056 \mathrm{E}+00 \\
1.2307 \mathrm{E}+00 \\
1.2372 \mathrm{E}+00 \\
1.2249 \mathrm{E}+00 \\
1.1939 \mathrm{E}+00 \\
1.1451 \mathrm{E}+00 \\
1.0800 \mathrm{E}+00 \\
9.9923 \mathrm{E}-01 \\
9.0512 \mathrm{E}-01 \\
8.0002 \mathrm{E}-01 \\
6.8595 \mathrm{E}-01 \\
5.6832 \mathrm{E}-01 \\
4.9079 \mathrm{E}-01 \\
4.1923 \mathrm{E}-01 \\
3.3134 \mathrm{E}-01 \\
2.5374 \mathrm{E}-01 \\
2.0336 \mathrm{E}-01 \\
1.7279 \mathrm{E}-01 \\
1.2945 \mathrm{E}-01 \\
8.3013 \mathrm{E}-02 \\
4.7156 \mathrm{E}-02 \\
1.9979 \mathrm{E}-02\end{array}$ & $\begin{array}{c}\text { Flux }>0.1 \mathrm{MeV} \\
1.3318 \mathrm{E}-02 \\
2.5714 \mathrm{E}-02 \\
4.1172 \mathrm{E}-02 \\
6.1493 \mathrm{E}-02 \\
8.8954 \mathrm{E}-02 \\
1.2487 \mathrm{E}-01 \\
1.7137 \mathrm{E}-01 \\
2.3529 \mathrm{E}-01 \\
3.2012 \mathrm{E}-01 \\
4.3106 \mathrm{E}-01 \\
5.3585 \mathrm{E}-01 \\
6.5437 \mathrm{E}-01 \\
7.9879 \mathrm{E}-01 \\
9.2176 \mathrm{E}-01 \\
1.0236 \mathrm{E}+00 \\
1.1066 \mathrm{E}+00 \\
1.1704 \mathrm{E}+00 \\
1.2144 \mathrm{E}+00 \\
1.2399 \mathrm{E}+00 \\
1.2467 \mathrm{E}+00 \\
1.2350 \mathrm{E}+00 \\
1.2044 \mathrm{E}+00 \\
1.1558 \mathrm{E}+00 \\
1.0909 \mathrm{E}+00 \\
1.0090 \mathrm{E}+00 \\
9.1201 \mathrm{E}-01 \\
8.0160 \mathrm{E}-01 \\
6.7647 \mathrm{E}-01 \\
5.3811 \mathrm{E}-01 \\
4.3309 \mathrm{E}-01 \\
3.4253 \mathrm{E}-01 \\
2.4775 \mathrm{E}-01 \\
1.7425 \mathrm{E}-01 \\
1.2985 \mathrm{E}-01 \\
1.0569 \mathrm{E}-01 \\
7.3528 \mathrm{E}-02 \\
4.3292 \mathrm{E}-02 \\
2.3148 \mathrm{E}-02 \\
1.0180 \mathrm{E}-02\end{array}$ & $\begin{array}{c}\text { Power } \\
0.0000 E+00 \\
0.0000 E+00 \\
0.0000 E+00 \\
0.0000 E+00 \\
0.0000 E+00 \\
0.0000 E+00 \\
0.0000 E+00 \\
6.6934 E-02 \\
7.9371 E-02 \\
9.6739 E-02 \\
1.1549 E-01 \\
7.5801 E-01 \\
8.4853 E-01 \\
9.5091 E-01 \\
1.0110 E+00 \\
1.0879 E+00 \\
1.1483 E+00 \\
1.1713 E+00 \\
1.1954 E+00 \\
1.2015 E+00 \\
1.1944 E+00 \\
1.1642 E+00 \\
1.1168 E+00 \\
1.0785 E+00 \\
9.9848 E-01 \\
9.0546 E-01 \\
8.3257 E-01 \\
7.2015 E-01 \\
6.1653 E-01 \\
8.1691 E-02 \\
6.4479 E-02 \\
4.8433 E-02 \\
3.6735 E-02 \\
0.0000 E+00 \\
0.0000 E+00 \\
0.0000 E+00 \\
0.0000 E+00 \\
0.0000 E+00 \\
0.0000 E+00\end{array}$ \\
\hline
\end{tabular}


HNF-SD-FF-ANAL-009 Rev. 1

Table BllA-1.5. Fission Power Distribution by Pin in MFA-1 at BOC 11A-1 CORE POSITION 1404

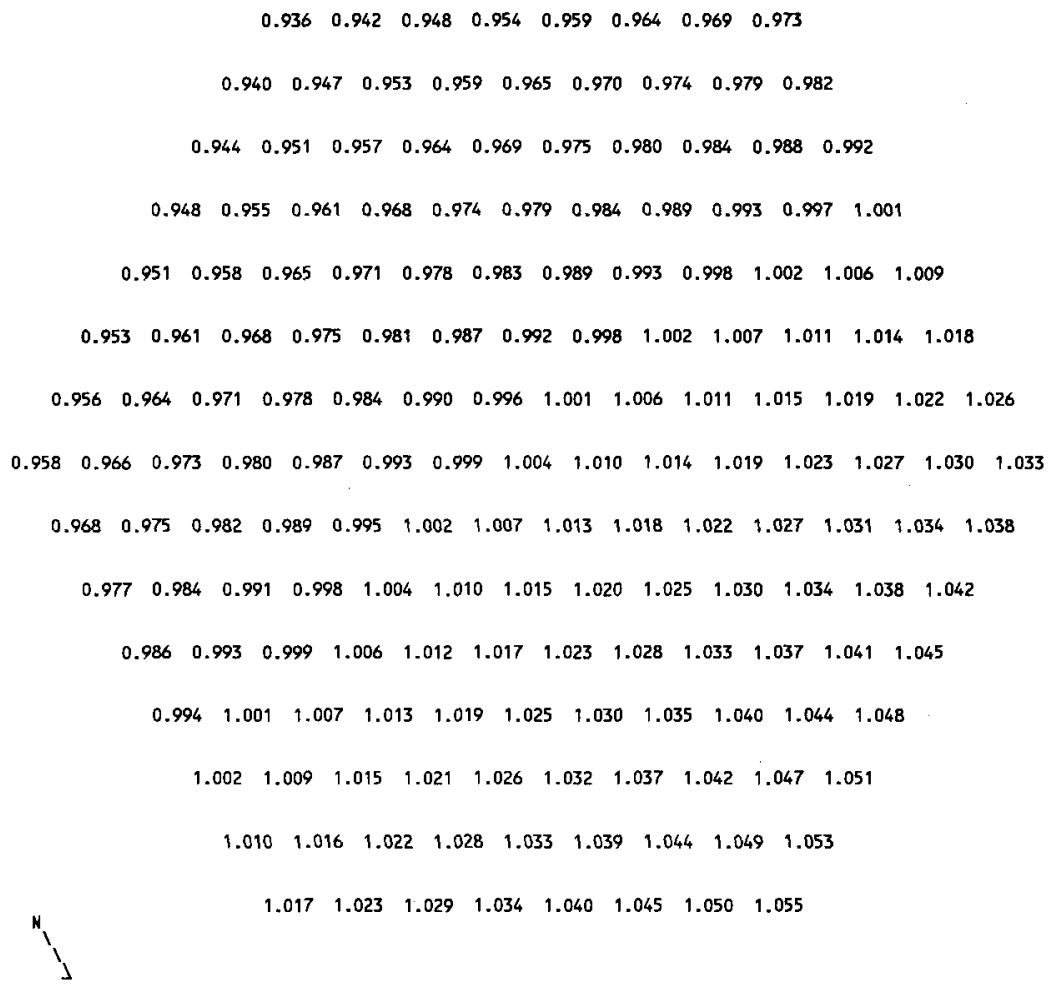


HNF-SD-FF-ANAL-009 Rev. 1

Table B11A-1.6. Fission Power Distribution by Pin in MFA-2 at BOC 11A-1 CORE POSITION 2506

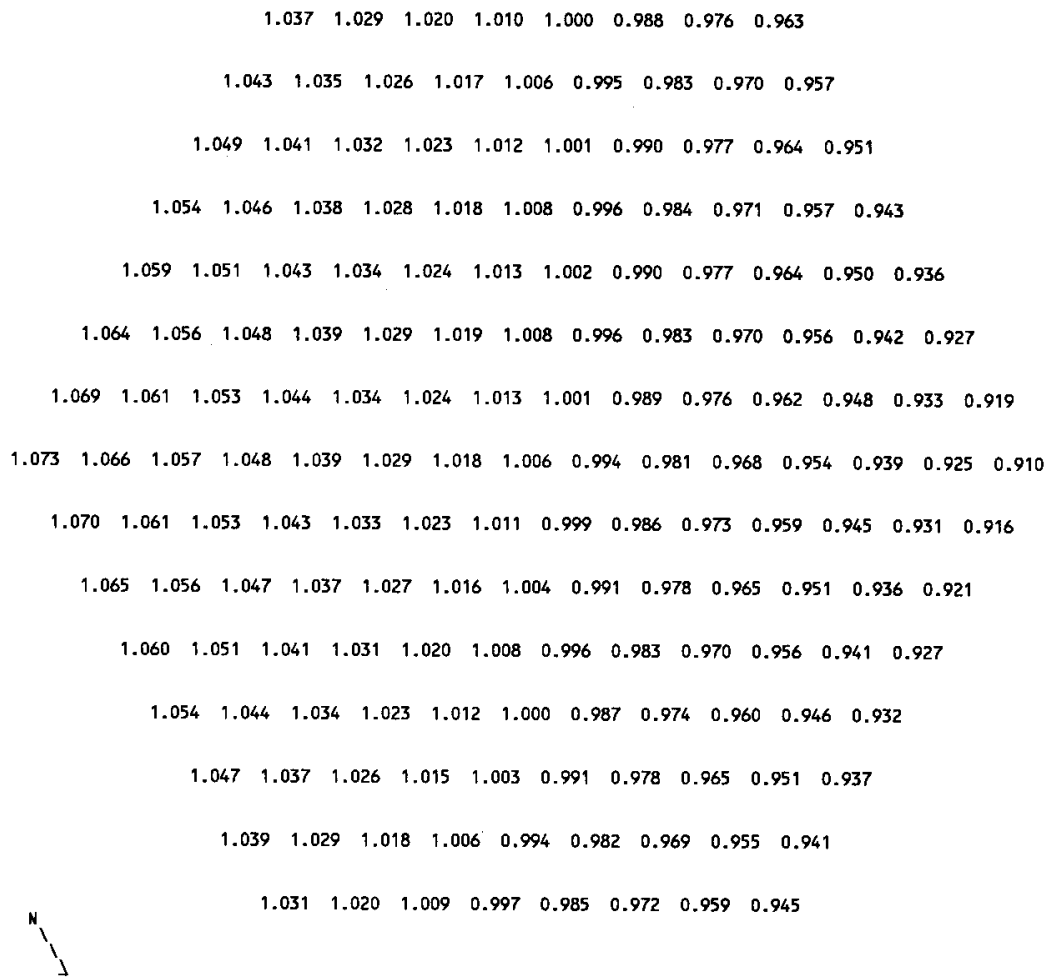


Table B11A-1.7. Fast F7ux Distribution by Pin in MFA-1 at BOC 11A-1 CORE POSITION 1404

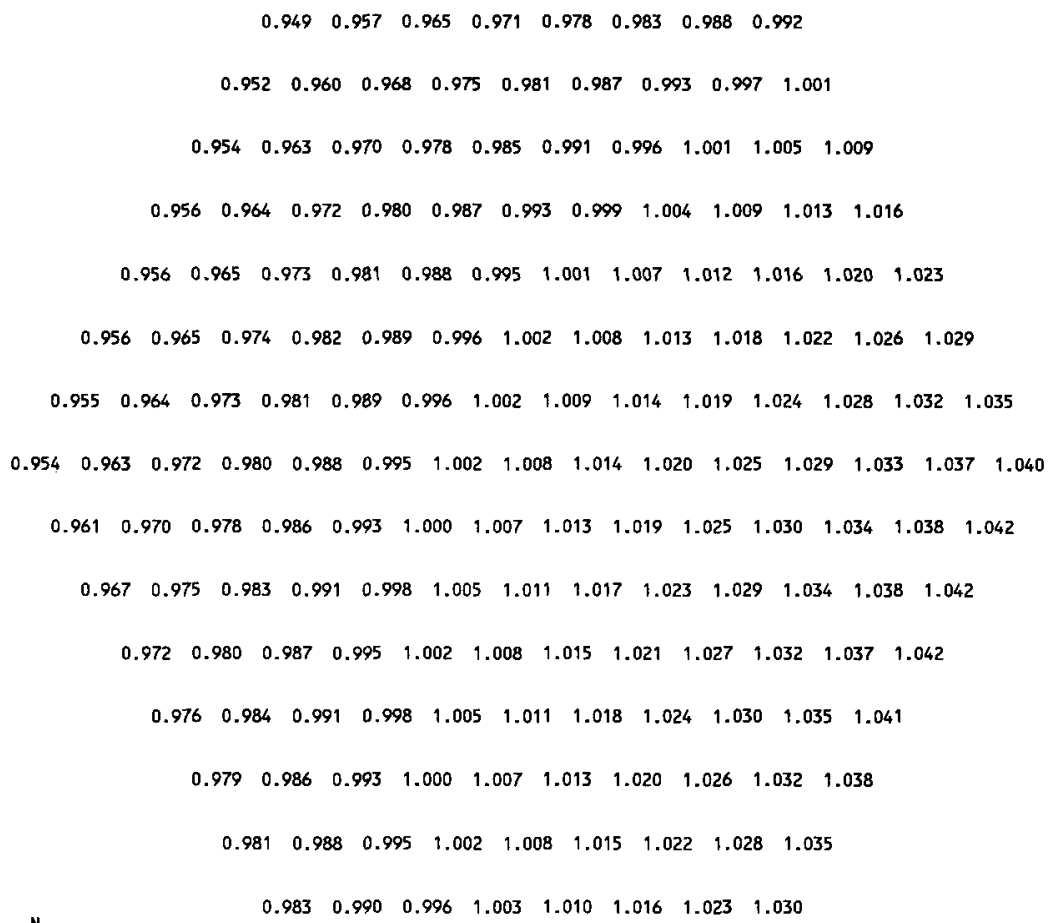


HNF-SD-FF-ANAL-009 Rev. 1

Table B11A-1.8. Fast Flux Distribution by Pin in MFA-2 at BOC $11 \mathrm{~A}-1$

CORE POSITION 2506

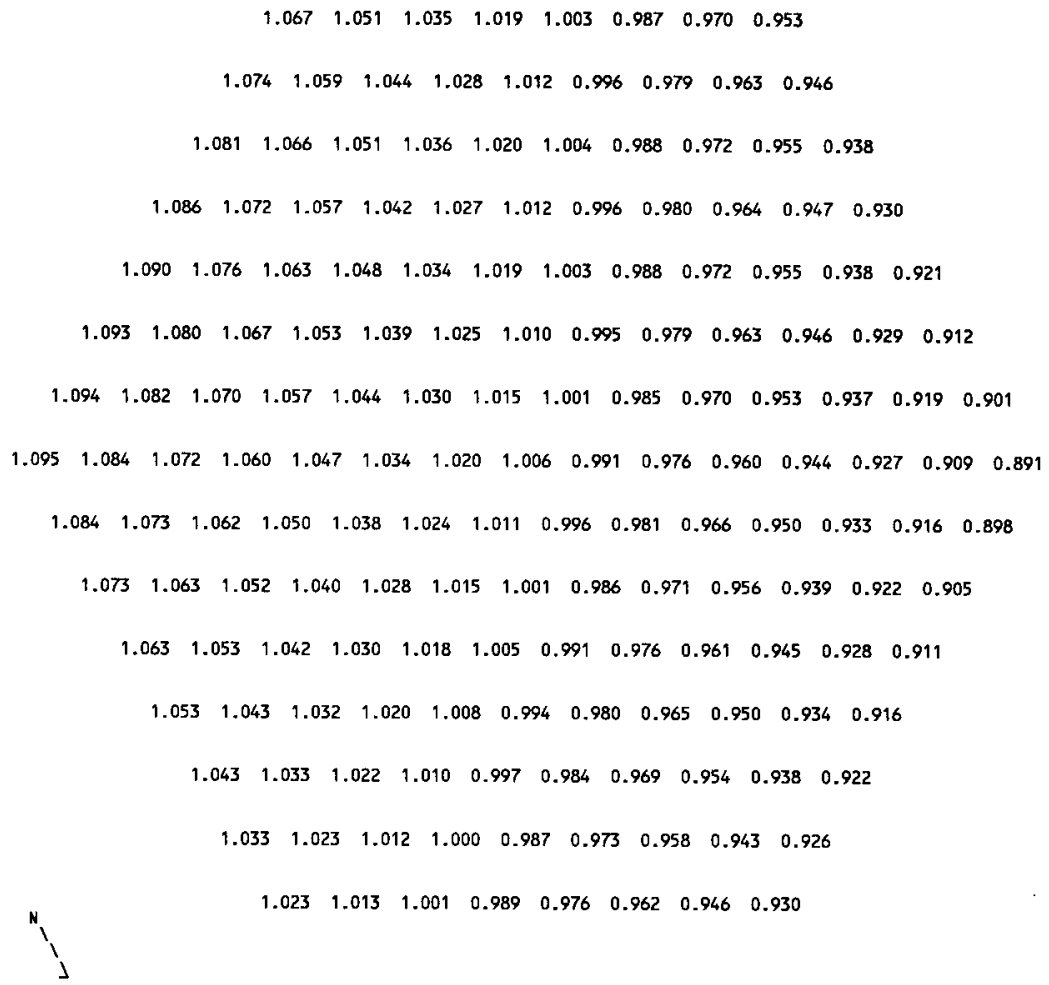


Table B11A-1.9. MFA-1 and MFA-2 Duct Wall Fast Flux Data at BOC 11A-1

$\begin{array}{ccc}\text { Side } & \begin{array}{c}\text { Flux }>0.1 \\ \text { MFA-1 }\end{array} & \begin{array}{c}\text { MeV }\left(\mathrm{n} / \mathrm{cm}^{2}-\mathrm{sec}\right) \\ \text { MFA-2 }\end{array} \\ \text { E } & 1.9844 \mathrm{E}+15 & 2.2273 \mathrm{E}+15 \\ \text { SE } & 1.9276 \mathrm{E}+15 & 2.3130 \mathrm{E}+15 \\ \text { SW } & 1.9732 \mathrm{E}+15 & 2.1096 \mathrm{E}+15 \\ \text { W } & 2.0858 \mathrm{E}+15 & 1.8996 \mathrm{E}+15 \\ \text { NW } & 2.1514 \mathrm{E}+15 & 1.8664 \mathrm{E}+15 \\ \text { NE } & 2.0586 \mathrm{E}+15 & 2.0465 \mathrm{E}+15\end{array}$

Table B11A-1.10. Assembly Outlet Temperatures and Flow Rates at BOC 11A-1

$\begin{array}{lcl}\text { CALCULATED } & \\ \text { CORE } & \begin{array}{c}\text { OUTLET TEMP. } \\ \text { POS. }\end{array} & \begin{array}{l}\text { FLOW RATE } \\ \text { (DEG F) }\end{array} \\ \text { (LB/H) } \\ 1404 & 1066 & 113570 \\ 1303 & 981 & 68360 \\ 1304 & 916 & 205640 \\ 1403 & 858 & 205660 \\ 1405 & 886 & 205660 \\ 1505 & 893 & 195550 \\ 1506 & 887 & 205640 \\ 2506 & 1059 & 113570 \\ 2404 & 1055 & 202240 \\ 2405 & 882 & 205660 \\ 2505 & 898 & 205640 \\ 2507 & 899 & 205640 \\ 2606 & 937 & 168640 \\ 2607 & 917 & 161730\end{array}$

Assembly flows are based on a calculated core pressure drop of $113.3 \mathrm{psi}$ at a total reactor flow rate of $16.67 \mathrm{E}+06 \mathrm{lb} / \mathrm{hr}$. 
temperature map for bundle 23 ASSEMBLY NO. 1484 MFA-1
axial hoight 44.860 in

$893 \quad 897 \quad 899 \quad 981$

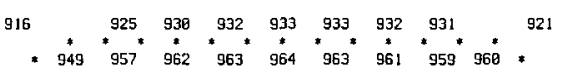

$\mathbf{8 8 1}$

$1010 \quad 1026 \quad 10301032 \quad 1033 \quad 10311019$

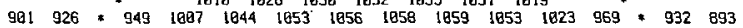

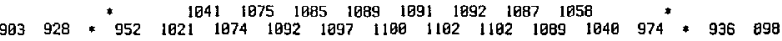

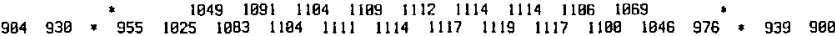

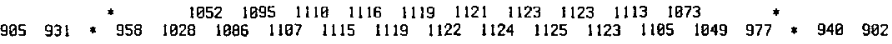

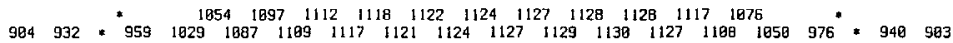

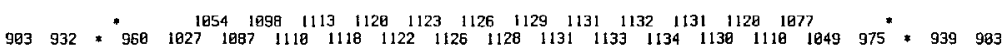

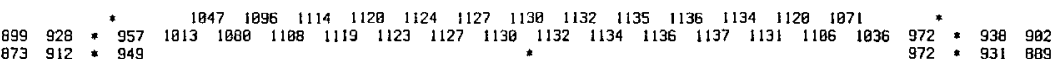

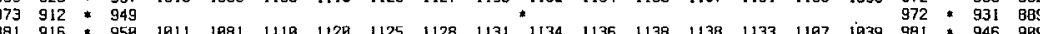

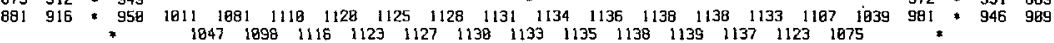

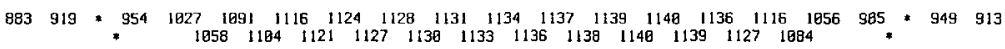

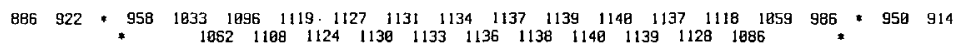

$888 \quad 925 \quad * 962 \quad 1037 \quad 1899 \quad 1122 \quad 1139 \quad 1133 \quad 1136 \quad 1138 \cdot 1146 \quad 1137 \quad 1119 \quad 1859 \quad 985$ * $950 \quad 915$

- $889 \quad 928 * 965 \quad 1848 \quad 1188 \quad 1122 \quad 1129 \quad 1132 \quad 1135 \quad 1137 \quad 1135 \quad 1117 \quad 1858 \quad 982 *{ }^{*} \quad 949 \quad 915$

$\%$

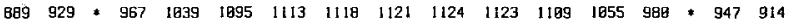

$888 \quad 927 * 965 \quad 1026 \quad 1063 \quad 1073 \quad 1077 \quad 1080 \quad 1082 \quad 1076 \quad 1048 \quad 977$ * $945 \quad 913$

- $959964970976 \quad 988984986 \quad 984975$ *

933

* * 956 "

$\begin{array}{lllllllll}906 & 947 & 951 & 954 & 956 & 957 & 956 & 954 & 923\end{array}$ 
Table B11A-1.12. MFA-2 Sodium Subchanne1 Temperatures at Top of Core Elevation for BOC 11A-1

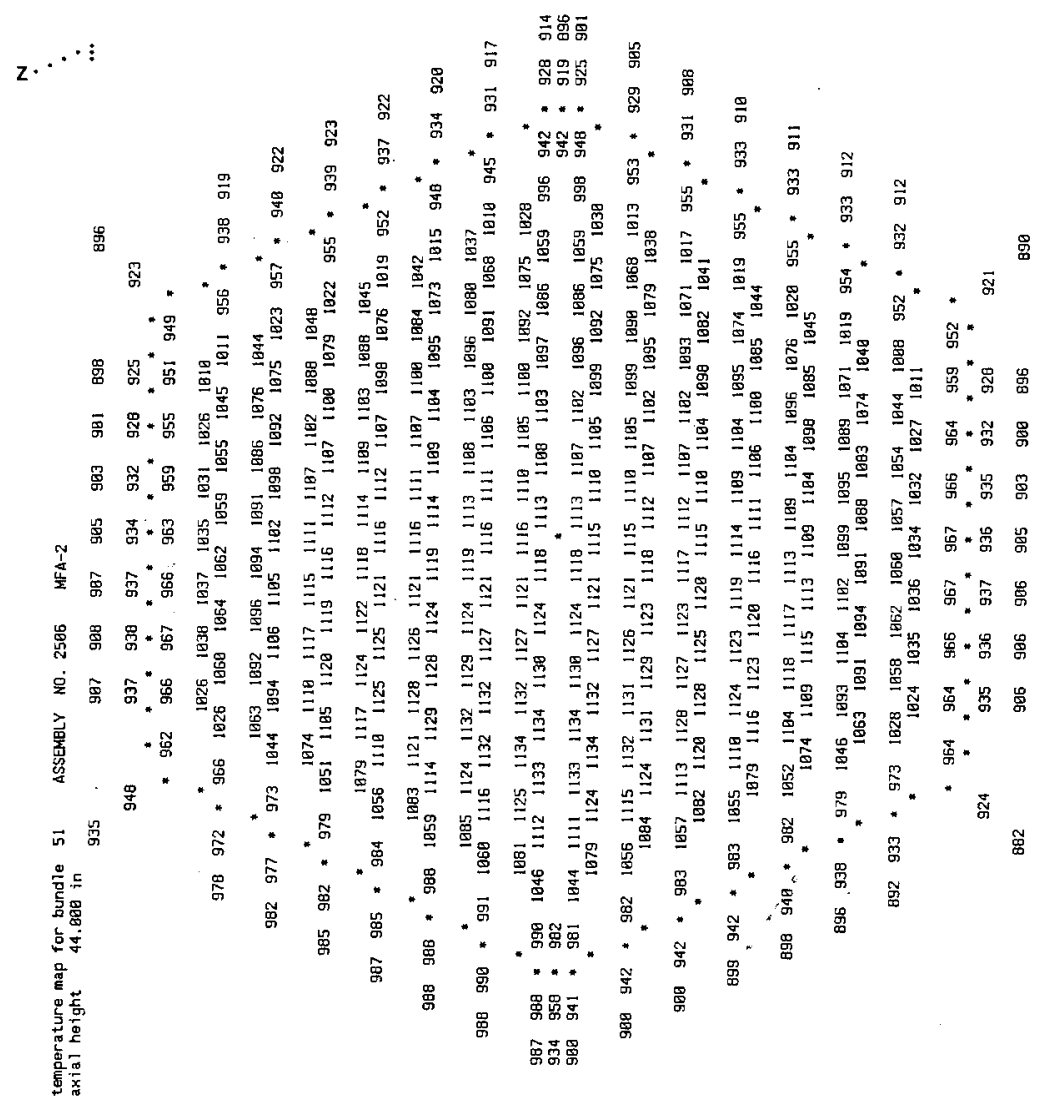


Table B11A-1.13. MFA-1 Sodium Subchannel Temperatures at Elevation of Upper Axial Blanket for BOC 11A-1

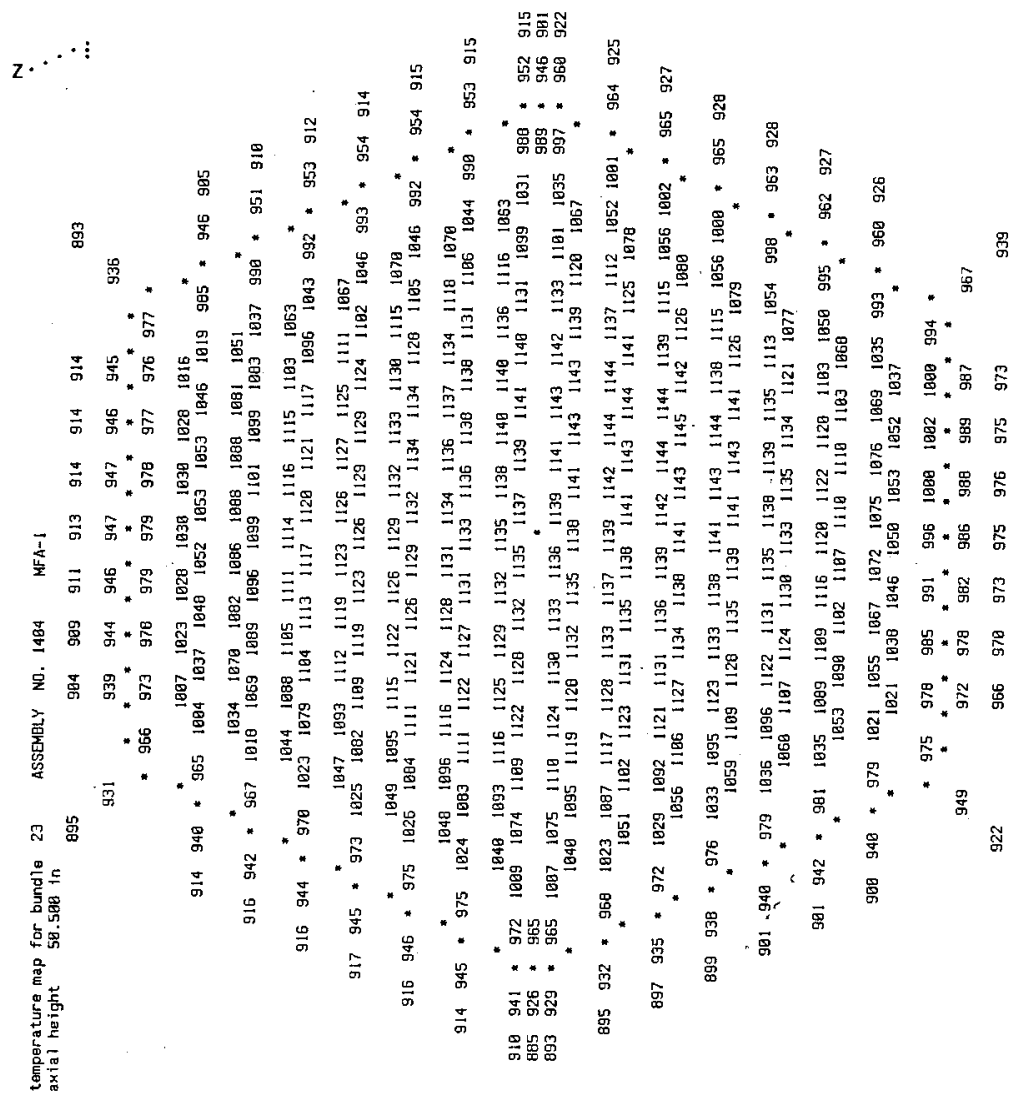


Table B11A-1.14. MFA-2 Sodium Subchannel Temperatures at Elevation of Upper Axial Blanket for BOC 11A-1

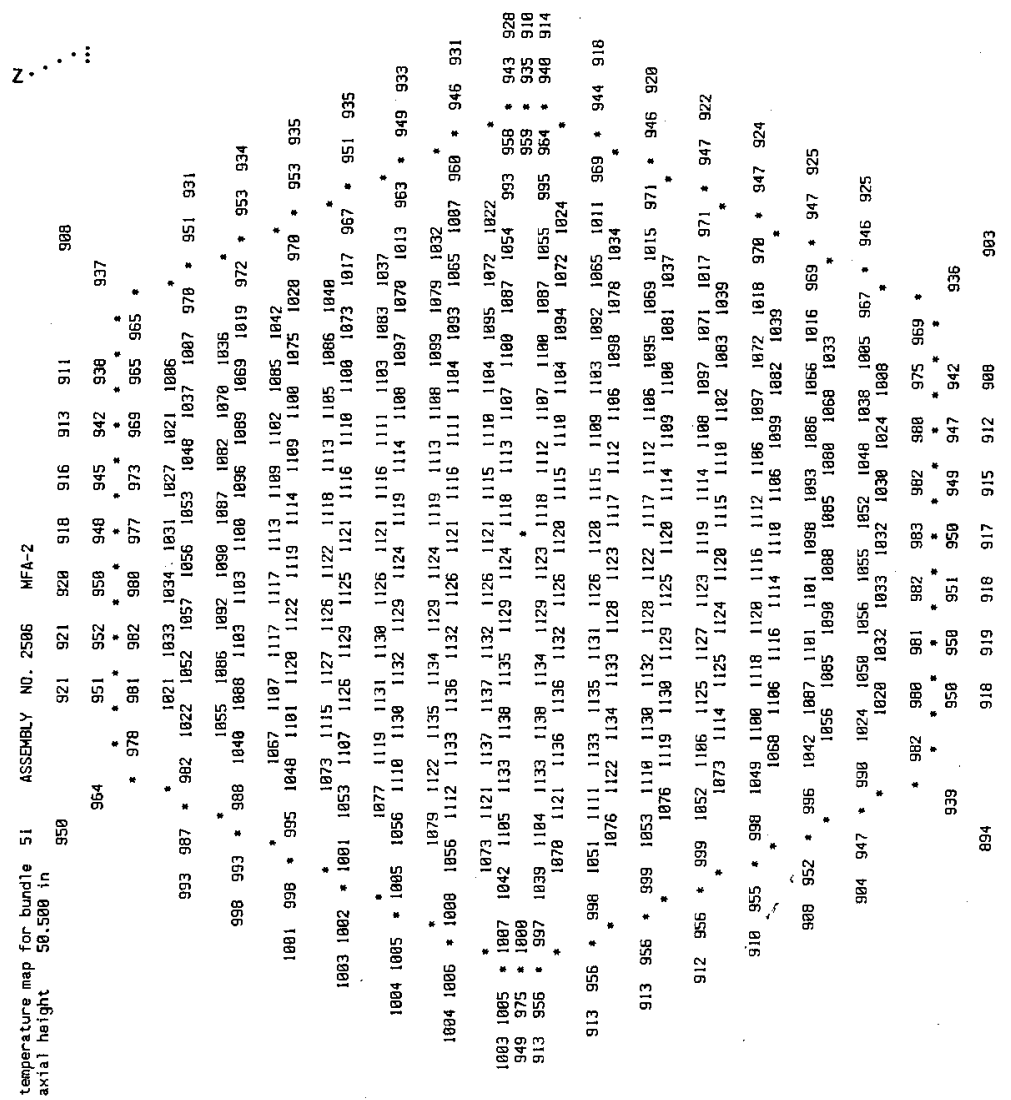


Table B11A-1.15. MFA-1 Sodium Subchannel Temperatures at Top of Fuel Pin Bundle for BOC 11A-1

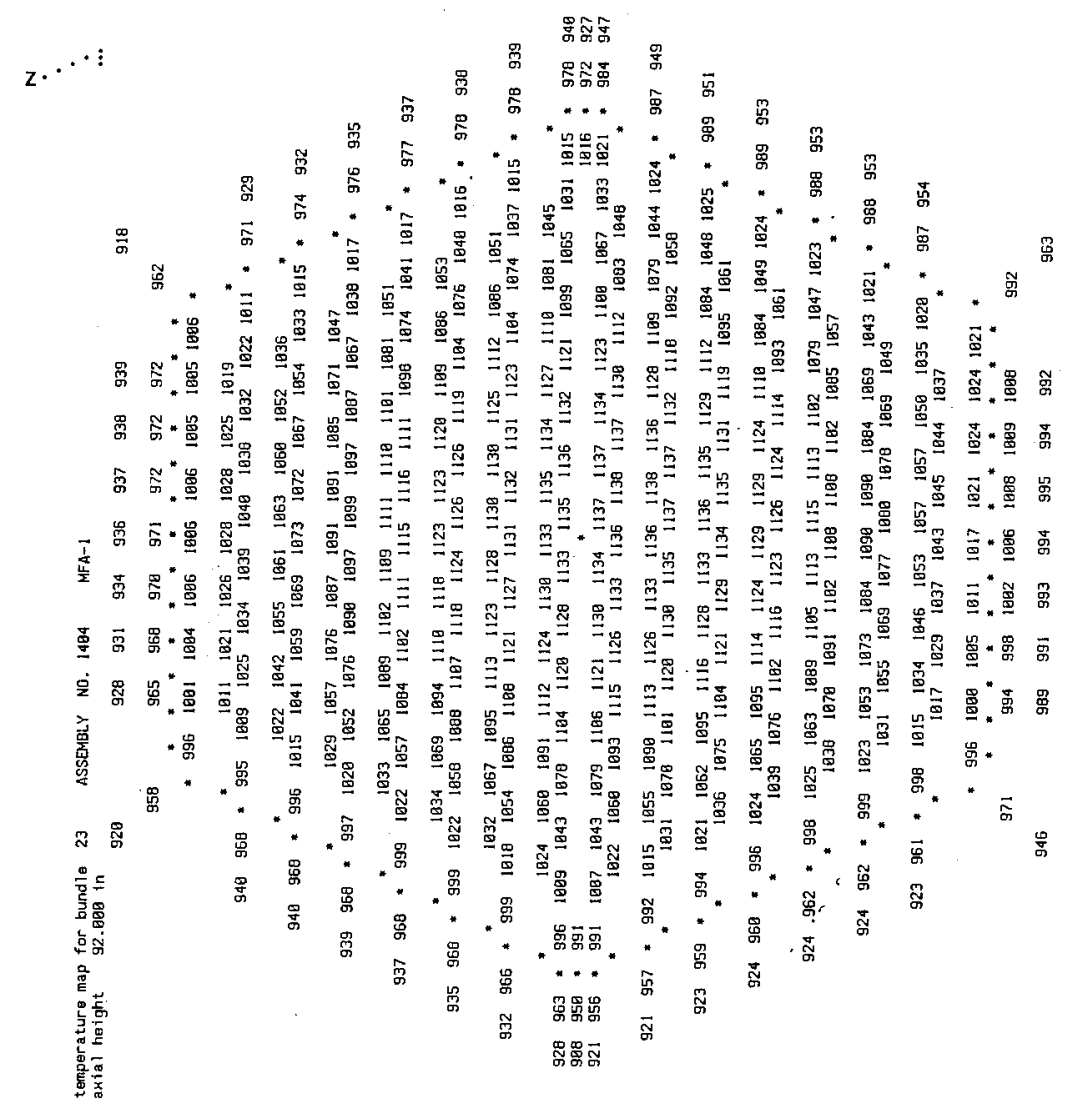


temperature map for bundle 51 ASSEMBLY NO. 2586 MFA-2

$\begin{array}{lllllllllll}\text { axial height } & 92.898 & \text { in } & & & & & & & & \\ & & 979 & 948 & 948 & 947 & 946 & 944 & 942 & 941 & 935\end{array}$

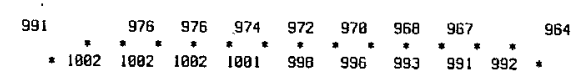

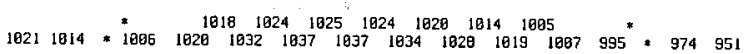

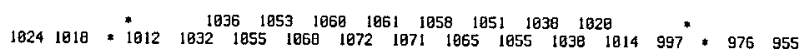

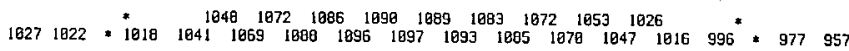

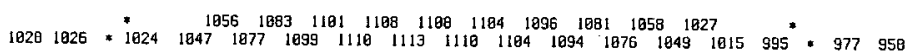

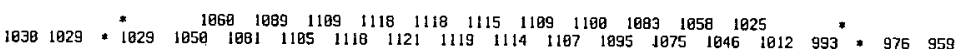

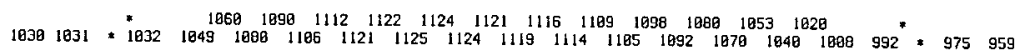

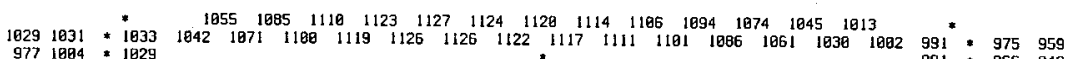

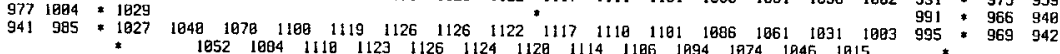

$\begin{array}{ccccccccccccccccccc}540 \quad 983 * & * & 1026 & 1045 & 1078 & 1105 & 1120 & 1125 & 1123 & 1119 & 1113 & 1185 & 1091 & 1071 & 1042 & 1012 & 998 & * 971 & 945\end{array}$

$939 \quad 982 * 1025 \quad 1947 \quad 1880 \quad 1104 \quad 1117 \quad 1120 \quad 1118 \quad 1113 \quad 1105 \quad 1894 \quad 1875 \quad 1847 \quad 1816 \quad 999 * 973 \quad 947$

$937 \quad 981 * 1825 \quad 1846 \quad 1877 \quad 1899 \quad 1189 \quad 1111 \quad 1109 \quad 1102 \quad 1092 \quad 1075 \quad 1049 \quad 1018 \quad 999 * 974 \quad 948$

$935 * 980 * 1023 \quad 1044 \quad 1070 \quad 1868 \quad 1895 \quad 1896 \quad 1892 \quad 1084 \quad 1869 \quad 1046 \quad 1018 \quad 999 \quad * 975 \quad 958$

$\begin{array}{lllllllllllllll}933 \quad 977 * 1021 \quad 1037 \quad 1057 \quad 1069 \quad 1072 \quad 1071 \quad 1066 \quad 1056 \quad 1039 & 1015 \quad 998 * & 975 & 951\end{array}$

$939 \quad 974 * 1016 \quad 1027 \quad 1036 \quad 1040 \quad 1841 \quad 1040 \quad 1835 \quad 1025 \quad 1010 \quad 998 * 975 \quad 951$

* $1011 \quad 1010100910101010 \quad 16091087 \quad 1003 \quad 999 *$

966 * " 977 "

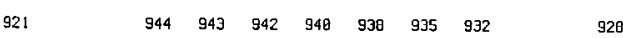


HNF-SD-FF-ANAL-009 Rev. 1

Table E11A-1.17. Composition of MFA-1 at EOC 11A-1

\begin{tabular}{|c|c|c|c|c|c|}
\hline sial & Axial & ge $(c m)$ & & Atom Den & $(a / b-c m)$ \\
\hline Level & $\begin{array}{r}\text { Lower } \\
-128.05\end{array}$ & Upper & Constituent & Fresh & nd of cycle \\
\hline & & & $\begin{array}{l}\mathrm{Na}-23 \\
\mathrm{SS}-316\end{array}$ & $\begin{array}{l}5.3150 E-03 \\
6.4090 E-02\end{array}$ & $\begin{array}{l}5.3150 E-03 \\
6.4090 E-02\end{array}$ \\
\hline 2 & -75.28 & -62.78 & & & \\
\hline & & & $\begin{array}{l}\mathrm{Na}-23 \\
\mathrm{SS}-316\end{array}$ & $\begin{array}{l}1.3177 \mathrm{E}-02 \\
3.4175 \mathrm{E}-02\end{array}$ & $\begin{array}{l}1.3177 \mathrm{E}-02 \\
3.4175 \mathrm{E}-02\end{array}$ \\
\hline 3 & -62.78 & -46.14 & & & \\
\hline & & & $\begin{array}{l}\mathrm{U}-235 \\
\mathrm{U}-238 \\
\mathrm{Pu}-239 \\
\mathrm{Pu}-240 \\
\mathrm{FP} \\
0-16 \\
\mathrm{Na}-23 \\
\mathrm{SS}-316\end{array}$ & $\begin{array}{l}1.4143 \mathrm{E}-05 \\
7.0573 \mathrm{E}-03 \\
0.0000 \mathrm{E}+00 \\
0.0000 \mathrm{E}+00 \\
0.0000 \mathrm{E}+00 \\
1.4143 \mathrm{E}-02 \\
9.7416 \mathrm{E}-03 \\
1.9073 \mathrm{E}-02\end{array}$ & $\begin{array}{l}1.1443 \mathrm{E}-05 \\
6.9115 \mathrm{E}-03 \\
1.2528 \mathrm{E}-04 \\
3.7604 \mathrm{E}-06 \\
1.8643 \mathrm{E}-05 \\
1.4143 \mathrm{E}-02 \\
9.7416 \mathrm{E}-03 \\
1.9073 \mathrm{E}-02\end{array}$ \\
\hline 4 & -46.14 & -30.76 & & & \\
\hline & & & $\begin{array}{l}\mathrm{U}-235 \\
\mathrm{U}-238 \\
\mathrm{Pu}-239 \\
\mathrm{Pu}-240 \\
\mathrm{Pu}-241 \\
\mathrm{Pu}-242 \\
\mathrm{Am}-241 \\
\mathrm{FP} \\
0-16 \\
\mathrm{Na}-23 \\
\mathrm{SS}-316\end{array}$ & $\begin{array}{l}1.0840 \mathrm{E}-05 \\
4.4499 \mathrm{E}-03 \\
1.7520 \mathrm{E}-03 \\
2.2340 \mathrm{E}-04 \\
1.9280 \mathrm{E}-05 \\
4.7430 \mathrm{E}-06 \\
7.6680 \mathrm{E}-07 \\
0.0000 \mathrm{E}+00 \\
1.2670 \mathrm{E}-02 \\
9.8100 \mathrm{E}-03 \\
1.8990 \mathrm{E}-02\end{array}$ & $\begin{array}{l}8.9775 \mathrm{E}-06 \\
4.3400 \mathrm{E}-03 \\
1.5398 \mathrm{E}-03 \\
2.6346 \mathrm{E}-04 \\
2.2098 \mathrm{E}-05 \\
4.9950 \mathrm{E}-06 \\
1.9866 \mathrm{E}-06 \\
2.7877 \mathrm{E}-04 \\
1.2670 \mathrm{E}-02 \\
9.8100 \mathrm{E}-03 \\
1.8990 \mathrm{E}-02\end{array}$ \\
\hline 5 & -30.76 & -15.38 & & & \\
\hline & & & $\begin{array}{l}\mathrm{U}-235 \\
\mathrm{U}-238 \\
\mathrm{Pu}-239 \\
\mathrm{Pu}-240 \\
\mathrm{Pu}-241 \\
\mathrm{Pu}-242 \\
\mathrm{Am}-241 \\
\mathrm{FP} \\
0-16 \\
\mathrm{Na}-23 \\
\mathrm{SS}-316\end{array}$ & $\begin{array}{l}1.0840 \mathrm{E}-05 \\
4.4499 \mathrm{E}-03 \\
1.7520 \mathrm{E}-03 \\
2.2340 \mathrm{E}-04 \\
1.9280 \mathrm{E}-05 \\
4.7430 \mathrm{E}-06 \\
7.6680 \mathrm{E}-07 \\
0.0000 \mathrm{E}+00 \\
1.2670 \mathrm{E}-02 \\
9.8100 \mathrm{E}-03 \\
1.8990 \mathrm{E}-02\end{array}$ & $\begin{array}{l}8.5737 \mathrm{E}-06 \\
4.3113 \mathrm{E}-03 \\
1.4861 \mathrm{E}-03 \\
2.6588 \mathrm{E}-04 \\
2.2286 \mathrm{E}-05 \\
4.9976 \mathrm{E}-06 \\
1.9536 \mathrm{E}-06 \\
3.5896 \mathrm{E}-04 \\
1.2670 \mathrm{E}-02 \\
9.8100 \mathrm{E}-03 \\
1.8990 \mathrm{E}-02\end{array}$ \\
\hline 6 & -15.38 & 0.00 & & & \\
\hline & & & $\begin{array}{l}\mathrm{U}-235 \\
\mathrm{U}-238 \\
\mathrm{Pu}-239 \\
\mathrm{Pu}-240 \\
\mathrm{Pu}-241 \\
\mathrm{Pu}-242 \\
\mathrm{Am}-241 \\
\mathrm{FP} \\
0-16 \\
\mathrm{Na}-23 \\
\mathrm{SS}-316\end{array}$ & $\begin{array}{l}1.0840 \mathrm{E}-05 \\
4.4499 \mathrm{E}-03 \\
1.7520 \mathrm{E}-03 \\
2.2340 \mathrm{E}-04 \\
1.9280 \mathrm{E}-05 \\
4.7430 \mathrm{E}-06 \\
7.6680 \mathrm{E}-07 \\
0.0000 \mathrm{E}+00 \\
1.2670 \mathrm{E}-02 \\
9.8100 \mathrm{E}-03 \\
1.8990 \mathrm{E}-02\end{array}$ & $\begin{array}{l}8.3537 \mathrm{E}-06 \\
4.2961 \mathrm{E}-03 \\
1.4595 \mathrm{E}-03 \\
2.6936 \mathrm{E}-04 \\
2.2735 \mathrm{E}-05 \\
5.0297 \mathrm{E}-06 \\
1.9464 \mathrm{E}-06 \\
3.9678 \mathrm{E}-04 \\
1.2670 \mathrm{E}-02 \\
9.8100 \mathrm{E}-03 \\
1.8990 \mathrm{E}-02\end{array}$ \\
\hline
\end{tabular}


HNF-SD-FF-ANAL-009 Rev. I

$\begin{array}{lll}7 & 0.00 & 15.38\end{array}$

$\begin{array}{lll}\mathrm{U}-235 & 1.0840 \mathrm{E}-05 & 8.4260 \mathrm{E}-06 \\ \mathrm{U}-238 & 4.4499 \mathrm{E}-03 & 4.3011 \mathrm{E}-03 \\ \mathrm{Pu}-239 & 1.7520 \mathrm{E}-03 & 1.4679 \mathrm{E}-03 \\ \mathrm{Pu}-240 & 2.2340 \mathrm{E}-04 & 2.6797 \mathrm{E}-04 \\ \mathrm{Pu}-24 \mathrm{I} & 1.9280 \mathrm{E}-05 & 2.2556 \mathrm{E}-05 \\ \mathrm{Pu}-242 & 4.7430 \mathrm{E}-06 & 5.0164 \mathrm{E}-06 \\ \mathrm{Am}-241 & 7.6680 \mathrm{E}-07 & 1.9489 \mathrm{E}-06 \\ \mathrm{FP} & 0.0000 \mathrm{E}+00 & 3.8482 \mathrm{E}-04 \\ 0-16 & 1.2670 \mathrm{E}-02 & 1.2670 \mathrm{E}-02 \\ \mathrm{Na}-23 & 9.8100 \mathrm{E}-03 & 9.8100 \mathrm{E}-03 \\ \mathrm{SS}-316 & 1.8990 \mathrm{E}-02 & 1.8990 \mathrm{E}-02\end{array}$

$\begin{array}{lll}8 & 15.38 & 30.76\end{array}$

U-235 $\quad 1.0840 E-05 \quad 8.7772 E-06$

$\mathrm{U}-238 \quad 4.4499 \mathrm{E}-03 \quad 4.3248 \mathrm{E}-03$

$\mathrm{Pu}-239 \quad 1.7520 \mathrm{E}-03 \quad 1.5097 \mathrm{E}-03$

$\mathrm{Pu}-240 \quad 2.2340 \mathrm{E}-04 \quad 2.6191 \mathrm{E}-04$

Pu-24l 1.9280E-05 2.1797E-05

$\mathrm{Pu}-242 \quad 4.7430 \mathrm{E}-06 \quad 4.9631 \mathrm{E}-06$

Am-241 7.6680E-07 $1.9604 \mathrm{E}-06$

FP $\quad 0.0000 E+00 \quad 3.2626 \mathrm{E}-04$

0-16 $1.2670 \mathrm{E}-02 \quad 1.2670 \mathrm{E}-02$

$\mathrm{Na}-23 \quad 9.8100 \mathrm{E}-03 \quad 9.8100 \mathrm{E}-03$

SS-316 $1.8990 \mathrm{E}-02 \quad 1.8990 \mathrm{E}-02$

$\begin{array}{lll}9 & 30.76 & 46.14\end{array}$

$\begin{array}{lll}\mathrm{U}-235 & 1.0840 \mathrm{E}-05 & 9.2848 \mathrm{E}-06 \\ \mathrm{U}-238 & 4.4499 \mathrm{E}-03 & 4.3592 \mathrm{E}-03 \\ \mathrm{Pu}-239 & 1.7520 \mathrm{E}-03 & 1.5740 \mathrm{E}-03 \\ \mathrm{Pu}-240 & 2.2340 \mathrm{E}-04 & 2.5610 \mathrm{E}-04 \\ \mathrm{Pu}-241 & 1.9280 \mathrm{E}-05 & 2.1192 \mathrm{E}-05 \\ \mathrm{Pu}-242 & 4.7430 \mathrm{E}-06 & 4.9317 \mathrm{E}-06 \\ \mathrm{Am}-241 & 7.6680 \mathrm{E}-07 & 1.9908 \mathrm{E}-06 \\ \mathrm{FP} & 0.0000 \mathrm{E}+00 & 2.3354 \mathrm{E}-04 \\ 0-16 & 1.2670 \mathrm{E}-02 & 1.2670 \mathrm{E}-02 \\ \mathrm{Na}-23 & 9.8100 \mathrm{E}-03 & 9.8100 \mathrm{E}-03 \\ \mathrm{SS}-316 & 1.8990 \mathrm{E}-02 & 1.8990 \mathrm{E}-02\end{array}$

$\begin{array}{lll}10 & 46.14 & 62.78\end{array}$

$\begin{array}{lll}\mathrm{U}-235 & 1.4143 \mathrm{E}-05 & 1.2284 \mathrm{E}-05 \\ \mathrm{U}-238 & 7.0573 \mathrm{E}-03 & 6.9558 \mathrm{E}-03 \\ \mathrm{Pu}-239 & 0.0000 \mathrm{E}+00 & 8.9044 \mathrm{E}-05 \\ \mathrm{Pu}-240 & 0.0000 \mathrm{E}+00 & 1.6979 \mathrm{E}-06 \\ \mathrm{FP} & 0.0000 \mathrm{E}+00 & 1.2085 \mathrm{E}-05 \\ 0-16 & 1.4143 \mathrm{E}-02 & 1.4143 \mathrm{E}-02 \\ \mathrm{Na}-23 & 9.7416 \mathrm{E}-03 & 9.7416 \mathrm{E}-03 \\ \mathrm{SS}-316 & 1.9073 \mathrm{E}-02 & 1.9073 \mathrm{E}-02\end{array}$

$\begin{array}{lll}11 & 62.78 & 172.84\end{array}$

$\begin{array}{lll}\mathrm{Na}-23 & 8.2400 \mathrm{E}-03 & 8.2400 \mathrm{E}-03 \\ \mathrm{SS}-316 & 2.5562 \mathrm{E}-02 & 2.5562 \mathrm{E}-02\end{array}$


Table E11A-1.18. Composition of MFA-2 at EOC 11A-1

\begin{tabular}{|c|c|c|c|c|c|}
\hline ial & Axial & ge $(\mathrm{cm})$ & & Atom Densi & (b-cm) \\
\hline $\begin{array}{c}\text { vel } \\
1\end{array}$ & $\begin{array}{r}\text { Lower } \\
-128.05\end{array}$ & $\begin{array}{r}\text { Upper } \\
-75.28\end{array}$ & Constituent & Fresh & End of cycle \\
\hline & & & $\begin{array}{l}\mathrm{Na}-23 \\
\mathrm{SS}-316\end{array}$ & $\begin{array}{l}5.3150 \mathrm{E}-03 \\
6.4090 \mathrm{E}-02\end{array}$ & $\begin{array}{l}5.3150 E-03 \\
6.4090 E-02\end{array}$ \\
\hline 2 & -75.28 & -62.78 & $\begin{array}{l}\mathrm{Na}-23 \\
\mathrm{SS}-316\end{array}$ & $\begin{array}{l}1.3177 \mathrm{E}-02 \\
3.4175 \mathrm{E}-02\end{array}$ & $\begin{array}{l}1.3177 \mathrm{E}-02 \\
3.4175 \mathrm{E}-02\end{array}$ \\
\hline 3 & -62.78 & -46.14 & $\begin{array}{l}U-235 \\
U-238 \\
P u-239 \\
P u-240 \\
\text { FP } \\
0-16 \\
\mathrm{Na}-23 \\
\text { SS-316 }\end{array}$ & $\begin{array}{l}1.4143 \mathrm{E}-05 \\
7.0573 \mathrm{E}-03 \\
0.0000 \mathrm{E}+00 \\
0.0000 \mathrm{E}+00 \\
0.0000 \mathrm{E}+00 \\
1.4143 \mathrm{E}-02 \\
9.7416 \mathrm{E}-03 \\
1.9073 \mathrm{E}-02\end{array}$ & $\begin{array}{l}1.1346 \mathrm{E}-05 \\
6.9069 \mathrm{E}-03 \\
1.2877 \mathrm{E}-04 \\
4.0661 \mathrm{E}-06 \\
1.9589 \mathrm{E}-05 \\
1.4143 \mathrm{E}-02 \\
9.7416 \mathrm{E}-03 \\
1.9073 \mathrm{E}-02\end{array}$ \\
\hline 4 & -46.14 & -30.76 & $\begin{array}{l}\text { U-235 } \\
U-238 \\
P u-239 \\
P u-240 \\
P u-241 \\
P u-242 \\
\text { Am-241 } \\
\text { FP } \\
0-16 \\
\mathrm{Na}-23 \\
\text { SS-316 }\end{array}$ & $\begin{array}{l}1.0160 \mathrm{E}-05 \\
4.4270 \mathrm{E}-03 \\
1.7483 \mathrm{E}-03 \\
2.2290 \mathrm{E}-04 \\
1.9040 \mathrm{E}-05 \\
4.7330 \mathrm{E}-06 \\
7.5700 \mathrm{E}-07 \\
0.0000 \mathrm{E}+00 \\
1.2760 \mathrm{E}-02 \\
9.8100 \mathrm{E}-03 \\
1.8990 \mathrm{E}-02\end{array}$ & $\begin{array}{l}8.3228 \mathrm{E}-06 \\
4.3112 \mathrm{E}-03 \\
1.5261 \mathrm{E}-03 \\
2.6565 \mathrm{E}-04 \\
2.2262 \mathrm{E}-05 \\
5.0041 \mathrm{E}-06 \\
1.9625 \mathrm{E}-06 \\
2.9157 \mathrm{E}-04 \\
1.2760 \mathrm{E}-02 \\
9.8100 \mathrm{E}-03 \\
1.8990 \mathrm{E}-02\end{array}$ \\
\hline 5 & -30.76 & -15.38 & $\begin{array}{l}\text { U-235 } \\
\mathrm{U}-238 \\
\mathrm{Pu}-239 \\
\mathrm{Pu}-240 \\
\mathrm{Pu}-241 \\
\mathrm{Pu}-242 \\
\mathrm{Am}-241 \\
\mathrm{FP} \\
0-16 \\
\mathrm{Na}-23 \\
\mathrm{SS}-316\end{array}$ & $\begin{array}{l}1.0160 \mathrm{E}-05 \\
4.4270 \mathrm{E}-03 \\
1.7483 \mathrm{E}-03 \\
2.2290 \mathrm{E}-04 \\
1.9040 \mathrm{E}-05 \\
4.7330 \mathrm{E}-06 \\
7.5700 \mathrm{E}-07 \\
0.0000 \mathrm{E}+00 \\
1.2760 \mathrm{E}-02 \\
9.8100 \mathrm{E}-03 \\
1.8990 \mathrm{E}-02\end{array}$ & $\begin{array}{l}7.9069 \mathrm{E}-06 \\
4.2792 \mathrm{E}-03 \\
1.4677 \mathrm{E}-03 \\
2.6861 \mathrm{E}-04 \\
2.2529 \mathrm{E}-05 \\
5.0113 \mathrm{E}-06 \\
1.9274 \mathrm{E}-06 \\
3.7900 \mathrm{E}-04 \\
1.2760 \mathrm{E}-02 \\
9.8100 \mathrm{E}-03 \\
1.8990 \mathrm{E}-02\end{array}$ \\
\hline 6 & 15.38 & 0.00 & $\begin{array}{l}\text { U-235 } \\
U-238 \\
\mathrm{Pu}-239 \\
\mathrm{Pu}-240 \\
\mathrm{Pu}-241 \\
\mathrm{Pu}-242 \\
\mathrm{Am}-241 \\
\mathrm{FP} \\
\mathrm{O}-16 \\
\mathrm{Na}-23 \\
\mathrm{SS}-316\end{array}$ & $\begin{array}{l}1.0160 \mathrm{E}-05 \\
4.4270 \mathrm{E}-03 \\
1.7483 \mathrm{E}-03 \\
2.2290 \mathrm{E}-04 \\
1.9040 \mathrm{E}-05 \\
4.7330 \mathrm{E}-06 \\
7.5700 \mathrm{E}-07 \\
0.0000 \mathrm{E}+00 \\
1.2760 \mathrm{E}-02 \\
9.8100 \mathrm{E}-03 \\
1.8990 \mathrm{E}-02\end{array}$ & $\begin{array}{l}7.6750 \mathrm{E}-06 \\
4.2620 \mathrm{E}-03 \\
1.4381 \mathrm{E}-03 \\
2.7257 \mathrm{E}-04 \\
2.3072 \mathrm{E}-05 \\
5.0505 \mathrm{E}-06 \\
1.9194 \mathrm{E}-06 \\
4.2145 \mathrm{E}-04 \\
1.2760 \mathrm{E}-02 \\
9.8100 \mathrm{E}-03 \\
1.8990 \mathrm{E}-02\end{array}$ \\
\hline
\end{tabular}


HNF-SD-FF-ANAL-009 Rev. I

7

$0.00 \quad 15.38$

8

9

$30.76 \quad 46.14$

10

46.14

62.78

11

$15.38 \quad 30.76$

\section{$\mathrm{U}-235$ \\ $\mathrm{U}-238$ \\ $\mathrm{Pu}-239$ \\ $\mathrm{Pu}-240$ \\ $\mathrm{Pu}-241$ \\ $\mathrm{Pu}-242$ \\ Am-241 \\ FP}

$0-16$

$\mathrm{Na}-23$

SS -316

$\mathrm{U}-235$

$\mathrm{U}-238$

$\mathrm{Pu}-239$

$\mathrm{Pu}-240$

$\mathrm{Pu}-241$

$\mathrm{Pu}-242$

Am-241

FP

$0-16$

$\mathrm{Na}-23$

SS-316

\section{U-235}

$\mathrm{U}-238$

$\mathrm{Pu}-239$

$\mathrm{Pu}-240$

$\mathrm{Pu}-241$

$\mathrm{Pu}-242$

Am-24l

FP

0-16

$\mathrm{Na}-23$

SS-316
1. $0160 \mathrm{E}-05$

4. 4270E-03

1. $7483 \mathrm{E}-03$

2. $2290 \mathrm{E}-04$

1. $9040 \mathrm{E}-05$

4.7330E-06

$7.5700 \mathrm{E}-07$

$0.0000 \mathrm{E}+00$

1. $2760 \mathrm{E}-02$

9. $8100 \mathrm{E}-03$

1. 8990E-02

1.0160E-05

4. $4270 \mathrm{E}-03$

$1.7483 \mathrm{E}-03$

2. $2290 \mathrm{E}-04$

1. $9040 \mathrm{E}-05$

4. $7330 \mathrm{E}-06$

$7.5700 \mathrm{E}-07$

$0.0000 E+00$

1.2760E-02

$9.8100 \mathrm{E}-03$

1. $8990 \mathrm{E}-02$

1. $0160 \mathrm{E}-05$

4. $4270 \mathrm{E}-03$

$1.7483 \mathrm{E}-03$

$2.2290 \mathrm{E}-04$

1. $9040 \mathrm{E}-05$

4.7330E-06

7.5700E-07

$0.0000 E+00$

1.2760E-02

9.8100E-03

1.8990E-02
7.7370E-06

4. $2666 \mathrm{E}-03$

$1.4456 \mathrm{E}-03$

2.7134E-04

2.2901E-05

5.0376E-06

1.9215E-06

4.1054E-04

1. $2760 \mathrm{E}-02$

9.8100E-03

1. $8990 \mathrm{E}-02$

8.0748E-06

$4.2913 \mathrm{E}-03$

$1.4885 \mathrm{E}-03$

2. $6517 \mathrm{E}-04$

2. $2088 \mathrm{E}-05$

4. $9800 \mathrm{E}-06$

$1.9328 \mathrm{E}-06$

3. $5000 \mathrm{E}-04$

1. $2760 \mathrm{E}-02$

$9.8100 \mathrm{E}-03$

1.8990E-02

8. 5755E-06

4.3280E-03

1. $5559 \mathrm{E}-03$

2.5921E-04

2. $1437 \mathrm{E}-05$

4. $9463 \mathrm{E}-06$

1. $9650 \mathrm{E}-06$

2.5211E-04

1.2760E-02

9.8100E-03

1. 8990E-02

U-235 $\quad 1.4143 \mathrm{E}-05$

U-238 7.0573E-03

$\mathrm{Pu}-239 \quad 0.0000 \mathrm{E}+00$

$\mathrm{Pu}-240 \quad 0.0000 \mathrm{E}+00$

FP $\quad 0.0000 \mathrm{E}+00$

0-16 $\quad 1.4143 \mathrm{E}-02$

$\mathrm{Na}-23 \quad 9.7416 \mathrm{E}-03$

SS-316 $1.9073 \mathrm{E}-02$

1.2081E-05

$6.9459 \mathrm{E}-03$

9.7223E-05

$2.1254 \mathrm{E}-06$

$1.3579 \mathrm{E}-05$

$1.4143 \mathrm{E}-02$

$9.7416 \mathrm{E}-03$

$1.9073 \mathrm{E}-02$
$\mathrm{Na}-23 \quad 8.2400 \mathrm{E}-03$

8. $2400 \mathrm{E}-03$

2. $5562 \mathrm{E}-02$ 


\subsection{Cycle 11A-2}

Table B11A-2.1. Fission Power Generated in MFA-1, MFA-2 and Neighboring Assemblies at BOC 11A-2

\begin{tabular}{lccccc} 
CORE & $* * * * * *$ & \multicolumn{2}{c}{ POWER IN MEGAWATTS } & $* * * * * *$ \\
POS. & BELOW CORE & \multicolumn{2}{c}{ IN CORE } & ABOVE CORE & TOTAL PWR \\
1404 & $6.257 \mathrm{E}-02$ & $4.130 \mathrm{E}+00$ & $3.495 \mathrm{E}-02$ & $4.228 \mathrm{E}+00$ \\
1303 & $1.427 \mathrm{E}-01$ & $1.778 \mathrm{E}+00$ & $6.596 \mathrm{E}-02$ & $1.987 \mathrm{E}+00$ \\
1304 & $9.757 \mathrm{E}-03$ & $4.480 \mathrm{E}+00$ & $7.084 \mathrm{E}-03$ & $4.497 \mathrm{E}+00$ \\
1403 & $1.408 \mathrm{E}-02$ & $3.388 \mathrm{E}+00$ & $7.411 \mathrm{E}-03$ & $3.409 \mathrm{E}+00$ \\
1405 & $7.304 \mathrm{E}-03$ & $3.845 \mathrm{E}+00$ & $5.647 \mathrm{E}-03$ & $3.858 \mathrm{E}+00$ \\
1505 & $6.530 \mathrm{E}-03$ & $3.940 \mathrm{E}+00$ & $4.374 \mathrm{E}-03$ & $3.951 \mathrm{E}+00$ \\
1506 & $8.972 \mathrm{E}-03$ & $3.921 \mathrm{E}+00$ & $6.327 \mathrm{E}-03$ & $3.936 \mathrm{E}+00$ \\
2506 & $6.135 \mathrm{E}-02$ & $3.908 \mathrm{E}+00$ & $3.862 \mathrm{E}-02$ & $4.008 \mathrm{E}+00$ \\
2404 & $0.000 \mathrm{E}+00$ & $7.226 \mathrm{E}+00$ & $0.000 \mathrm{E}+00$ & $7.226 \mathrm{E}+00$ \\
2505 & $1.310 \mathrm{E}-02$ & $4.113 \mathrm{E}+00$ & $6.986 \mathrm{E}-03$ & $4.133 \mathrm{E}+00$ \\
2405 & $1.435 \mathrm{E}-02$ & $3.674 \mathrm{E}+00$ & $1.005 \mathrm{E}-02$ & $3.698 \mathrm{E}+00$ \\
2606 & $6.243 \mathrm{E}-03$ & $3.979 \mathrm{E}+00$ & $4.798 \mathrm{E}-03$ & $3.990 \mathrm{E}+00$ \\
2607 & $1.349 \mathrm{E}-02$ & $3.443 \mathrm{E}+00$ & $7.765 \mathrm{E}-03$ & $3.464 \mathrm{E}+00$ \\
2507 & $9.545 \mathrm{E}-03$ & $4.056 \mathrm{E}+00$ & $7.291 \mathrm{E}-03$ & $4.073 \mathrm{E}+00$
\end{tabular}

Table B11A-2.2. Assembly Averaged Total and Fast Flux in MFA-1 and MFA-2 at BOC $11 \mathrm{~A}-2$

\begin{tabular}{cccr} 
& Core & \multicolumn{2}{c}{ Flux $\left(\mathrm{n} / \mathrm{cm}^{2}-\mathrm{sec}\right)$} \\
Assembly & Pos. & Total & $>0.1 \mathrm{MeV}$ \\
MFA-1 & 1404 & $3.372 \mathrm{E}+15$ & $2.080 \mathrm{E}+15$ \\
MFA-2 & 2506 & $3.239 \mathrm{E}+15$ & $2.117 \mathrm{E}+15$
\end{tabular}


Table B11A-2.3. Axial Distribution of Total Flux, Fast Flux and Power in MFA-1 at BOC $11 \mathrm{~A}-2$

$\begin{array}{rccc}z(\mathrm{~cm}) & \text { Total Flux } & \text { Flux }>0.1 \text { MeV } & \text { Power } \\ -97.78 & 2.8883 \mathrm{E}-02 & 1.5414 \mathrm{E}-02 & 0.0000 \mathrm{E}+00 \\ -92.78 & 6.1995 \mathrm{E}-02 & 2.9706 \mathrm{E}-02 & 0.0000 \mathrm{E}+00 \\ -87.78 & 9.9294 \mathrm{E}-02 & 4.7418 \mathrm{E}-02 & 0.0000 \mathrm{E}+00 \\ -82.78 & 1.4354 \mathrm{E}-01 & 7.0510 \mathrm{E}-02 & 0.0000 \mathrm{E}+00 \\ -77.78 & 1.9750 \mathrm{E}-01 & 1.0138 \mathrm{E}-01 & 0.0000 \mathrm{E}+00 \\ -72.15 & 2.5833 \mathrm{E}-01 & 1.4105 \mathrm{E}-01 & 0.0000 \mathrm{E}+00 \\ -65.90 & 3.1899 \mathrm{E}-01 & 1.9040 \mathrm{E}-01 & 0.0000 \mathrm{E}+00 \\ -60.35 & 3.8210 \mathrm{E}-01 & 2.5593 \mathrm{E}-01 & 6.6848 \mathrm{E}-02 \\ -55.49 & 4.6462 \mathrm{E}-01 & 3.4136 \mathrm{E}-01 & 7.8429 \mathrm{E}-02 \\ -50.62 & 5.5953 \mathrm{E}-01 & 4.5056 \mathrm{E}-01 & 9.4837 \mathrm{E}-02 \\ -47.17 & 6.3493 \mathrm{E}-01 & 5.5251 \mathrm{E}-01 & 1.1240 \mathrm{E}-01 \\ -43.58 & 7.1413 \mathrm{E}-01 & 6.6863 \mathrm{E}-01 & 7.7002 \mathrm{E}-01 \\ -38.45 & 8.2853 \mathrm{E}-01 & 8.0963 \mathrm{E}-01 & 8.6054 \mathrm{E}-01 \\ -33.32 & 9.3487 \mathrm{E}-01 & 9.3040 \mathrm{E}-01 & 9.5983 \mathrm{E}-01 \\ -28.20 & 1.0279 \mathrm{E}+00 & 1.0311 \mathrm{E}+00 & 1.0186 \mathrm{E}+00 \\ -23.07 & 1.1058 \mathrm{E}+00 & 1.1135 \mathrm{E}+00 & 1.0936 \mathrm{E}+00 \\ -17.94 & 1.1666 \mathrm{E}+00 & 1.1767 \mathrm{E}+00 & 1.1525 \mathrm{E}+00 \\ -12.82 & 1.2091 \mathrm{E}+00 & 1.2202 \mathrm{E}+00 & 1.1759 \mathrm{E}+00 \\ -7.69 & 1.2332 \mathrm{E}+00 & 1.2447 \mathrm{E}+00 & 1.1989 \mathrm{E}+00 \\ -2.56 & 1.2383 \mathrm{E}+00 & 1.2500 \mathrm{E}+00 & 1.2036 \mathrm{E}+00 \\ 2.56 & 1.2243 \mathrm{E}+00 & 1.2360 \mathrm{E}+00 & 1.1956 \mathrm{E}+00 \\ 7.69 & 1.1914 \mathrm{E}+00 & 1.2027 \mathrm{E}+00 & 1.1633 \mathrm{E}+00 \\ 12.82 & 1.1403 \mathrm{E}+00 & 1.1510 \mathrm{E}+00 & 1.1134 \mathrm{E}+00 \\ 17.94 & 1.0727 \mathrm{E}+00 & 1.0827 \mathrm{E}+00 & 1.0723 \mathrm{E}+00 \\ 23.07 & 9.9016 \mathrm{E}-01 & 9.9817 \mathrm{E}-01 & 9.9025 \mathrm{E}-01 \\ 28.20 & 8.9510 \mathrm{E}-01 & 8.9963 \mathrm{E}-01 & 8.9616 \mathrm{E}-01 \\ 33.32 & 7.8996 \mathrm{E}-01 & 7.8893 \mathrm{E}-01 & 8.2206 \mathrm{E}-01 \\ 38.45 & 6.7692 \mathrm{E}-01 & 6.6545 \mathrm{E}-01 & 7.0977 \mathrm{E}-01 \\ 43.58 & 5.6076 \mathrm{E}-01 & 5.3057 \mathrm{E}-01 & 6.0355 \mathrm{E}-01 \\ 47.17 & 4.8226 \mathrm{E}-01 & 4.2705 \mathrm{E}-01 & 7.3145 \mathrm{E}-02 \\ 50.62 & 4.0821 \mathrm{E}-01 & 3.3649 \mathrm{E}-01 & 5.7010 \mathrm{E}-02 \\ 55.49 & 3.1676 \mathrm{E}-01 & 2.4099 \mathrm{E}-01 & 4.1822 \mathrm{E}-02 \\ 60.35 & 2.3788 \mathrm{E}-01 & 1.6766 \mathrm{E}-01 & 3.0891 \mathrm{E}-02 \\ 64.98 & 1.8907 \mathrm{E}-01 & 1.2539 \mathrm{E}-01 & 0.0000 \mathrm{E}+00 \\ 69.39 & 1.6095 \mathrm{E}-01 & 1.0315 \mathrm{E}-01 & 0.0000 \mathrm{E}+00 \\ 76.46 & 1.2151 \mathrm{E}-01 & 7.3336 \mathrm{E}-02 & 0.0000 \mathrm{E}+00 \\ 86.80 & 7.8859 \mathrm{E}-02 & 4.4308 \mathrm{E}-02 & 0.0000 \mathrm{E}+00 \\ 98.14 & 4.5387 \mathrm{E}-02 & 2.4236 \mathrm{E}-02 & 0.0000 \mathrm{E}+00 \\ 110.00 & 1.9456 \mathrm{E}-02 & 1.0840 \mathrm{E}-02 & 0.0000 \mathrm{E}+00\end{array}$


Table BllA-2.4. Axial Distribution of Total Flux, Fast Flux and Power in MFA-2 at BOC 11A-2

$\begin{array}{rccc}\text { Z }(\mathrm{cm}) & \text { Total Flux } & \text { Flux }>0.1 \text { MeV } & \text { Power } \\ -97.78 & 2.6242 \mathrm{E}-02 & 1.3319 \mathrm{E}-02 & 0.0000 \mathrm{E}+00 \\ -92.78 & 5.6314 \mathrm{E}-02 & 2.5716 \mathrm{E}-02 & 0.0000 \mathrm{E}+00 \\ -87.78 & 9.0297 \mathrm{E}-02 & 4.1175 \mathrm{E}-02 & 0.0000 \mathrm{E}+00 \\ -82.78 & 1.3087 \mathrm{E}-01 & 6.1499 \mathrm{E}-02 & 0.0000 \mathrm{E}+00 \\ -77.78 & 1.8097 \mathrm{E}-01 & 8.8964 \mathrm{E}-02 & 0.0000 \mathrm{E}+00 \\ -72.15 & 2.3880 \mathrm{E}-01 & 1.2489 \mathrm{E}-01 & 0.0000 \mathrm{E}+00 \\ -65.90 & 2.9874 \mathrm{E}-01 & 1.7143 \mathrm{E}-01 & 0.0000 \mathrm{E}+00 \\ -60.35 & 3.6287 \mathrm{E}-01 & 2.3544 \mathrm{E}-01 & 6.8538 \mathrm{E}-02 \\ -55.49 & 4.4602 \mathrm{E}-01 & 3.2032 \mathrm{E}-01 & 8.1136 \mathrm{E}-02 \\ -50.62 & 5.4184 \mathrm{E}-01 & 4.3129 \mathrm{E}-01 & 9.8654 \mathrm{E}-02 \\ -47.17 & 6.1840 \mathrm{E}-01 & 5.3605 \mathrm{E}-01 & 1.1751 \mathrm{E}-01 \\ -43.58 & 6.9900 \mathrm{E}-01 & 6.5459 \mathrm{E}-01 & 7.5837 \mathrm{E}-01 \\ -38.45 & 8.1648 \mathrm{E}-01 & 7.9894 \mathrm{E}-01 & 8.4908 \mathrm{E}-01 \\ -33.32 & 9.2555 \mathrm{E}-01 & 9.2180 \mathrm{E}-01 & 9.5151 \mathrm{E}-01 \\ -28.20 & 1.0206 \mathrm{E}+00 & 1.0234 \mathrm{E}+00 & 1.0106 \mathrm{E}+00 \\ -23.07 & 1.0999 \mathrm{E}+00 & 1.1064 \mathrm{E}+00 & 1.0874 \mathrm{E}+00 \\ -17.94 & 1.1617 \mathrm{E}+00 & 1.1700 \mathrm{E}+00 & 1.1476 \mathrm{E}+00 \\ -12.82 & 1.2052 \mathrm{E}+00 & 1.2138 \mathrm{E}+00 & 1.1701 \mathrm{E}+00 \\ -7.69 & 1.2303 \mathrm{E}+00 & 1.2393 \mathrm{E}+00 & 1.1941 \mathrm{E}+00 \\ -2.56 & 1.2367 \mathrm{E}+00 & 1.2461 \mathrm{E}+00 & 1.2003 \mathrm{E}+00 \\ 2.56 & 1.2245 \mathrm{E}+00 & 1.2345 \mathrm{E}+00 & 1.1934 \mathrm{E}+00 \\ 7.69 & 1.1937 \mathrm{E}+00 & 1.2040 \mathrm{E}+00 & 1.1635 \mathrm{E}+00 \\ 12.82 & 1.1451 \mathrm{E}+00 & 1.1557 \mathrm{E}+00 & 1.1162 \mathrm{E}+00 \\ 17.94 & 1.0801 \mathrm{E}+00 & 1.0911 \mathrm{E}+00 & 1.0789 \mathrm{E}+00 \\ 23.07 & 9.9958 \mathrm{E}-01 & 1.0094 \mathrm{E}+00 & 9.9902 \mathrm{E}-01 \\ 28.20 & 9.0559 \mathrm{E}-01 & 9.1260 \mathrm{E}-01 & 9.0614 \mathrm{E}-01 \\ 33.32 & 8.0062 \mathrm{E}-01 & 8.0233 \mathrm{E}-01 & 8.3426 \mathrm{E}-01 \\ 38.45 & 6.8654 \mathrm{E}-01 & 6.7723 \mathrm{E}-01 & 7.2167 \mathrm{E}-01 \\ 43.58 & 5.6885 \mathrm{E}-01 & 5.3875 \mathrm{E}-01 & 6.1781 \mathrm{E}-01 \\ 47.17 & 4.9122 \mathrm{E}-01 & 4.3364 \mathrm{E}-01 & 8.2952 \mathrm{E}-02 \\ 50.62 & 4.1956 \mathrm{E}-01 & 3.4299 \mathrm{E}-01 & 6.5606 \mathrm{E}-02 \\ 55.49 & 3.3157 \mathrm{E}-01 & 2.4809 \mathrm{E}-01 & 4.9392 \mathrm{E}-02 \\ 60.35 & 2.5389 \mathrm{E}-01 & 1.7448 \mathrm{E}-01 & 3.7529 \mathrm{E}-02 \\ 64.98 & 2.0347 \mathrm{E}-01 & 1.3000 \mathrm{E}-01 & 0.0000 \mathrm{E}+00 \\ 69.39 & 1.7288 \mathrm{E}-01 & 1.0580 \mathrm{E}-01 & 0.0000 \mathrm{E}+00 \\ 76.46 & 1.2951 \mathrm{E}-01 & 7.3599 \mathrm{E}-02 & 0.0000 \mathrm{E}+00 \\ 86.80 & 8.3055 \mathrm{E}-02 & 4.3336 \mathrm{E}-02 & 0.0000 \mathrm{E}+00 \\ 98.14 & 4.7184 \mathrm{E}-02 & 2.3173 \mathrm{E}-02 & 0.0000 \mathrm{E}+00 \\ 110.00 & 1.9993 \mathrm{E}-02 & 1.0192 \mathrm{E}-02 & 0.0000 \mathrm{E}+00\end{array}$


HNF-SD-FF-ANAL-009 Rev. 1

Table BllA-2.5. Fission Power Distribution by Pin in MFA-1 at BOC $11 A-2$ CORE POSITION 1404

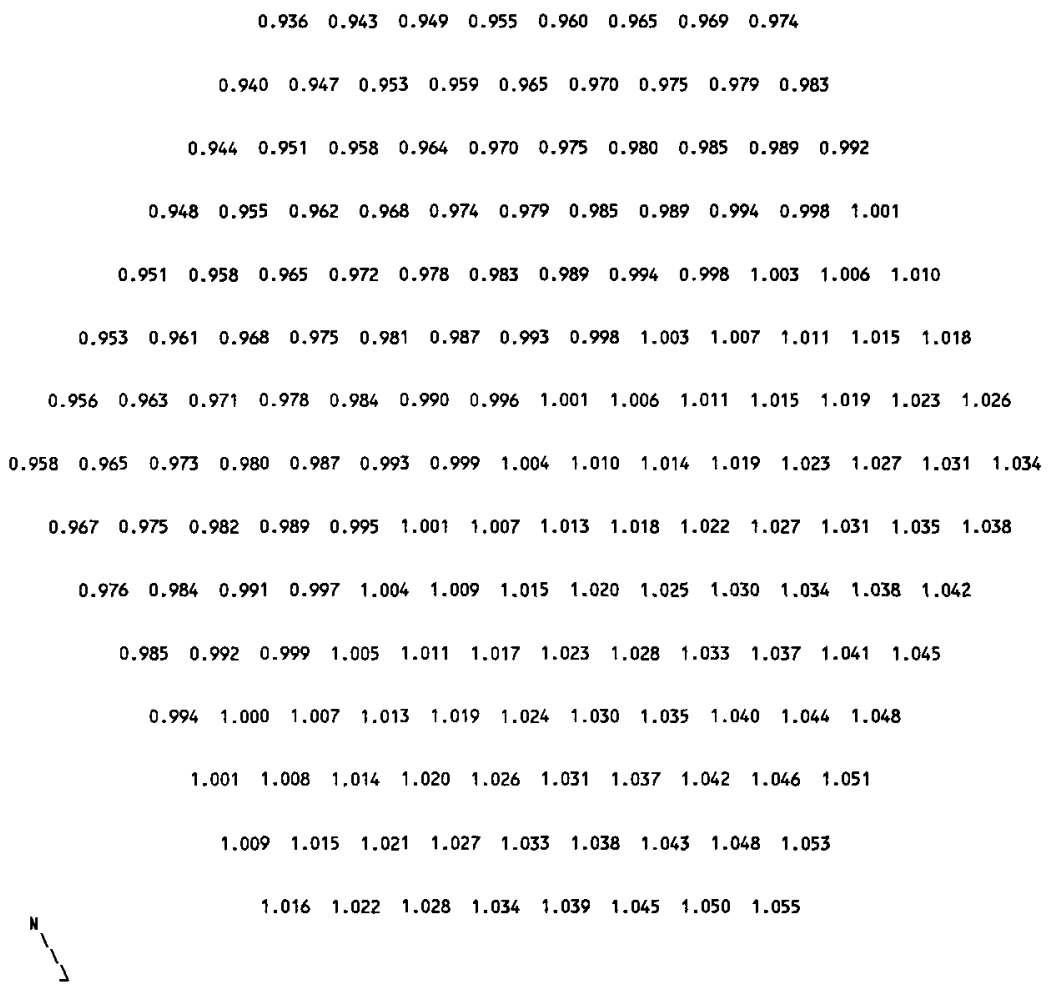


HNF-SD-FF-ANAL-009 Rev. 1

Table B11A-2.6. Fission Power Distribution by Pin in MFA-2 at BOC 11A-2 CORE POSITION 2506

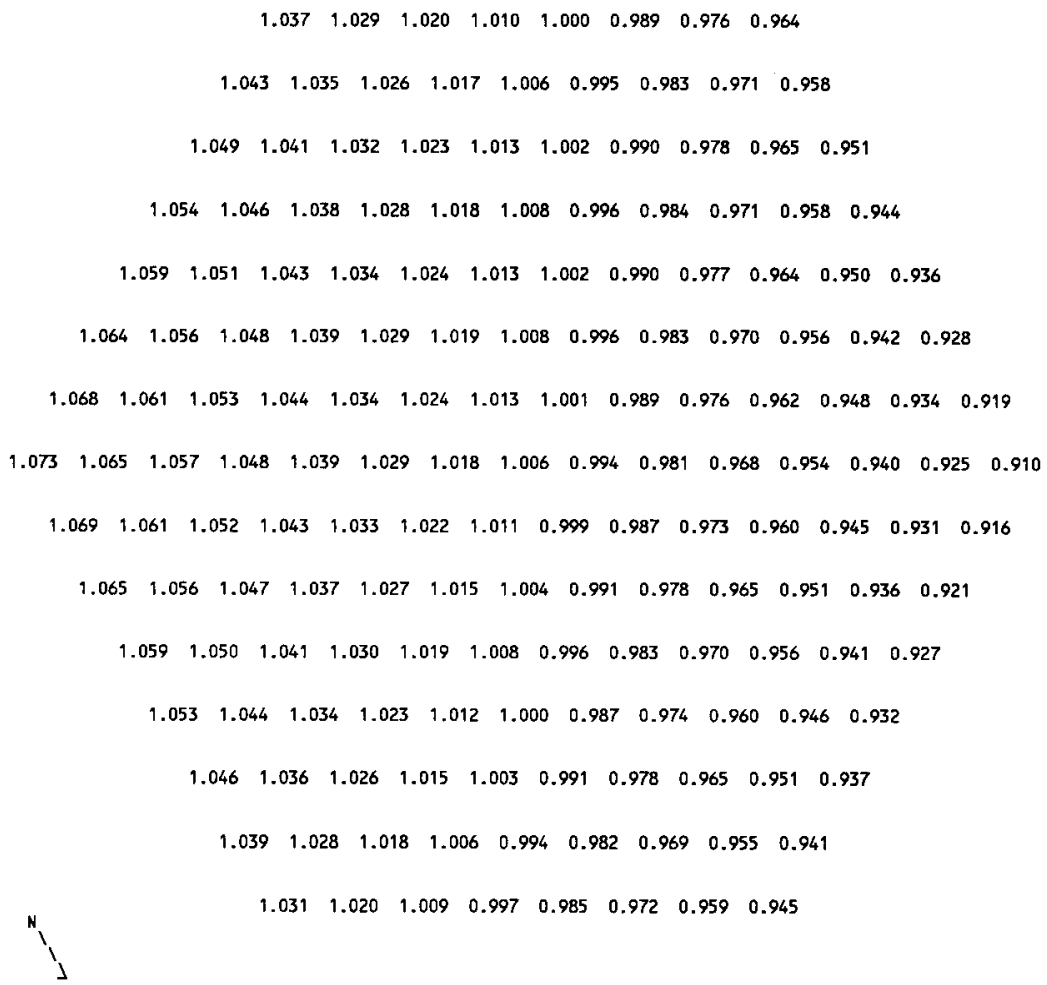




\section{HNF-SD-FF-ANAL-009 Rev. 1}

Table Bl1A-2.7. Fast Flux Distribution by Pin in MFA-1 at BOC 11A-2 CORE POSITION 1404

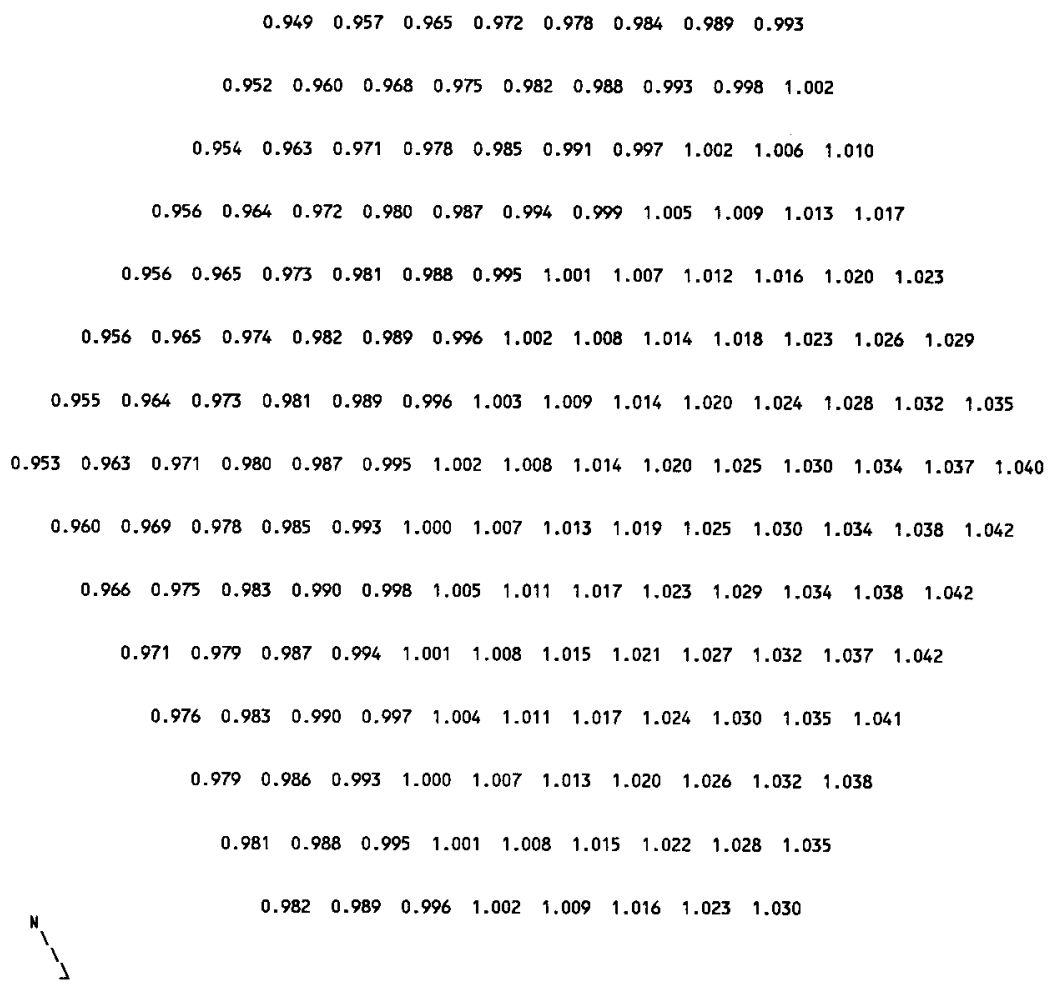




\section{HNF-SD-FF-ANAL-009 Rev. 1}

Table BllA-2.8. Fast Flux Distribution by Pin in MFA-2 at BOC $11 A-2$

CORE POSITION 2506

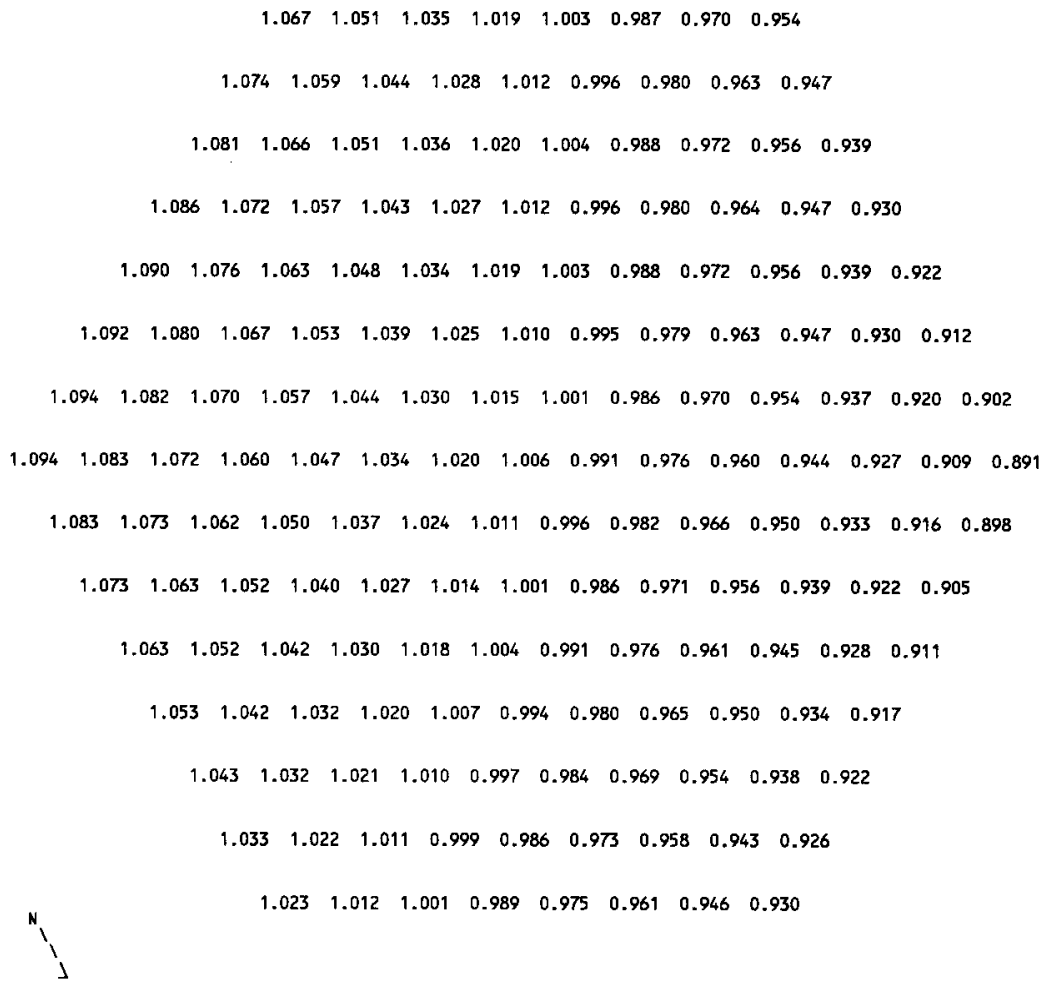


HNF-SD-FF-ANAL-009 Rev. 1

Table B11A-2.9. MFA-1 and MFA-2 Duct Wall Fast Flux Data at BOC 11A-2

$\begin{array}{ccc}\text { Side } & \text { Flux }>0.1 \mathrm{MeV} & \begin{array}{c}\text { MFA-1 } \\ \left(\mathrm{n} / \mathrm{cm}^{2}-\mathrm{sec}\right) \\ \text { MFA-2 }\end{array} \\ \text { E } & 2.0016 \mathrm{E}+15 & 2.2460 \mathrm{E}+15 \\ \text { SE } & 1.9456 \mathrm{E}+15 & 2.3331 \mathrm{E}+15 \\ \text { SW } & 1.9927 \mathrm{E}+15 & 2.1292 \mathrm{E}+15 \\ \text { W } & 2.1063 \mathrm{E}+15 & 1.9175 \mathrm{E}+15 \\ \text { NW } & 2.1714 \mathrm{E}+15 & 1.8834 \mathrm{E}+15 \\ \text { NE } & 2.0767 \mathrm{E}+15 & 2.0641 \mathrm{E}+15\end{array}$


Table E11A-2.17. Composition of MFA-1 at EOC 11A-2

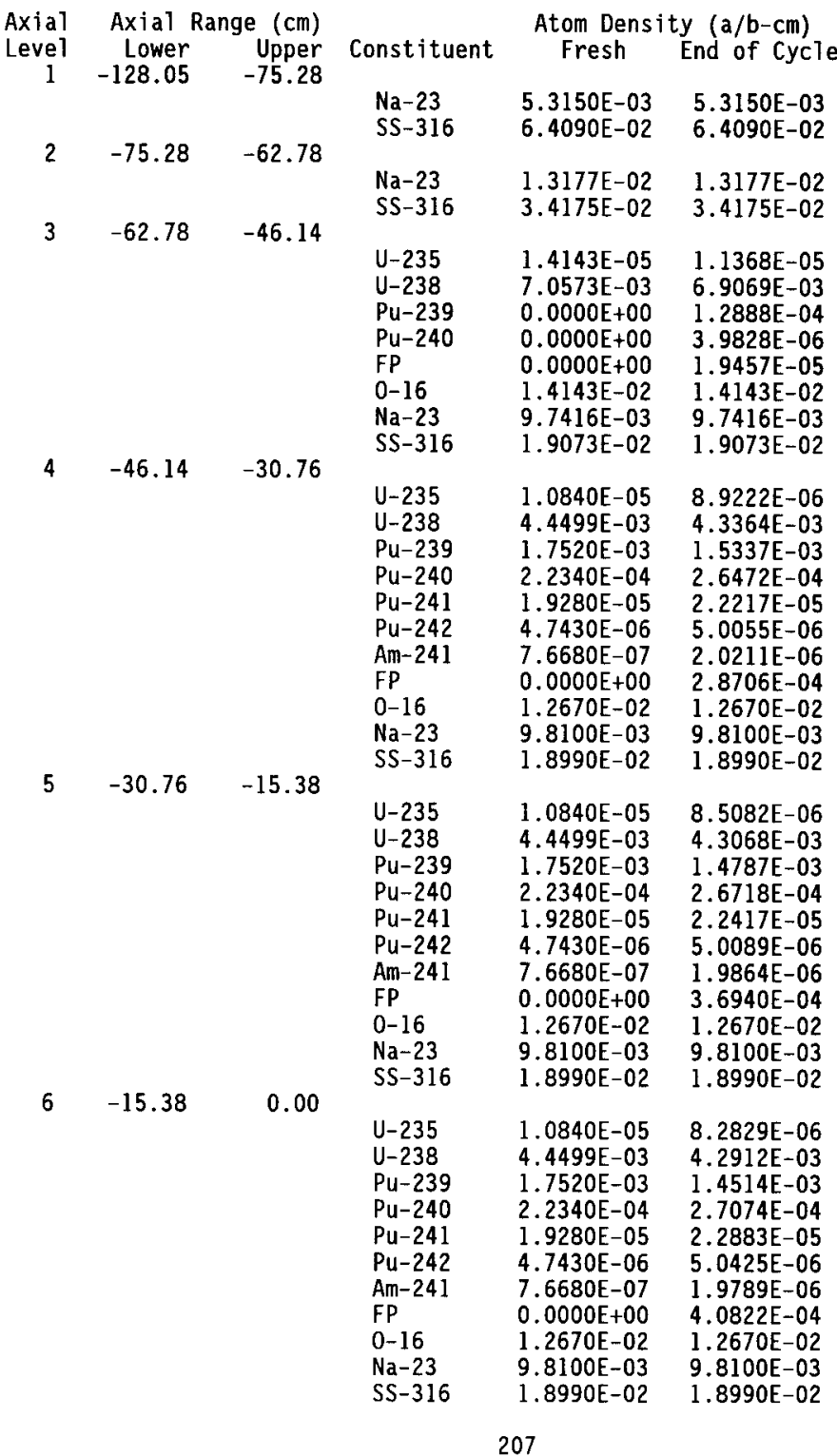




\begin{tabular}{|c|c|c|c|c|c|}
\hline \multirow{2}{*}{7} & 0.00 & 15.38 & & & \\
\hline & & & $\begin{array}{l}\mathrm{U}-235 \\
\mathrm{U}-238 \\
\mathrm{Pu}-239 \\
\mathrm{Pu}-240 \\
\mathrm{Pu}-241 \\
\mathrm{Pu}-242 \\
\mathrm{Am}-241 \\
\mathrm{FP} \\
\mathrm{O}-16 \\
\mathrm{Na}-23 \\
\mathrm{SS}-316\end{array}$ & $\begin{array}{l}1.0840 \mathrm{E}-05 \\
4.4499 \mathrm{E}-03 \\
1.7520 \mathrm{E}-03 \\
2.2340 \mathrm{E}-04 \\
1.9280 \mathrm{E}-05 \\
4.7430 \mathrm{E}-06 \\
7.6680 \mathrm{E}-07 \\
0.0000 \mathrm{E}+00 \\
1.2670 \mathrm{E}-02 \\
9.8100 \mathrm{E}-03 \\
1.8990 \mathrm{E}-02\end{array}$ & $\begin{array}{l}8.3570 \mathrm{E}-06 \\
4.2963 \mathrm{E}-03 \\
1.4601 \mathrm{E}-03 \\
2.6932 \mathrm{E}-04 \\
2.2697 \mathrm{E}-05 \\
5.0286 \mathrm{E}-06 \\
1.9815 \mathrm{E}-06 \\
3.9592 \mathrm{E}-04 \\
1.2670 \mathrm{E}-02 \\
9.8100 \mathrm{E}-03 \\
1.8990 \mathrm{E}-02\end{array}$ \\
\hline \multirow[t]{2}{*}{8} & 15.38 & 30.76 & & & \\
\hline & & & $\begin{array}{l}U-235 \\
U-238 \\
P u-239 \\
P u-240 \\
P u-241 \\
P u-242 \\
\text { Am-241 } \\
\text { FP } \\
0-16 \\
\mathrm{Na}-23 \\
\mathrm{SS}-316\end{array}$ & $\begin{array}{l}1.0840 \mathrm{E}-05 \\
4.4499 \mathrm{E}-03 \\
1.7520 \mathrm{E}-03 \\
2.2340 \mathrm{E}-04 \\
1.9280 \mathrm{E}-05 \\
4.7430 \mathrm{E}-06 \\
7.6680 \mathrm{E}-07 \\
0.0000 \mathrm{E}+00 \\
1.2670 \mathrm{E}-02 \\
9.8100 \mathrm{E}-03 \\
1.8990 \mathrm{E}-02\end{array}$ & $\begin{array}{l}8.7173 \mathrm{E}-06 \\
4.3208 \mathrm{E}-03 \\
1.5029 \mathrm{E}-03 \\
2.6311 \mathrm{E}-04 \\
2.1908 \mathrm{E}-05 \\
4.9726 \mathrm{E}-06 \\
1.9935 \mathrm{E}-06 \\
3.3572 \mathrm{E}-04 \\
1.2670 \mathrm{E}-02 \\
9.8100 \mathrm{E}-03 \\
1.8990 \mathrm{E}-02\end{array}$ \\
\hline \multirow[t]{2}{*}{9} & 30.76 & 46.14 & & & \\
\hline & & & $\begin{array}{l}U-235 \\
U-238 \\
P u-239 \\
P u-240 \\
P u-241 \\
P u-242 \\
\mathrm{Am}-241 \\
\mathrm{FP} \\
0-16 \\
\mathrm{Na}-23 \\
\mathrm{SS}-316\end{array}$ & $\begin{array}{l}1.0840 \mathrm{E}-05 \\
4.4499 \mathrm{E}-03 \\
1.7520 \mathrm{E}-03 \\
2.2340 \mathrm{E}-04 \\
1.9280 \mathrm{E}-05 \\
4.7430 \mathrm{E}-06 \\
7.6680 \mathrm{E}-07 \\
0.0000 \mathrm{E}+00 \\
1.2670 \mathrm{E}-02 \\
9.8100 \mathrm{E}-03 \\
1.8990 \mathrm{E}-02\end{array}$ & $\begin{array}{l}9.2390 \mathrm{E}-06 \\
4.3563 \mathrm{E}-03 \\
1.5689 \mathrm{E}-03 \\
2.5712 \mathrm{E}-04 \\
2.1272 \mathrm{E}-05 \\
4.9391 \mathrm{E}-06 \\
2.0254 \mathrm{E}-06 \\
2.4037 \mathrm{E}-04 \\
1.2670 \mathrm{E}-02 \\
9.8100 \mathrm{E}-03 \\
1.8990 \mathrm{E}-02\end{array}$ \\
\hline \multirow[t]{2}{*}{10} & 46.14 & 62.78 & & & \\
\hline & & & $\begin{array}{l}U-235 \\
U-238 \\
\mathrm{Pu}-239 \\
\mathrm{Pu}-240 \\
\mathrm{FP} \\
\mathrm{O}-16 \\
\mathrm{Na}-23 \\
\mathrm{SS}-316\end{array}$ & $\begin{array}{l}1.4143 \mathrm{E}-05 \\
7.0573 \mathrm{E}-03 \\
0.0000 \mathrm{E}+00 \\
0.0000 \mathrm{E}+00 \\
0.0000 \mathrm{E}+00 \\
1.4143 \mathrm{E}-02 \\
9.7416 \mathrm{E}-03 \\
1.9073 \mathrm{E}-02\end{array}$ & $\begin{array}{l}1.2235 \mathrm{E}-05 \\
6.9528 \mathrm{E}-03 \\
9.1512 \mathrm{E}-05 \\
1.7911 \mathrm{E}-06 \\
1.2539 \mathrm{E}-05 \\
1.4143 \mathrm{E}-02 \\
9.7416 \mathrm{E}-03 \\
1.9073 \mathrm{E}-02\end{array}$ \\
\hline \multirow[t]{2}{*}{11} & 62.78 & 172.84 & & & \\
\hline & & & $\begin{array}{l}\mathrm{Na}-23 \\
\mathrm{SS}-316\end{array}$ & $\begin{array}{l}8.2400 \mathrm{E}-03 \\
2.5562 \mathrm{E}-02\end{array}$ & $\begin{array}{l}8.2400 E-03 \\
2.5562 E-02\end{array}$ \\
\hline
\end{tabular}


Table El1A-2.18. Composition of MFA-2 at EOC 11A-2

\begin{tabular}{|c|c|c|c|c|c|}
\hline ial & Axial & ge $(\mathrm{cm})$ & & Atom Dens & $(a / b-c m)$ \\
\hline $\begin{array}{c}\text { Level } \\
1\end{array}$ & $\begin{array}{r}\text { Lower } \\
-128.05\end{array}$ & $\begin{array}{r}\text { Upper } \\
-75.28\end{array}$ & Constituent & Fresh & End of Cycle \\
\hline & & & $\begin{array}{l}\mathrm{Na}-23 \\
\mathrm{SS}-316\end{array}$ & $\begin{array}{l}5.3150 \mathrm{E}-03 \\
6.4090 \mathrm{E}-02\end{array}$ & $\begin{array}{l}5.3150 \mathrm{E}-03 \\
6.4090 \mathrm{E}-02\end{array}$ \\
\hline 2 & -75.28 & -62.78 & & & \\
\hline & & & $\begin{array}{l}\mathrm{Na}-23 \\
\mathrm{SS}-316\end{array}$ & $\begin{array}{l}1.3177 \mathrm{E}-02 \\
3.4175 \mathrm{E}-02\end{array}$ & $\begin{array}{l}1.3177 \mathrm{E}-02 \\
3.4175 \mathrm{E}-02\end{array}$ \\
\hline 3 & -62.78 & -46.14 & $\begin{array}{l}U-235 \\
U-238 \\
\mathrm{Pu}-239 \\
\mathrm{Pu}-240 \\
\mathrm{FP} \\
\mathrm{O}-16 \\
\mathrm{Na}-23 \\
\mathrm{SS}-316\end{array}$ & $\begin{array}{l}1.4143 \mathrm{E}-05 \\
7.0573 \mathrm{E}-03 \\
0.0000 \mathrm{E}+00 \\
0.0000 \mathrm{E}+00 \\
0.0000 \mathrm{E}+00 \\
1.4143 \mathrm{E}-02 \\
9.7416 \mathrm{E}-03 \\
1.9073 \mathrm{E}-02\end{array}$ & $\begin{array}{l}1.1274 \mathrm{E}-05 \\
6.9026 \mathrm{E}-03 \\
1.3208 \mathrm{E}-04 \\
4.2904 \mathrm{E}-06 \\
2.0390 \mathrm{E}-05 \\
1.4143 \mathrm{E}-02 \\
9.7416 \mathrm{E}-03 \\
1.9073 \mathrm{E}-02\end{array}$ \\
\hline 4 & -46.14 & -30.76 & $\begin{array}{l}U-235 \\
U-238 \\
P u-239 \\
P u-240 \\
P u-241 \\
P u-242 \\
A m-241 \\
F P \\
O-16 \\
\mathrm{Na}-23 \\
S S-316\end{array}$ & $\begin{array}{l}1.0160 \mathrm{E}-05 \\
4.4270 \mathrm{E}-03 \\
1.7483 \mathrm{E}-03 \\
2.2290 \mathrm{E}-04 \\
1.9040 \mathrm{E}-05 \\
4.7330 \mathrm{E}-06 \\
7.5700 \mathrm{E}-07 \\
0.0000 \mathrm{E}+00 \\
1.2760 \mathrm{E}-02 \\
9.8100 \mathrm{E}-03 \\
1.8990 \mathrm{E}-02\end{array}$ & $\begin{array}{l}8.2764 \mathrm{E}-06 \\
4.3080 \mathrm{E}-03 \\
1.5204 \mathrm{E}-03 \\
2.6665 \mathrm{E}-04 \\
2.2356 \mathrm{E}-05 \\
5.0126 \mathrm{E}-06 \\
1.9983 \mathrm{E}-06 \\
2.9935 \mathrm{E}-04 \\
1.2760 \mathrm{E}-02 \\
9.8100 \mathrm{E}-03 \\
1.8990 \mathrm{E}-02\end{array}$ \\
\hline 5 & -30.76 & -15.38 & $\begin{array}{l}\text { U-235 } \\
\text { U-238 } \\
\text { Pu-239 } \\
\text { Pu-240 } \\
\text { Pu-241 } \\
\text { Pu-242 } \\
\text { Am-241 } \\
\text { FP } \\
0-16 \\
\text { Na-23 } \\
\text { SS-316 }\end{array}$ & $\begin{array}{l}1.0160 \mathrm{E}-05 \\
4.4270 \mathrm{E}-03 \\
1.7483 \mathrm{E}-03 \\
2.2290 \mathrm{E}-04 \\
1.9040 \mathrm{E}-05 \\
4.7330 \mathrm{E}-06 \\
7.5700 \mathrm{E}-07 \\
0.0000 \mathrm{E}+00 \\
1.2760 \mathrm{E}-02 \\
9.8100 \mathrm{E}-03 \\
1.8990 \mathrm{E}-02\end{array}$ & $\begin{array}{l}7.8520 \mathrm{E}-06 \\
4.2752 \mathrm{E}-03 \\
1.4607 \mathrm{E}-03 \\
2.6961 \mathrm{E}-04 \\
2.2627 \mathrm{E}-05 \\
5.0201 \mathrm{E}-06 \\
1.9618 \mathrm{E}-06 \\
3.8886 \mathrm{E}-04 \\
1.2760 \mathrm{E}-02 \\
9.8100 \mathrm{E}-03 \\
1.8990 \mathrm{E}-02\end{array}$ \\
\hline 6 & -15.38 & 0.00 & $\begin{array}{l}U-235 \\
U-238 \\
P u-239 \\
P u-240 \\
P u-241 \\
P u-242 \\
\text { Am-241 } \\
F P \\
0-16 \\
\mathrm{Na}-23 \\
\text { SS }-316\end{array}$ & $\begin{array}{l}1.0160 \mathrm{E}-05 \\
4.4270 \mathrm{E}-03 \\
1.7483 \mathrm{E}-03 \\
2.2290 \mathrm{E}-04 \\
1.9040 \mathrm{E}-05 \\
4.7330 \mathrm{E}-06 \\
7.5700 \mathrm{E}-07 \\
0.0000 \mathrm{E}+00 \\
1.2760 \mathrm{E}-02 \\
9.8100 \mathrm{E}-03 \\
1.8990 \mathrm{E}-02\end{array}$ & $\begin{array}{l}7.6157 \mathrm{E}-06 \\
4.2575 \mathrm{E}-03 \\
1.4305 \mathrm{E}-03 \\
2.7362 \mathrm{E}-04 \\
2.3185 \mathrm{E}-05 \\
5.0607 \mathrm{E}-06 \\
1.9538 \mathrm{E}-06 \\
4.3228 \mathrm{E}-04 \\
1.2760 \mathrm{E}-02 \\
9.8100 \mathrm{E}-03 \\
1.8990 \mathrm{E}-02\end{array}$ \\
\hline & & & & & \\
\hline
\end{tabular}


HNF-SD-FF-ANAL-009 Rev. 1

\begin{tabular}{|c|c|c|c|c|c|}
\hline \multirow[t]{2}{*}{7} & 0.00 & 15.38 & & & \\
\hline & & & $\begin{array}{l}U-235 \\
U-238 \\
P u-239 \\
P u-240 \\
P u-241 \\
P u-242 \\
\mathrm{Am}-241 \\
\mathrm{FP} \\
0-16 \\
\mathrm{Na}-23 \\
\mathrm{SS}-316\end{array}$ & $\begin{array}{l}1.0160 \mathrm{E}-05 \\
4.4270 \mathrm{E}-03 \\
1.7483 \mathrm{E}-03 \\
2.2290 \mathrm{E}-04 \\
1.9040 \mathrm{E}-05 \\
4.7330 \mathrm{E}-06 \\
7.5700 \mathrm{E}-07 \\
0.0000 \mathrm{E}+00 \\
1.2760 \mathrm{E}-02 \\
9.8100 \mathrm{E}-03 \\
1.8990 \mathrm{E}-02\end{array}$ & $\begin{array}{l}7.6790 \mathrm{E}-06 \\
4.2623 \mathrm{E}-03 \\
1.4382 \mathrm{E}-03 \\
2.7237 \mathrm{E}-04 \\
2.3008 \mathrm{E}-05 \\
5.0473 \mathrm{E}-06 \\
1.9560 \mathrm{E}-06 \\
4.2109 \mathrm{E}-04 \\
1.2760 \mathrm{E}-02 \\
9.8100 \mathrm{E}-03 \\
1.8990 \mathrm{E}-02\end{array}$ \\
\hline \multirow[t]{2}{*}{8} & 15.38 & 30.76 & & & \\
\hline & & & $\begin{array}{l}\mathrm{U}-235 \\
\mathrm{U}-238 \\
\mathrm{Pu}-239 \\
\mathrm{Pu}-240 \\
\mathrm{Pu}-241 \\
\mathrm{Pu}-242 \\
\mathrm{Am}-241 \\
\mathrm{FP} \\
0-16 \\
\mathrm{Na}-23 \\
\mathrm{SS}-316\end{array}$ & $\begin{array}{l}1.0160 \mathrm{E}-05 \\
4.4270 \mathrm{E}-03 \\
1.7483 \mathrm{E}-03 \\
2.2290 \mathrm{E}-04 \\
1.9040 \mathrm{E}-05 \\
4.7330 \mathrm{E}-06 \\
7.5700 \mathrm{E}-07 \\
0.0000 \mathrm{E}+00 \\
1.2760 \mathrm{E}-02 \\
9.8100 \mathrm{E}-03 \\
1.8990 \mathrm{E}-02\end{array}$ & $\begin{array}{l}8.0241 \mathrm{E}-06 \\
4.2876 \mathrm{E}-03 \\
1.4821 \mathrm{E}-03 \\
2.6609 \mathrm{E}-04 \\
2.2172 \mathrm{E}-05 \\
4.9875 \mathrm{E}-06 \\
1.9673 \mathrm{E}-06 \\
3.5907 \mathrm{E}-04 \\
1.2760 \mathrm{E}-02 \\
9.8100 \mathrm{E}-03 \\
1.8990 \mathrm{E}-02\end{array}$ \\
\hline \multirow[t]{2}{*}{9} & 30.76 & 46.14 & & & \\
\hline & & & $\begin{array}{l}\mathrm{U}-235 \\
\mathrm{U}-238 \\
\mathrm{Pu}-239 \\
\mathrm{Pu}-240 \\
\mathrm{Pu}-241 \\
\mathrm{Pu}-242 \\
\mathrm{Am}-241 \\
\mathrm{FP} \\
\mathrm{O}-16 \\
\mathrm{Na}-23 \\
\mathrm{SS}-316\end{array}$ & $\begin{array}{l}1.0160 \mathrm{E}-05 \\
4.4270 \mathrm{E}-03 \\
1.7483 \mathrm{E}-03 \\
2.2290 \mathrm{E}-04 \\
1.9040 \mathrm{E}-05 \\
4.7330 \mathrm{E}-06 \\
7.5700 \mathrm{E}-07 \\
0.0000 \mathrm{E}+00 \\
1.2760 \mathrm{E}-02 \\
9.8100 \mathrm{E}-03 \\
1.8990 \mathrm{E}-02\end{array}$ & $\begin{array}{l}8.5361 \mathrm{E}-06 \\
4.3253 \mathrm{E}-03 \\
1.5510 \mathrm{E}-03 \\
2.6005 \mathrm{E}-04 \\
2.1501 \mathrm{E}-05 \\
4.9524 \mathrm{E}-06 \\
2.0006 \mathrm{E}-06 \\
2.5872 \mathrm{E}-04 \\
1.2760 \mathrm{E}-02 \\
9.8100 \mathrm{E}-03 \\
1.8990 \mathrm{E}-02\end{array}$ \\
\hline \multirow[t]{2}{*}{10} & 46.14 & 62.78 & & & \\
\hline & & & $\begin{array}{l}U-235 \\
U-238 \\
P u-239 \\
P u-240 \\
F P \\
0-16 \\
\mathrm{Na}-23 \\
\mathrm{SS}-316\end{array}$ & $\begin{array}{l}1.4143 \mathrm{E}-05 \\
7.0573 \mathrm{E}-03 \\
0.0000 \mathrm{E}+00 \\
0.0000 \mathrm{E}+00 \\
0.0000 \mathrm{E}+00 \\
1.4143 \mathrm{E}-02 \\
9.7416 \mathrm{E}-03 \\
1.9073 \mathrm{E}-02\end{array}$ & $\begin{array}{l}1.2030 \mathrm{E}-05 \\
6.9429 \mathrm{E}-03 \\
9.9658 \mathrm{E}-05 \\
2.2345 \mathrm{E}-06 \\
1.4082 \mathrm{E}-05 \\
1.4143 \mathrm{E}-02 \\
9.7416 \mathrm{E}-03 \\
1.9073 \mathrm{E}-02\end{array}$ \\
\hline 11 & 62.78 & 172.84 & $\begin{array}{l}\mathrm{Na}-23 \\
\mathrm{SS}-316\end{array}$ & $\begin{array}{l}8.2400 \mathrm{E}-03 \\
2.5562 \mathrm{E}-02\end{array}$ & $\begin{array}{l}8.2400 \mathrm{E}-03 \\
2.5562 \mathrm{E}-02\end{array}$ \\
\hline
\end{tabular}




\subsection{Cycle 11A-3}

Table B11A-3.1. Fission Power Generated in MFA-1, MFA-2 and Neighboring Assemblies at BOC 11A-3

\begin{tabular}{lcrrrr} 
CORE & $* * * * * *$ & \multicolumn{2}{c}{ POWER IN MEGAWATTS } & $* * * * * *$ \\
POS. & BELOW CORE & IN CORE & ABOVE CORE & TOTAL PWR \\
1404 & $6.355 \mathrm{E}-02$ & $4.106 \mathrm{E}+00$ & $3.539 \mathrm{E}-02$ & $4.205 \mathrm{E}+00$ \\
1303 & $1.438 \mathrm{E}-01$ & $1.788 \mathrm{E}+00$ & $6.652 \mathrm{E}-02$ & $1.999 \mathrm{E}+00$ \\
1304 & $9.915 \mathrm{E}-03$ & $4.452 \mathrm{E}+00$ & $7.178 \mathrm{E}-03$ & $4.469 \mathrm{E}+00$ \\
1403 & $1.422 \mathrm{E}-02$ & $3.379 \mathrm{E}+00$ & $7.473 \mathrm{E}-03$ & $3.401 \mathrm{E}+00$ \\
1405 & $7.439 \mathrm{E}-03$ & $3.820 \mathrm{E}+00$ & $5.723 \mathrm{E}-03$ & $3.834 \mathrm{E}+00$ \\
1505 & $6.651 \mathrm{E}-03$ & $3.922 \mathrm{E}+00$ & $4.426 \mathrm{E}-03$ & $3.933 \mathrm{E}+00$ \\
1506 & $9.053 \mathrm{E}-03$ & $3.897 \mathrm{E}+00$ & $6.377 \mathrm{E}-03$ & $3.912 \mathrm{E}+00$ \\
2506 & $6.199 \mathrm{E}-02$ & $3.870 \mathrm{E}+00$ & $3.885 \mathrm{E}-02$ & $3.971 \mathrm{E}+00$ \\
2404 & $0.000 \mathrm{E}+00$ & $7.160 \mathrm{E}+00$ & $0.000 \mathrm{E}+00$ & $7.160 \mathrm{E}+00$ \\
2505 & $1.314 \mathrm{E}-02$ & $4.074 \mathrm{E}+00$ & $7.002 \mathrm{E}-03$ & $4.094 \mathrm{E}+00$ \\
2405 & $1.444 \mathrm{E}-02$ & $3.648 \mathrm{E}+00$ & $1.010 \mathrm{E}-02$ & $3.672 \mathrm{E}+00$ \\
2606 & $6.318 \mathrm{E}-03$ & $3.939 \mathrm{E}+00$ & $4.819 \mathrm{E}-03$ & $3.950 \mathrm{E}+00$ \\
2607 & $1.111 \mathrm{E}-02$ & $3.384 \mathrm{E}+00$ & $7.128 \mathrm{E}-03$ & $3.402 \mathrm{E}+00$ \\
2507 & $9.606 \mathrm{E}-03$ & $4.020 \mathrm{E}+00$ & $7.310 \mathrm{E}-03$ & $4.037 \mathrm{E}+00$
\end{tabular}

Table BllA-3.2. Assembly Averaged Total and Fast Flux in MFA-1 and MFA-2 at BOC $11 \mathrm{~A}-3$

\begin{tabular}{cccc} 
& Core & \multicolumn{2}{c}{ Flux $\left(\mathrm{n} / \mathrm{cm}^{2}-\mathrm{sec}\right)$} \\
Assembly & Pos, & Total & $>0.1 \mathrm{MeV}$ \\
MFA-1 & 1404 & $3.365 \mathrm{E}+15$ & $2.075 \mathrm{E}+15$ \\
MFA-2 & 2506 & $3.220 \mathrm{E}+15$ & $2.102 \mathrm{E}+15$
\end{tabular}


Table B11A-3.3. Axial Distribution of Total Flux, Fast Flux and Power in MFA-1 at BOC $11 \mathrm{~A}-3$

$\begin{array}{rccc}z(c m) & \text { Total Flux } & \text { Flux }>0.1 \text { MeV } & \text { Power } \\ -97.78 & 2.8927 \mathrm{E}-02 & 1.5450 \mathrm{E}-02 & 0.0000 \mathrm{E}+00 \\ -92.78 & 6.2092 \mathrm{E}-02 & 2.9775 \mathrm{E}-02 & 0.0000 \mathrm{E}+00 \\ -87.78 & 9.9443 \mathrm{E}-02 & 4.7526 \mathrm{E}-02 & 0.0000 \mathrm{E}+00 \\ -82.78 & 1.4375 \mathrm{E}-01 & 7.0669 \mathrm{E}-02 & 0.0000 \mathrm{E}+00 \\ -77.78 & 1.9777 \mathrm{E}-01 & 1.0160 \mathrm{E}-01 & 0.0000 \mathrm{E}+00 \\ -72.15 & 2.5868 \mathrm{E}-01 & 1.4136 \mathrm{E}-01 & 0.0000 \mathrm{E}+00 \\ -65.90 & 3.1937 \mathrm{E}-01 & 1.9080 \mathrm{E}-01 & 0.0000 \mathrm{E}+00 \\ -60.35 & 3.8249 \mathrm{E}-01 & 2.5646 \mathrm{E}-01 & 6.8505 \mathrm{E}-02 \\ -55.49 & 4.6501 \mathrm{E}-01 & 3.4194 \mathrm{E}-01 & 8.0215 \mathrm{E}-02 \\ -50.62 & 5.5989 \mathrm{E}-01 & 4.5112 \mathrm{E}-01 & 9.6743 \mathrm{E}-02 \\ -47.17 & 6.3522 \mathrm{E}-01 & 5.5300 \mathrm{E}-01 & 1.1436 \mathrm{E}-01 \\ -43.58 & 7.1434 \mathrm{E}-01 & 6.6893 \mathrm{E}-01 & 7.7067 \mathrm{E}-01 \\ -38.45 & 8.2859 \mathrm{E}-01 & 8.0977 \mathrm{E}-01 & 8.6118 \mathrm{E}-01 \\ -33.32 & 9.3480 \mathrm{E}-01 & 9.3041 \mathrm{E}-01 & 9.6040 \mathrm{E}-01 \\ -28.20 & 1.0277 \mathrm{E}+00 & 1.0309 \mathrm{E}+00 & 1.0182 \mathrm{E}+00 \\ -23.07 & 1.1055 \mathrm{E}+00 & 1.1131 \mathrm{E}+00 & 1.0931 \mathrm{E}+00 \\ -17.94 & 1.1662 \mathrm{E}+00 & 1.1762 \mathrm{E}+00 & 1.1519 \mathrm{E}+00 \\ -12.82 & 1.2087 \mathrm{E}+00 & 1.2196 \mathrm{E}+00 & 1.1747 \mathrm{E}+00 \\ -7.69 & 1.2327 \mathrm{E}+00 & 1.2441 \mathrm{E}+00 & 1.1976 \mathrm{E}+00 \\ -2.56 & 1.2379 \mathrm{E}+00 & 1.2494 \mathrm{E}+00 & 1.2024 \mathrm{E}+00 \\ 2.56 & 1.2240 \mathrm{E}+00 & 1.2355 \mathrm{E}+00 & 1.1947 \mathrm{E}+00 \\ 7.69 & 1.1912 \mathrm{E}+00 & 1.2024 \mathrm{E}+00 & 1.1626 \mathrm{E}+00 \\ 12.82 & 1.1402 \mathrm{E}+00 & 1.1509 \mathrm{E}+00 & 1.1128 \mathrm{E}+00 \\ 17.94 & 1.0728 \mathrm{E}+00 & 1.0829 \mathrm{E}+00 & 1.0726 \mathrm{E}+00 \\ 23.07 & 9.9046 \mathrm{E}-01 & 9.9854 \mathrm{E}-01 & 9.9071 \mathrm{E}-01 \\ 28.20 & 8.9552 \mathrm{E}-01 & 9.0013 \mathrm{E}-01 & 8.9675 \mathrm{E}-01 \\ 33.32 & 7.9049 \mathrm{E}-01 & 7.8958 \mathrm{E}-01 & 8.2362 \mathrm{E}-01 \\ 38.45 & 6.7749 \mathrm{E}-01 & 6.6617 \mathrm{E}-01 & 7.1125 \mathrm{E}-01 \\ 43.58 & 5.6133 \mathrm{E}-01 & 5.3129 \mathrm{E}-01 & 6.0486 \mathrm{E}-01 \\ 47.17 & 4.8280 \mathrm{E}-01 & 4.2771 \mathrm{E}-01 & 7.4251 \mathrm{E}-02 \\ 50.62 & 4.0870 \mathrm{E}-01 & 3.3709 \mathrm{E}-01 & 5.8004 \mathrm{E}-02 \\ 55.49 & 3.1720 \mathrm{E}-01 & 2.4149 \mathrm{E}-01 & 4.2652 \mathrm{E}-02 \\ 60.35 & 2.3824 \mathrm{E}-01 & 1.6805 \mathrm{E}-01 & 3.1568 \mathrm{E}-02 \\ 64.98 & 1.8938 \mathrm{E}-01 & 1.2568 \mathrm{E}-01 & 0.0000 \mathrm{E}+00 \\ 69.39 & 1.6122 \mathrm{E}-01 & 1.0340 \mathrm{E}-01 & 0.0000 \mathrm{E}+00 \\ 76.46 & 1.2172 \mathrm{E}-01 & 7.3516 \mathrm{E}-02 & 0.0000 \mathrm{E}+00 \\ 86.80 & 7.8996 \mathrm{E}-02 & 4.4418 \mathrm{E}-02 & 0.0000 \mathrm{E}+00 \\ 98.14 & 4.5465 \mathrm{E}-02 & 2.4295 \mathrm{E}-02 & 0.0000 \mathrm{E}+00 \\ 110.00 & 1.9489 \mathrm{E}-02 & 1.0866 \mathrm{E}-02 & 0.0000 \mathrm{E}+00\end{array}$


HNF-SD-FF-ANAL-009 Rev. 1

Table BllA-3.4. Axial Distribution of Total Flux, Fast Flux and Power in MFA-2 at BOC 11A-3

$\begin{array}{rccc}\text { z }(\mathrm{cm}) & \text { Total Flux } & \text { Flux }>0.1 \text { MeV } & \text { Power } \\ -97.78 & 2.6321 \mathrm{E}-02 & 1.3370 \mathrm{E}-02 & 0.0000 \mathrm{E}+00 \\ -92.78 & 5.6484 \mathrm{E}-02 & 2.5814 \mathrm{E}-02 & 0.0000 \mathrm{E}+00 \\ -87.78 & 9.0567 \mathrm{E}-02 & 4.1328 \mathrm{E}-02 & 0.0000 \mathrm{E}+00 \\ -82.78 & 1.3125 \mathrm{E}-01 & 6.1720 \mathrm{E}-02 & 0.0000 \mathrm{E}+00 \\ -77.78 & 1.8147 \mathrm{E}-01 & 8.9272 \mathrm{E}-02 & 0.0000 \mathrm{E}+00 \\ -72.15 & 2.3944 \mathrm{E}-01 & 1.2530 \mathrm{E}-01 & 0.0000 \mathrm{E}+00 \\ -65.90 & 2.9947 \mathrm{E}-01 & 1.7195 \mathrm{E}-01 & 0.0000 \mathrm{E}+00 \\ -60.35 & 3.6362 \mathrm{E}-01 & 2.3611 \mathrm{E}-01 & 7.0187 \mathrm{E}-02 \\ -55.49 & 4.4681 \mathrm{E}-01 & 3.2108 \mathrm{E}-01 & 8.2905 \mathrm{E}-02 \\ -50.62 & 5.4263 \mathrm{E}-01 & 4.3210 \mathrm{E}-01 & 1.0051 \mathrm{E}-01 \\ -47.17 & 6.1921 \mathrm{E}-01 & 5.3690 \mathrm{E}-01 & 1.1940 \mathrm{E}-01 \\ -43.58 & 6.9980 \mathrm{E}-01 & 6.5545 \mathrm{E}-01 & 7.5984 \mathrm{E}-01 \\ -38.45 & 8.1725 \mathrm{E}-01 & 7.9988 \mathrm{E}-01 & 8.5052 \mathrm{E}-01 \\ -33.32 & 9.2627 \mathrm{E}-01 & 9.2273 \mathrm{E}-01 & 9.5291 \mathrm{E}-01 \\ -28.20 & 1.0212 \mathrm{E}+00 & 1.0242 \mathrm{E}+00 & 1.0110 \mathrm{E}+00 \\ -23.07 & 1.1004 \mathrm{E}+00 & 1.1070 \mathrm{E}+00 & 1.0876 \mathrm{E}+00 \\ -17.94 & 1.1621 \mathrm{E}+00 & 1.1704 \mathrm{E}+00 & 1.1477 \mathrm{E}+00 \\ -12.82 & 1.2054 \mathrm{E}+00 & 1.2141 \mathrm{E}+00 & 1.1695 \mathrm{E}+00 \\ -7.69 & 1.2303 \mathrm{E}+00 & 1.2394 \mathrm{E}+00 & 1.1934 \mathrm{E}+00 \\ -2.56 & 1.2366 \mathrm{E}+00 & 1.2460 \mathrm{E}+00 & 1.1993 \mathrm{E}+00 \\ 2.56 & 1.2242 \mathrm{E}+00 & 1.2341 \mathrm{E}+00 & 1.1925 \mathrm{E}+00 \\ 7.69 & 1.1933 \mathrm{E}+00 & 1.2034 \mathrm{E}+00 & 1.1624 \mathrm{E}+00 \\ 12.82 & 1.1445 \mathrm{E}+00 & 1.1550 \mathrm{E}+00 & 1.1151 \mathrm{E}+00 \\ 17.94 & 1.0795 \mathrm{E}+00 & 1.0903 \mathrm{E}+00 & 1.0784 \mathrm{E}+00 \\ 23.07 & 9.9904 \mathrm{E}-01 & 1.0087 \mathrm{E}+00 & 9.9858 \mathrm{E}-01 \\ 28.20 & 9.0509 \mathrm{E}-01 & 9.1196 \mathrm{E}-01 & 9.0577 \mathrm{E}-01 \\ 33.32 & 8.0020 \mathrm{E}-01 & 8.0184 \mathrm{E}-01 & 8.3479 \mathrm{E}-01 \\ 38.45 & 6.8627 \mathrm{E}-01 & 6.7689 \mathrm{E}-01 & 7.2225 \mathrm{E}-01 \\ 43.58 & 5.6872 \mathrm{E}-01 & 5.3859 \mathrm{E}-01 & 6.1847 \mathrm{E}-01 \\ 47.17 & 4.9122 \mathrm{E}-01 & 4.3361 \mathrm{E}-01 & 8.3922 \mathrm{E}-02 \\ 50.62 & 4.1967 \mathrm{E}-01 & 3.4308 \mathrm{E}-01 & 6.6549 \mathrm{E}-02 \\ 55.49 & 3.3178 \mathrm{E}-01 & 2.4828 \mathrm{E}-01 & 5.0253 \mathrm{E}-02 \\ 60.35 & 2.5414 \mathrm{E}-01 & 1.7471 \mathrm{E}-01 & 3.8277 \mathrm{E}-02 \\ 64.98 & 2.0374 \mathrm{E}-01 & 1.3021 \mathrm{E}-01 & 0.0000 \mathrm{E}+00 \\ 69.39 & 1.7314 \mathrm{E}-01 & 1.0600 \mathrm{E}-01 & 0.0000 \mathrm{E}+00 \\ 76.46 & 1.2975 \mathrm{E}-01 & 7.3766 \mathrm{E}-02 & 0.0000 \mathrm{E}+00 \\ 86.80 & 8.3229 \mathrm{E}-02 & 4.3450 \mathrm{E}-02 & 0.0000 \mathrm{E}+00 \\ 98.14 & 4.7291 \mathrm{E}-02 & 2.3241 \mathrm{E}-02 & 0.0000 \mathrm{E}+00 \\ 110.00 & 2.0039 \mathrm{E}-02 & 1.0223 \mathrm{E}-02 & 0.0000 \mathrm{E}+00\end{array}$


HNF-SD-FF-ANAL-009 Rev. 1

Table B11A-3.5. Fission Power Distribution by Pin in MFA-1 at BOC 11A-3

CORE POSITION 1404

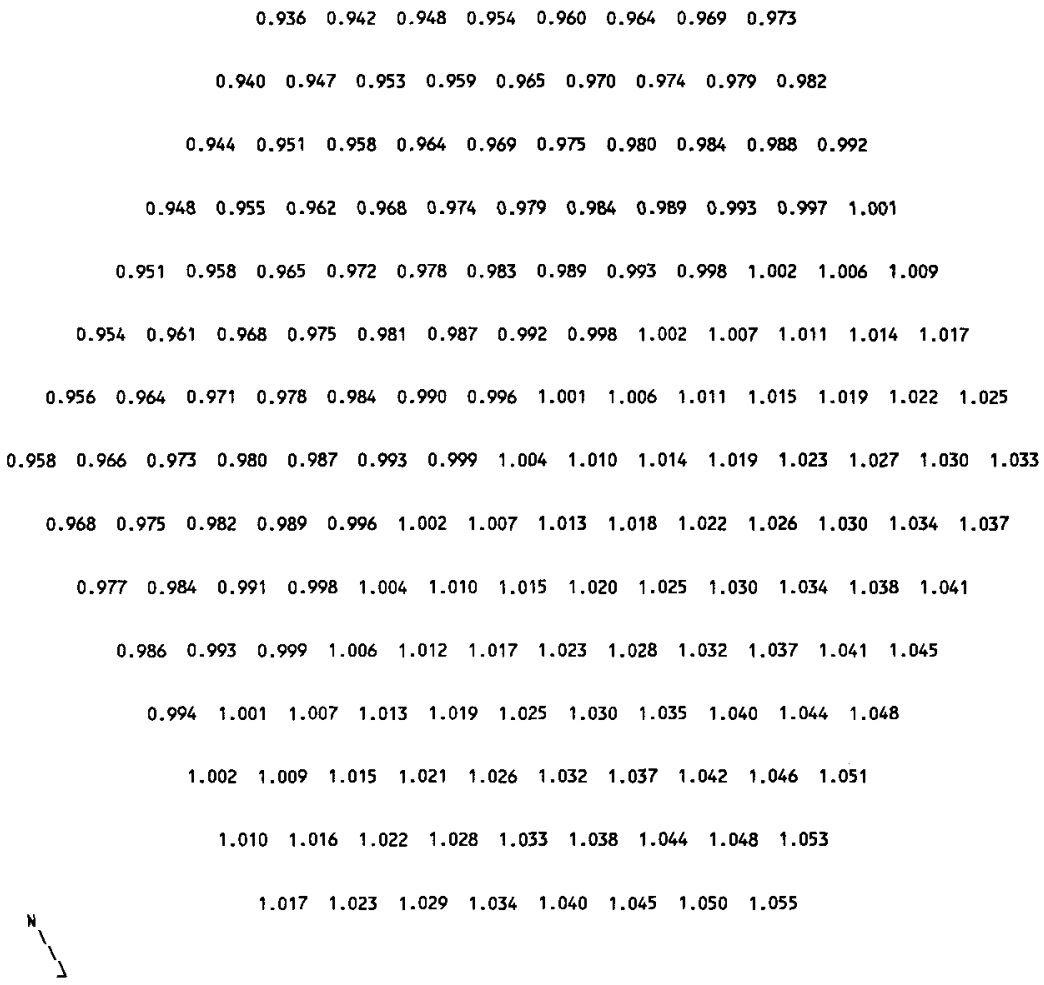


HNF-SD-FF-ANAL-009 Rev. 1

Table B11A-3.6. Fission Power Distribution by Pin in MFA-2 at BOC 11A-3

CORE POSITION 2506

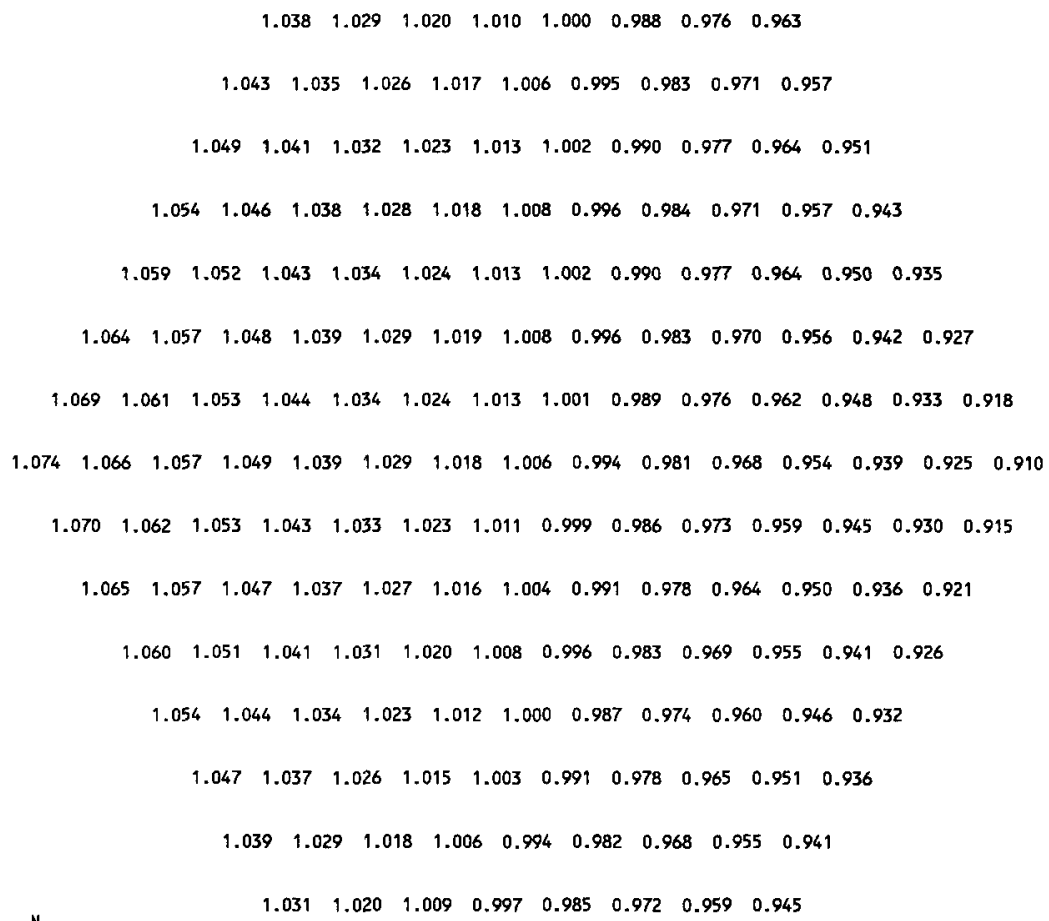


HNF-SD-FF-ANAL-009 Rev. 1

Table B11A-3.7. Fast Flux Distribution by Pin in MFA-1 at BOC 11A-3 CORE POSITION 1404

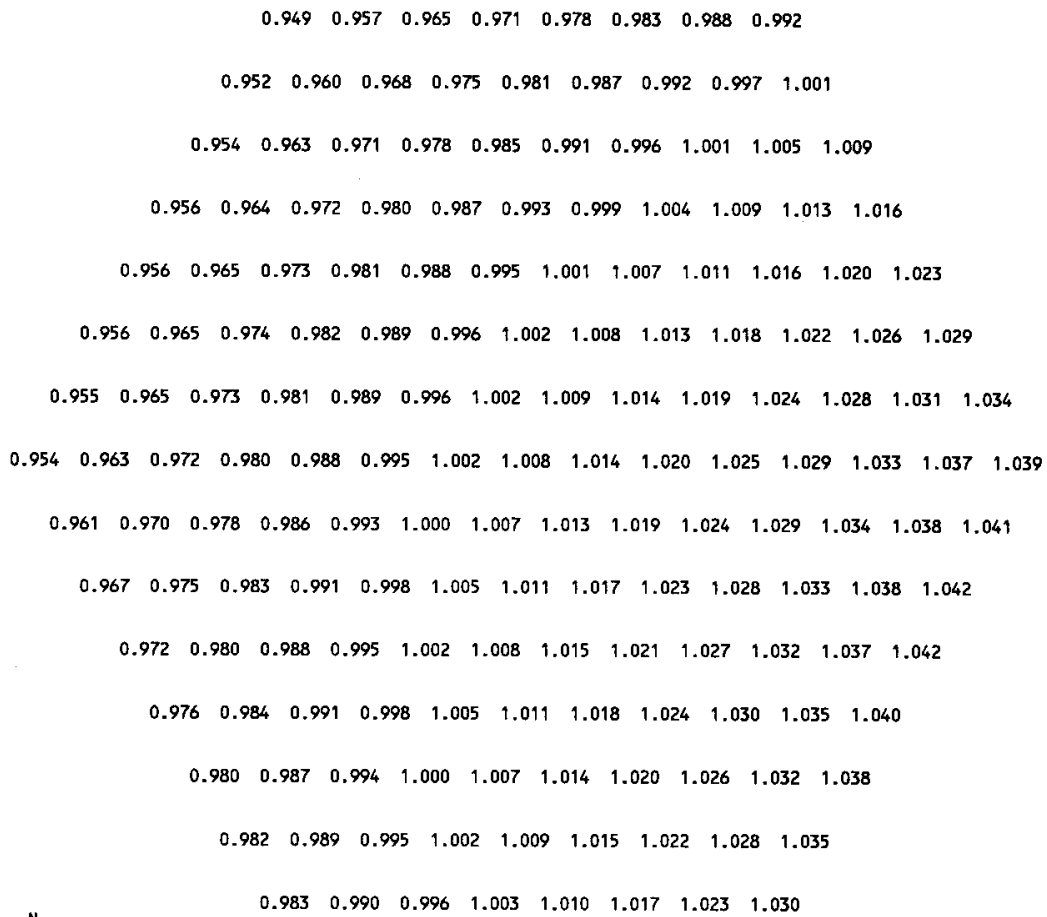


HNF-SD-FF-ANAL-009 Rev. 1

Table Bl1A-3.8. Fast Flux Distribution by Pin in MFA-2 at BOC $11 \mathrm{~A}-3$ CORE POSITION 2506

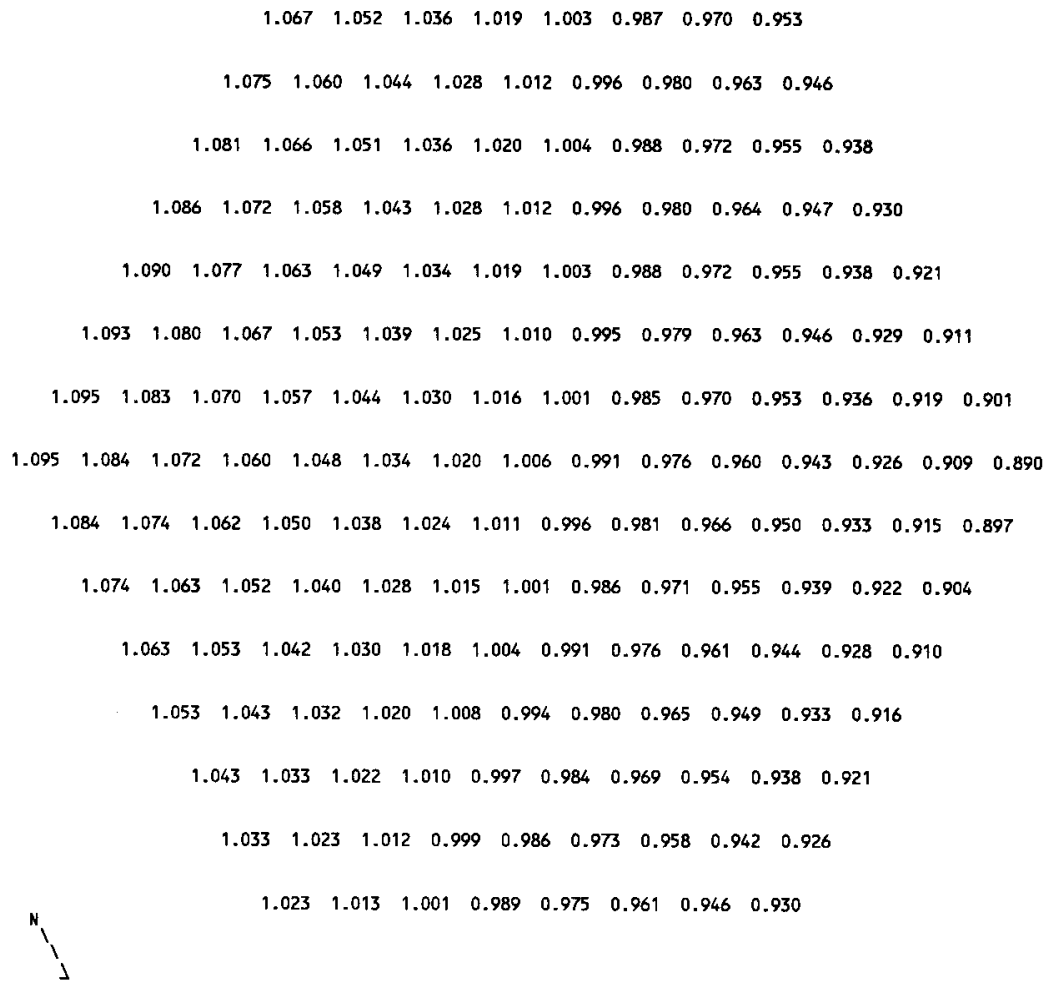


Table B11A-3.9. MFA-1 and MFA-2 Duct Wall Fast Flux Data at BOC $11 \mathrm{~A}-3$

$\begin{array}{ccc}\text { Side } & \begin{array}{c}\text { Flux }>0.1 \\ \text { MFA-1 }\end{array} & \begin{array}{c}\mathrm{MeV} \\ \left(\mathrm{n} / \mathrm{cm}^{2}-\mathrm{sec}\right) \\ \text { MFA-2 }\end{array} \\ \text { E } & 1.9985 E+15 & 2.2320 \mathrm{E}+15 \\ \text { SE } & 1.9412 \mathrm{E}+15 & 2.3183 \mathrm{E}+15 \\ \text { SW } & 1.9866 \mathrm{E}+15 & 2.1142 \mathrm{E}+15 \\ \text { W } & 2.0993 \mathrm{E}+15 & 1.9028 \mathrm{E}+15 \\ \text { NW } & 2.1652 \mathrm{E}+15 & 1.8682 \mathrm{E}+15 \\ \text { NE } & 2.0733 E+15 & 2.0498 \mathrm{E}+15\end{array}$

Table E11A-3.1. Fission Power Generated in MFA-1, MFA-2 and Neighboring Assemblies at EOC 11A-3

\begin{tabular}{|c|c|c|c|c|}
\hline $\begin{array}{l}\text { CORE } \\
\text { POS. } \\
1404 \\
1303 \\
1304 \\
1403 \\
1405 \\
1505 \\
1506 \\
2506 \\
2404 \\
2505 \\
2405 \\
2606 \\
2607 \\
2507\end{array}$ & $\begin{array}{c}\star \star \star \star \star \star \star \\
\text { BELOW CORE } \\
6.997 \mathrm{E}-02 \\
1.477 \mathrm{E}-01 \\
1.101 \mathrm{E}-02 \\
1.479 \mathrm{E}-02 \\
8.456 \mathrm{E}-03 \\
7.502 \mathrm{E}-03 \\
9.575 \mathrm{E}-03 \\
6.644 \mathrm{E}-02 \\
0.000 \mathrm{E}+00 \\
1.344 \mathrm{E}-02 \\
1.477 \mathrm{E}-02 \\
6.942 \mathrm{E}-03 \\
1.114 \mathrm{E}-02 \\
9.953 \mathrm{E}-03\end{array}$ & $\begin{array}{l}\text { POWER IN } \\
\text { IN CORE } \\
4.035 E+00 \\
1.902 E+00 \\
4.343 E+00 \\
3.420 E+00 \\
3.759 E+00 \\
3.980 E+00 \\
3.854 E+00 \\
3.738 E+00 \\
6.888 E+00 \\
4.050 E+00 \\
3.536 E+00 \\
3.875 E+00 \\
3.286 E+00 \\
3.867 E+00\end{array}$ & $\begin{array}{r}\text { MEGAWATTS } \\
\text { ABOVE CORE } \\
4.503 \mathrm{E}-02 \\
7.998 \mathrm{E}-02 \\
8.932 \mathrm{E}-03 \\
9.595 \mathrm{E}-03 \\
7.221 \mathrm{E}-03 \\
5.934 \mathrm{E}-03 \\
7.807 \mathrm{E}-03 \\
4.783 \mathrm{E}-02 \\
0.000 \mathrm{E}+00 \\
8.825 \mathrm{E}-03 \\
1.183 \mathrm{E}-02 \\
6.096 \mathrm{E}-03 \\
8.301 \mathrm{E}-03 \\
8.576 \mathrm{E}-03\end{array}$ & $\begin{array}{l}* \star \star \star * \\
\text { TOTAL PWR } \\
4.150 \mathrm{E}+00 \\
2.130 \mathrm{E}+00 \\
4.363 \mathrm{E}+00 \\
3.445 \mathrm{E}+00 \\
3.774 \mathrm{E}+00 \\
3.994 \mathrm{E}+00 \\
3.871 \mathrm{E}+00 \\
3.852 \mathrm{E}+00 \\
6.888 \mathrm{E}+00 \\
4.072 \mathrm{E}+00 \\
3.562 \mathrm{E}+00 \\
3.888 \mathrm{E}+00 \\
3.305 \mathrm{E}+00 \\
3.885 \mathrm{E}+00\end{array}$ \\
\hline
\end{tabular}

Table E11A-3.2. Assembly Averaged Total and Fast Flux in MFA-1 and MFA-2 at EOC $11 \mathrm{~A}-3$

\begin{tabular}{|c|c|c|c|}
\hline & Core & Flux & $\left.m^{2}-\sec \right)$ \\
\hline $\begin{array}{l}\text { Assembly } \\
\text { MFA-1 } \\
\text { MFA-2 }\end{array}$ & $\begin{array}{l}\text { Pos. } \\
1404 \\
2506\end{array}$ & $\begin{array}{c}\text { Total } \\
3.422 \mathrm{E}+15 \\
3.213 \mathrm{E}+15\end{array}$ & $\begin{array}{l}>0.1 \mathrm{MeV} \\
2.096 \mathrm{E}+15 \\
2.080 \mathrm{E}+15\end{array}$ \\
\hline
\end{tabular}


Table EllA-3.3. Axial Distribution of Total Flux, Fast Flux and Power in MFA-1 at EOC $11 \mathrm{~A}-3$

$\begin{array}{rccc}\text { z }(\mathrm{cm}) & \text { Total Flux } & \text { Flux }>0.1 \text { MeV } & \text { Power } \\ -97.78 & 2.7358 \mathrm{E}-02 & 1.4721 \mathrm{E}-02 & 0.0000 \mathrm{E}+00 \\ -92.78 & 5.8722 \mathrm{E}-02 & 2.8371 \mathrm{E}-02 & 0.0000 \mathrm{E}+00 \\ -87.78 & 9.4054 \mathrm{E}-02 & 4.5290 \mathrm{E}-02 & 0.0000 \mathrm{E}+00 \\ -82.78 & 1.3597 \mathrm{E}-01 & 6.7356 \mathrm{E}-02 & 0.0000 \mathrm{E}+00 \\ -77.78 & 1.8709 \mathrm{E}-01 & 9.6861 \mathrm{E}-02 & 0.0000 \mathrm{E}+00 \\ -72.15 & 2.4472 \mathrm{E}-01 & 1.3480 \mathrm{E}-01 & 0.0000 \mathrm{E}+00 \\ -65.90 & 3.0217 \mathrm{E}-01 & 1.8208 \mathrm{E}-01 & 0.0000 \mathrm{E}+00 \\ -60.35 & 3.6193 \mathrm{E}-01 & 2.4512 \mathrm{E}-01 & 7.8423 \mathrm{E}-02 \\ -55.49 & 4.4013 \mathrm{E}-01 & 3.2639 \mathrm{E}-01 & 9.0639 \mathrm{E}-02 \\ -50.62 & 5.3003 \mathrm{E}-01 & 4.2961 \mathrm{E}-01 & 1.0740 \mathrm{E}-01 \\ -47.17 & 6.0130 \mathrm{E}-01 & 5.2549 \mathrm{E}-01 & 1.2466 \mathrm{E}-01 \\ -43.58 & 6.7622 \mathrm{E}-01 & 6.3450 \mathrm{E}-01 & 7.3208 \mathrm{E}-01 \\ -38.45 & 7.8525 \mathrm{E}-01 & 7.6814 \mathrm{E}-01 & 8.1969 \mathrm{E}-01 \\ -33.32 & 8.8765 \mathrm{E}-01 & 8.8379 \mathrm{E}-01 & 9.1616 \mathrm{E}-01 \\ -28.20 & 9.7867 \mathrm{E}-01 & 9.8148 \mathrm{E}-01 & 9.6651 \mathrm{E}-01 \\ -23.07 & 1.0569 \mathrm{E}+00 & 1.0636 \mathrm{E}+00 & 1.0417 \mathrm{E}+00 \\ -17.94 & 1.1204 \mathrm{E}+00 & 1.1292 \mathrm{E}+00 & 1.1032 \mathrm{E}+00 \\ -12.82 & 1.1683 \mathrm{E}+00 & 1.1779 \mathrm{E}+00 & 1.1276 \mathrm{E}+00 \\ -7.69 & 1.2004 \mathrm{E}+00 & 1.2105 \mathrm{E}+00 & 1.1582 \mathrm{E}+00 \\ -2.56 & 1.2162 \mathrm{E}+00 & 1.2267 \mathrm{E}+00 & 1.1733 \mathrm{E}+00 \\ 2.56 & 1.2155 \mathrm{E}+00 & 1.2263 \mathrm{E}+00 & 1.1799 \mathrm{E}+00 \\ 7.69 & 1.1977 \mathrm{E}+00 & 1.2087 \mathrm{E}+00 & 1.1628 \mathrm{E}+00 \\ 12.82 & 1.1630 \mathrm{E}+00 & 1.1739 \mathrm{E}+00 & 1.1294 \mathrm{E}+00 \\ 17.94 & 1.1115 \mathrm{E}+00 & 1.1223 \mathrm{E}+00 & 1.1129 \mathrm{E}+00 \\ 23.07 & 1.0426 \mathrm{E}+00 & 1.0518 \mathrm{E}+00 & 1.0447 \mathrm{E}+00 \\ 28.20 & 9.5720 \mathrm{E}-01 & 9.6296 \mathrm{E}-01 & 9.6039 \mathrm{E}-01 \\ 33.32 & 8.5696 \mathrm{E}-01 & 8.5688 \mathrm{E}-01 & 9.0334 \mathrm{E}-01 \\ 38.45 & 7.4345 \mathrm{E}-01 & 7.3186 \mathrm{E}-01 & 7.8973 \mathrm{E}-01 \\ 43.58 & 6.2218 \mathrm{E}-01 & 5.8959 \mathrm{E}-01 & 6.7842 \mathrm{E}-01 \\ 47.17 & 5.3794 \mathrm{E}-01 & 4.7743 \mathrm{E}-01 & 9.2994 \mathrm{E}-02 \\ 50.62 & 4.5774 \mathrm{E}-01 & 3.7865 \mathrm{E}-01 & 7.4322 \mathrm{E}-02 \\ 55.49 & 3.5705 \mathrm{E}-01 & 2.7295 \mathrm{E}-01 & 5.5980 \mathrm{E}-02 \\ 60.35 & 2.6927 \mathrm{E}-01 & 1.9082 \mathrm{E}-01 & 4.2238 \mathrm{E}-02 \\ 64.98 & 2.1473 \mathrm{E}-01 & 1.4299 \mathrm{E}-01 & 0.0000 \mathrm{E}+00 \\ 69.39 & 1.8308 \mathrm{E}-01 & 1.1774 \mathrm{E}-01 & 0.0000 \mathrm{E}+00 \\ 76.46 & 1.3845 \mathrm{E}-01 & 8.3812 \mathrm{E}-02 & 0.0000 \mathrm{E}+00 \\ 86.80 & 8.9917 \mathrm{E}-02 & 5.0664 \mathrm{E}-02 & 0.0000 \mathrm{E}+00 \\ 98.14 & 5.1718 \mathrm{E}-02 & 2.7702 \mathrm{E}-02 & 0.0000 \mathrm{E}+00 \\ 110.00 & 2.2097 \mathrm{E}-02 & 1.2362 \mathrm{E}-02 & 0.0000 \mathrm{E}+00\end{array}$


HNF-SD-FF-ANAL-009 Rev. 1

Table EllA-3.4. Axial Distribution of Total Flux, Fast Flux and Power in MFA-2 at EOC $11 \mathrm{~A}-3$

$\begin{array}{rccc}\text { Z (cm) } & \text { Total Flux } & \text { Flux }>0.1 \mathrm{MeV} & \text { Power } \\ -97.78 & 2.5095 \mathrm{E}-02 & 1.2838 \mathrm{E}-02 & 0.0000 \mathrm{E}+00 \\ -92.78 & 5.3859 \mathrm{E}-02 & 2.4782 \mathrm{E}-02 & 0.0000 \mathrm{E}+00 \\ -87.78 & 8.6353 \mathrm{E}-02 & 3.9667 \mathrm{E}-02 & 0.0000 \mathrm{E}+00 \\ -82.78 & 1.2514 \mathrm{E}-01 & 5.9224 \mathrm{E}-02 & 0.0000 \mathrm{E}+00 \\ -77.78 & 1.7301 \mathrm{E}-01 & 8.5636 \mathrm{E}-02 & 0.0000 \mathrm{E}+00 \\ -72.15 & 2.2825 \mathrm{E}-01 & 1.2017 \mathrm{E}-01 & 0.0000 \mathrm{E}+00 \\ -65.90 & 2.8533 \mathrm{E}-01 & 1.6487 \mathrm{E}-01 & 0.0000 \mathrm{E}+00 \\ -60.35 & 3.4614 \mathrm{E}-01 & 2.2650 \mathrm{E}-01 & 7.9755 \mathrm{E}-02 \\ -55.49 & 4.2511 \mathrm{E}-01 & 3.0746 \mathrm{E}-01 & 9.2883 \mathrm{E}-02 \\ -50.62 & 5.1602 \mathrm{E}-01 & 4.1280 \mathrm{E}-01 & 1.1043 \mathrm{E}-01 \\ -47.17 & 5.8859 \mathrm{E}-01 & 5.1193 \mathrm{E}-01 & 1.2864 \mathrm{E}-01 \\ -43.58 & 6.6499 \mathrm{E}-01 & 6.2398 \mathrm{E}-01 & 7.2531 \mathrm{E}-01 \\ -38.45 & 7.7715 \mathrm{E}-01 & 7.6153 \mathrm{E}-01 & 8.1277 \mathrm{E}-01 \\ -33.32 & 8.8227 \mathrm{E}-01 & 8.7954 \mathrm{E}-01 & 9.1217 \mathrm{E}-01 \\ -28.20 & 9.7518 \mathrm{E}-01 & 9.7826 \mathrm{E}-01 & 9.6282 \mathrm{E}-01 \\ -23.07 & 1.0546 \mathrm{E}+00 & 1.0608 \mathrm{E}+00 & 1.0396 \mathrm{E}+00 \\ -17.94 & 1.1191 \mathrm{E}+00 & 1.1266 \mathrm{E}+00 & 1.1022 \mathrm{E}+00 \\ -12.82 & 1.1675 \mathrm{E}+00 & 1.1752 \mathrm{E}+00 & 1.1255 \mathrm{E}+00 \\ -7.69 & 1.2002 \mathrm{E}+00 & 1.2081 \mathrm{E}+00 & 1.1568 \mathrm{E}+00 \\ -2.56 & 1.2168 \mathrm{E}+00 & 1.2251 \mathrm{E}+00 & 1.1726 \mathrm{E}+00 \\ 2.56 & 1.2169 \mathrm{E}+00 & 1.2259 \mathrm{E}+00 & 1.1791 \mathrm{E}+00 \\ 7.69 & 1.2000 \mathrm{E}+00 & 1.2097 \mathrm{E}+00 & 1.1630 \mathrm{E}+00 \\ 12.82 & 1.1662 \mathrm{E}+00 & 1.1766 \mathrm{E}+00 & 1.1305 \mathrm{E}+00 \\ 17.94 & 1.1156 \mathrm{E}+00 & 1.1269 \mathrm{E}+00 & 1.1153 \mathrm{E}+00 \\ 23.07 & 1.0472 \mathrm{E}+00 & 1.0577 \mathrm{E}+00 & 1.0477 \mathrm{E}+00 \\ 28.20 & 9.6192 \mathrm{E}-01 & 9.6974 \mathrm{E}-01 & 9.6368 \mathrm{E}-01 \\ 33.32 & 8.6151 \mathrm{E}-01 & 8.6398 \mathrm{E}-01 & 9.0804 \mathrm{E}-01 \\ 38.45 & 7.4733 \mathrm{E}-01 & 7.3769 \mathrm{E}-01 & 7.9489 \mathrm{E}-01 \\ 43.58 & 6.2552 \mathrm{E}-01 & 5.9267 \mathrm{E}-01 & 6.8795 \mathrm{E}-01 \\ 47.17 & 5.4343 \mathrm{E}-01 & 4.7990 \mathrm{E}-01 & 1.0321 \mathrm{E}-01 \\ 50.62 & 4.6703 \mathrm{E}-01 & 3.8215 \mathrm{E}-01 & 8.3803 \mathrm{E}-02 \\ 55.49 & 3.7155 \mathrm{E}-01 & 2.7847 \mathrm{E}-01 & 6.4955 \mathrm{E}-02 \\ 60.35 & 2.8630 \mathrm{E}-01 & 1.9716 \mathrm{E}-01 & 5.0556 \mathrm{E}-02 \\ 64.98 & 2.3072 \mathrm{E}-01 & 1.4754 \mathrm{E}-01 & 0.0000 \mathrm{E}+00 \\ 69.39 & 1.9664 \mathrm{E}-01 & 1.2041 \mathrm{E}-01 & 0.0000 \mathrm{E}+00 \\ 76.46 & 1.4792 \mathrm{E}-01 & 8.4113 \mathrm{E}-02 & 0.0000 \mathrm{E}+00 \\ 86.80 & 9.5175 \mathrm{E}-02 & 4.9717 \mathrm{E}-02 & 0.0000 \mathrm{E}+00 \\ 98.14 & 5.4147 \mathrm{E}-02 & 2.6645 \mathrm{E}-02 & 0.0000 \mathrm{E}+00 \\ 110.00 & 2.2909 \mathrm{E}-02 & 1.1716 \mathrm{E}-02 & 0.0000 \mathrm{E}+00 \\ & & & \end{array}$


HNF-SD-FF-ANAL-009 Rev. 1

Table E11A-3.5. Fission Power Distribution by Pin in MFA-1 at EOC 11A-3 CORE POSITION 1404

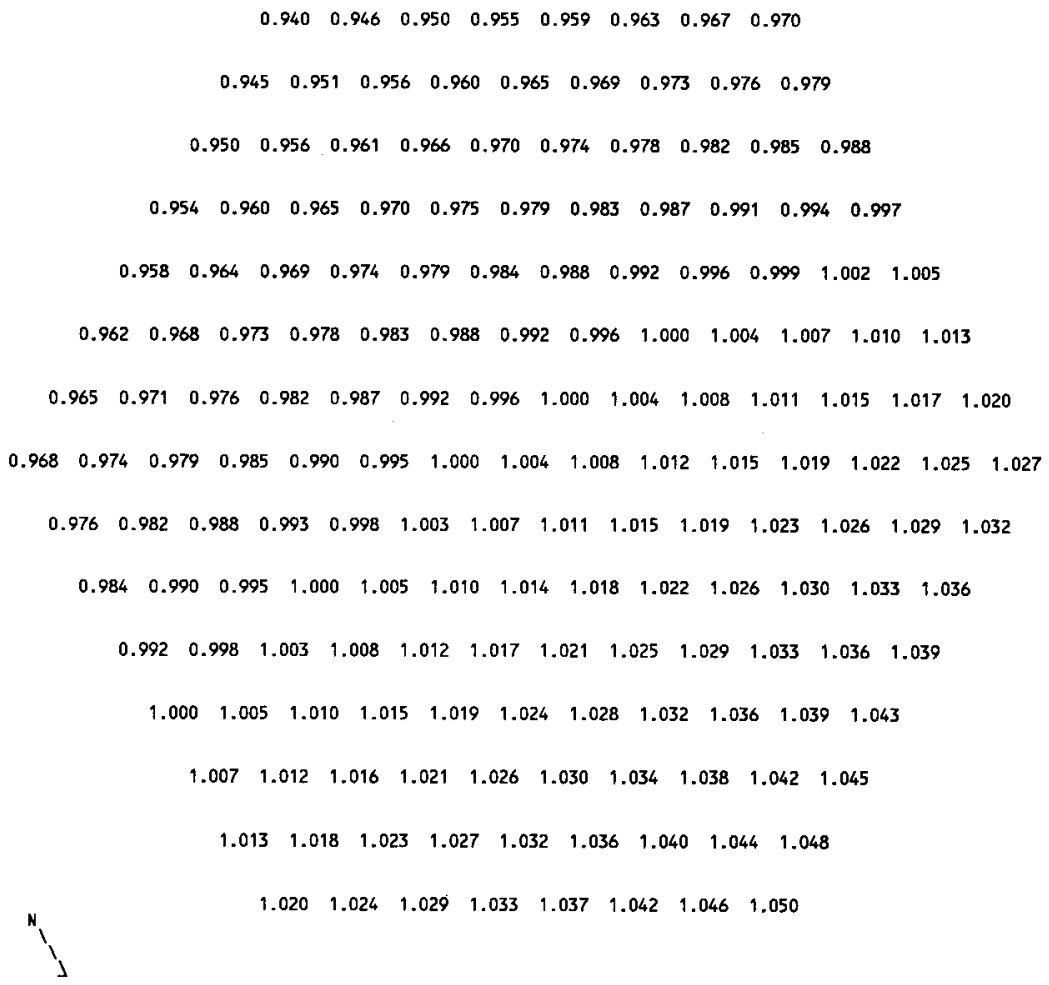


HNF-SD-FF-ANAL-009 Rev. 1

Table E11A-3.6. Fission Power Distribution by Pin in MFA-2 at EOC 11A-3 CORE POSITION 2506

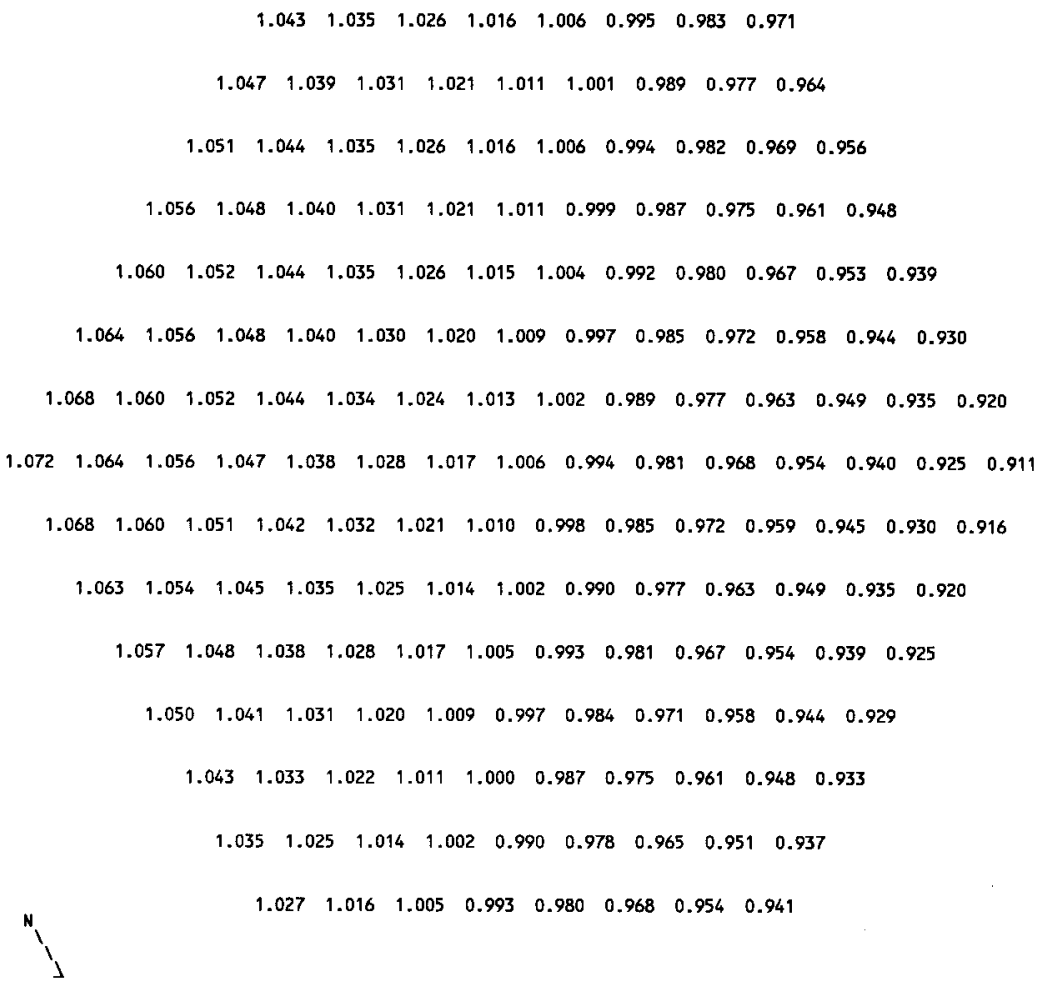




\section{HNF-SD-FF-ANAL-009 Rev. 1}

Table El1A-3.7. Fast Flux Distribution by Pin in MFA-1 at EOC 11A-3 CORE POSITION 1404

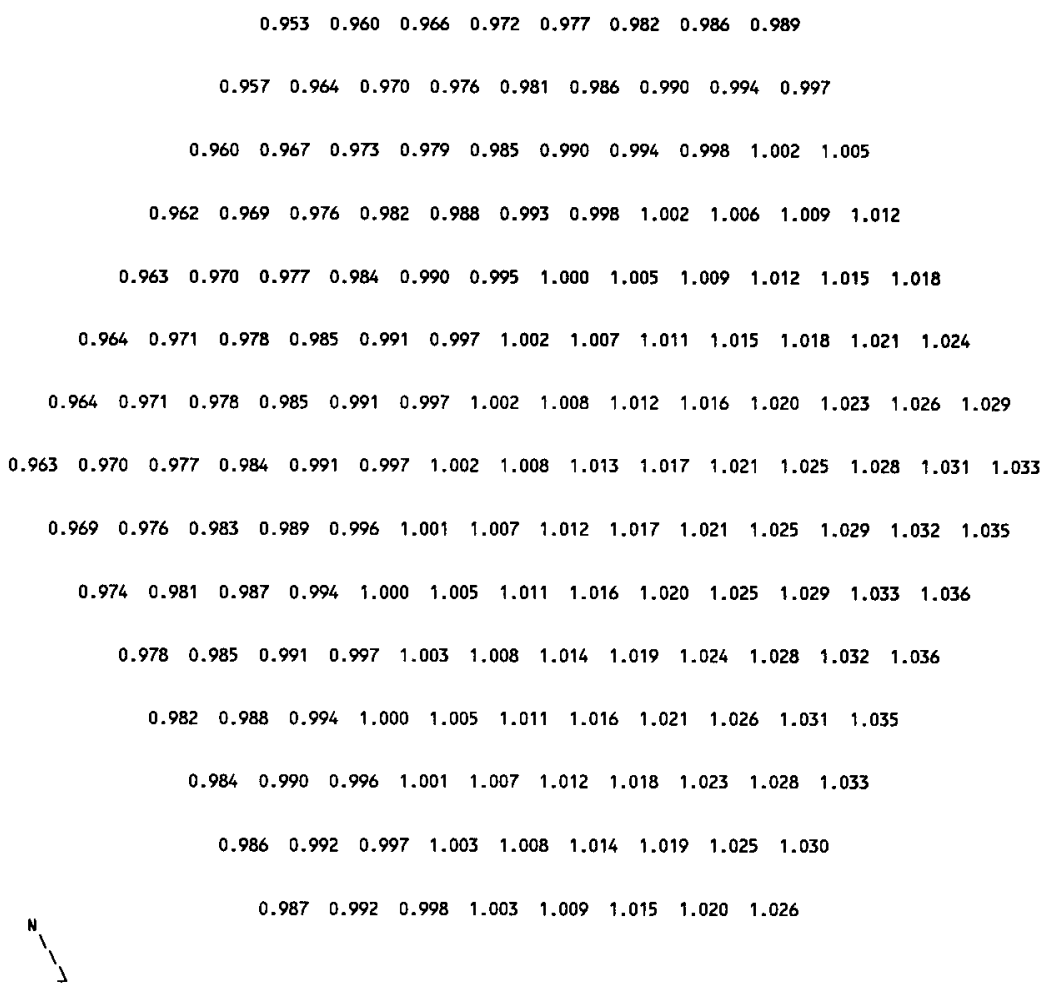




\section{HNF-SD-FF-ANAL-009 Rev. 1}

Table EllA-3.8. Fast Flux Distribution by Pin in MFA-2 at EOC 11A-3 CORE POSITION 2506

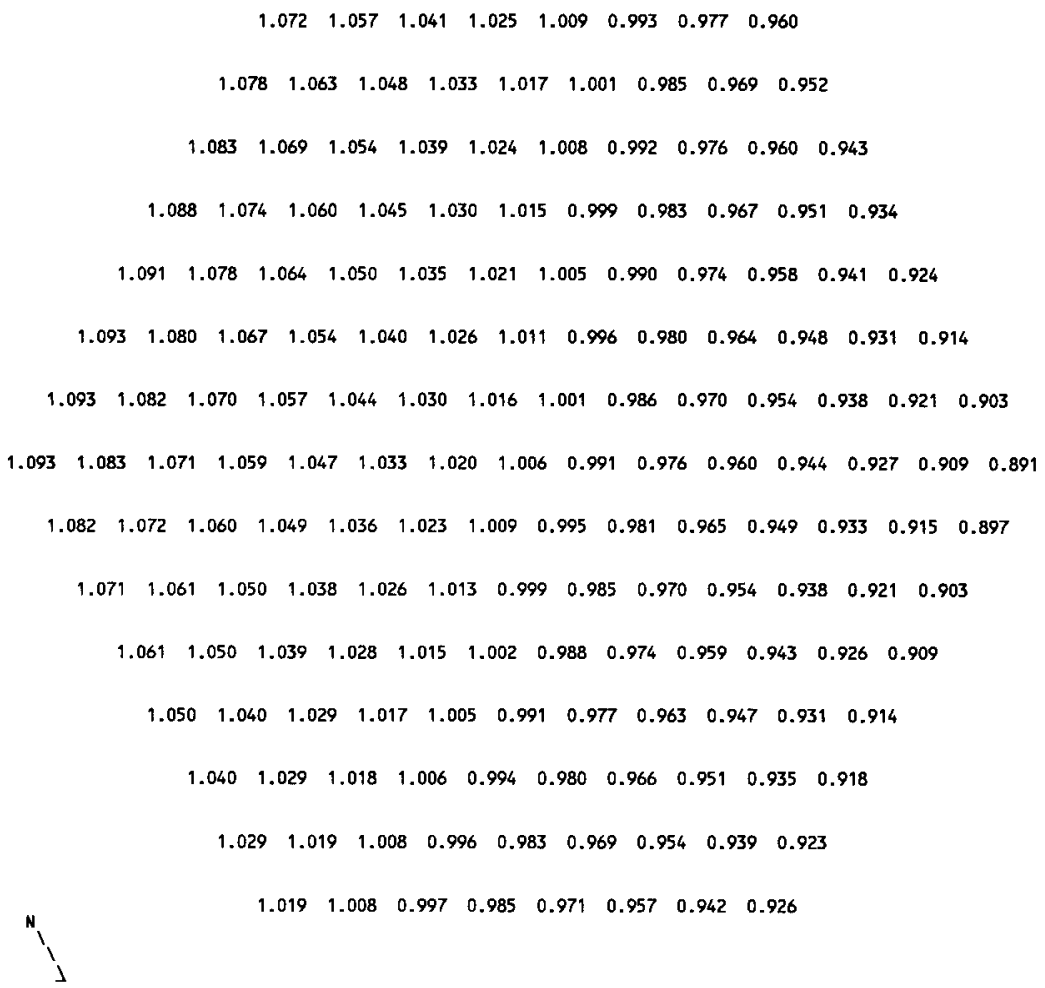


Table EIIA-3.9. MFA-1 and MFA-2 Duct Wall

Fast Flux Data at EOC 11A-3

$\begin{array}{ccc}\text { Side } & \text { Flux }>0.1 \mathrm{MeV}\left(\mathrm{n} / \mathrm{cm}^{2}-\mathrm{sec}\right) \\ \text { MFA-1 } & \text { MFA-2 } \\ \text { E } & 2.0341 \mathrm{E}+15 & 2.2026 \mathrm{E}+15 \\ \text { SE } & 1.9788 \mathrm{1}+15 & 2.2969 \mathrm{E}+15 \\ \text { SW } & 2.0075 \mathrm{E}+15 & 2.1089 \mathrm{E}+15 \\ \text { W } & 2.1108 \mathrm{1}+15 & 1.8927 \mathrm{E}+15 \\ \text { NW } & 2.1746 \mathrm{E}+15 & 1.8453 \mathrm{E}+15 \\ \text { NE } & 2.0938 \mathrm{E}+15 & 2.0195 \mathrm{E}+15\end{array}$

Table E11A-3.10. Assembly Outlet Temperatures and Flow Rates at EOC $11 \mathrm{~A}-3$

$\begin{array}{lcl}\text { CALCULATED } \\ \text { CORE } & \begin{array}{c}\text { OUTLET TEMP. } \\ \text { (DEG F) }\end{array} & \begin{array}{l}\text { FLOW RATE } \\ \text { (LB/H) }\end{array} \\ \text { POS. } & \text { (DES } \\ 1404 & 1059 & 113570 \\ 1303 & 999 & 68360 \\ 1304 & 910 & 205640 \\ 1403 & 862 & 205660 \\ 1405 & 881 & 205660 \\ 1505 & 899 & 195550 \\ 1506 & 884 & 205640 \\ 2506 & 1043 & 113570 \\ 2404 & 1037 & 202240 \\ 2405 & 874 & 205660 \\ 2505 & 897 & 205640 \\ 2507 & 889 & 205640 \\ 2606 & 931 & 168640 \\ 2607 & 908 & 161730 \\ & & \end{array}$

Assembly flows are based on a calculated core pressure drop of $113.3 \mathrm{psi}$ at a total reactor flow rate of 16.67E+06 1b/hr. 


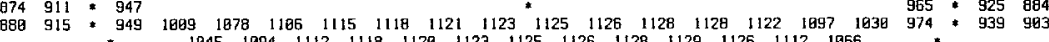
* $\quad \begin{array}{lllllllllllll}1845 & 1894 & 1112 & 1118 & 1128 & 1123 & 1125 & 1126 & 1128 & 1129 & 1126 & 1112 & 1866\end{array}$

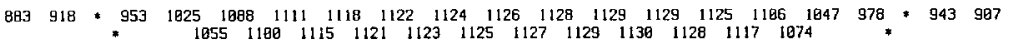

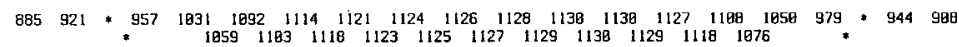

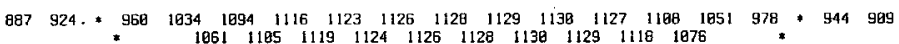

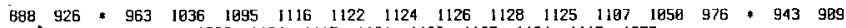
$\begin{array}{llllllllll}* & 1862 & 1104 & 1117 & 1121 & 1123 & 1125 & 1124 & 1115 & 1075\end{array}$

889927 * 9641035 1889 I1घ5 $111111113 \quad 1115 \quad 1114 \quad 1106 \quad 1946 \quad 974$ * 942989

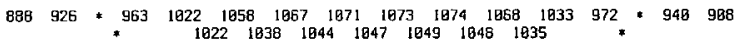

$$
\begin{aligned}
& \text { *957 961 968 } 573 \text { 977 } 988 \text { 981 } 979.972 * \\
& 934 \text { " " }
\end{aligned}
$$

$\begin{array}{lllllllll}911 & 956 & 959 & 962 & 964 & 964 & 963 & 968 & 924\end{array}$


Table EllA-3.12. MFA-2 Sodium Subchannel Temperatures at Top of Core Elevation for EOC 11A-3

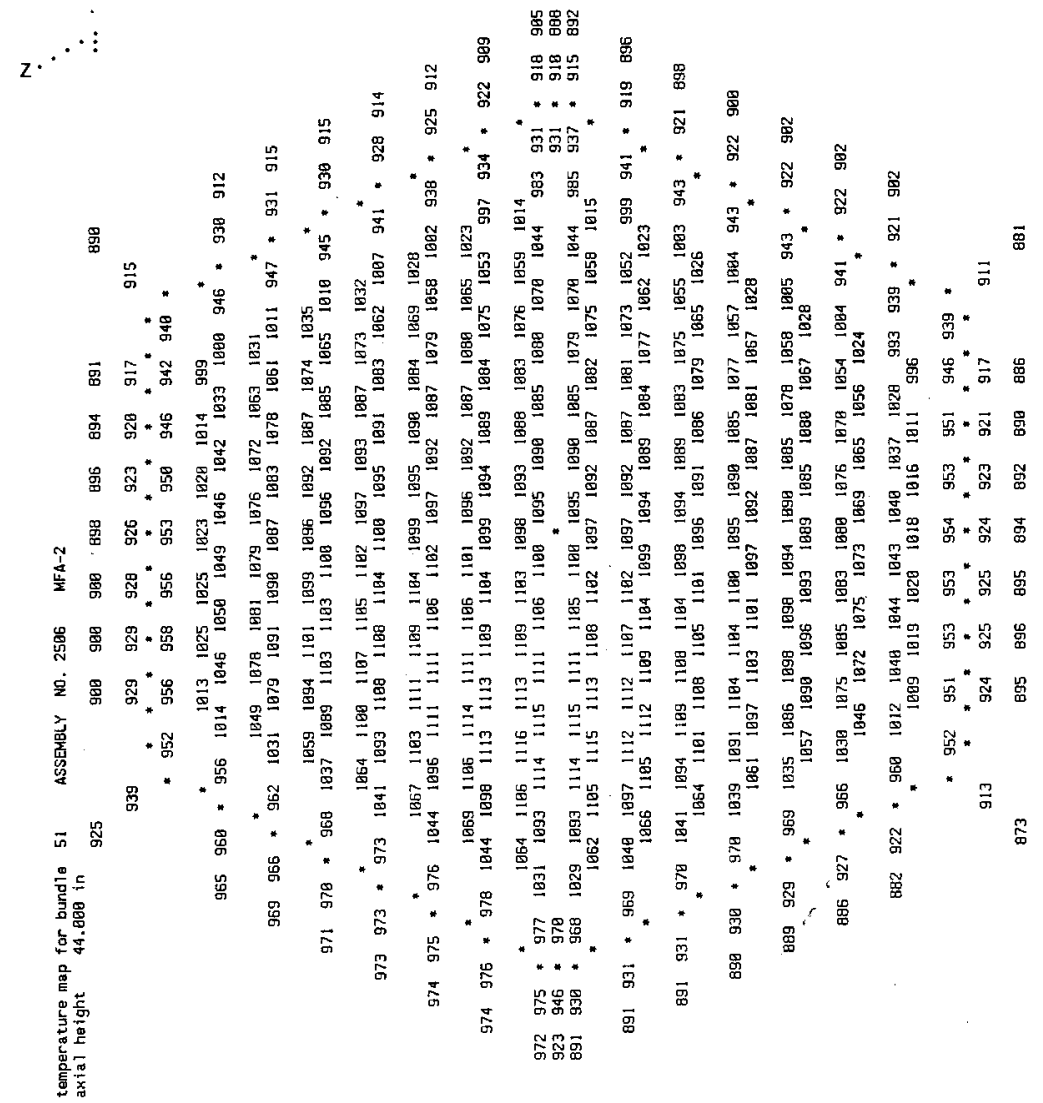


Table EllA-3.13. MFA-1 Sodium Subchanne1 Temperatures at Elevation of Upper Axial Blanket for EOC 11A-3

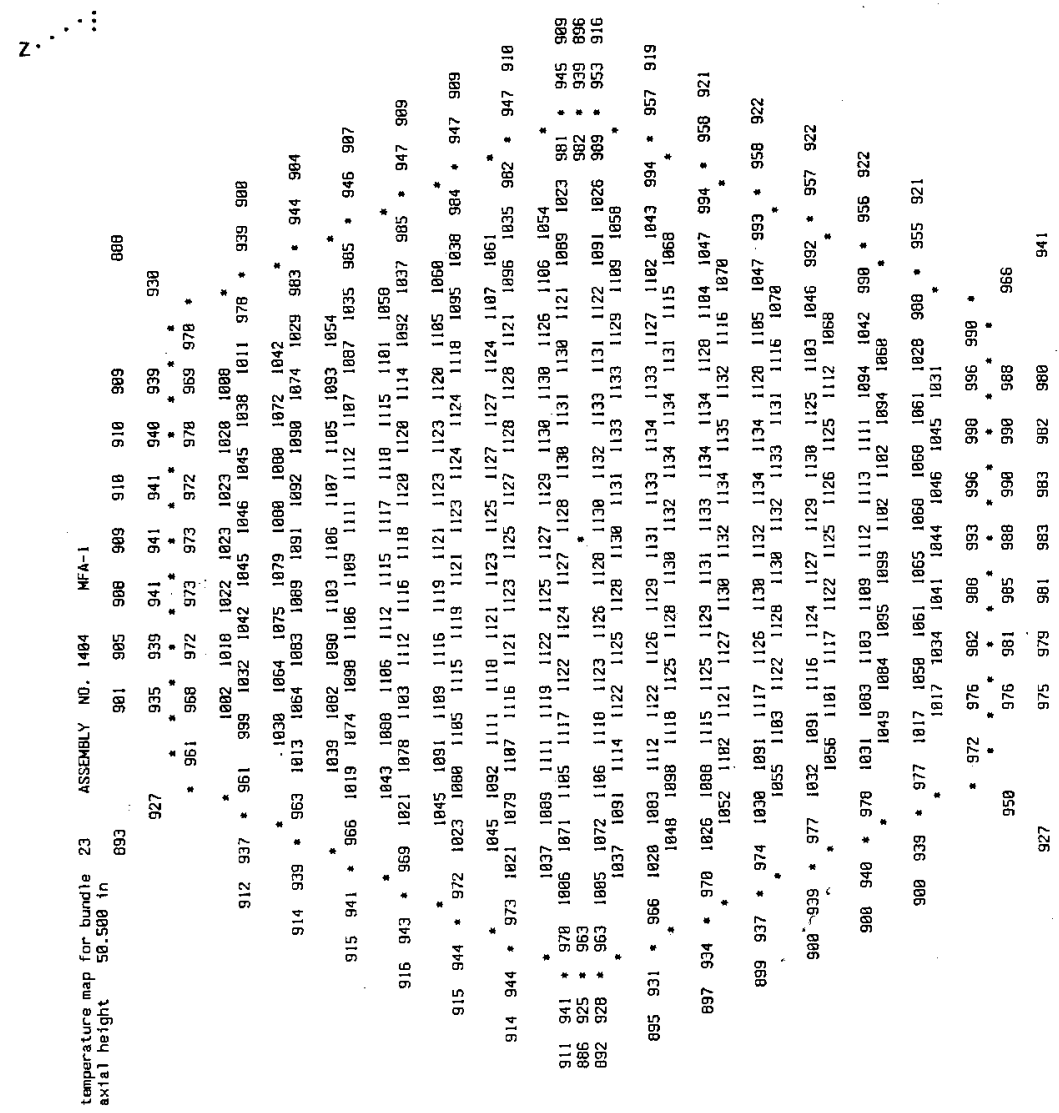


Table E11A-3.14. MFA-2 Sodium Subchannel Temperatures at Elevation of Upper Axial Blanket for EOC 11A-3

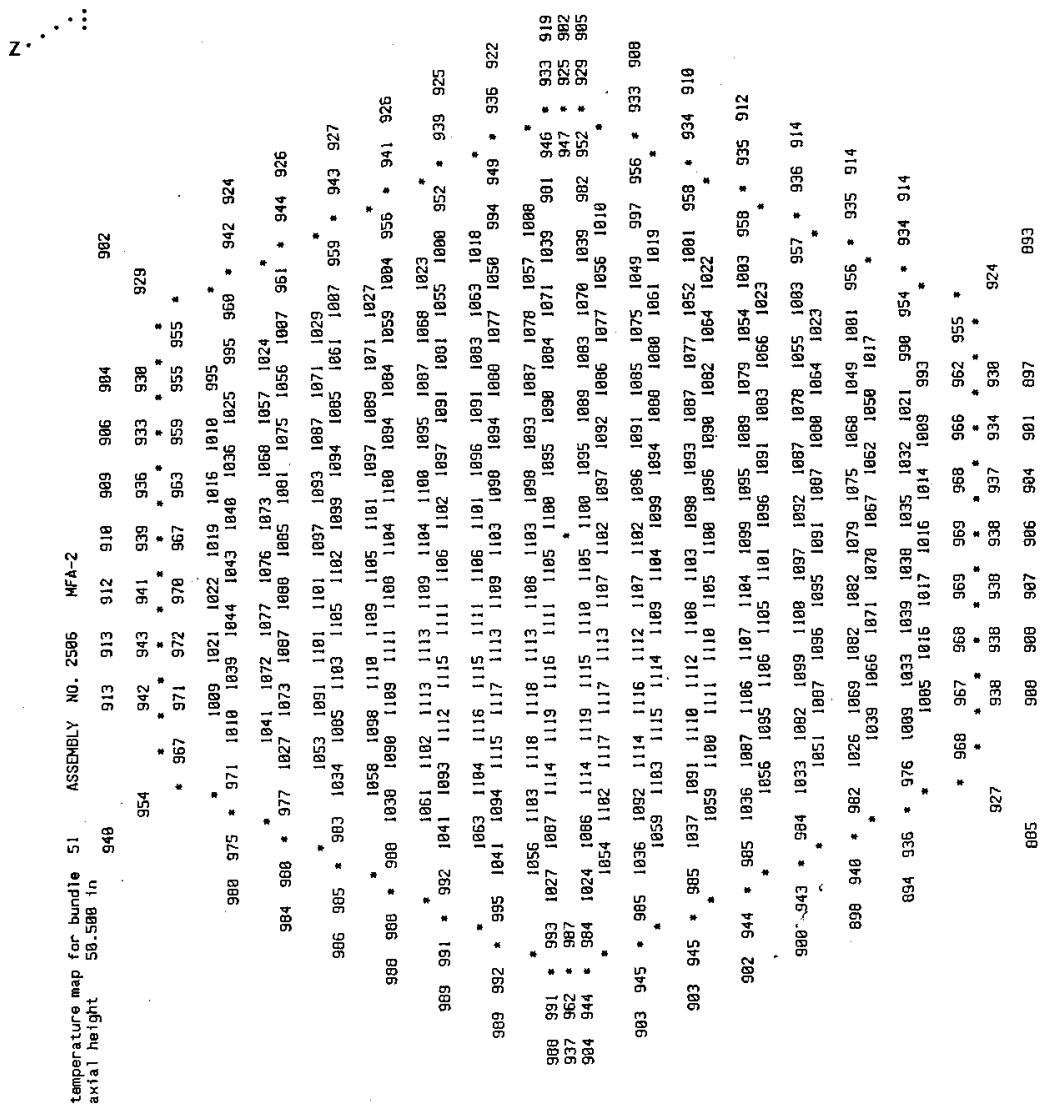


Table E11A-3.15. MFA-1 Sodium Subchannel Temperatures at Top of Fuel Pin Bundle for EOC $11 \mathrm{~A}-3$

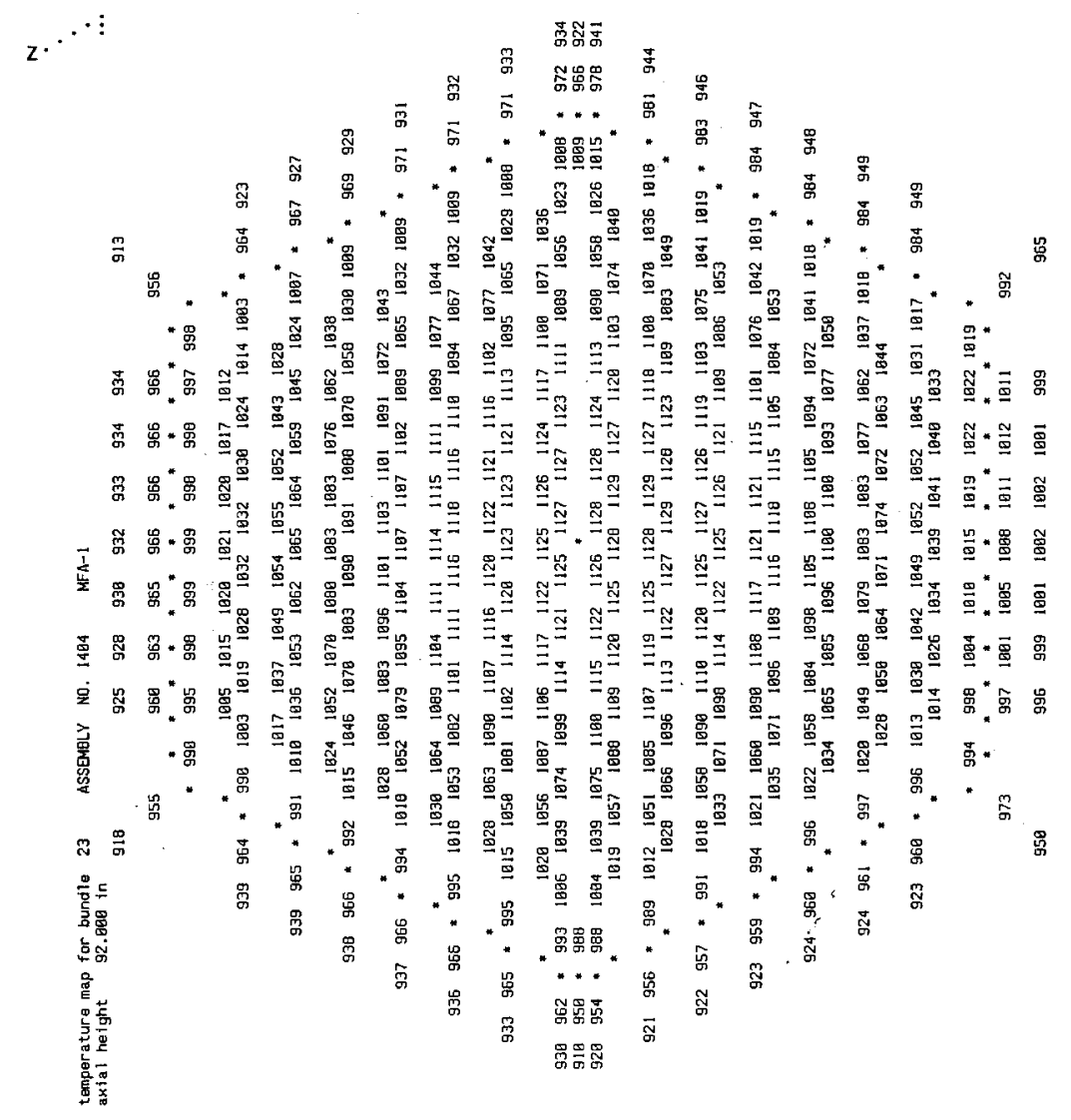


Table E11A-3.16. MFA-2 Sodium Subchannel Temperatures at Top of Fuel Pin Bundle for EOC $11 \mathrm{~A}-3$

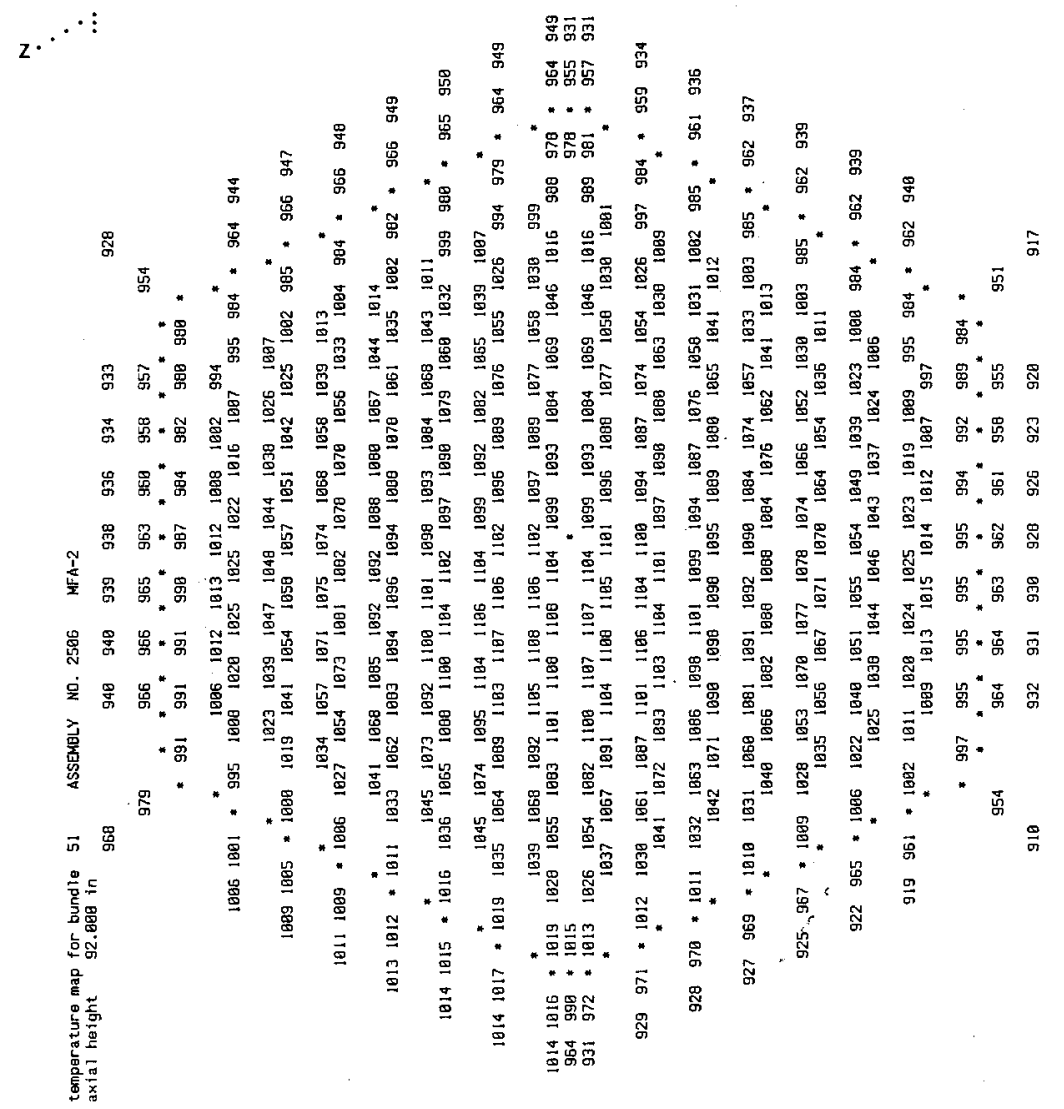


Table El1A-3.17. Composition of MFA-1 at EOC 11A-3

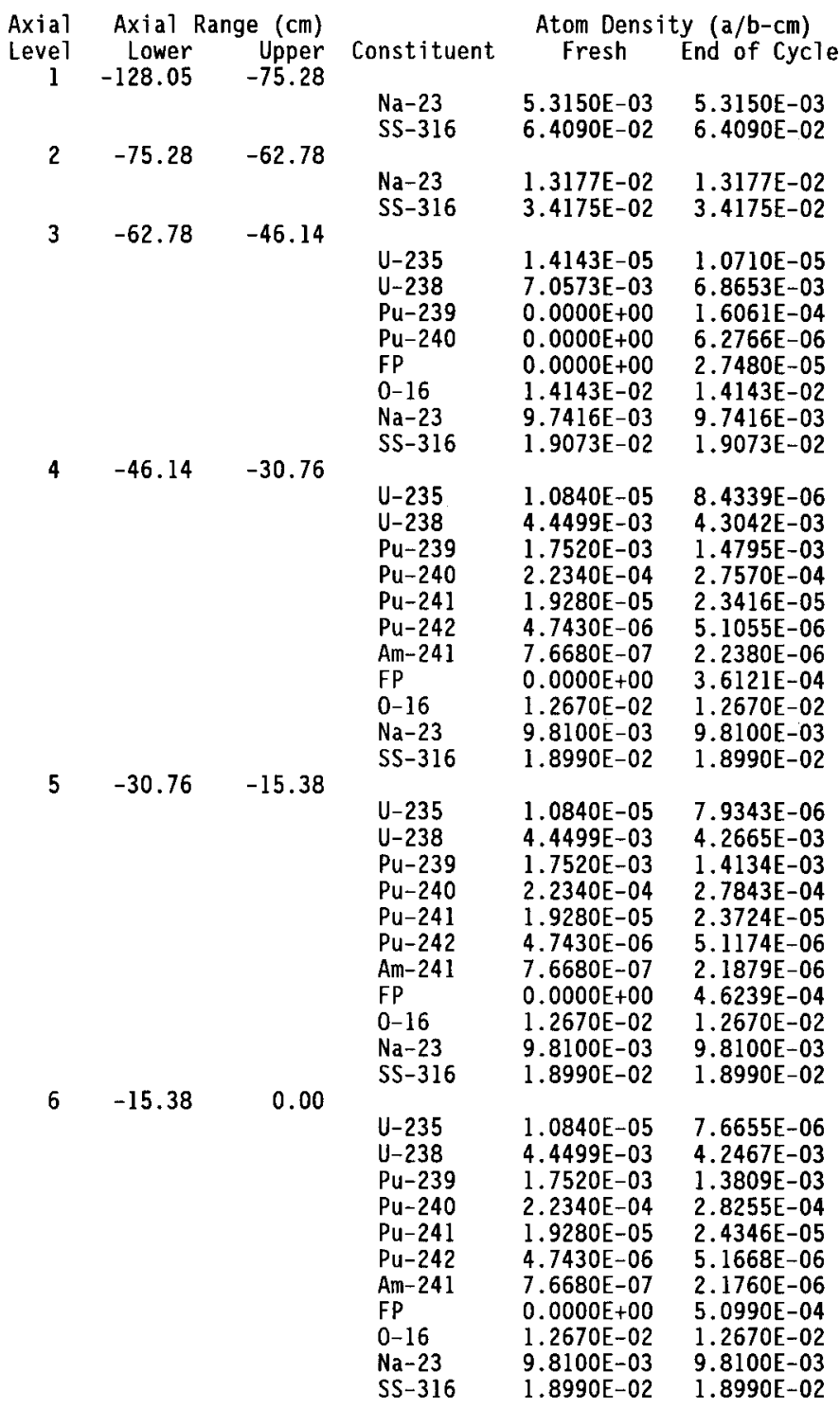


HNF-SD-FF-ANAL-009 Rev, 1

\begin{tabular}{|c|c|c|c|c|c|}
\hline 7 & 0.00 & 15.38 & & & \\
\hline & & & $\begin{array}{l}\mathrm{U}-235 \\
\mathrm{U}-238 \\
\mathrm{Pu}-239 \\
\mathrm{Pu}-240 \\
\mathrm{Pu}-241 \\
\mathrm{Pu}-242 \\
\mathrm{Am}-241 \\
\mathrm{FP} \\
0-16 \\
\mathrm{Na}-23 \\
\mathrm{SS}-316\end{array}$ & $\begin{array}{l}1.0840 \mathrm{E}-05 \\
4.4499 \mathrm{E}-03 \\
1.7520 \mathrm{E}-03 \\
2.2340 \mathrm{E}-04 \\
1.9280 \mathrm{E}-05 \\
4.7430 \mathrm{E}-06 \\
7.6680 \mathrm{E}-07 \\
0.0000 \mathrm{E}+00 \\
1.2670 \mathrm{E}-02 \\
9.8100 \mathrm{E}-03 \\
1.8990 \mathrm{E}-02\end{array}$ & $\begin{array}{l}7.7547 \mathrm{E}-06 \\
4.2532 \mathrm{E}-03 \\
1.3914 \mathrm{E}-03 \\
2.8089 \mathrm{E}-04 \\
2.4097 \mathrm{E}-05 \\
5.1465 \mathrm{E}-06 \\
2.1798 \mathrm{E}-06 \\
4.9469 \mathrm{E}-04 \\
1.2670 \mathrm{E}-02 \\
9.8100 \mathrm{E}-03 \\
1.8990 \mathrm{E}-02\end{array}$ \\
\hline 8 & 15.38 & 30.76 & & & \\
\hline & & & $\begin{array}{l}\mathrm{U}-235 \\
\mathrm{U}-238 \\
\mathrm{Pu}-239 \\
\mathrm{Pu}-240 \\
\mathrm{Pu}-241 \\
\mathrm{Pu}-242 \\
\mathrm{Am}-241 \\
\mathrm{FP} \\
0-16 \\
\mathrm{Na}-23 \\
\mathrm{SS}-316\end{array}$ & $\begin{array}{l}1.0840 \mathrm{E}-05 \\
4.4499 \mathrm{E}-03 \\
1.7520 \mathrm{E}-03 \\
2.2340 \mathrm{E}-04 \\
1.9280 \mathrm{E}-05 \\
4.7430 \mathrm{E}-06 \\
7.6680 \mathrm{E}-07 \\
0.0000 \mathrm{E}+00 \\
1.2670 \mathrm{E}-02 \\
9.8100 \mathrm{E}-03 \\
1.8990 \mathrm{E}-02\end{array}$ & $\begin{array}{l}8.1903 \mathrm{E}-06 \\
4.2847 \mathrm{E}-03 \\
1.4430 \mathrm{E}-03 \\
2.7349 \mathrm{E}-04 \\
2.3030 \mathrm{E}-05 \\
5.0641 \mathrm{E}-06 \\
2.1987 \mathrm{E}-06 \\
4.2023 \mathrm{E}-04 \\
1.2670 \mathrm{E}-02 \\
9.8100 \mathrm{E}-03 \\
1.8990 \mathrm{E}-02\end{array}$ \\
\hline 9 & 30.76 & 46.14 & & & \\
\hline & & & $\begin{array}{l}\mathrm{U}-235 \\
\mathrm{U}-238 \\
\mathrm{Pu}-239 \\
\mathrm{Pu}-240 \\
\mathrm{Pu}-241 \\
\mathrm{Pu}-242 \\
\mathrm{Am}-241 \\
\mathrm{FP} \\
\mathrm{O}-16 \\
\mathrm{Na}-23 \\
\mathrm{SS}-316\end{array}$ & $\begin{array}{l}1.0840 \mathrm{E}-05 \\
4.4499 \mathrm{E}-03 \\
1.7520 \mathrm{E}-03 \\
2.2340 \mathrm{E}-04 \\
1.9280 \mathrm{E}-05 \\
4.7430 \mathrm{E}-06 \\
7.6680 \mathrm{E}-07 \\
0.0000 \mathrm{E}+00 \\
1.2670 \mathrm{E}-02 \\
9.8100 \mathrm{E}-03 \\
1.8990 \mathrm{E}-02\end{array}$ & $\begin{array}{l}8.8321 \mathrm{E}-06 \\
4.3302 \mathrm{E}-03 \\
1.5237 \mathrm{E}-03 \\
2.6611 \mathrm{E}-04 \\
2.2112 \mathrm{E}-05 \\
5.0090 \mathrm{E}-06 \\
2.2463 \mathrm{E}-06 \\
3.0174 \mathrm{E}-04 \\
1.2670 \mathrm{E}-02 \\
9.8100 \mathrm{E}-03 \\
1.8990 \mathrm{E}-02\end{array}$ \\
\hline 10 & 46.14 & 62.78 & & & \\
\hline & & & $\begin{array}{l}\mathrm{U}-235 \\
\mathrm{U}-238 \\
\mathrm{Pu}-239 \\
\mathrm{Pu}-240 \\
\mathrm{FP} \\
0-16 \\
\mathrm{Na}-23 \\
\mathrm{SS}-316\end{array}$ & $\begin{array}{l}1.4143 \mathrm{E}-05 \\
7.0573 \mathrm{E}-03 \\
0.0000 \mathrm{E}+00 \\
0.0000 \mathrm{E}+00 \\
0.0000 \mathrm{E}+00 \\
1.4143 \mathrm{E}-02 \\
9.7416 \mathrm{E}-03 \\
1.9073 \mathrm{E}-02\end{array}$ & $\begin{array}{l}1.1796 \mathrm{E}-05 \\
6.9257 \mathrm{E}-03 \\
1.1354 \mathrm{E}-04 \\
2.7526 \mathrm{E}-06 \\
1.6943 \mathrm{E}-05 \\
1.4143 \mathrm{E}-02 \\
9.7416 \mathrm{E}-03 \\
1.9073 \mathrm{E}-02\end{array}$ \\
\hline 11 & 62.78 & 17 & $\begin{array}{l}\mathrm{Na}-23 \\
\mathrm{SS}-316\end{array}$ & $\begin{array}{l}8.2400 \mathrm{E}-03 \\
2.5562 \mathrm{E}-02\end{array}$ & $\begin{array}{l}8.2400 E-03 \\
2.5562 E-02\end{array}$ \\
\hline
\end{tabular}


Table EllA-3.18. Composition of MFA-2 at EOC 11A-3

\begin{tabular}{|c|c|c|c|c|c|}
\hline ial & Axial & ge $(\mathrm{cm})$ & & Atom Dens & $(a / b-c m)$ \\
\hline Leve1 & Lower & Upper & Constituent & Fresh & ind of cycle \\
\hline & & & $\begin{array}{l}\mathrm{Na}-23 \\
\mathrm{SS}-316\end{array}$ & $\begin{array}{l}5.3150 \mathrm{E}-03 \\
6.4090 \mathrm{E}-02\end{array}$ & $\begin{array}{l}5.3150 \mathrm{E}-03 \\
6.4090 \mathrm{E}-02\end{array}$ \\
\hline 2 & -75.28 & -62.78 & & & \\
\hline & & & $\begin{array}{l}\mathrm{Na}-23 \\
\mathrm{SS}-316\end{array}$ & $\begin{array}{l}1.3177 \mathrm{E}-02 \\
3.4175 \mathrm{E}-02\end{array}$ & $\begin{array}{l}1.3177 \mathrm{E}-02 \\
3.4175 \mathrm{E}-02\end{array}$ \\
\hline 3 & -62.78 & -46.14 & $\begin{array}{l}U-235 \\
U-238 \\
\mathrm{Pu}-239 \\
\mathrm{Pu}-240 \\
\mathrm{FP} \\
0-16 \\
\mathrm{Na}-23 \\
\mathrm{SS}-316\end{array}$ & $\begin{array}{l}1.4143 \mathrm{E}-05 \\
7.0573 \mathrm{E}-03 \\
0.0000 \mathrm{E}+00 \\
0.0000 \mathrm{E}+00 \\
0.0000 \mathrm{E}+00 \\
1.4143 \mathrm{E}-02 \\
9.7416 \mathrm{E}-03 \\
1.9073 \mathrm{E}-02\end{array}$ & $\begin{array}{l}1.0640 \mathrm{E}-05 \\
6.8638 \mathrm{E}-03 \\
1.6120 \mathrm{E}-04 \\
6.5696 \mathrm{E}-06 \\
2.8194 \mathrm{E}-05 \\
1.4143 \mathrm{E}-02 \\
9.7416 \mathrm{E}-03 \\
1.9073 \mathrm{E}-02\end{array}$ \\
\hline 4 & -46.14 & -30.76 & $\begin{array}{l}U-235 \\
U-238 \\
P u-239 \\
P u-240 \\
P u-241 \\
P u-242 \\
\text { Am-241 } \\
\text { FP } \\
0-16 \\
\mathrm{Na}-23 \\
\text { SS-316 }\end{array}$ & $\begin{array}{l}1.0160 \mathrm{E}-05 \\
4.4270 \mathrm{E}-03 \\
1.7483 \mathrm{E}-03 \\
2.2290 \mathrm{E}-04 \\
1.9040 \mathrm{E}-05 \\
4.7330 \mathrm{E}-06 \\
7.5700 \mathrm{E}-07 \\
0.0000 \mathrm{E}+00 \\
1.2760 \mathrm{E}-02 \\
9.8100 \mathrm{E}-03 \\
1.8990 \mathrm{E}-02\end{array}$ & $\begin{array}{l}7.8673 \mathrm{E}-06 \\
4.2792 \mathrm{E}-03 \\
1.4699 \mathrm{E}-03 \\
2.7542 \mathrm{E}-04 \\
2.3313 \mathrm{E}-05 \\
5.0929 \mathrm{E}-06 \\
2.2254 \mathrm{E}-06 \\
3.6880 \mathrm{E}-04 \\
1.2760 \mathrm{E}-02 \\
9.8100 \mathrm{E}-03 \\
1.8990 \mathrm{E}-02\end{array}$ \\
\hline 5 & -30.76 & -15.38 & $\begin{array}{l}U-235 \\
U-238 \\
P u-239 \\
P u-240 \\
P u-241 \\
P u-242 \\
\text { Am-241 } \\
\text { FP } \\
\text { O-16 } \\
\mathrm{Na}-23 \\
\text { SS-316 }\end{array}$ & $\begin{array}{l}1.0160 \mathrm{E}-05 \\
4.4270 \mathrm{E}-03 \\
1.7483 \mathrm{E}-03 \\
2.2290 \mathrm{E}-04 \\
1.9040 \mathrm{E}-05 \\
4.7330 \mathrm{E}-06 \\
7.5700 \mathrm{E}-07 \\
0.0000 \mathrm{E}+00 \\
1.2760 \mathrm{E}-02 \\
9.8100 \mathrm{E}-03 \\
1.8990 \mathrm{E}-02\end{array}$ & $\begin{array}{l}7.3712 \mathrm{E}-06 \\
4.2389 \mathrm{E}-03 \\
1.3997 \mathrm{E}-03 \\
2.7822 \mathrm{E}-04 \\
2.3631 \mathrm{E}-05 \\
5.1041 \mathrm{E}-06 \\
2.1757 \mathrm{E}-06 \\
4.7650 \mathrm{E}-04 \\
1.2760 \mathrm{E}-02 \\
9.8100 \mathrm{E}-03 \\
1.8990 \mathrm{E}-02\end{array}$ \\
\hline 6 & -15.38 & 0.00 & $\begin{array}{l}U-235 \\
U-238 \\
P u-239 \\
P u-240 \\
P u-241 \\
P u-242 \\
\text { Am-241 } \\
\text { FP } \\
0-16 \\
\mathrm{Na}-23 \\
S S-316\end{array}$ & $\begin{array}{l}1.0160 \mathrm{E}-05 \\
4.4270 \mathrm{E}-03 \\
1.7483 \mathrm{E}-03 \\
2.2290 \mathrm{E}-04 \\
1.9040 \mathrm{E}-05 \\
4.7330 \mathrm{E}-06 \\
7.5700 \mathrm{E}-07 \\
0.0000 \mathrm{E}+00 \\
1.2760 \mathrm{E}-02 \\
9.8100 \mathrm{E}-03 \\
1.8990 \mathrm{E}-02\end{array}$ & $\begin{array}{l}7.0980 \mathrm{E}-06 \\
4.2173 \mathrm{E}-03 \\
1.3645 \mathrm{E}-03 \\
2.8262 \mathrm{E}-04 \\
2.4318 \mathrm{E}-05 \\
5.1586 \mathrm{E}-06 \\
2.1652 \mathrm{E}-06 \\
5.2827 \mathrm{E}-04 \\
1.2760 \mathrm{E}-02 \\
9.8100 \mathrm{E}-03 \\
1.8990 \mathrm{E}-02\end{array}$ \\
\hline
\end{tabular}


HNF-SD-FF-ANAL-009 Rev, 1

\begin{tabular}{|c|c|c|c|c|c|}
\hline \multirow[t]{2}{*}{7} & 0.00 & 15.38 & & & \\
\hline & & & $\begin{array}{l}U-235 \\
U-238 \\
\mathrm{Pu}-239 \\
\mathrm{Pu}-240 \\
\mathrm{Pu}-241 \\
\mathrm{Pu}-242 \\
\mathrm{Am}-241 \\
\mathrm{FP} \\
\mathrm{O}-16 \\
\mathrm{Na}-23 \\
\mathrm{SS}-316\end{array}$ & $\begin{array}{l}1.0160 \mathrm{E}-05 \\
4.4270 \mathrm{E}-03 \\
1.7483 \mathrm{E}-03 \\
2.2290 \mathrm{E}-04 \\
1.9040 \mathrm{E}-05 \\
4.7330 \mathrm{E}-06 \\
7.5700 \mathrm{E}-07 \\
0.0000 \mathrm{E}+00 \\
1.2760 \mathrm{E}-02 \\
9.8100 \mathrm{E}-03 \\
1.8990 \mathrm{E}-02\end{array}$ & $\begin{array}{l}7.1728 \mathrm{E}-06 \\
4.2233 \mathrm{E}-03 \\
1.3737 \mathrm{E}-03 \\
2.8118 \mathrm{E}-04 \\
2.4093 \mathrm{E}-05 \\
5.1401 \mathrm{E}-06 \\
2.1680 \mathrm{E}-06 \\
5.1464 \mathrm{E}-04 \\
1.2760 \mathrm{E}-02 \\
9.8100 \mathrm{E}-03 \\
1.8990 \mathrm{E}-02\end{array}$ \\
\hline \multirow{2}{*}{8} & 15.38 & 30.76 & & & \\
\hline & & & $\begin{array}{l}U-235 \\
U-238 \\
\mathrm{Pu}-239 \\
\mathrm{Pu}-240 \\
\mathrm{Pu}-241 \\
\mathrm{Pu}-242 \\
\mathrm{Am}-241 \\
\mathrm{FP} \\
\mathrm{O}-16 \\
\mathrm{Na}-23 \\
\mathrm{SS}-316\end{array}$ & $\begin{array}{l}1.0160 \mathrm{E}-05 \\
4.4270 \mathrm{E}-03 \\
1.7483 \mathrm{E}-03 \\
2.2290 \mathrm{E}-04 \\
1.9040 \mathrm{E}-05 \\
4.7330 \mathrm{E}-06 \\
7.5700 \mathrm{E}-07 \\
0.0000 \mathrm{E}+00 \\
1.2760 \mathrm{E}-02 \\
9.8100 \mathrm{E}-03 \\
1.8990 \mathrm{E}-02\end{array}$ & $\begin{array}{l}7.5786 \mathrm{E}-06 \\
4.2547 \mathrm{E}-03 \\
1.4255 \mathrm{E}-03 \\
2.7408 \mathrm{E}-04 \\
2.3040 \mathrm{E}-05 \\
5.0588 \mathrm{E}-06 \\
2.1837 \mathrm{E}-06 \\
4.3971 \mathrm{E}-04 \\
1.2760 \mathrm{E}-02 \\
9.8100 \mathrm{E}-03 \\
1.8990 \mathrm{E}-02\end{array}$ \\
\hline \multirow[t]{2}{*}{9} & 30.76 & 46.14 & & & \\
\hline & & & $\begin{array}{l}U-235 \\
U-238 \\
P u-239 \\
P u-240 \\
P u-241 \\
P U-242 \\
A m-241 \\
F P \\
O-16 \\
N a-23 \\
\text { SS-316 }\end{array}$ & $\begin{array}{l}1.0160 \mathrm{E}-05 \\
4.4270 \mathrm{E}-03 \\
1.7483 \mathrm{E}-03 \\
2.2290 \mathrm{E}-04 \\
1.9040 \mathrm{E}-05 \\
4.7330 \mathrm{E}-06 \\
7.5700 \mathrm{E}-07 \\
0.0000 \mathrm{E}+00 \\
1.2760 \mathrm{E}-02 \\
9.8100 \mathrm{E}-03 \\
1.8990 \mathrm{E}-02\end{array}$ & $\begin{array}{l}8.1868 \mathrm{E}-06 \\
4.3013 \mathrm{E}-03 \\
1.5078 \mathrm{E}-03 \\
2.6740 \mathrm{E}-04 \\
2.2193 \mathrm{E}-05 \\
5.0102 \mathrm{E}-06 \\
2.2295 \mathrm{E}-06 \\
3.1779 \mathrm{E}-04 \\
1.2760 \mathrm{E}-02 \\
9.8100 \mathrm{E}-03 \\
1.8990 \mathrm{E}-02\end{array}$ \\
\hline \multirow[t]{2}{*}{10} & 46.14 & 62.78 & & & \\
\hline & & & $\begin{array}{l}\mathrm{U}-235 \\
\mathrm{U}-238 \\
\mathrm{Pu}-239 \\
\mathrm{Pu}-240 \\
\mathrm{FP} \\
\mathrm{O}-16 \\
\mathrm{Na}-23 \\
\mathrm{SS}-316\end{array}$ & $\begin{array}{l}1.4143 \mathrm{E}-05 \\
7.0573 \mathrm{E}-03 \\
0.0000 \mathrm{E}+00 \\
0.0000 \mathrm{E}+00 \\
0.0000 \mathrm{E}+00 \\
1.4143 \mathrm{E}-02 \\
9.7416 \mathrm{E}-03 \\
1.9073 \mathrm{E}-02\end{array}$ & $\begin{array}{l}1.1576 \mathrm{E}-05 \\
6.9156 \mathrm{E}-03 \\
1.2129 \mathrm{E}-04 \\
3.3420 \mathrm{E}-06 \\
1.8917 \mathrm{E}-05 \\
1.4143 \mathrm{E}-02 \\
9.7416 \mathrm{E}-03 \\
1.9073 \mathrm{E}-02\end{array}$ \\
\hline \multirow[t]{2}{*}{11} & 62.78 & 172.84 & & & \\
\hline & & & $\begin{array}{l}\mathrm{Na}-23 \\
\mathrm{SS}-316\end{array}$ & $\begin{array}{l}8.2400 E-03 \\
2.5562 E-02\end{array}$ & $\begin{array}{l}8.2400 \mathrm{E}-03 \\
2.5562 \mathrm{E}-02\end{array}$ \\
\hline
\end{tabular}




\subsection{Cycle 11B-1}

Table B11B-1.1. Fission Power Generated in MFA-1, MFA-2 and Neighboring Assemblies at BOC 11B-1

\begin{tabular}{lcccc} 
CORE & $* * * * * *$ & \multicolumn{2}{c}{ POWER IN MEGAWATTS } & $* * * * * *$ \\
POS. & BELOW CORE & \multicolumn{1}{c}{ IN CORE } & ABOVE CORE & TOTAL PWR \\
1404 & $7.484 \mathrm{E}-02$ & $4.002 \mathrm{E}+00$ & $4.082 \mathrm{E}-02$ & $4.117 \mathrm{E}+00$ \\
1303 & $1.569 \mathrm{E}-01$ & $1.912 \mathrm{E}+00$ & $7.264 \mathrm{E}-02$ & $2.142 \mathrm{E}+00$ \\
1304 & $0.000 \mathrm{E}+00$ & $7.464 \mathrm{E}+00$ & $0.000 \mathrm{E}+00$ & $7.464 \mathrm{E}+00$ \\
1403 & $1.099 \mathrm{E}-02$ & $3.844 \mathrm{E}+00$ & $6.432 \mathrm{E}-03$ & $3.861 \mathrm{E}+00$ \\
1405 & $9.165 \mathrm{E}-03$ & $3.758 \mathrm{E}+00$ & $6.694 \mathrm{E}-03$ & $3.774 \mathrm{E}+00$ \\
1505 & $7.910 \mathrm{E}-03$ & $3.856 \mathrm{E}+00$ & $5.002 \mathrm{E}-03$ & $3.869 \mathrm{E}+00$ \\
1506 & $1.018 \mathrm{E}-02$ & $3.856 \mathrm{E}+00$ & $7.066 \mathrm{E}-03$ & $3.873 \mathrm{E}+00$ \\
2506 & $7.139 \mathrm{E}-02$ & $3.795 \mathrm{E}+00$ & $4.377 \mathrm{E}-02$ & $3.911 \mathrm{E}+00$ \\
2404 & $0.000 \mathrm{E}+00$ & $6.924 \mathrm{E}+00$ & $0.000 \mathrm{E}+00$ & $6.924 \mathrm{E}+00$ \\
2505 & $1.427 \mathrm{E}-02$ & $3.972 \mathrm{E}+00$ & $7.562 \mathrm{E}-03$ & $3.993 \mathrm{E}+00$ \\
2405 & $8.214 \mathrm{E}-03$ & $5.360 \mathrm{E}+00$ & $6.535 \mathrm{E}-03$ & $5.374 \mathrm{E}+00$ \\
2606 & $7.378 \mathrm{E}-03$ & $3.841 \mathrm{E}+00$ & $5.421 \mathrm{E}-03$ & $3.854 \mathrm{E}+00$ \\
2607 & $1.193 \mathrm{E}-02$ & $3.318 \mathrm{E}+00$ & $7.725 \mathrm{E}-03$ & $3.338 \mathrm{E}+00$ \\
2507 & $1.081 \mathrm{E}-02$ & $3.972 \mathrm{E}+00$ & $8.099 \mathrm{E}-03$ & $3.991 \mathrm{E}+00$
\end{tabular}

Table B1lB-1.2. Assembly Averaged Total and Fast Flux in MFA-1 and MFA-2 at BOC $11 \mathrm{~B}-1$

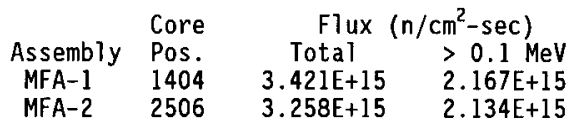


Table Bl1B-1.3. Axial Distribution of Total Flux, Fast Flux and Power in MFA-1 at BOC 11B-1

$\begin{array}{rccc}\text { z }(\mathrm{cm}) & \text { Total Flux } & \text { Flux }>0.1 \text { MeV } & \text { Power } \\ -97.78 & 2.9486 \mathrm{E}-02 & 1.5457 \mathrm{E}-02 & 0.0000 \mathrm{E}+00 \\ -92.78 & 6.3230 \mathrm{E}-02 & 2.9794 \mathrm{E}-02 & 0.0000 \mathrm{E}+00 \\ -87.78 & 1.0120 \mathrm{E}-01 & 4.7582 \mathrm{E}-02 & 0.0000 \mathrm{E}+00 \\ -82.78 & 1.4619 \mathrm{E}-01 & 7.0801 \mathrm{E}-02 & 0.0000 \mathrm{E}+00 \\ -77.78 & 2.0093 \mathrm{E}-01 & 1.0188 \mathrm{E}-01 & 0.0000 \mathrm{E}+00 \\ -72.15 & 2.6248 \mathrm{E}-01 & 1.4189 \mathrm{E}-01 & 0.0000 \mathrm{E}+00 \\ -65.90 & 3.2386 \mathrm{E}-01 & 1.9186 \mathrm{E}-01 & 0.0000 \mathrm{E}+00 \\ -60.35 & 3.8774 \mathrm{E}-01 & 2.5871 \mathrm{E}-01 & 8.4319 \mathrm{E}-02 \\ -55.49 & 4.7086 \mathrm{E}-01 & 3.4517 \mathrm{E}-01 & 9.7617 \mathrm{E}-02 \\ -50.62 & 5.6590 \mathrm{E}-01 & 4.5532 \mathrm{E}-01 & 1.1600 \mathrm{E}-01 \\ -47.17 & 6.4070 \mathrm{E}-01 & 5.5746 \mathrm{E}-01 & 1.3503 \mathrm{E}-01 \\ -43.58 & 7.1891 \mathrm{E}-01 & 6.7296 \mathrm{E}-01 & 7.7788 \mathrm{E}-01 \\ -38.45 & 8.3203 \mathrm{E}-01 & 8.1321 \mathrm{E}-01 & 8.6861 \mathrm{E}-01 \\ -33.32 & 9.3709 \mathrm{E}-01 & 9.3277 \mathrm{E}-01 & 9.6772 \mathrm{E}-01 \\ -28.20 & 1.0289 \mathrm{E}+00 & 1.0320 \mathrm{E}+00 & 1.0172 \mathrm{E}+00 \\ -23.07 & 1.1056 \mathrm{E}+00 & 1.1130 \mathrm{E}+00 & 1.0910 \mathrm{E}+00 \\ -17.94 & 1.1654 \mathrm{E}+00 & 1.1752 \mathrm{E}+00 & 1.1489 \mathrm{E}+00 \\ -12.82 & 1.2072 \mathrm{E}+00 & 1.2177 \mathrm{E}+00 & 1.1670 \mathrm{E}+00 \\ -7.69 & 1.2308 \mathrm{E}+00 & 1.2418 \mathrm{E}+00 & 1.1894 \mathrm{E}+00 \\ -2.56 & 1.2358 \mathrm{E}+00 & 1.2470 \mathrm{E}+00 & 1.1940 \mathrm{E}+00 \\ 2.56 & 1.2221 \mathrm{E}+00 & 1.2334 \mathrm{E}+00 & 1.1880 \mathrm{E}+00 \\ 7.69 & 1.1896 \mathrm{E}+00 & 1.2007 \mathrm{E}+00 & 1.1564 \mathrm{E}+00 \\ 12.82 & 1.1391 \mathrm{E}+00 & 1.1499 \mathrm{E}+00 & 1.1073 \mathrm{E}+00 \\ 17.94 & 1.0723 \mathrm{E}+00 & 1.0827 \mathrm{E}+00 & 1.0743 \mathrm{E}+00 \\ 23.07 & 9.9044 \mathrm{E}-01 & 9.9900 \mathrm{E}-01 & 9.9268 \mathrm{E}-01 \\ 28.20 & 8.9577 \mathrm{E}-01 & 9.0100 \mathrm{E}-01 & 8.9871 \mathrm{E}-01 \\ 33.32 & 7.9076 \mathrm{E}-01 & 7.9047 \mathrm{E}-01 & 8.3305 \mathrm{E}-01 \\ 38.45 & 6.7741 \mathrm{E}-01 & 6.6640 \mathrm{E}-01 & 7.1852 \mathrm{E}-01 \\ 43.58 & 5.6096 \mathrm{E}-01 & 5.3075 \mathrm{E}-01 & 6.0927 \mathrm{E}-01 \\ 47.17 & 4.8391 \mathrm{E}-01 & 4.2883 \mathrm{E}-01 & 8.5129 \mathrm{E}-02 \\ 50.62 & 4.1269 \mathrm{E}-01 & 3.4096 \mathrm{E}-01 & 6.7771 \mathrm{E}-02 \\ 55.49 & 3.2519 \mathrm{E}-01 & 2.4828 \mathrm{E}-01 & 5.1119 \mathrm{E}-02 \\ 60.35 & 2.4858 \mathrm{E}-01 & 1.7596 \mathrm{E}-01 & 3.8789 \mathrm{E}-02 \\ 64.98 & 1.9896 \mathrm{E}-01 & 1.3231 \mathrm{E}-01 & 0.0000 \mathrm{E}+00 \\ 69.39 & 1.6942 \mathrm{E}-01 & 1.0859 \mathrm{E}-01 & 0.0000 \mathrm{E}+00 \\ 76.46 & 1.2764 \mathrm{E}-01 & 7.6709 \mathrm{E}-02 & 0.0000 \mathrm{E}+00 \\ 86.80 & 8.2660 \mathrm{E}-02 & 4.6073 \mathrm{E}-02 & 0.0000 \mathrm{E}+00 \\ 98.14 & 4.7519 \mathrm{E}-02 & 2.5108 \mathrm{E}-02 & 0.0000 \mathrm{E}+00 \\ 110.00 & 2.0377 \mathrm{E}-02 & 1.1214 \mathrm{E}-02 & 0.0000 \mathrm{E}+00\end{array}$


Table BllB-1.4. Axial Distribution of Total Flux, Fast Flux and Power in MFA-2 at BOC 11B-1

$\begin{array}{rccc}\text { z }(\mathrm{cm}) & \text { Total Flux } & \text { Flux }>0.1 \text { MeV } & \text { Power } \\ -97.78 & 2.6753 \mathrm{E}-02 & 1.3607 \mathrm{E}-02 & 0.0000 \mathrm{E}+00 \\ -92.78 & 5.7376 \mathrm{E}-02 & 2.6261 \mathrm{E}-02 & 0.0000 \mathrm{E}+00 \\ -87.78 & 9.1949 \mathrm{E}-02 & 4.2025 \mathrm{E}-02 & 0.0000 \mathrm{E}+00 \\ -82.78 & 1.3318 \mathrm{E}-01 & 6.2728 \mathrm{E}-02 & 0.0000 \mathrm{E}+00 \\ -77.78 & 1.8401 \mathrm{E}-01 & 9.0670 \mathrm{E}-02 & 0.0000 \mathrm{E}+00 \\ -72.15 & 2.4255 \mathrm{E}-01 & 1.2715 \mathrm{E}-01 & 0.0000 \mathrm{E}+00 \\ -65.90 & 3.0288 \mathrm{E}-01 & 1.7430 \mathrm{E}-01 & 0.0000 \mathrm{E}+00 \\ -60.35 & 3.6699 \mathrm{E}-01 & 2.3920 \mathrm{E}-01 & 8.4172 \mathrm{E}-02 \\ -55.49 & 4.5022 \mathrm{E}-01 & 3.2454 \mathrm{E}-01 & 9.8142 \mathrm{E}-02 \\ -50.62 & 5.4601 \mathrm{E}-01 & 4.3572 \mathrm{E}-01 & 1.1698 \mathrm{E}-01 \\ -47.17 & 6.2250 \mathrm{E}-01 & 5.4041 \mathrm{E}-01 & 1.3671 \mathrm{E}-01 \\ -43.58 & 7.0300 \mathrm{E}-01 & 6.5868 \mathrm{E}-01 & 7.6570 \mathrm{E}-01 \\ -38.45 & 8.2011 \mathrm{E}-01 & 8.0282 \mathrm{E}-01 & 8.5735 \mathrm{E}-01 \\ -33.32 & 9.2846 \mathrm{E}-01 & 9.2501 \mathrm{E}-01 & 9.5995 \mathrm{E}-01 \\ -28.20 & 1.0225 \mathrm{E}+00 & 1.0255 \mathrm{E}+00 & 1.0106 \mathrm{E}+00 \\ -23.07 & 1.1008 \mathrm{E}+00 & 1.1072 \mathrm{E}+00 & 1.0862 \mathrm{E}+00 \\ -17.94 & 1.1616 \mathrm{E}+00 & 1.1697 \mathrm{E}+00 & 1.1453 \mathrm{E}+00 \\ -12.82 & 1.2040 \mathrm{E}+00 & 1.2125 \mathrm{E}+00 & 1.1624 \mathrm{E}+00 \\ -7.69 & 1.2284 \mathrm{E}+00 & 1.2370 \mathrm{E}+00 & 1.1855 \mathrm{E}+00 \\ -2.56 & 1.2343 \mathrm{E}+00 & 1.2433 \mathrm{E}+00 & 1.1911 \mathrm{E}+00 \\ 2.56 & 1.2218 \mathrm{E}+00 & 1.2314 \mathrm{E}+00 & 1.1856 \mathrm{E}+00 \\ 7.69 & 1.1910 \mathrm{E}+00 & 1.2010 \mathrm{E}+00 & 1.1558 \mathrm{E}+00 \\ 12.82 & 1.1427 \mathrm{E}+00 & 1.1531 \mathrm{E}+00 & 1.1091 \mathrm{E}+00 \\ 17.94 & 1.0784 \mathrm{E}+00 & 1.0893 \mathrm{E}+00 & 1.0788 \mathrm{E}+00 \\ 23.07 & 9.9855 \mathrm{E}-01 & 1.0084 \mathrm{E}+00 & 9.9954 \mathrm{E}-01 \\ 28.20 & 9.0533 \mathrm{E}-01 & 9.1256 \mathrm{E}-01 & 9.0729 \mathrm{E}-01 \\ 33.32 & 8.0112 \mathrm{E}-01 & 8.0333 \mathrm{E}-01 & 8.4390 \mathrm{E}-01 \\ 38.45 & 6.8766 \mathrm{E}-01 & 6.7878 \mathrm{E}-01 & 7.3050 \mathrm{E}-01 \\ 43.58 & 5.7031 \mathrm{E}-01 & 5.4059 \mathrm{E}-01 & 6.2534 \mathrm{E}-01 \\ 47.17 & 4.9283 \mathrm{E}-01 & 4.3571 \mathrm{E}-01 & 9.4294 \mathrm{E}-02 \\ 50.62 & 4.2132 \mathrm{E}-01 & 3.4515 \mathrm{E}-01 & 7.5864 \mathrm{E}-02 \\ 55.49 & 3.3350 \mathrm{E}-01 & 2.5021 \mathrm{E}-01 & 5.8233 \mathrm{E}-02 \\ 60.35 & 2.5594 \mathrm{E}-01 & 1.7643 \mathrm{E}-01 & 4.5000 \mathrm{E}-02 \\ 64.98 & 2.0559 \mathrm{E}-01 & 1.3164 \mathrm{E}-01 & 0.0000 \mathrm{E}+00 \\ 69.39 & 1.7496 \mathrm{E}-01 & 1.0729 \mathrm{E}-01 & 0.0000 \mathrm{E}+00 \\ 76.46 & 1.3138 \mathrm{E}-01 & 7.4836 \mathrm{E}-02 & 0.0000 \mathrm{E}+00 \\ 86.80 & 8.4382 \mathrm{E}-02 & 4.4182 \mathrm{E}-02 & 0.0000 \mathrm{E}+00 \\ 98.14 & 4.7975 \mathrm{E}-02 & 2.3688 \mathrm{E}-02 & 0.0000 \mathrm{E}+00 \\ 110.00 & 2.0389 \mathrm{E}-02 & 1.0458 \mathrm{E}-02 & 0.0000 \mathrm{E}+00\end{array}$


HNF-SD-FF-ANAL-009 Rev. 1

Table B11B-1.5. Fission Power Distribution by Pin in MFA-1 at BOC $11 \mathrm{~B}-1$ CORE POSITION 1404

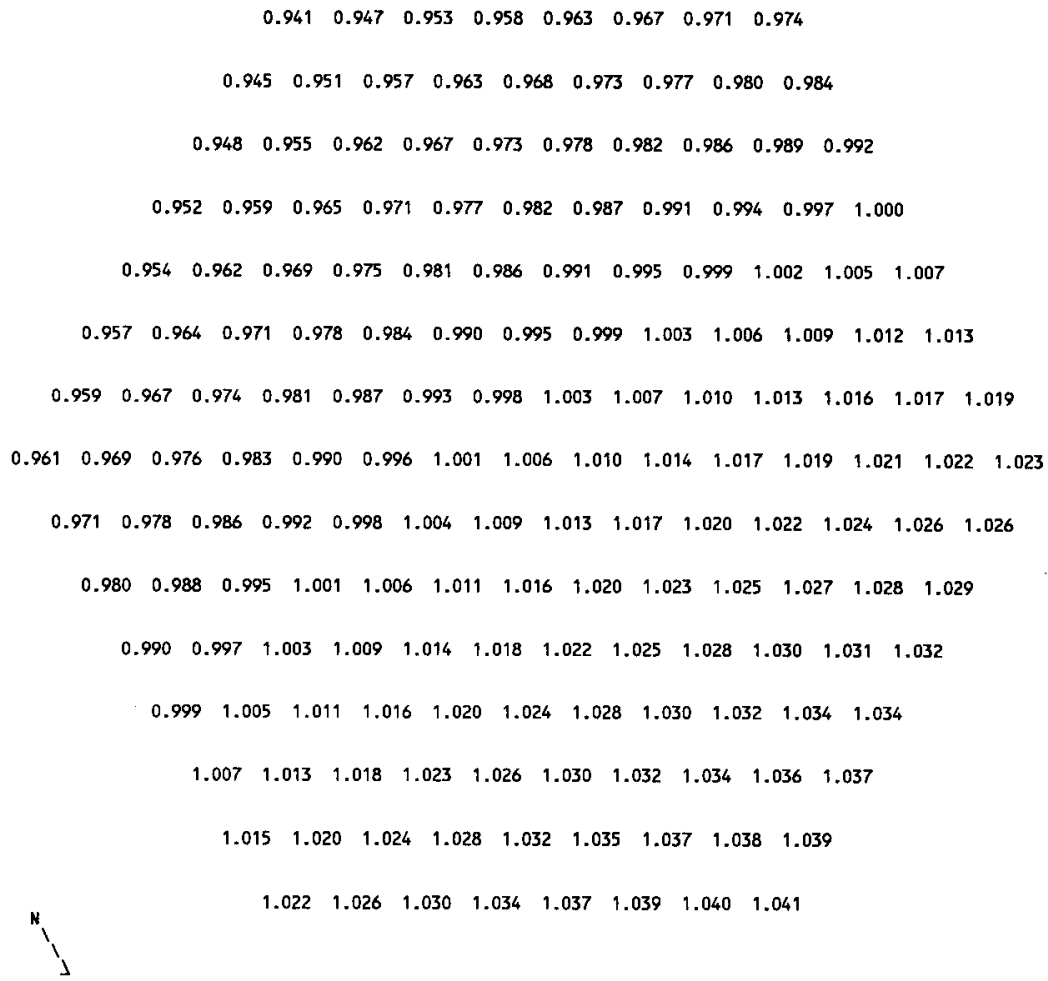


Table B11B-1.6. Fission Power Distribution by Pin in MFA-2 at BOC 11B-1 CORE POSITION 2506

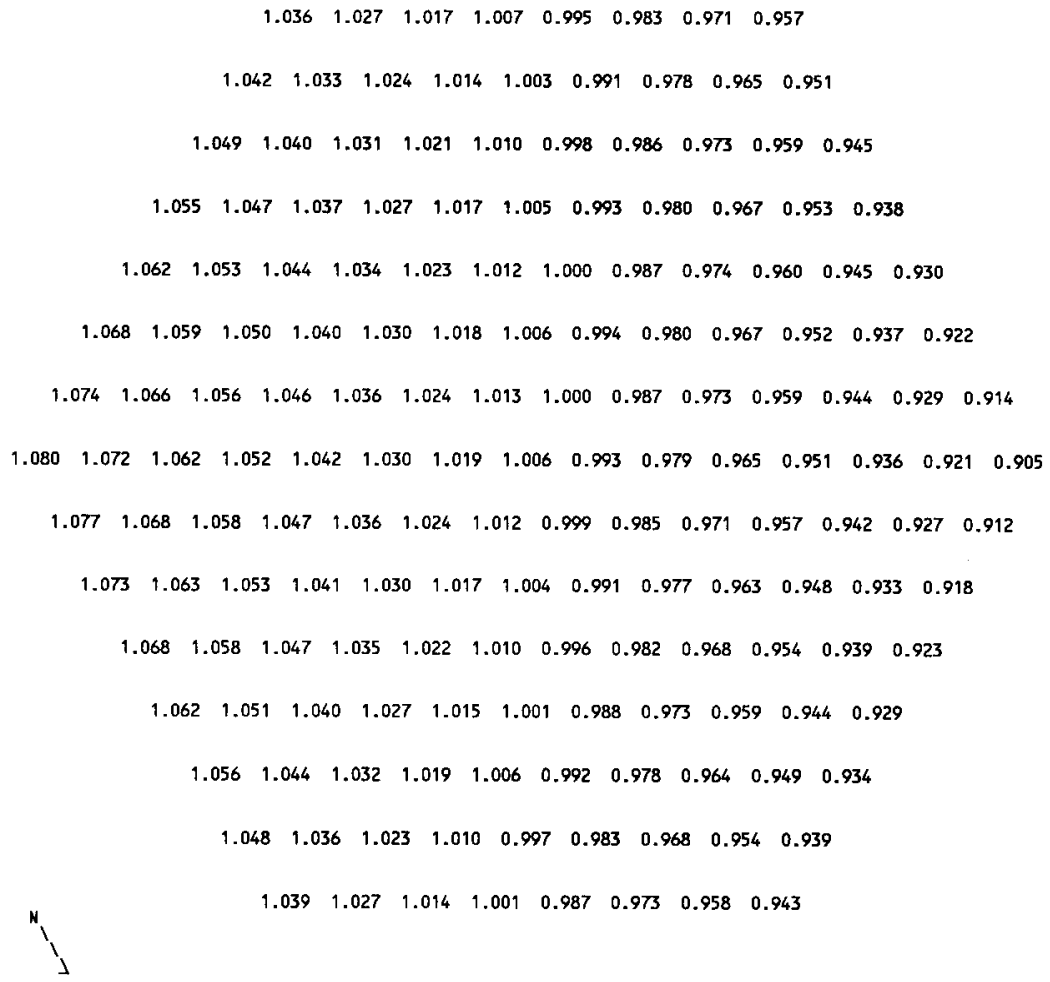


HNF-SD-FF-ANAL-009 Rev. 1

Table BI1B-1.7. Fast Flux Distribution by Pin in MFA-1 at BOC 11B-1 CORE POSITION 1404

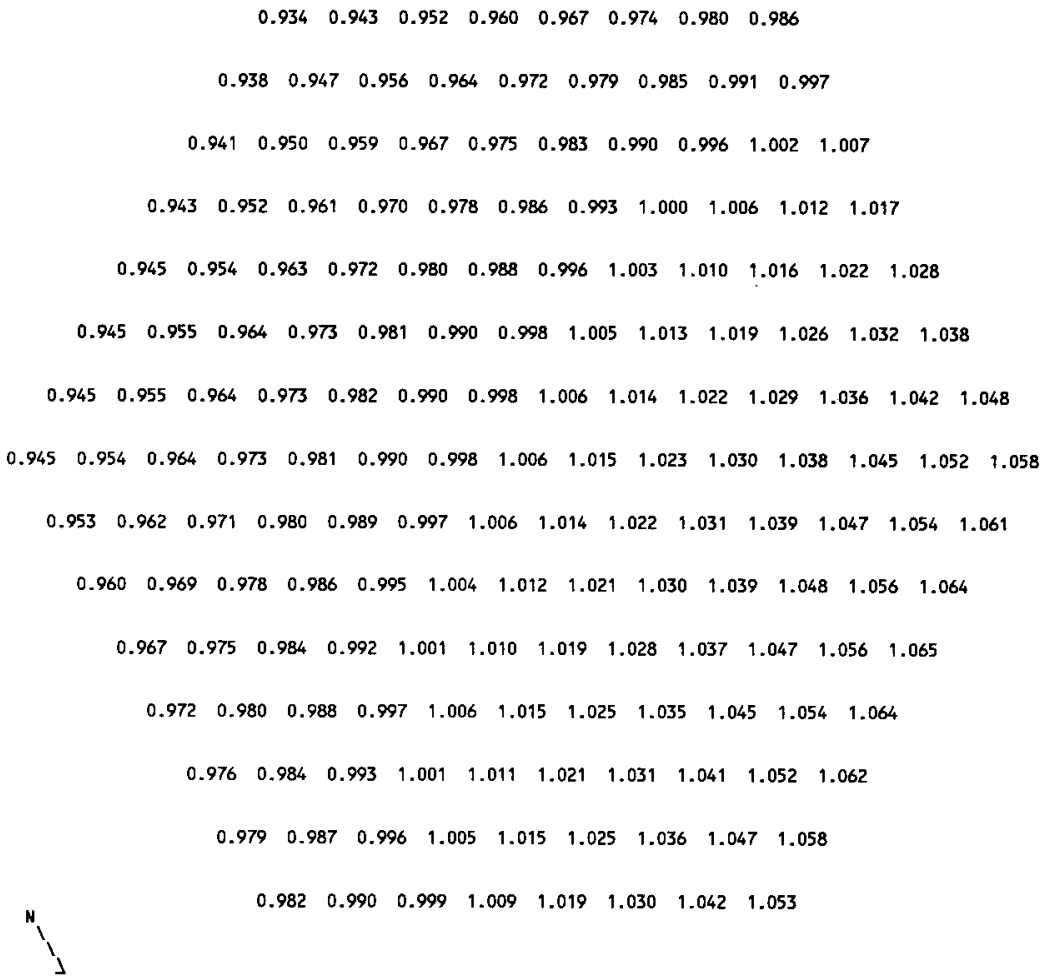


HNF-SD-FF-ANAL-009 Rev. 1

Table B11B-1.8. Fast Flux Distribution by Pin in MFA-2 at BOC 11B-1 CORE POSITION 2506

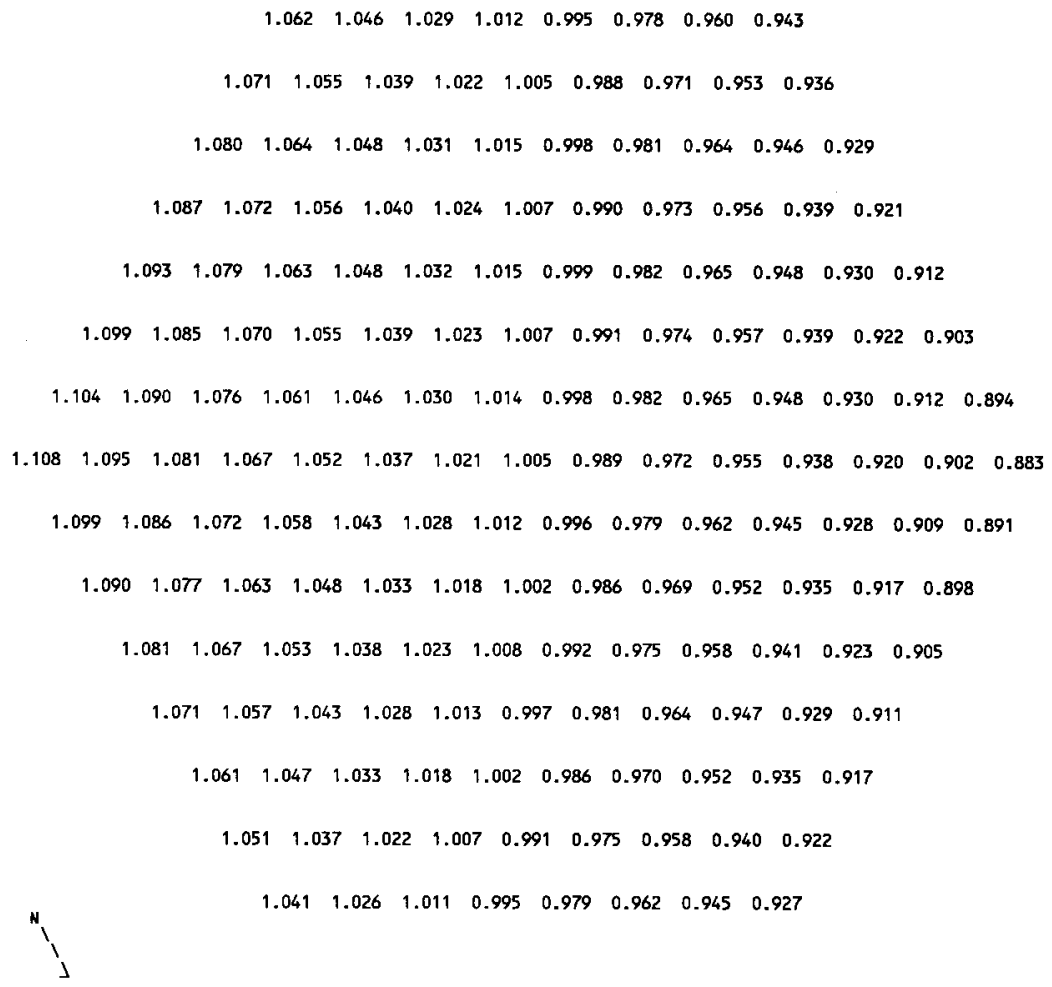


Table B11B-1.9. MFA-1 and MFA-2 Duct Wa11

Fast Flux Data at BOC 11B-1

$\begin{array}{ccc}\text { Side } & \begin{array}{c}\text { Flux }>0.1 \mathrm{MeV}\left(\mathrm{n} / \mathrm{cm}^{2}-\mathrm{sec}\right) \\ \text { MFA-1 }\end{array} & \begin{array}{c}\text { MFA-2 } \\ \text { E }\end{array} \\ 2.0774 \mathrm{E}+15 & 2.3135 \mathrm{E}+15 \\ \text { SE } & 1.9983 \mathrm{E}+15 & 2.3555 \mathrm{E}+15 \\ \text { SW } & 2.0500 \mathrm{E}+15 & 2.1278 \mathrm{E}+15 \\ \text { W } & 2.1977 \mathrm{E}+15 & 1.9105 \mathrm{E}+15 \\ \text { NW } & 2.3235 \mathrm{E}+15 & 1.8854 \mathrm{E}+15 \\ \text { NE } & 2.1810 \mathrm{E}+15 & 2.0916 \mathrm{E}+15\end{array}$

Table BlIB-1.10. Assembly Outlet Temperatures and Flow Rates at BOC 11B-1

\begin{tabular}{lcc}
\multicolumn{3}{c}{ CALCULATED } \\
CORE & OUTLET TEMP. & FLOW RATE \\
POS. & (DEG F) & (LB/H) \\
1404 & 1048 & 114690 \\
1303 & 1002 & 69040 \\
1304 & 1059 & 200400 \\
1403 & 879 & 207670 \\
1405 & 880 & 207700 \\
1505 & 890 & 197480 \\
1506 & 880 & 207670 \\
2506 & 1046 & 114690 \\
2404 & 1037 & 204240 \\
2405 & 1016 & 171220 \\
2505 & 890 & 207670 \\
2507 & 893 & 207670 \\
2606 & 926 & 170310 \\
2607 & 899 & 170310
\end{tabular}

Assembly flows are based on a calculated core pressure drop of $110.1 \mathrm{psi}$ at a total reactor flow rate of $16.74 \mathrm{E}+06 \mathrm{lb} / \mathrm{hr}$. 
1 temperature map for bund1e 23 ASSEMBLY NO. 1484 MFA-1

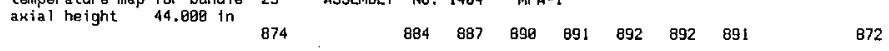

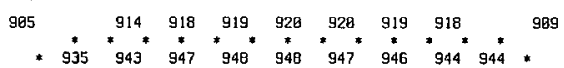

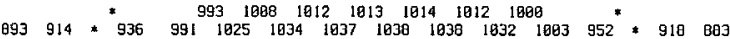

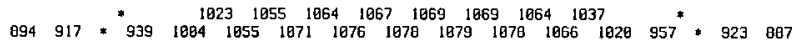

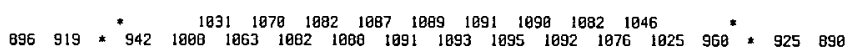

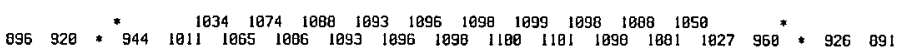

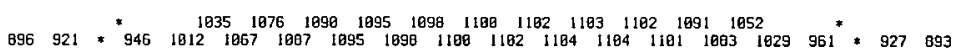

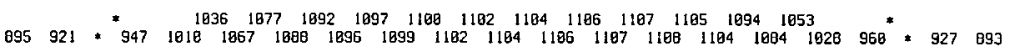

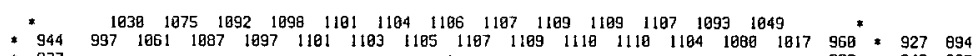

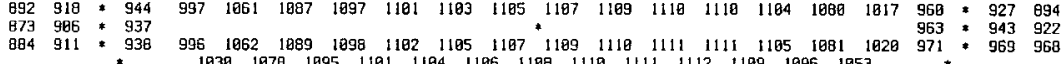

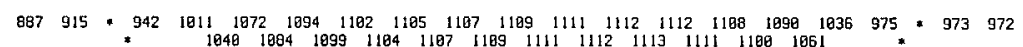

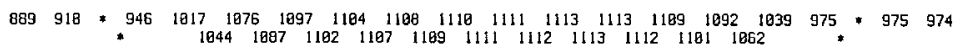

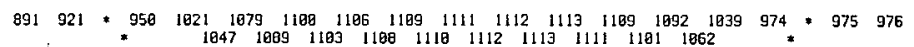

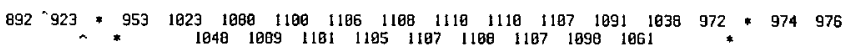

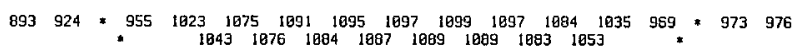

$892 \quad 923$ * $953 \quad 1818 \quad 1045 \quad 1054 \quad 1657 \quad 1059 \quad 1859 \quad 1954 \quad 1921 \quad 966$ * $970 \quad 975$

* 948 $953950 \quad 963967 \quad 970971969 \quad 965$ *

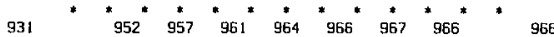

$\begin{array}{llllllllll}913 & 952 & 956 & 960 & 962 & 963 & 963 & 962 & 968\end{array}$ 
temperature map for bundle 51 ASSEMELY NO. 2586 MFA-2

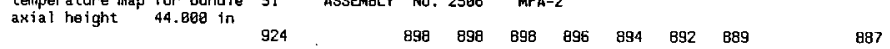

$$
\begin{aligned}
& 937 \quad 927 \quad 928 \quad 926 \quad 924 \quad 921 \quad 918,915,913
\end{aligned}
$$

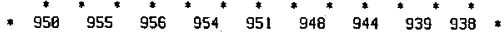

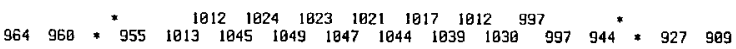

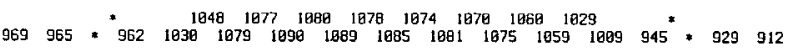

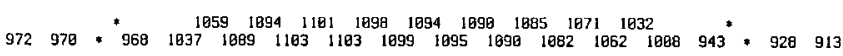

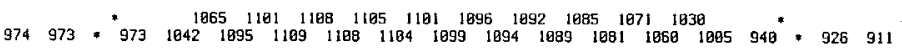

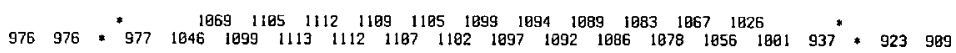

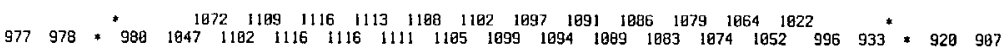

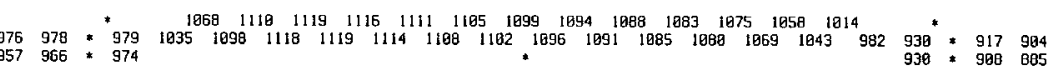

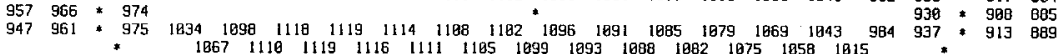

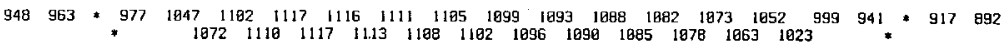

$948 \quad 964 * 979 \quad 1848 \quad 1108 \quad 1114 \quad 1112 \quad 1107 \quad 1101 \quad 1096 \quad 1098 \quad 1085 \quad 1076 \quad 1055 \quad 1003 \quad 943 *$ * 919895

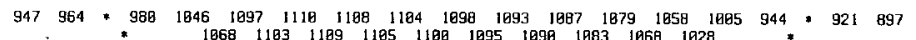

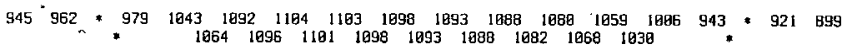

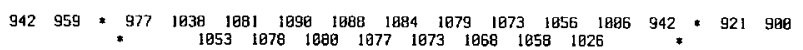

$937 \quad 554 * 971 \quad 1821 \quad 1847 \quad 1858 \quad 1847 \quad 1844 \quad 1848 \quad 1038995 \quad 941 * 921908$

* $963969960959958957954 \quad 949942$ *

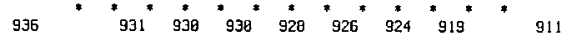

097

$901 \quad 909 \quad 899 \quad 897 \quad 095 \quad 092 \quad 808$

880 
Table B1lB-1.13. MFA-1 Sodium Subchannel Temperatures

at Elevation of Upper Axial Blanket for BOC 11B-1

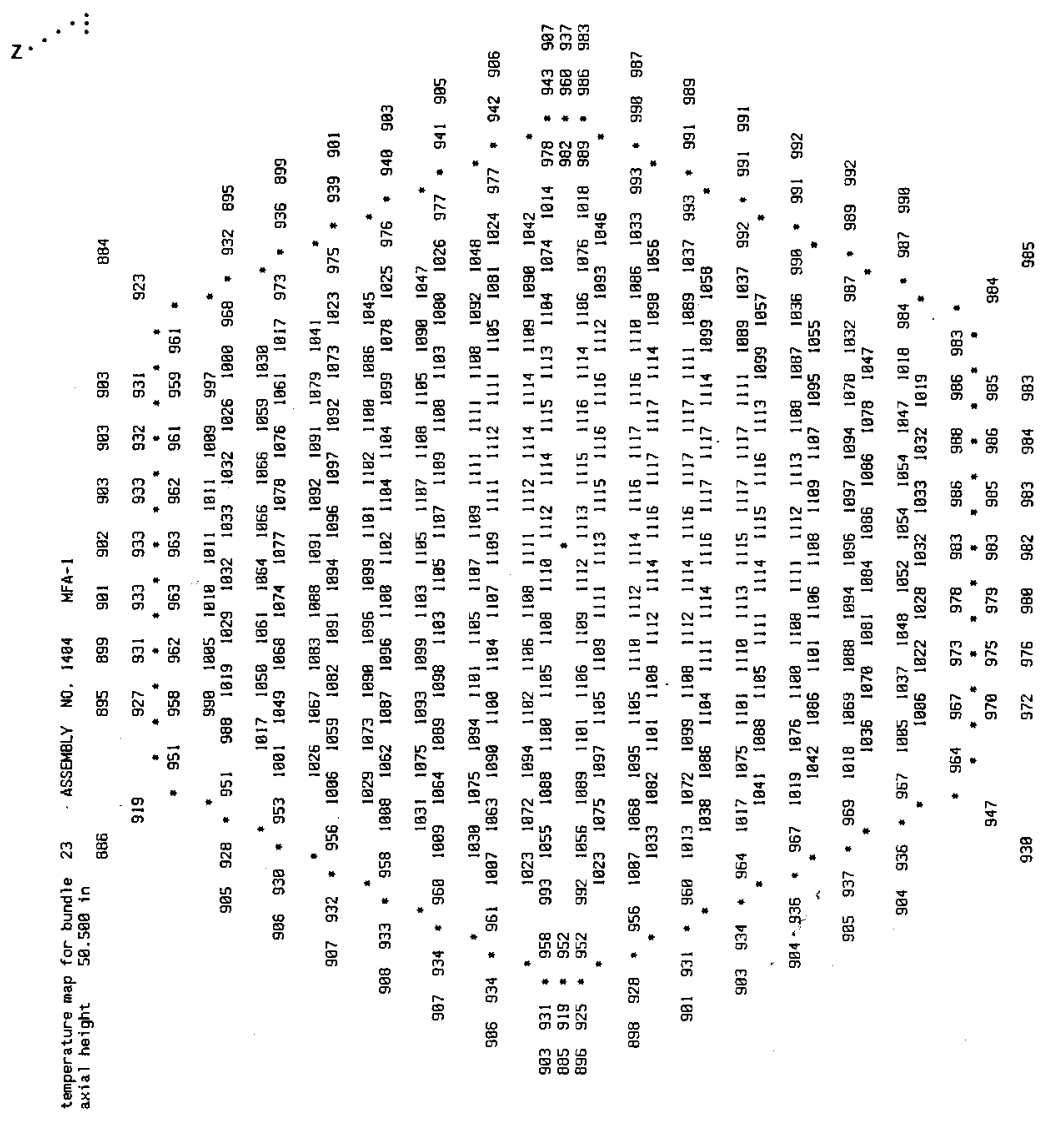


Table B1lB-1.14. MFA-2 Sodium Subchannel Temperatures

at Elevation of Upper Axial Blanket for BOC IlB-1

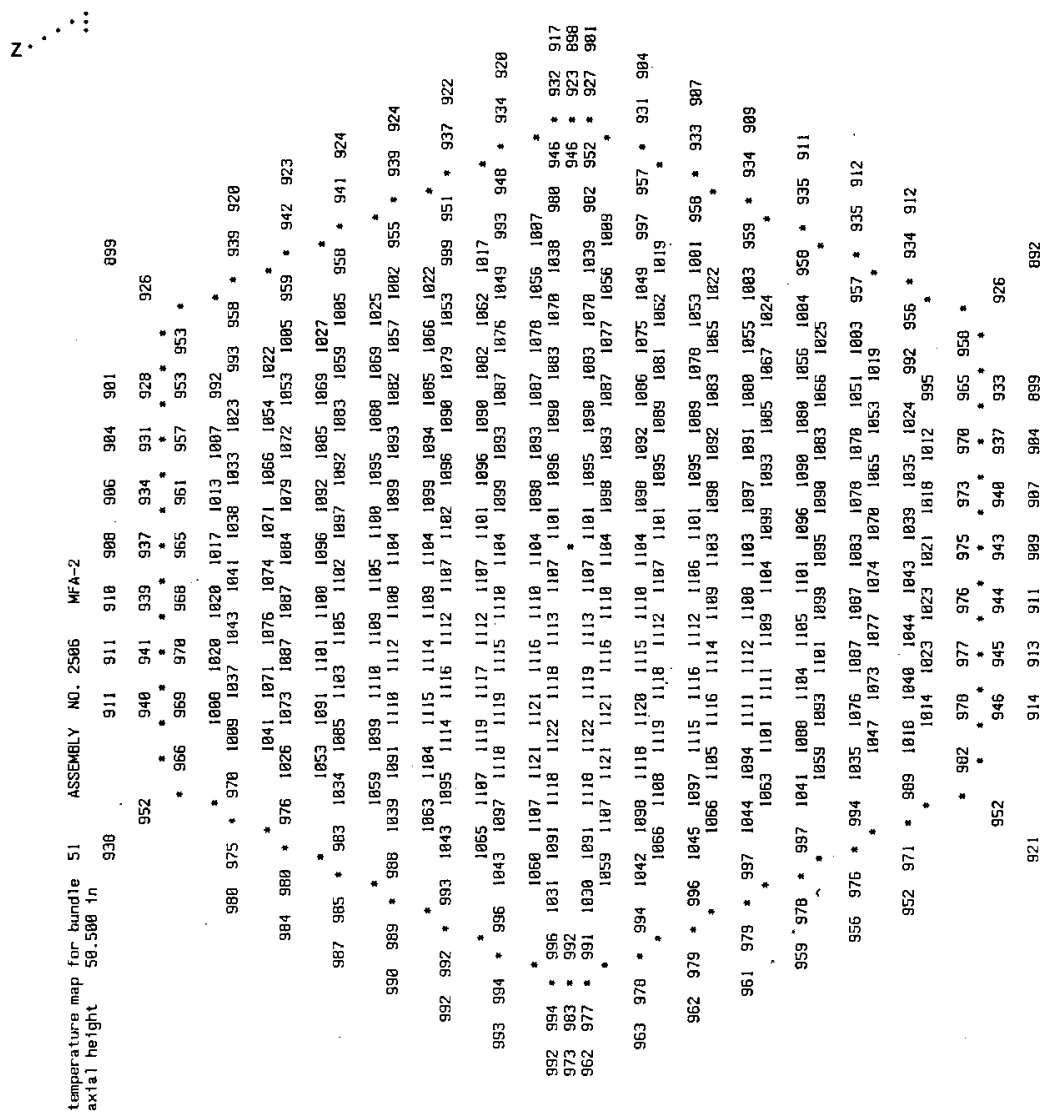


Table Bl1B-1.15. MFA-l Sodium Subchannel Temperatures

at Top of Fuel Pin Bundle for BOC 11B-1

$z^{\cdot \cdot \cdot}$

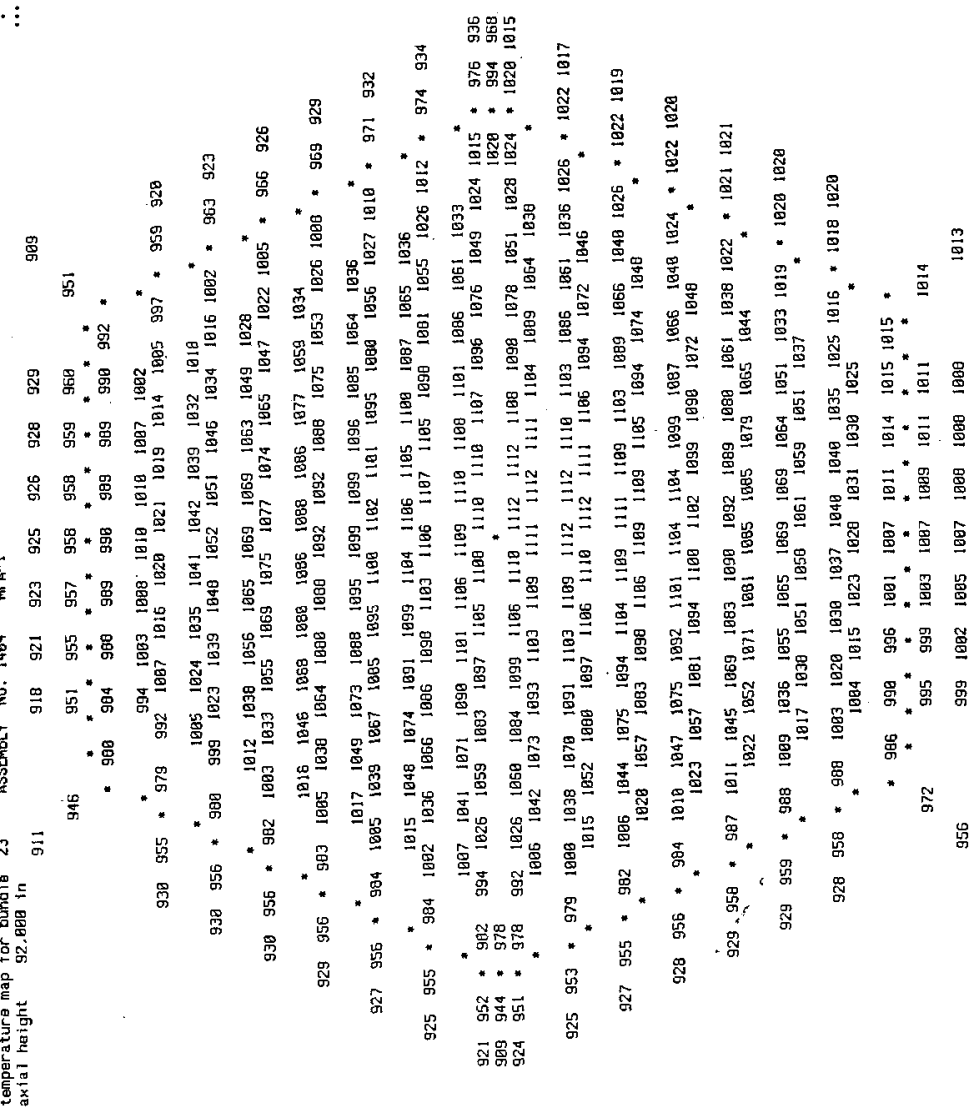


Table B11B-1.16. MFA-2 Sodium Subchannel Temperatures at Top of Fuel Pin Bundle for BOC 11B-1

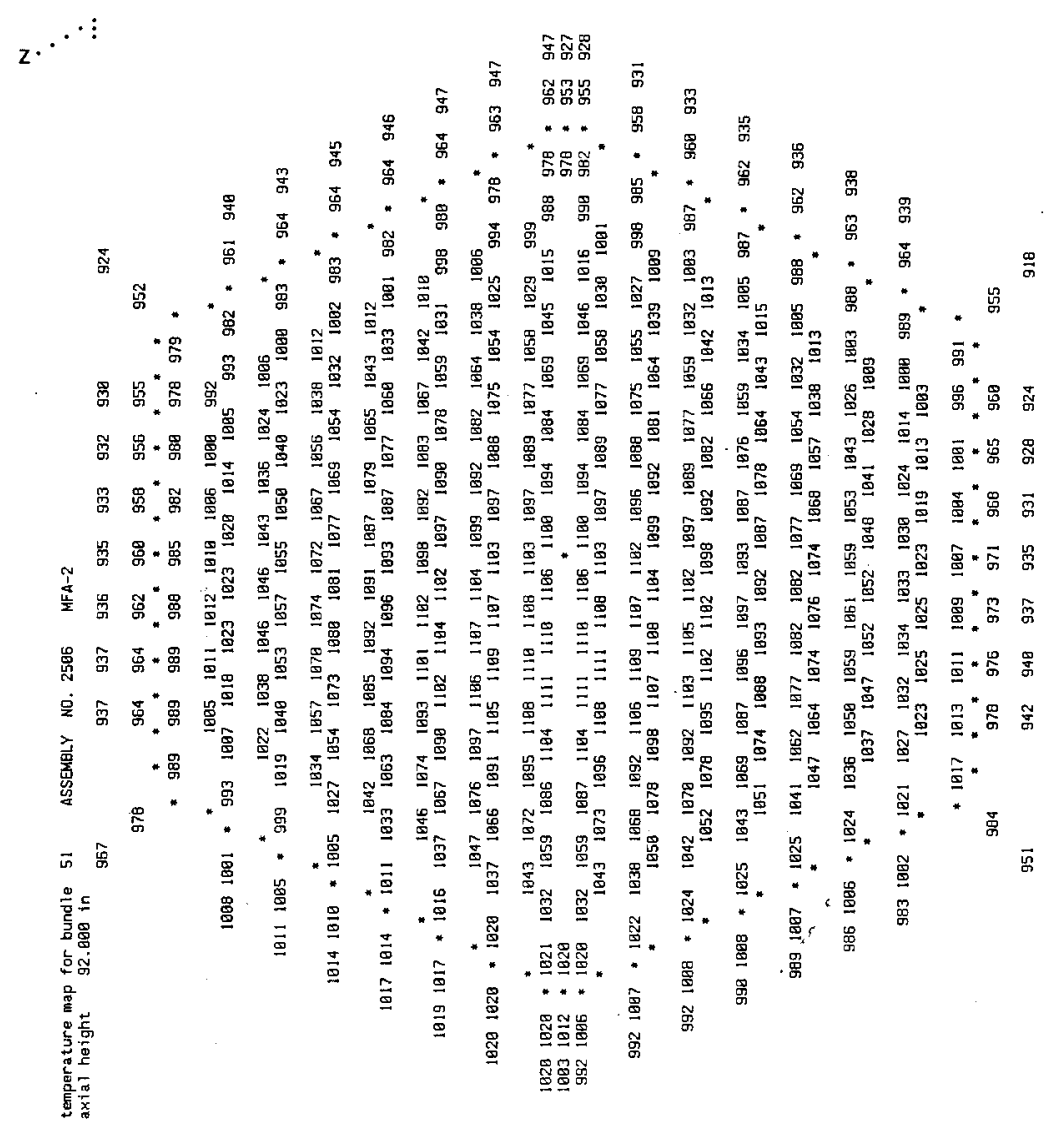




\section{HNF-SD-FF-ANAL-009 Rev. 1}

Table E11B-1.1. Fission Power Generated in MFA-1, MFA-2 and Neighboring Assemblies at EOC 11B-1

\begin{tabular}{lcccc} 
CORE & $* * * * * *$ & \multicolumn{2}{c}{ POWER IN MEGAWATTS } & $* * * * * *$ \\
POS. & BELOW CORE & IN CORE & ABOVE CORE & \multicolumn{1}{c}{ TOTAL PWR } \\
1404 & $7.893 \mathrm{E}-02$ & $3.912 \mathrm{E}+00$ & $4.998 \mathrm{E}-02$ & $4.041 \mathrm{E}+00$ \\
1303 & $1.578 \mathrm{E}-01$ & $1.991 \mathrm{E}+00$ & $8.538 \mathrm{E}-02$ & $2.234 \mathrm{E}+00$ \\
1304 & $0.000 \mathrm{E}+00$ & $7.196 \mathrm{E}+00$ & $0.000 \mathrm{E}+00$ & $7.196 \mathrm{E}+00$ \\
1403 & $1.166 \mathrm{E}-02$ & $3.846 \mathrm{E}+00$ & $8.208 \mathrm{E}-03$ & $3.866 \mathrm{E}+00$ \\
1405 & $9.841 \mathrm{E}-03$ & $3.674 \mathrm{E}+00$ & $8.068 \mathrm{E}-03$ & $3.692 \mathrm{E}+00$ \\
1505 & $8.501 \mathrm{E}-03$ & $3.885 \mathrm{E}+00$ & $6.482 \mathrm{E}-03$ & $3.900 \mathrm{E}+00$ \\
1506 & $1.046 \mathrm{E}-02$ & $3.786 \mathrm{E}+00$ & $8.409 \mathrm{E}-03$ & $3.805 \mathrm{E}+00$ \\
2506 & $7.427 \mathrm{E}-02$ & $3.673 \mathrm{E}+00$ & $5.263 \mathrm{E}-02$ & $3.800 \mathrm{E}+00$ \\
2404 & $0.000 \mathrm{E}+00$ & $6.682 \mathrm{E}+00$ & $0.000 \mathrm{E}+00$ & $6.682 \mathrm{E}+00$ \\
2505 & $1.435 \mathrm{E}-02$ & $3.950 \mathrm{E}+00$ & $9.364 \mathrm{E}-03$ & $3.974 \mathrm{E}+00$ \\
2405 & $9.120 \mathrm{E}-03$ & $5.150 \mathrm{E}+00$ & $7.990 \mathrm{E}-03$ & $5.167 \mathrm{E}+00$ \\
2606 & $7.820 \mathrm{E}-03$ & $3.782 \mathrm{E}+00$ & $6.682 \mathrm{E}-03$ & $3.797 \mathrm{E}+00$ \\
2507 & $1.182 \mathrm{E}-02$ & $3.227 \mathrm{E}+00$ & $8.870 \mathrm{E}-03$ & $3.248 \mathrm{E}+00$ \\
2507 & $1.097 \mathrm{E}-02$ & $3.831 \mathrm{E}+00$ & $9.339 \mathrm{E}-03$ & $3.852 \mathrm{E}+00$
\end{tabular}

Table EllB-1.2. Assembly Averaged Total and Fast Flux in MFA-1 and MFA-2 at EOC 11B-1

\begin{tabular}{|c|c|c|c|}
\hline & Core & Flux & $\left.m^{2}-s e c\right)$ \\
\hline $\begin{array}{l}\text { Assembly } \\
\text { MFA-1 } \\
\text { MFA-2 }\end{array}$ & $\begin{array}{l}\text { Pos. } \\
1404 \\
2506\end{array}$ & $\begin{array}{c}\text { Total } \\
3.446 \mathrm{E}+15 \\
3.247 \mathrm{E}+15\end{array}$ & $\begin{array}{l}>0.1 \mathrm{MeV} \\
2.168 \mathrm{E}+15 \\
2.109 \mathrm{E}+15\end{array}$ \\
\hline
\end{tabular}


Table El1B-1.3. Axial Distribution of Total Flux, Fast Flux and Power in MFA-1 at EOC 11B-1

$\begin{array}{rccc}z(\mathrm{~cm}) & \text { Total Flux } & \text { Flux }>0.1 \mathrm{MeV} & \text { Power } \\ -97.78 & 2.7933 \mathrm{E}-02 & 1.4749 \mathrm{E}-02 & 0.0000 \mathrm{E}+00 \\ -92.78 & 5.9903 \mathrm{E}-02 & 2.8429 \mathrm{E}-02 & 0.0000 \mathrm{E}+00 \\ -87.78 & 9.5890 \mathrm{E}-02 & 4.5403 \mathrm{E}-02 & 0.0000 \mathrm{E}+00 \\ -82.78 & 1.3853 \mathrm{E}-01 & 6.7568 \mathrm{E}-02 & 0.0000 \mathrm{E}+00 \\ -77.78 & 1.9042 \mathrm{E}-01 & 9.7240 \mathrm{E}-02 & 0.0000 \mathrm{E}+00 \\ -72.15 & 2.4878 \mathrm{E}-01 & 1.3544 \mathrm{E}-01 & 0.0000 \mathrm{E}+00 \\ -65.90 & 3.0698 \mathrm{E}-01 & 1.8322 \mathrm{E}-01 & 0.0000 \mathrm{E}+00 \\ -60.35 & 3.6747 \mathrm{E}-01 & 2.4731 \mathrm{E}-01 & 9.2332 \mathrm{E}-02 \\ -55.49 & 4.4630 \mathrm{E}-01 & 3.2953 \mathrm{E}-01 & 1.0594 \mathrm{E}-01 \\ -50.62 & 5.3640 \mathrm{E}-01 & 4.3381 \mathrm{E}-01 & 1.2435 \mathrm{E}-01 \\ -47.17 & 6.0723 \mathrm{E}-01 & 5.3017 \mathrm{E}-01 & 1.4286 \mathrm{E}-01 \\ -43.58 & 6.8136 \mathrm{E}-01 & 6.3900 \mathrm{E}-01 & 7.3944 \mathrm{E}-01 \\ -38.45 & 7.8943 \mathrm{E}-01 & 7.7238 \mathrm{E}-01 & 8.2705 \mathrm{E}-01 \\ -33.32 & 8.9094 \mathrm{E}-01 & 8.8732 \mathrm{E}-01 & 9.2338 \mathrm{E}-01 \\ -28.20 & 9.8101 \mathrm{E}-01 & 9.8406 \mathrm{E}-01 & 9.6686 \mathrm{E}-01 \\ -23.07 & 1.0583 \mathrm{E}+00 & 1.0652 \mathrm{E}+00 & 1.0411 \mathrm{E}+00 \\ -17.94 & 1.1210 \mathrm{E}+00 & 1.1299 \mathrm{E}+00 & 1.1017 \mathrm{E}+00 \\ -12.82 & 1.1682 \mathrm{E}+00 & 1.1777 \mathrm{E}+00 & 1.1222 \mathrm{E}+00 \\ -7.69 & 1.1996 \mathrm{E}+00 & 1.2097 \mathrm{E}+00 & 1.1520 \mathrm{E}+00 \\ -2.56 & 1.2150 \mathrm{E}+00 & 1.2254 \mathrm{E}+00 & 1.1666 \mathrm{E}+00 \\ 2.56 & 1.2139 \mathrm{E}+00 & 1.2247 \mathrm{E}+00 & 1.1742 \mathrm{E}+00 \\ 7.69 & 1.1960 \mathrm{E}+00 & 1.2070 \mathrm{E}+00 & 1.1570 \mathrm{E}+00 \\ 12.82 & 1.1613 \mathrm{E}+00 & 1.1723 \mathrm{E}+00 & 1.1237 \mathrm{E}+00 \\ 17.94 & 1.1099 \mathrm{E}+00 & 1.1209 \mathrm{E}+00 & 1.1131 \mathrm{E}+00 \\ 23.07 & 1.0411 \mathrm{E}+00 & 1.0504 \mathrm{E}+00 & 1.0449 \mathrm{E}+00 \\ 28.20 & 9.5569 \mathrm{E}-01 & 9.6164 \mathrm{E}-01 & 9.6037 \mathrm{E}-01 \\ 33.32 & 8.5539 \mathrm{E}-01 & 8.5551 \mathrm{E}-01 & 9.1045 \mathrm{E}-01 \\ 38.45 & 7.4162 \mathrm{E}-01 & 7.2987 \mathrm{E}-01 & 7.9488 \mathrm{E}-01 \\ 43.58 & 6.2027 \mathrm{E}-01 & 5.8705 \mathrm{E}-01 & 6.8100 \mathrm{E}-01 \\ 47.17 & 5.3777 \mathrm{E}-01 & 4.7686 \mathrm{E}-01 & 1.0388 \mathrm{E}-01 \\ 50.62 & 4.6083 \mathrm{E}-01 & 3.8128 \mathrm{E}-01 & 8.4183 \mathrm{E}-02 \\ 55.49 & 3.6477 \mathrm{E}-01 & 2.7914 \mathrm{E}-01 & 6.4669 \mathrm{E}-02 \\ 60.35 & 2.7987 \mathrm{E}-01 & 1.9864 \mathrm{E}-01 & 4.9800 \mathrm{E}-02 \\ 64.98 & 2.2476 \mathrm{E}-01 & 1.4972 \mathrm{E}-01 & 0.0000 \mathrm{E}+00 \\ 69.39 & 1.9171 \mathrm{E}-01 & 1.2304 \mathrm{E}-01 & 0.0000 \mathrm{E}+00 \\ 76.46 & 1.4474 \mathrm{E}-01 & 8.7086 \mathrm{E}-02 & 0.0000 \mathrm{E}+00 \\ 86.80 & 9.3858 \mathrm{E}-02 & 5.2375 \mathrm{E}-02 & 0.0000 \mathrm{E}+00 \\ 98.14 & 5.3952 \mathrm{E}-02 & 2.8555 \mathrm{E}-02 & 0.0000 \mathrm{E}+00 \\ 110.00 & 2.3069 \mathrm{E}-02 & 1.2731 \mathrm{E}-02 & 0.0000 \mathrm{E}+00\end{array}$


Table EllB-1.4. Axial Distribution of Total Flux, Fast Flux and Power in MFA-2 at EOC $11 \mathrm{~B}-1$

$\begin{array}{rccc}\text { Z }(\mathrm{cm}) & \text { Total Flux } & \text { Flux }>0.1 \text { MeV } & \text { Power } \\ -97.78 & 2.5461 \mathrm{E}-02 & 1.3033 \mathrm{E}-02 & 0.0000 \mathrm{E}+00 \\ -92.78 & 5.4615 \mathrm{E}-02 & 2.5150 \mathrm{E}-02 & 0.0000 \mathrm{E}+00 \\ -87.78 & 8.7529 \mathrm{E}-02 & 4.0241 \mathrm{E}-02 & 0.0000 \mathrm{E}+00 \\ -82.78 & 1.2679 \mathrm{E}-01 & 6.0050 \mathrm{E}-02 & 0.0000 \mathrm{E}+00 \\ -77.78 & 1.7518 \mathrm{E}-01 & 8.6784 \mathrm{E}-02 & 0.0000 \mathrm{E}+00 \\ -72.15 & 2.3090 \mathrm{E}-01 & 1.2168 \mathrm{E}-01 & 0.0000 \mathrm{E}+00 \\ -65.90 & 2.8824 \mathrm{E}-01 & 1.6678 \mathrm{E}-01 & 0.0000 \mathrm{E}+00 \\ -60.35 & 3.4899 \mathrm{E}-01 & 2.2899 \mathrm{E}-01 & 9.2010 \mathrm{E}-02 \\ -55.49 & 4.2796 \mathrm{E}-01 & 3.1025 \mathrm{E}-01 & 1.0622 \mathrm{E}-01 \\ -50.62 & 5.1885 \mathrm{E}-01 & 4.1571 \mathrm{E}-01 & 1.2484 \mathrm{E}-01 \\ -47.17 & 5.9131 \mathrm{E}-01 & 5.1476 \mathrm{E}-01 & 1.4380 \mathrm{E}-01 \\ -43.58 & 6.6762 \mathrm{E}-01 & 6.2658 \mathrm{E}-01 & 7.2991 \mathrm{E}-01 \\ -38.45 & 7.7953 \mathrm{E}-01 & 7.6391 \mathrm{E}-01 & 8.1823 \mathrm{E}-01 \\ -33.32 & 8.8417 \mathrm{E}-01 & 8.8146 \mathrm{E}-01 & 9.1785 \mathrm{E}-01 \\ -28.20 & 9.7642 \mathrm{E}-01 & 9.7947 \mathrm{E}-01 & 9.6251 \mathrm{E}-01 \\ -23.07 & 1.0552 \mathrm{E}+00 & 1.0613 \mathrm{E}+00 & 1.0385 \mathrm{E}+00 \\ -17.94 & 1.1190 \mathrm{E}+00 & 1.1264 \mathrm{E}+00 & 1.1004 \mathrm{E}+00 \\ -12.82 & 1.1668 \mathrm{E}+00 & 1.1743 \mathrm{E}+00 & 1.1199 \mathrm{E}+00 \\ -7.69 & 1.1991 \mathrm{E}+00 & 1.2067 \mathrm{E}+00 & 1.1505 \mathrm{E}+00 \\ -2.56 & 1.2153 \mathrm{E}+00 & 1.2233 \mathrm{E}+00 & 1.1660 \mathrm{E}+00 \\ 2.56 & 1.2152 \mathrm{E}+00 & 1.2240 \mathrm{E}+00 & 1.1736 \mathrm{E}+00 \\ 7.69 & 1.1984 \mathrm{E}+00 & 1.2079 \mathrm{E}+00 & 1.1575 \mathrm{E}+00 \\ 12.82 & 1.1647 \mathrm{E}+00 & 1.1750 \mathrm{E}+00 & 1.1254 \mathrm{E}+00 \\ 17.94 & 1.1144 \mathrm{E}+00 & 1.1258 \mathrm{E}+00 & 1.1155 \mathrm{E}+00 \\ 23.07 & 1.0464 \mathrm{E}+00 & 1.0571 \mathrm{E}+00 & 1.0482 \mathrm{E}+00 \\ 28.20 & 9.6163 \mathrm{E}-01 & 9.6967 \mathrm{E}-01 & 9.6453 \mathrm{E}-01 \\ 33.32 & 8.6175 \mathrm{E}-01 & 8.6457 \mathrm{E}-01 & 9.1586 \mathrm{E}-01 \\ 38.45 & 7.4798 \mathrm{E}-01 & 7.3872 \mathrm{E}-01 & 8.0193 \mathrm{E}-01 \\ 43.58 & 6.2643 \mathrm{E}-01 & 5.9388 \mathrm{E}-01 & 6.9373 \mathrm{E}-01 \\ 47.17 & 5.4446 \mathrm{E}-01 & 4.8131 \mathrm{E}-01 & 1.1368 \mathrm{E}-01 \\ 50.62 & 4.6818 \mathrm{E}-01 & 3.8365 \mathrm{E}-01 & 9.3287 \mathrm{E}-02 \\ 55.49 & 3.7287 \mathrm{E}-01 & 2.7997 \mathrm{E}-01 & 7.3144 \mathrm{E}-02 \\ 60.35 & 2.8779 \mathrm{E}-01 & 1.9859 \mathrm{E}-01 & 5.7510 \mathrm{E}-02 \\ 64.98 & 2.3233 \mathrm{E}-01 & 1.4877 \mathrm{E}-01 & 0.0000 \mathrm{E}+00 \\ 69.39 & 1.9824 \mathrm{E}-01 & 1.2155 \mathrm{E}-01 & 0.0000 \mathrm{E}+00 \\ 76.46 & 1.4938 \mathrm{E}-01 & 8.5086 \mathrm{E}-02 & 0.0000 \mathrm{E}+00 \\ 86.80 & 9.6194 \mathrm{E}-02 & 5.0394 \mathrm{E}-02 & 0.0000 \mathrm{E}+00 \\ 98.14 & 5.4746 \mathrm{E}-02 & 2.7066 \mathrm{E}-02 & 0.0000 \mathrm{E}+00 \\ 110.00 & 2.3229 \mathrm{E}-02 & 1.1943 \mathrm{E}-02 & 0.0000 \mathrm{E}+00\end{array}$


HNF-SD-FF-ANAL-009 Rev. 1

Table E11B-1.5. Fission Power Distribution by Pin in MFA-1 at EOC 11B-1 CORE POSITION 1404

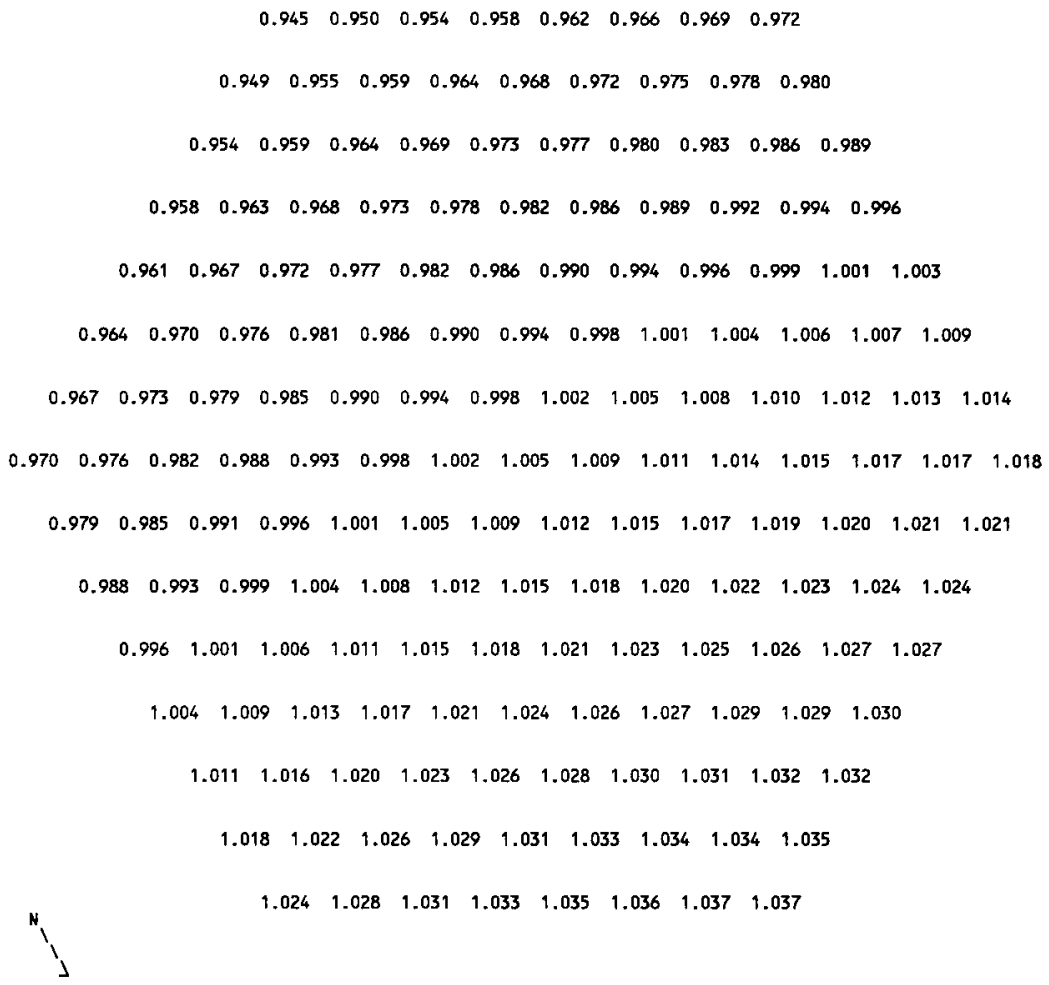




\section{HNF-SD-FF-ANAL-009 Rev. 1}

Table E11B-1.6. Fission Power Distribution by Pin in MFA-2 at EOC 11B-1 CORE POSITION 2506

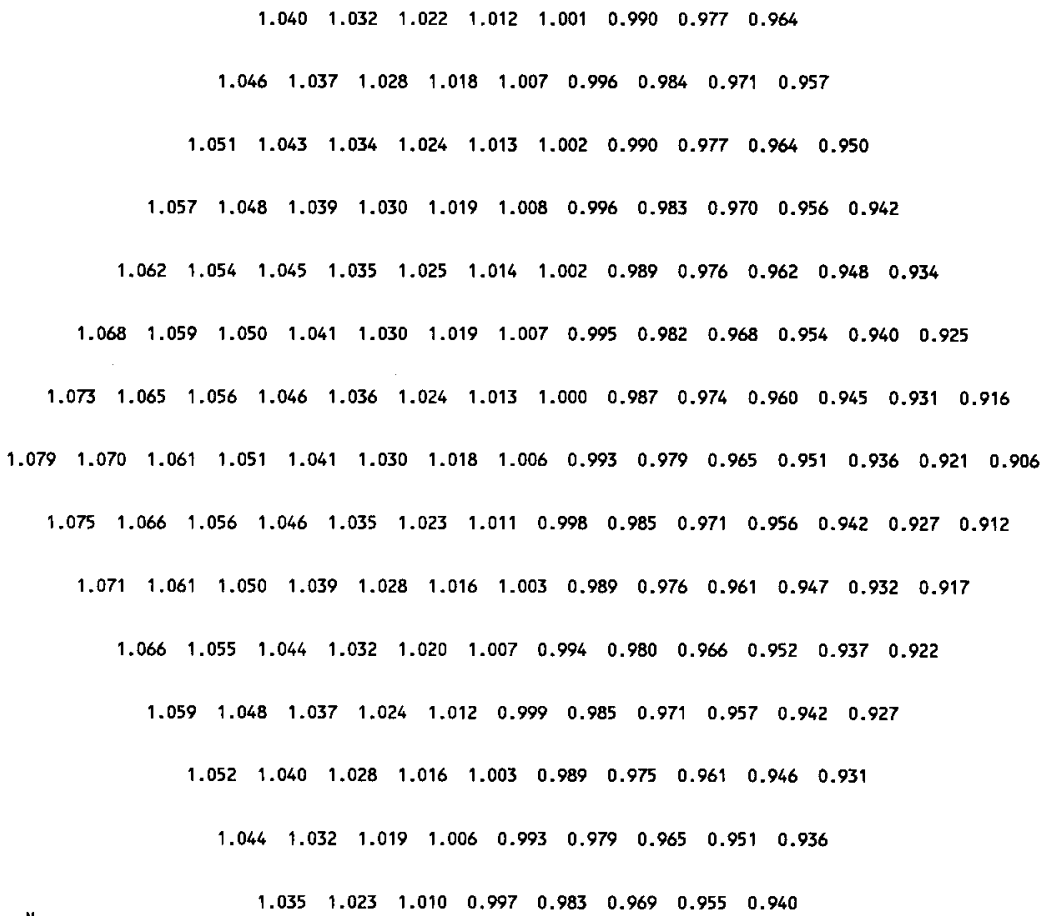


Table El1B-1.7. Fast Flux Distribution by Pin in MFA-1 at EOC 11B-1 CORE POSITION 1404

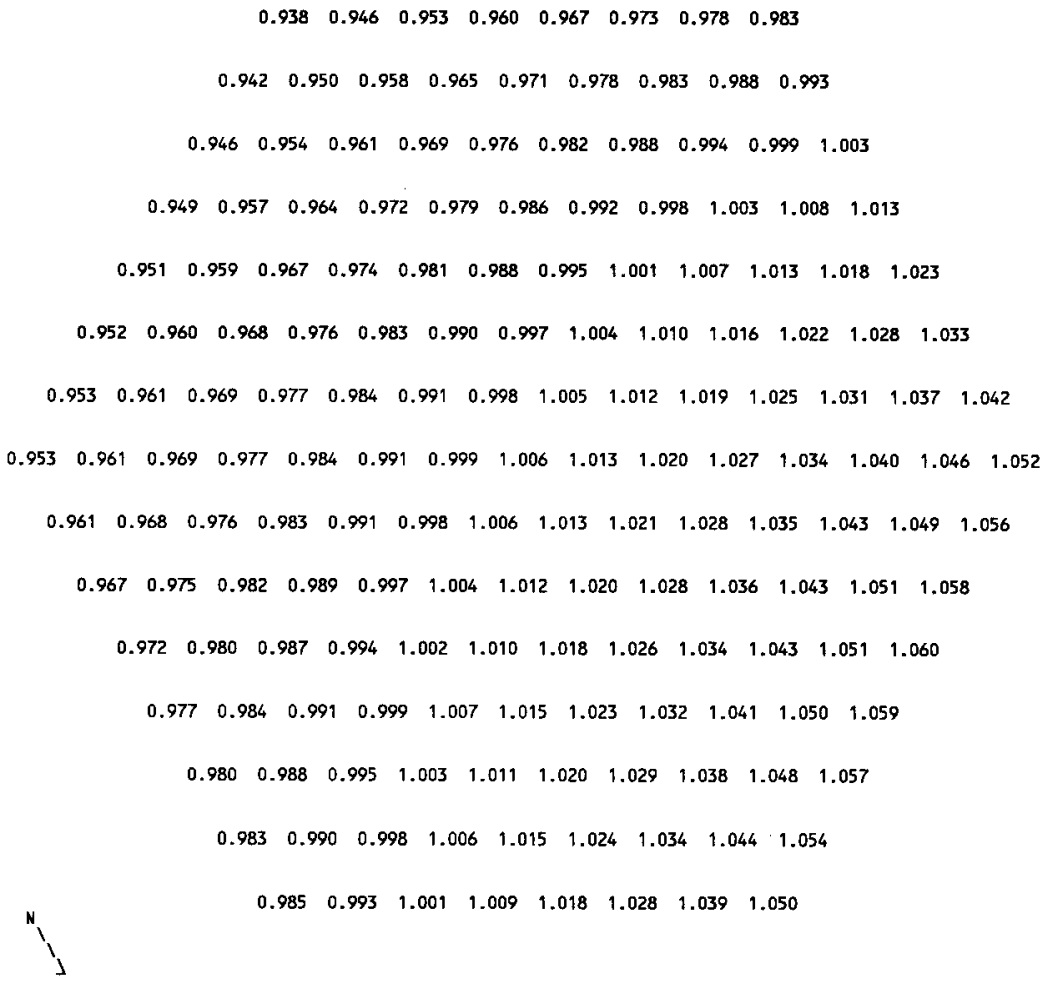




\section{HNF-SD-FF-ANAL-009 Rev. 1}

Table El1B-1.8. Fast Flux Distribution by Pin in MFA-2 at EOC 11B-1 CORE POSITION 2506

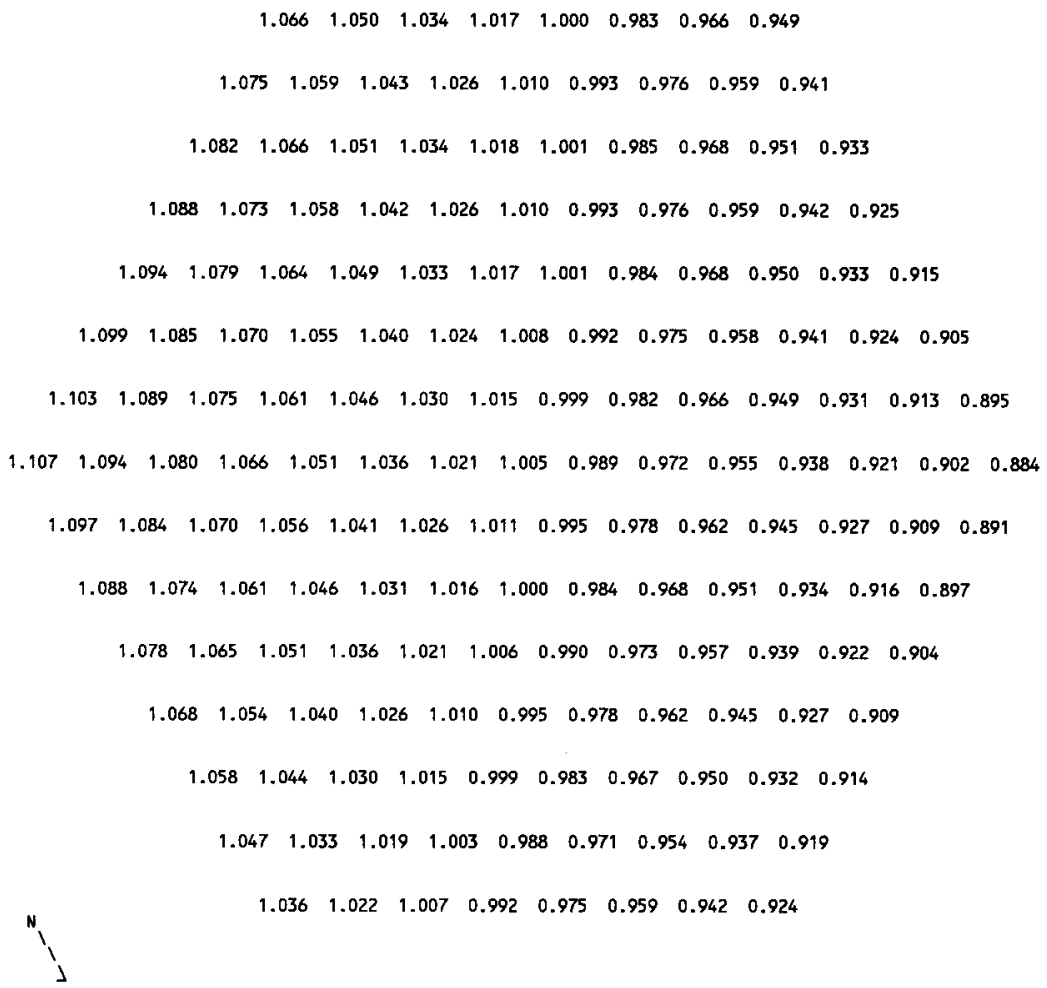


Table EllB-1.9. MFA-1 and MFA-2 Duct Wall Fast Flux Data at EOC 11B-1

$\begin{array}{ccc}\text { Side } & \begin{array}{c}\text { Flux }>0.1 \\ \text { MFA-1 }\end{array} & \begin{array}{c}\mathrm{MeV}\left(\mathrm{n} / \mathrm{cm}^{2}-\mathrm{sec}\right) \\ \text { MFA-2 }\end{array} \\ \text { E } & 2.0929 E+15 & 2.2804 \mathrm{E}+15 \\ \text { SE } & 2.0158 E+15 & 2.3310 E+15 \\ \text { SW } & 2.0504 \mathrm{1}+15 & 2.1187 \mathrm{E}+15 \\ \text { W } & 2.1881 \mathrm{E}+15 & 1.8972 \mathrm{E}+15 \\ \text { NW } & 2.3113 \mathrm{E}+15 & 1.8601 \mathrm{E}+15 \\ \text { NE } & 2.1812 \mathrm{E}+15 & 2.0589 \mathrm{1})+15\end{array}$

Table EllB-1.10. Assembly Outlet Temperatures and Flow Rates at EOC 11B-1

$\begin{array}{cc}\begin{array}{c}\text { CALCULATED } \\ \text { OUTLET TEMP. }\end{array} & \text { FLOW RATE } \\ \text { (DEG F) } & \text { (LB/H) } \\ 1051 & 114690 \\ 1015 & 69040 \\ 1049 & 200400 \\ 881 & 207670 \\ 877 & 207700 \\ 893 & 197480 \\ 879 & 207670 \\ 1036 & 114690 \\ 1025 & 204240 \\ 1004 & 171220 \\ 889 & 207670 \\ 886 & 207670 \\ 923 & 170310 \\ 893 & 170310\end{array}$

Assembly flows are based on a calculated core pressure drop of $110.1 \mathrm{psi}$ at a total reactor flow rate of $16.74 \mathrm{E}+06 \mathrm{lb} / \mathrm{hr}$. 
Table E11B-1.11. MFA-1 Sodium Subchannel Temperatures at Top of Core Elevation for EOC 11B-1

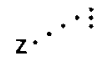

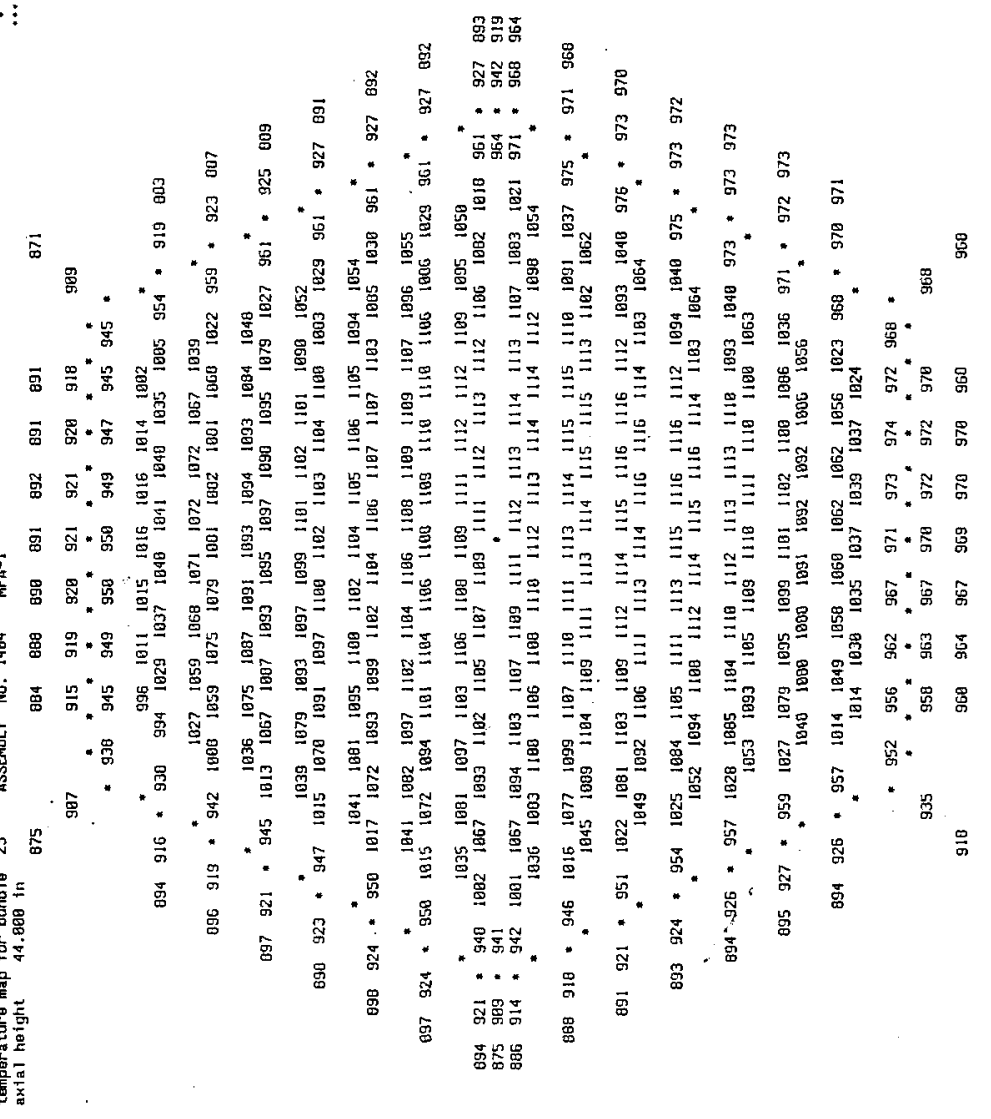


Table E11B-1.12. MFA-2 Sodium Subchanne1 Temperatures at Top of Core Elevation for EOC 11B-1

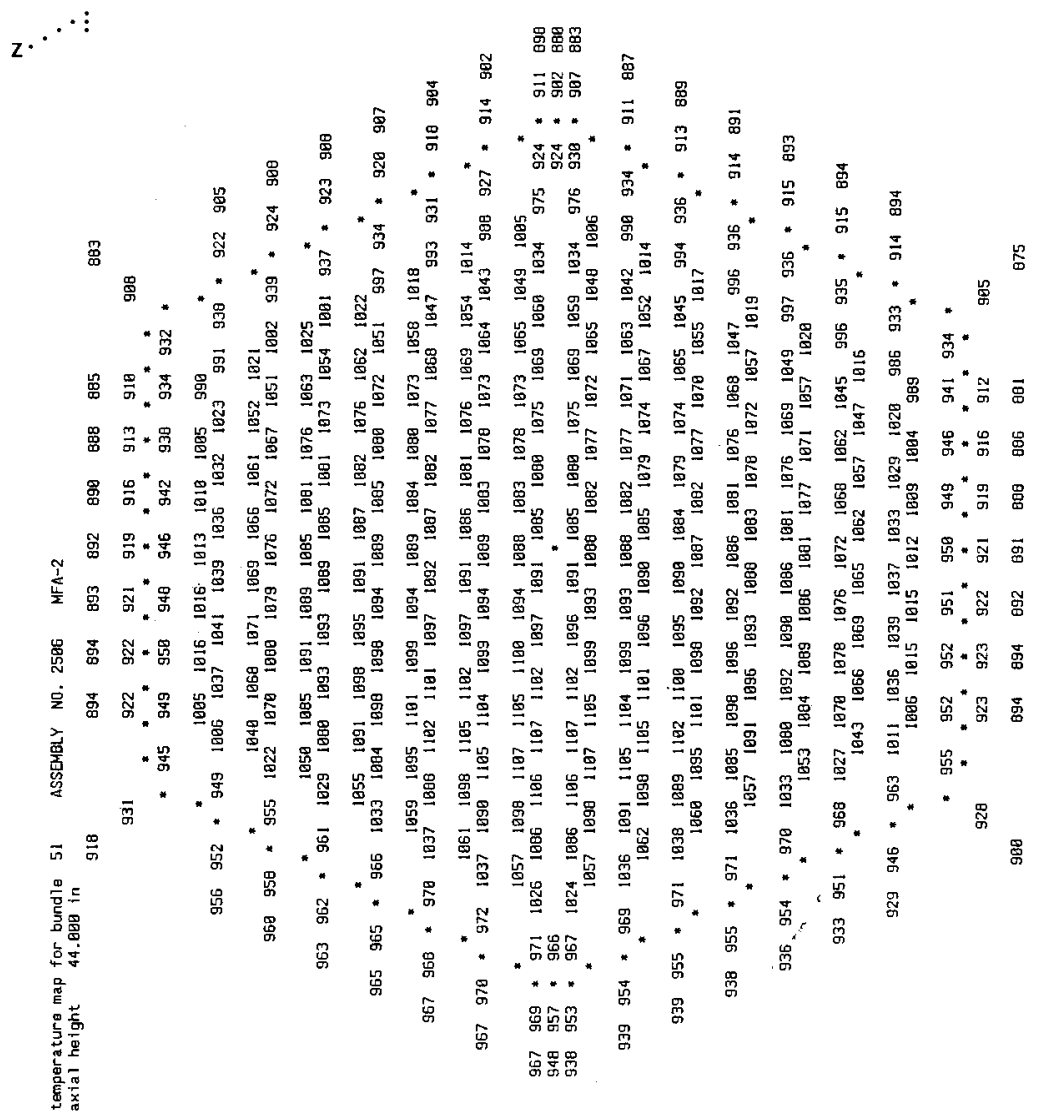


Table El1B-1.13. MFA-1 Sodium Subchannel Temperatures at Elevation of Upper Axial Blanket for EOC 11B-1

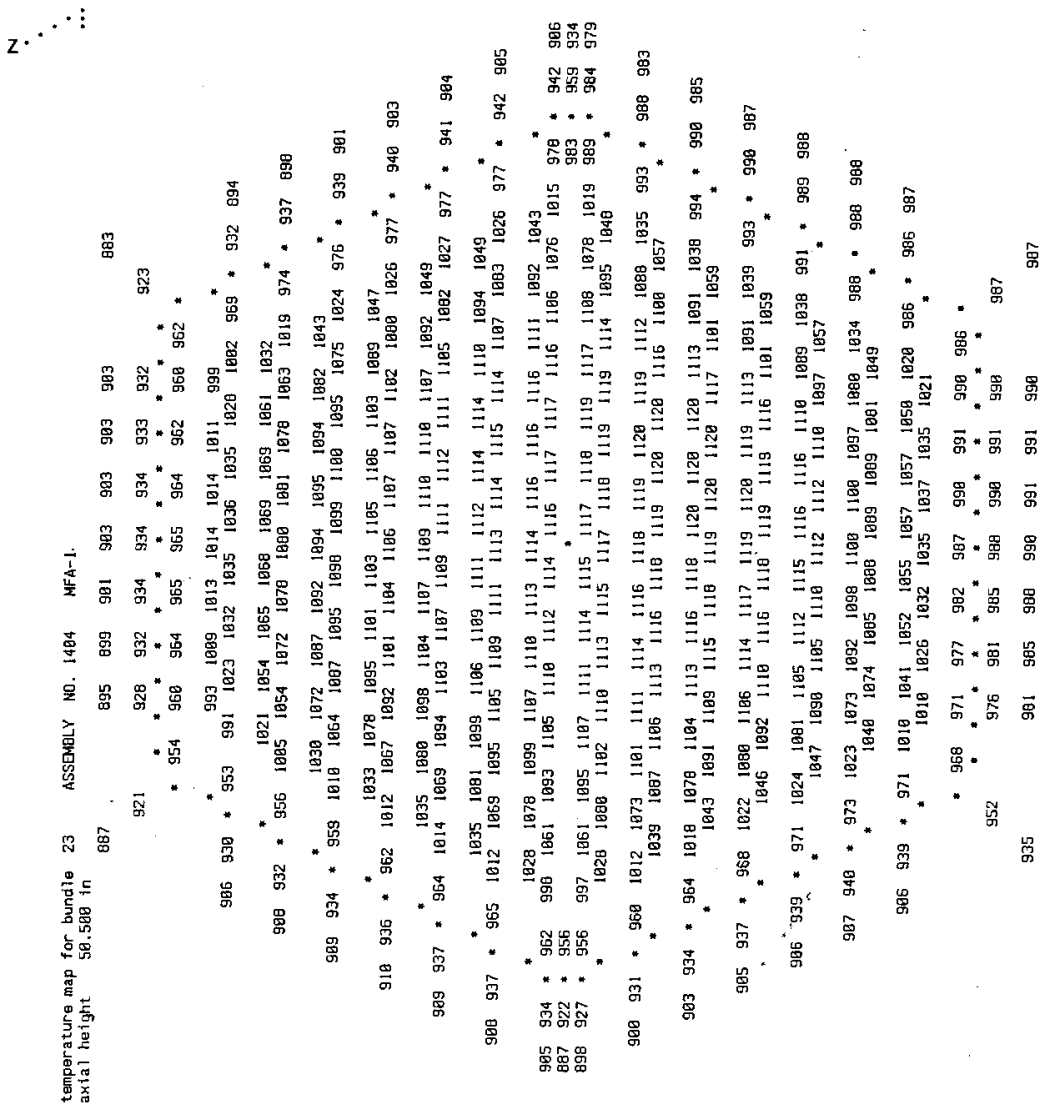


Table E11B-1.14. MFA-2 Sodium Subchannel Temperatures at Elevation of Upper Axial Blanket for EOC 11B-1

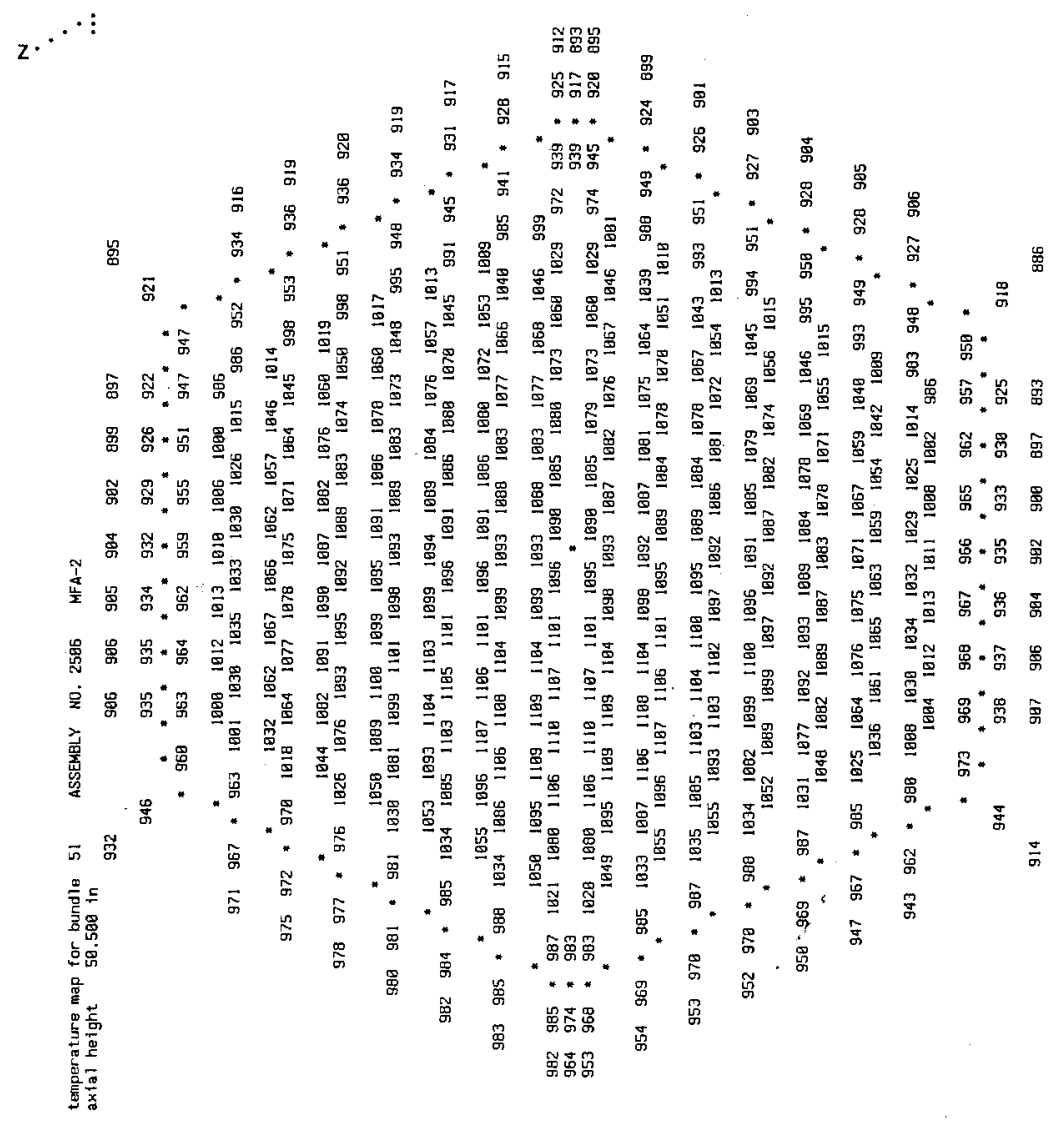


Table E11B-1.15. MFA-1 Sodium Subchanne1 Temperatures at Top of Fuel Pin Bundle for EOC 11B-1

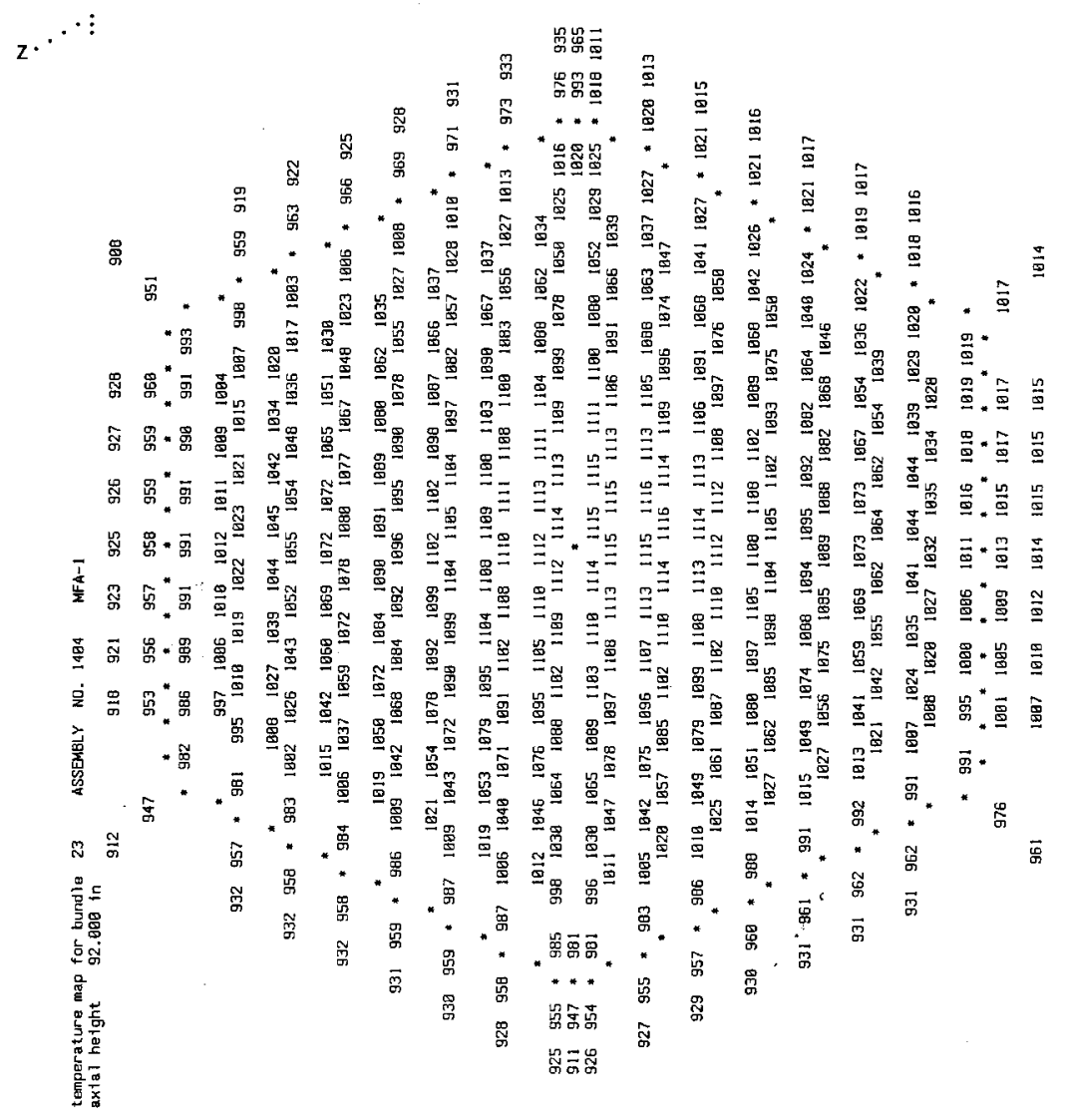


Table El1B-1.16. MFA-2 Sodium Subchannel Temperatures at Top of Fuel Pin Bundle for EOC $118-1$

$z^{\cdot \cdot \cdot !}$

ถั้

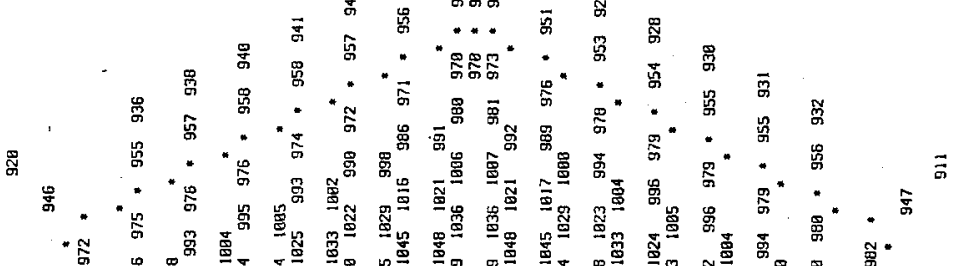

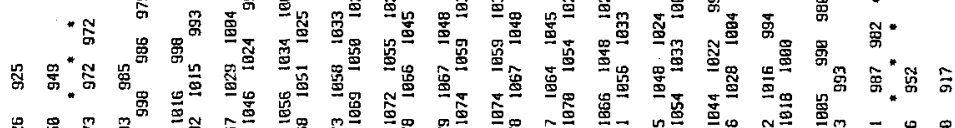

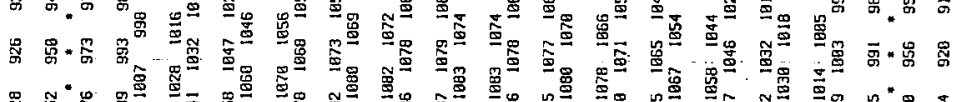

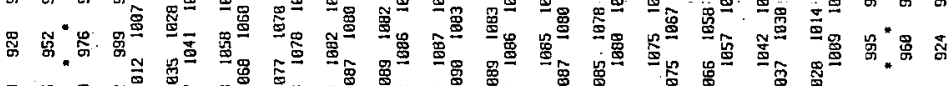

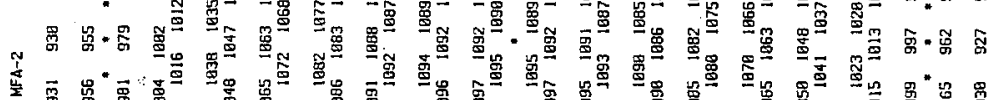

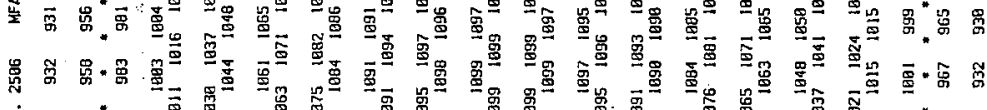

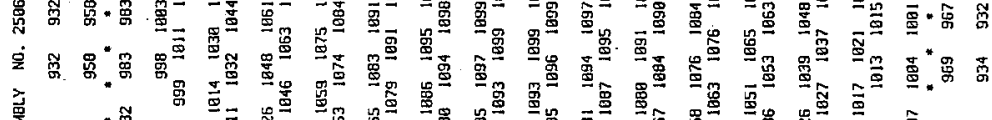
票

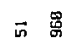

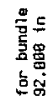

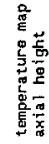

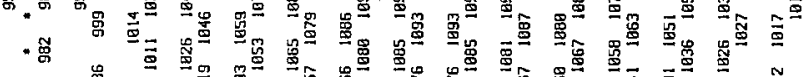
.

EN⿱ 
Table EllB-1.17. Composition of MFA-1 at EOC 11B-1

\begin{tabular}{|c|c|c|c|c|c|}
\hline Axial & Axial R & ge $(\mathrm{cm})$ & & Atom Dens & $y(a / b-c m)$ \\
\hline Level & $\begin{array}{r}\text { Lower } \\
-12805\end{array}$ & $\begin{array}{l}\text { Upper } \\
-75\end{array}$ & Constituent & Fresh & End of cycle \\
\hline & & & $\begin{array}{l}\mathrm{Na}-23 \\
\mathrm{SS}-316\end{array}$ & $\begin{array}{l}5.3150 \mathrm{E}-03 \\
6.4090 \mathrm{E}-02\end{array}$ & $\begin{array}{l}5.3150 \mathrm{E}-03 \\
6.4090 \mathrm{E}-02\end{array}$ \\
\hline 2 & -75.28 & -62.78 & $\begin{array}{l}\mathrm{Na}-23 \\
\mathrm{SS}-316\end{array}$ & $\begin{array}{l}1.3177 \mathrm{E}-02 \\
3.4175 \mathrm{E}-02\end{array}$ & $\begin{array}{l}1.3177 \mathrm{E}-02 \\
3.4175 \mathrm{E}-02\end{array}$ \\
\hline 3 & -62.78 & -46.14 & $\begin{array}{l}U-235 \\
U-238 \\
P u-239 \\
\text { Pu-240 } \\
F P \\
0-16 \\
\mathrm{Na}-23 \\
\text { SS-316 }\end{array}$ & $\begin{array}{l}1.4143 \mathrm{E}-05 \\
7.0573 \mathrm{E}-03 \\
0.0000 \mathrm{E}+00 \\
0.0000 \mathrm{E}+00 \\
0.0000 \mathrm{E}+00 \\
1.4143 \mathrm{E}-02 \\
9.7416 \mathrm{E}-03 \\
1.9073 \mathrm{E}-02\end{array}$ & $\begin{array}{l}1.0147 \mathrm{E}-05 \\
6.8276 \mathrm{E}-03 \\
1.8778 \mathrm{E}-04 \\
8.7336 \mathrm{E}-06 \\
3.5874 \mathrm{E}-05 \\
1.4143 \mathrm{E}-02 \\
9.7416 \mathrm{E}-03 \\
1.9073 \mathrm{E}-02\end{array}$ \\
\hline 4 & -46.14 & -30.76 & $\begin{array}{l}U-235 \\
U-238 \\
P u-239 \\
P u-240 \\
P u-241 \\
P u-242 \\
\text { Am-241 } \\
F P \\
O-16 \\
N a-23 \\
S S-316\end{array}$ & $\begin{array}{l}1.0840 \mathrm{E}-05 \\
4.4499 \mathrm{E}-03 \\
1.7520 \mathrm{E}-03 \\
2.2340 \mathrm{E}-04 \\
1.9280 \mathrm{E}-05 \\
4.7430 \mathrm{E}-06 \\
7.6680 \mathrm{E}-07 \\
0.0000 \mathrm{E}+00 \\
1.2670 \mathrm{E}-02 \\
9.8100 \mathrm{E}-03 \\
1.8990 \mathrm{E}-02\end{array}$ & $\begin{array}{l}8.0210 \mathrm{E}-06 \\
4.2757 \mathrm{E}-03 \\
1.4323 \mathrm{E}-03 \\
2.8424 \mathrm{E}-04 \\
2.4175 \mathrm{E}-05 \\
5.1940 \mathrm{E}-06 \\
2.6996 \mathrm{E}-06 \\
4.2713 \mathrm{E}-04 \\
1.2670 \mathrm{E}-02 \\
9.8100 \mathrm{E}-03 \\
1.8990 \mathrm{E}-02\end{array}$ \\
\hline 5 & -30.76 & -15.38 & $\begin{array}{l}U-235 \\
U-238 \\
P u-239 \\
P U-240 \\
P U-241 \\
P U-242 \\
\text { Am-241 } \\
\text { FP } \\
\text { O-16 } \\
\mathrm{Na}-23 \\
\text { SS-316 }\end{array}$ & $\begin{array}{l}1.0840 \mathrm{E}-05 \\
4.4499 \mathrm{E}-03 \\
1.7520 \mathrm{E}-03 \\
2.2340 \mathrm{E}-04 \\
1.9280 \mathrm{E}-05 \\
4.7430 \mathrm{E}-06 \\
7.6680 \mathrm{E}-07 \\
0.0000 \mathrm{E}+00 \\
1.2670 \mathrm{E}-02 \\
9.8100 \mathrm{E}-03 \\
1.8990 \mathrm{E}-02\end{array}$ & $\begin{array}{l}7.4593 \mathrm{E}-06 \\
4.2310 \mathrm{E}-03 \\
1.3577 \mathrm{E}-03 \\
2.8674 \mathrm{E}-04 \\
2.4535 \mathrm{E}-05 \\
5.2116 \mathrm{E}-06 \\
2.6375 \mathrm{E}-06 \\
5.4400 \mathrm{E}-04 \\
1.2670 \mathrm{E}-02 \\
9.8100 \mathrm{E}-03 \\
1.8990 \mathrm{E}-02\end{array}$ \\
\hline 6 & -15.38 & 0.00 & $\begin{array}{l}U-235 \\
U-238 \\
\mathrm{Pu}-239 \\
\mathrm{Pu}-240 \\
\mathrm{Pu}-241 \\
\mathrm{Pu}-242 \\
\mathrm{Am}-241 \\
\mathrm{FP} \\
0-16 \\
\mathrm{Na}-23 \\
\mathrm{SS}-316\end{array}$ & $\begin{array}{l}1.0840 \mathrm{E}-05 \\
4.4499 \mathrm{E}-03 \\
1.7520 \mathrm{E}-03 \\
2.2340 \mathrm{E}-04 \\
1.9280 \mathrm{E}-05 \\
4.7430 \mathrm{E}-06 \\
7.6680 \mathrm{E}-07 \\
0.0000 \mathrm{E}+00 \\
1.2670 \mathrm{E}-02 \\
9.8100 \mathrm{E}-03 \\
1.8990 \mathrm{E}-02\end{array}$ & $\begin{array}{l}7.1596 \mathrm{E}-06 \\
4.2081 \mathrm{E}-03 \\
1.3216 \mathrm{E}-03 \\
2.9107 \mathrm{E}-04 \\
2.5266 \mathrm{E}-05 \\
5.2750 \mathrm{E}-06 \\
2.6285 \mathrm{E}-06 \\
5.9836 \mathrm{E}-04 \\
1.2670 \mathrm{E}-02 \\
9.8100 \mathrm{E}-03 \\
1.8990 \mathrm{E}-02\end{array}$ \\
\hline & & & & & \\
\hline
\end{tabular}


HNF-SD-FF-ANAL-009 Rev. 1
7
$0.00 \quad 15.38$

$\begin{array}{lll}8 & 15.38 & 30.76\end{array}$

$\begin{array}{lll}\mathrm{U}-235 & 1.0840 \mathrm{E}-05 & 7.2644 \mathrm{E}-06 \\ \mathrm{U}-238 & 4.4499 \mathrm{E}-03 & 4.2161 \mathrm{E}-03 \\ \mathrm{Pu}-239 & 1.7520 \mathrm{E}-03 & 1.3336 \mathrm{E}-03 \\ \mathrm{Pu}-240 & 2.2340 \mathrm{E}-04 & 2.8920 \mathrm{E}-04 \\ \mathrm{Pu}-241 & 1.9280 \mathrm{E}-05 & 2.4958 \mathrm{E}-05 \\ \mathrm{Pu}-242 & 4.7430 \mathrm{E}-06 & 5.2479 \mathrm{E}-06 \\ \mathrm{Am}-241 & 7.6680 \mathrm{E}-07 & 2.6309 \mathrm{E}-06 \\ \mathrm{FP} & 0.0000 \mathrm{E}+00 & 5.8016 \mathrm{E}-04 \\ \mathrm{O}-16 & 1.2670 \mathrm{E}-02 & 1.2670 \mathrm{E}-02 \\ \mathrm{Na}-23 & 9.8100 \mathrm{E}-03 & 9.8100 \mathrm{E}-03 \\ \mathrm{SS}-316 & 1.8990 \mathrm{E}-02 & 1.8990 \mathrm{E}-02\end{array}$

$\begin{array}{lll}\mathrm{U}-235 & 1.0840 \mathrm{E}-05 & 7.7581 \mathrm{E}-06 \\ \mathrm{U}-238 & 4.4499 \mathrm{E}-03 & 4.2536 \mathrm{E}-03 \\ \mathrm{Pu}-239 & 1.7520 \mathrm{E}-03 & 1.3923 \mathrm{E}-03 \\ \mathrm{Pu}-240 & 2.2340 \mathrm{E}-04 & 2.8106 \mathrm{E}-04 \\ \mathrm{Pu}-241 & 1.9280 \mathrm{E}-05 & 2.3670 \mathrm{E}-05 \\ \mathrm{Pu}-242 & 4.7430 \mathrm{E}-06 & 5.1404 \mathrm{E}-06 \\ \mathrm{Am}-241 & 7.6680 \mathrm{E}-07 & 2.6440 \mathrm{E}-06 \\ \mathrm{FP} & 0.0000 \mathrm{E}+00 & 4.9337 \mathrm{E}-04 \\ \mathrm{O}-16 & 1.2670 \mathrm{E}-02 & 1.2670 \mathrm{E}-02 \\ \mathrm{Na}-23 & 9.8100 \mathrm{E}-03 & 9.8100 \mathrm{E}-03 \\ \mathrm{SS}-316 & 1.8990 \mathrm{E}-02 & 1.8990 \mathrm{E}-02\end{array}$

$9 \quad 30.76 \quad 46.14$

$\begin{array}{lll}\mathrm{U}-235 & 1.0840 \mathrm{E}-05 & 8.4963 \mathrm{E}-06 \\ \mathrm{U}-238 & 4.4499 \mathrm{E}-03 & 4.3077 \mathrm{E}-03 \\ \mathrm{Pu}-239 & 1.7520 \mathrm{E}-03 & 1.4853 \mathrm{E}-03 \\ \mathrm{Pu}-240 & 2.2340 \mathrm{E}-04 & 2.7295 \mathrm{E}-04 \\ \mathrm{Pu}-241 & 1.9280 \mathrm{E}-05 & 2.2538 \mathrm{E}-05 \\ \mathrm{Pu}-242 & 4.7430 \mathrm{E}-06 & 5.0663 \mathrm{E}-06 \\ \mathrm{Am}-241 & 7.6680 \mathrm{E}-07 & 2.6969 \mathrm{E}-06 \\ \mathrm{FP} & 0.0000 \mathrm{E}+00 & 3.5497 \mathrm{E}-04 \\ 0-16 & 1.2670 \mathrm{E}-02 & 1.2670 \mathrm{E}-02 \\ \mathrm{Na}-23 & 9.8100 \mathrm{E}-03 & 9.8100 \mathrm{E}-03 \\ \mathrm{SS}-316 & 1.8990 \mathrm{E}-02 & 1.8990 \mathrm{E}-02\end{array}$

$\begin{array}{lll}10 & 46.14 \quad 62.78\end{array}$

$\begin{array}{lll}\mathrm{U}-235 & 1.4143 \mathrm{E}-05 & 1.1431 \mathrm{E}-05 \\ \mathrm{U}-238 & 7.0573 \mathrm{E}-03 & 6.9021 \mathrm{E}-03 \\ \mathrm{Pu}-239 & 0.0000 \mathrm{E}+00 & 1.3198 \mathrm{E}-04 \\ \mathrm{PU}-240 & 0.0000 \mathrm{E}+00 & 3.7147 \mathrm{E}-06 \\ \mathrm{FP} & 0.0000 \mathrm{E}+00 & 2.1337 \mathrm{E}-05 \\ 0-16 & 1.4143 \mathrm{E}-02 & 1.4143 \mathrm{E}-02 \\ \mathrm{Na}-23 & 9.7416 \mathrm{E}-03 & 9.7416 \mathrm{E}-03 \\ \mathrm{SS}-316 & 1.9073 \mathrm{E}-02 & 1.9073 \mathrm{E}-02\end{array}$

$\begin{array}{lll}11 & 62.78 & 172.84\end{array}$

$\begin{array}{lll}\mathrm{Na}-23 & 8.2400 \mathrm{E}-03 & 8.2400 \mathrm{E}-03 \\ \mathrm{SS}-316 & 2.5562 \mathrm{E}-02 & 2.5562 \mathrm{E}-02\end{array}$


Table EllB-1.18. Composition of MFA-2 at EOC 11B-1

\begin{tabular}{|c|c|c|c|c|c|}
\hline Axial & Axial $R$ & ge $(\mathrm{cm})$ & & Atom Densi & $(a / b-c m)$ \\
\hline $\begin{array}{c}\text { Level } \\
1\end{array}$ & $\begin{array}{r}\text { Lower } \\
-128.05\end{array}$ & $\begin{array}{r}\text { Upper } \\
-75.28\end{array}$ & Constituent & Fresh & End of Cycle \\
\hline & & & $\begin{array}{l}\mathrm{Na}-23 \\
\text { SS-316 }\end{array}$ & $\begin{array}{l}5.3150 \mathrm{E}-03 \\
6.4090 \mathrm{E}-02\end{array}$ & $\begin{array}{l}5.3150 E-03 \\
6.4090 E-02\end{array}$ \\
\hline 2 & -75.28 & -62.78 & & & \\
\hline & & & $\begin{array}{l}\mathrm{Na}-23 \\
\text { SS-316 }\end{array}$ & $\begin{array}{l}1.3177 \mathrm{E}-02 \\
3.4175 \mathrm{E}-02\end{array}$ & $\begin{array}{l}1.3177 \mathrm{E}-02 \\
3.4175 \mathrm{E}-02\end{array}$ \\
\hline 3 & -62.78 & -46.14 & $\begin{array}{l}U-235 \\
U-238 \\
\text { Pu-239 } \\
\text { Pu-240 } \\
\text { FP } \\
0-16 \\
\mathrm{Na}-23 \\
\text { SS-316 }\end{array}$ & $\begin{array}{l}1.4143 \mathrm{E}-05 \\
7.0573 \mathrm{E}-03 \\
0.0000 \mathrm{E}+00 \\
0.0000 \mathrm{E}+00 \\
0.0000 \mathrm{E}+00 \\
1.4143 \mathrm{E}-02 \\
9.7416 \mathrm{E}-03 \\
1.9073 \mathrm{E}-02\end{array}$ & $\begin{array}{l}1.0099 \mathrm{E}-05 \\
6.8293 \mathrm{E}-03 \\
1.8582 \mathrm{E}-04 \\
8.9796 \mathrm{E}-06 \\
3.5892 \mathrm{E}-05 \\
1.4143 \mathrm{E}-02 \\
9.7416 \mathrm{E}-03 \\
1.9073 \mathrm{E}-02\end{array}$ \\
\hline 4 & -46.14 & -30.76 & $\begin{array}{l}U-235 \\
U-238 \\
P u-239 \\
P u-240 \\
P u-241 \\
P u-242 \\
A m-241 \\
F P \\
0-16 \\
\mathrm{Na}-23 \\
\mathrm{SS}-316\end{array}$ & $\begin{array}{l}1.0160 \mathrm{E}-05 \\
4.4270 \mathrm{E}-03 \\
1.7483 \mathrm{E}-03 \\
2.2290 \mathrm{E}-04 \\
1.9040 \mathrm{E}-05 \\
4.7330 \mathrm{E}-06 \\
7.5700 \mathrm{E}-07 \\
0.0000 \mathrm{E}+00 \\
1.2760 \mathrm{E}-02 \\
9.8100 \mathrm{E}-03 \\
1.8990 \mathrm{E}-02\end{array}$ & $\begin{array}{l}7.5263 \mathrm{E}-06 \\
4.2542 \mathrm{E}-03 \\
1.4276 \mathrm{E}-03 \\
2.8266 \mathrm{E}-04 \\
2.3918 \mathrm{E}-05 \\
5.1674 \mathrm{E}-06 \\
2.6907 \mathrm{E}-06 \\
4.2756 \mathrm{E}-04 \\
1.2760 \mathrm{E}-02 \\
9.8100 \mathrm{E}-03 \\
1.8990 \mathrm{E}-02\end{array}$ \\
\hline 5 & -30.76 & -15.38 & $\begin{array}{l}U-235 \\
U-238 \\
\mathrm{Pu}-239 \\
\mathrm{Pu}-240 \\
\mathrm{Pu}-241 \\
\mathrm{Pu}-242 \\
\mathrm{Am}-241 \\
\mathrm{FP} \\
\mathrm{O}-16 \\
\mathrm{Na}-23 \\
\mathrm{SS}-316\end{array}$ & $\begin{array}{l}1.0160 \mathrm{E}-05 \\
4.4270 \mathrm{E}-03 \\
1.7483 \mathrm{E}-03 \\
2.2290 \mathrm{E}-04 \\
1.9040 \mathrm{E}-05 \\
4.7330 \mathrm{E}-06 \\
7.5700 \mathrm{E}-07 \\
0.0000 \mathrm{E}+00 \\
1.2760 \mathrm{E}-02 \\
9.8100 \mathrm{E}-03 \\
1.8990 \mathrm{E}-02\end{array}$ & $\begin{array}{l}6.9789 \mathrm{E}-06 \\
4.2078 \mathrm{E}-03 \\
1.3500 \mathrm{E}-03 \\
2.8510 \mathrm{E}-04 \\
2.4259 \mathrm{E}-05 \\
5.1816 \mathrm{E}-06 \\
2.6300 \mathrm{E}-06 \\
5.4967 \mathrm{E}-04 \\
1.2760 \mathrm{E}-02 \\
9.8100 \mathrm{E}-03 \\
1.8990 \mathrm{E}-02\end{array}$ \\
\hline 6 & -15.38 & 0.00 & $\begin{array}{l}U-235 \\
U-238 \\
P u-239 \\
P u-240 \\
P u-241 \\
P u-242 \\
\text { Am-241 } \\
\text { FP } \\
0-16 \\
\mathrm{Na}-23 \\
\text { SS-316 }\end{array}$ & $\begin{array}{l}1.0160 \mathrm{E}-05 \\
4.4270 \mathrm{E}-03 \\
1.7483 \mathrm{E}-03 \\
2.2290 \mathrm{E}-04 \\
1.9040 \mathrm{E}-05 \\
4.7330 \mathrm{E}-06 \\
7.5700 \mathrm{E}-07 \\
0.0000 \mathrm{E}+00 \\
1.2760 \mathrm{E}-02 \\
9.8100 \mathrm{E}-03 \\
1.8990 \mathrm{E}-02\end{array}$ & $\begin{array}{l}6.6804 \mathrm{E}-06 \\
4.1831 \mathrm{E}-03 \\
1.3113 \mathrm{E}-03 \\
2.8969 \mathrm{E}-04 \\
2.5037 \mathrm{E}-05 \\
5.2483 \mathrm{E}-06 \\
2.6236 \mathrm{E}-06 \\
6.0765 \mathrm{E}-04 \\
1.2760 \mathrm{E}-02 \\
9.8100 \mathrm{E}-03 \\
1.8990 \mathrm{E}-02\end{array}$ \\
\hline & & & & & \\
\hline
\end{tabular}


HNF-SD-FF-ANAL-009 Rev. 1
7
$0.00 \quad 15.38$

8

$15.38 \quad 30.76$

$\begin{array}{ll}\mathrm{U}-235 & 1.0160 \mathrm{E}-05 \\ \mathrm{U}-238 & 4.4270 \mathrm{E}-03 \\ \mathrm{Pu}-239 & 1.7483 \mathrm{E}-03 \\ \mathrm{Pu}-240 & 2.2290 \mathrm{E}-04 \\ \mathrm{Pu}-241 & 1.9040 \mathrm{E}-05 \\ \mathrm{Pu}-242 & 4.7330 \mathrm{E}-06 \\ \mathrm{Am}-241 & 7.5700 \mathrm{E}-07 \\ \mathrm{FP} & 0.0000 \mathrm{E}+00 \\ 0-16 & 1.2760 \mathrm{E}-02 \\ \mathrm{Na}-23 & 9.8100 \mathrm{E}-03 \\ \mathrm{SS}-316 & 1.8990 \mathrm{E}-02\end{array}$

6.7667E-06

4.1901E-03

1. 3220E-03

$2.8805 E-04$

$2.4768 \mathrm{E}-05$

5. $2244 \mathrm{E}-06$

2. $6250 \mathrm{E}-06$

$5.9163 \mathrm{E}-04$

1. $2760 \mathrm{E}-02$

9.8100E-03

1.8990E-02

$\begin{array}{lll}\mathrm{U}-235 & 1.0160 \mathrm{E}-05 & 7.2198 \mathrm{E}-06 \\ \mathrm{U}-238 & 4.4270 \mathrm{E}-03 & 4.2270 \mathrm{E}-03 \\ \mathrm{Pu}-239 & 1.7483 \mathrm{E}-03 & 1.3797 \mathrm{E}-03 \\ \mathrm{Pu}-240 & 2.2290 \mathrm{E}-04 & 2.8033 \mathrm{E}-04 \\ \mathrm{Pu}-241 & 1.9040 \mathrm{E}-05 & 2.3527 \mathrm{E}-05 \\ \mathrm{Pu}-242 & 4.7330 \mathrm{E}-06 & 5.1220 \mathrm{E}-06 \\ \mathrm{Am}-241 & 7.5700 \mathrm{E}-07 & 2.6343 \mathrm{E}-06 \\ \mathrm{FP} & 0.0000 \mathrm{E}+00 & 5.0599 \mathrm{E}-04 \\ \mathrm{O}-16 & 1.2760 \mathrm{E}-02 & 1.2760 \mathrm{E}-02 \\ \mathrm{Na}-23 & 9.8100 \mathrm{E}-03 & 9.8100 \mathrm{E}-03 \\ \mathrm{SS}-316 & 1.8990 \mathrm{E}-02 & 1.8990 \mathrm{E}-02\end{array}$

$\begin{array}{lll}9 & 30.76 \quad 46.14\end{array}$

$\begin{array}{lll}\mathrm{U}-235 & 1.0160 \mathrm{E}-05 & 7.9012 \mathrm{E}-06 \\ \mathrm{U}-238 & 4.4270 \mathrm{E}-03 & 4.2812 \mathrm{E}-03 \\ \mathrm{Pu}-239 & 1.7483 \mathrm{E}-03 & 1.4723 \mathrm{E}-03 \\ \mathrm{Pu}-240 & 2.2290 \mathrm{E}-04 & 2.7330 \mathrm{E}-04 \\ \mathrm{Pu}-241 & 1.9040 \mathrm{E}-05 & 2.2542 \mathrm{E}-05 \\ \mathrm{Pu}-242 & 4.7330 \mathrm{E}-06 & 5.0610 \mathrm{E}-06 \\ \mathrm{Am}-241 & 7.5700 \mathrm{E}-07 & 2.6845 \mathrm{E}-06 \\ \mathrm{FP} & 0.0000 \mathrm{E}+00 & 3.6677 \mathrm{E}-04 \\ \mathrm{O}-16 & 1.2760 \mathrm{E}-02 & 1.2760 \mathrm{E}-02 \\ \mathrm{Na}-23 & 9.8100 \mathrm{E}-03 & 9.8100 \mathrm{E}-03 \\ \mathrm{SS}-316 & 1.8990 \mathrm{E}-02 & 1.8990 \mathrm{E}-02\end{array}$

$\begin{array}{lll}10 & 46.14 & 62.78\end{array}$

$\begin{array}{lll}\text { U-235 } & 1.4143 \mathrm{E}-05 & 1.1192 \mathrm{E}-05 \\ \mathrm{U}-238 & 7.0573 \mathrm{E}-03 & 6.8922 \mathrm{E}-03 \\ \mathrm{Pu}-239 & 0.0000 \mathrm{E}+00 & 1.3937 \mathrm{E}-04 \\ \mathrm{Pu}-240 & 0.0000 \mathrm{E}+00 & 4.4735 \mathrm{E}-06 \\ \mathrm{FP} & 0.0000 \mathrm{E}+00 & 2.3461 \mathrm{E}-05 \\ 0-16 & 1.4143 \mathrm{E}-02 & 1.4143 \mathrm{E}-02 \\ \mathrm{Na}-23 & 9.7416 \mathrm{E}-03 & 9.7416 \mathrm{E}-03 \\ \mathrm{SS}-316 & 1.9073 \mathrm{E}-02 & 1.9073 \mathrm{E}-02\end{array}$

$\begin{array}{lll}11 & 62.78 & 172.84\end{array}$

$\begin{array}{lll}\mathrm{Na}-23 & 8.2400 \mathrm{E}-03 & 8.2400 \mathrm{E}-03 \\ \mathrm{SS}-316 & 2.5562 \mathrm{E}-02 & 2.5562 \mathrm{E}-02\end{array}$




\subsection{Cycle 11B-2}

Table BllB-2.1. Fission Power Generated in MFA-1, MFA-2 and Neighboring Assemblies at BOC 11B-2

\begin{tabular}{lcccc} 
CORE & $* \star * * * * *$ & \multicolumn{2}{c}{ POWER IN MEGAWATTS } & $* * * * * *$ \\
POS. & BELOW CORE & IN CORE & ABOVE CORE & TOTAL PWR \\
1405 & $8.611 \mathrm{E}-02$ & $3.818 \mathrm{E}+00$ & $4.807 \mathrm{E}-02$ & $3.952 \mathrm{E}+00$ \\
1304 & $0.000 \mathrm{E}+00$ & $7.376 \mathrm{E}+00$ & $0.000 \mathrm{E}+00$ & $7.376 \mathrm{E}+00$ \\
1406 & $0.000 \mathrm{E}+00$ & $0.000 \mathrm{E}+00$ & $0.000 \mathrm{E}+00$ & $0.000 \mathrm{E}+00$ \\
1404 & $0.000 \mathrm{E}+00$ & $6.381 \mathrm{E}+00$ & $0.000 \mathrm{E}+00$ & $6.381 \mathrm{E}+00$ \\
1508 & $0.000 \mathrm{E}+00$ & $5.184 \mathrm{E}+00$ & $0.000 \mathrm{E}+00$ & $5.184 \mathrm{E}+00$ \\
1506 & $1.198 \mathrm{E}-02$ & $3.769 \mathrm{E}+00$ & $7.625 \mathrm{E}-03$ & $3.789 \mathrm{E}+00$ \\
1507 & $1.099 \mathrm{E}-02$ & $3.237 \mathrm{E}+00$ & $7.404 \mathrm{E}-03$ & $3.256 \mathrm{E}+00$ \\
2506 & $7.776 \mathrm{E}-02$ & $3.512 \mathrm{E}+00$ & $4.595 \mathrm{E}-02$ & $3.635 \mathrm{E}+00$ \\
2404 & $0.000 \mathrm{E}+00$ & $6.547 \mathrm{E}+00$ & $0.000 \mathrm{E}+00$ & $6.547 \mathrm{E}+00$ \\
2505 & $1.479 \mathrm{E}-02$ & $3.617 \mathrm{E}+00$ & $7.584 \mathrm{E}-03$ & $3.640 \mathrm{E}+00$ \\
2405 & $9.743 \mathrm{E}-03$ & $5.080 \mathrm{E}+00$ & $7.258 \mathrm{E}-03$ & $5.097 \mathrm{E}+00$ \\
2606 & $1.351 \mathrm{E}-02$ & $3.416 \mathrm{E}+00$ & $7.226 \mathrm{E}-03$ & $3.437 \mathrm{E}+00$ \\
2607 & $1.207 \mathrm{E}-02$ & $3.053 \mathrm{E}+00$ & $7.700 \mathrm{E}-03$ & $3.072 \mathrm{E}+00$ \\
2507 & $1.137 \mathrm{E}-02$ & $3.694 \mathrm{E}+00$ & $8.269 \mathrm{E}-03$ & $3.713 \mathrm{E}+00$
\end{tabular}

Table B11B-2.2. Assembly Averaged Total and Fast Flux in MFA-1 and MFA-2 at BOC 11B-2

\begin{tabular}{|c|c|c|}
\hline & Core & $\mathrm{Fl}$ \\
\hline $\begin{array}{l}\text { sssembly } \\
\text { MFA-1 } \\
\text { MFA-2 }\end{array}$ & $\begin{array}{l}\text { Pos. } \\
1405 \\
2506\end{array}$ & $\begin{array}{c}\text { Total } \\
3.382 \mathrm{E}+1 \\
3.108 \mathrm{E}+1\end{array}$ \\
\hline
\end{tabular}


Table B11B-2.3. Axial Distribution of Total Flux, Fast Flux and Power in MFA-1 at BOC 11B-2

$\begin{array}{rccc}z(\mathrm{~cm}) & \text { Total Flux } & \text { Flux }>0.1 \text { MeV } & \text { Power } \\ -97.78 & 2.8759 \mathrm{E}-02 & 1.4378 \mathrm{E}-02 & 0.0000 \mathrm{E}+00 \\ -92.78 & 6.1805 \mathrm{E}-02 & 2.7849 \mathrm{E}-02 & 0.0000 \mathrm{E}+00 \\ -87.78 & 9.9166 \mathrm{E}-02 & 4.4689 \mathrm{E}-02 & 0.0000 \mathrm{E}+00 \\ -82.78 & 1.4377 \mathrm{E}-01 & 6.6884 \mathrm{E}-02 & 0.0000 \mathrm{E}+00 \\ -77.78 & 1.9881 \mathrm{E}-01 & 9.6932 \mathrm{E}-02 & 0.0000 \mathrm{E}+00 \\ -72.15 & 2.6200 \mathrm{E}-01 & 1.3615 \mathrm{E}-01 & 0.0000 \mathrm{E}+00 \\ -65.90 & 3.2639 \mathrm{E}-01 & 1.8663 \mathrm{E}-01 & 0.0000 \mathrm{E}+00 \\ -60.35 & 3.9385 \mathrm{E}-01 & 2.5564 \mathrm{E}-01 & 1.0401 \mathrm{E}-01 \\ -55.49 & 4.7902 \mathrm{E}-01 & 3.4419 \mathrm{E}-01 & 1.1900 \mathrm{E}-01 \\ -50.62 & 5.7502 \mathrm{E}-01 & 4.5766 \mathrm{E}-01 & 1.3848 \mathrm{E}-01 \\ -47.17 & 6.4931 \mathrm{E}-01 & 5.6211 \mathrm{E}-01 & 1.5781 \mathrm{E}-01 \\ -43.58 & 7.2638 \mathrm{E}-01 & 6.7913 \mathrm{E}-01 & 7.9699 \mathrm{E}-01 \\ -38.45 & 8.3965 \mathrm{E}-01 & 8.2107 \mathrm{E}-01 & 8.8159 \mathrm{E}-01 \\ -33.32 & 9.4483 \mathrm{E}-01 & 9.4094 \mathrm{E}-01 & 9.7925 \mathrm{E}-01 \\ -28.20 & 1.0358 \mathrm{E}+00 & 1.0388 \mathrm{E}+00 & 1.0206 \mathrm{E}+00 \\ -23.07 & 1.1107 \mathrm{E}+00 & 1.1175 \mathrm{E}+00 & 1.0922 \mathrm{E}+00 \\ -17.94 & 1.1677 \mathrm{E}+00 & 1.1763 \mathrm{E}+00 & 1.1471 \mathrm{E}+00 \\ -12.82 & 1.2058 \mathrm{E}+00 & 1.2148 \mathrm{E}+00 & 1.1579 \mathrm{E}+00 \\ -7.69 & 1.2255 \mathrm{E}+00 & 1.2348 \mathrm{E}+00 & 1.1764 \mathrm{E}+00 \\ -2.56 & 1.2270 \mathrm{E}+00 & 1.2366 \mathrm{E}+00 & 1.1776 \mathrm{E}+00 \\ 2.56 & 1.2109 \mathrm{E}+00 & 1.2211 \mathrm{E}+00 & 1.1706 \mathrm{E}+00 \\ 7.69 & 1.1781 \mathrm{E}+00 & 1.1887 \mathrm{E}+00 & 1.1389 \mathrm{E}+00 \\ 12.82 & 1.1294 \mathrm{E}+00 & 1.1405 \mathrm{E}+00 & 1.0922 \mathrm{E}+00 \\ 17.94 & 1.0663 \mathrm{E}+00 & 1.0778 \mathrm{E}+00 & 1.0684 \mathrm{E}+00 \\ 23.07 & 9.8859 \mathrm{E}-01 & 9.9888 \mathrm{E}-01 & 9.9132 \mathrm{E}-01 \\ 28.20 & 8.9758 \mathrm{E}-01 & 9.0503 \mathrm{E}-01 & 9.0140 \mathrm{E}-01 \\ 33.32 & 7.9533 \mathrm{E}-01 & 7.9742 \mathrm{E}-01 & 8.4609 \mathrm{E}-01 \\ 38.45 & 6.8326 \mathrm{E}-01 & 6.7397 \mathrm{E}-01 & 7.3344 \mathrm{E}-01 \\ 43.58 & 5.6713 \mathrm{E}-01 & 5.3680 \mathrm{E}-01 & 6.2804 \mathrm{E}-01 \\ 47.17 & 4.9231 \mathrm{E}-01 & 4.3551 \mathrm{E}-01 & 9.9219 \mathrm{E}-02 \\ 50.62 & 4.2605 \mathrm{E}-01 & 3.5110 \mathrm{E}-01 & 8.1484 \mathrm{E}-02 \\ 55.49 & 3.4576 \mathrm{E}-01 & 2.6248 \mathrm{E}-01 & 6.4358 \mathrm{E}-02 \\ 60.35 & 2.7365 \mathrm{E}-01 & 1.9181 \mathrm{E}-01 & 5.1241 \mathrm{E}-02 \\ 64.98 & 2.2342 \mathrm{E}-01 & 1.4577 \mathrm{E}-01 & 0.0000 \mathrm{E}+00 \\ 69.39 & 1.9011 \mathrm{E}-01 & 1.1851 \mathrm{E}-01 & 0.0000 \mathrm{E}+00 \\ 76.46 & 1.4212 \mathrm{E}-01 & 8.1832 \mathrm{E}-02 & 0.0000 \mathrm{E}+00 \\ 86.80 & 9.1567 \mathrm{E}-02 & 4.8087 \mathrm{E}-02 & 0.0000 \mathrm{E}+00 \\ 98.14 & 5.2687 \mathrm{E}-02 & 2.5845 \mathrm{E}-02 & 0.0000 \mathrm{E}+00 \\ 110.00 & 2.2472 \mathrm{E}-02 & 1.1398 \mathrm{E}-02 & 0.0000 \mathrm{E}+00\end{array}$


Table B11B-2.4. Axial Distribution of Total Flux, Fast Flux and Power in MFA-2 at BOC 11B-2

$\begin{array}{rccc}z(\mathrm{~cm}) & \text { Total Flux } & \text { Flux }>0.1 \text { MeV } & \text { Power } \\ -97.78 & 2.7763 \mathrm{E}-02 & 1.4142 \mathrm{E}-02 & 0.0000 \mathrm{E}+00 \\ -92.78 & 5.9557 \mathrm{E}-02 & 2.7301 \mathrm{E}-02 & 0.0000 \mathrm{E}+00 \\ -87.78 & 9.5445 \mathrm{E}-02 & 4.3686 \mathrm{E}-02 & 0.0000 \mathrm{E}+00 \\ -82.78 & 1.3827 \mathrm{E}-01 & 6.5189 \mathrm{E}-02 & 0.0000 \mathrm{E}+00 \\ -77.78 & 1.9117 \mathrm{E}-01 & 9.4200 \mathrm{E}-02 & 0.0000 \mathrm{E}+00 \\ -72.15 & 2.5227 \mathrm{E}-01 & 1.3205 \mathrm{E}-01 & 0.0000 \mathrm{E}+00 \\ -65.90 & 3.1459 \mathrm{E}-01 & 1.8085 \mathrm{E}-01 & 0.0000 \mathrm{E}+00 \\ -60.35 & 3.7962 \mathrm{E}-01 & 2.4800 \mathrm{E}-01 & 1.0175 \mathrm{E}-01 \\ -55.49 & 4.6422 \mathrm{E}-01 & 3.3549 \mathrm{E}-01 & 1.1665 \mathrm{E}-01 \\ -50.62 & 5.6116 \mathrm{E}-01 & 4.4874 \mathrm{E}-01 & 1.3608 \mathrm{E}-01 \\ -47.17 & 6.3802 \mathrm{E}-01 & 5.5465 \mathrm{E}-01 & 1.5588 \mathrm{E}-01 \\ -43.58 & 7.1869 \mathrm{E}-01 & 6.7396 \mathrm{E}-01 & 7.8858 \mathrm{E}-01 \\ -38.45 & 8.3602 \mathrm{E}-01 & 8.1870 \mathrm{E}-01 & 8.7884 \mathrm{E}-01 \\ -33.32 & 9.4401 \mathrm{E}-01 & 9.4044 \mathrm{E}-01 & 9.8086 \mathrm{E}-01 \\ -28.20 & 1.0366 \mathrm{E}+00 & 1.0393 \mathrm{E}+00 & 1.0227 \mathrm{E}+00 \\ -23.07 & 1.1126 \mathrm{E}+00 & 1.1185 \mathrm{E}+00 & 1.0957 \mathrm{E}+00 \\ -17.94 & 1.1700 \mathrm{E}+00 & 1.1774 \mathrm{E}+00 & 1.1513 \mathrm{E}+00 \\ -12.82 & 1.2080 \mathrm{E}+00 & 1.2155 \mathrm{E}+00 & 1.1600 \mathrm{E}+00 \\ -7.69 & 1.2272 \mathrm{E}+00 & 1.2349 \mathrm{E}+00 & 1.1780 \mathrm{E}+00 \\ -2.56 & 1.2277 \mathrm{E}+00 & 1.2359 \mathrm{E}+00 & 1.1783 \mathrm{E}+00 \\ 2.56 & 1.2107 \mathrm{E}+00 & 1.2197 \mathrm{E}+00 & 1.1694 \mathrm{E}+00 \\ 7.69 & 1.1770 \mathrm{E}+00 & 1.1866 \mathrm{E}+00 & 1.1369 \mathrm{E}+00 \\ 12.82 & 1.1280 \mathrm{E}+00 & 1.1384 \mathrm{E}+00 & 1.0898 \mathrm{E}+00 \\ 17.94 & 1.0649 \mathrm{E}+00 & 1.0762 \mathrm{E}+00 & 1.0658 \mathrm{E}+00 \\ 23.07 & 9.8773 \mathrm{E}-01 & 9.9830 \mathrm{E}-01 & 9.8924 \mathrm{E}-01 \\ 28.20 & 8.9760 \mathrm{E}-01 & 9.0573 \mathrm{E}-01 & 9.0015 \mathrm{E}-01 \\ 33.32 & 7.9657 \mathrm{E}-01 & 7.9993 \mathrm{E}-01 & 8.4640 \mathrm{E}-01 \\ 38.45 & 6.8583 \mathrm{E}-01 & 6.7829 \mathrm{E}-01 & 7.3528 \mathrm{E}-01 \\ 43.58 & 5.7084 \mathrm{E}-01 & 5.4235 \mathrm{E}-01 & 6.3283 \mathrm{E}-01 \\ 47.17 & 4.9499 \mathrm{E}-01 & 4.3880 \mathrm{E}-01 & 1.0377 \mathrm{E}-01 \\ 50.62 & 4.2480 \mathrm{E}-01 & 3.4920 \mathrm{E}-01 & 8.5116 \mathrm{E}-02 \\ 55.49 & 3.3790 \mathrm{E}-01 & 2.5461 \mathrm{E}-01 & 6.6803 \mathrm{E}-02 \\ 60.35 & 2.6067 \mathrm{E}-01 & 1.8064 \mathrm{E}-01 & 5.2621 \mathrm{E}-02 \\ 64.98 & 2.1042 \mathrm{E}-01 & 1.3539 \mathrm{E}-01 & 0.0000 \mathrm{E}+00 \\ 69.39 & 1.7962 \mathrm{E}-01 & 1.1069 \mathrm{E}-01 & 0.0000 \mathrm{E}+00 \\ 76.46 & 1.3558 \mathrm{E}-01 & 7.7655 \mathrm{E}-02 & 0.0000 \mathrm{E}+00 \\ 86.80 & 8.7838 \mathrm{E}-02 & 4.6195 \mathrm{E}-02 & 0.0000 \mathrm{E}+00 \\ 98.14 & 5.0651 \mathrm{E}-02 & 2.5004 \mathrm{E}-02 & 0.0000 \mathrm{E}+00 \\ 110.00 & 2.1754 \mathrm{E}-02 & 1.1130 \mathrm{E}-02 & 0.0000 \mathrm{E}+00\end{array}$


HNF-SD-FF-ANAL-009 Rev. 1

Tabie B11B-2.5. Fission Power Distribution by Pin in MFA-1 at BOC $11 B-2$ CORE POSIIION 1405

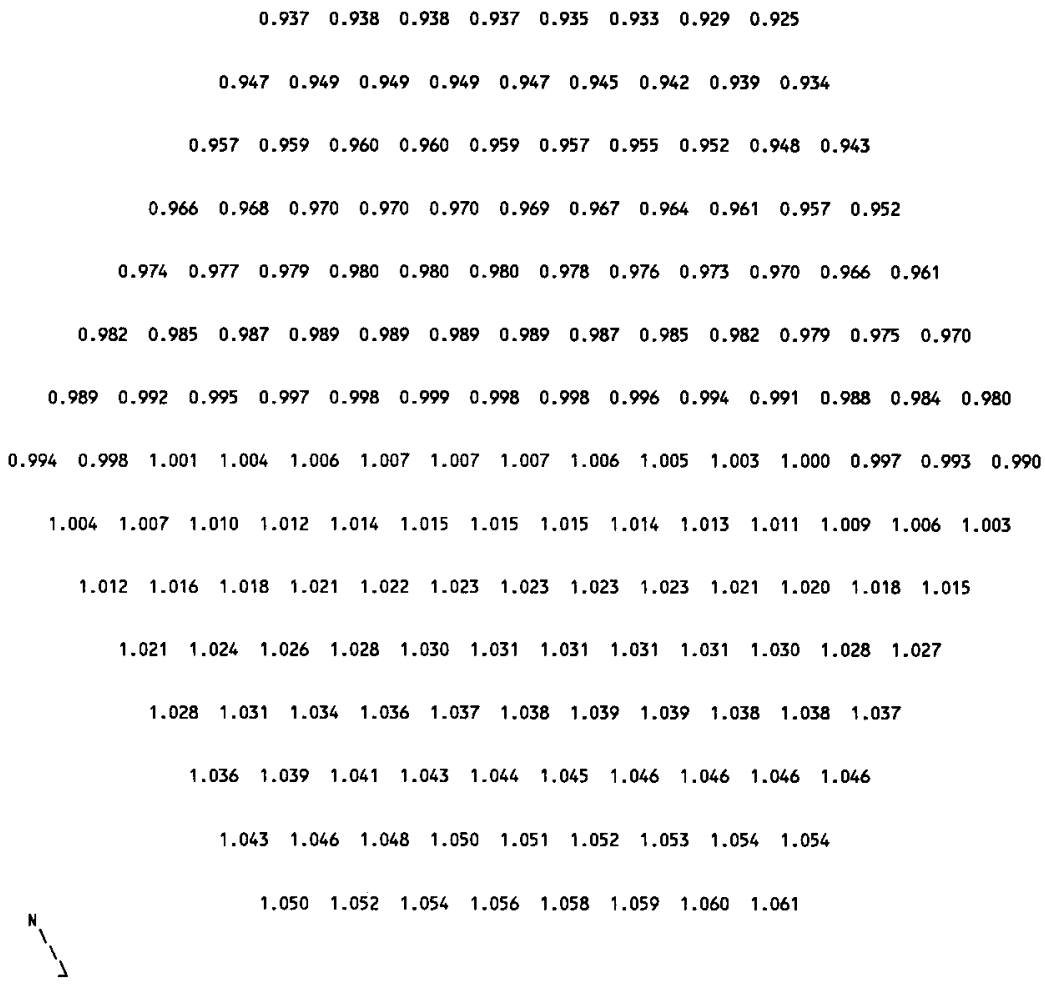


HNF-SD-FF-ANAL-009 Rev. 1

Table B11B-2.6. Fission Power Distribution by Pin in MFA-2 at BOC $11 \mathrm{~B}-2$ CORE POSITION 2506

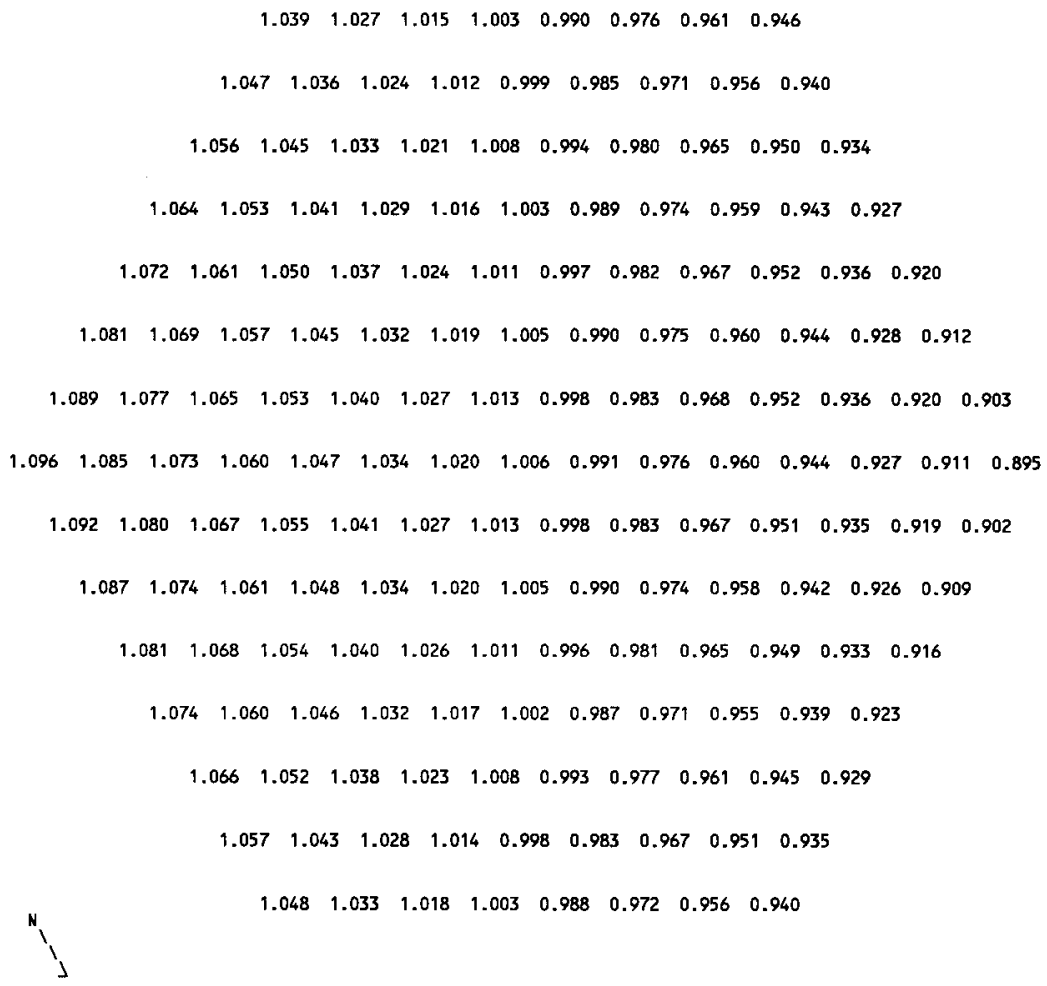


Table B11B-2.7. Fast Flux Distribution by Pin in MFA-1 at BOC 11B-2 CORE POSITION 1404

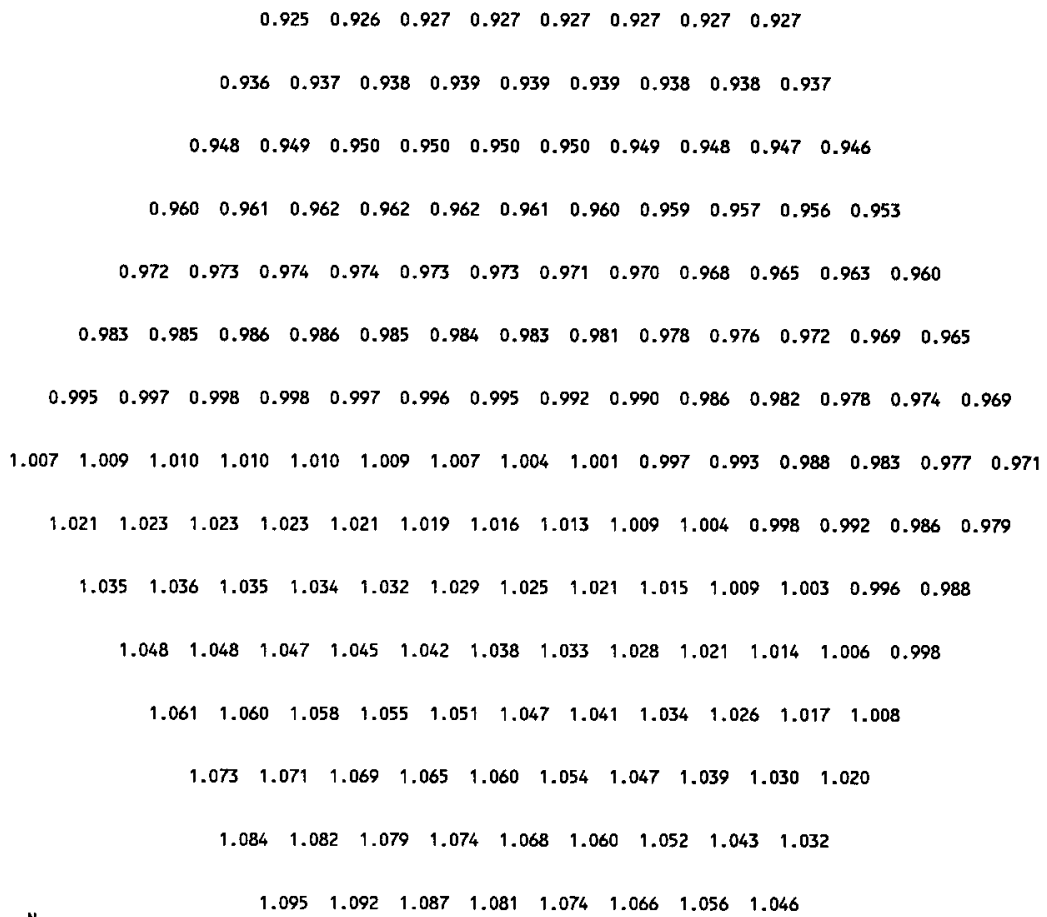


HNF-SD-FF-ANAL-009 Rev. 1

Table Bl1B-2.8. Fast Flux Distribution by Pin in MFA-2 at BOC 11B-2

CORE POSITION 2506

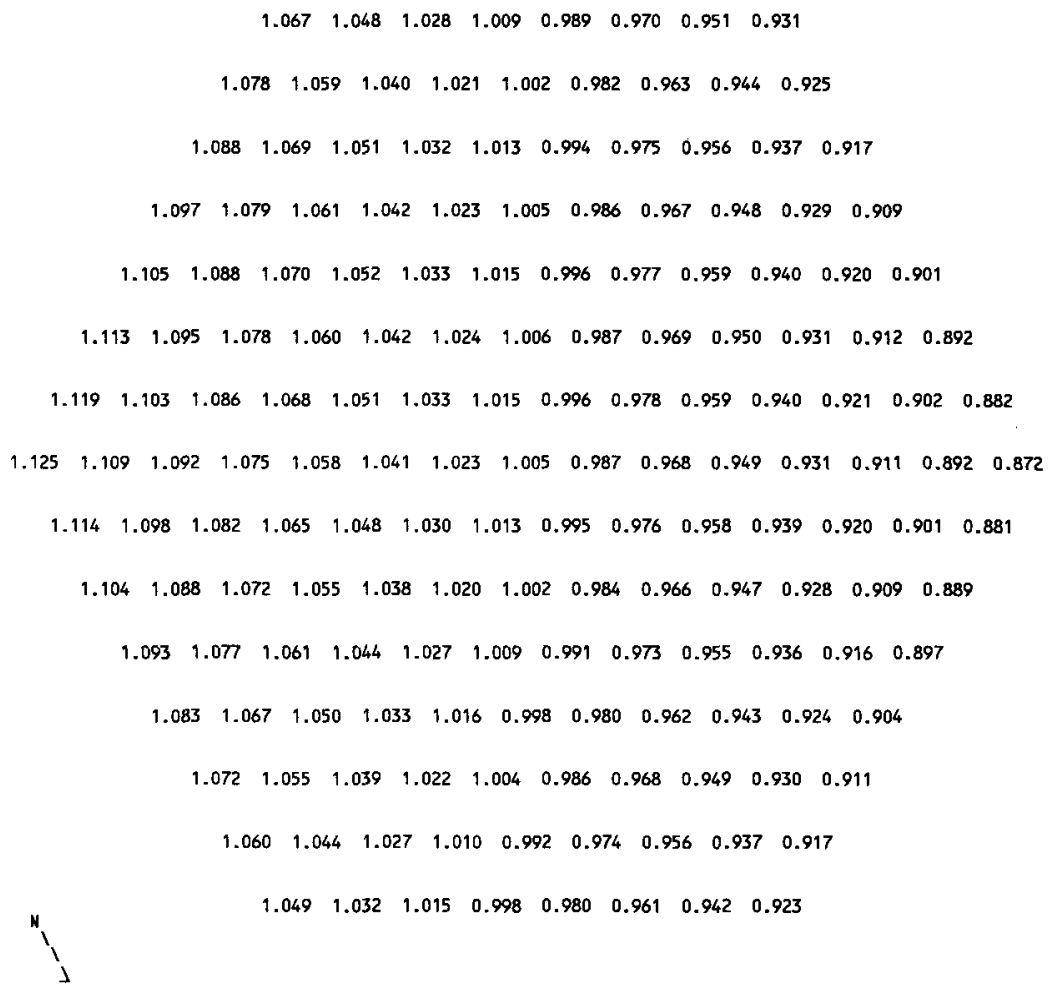


Table B11B-2.9. MFA-1 and MFA-2 Duct Wall

Fast Flux Data at BOC $11 \mathrm{~B}-2$

$\begin{array}{ccc}\text { Side } & \text { Flux }>0.1 \mathrm{MeV} & \begin{array}{c}\text { MFA-1 } \\ \left(\mathrm{n} / \mathrm{cm}^{2}-\mathrm{sec}\right) \\ \text { MFA-2 }\end{array} \\ \text { E } & 2.3793 \mathrm{E}+15 & 2.2245 \mathrm{E}+15 \\ \text { SE } & 2.1408 \mathrm{E}+15 & 2.2631 \mathrm{E}+15 \\ \text { SW } & 2.0410 \mathrm{E}+15 & 2.0069 \mathrm{E}+15 \\ \text { W } & 2.1304 \mathrm{E}+15 & 1.7854 \mathrm{E}+15 \\ \text { NW } & 2.2338 \mathrm{E}+15 & 1.7720 \mathrm{E}+15 \\ \text { NE } & 2.4536 \mathrm{E}+15 & 1.9867 \mathrm{E}+15\end{array}$

Table B11B-2.10. Assembly Outlet Temperatures and Flow Rates at BOC 11B-2

$\begin{array}{lcl} & \text { CALCULATED } \\ \text { CORE } & \text { OUTLET TEMP. } & \text { FLOW RATE } \\ \text { POS. } & \text { (DEG F) } & (\text { LB } / H) \\ 1405 & 1058 & 111160 \\ 1304 & 1078 & 194240 \\ 1404 & 1020 & 196630 \\ 1406 & 860 & 14170 \\ 1506 & 888 & 201290 \\ 1507 & 869 & 191410 \\ 1508 & 978 & 181190 \\ 2506 & 1031 & 111160 \\ 2404 & 1029 & 197960 \\ 2405 & 1010 & 165960 \\ 2505 & 877 & 201290 \\ 2507 & 885 & 201290 \\ 2606 & 906 & 165070 \\ 2607 & 888 & 165070\end{array}$

Assembly flows are based on a calculated core pressure drop of $103.4 \mathrm{psi}$ at a total reactor flow rate of 16. $74 \mathrm{E}+06 \mathrm{~Tb} / \mathrm{hr}$. 
Table B11B-2.11. MFA-1 Sodium Subchannel Temperatures at Top of Core Elevation for BOC 11B-2

$$
z^{\cdot \cdot \cdot \cdot}
$$

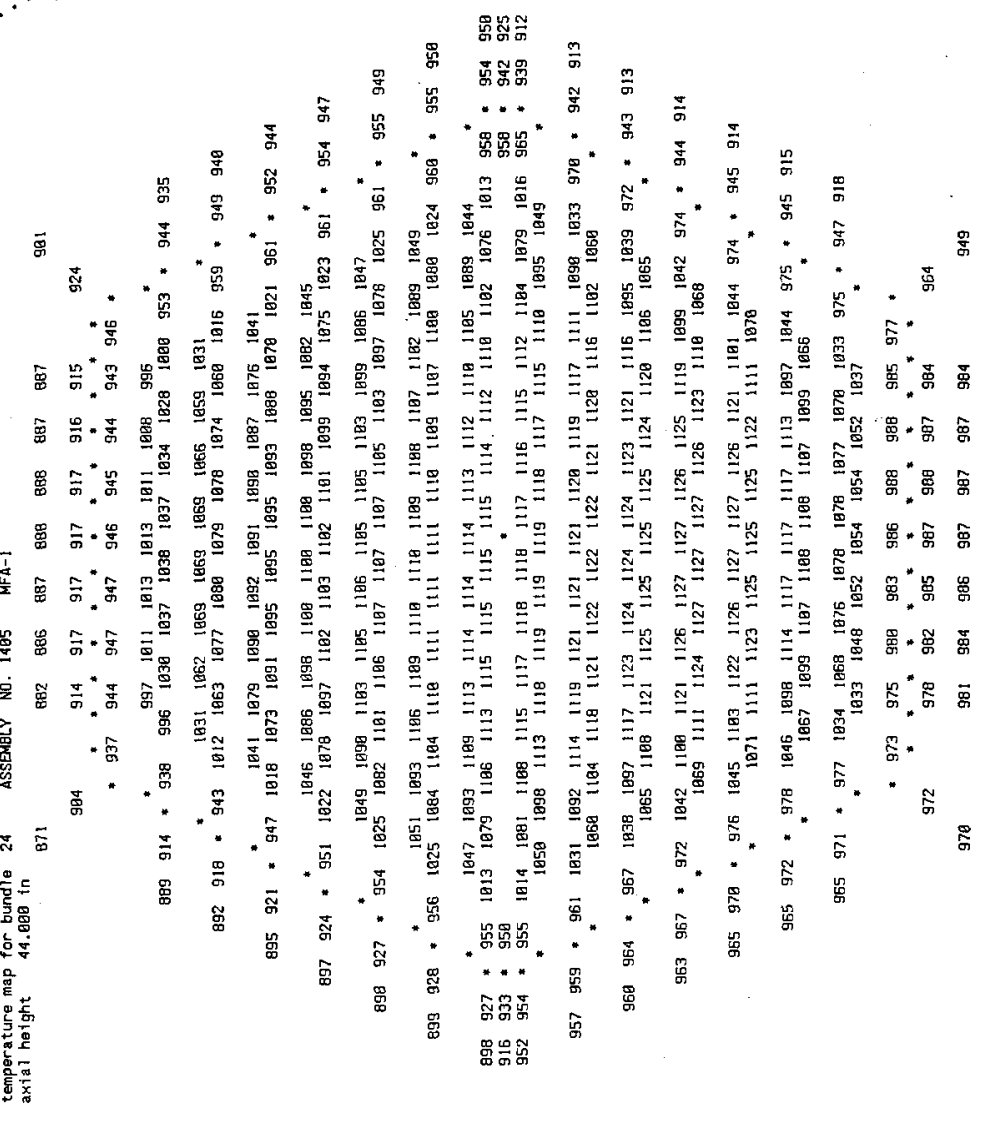


Table B11B-2.12. MFA-2 Sodium Subchannel Temperatures at Top of Core Elevation for BOC 11B-2

$$
\begin{aligned}
& z \cdot \cdot
\end{aligned}
$$

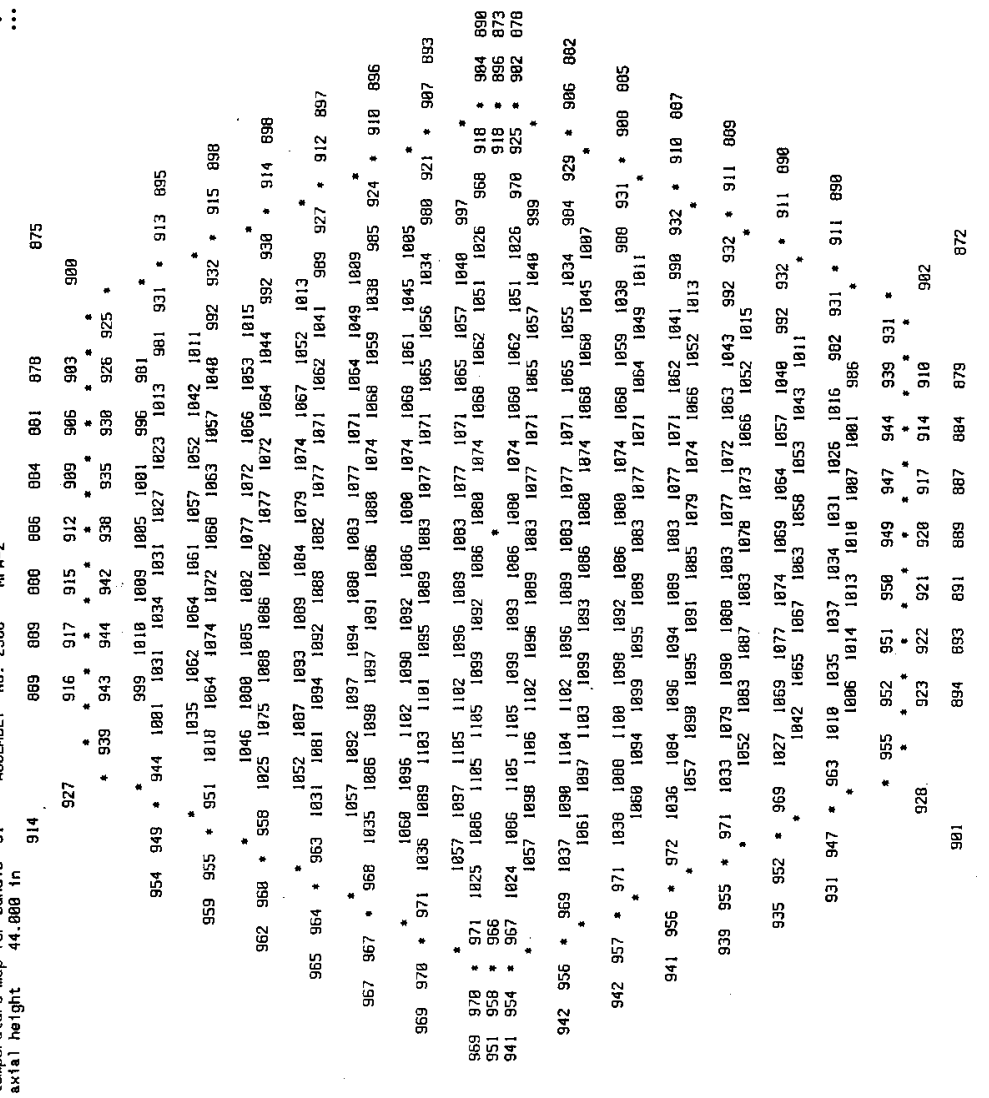


temperature map for bundle 24 ASSEMBLY NO. I485 MFA-1

axial height 58.588 in

883

$\begin{array}{lllllll}893 & 896 & 898 & 899 & 908 & 988 & 900\end{array}$

914

$\begin{array}{lllllllll}918 & 926 & 930 & 931 & 931 & 938 & 930 & 930 & 940\end{array}$

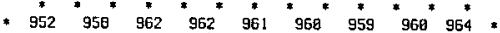

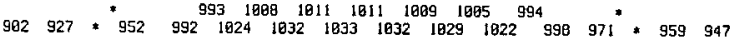

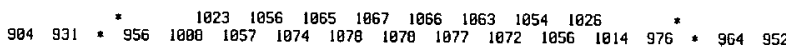

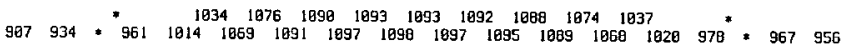

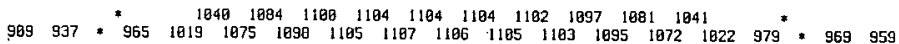

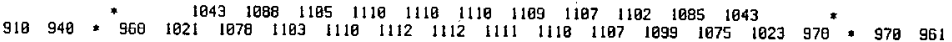

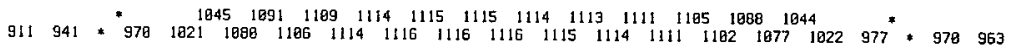

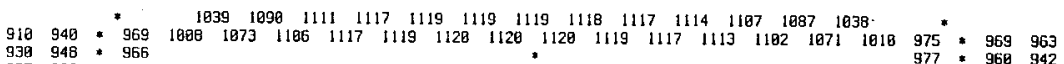

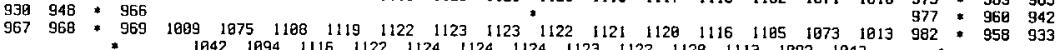

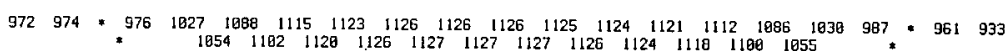

$\begin{array}{cccccccccccccccccccccccc}976 & 979 & * & 982 & 1034 & 1094 & 1119 & 1127 & 1129 & 1129 & 1129 & 1128 & 1126 & 1117 & 1892 & 1037 & 998 & * & 962 & 939\end{array}$

$979 \quad 983 * 987 \quad 1839 \quad 1097 \quad 1122 \quad 1129 \quad 1131 \quad 131 \quad 1131 \quad 1129 \quad 1121 \quad 1096 \quad 1040 \quad 991 \quad * \quad 963 \quad 934$

$980^{*}, 986 * 992 \quad 1043 \quad 1099 \quad 1122 \quad 1128 \quad 1130 \quad 1130 \quad 1128 \quad 1121 \quad 1997 \quad 1841 \quad 992 \quad * 963934$

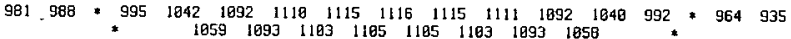

$981987 * \begin{array}{cccccccccccccc}994 & 1030 & 1060 & 1070 & 1073 \quad 1873 \quad 1872 & 10663 & 1030 & 993 & * & 966 & 939\end{array}$

* 991 $992996 \quad 1801,1904 \quad 100610061003 \quad 997 *$

989 * * * * * * * * * * * * * * * *

$\begin{array}{lllllllll}997 & 997 & 1091 & 1003 & 1004 & 1084 & 1803 & 1081 & 978\end{array}$ 
Table Bl1B-2.14. MFA-2 Sodium Subchannel Temperatures at Elevation of Upper Axial Blanket for BOC 11B-2

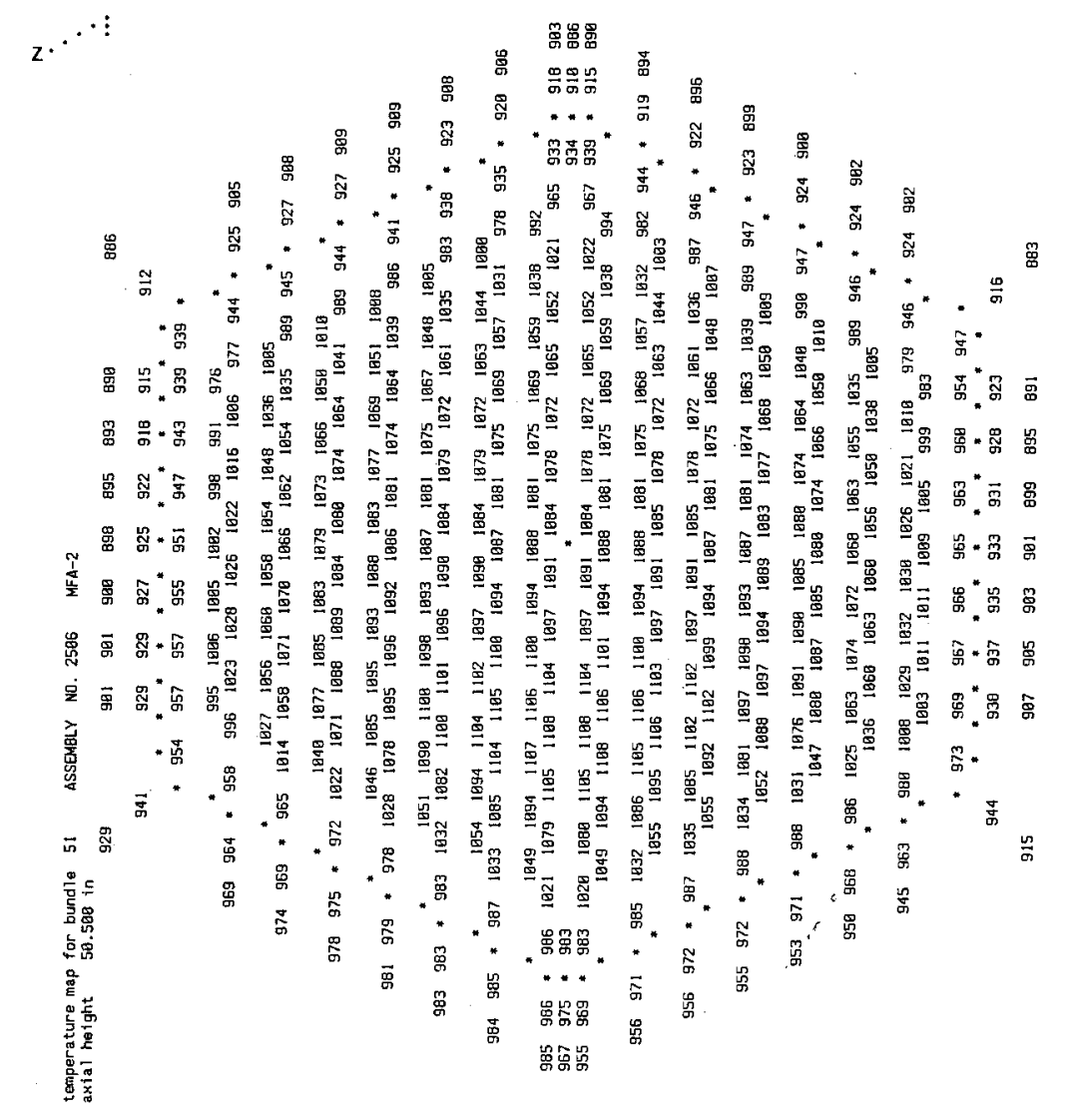


Table B11B-2.15. MFA-1 Sodium Subchannel Temperatures at Top of Fuel Pin Bundle for BOC 11B-2

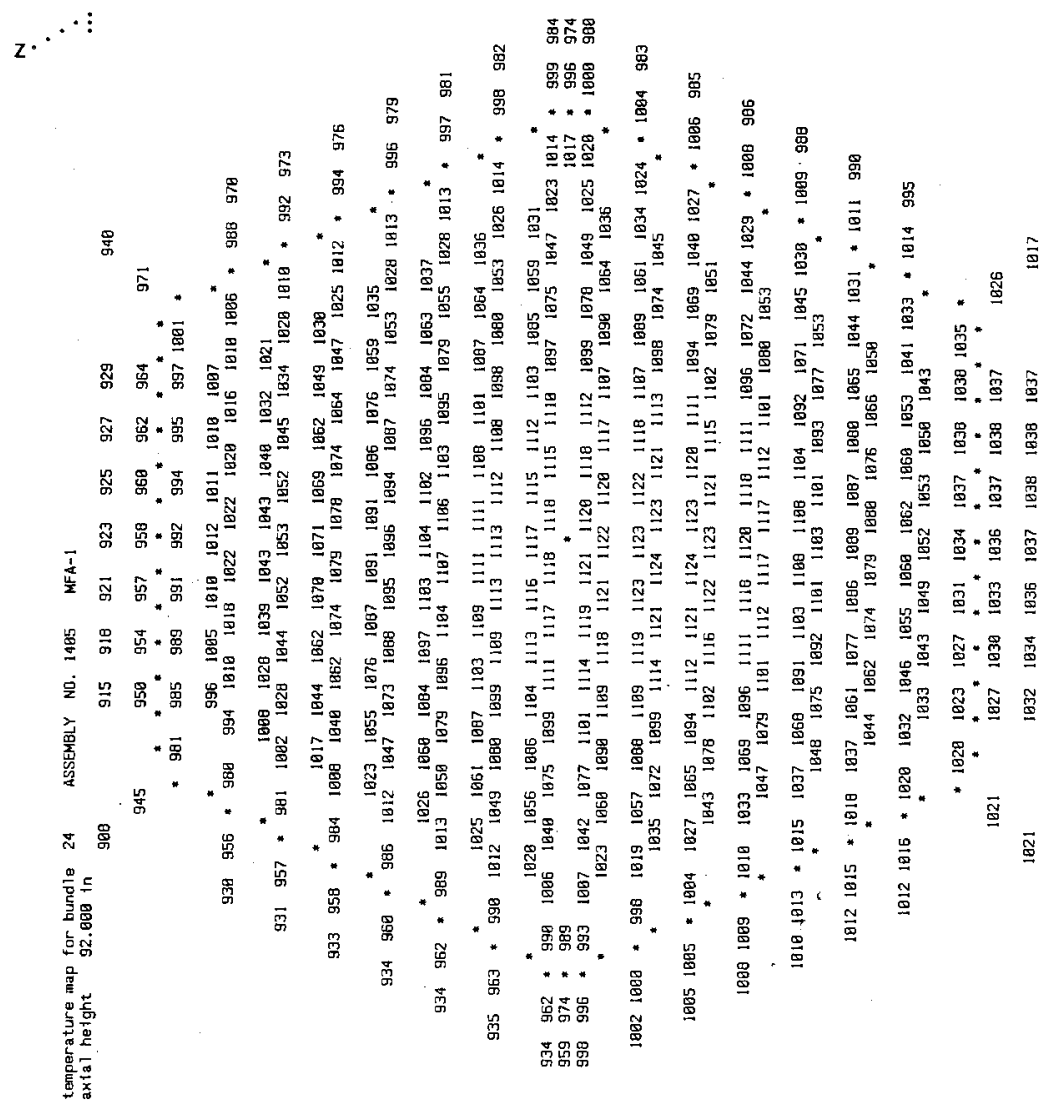


Table B11B-2.16. MFA-2 Sodium Subchannel Temperatures at Top of Fuel Pin Bundle for BOC 11B-2

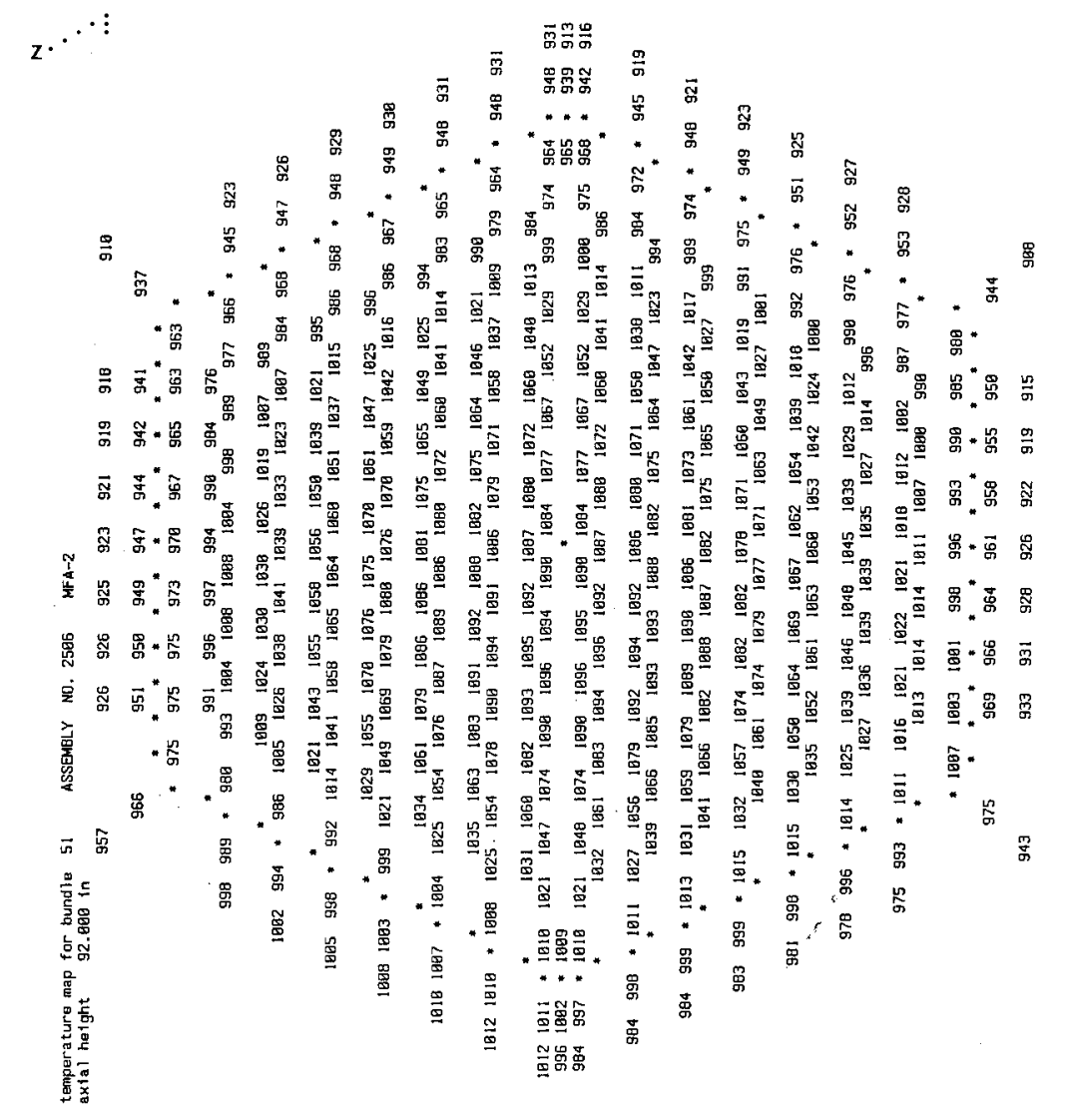


Table E11B-2.1. Fission Power Generated in MFA-1, MFA-2 and Neighboring Assemblies at EOC 11B-2

\begin{tabular}{lcccc} 
CORE & $* * * * * *$ & \multicolumn{2}{c}{ POWER IN MEGAWATTS } & $* * * * * *$ \\
POS. & BELOW CORE & IN CORE & ABOVE CORE & TOTAL PWR \\
1405 & $9.229 \mathrm{E}-02$ & $3.655 \mathrm{E}+00$ & $6.099 \mathrm{E}-02$ & $3.808 \mathrm{E}+00$ \\
1304 & $0.000 \mathrm{E}+00$ & $6.926 \mathrm{E}+00$ & $0.000 \mathrm{E}+00$ & $6.926 \mathrm{E}+00$ \\
1406 & $0.000 \mathrm{E}+00$ & $0.000 \mathrm{E}+00$ & $0.000 \mathrm{E}+00$ & $0.000 \mathrm{E}+00$ \\
1404 & $0.000 \mathrm{E}+00$ & $6.129 \mathrm{E}+00$ & $0.000 \mathrm{E}+00$ & $6.129 \mathrm{E}+00$ \\
1508 & $0.000 \mathrm{E}+00$ & $5.040 \mathrm{E}+00$ & $0.000 \mathrm{E}+00$ & $5.040 \mathrm{E}+00$ \\
1506 & $1.238 \mathrm{E}-02$ & $3.678 \mathrm{E}+00$ & $9.470 \mathrm{E}-03$ & $3.700 \mathrm{E}+00$ \\
1507 & $1.127 \mathrm{E}-02$ & $3.145 \mathrm{E}+00$ & $8.980 \mathrm{E}-03$ & $3.165 \mathrm{E}+00$ \\
2506 & $8.217 \mathrm{E}-02$ & $3.367 \mathrm{E}+00$ & $5.800 \mathrm{E}-02$ & $3.507 \mathrm{E}+00$ \\
2404 & $0.000 \mathrm{E}+00$ & $6.206 \mathrm{E}+00$ & $0.000 \mathrm{E}+00$ & $6.206 \mathrm{E}+00$ \\
2505 & $1.507 \mathrm{E}-02$ & $3.648 \mathrm{E}+00$ & $9.882 \mathrm{E}-03$ & $3.673 \mathrm{E}+00$ \\
2405 & $1.110 \mathrm{E}-02$ & $4.781 \mathrm{E}+00$ & $9.380 \mathrm{E}-03$ & $4.802 \mathrm{E}+00$ \\
2606 & $1.349 \mathrm{E}-02$ & $3.396 \mathrm{E}+00$ & $9.118 \mathrm{E}-03$ & $3.419 \mathrm{E}+00$ \\
2607 & $1.202 \mathrm{E}-02$ & $2.956 \mathrm{E}+00$ & $9.207 \mathrm{E}-03$ & $2.977 \mathrm{E}+00$ \\
2507 & $1.165 \mathrm{E}-02$ & $3.518 \mathrm{E}+00$ & $9.921 \mathrm{E}-03$ & $3.540 \mathrm{E}+00$
\end{tabular}

Table E11B-2.2. Assembly Averaged Total and Fast Flux in MFA-1 and MFA-2 at EOC $11 \mathrm{~B}-2$

\begin{tabular}{|c|c|c|c|}
\hline & Core & Flux & $\left.m^{2}-s e c\right)$ \\
\hline $\begin{array}{l}\text { Assembly } \\
\text { MFA-1 } \\
\text { MFA-2 }\end{array}$ & $\begin{array}{l}\text { Pos. } \\
1405 \\
2506\end{array}$ & $\begin{array}{c}\text { Total } \\
3.398 \mathrm{E}+15 \\
3.116 \mathrm{E}+15\end{array}$ & $\begin{array}{l}>0.1 \mathrm{MeV} \\
2.237 \mathrm{E}+15 \\
2.002 \mathrm{E}+15\end{array}$ \\
\hline
\end{tabular}


Table E11B-2.3. Axial Distribution of Total Flux, Fast Flux and Power in MFA-1 at EOC 11B-2

$\begin{array}{rccc}\text { Z (cm) } & \text { Total Flux } & \text { Flux }>0.1 \text { MeV } & \text { Power } \\ -97.78 & 2.6781 \mathrm{E}-02 & 1.3523 \mathrm{E}-02 & 0.0000 \mathrm{E}+00 \\ -92.78 & 5.7582 \mathrm{E}-02 & 2.6196 \mathrm{E}-02 & 0.0000 \mathrm{E}+00 \\ -87.78 & 9.2424 \mathrm{E}-02 & 4.2041 \mathrm{E}-02 & 0.0000 \mathrm{E}+00 \\ -82.78 & 1.3407 \mathrm{E}-01 & 6.2927 \mathrm{E}-02 & 0.0000 \mathrm{E}+00 \\ -77.78 & 1.8548 \mathrm{E}-01 & 9.1211 \mathrm{E}-02 & 0.0000 \mathrm{E}+00 \\ -72.15 & 2.4455 \mathrm{E}-01 & 1.2814 \mathrm{E}-01 & 0.0000 \mathrm{E}+00 \\ -65.90 & 3.0467 \mathrm{E}-01 & 1.7574 \mathrm{E}-01 & 0.0000 \mathrm{E}+00 \\ -60.35 & 3.6746 \mathrm{E}-01 & 2.4107 \mathrm{E}-01 & 1.1865 \mathrm{E}-01 \\ -55.49 & 4.4700 \mathrm{E}-01 & 3.2408 \mathrm{E}-01 & 1.3423 \mathrm{E}-01 \\ -50.62 & 5.3672 \mathrm{E}-01 & 4.2988 \mathrm{E}-01 & 1.5372 \mathrm{E}-01 \\ -47.17 & 6.0608 \mathrm{E}-01 & 5.2691 \mathrm{E}-01 & 1.7212 \mathrm{E}-01 \\ -43.58 & 6.7809 \mathrm{E}-01 & 6.3563 \mathrm{E}-01 & 7.4882 \mathrm{E}-01 \\ -38.45 & 7.8545 \mathrm{E}-01 & 7.6943 \mathrm{E}-01 & 8.3026 \mathrm{E}-01 \\ -33.32 & 8.8697 \mathrm{E}-01 & 8.8429 \mathrm{E}-01 & 9.2545 \mathrm{E}-01 \\ -28.20 & 9.7712 \mathrm{E}-01 & 9.8029 \mathrm{E}-01 & 9.5919 \mathrm{E}-01 \\ -23.07 & 1.0546 \mathrm{E}+00 & 1.0608 \mathrm{E}+00 & 1.0331 \mathrm{E}+00 \\ -17.94 & 1.1176 \mathrm{E}+00 & 1.1251 \mathrm{E}+00 & 1.0937 \mathrm{E}+00 \\ -12.82 & 1.1651 \mathrm{E}+00 & 1.1726 \mathrm{E}+00 & 1.1090 \mathrm{E}+00 \\ -7.69 & 1.1972 \mathrm{E}+00 & 1.2048 \mathrm{E}+00 & 1.1392 \mathrm{E}+00 \\ -2.56 & 1.2135 \mathrm{E}+00 & 1.2215 \mathrm{E}+00 & 1.1546 \mathrm{E}+00 \\ 2.56 & 1.2136 \mathrm{E}+00 & 1.2224 \mathrm{E}+00 & 1.1647 \mathrm{E}+00 \\ 7.69 & 1.1969 \mathrm{E}+00 & 1.2065 \mathrm{E}+00 & 1.1489 \mathrm{E}+00 \\ 12.82 & 1.1633 \mathrm{E}+00 & 1.1738 \mathrm{E}+00 & 1.1172 \mathrm{E}+00 \\ 17.94 & 1.1132 \mathrm{E}+00 & 1.1249 \mathrm{E}+00 & 1.1163 \mathrm{E}+00 \\ 23.07 & 1.0452 \mathrm{E}+00 & 1.0562 \mathrm{E}+00 & 1.0491 \mathrm{E}+00 \\ 28.20 & 9.6026 \mathrm{E}-01 & 9.6868 \mathrm{E}-01 & 9.6548 \mathrm{E}-01 \\ 33.32 & 8.6025 \mathrm{E}-01 & 8.6340 \mathrm{E}-01 & 9.2831 \mathrm{E}-01 \\ 38.45 & 7.4638 \mathrm{E}-01 & 7.3711 \mathrm{E}-01 & 8.1309 \mathrm{E}-01 \\ 43.58 & 6.2533 \mathrm{E}-01 & 5.9244 \mathrm{E}-01 & 7.0350 \mathrm{E}-01 \\ 47.17 & 5.4622 \mathrm{E}-01 & 4.8359 \mathrm{E}-01 & 1.2672 \mathrm{E}-01 \\ 50.62 & 4.7555 \mathrm{E}-01 & 3.9245 \mathrm{E}-01 & 1.0665 \mathrm{E}-01 \\ 55.49 & 3.8825 \mathrm{E}-01 & 2.9541 \mathrm{E}-01 & 8.6388 \mathrm{E}-02 \\ 60.35 & 3.0880 \mathrm{E}-01 & 2.1710 \mathrm{E}-01 & 7.0190 \mathrm{E}-02 \\ 64.98 & 2.5315 \mathrm{E}-01 & 1.6552 \mathrm{E}-01 & 0.0000 \mathrm{E}+00 \\ 69.39 & 2.1587 \mathrm{E}-01 & 1.3485 \mathrm{E}-01 & 0.0000 \mathrm{E}+00 \\ 76.46 & 1.6175 \mathrm{E}-01 & 9.3410 \mathrm{E}-02 & 0.0000 \mathrm{E}+00 \\ 86.80 & 1.0412 \mathrm{E}-01 & 5.4978 \mathrm{E}-02 & 0.0000 \mathrm{E}+00 \\ 98.14 & 5.9538 \mathrm{E}-02 & 2.9483 \mathrm{E}-02 & 0.0000 \mathrm{E}+00 \\ 110.00 & 2.5210 \mathrm{E}-02 & 1.2942 \mathrm{E}-02 & 0.0000 \mathrm{E}+00\end{array}$


Table E11B-2.4. Axial Distribution of Total Flux, Fast Flux and Power in MFA-2 at EOC 11B-2

$\begin{array}{rccc}\text { z }(\mathrm{cm}) & \text { Total Flux } & \text { Flux }>0.1 \text { MeV } & \text { Power } \\ -97.78 & 2.5769 \mathrm{E}-02 & 1.3247 \mathrm{E}-02 & 0.0000 \mathrm{E}+00 \\ -92.78 & 5.5307 \mathrm{E}-02 & 2.5576 \mathrm{E}-02 & 0.0000 \mathrm{E}+00 \\ -87.78 & 8.8670 \mathrm{E}-02 & 4.0929 \mathrm{E}-02 & 0.0000 \mathrm{E}+00 \\ -82.78 & 1.2852 \mathrm{E}-01 & 6.1083 \mathrm{E}-02 & 0.0000 \mathrm{E}+00 \\ -77.78 & 1.7777 \mathrm{E}-01 & 8.8273 \mathrm{E}-02 & 0.0000 \mathrm{E}+00 \\ -72.15 & 2.3470 \mathrm{E}-01 & 1.2377 \mathrm{E}-01 & 0.0000 \mathrm{E}+00 \\ -65.90 & 2.9264 \mathrm{E}-01 & 1.6959 \mathrm{E}-01 & 0.0000 \mathrm{E}+00 \\ -60.35 & 3.5287 \mathrm{E}-01 & 2.3291 \mathrm{E}-01 & 1.1431 \mathrm{E}-01 \\ -55.49 & 4.3150 \mathrm{E}-01 & 3.1460 \mathrm{E}-01 & 1.2957 \mathrm{E}-01 \\ -50.62 & 5.2158 \mathrm{E}-01 & 4.1978 \mathrm{E}-01 & 1.4870 \mathrm{E}-01 \\ -47.17 & 5.9292 \mathrm{E}-01 & 5.1776 \mathrm{E}-01 & 1.6738 \mathrm{E}-01 \\ -43.58 & 6.6785 \mathrm{E}-01 & 6.2808 \mathrm{E}-01 & 7.3710 \mathrm{E}-01 \\ -38.45 & 7.7840 \mathrm{E}-01 & 7.6371 \mathrm{E}-01 & 8.2333 \mathrm{E}-01 \\ -33.32 & 8.8197 \mathrm{E}-01 & 8.7974 \mathrm{E}-01 & 9.2213 \mathrm{E}-01 \\ -28.20 & 9.7329 \mathrm{E}-01 & 9.7620 \mathrm{E}-01 & 9.5667 \mathrm{E}-01 \\ -23.07 & 1.0516 \mathrm{E}+00 & 1.0570 \mathrm{E}+00 & 1.0318 \mathrm{E}+00 \\ -17.94 & 1.1151 \mathrm{E}+00 & 1.1215 \mathrm{E}+00 & 1.0932 \mathrm{E}+00 \\ -12.82 & 1.1629 \mathrm{E}+00 & 1.1690 \mathrm{E}+00 & 1.1075 \mathrm{E}+00 \\ -7.69 & 1.1955 \mathrm{E}+00 & 1.2017 \mathrm{E}+00 & 1.1382 \mathrm{E}+00 \\ -2.56 & 1.2124 \mathrm{E}+00 & 1.2190 \mathrm{E}+00 & 1.1542 \mathrm{E}+00 \\ 2.56 & 1.2132 \mathrm{E}+00 & 1.2207 \mathrm{E}+00 & 1.1641 \mathrm{E}+00 \\ 7.69 & 1.1975 \mathrm{E}+00 & 1.2059 \mathrm{E}+00 & 1.1492 \mathrm{E}+00 \\ 12.82 & 1.1650 \mathrm{E}+00 & 1.1747 \mathrm{E}+00 & 1.1185 \mathrm{E}+00 \\ 17.94 & 1.1161 \mathrm{E}+00 & 1.1275 \mathrm{E}+00 & 1.1180 \mathrm{E}+00 \\ 23.07 & 1.0494 \mathrm{E}+00 & 1.0605 \mathrm{E}+00 & 1.0520 \mathrm{E}+00 \\ 28.20 & 9.6574 \mathrm{E}-01 & 9.7473 \mathrm{E}-01 & 9.6952 \mathrm{E}-01 \\ 33.32 & 8.6697 \mathrm{E}-01 & 8.7133 \mathrm{E}-01 & 9.3331 \mathrm{E}-01 \\ 38.45 & 7.5399 \mathrm{E}-01 & 7.4641 \mathrm{E}-01 & 8.1925 \mathrm{E}-01 \\ 43.58 & 6.3311 \mathrm{E}-01 & 6.0218 \mathrm{E}-01 & 7.1191 \mathrm{E}-01 \\ 47.17 & 5.5196 \mathrm{E}-01 & 4.8993 \mathrm{E}-01 & 1.3183 \mathrm{E}-01 \\ 50.62 & 4.7624 \mathrm{E}-01 & 3.9230 \mathrm{E}-01 & 1.1075 \mathrm{E}-01 \\ 55.49 & 3.8085 \mathrm{E}-01 & 2.8788 \mathrm{E}-01 & 8.9068 \mathrm{E}-02 \\ 60.35 & 2.9517 \mathrm{E}-01 & 2.0532 \mathrm{E}-01 & 7.1497 \mathrm{E}-02 \\ 64.98 & 2.3924 \mathrm{E}-01 & 1.5431 \mathrm{E}-01 & 0.0000 \mathrm{E}+00 \\ 69.39 & 2.0459 \mathrm{E}-01 & 1.2637 \mathrm{E}-01 & 0.0000 \mathrm{E}+00 \\ 76.46 & 1.5463 \mathrm{E}-01 & 8.8834 \mathrm{E}-02 & 0.0000 \mathrm{E}+00 \\ 86.80 & 9.9902 \mathrm{E}-02 & 5.2842 \mathrm{E}-02 & 0.0000 \mathrm{E}+00 \\ 98.14 & 5.7002 \mathrm{E}-02 & 2.8460 \mathrm{E}-02 & 0.0000 \mathrm{E}+00 \\ 110.00 & 2.4224 \mathrm{E}-02 & 1.2571 \mathrm{E}-02 & 0.0000 \mathrm{E}+00 \\ & & & \end{array}$


HNF-SD-FF-ANAL-009 Rev. 1

Table El1B-2.5. Fission Power Distribution by Pin in MFA-1 at EOC 11B-2 CORE POSITION 1405

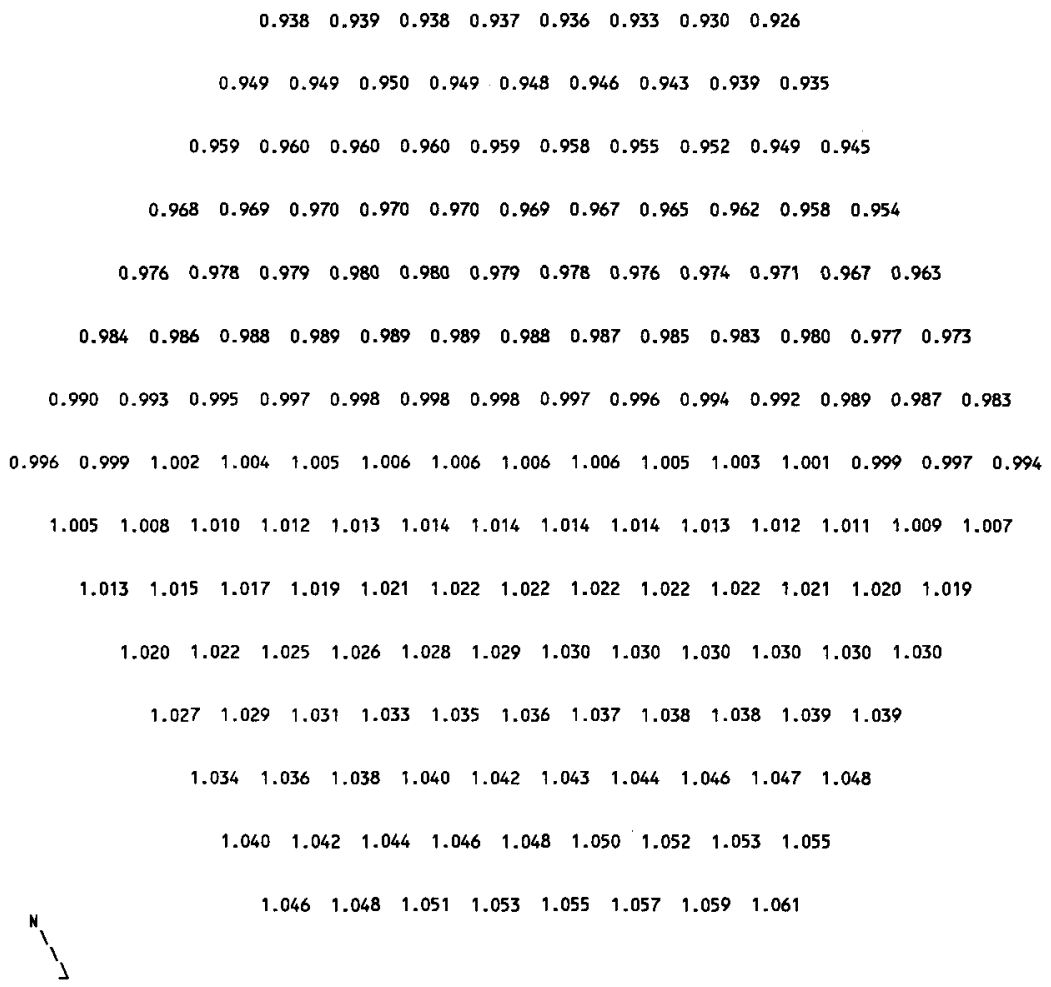


HNF-SD-FF-ANAL-009 Rev, 1

Table EllB-2.6. Fission Power Distribution by Pin in MFA-2 at EOC 11B-2 CORE POSITION 2506

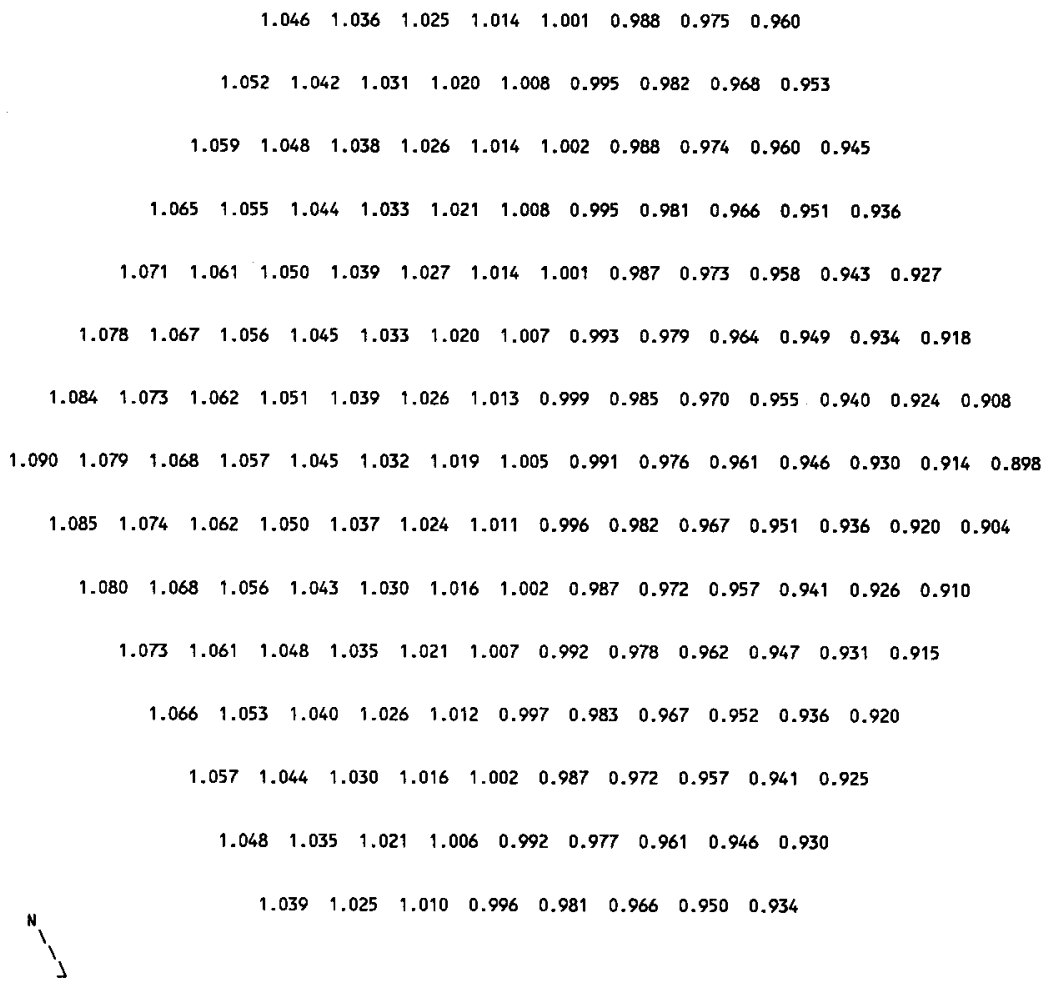


HNF-SD-FF-ANAL-009 Rev. 1

Table E11B-2.7. Fast Flux Distribution by Pin in MFA-1 at EOC 11B-2 CORE POSITION 1404

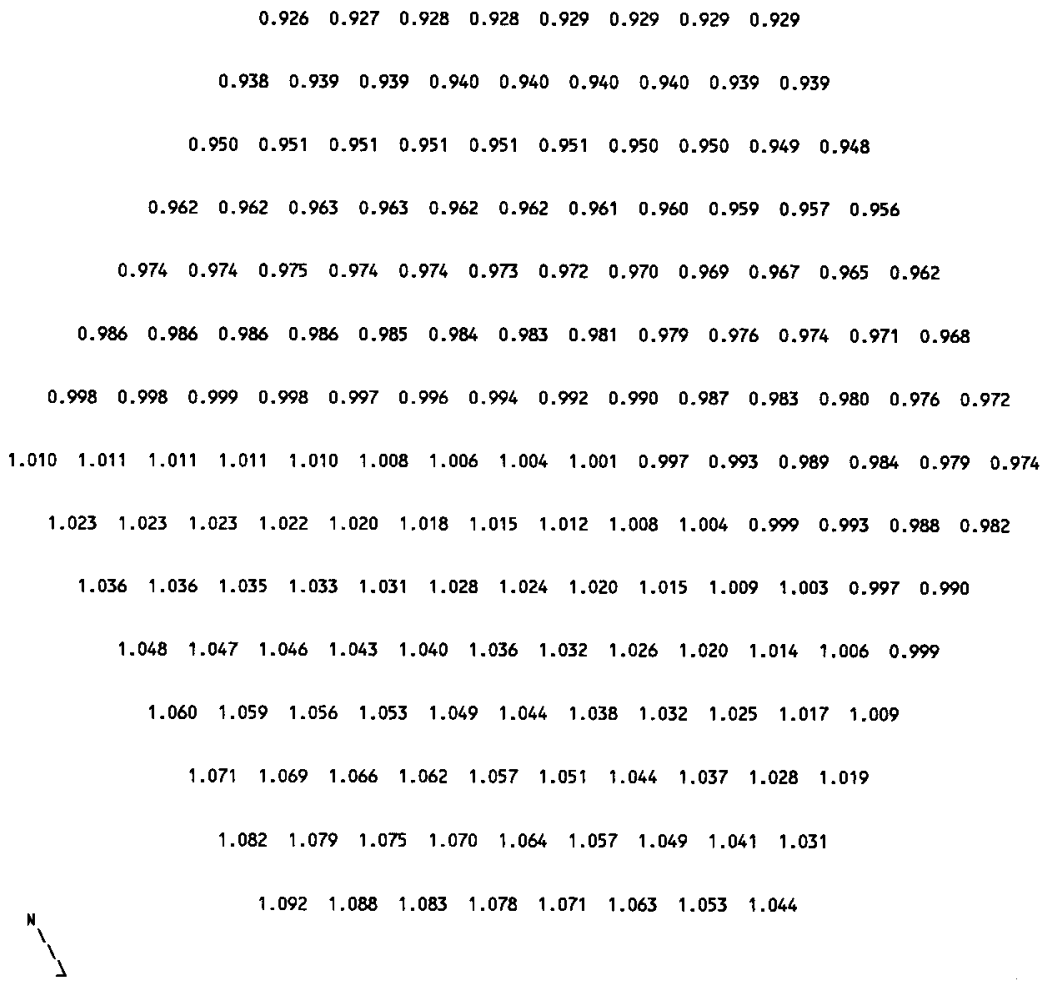


Table EllB-2.8. Fast Flux Distribution by Pin in MFA-2 at EOC 11B-2 CORE POSITION 2506

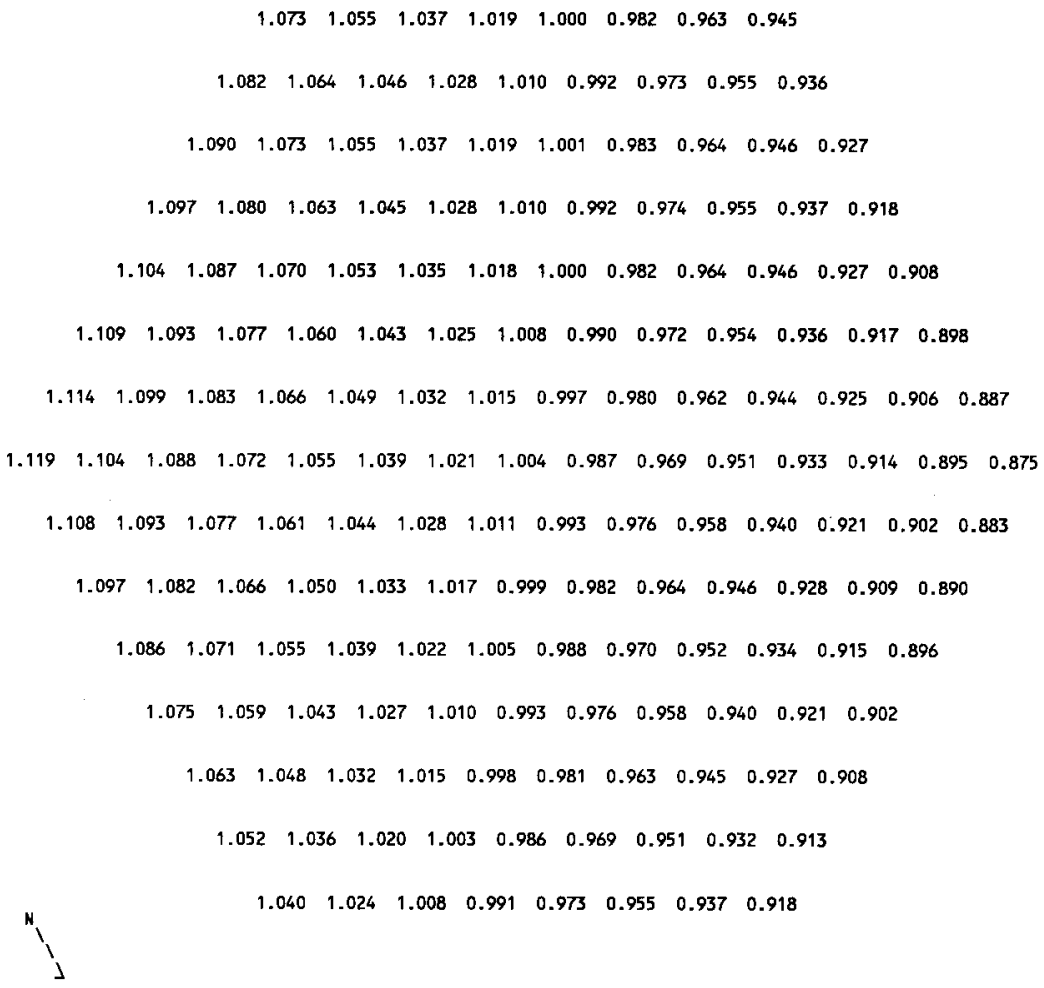


Table E11B-2.9. MFA-1 and MFA-2 Duct Wall

Fast Flux Data at EOC 11B-2

$\begin{array}{ccc}\text { Side } & \begin{array}{c}\text { Flux }>0.1 \\ \text { MFA-1 }\end{array} & \begin{array}{c}\text { MeV }\left(\mathrm{n} / \mathrm{cm}^{2}-\mathrm{sec}\right) \\ \text { MFA-2 }\end{array} \\ \text { E } & 2.3605 \mathrm{E}+15 & 2.1837 \mathrm{E}+15 \\ \text { SE } & 2.1303 \mathrm{E}+15 & 2.2372 \mathrm{E}+15 \\ \text { SW } & 2.0275 \mathrm{E}+15 & 2.0132 \mathrm{E}+15 \\ \text { W } & 2.1205 \mathrm{E}+15 & 1.7862 \mathrm{E}+15 \\ \text { NW } & 2.2196 \mathrm{E}+15 & 1.7506 \mathrm{E}+15 \\ \text { NE } & 2.4228 \mathrm{E}+15 & 1.9510 \mathrm{E}+15\end{array}$

Table E11B-2.10. Assembly Outlet Temperatures and Flow Rates at EOC 11B-2

$\begin{array}{lcl}\text { CORE } & \begin{array}{c}\text { CALCULATED } \\ \text { OUTLET TEMP. }\end{array} & \begin{array}{l}\text { FLOW RATE } \\ \text { (LB/H) }\end{array} \\ \text { POS. } & \text { (DEG F) } & 111160 \\ 1405 & 1044 & 1194240 \\ 1304 & 1052 & 194240 \\ 1404 & 1005 & 196630 \\ 1406 & 857 & 14170 \\ 1506 & 883 & 201290 \\ 1507 & 863 & 191410 \\ 1508 & 970 & 181190 \\ 2506 & 1020 & 111160 \\ 2404 & 1011 & 197960 \\ 2405 & 990 & 165960 \\ 2505 & 879 & 201290 \\ 2507 & 875 & 201290 \\ 2606 & 906 & 165070 \\ 2607 & 881 & 165070\end{array}$

Assembly flows are based on a calculated core pressure drop of $103.4 \mathrm{psi}$ at a total reactor flow rate of $16.74 \mathrm{E}+06 \mathrm{lb} / \mathrm{hr}$. 
Table E11B-2.11. MFA-1 Sodium Subchannel Temperatures

at Top of Core Elevation for EOC 11B-2

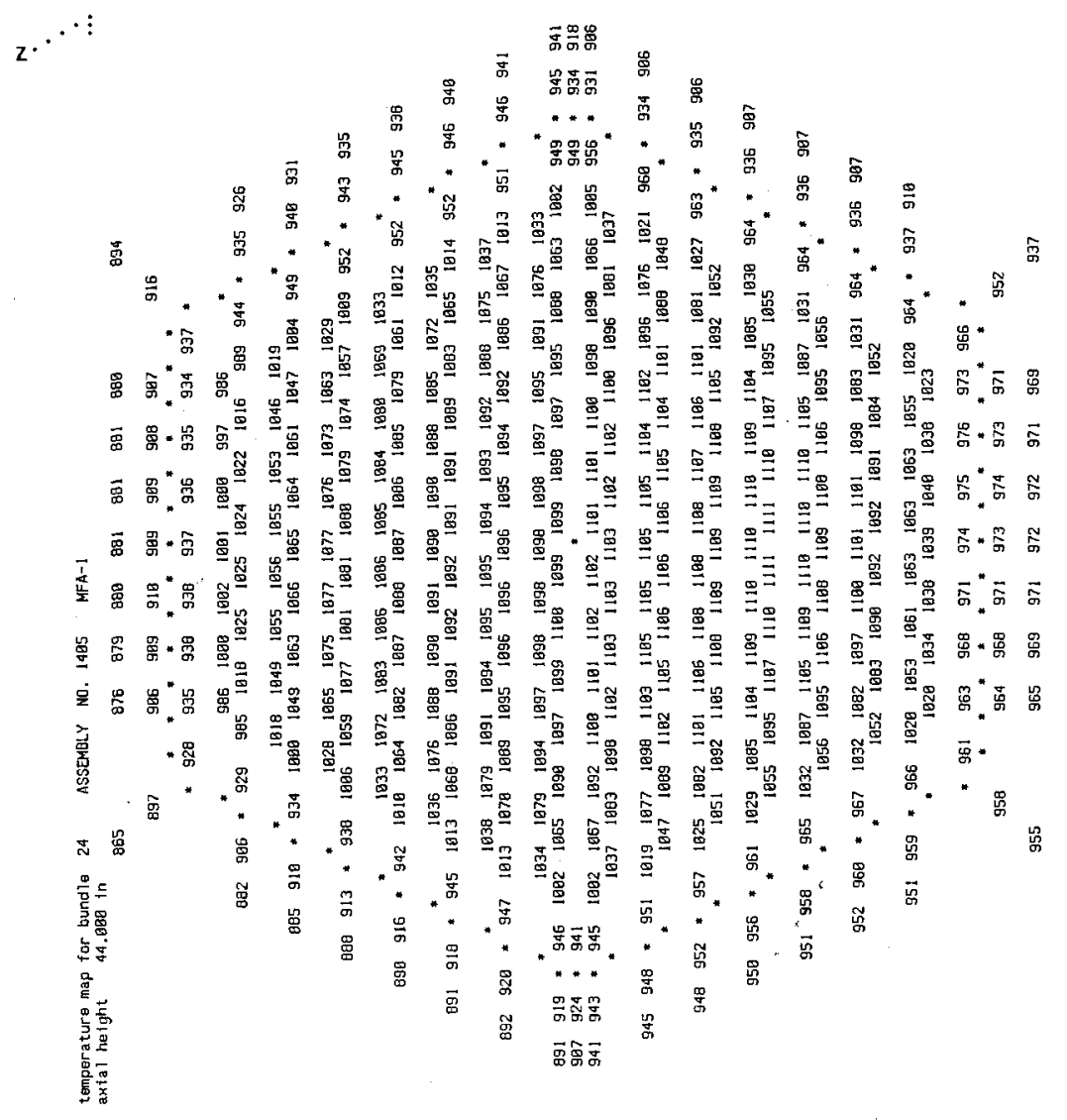


Table El1B-2.12. MFA-2 Sodium Subchannel Temperatures at Top of Core Elevation for EOC 11B-2

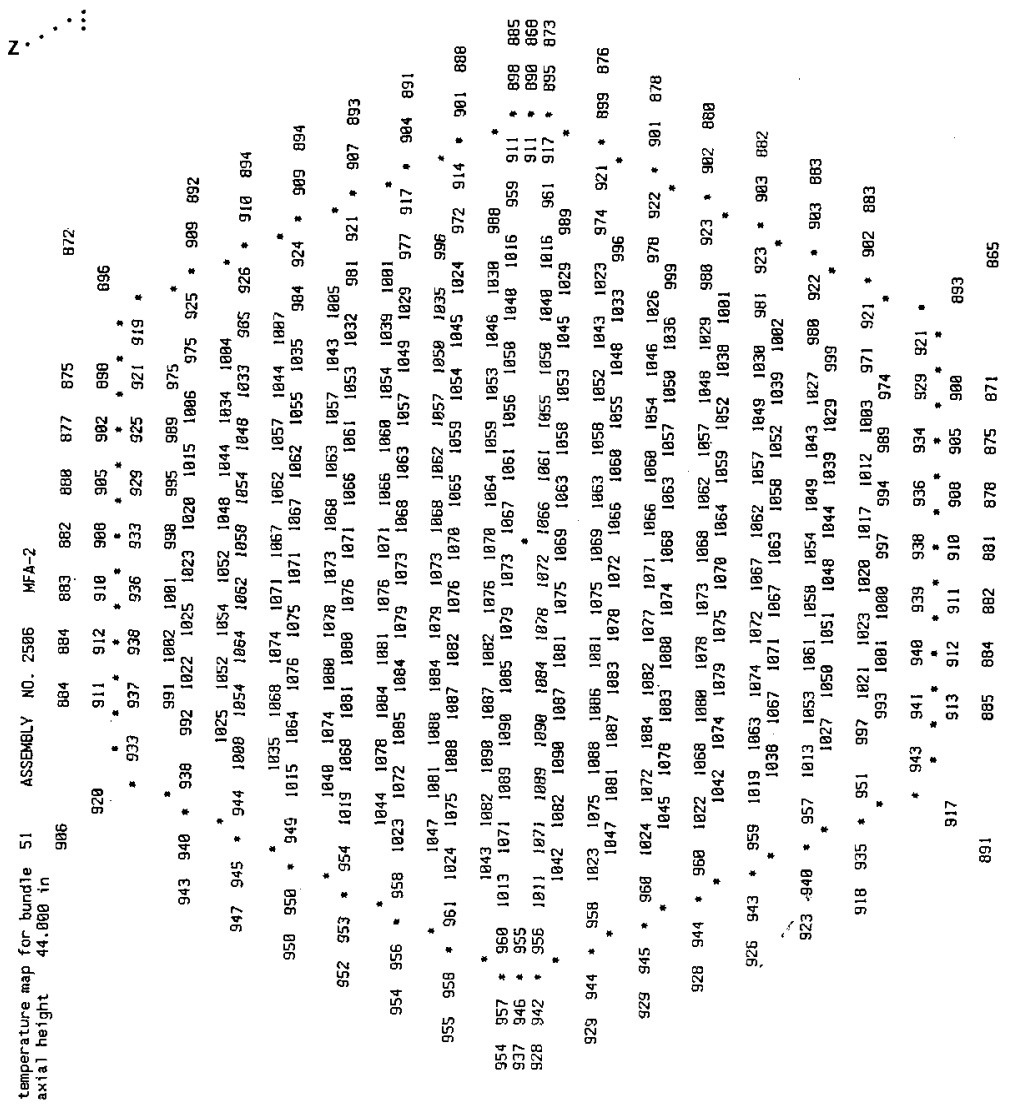


Table E11B-2.13. MFA-1 Sodium Subchannel Temperatures at Elevation of Upper Axial B1anket for EOC 11B-2

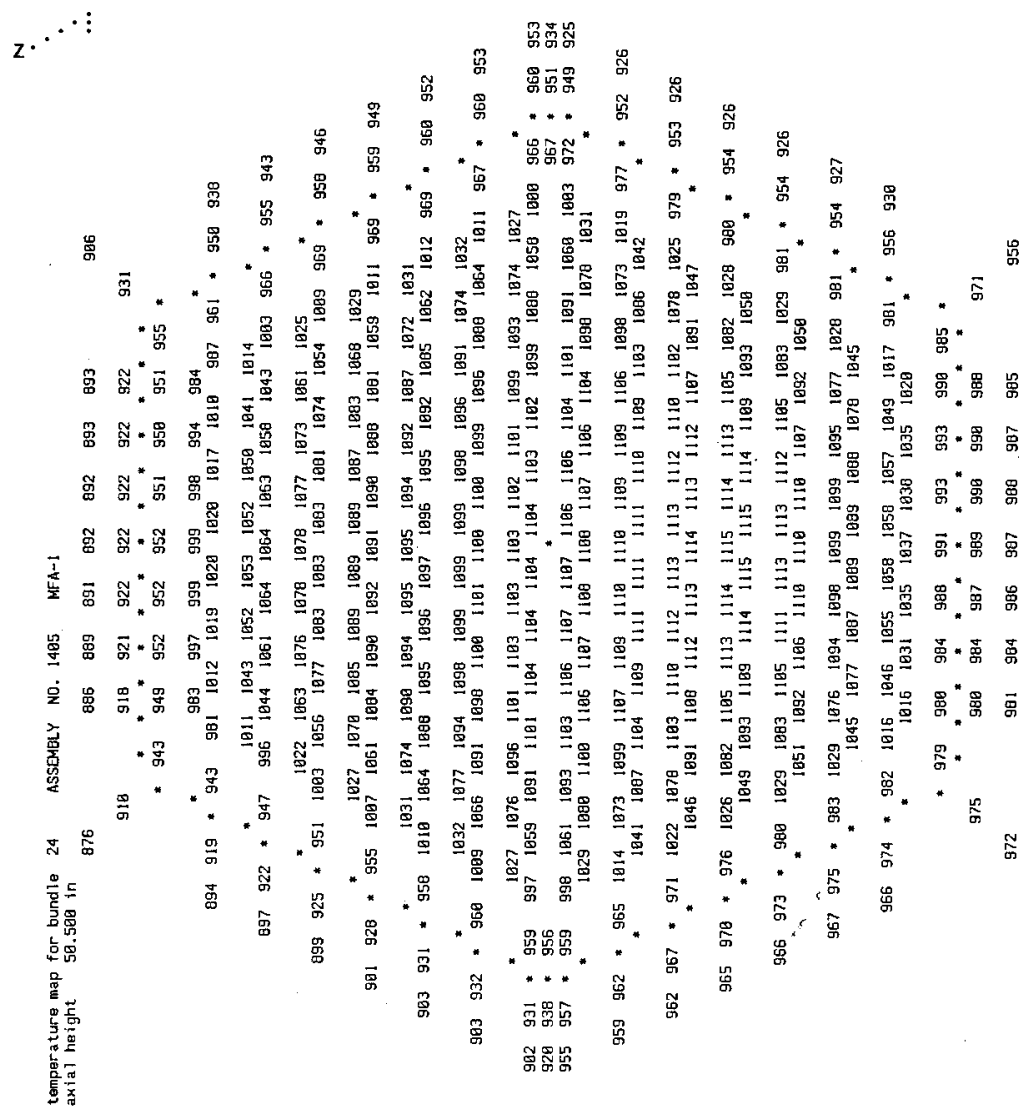


Table El1B-2.14. MFA-2 Sodium Subchannel Temperatures at Elevation of Upper Axial Blanket for EOC 11B-2

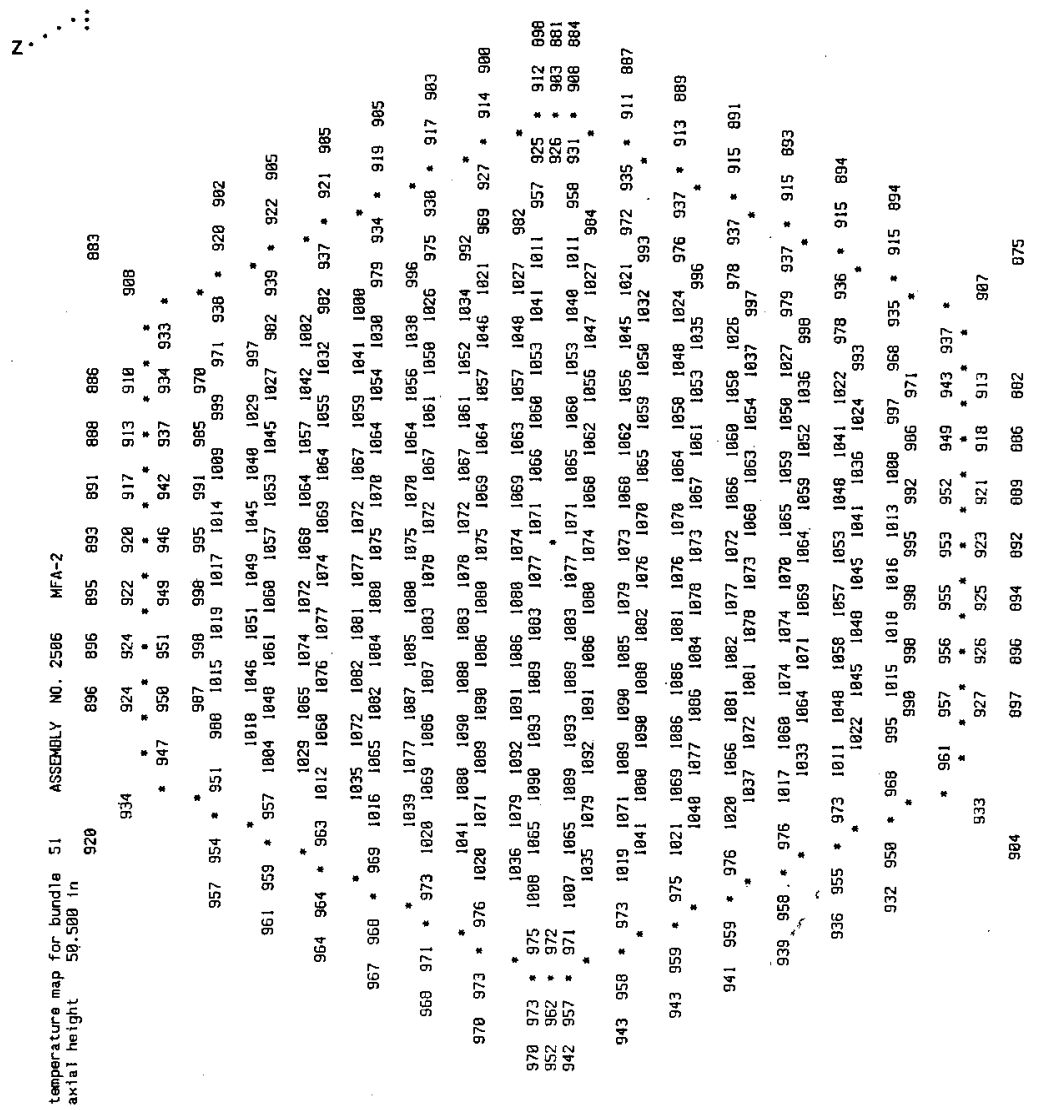


Table E11B-2.15. MFA-1 Sodium Subchannel Temperatures

$$
\text { at Top of Fuel Pin Bundle for EOC 11B-2 }
$$

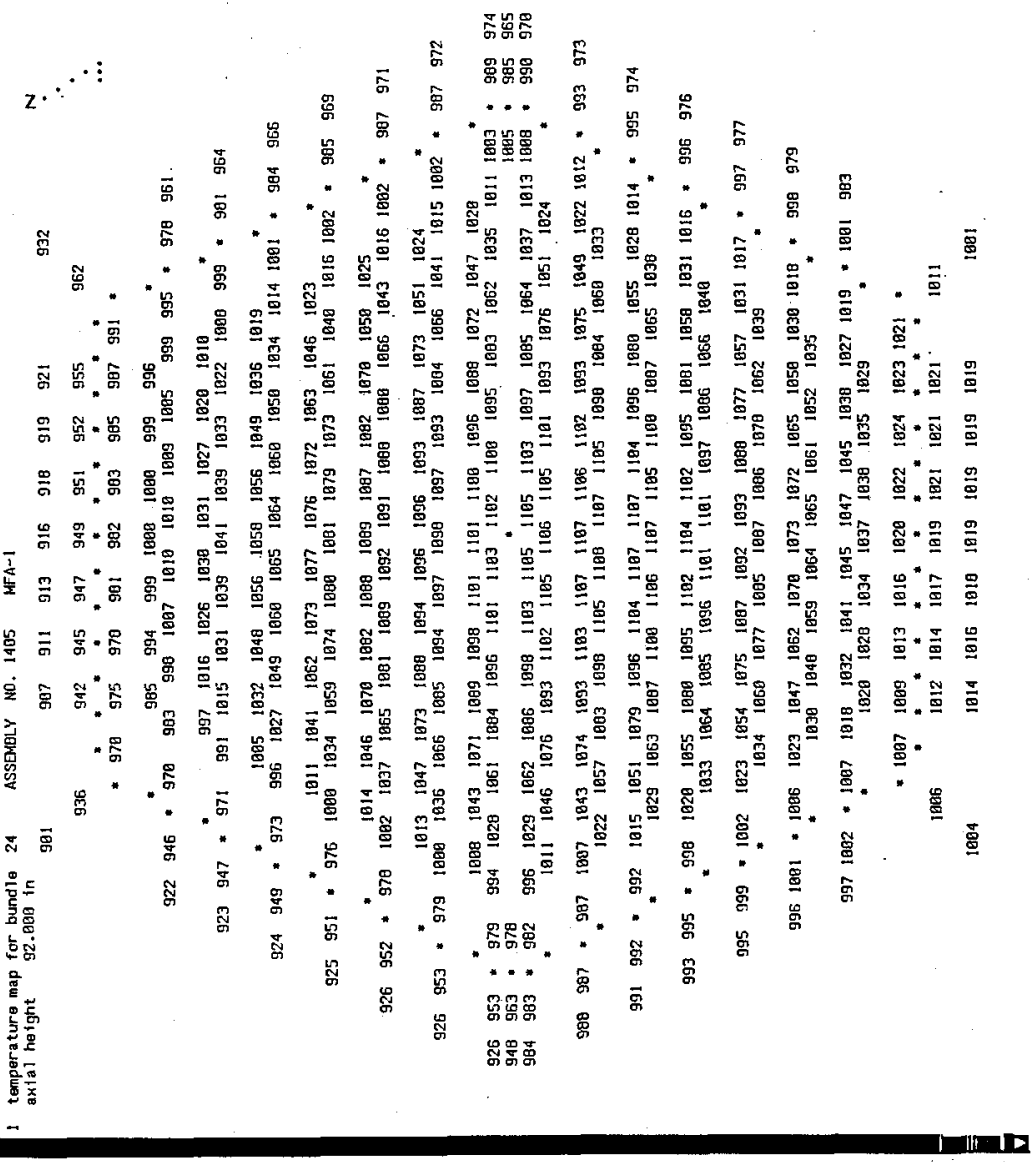


Table E11B-2.16. MFA-2 Sodium Subchannel Temperatures at Top of Fuel Pin Bundle for EOC 11B-2

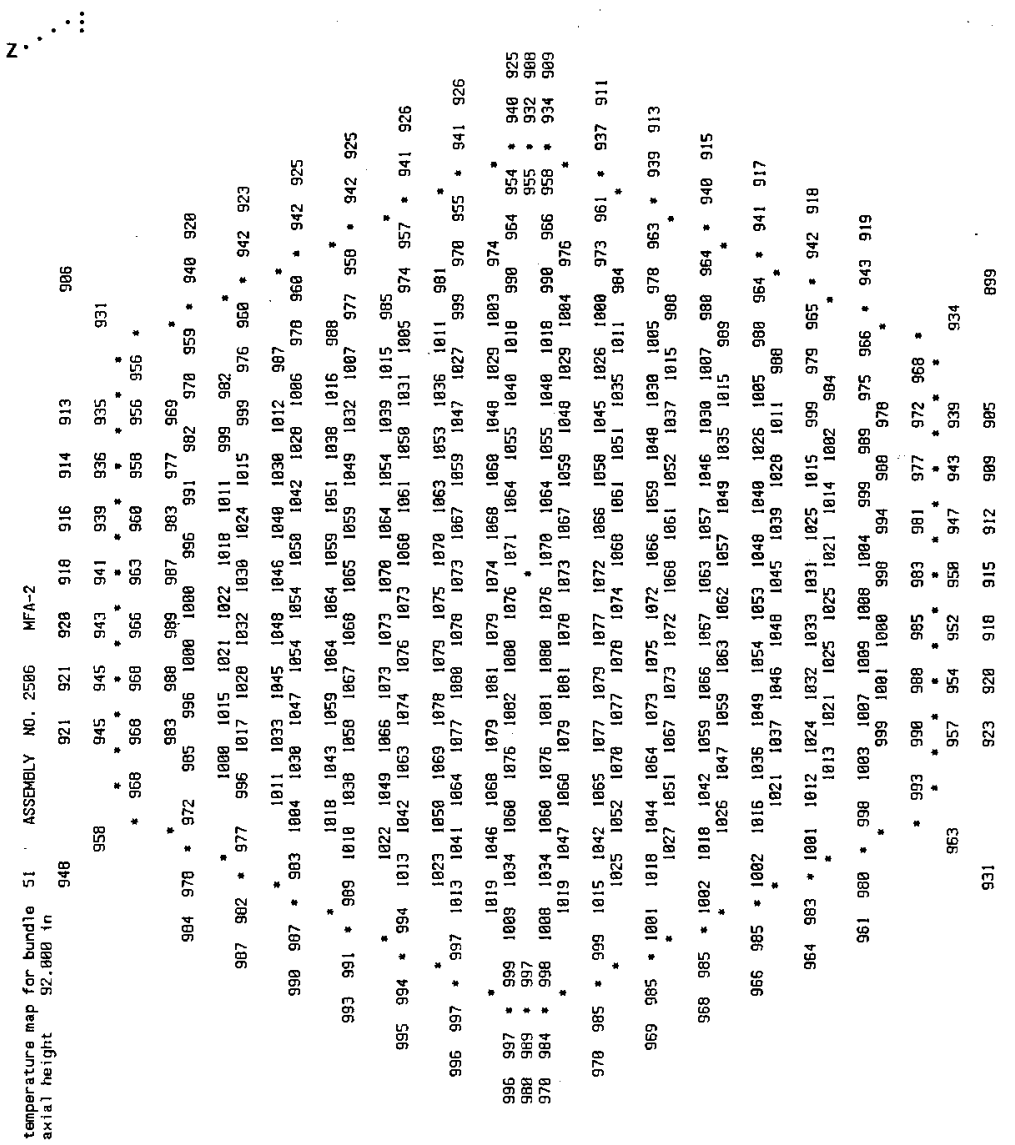


Table E11B-2.17. Composition of MFA-1 at EOC 11B-2

\begin{tabular}{|c|c|c|c|c|c|}
\hline ial & Axial $R$ & $\mathrm{ge}(\mathrm{cm})$ & & Atom Densi & $(a / b-c m)$ \\
\hline vel & $\begin{array}{r}\text { Lower } \\
-12805\end{array}$ & Upper & Constituent & Fresh & nd of cycle \\
\hline & & & $\begin{array}{l}\mathrm{Na}-23 \\
\mathrm{SS}-316\end{array}$ & $\begin{array}{l}5.3150 \mathrm{E}-03 \\
6.4090 \mathrm{E}-02\end{array}$ & $\begin{array}{l}5.3150 \mathrm{E}-03 \\
6.4090 \mathrm{E}-02\end{array}$ \\
\hline 2 & -75.28 & -62.78 & & & \\
\hline & & & $\begin{array}{l}\mathrm{Na}-23 \\
\text { SS-316 }\end{array}$ & $\begin{array}{l}1.3177 \mathrm{E}-02 \\
3.4175 \mathrm{E}-02\end{array}$ & $\begin{array}{l}1.3177 \mathrm{E}-02 \\
3.4175 \mathrm{E}-02\end{array}$ \\
\hline 3 & -62.78 & -46.14 & $\begin{array}{l}\text { U-235 } \\
U-238 \\
\mathrm{Pu}-239 \\
\mathrm{Pu}-240 \\
\mathrm{FP} \\
\mathrm{O}-16 \\
\mathrm{Na}-23 \\
\mathrm{SS}-316\end{array}$ & $\begin{array}{l}1.4143 \mathrm{E}-05 \\
7.0573 \mathrm{E}-03 \\
0.0000 \mathrm{E}+00 \\
0.0000 \mathrm{E}+00 \\
0.0000 \mathrm{E}+00 \\
1.4143 \mathrm{E}-02 \\
9.7416 \mathrm{E}-03 \\
1.9073 \mathrm{E}-02\end{array}$ & $\begin{array}{l}9.2580 \mathrm{E}-06 \\
6.7668 \mathrm{E}-03 \\
2.2827 \mathrm{E}-04 \\
1.3736 \mathrm{E}-05 \\
5.1535 \mathrm{E}-05 \\
1.4143 \mathrm{E}-02 \\
9.7416 \mathrm{E}-03 \\
1.9073 \mathrm{E}-02\end{array}$ \\
\hline 4 & -46.14 & -30.76 & $\begin{array}{l}\text { U-235 } \\
\text { U-238 } \\
\text { Pu-239 } \\
\text { Pu-240 } \\
\text { Pu-241 } \\
\text { Pu-242 } \\
\text { Am-241 } \\
\text { FP } \\
0-16 \\
\mathrm{Na}-23 \\
\text { SS-316 }\end{array}$ & $\begin{array}{l}1.0840 \mathrm{E}-05 \\
4.4499 \mathrm{E}-03 \\
1.7520 \mathrm{E}-03 \\
2.2340 \mathrm{E}-04 \\
1.9280 \mathrm{E}-05 \\
4.7430 \mathrm{E}-06 \\
7.6680 \mathrm{E}-07 \\
0.0000 \mathrm{E}+00 \\
1.2670 \mathrm{E}-02 \\
9.8100 \mathrm{E}-03 \\
1.8990 \mathrm{E}-02\end{array}$ & $\begin{array}{l}7.4220 \mathrm{E}-06 \\
4.2325 \mathrm{E}-03 \\
1.3614 \mathrm{E}-03 \\
2.9540 \mathrm{E}-04 \\
2.5584 \mathrm{E}-05 \\
5.3330 \mathrm{E}-06 \\
3.1203 \mathrm{E}-06 \\
5.2835 \mathrm{E}-04 \\
1.2670 \mathrm{E}-02 \\
9.8100 \mathrm{E}-03 \\
1.8990 \mathrm{E}-02\end{array}$ \\
\hline 5 & -30.76 & -15.38 & $\begin{array}{l}U-235 \\
U-238 \\
P u-239 \\
P u-240 \\
P u-241 \\
P u-242 \\
\text { Am-241 } \\
\text { FP } \\
\text { O-16 } \\
\mathrm{Na}-23 \\
\text { SS }-316\end{array}$ & $\begin{array}{l}1.0840 \mathrm{E}-05 \\
4.4499 \mathrm{E}-03 \\
1.7520 \mathrm{E}-03 \\
2.2340 \mathrm{E}-04 \\
1.9280 \mathrm{E}-05 \\
4.7430 \mathrm{E}-06 \\
7.6680 \mathrm{E}-07 \\
0.0000 \mathrm{E}+00 \\
1.2670 \mathrm{E}-02 \\
9.8100 \mathrm{E}-03 \\
1.8990 \mathrm{E}-02\end{array}$ & $\begin{array}{l}6.7839 \mathrm{E}-06 \\
4.1777 \mathrm{E}-03 \\
1.2751 \mathrm{E}-03 \\
2.9663 \mathrm{E}-04 \\
2.5886 \mathrm{E}-05 \\
5.3521 \mathrm{E}-06 \\
3.0355 \mathrm{E}-06 \\
6.6834 \mathrm{E}-04 \\
1.2670 \mathrm{E}-02 \\
9.8100 \mathrm{E}-03 \\
1.8990 \mathrm{E}-02\end{array}$ \\
\hline 6 & -15.38 & 0.00 & $\begin{array}{l}U-235 \\
U-238 \\
P u-239 \\
P u-240 \\
P u-241 \\
P u-242 \\
\text { Am-241 } \\
\text { FP } \\
\text { O-16 } \\
\mathrm{Na}-23 \\
\text { SS }-316\end{array}$ & $\begin{array}{l}1.0840 \mathrm{E}-05 \\
4.4499 \mathrm{E}-03 \\
1.7520 \mathrm{E}-03 \\
2.2340 \mathrm{E}-04 \\
1.9280 \mathrm{E}-05 \\
4.7430 \mathrm{E}-06 \\
7.6680 \mathrm{E}-07 \\
0.0000 \mathrm{E}+00 \\
1.2670 \mathrm{E}-02 \\
9.8100 \mathrm{E}-03 \\
1.8990 \mathrm{E}-02\end{array}$ & $\begin{array}{l}6.4432 \mathrm{E}-06 \\
4.1493 \mathrm{E}-03 \\
1.2335 \mathrm{E}-03 \\
3.0106 \mathrm{E}-04 \\
2.6766 \mathrm{E}-05 \\
5.4386 \mathrm{E}-06 \\
3.0231 \mathrm{E}-06 \\
7.3343 \mathrm{E}-04 \\
1.2670 \mathrm{E}-02 \\
9.8100 \mathrm{E}-03 \\
1.8990 \mathrm{E}-02\end{array}$ \\
\hline & & & & & \\
\hline
\end{tabular}


HNF-SD-FF-ANAL-009 Rev. I

$\begin{array}{lll}7 & 0.00 & 15.38\end{array}$

$\begin{array}{lll}8 & 15.38 & 30.76\end{array}$

U-235 $\quad 1.0840 E-05 \quad 6.5587 \mathrm{E}-06$

$\mathrm{U}-238 \quad 4.4499 \mathrm{E}-03 \quad 4.1590 \mathrm{E}-03$

$\mathrm{Pu}-239 \quad 1.7520 \mathrm{E}-03 \quad 1.2469 \mathrm{E}-03$

Pu-240 2.2340E-04 2.9911E-04

$\mathrm{Pu}-241 \quad 1.9280 \mathrm{E}-05 \quad 2.6400 \mathrm{E}-05$

Pu-242 4.7430E-06 5.4020E-06

Am-241 7.6680E-07 3.0246E-06

FP $\quad 0.0000 \mathrm{E}+00 \quad 7.1231 \mathrm{E}-04$

$0-16 \quad 1.2670 \mathrm{E}-02 \quad 1.2670 \mathrm{E}-02$

$\mathrm{Na}-23 \quad 9.8100 \mathrm{E}-03 \quad 9.8100 \mathrm{E}-03$

SS-316 $1.8990 \mathrm{E}-02 \quad 1.8990 \mathrm{E}-02$

U-235 1.0840E-05 7.1196E-06

U-238 4.4499E-03 4.2051E-03

$\mathrm{Pu}-239 \quad 1.7520 \mathrm{E}-03 \quad 1.3143 \mathrm{E}-03$

Pu-240 2.2340E-04 2.9056E-04

$\mathrm{Pu}-241 \quad 1.9280 \mathrm{E}-05 \quad 2.4843 \mathrm{E}-05$

$\mathrm{Pu}-242 \quad 4.7430 \mathrm{E}-06 \quad 5.2564 \mathrm{E}-06$

Am-241 7.6680E-07 3.0393E-06

FP $\quad 0.0000 E+00 \quad 6.0899 \mathrm{E}-04$

0-16 $1.2670 \mathrm{E}-02 \quad 1.2670 \mathrm{E}-02$

$\mathrm{Na}-23 \quad 9.8100 \mathrm{E}-03 \quad 9.8100 \mathrm{E}-03$

SS-316 $1.8990 \mathrm{E}-02 \quad 1.8990 \mathrm{E}-02$

$\begin{array}{lll}9 & 30.76 & 46.14\end{array}$

U-235 $\quad 1.0840 \mathrm{E}-05 \quad 7.9765 \mathrm{E}-06$

$\mathrm{U}-238 \quad 4.4499 \mathrm{E}-03 \quad 4.2720 \mathrm{E}-03$

Pu-239 1.7520E-03 1.4237E-03

$\mathrm{Pu}-240 \quad 2.2340 \mathrm{E}-04 \quad 2.8250 \mathrm{E}-04$

Pu-241 1.9280E-05 2.3508E-05

$\mathrm{Pu}-242 \quad 4.7430 \mathrm{E}-06 \quad 5.1583 \mathrm{E}-06$

Am-241 7.6680E-07 3.1091E-06

FP $\quad 0.0000 \mathrm{E}+00 \quad 4.4145 \mathrm{E}-04$

0-16 $1.2670 \mathrm{E}-02 \quad 1.2670 \mathrm{E}-02$

$\mathrm{Na}-23 \quad 9.8100 \mathrm{E}-03 \quad 9.8100 \mathrm{E}-03$

SS-316 $1.8990 \mathrm{E}-02 \quad 1.8990 \mathrm{E}-02$

$\begin{array}{lll}10 & 46.14 & 62.78\end{array}$

$\begin{array}{lll}\mathrm{U}-235 & 1.4143 \mathrm{E}-05 & 1.0785 \mathrm{E}-05 \\ \mathrm{U}-238 & 7.0573 \mathrm{E}-03 & 6.8605 \mathrm{E}-03 \\ \mathrm{PU}-239 & 0.0000 \mathrm{E}+00 & 1.6292 \mathrm{E}-04 \\ \mathrm{PU}-240 & 0.0000 \mathrm{E}+00 & 5.8761 \mathrm{E}-06 \\ \mathrm{FP} & 0.0000 \mathrm{E}+00 & 3.0217 \mathrm{E}-05 \\ \mathrm{O}-16 & 1.4143 \mathrm{E}-02 & 1.4143 \mathrm{E}-02 \\ \mathrm{Na}-23 & 9.7416 \mathrm{E}-03 & 9.7416 \mathrm{E}-03 \\ \mathrm{SS}-316 & 1.9073 \mathrm{E}-02 & 1.9073 \mathrm{E}-02\end{array}$

$11 \quad 62.78 \quad 172.84$

$\begin{array}{lll}\mathrm{Na}-23 & 8.2400 \mathrm{E}-03 & 8.2400 \mathrm{E}-03 \\ \mathrm{SS}-316 & 2.5562 \mathrm{E}-02 & 2.5562 \mathrm{E}-02\end{array}$


HNF-SD-FF-ANAL-009 Rev. 1

Table El1B-2.18. Composition of MFA-2 at EOC 11B-2

\begin{tabular}{|c|c|c|c|c|c|}
\hline Axial & Axial R & $e(\mathrm{~cm})$ & & Atom Den & $(\mathrm{a} / \mathrm{b}-\mathrm{cm})$ \\
\hline $\begin{array}{c}\text { evel } \\
1\end{array}$ & $\begin{array}{r}\text { Lower } \\
-128.05\end{array}$ & $\begin{array}{l}\text { Upper } \\
-75.28\end{array}$ & Constituent & Fresh & id of Cycle \\
\hline & & & $\begin{array}{l}\mathrm{Na}-23 \\
\mathrm{SS}-316\end{array}$ & $\begin{array}{l}5.3150 \mathrm{E}-03 \\
6.4090 \mathrm{E}-02\end{array}$ & $\begin{array}{l}5.3150 E-03 \\
6.4090 E-02\end{array}$ \\
\hline 2 & -75.28 & -62.78 & & & \\
\hline & & & $\begin{array}{l}\mathrm{Na}-23 \\
\mathrm{SS}-316\end{array}$ & $\begin{array}{l}1.3177 \mathrm{E}-02 \\
3.4175 \mathrm{E}-02\end{array}$ & $\begin{array}{l}1.3177 \mathrm{E}-02 \\
3.4175 \mathrm{E}-02\end{array}$ \\
\hline 3 & -62.78 & -46.14 & & & \\
\hline & & & $\begin{array}{l}U-235 \\
U-238 \\
P U-239 \\
P u-240 \\
F P \\
0-16 \\
\mathrm{Na}-23 \\
\mathrm{SS}-316\end{array}$ & $\begin{array}{l}1.4143 \mathrm{E}-05 \\
7.0573 \mathrm{E}-03 \\
0.0000 \mathrm{E}+00 \\
0.0000 \mathrm{E}+00 \\
0.0000 \mathrm{E}+00 \\
1.4143 \mathrm{E}-02 \\
9.7416 \mathrm{E}-03 \\
1.9073 \mathrm{E}-02\end{array}$ & $\begin{array}{l}9.2743 \mathrm{E}-06 \\
6.7733 \mathrm{E}-03 \\
2.2335 \mathrm{E}-04 \\
1.3590 \mathrm{E}-05 \\
5.0079 \mathrm{E}-05 \\
1.4143 \mathrm{E}-02 \\
9.7416 \mathrm{E}-03 \\
1.9073 \mathrm{E}-02\end{array}$ \\
\hline 4 & -46.14 & -30.76 & & & \\
\hline & & & $\begin{array}{l}U-235 \\
U-238 \\
\mathrm{Pu}-239 \\
\mathrm{Pu}-240 \\
\mathrm{Pu}-241 \\
\mathrm{Pu}-242 \\
\mathrm{Am}-241 \\
\mathrm{FP} \\
0-16 \\
\mathrm{Na}-23 \\
\mathrm{SS}-316\end{array}$ & $\begin{array}{l}1.0160 \mathrm{E}-05 \\
4.4270 \mathrm{E}-03 \\
1.7483 \mathrm{E}-03 \\
2.2290 \mathrm{E}-04 \\
1.9040 \mathrm{E}-05 \\
4.7330 \mathrm{E}-06 \\
7.5700 \mathrm{E}-07 \\
0.0000 \mathrm{E}+00 \\
1.2760 \mathrm{E}-02 \\
9.8100 \mathrm{E}-03 \\
1.8990 \mathrm{E}-02\end{array}$ & $\begin{array}{l}6.9980 \mathrm{E}-06 \\
4.2135 \mathrm{E}-03 \\
1.3621 \mathrm{E}-03 \\
2.9356 \mathrm{E}-04 \\
2.5262 \mathrm{E}-05 \\
5.2993 \mathrm{E}-06 \\
3.1159 \mathrm{E}-06 \\
5.2110 \mathrm{E}-04 \\
1.2760 \mathrm{E}-02 \\
9.8100 \mathrm{E}-03 \\
1.8990 \mathrm{E}-02\end{array}$ \\
\hline 5 & -30.76 & -15.38 & & & \\
\hline & & & $\begin{array}{l}\mathrm{U}-235 \\
\mathrm{U}-238 \\
\mathrm{Pu}-239 \\
\mathrm{Pu}-240 \\
\mathrm{Pu}-241 \\
\mathrm{Pu}-242 \\
\mathrm{Am}-241 \\
\mathrm{FP} \\
0-16 \\
\mathrm{Na}-23 \\
\mathrm{SS}-316\end{array}$ & $\begin{array}{l}1.0160 \mathrm{E}-05 \\
4.4270 \mathrm{E}-03 \\
1.7483 \mathrm{E}-03 \\
2.2290 \mathrm{E}-04 \\
1.9040 \mathrm{E}-05 \\
4.7330 \mathrm{E}-06 \\
7.5700 \mathrm{E}-07 \\
0.0000 \mathrm{E}+00 \\
1.2760 \mathrm{E}-02 \\
9.8100 \mathrm{E}-03 \\
1.8990 \mathrm{E}-02\end{array}$ & $\begin{array}{l}6.3765 \mathrm{E}-06 \\
4.1570 \mathrm{E}-03 \\
1.2733 \mathrm{E}-03 \\
2.9522 \mathrm{E}-04 \\
2.5640 \mathrm{E}-05 \\
5.3213 \mathrm{E}-06 \\
3.0314 \mathrm{E}-06 \\
6.6538 \mathrm{E}-04 \\
1.2760 \mathrm{E}-02 \\
9.8100 \mathrm{E}-03 \\
1.8990 \mathrm{E}-02\end{array}$ \\
\hline 6 & -15.38 & 0.00 & & & \\
\hline & & & $\begin{array}{l}\mathrm{U}-235 \\
\mathrm{U}-238 \\
\mathrm{Pu}-239 \\
\mathrm{Pu}-240 \\
\mathrm{Pu}-241 \\
\mathrm{Pu}-242 \\
\mathrm{Am}-241 \\
\mathrm{FP} \\
0-16 \\
\mathrm{Na}-23 \\
\mathrm{SS}-316\end{array}$ & $\begin{array}{l}1.0160 \mathrm{E}-05 \\
4.4270 \mathrm{E}-03 \\
1.7483 \mathrm{E}-03 \\
2.2290 \mathrm{E}-04 \\
1.9040 \mathrm{E}-05 \\
4.7330 \mathrm{E}-06 \\
7.5700 \mathrm{E}-07 \\
0.0000 \mathrm{E}+00 \\
1.2760 \mathrm{E}-02 \\
9.8100 \mathrm{E}-03 \\
1.8990 \mathrm{E}-02\end{array}$ & $\begin{array}{l}6.0417 \mathrm{E}-06 \\
4.1270 \mathrm{E}-03 \\
1.2296 \mathrm{E}-03 \\
2.9996 \mathrm{E}-04 \\
2.6583 \mathrm{E}-05 \\
5.4125 \mathrm{E}-06 \\
3.0233 \mathrm{E}-06 \\
7.3333 \mathrm{E}-04 \\
1.2760 \mathrm{E}-02 \\
9.8100 \mathrm{E}-03 \\
1.8990 \mathrm{E}-02\end{array}$ \\
\hline & & & & & \\
\hline
\end{tabular}


HNF-SD-FF-ANAL-009 Rev. I

$0.00 \quad 15.38$

$\begin{array}{lll}8 & 15.38 & 30.76\end{array}$

$\begin{array}{lll}\mathrm{U}-235 & 1.0160 \mathrm{E}-05 & 6.1390 \mathrm{E}-06 \\ \mathrm{U}-238 & 4.4270 \mathrm{E}-03 & 4.1356 \mathrm{E}-03 \\ \mathrm{Pu}-239 & 1.7483 \mathrm{E}-03 & 1.2417 \mathrm{E}-03 \\ \mathrm{Pu}-240 & 2.2290 \mathrm{E}-04 & 2.9819 \mathrm{E}-04 \\ \mathrm{Pu}-241 & 1.9040 \mathrm{E}-05 & 2.6250 \mathrm{E}-05 \\ \mathrm{Pu}-242 & 4.7330 \mathrm{E}-06 & 5.3794 \mathrm{E}-06 \\ \mathrm{Am}-241 & 7.5700 \mathrm{E}-07 & 3.0244 \mathrm{E}-06 \\ \mathrm{FP} & 0.0000 \mathrm{E}+00 & 7.1448 \mathrm{E}-04 \\ 0-16 & 1.2760 \mathrm{E}-02 & 1.2760 \mathrm{E}-02 \\ \mathrm{Na}-23 & 9.8100 \mathrm{E}-03 & 9.8100 \mathrm{E}-03 \\ \mathrm{SS}-316 & 1.8990 \mathrm{E}-02 & 1.8990 \mathrm{E}-02\end{array}$

U-235 $\quad 1.0160 E-05 \quad 6.6538 E-06$

U-238 4.4270E-03 4.1808E-03

$\mathrm{Pu}-239 \quad 1.7483 \mathrm{E}-03 \quad 1.3075 \mathrm{E}-03$

$\mathrm{Pu}-240 \quad 2.2290 \mathrm{E}-04 \quad 2.8990 \mathrm{E}-04$

$\mathrm{Pu}-241 \quad 1.9040 \mathrm{E}-05 \quad 2.4719 \mathrm{E}-05$

$\mathrm{Pu}-242 \quad 4.7330 \mathrm{E}-06 \quad 5.2385 \mathrm{E}-06$

Am-241 7.5700E-07 3.0354E-06

FP $\quad 0.0000 E+00 \quad 6.1331 E-04$

$0-16 \quad 1.2760 \mathrm{E}-02 \quad 1.2760 \mathrm{E}-02$

$\mathrm{Na}-23 \quad 9.8100 \mathrm{E}-03 \quad 9.8100 \mathrm{E}-03$

SS-316 $1.8990 \mathrm{E}-02 \quad 1.8990 \mathrm{E}-02$

$\begin{array}{lll}9 & 30.76 & 46.14\end{array}$

$\begin{array}{lll}\mathrm{U}-235 & 1.0160 \mathrm{E}-05 & 7.4406 \mathrm{E}-06 \\ \mathrm{U}-238 & 4.4270 \mathrm{E}-03 & 4.2472 \mathrm{E}-03 \\ \mathrm{Pu}-239 & 1.7483 \mathrm{E}-03 & 1.4151 \mathrm{E}-03 \\ \mathrm{Pu}-240 & 2.2290 \mathrm{E}-04 & 2.8269 \mathrm{E}-04 \\ \mathrm{Pu}-241 & 1.9040 \mathrm{E}-05 & 2.3505 \mathrm{E}-05 \\ \mathrm{Pu}-242 & 4.7330 \mathrm{E}-06 & 5.1530 \mathrm{E}-06 \\ \mathrm{Am}-241 & 7.5700 \mathrm{E}-07 & 3.1037 \mathrm{E}-06 \\ \mathrm{FP} & 0.0000 \mathrm{E}+00 & 4.4731 \mathrm{E}-04 \\ 0-16 & 1.2760 \mathrm{E}-02 & 1.2760 \mathrm{E}-02 \\ \mathrm{Na}-23 & 9.8100 \mathrm{E}-03 & 9.8100 \mathrm{E}-03 \\ \mathrm{SS}-316 & 1.8990 \mathrm{E}-02 & 1.8990 \mathrm{E}-02\end{array}$

$10 \quad 46.14 \quad 62.78$

$\begin{array}{lll}\mathrm{U}-235 & 1.4143 \mathrm{E}-05 & 1.0571 \mathrm{E}-05 \\ \mathrm{U}-238 & 7.0573 \mathrm{E}-03 & 6.8525 \mathrm{E}-03 \\ \mathrm{Pu}-239 & 0.0000 \mathrm{E}+00 & 1.6861 \mathrm{E}-04 \\ \mathrm{Pu}-240 & 0.0000 \mathrm{E}+00 & 6.7456 \mathrm{E}-06 \\ \mathrm{FP} & 0.0000 \mathrm{E}+00 & 3.1990 \mathrm{E}-05 \\ \mathrm{O}-16 & 1.4143 \mathrm{E}-02 & 1.4143 \mathrm{E}-02 \\ \mathrm{Na}-23 & 9.7416 \mathrm{E}-03 & 9.7416 \mathrm{E}-03 \\ \mathrm{SS}-316 & 1.9073 \mathrm{E}-02 & 1.9073 \mathrm{E}-02\end{array}$

$11 \quad 62.78 \quad 172.84$

$\begin{array}{lll}\mathrm{Na}-23 & 8.2400 \mathrm{E}-03 & 8.2400 \mathrm{E}-03 \\ \mathrm{SS}-316 & 2.5562 \mathrm{E}-02 & 2.5562 \mathrm{E}-02\end{array}$




\subsection{Cycle 11C}

Table Bl1C.1. Fission Power Generated in MFA-1, MFA-2 and Neighboring Assemblies at BOC 11C

\begin{tabular}{lcccc} 
CORE & $* * * * * *$ & \multicolumn{2}{c}{ POWER IN MEGAWATTS } & $* * * * * * *$ \\
POS. & BELOW CORE & IN CORE & ABOVE CORE & TOTAL PWR \\
1405 & $9.643 \mathrm{E}-02$ & $3.625 \mathrm{E}+00$ & $5.495 \mathrm{E}-02$ & $3.776 \mathrm{E}+00$ \\
1304 & $0.000 \mathrm{E}+00$ & $6.886 \mathrm{E}+00$ & $0.000 \mathrm{E}+00$ & $6.886 \mathrm{E}+00$ \\
1406 & $0.000 \mathrm{E}+00$ & $0.000 \mathrm{E}+00$ & $0.000 \mathrm{E}+00$ & $0.000 \mathrm{E}+00$ \\
1404 & $0.000 \mathrm{E}+00$ & $5.997 \mathrm{E}+00$ & $0.000 \mathrm{E}+00$ & $5.997 \mathrm{E}+00$ \\
1508 & $0.000 \mathrm{E}+00$ & $5.002 \mathrm{E}+00$ & $0.000 \mathrm{E}+00$ & $5.002 \mathrm{E}+00$ \\
1506 & $1.285 \mathrm{E}-02$ & $3.601 \mathrm{E}+00$ & $8.443 \mathrm{E}-03$ & $3.622 \mathrm{E}+00$ \\
1507 & $1.041 \mathrm{E}-02$ & $3.512 \mathrm{E}+00$ & $7.524 \mathrm{E}-03$ & $3.529 \mathrm{E}+00$ \\
2506 & $8.629 \mathrm{E}-02$ & $3.350 \mathrm{E}+00$ & $5.176 \mathrm{E}-02$ & $3.488 \mathrm{E}+00$ \\
2404 & $0.000 \mathrm{E}+00$ & $6.171 \mathrm{E}+00$ & $0.000 \mathrm{E}+00$ & $6.171 \mathrm{E}+00$ \\
2505 & $7.102 \mathrm{E}-03$ & $4.430 \mathrm{E}+00$ & $5.018 \mathrm{E}-03$ & $4.442 \mathrm{E}+00$ \\
2405 & $1.641 \mathrm{E}-02$ & $3.569 \mathrm{E}+00$ & $1.093 \mathrm{E}-02$ & $3.596 \mathrm{E}+00$ \\
2606 & $1.432 \mathrm{E}-02$ & $3.375 \mathrm{E}+00$ & $8.043 \mathrm{E}-03$ & $3.398 \mathrm{E}+00$ \\
2607 & $5.557 \mathrm{E}-03$ & $3.832 \mathrm{E}+00$ & $4.598 \mathrm{E}-03$ & $3.842 \mathrm{E}+00$ \\
2507 & $1.173 \mathrm{E}-02$ & $3.601 \mathrm{E}+00$ & $8.216 \mathrm{E}-03$ & $3.621 \mathrm{E}+00$
\end{tabular}

Table Bl1C.2. Assembly Averaged Total and Fast Flux in MFA-1 and MFA-2 at BOC 11C

\begin{tabular}{|c|c|c|c|}
\hline & Core & Flux & $\left(\mathrm{n} / \mathrm{cm}^{2}-\mathrm{sec}\right)$ \\
\hline $\begin{array}{l}\text { sssembly } \\
\text { MFA-1 } \\
\text { MFA-2 }\end{array}$ & $\begin{array}{l}\text { Pos. } \\
1405 \\
2506\end{array}$ & $\begin{array}{c}\text { Total } \\
3.376 E+15 \\
3.103 E+15\end{array}$ & $\begin{array}{l}>0.1 \mathrm{MeV} \\
2.233 \mathrm{E}+15 \\
2.005 \mathrm{E}+15\end{array}$ \\
\hline
\end{tabular}


Table B1lC.3. Axial Distribution of Total Flux, Fast Flux and Power in MFA-1 at BOC $11 \mathrm{C}$

$\begin{array}{rccc}\text { z }(\mathrm{cm}) & \text { Total Flux } & \text { Flux }>0.1 \text { MeV } & \text { Power } \\ -97.78 & 2.8268 \mathrm{E}-02 & 1.4252 \mathrm{E}-02 & 0.0000 \mathrm{E}+00 \\ -92.78 & 6.0758 \mathrm{E}-02 & 2.7607 \mathrm{E}-02 & 0.0000 \mathrm{E}+00 \\ -87.78 & 9.7507 \mathrm{E}-02 & 4.4307 \mathrm{E}-02 & 0.0000 \mathrm{E}+00 \\ -82.78 & 1.4141 \mathrm{E}-01 & 6.6325 \mathrm{E}-02 & 0.0000 \mathrm{E}+00 \\ -77.78 & 1.9558 \mathrm{E}-01 & 9.6145 \mathrm{E}-02 & 0.0000 \mathrm{E}+00 \\ -72.15 & 2.5778 \mathrm{E}-01 & 1.3507 \mathrm{E}-01 & 0.0000 \mathrm{E}+00 \\ -65.90 & 3.2110 \mathrm{E}-01 & 1.8521 \mathrm{E}-01 & 0.0000 \mathrm{E}+00 \\ -60.35 & 3.8730 \mathrm{E}-01 & 2.5394 \mathrm{E}-01 & 1.2496 \mathrm{E}-01 \\ -55.49 & 4.7094 \mathrm{E}-01 & 3.4124 \mathrm{E}-01 & 1.4140 \mathrm{E}-01 \\ -50.62 & 5.6506 \mathrm{E}-01 & 4.5232 \mathrm{E}-01 & 1.6198 \mathrm{E}-01 \\ -47.17 & 6.3758 \mathrm{E}-01 & 5.5391 \mathrm{E}-01 & 1.8137 \mathrm{E}-01 \\ -43.58 & 7.1271 \mathrm{E}-01 & 6.6746 \mathrm{E}-01 & 7.8746 \mathrm{E}-01 \\ -38.45 & 8.2412 \mathrm{E}-01 & 8.0671 \mathrm{E}-01 & 8.7193 \mathrm{E}-01 \\ -33.32 & 9.2848 \mathrm{E}-01 & 9.2525 \mathrm{E}-01 & 9.6975 \mathrm{E}-01 \\ -28.20 & 1.0197 \mathrm{E}+00 & 1.0228 \mathrm{E}+00 & 1.0021 \mathrm{E}+00 \\ -23.07 & 1.0962 \mathrm{E}+00 & 1.1027 \mathrm{E}+00 & 1.0751 \mathrm{E}+00 \\ -17.94 & 1.1561 \mathrm{E}+00 & 1.1640 \mathrm{E}+00 & 1.1326 \mathrm{E}+00 \\ -12.82 & 1.1981 \mathrm{E}+00 & 1.2060 \mathrm{E}+00 & 1.1417 \mathrm{E}+00 \\ -7.69 & 1.2225 \mathrm{E}+00 & 1.2306 \mathrm{E}+00 & 1.1646 \mathrm{E}+00 \\ -2.56 & 1.2290 \mathrm{E}+00 & 1.2374 \mathrm{E}+00 & 1.1706 \mathrm{E}+00 \\ 2.56 & 1.2177 \mathrm{E}+00 & 1.2266 \mathrm{E}+00 & 1.1698 \mathrm{E}+00 \\ 7.69 & 1.1884 \mathrm{E}+00 & 1.1980 \mathrm{E}+00 & 1.1418 \mathrm{E}+00 \\ 12.82 & 1.1420 \mathrm{E}+00 & 1.1523 \mathrm{E}+00 & 1.0976 \mathrm{E}+00 \\ 17.94 & 1.0798 \mathrm{E}+00 & 1.0910 \mathrm{E}+00 & 1.0835 \mathrm{E}+00 \\ 23.07 & 1.0017 \mathrm{E}+00 & 1.0122 \mathrm{E}+00 & 1.0060 \mathrm{E}+00 \\ 28.20 & 9.0963 \mathrm{E}-01 & 9.1764 \mathrm{E}-01 & 9.1493 \mathrm{E}-01 \\ 33.32 & 8.0612 \mathrm{E}-01 & 8.0923 \mathrm{E}-01 & 8.7003 \mathrm{E}-01 \\ 38.45 & 6.9260 \mathrm{E}-01 & 6.8436 \mathrm{E}-01 & 7.5430 \mathrm{E}-01 \\ 43.58 & 5.7522 \mathrm{E}-01 & 5.4573 \mathrm{E}-01 & 6.4611 \mathrm{E}-01 \\ 47.17 & 4.9989 \mathrm{E}-01 & 4.4378 \mathrm{E}-01 & 1.1625 \mathrm{E}-01 \\ 50.62 & 4.3307 \mathrm{E}-01 & 3.5864 \mathrm{E}-01 & 9.7242 \mathrm{E}-02 \\ 55.49 & 3.5171 \mathrm{E}-01 & 2.6871 \mathrm{E}-01 & 7.8223 \mathrm{E}-02 \\ 60.35 & 2.7839 \mathrm{E}-01 & 1.9662 \mathrm{E}-01 & 6.3172 \mathrm{E}-02 \\ 64.98 & 2.2728 \mathrm{E}-01 & 1.4936 \mathrm{E}-01 & 0.0000 \mathrm{E}+00 \\ 69.39 & 1.9332 \mathrm{E}-01 & 1.2140 \mathrm{E}-01 & 0.0000 \mathrm{E}+00 \\ 76.46 & 1.4431 \mathrm{E}-01 & 8.3768 \mathrm{E}-02 & 0.0000 \mathrm{E}+00 \\ 86.80 & 9.2547 \mathrm{E}-02 & 4.9108 \mathrm{E}-02 & 0.0000 \mathrm{E}+00 \\ 98.14 & 5.2789 \mathrm{E}-02 & 2.6260 \mathrm{E}-02 & 0.0000 \mathrm{E}+00 \\ 110.00 & 2.2354 \mathrm{E}-02 & 1.1518 \mathrm{E}-02 & 0.0000 \mathrm{E}+00\end{array}$


Table B11C.4. Axial Distribution of Total Flux, Fast Flux and Power in MFA-2 at BOC $11 \mathrm{C}$

\begin{tabular}{|c|c|c|c|}
\hline $\begin{array}{l}z(\mathrm{~cm}) \\
-97.78 \\
-92.78 \\
-87.78 \\
-82.78 \\
-77.78 \\
-72.15 \\
-65.90 \\
-60.35 \\
-55.49 \\
-50.62 \\
-47.17 \\
-43.58 \\
-38.45 \\
-33.32 \\
-28.20 \\
-23.07 \\
-17.94 \\
-12.82 \\
-7.69 \\
-2.56 \\
2.56 \\
7.69 \\
12.82 \\
17.94 \\
23.07 \\
28.20 \\
33.32 \\
38.45 \\
43.58 \\
47.17 \\
50.62 \\
55.49 \\
60.35 \\
64.98 \\
69.39 \\
76.46 \\
86.80 \\
98.14 \\
110.00\end{array}$ & $\begin{array}{l}\text { Total Flux } \\
2.7199 \mathrm{E}-02 \\
5.8357 \mathrm{E}-02 \\
9.3541 \mathrm{E}-02 \\
1.3554 \mathrm{E}-01 \\
1.8743 \mathrm{E}-01 \\
2.4742 \mathrm{E}-01 \\
3.0861 \mathrm{E}-01 \\
3.7232 \mathrm{E}-01 \\
4.5535 \mathrm{E}-01 \\
5.5041 \mathrm{E}-01 \\
6.2552 \mathrm{E}-01 \\
7.0421 \mathrm{E}-01 \\
8.1968 \mathrm{E}-01 \\
9.2704 \mathrm{E}-01 \\
1.0204 \mathrm{E}+00 \\
1.0986 \mathrm{E}+00 \\
1.1595 \mathrm{E}+00 \\
1.2022 \mathrm{E}+00 \\
1.2269 \mathrm{E}+00 \\
1.2332 \mathrm{E}+00 \\
1.2212 \mathrm{E}+00 \\
1.1907 \mathrm{E}+00 \\
1.1427 \mathrm{E}+00 \\
1.0787 \mathrm{E}+00 \\
9.9929 \mathrm{E}-01 \\
9.0659 \mathrm{E}-01 \\
8.0326 \mathrm{E}-01 \\
6.9080 \mathrm{E}-01 \\
5.7485 \mathrm{E}-01 \\
4.9892 \mathrm{E}-01 \\
4.2850 \mathrm{E}-01 \\
3.4099 \mathrm{E}-01 \\
2.6310 \mathrm{E}-01 \\
2.1241 \mathrm{E}-01 \\
1.8127 \mathrm{E}-01 \\
1.3665 \mathrm{E}-01 \\
8.8113 \mathrm{E}-02 \\
5.0253 \mathrm{E}-02 \\
2.1396 \mathrm{E}-02\end{array}$ & $\begin{array}{c}\text { Flux }>0.1 \mathrm{MeV} \\
1.3951 \mathrm{E}-02 \\
2.6933 \mathrm{E}-02 \\
4.3102 \mathrm{E}-02 \\
6.4326 \mathrm{E}-02 \\
9.2971 \mathrm{E}-02 \\
1.3038 \mathrm{E}-01 \\
1.7871 \mathrm{E}-01 \\
2.4544 \mathrm{E}-01 \\
3.3152 \mathrm{E}-01 \\
4.4228 \mathrm{E}-01 \\
5.4528 \mathrm{E}-01 \\
6.6107 \mathrm{E}-01 \\
8.0322 \mathrm{E}-01 \\
9.2412 \mathrm{E}-01 \\
1.0236 \mathrm{E}+00 \\
1.1049 \mathrm{E}+00 \\
1.1673 \mathrm{E}+00 \\
1.2100 \mathrm{E}+00 \\
1.2349 \mathrm{E}+00 \\
1.2415 \mathrm{E}+00 \\
1.2299 \mathrm{E}+00 \\
1.1999 \mathrm{E}+00 \\
1.1525 \mathrm{E}+00 \\
1.0893 \mathrm{E}+00 \\
1.0091 \mathrm{E}+00 \\
9.1401 \mathrm{E}-01 \\
8.0602 \mathrm{E}-01 \\
6.8267 \mathrm{E}-01 \\
5.4592 \mathrm{E}-01 \\
4.4257 \mathrm{E}-01 \\
3.5301 \mathrm{E}-01 \\
2.5796 \mathrm{E}-01 \\
1.8326 \mathrm{E}-01 \\
1.3728 \mathrm{E}-01 \\
1.1222 \mathrm{E}-01 \\
7.8678 \mathrm{E}-02 \\
4.6701 \mathrm{E}-02 \\
2.5129 \mathrm{E}-02 \\
1.1112 \mathrm{E}-02\end{array}$ & $\begin{array}{c}\text { Power } \\
0.0000 \mathrm{E}+00 \\
0.0000 \mathrm{E}+00 \\
0.0000 \mathrm{E}+00 \\
0.0000 \mathrm{E}+00 \\
0.0000 \mathrm{E}+00 \\
0.0000 \mathrm{E}+00 \\
0.0000 \mathrm{E}+00 \\
1.2047 \mathrm{E}-01 \\
1.3669 \mathrm{E}-01 \\
1.5708 \mathrm{E}-01 \\
1.7696 \mathrm{E}-01 \\
7.7838 \mathrm{E}-01 \\
8.6818 \mathrm{E}-01 \\
9.7042 \mathrm{E}-01 \\
1.0043 \mathrm{E}+00 \\
1.0794 \mathrm{E}+00 \\
1.1383 \mathrm{E}+00 \\
1.1465 \mathrm{E}+00 \\
1.1697 \mathrm{E}+00 \\
1.1756 \mathrm{E}+00 \\
1.1731 \mathrm{E}+00 \\
1.1438 \mathrm{E}+00 \\
1.0980 \mathrm{E}+00 \\
1.0811 \mathrm{E}+00 \\
1.0021 \mathrm{E}+00 \\
9.1028 \mathrm{E}-01 \\
8.6470 \mathrm{E}-01 \\
7.5043 \mathrm{E}-01 \\
6.4583 \mathrm{E}-01 \\
1.1928 \mathrm{E}-01 \\
9.9660 \mathrm{E}-02 \\
7.9650 \mathrm{E}-02 \\
6.3599 \mathrm{E}-02 \\
0.0000 \mathrm{E}+00 \\
0.0000 \mathrm{E}+00 \\
0.0000 \mathrm{E}+00 \\
0.0000 \mathrm{E}+00 \\
0.0000 \mathrm{E}+00 \\
0.0000 \mathrm{E}+00\end{array}$ \\
\hline
\end{tabular}


HNF-SD-FF-ANAL-009 Rev. 1

Table B11C.5. Fission Power Distribution by Pin in MFA-1 at BOC 11C CORE POSITION 1405

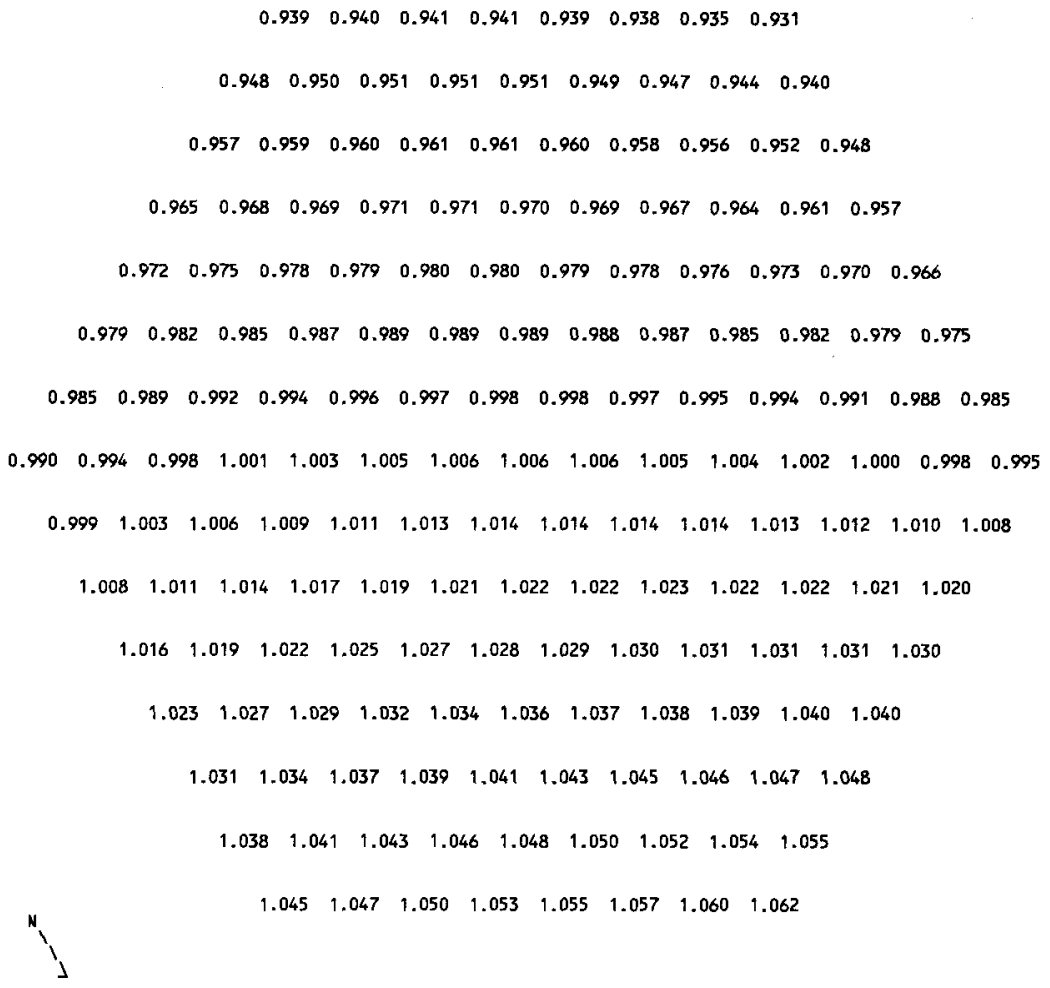


HNF-SD-FF-ANAL-009 Rev. I

Table B11C.6. Fission Power Distribution by Pin in MFA-2 at BOC $11 \mathrm{C}$ CORE POSITION 2506

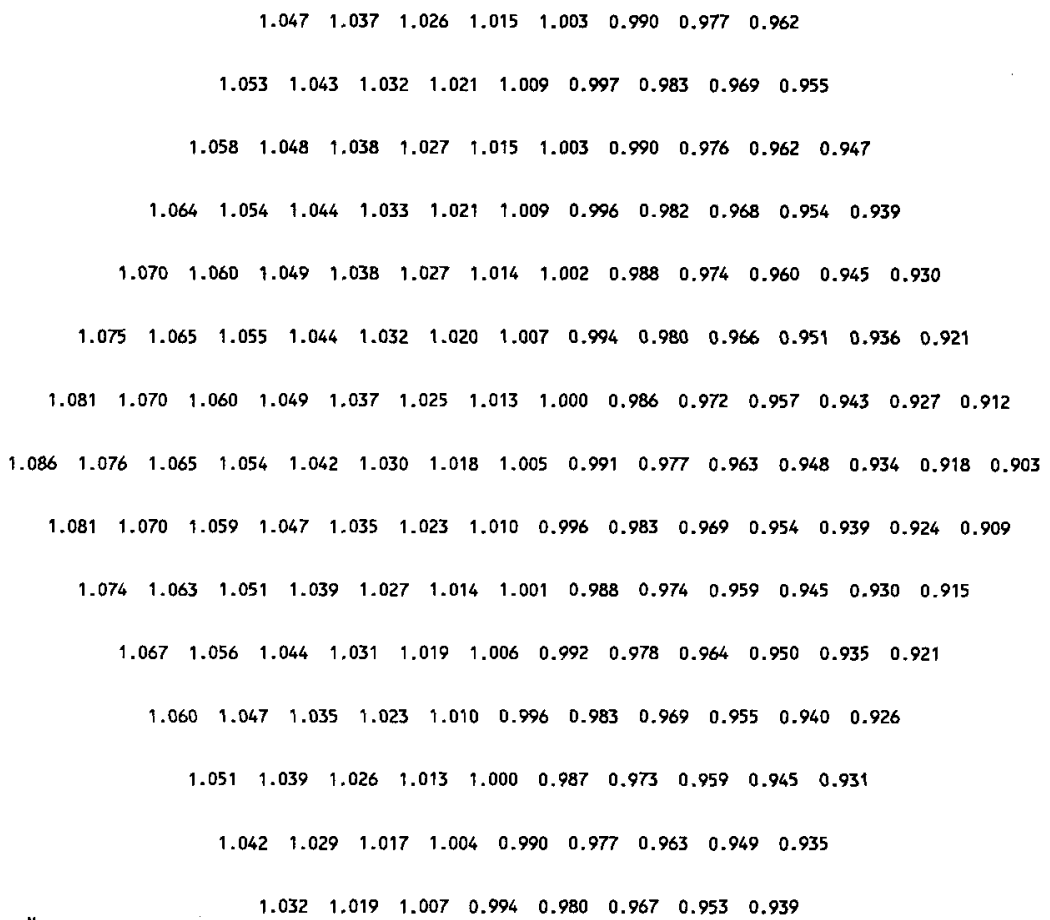


HNF-SD-FF-ANAL-009 Rev. 1

Table BllC.7. Fast Flux Distribution by Pin in MFA-1 at BOC $11 C$ CORE POSITION 1404

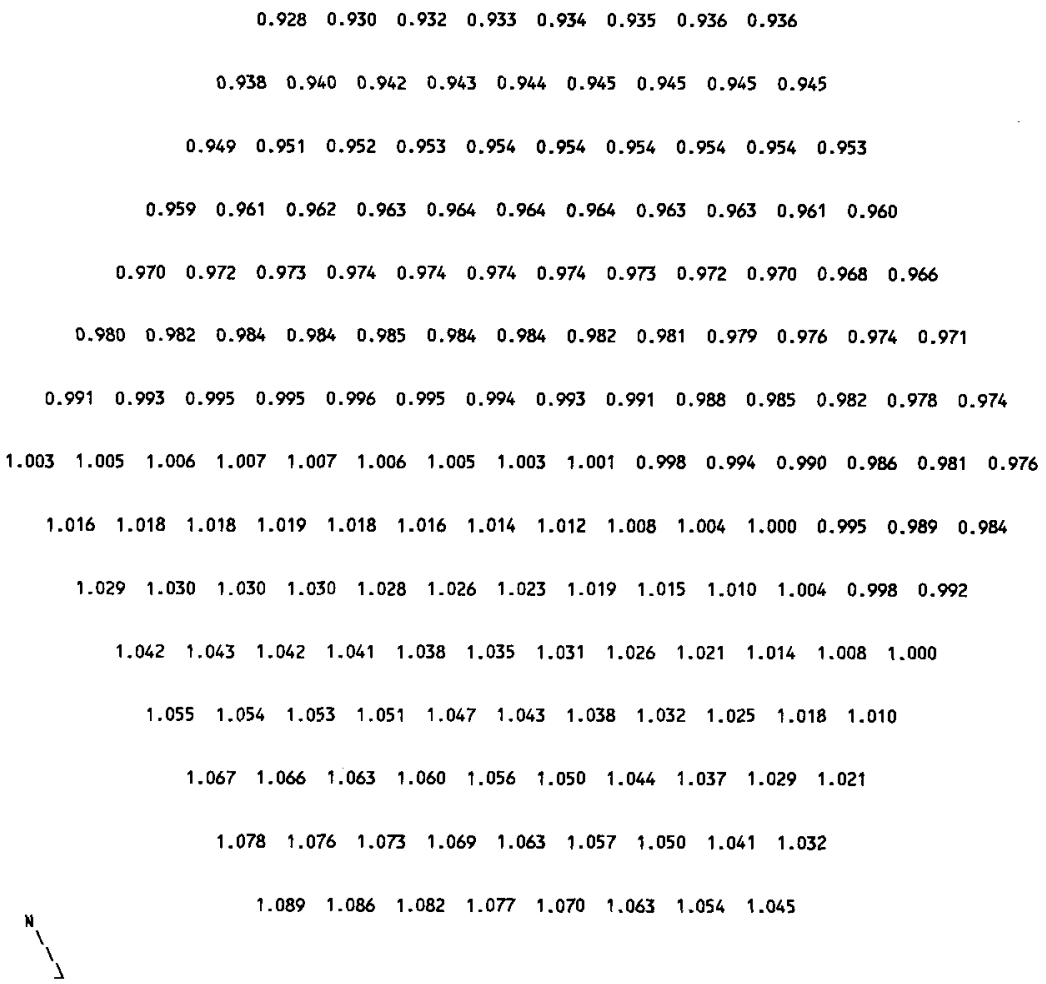


HNF-SD-FF-ANAL-009 Rev. 1

Table B11C.8. Fast Flux Distribution by Pin in MFA-2 at BOC $11 C$ CORE POSITION 2506

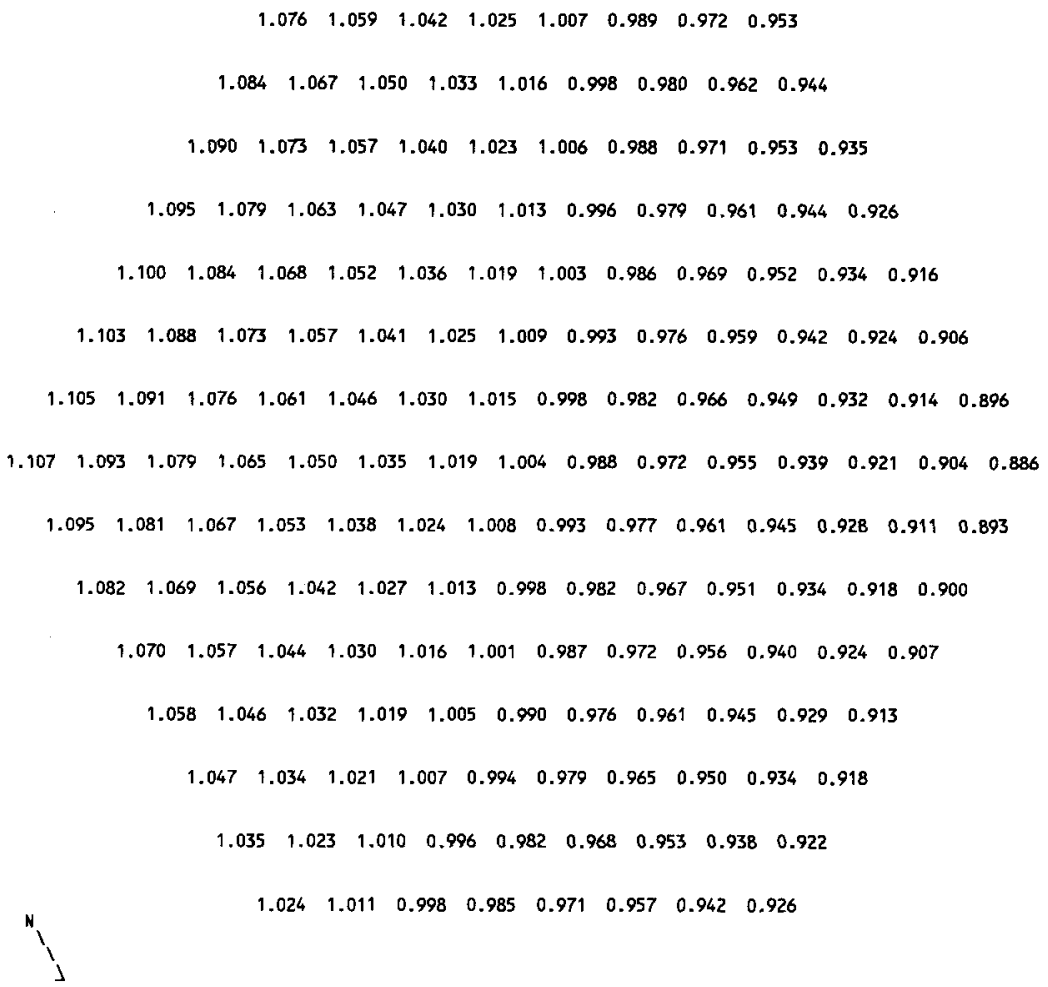


Table B11C.9. MFA-1 and MFA-2 Duct Wall

Fast Flux Data at BOC $11 \mathrm{C}$

$\begin{array}{ccc}\text { Side } & \begin{array}{c}\text { Flux }>0.1 \\ \text { MFA-1 }\end{array} & \begin{array}{c}\text { MeV } \\ \left(\mathrm{n} / \mathrm{cm}^{2}-\mathrm{sec}\right) \\ \text { MFA-2 }\end{array} \\ \text { E } & 2.3414 \mathrm{E}+15 & 2.1482 E+15 \\ \text { SE } & 2.1158 E+15 & 2.2345 E+15 \\ \text { SW } & 2.0395 E+15 & 2.0307 E+15 \\ \text { W } & 2.1254 E+15 & 1.8026 E+15 \\ \text { NW } & 2.2186 E+15 & 1.7797 E+15 \\ \text { NE } & 2.4182 E+15 & 1.9447 E+15\end{array}$

Table B11C.10. Assembly Outlet Temperatures and Flow Rates at BOC 11C

$\begin{array}{lcl} & \text { CALCULATED } \\ \text { CORE } & \text { OUTLET TEMP. } & \text { FLOW RATE } \\ \text { POS. } & \text { (DEG F) } & (\text { LB/H) } \\ 1405 & 1045 & 111070 \\ 1304 & 1056 & 194070 \\ 1404 & 1002 & 197790 \\ 1406 & 858 & 14150 \\ 1506 & 881 & 201110 \\ 1507 & 874 & 201110 \\ 1508 & 970 & 181030 \\ 2506 & 1019 & 111070 \\ 2404 & 1011 & 197790 \\ 2405 & 881 & 191240 \\ 2505 & 915 & 201110 \\ 2507 & 878 & 201110 \\ 2606 & 905 & 164920 \\ 2607 & 943 & 158170\end{array}$

Assembly flows are based on a calculated core pressure drop of 103.3 psi at a total reactor flow rate of $16.74 \mathrm{E}+06 \mathrm{lb} / \mathrm{hr}$. 
Table B11C.11. MFA-1 Sodium Subchannel Temperatures at Top of Core Elevation for BOC 11C

$$
\begin{aligned}
& z^{. \cdot} \cdot \\
& \text { 章 }
\end{aligned}
$$


Table B11C.12. MFA-2 Sodium Subchannel Temperatures at Top of Core Elevation for BOC 11C

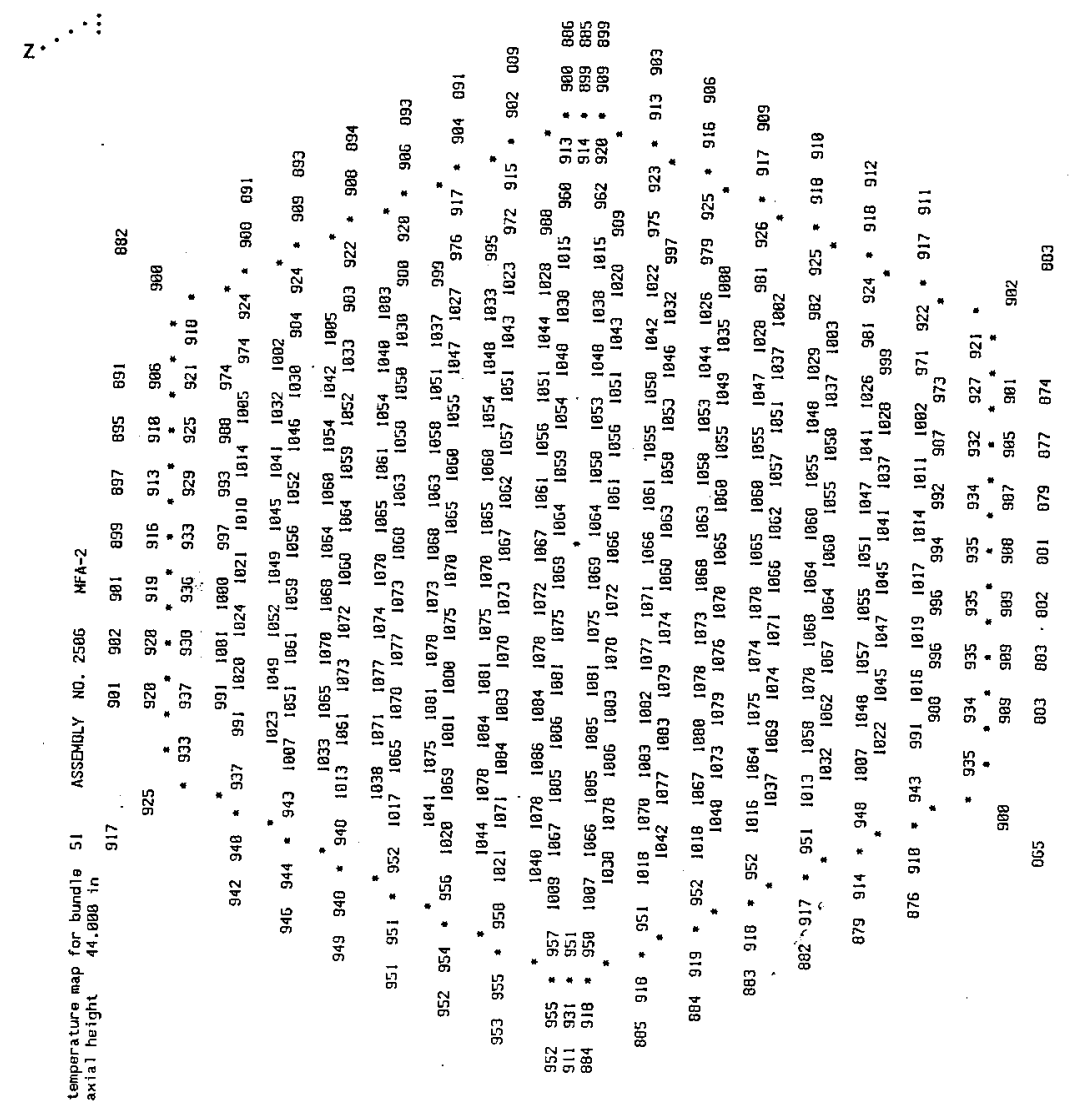


1 texperature map for bundle 24 ASSEMBLY NO. 1485 MFA-1

$\begin{array}{lllllllll}879 & 891 & 894 & 896 & 897 & 897 & 897 & 897 & 910\end{array}$

$912, *^{921} \cdot 924,925,925,925,925,925,933$

* $944950953954953952951951955 *$

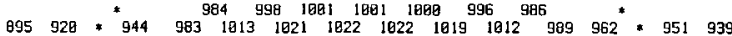

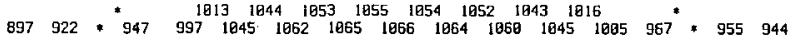

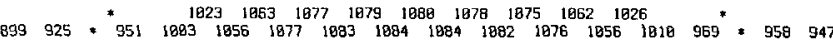

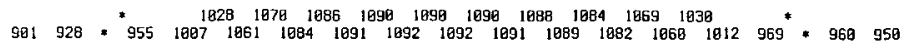

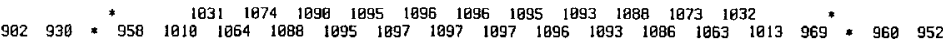

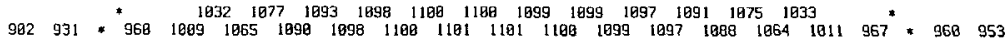

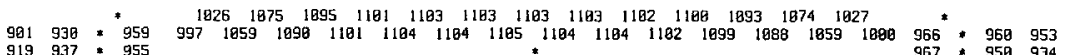

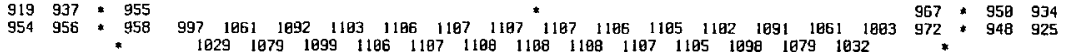

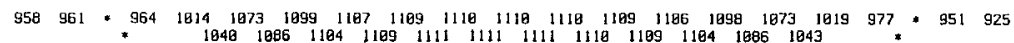

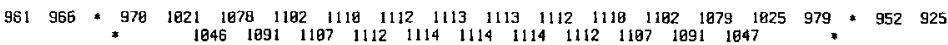

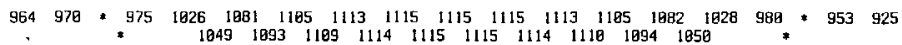

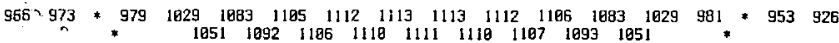

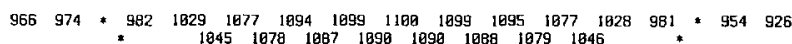

$966 \quad 974 * 981 \quad 1817 \quad 1846 \quad 1856 \quad 1858 \quad 1859 \quad 1858 \quad 1858 \quad 1818 \quad 981 * 956929$

옥죾

등

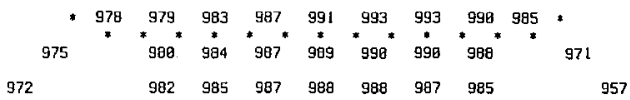


Table B11C.14. MFA-2 Sodium Subchannel Temperatures

at Elevation of Upper Axial Blanket for BOC 11C

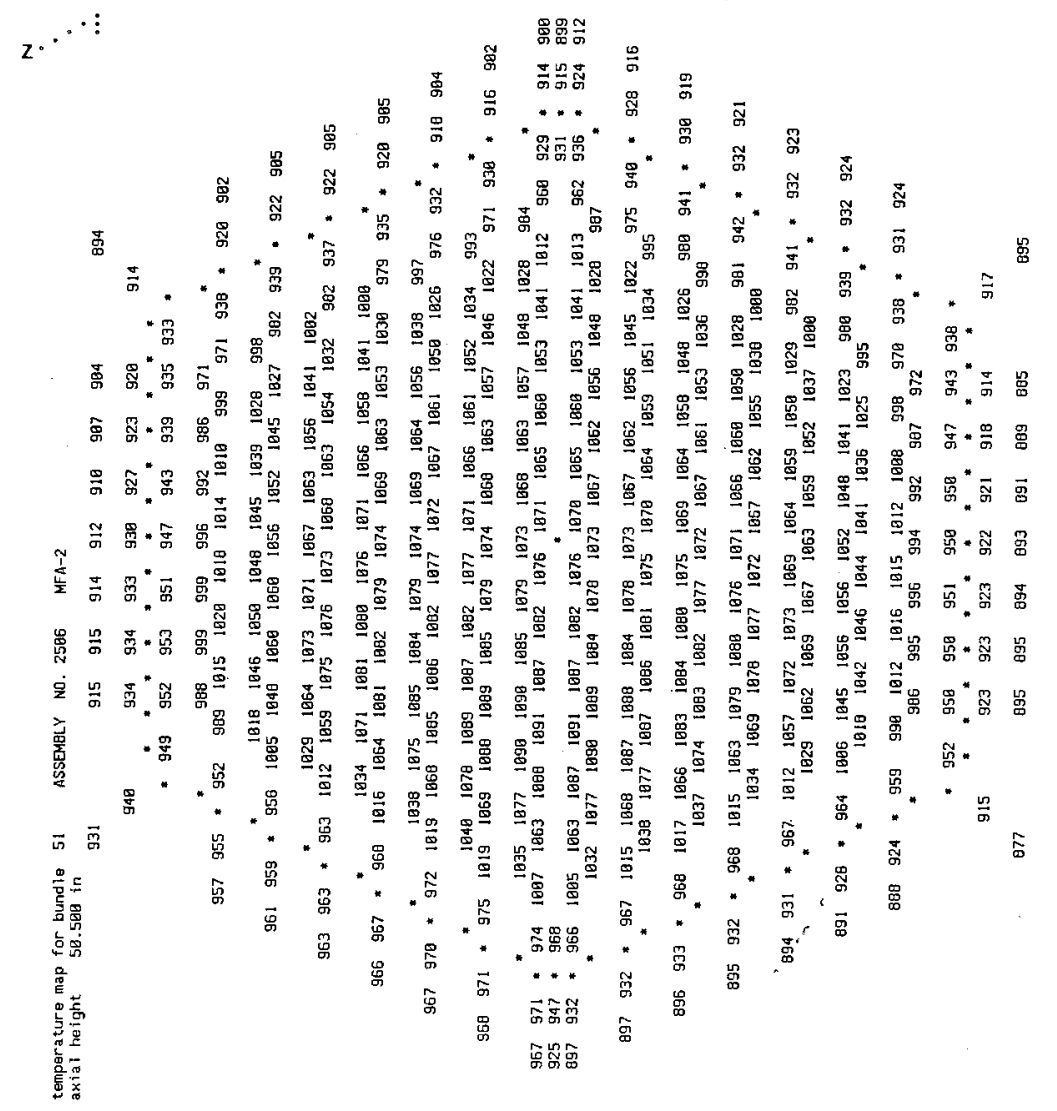


Table Bl1C.15. MFA-1 Sodium Subchannel Temperatures

at Top of Fuel Pin Bundle for BOC 11C

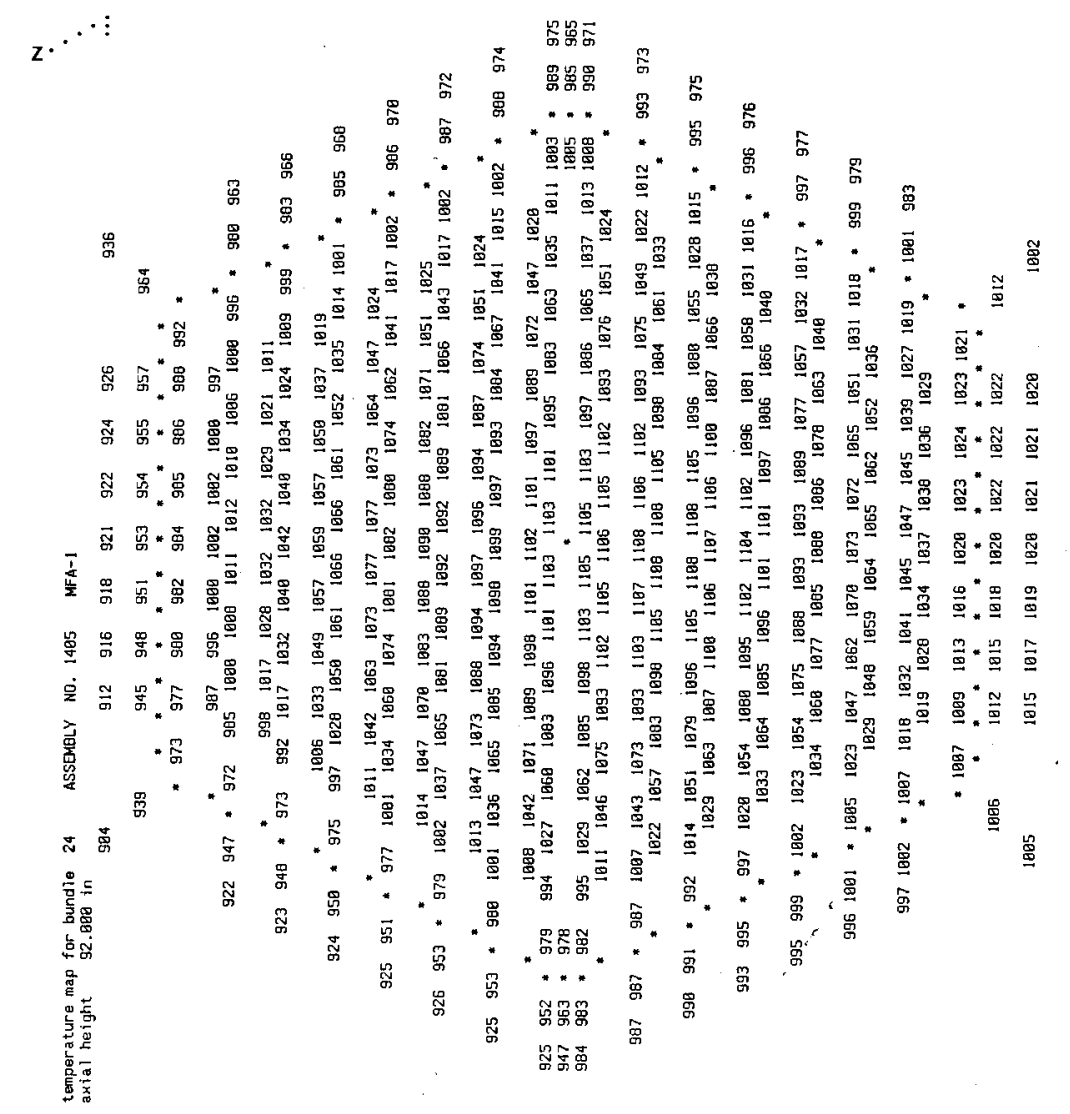


Table B11C.16. MFA-2 Sodium Subchanne1 Temperatures at Top of Fuel Pin Bundle for BOC $11 \mathrm{C}$

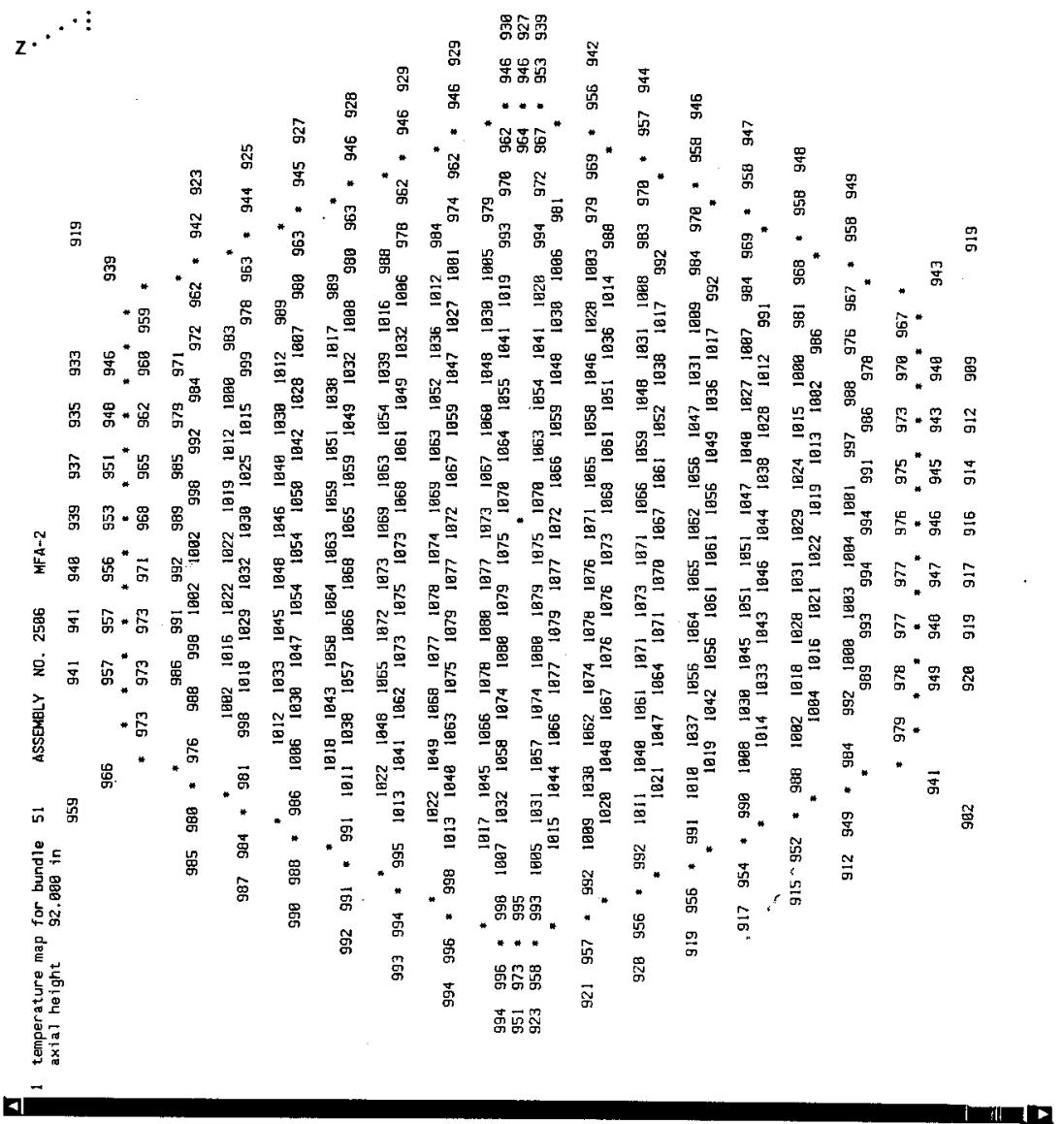


Table El1C.1. Fission Power Generated in MFA-1, MFA-2 and Neighboring Assemblies at EOC $11 \mathrm{C}$

\begin{tabular}{lcccc} 
CORE & $* * * * * *$ & \multicolumn{2}{c}{ POWER IN MEGAWATTS } & $* * * * * *$ \\
POS. & BELOW CORE & IN CORE & ABOVE CORE & TOTAL PWR \\
1405 & $1.016 \mathrm{E}-01$ & $3.569 \mathrm{E}+00$ & $6.232 \mathrm{E}-02$ & $3.733 \mathrm{E}+00$ \\
1304 & $0.000 \mathrm{E}+00$ & $6.699 \mathrm{E}+00$ & $0.000 \mathrm{E}+00$ & $6.699 \mathrm{E}+00$ \\
1406 & $0.000 \mathrm{E}+00$ & $0.000 \mathrm{E}+00$ & $0.000 \mathrm{E}+00$ & $0.000 \mathrm{E}+00$ \\
1404 & $0.000 \mathrm{E}+00$ & $5.895 \mathrm{E}+00$ & $0.000 \mathrm{E}+00$ & $5.895 \mathrm{E}+00$ \\
1508 & $0.000 \mathrm{E}+00$ & $4.950 \mathrm{E}+00$ & $0.000 \mathrm{E}+00$ & $4.950 \mathrm{E}+00$ \\
1506 & $1.334 \mathrm{E}-02$ & $3.578 \mathrm{E}+00$ & $9.476 \mathrm{E}-03$ & $3.600 \mathrm{E}+00$ \\
1507 & $1.091 \mathrm{E}-02$ & $3.478 \mathrm{E}+00$ & $8.437 \mathrm{E}-03$ & $3.497 \mathrm{E}+00$ \\
2506 & $9.041 \mathrm{E}-02$ & $3.301 \mathrm{E}+00$ & $5.853 \mathrm{E}-02$ & $3.450 \mathrm{E}+00$ \\
2404 & $0.000 \mathrm{E}+00$ & $6.027 \mathrm{E}+00$ & $0.000 \mathrm{E}+00$ & $6.027 \mathrm{E}+00$ \\
2505 & $8.136 \mathrm{E}-03$ & $4.421 \mathrm{E}+00$ & $5.964 \mathrm{E}-03$ & $4.435 \mathrm{E}+00$ \\
2405 & $1.705 \mathrm{E}-02$ & $3.517 \mathrm{E}+00$ & $1.223 \mathrm{E}-02$ & $3.546 \mathrm{E}+00$ \\
2606 & $1.458 \mathrm{E}-02$ & $3.374 \mathrm{E}+00$ & $9.055 \mathrm{E}-03$ & $3.398 \mathrm{E}+00$ \\
2607 & $6.212 \mathrm{E}-03$ & $3.780 \mathrm{E}+00$ & $5.389 \mathrm{E}-03$ & $3.792 \mathrm{E}+00$ \\
2507 & $1.218 \mathrm{E}-02$ & $3.544 \mathrm{E}+00$ & $9.180 \mathrm{E}-03$ & $3.566 \mathrm{E}+00$
\end{tabular}

Table EllC.2. Assembly Averaged Total and Fast Flux in MFA-1 and MFA-2 at EOC $11 \mathrm{C}$

\begin{tabular}{|c|c|c|c|}
\hline & Cor & Flux & $\left.m^{2}-s e c\right)$ \\
\hline $\begin{array}{l}\text { Assembly } \\
\text { MFA-1 } \\
\text { MFA-2 }\end{array}$ & $\begin{array}{l}\text { Pos. } \\
1405 \\
2506\end{array}$ & $\begin{array}{c}\text { Total } \\
3.421 \mathrm{E}+15 \\
3.141 \mathrm{E}+15\end{array}$ & $\begin{array}{l}>0.1 \mathrm{MeV} \\
2.249 \mathrm{E}+15 \\
2.017 \mathrm{E}+15\end{array}$ \\
\hline
\end{tabular}


Table E11C.3. Axial Distribution of Total Flux, Fast Flux and Power in MFA-1 at EOC $11 \mathrm{C}$

\begin{tabular}{|c|c|c|c|}
\hline $\begin{array}{r}z(c m) \\
-97.78 \\
-92.78 \\
-87.78 \\
-82.78 \\
-77.78 \\
-72.15 \\
-65.90 \\
-60.35 \\
-55.49 \\
-50.62 \\
-47.17 \\
-43.58 \\
-38.45 \\
-33.32 \\
-28.20 \\
-23.07 \\
-17.94 \\
-12.82 \\
-7.69 \\
-2.56 \\
2.56 \\
7.69 \\
12.82 \\
17.94 \\
23.07 \\
28.20 \\
33.32 \\
38.45 \\
43.58 \\
47.17 \\
50.62 \\
55.49 \\
60.35 \\
64.98 \\
69.39 \\
76.46 \\
86.80 \\
98.14 \\
110.00\end{array}$ & $\begin{array}{l}\text { Total Flux } \\
2.7431 \mathrm{E}-02 \\
5.8969 \mathrm{E}-02 \\
9.4648 \mathrm{E}-02 \\
1.3729 \mathrm{E}-01 \\
1.8992 \mathrm{E}-01 \\
2.5037 \mathrm{E}-01 \\
3.1191 \mathrm{E}-01 \\
3.7620 \mathrm{E}-01 \\
4.5756 \mathrm{E}-01 \\
5.4914 \mathrm{E}-01 \\
6.1973 \mathrm{E}-01 \\
6.9285 \mathrm{E}-01 \\
8.0176 \mathrm{E}-01 \\
9.0435 \mathrm{E}-01 \\
9.9474 \mathrm{E}-01 \\
1.0718 \mathrm{E}+00 \\
1.1335 \mathrm{E}+00 \\
1.1788 \mathrm{E}+00 \\
1.2081 \mathrm{E}+00 \\
1.2207 \mathrm{E}+00 \\
1.2165 \mathrm{E}+00 \\
1.1950 \mathrm{E}+00 \\
1.1563 \mathrm{E}+00 \\
1.1010 \mathrm{E}+00 \\
1.0280 \mathrm{E}+00 \\
9.3889 \mathrm{E}-01 \\
8.3604 \mathrm{E}-01 \\
7.2101 \mathrm{E}-01 \\
6.0061 \mathrm{E}-01 \\
5.2289 \mathrm{E}-01 \\
4.5375 \mathrm{E}-01 \\
3.6910 \mathrm{E}-01 \\
2.9253 \mathrm{E}-01 \\
2.3912 \mathrm{E}-01 \\
2.0354 \mathrm{E}-01 \\
1.5208 \mathrm{E}-01 \\
9.7586 \mathrm{E}-02 \\
5.5653 \mathrm{E}-02 \\
2.3537 \mathrm{E}-02\end{array}$ & $\begin{array}{c}\text { Flux }>0.1 \mathrm{MeV} \\
1.3900 \mathrm{E}-02 \\
2.6927 \mathrm{E}-02 \\
4.3220 \mathrm{E}-02 \\
6.4701 \mathrm{E}-02 \\
9.3803 \mathrm{E}-02 \\
1.3180 \mathrm{E}-01 \\
1.8081 \mathrm{E}-01 \\
2.4819 \mathrm{E}-01 \\
3.3328 \mathrm{E}-01 \\
4.4121 \mathrm{E}-01 \\
5.3974 \mathrm{E}-01 \\
6.4982 \mathrm{E}-01 \\
7.8556 \mathrm{E}-01 \\
9.0166 \mathrm{E}-01 \\
9.9790 \mathrm{E}-01 \\
1.0779 \mathrm{E}+00 \\
1.1408 \mathrm{E}+00 \\
1.1859 \mathrm{E}+00 \\
1.2152 \mathrm{E}+00 \\
1.2281 \mathrm{E}+00 \\
1.2247 \mathrm{E}+00 \\
1.2040 \mathrm{E}+00 \\
1.1663 \mathrm{E}+00 \\
1.1123 \mathrm{E}+00 \\
1.0389 \mathrm{E}+00 \\
9.4747 \mathrm{E}-01 \\
8.3989 \mathrm{E}-01 \\
7.1316 \mathrm{E}-01 \\
5.7049 \mathrm{E}-01 \\
4.6484 \mathrm{E}-01 \\
3.7651 \mathrm{E}-01 \\
2.8272 \mathrm{E}-01 \\
2.0721 \mathrm{E}-01 \\
1.5748 \mathrm{E}-01 \\
1.2806 \mathrm{E}-01 \\
8.8444 \mathrm{E}-02 \\
5.1887 \mathrm{E}-02 \\
2.7753 \mathrm{E}-02 \\
1.2164 \mathrm{E}-02 \\
\end{array}$ & $\begin{array}{l}\text { Power } \\
0.0000 \mathrm{E}+00 \\
0.0000 \mathrm{E}+00 \\
0.0000 \mathrm{E}+00 \\
0.0000 \mathrm{E}+00 \\
0.0000 \mathrm{E}+00 \\
0.0000 \mathrm{E}+00 \\
0.0000 \mathrm{E}+00 \\
1.3481 \mathrm{E}-01 \\
1.5185 \mathrm{E}-01 \\
1.7281 \mathrm{E}-01 \\
1.9206 \mathrm{E}-01 \\
7.6828 \mathrm{E}-01 \\
8.5165 \mathrm{E}-01 \\
9.4829 \mathrm{E}-01 \\
9.7563 \mathrm{E}-01 \\
1.0490 \mathrm{E}+00 \\
1.1081 \mathrm{E}+00 \\
1.1179 \mathrm{E}+00 \\
1.1451 \mathrm{E}+00 \\
1.1570 \mathrm{E}+00 \\
1.1639 \mathrm{E}+00 \\
1.1435 \mathrm{E}+00 \\
1.1070 \mathrm{E}+00 \\
1.1052 \mathrm{E}+00 \\
1.0329 \mathrm{E}+00 \\
9.4488 \mathrm{E}-01 \\
9.0962 \mathrm{E}-01 \\
7.9166 \mathrm{E}-01 \\
6.8027 \mathrm{E}-01 \\
1.3186 \mathrm{E}-01 \\
1.1144 \mathrm{E}-01 \\
9.0575 \mathrm{E}-02 \\
7.3750 \mathrm{E}-02 \\
0.0000 \mathrm{E}+00 \\
0.0000 \mathrm{E}+00 \\
0.0000 \mathrm{E}+00 \\
0.0000 \mathrm{E}+00 \\
0.0000 \mathrm{E}+00 \\
0.0000 \mathrm{E}+00\end{array}$ \\
\hline
\end{tabular}


Table E11C.4. Axial Distribution of Total Flux, Fast Flux and Power in MFA-2 at EOC $11 \mathrm{C}$

$\begin{array}{rccc}\text { z }(\mathrm{cm}) & \text { Total Flux } & \text { Flux }>0.1 \text { MeV } & \text { Power } \\ -97.78 & 2.6358 \mathrm{E}-02 & 1.3579 \mathrm{E}-02 & 0.0000 \mathrm{E}+00 \\ -92.78 & 5.6564 \mathrm{E}-02 & 2.6218 \mathrm{E}-02 & 0.0000 \mathrm{E}+00 \\ -87.78 & 9.0678 \mathrm{E}-02 & 4.1959 \mathrm{E}-02 & 0.0000 \mathrm{E}+00 \\ -82.78 & 1.3142 \mathrm{E}-01 & 6.2627 \mathrm{E}-02 & 0.0000 \mathrm{E}+00 \\ -77.78 & 1.8177 \mathrm{E}-01 & 9.0523 \mathrm{E}-02 & 0.0000 \mathrm{E}+00 \\ -72.15 & 2.3999 \mathrm{E}-01 & 1.2698 \mathrm{E}-01 & 0.0000 \mathrm{E}+00 \\ -65.90 & 2.9935 \mathrm{E}-01 & 1.7413 \mathrm{E}-01 & 0.0000 \mathrm{E}+00 \\ -60.35 & 3.6110 \mathrm{E}-01 & 2.3944 \mathrm{E}-01 & 1.2907 \mathrm{E}-01 \\ -55.49 & 4.4170 \mathrm{E}-01 & 3.2321 \mathrm{E}-01 & 1.4578 \mathrm{E}-01 \\ -50.62 & 5.3397 \mathrm{E}-01 & 4.3069 \mathrm{E}-01 & 1.6639 \mathrm{E}-01 \\ -47.17 & 6.0687 \mathrm{E}-01 & 5.3042 \mathrm{E}-01 & 1.8604 \mathrm{E}-01 \\ -43.58 & 6.8331 \mathrm{E}-01 & 6.4246 \mathrm{E}-01 & 7.5773 \mathrm{E}-01 \\ -38.45 & 7.9588 \mathrm{E}-01 & 7.8065 \mathrm{E}-01 & 8.4607 \mathrm{E}-01 \\ -33.32 & 9.0111 \mathrm{E}-01 & 8.9875 \mathrm{E}-01 & 9.4680 \mathrm{E}-01 \\ -28.20 & 9.9340 \mathrm{E}-01 & 9.9655 \mathrm{E}-01 & 9.7575 \mathrm{E}-01 \\ -23.07 & 1.0719 \mathrm{E}+00 & 1.0779 \mathrm{E}+00 & 1.0510 \mathrm{E}+00 \\ -17.94 & 1.1346 \mathrm{E}+00 & 1.1418 \mathrm{E}+00 & 1.1116 \mathrm{E}+00 \\ -12.82 & 1.1807 \mathrm{E}+00 & 1.1876 \mathrm{E}+00 & 1.1207 \mathrm{E}+00 \\ -7.69 & 1.2104 \mathrm{E}+00 & 1.2173 \mathrm{E}+00 & 1.1485 \mathrm{E}+00 \\ -2.56 & 1.2232 \mathrm{E}+00 & 1.2305 \mathrm{E}+00 & 1.1606 \mathrm{E}+00 \\ 2.56 & 1.2191 \mathrm{E}+00 & 1.2270 \mathrm{E}+00 & 1.1666 \mathrm{E}+00 \\ 7.69 & 1.1974 \mathrm{E}+00 & 1.2060 \mathrm{E}+00 & 1.1460 \mathrm{E}+00 \\ 12.82 & 1.1584 \mathrm{E}+00 & 1.1679 \mathrm{E}+00 & 1.1090 \mathrm{E}+00 \\ 17.94 & 1.1026 \mathrm{E}+00 & 1.1132 \mathrm{E}+00 & 1.1055 \mathrm{E}+00 \\ 23.07 & 1.0290 \mathrm{E}+00 & 1.0392 \mathrm{E}+00 & 1.0325 \mathrm{E}+00 \\ 28.20 & 9.3936 \mathrm{E}-01 & 9.4733 \mathrm{E}-01 & 9.4374 \mathrm{E}-01 \\ 33.32 & 8.3626 \mathrm{E}-01 & 8.3977 \mathrm{E}-01 & 9.0692 \mathrm{E}-01 \\ 38.45 & 7.2160 \mathrm{E}-01 & 7.1388 \mathrm{E}-01 & 7.8973 \mathrm{E}-01 \\ 43.58 & 6.0192 \mathrm{E}-01 & 5.7237 \mathrm{E}-01 & 6.8131 \mathrm{E}-01 \\ 47.17 & 5.2306 \mathrm{E}-01 & 4.6481 \mathrm{E}-01 & 1.3488 \mathrm{E}-01 \\ 50.62 & 4.4982 \mathrm{E}-01 & 3.7143 \mathrm{E}-01 & 1.1380 \mathrm{E}-01 \\ 55.49 & 3.5840 \mathrm{E}-01 & 2.7193 \mathrm{E}-01 & 9.1861 \mathrm{E}-02 \\ 60.35 & 2.7686 \mathrm{E}-01 & 1.9345 \mathrm{E}-01 & 7.3897 \mathrm{E}-02 \\ 64.98 & 2.2379 \mathrm{E}-01 & 1.4494 \mathrm{E}-01 & 0.0000 \mathrm{E}+00 \\ 69.39 & 1.9109 \mathrm{E}-01 & 1.1851 \mathrm{E}-01 & 0.0000 \mathrm{E}+00 \\ 76.46 & 1.4416 \mathrm{E}-01 & 8.3133 \mathrm{E}-02 & 0.0000 \mathrm{E}+00 \\ 86.80 & 9.2981 \mathrm{E}-02 & 4.9364 \mathrm{E}-02 & 0.0000 \mathrm{E}+00 \\ 98.14 & 5.3002 \mathrm{E}-02 & 2.6558 \mathrm{E}-02 & 0.0000 \mathrm{E}+00 \\ 110.00 & 2.2515 \mathrm{E}-02 & 1.1724 \mathrm{E}-02 & 0.0000 \mathrm{E}+00\end{array}$




\section{HNF-SD-FF-ANAL-009 Rev. 1}

Table E11C.5. Fission Power Distribution by Pin in MFA-1 at EOC $11 \mathrm{C}$ CORE POSITION 1405

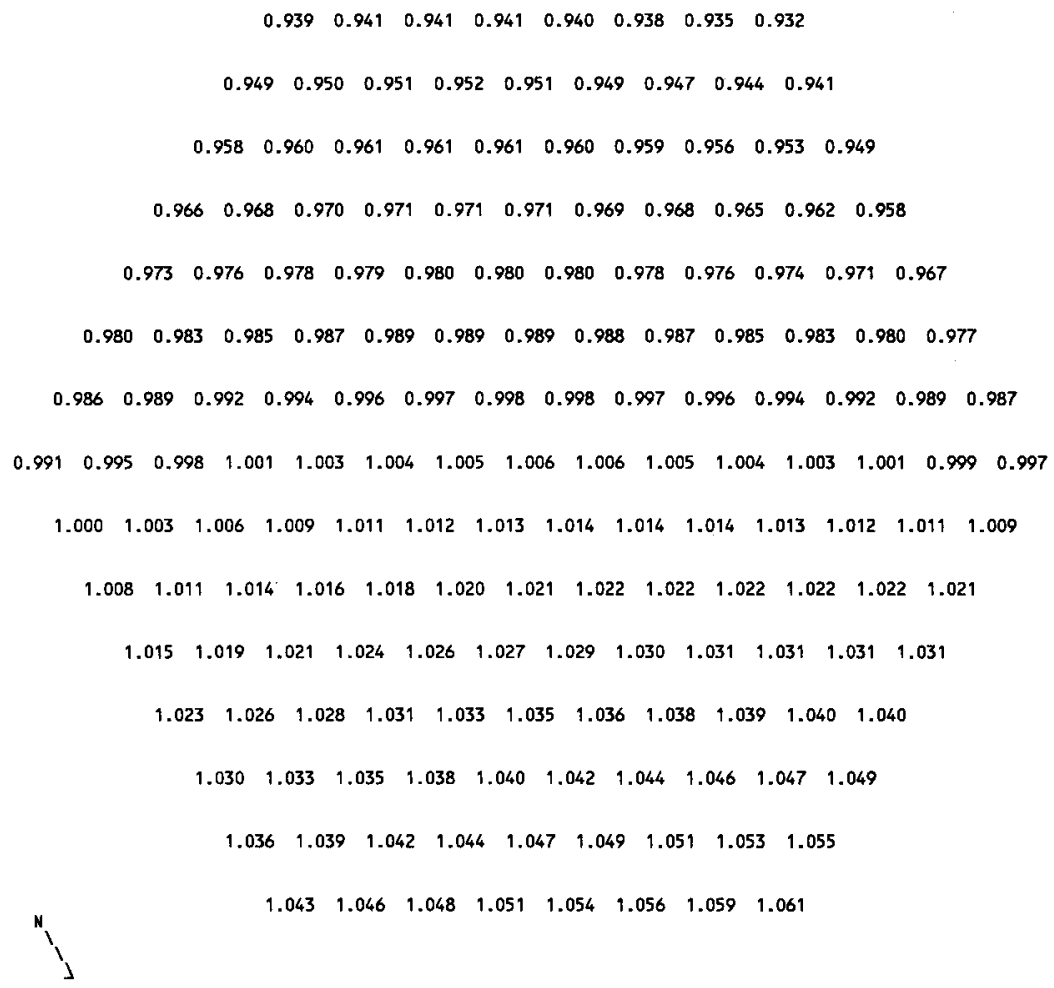


HNF-SD-FF-ANAL-009 Rev. 1

Table El1C.6. Fission Power Distribution by Pin in MFA-2 at EOC 11C CORE POSITION 2506

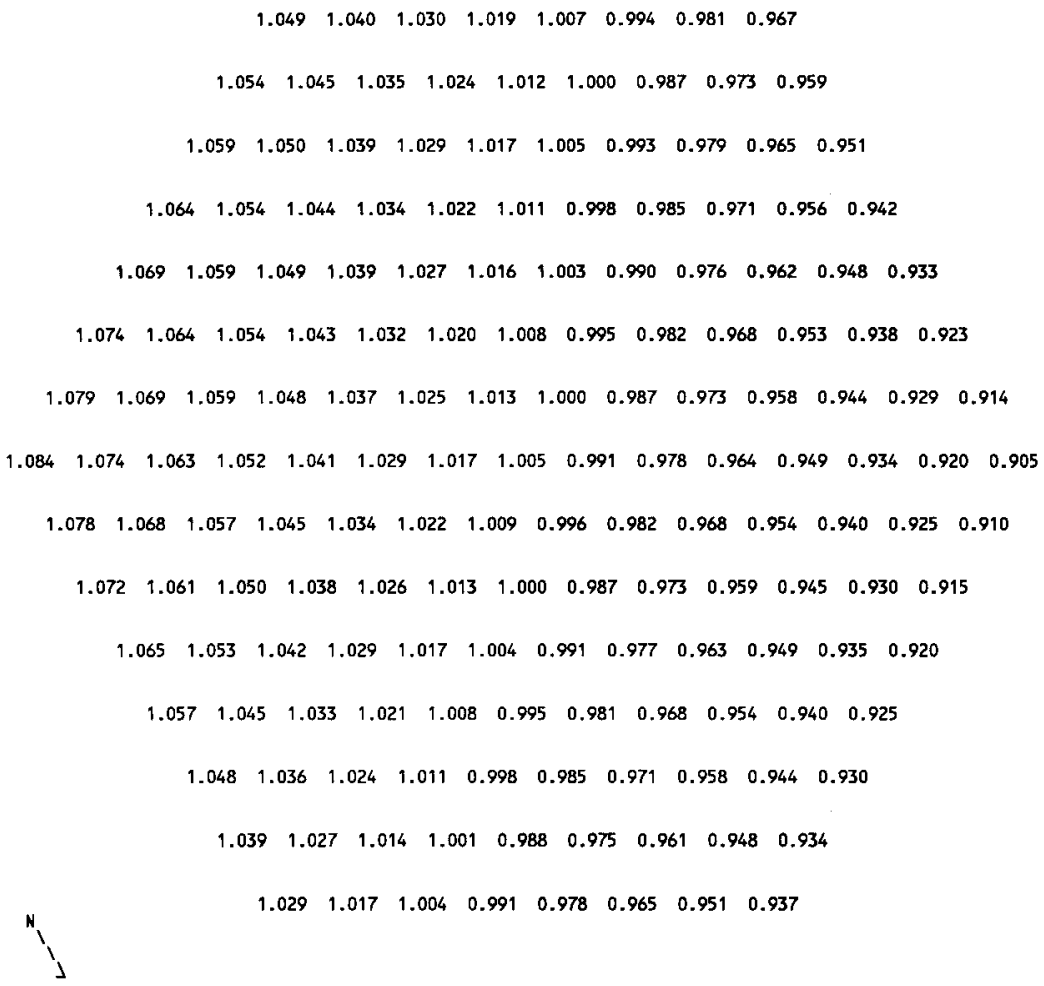


HNF-SD-FF-ANAL-009 Rev. 1

Table EllC.7. Fast Flux Distribution by Pin in MFA-1 at EOC 11C CORE POSITION 1604

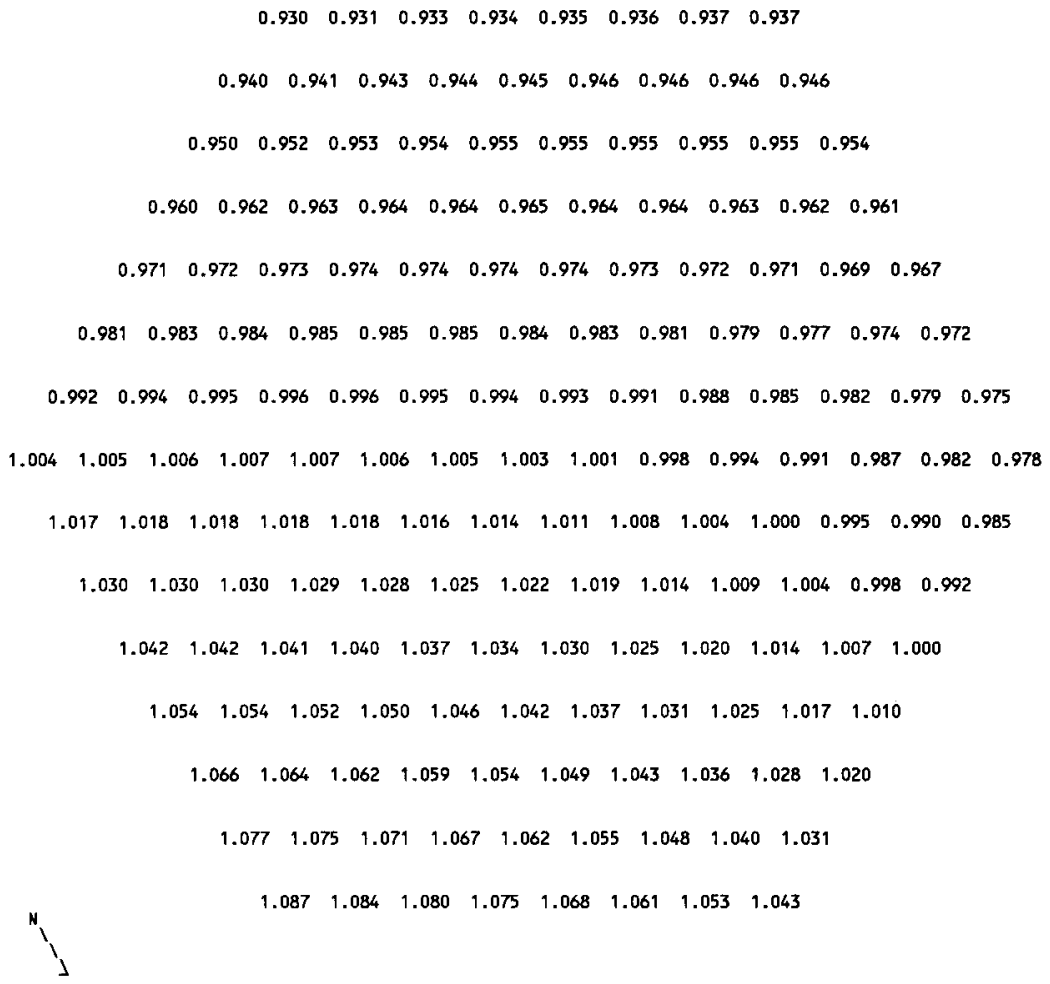


HNF-SD-FF-ANAL-009 Rev. 1

Table E11C.8. Fast Flux Distribution by Pin in MFA-2 at EOC 11C CORE POSITION 2506

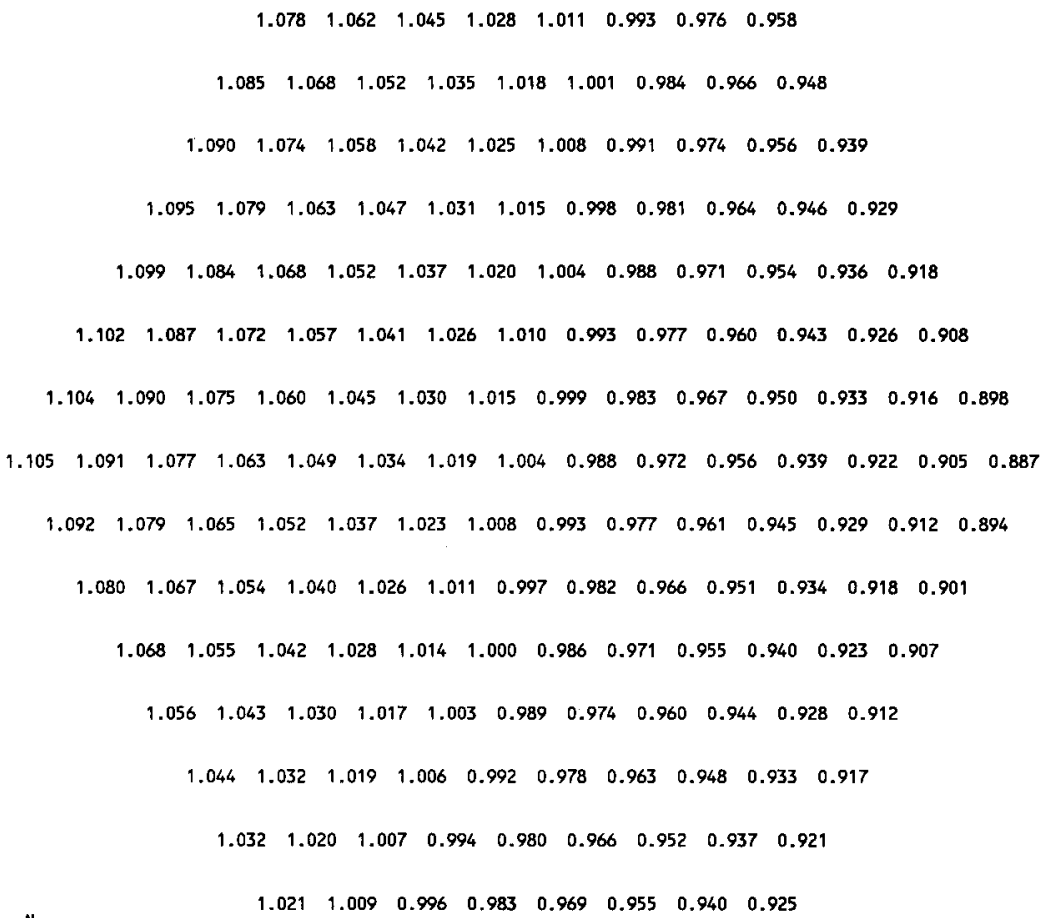


Table E11C.9. MFA-1 and MFA-2 Duct Wall

Fast Flux Data at EOC 11C

$\begin{array}{ccc}\text { Side } & \begin{array}{c}\text { Flux }>0.1 \\ \text { MFA-1 }\end{array} & \begin{array}{c}\text { MeV }\left(\mathrm{n} / \mathrm{cm}^{2}-\mathrm{sec}\right) \\ \text { MFA-2 }\end{array} \\ \text { E } & 2.3584 E+15 & 2.1559 \mathrm{E}+15 \\ \text { SE } & 2.1348 E+15 & 2.2469 \mathrm{E}+15 \\ \text { SW } & 2.0568 E+15 & 2.0527 \mathrm{E}+15 \\ \text { W } & 2.14444 \mathrm{1}+15 & 1.8210 \mathrm{E}+15 \\ \text { NW } & 2.2356 \mathrm{E}+15 & 1.7901 \mathrm{E}+15 \\ \text { NE } & 2.4305 \mathrm{E}+15 & 1.9524 \mathrm{E}+15\end{array}$

Table E11C.10. Assembly Outlet Temperatures and Flow Rates at EOC 11C

$\begin{array}{lcl} & \text { CALCULATED } \\ \text { CORE } & \text { OUTLET TEMP. } & \text { FLOW RATE } \\ \text { POS. } & \text { (DEG F) } & \text { (LB/H) } \\ 1405 & 1035 & 111070 \\ 1304 & 1040 & 194070 \\ 1404 & 993 & 197790 \\ 1406 & 855 & 14150 \\ 1506 & 877 & 201110 \\ 1507 & 869 & 201110 \\ 1508 & 964 & 181030 \\ 2506 & 1013 & 111070 \\ 2404 & 1001 & 197790 \\ 2405 & 876 & 191240 \\ 2505 & 918 & 201110 \\ 2507 & 872 & 201110 \\ 2606 & 905 & 164920 \\ 2607 & 937 & 158170\end{array}$

Assembly flows are based on a calculated core pressure drop of $103.3 \mathrm{psi}$ at a total reactor flow rate of $16.74 \mathrm{E}+06 \mathrm{lb} / \mathrm{hr}$. 
Table E11C.11. MFA-1 Sodium Subchannel Temperatures at Top of Core Elevation for EOC $11 \mathrm{C}$

$$
\begin{aligned}
& { }_{z} . \cdot \cdot
\end{aligned}
$$

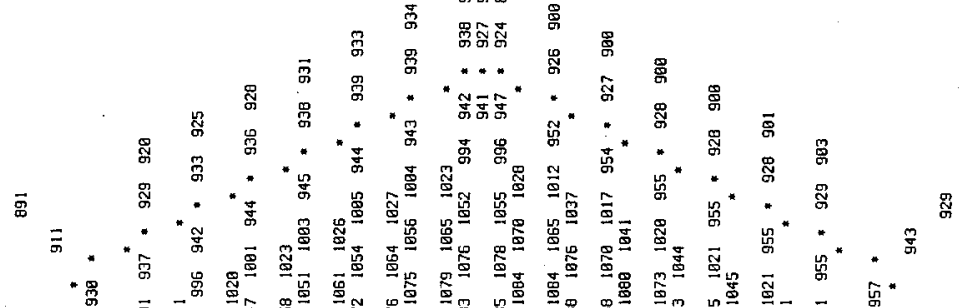

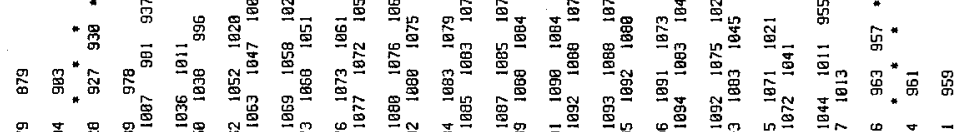

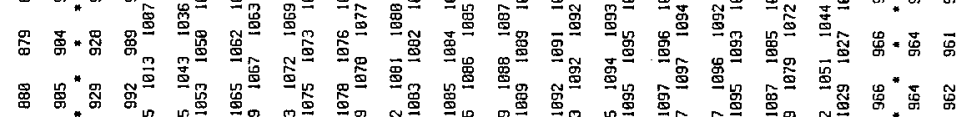

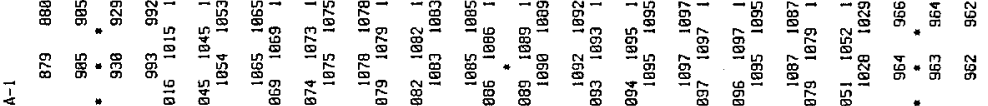

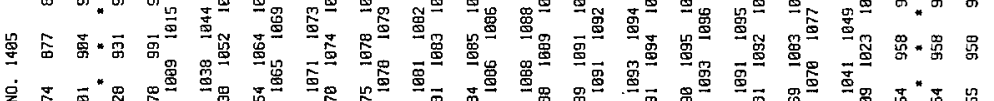

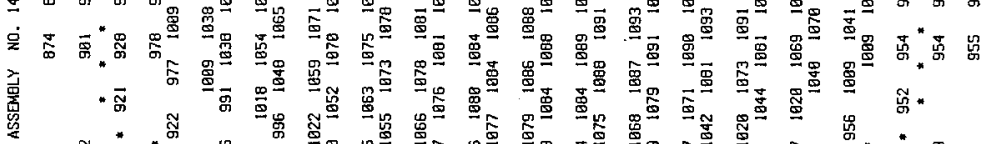

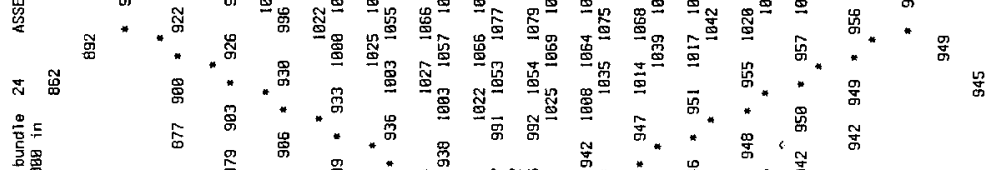

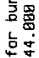

$$
\begin{aligned}
& \text { 迹离 }
\end{aligned}
$$

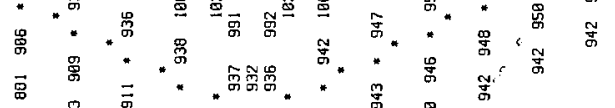


Table El1C.12. MFA-2 Sodium Subchannel Temperatures at Top of Core Elevation for EOC 11C

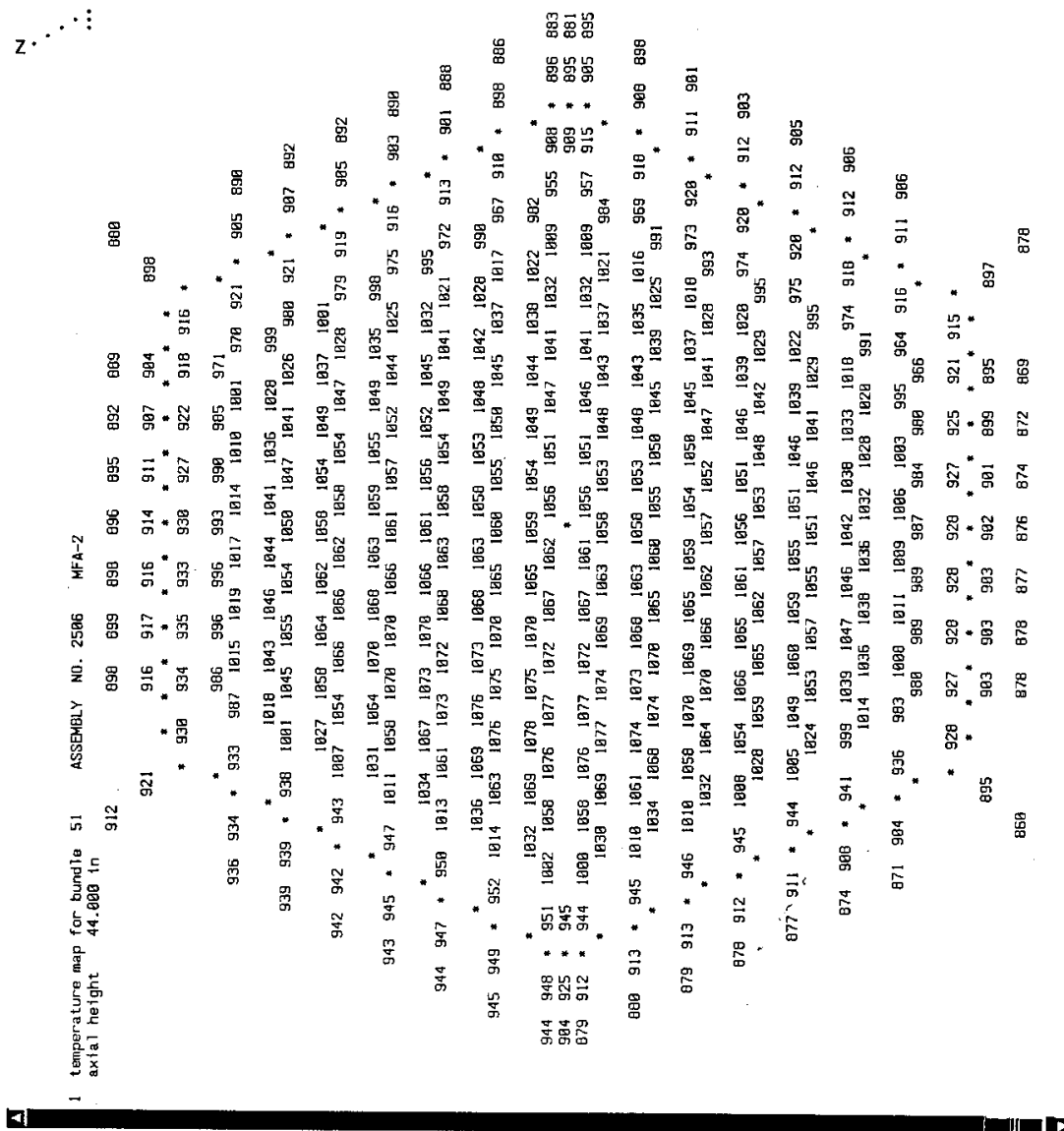


Table EllC.13. MFA-1 Sodium Subchannel Temperatures at Elevation of Upper Axial Blanket for EOC 11C

$$
\begin{aligned}
& z \cdot \cdot \cdot \\
& \text { s. }
\end{aligned}
$$

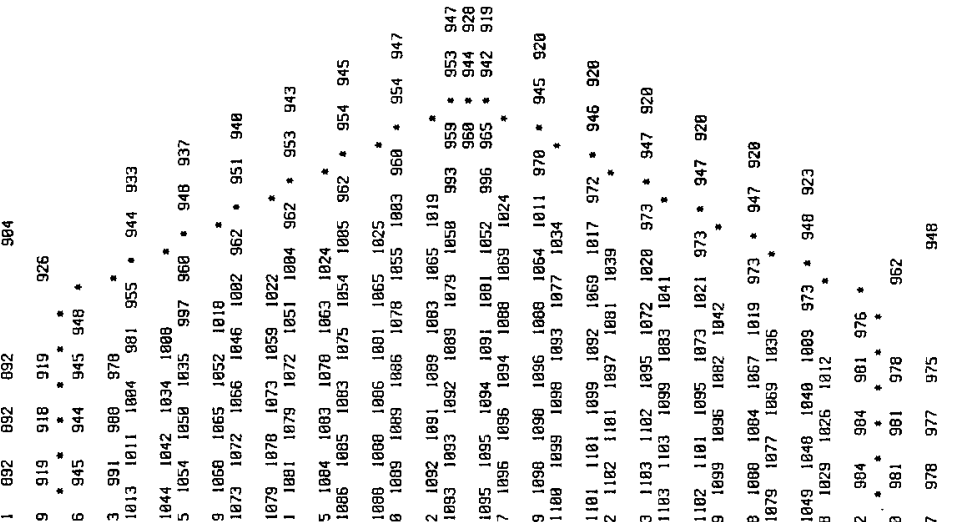

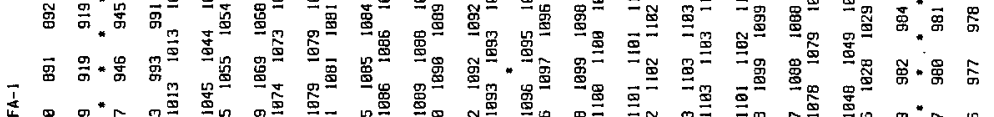

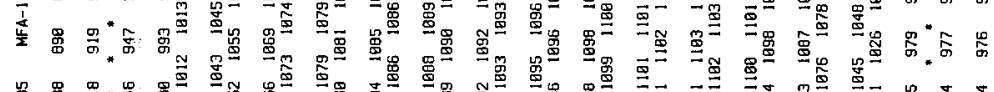

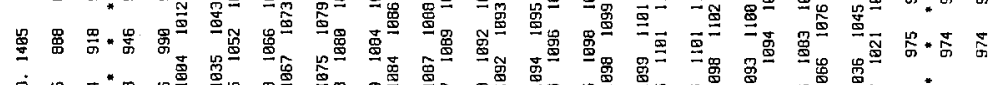

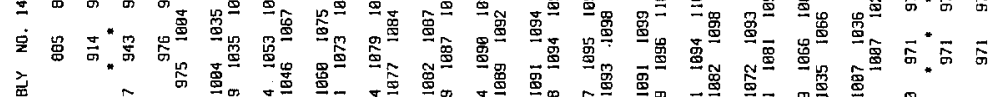

$$
\begin{aligned}
& \text { 嚆 } \\
& \approx \text { : }
\end{aligned}
$$

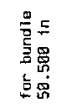

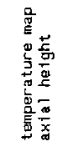

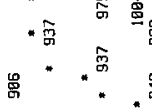

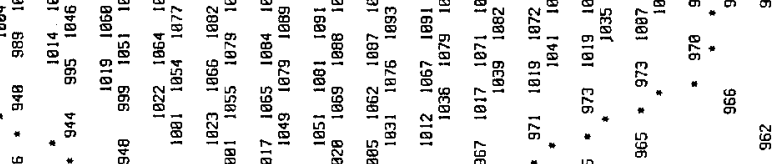

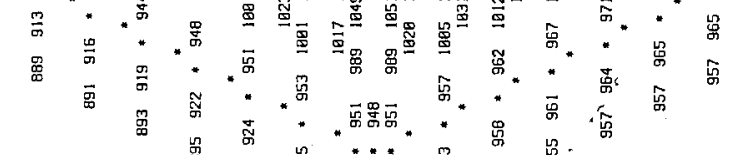


Table E11C.14. MFA-2 Sodium Subchannel Temperatures at Elevation of Upper Axial Blanket for EOC $11 \mathrm{C}$

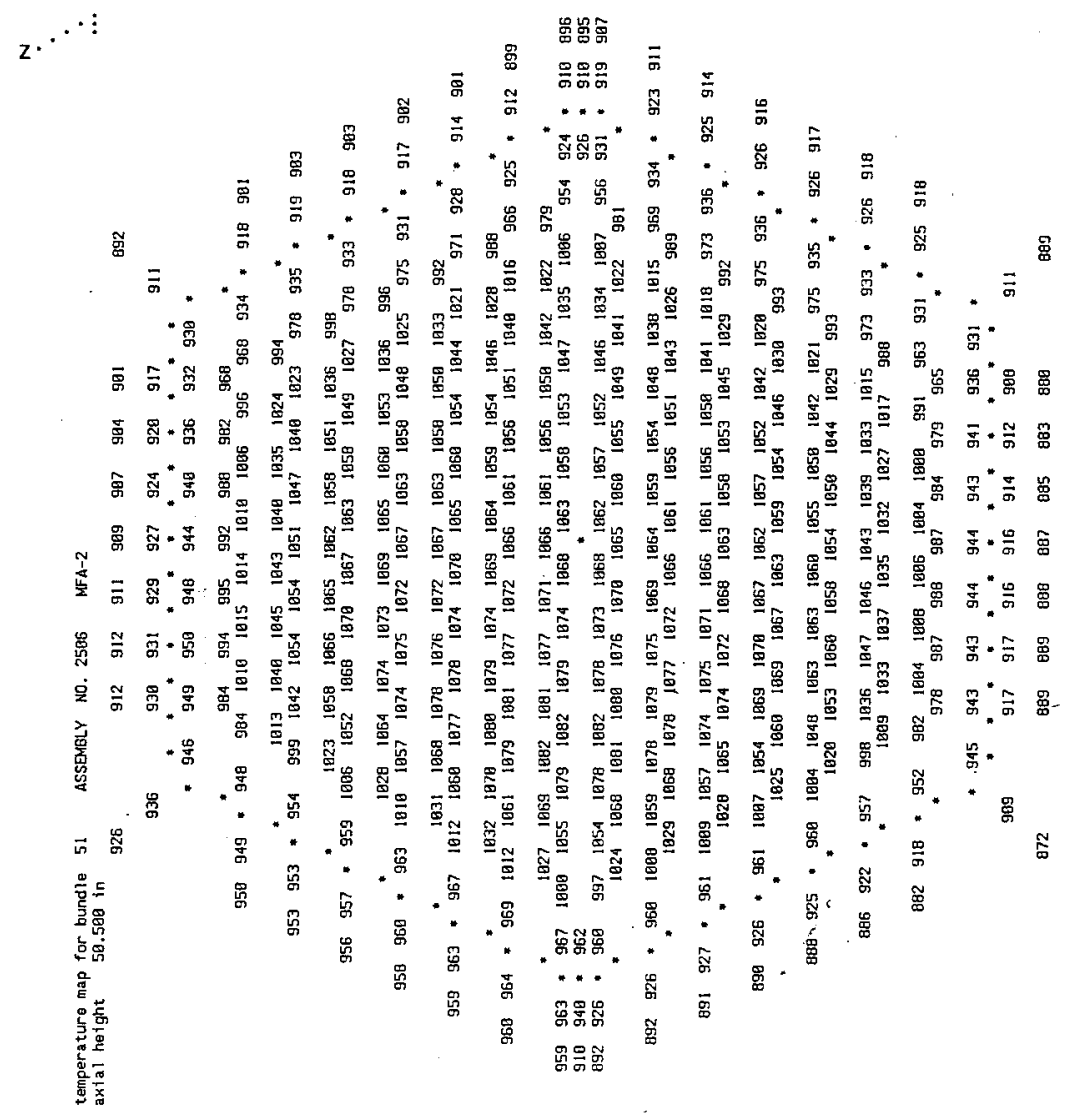


Table EllC.15. MFA-1 Sodium Subchannel Temperatures at Top of Fuel Pin Bundle for EOC 11C

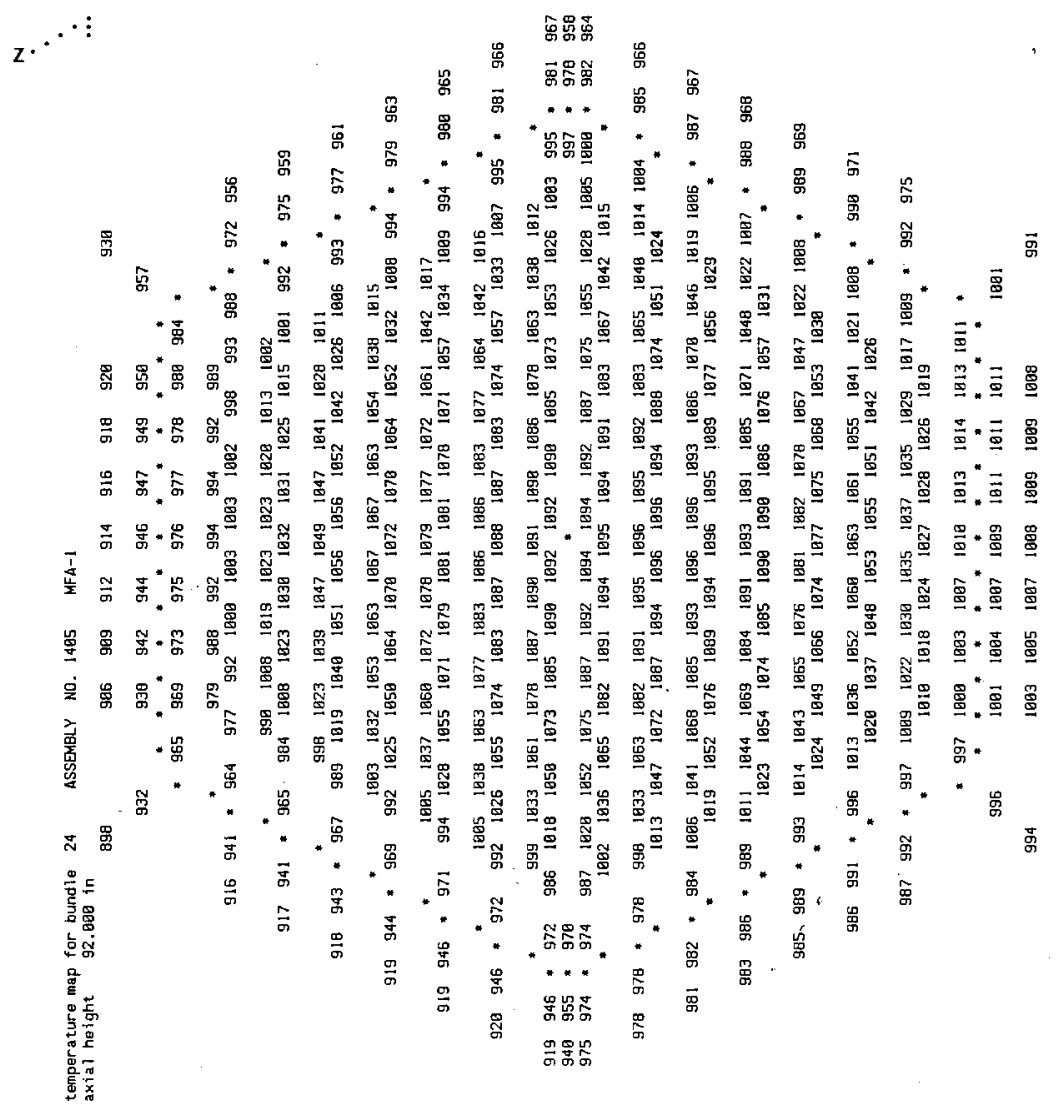


Table E11C.16. MFA-2 Sodium Subchannel Temperatures at Top of Fuel Pin Bundle for EOC $11 \mathrm{C}$

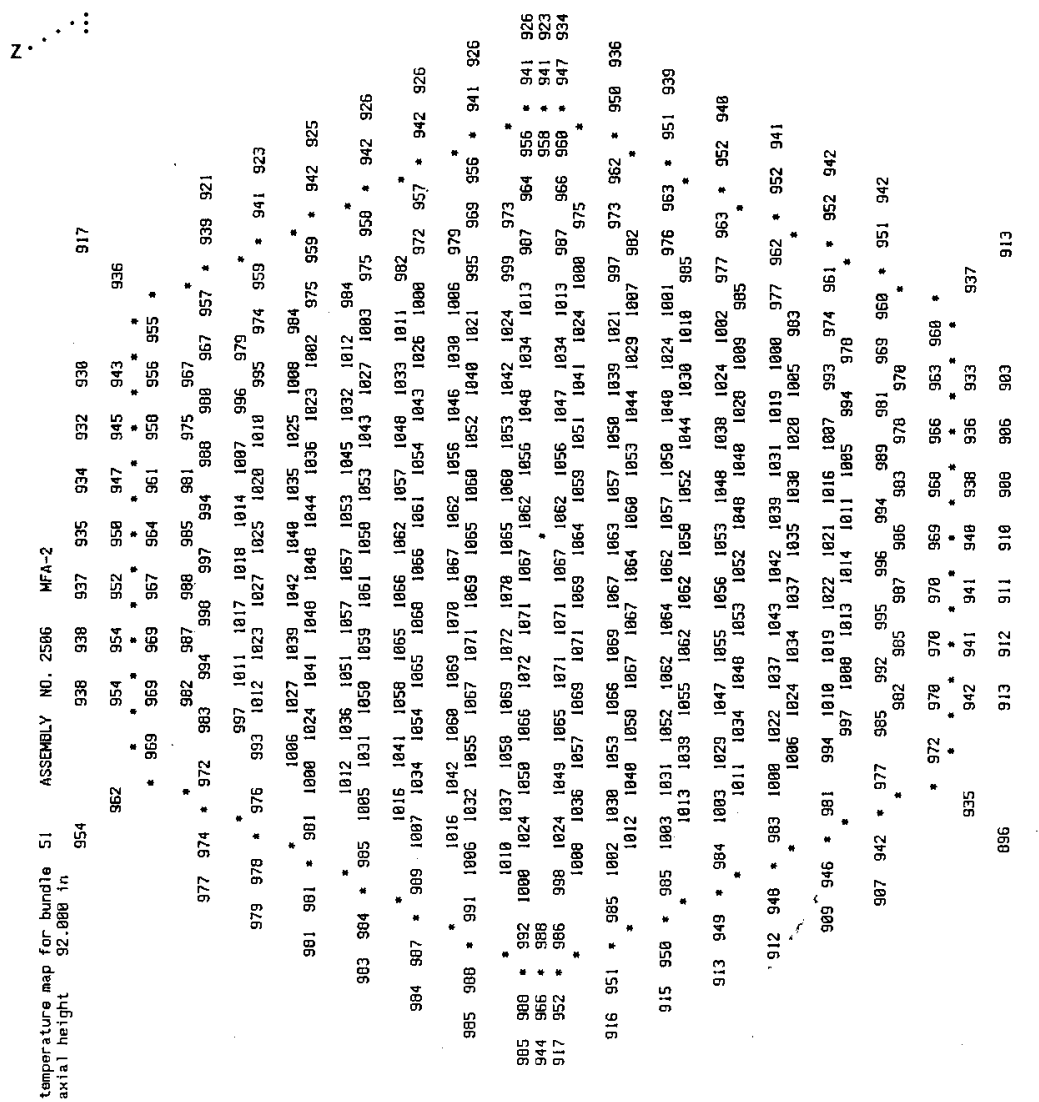


Table EllC.17. Composition of MFA-1 at EOC 11C

\begin{tabular}{|c|c|c|c|c|c|}
\hline ial & Axial & ge $(\mathrm{cm})$ & & Atom Dens & $\mathrm{cm})$ \\
\hline vel & $\begin{array}{l}\text { Lower } \\
-12805\end{array}$ & $\begin{array}{l}\text { Upper } \\
-75\end{array}$ & Constituent & Fresh & End of Cycle \\
\hline & & & $\begin{array}{l}\mathrm{Na}-23 \\
\mathrm{SS}-316\end{array}$ & $\begin{array}{l}5.3150 \mathrm{E}-03 \\
6.4090 \mathrm{E}-02\end{array}$ & $\begin{array}{l}5.3150 \mathrm{E}-03 \\
6.4090 \mathrm{E}-02\end{array}$ \\
\hline 2 & -75.28 & -62.78 & & & \\
\hline & & & $\begin{array}{l}\mathrm{Na}-23 \\
\text { SS-316 }\end{array}$ & $\begin{array}{l}1.3177 \mathrm{E}-02 \\
3.4175 \mathrm{E}-02\end{array}$ & $\begin{array}{l}1.3177 \mathrm{E}-02 \\
3.4175 \mathrm{E}-02\end{array}$ \\
\hline 3 & -62.78 & -46.14 & $\begin{array}{l}\text { U-235 } \\
\text { U-238 } \\
\text { Pu-239 } \\
\text { Pu-240 } \\
\text { FP } \\
\text { O-16 } \\
\text { Na-23 } \\
\text { SS-316 }\end{array}$ & $\begin{array}{l}1.4143 \mathrm{E}-05 \\
7.0573 \mathrm{E}-03 \\
0.0000 \mathrm{E}+00 \\
0.0000 \mathrm{E}+00 \\
0.0000 \mathrm{E}+00 \\
1.4143 \mathrm{E}-02 \\
9.7416 \mathrm{E}-03 \\
1.9073 \mathrm{E}-02\end{array}$ & $\begin{array}{l}8.7575 \mathrm{E}-06 \\
6.7301 \mathrm{E}-03 \\
2.5115 \mathrm{E}-04 \\
1.7172 \mathrm{E}-05 \\
6.2090 \mathrm{E}-05 \\
1.4143 \mathrm{E}-02 \\
9.7416 \mathrm{E}-03 \\
1.9073 \mathrm{E}-02\end{array}$ \\
\hline 4 & -46.14 & -30.76 & $\begin{array}{l}\text { U-235 } \\
\mathrm{U}-238 \\
\mathrm{Pu}-239 \\
\mathrm{Pu}-240 \\
\mathrm{Pu}-241 \\
\mathrm{Pu}-242 \\
\mathrm{Am}-241 \\
\mathrm{FP} \\
0-16 \\
\mathrm{Na}-23 \\
\mathrm{SS}-316\end{array}$ & $\begin{array}{l}1.0840 \mathrm{E}-05 \\
4.4499 \mathrm{E}-03 \\
1.7520 \mathrm{E}-03 \\
2.2340 \mathrm{E}-04 \\
1.9280 \mathrm{E}-05 \\
4.7430 \mathrm{E}-06 \\
7.6680 \mathrm{E}-07 \\
0.0000 \mathrm{E}+00 \\
1.2670 \mathrm{E}-02 \\
9.8100 \mathrm{E}-03 \\
1.8990 \mathrm{E}-02\end{array}$ & $\begin{array}{l}7.0773 \mathrm{E}-06 \\
4.2063 \mathrm{E}-03 \\
1.3205 \mathrm{E}-03 \\
3.0166 \mathrm{E}-04 \\
2.6397 \mathrm{E}-05 \\
5.4252 \mathrm{E}-06 \\
3.4356 \mathrm{E}-06 \\
5.8808 \mathrm{E}-04 \\
1.2670 \mathrm{E}-02 \\
9.8100 \mathrm{E}-03 \\
1.8990 \mathrm{E}-02\end{array}$ \\
\hline 5 & -30.76 & -15.38 & $\begin{array}{l}U-235 \\
U-238 \\
P u-239 \\
P u-240 \\
P u-241 \\
P u-242 \\
\mathrm{Am}-241 \\
\mathrm{FP} \\
0-16 \\
\mathrm{Na}-23 \\
\mathrm{SS}-316\end{array}$ & $\begin{array}{l}1.0840 \mathrm{E}-05 \\
4.4499 \mathrm{E}-03 \\
1.7520 \mathrm{E}-03 \\
2.2340 \mathrm{E}-04 \\
1.9280 \mathrm{E}-05 \\
4.7430 \mathrm{E}-06 \\
7.6680 \mathrm{E}-07 \\
0.0000 \mathrm{E}+00 \\
1.2670 \mathrm{E}-02 \\
9.8100 \mathrm{E}-03 \\
1.8990 \mathrm{E}-02\end{array}$ & $\begin{array}{l}6.4004 \mathrm{E}-06 \\
4.1454 \mathrm{E}-03 \\
1.2282 \mathrm{E}-03 \\
3.0202 \mathrm{E}-04 \\
2.6662 \mathrm{E}-05 \\
5.4463 \mathrm{E}-06 \\
3.3349 \mathrm{E}-06 \\
7.4104 \mathrm{E}-04 \\
1.2670 \mathrm{E}-02 \\
9.8100 \mathrm{E}-03 \\
1.8990 \mathrm{E}-02\end{array}$ \\
\hline b & -15.38 & 0.00 & $\begin{array}{l}U-235 \\
U-238 \\
P u-239 \\
P u-240 \\
P u-241 \\
P u-242 \\
\text { Am-241 } \\
\mathrm{FP} \\
0-16 \\
\mathrm{Na}-23 \\
\mathrm{SS}-316\end{array}$ & $\begin{array}{l}1.0840 \mathrm{E}-05 \\
4.4499 \mathrm{E}-03 \\
1.7520 \mathrm{E}-03 \\
2.2340 \mathrm{E}-04 \\
1.9280 \mathrm{E}-05 \\
4.7430 \mathrm{E}-06 \\
7.6680 \mathrm{E}-07 \\
0.0000 \mathrm{E}+00 \\
1.2670 \mathrm{E}-02 \\
9.8100 \mathrm{E}-03 \\
1.8990 \mathrm{E}-02\end{array}$ & $\begin{array}{l}6.0398 \mathrm{E}-06 \\
4.1138 \mathrm{E}-03 \\
1.1839 \mathrm{E}-03 \\
3.0641 \mathrm{E}-04 \\
2.7625 \mathrm{E}-05 \\
5.5487 \mathrm{E}-06 \\
3.3225 \mathrm{E}-06 \\
8.1206 \mathrm{E}-04 \\
1.2670 \mathrm{E}-02 \\
9.8100 \mathrm{E}-03 \\
1.8990 \mathrm{E}-02\end{array}$ \\
\hline
\end{tabular}


HNF-SD-FF-ANAL-009 Rev . 1

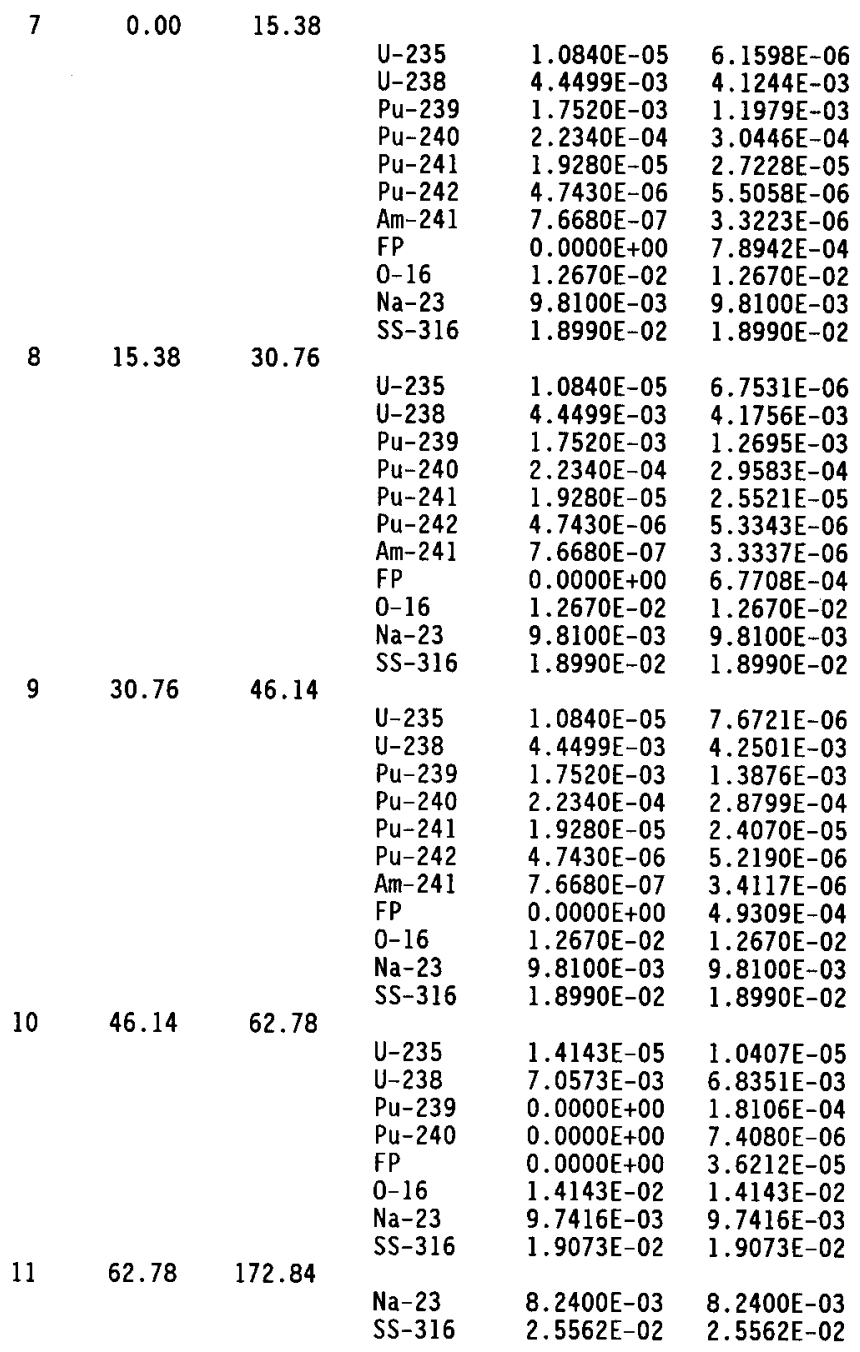


HNF-SD-FF-ANAL-009 Rev. 1

Table EllC.18. Composition of MFA-2 at EOC $11 \mathrm{C}$

\begin{tabular}{|c|c|c|c|c|c|}
\hline Axial & Axial & le $(\mathrm{cm})$ & & Atom Den & $y(a / b-c m)$ \\
\hline $\begin{array}{c}\text { Level } \\
1\end{array}$ & $\begin{array}{r}\text { Lower } \\
-128.05\end{array}$ & $\begin{array}{r}\text { Upper } \\
-75.28\end{array}$ & Constituent & Fresh & nd of cycle \\
\hline & & & $\begin{array}{l}\mathrm{Na}-23 \\
\mathrm{SS}-316\end{array}$ & $\begin{array}{l}5.3150 E-03 \\
6.4090 E-02\end{array}$ & $\begin{array}{l}5.3150 \mathrm{E}-03 \\
6.4090 \mathrm{E}-02\end{array}$ \\
\hline 2 & -75.28 & -62.78 & & & \\
\hline & & & $\begin{array}{l}\mathrm{Na}-23 \\
\mathrm{SS}-316\end{array}$ & $\begin{array}{l}1.3177 \mathrm{E}-02 \\
3.4175 \mathrm{E}-02\end{array}$ & $\begin{array}{l}1.3177 \mathrm{E}-02 \\
3.4175 \mathrm{E}-02\end{array}$ \\
\hline 3 & -62.78 & -46.14 & & & \\
\hline & & & $\begin{array}{l}\mathrm{U}-235 \\
\mathrm{U}-238 \\
\mathrm{Pu}-239 \\
\mathrm{Pu}-240 \\
\mathrm{FP} \\
0-16 \\
\mathrm{Na}-23 \\
\mathrm{SS}-316\end{array}$ & $\begin{array}{l}1.4143 E-05 \\
7.0573 E-03 \\
0.0000 E+00 \\
0.0000 E+00 \\
0.0000 E+00 \\
1.4143 E-02 \\
9.7416 E-03 \\
1.9073 E-02\end{array}$ & $\begin{array}{l}8.8072 \mathrm{E}-06 \\
6.7395 \mathrm{E}-03 \\
2.4468 \mathrm{E}-04 \\
1.6742 \mathrm{E}-05 \\
5.9589 \mathrm{E}-05 \\
1.4143 \mathrm{E}-02 \\
9.7416 \mathrm{E}-03 \\
1.9073 \mathrm{E}-02\end{array}$ \\
\hline 4 & -46.14 & -30.76 & & & \\
\hline & & & $\begin{array}{l}\mathrm{U}-235 \\
\mathrm{U}-238 \\
\mathrm{Pu}-239 \\
\mathrm{Pu}-240 \\
\mathrm{Pu}-241 \\
\mathrm{Pu}-242 \\
\mathrm{Am}-241 \\
\mathrm{FP} \\
0-16 \\
\mathrm{Na}-23 \\
\mathrm{SS}-316\end{array}$ & $\begin{array}{l}1.0160 \mathrm{E}-05 \\
4.4270 \mathrm{E}-03 \\
1.7483 \mathrm{E}-03 \\
2.2290 \mathrm{E}-04 \\
1.9040 \mathrm{E}-05 \\
4.7330 \mathrm{E}-06 \\
7.5700 \mathrm{E}-07 \\
0.0000 \mathrm{E}+00 \\
1.2760 \mathrm{E}-02 \\
9.8100 \mathrm{E}-03 \\
1.8990 \mathrm{E}-02\end{array}$ & $\begin{array}{l}6.6925 \mathrm{E}-06 \\
4.1888 \mathrm{E}-03 \\
1.3242 \mathrm{E}-03 \\
2.9974 \mathrm{E}-04 \\
2.6042 \mathrm{E}-05 \\
5.3867 \mathrm{E}-06 \\
3.4329 \mathrm{E}-06 \\
5.7651 \mathrm{E}-04 \\
1.2760 \mathrm{E}-02 \\
9.8100 \mathrm{E}-03 \\
1.8990 \mathrm{E}-02\end{array}$ \\
\hline 5 & -30.76 & -15.38 & & & \\
\hline & & & $\begin{array}{l}\mathrm{U}-235 \\
\mathrm{U}-238 \\
\mathrm{Pu}-239 \\
\mathrm{Pu}-240 \\
\mathrm{Pu}-241 \\
\mathrm{Pu}-242 \\
\mathrm{Am}-241 \\
\mathrm{FP} \\
0-16 \\
\mathrm{Na}-23 \\
\mathrm{SS}-316\end{array}$ & $\begin{array}{l}1.0160 \mathrm{E}-05 \\
4.4270 \mathrm{E}-03 \\
1.7483 \mathrm{E}-03 \\
2.2290 \mathrm{E}-04 \\
1.9040 \mathrm{E}-05 \\
4.7330 \mathrm{E}-06 \\
7.5700 \mathrm{E}-07 \\
0.0000 \mathrm{E}+00 \\
1.2760 \mathrm{E}-02 \\
9.8100 \mathrm{E}-03 \\
1.8990 \mathrm{E}-02\end{array}$ & $\begin{array}{l}6.0331 \mathrm{E}-06 \\
4.1262 \mathrm{E}-03 \\
1.2295 \mathrm{E}-03 \\
3.0078 \mathrm{E}-04 \\
2.6432 \mathrm{E}-05 \\
5.4146 \mathrm{E}-06 \\
3.3330 \mathrm{E}-06 \\
7.3338 \mathrm{E}-04 \\
1.2760 \mathrm{E}-02 \\
9.8100 \mathrm{E}-03 \\
1.8990 \mathrm{E}-02\end{array}$ \\
\hline 6 & -15.38 & 0.00 & & & \\
\hline & & & $\begin{array}{l}\mathrm{U}-235 \\
\mathrm{U}-238 \\
\mathrm{Pu}-239 \\
\mathrm{Pu}-240 \\
\mathrm{Pu}-241 \\
\mathrm{Pu}-242 \\
\mathrm{Am}-241 \\
\mathrm{FP} \\
0-16 \\
\mathrm{Na}-23 \\
\mathrm{SS}-316\end{array}$ & $\begin{array}{l}1.0160 \mathrm{E}-05 \\
4.4270 \mathrm{E}-03 \\
1.7483 \mathrm{E}-03 \\
2.2290 \mathrm{E}-04 \\
1.9040 \mathrm{E}-05 \\
4.7330 \mathrm{E}-06 \\
7.5700 \mathrm{E}-07 \\
0.0000 \mathrm{E}+00 \\
1.2760 \mathrm{E}-02 \\
9.8100 \mathrm{E}-03 \\
1.8990 \mathrm{E}-02\end{array}$ & $\begin{array}{l}5.6805 \mathrm{E}-06 \\
4.0930 \mathrm{E}-03 \\
1.1833 \mathrm{E}-03 \\
3.0550 \mathrm{E}-04 \\
2.7466 \mathrm{E}-05 \\
5.5223 \mathrm{E}-06 \\
3.3262 \mathrm{E}-06 \\
8.0692 \mathrm{E}-04 \\
1.2760 \mathrm{E}-02 \\
9.8100 \mathrm{E}-03 \\
1.8990 \mathrm{E}-02\end{array}$ \\
\hline & & & & & \\
\hline
\end{tabular}


HNF-SD-FF-ANAL-009 Rev. 1

\begin{tabular}{|c|c|c|c|c|c|}
\hline 7 & 0.00 & 15.38 & & & \\
\hline & & & $\begin{array}{l}U-235 \\
U-238 \\
P u-239 \\
P u-240 \\
P u-241 \\
P u-242 \\
\text { Am-241 } \\
\text { FP } \\
0-16 \\
\mathrm{Na}-23 \\
\text { SS-316 }\end{array}$ & $\begin{array}{l}1.0160 \mathrm{E}-05 \\
4.4270 \mathrm{E}-03 \\
1.7483 \mathrm{E}-03 \\
2.2290 \mathrm{E}-04 \\
1.9040 \mathrm{E}-05 \\
4.7330 \mathrm{E}-06 \\
7.5700 \mathrm{E}-07 \\
0.0000 \mathrm{E}+00 \\
1.2760 \mathrm{E}-02 \\
9.8100 \mathrm{E}-03 \\
1.8990 \mathrm{E}-02\end{array}$ & $\begin{array}{l}5.7831 \mathrm{E}-06 \\
4.1026 \mathrm{E}-03 \\
1.1961 \mathrm{E}-03 \\
3.0370 \mathrm{E}-04 \\
2.7099 \mathrm{E}-05 \\
5.4832 \mathrm{E}-06 \\
3.3262 \mathrm{E}-06 \\
7.8647 \mathrm{E}-04 \\
1.2760 \mathrm{E}-02 \\
9.8100 \mathrm{E}-03 \\
1.8990 \mathrm{E}-02\end{array}$ \\
\hline 8 & 15.38 & 30.76 & $\begin{array}{l}\mathrm{U}-235 \\
\mathrm{U}-238 \\
\mathrm{Pu}-239 \\
\mathrm{Pu}-240 \\
\mathrm{Pu}-241 \\
\mathrm{Pu}-242 \\
\mathrm{Am}-241 \\
\mathrm{FP} \\
0-16 \\
\mathrm{Na}-23 \\
\mathrm{SS}-316\end{array}$ & $\begin{array}{l}1.0160 \mathrm{E}-05 \\
4.4270 \mathrm{E}-03 \\
1.7483 \mathrm{E}-03 \\
2.2290 \mathrm{E}-04 \\
1.9040 \mathrm{E}-05 \\
4.7330 \mathrm{E}-06 \\
7.5700 \mathrm{E}-07 \\
0.0000 \mathrm{E}+00 \\
1.2760 \mathrm{E}-02 \\
9.8100 \mathrm{E}-03 \\
1.8990 \mathrm{E}-02\end{array}$ & $\begin{array}{l}6.3286 \mathrm{E}-06 \\
4.1527 \mathrm{E}-03 \\
1.2660 \mathrm{E}-03 \\
2.9525 \mathrm{E}-04 \\
2.5407 \mathrm{E}-05 \\
5.3163 \mathrm{E}-06 \\
3.3341 \mathrm{E}-06 \\
6.7654 \mathrm{E}-04 \\
1.2760 \mathrm{E}-02 \\
9.8100 \mathrm{E}-03 \\
1.8990 \mathrm{E}-02\end{array}$ \\
\hline 9 & 30.76 & 46.14 & $\begin{array}{l}U-235 \\
U-238 \\
\text { Pu-239 } \\
\text { Pu-240 } \\
\text { Pu-241 } \\
\text { Pu-242 } \\
\text { Am-241 } \\
\mathrm{FP} \\
0-16 \\
\mathrm{Na}-23 \\
\mathrm{SS}-316\end{array}$ & $\begin{array}{l}1.0160 \mathrm{E}-05 \\
4.4270 \mathrm{E}-03 \\
1.7483 \mathrm{E}-03 \\
2.2290 \mathrm{E}-04 \\
1.9040 \mathrm{E}-05 \\
4.7330 \mathrm{E}-06 \\
7.5700 \mathrm{E}-07 \\
0.0000 \mathrm{E}+00 \\
1.2760 \mathrm{E}-02 \\
9.8100 \mathrm{E}-03 \\
1.8990 \mathrm{E}-02\end{array}$ & $\begin{array}{l}7.1711 \mathrm{E}-06 \\
4.2265 \mathrm{E}-03 \\
1.3816 \mathrm{E}-03 \\
2.8812 \mathrm{E}-04 \\
2.4063 \mathrm{E}-05 \\
5.2137 \mathrm{E}-06 \\
3.4110 \mathrm{E}-06 \\
4.9524 \mathrm{E}-04 \\
1.2760 \mathrm{E}-02 \\
9.8100 \mathrm{E}-03 \\
1.8990 \mathrm{E}-02\end{array}$ \\
\hline 10 & 46.14 & 62.78 & $\begin{array}{l}U-235 \\
U-238 \\
\mathrm{Pu}-239 \\
\mathrm{Pu}-240 \\
\mathrm{FP} \\
0-16 \\
\mathrm{Na}-23 \\
\mathrm{SS}-316\end{array}$ & $\begin{array}{l}1.4143 \mathrm{E}-05 \\
7.0573 \mathrm{E}-03 \\
0.0000 \mathrm{E}+00 \\
0.0000 \mathrm{E}+00 \\
0.0000 \mathrm{E}+00 \\
1.4143 \mathrm{E}-02 \\
9.7416 \mathrm{E}-03 \\
1.9073 \mathrm{E}-02\end{array}$ & $\begin{array}{l}1.0209 \mathrm{E}-05 \\
6.8283 \mathrm{E}-03 \\
1.8570 \mathrm{E}-04 \\
8.3289 \mathrm{E}-06 \\
3.7677 \mathrm{E}-05 \\
1.4143 \mathrm{E}-02 \\
9.7416 \mathrm{E}-03 \\
1.9073 \mathrm{E}-02\end{array}$ \\
\hline 11 & 62.78 & 172.84 & $\begin{array}{l}\mathrm{Na}-23 \\
\text { SS-316 }\end{array}$ & $\begin{array}{l}8.2400 \mathrm{E}-03 \\
2.5562 \mathrm{E}-02\end{array}$ & $\begin{array}{l}8.2400 \mathrm{E}-03 \\
2.5562 \mathrm{E}-02\end{array}$ \\
\hline
\end{tabular}




\subsection{Cycle 12A-1}

Table B12A-1.1. Fission Power Generated in MFA-1, MFA-2 and Neighboring Assemblies at BOC 12A-1

\begin{tabular}{lcccc} 
CORE & $* * * * \star *$ & \multicolumn{2}{c}{ POWER IN MEGAWATTS } & $* * * * * *$ \\
POS. & BELOW CORE & IN CORE & ABOVE CORE & TOTAL PWR \\
1405 & $1.109 \mathrm{E}-01$ & $3.919 \mathrm{E}+00$ & $6.464 \mathrm{E}-02$ & $4.095 \mathrm{E}+00$ \\
1304 & $0.000 \mathrm{E}+00$ & $7.031 \mathrm{E}+00$ & $0.000 \mathrm{E}+00$ & $7.031 \mathrm{E}+00$ \\
1406 & $1.070 \mathrm{E}-02$ & $4.752 \mathrm{E}+00$ & $7.834 \mathrm{E}-03$ & $4.770 \mathrm{E}+00$ \\
1404 & $0.000 \mathrm{E}+00$ & $6.214 \mathrm{E}+00$ & $0.000 \mathrm{E}+00$ & $6.214 \mathrm{E}+00$ \\
1508 & $0.000 \mathrm{E}+00$ & $5.408 \mathrm{E}+00$ & $0.000 \mathrm{E}+00$ & $5.408 \mathrm{E}+00$ \\
1506 & $1.520 \mathrm{E}-02$ & $3.936 \mathrm{E}+00$ & $1.021 \mathrm{E}-02$ & $3.962 \mathrm{E}+00$ \\
1507 & $1.238 \mathrm{E}-02$ & $3.893 \mathrm{E}+00$ & $9.092 \mathrm{E}-03$ & $3.915 \mathrm{E}+00$ \\
2506 & $8.925 \mathrm{E}-02$ & $3.128 \mathrm{E}+00$ & $5.334 \mathrm{E}-02$ & $3.271 \mathrm{E}+00$ \\
2404 & $0.000 \mathrm{E}+00$ & $5.785 \mathrm{E}+00$ & $0.000 \mathrm{E}+00$ & $5.785 \mathrm{E}+00$ \\
2505 & $8.139 \mathrm{E}-03$ & $4.214 \mathrm{E}+00$ & $5.409 \mathrm{E}-03$ & $4.227 \mathrm{E}+00$ \\
2405 & $1.665 \mathrm{E}-02$ & $3.320 \mathrm{E}+00$ & $1.114 \mathrm{E}-02$ & $3.348 \mathrm{E}+00$ \\
2606 & $1.444 \mathrm{E}-02$ & $3.192 \mathrm{E}+00$ & $8.182 \mathrm{E}-03$ & $3.215 \mathrm{E}+00$ \\
2607 & $6.058 \mathrm{E}-03$ & $3.562 \mathrm{E}+00$ & $4.890 \mathrm{E}-03$ & $3.573 \mathrm{E}+00$ \\
2507 & $1.182 \mathrm{E}-02$ & $3.324 \mathrm{E}+00$ & $8.315 \mathrm{E}-03$ & $3.344 \mathrm{E}+00$
\end{tabular}

Table B12A-1.2. Assembly Averaged Total and Fast Flux in MFA-1 and MFA-2 at BOC $12 \mathrm{~A}-1$

\begin{tabular}{cccc} 
& Core & \multicolumn{2}{c}{ Flux $\left(\mathrm{n} / \mathrm{cm}^{2}-\mathrm{sec}\right)$} \\
Assembly & Pos. & Total & $>0.1 \mathrm{MeV}$ \\
MFA-1 & 1405 & $3.772 \mathrm{E}+15$ & $2.527 \mathrm{E}+15$ \\
MFA-2 & 2506 & $2.979 \mathrm{E}+15$ & $1.912 \mathrm{E}+15$
\end{tabular}


Table B12A-1.3. Axial Distribution of Total Flux, Fast Flux and Power in MFA-1 at BOC 12A-1

$\begin{array}{rccc}z(c m) & \text { Total Flux } & \text { Flux }>0.1 \text { MeV } & \text { Power } \\ -97.78 & 2.6833 \mathrm{E}-02 & 1.3450 \mathrm{E}-02 & 0.0000 \mathrm{E}+00 \\ -92.78 & 5.7474 \mathrm{E}-02 & 2.5957 \mathrm{E}-02 & 0.0000 \mathrm{E}+00 \\ -87.78 & 9.2005 \mathrm{E}-02 & 4.1580 \mathrm{E}-02 & 0.0000 \mathrm{E}+00 \\ -82.78 & 1.3308 \mathrm{E}-01 & 6.2106 \mathrm{E}-02 & 0.0000 \mathrm{E}+00 \\ -77.78 & 1.8358 \mathrm{E}-01 & 8.9861 \mathrm{E}-02 & 0.0000 \mathrm{E}+00 \\ -72.15 & 2.4176 \mathrm{E}-01 & 1.2626 \mathrm{E}-01 & 0.0000 \mathrm{E}+00 \\ -65.90 & 3.0199 \mathrm{E}-01 & 1.7396 \mathrm{E}-01 & 0.0000 \mathrm{E}+00 \\ -60.35 & 3.6638 \mathrm{E}-01 & 2.4095 \mathrm{E}-01 & 1.3206 \mathrm{E}-01 \\ -55.49 & 4.5011 \mathrm{E}-01 & 3.2754 \mathrm{E}-01 & 1.5013 \mathrm{E}-01 \\ -50.62 & 5.4573 \mathrm{E}-01 & 4.3895 \mathrm{E}-01 & 1.7281 \mathrm{E}-01 \\ -47.17 & 6.2060 \mathrm{E}-01 & 5.4162 \mathrm{E}-01 & 1.9434 \mathrm{E}-01 \\ -43.58 & 6.9875 \mathrm{E}-01 & 6.5641 \mathrm{E}-01 & 7.6923 \mathrm{E}-01 \\ -38.45 & 8.1414 \mathrm{E}-01 & 7.9845 \mathrm{E}-01 & 8.6201 \mathrm{E}-01 \\ -33.32 & 9.2159 \mathrm{E}-01 & 9.1922 \mathrm{E}-01 & 9.6570 \mathrm{E}-01 \\ -28.20 & 1.0150 \mathrm{E}+00 & 1.0183 \mathrm{E}+00 & 9.9638 \mathrm{E}-01 \\ -23.07 & 1.0932 \mathrm{E}+00 & 1.0993 \mathrm{E}+00 & 1.0715 \mathrm{E}+00 \\ -17.94 & 1.1544 \mathrm{E}+00 & 1.1615 \mathrm{E}+00 & 1.1304 \mathrm{E}+00 \\ -12.82 & 1.1975 \mathrm{E}+00 & 1.2042 \mathrm{E}+00 & 1.1378 \mathrm{E}+00 \\ -7.69 & 1.2231 \mathrm{E}+00 & 1.2297 \mathrm{E}+00 & 1.1617 \mathrm{E}+00 \\ -2.56 & 1.2308 \mathrm{E}+00 & 1.2377 \mathrm{E}+00 & 1.1689 \mathrm{E}+00 \\ 2.56 & 1.2208 \mathrm{E}+00 & 1.2284 \mathrm{E}+00 & 1.1703 \mathrm{E}+00 \\ 7.69 & 1.1929 \mathrm{E}+00 & 1.2012 \mathrm{E}+00 & 1.1437 \mathrm{E}+00 \\ 12.82 & 1.1478 \mathrm{E}+00 & 1.1571 \mathrm{E}+00 & 1.1009 \mathrm{E}+00 \\ 17.94 & 1.0865 \mathrm{E}+00 & 1.0972 \mathrm{E}+00 & 1.0924 \mathrm{E}+00 \\ 23.07 & 1.0087 \mathrm{E}+00 & 1.0191 \mathrm{E}+00 & 1.0151 \mathrm{E}+00 \\ 28.20 & 9.1615 \mathrm{E}-01 & 9.2450 \mathrm{E}-01 & 9.2309 \mathrm{E}-01 \\ 33.32 & 8.1105 \mathrm{E}-01 & 8.1502 \mathrm{E}-01 & 8.8254 \mathrm{E}-01 \\ 38.45 & 6.9459 \mathrm{E}-01 & 6.8761 \mathrm{E}-01 & 7.6164 \mathrm{E}-01 \\ 43.58 & 5.7304 \mathrm{E}-01 & 5.4510 \mathrm{E}-01 & 6.4663 \mathrm{E}-01 \\ 47.17 & 4.9448 \mathrm{E}-01 & 4.4035 \mathrm{E}-01 & 1.2726 \mathrm{E}-01 \\ 50.62 & 4.2518 \mathrm{E}-01 & 3.5307 \mathrm{E}-01 & 1.0612 \mathrm{E}-01 \\ 55.49 & 3.4192 \mathrm{E}-01 & 2.6175 \mathrm{E}-01 & 8.4999 \mathrm{E}-02 \\ 60.35 & 2.6760 \mathrm{E}-01 & 1.8925 \mathrm{E}-01 & 6.8208 \mathrm{E}-02 \\ 64.98 & 2.1693 \mathrm{E}-01 & 1.4272 \mathrm{E}-01 & 0.0000 \mathrm{E}+00 \\ 69.39 & 1.8428 \mathrm{E}-01 & 1.1597 \mathrm{E}-01 & 0.0000 \mathrm{E}+00 \\ 76.46 & 1.3750 \mathrm{E}-01 & 8.0165 \mathrm{E}-02 & 0.0000 \mathrm{E}+00 \\ 86.80 & 8.8108 \mathrm{E}-02 & 4.7069 \mathrm{E}-02 & 0.0000 \mathrm{E}+00 \\ 98.14 & 5.0228 \mathrm{E}-02 & 2.5197 \mathrm{E}-02 & 0.0000 \mathrm{E}+00 \\ 110.00 & 2.1359 \mathrm{E}-02 & 1.1101 \mathrm{E}-02 & 0.0000 \mathrm{E}+00 \\ -15 & & \end{array}$


Table Bl2A-1.4. Axial Distribution of Total Flux, Fast Flux and Power in MFA-2 at BOC $12 \mathrm{~A}-1$

$\begin{array}{rccc}z(\mathrm{~cm}) & \text { Total Flux } & \text { Flux }>0.1 \text { MeV } & \text { Power } \\ -97.78 & 2.7692 \mathrm{E}-02 & 1.4268 \mathrm{E}-02 & 0.0000 \mathrm{E}+00 \\ -92.78 & 5.9408 \mathrm{E}-02 & 2.7535 \mathrm{E}-02 & 0.0000 \mathrm{E}+00 \\ -87.78 & 9.5190 \mathrm{E}-02 & 4.4037 \mathrm{E}-02 & 0.0000 \mathrm{E}+00 \\ -82.78 & 1.3786 \mathrm{E}-01 & 6.5672 \mathrm{E}-02 & 0.0000 \mathrm{E}+00 \\ -77.78 & 1.9054 \mathrm{E}-01 & 9.4822 \mathrm{E}-02 & 0.0000 \mathrm{E}+00 \\ -72.15 & 2.5134 \mathrm{E}-01 & 1.3285 \mathrm{E}-01 & 0.0000 \mathrm{E}+00 \\ -65.90 & 3.1304 \mathrm{E}-01 & 1.8187 \mathrm{E}-01 & 0.0000 \mathrm{E}+00 \\ -60.35 & 3.7672 \mathrm{E}-01 & 2.4953 \mathrm{E}-01 & 1.3491 \mathrm{E}-01 \\ -55.49 & 4.5983 \mathrm{E}-01 & 3.3613 \mathrm{E}-01 & 1.5201 \mathrm{E}-01 \\ -50.62 & 5.5470 \mathrm{E}-01 & 4.4701 \mathrm{E}-01 & 1.7304 \mathrm{E}-01 \\ -47.17 & 6.2936 \mathrm{E}-01 & 5.4970 \mathrm{E}-01 & 1.9307 \mathrm{E}-01 \\ -43.58 & 7.0752 \mathrm{E}-01 & 6.6496 \mathrm{E}-01 & 7.8536 \mathrm{E}-01 \\ -38.45 & 8.2215 \mathrm{E}-01 & 8.0620 \mathrm{E}-01 & 8.7456 \mathrm{E}-01 \\ -33.32 & 9.2845 \mathrm{E}-01 & 9.2594 \mathrm{E}-01 & 9.7609 \mathrm{E}-01 \\ -28.20 & 1.0207 \mathrm{E}+00 & 1.0239 \mathrm{E}+00 & 1.0030 \mathrm{E}+00 \\ -23.07 & 1.0977 \mathrm{E}+00 & 1.1039 \mathrm{E}+00 & 1.0768 \mathrm{E}+00 \\ -17.94 & 1.1578 \mathrm{E}+00 & 1.1650 \mathrm{E}+00 & 1.1347 \mathrm{E}+00 \\ -12.82 & 1.1997 \mathrm{E}+00 & 1.2067 \mathrm{E}+00 & 1.1392 \mathrm{E}+00 \\ -7.69 & 1.2240 \mathrm{E}+00 & 1.2310 \mathrm{E}+00 & 1.1618 \mathrm{E}+00 \\ -2.56 & 1.2302 \mathrm{E}+00 & 1.2374 \mathrm{E}+00 & 1.1675 \mathrm{E}+00 \\ 2.56 & 1.2184 \mathrm{E}+00 & 1.2262 \mathrm{E}+00 & 1.1661 \mathrm{E}+00 \\ 7.69 & 1.1884 \mathrm{E}+00 & 1.1970 \mathrm{E}+00 & 1.1376 \mathrm{E}+00 \\ 12.82 & 1.1412 \mathrm{E}+00 & 1.1506 \mathrm{E}+00 & 1.0926 \mathrm{E}+00 \\ 17.94 & 1.0782 \mathrm{E}+00 & 1.0888 \mathrm{E}+00 & 1.0811 \mathrm{E}+00 \\ 23.07 & 9.9977 \mathrm{E}-01 & 1.0100 \mathrm{E}+00 & 1.0032 \mathrm{E}+00 \\ 28.20 & 9.0807 \mathrm{E}-01 & 9.1611 \mathrm{E}-01 & 9.1236 \mathrm{E}-01 \\ 33.32 & 8.0566 \mathrm{E}-01 & 8.0939 \mathrm{E}-01 & 8.7387 \mathrm{E}-01 \\ 38.45 & 6.9384 \mathrm{E}-01 & 6.8672 \mathrm{E}-01 & 7.5953 \mathrm{E}-01 \\ 43.58 & 5.7829 \mathrm{E}-01 & 5.5021 \mathrm{E}-01 & 6.5470 \mathrm{E}-01 \\ 47.17 & 5.0261 \mathrm{E}-01 & 4.4687 \mathrm{E}-01 & 1.2961 \mathrm{E}-01 \\ 50.62 & 4.3235 \mathrm{E}-01 & 3.5721 \mathrm{E}-01 & 1.0939 \mathrm{E}-01 \\ 55.49 & 3.4471 \mathrm{E}-01 & 2.6171 \mathrm{E}-01 & 8.8360 \mathrm{E}-02 \\ 60.35 & 2.6650 \mathrm{E}-01 & 1.8637 \mathrm{E}-01 & 7.1142 \mathrm{E}-02 \\ 64.98 & 2.1555 \mathrm{E}-01 & 1.3976 \mathrm{E}-01 & 0.0000 \mathrm{E}+00 \\ 69.39 & 1.8415 \mathrm{E}-01 & 1.1435 \mathrm{E}-01 & 0.0000 \mathrm{E}+00 \\ 76.46 & 1.3905 \mathrm{E}-01 & 8.0332 \mathrm{E}-02 & 0.0000 \mathrm{E}+00 \\ 86.80 & 8.9802 \mathrm{E}-02 & 4.7788 \mathrm{E}-02 & 0.0000 \mathrm{E}+00 \\ 98.14 & 5.1268 \mathrm{E}-02 & 2.5767 \mathrm{E}-02 & 0.0000 \mathrm{E}+00 \\ 110.00 & 2.1852 \mathrm{E}-02 & 1.1415 \mathrm{E}-02 & 0.0000 \mathrm{E}+00\end{array}$


HNF-SD-FF-ANAL-009 Rev. 1

Table Bl2A-1.5. Fission Power Distribution by Pin in MFA-1 at BOC 12A-1 CORE POSITION 1405

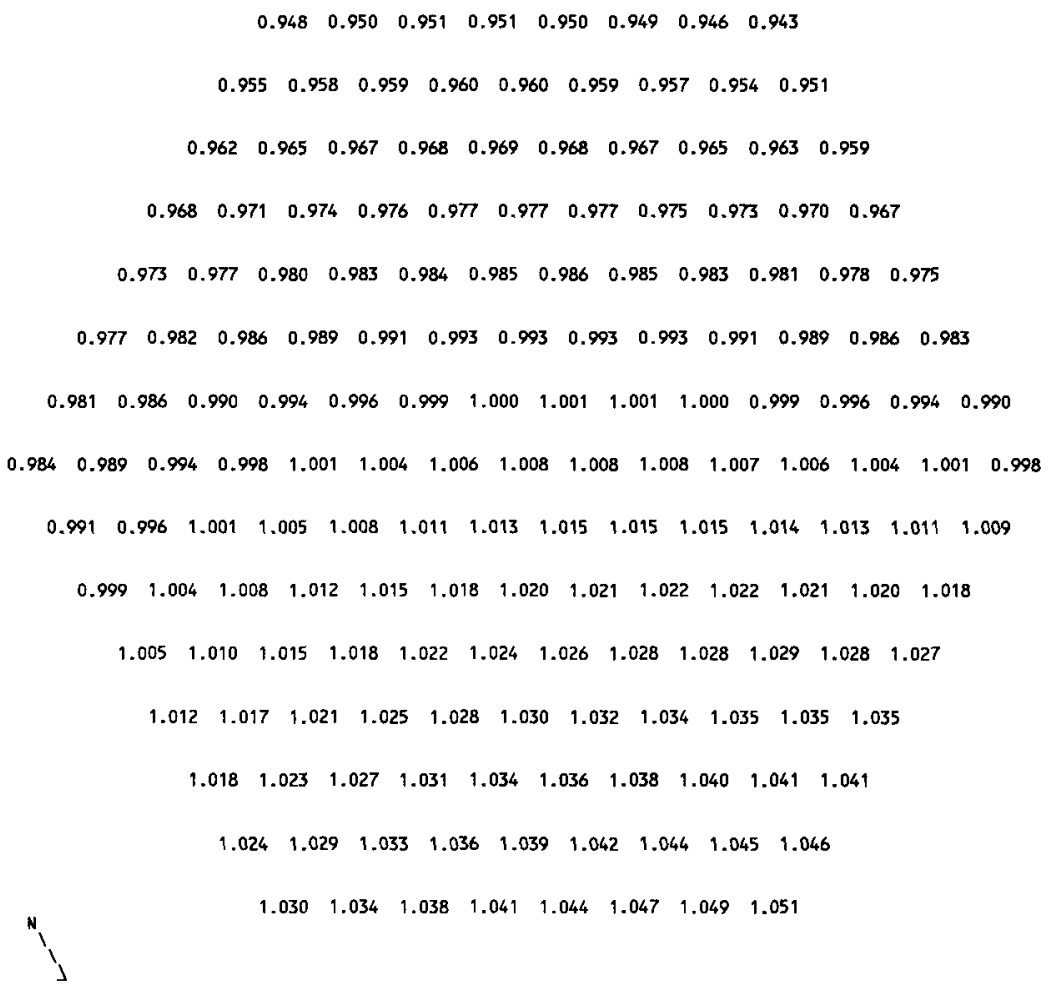


HNF-SD-FF-ANAL-009 Rev. 1

Table B12A-1.6. Fission Power Distribution by Pin in MFA-2 at BOC 12A-1 CORE POSITION 2506

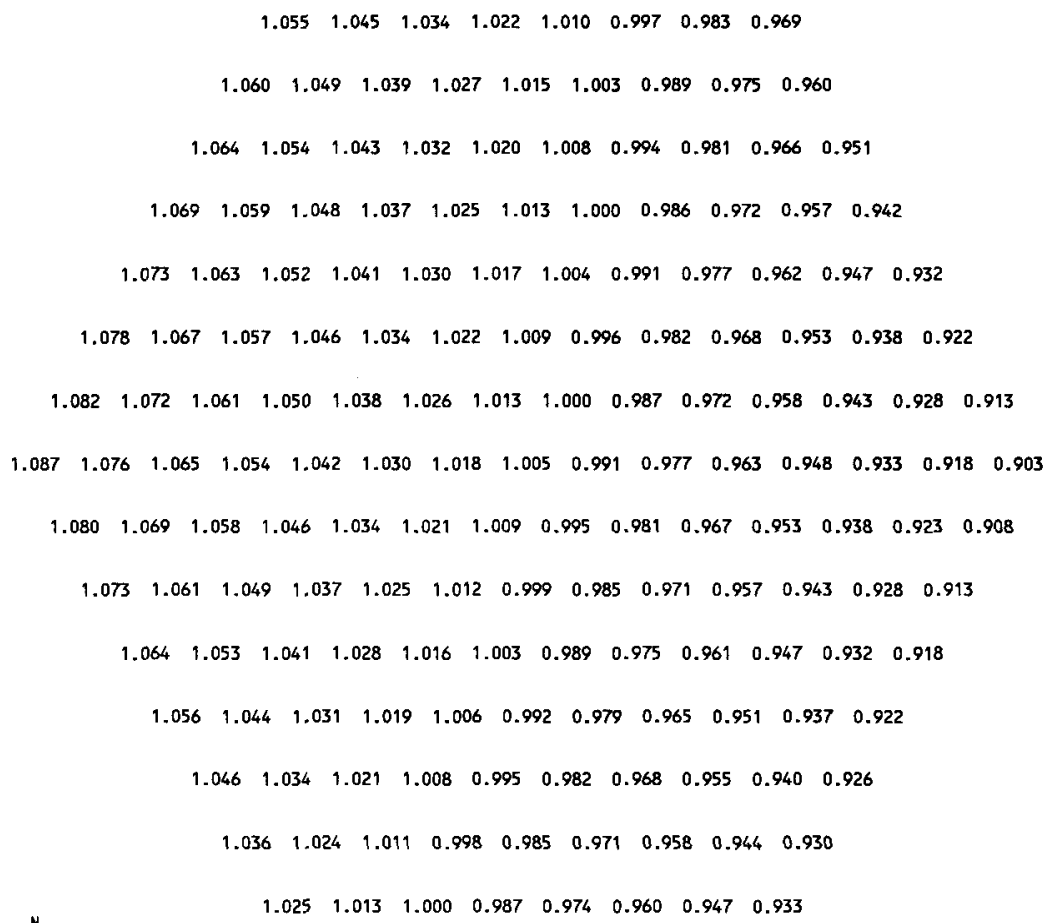


HNF-SD-FF-ANAL-009 Rev. I

Table B12A-1.7. Fast Flux Distribution by Pin in MFA-1 at BOC 12A-1 CORE POSITION 1404

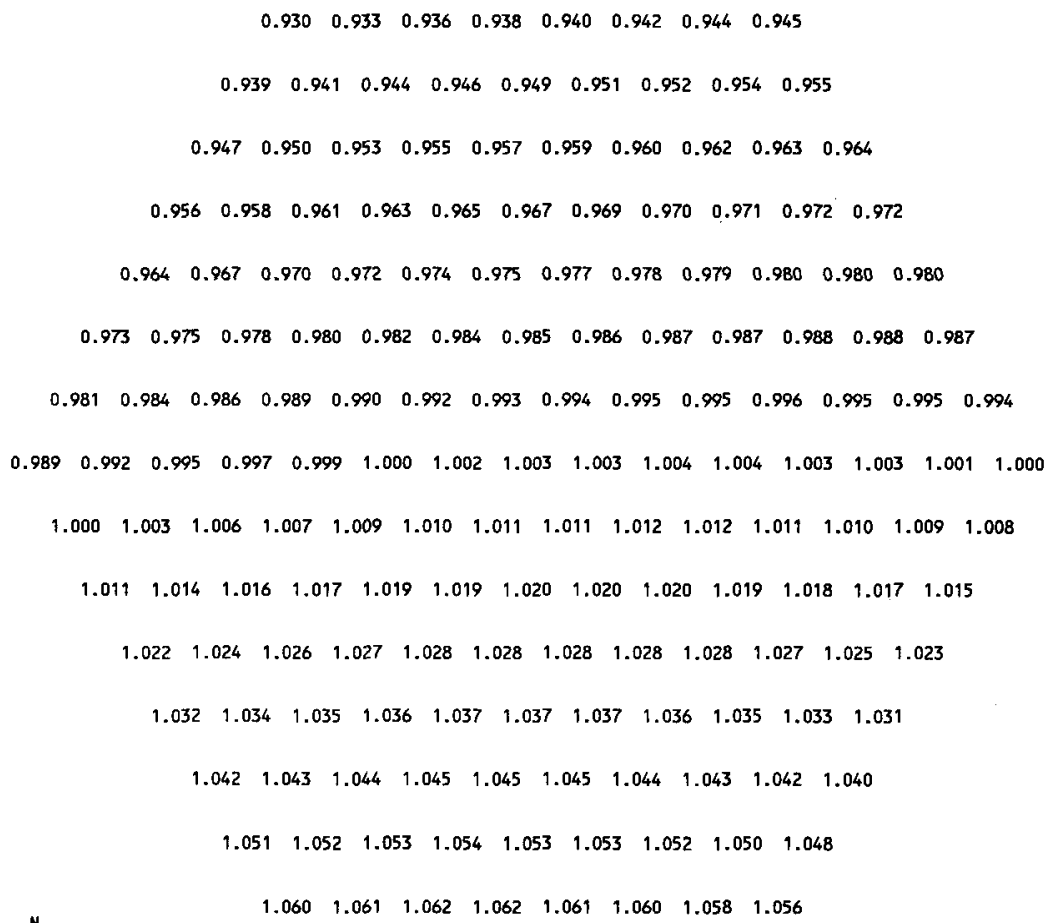


HNF-SD-FF-ANAL-009 Rev. 1

Table B12A-1.8. Fast Flux Distribution by Pin in MFA-2 at BOC $12 A-1$

CORE POSITION 2506

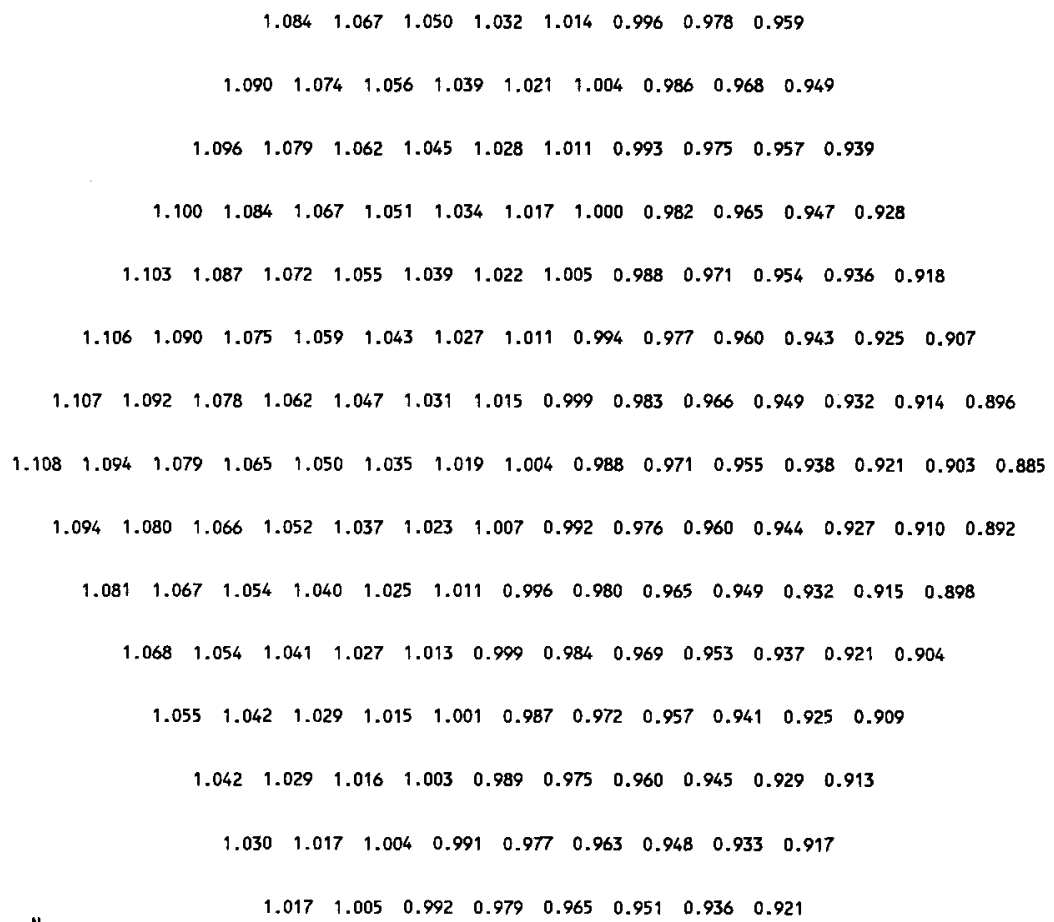


Table B12A-1.9. MFA-1 and MFA-2 Duct Wall

Fast Flux Data at BOC $12 \mathrm{~A}-1$

$\begin{array}{ccc}\text { Side } & \begin{array}{c}\text { Flux }>0.1 \\ \text { MFA-1 }\end{array} & \begin{array}{c}\text { MeV }\left(\mathrm{n} / \mathrm{cm}^{2}-\mathrm{sec}\right) \\ \text { MFA-2 }\end{array} \\ \text { E } & 2.5860 E+15 & 2.0415 \mathrm{E}+15 \\ \text { SE } & 2.3848 \mathrm{E}+15 & 2.1419 \mathrm{E}+15 \\ \text { SW } & 2.3236 \mathrm{E}+15 & 1.9538 \mathrm{E}+15 \\ \text { W } & 2.4376 \mathrm{E}+15 & 1.7251 \mathrm{E}+15 \\ \text { NW } & 2.5851 \mathrm{E}+15 & 1.6905 \mathrm{E}+15 \\ \text { NE } & 2.6934 \mathrm{E}+15 & 1.8413 \mathrm{E}+15\end{array}$

Table B12A-1.10. Assembly Outlet Temperatures and Flow Rates at BOC 12A-1

$\begin{array}{lcl}\text { CALCULATED } & \\ \text { CORE } & \begin{array}{c}\text { OUTLET TEMP. } \\ \text { POS. }\end{array} & \begin{array}{l}\text { FLOW RATE } \\ \text { (DEG F) }\end{array} \\ \text { (LB/H) } \\ 1405 & 1085 & 108910 \\ 1304 & 1071 & 190310 \\ 1404 & 1020 & 192650 \\ 1406 & 948 & 197210 \\ 1506 & 902 & 197210 \\ 1507 & 898 & 197210 \\ 1508 & 1002 & 177530 \\ 2506 & 1003 & 108910 \\ 2404 & 995 & 193950 \\ 2405 & 869 & 197230 \\ 2505 & 909 & 197210 \\ 2507 & 865 & 197210 \\ 2606 & 897 & 161730 \\ 2607 & 927 & 155100\end{array}$

Assembly flows are based on a calculated core pressure drop of 99.3 psi at a total reactor flow rate of $16.74 \mathrm{E}+06 \mathrm{ib} / \mathrm{hr}$. 
Table B12A-1.11. MFA-1 Sodium Subchanne1 Temperatures at Top of Core Elevation for BOC 12A-1

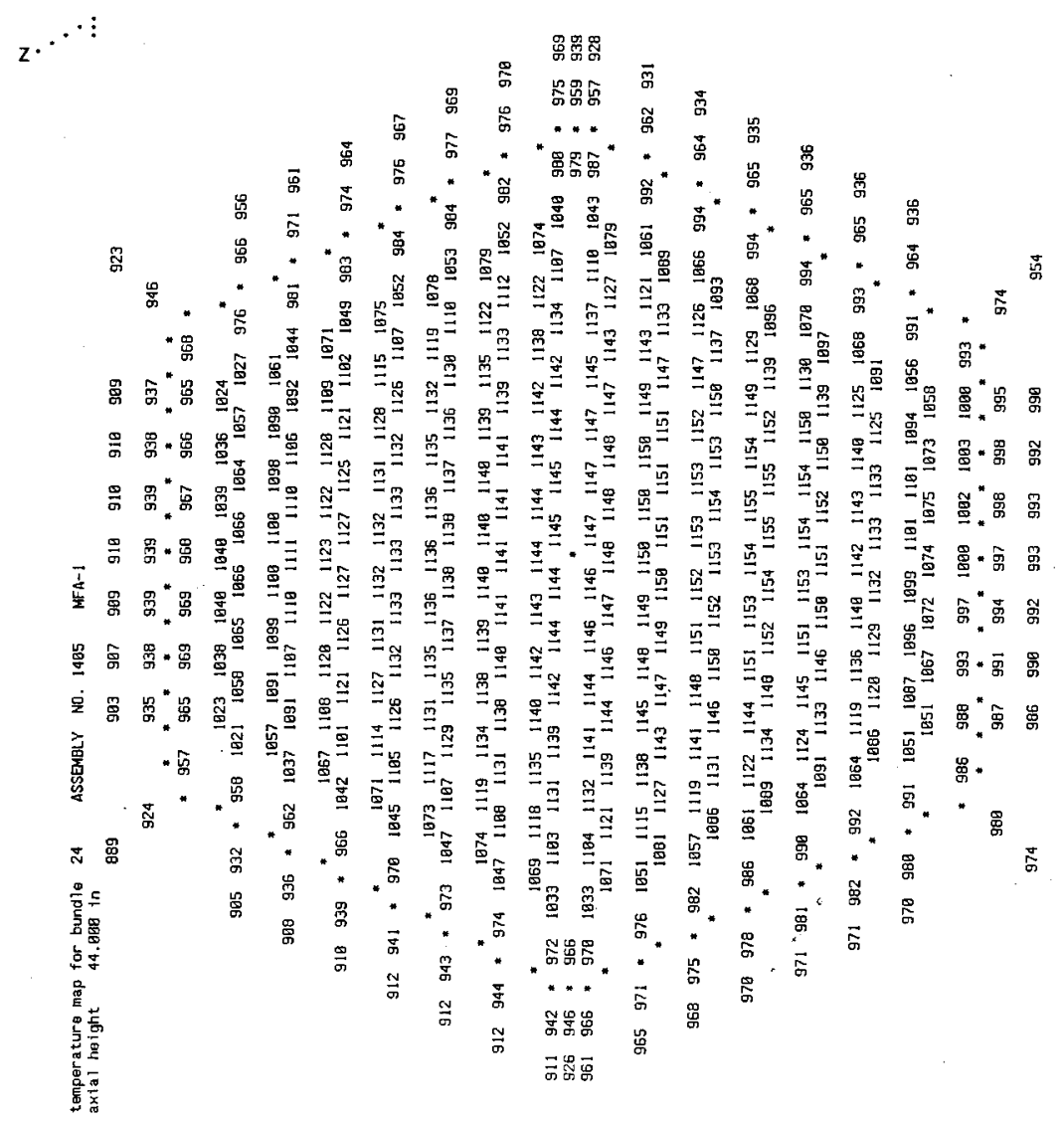


Table B12A-1.12. MFA-2 Sodium Subchanne1 Temperatures at Top of Core Elevation for BOC 12A-1

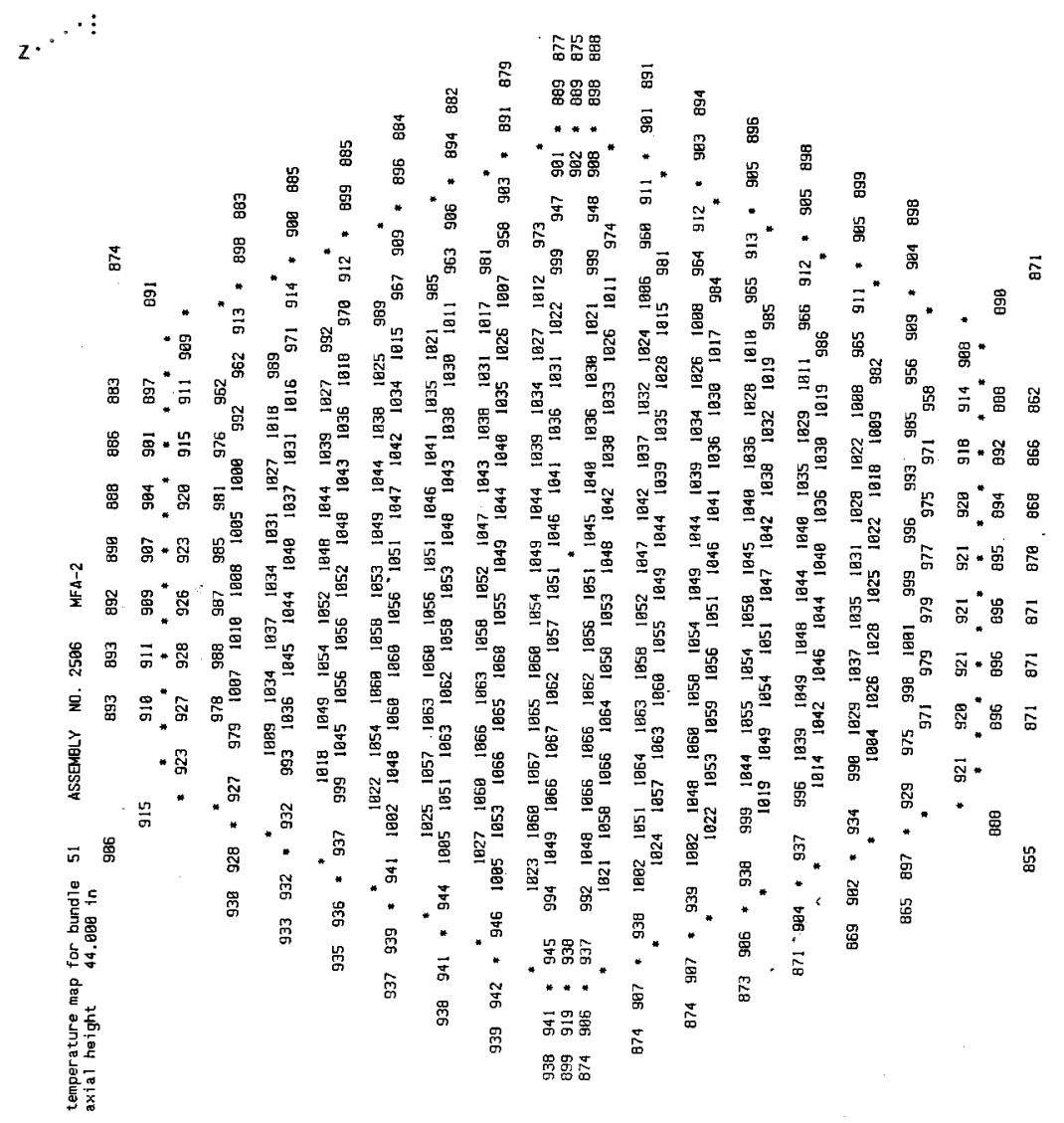


1 temperature map for bundle 24 ASSEABLY NO. 1485 MFA-1

$\begin{array}{llllllllllll}\text { axial height } & 50.580 & \text { in } & 982 & 916 & 920 & 922 & 923 & 924 & 924 & 924 & 938\end{array}$

$\because$

$939 \quad 958 \quad 953955,955 \quad 955,955,955,964$

. 975 * 982 "

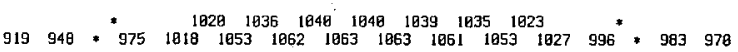

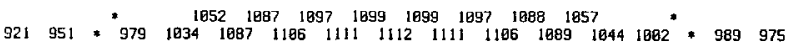

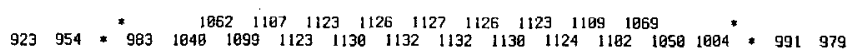

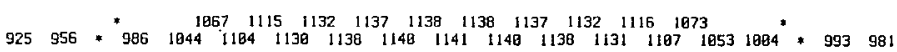

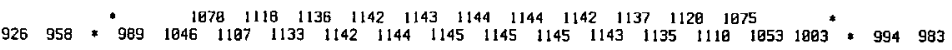

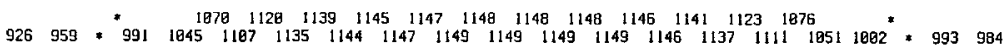

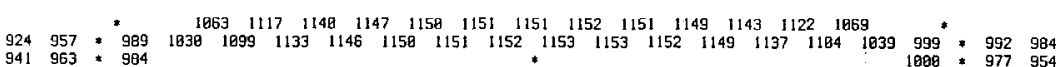

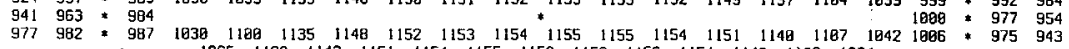

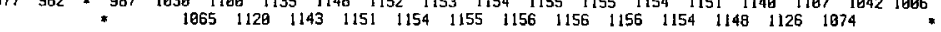

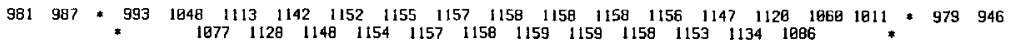

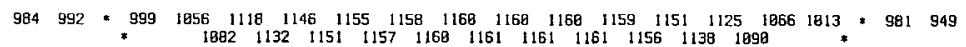

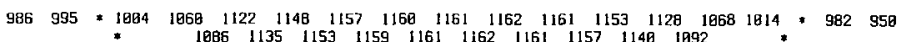

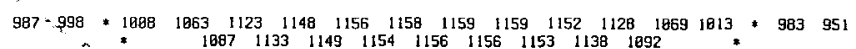

$988 \quad 999 * 1611 \quad 1052 \quad 1115 \quad 1135 \quad 1141 \quad 1143 \quad 1144 \quad 1140 \quad 1121 \quad 10661012 *$ * 982952

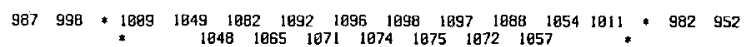

* $1006 \quad 1087 \quad 1012 \quad 1816 \quad 1028 \quad 1023 \quad 1023 \quad 1021 \quad 1814$ *

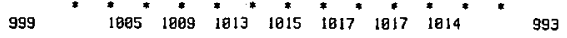

$\begin{array}{llllllllll}992 & 1004 & 1087 & 1009 & 1011 & 1011 & 1010 & 1087 & 971\end{array}$ 
Table B12A-1.14. MFA-2 Sodium Subchannel Temperatures at Elevation of Upper Axial Blanket for BOC 12A-1

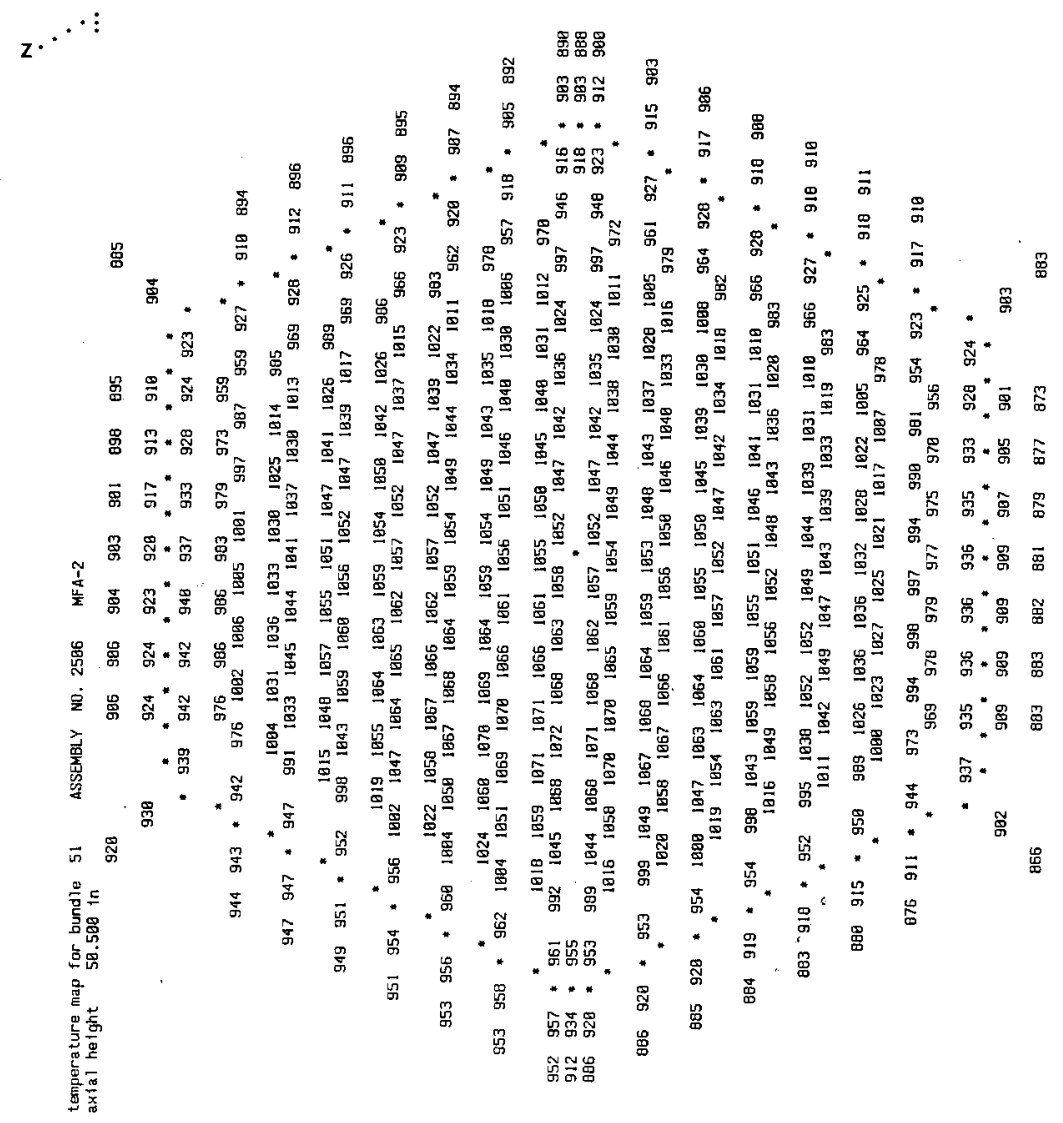


Table B12A-1.15. MFA-1 Sodium Subchannel Temperatures at Top of Fuel Pin Bundle for BOC 12A-1

$$
\begin{aligned}
& z^{\cdot \cdot \cdot} \cdot
\end{aligned}
$$

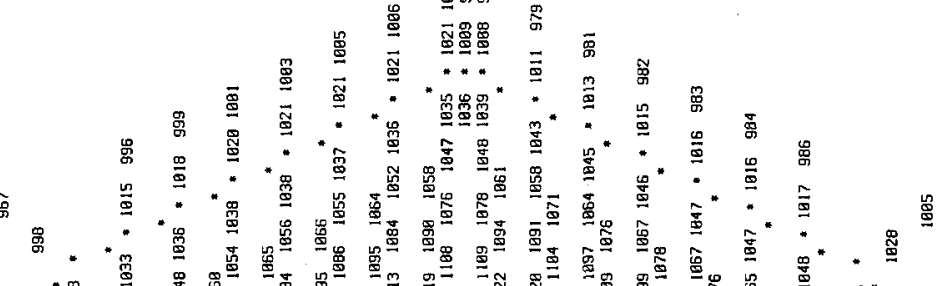

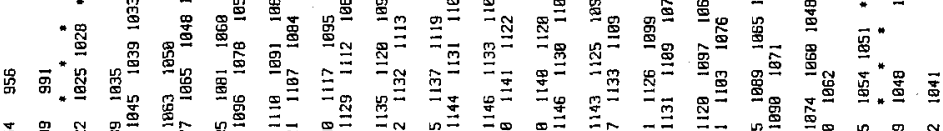

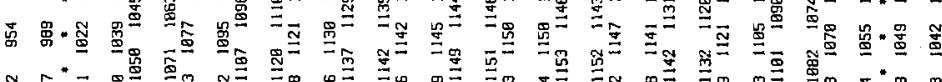

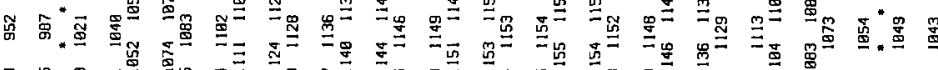

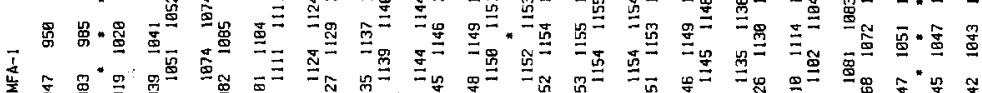

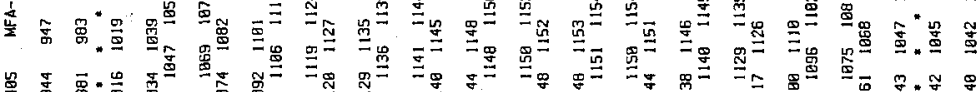

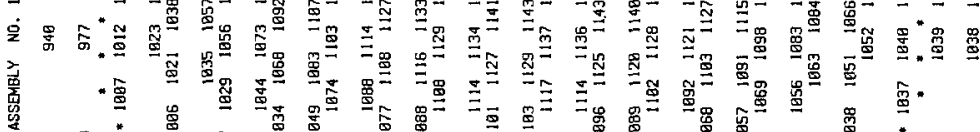

$$
\begin{aligned}
& \text { 蝒 } \\
& \text { 密. }
\end{aligned}
$$

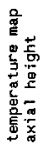

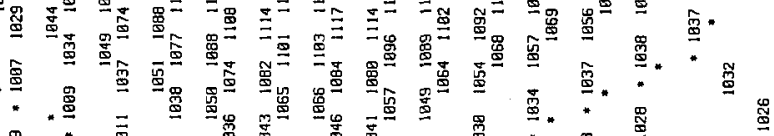

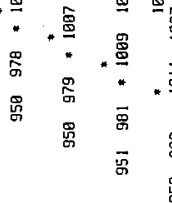


Table B12A-1.16. MFA-2 Sodium Subchannel Temperatures at Top of Fue 1 Pin Bundle for BOC 12A-1

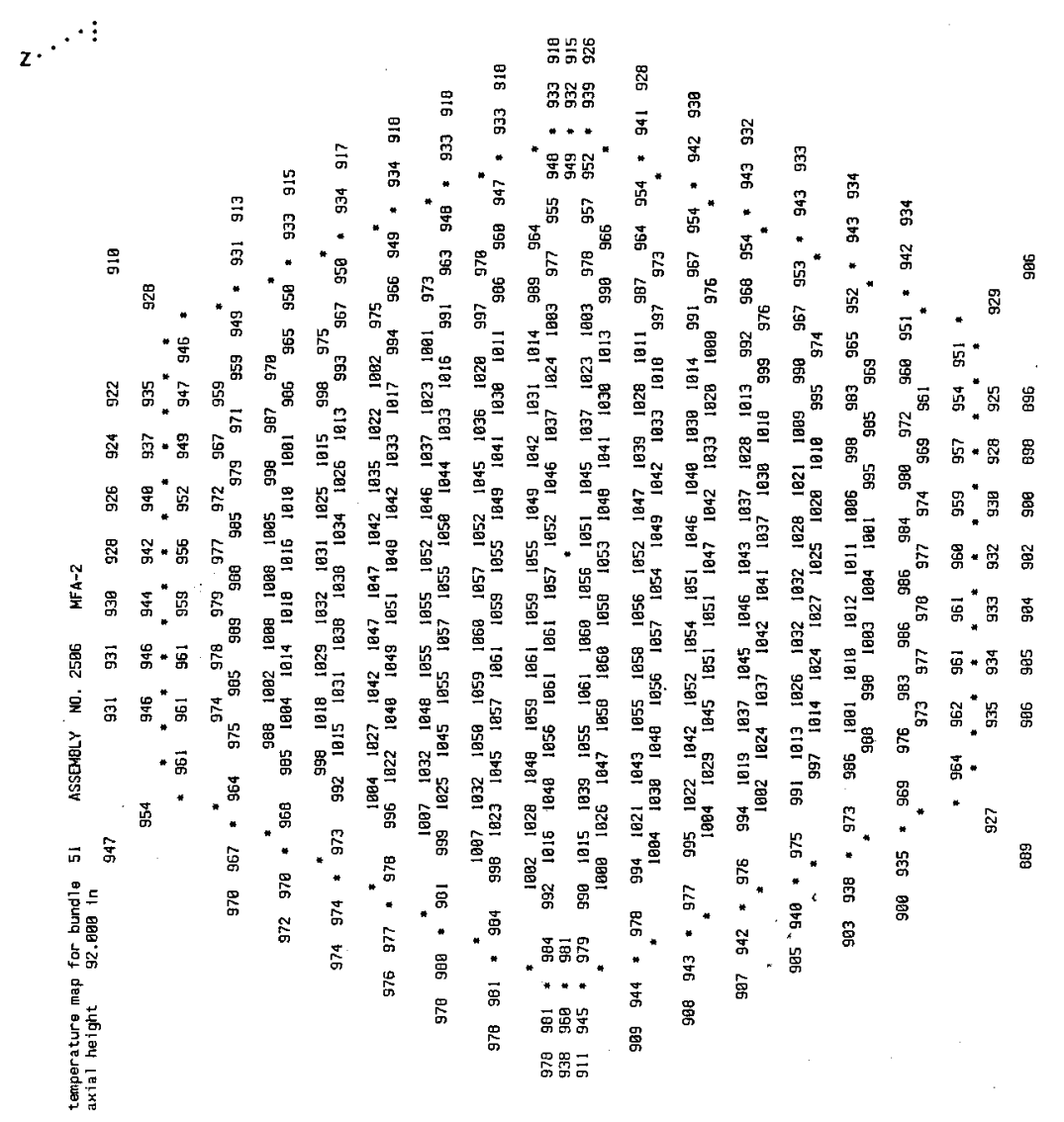


Table E12A-1.1. Fission Power Generated in MFA-1, MFA-2 and Neighboring Assemblies at EOC 12A-1

\begin{tabular}{|c|c|c|c|c|}
\hline $\begin{array}{l}\text { CORE } \\
\text { POS. } \\
1405 \\
1304 \\
1406 \\
1404 \\
1508 \\
1506 \\
1507 \\
2506 \\
2404 \\
2505 \\
2405 \\
2606\end{array}$ & $\begin{array}{l}\star \star \star \star \star \star \star \\
\text { BELOW CORE } \\
1.119 \mathrm{E}-01 \\
0.000 \mathrm{E}+00 \\
1.129 \mathrm{E}-02 \\
0.000 \mathrm{E}+00 \\
0.000 \mathrm{E}+00 \\
1.520 \mathrm{E}-02 \\
1.244 \mathrm{E}-02 \\
9.017 \mathrm{E}-02 \\
0.000 \mathrm{E}+00 \\
8.598 \mathrm{E}-03 \\
1.675 \mathrm{E}-02 \\
1.437 \mathrm{E}-02 \\
6.323 \mathrm{E}-03 \\
1.189 \mathrm{E}-02\end{array}$ & $\begin{array}{c}\text { POWER IN } \\
\text { IN CORE } \\
3.826 E+00 \\
6.838 E+00 \\
4.653 E+00 \\
6.087 E+00 \\
5.298 E+00 \\
3.867 E+00 \\
3.807 E+00 \\
3.102 E+00 \\
5.700 E+00 \\
4.230 E+00 \\
3.289 E+00 \\
3.203 E+00 \\
3.533 E+00 \\
3.290 E+00\end{array}$ & $\begin{array}{r}\text { MEGAWATTS } \\
\text { ABOVE CORE } \\
6.999 E-02 \\
0.000 E+00 \\
8.698 E-03 \\
0.000 E+00 \\
0.000 E+00 \\
1.099 E-02 \\
9.748 E-03 \\
5.823 E-02 \\
0.000 E+00 \\
6.109 E-03 \\
1.205 E-02 \\
8.960 E-03 \\
5.420 E-03 \\
8.983 E-03\end{array}$ & $\begin{array}{l}\star \star \star \star \\
\text { TOTAL PWF } \\
4.008 \mathrm{E}+00 \\
6.838 \mathrm{E}+00 \\
4.673 \mathrm{E}+0 \mathrm{C} \\
6.087 \mathrm{E}+00 \\
5.298 \mathrm{E}+0 \mathrm{OC} \\
3.893 \mathrm{E}+00 \\
3.829 \mathrm{E}+00 \\
3.250 \mathrm{E}+00 \\
5.700 \mathrm{E}+00 \\
4.244 \mathrm{E}+00\end{array}$ \\
\hline
\end{tabular}

Table E12A-1.2. Assembly Averaged Total and Fast Flux in MFA-1 and MFA-2 at EOC 12A-1

\begin{tabular}{|c|c|c|c|}
\hline & Core & Flux & $m^{2}-\sec$ ) \\
\hline $\begin{array}{l}\text { Assembly } \\
\text { MFA-1 } \\
\text { MFA-2 }\end{array}$ & $\begin{array}{l}\text { Pos. } \\
1405 \\
2506\end{array}$ & $\begin{array}{c}\text { Total } \\
3.756 \mathrm{E}+15 \\
2.999 \mathrm{E}+15\end{array}$ & $\begin{array}{l}>0.1 \mathrm{MeV} \\
2.503 \mathrm{E}+15 \\
1.917 \mathrm{E}+15\end{array}$ \\
\hline
\end{tabular}


Table E12A-1.3. Axial Distribution of Total Flux, Fast Flux and Power in MFA-1 at EOC 12A-1

$\begin{array}{rccc}z(\mathrm{~cm}) & \text { Total Flux } & \text { Flux }>0.1 \mathrm{MeV} & \text { Power } \\ -97.78 & 2.6150 \mathrm{E}-02 & 1.3153 \mathrm{E}-02 & 0.0000 \mathrm{E}+00 \\ -92.78 & 5.6022 \mathrm{E}-02 & 2.5393 \mathrm{E}-02 & 0.0000 \mathrm{E}+00 \\ -87.78 & 8.9688 \mathrm{E}-02 & 4.0660 \mathrm{E}-02 & 0.0000 \mathrm{E}+00 \\ -82.78 & 1.2974 \mathrm{E}-01 & 6.0730 \mathrm{E}-02 & 0.0000 \mathrm{E}+00 \\ -77.78 & 1.7898 \mathrm{E}-01 & 8.7860 \mathrm{E}-02 & 0.0000 \mathrm{E}+00 \\ -72.15 & 2.3573 \mathrm{E}-01 & 1.2344 \mathrm{E}-01 & 0.0000 \mathrm{E}+00 \\ -65.90 & 2.9439 \mathrm{E}-01 & 1.7008 \mathrm{E}-01 & 0.0000 \mathrm{E}+00 \\ -60.35 & 3.5700 \mathrm{E}-01 & 2.3562 \mathrm{E}-01 & 1.3718 \mathrm{E}-01 \\ -55.49 & 4.3850 \mathrm{E}-01 & 3.2006 \mathrm{E}-01 & 1.5545 \mathrm{E}-01 \\ -50.62 & 5.3154 \mathrm{E}-01 & 4.2847 \mathrm{E}-01 & 1.7810 \mathrm{E}-01 \\ -47.17 & 6.0438 \mathrm{E}-01 & 5.2824 \mathrm{E}-01 & 1.9927 \mathrm{E}-01 \\ -43.58 & 6.8041 \mathrm{E}-01 & 6.3978 \mathrm{E}-01 & 7.5118 \mathrm{E}-01 \\ -38.45 & 7.9318 \mathrm{E}-01 & 7.7839 \mathrm{E}-01 & 8.4228 \mathrm{E}-01 \\ -33.32 & 8.9885 \mathrm{E}-01 & 8.9691 \mathrm{E}-01 & 9.4456 \mathrm{E}-01 \\ -28.20 & 9.9156 \mathrm{E}-01 & 9.9483 \mathrm{E}-01 & 9.7190 \mathrm{E}-01 \\ -23.07 & 1.0703 \mathrm{E}+00 & 1.0761 \mathrm{E}+00 & 1.0474 \mathrm{E}+00 \\ -17.94 & 1.1333 \mathrm{E}+00 & 1.1400 \mathrm{E}+00 & 1.1081 \mathrm{E}+00 \\ -12.82 & 1.1796 \mathrm{E}+00 & 1.1858 \mathrm{E}+00 & 1.1169 \mathrm{E}+00 \\ -7.69 & 1.2098 \mathrm{E}+00 & 1.2158 \mathrm{E}+00 & 1.1451 \mathrm{E}+00 \\ -2.56 & 1.2233 \mathrm{E}+00 & 1.2296 \mathrm{E}+00 & 1.1578 \mathrm{E}+00 \\ 2.56 & 1.2200 \mathrm{E}+00 & 1.2270 \mathrm{E}+00 & 1.1659 \mathrm{E}+00 \\ 7.69 & 1.1993 \mathrm{E}+00 & 1.2073 \mathrm{E}+00 & 1.1464 \mathrm{E}+00 \\ 12.82 & 1.1613 \mathrm{E}+00 & 1.1704 \mathrm{E}+00 & 1.1106 \mathrm{E}+00 \\ 17.94 & 1.1063 \mathrm{E}+00 & 1.1171 \mathrm{E}+00 & 1.1123 \mathrm{E}+00 \\ 23.07 & 1.0333 \mathrm{E}+00 & 1.0440 \mathrm{E}+00 & 1.0397 \mathrm{E}+00 \\ 28.20 & 9.4334 \mathrm{E}-01 & 9.5209 \mathrm{E}-01 & 9.5054 \mathrm{E}-01 \\ 33.32 & 8.3880 \mathrm{E}-01 & 8.4324 \mathrm{E}-01 & 9.1747 \mathrm{E}-01 \\ 38.45 & 7.2089 \mathrm{E}-01 & 7.1402 \mathrm{E}-01 & 7.9473 \mathrm{E}-01 \\ 43.58 & 5.9654 \mathrm{E}-01 & 5.6771 \mathrm{E}-01 & 6.7702 \mathrm{E}-01 \\ 47.17 & 5.1581 \mathrm{E}-01 & 4.5961 \mathrm{E}-01 & 1.3950 \mathrm{E}-01 \\ 50.62 & 4.4444 \mathrm{E}-01 & 3.6931 \mathrm{E}-01 & 1.1723 \mathrm{E}-01 \\ 55.49 & 3.5813 \mathrm{E}-01 & 2.7442 \mathrm{E}-01 & 9.4642 \mathrm{E}-02 \\ 60.35 & 2.8079 \mathrm{E}-01 & 1.9880 \mathrm{E}-01 & 7.6437 \mathrm{E}-02 \\ 64.98 & 2.2800 \mathrm{E}-01 & 1.5008 \mathrm{E}-01 & 0.0000 \mathrm{E}+00 \\ 69.39 & 1.9387 \mathrm{E}-01 & 1.2204 \mathrm{E}-01 & 0.0000 \mathrm{E}+00 \\ 76.46 & 1.4484 \mathrm{E}-01 & 8.4474 \mathrm{E}-02 & 0.0000 \mathrm{E}+00 \\ 86.80 & 9.2905 \mathrm{E}-02 & 4.9663 \mathrm{E}-02 & 0.0000 \mathrm{E}+00 \\ 98.14 & 5.2973 \mathrm{E}-02 & 2.6600 \mathrm{E}-02 & 0.0000 \mathrm{E}+00 \\ 110.00 & 2.2503 \mathrm{E}-02 & 1.1714 \mathrm{E}-02 & 0.0000 \mathrm{E}+00\end{array}$


Table El2A-1.4. Axial Distribution of Total Flux, Fast Flux and Power in MFA-2 at EOC 12A-1

$\begin{array}{rccc}z(\mathrm{~cm}) & \text { Total Flux } & \text { Flux }>0.1 \text { MeV } & \text { Power } \\ -97.78 & 2.6777 \mathrm{E}-02 & 1.3839 \mathrm{E}-02 & 0.0000 \mathrm{E}+00 \\ -92.78 & 5.7456 \mathrm{E}-02 & 2.6709 \mathrm{E}-02 & 0.0000 \mathrm{E}+00 \\ -87.78 & 9.2080 \mathrm{E}-02 & 4.2718 \mathrm{E}-02 & 0.0000 \mathrm{E}+00 \\ -82.78 & 1.3339 \mathrm{E}-01 & 6.3710 \mathrm{E}-02 & 0.0000 \mathrm{E}+00 \\ -77.78 & 1.8440 \mathrm{E}-01 & 9.2006 \mathrm{E}-02 & 0.0000 \mathrm{E}+00 \\ -72.15 & 2.4330 \mathrm{E}-01 & 1.2893 \mathrm{E}-01 & 0.0000 \mathrm{E}+00 \\ -65.90 & 3.0306 \mathrm{E}-01 & 1.7657 \mathrm{E}-01 & 0.0000 \mathrm{E}+00 \\ -60.35 & 3.6474 \mathrm{E}-01 & 2.4243 \mathrm{E}-01 & 1.3802 \mathrm{E}-01 \\ -55.49 & 4.4529 \mathrm{E}-01 & 3.2648 \mathrm{E}-01 & 1.5515 \mathrm{E}-01 \\ -50.62 & 5.3729 \mathrm{E}-01 & 4.3395 \mathrm{E}-01 & 1.7599 \mathrm{E}-01 \\ -47.17 & 6.0973 \mathrm{E}-01 & 5.3337 \mathrm{E}-01 & 1.9556 \mathrm{E}-01 \\ -43.58 & 6.8556 \mathrm{E}-01 & 6.4495 \mathrm{E}-01 & 7.6222 \mathrm{E}-01 \\ -38.45 & 7.9737 \mathrm{E}-01 & 7.8240 \mathrm{E}-01 & 8.4975 \mathrm{E}-01 \\ -33.32 & 9.0184 \mathrm{E}-01 & 8.9968 \mathrm{E}-01 & 9.4979 \mathrm{E}-01 \\ -28.20 & 9.9337 \mathrm{E}-01 & 9.9653 \mathrm{E}-01 & 9.7481 \mathrm{E}-01 \\ -23.07 & 1.0712 \mathrm{E}+00 & 1.0770 \mathrm{E}+00 & 1.0493 \mathrm{E}+00 \\ -17.94 & 1.1334 \mathrm{E}+00 & 1.1402 \mathrm{E}+00 & 1.1092 \mathrm{E}+00 \\ -12.82 & 1.1790 \mathrm{E}+00 & 1.1855 \mathrm{E}+00 & 1.1163 \mathrm{E}+00 \\ -7.69 & 1.2085 \mathrm{E}+00 & 1.2150 \mathrm{E}+00 & 1.1439 \mathrm{E}+00 \\ -2.56 & 1.2214 \mathrm{E}+00 & 1.2281 \mathrm{E}+00 & 1.1559 \mathrm{E}+00 \\ 2.56 & 1.2175 \mathrm{E}+00 & 1.2249 \mathrm{E}+00 & 1.1626 \mathrm{E}+00 \\ 7.69 & 1.1961 \mathrm{E}+00 & 1.2043 \mathrm{E}+00 & 1.1424 \mathrm{E}+00 \\ 12.82 & 1.1576 \mathrm{E}+00 & 1.1668 \mathrm{E}+00 & 1.1060 \mathrm{E}+00 \\ 17.94 & 1.1023 \mathrm{E}+00 & 1.1130 \mathrm{E}+00 & 1.1055 \mathrm{E}+00 \\ 23.07 & 1.0293 \mathrm{E}+00 & 1.0398 \mathrm{E}+00 & 1.0331 \mathrm{E}+00 \\ 28.20 & 9.4022 \mathrm{E}-01 & 9.4864 \mathrm{E}-01 & 9.4488 \mathrm{E}-01 \\ 33.32 & 8.3766 \mathrm{E}-01 & 8.4173 \mathrm{E}-01 & 9.1234 \mathrm{E}-01 \\ 38.45 & 7.2342 \mathrm{E}-01 & 7.1629 \mathrm{E}-01 & 7.9518 \mathrm{E}-01 \\ 43.58 & 6.0412 \mathrm{E}-01 & 5.7505 \mathrm{E}-01 & 6.8691 \mathrm{E}-01 \\ 47.17 & 5.2567 \mathrm{E}-01 & 4.6762 \mathrm{E}-01 & 1.4143 \mathrm{E}-01 \\ 50.62 & 4.5267 \mathrm{E}-01 & 3.7431 \mathrm{E}-01 & 1.2009 \mathrm{E}-01 \\ 55.49 & 3.6127 \mathrm{E}-01 & 2.7460 \mathrm{E}-01 & 9.7577 \mathrm{E}-02 \\ 60.35 & 2.7956 \mathrm{E}-01 & 1.9574 \mathrm{E}-01 & 7.8927 \mathrm{E}-02 \\ 64.98 & 2.2634 \mathrm{E}-01 & 1.4684 \mathrm{E}-01 & 0.0000 \mathrm{E}+00 \\ 69.39 & 1.9345 \mathrm{E}-01 & 1.2018 \mathrm{E}-01 & 0.0000 \mathrm{E}+00 \\ 76.46 & 1.4614 \mathrm{E}-01 & 8.4450 \mathrm{E}-02 & 0.0000 \mathrm{E}+00 \\ 86.80 & 9.4377 \mathrm{E}-02 & 5.0244 \mathrm{E}-02 & 0.0000 \mathrm{E}+00 \\ 98.14 & 5.3834 \mathrm{E}-02 & 2.7077 \mathrm{E}-02 & 0.0000 \mathrm{E}+00 \\ 110.00 & 2.2888 \mathrm{E}-02 & 1.1972 \mathrm{E}-02 & 0.0000 \mathrm{E}+00\end{array}$


HNF-SD-FF-ANAL-009 Rev. 1

Table E12A-1.5. Fission Power Distribution by Pin in MFA-1 at EOC 12A-1 CORE POSITION 1405

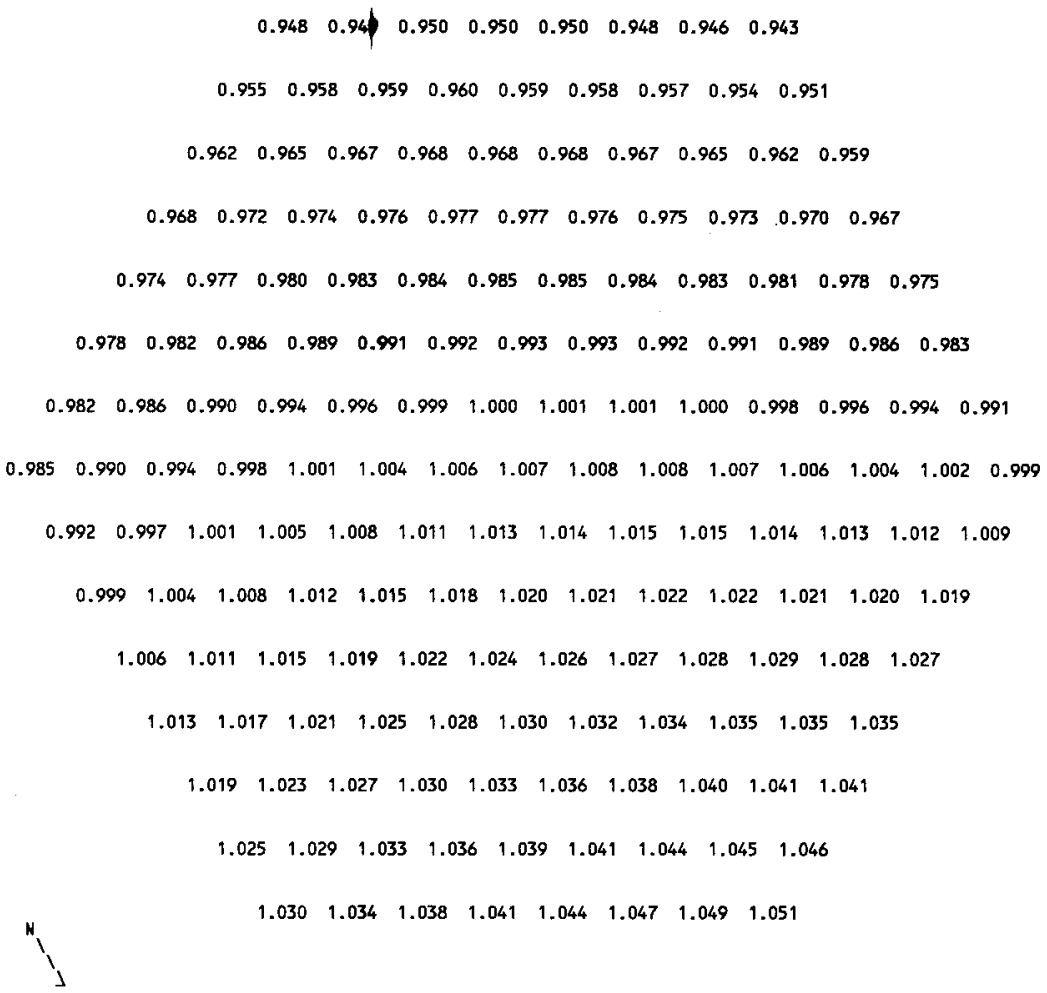


HNF-SD-FF-ANAL-009 Rev. 1

Table E12A-1.6. Fission Power Distribution by Pin in MFA-2 at EOC 12A-1 CORE POSITION 2506

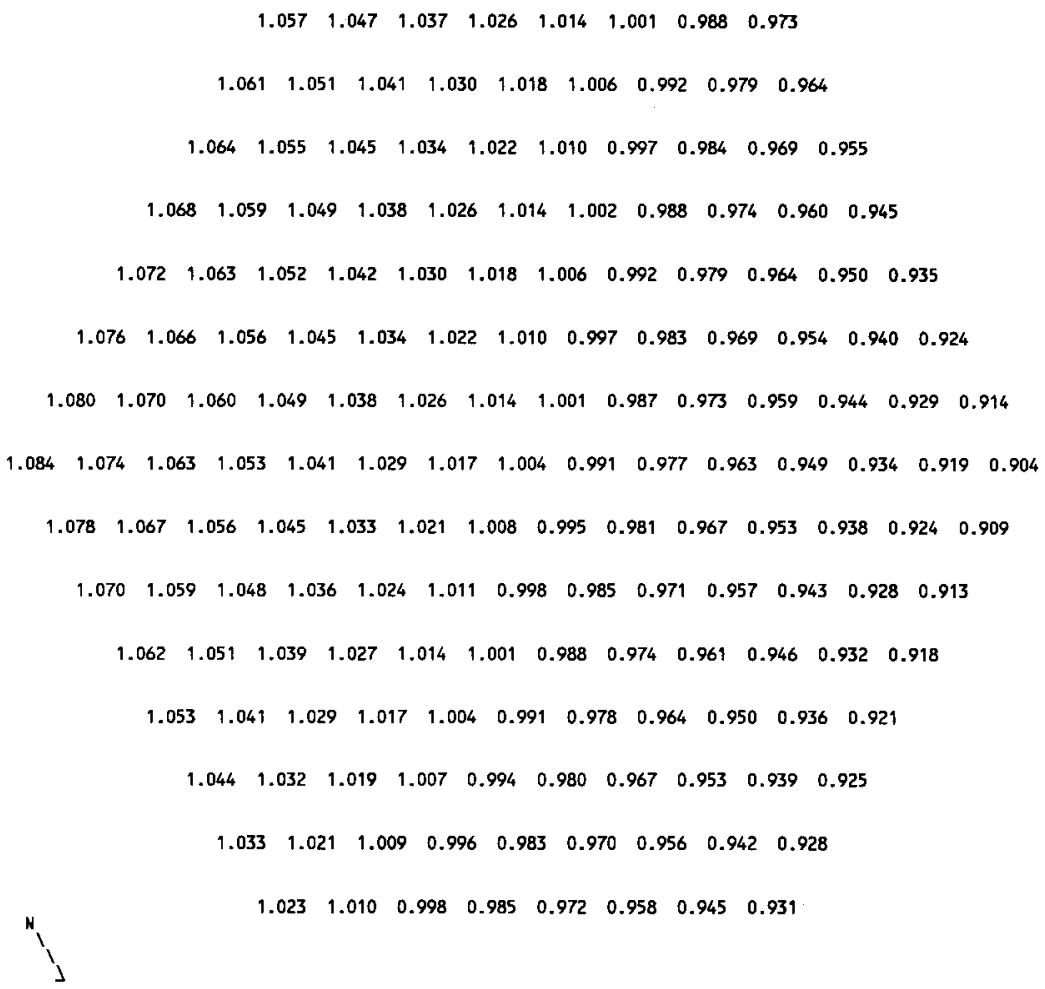


HNF-SD-FF-ANAL-009 Rev. 1

Table E12A-1.7. Fast Flux Distribution by Pin in MFA-1 at EOC 12A-1 CORE POSITION 1404

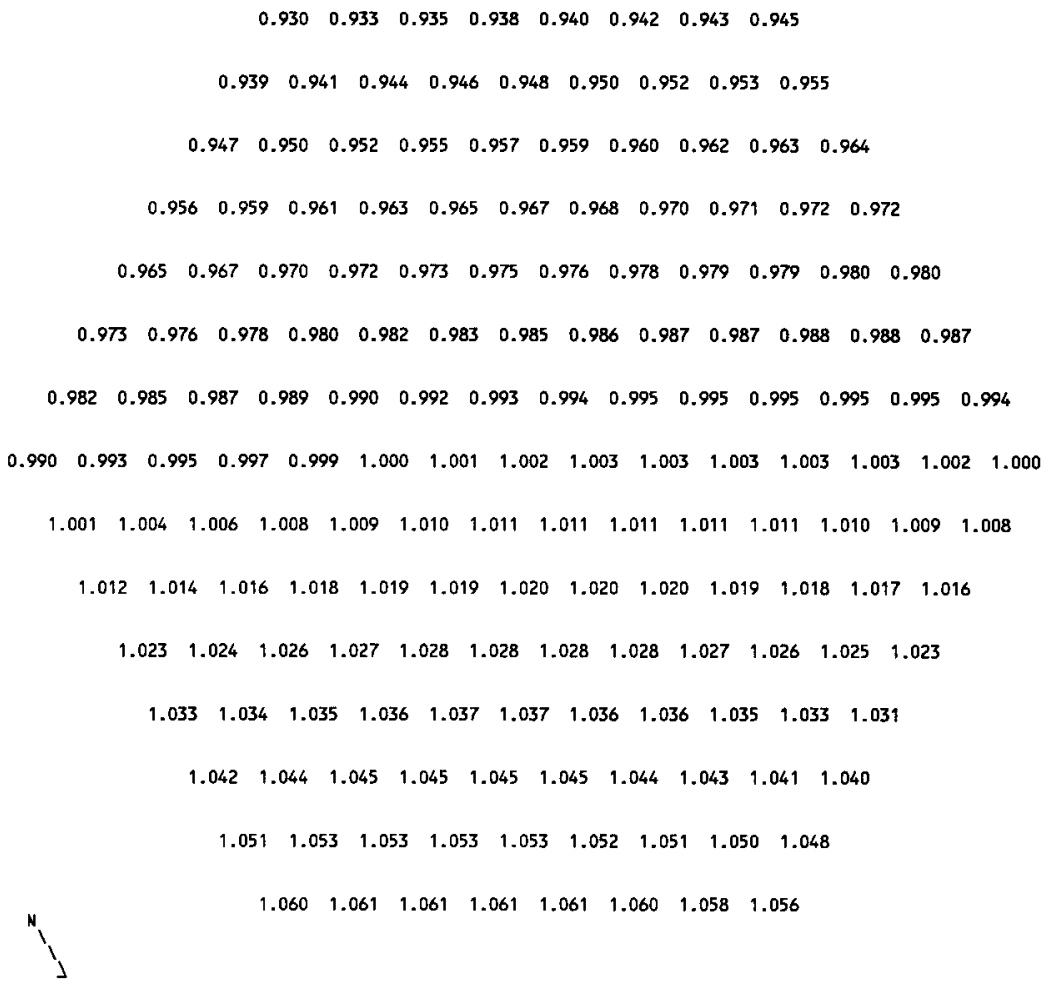


HNF-SD-FF-ANAL-009 Rev. 1

Table E12A-1.8. Fast Flux Distribution by Pin in MFA-2 at EOC 12A-1 CORE POSITION 2506

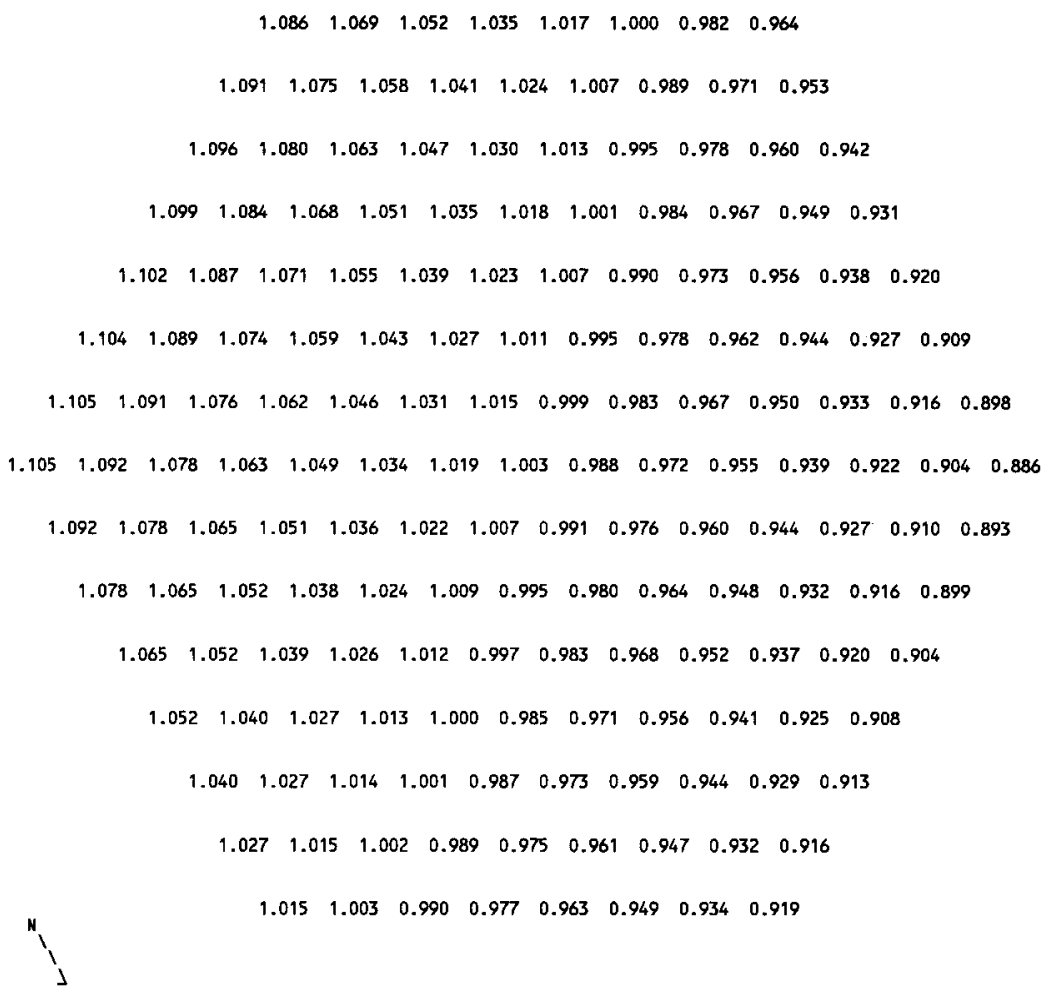


Table E12A-1.9. MFA-1 and MFA-2 Duct Wa11

Fast Flux Data at EOC 12A-1

$\begin{array}{ccc}\text { Side } & \begin{array}{c}\text { Flux }>0.1 \\ \text { MFA-1 }\end{array} & \begin{array}{c}\text { MeV }\left(\mathrm{n} / \mathrm{cm}^{2}-\mathrm{sec}\right) \\ \text { MFA-2 }\end{array} \\ \text { E } & 2.5646 \mathrm{E}+15 & 2.0417 \mathrm{E}+15 \\ \text { SE } & 2.3645 \mathrm{E}+15 & 2.1455 \mathrm{E}+15 \\ \text { SW } & 2.3006 \mathrm{E}+15 & 1.9670 \mathrm{E}+15 \\ \text { W } & 2.4155 \mathrm{E}+15 & 1.7359 \mathrm{E}+15 \\ \text { NW } & 2.5619 \mathrm{E}+15 & 1.6943 \mathrm{E}+15 \\ \text { NE } & 2.6675 \mathrm{E}+15 & 1.8421 \mathrm{E}+15\end{array}$

Table E12A-1.10. Assembly Outlet Temperatures and Flow Rates at EOC 12A-1

\begin{tabular}{lcl}
\multicolumn{3}{c}{ CALCULATED } \\
CORE & $\begin{array}{c}\text { OUTLET TEMP. } \\
\text { (DEG F) }\end{array}$ & $\begin{array}{l}\text { FLOW RATE } \\
\text { (LB/H) }\end{array}$ \\
POS. & 108910 \\
1405 & 1073 & 105 \\
1304 & 1058 & 190310 \\
1404 & 1011 & 192650 \\
1406 & 941 & 197210 \\
1506 & 897 & 197210 \\
1507 & 892 & 197210 \\
1508 & 993 & 177530 \\
2506 & 1000 & 108910 \\
2404 & 990 & 193950 \\
2405 & 866 & 197230 \\
2505 & 912 & 197210 \\
2507 & 862 & 197210 \\
2606 & 898 & 161730 \\
2607 & 924 & 155100
\end{tabular}

Assembly flows are based on a calculated core pressure drop of $99.3 \mathrm{psi}$ at a total reactor flow rate of $16.74 \mathrm{E}+06 \mathrm{lb} / \mathrm{hr}$. 
Table E12A-1.11. MFA-1 Sodium Subchannel Temperatures at Top of Core Elevation for EOC 12A-1

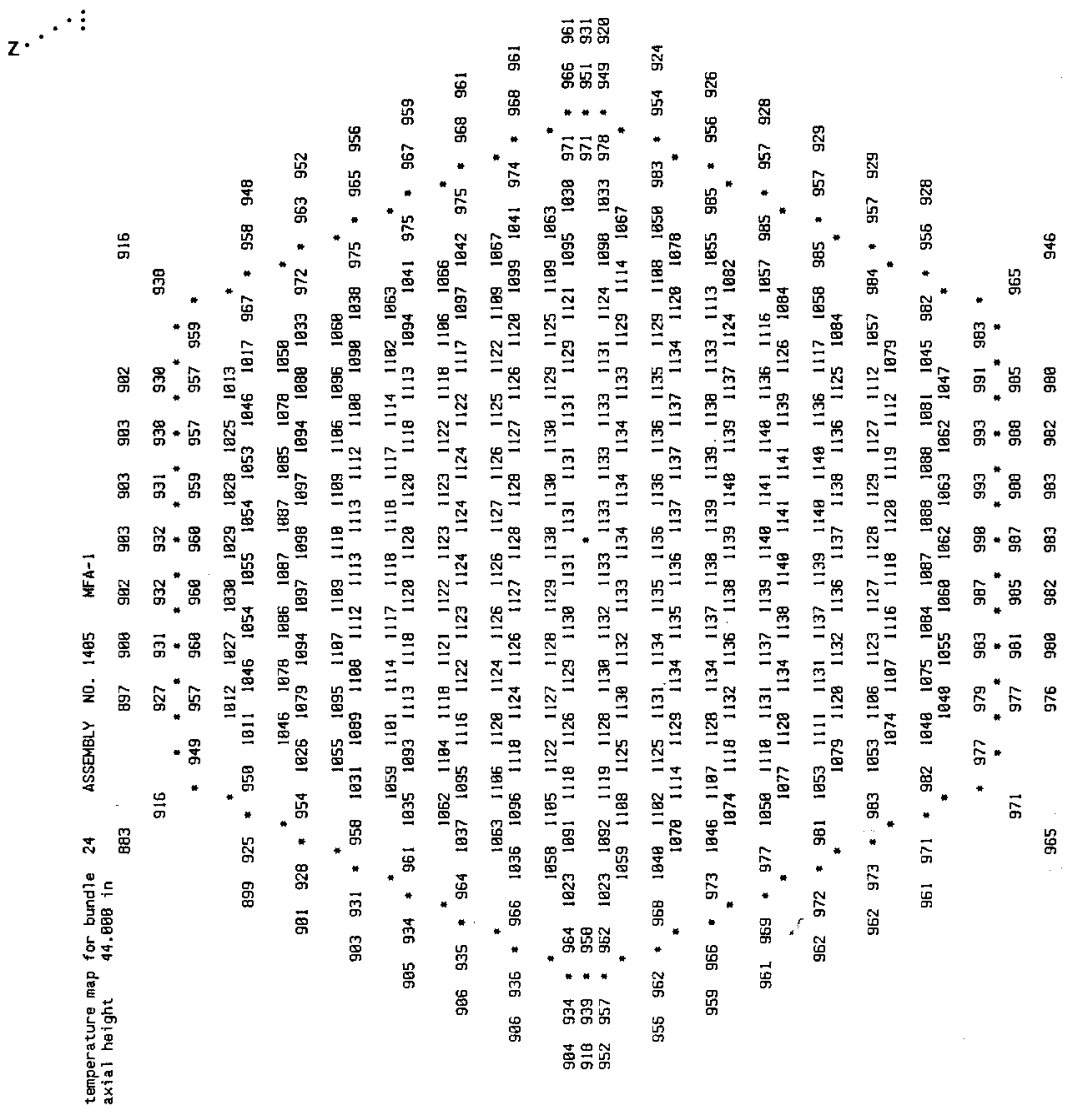


Table E12A-1.12. MFA-2 Sodium Subchannel Temperatures at Top of Core Elevation for EOC 12A-1

${ }_{2} . \cdot \cdots$

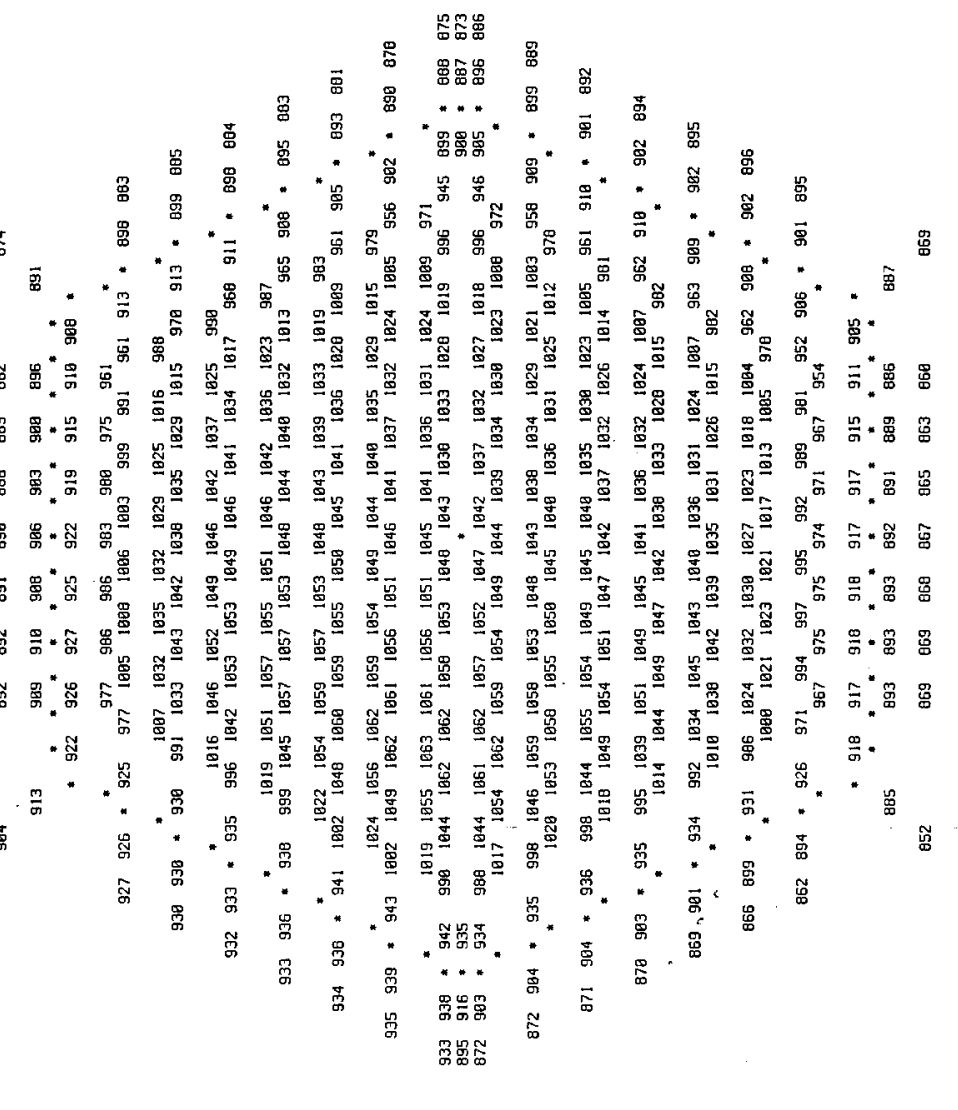


Table E12A-1.13. MFA-l Sodium Subchannel Temperatures at Elevation of Upper Axial Blanket for EOC 12A-1

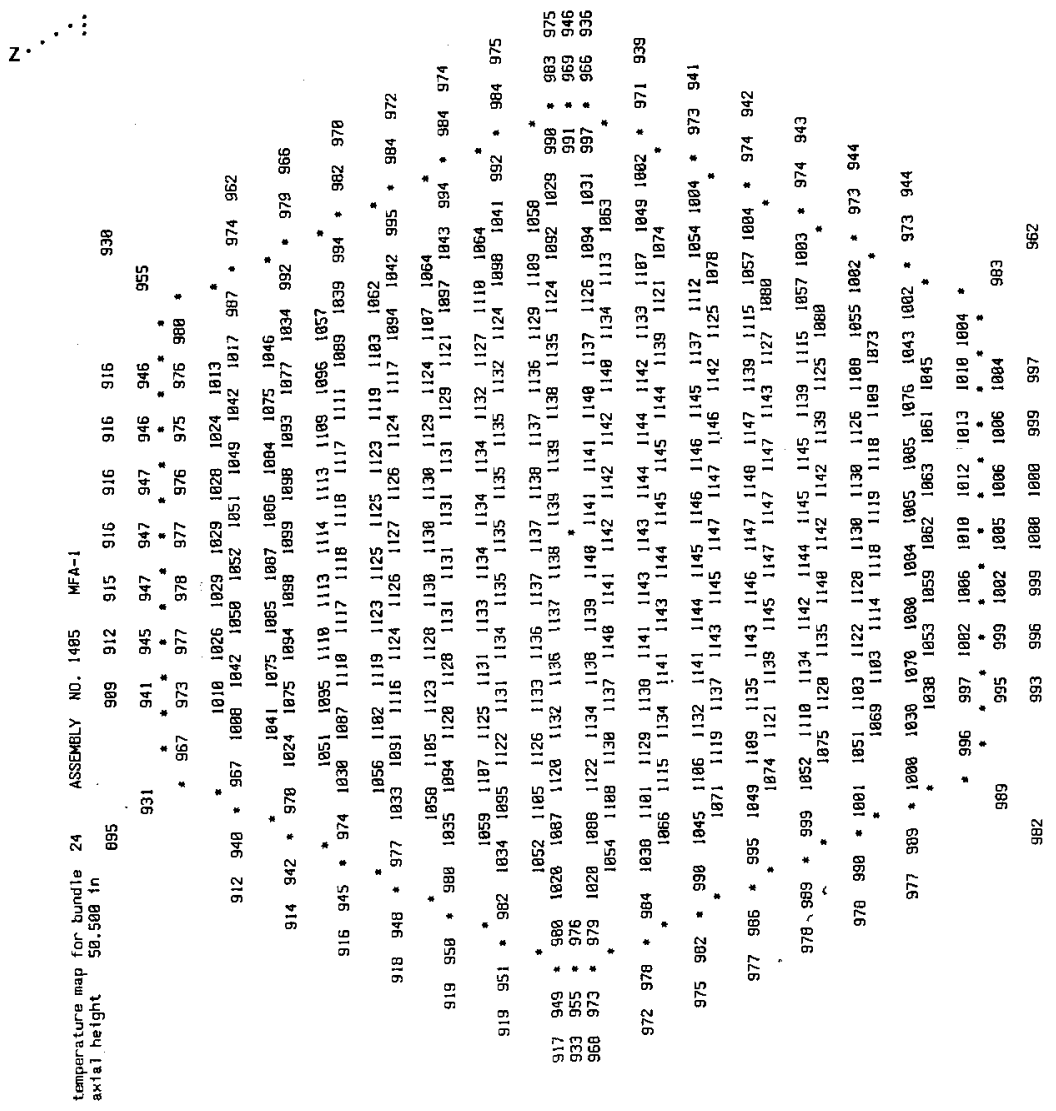


Table E12A-1.14. MFA-2 Sodium Subchannel Temperatures at Elevation of Upper Axial Blanket for EOC 12A-1

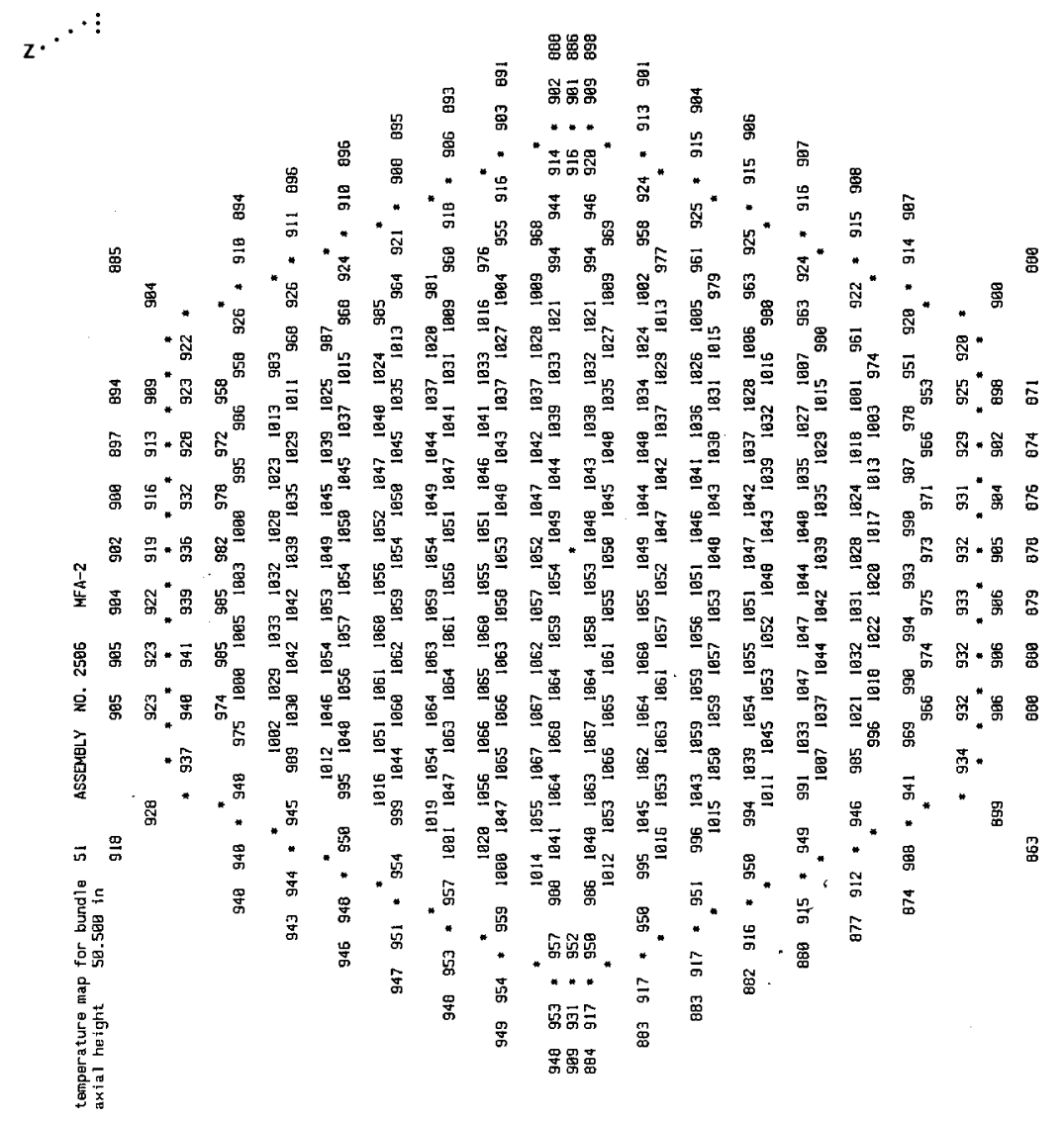


Table E12A-1.15. MFA-1 Sodium Subchannel Temperatures at Top of Fuel Pin Bundle for EOC 12A-1

$$
\begin{aligned}
& z \cdot \cdot \cdot
\end{aligned}
$$

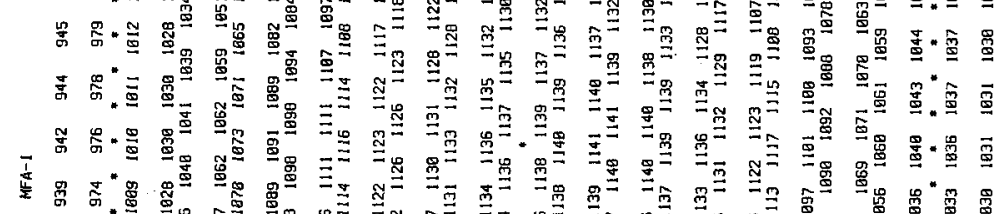

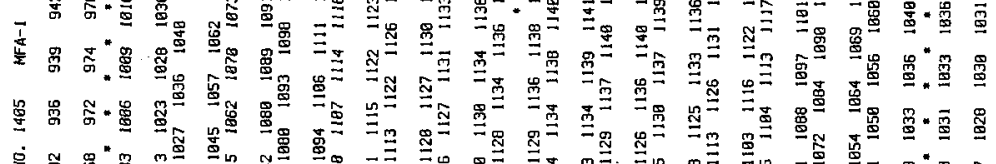

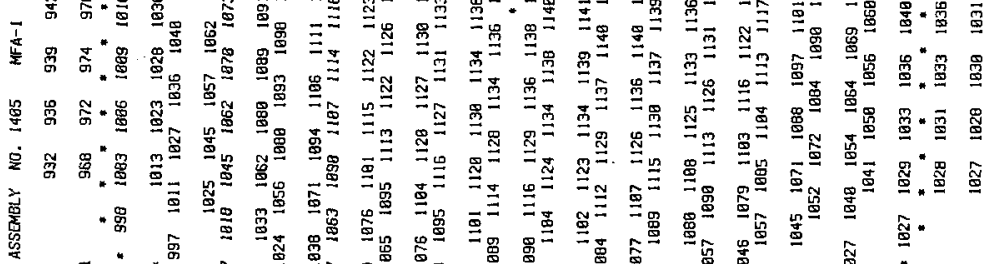

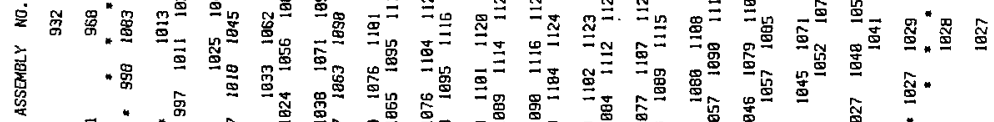

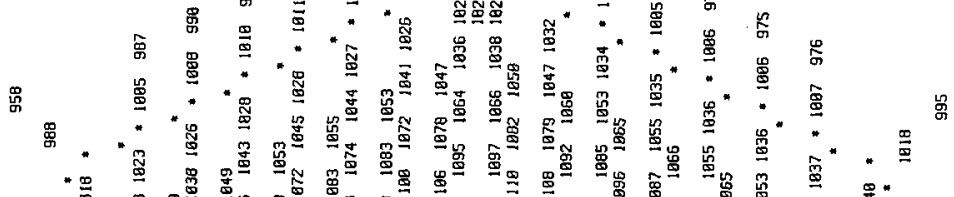

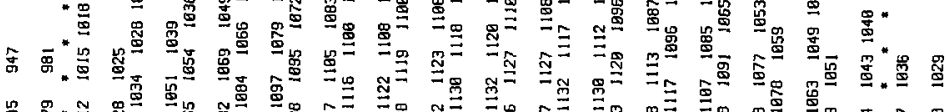

$$
\begin{aligned}
& \text { 品 } \\
& \text { 㠃 }
\end{aligned}
$$

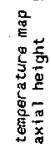

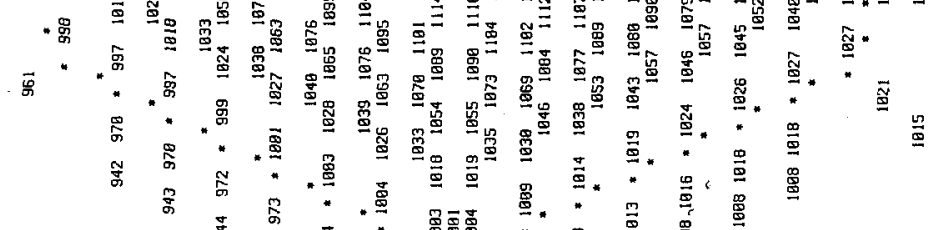


Table E12A-1.16. MFA-2 Sodium Subchanne1 Temperatures at Top of Fuel Pin Bundle for EOC 12A-1

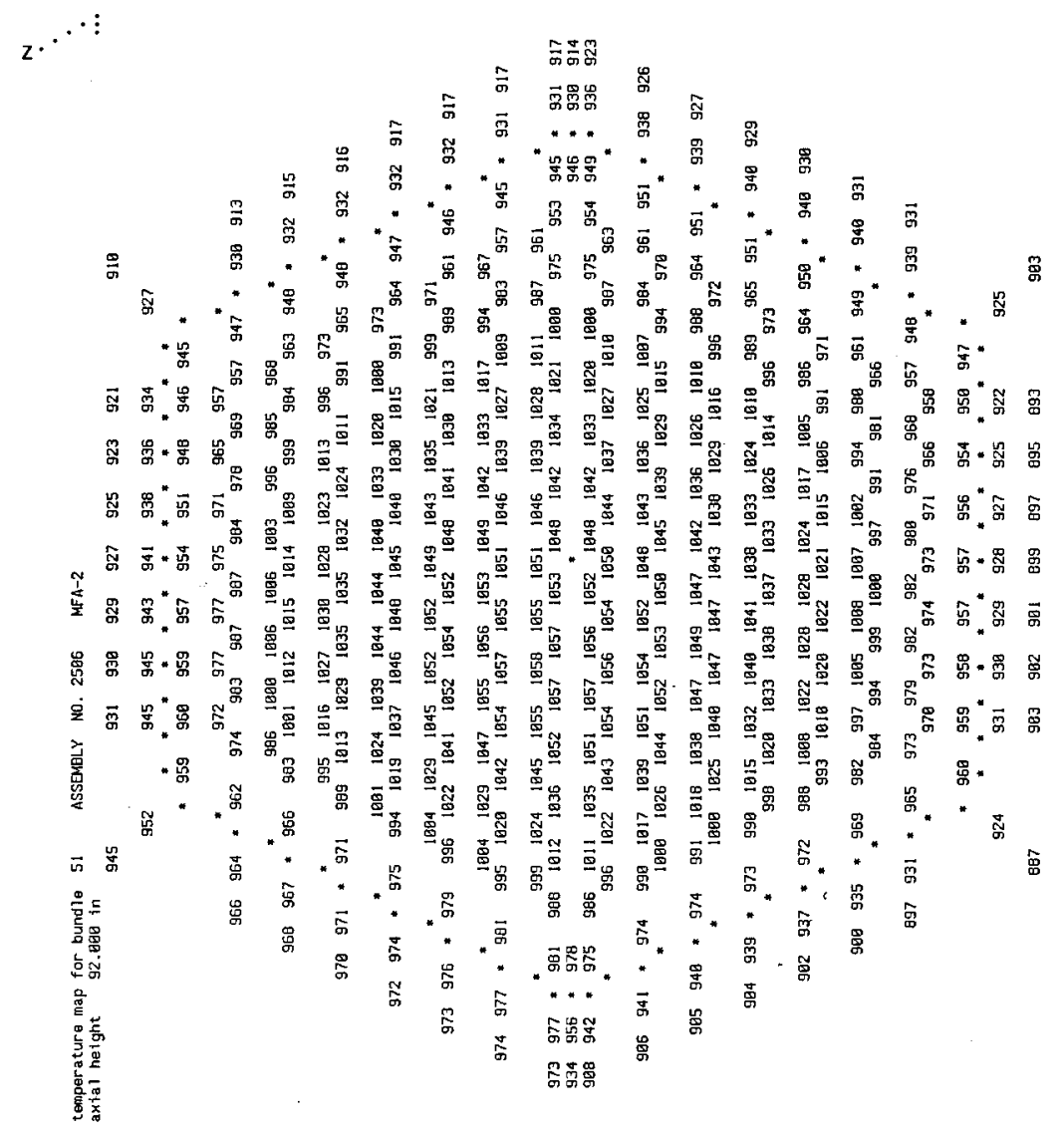


Table E12A-1.17. Composition of MFA-1 at EOC 12A-1

Axial Axial Range (cm) Leve1 Lower Upper $1-128.05 \quad-75.28$

$\begin{array}{lll}2 & -75.28 & -62.78\end{array}$

$3 \quad-62.78$

$-46.14$

4 $-46.14 \quad-30.76$

$5 \quad-30.76 \quad-15.38$

$6 \quad-15.38$

0.00

$$
\text { Atom Density }(\mathrm{a} / \mathrm{b}-\mathrm{cm})
$$

Fresh End of Cycle

$5.3150 \mathrm{E}-03 \quad 5.3150 \mathrm{E}-03$

$6.4090 \mathrm{E}-02 \quad 6.4090 \mathrm{E}-02$

SS-316 6.4090E-02

$\begin{array}{lll}\mathrm{Na}-23 & 1.3177 \mathrm{E}-02 & 1.3177 \mathrm{E}-02 \\ \mathrm{SS}-316 & 3.4175 \mathrm{E}-02 & 3.4175 \mathrm{E}-02\end{array}$

$\begin{array}{lll}\mathrm{U}-235 & 1.4143 \mathrm{E}-05 \quad 8.4474 \mathrm{E}-06\end{array}$

U-238 $\quad 7.0573 \mathrm{E}-03 \quad 6.7063 \mathrm{E}-03$

$\mathrm{Pu}-239 \quad 0.0000 \mathrm{E}+00 \quad 2.6525 \mathrm{E}-04$

$\mathrm{Pu}-240 \quad 0.0000 \mathrm{E}+00 \quad 1.9530 \mathrm{E}-05$

$\begin{array}{lll}\mathrm{FP} & 0.0000 \mathrm{E}+00 \quad 6.9474 \mathrm{E}-05\end{array}$

$\begin{array}{lll}0-16 & 1.4143 \mathrm{E}-02 & 1.4143 \mathrm{E}-02\end{array}$

$\mathrm{Na}-23 \quad 9.7416 \mathrm{E}-03 \quad 9.7416 \mathrm{E}-03$

SS-316 $\quad 1.9073 \mathrm{E}-02 \quad 1.9073 \mathrm{E}-02$

$\begin{array}{lll}\mathrm{U}-235 & 1.0840 \mathrm{E}-05 & 6.8588 \mathrm{E}-06\end{array}$

U-238 $\quad 4.4499 \mathrm{E}-03 \quad 4.1889 \mathrm{E}-03$

$\begin{array}{lll}\mathrm{Pu}-239 \quad 1.7520 \mathrm{E}-03 & 1.2941 \mathrm{E}-03\end{array}$

Pu-240 2.2340E-04 3.0520E-04

Pu-241 $1.9280 \mathrm{E}-05 \quad 2.6738 \mathrm{E}-05$

Pu-242 4.7430E-06 5.4830E-06

Am-241 7.6680E-07 3.7805E-06

$\begin{array}{lll}\mathrm{FP} & 0.0000 \mathrm{E}+00 & 6.2765 \mathrm{E}-04\end{array}$

0-16 $1.2670 \mathrm{E}-02 \quad 1.2670 \mathrm{E}-02$

$\begin{array}{lll}\mathrm{Na}-23 & 9.8100 \mathrm{E}-03 & 9.8100 \mathrm{E}-03\end{array}$

SS-316 $1.8990 \mathrm{E}-02 \quad 1.8990 \mathrm{E}-02$

U-235 $1.0840 \mathrm{E}-05 \quad 6.1553 \mathrm{E}-06$

U-238 4.4499E-03 4.1238E-03

Pu-239 $1.7520 \mathrm{E}-03 \quad 1.1976 \mathrm{E}-03$

Pu-240 2.2340E-04 3.0496E-04

Pu-241 1.9280E-05 2.6989E-05

Pu-242 4.7430E-06 5.5062E-06

Am-241 7.6680E-07 3.6667E-06

$\begin{array}{lll}\text { FP } & 0.0000 \mathrm{E}+00 \quad 7.8967 \mathrm{E}-04\end{array}$

0-16 $1.2670 \mathrm{E}-02 \quad 1.2670 \mathrm{E}-02$

$\mathrm{Na}-23 \quad 9.8100 \mathrm{E}-03 \quad 9.8100 \mathrm{E}-03$

SS-316 $\quad 1.8990 \mathrm{E}-02 \quad 1.8990 \mathrm{E}-02$

U-235 $\quad 1.0840 \mathrm{E}-05 \quad 5.7821 \mathrm{E}-06$

U-238 $4.4499 \mathrm{E}-03 \quad 4.0899 \mathrm{E}-03$

Pu-239 $1.7520 \mathrm{E}-03 \quad 1.1516 \mathrm{E}-03$

Pu-240 2.2340E-04 3.0928E-04

Pu-241 $1.9280 \mathrm{E}-05 \quad 2.8000 \mathrm{E}-05$

Pu-242 4.7430E-06 5.6196E-06

Am-241 7.6680E-07 3.6580E-06

$\begin{array}{lll}\text { FP } & 0.0000 \mathrm{E}+00 \quad 8.6475 \mathrm{E}-04\end{array}$

$0-16 \quad 1.2670 \mathrm{E}-02 \quad 1.2670 \mathrm{E}-02$

$\mathrm{Na}-23 \quad 9.8100 \mathrm{E}-03 \quad 9.8100 \mathrm{E}-03$

SS-316 $1.8990 \mathrm{E}-02 \quad 1.8990 \mathrm{E}-02$ 
$\begin{array}{lll}7 & 0.00 & 15.38\end{array}$

$\begin{array}{lll}8 & 15.38 & 30.76\end{array}$

$\begin{array}{lll}\text { U-235 } & 1.0840 \mathrm{E}-05 & 5.9037 \mathrm{E}-06 \\ \mathrm{U}-238 & 4.4499 \mathrm{E}-03 & 4.1010 \mathrm{E}-03 \\ \mathrm{Pu}-239 & 1.7520 \mathrm{E}-03 & 1.1658 \mathrm{E}-03 \\ \mathrm{Pu}-240 & 2.2340 \mathrm{E}-04 & 3.0735 \mathrm{E}-04 \\ \mathrm{Pu}-241 & 1.9280 \mathrm{E}-05 & 2.7591 \mathrm{E}-05 \\ \mathrm{Pu}-242 & 4.7430 \mathrm{E}-06 & 5.5728 \mathrm{E}-06 \\ \mathrm{Am}-241 & 7.6680 \mathrm{E}-07 & 3.6544 \mathrm{E}-06 \\ \mathrm{FP} & 0.0000 \mathrm{E}+00 & 8.4132 \mathrm{E}-04 \\ 0-16 & 1.2670 \mathrm{E}-02 & 1.2670 \mathrm{E}-02 \\ \mathrm{Na}-23 & 9.8100 \mathrm{E}-03 & 9.8100 \mathrm{E}-03 \\ \text { SS-316 } & 1.8990 \mathrm{E}-02 & 1.8990 \mathrm{E}-02\end{array}$

$\begin{array}{lll}\text { U-235 } & 1.0840 \mathrm{E}-05 & 6.5153 \mathrm{E}-06 \\ \mathrm{U}-238 & 4.4499 \mathrm{E}-03 & 4.1556 \mathrm{E}-03 \\ \text { Pu-239 } & 1.7520 \mathrm{E}-03 & 1.2399 \mathrm{E}-03 \\ \text { Pu-240 } & 2.2340 \mathrm{E}-04 & 2.9878 \mathrm{E}-04 \\ \mathrm{Pu}-241 & 1.9280 \mathrm{E}-05 & 2.5806 \mathrm{E}-05 \\ \mathrm{Pu}-242 & 4.7430 \mathrm{E}-06 & 5.3841 \mathrm{E}-06 \\ \mathrm{Am}-241 & 7.6680 \mathrm{E}-07 & 3.6558 \mathrm{E}-06 \\ \text { FP } & 0.0000 \mathrm{E}+00 & 7.2320 \mathrm{E}-04 \\ 0-16 & 1.2670 \mathrm{E}-02 & 1.2670 \mathrm{E}-02 \\ \mathrm{Na}-23 & 9.8100 \mathrm{E}-03 & 9.8100 \mathrm{E}-03 \\ \text { SS-316 } & 1.8990 \mathrm{E}-02 & 1.8990 \mathrm{E}-02\end{array}$

$\begin{array}{lll}9 & 30.76 & 46.14\end{array}$

$\begin{array}{lll}\text { U-235 } & 1.0840 \mathrm{E}-05 & 7.4738 \mathrm{E}-06 \\ \mathrm{U}-238 & 4.4499 \mathrm{E}-03 & 4.2354 \mathrm{E}-03 \\ \text { Pu-239 } & 1.7520 \mathrm{E}-03 & 1.3636 \mathrm{E}-03 \\ \text { Pu-240 } & 2.2340 \mathrm{E}-04 & 2.9122 \mathrm{E}-04 \\ \text { Pu-241 } & 1.9280 \mathrm{E}-05 & 2.4293 \mathrm{E}-05 \\ \text { Pu-242 } & 4.7430 \mathrm{E}-06 & 5.2575 \mathrm{E}-06 \\ \text { Am-241 } & 7.6680 \mathrm{E}-07 & 3.7340 \mathrm{E}-06 \\ \text { FP } & 0.0000 \mathrm{E}+00 & 5.2805 \mathrm{E}-04 \\ 0-16 & 1.2670 \mathrm{E}-02 & 1.2670 \mathrm{E}-02 \\ \mathrm{Na}-23 & 9.8100 \mathrm{E}-03 & 9.8100 \mathrm{E}-03 \\ \text { SS-316 } & 1.8990 \mathrm{E}-02 & 1.8990 \mathrm{E}-02\end{array}$

10

$\begin{array}{ll}46.14 & 62.78\end{array}$

$\begin{array}{lll}\text { U-235 } & 1.4143 \mathrm{E}-05 & 1.0163 \mathrm{E}-05 \\ \mathrm{U}-238 & 7.0573 \mathrm{E}-03 & 6.8182 \mathrm{E}-03 \\ \text { Pu-239 } & 0.0000 \mathrm{E}+00 & 1.9268 \mathrm{E}-04 \\ \text { PU-240 } & 0.0000 \mathrm{E}+00 & 8.5020 \mathrm{E}-06 \\ \text { FP } & 0.0000 \mathrm{E}+00 & 4.0515 \mathrm{E}-05 \\ 0-16 & 1.4143 \mathrm{E}-02 & 1.4143 \mathrm{E}-02 \\ \mathrm{Na}-23 & 9.7416 \mathrm{E}-03 & 9.7416 \mathrm{E}-03 \\ \text { SS-316 } & 1.9073 \mathrm{E}-02 & 1.9073 \mathrm{E}-02\end{array}$

$\begin{array}{lll}11 & 62.78 & 172.84\end{array}$

$\begin{array}{lll}\mathrm{Na}-23 & 8.2400 \mathrm{E}-03 & 8.2400 \mathrm{E}-03 \\ \mathrm{SS}-316 & 2.5562 \mathrm{E}-02 & 2.5562 \mathrm{E}-02\end{array}$


Table E12A-1.18. Composition of MFA-2 at EOC 12A-1

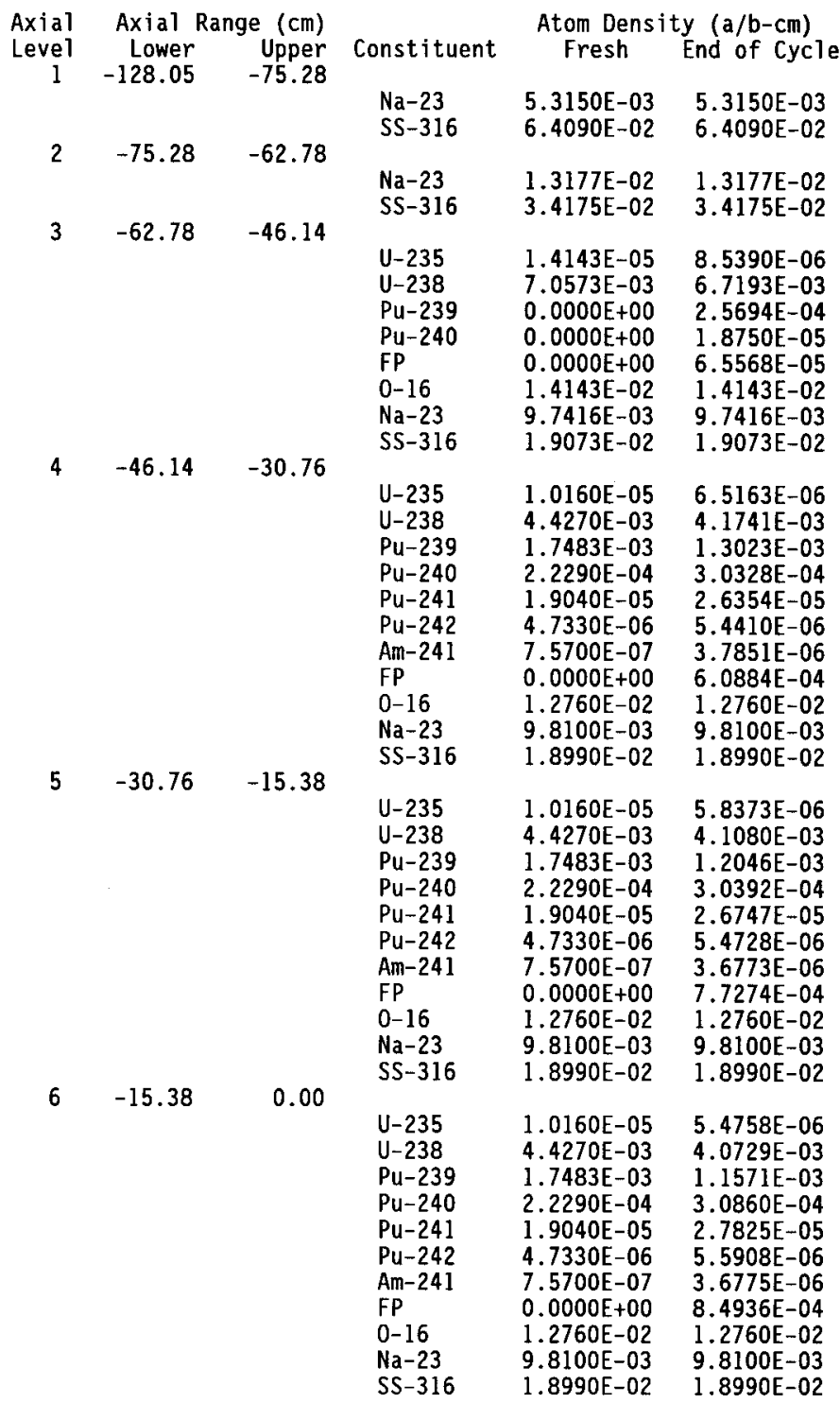




\begin{tabular}{|c|c|c|c|c|c|}
\hline \multirow{2}{*}{7} & 0.00 & 15.38 & & & \\
\hline & & & $\begin{array}{l}\mathrm{U}-235 \\
\mathrm{U}-238 \\
\mathrm{Pu}-239 \\
\mathrm{Pu}-240 \\
\mathrm{Pu}-241 \\
\mathrm{Pu}-242 \\
\mathrm{Am}-241 \\
\mathrm{FP} \\
\mathrm{O}-16 \\
\mathrm{Na}-23 \\
\mathrm{SS}-316\end{array}$ & $\begin{array}{l}1.0160 \mathrm{E}-05 \\
4.4270 \mathrm{E}-03 \\
1.7483 \mathrm{E}-03 \\
2.2290 \mathrm{E}-04 \\
1.9040 \mathrm{E}-05 \\
4.7330 \mathrm{E}-06 \\
7.5700 \mathrm{E}-07 \\
0.0000 \mathrm{E}+00 \\
1.2760 \mathrm{E}-02 \\
9.8100 \mathrm{E}-03 \\
1.8990 \mathrm{E}-02\end{array}$ & $\begin{array}{l}5.5810 \mathrm{E}-06 \\
4.0831 \mathrm{E}-03 \\
1.1703 \mathrm{E}-03 \\
3.0679 \mathrm{E}-04 \\
2.7442 \mathrm{E}-05 \\
5.5479 \mathrm{E}-06 \\
3.6746 \mathrm{E}-06 \\
8.2804 \mathrm{E}-04 \\
1.2760 \mathrm{E}-02 \\
9.8100 \mathrm{E}-03 \\
1.8990 \mathrm{E}-02\end{array}$ \\
\hline \multirow[t]{2}{*}{8} & 15.38 & 30.76 & & & \\
\hline & & & $\begin{array}{l}U-235 \\
U-238 \\
P u-239 \\
P u-240 \\
P u-241 \\
P u-242 \\
A m-241 \\
F P \\
O-16 \\
N a-23 \\
S S-316\end{array}$ & $\begin{array}{l}1.0160 \mathrm{E}-05 \\
4.4270 \mathrm{E}-03 \\
1.7483 \mathrm{E}-03 \\
2.2290 \mathrm{E}-04 \\
1.9040 \mathrm{E}-05 \\
4.7330 \mathrm{E}-06 \\
7.5700 \mathrm{E}-07 \\
0.0000 \mathrm{E}+00 \\
1.2760 \mathrm{E}-02 \\
9.8100 \mathrm{E}-03 \\
1.8990 \mathrm{E}-02\end{array}$ & $\begin{array}{l}6.1422 \mathrm{E}-06 \\
4.1360 \mathrm{E}-03 \\
1.2423 \mathrm{E}-03 \\
2.9829 \mathrm{E}-04 \\
2.5666 \mathrm{E}-05 \\
5.3647 \mathrm{E}-06 \\
3.6702 \mathrm{E}-06 \\
7.1327 \mathrm{E}-04 \\
1.2760 \mathrm{E}-02 \\
9.8100 \mathrm{E}-03 \\
1.8990 \mathrm{E}-02\end{array}$ \\
\hline \multirow[t]{2}{*}{9} & 30.76 & 46.14 & & & \\
\hline & & & $\begin{array}{l}U-235 \\
U-238 \\
P U-239 \\
P u-240 \\
P u-241 \\
P u-242 \\
A m-241 \\
F P \\
0-16 \\
N a-23 \\
S S-316\end{array}$ & $\begin{array}{l}1.0160 \mathrm{E}-05 \\
4.4270 \mathrm{E}-03 \\
1.7483 \mathrm{E}-03 \\
2.2290 \mathrm{E}-04 \\
1.9040 \mathrm{E}-05 \\
4.7330 \mathrm{E}-06 \\
7.5700 \mathrm{E}-07 \\
0.0000 \mathrm{E}+00 \\
1.2760 \mathrm{E}-02 \\
9.8100 \mathrm{E}-03 \\
1.8990 \mathrm{E}-02\end{array}$ & $\begin{array}{l}7.0145 \mathrm{E}-06 \\
4.2141 \mathrm{E}-03 \\
1.3622 \mathrm{E}-03 \\
2.9127 \mathrm{E}-04 \\
2.4255 \mathrm{E}-05 \\
5.2512 \mathrm{E}-06 \\
3.7445 \mathrm{E}-06 \\
5.2335 \mathrm{E}-04 \\
1.2760 \mathrm{E}-02 \\
9.8100 \mathrm{E}-03 \\
1.8990 \mathrm{E}-02\end{array}$ \\
\hline \multirow[t]{2}{*}{10} & 46.14 & 62.78 & & & \\
\hline & & & $\begin{array}{l}U-235 \\
U-238 \\
\mathrm{Pu}-239 \\
\mathrm{Pu}-240 \\
\mathrm{FP} \\
0-16 \\
\mathrm{Na}-23 \\
\mathrm{SS}-316\end{array}$ & $\begin{array}{l}1.4143 \mathrm{E}-05 \\
7.0573 \mathrm{E}-03 \\
0.0000 \mathrm{E}+00 \\
0.0000 \mathrm{E}+00 \\
0.0000 \mathrm{E}+00 \\
1.4143 \mathrm{E}-02 \\
9.7416 \mathrm{E}-03 \\
1.9073 \mathrm{E}-02\end{array}$ & $\begin{array}{l}9.9976 \mathrm{E}-06 \\
6.8138 \mathrm{E}-03 \\
1.9569 \mathrm{E}-04 \\
9.3481 \mathrm{E}-06 \\
4.1249 \mathrm{E}-05 \\
1.4143 \mathrm{E}-02 \\
9.7416 \mathrm{E}-03 \\
1.9073 \mathrm{E}-02\end{array}$ \\
\hline \multirow[t]{2}{*}{11} & 62.78 & 172.84 & & & \\
\hline & & & $\begin{array}{l}\mathrm{Na}-23 \\
\mathrm{SS}-316\end{array}$ & $\begin{array}{l}8.2400 \mathrm{E}-03 \\
2.5562 \mathrm{E}-02\end{array}$ & $\begin{array}{l}8.2400 \mathrm{E}-03 \\
2.5562 \mathrm{E}-02\end{array}$ \\
\hline
\end{tabular}




\subsection{Cycle 12A-2}

Table B12A-2.1. Fission Power Generated in MFA-1, MFA-2 and Neighboring Assemblies at BOC 12A-2

\begin{tabular}{lcccc} 
CORE & $* * * * * *$ & \multicolumn{2}{c}{ POWER IN MEGAWATTS } & $* * * * * *$ \\
POS. & BELOW CORE & IN CORE & ABOVE CORE & TOTAL PWR \\
1405 & $1.158 \mathrm{E}-01$ & $3.858 \mathrm{E}+00$ & $6.757 \mathrm{E}-02$ & $4.042 \mathrm{E}+00$ \\
1304 & $0.000 \mathrm{E}+00$ & $6.885 \mathrm{E}+00$ & $0.000 \mathrm{E}+00$ & $6.885 \mathrm{E}+00$ \\
1406 & $1.165 \mathrm{E}-02$ & $4.654 \mathrm{E}+00$ & $8.364 \mathrm{E}-03$ & $4.674 \mathrm{E}+00$ \\
1404 & $0.000 \mathrm{E}+00$ & $6.099 \mathrm{E}+00$ & $0.000 \mathrm{E}+00$ & $6.099 \mathrm{E}+00$ \\
1508 & $0.000 \mathrm{E}+00$ & $5.303 \mathrm{E}+00$ & $0.000 \mathrm{E}+00$ & $5.303 \mathrm{E}+00$ \\
1506 & $1.578 \mathrm{E}-02$ & $3.894 \mathrm{E}+00$ & $1.061 \mathrm{E}-02$ & $3.920 \mathrm{E}+00$ \\
1507 & $7.069 \mathrm{E}-03$ & $4.613 \mathrm{E}+00$ & $5.912 \mathrm{E}-03$ & $4.626 \mathrm{E}+00$ \\
2506 & $9.201 \mathrm{E}-02$ & $3.054 \mathrm{E}+00$ & $5.507 \mathrm{E}-02$ & $3.201 \mathrm{E}+00$ \\
2404 & $0.000 \mathrm{E}+00$ & $5.656 \mathrm{E}+00$ & $0.000 \mathrm{E}+00$ & $5.656 \mathrm{E}+00$ \\
2505 & $8.733 \mathrm{E}-03$ & $4.100 \mathrm{E}+00$ & $5.667 \mathrm{E}-03$ & $4.114 \mathrm{E}+00$ \\
2405 & $1.720 \mathrm{E}-02$ & $3.279 \mathrm{E}+00$ & $1.154 \mathrm{E}-02$ & $3.308 \mathrm{E}+00$ \\
2606 & $1.455 \mathrm{E}-02$ & $3.107 \mathrm{E}+00$ & $8.320 \mathrm{E}-03$ & $3.130 \mathrm{E}+00$ \\
2607 & $6.407 \mathrm{E}-03$ & $3.466 \mathrm{E}+00$ & $5.120 \mathrm{E}-03$ & $3.477 \mathrm{E}+00$ \\
2507 & $1.212 \mathrm{E}-02$ & $3.251 \mathrm{E}+00$ & $8.547 \mathrm{E}-03$ & $3.271 \mathrm{E}+00$
\end{tabular}

Table B12A-2.2. Assembly Averaged Total and Fast Flux in MFA-1 and MFA-2 at BOC $12 \mathrm{~A}-2$

\begin{tabular}{|c|c|c|c|}
\hline & Core & Flux & $\left.m^{2}-s e c\right)$ \\
\hline $\begin{array}{l}\text { Assembly } \\
\text { MFA-1 } \\
\text { MFA-2 }\end{array}$ & $\begin{array}{l}\text { Pos. } \\
1405 \\
2506\end{array}$ & $\begin{array}{c}\text { Total } \\
3.787 \mathrm{E}+15 \\
2.955 \mathrm{E}+15\end{array}$ & $\begin{array}{l}>0.1 \mathrm{MeV} \\
2.535 \mathrm{E}+15 \\
1.890 \mathrm{E}+15\end{array}$ \\
\hline
\end{tabular}


Table B12A-2.3. Axial Distribution of Total Flux, Fast Flux and Power in MFA-1 at BOC 12A-2

$\begin{array}{rccc}z(\mathrm{~cm}) & \text { Total Flux } & \text { F Tux }>0.1 \text { MeV } & \text { Power } \\ -97.78 & 2.6854 \mathrm{E}-02 & 1.3475 \mathrm{E}-02 & 0.0000 \mathrm{E}+00 \\ -92.78 & 5.7513 \mathrm{E}-02 & 2.6015 \mathrm{E}-02 & 0.0000 \mathrm{E}+00 \\ -87.78 & 9.2067 \mathrm{E}-02 & 4.1658 \mathrm{E}-02 & 0.0000 \mathrm{E}+00 \\ -82.78 & 1.3316 \mathrm{E}-01 & 6.2219 \mathrm{E}-02 & 0.0000 \mathrm{E}+00 \\ -77.78 & 1.8368 \mathrm{E}-01 & 9.0027 \mathrm{E}-02 & 0.0000 \mathrm{E}+00 \\ -72.15 & 2.4188 \mathrm{E}-01 & 1.2650 \mathrm{E}-01 & 0.0000 \mathrm{E}+00 \\ -65.90 & 3.0211 \mathrm{E}-01 & 1.7431 \mathrm{E}-01 & 0.0000 \mathrm{E}+00 \\ -60.35 & 3.6646 \mathrm{E}-01 & 2.4155 \mathrm{E}-01 & 1.4064 \mathrm{E}-01 \\ -55.49 & 4.5018 \mathrm{E}-01 & 3.2821 \mathrm{E}-01 & 1.5948 \mathrm{E}-01 \\ -50.62 & 5.4578 \mathrm{E}-01 & 4.3950 \mathrm{E}-01 & 1.8292 \mathrm{E}-01 \\ -47.17 & 6.2057 \mathrm{E}-01 & 5.4190 \mathrm{E}-01 & 2.0489 \mathrm{E}-01 \\ -43.58 & 6.9860 \mathrm{E}-01 & 6.5634 \mathrm{E}-01 & 7.7120 \mathrm{E}-01 \\ -38.45 & 8.1388 \mathrm{E}-01 & 7.9823 \mathrm{E}-01 & 8.6446 \mathrm{E}-01 \\ -33.32 & 9.2127 \mathrm{E}-01 & 9.1895 \mathrm{E}-01 & 9.6847 \mathrm{E}-01 \\ -28.20 & 1.0147 \mathrm{E}+00 & 1.0179 \mathrm{E}+00 & 9.9510 \mathrm{E}-01 \\ -23.07 & 1.0929 \mathrm{E}+00 & 1.0990 \mathrm{E}+00 & 1.0702 \mathrm{E}+00 \\ -17.94 & 1.1541 \mathrm{E}+00 & 1.1612 \mathrm{E}+00 & 1.1291 \mathrm{E}+00 \\ -12.82 & 1.1972 \mathrm{E}+00 & 1.2040 \mathrm{E}+00 & 1.1344 \mathrm{E}+00 \\ -7.69 & 1.2229 \mathrm{E}+00 & 1.2295 \mathrm{E}+00 & 1.1583 \mathrm{E}+00 \\ -2.56 & 1.2307 \mathrm{E}+00 & 1.2376 \mathrm{E}+00 & 1.1656 \mathrm{E}+00 \\ 2.56 & 1.2207 \mathrm{E}+00 & 1.2283 \mathrm{E}+00 & 1.1674 \mathrm{E}+00 \\ 7.69 & 1.1929 \mathrm{E}+00 & 1.2012 \mathrm{E}+00 & 1.1410 \mathrm{E}+00 \\ 12.82 & 1.1479 \mathrm{E}+00 & 1.1572 \mathrm{E}+00 & 1.0983 \mathrm{E}+00 \\ 17.94 & 1.0867 \mathrm{E}+00 & 1.0973 \mathrm{E}+00 & 1.0929 \mathrm{E}+00 \\ 23.07 & 1.0090 \mathrm{E}+00 & 1.0194 \mathrm{E}+00 & 1.0156 \mathrm{E}+00 \\ 28.20 & 9.1647 \mathrm{E}-01 & 9.2479 \mathrm{E}-01 & 9.2370 \mathrm{E}-01 \\ 33.32 & 8.1148 \mathrm{E}-01 & 8.1546 \mathrm{E}-01 & 8.8763 \mathrm{E}-01 \\ 38.45 & 6.9508 \mathrm{E}-01 & 6.8811 \mathrm{E}-01 & 7.6616 \mathrm{E}-01 \\ 43.58 & 5.7357 \mathrm{E}-01 & 5.4564 \mathrm{E}-01 & 6.5053 \mathrm{E}-01 \\ 47.17 & 4.9507 \mathrm{E}-01 & 4.4106 \mathrm{E}-01 & 1.3414 \mathrm{E}-01 \\ 50.62 & 4.2581 \mathrm{E}-01 & 3.5383 \mathrm{E}-01 & 1.1240 \mathrm{E}-01 \\ 55.49 & 3.4252 \mathrm{E}-01 & 2.6245 \mathrm{E}-01 & 9.0464 \mathrm{E}-02 \\ 60.35 & 2.6809 \mathrm{E}-01 & 1.8982 \mathrm{E}-01 & 7.2875 \mathrm{E}-02 \\ 64.98 & 2.1736 \mathrm{E}-01 & 1.4311 \mathrm{E}-01 & 0.0000 \mathrm{E}+00 \\ 69.39 & 1.8467 \mathrm{E}-01 & 1.1628 \mathrm{E}-01 & 0.0000 \mathrm{E}+00 \\ 76.46 & 1.3780 \mathrm{E}-01 & 8.0388 \mathrm{E}-02 & 0.0000 \mathrm{E}+00 \\ 86.80 & 8.8308 \mathrm{E}-02 & 4.7204 \mathrm{E}-02 & 0.0000 \mathrm{E}+00 \\ 98.14 & 5.0342 \mathrm{E}-02 & 2.5272 \mathrm{E}-02 & 0.0000 \mathrm{E}+00 \\ 110.00 & 2.1407 \mathrm{E}-02 & 1.1133 \mathrm{E}-02 & 0.0000 \mathrm{E}+00 \\ & & & \end{array}$


Table B12A-2.4. Axial Distribution of Total Flux, Fast Flux and Power in MFA-2 at BOC $12 A-2$

$\begin{array}{rccc}\text { Z }(\mathrm{cm}) & \text { Total Flux } & \text { Flux }>0.1 \text { MeV } & \text { Power } \\ -97.78 & 2.7894 \mathrm{E}-02 & 1.4419 \mathrm{E}-02 & 0.0000 \mathrm{E}+00 \\ -92.78 & 5.9838 \mathrm{E}-02 & 2.7822 \mathrm{E}-02 & 0.0000 \mathrm{E}+00 \\ -87.78 & 9.5870 \mathrm{E}-02 & 4.4486 \mathrm{E}-02 & 0.0000 \mathrm{E}+00 \\ -82.78 & 1.3882 \mathrm{E}-01 & 6.6318 \mathrm{E}-02 & 0.0000 \mathrm{E}+00 \\ -77.78 & 1.9181 \mathrm{E}-01 & 9.5726 \mathrm{E}-02 & 0.0000 \mathrm{E}+00 \\ -72.15 & 2.5293 \mathrm{E}-01 & 1.3406 \mathrm{E}-01 & 0.0000 \mathrm{E}+00 \\ -65.90 & 3.1482 \mathrm{E}-01 & 1.8343 \mathrm{E}-01 & 0.0000 \mathrm{E}+00 \\ -60.35 & 3.7850 \mathrm{E}-01 & 2.5154 \mathrm{E}-01 & 1.4328 \mathrm{E}-01 \\ -55.49 & 4.6158 \mathrm{E}-01 & 3.3834 \mathrm{E}-01 & 1.6088 \mathrm{E}-01 \\ -50.62 & 5.5626 \mathrm{E}-01 & 4.4912 \mathrm{E}-01 & 1.8228 \mathrm{E}-01 \\ -47.17 & 6.3058 \mathrm{E}-01 & 5.5141 \mathrm{E}-01 & 2.0233 \mathrm{E}-01 \\ -43.58 & 7.0831 \mathrm{E}-01 & 6.6609 \mathrm{E}-01 & 7.8801 \mathrm{E}-01 \\ -38.45 & 8.2243 \mathrm{E}-01 & 8.0681 \mathrm{E}-01 & 8.7693 \mathrm{E}-01 \\ -33.32 & 9.2829 \mathrm{E}-01 & 9.2597 \mathrm{E}-01 & 9.7818 \mathrm{E}-01 \\ -28.20 & 1.0200 \mathrm{E}+00 & 1.0232 \mathrm{E}+00 & 1.0015 \mathrm{E}+00 \\ -23.07 & 1.0968 \mathrm{E}+00 & 1.1028 \mathrm{E}+00 & 1.0749 \mathrm{E}+00 \\ -17.94 & 1.1565 \mathrm{E}+00 & 1.1636 \mathrm{E}+00 & 1.1324 \mathrm{E}+00 \\ -12.82 & 1.1983 \mathrm{E}+00 & 1.2049 \mathrm{E}+00 & 1.1350 \mathrm{E}+00 \\ -7.69 & 1.2225 \mathrm{E}+00 & 1.2291 \mathrm{E}+00 & 1.1575 \mathrm{E}+00 \\ -2.56 & 1.2288 \mathrm{E}+00 & 1.2357 \mathrm{E}+00 & 1.1633 \mathrm{E}+00 \\ 2.56 & 1.2172 \mathrm{E}+00 & 1.2247 \mathrm{E}+00 & 1.1627 \mathrm{E}+00 \\ 7.69 & 1.1877 \mathrm{E}+00 & 1.1959 \mathrm{E}+00 & 1.1346 \mathrm{E}+00 \\ 12.82 & 1.1410 \mathrm{E}+00 & 1.1501 \mathrm{E}+00 & 1.0902 \mathrm{E}+00 \\ 17.94 & 1.0785 \mathrm{E}+00 & 1.0891 \mathrm{E}+00 & 1.0817 \mathrm{E}+00 \\ 23.07 & 1.0006 \mathrm{E}+00 & 1.0109 \mathrm{E}+00 & 1.0043 \mathrm{E}+00 \\ 28.20 & 9.0934 \mathrm{E}-01 & 9.1764 \mathrm{E}-01 & 9.1400 \mathrm{E}-01 \\ 33.32 & 8.0738 \mathrm{E}-01 & 8.1155 \mathrm{E}-01 & 8.7955 \mathrm{E}-01 \\ 38.45 & 6.9585 \mathrm{E}-01 & 6.8921 \mathrm{E}-01 & 7.6507 \mathrm{E}-01 \\ 43.58 & 5.8053 \mathrm{E}-01 & 5.5281 \mathrm{E}-01 & 6.6012 \mathrm{E}-01 \\ 47.17 & 5.0504 \mathrm{E}-01 & 4.4959 \mathrm{E}-01 & 1.3591 \mathrm{E}-01 \\ 50.62 & 4.3491 \mathrm{E}-01 & 3.5995 \mathrm{E}-01 & 1.1537 \mathrm{E}-01 \\ 55.49 & 3.4713 \mathrm{E}-01 & 2.6417 \mathrm{E}-01 & 9.3724 \mathrm{E}-02 \\ 60.35 & 2.6866 \mathrm{E}-01 & 1.8841 \mathrm{E}-01 & 7.5795 \mathrm{E}-02 \\ 64.98 & 2.1749 \mathrm{E}-01 & 1.4139 \mathrm{E}-01 & 0.0000 \mathrm{E}+00 \\ 69.39 & 1.8590 \mathrm{E}-01 & 1.1574 \mathrm{E}-01 & 0.0000 \mathrm{E}+00 \\ 76.46 & 1.4048 \mathrm{E}-01 & 8.1373 \mathrm{E}-02 & 0.0000 \mathrm{E}+00 \\ 86.80 & 9.0781 \mathrm{E}-02 & 4.8452 \mathrm{E}-02 & 0.0000 \mathrm{E}+00 \\ 98.14 & 5.1849 \mathrm{E}-02 & 2.6141 \mathrm{E}-02 & 0.0000 \mathrm{E}+00 \\ 110.00 & 2.2104 \mathrm{E}-02 & 1.1585 \mathrm{E}-02 & 0.0000 \mathrm{E}+00\end{array}$




\section{HNF-SD-FF-ANAL-009 Rev. I}

Table B12A-2.5. Fission Power Distribution by Pin in MFA-1 at BOC 12A-2 CORE POSITION 1405

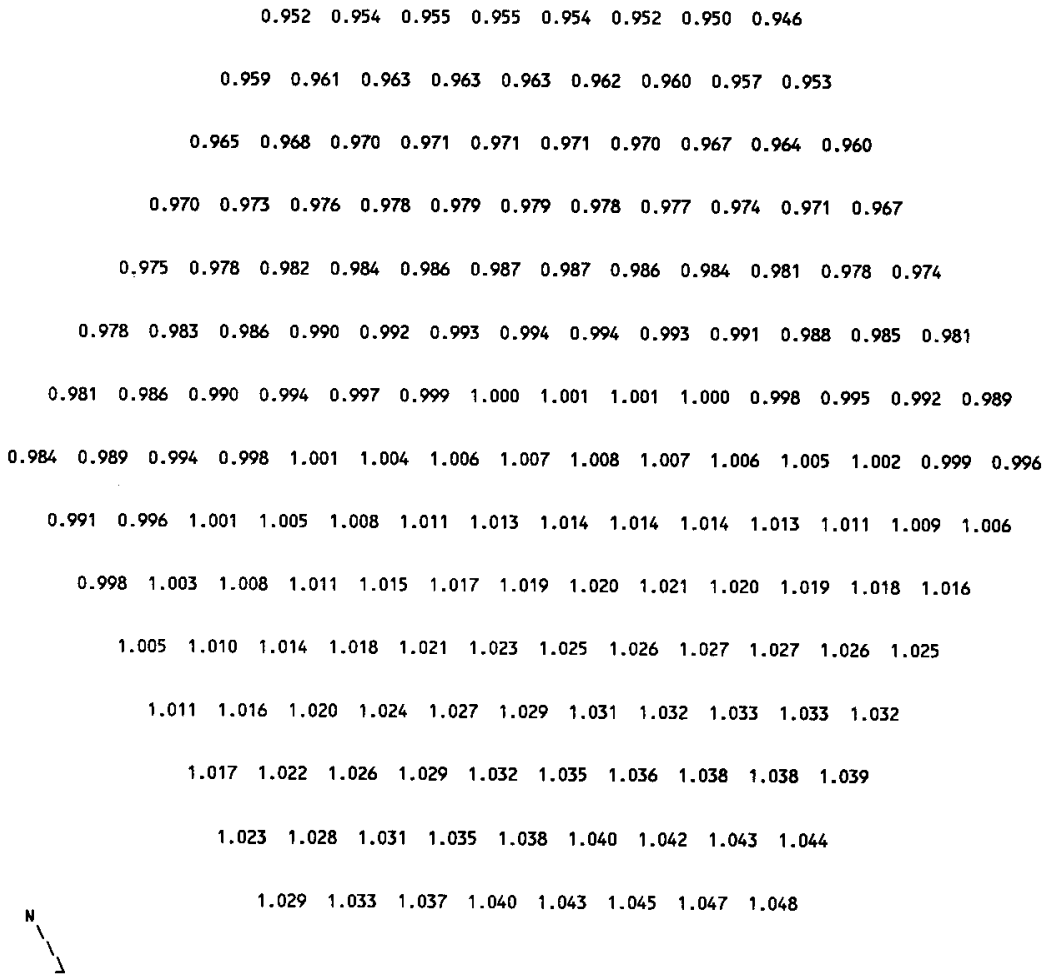




\section{HNF-SD-FF-ANAL-009 Rev. 1}

Table B12A-2.6. Fission Power Distribution by Pin in MFA-2 at BOC $12 A-2$ CORE POSITION 2506

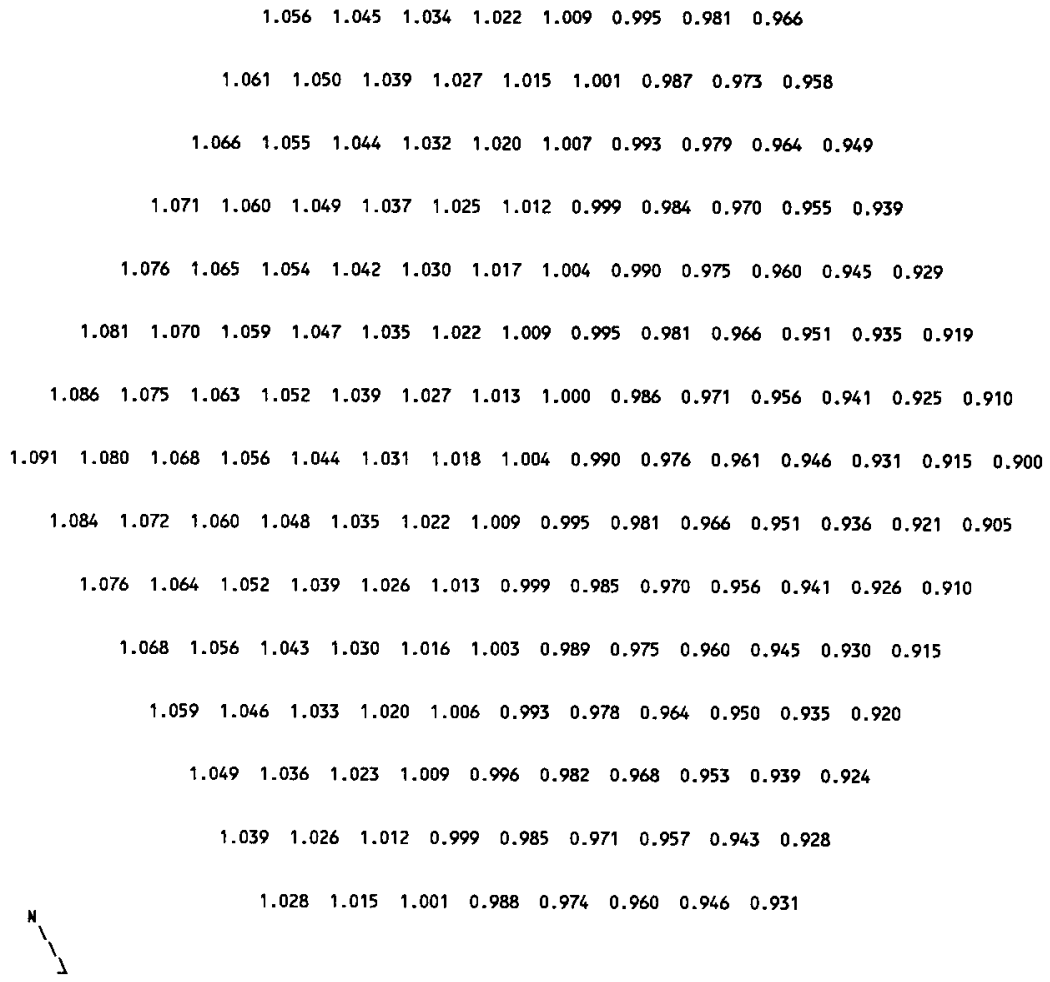


HNF-SD-FF-ANAL-009 Rev. 1

Table B12A-2.7. Fast Flux Distribution by Pin in MFA-1 at BOC 12A-2 CORE POSITION 1404

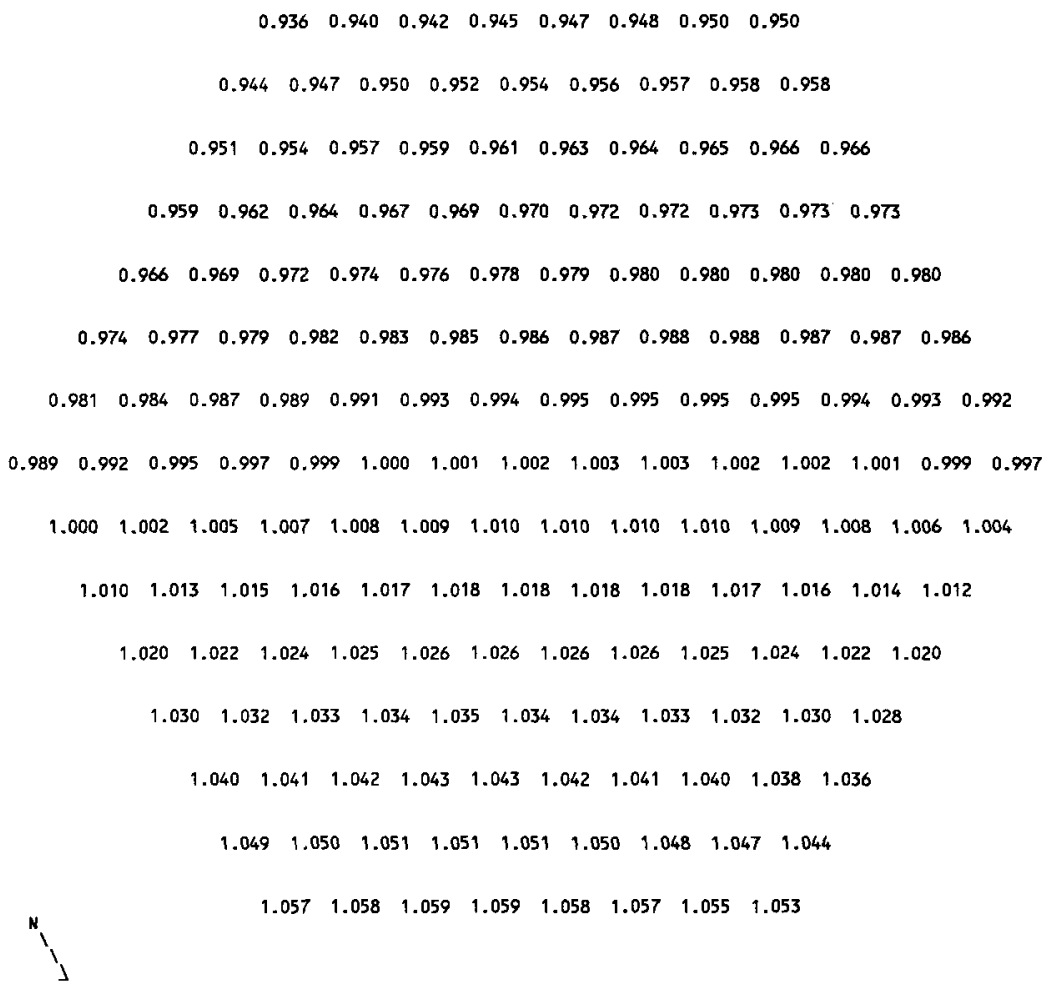


HNF-SD-FF-ANAL-009 Rev. 1

Table B12A-2.8. Fast Flux Distribution by Pin in MFA-2 at BOC 12A-2 CORE POSIIION 2506

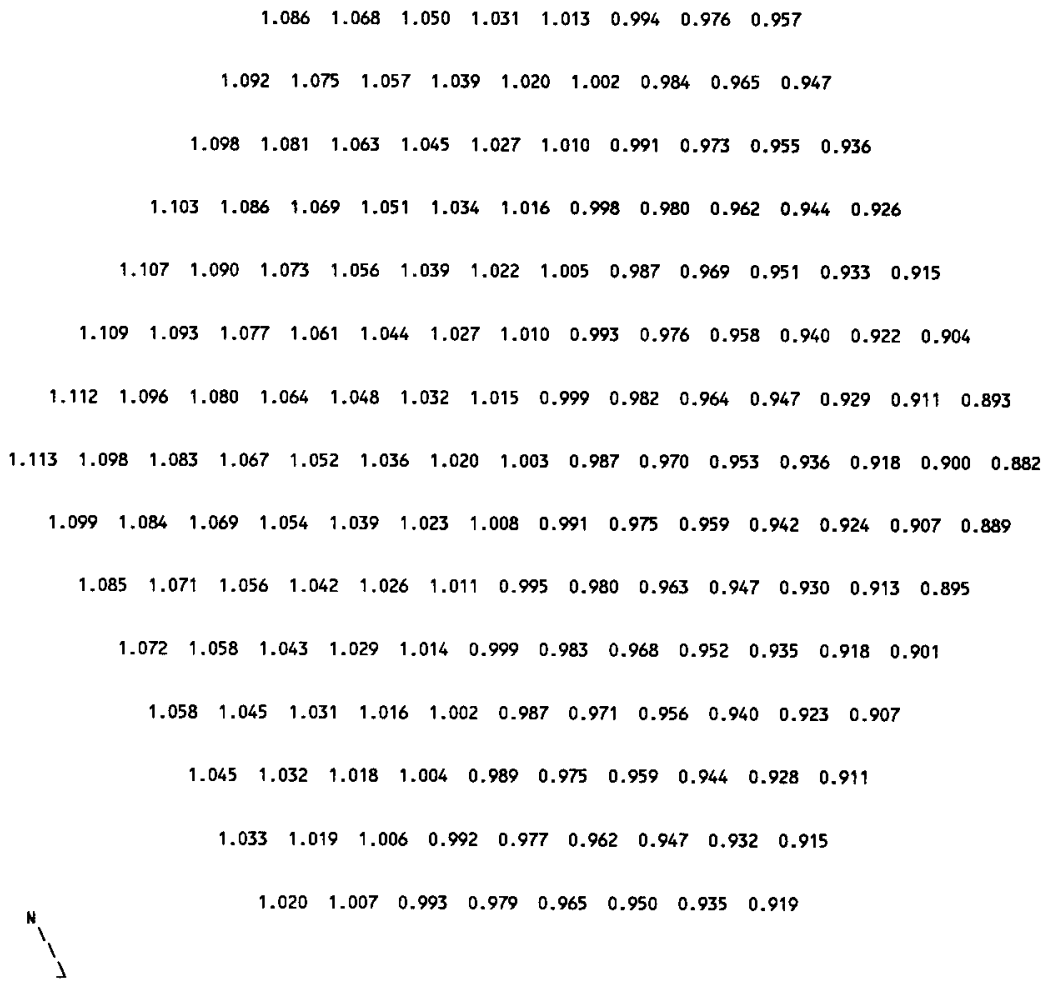


Table B12A-2.9. MFA-1 and MFA-2 Duct Wall

Fast Flux Data at BOC $12 \mathrm{~A}-2$

$\begin{array}{ccc}\text { Side } & \begin{array}{c}\text { Flux }>0.1 \\ \text { MFA-1 }\end{array} & \begin{array}{c}\text { MeV } \\ \left(\mathrm{n} / \mathrm{cm}^{2}-\mathrm{sec}\right) \\ \text { MFA-2 }\end{array} \\ \text { E } & 2.5897 \mathrm{E}+15 & 2.0273 \mathrm{E}+15 \\ \text { SE } & 2.3979 \mathrm{E}+15 & 2.1247 \mathrm{E}+15 \\ \text { SW } & 2.3524 \mathrm{E}+15 & 1.9289 \mathrm{E}+15 \\ \text { W } & 2.4443 \mathrm{E}+15 & 1.6989 \mathrm{E}+15 \\ \text { NW } & 2.5833 \mathrm{E}+15 & 1.6656 \mathrm{E}+15 \\ \text { NE } & 2.6945 \mathrm{E}+15 & 1.8206 \mathrm{E}+15\end{array}$

Table B12A-2.10. Assembly 0utlet Temperatures and Flow Rates at BOC 12A-2

$\begin{array}{lcl}\text { CALCULATED } & \\ \text { CORE } & \text { OUTLET TEMP. } & \text { FLOW RATE } \\ \text { POS. } & \text { (DEG F) } & \text { (LB/H) } \\ 1405 & 1082 & 108830 \\ 1304 & 1065 & 190160 \\ 1404 & 1014 & 192500 \\ 1406 & 944 & 197060 \\ 1506 & 901 & 197060 \\ 1507 & 935 & 197060 \\ 1508 & 997 & 177390 \\ 2506 & 997 & 108830 \\ 2404 & 989 & 193800 \\ 2405 & 867 & 197080 \\ 2505 & 903 & 197060 \\ 2507 & 861 & 197060 \\ 2606 & 891 & 161600 \\ 2607 & 921 & 154980\end{array}$

Assembly flows are based on a calculated core pressure drop of 99.1 psi at a total reactor flow rate of 16.74E+06 $1 \mathrm{~b} / \mathrm{hr}$. 
Table B12A-2.11. MFA-1 Sodium Subchannel Temperatures

at Top of Core Elevation for BOC 12A-2

$$
\begin{aligned}
& \mathrm{z}^{\cdot \cdot \cdot !}
\end{aligned}
$$

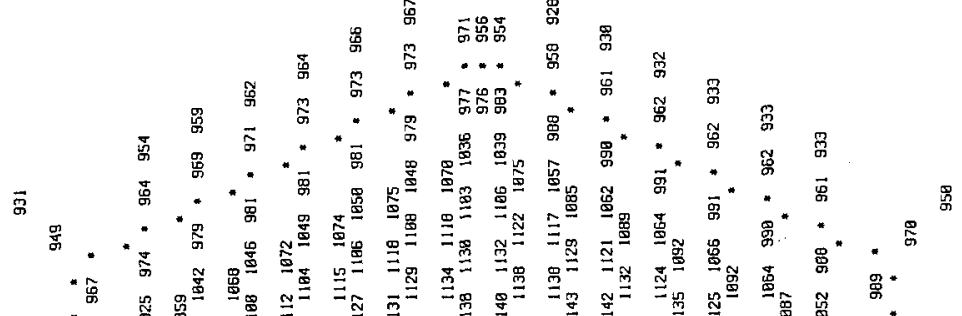

$$
\begin{aligned}
& \text { 吕品哭 }
\end{aligned}
$$

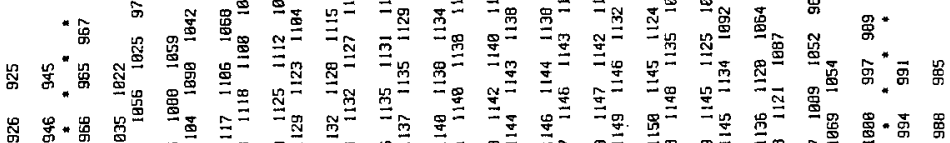

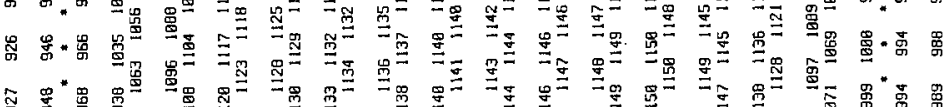

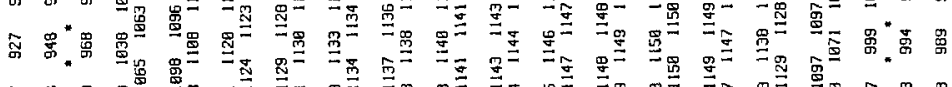

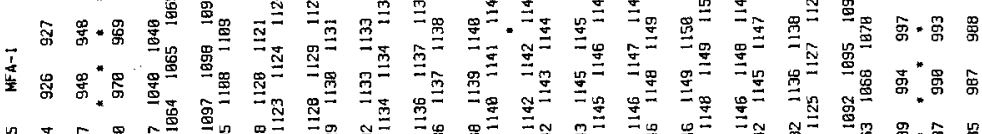

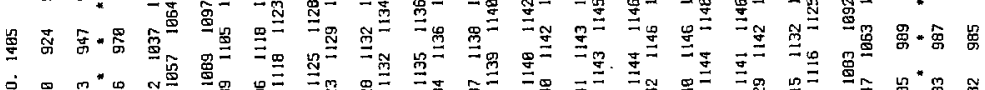

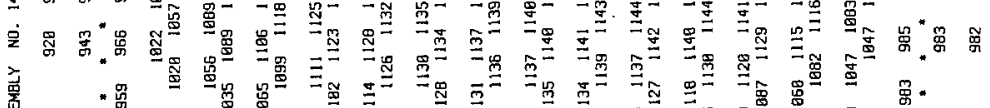

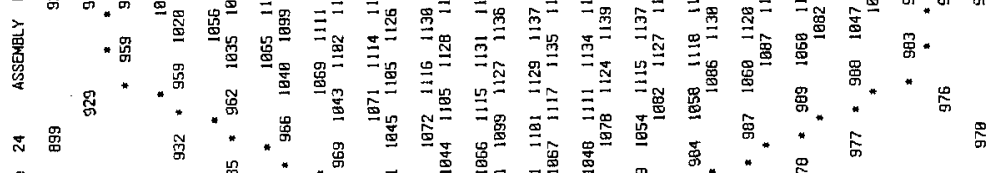

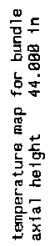

$$
\begin{aligned}
& \text { 兽 }
\end{aligned}
$$

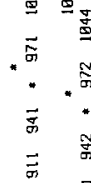

$$
\begin{aligned}
& \text { 兽要曋 }
\end{aligned}
$$

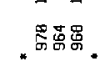

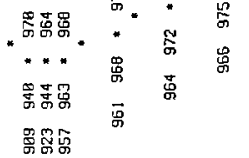


Table B12A-2.12. MFA-2 Sodium Subchannel Temperatures at Top of Core Elevation for BOC 12A-2

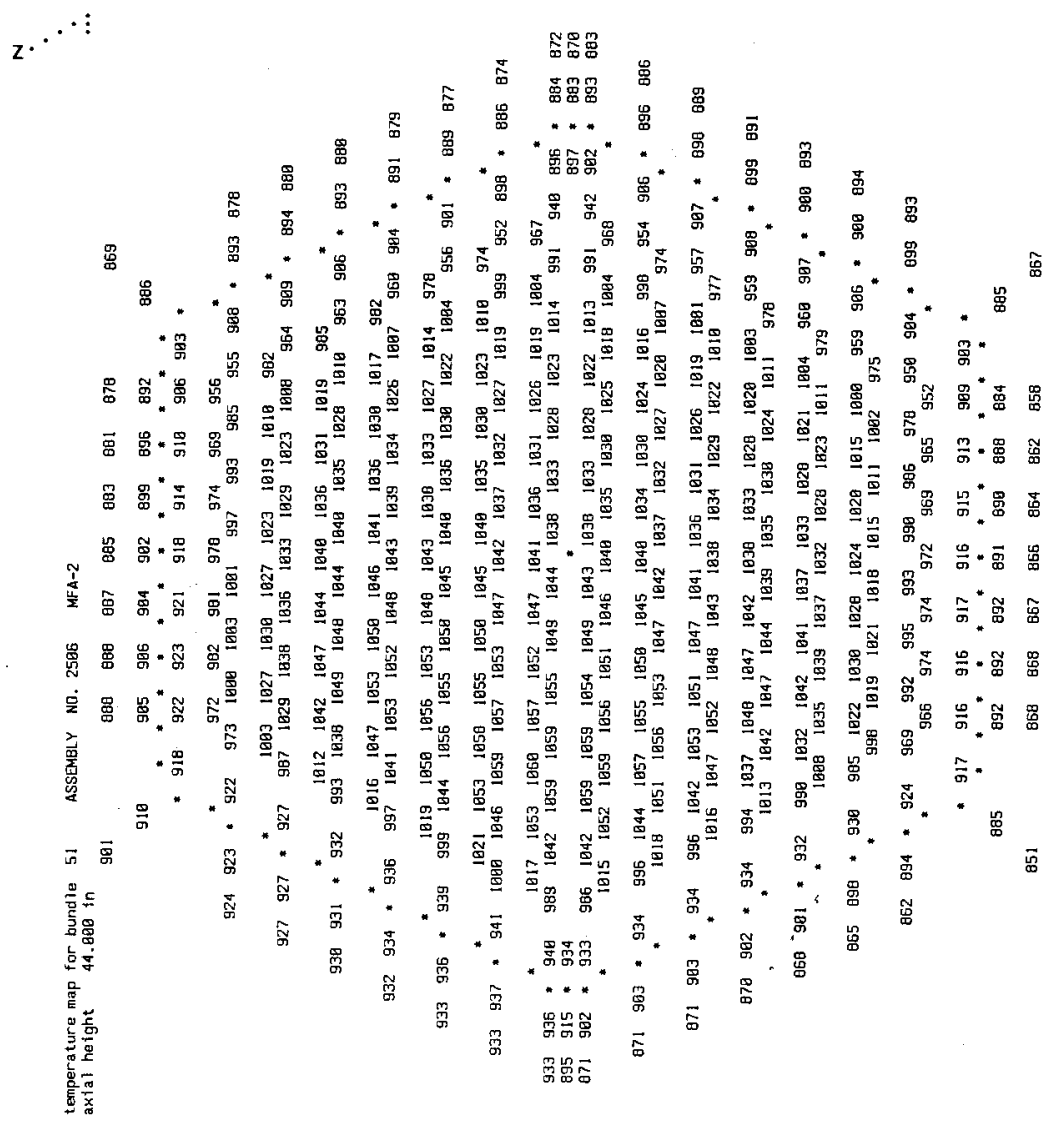


Table B12A-2.13. MFA-1 Sodium Subchanne1 Temperatures at Elevation of Upper Axial Blanket for BOC $12 \mathrm{~A}-2$

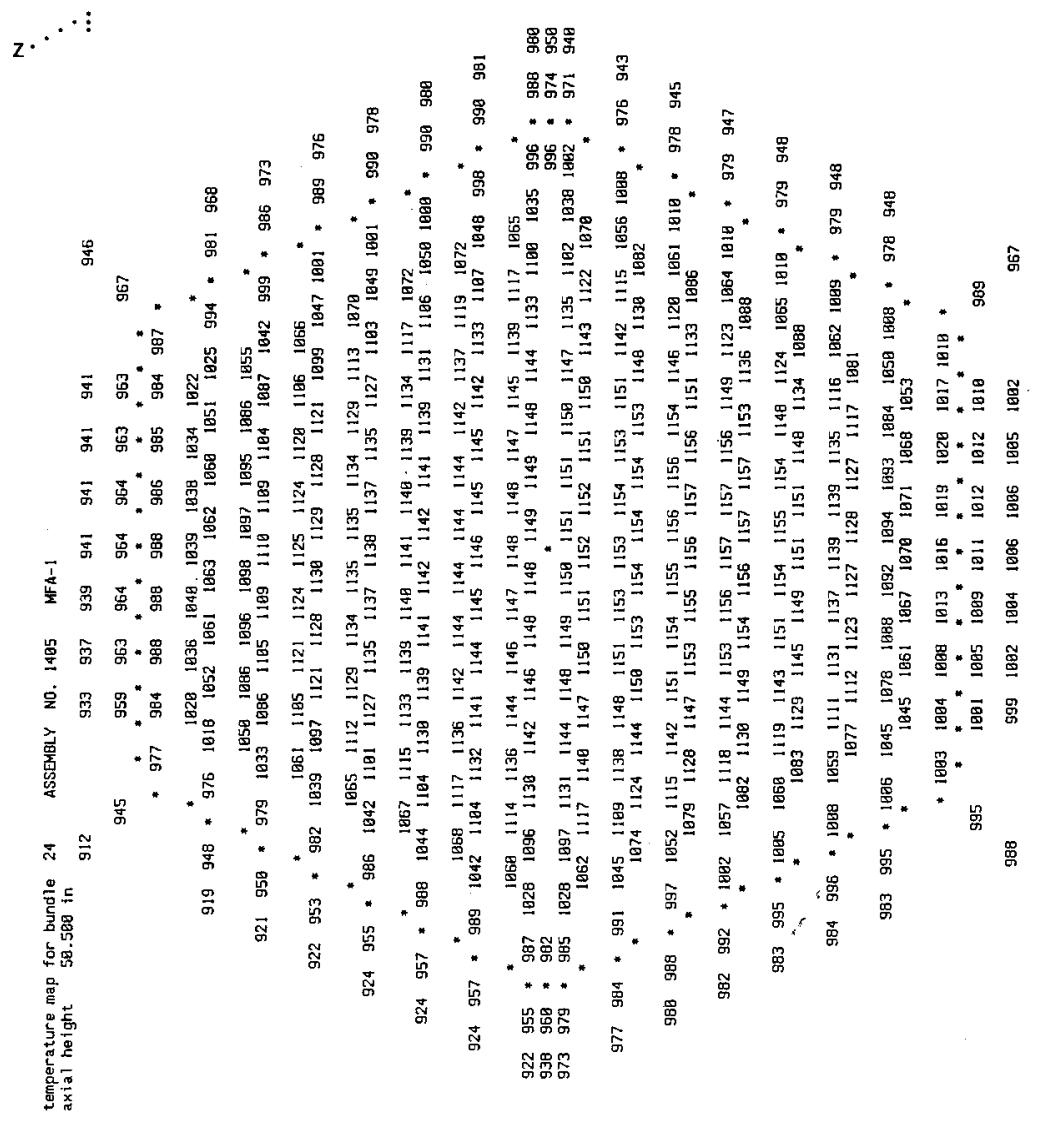


Table B12A-2.14. MFA-2 Sodium Subchanne1 Temperatures at Elevation of Upper Axial Blanket for BOC 12A-2

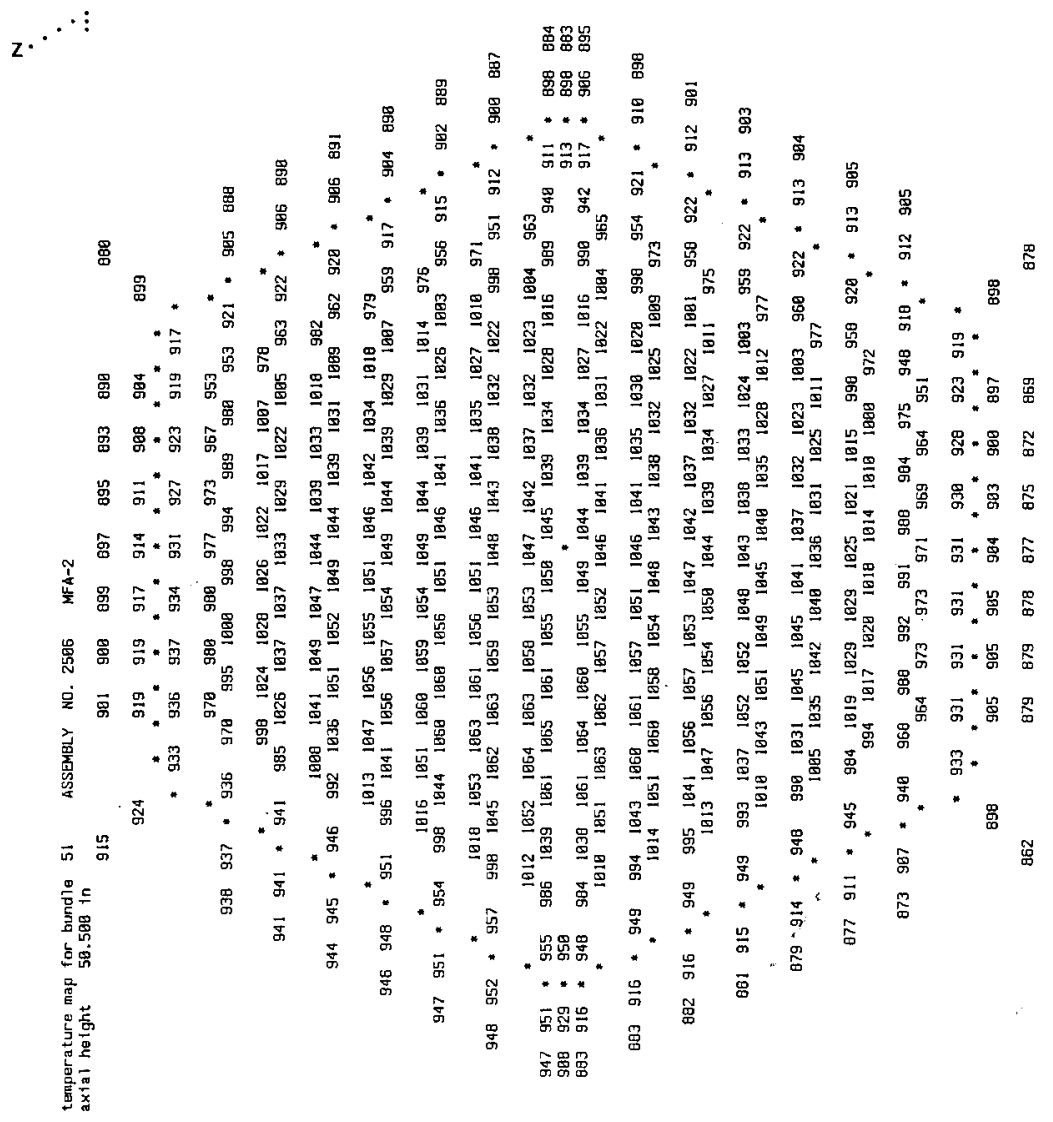


Table B12A-2.15. MFA-1 Sodium Subchannel Temperatures at Top of Fuel Pin Bundle for BOC 12A-2

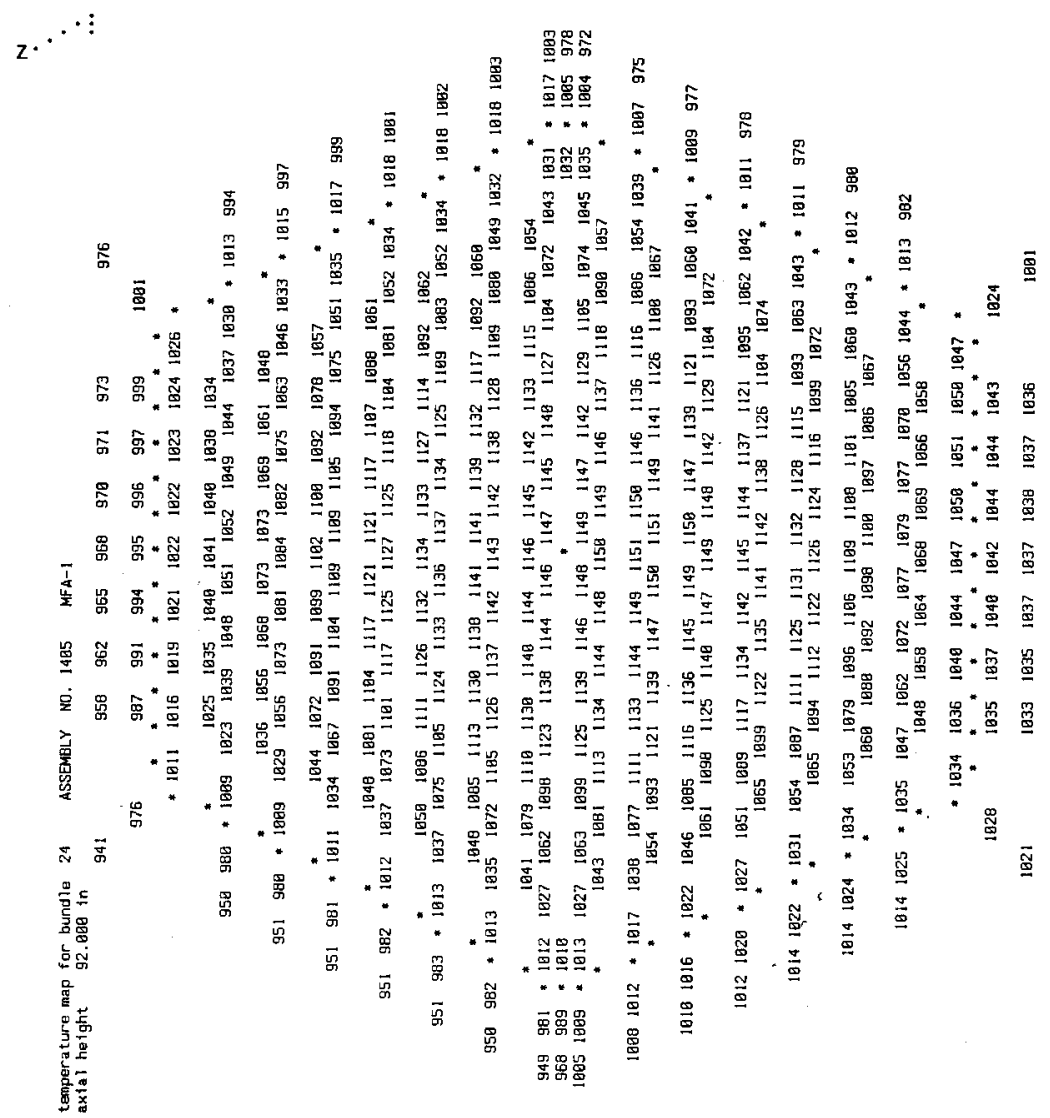


Table B12A-2.16. MFA-2 Sodium Subchannel Temperatures at Top of Fuel Pin Bundle for BOC 12A-2

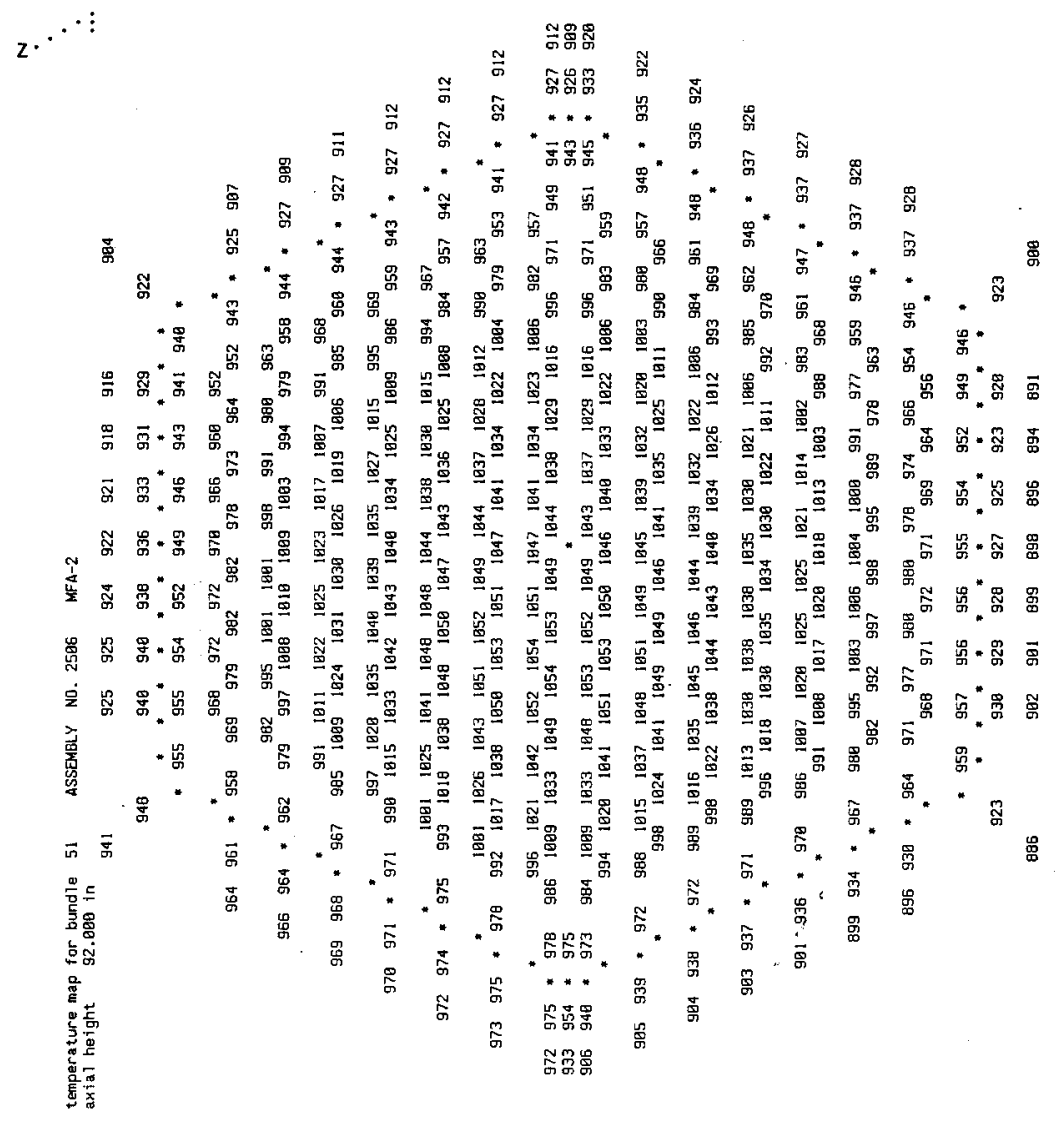


Table E12A-2.1. Fission Power Generated in MFA-1, MFA-2 and Neighboring Assemblies at EOC 12A-2

\begin{tabular}{lcrrrr} 
CORE & $* * * * * *$ & \multicolumn{2}{c}{ POWER IN MEGAWATTS } & $* * * * * *$ \\
POS. & BELOW CORE & \multicolumn{1}{c}{ IN CORE } & ABOVE CORE & TOTAL PWR \\
1405 & $1.164 \mathrm{E}-01$ & $3.771 \mathrm{E}+00$ & $7.276 \mathrm{E}-02$ & $3.961 \mathrm{E}+00$ \\
1304 & $0.000 \mathrm{E}+00$ & $6.704 \mathrm{E}+00$ & $0.000 \mathrm{E}+00$ & $6.704 \mathrm{E}+00$ \\
1406 & $1.215 \mathrm{E}-02$ & $4.562 \mathrm{E}+00$ & $9.199 \mathrm{E}-03$ & $\mathbf{4 . 5 8 4 \mathrm { E } + 0 0}$ \\
1404 & $0.000 \mathrm{E}+00$ & $5.982 \mathrm{E}+00$ & $0.000 \mathrm{E}+00$ & $5.982 \mathrm{E}+00$ \\
1508 & $0.000 \mathrm{E}+00$ & $5.201 \mathrm{E}+00$ & $0.000 \mathrm{E}+00$ & $5.201 \mathrm{E}+00$ \\
1506 & $1.575 \mathrm{E}-02$ & $3.829 \mathrm{E}+00$ & $1.137 \mathrm{E}-02$ & $3.856 \mathrm{E}+00$ \\
1507 & $7.424 \mathrm{E}-03$ & $4.505 \mathrm{E}+00$ & $6.495 \mathrm{E}-03$ & $4.519 \mathrm{E}+00$ \\
2506 & $9.265 \mathrm{E}-02$ & $3.029 \mathrm{E}+00$ & $5.978 \mathrm{E}-02$ & $3.182 \mathrm{E}+00$ \\
2404 & $0.000 \mathrm{E}+00$ & $5.575 \mathrm{E}+00$ & $0.000 \mathrm{E}+00$ & $5.575 \mathrm{E}+00$ \\
2505 & $9.128 \mathrm{E}-03$ & $4.118 \mathrm{E}+00$ & $6.350 \mathrm{E}-03$ & $4.134 \mathrm{E}+00$ \\
2405 & $1.725 \mathrm{E}-02$ & $3.248 \mathrm{E}+00$ & $1.241 \mathrm{E}-02$ & $3.277 \mathrm{E}+00$ \\
2606 & $1.448 \mathrm{E}-02$ & $3.120 \mathrm{E}+00$ & $9.073 \mathrm{E}-03$ & $3.143 \mathrm{E}+00$ \\
2607 & $6.633 \mathrm{E}-03$ & $3.440 \mathrm{E}+00$ & $5.628 \mathrm{E}-03$ & $3.453 \mathrm{E}+00$ \\
2507 & $1.216 \mathrm{E}-02$ & $3.219 \mathrm{E}+00$ & $9.185 \mathrm{E}-03$ & $3.241 \mathrm{E}+00$
\end{tabular}

Table E12A-2.2. Assembly Averaged Total and Fast Flux in MFA-1 and MFA-2 at EOC $12 A-2$

\begin{tabular}{|c|c|c|}
\hline & Core & Flux $\left(\mathrm{n} / \mathrm{cm}^{2}-\right.$ \\
\hline $\begin{array}{l}\text { Assembly } \\
\text { MFA-1 } \\
\text { MFA-2 }\end{array}$ & $\begin{array}{l}\text { Pos. } \\
1405 \\
2506\end{array}$ & $\begin{array}{cc}\text { Total } & >0.1 \mathrm{MeV} \\
3.770 \mathrm{E}+15 & 2.511 \mathrm{E}+15 \\
2.973 \mathrm{E}+15 & 1.894 \mathrm{E}+15\end{array}$ \\
\hline
\end{tabular}


Table E12A-2.3. Axial Distribution of Total Flux, Fast Flux and Power in MFA-1 at EOC $12 \mathrm{~A}-2$

$\begin{array}{rccc}\text { Z }(\mathrm{cm}) & \text { Total Flux } & \text { Flux }>0.1 \text { MeV } & \text { Power } \\ -97.78 & 2.6206 \mathrm{E}-02 & 1.3195 \mathrm{E}-02 & 0.0000 \mathrm{E}+00 \\ -92.78 & 5.6136 \mathrm{E}-02 & 2.5474 \mathrm{E}-02 & 0.0000 \mathrm{E}+00 \\ -87.78 & 8.9866 \mathrm{E}-02 & 4.0786 \mathrm{E}-02 & 0.0000 \mathrm{E}+00 \\ -82.78 & 1.2999 \mathrm{E}-01 & 6.0917 \mathrm{E}-02 & 0.0000 \mathrm{E}+00 \\ -77.78 & 1.7931 \mathrm{E}-01 & 8.8129 \mathrm{E}-02 & 0.0000 \mathrm{E}+00 \\ -72.15 & 2.3613 \mathrm{E}-01 & 1.2382 \mathrm{E}-01 & 0.0000 \mathrm{E}+00 \\ -65.90 & 2.9484 \mathrm{E}-01 & 1.7060 \mathrm{E}-01 & 0.0000 \mathrm{E}+00 \\ -60.35 & 3.5750 \mathrm{E}-01 & 2.3646 \mathrm{E}-01 & 1.4530 \mathrm{E}-01 \\ -55.49 & 4.3907 \mathrm{E}-01 & 3.2104 \mathrm{E}-01 & 1.6430 \mathrm{E}-01 \\ -50.62 & 5.3222 \mathrm{E}-01 & 4.2945 \mathrm{E}-01 & 1.8770 \mathrm{E}-01 \\ -47.17 & 6.0507 \mathrm{E}-01 & 5.2911 \mathrm{E}-01 & 2.0932 \mathrm{E}-01 \\ -43.58 & 6.8106 \mathrm{E}-01 & 6.4044 \mathrm{E}-01 & 7.5381 \mathrm{E}-01 \\ -38.45 & 7.9382 \mathrm{E}-01 & 7.7909 \mathrm{E}-01 & 8.4538 \mathrm{E}-01 \\ -33.32 & 8.9950 \mathrm{E}-01 & 8.9758 \mathrm{E}-01 & 9.4802 \mathrm{E}-01 \\ -28.20 & 9.9215 \mathrm{E}-01 & 9.9550 \mathrm{E}-01 & 9.7169 \mathrm{E}-01 \\ -23.07 & 1.0709 \mathrm{E}+00 & 1.0767 \mathrm{E}+00 & 1.0471 \mathrm{E}+00 \\ -17.94 & 1.1338 \mathrm{E}+00 & 1.1406 \mathrm{E}+00 & 1.1077 \mathrm{E}+00 \\ -12.82 & 1.1800 \mathrm{E}+00 & 1.1862 \mathrm{E}+00 & 1.1145 \mathrm{E}+00 \\ -7.69 & 1.2100 \mathrm{E}+00 & 1.2161 \mathrm{E}+00 & 1.1423 \mathrm{E}+00 \\ -2.56 & 1.2234 \mathrm{E}+00 & 1.2297 \mathrm{E}+00 & 1.1549 \mathrm{E}+00 \\ 2.56 & 1.2199 \mathrm{E}+00 & 1.2269 \mathrm{E}+00 & 1.1632 \mathrm{E}+00 \\ 7.69 & 1.1990 \mathrm{E}+00 & 1.2069 \mathrm{E}+00 & 1.1435 \mathrm{E}+00 \\ 12.82 & 1.1608 \mathrm{E}+00 & 1.1699 \mathrm{E}+00 & 1.1076 \mathrm{E}+00 \\ 17.94 & 1.1057 \mathrm{E}+00 & 1.1164 \mathrm{E}+00 & 1.1119 \mathrm{E}+00 \\ 23.07 & 1.0326 \mathrm{E}+00 & 1.0432 \mathrm{E}+00 & 1.0393 \mathrm{E}+00 \\ 28.20 & 9.4263 \mathrm{E}-01 & 9.5132 \mathrm{E}-01 & 9.5003 \mathrm{E}-01 \\ 33.32 & 8.3815 \mathrm{E}-01 & 8.4258 \mathrm{E}-01 & 9.2126 \mathrm{E}-01 \\ 38.45 & 7.2039 \mathrm{E}-01 & 7.1346 \mathrm{E}-01 & 7.9803 \mathrm{E}-01 \\ 43.58 & 5.9624 \mathrm{E}-01 & 5.6739 \mathrm{E}-01 & 6.7985 \mathrm{E}-01 \\ 47.17 & 5.1566 \mathrm{E}-01 & 4.5955 \mathrm{E}-01 & 1.4620 \mathrm{E}-01 \\ 50.62 & 4.4442 \mathrm{E}-01 & 3.6946 \mathrm{E}-01 & 1.2337 \mathrm{E}-01 \\ 55.49 & 3.5818 \mathrm{E}-01 & 2.7465 \mathrm{E}-01 & 1.0001 \mathrm{E}-01 \\ 60.35 & 2.8085 \mathrm{E}-01 & 1.9901 \mathrm{E}-01 & 8.1037 \mathrm{E}-02 \\ 64.98 & 2.2809 \mathrm{E}-01 & 1.5021 \mathrm{E}-01 & 0.0000 \mathrm{E}+00 \\ 69.39 & 1.9396 \mathrm{E}-01 & 1.2214 \mathrm{E}-01 & 0.0000 \mathrm{E}+00 \\ 76.46 & 1.4492 \mathrm{E}-01 & 8.4545 \mathrm{E}-02 & 0.0000 \mathrm{E}+00 \\ 86.80 & 9.2963 \mathrm{E}-02 & 4.9704 \mathrm{E}-02 & 0.0000 \mathrm{E}+00 \\ 98.14 & 5.3005 \mathrm{E}-02 & 2.6625 \mathrm{E}-02 & 0.0000 \mathrm{E}+00 \\ 110.00 & 2.2515 \mathrm{E}-02 & 1.1725 \mathrm{E}-02 & 0.0000 \mathrm{E}+00\end{array}$


Table E12A-2.4. Axial Distribution of Total Flux, Fast Flux and Power in MFA-2 at EOC 12A-2

$\begin{array}{rccc}\text { z }(\mathrm{cm}) & \text { Total Flux } & \text { Flux }>0.1 \mathrm{MeV} & \text { Power } \\ -97.78 & 2.7020 \mathrm{E}-02 & 1.4009 \mathrm{E}-02 & 0.0000 \mathrm{E}+00 \\ -92.78 & 5.7972 \mathrm{E}-02 & 2.7032 \mathrm{E}-02 & 0.0000 \mathrm{E}+00 \\ -87.78 & 9.2888 \mathrm{E}-02 & 4.3224 \mathrm{E}-02 & 0.0000 \mathrm{E}+00 \\ -82.78 & 1.3452 \mathrm{E}-01 & 6.4443 \mathrm{E}-02 & 0.0000 \mathrm{E}+00 \\ -77.78 & 1.8591 \mathrm{E}-01 & 9.3018 \mathrm{E}-02 & 0.0000 \mathrm{E}+00 \\ -72.15 & 2.4519 \mathrm{E}-01 & 1.3029 \mathrm{E}-01 & 0.0000 \mathrm{E}+00 \\ -65.90 & 3.0521 \mathrm{E}-01 & 1.7831 \mathrm{E}-01 & 0.0000 \mathrm{E}+00 \\ -60.35 & 3.6693 \mathrm{E}-01 & 2.4468 \mathrm{E}-01 & 1.4592 \mathrm{E}-01 \\ -55.49 & 4.4758 \mathrm{E}-01 & 3.2903 \mathrm{E}-01 & 1.6352 \mathrm{E}-01 \\ -50.62 & 5.3951 \mathrm{E}-01 & 4.3654 \mathrm{E}-01 & 1.8472 \mathrm{E}-01 \\ -47.17 & 6.1169 \mathrm{E}-01 & 5.3571 \mathrm{E}-01 & 2.0434 \mathrm{E}-01 \\ -43.58 & 6.8721 \mathrm{E}-01 & 6.4687 \mathrm{E}-01 & 7.6568 \mathrm{E}-01 \\ -38.45 & 7.9866 \mathrm{E}-01 & 7.8395 \mathrm{E}-01 & 8.5296 \mathrm{E}-01 \\ -33.32 & 9.0271 \mathrm{E}-01 & 9.0078 \mathrm{E}-01 & 9.5275 \mathrm{E}-01 \\ -28.20 & 9.9381 \mathrm{E}-01 & 9.9703 \mathrm{E}-01 & 9.7444 \mathrm{E}-01 \\ -23.07 & 1.0712 \mathrm{E}+00 & 1.0770 \mathrm{E}+00 & 1.0485 \mathrm{E}+00 \\ -17.94 & 1.1330 \mathrm{E}+00 & 1.1397 \mathrm{E}+00 & 1.1079 \mathrm{E}+00 \\ -12.82 & 1.1783 \mathrm{E}+00 & 1.1845 \mathrm{E}+00 & 1.1131 \mathrm{E}+00 \\ -7.69 & 1.2076 \mathrm{E}+00 & 1.2136 \mathrm{E}+00 & 1.1403 \mathrm{E}+00 \\ -2.56 & 1.2203 \mathrm{E}+00 & 1.2266 \mathrm{E}+00 & 1.1522 \mathrm{E}+00 \\ 2.56 & 1.2163 \mathrm{E}+00 & 1.2233 \mathrm{E}+00 & 1.1592 \mathrm{E}+00 \\ 7.69 & 1.1950 \mathrm{E}+00 & 1.2029 \mathrm{E}+00 & 1.1392 \mathrm{E}+00 \\ 12.82 & 1.1567 \mathrm{E}+00 & 1.1657 \mathrm{E}+00 & 1.1030 \mathrm{E}+00 \\ 17.94 & 1.1017 \mathrm{E}+00 & 1.1124 \mathrm{E}+00 & 1.1051 \mathrm{E}+00 \\ 23.07 & 1.0291 \mathrm{E}+00 & 1.0396 \mathrm{E}+00 & 1.0331 \mathrm{E}+00 \\ 28.20 & 9.4036 \mathrm{E}-01 & 9.4898 \mathrm{E}-01 & 9.4534 \mathrm{E}-01 \\ 33.32 & 8.3824 \mathrm{E}-01 & 8.4270 \mathrm{E}-01 & 9.1655 \mathrm{E}-01 \\ 38.45 & 7.2440 \mathrm{E}-01 & 7.1762 \mathrm{E}-01 & 7.9942 \mathrm{E}-01 \\ 43.58 & 6.0547 \mathrm{E}-01 & 5.7674 \mathrm{E}-01 & 6.9117 \mathrm{E}-01 \\ 47.17 & 5.2730 \mathrm{E}-01 & 4.6959 \mathrm{E}-01 & 1.4750 \mathrm{E}-01 \\ 50.62 & 4.5453 \mathrm{E}-01 & 3.7643 \mathrm{E}-01 & 1.2590 \mathrm{E}-01 \\ 55.49 & 3.6316 \mathrm{E}-01 & 2.7662 \mathrm{E}-01 & 1.0282 \mathrm{E}-01 \\ 60.35 & 2.8132 \mathrm{E}-01 & 1.9747 \mathrm{E}-01 & 8.3502 \mathrm{E}-02 \\ 64.98 & 2.2797 \mathrm{E}-01 & 1.4824 \mathrm{E}-01 & 0.0000 \mathrm{E}+00 \\ 69.39 & 1.9494 \mathrm{E}-01 & 1.2137 \mathrm{E}-01 & 0.0000 \mathrm{E}+00 \\ 76.46 & 1.4737 \mathrm{E}-01 & 8.5361 \mathrm{E}-02 & 0.0000 \mathrm{E}+00 \\ 86.80 & 9.5231 \mathrm{E}-02 & 5.0828 \mathrm{E}-02 & 0.0000 \mathrm{E}+00 \\ 98.14 & 5.4345 \mathrm{E}-02 & 2.7409 \mathrm{E}-02 & 0.0000 \mathrm{E}+00 \\ 110.00 & 2.3109 \mathrm{E}-02 & 1.2123 \mathrm{E}-02 & 0.0000 \mathrm{E}+00\end{array}$


HNF-SD-FF-ANAL-009 Rev. 1

Table E12A-2.5. Fission Power Distribution by Pin in MFA-1 at EOC 12A-2 CORE POSITION 1405

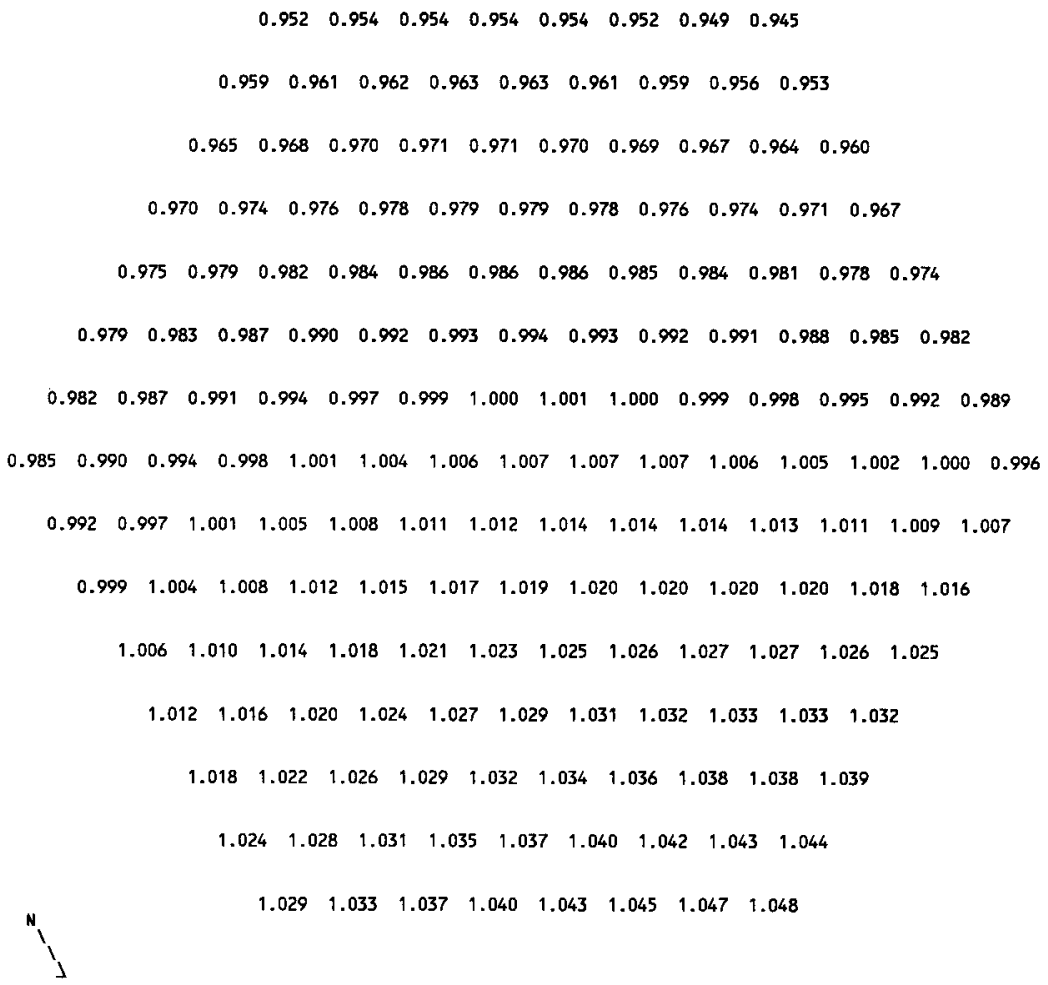


HNF-SD-FF-ANAL-009 Rev. 1

Table E12A-2.6. Fission Power Distribution by Pin in MFA-2 at EOC 12A-2 CORE POSITION 2506

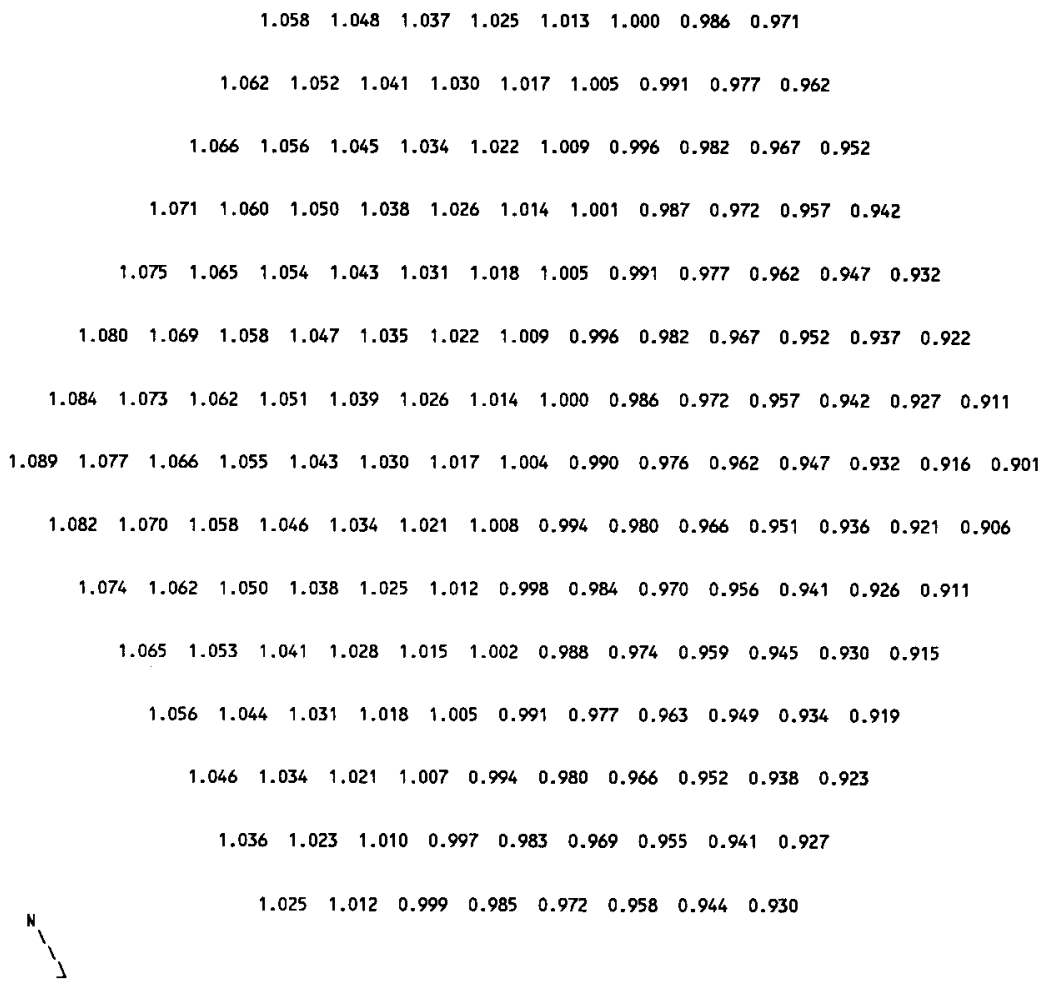


HNF-SD-FF-ANAL-009 Rev. 1

Table E12A-2.7. Fast Flux Distribution by Pin in MFA-1 at EOC 12A-2

CORE POSITION 1404

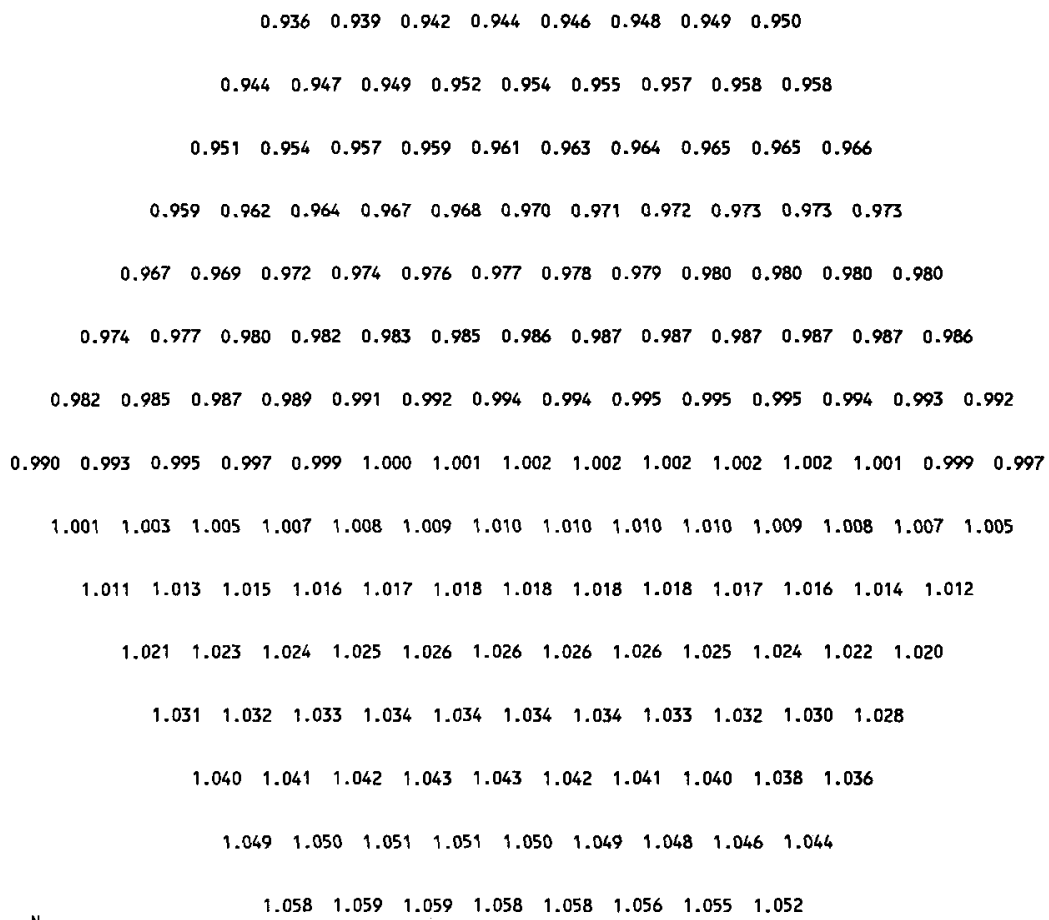


HNF-SD-FF-ANAL-009 Rev. 1

Table E12A-2.8. Fast Flux Distribution by Pin in MFA-2 at EOC $12 \mathrm{~A}-2$

CORE POSITION 2506

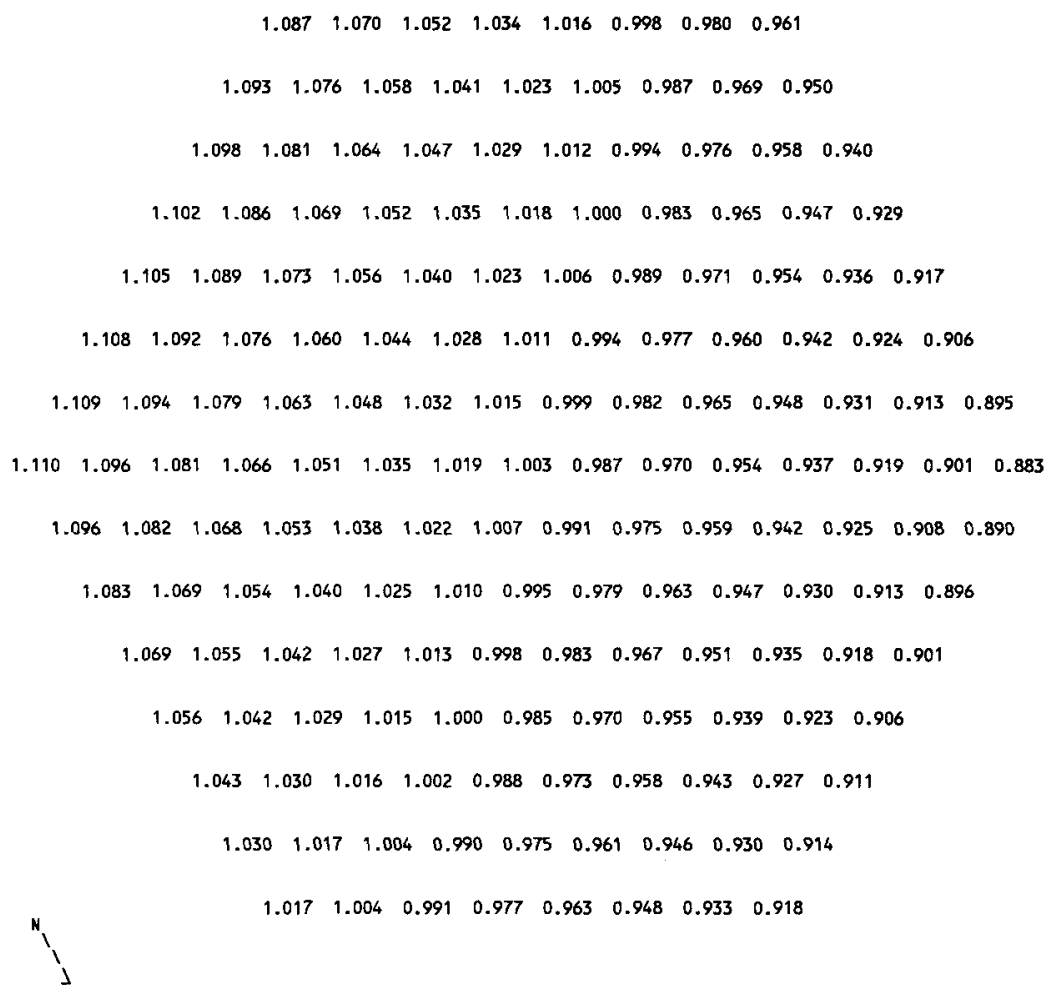


Table E12A-2.9. MFA-1 and MFA-2 Duct Wall

Fast Flux Data at EOC 12A-2

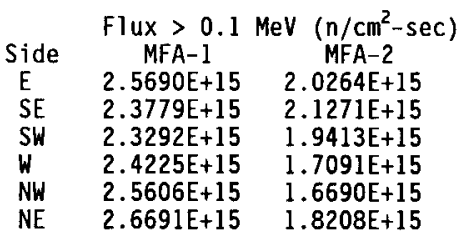

Table E12A-2.10. Assembly Outlet Temperatures and Flow Rates at EOC 12A-2

\begin{tabular}{lcl} 
CORE & $\begin{array}{c}\text { CALCULATED } \\
\text { OUTLET TEMP. } \\
\text { (DEG F) }\end{array}$ & $\begin{array}{l}\text { FLOW RATE } \\
(\text { LB/H) }\end{array}$ \\
POS. & (DEG9 & 108830 \\
1405 & 1069 & 190160 \\
1304 & 1051 & 1900 \\
1404 & 1005 & 192500 \\
1406 & 937 & 197060 \\
1506 & 895 & 197060 \\
1507 & 926 & 197060 \\
1508 & 988 & 177390 \\
2506 & 993 & 108830 \\
2404 & 983 & 193800 \\
2405 & 864 & 197080 \\
2505 & 906 & 197060 \\
2507 & 858 & 197060 \\
2606 & 892 & 161600 \\
2607 & 918 & 154980 \\
\multicolumn{3}{|c}{} \\
Assembly flows are based on a calculated core pressure \\
drop of 99.1 psi at a total reactor flow rate of \\
l6.74E+06 ib/hr.
\end{tabular}


Table E12A-2.11. MFA-1 Sodium Subchannel Temperatures at Top of Core Elevation for EOC $12 \mathrm{~A}-2$

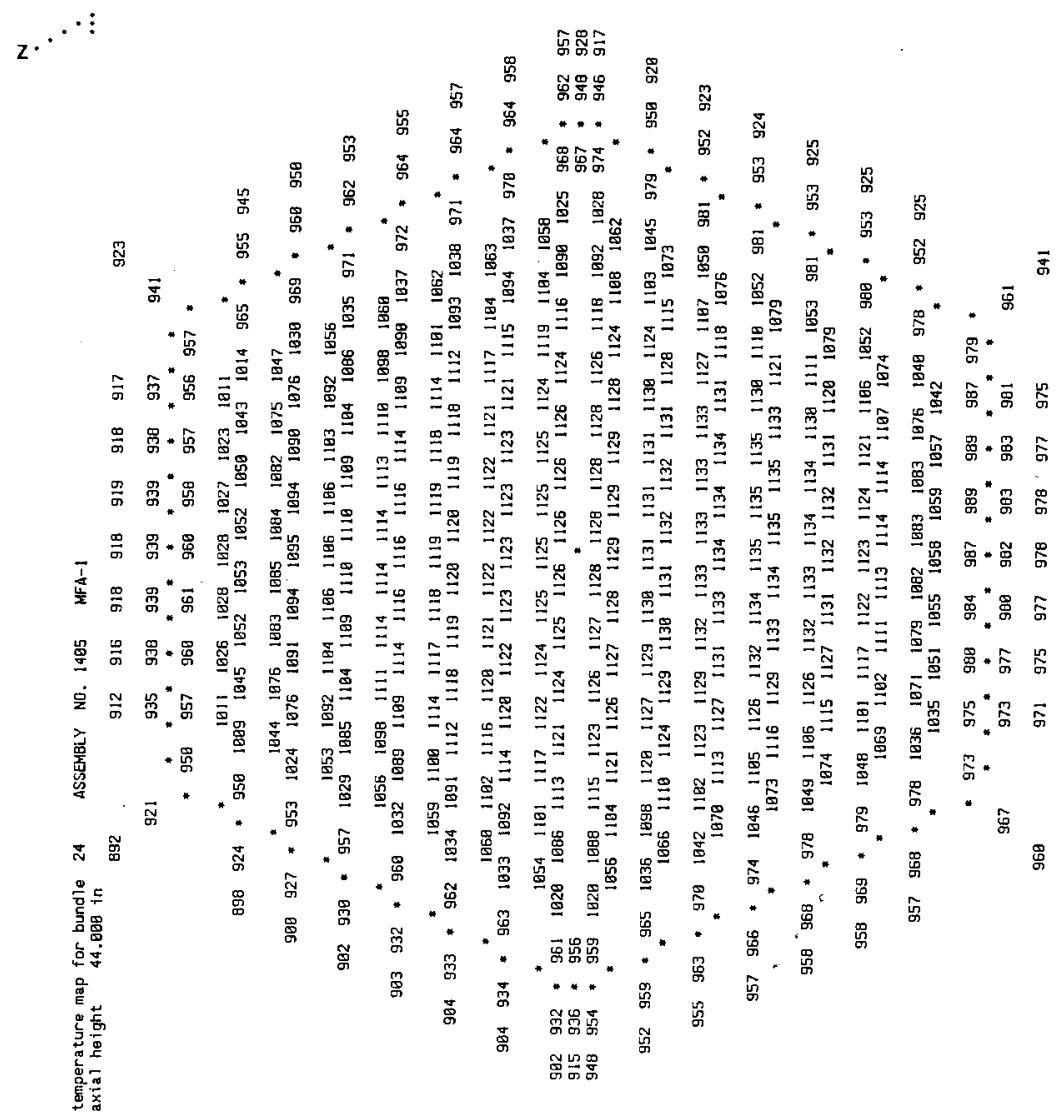


Table E12A-2.12. MFA-2 Sodium Subchannel Temperatures at Top of Core Elevation for EOC 12A-2

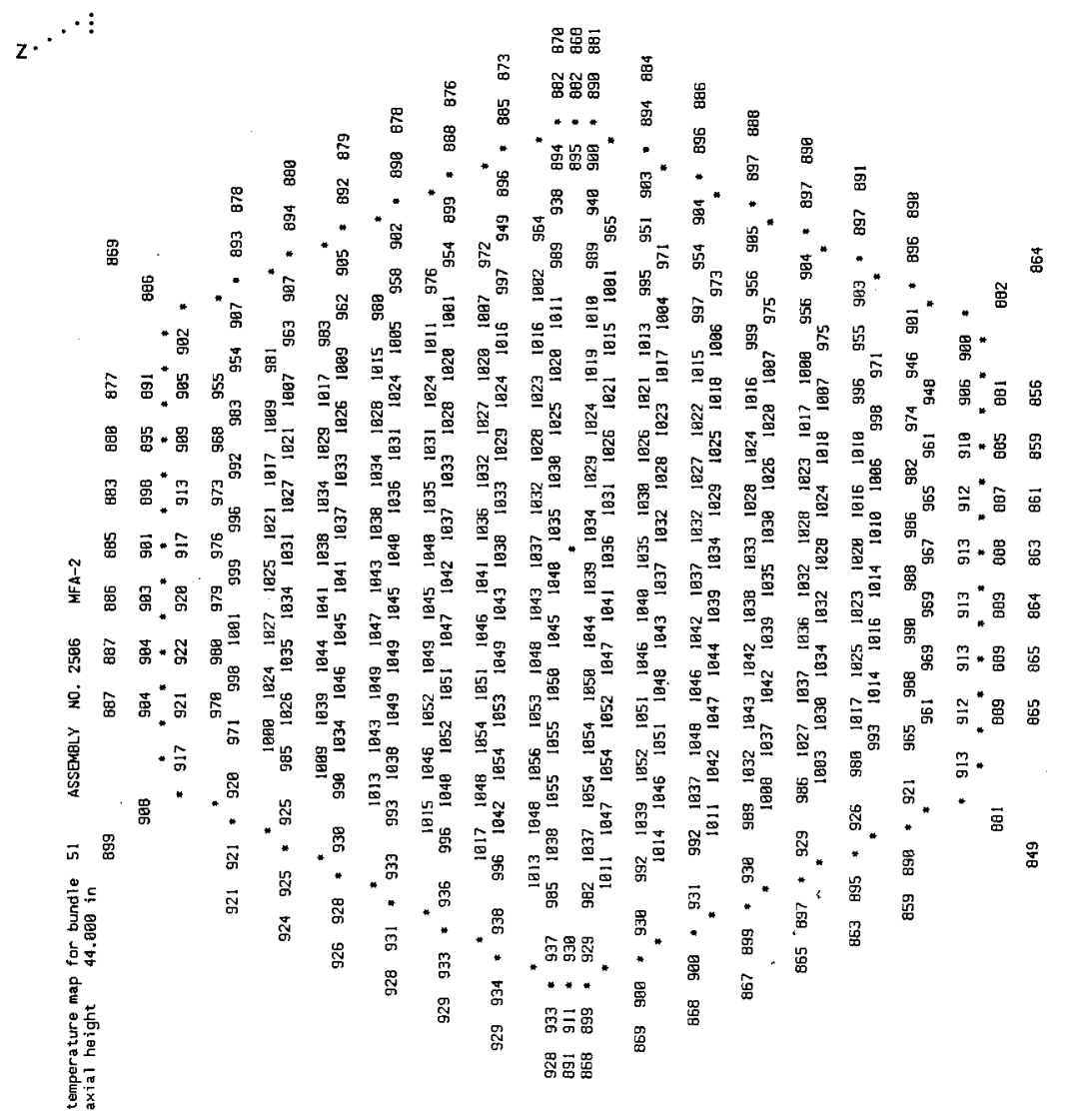


Table E12A-2:13. MFA-1 Sodium Subchannel Temperatures

at Elevation of Upper Axial Blanket for EOC $12 \mathrm{~A}-2$

$$
\begin{aligned}
& { }_{2} . \cdot \cdot
\end{aligned}
$$

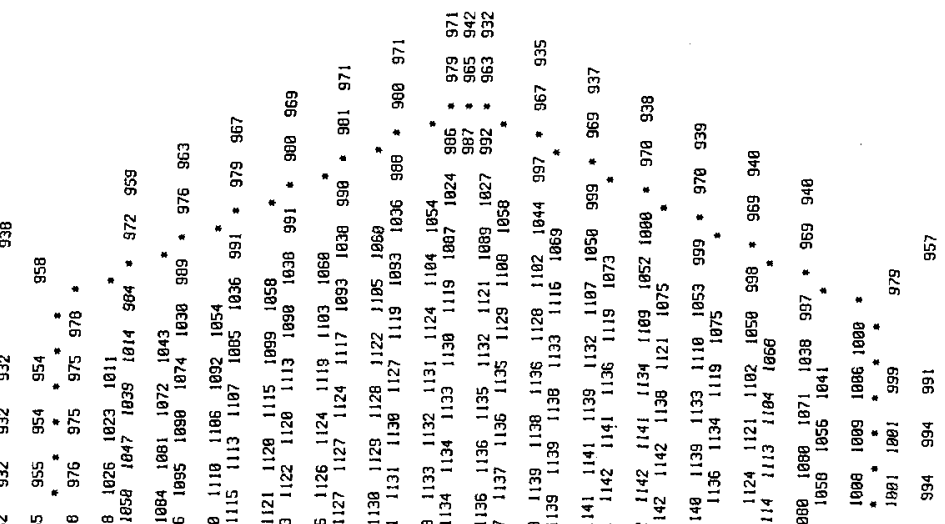

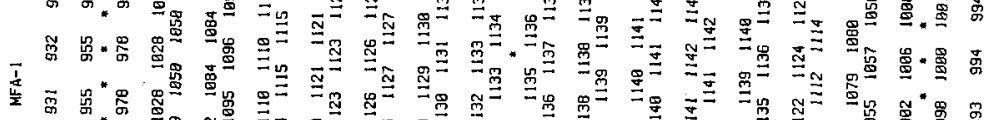

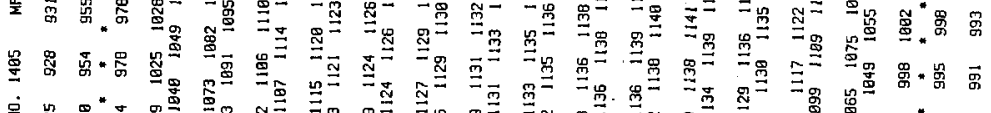

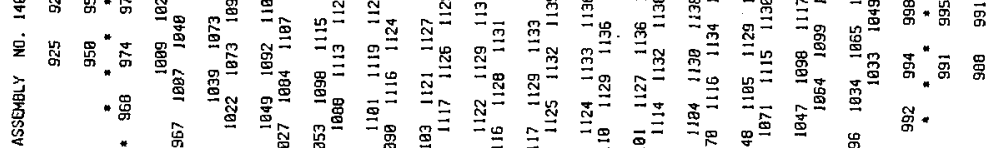

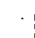

$$
\begin{aligned}
& \text { 芩 } \\
& \text { 产: } \\
& \text { 占品 } \\
& \text { 营 } \\
& \text { 点 } \\
& \text { 高焉 }
\end{aligned}
$$

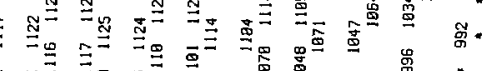

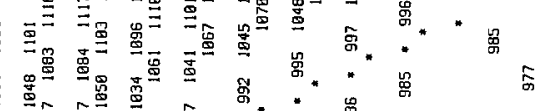


Table E12A-2.14. MFA-2 Sodium Subchannel Temperatures at Elevation of Upper Axial Blanket for EOC $12 A-2$

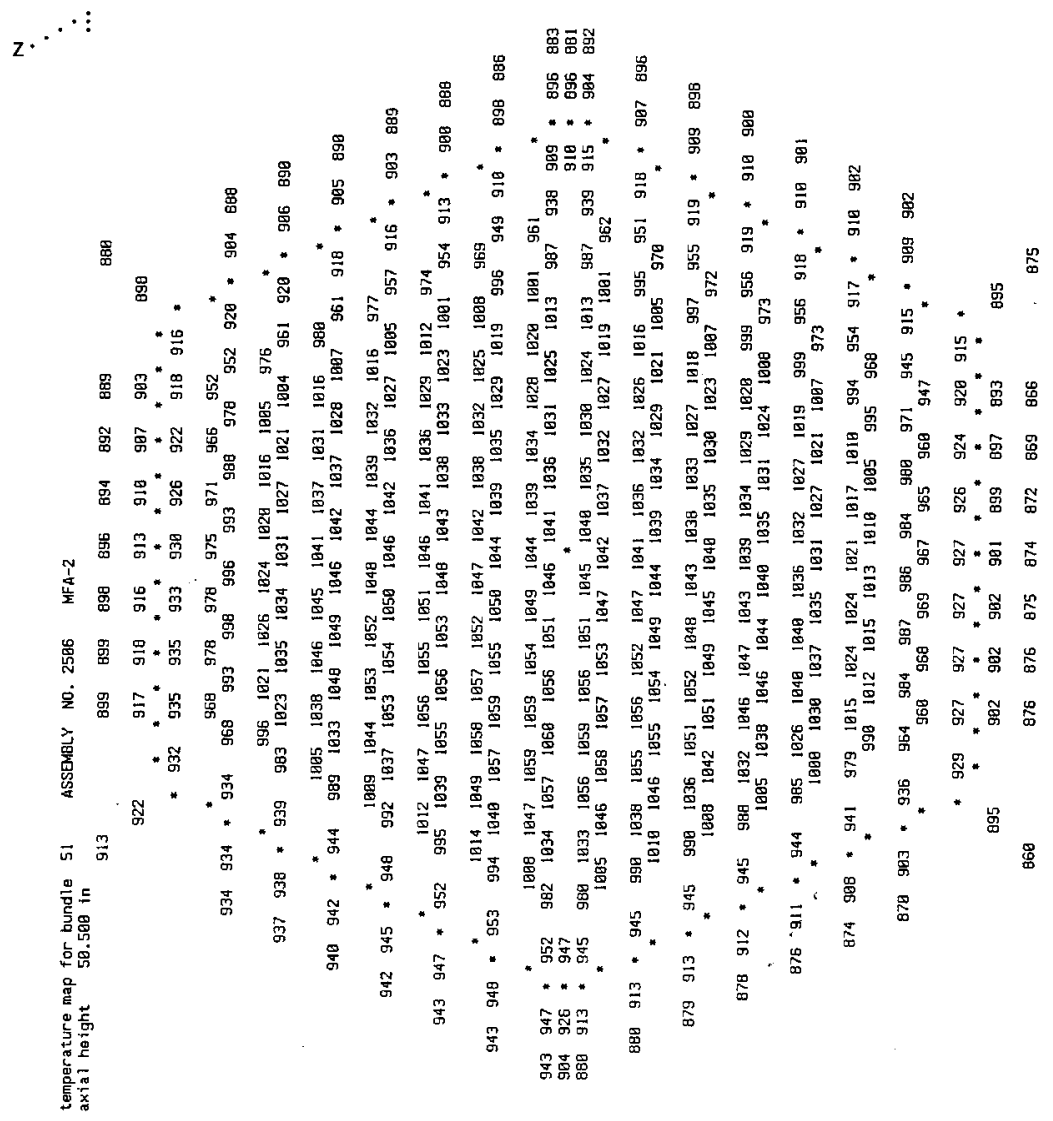


Table E12A-2.15. MFA-1 Sodium Subchannel Temperatures at Top of Fuel Pin Bundle for EOC 12A-2

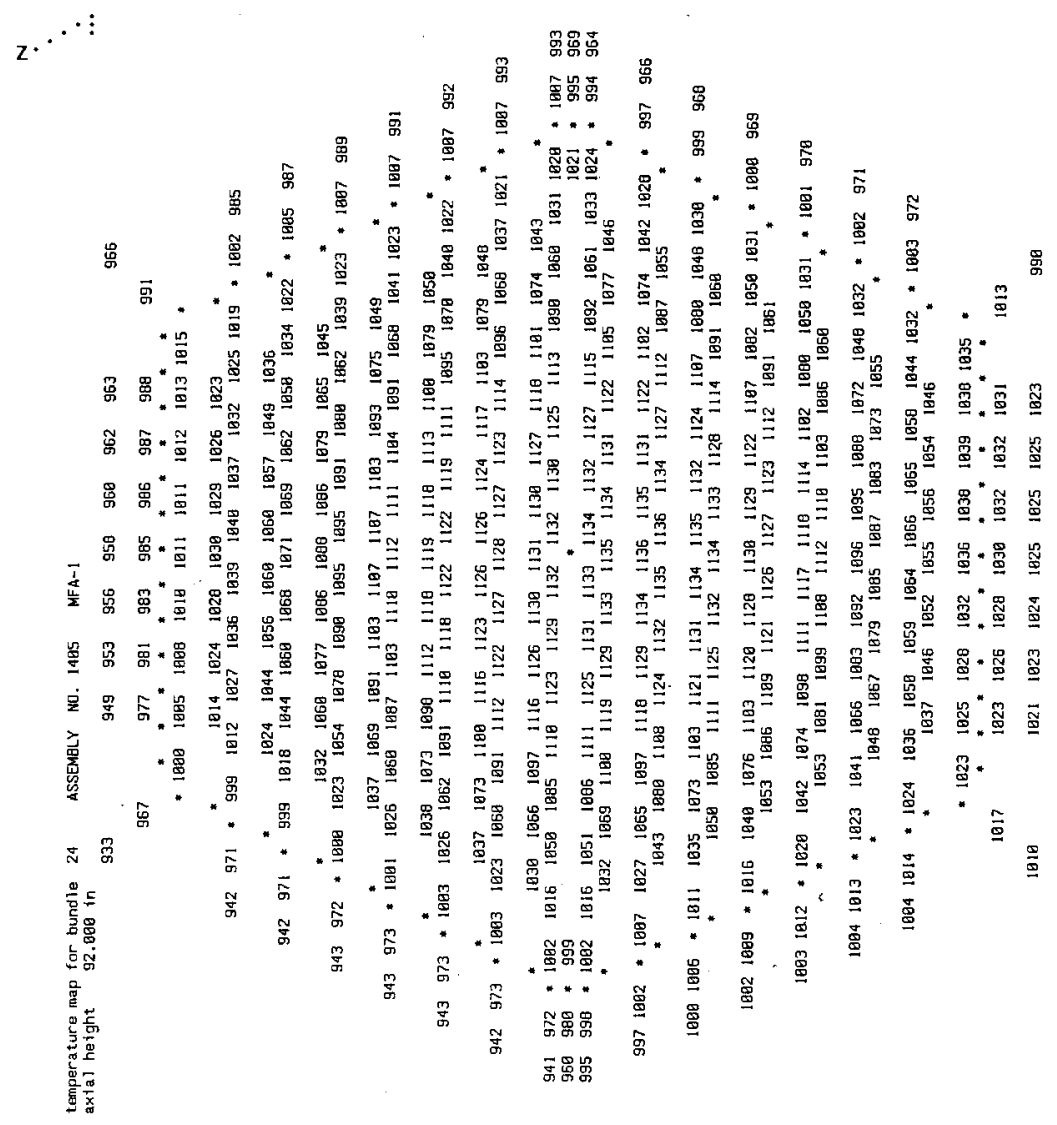


Table E12A-2.16. MFA-2 Sodium Subchanne1 Temperatures at Top of Fuel Pin Bundle for EOC $12 A-2$

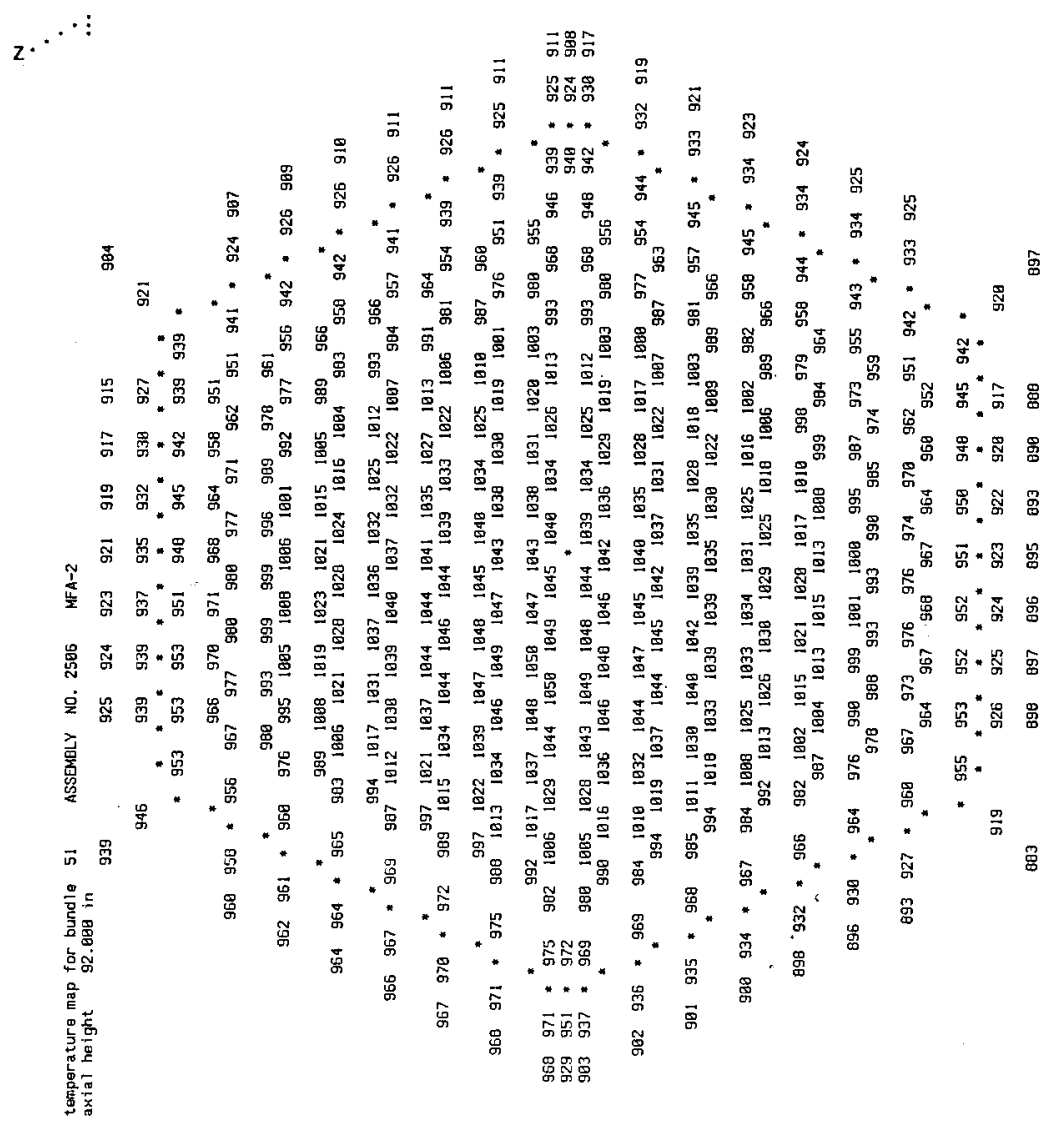


Table E12A-2.17. Composition of MFA-1 at EOC 12A-2

\begin{tabular}{|c|c|c|c|c|c|}
\hline ial & Axial $R$ & ge $(\mathrm{cm})$ & & Atom Densi & $(a / b-c m)$ \\
\hline Level & $\begin{array}{r}\text { Lower } \\
-12805\end{array}$ & $\begin{array}{l}\text { Upper } \\
-75\end{array}$ & Constituent & Fresh & End of cycle \\
\hline & & & $\begin{array}{l}\mathrm{Na}-23 \\
\text { SS-316 }\end{array}$ & $\begin{array}{l}5.3150 \mathrm{E}-03 \\
6.4090 \mathrm{E}-02\end{array}$ & $\begin{array}{l}5.3150 E-03 \\
6.4090 E-02\end{array}$ \\
\hline 2 & -75.28 & -62.78 & & & \\
\hline & & & $\begin{array}{l}\mathrm{Na}-23 \\
\mathrm{SS}-316\end{array}$ & $\begin{array}{l}1.3177 \mathrm{E}-02 \\
3.4175 \mathrm{E}-02\end{array}$ & $\begin{array}{l}1.3177 \mathrm{E}-02 \\
3.4175 \mathrm{E}-02\end{array}$ \\
\hline 3 & -62.78 & $-4 b .14$ & $\begin{array}{l}U-235 \\
U-238 \\
\text { Pu-239 } \\
\text { Pu-240 } \\
\text { FP } \\
\text { O-16 } \\
\mathrm{Na}-23 \\
\text { SS }-316\end{array}$ & $\begin{array}{l}1.4143 \mathrm{E}-05 \\
7.0573 \mathrm{E}-03 \\
0.0000 \mathrm{E}+00 \\
0.0000 \mathrm{E}+00 \\
0.0000 \mathrm{E}+00 \\
1.4143 \mathrm{E}-02 \\
9.7416 \mathrm{E}-03 \\
1.9073 \mathrm{E}-02\end{array}$ & $\begin{array}{l}8.1637 \mathrm{E}-06 \\
6.6838 \mathrm{E}-03 \\
2.7816 \mathrm{E}-04 \\
2.1864 \mathrm{E}-05 \\
7.6754 \mathrm{E}-05 \\
1.4143 \mathrm{E}-02 \\
9.7416 \mathrm{E}-03 \\
1.9073 \mathrm{E}-02\end{array}$ \\
\hline 4 & -46.14 & -30.76 & $\begin{array}{l}U-235 \\
U-238 \\
P u-239 \\
P u-240 \\
P u-241 \\
P u-242 \\
\text { Am-241 } \\
F P \\
O-16 \\
\text { Na-23 } \\
\text { SS }-316\end{array}$ & $\begin{array}{l}1.0840 \mathrm{E}-05 \\
4.4499 \mathrm{E}-03 \\
1.7520 \mathrm{E}-03 \\
2.2340 \mathrm{E}-04 \\
1.9280 \mathrm{E}-05 \\
4.7430 \mathrm{E}-06 \\
7.6680 \mathrm{E}-07 \\
0.0000 \mathrm{E}+00 \\
1.2670 \mathrm{E}-02 \\
9.8100 \mathrm{E}-03 \\
1.8990 \mathrm{E}-02\end{array}$ & $\begin{array}{l}6.6573 \mathrm{E}-06 \\
4.1725 \mathrm{E}-03 \\
1.2697 \mathrm{E}-03 \\
3.0840 \mathrm{E}-04 \\
2.7272 \mathrm{E}-05 \\
5.5399 \mathrm{E}-06 \\
3.8950 \mathrm{E}-06 \\
6.6464 \mathrm{E}-04 \\
1.2670 \mathrm{E}-02 \\
9.8100 \mathrm{E}-03 \\
1.8990 \mathrm{E}-02\end{array}$ \\
\hline 5 & -30.76 & -15.38 & $\begin{array}{l}U-235 \\
U-238 \\
\mathrm{Pu}-239 \\
\mathrm{Pu}-240 \\
\mathrm{Pu}-241 \\
\mathrm{Pu}-242 \\
\mathrm{Am}-241 \\
\mathrm{FP} \\
0-16 \\
\mathrm{Na}-23 \\
\mathrm{SS}-316\end{array}$ & $\begin{array}{l}1.0840 \mathrm{E}-05 \\
4.4499 \mathrm{E}-03 \\
1.7520 \mathrm{E}-03 \\
2.2340 \mathrm{E}-04 \\
1.9280 \mathrm{E}-05 \\
4.7430 \mathrm{E}-06 \\
7.6680 \mathrm{E}-07 \\
0.0000 \mathrm{E}+00 \\
1.2670 \mathrm{E}-02 \\
9.8100 \mathrm{E}-03 \\
1.8990 \mathrm{E}-02\end{array}$ & $\begin{array}{l}5.9310 \mathrm{E}-06 \\
4.1033 \mathrm{E}-03 \\
1.1696 \mathrm{E}-03 \\
3.0755 \mathrm{E}-04 \\
2.7508 \mathrm{E}-05 \\
5.5653 \mathrm{E}-06 \\
3.7663 \mathrm{E}-06 \\
8.3495 \mathrm{E}-04 \\
1.2670 \mathrm{E}-02 \\
9.8100 \mathrm{E}-03 \\
1.8990 \mathrm{E}-02\end{array}$ \\
\hline 6 & -15.38 & 0.00 & $\begin{array}{l}U-235 \\
U-238 \\
P u-239 \\
P u-240 \\
P u-241 \\
P u-242 \\
\text { Am-241 } \\
\text { FP } \\
0-16 \\
\text { Na-23 } \\
\text { SS-316 }\end{array}$ & $\begin{array}{l}1.0840 \mathrm{E}-05 \\
4.4499 \mathrm{E}-03 \\
1.7520 \mathrm{E}-03 \\
2.2340 \mathrm{E}-04 \\
1.9280 \mathrm{E}-05 \\
4.7430 \mathrm{E}-06 \\
7.6680 \mathrm{E}-07 \\
0.0000 \mathrm{E}+00 \\
1.2670 \mathrm{E}-02 \\
9.8100 \mathrm{E}-03 \\
1.8990 \mathrm{E}-02\end{array}$ & $\begin{array}{l}5.5473 \mathrm{E}-06 \\
4.0673 \mathrm{E}-03 \\
1.1221 \mathrm{E}-03 \\
3.1177 \mathrm{E}-04 \\
2.8567 \mathrm{E}-05 \\
5.6895 \mathrm{E}-06 \\
3.7536 \mathrm{E}-06 \\
9.1372 \mathrm{E}-04 \\
1.2670 \mathrm{E}-02 \\
9.8100 \mathrm{E}-03 \\
1.8990 \mathrm{E}-02\end{array}$ \\
\hline
\end{tabular}




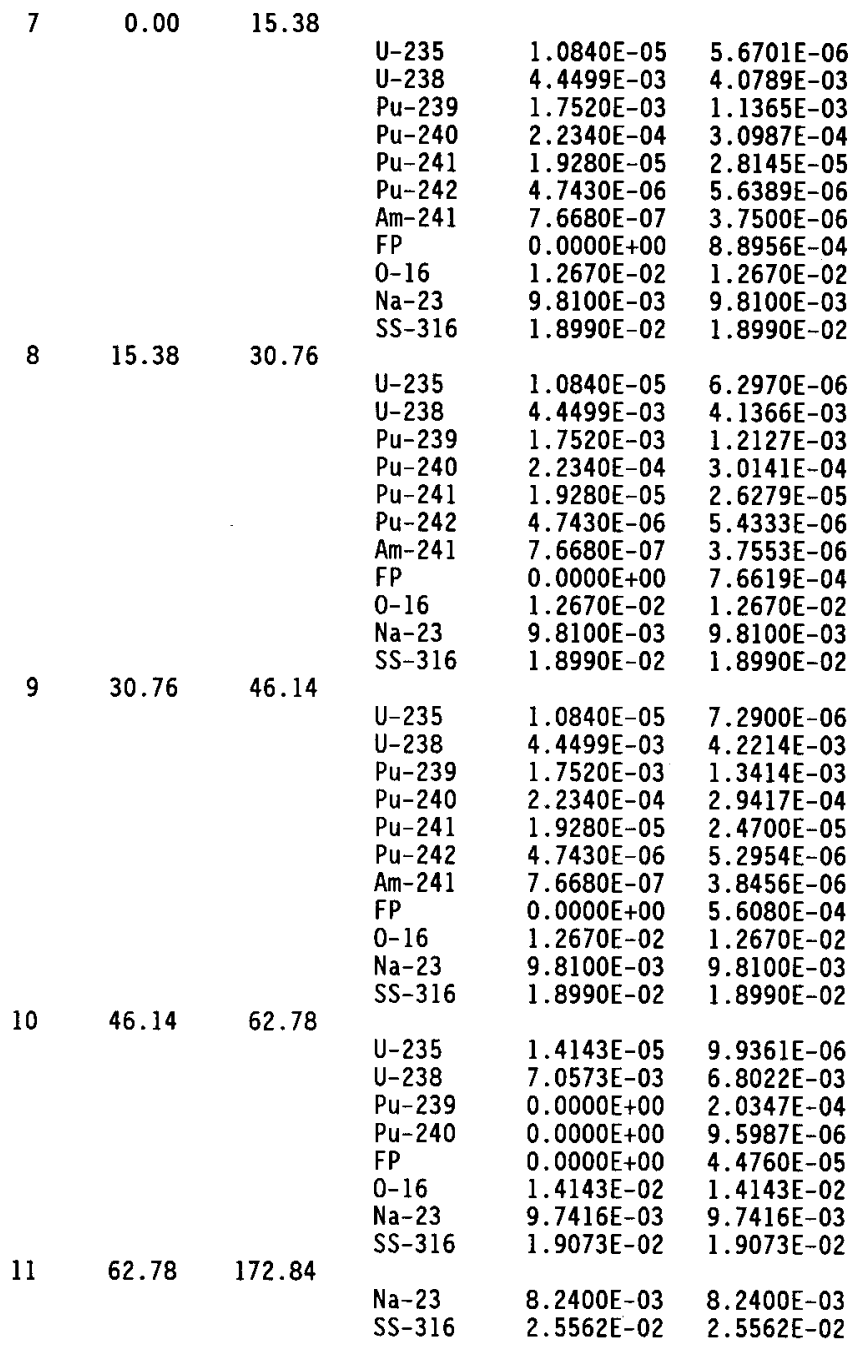


Table El2A-2.18. Composition of MFA-2 at EOC 12A-2

\begin{tabular}{|c|c|c|c|c|c|}
\hline Axial & Axial R & ge $(\mathrm{cm})$ & & Atom Dens & $(a / b-c m)$ \\
\hline $\begin{array}{c}\text { Level } \\
1\end{array}$ & $\begin{array}{r}\text { Lower } \\
-128.05\end{array}$ & $\begin{array}{r}\text { Upper } \\
-75.28\end{array}$ & Constituent & Fresh & End of Cycle \\
\hline & & & $\begin{array}{l}\mathrm{Na}-23 \\
\text { SS-316 }\end{array}$ & $\begin{array}{l}5.3150 \mathrm{E}-03 \\
6.4090 \mathrm{E}-02\end{array}$ & $\begin{array}{l}5.3150 E-03 \\
6.4090 E-02\end{array}$ \\
\hline 2 & -15.28 & -62.18 & $\begin{array}{l}\mathrm{Na}-23 \\
\mathrm{SS}-316\end{array}$ & $\begin{array}{l}1.3177 \mathrm{E}-02 \\
3.4175 \mathrm{E}-02\end{array}$ & $\begin{array}{l}1.3177 \mathrm{E}-02 \\
3.4175 \mathrm{E}-02\end{array}$ \\
\hline 3 & -62.78 & -46.14 & $\begin{array}{l}U-235 \\
U-238 \\
\mathrm{Pu}-239 \\
\mathrm{Pu}-240 \\
\mathrm{FP} \\
\mathrm{O}-16 \\
\mathrm{Na}-23 \\
\mathrm{SS}-316\end{array}$ & $\begin{array}{l}1.4143 \mathrm{E}-05 \\
7.0573 \mathrm{E}-03 \\
0.0000 \mathrm{E}+00 \\
0.0000 \mathrm{E}+00 \\
0.0000 \mathrm{E}+00 \\
1.4143 \mathrm{E}-02 \\
9.7416 \mathrm{E}-03 \\
1.9073 \mathrm{E}-02\end{array}$ & $\begin{array}{l}8.2937 \mathrm{E}-06 \\
6.7003 \mathrm{E}-03 \\
2.6816 \mathrm{E}-04 \\
2.0721 \mathrm{E}-05 \\
7.1396 \mathrm{E}-05 \\
1.4143 \mathrm{E}-02 \\
9.7416 \mathrm{E}-03 \\
1.9073 \mathrm{E}-02\end{array}$ \\
\hline 4 & -46.14 & -30.76 & $\begin{array}{l}U-235 \\
U-238 \\
P u-239 \\
P u-240 \\
P u-241 \\
P u-242 \\
\text { Am-241 } \\
\text { FP } \\
0-16 \\
\text { Na-23 } \\
\text { SS-316 }\end{array}$ & $\begin{array}{l}1.0160 \mathrm{E}-05 \\
4.4270 \mathrm{E}-03 \\
1.7483 \mathrm{E}-03 \\
2.2290 \mathrm{E}-04 \\
1.9040 \mathrm{E}-05 \\
4.7330 \mathrm{E}-06 \\
7.5700 \mathrm{E}-07 \\
0.0000 \mathrm{E}+00 \\
1.2760 \mathrm{E}-02 \\
9.8100 \mathrm{E}-03 \\
1.8990 \mathrm{E}-02\end{array}$ & $\begin{array}{l}6.3545 \mathrm{E}-06 \\
4.1603 \mathrm{E}-03 \\
1.2822 \mathrm{E}-03 \\
3.0651 \mathrm{E}-04 \\
2.6856 \mathrm{E}-05 \\
5.4941 \mathrm{E}-06 \\
3.9100 \mathrm{E}-06 \\
6.3884 \mathrm{E}-04 \\
1.2760 \mathrm{E}-02 \\
9.8100 \mathrm{E}-03 \\
1.8990 \mathrm{E}-02\end{array}$ \\
\hline 5 & -30.76 & -15.38 & $\begin{array}{l}U-235 \\
U-238 \\
\mathrm{Pu}-239 \\
\mathrm{Pu}-240 \\
\mathrm{Pu}-241 \\
\mathrm{Pu}-242 \\
\mathrm{Am}-241 \\
\mathrm{FP} \\
\mathrm{O}-16 \\
\mathrm{Na}-23 \\
\mathrm{SS}-316\end{array}$ & $\begin{array}{l}1.0160 \mathrm{E}-05 \\
4.4270 \mathrm{E}-03 \\
1.7483 \mathrm{E}-03 \\
2.2290 \mathrm{E}-04 \\
1.9040 \mathrm{E}-05 \\
4.7330 \mathrm{E}-06 \\
7.5700 \mathrm{E}-07 \\
0.0000 \mathrm{E}+00 \\
1.2760 \mathrm{E}-02 \\
9.8100 \mathrm{E}-03 \\
1.8990 \mathrm{E}-02\end{array}$ & $\begin{array}{l}5.6586 \mathrm{E}-06 \\
4.0909 \mathrm{E}-03 \\
1.1819 \mathrm{E}-03 \\
3.0675 \mathrm{E}-04 \\
2.7253 \mathrm{E}-05 \\
5.5299 \mathrm{E}-06 \\
3.7916 \mathrm{E}-06 \\
8.0910 \mathrm{E}-04 \\
1.2760 \mathrm{E}-02 \\
9.8100 \mathrm{E}-03 \\
1.8990 \mathrm{E}-02\end{array}$ \\
\hline 6 & -15.38 & 0.00 & $\begin{array}{l}\text { U-235 } \\
\text { U-238 } \\
\mathrm{Pu}-239 \\
\mathrm{Pu}-240 \\
\mathrm{Pu}-241 \\
\mathrm{Pu}-242 \\
\mathrm{Am}-241 \\
\mathrm{FP} \\
0-16 \\
\mathrm{Na}-23 \\
\mathrm{SS}-316\end{array}$ & $\begin{array}{l}1.0160 \mathrm{E}-05 \\
4.4270 \mathrm{E}-03 \\
1.7483 \mathrm{E}-03 \\
2.2290 \mathrm{E}-04 \\
1.9040 \mathrm{E}-05 \\
4.7330 \mathrm{E}-06 \\
7.5700 \mathrm{E}-07 \\
0.0000 \mathrm{E}+00 \\
1.2760 \mathrm{E}-02 \\
9.8100 \mathrm{E}-03 \\
1.8990 \mathrm{E}-02\end{array}$ & $\begin{array}{l}5.2897 \mathrm{E}-06 \\
4.0541 \mathrm{E}-03 \\
1.1333 \mathrm{E}-03 \\
3.1137 \mathrm{E}-04 \\
2.8380 \mathrm{E}-05 \\
5.6581 \mathrm{E}-06 \\
3.7902 \mathrm{E}-06 \\
8.8849 \mathrm{E}-04 \\
1.2760 \mathrm{E}-02 \\
9.8100 \mathrm{E}-03 \\
1.8990 \mathrm{E}-02\end{array}$ \\
\hline & & & & & \\
\hline
\end{tabular}


HNF-SD-FF-ANAL-009 Rev. 1

\begin{tabular}{|c|c|c|c|c|c|}
\hline \multirow{2}{*}{7} & 0.00 & 15.38 & & & \\
\hline & & & $\begin{array}{l}U-235 \\
U-238 \\
P u-239 \\
P u-240 \\
P u-241 \\
P u-242 \\
A m-241 \\
F P \\
O-16 \\
N a-23 \\
S S-316\end{array}$ & $\begin{array}{l}1.0160 \mathrm{E}-05 \\
4.4270 \mathrm{E}-03 \\
1.7483 \mathrm{E}-03 \\
2.2290 \mathrm{E}-04 \\
1.9040 \mathrm{E}-05 \\
4.7330 \mathrm{E}-06 \\
7.5700 \mathrm{E}-07 \\
0.0000 \mathrm{E}+00 \\
1.2760 \mathrm{E}-02 \\
9.8100 \mathrm{E}-03 \\
1.8990 \mathrm{E}-02\end{array}$ & $\begin{array}{l}5.3970 \mathrm{E}-06 \\
4.0648 \mathrm{E}-03 \\
1.1468 \mathrm{E}-03 \\
3.0956 \mathrm{E}-04 \\
2.7978 \mathrm{E}-05 \\
5.6115 \mathrm{E}-06 \\
3.7874 \mathrm{E}-06 \\
8.6640 \mathrm{E}-04 \\
1.2760 \mathrm{E}-02 \\
9.8100 \mathrm{E}-03 \\
1.8990 \mathrm{E}-02\end{array}$ \\
\hline \multirow[t]{2}{*}{8} & 15.38 & 30.76 & & & \\
\hline & & & $\begin{array}{l}U-235 \\
U-238 \\
P u-239 \\
P u-240 \\
P u-241 \\
P u-242 \\
\mathrm{Am}-241 \\
\mathrm{FP} \\
\mathrm{O}-16 \\
\mathrm{Na}-23 \\
\mathrm{SS}-316\end{array}$ & $\begin{array}{l}1.0160 \mathrm{E}-05 \\
4.4270 \mathrm{E}-03 \\
1.7483 \mathrm{E}-03 \\
2.2290 \mathrm{E}-04 \\
1.9040 \mathrm{E}-05 \\
4.7330 \mathrm{E}-06 \\
7.5700 \mathrm{E}-07 \\
0.0000 \mathrm{E}+00 \\
1.2760 \mathrm{E}-02 \\
9.8100 \mathrm{E}-03 \\
1.8990 \mathrm{E}-02\end{array}$ & $\begin{array}{l}5.9714 \mathrm{E}-06 \\
4.1204 \mathrm{E}-03 \\
1.2206 \mathrm{E}-03 \\
3.0105 \mathrm{E}-04 \\
2.6114 \mathrm{E}-05 \\
5.4123 \mathrm{E}-06 \\
3.7846 \mathrm{E}-06 \\
7.4728 \mathrm{E}-04 \\
1.2760 \mathrm{E}-02 \\
9.8100 \mathrm{E}-03 \\
1.8990 \mathrm{E}-02\end{array}$ \\
\hline \multirow[t]{2}{*}{9} & 30.76 & 46.14 & & & \\
\hline & & & $\begin{array}{l}U-235 \\
U-238 \\
P u-239 \\
P u-240 \\
P u-241 \\
P u-242 \\
\mathrm{Am}-241 \\
\mathrm{FP} \\
0-16 \\
\mathrm{Na}-23 \\
\mathrm{SS}-316\end{array}$ & $\begin{array}{l}1.0160 \mathrm{E}-05 \\
4.4270 \mathrm{E}-03 \\
1.7483 \mathrm{E}-03 \\
2.2290 \mathrm{E}-04 \\
1.9040 \mathrm{E}-05 \\
4.7330 \mathrm{E}-06 \\
7.5700 \mathrm{E}-07 \\
0.0000 \mathrm{E}+00 \\
1.2760 \mathrm{E}-02 \\
9.8100 \mathrm{E}-03 \\
1.8990 \mathrm{E}-02\end{array}$ & $\begin{array}{l}6.8697 \mathrm{E}-06 \\
4.2025 \mathrm{E}-03 \\
1.3442 \mathrm{E}-03 \\
2.9417 \mathrm{E}-04 \\
2.4632 \mathrm{E}-05 \\
5.2878 \mathrm{E}-06 \\
3.8671 \mathrm{E}-06 \\
5.4953 \mathrm{E}-04 \\
1.2760 \mathrm{E}-02 \\
9.8100 \mathrm{E}-03 \\
1.8990 \mathrm{E}-02\end{array}$ \\
\hline \multirow[t]{2}{*}{10} & 46.14 & 62.78 & & & \\
\hline & & & $\begin{array}{l}\mathrm{U}-235 \\
\mathrm{U}-238 \\
\mathrm{Pu}-239 \\
\mathrm{Pu}-240 \\
\mathrm{FP} \\
\mathrm{O}-16 \\
\mathrm{Na}-23 \\
\mathrm{SS}-316\end{array}$ & $\begin{array}{l}1.4143 \mathrm{E}-05 \\
7.0573 \mathrm{E}-03 \\
0.0000 \mathrm{E}+00 \\
0.0000 \mathrm{E}+00 \\
0.0000 \mathrm{E}+00 \\
1.4143 \mathrm{E}-02 \\
9.7416 \mathrm{E}-03 \\
1.9073 \mathrm{E}-02\end{array}$ & $\begin{array}{l}9.8017 \mathrm{E}-06 \\
6.8001 \mathrm{E}-03 \\
2.0495 \mathrm{E}-04 \\
1.0357 \mathrm{E}-05 \\
4.4734 \mathrm{E}-05 \\
1.4143 \mathrm{E}-02 \\
9.7416 \mathrm{E}-03 \\
1.9073 \mathrm{E}-02\end{array}$ \\
\hline \multirow[t]{2}{*}{11} & 62.78 & 172.84 & & & \\
\hline & & & $\begin{array}{l}\mathrm{Na}-23 \\
\mathrm{SS}-316\end{array}$ & $\begin{array}{l}8.2400 \mathrm{E}-03 \\
2.5562 \mathrm{E}-02\end{array}$ & $\begin{array}{l}8.2400 \mathrm{E}-03 \\
2.5562 \mathrm{E}-02\end{array}$ \\
\hline
\end{tabular}




\subsection{Cycle 12B-1}

Table B12B-1.1. Fission Power Generated in MFA-2 and Neighboring Assemblies at BOC 12B-1

\begin{tabular}{lccccc} 
CORE & $* \star * * * * *$ & \multicolumn{2}{c}{ POWER IN MEGAWATTS } & $* * * * * *$ \\
POS. & BELOW CORE & IN CORE & ABOVE CORE & TOTAL PWR \\
2405 & $1.119 \mathrm{E}-01$ & $3.575 \mathrm{E}+00$ & $6.707 \mathrm{E}-02$ & $3.754 \mathrm{E}+00$ \\
2304 & $1.295 \mathrm{E}-02$ & $4.818 \mathrm{E}+00$ & $9.428 \mathrm{E}-03$ & $4.840 \mathrm{E}+00$ \\
2404 & $0.000 \mathrm{E}+00$ & $5.813 \mathrm{E}+00$ & $0.000 \mathrm{E}+00$ & $5.813 \mathrm{E}+00$ \\
2406 & $0.000 \mathrm{E}+00$ & $0.000 \mathrm{E}+00$ & $0.000 \mathrm{E}+00$ & $0.000 \mathrm{E}+00$ \\
2506 & $1.244 \mathrm{E}-02$ & $3.806 \mathrm{E}+00$ & $8.897 \mathrm{E}-03$ & $3.827 \mathrm{E}+00$ \\
2507 & $1.495 \mathrm{E}-02$ & $3.300 \mathrm{E}+00$ & $9.297 \mathrm{E}-03$ & $3.324 \mathrm{E}+00$ \\
2508 & $1.367 \mathrm{E}-02$ & $3.618 \mathrm{E}+00$ & $8.929 \mathrm{E}-03$ & $3.640 \mathrm{E}+00$
\end{tabular}

Table Bl2B-1.2. Assembly Averaged Total and Fast Flux in MFA-2 at BOC $12 \mathrm{~B}-1$

$\begin{array}{cccc} & \text { Core } & \text { Flux } & \left(\mathrm{n} / \mathrm{cm}^{2}-\mathrm{sec}\right) \\ \text { Assembly } & \text { Pos. } & \text { Total } & >0.1 \mathrm{MeV} \\ \text { MFA-2 } & 2405 & 3.501 \mathrm{E}+15 & 2.216 \mathrm{E}+15\end{array}$


Table B12B-1.4. Axial Distribution of Total Flux, Fast Flux and Power in MFA-2 at BOC 12B-1

$\begin{array}{rccc}z(\mathrm{~cm}) & \text { Total Flux } & \text { Flux }>0.1 \text { MeV } & \text { Power } \\ -97.78 & 2.7884 \mathrm{E}-02 & 1.4681 \mathrm{E}-02 & 0.0000 \mathrm{E}+00 \\ -92.78 & 5.9935 \mathrm{E}-02 & 2.8414 \mathrm{E}-02 & 0.0000 \mathrm{E}+00 \\ -87.78 & 9.6193 \mathrm{E}-02 & 4.5549 \mathrm{E}-02 & 0.0000 \mathrm{E}+00 \\ -82.78 & 1.3954 \mathrm{E}-01 & 6.8072 \mathrm{E}-02 & 0.0000 \mathrm{E}+00 \\ -77.78 & 1.9313 \mathrm{E}-01 & 9.8491 \mathrm{E}-02 & 0.0000 \mathrm{E}+00 \\ -72.15 & 2.5475 \mathrm{E}-01 & 1.3807 \mathrm{E}-01 & 0.0000 \mathrm{E}+00 \\ -65.90 & 3.1734 \mathrm{E}-01 & 1.8881 \mathrm{E}-01 & 0.0000 \mathrm{E}+00 \\ -60.35 & 3.8231 \mathrm{E}-01 & 2.5830 \mathrm{E}-01 & 1.4928 \mathrm{E}-01 \\ -55.49 & 4.6508 \mathrm{E}-01 & 3.4517 \mathrm{E}-01 & 1.6742 \mathrm{E}-01 \\ -50.62 & 5.5874 \mathrm{E}-01 & 4.5451 \mathrm{E}-01 & 1.8922 \mathrm{E}-01 \\ -47.17 & 6.3169 \mathrm{E}-01 & 5.5398 \mathrm{E}-01 & 2.0905 \mathrm{E}-01 \\ -43.58 & 7.0772 \mathrm{E}-01 & 6.6539 \mathrm{E}-01 & 7.9087 \mathrm{E}-01 \\ -38.45 & 8.1962 \mathrm{E}-01 & 8.0315 \mathrm{E}-01 & 8.7765 \mathrm{E}-01 \\ -33.32 & 9.2389 \mathrm{E}-01 & 9.2084 \mathrm{E}-01 & 9.7639 \mathrm{E}-01 \\ -28.20 & 1.0147 \mathrm{E}+00 & 1.0175 \mathrm{E}+00 & 9.9520 \mathrm{E}-01 \\ -23.07 & 1.0909 \mathrm{E}+00 & 1.0968 \mathrm{E}+00 & 1.0676 \mathrm{E}+00 \\ -17.94 & 1.1506 \mathrm{E}+00 & 1.1578 \mathrm{E}+00 & 1.1247 \mathrm{E}+00 \\ -12.82 & 1.1927 \mathrm{E}+00 & 1.1996 \mathrm{E}+00 & 1.1262 \mathrm{E}+00 \\ -7.69 & 1.2175 \mathrm{E}+00 & 1.2245 \mathrm{E}+00 & 1.1492 \mathrm{E}+00 \\ -2.56 & 1.2249 \mathrm{E}+00 & 1.2322 \mathrm{E}+00 & 1.1560 \mathrm{E}+00 \\ 2.56 & 1.2148 \mathrm{E}+00 & 1.2228 \mathrm{E}+00 & 1.1573 \mathrm{E}+00 \\ 7.69 & 1.1871 \mathrm{E}+00 & 1.1958 \mathrm{E}+00 & 1.1311 \mathrm{E}+00 \\ 12.82 & 1.1426 \mathrm{E}+00 & 1.1522 \mathrm{E}+00 & 1.0890 \mathrm{E}+00 \\ 17.94 & 1.0825 \mathrm{E}+00 & 1.0933 \mathrm{E}+00 & 1.0854 \mathrm{E}+00 \\ 23.07 & 1.0065 \mathrm{E}+00 & 1.0169 \mathrm{E}+00 & 1.0101 \mathrm{E}+00 \\ 28.20 & 9.1661 \mathrm{E}-01 & 9.2472 \mathrm{E}-01 & 9.2147 \mathrm{E}-01 \\ 33.32 & 8.1536 \mathrm{E}-01 & 8.1915 \mathrm{E}-01 & 8.9221 \mathrm{E}-01 \\ 38.45 & 7.0394 \mathrm{E}-01 & 6.9686 \mathrm{E}-01 & 7.7798 \mathrm{E}-01 \\ 43.58 & 5.8804 \mathrm{E}-01 & 5.6053 \mathrm{E}-01 & 6.7146 \mathrm{E}-01 \\ 47.17 & 5.1114 \mathrm{E}-01 & 4.5728 \mathrm{E}-01 & 1.4130 \mathrm{E}-01 \\ 50.62 & 4.3968 \mathrm{E}-01 & 3.6757 \mathrm{E}-01 & 1.2023 \mathrm{E}-01 \\ 55.49 & 3.5033 \mathrm{E}-01 & 2.7072 \mathrm{E}-01 & 9.7558 \mathrm{E}-02 \\ 60.35 & 2.7060 \mathrm{E}-01 & 1.9348 \mathrm{E}-01 & 7.8653 \mathrm{E}-02 \\ 64.98 & 2.1808 \mathrm{E}-01 & 1.4491 \mathrm{E}-01 & 0.0000 \mathrm{E}+00 \\ 69.39 & 1.8529 \mathrm{E}-01 & 1.1802 \mathrm{E}-01 & 0.0000 \mathrm{E}+00 \\ 76.46 & 1.3865 \mathrm{E}-01 & 8.2205 \mathrm{E}-02 & 0.0000 \mathrm{E}+00 \\ 86.80 & 8.9135 \mathrm{E}-02 & 4.8661 \mathrm{E}-02 & 0.0000 \mathrm{E}+00 \\ 98.14 & 5.0879 \mathrm{E}-02 & 2.6186 \mathrm{E}-02 & 0.0000 \mathrm{E}+00 \\ 110.00 & 2.1560 \mathrm{E}-02 & 1.1534 \mathrm{E}-02 & 0.0000 \mathrm{E}+00\end{array}$




\section{HNF-SD-FF-ANAL-009 Rev. 1}

Table B12B-1.6. Fission Power Distribution by Pin in MFA-2 at BOC 12B-1 CORE POSITION 2405

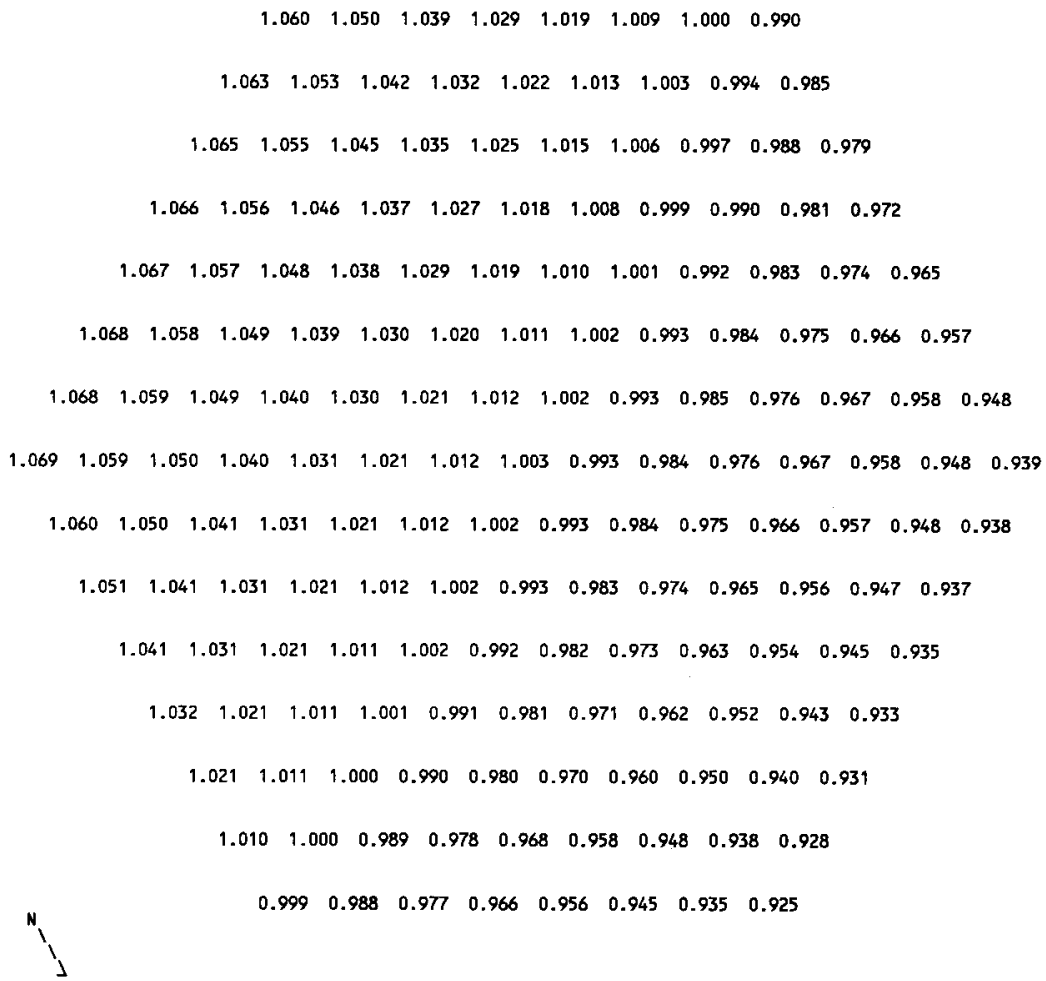




\section{HNF-SD-FF-ANAL-009 Rev. 1}

Table B12B-1.8. Fast Flux Distribution by Pin in MFA-2 at BOC 12B-1 CORE POSITION 2405

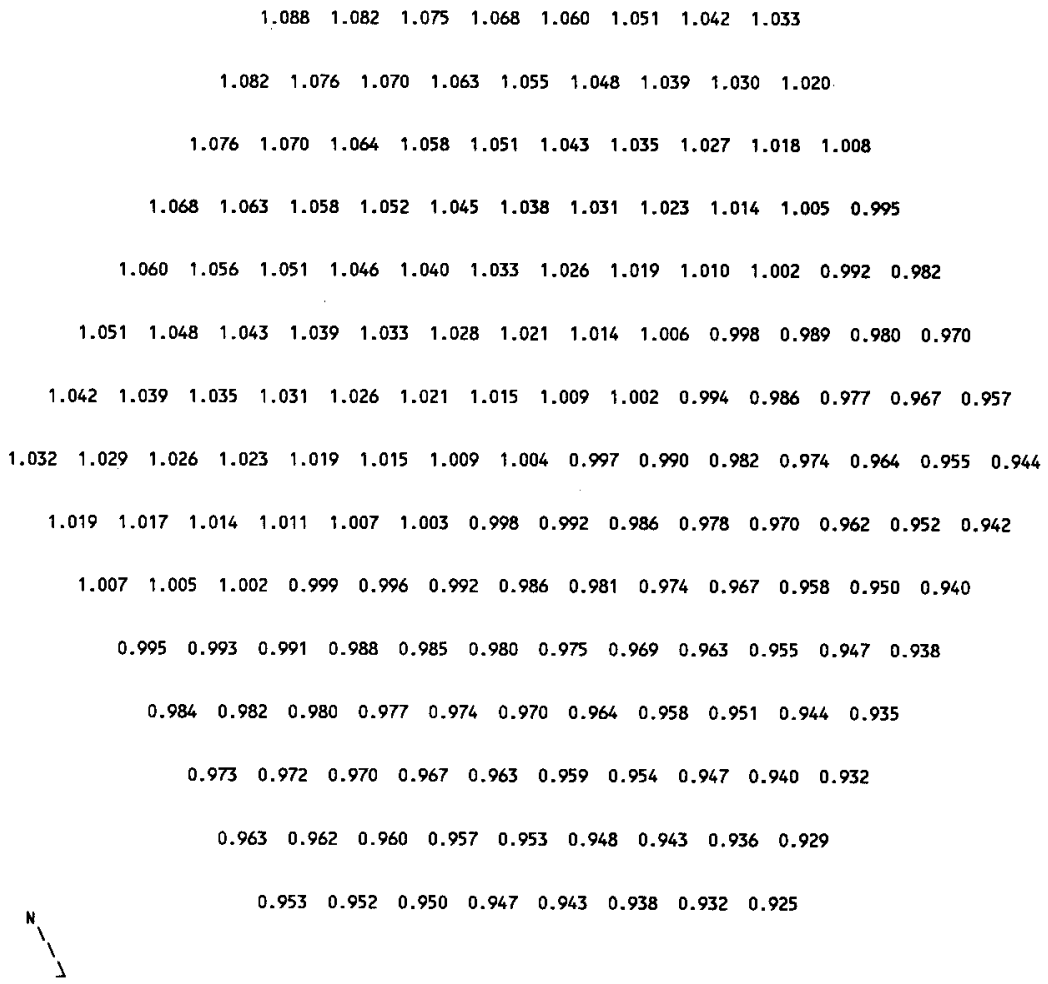


HNF-SD-FF-ANAL-009 Rev. 1

Table B12B-1.9. MFA-2 Duct Wall

Fast Flux Data at BOC 12B-1

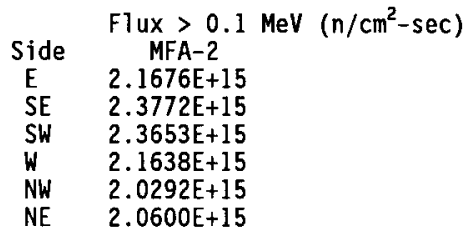

Table B12B-1.10. Assembly Outlet Temperatures and Flow Rates at BOC 12B-1

$\begin{array}{lcl}\text { CALCULATED } & \\ \text { CORE } & \begin{array}{c}\text { OUTLET TEMP. } \\ \text { (DEG F) }\end{array} & \begin{array}{l}\text { FLOW RATE } \\ \text { (LB/H) }\end{array} \\ \text { POS. } & \text { (DEG F } \\ 2405 & 1042 & 109980 \\ 2304 & 942 & 199130 \\ 2404 & 995 & 195840 \\ 2406 & 845 & 14020 \\ 2506 & 903 & 189360 \\ 2507 & 874 & 189360 \\ 2508 & 889 & 189360\end{array}$

Assembly flows are based on a calculated core pressure drop of $101.2 \mathrm{psi}$ at a total reactor flow rate of $16.74 \mathrm{E}+06 \mathrm{lb} / \mathrm{hr}$. 
Table B12B-1.12. MFA-2 Sodium Subchannel Temperatures at Top of Core Elevation for BOC 12B-1

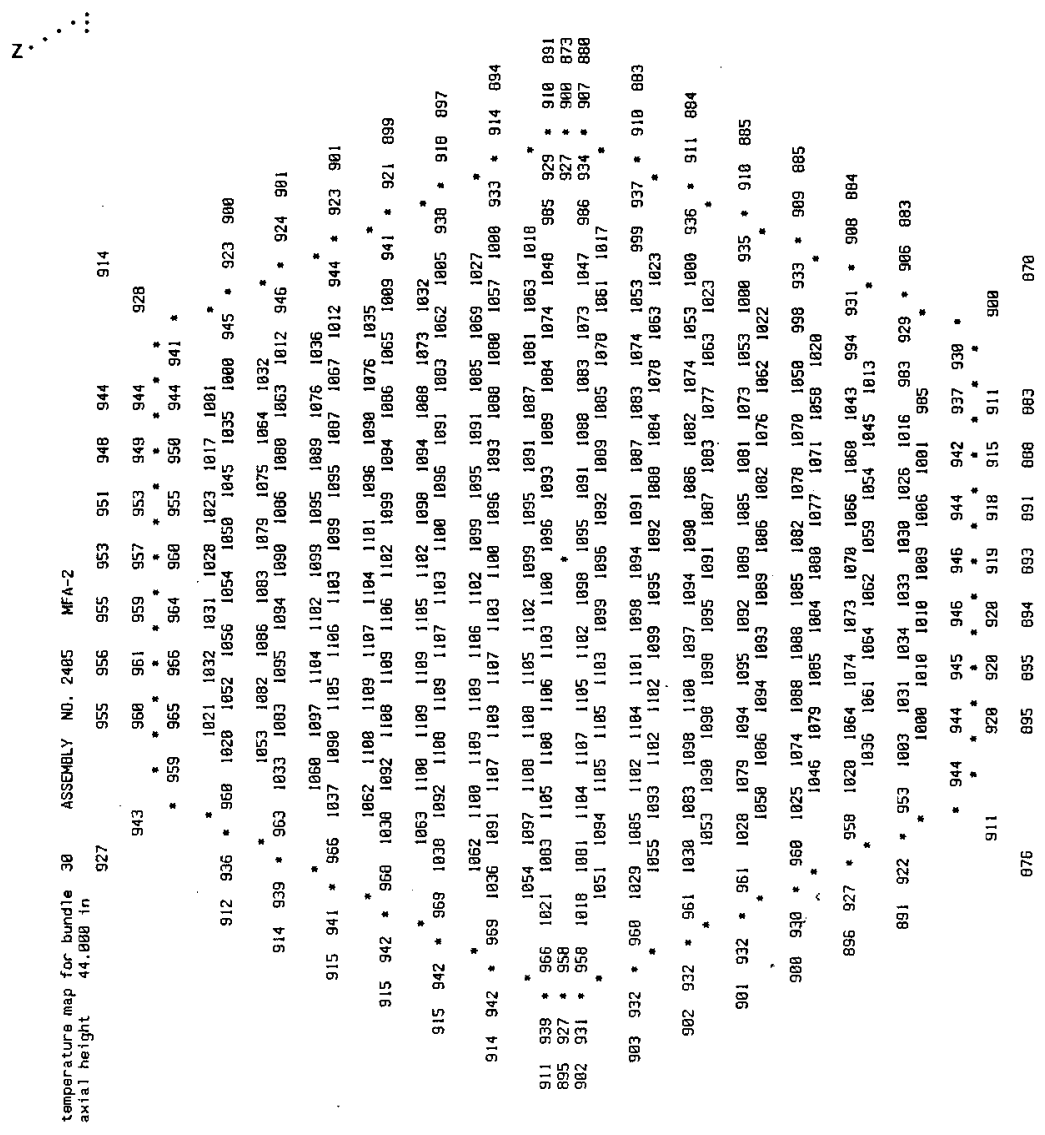


Table B12B-1.14. MFA-2 Sodium Subchannel Temperatures at Elevation of Upper Axial Blanket for BOC 12B-1

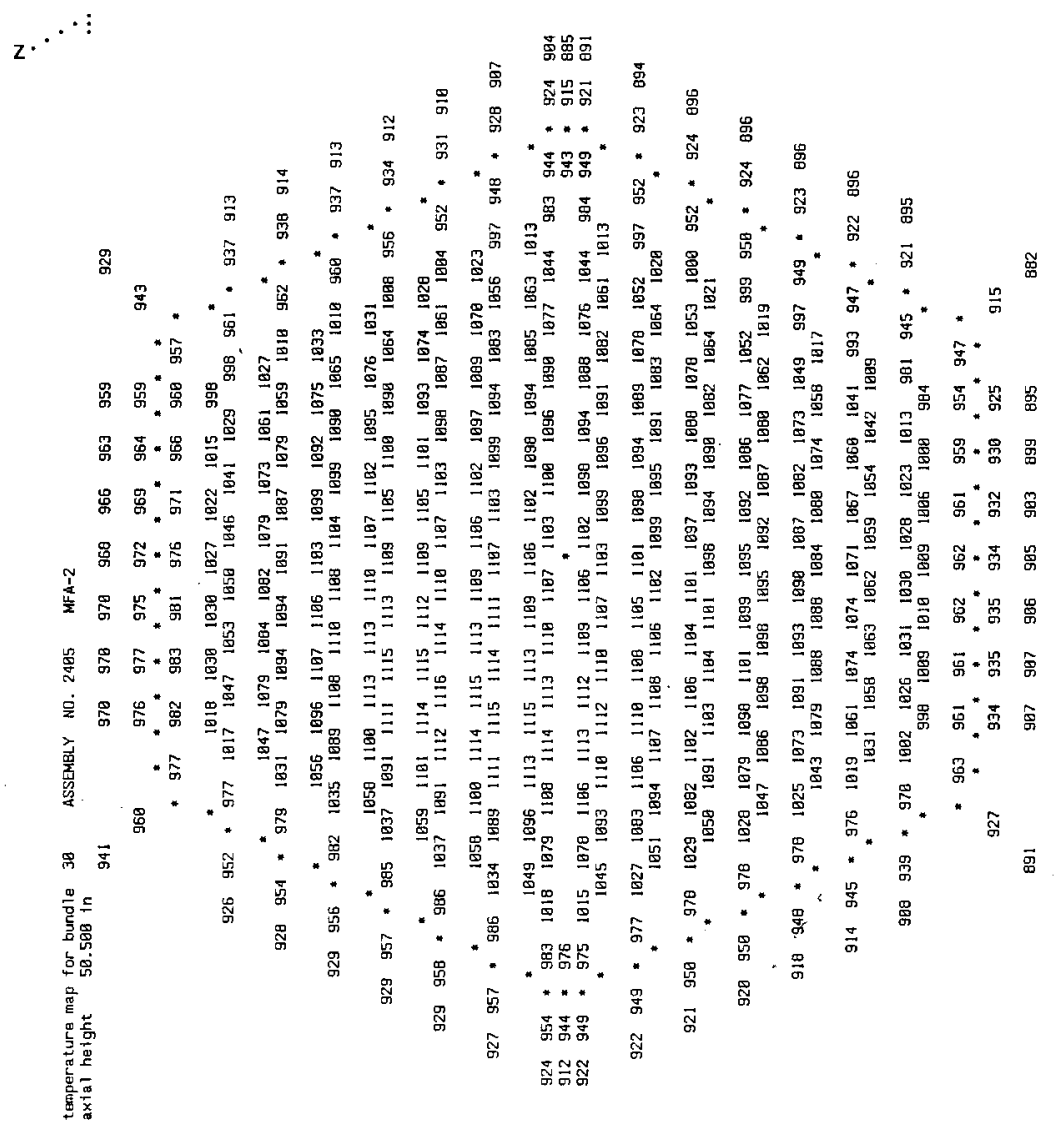

\section{4}


Table B12B-1.16. MFA-2 Sodium Subchanne1 Temperatures at Top of Fuel Pin Bundle for BOC 12B-1

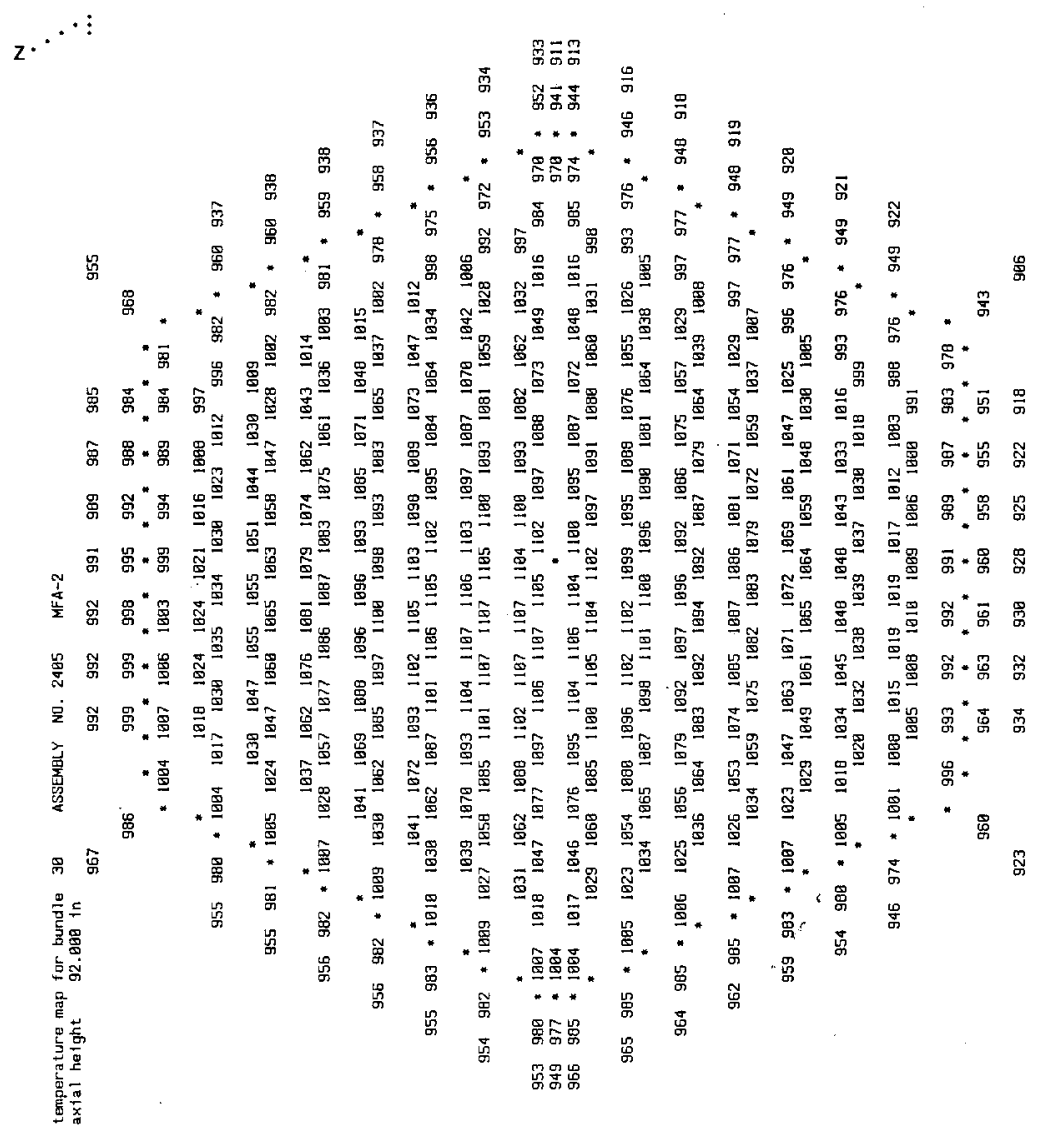


Table E12B-1.1. Fission Power Generated in MFA-2 and Neighboring Assemblies at EOC 12B-1

\begin{tabular}{lccccc} 
CORE & $* * * * * *$ & \multicolumn{2}{c}{ POWER IN MEGAWATTS } & $* * * * * *$ \\
POS. & BELOW CORE & IN CORE & ABOVE CORE & TOTAL PWR \\
2405 & $1.128 E-01$ & $3.513 E+00$ & $7.253 E-02$ & $3.698 E+00$ \\
2304 & $1.354 \mathrm{E}-02$ & $4.712 E+00$ & $1.038 E-02$ & $4.736 E+00$ \\
2404 & $0.000 E+00$ & $5.704 E+00$ & $0.000 E+00$ & $5.704 E+00$ \\
2406 & $0.000 E+00$ & $0.000 E+00$ & $0.000 E+00$ & $0.000 E+00$ \\
2506 & $1.258 E-02$ & $3.755 E+00$ & $9.636 E-03$ & $3.777 E+00$ \\
2507 & $1.486 E-02$ & $3.255 E+00$ & $9.946 E-03$ & $3.280 E+00$ \\
2508 & $1.377 E-02$ & $3.581 E+00$ & $9.684 E-03$ & $3.605 E+00$
\end{tabular}

Table E12B-1.2. Assembly Averaged Total and Fast Flux in MFA-2 at EOC $12 \mathrm{~B}-1$

$\begin{array}{cccc} & \text { Core } & \text { Flux } & \left(\mathrm{n} / \mathrm{cm}^{2}-\mathrm{sec}\right) \\ \text { Assembly } & \text { Pos. } & \text { Total } & >0.1 \mathrm{MeV} \\ \text { MFA-2 } & 2405 & 3.498 \mathrm{E}+15 & 2.204 \mathrm{E}+15\end{array}$


HNF-SD-FF-ANAL-009 Rev. 1

Table E12B-1.4. Axial Distribution of Total Flux, Fast Flux and Power in MFA-2 at EOC 12B-1

$\begin{array}{rccc}z(\mathrm{~cm}) & \text { Total Flux } & \text { Flux }>0.1 \text { MeV } & \text { Power } \\ -97.78 & 2.7150 \mathrm{E}-02 & 1.4344 \mathrm{E}-02 & 0.0000 \mathrm{E}+00 \\ -92.78 & 5.8369 \mathrm{E}-02 & 2.7760 \mathrm{E}-02 & 0.0000 \mathrm{E}+00 \\ -87.78 & 9.3686 \mathrm{E}-02 & 4.4497 \mathrm{E}-02 & 0.0000 \mathrm{E}+00 \\ -82.78 & 1.3592 \mathrm{E}-01 & 6.6500 \mathrm{E}-02 & 0.0000 \mathrm{E}+00 \\ -77.78 & 1.8813 \mathrm{E}-01 & 9.6213 \mathrm{E}-02 & 0.0000 \mathrm{E}+00 \\ -72.15 & 2.4819 \mathrm{E}-01 & 1.3487 \mathrm{E}-01 & 0.0000 \mathrm{E}+00 \\ -65.90 & 3.0914 \mathrm{E}-01 & 1.8446 \mathrm{E}-01 & 0.0000 \mathrm{E}+00 \\ -60.35 & 3.7232 \mathrm{E}-01 & 2.5246 \mathrm{E}-01 & 1.5371 \mathrm{E}-01 \\ -55.49 & 4.5291 \mathrm{E}-01 & 3.3718 \mathrm{E}-01 & 1.7199 \mathrm{E}-01 \\ -50.62 & 5.4413 \mathrm{E}-01 & 4.4363 \mathrm{E}-01 & 1.9375 \mathrm{E}-01 \\ -47.17 & 6.1512 \mathrm{E}-01 & 5.4031 \mathrm{E}-01 & 2.1325 \mathrm{E}-01 \\ -43.58 & 6.8917 \mathrm{E}-01 & 6.4855 \mathrm{E}-01 & 7.7158 \mathrm{E}-01 \\ -38.45 & 7.9865 \mathrm{E}-01 & 7.8304 \mathrm{E}-01 & 8.5694 \mathrm{E}-01 \\ -33.32 & 9.0128 \mathrm{E}-01 & 8.9858 \mathrm{E}-01 & 9.5443 \mathrm{E}-01 \\ -28.20 & 9.9150 \mathrm{E}-01 & 9.9422 \mathrm{E}-01 & 9.7115 \mathrm{E}-01 \\ -23.07 & 1.0683 \mathrm{E}+00 & 1.0740 \mathrm{E}+00 & 1.0441 \mathrm{E}+00 \\ -17.94 & 1.1299 \mathrm{E}+00 & 1.1366 \mathrm{E}+00 & 1.1030 \mathrm{E}+00 \\ -12.82 & 1.1752 \mathrm{E}+00 & 1.1816 \mathrm{E}+00 & 1.1065 \mathrm{E}+00 \\ -7.69 & 1.2045 \mathrm{E}+00 & 1.2109 \mathrm{E}+00 & 1.1337 \mathrm{E}+00 \\ -2.56 & 1.2176 \mathrm{E}+00 & 1.2243 \mathrm{E}+00 & 1.1459 \mathrm{E}+00 \\ 2.56 & 1.2141 \mathrm{E}+00 & 1.2216 \mathrm{E}+00 & 1.1537 \mathrm{E}+00 \\ 7.69 & 1.1934 \mathrm{E}+00 & 1.2018 \mathrm{E}+00 & 1.1343 \mathrm{E}+00 \\ 12.82 & 1.1559 \mathrm{E}+00 & 1.1654 \mathrm{E}+00 & 1.0991 \mathrm{E}+00 \\ 17.94 & 1.1020 \mathrm{E}+00 & 1.1130 \mathrm{E}+00 & 1.1049 \mathrm{E}+00 \\ 23.07 & 1.0306 \mathrm{E}+00 & 1.0414 \mathrm{E}+00 & 1.0343 \mathrm{E}+00 \\ 28.20 & 9.4338 \mathrm{E}-01 & 9.5191 \mathrm{E}-01 & 9.4838 \mathrm{E}-01 \\ 33.32 & 8.4274 \mathrm{E}-01 & 8.4695 \mathrm{E}-01 & 9.2603 \mathrm{E}-01 \\ 38.45 & 7.3006 \mathrm{E}-01 & 7.2303 \mathrm{E}-01 & 8.1028 \mathrm{E}-01 \\ 43.58 & 6.1156 \mathrm{E}-01 & 5.8320 \mathrm{E}-01 & 7.0153 \mathrm{E}-01 \\ 47.17 & 5.3255 \mathrm{E}-01 & 4.7667 \mathrm{E}-01 & 1.5398 \mathrm{E}-01 \\ 50.62 & 4.5898 \mathrm{E}-01 & 3.8401 \mathrm{E}-01 & 1.3187 \mathrm{E}-01 \\ 55.49 & 3.6644 \mathrm{E}-01 & 2.8345 \mathrm{E}-01 & 1.0771 \mathrm{E}-01 \\ 60.35 & 2.8356 \mathrm{E}-01 & 2.0295 \mathrm{E}-01 & 8.7293 \mathrm{E}-02 \\ 64.98 & 2.2889 \mathrm{E}-01 & 1.5216 \mathrm{E}-01 & 0.0000 \mathrm{E}+00 \\ 69.39 & 1.9466 \mathrm{E}-01 & 1.2400 \mathrm{E}-01 & 0.0000 \mathrm{E}+00 \\ 76.46 & 1.4585 \mathrm{E}-01 & 8.6466 \mathrm{E}-02 & 0.0000 \mathrm{E}+00 \\ 86.80 & 9.3839 \mathrm{E}-02 & 5.1235 \mathrm{E}-02 & 0.0000 \mathrm{E}+00 \\ 98.14 & 5.3566 \mathrm{E}-02 & 2.7581 \mathrm{E}-02 & 0.0000 \mathrm{E}+00 \\ 110.00 & 2.2672 \mathrm{E}-02 & 1.2140 \mathrm{E}-02 & 0.0000 \mathrm{E}+00 \\ & & & \end{array}$


Table E12B-1.6. Fission Power Distribution by Pin in MFA-2 at EOC 12B-1 CORE POSITION 2405

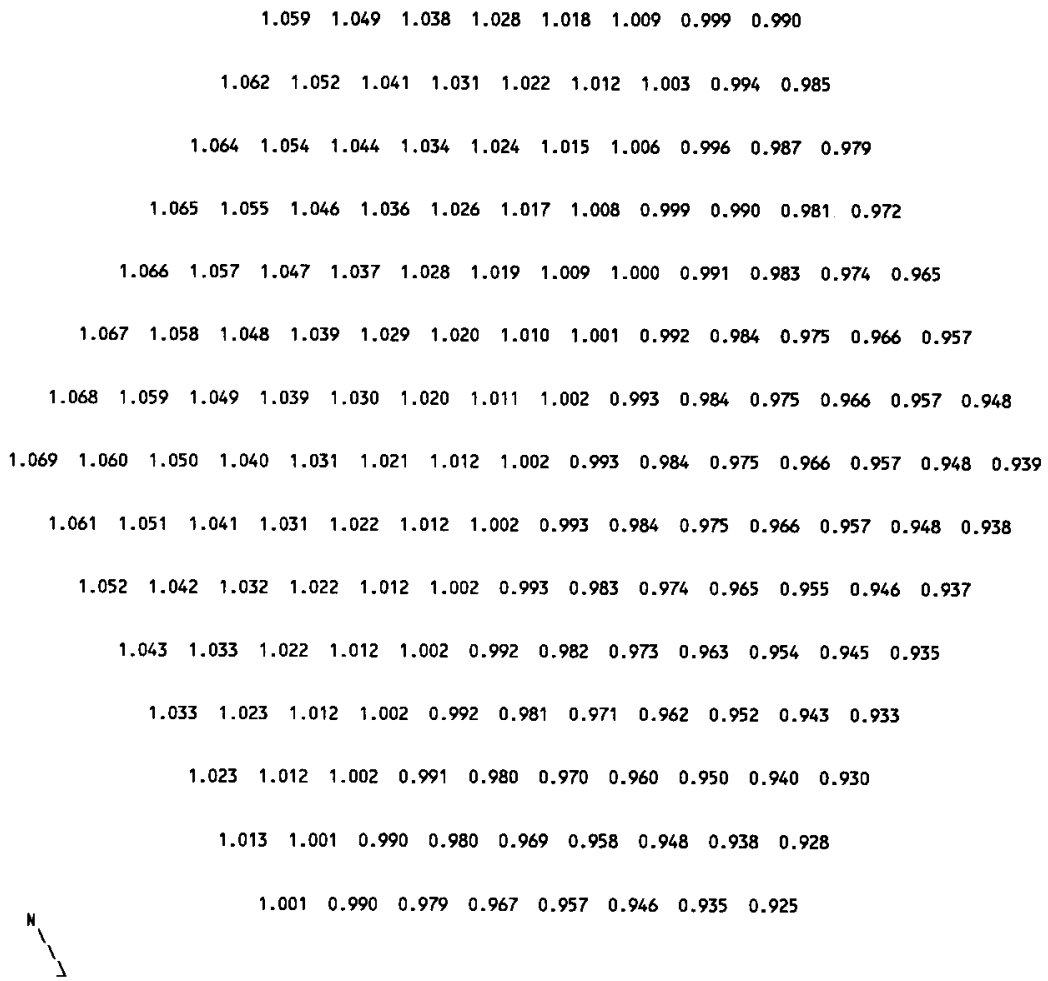


Table E12B-1.8. Fast Flux Distribution by Pin in MFA-2 at EOC 12B-1 CORE POSITION 2405

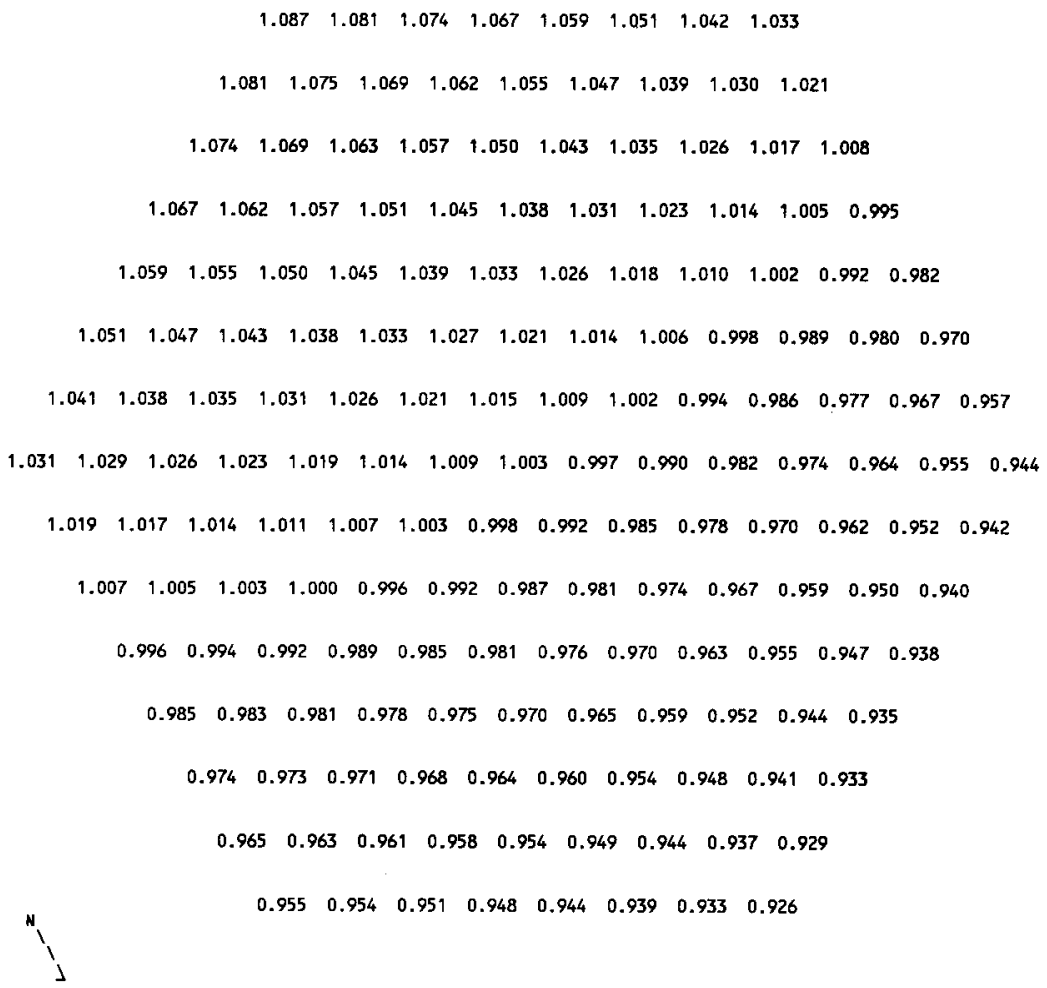


Table E12B-1.9. MFA-2 Duct Wall

Fast Flux Data at EOC 12B-1

$\begin{array}{cc}\text { Side } & \begin{array}{c}\text { Flux }>0.1 \mathrm{MeV}\left(\mathrm{n} / \mathrm{cm}^{2}-\mathrm{sec}\right) \\ \text { MFA-2 }\end{array} \\ \text { E } & 2.1594 \mathrm{E}+15 \\ \text { SE } & 2.3617 \mathrm{E}+15 \\ \text { SW } & 2.3521 \mathrm{E}+15 \\ \text { W } & 2.1533 \mathrm{E}+15 \\ \text { NW } & 2.0190 \mathrm{1}+15 \\ \text { NE } & 2.0525 \mathrm{E}+15\end{array}$

Table E12B-1.10. Assembly Outlet Temperatures and Flow Rates at EOC 12B-1

$\begin{array}{lcl}\text { COLCULATED } & \\ \text { CORE } & \begin{array}{c}\text { CUTLET TEMP. } \\ \text { OUTEW RATE }\end{array} & \begin{array}{l}\text { FLOW RAT } \\ \text { POS. }\end{array} \\ 2405 & 1036) & 10998) \\ 2304 & 936 & 199130 \\ 2404 & 989 & 195840 \\ 2406 & 844 & 14020 \\ 2506 & 900 & 189360 \\ 2507 & 871 & 189360 \\ 2508 & 886 & 189360\end{array}$

Assembly flows are based on a calculated core pressure drop of $101.2 \mathrm{psi}$ at a total reactor flow rate of $16.74 \mathrm{E}+06 \mathrm{lb} / \mathrm{hr}$. 
Table E12B-1.12. MFA-2 Sodium Subchannel Temperatures

at Top of Core Elevation for EOC 12B-1

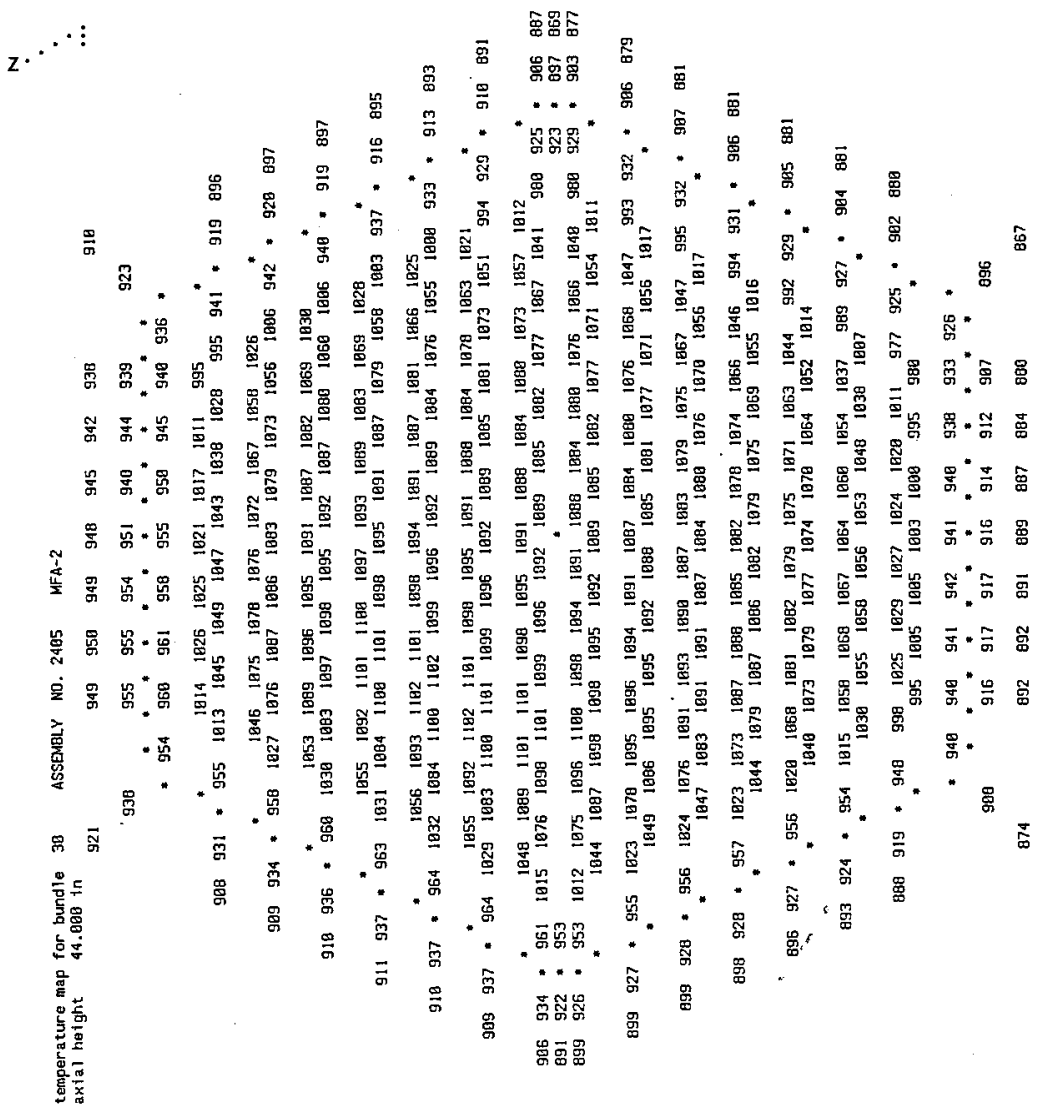


Table E12B-1.14. MFA-2 Sodium Subchannel Temperatures at Elevation of Upper Axial Blanket for EOC 12B-1

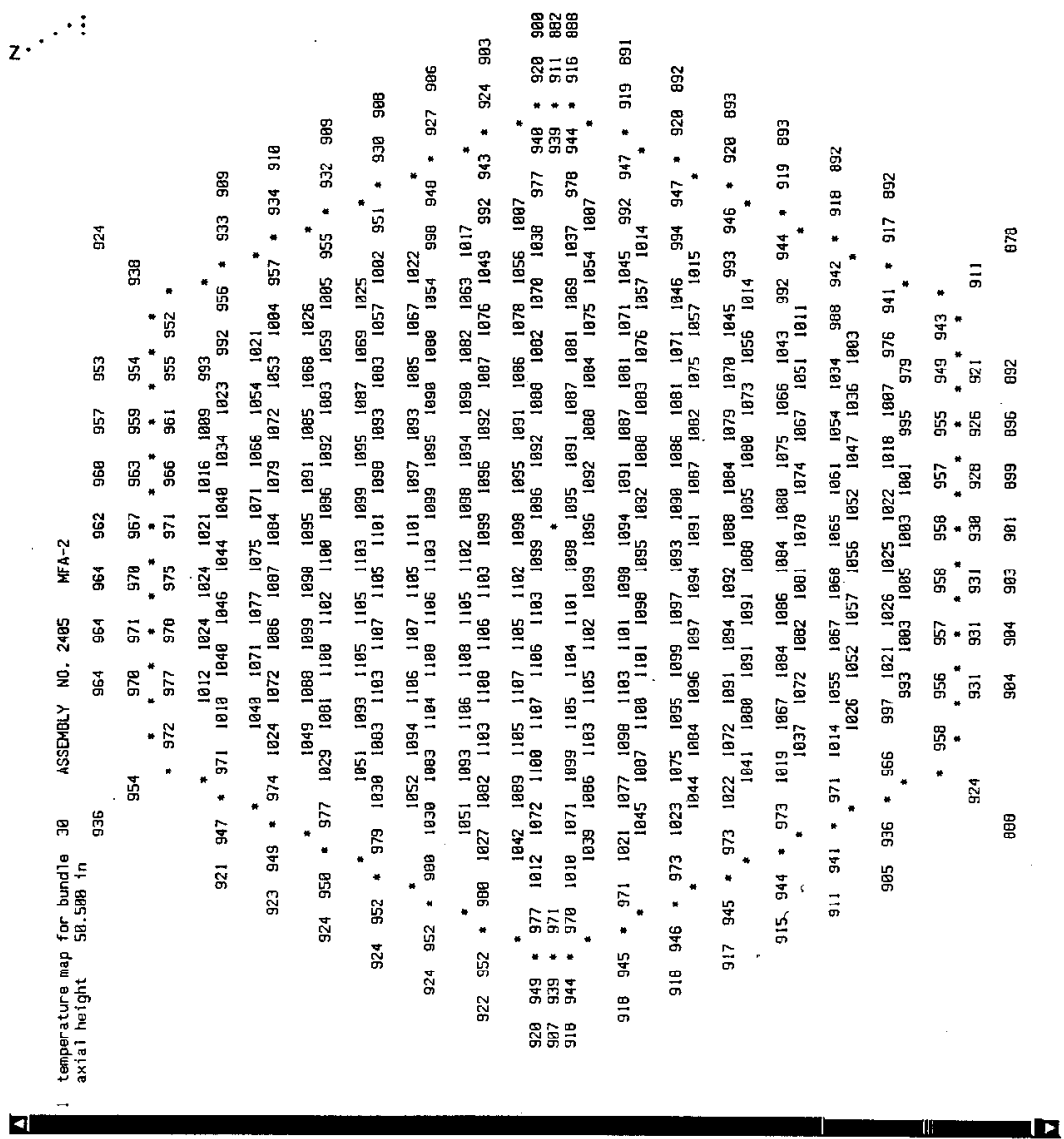


temperature map for bundie 38 ASSEMBLY No. 2485 MFA-2

$\begin{array}{lllllllllll}\text { axial height } & 92.080 \text { in } & 961 & 985 & 986 & 986 & 985 & 983 & 981 & 979 & 950\end{array}$

$988 \ldots 993,993,992,989,986982979 \quad 963$

* 990 * 1601 "1090 "997"

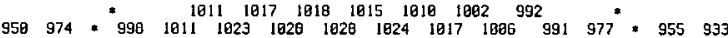

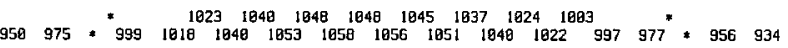

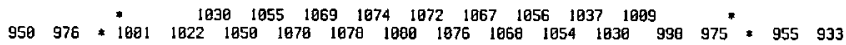

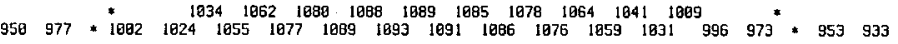

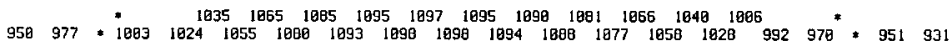

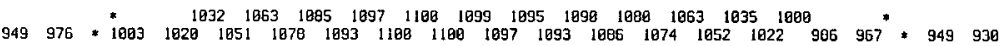

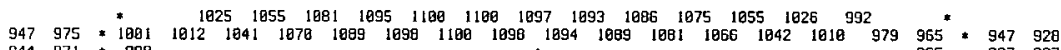

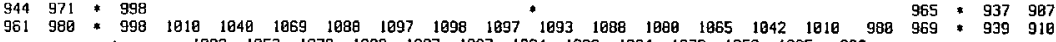

$960 \quad 580 \quad * 999 \quad 1016 \quad 1048 \quad 1074 \quad 1089 \quad 1095 \quad 1095 \quad 1092 \quad 1088 \quad 1081 \quad 1069 \quad 1049 \quad 1028$ 989 971 * $942 \quad 912$

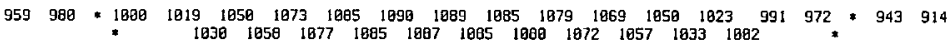

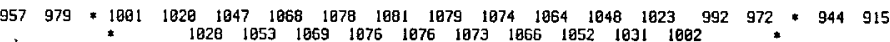

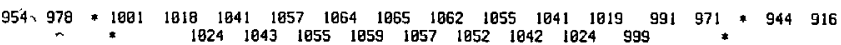

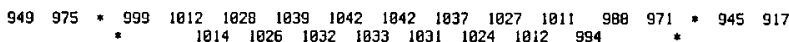

$942 \quad 969 * 996 \quad 1883 \quad 1889 \quad 1813 \quad 1814 \quad 1012 \quad 1007 \quad 998 \quad 983 \quad 972 \quad * \quad 945 \quad 918$

$\begin{array}{llllllllll} & 991 & 988 & 987 & 987 & 986 & 984 & 982 & 978 & 973\end{array}$

955 * " 959 *

$\begin{array}{lllllllll}919 & 930 & 928 & 926 & 924 & 921 & 918 & 915 & 983\end{array}$ 
Table E12B-1.18. Composition of MFA-2 at EOC 12B-1

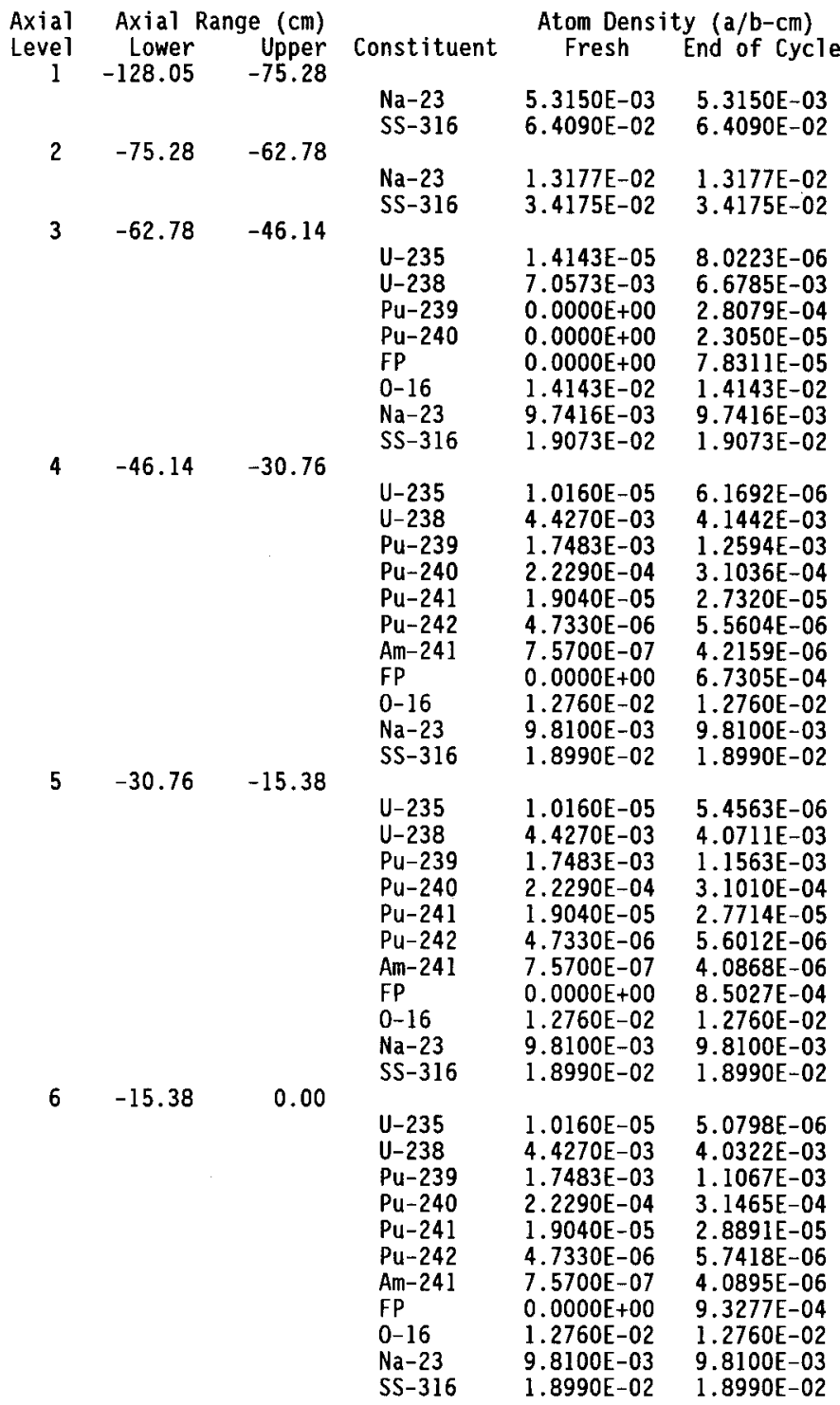


HNF-SD-FF-ANAL-009 Rev. 1

\begin{tabular}{|c|c|c|c|c|c|}
\hline \multirow[t]{2}{*}{7} & 0.00 & 15.38 & & & \\
\hline & & & $\begin{array}{l}\mathrm{U}-235 \\
\mathrm{U}-238 \\
\mathrm{Pu}-239 \\
\mathrm{Pu}-240 \\
\mathrm{Pu}-241 \\
\mathrm{Pu}-242 \\
\mathrm{Am}-241 \\
\mathrm{FP} \\
0-16 \\
\mathrm{Na}-23 \\
\mathrm{SS}-316\end{array}$ & $\begin{array}{l}1.0160 \mathrm{E}-05 \\
4.4270 \mathrm{E}-03 \\
1.7483 \mathrm{E}-03 \\
2.2290 \mathrm{E}-04 \\
1.9040 \mathrm{E}-05 \\
4.7330 \mathrm{E}-06 \\
7.5700 \mathrm{E}-07 \\
0.0000 \mathrm{E}+00 \\
1.2760 \mathrm{E}-02 \\
9.8100 \mathrm{E}-03 \\
1.8990 \mathrm{E}-02\end{array}$ & $\begin{array}{l}5.1882 \mathrm{E}-06 \\
4.0434 \mathrm{E}-03 \\
1.1204 \mathrm{E}-03 \\
3.1285 \mathrm{E}-04 \\
2.8473 \mathrm{E}-05 \\
5.6911 \mathrm{E}-06 \\
4.0842 \mathrm{E}-06 \\
9.1001 \mathrm{E}-04 \\
1.2760 \mathrm{E}-02 \\
9.8100 \mathrm{E}-03 \\
1.8990 \mathrm{E}-02\end{array}$ \\
\hline \multirow{2}{*}{8} & 15.38 & 30.76 & & & \\
\hline & & & $\begin{array}{l}U-235 \\
U-238 \\
P u-239 \\
P u-240 \\
P u-241 \\
P u-242 \\
\mathrm{Am}-241 \\
\mathrm{FP} \\
0-16 \\
\mathrm{Na}-23 \\
\mathrm{SS}-316\end{array}$ & $\begin{array}{l}1.0160 \mathrm{E}-05 \\
4.4270 \mathrm{E}-03 \\
1.7483 \mathrm{E}-03 \\
2.2290 \mathrm{E}-04 \\
1.9040 \mathrm{E}-05 \\
4.7330 \mathrm{E}-06 \\
7.5700 \mathrm{E}-07 \\
0.0000 \mathrm{E}+00 \\
1.2760 \mathrm{E}-02 \\
9.8100 \mathrm{E}-03 \\
1.8990 \mathrm{E}-02\end{array}$ & $\begin{array}{l}5.7751 \mathrm{E}-06 \\
4.1020 \mathrm{E}-03 \\
1.1959 \mathrm{E}-03 \\
3.0437 \mathrm{E}-04 \\
2.6523 \mathrm{E}-05 \\
5.4725 \mathrm{E}-06 \\
4.0722 \mathrm{E}-06 \\
7.8629 \mathrm{E}-04 \\
1.2760 \mathrm{E}-02 \\
9.8100 \mathrm{E}-03 \\
1.8990 \mathrm{E}-02\end{array}$ \\
\hline \multirow[t]{2}{*}{9} & 30.76 & 46.14 & & & \\
\hline & & & $\begin{array}{l}U-235 \\
U-238 \\
\mathrm{Pu}-239 \\
\mathrm{Pu}-240 \\
\mathrm{Pu}-241 \\
\mathrm{Pu}-242 \\
\mathrm{Am}-241 \\
\mathrm{FP} \\
\mathrm{O}-16 \\
\mathrm{Na}-23 \\
\mathrm{SS}-316\end{array}$ & $\begin{array}{l}1.0160 \mathrm{E}-05 \\
4.4270 \mathrm{E}-03 \\
1.7483 \mathrm{E}-03 \\
2.2290 \mathrm{E}-04 \\
1.9040 \mathrm{E}-05 \\
4.7330 \mathrm{E}-06 \\
7.5700 \mathrm{E}-07 \\
0.0000 \mathrm{E}+00 \\
1.2760 \mathrm{E}-02 \\
9.8100 \mathrm{E}-03 \\
1.8990 \mathrm{E}-02\end{array}$ & $\begin{array}{l}6.7010 \mathrm{E}-06 \\
4.1887 \mathrm{E}-03 \\
1.3236 \mathrm{E}-03 \\
2.9767 \mathrm{E}-04 \\
2.4962 \mathrm{E}-05 \\
5.3341 \mathrm{E}-06 \\
4.1564 \mathrm{E}-06 \\
5.7988 \mathrm{E}-04 \\
1.2760 \mathrm{E}-02 \\
9.8100 \mathrm{E}-03 \\
1.8990 \mathrm{E}-02\end{array}$ \\
\hline \multirow[t]{2}{*}{10} & 46.14 & 62.78 & & & \\
\hline & & & $\begin{array}{l}\mathrm{U}-235 \\
\mathrm{U}-238 \\
\mathrm{Pu}-239 \\
\mathrm{Pu}-240 \\
\mathrm{FP} \\
0-16 \\
\mathrm{Na}-23 \\
\mathrm{SS}-316\end{array}$ & $\begin{array}{l}1.4143 \mathrm{E}-05 \\
7.0573 \mathrm{E}-03 \\
0.0000 \mathrm{E}+00 \\
0.0000 \mathrm{E}+00 \\
0.0000 \mathrm{E}+00 \\
1.4143 \mathrm{E}-02 \\
9.7416 \mathrm{E}-03 \\
1.9073 \mathrm{E}-02\end{array}$ & $\begin{array}{l}9.5834 \mathrm{E}-06 \\
6.7844 \mathrm{E}-03 \\
2.1546 \mathrm{E}-04 \\
1.1550 \mathrm{E}-05 \\
4.8879 \mathrm{E}-05 \\
1.4143 \mathrm{E}-02 \\
9.7416 \mathrm{E}-03 \\
1.9073 \mathrm{E}-02\end{array}$ \\
\hline \multirow[t]{2}{*}{11} & 62.78 & 172.84 & & & \\
\hline & & & $\begin{array}{l}\mathrm{Na}-23 \\
\mathrm{SS}-316\end{array}$ & $\begin{array}{l}8.2400 E-03 \\
2.5562 E-02\end{array}$ & $\begin{array}{l}8.2400 \mathrm{E}-03 \\
2.5562 \mathrm{E}-02\end{array}$ \\
\hline
\end{tabular}


HNF-SD-FF-ANAL-009 Rev. 1

\subsection{Cycle 12B-2}

Table B12B-2.1. Fission Power Generated in MFA-2 and Neighboring Assemblies at BOC 12B-2

\begin{tabular}{lcccc} 
CORE & $* * * * \star * *$ & \multicolumn{2}{c}{ POWER IN MEGAWATTS } & $* * * * * *$ \\
POS. & BELOW CORE & IN CORE & ABOVE CORE & TOTAL PWR \\
2405 & $1.143 \mathrm{E}-01$ & $3.442 \mathrm{E}+00$ & $6.841 \mathrm{E}-02$ & $3.625 \mathrm{E}+00$ \\
2304 & $1.377 \mathrm{E}-02$ & $4.657 \mathrm{E}+00$ & $9.900 \mathrm{E}-03$ & $4.681 \mathrm{E}+00$ \\
2404 & $0.000 \mathrm{E}+00$ & $5.611 \mathrm{E}+00$ & $0.000 \mathrm{E}+00$ & $5.611 \mathrm{E}+00$ \\
2406 & $0.000 \mathrm{E}+00$ & $0.000 \mathrm{E}+00$ & $0.000 \mathrm{E}+00$ & $0.000 \mathrm{E}+00$ \\
2506 & $1.271 \mathrm{E}-02$ & $3.667 \mathrm{E}+00$ & $9.049 \mathrm{E}-03$ & $3.689 \mathrm{E}+00$ \\
2507 & $1.494 \mathrm{E}-02$ & $3.172 \mathrm{E}+00$ & $9.355 \mathrm{E}-03$ & $3.196 \mathrm{E}+00$ \\
2508 & $1.385 \mathrm{E}-02$ & $3.482 \mathrm{E}+00$ & $9.068 \mathrm{E}-03$ & $3.505 \mathrm{E}+00$
\end{tabular}

Table B12B-2.2. Assembly Averaged Total and Fast Flux in MFA-2 at BOC $12 \mathrm{~B}-2$

\begin{tabular}{cccc} 
& Core & \multicolumn{2}{c}{ Flux $\left(\mathrm{n} / \mathrm{cm}^{2}-\mathrm{sec}\right)$} \\
Assembly & Pos. & Total & $>0.1 \mathrm{MeV}$ \\
MFA-2 & 2405 & $3.431 \mathrm{E}+15$ & $2.163 \mathrm{E}+15$
\end{tabular}


Table B12B-2.4. Axial Distribution of Total Flux, Fast Flux and Power in MFA-2 at BOC 12B-2

$\begin{array}{rccc}\text { Z }(\mathrm{cm}) & \text { Total Flux } & \text { Flux }>0.1 \text { MeV } & \text { Power } \\ -97.78 & 2.8230 \mathrm{E}-02 & 1.4913 \mathrm{E}-02 & 0.0000 \mathrm{E}+00 \\ -92.78 & 6.0673 \mathrm{E}-02 & 2.8855 \mathrm{E}-02 & 0.0000 \mathrm{E}+00 \\ -87.78 & 9.7348 \mathrm{E}-02 & 4.6235 \mathrm{E}-02 & 0.0000 \mathrm{E}+00 \\ -82.78 & 1.4116 \mathrm{E}-01 & 6.9060 \mathrm{E}-02 & 0.0000 \mathrm{E}+00 \\ -77.78 & 1.9527 \mathrm{E}-01 & 9.9855 \mathrm{E}-02 & 0.0000 \mathrm{E}+00 \\ -72.15 & 2.5743 \mathrm{E}-01 & 1.3988 \mathrm{E}-01 & 0.0000 \mathrm{E}+00 \\ -65.90 & 3.2036 \mathrm{E}-01 & 1.9111 \mathrm{E}-01 & 0.0000 \mathrm{E}+00 \\ -60.35 & 3.8533 \mathrm{E}-01 & 2.6120 \mathrm{E}-01 & 1.5916 \mathrm{E}-01 \\ -55.49 & 4.6815 \mathrm{E}-01 & 3.4839 \mathrm{E}-01 & 1.7786 \mathrm{E}-01 \\ -50.62 & 5.6166 \mathrm{E}-01 & 4.5778 \mathrm{E}-01 & 2.0009 \mathrm{E}-01 \\ -47.17 & 6.3426 \mathrm{E}-01 & 5.5690 \mathrm{E}-01 & 2.1998 \mathrm{E}-01 \\ -43.58 & 7.0986 \mathrm{E}-01 & 6.6785 \mathrm{E}-01 & 7.9521 \mathrm{E}-01 \\ -38.45 & 8.2117 \mathrm{E}-01 & 8.0502 \mathrm{E}-01 & 8.8154 \mathrm{E}-01 \\ -33.32 & 9.2484 \mathrm{E}-01 & 9.2204 \mathrm{E}-01 & 9.7980 \mathrm{E}-01 \\ -28.20 & 1.0150 \mathrm{E}+00 & 1.0178 \mathrm{E}+00 & 9.9455 \mathrm{E}-01 \\ -23.07 & 1.0906 \mathrm{E}+00 & 1.0964 \mathrm{E}+00 & 1.0663 \mathrm{E}+00 \\ -17.94 & 1.1498 \mathrm{E}+00 & 1.1567 \mathrm{E}+00 & 1.1228 \mathrm{E}+00 \\ -12.82 & 1.1914 \mathrm{E}+00 & 1.1980 \mathrm{E}+00 & 1.1222 \mathrm{E}+00 \\ -7.69 & 1.2160 \mathrm{E}+00 & 1.2225 \mathrm{E}+00 & 1.1449 \mathrm{E}+00 \\ -2.56 & 1.2233 \mathrm{E}+00 & 1.2300 \mathrm{E}+00 & 1.1515 \mathrm{E}+00 \\ 2.56 & 1.2132 \mathrm{E}+00 & 1.2208 \mathrm{E}+00 & 1.1533 \mathrm{E}+00 \\ 7.69 & 1.1858 \mathrm{E}+00 & 1.1941 \mathrm{E}+00 & 1.1273 \mathrm{E}+00 \\ 12.82 & 1.1416 \mathrm{E}+00 & 1.1509 \mathrm{E}+00 & 1.0858 \mathrm{E}+00 \\ 17.94 & 1.0820 \mathrm{E}+00 & 1.0928 \mathrm{E}+00 & 1.0851 \mathrm{E}+00 \\ 23.07 & 1.0065 \mathrm{E}+00 & 1.0171 \mathrm{E}+00 & 1.0104 \mathrm{E}+00 \\ 28.20 & 9.1718 \mathrm{E}-01 & 9.2556 \mathrm{E}-01 & 9.2224 \mathrm{E}-01 \\ 33.32 & 8.1647 \mathrm{E}-01 & 8.2069 \mathrm{E}-01 & 8.9734 \mathrm{E}-01 \\ 38.45 & 7.0548 \mathrm{E}-01 & 6.9886 \mathrm{E}-01 & 7.8312 \mathrm{E}-01 \\ 43.58 & 5.8991 \mathrm{E}-01 & 5.6279 \mathrm{E}-01 & 6.7662 \mathrm{E}-01 \\ 47.17 & 5.1328 \mathrm{E}-01 & 4.5974 \mathrm{E}-01 & 1.4844 \mathrm{E}-01 \\ 50.62 & 4.4203 \mathrm{E}-01 & 3.7016 \mathrm{E}-01 & 1.2700 \mathrm{E}-01 \\ 55.49 & 3.5264 \mathrm{E}-01 & 2.7311 \mathrm{E}-01 & 1.0361 \mathrm{E}-01 \\ 60.35 & 2.7275 \mathrm{E}-01 & 1.9550 \mathrm{E}-01 & 8.3894 \mathrm{E}-02 \\ 64.98 & 2.2007 \mathrm{E}-01 & 1.4656 \mathrm{E}-01 & 0.0000 \mathrm{E}+00 \\ 69.39 & 1.8713 \mathrm{E}-01 & 1.1944 \mathrm{E}-01 & 0.0000 \mathrm{E}+00 \\ 76.46 & 1.4020 \mathrm{E}-01 & 8.3297 \mathrm{E}-02 & 0.0000 \mathrm{E}+00 \\ 86.80 & 9.0238 \mathrm{E}-02 & 4.9377 \mathrm{E}-02 & 0.0000 \mathrm{E}+00 \\ 98.14 & 5.1558 \mathrm{E}-02 & 2.6605 \mathrm{E}-02 & 0.0000 \mathrm{E}+00 \\ 110.00 & 2.1860 \mathrm{E}-02 & 1.1728 \mathrm{E}-02 & 0.0000 \mathrm{E}+00\end{array}$


HNF-SD-FF-ANAL-009 Rev. 1

Table B12B-2.6. Fission Power Distribution by Pin in MFA-2 at BOC $12 \mathrm{~B}-2$

CORE POSITION 2405

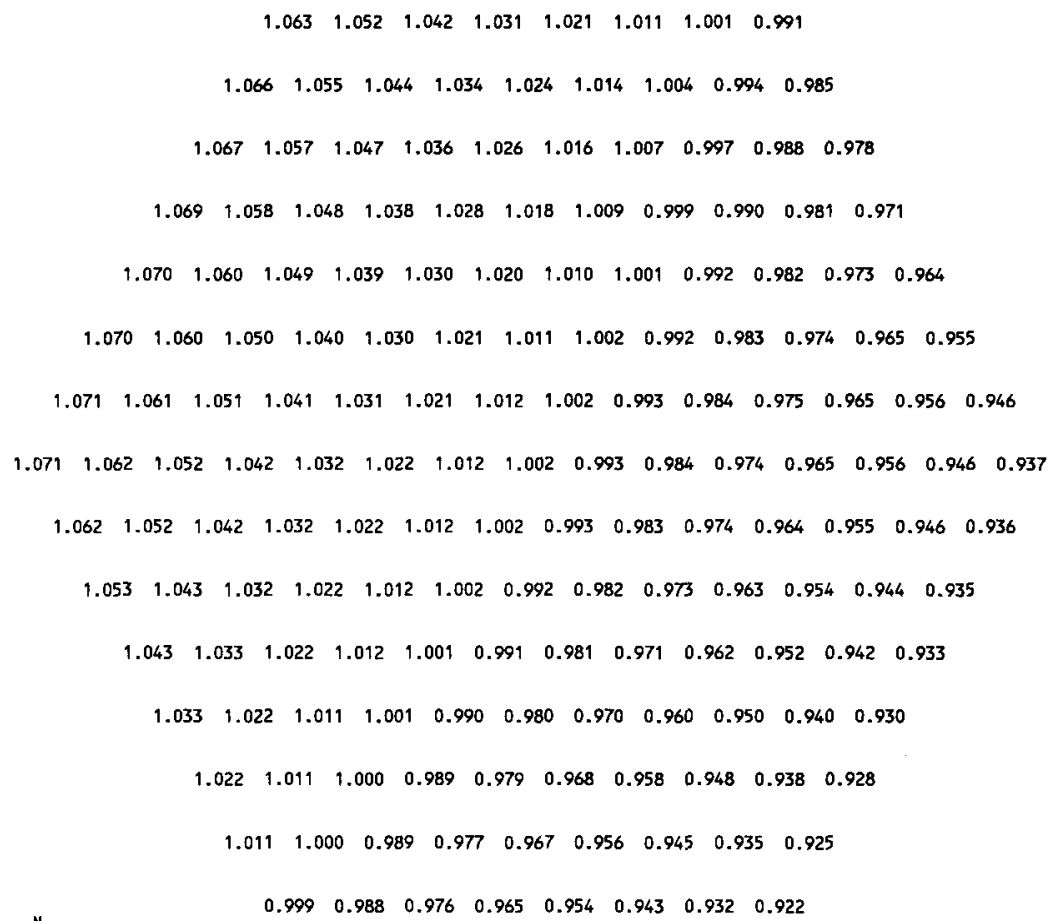


HNF-SD-FF-ANAL-009 Rev. 1

Table B12B-2.8. Fast Flux Distribution by Pin in MFA-2 at BOC $12 \mathrm{~B}-2$

CORE POSITION 2405

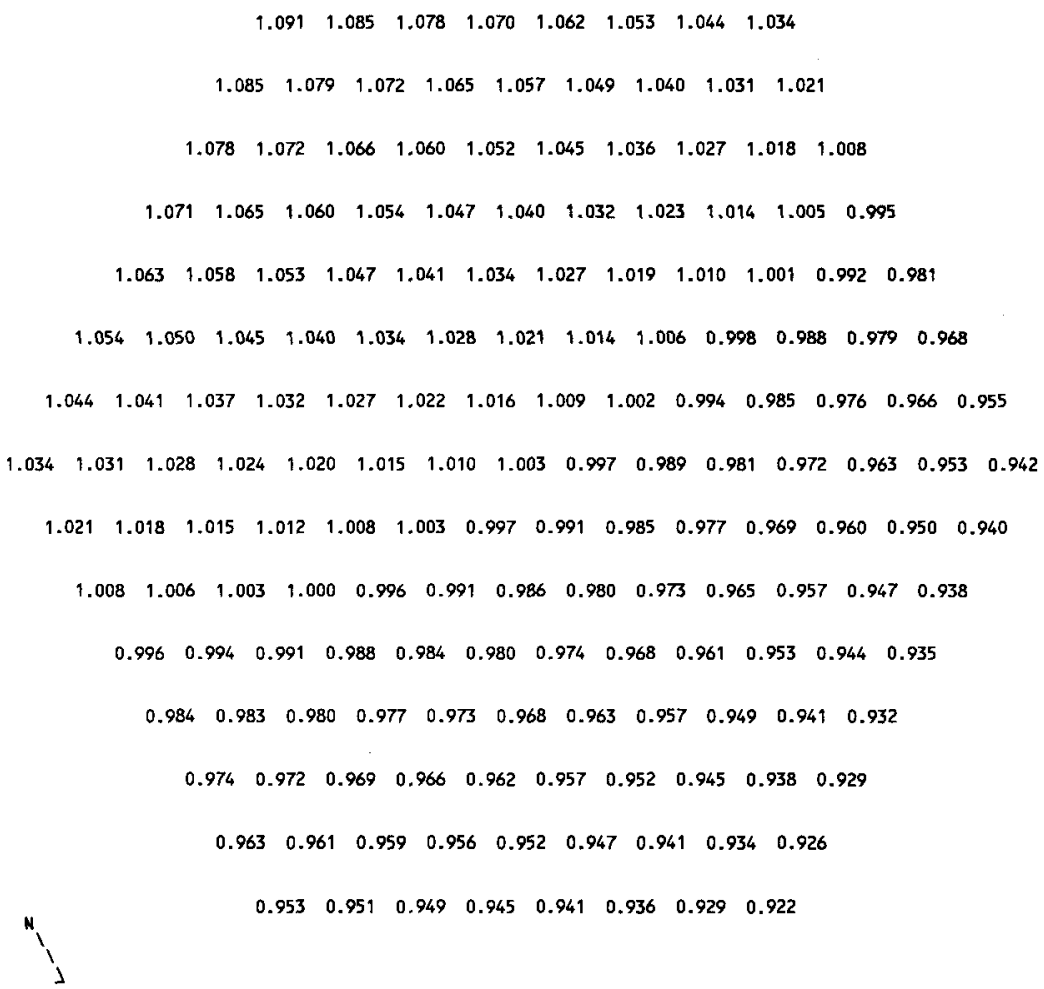


Table B12B-2.9. MFA-2 Duct Wall

Fast Flux Data at BOC 12B-2

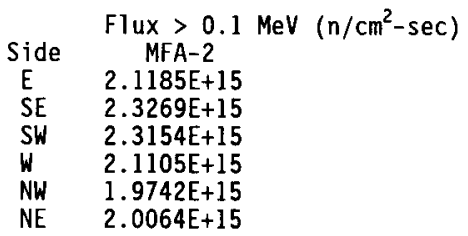

Table B12B-2.10. Assembly Outlet Temperatures and Flow Rates at BOC 12B-2

CALCULATED

CORE OUTLET TEMP. FLOW RATE

POS. (DEG F) (LB/H)

$2405 \quad 1030 \quad 109980$

$2304 \quad 934 \quad 199130$

$2404 \quad 985 \quad 195840$

$2406 \quad 841 \quad 14020$

$2506 \quad 896 \quad 189360$

$2507 \quad 867 \quad 189360$

$2508 \quad 881 \quad 189360$

Assembly flows are based on a calculated core pressure drop of $101.2 \mathrm{psi}$ at a total reactor flow rate of $16.74 \mathrm{E}+06 \mathrm{lb} / \mathrm{hr}$. 
Table B12B-2.12. MFA-2 Sodium Subchannel Temperatures at Top of Core Elevation for BOC 12B-2

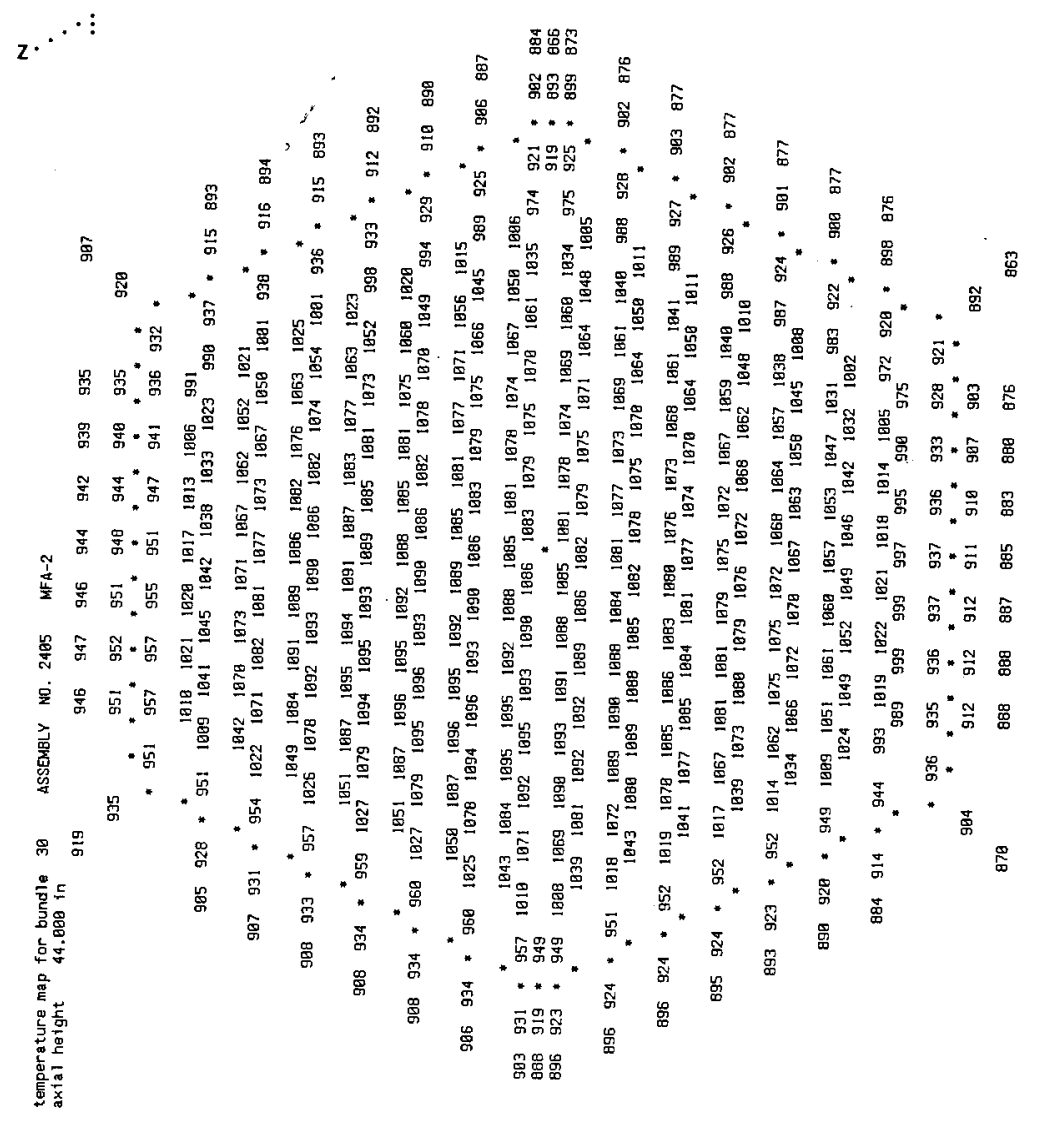


Table B12B-2.14. MFA-2 Sodium Subchannel Temperatures at Elevation of Upper Axial Blanket for BOC 12B-2

$$
\begin{aligned}
& { }_{2} . \cdot \cdot
\end{aligned}
$$

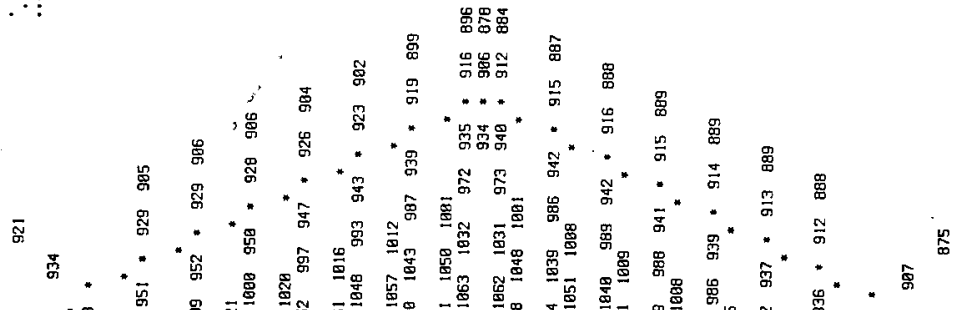

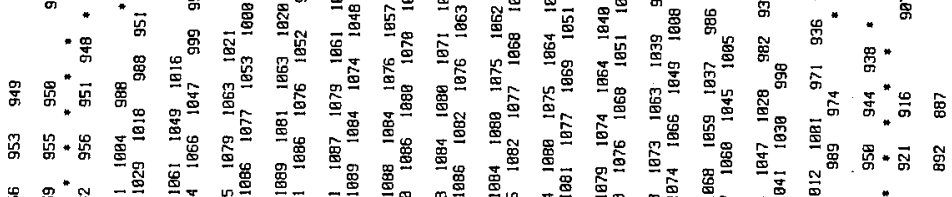

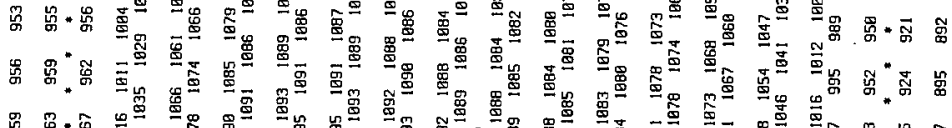

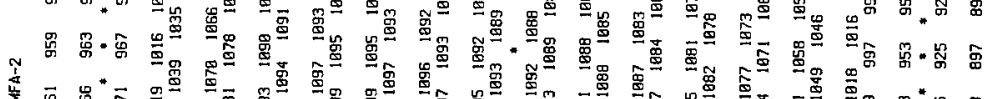

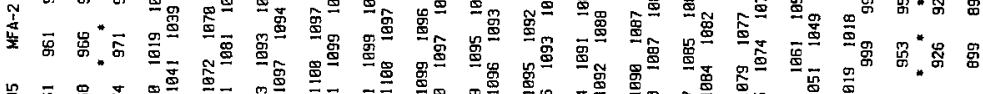

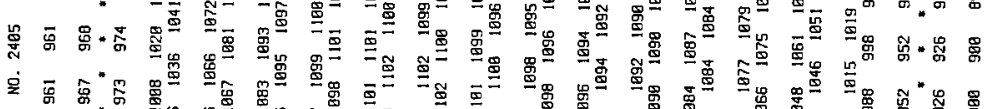

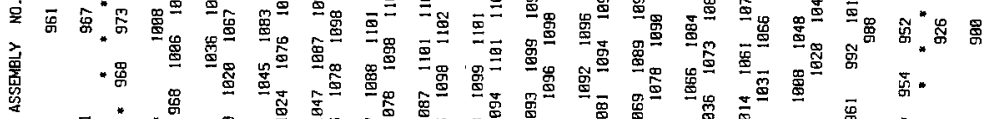

$$
\begin{aligned}
& \text { m 兽 }
\end{aligned}
$$

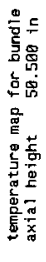

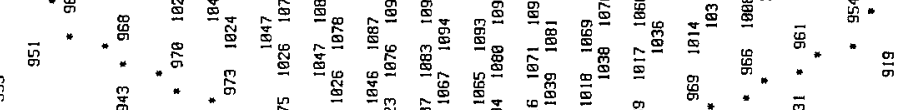

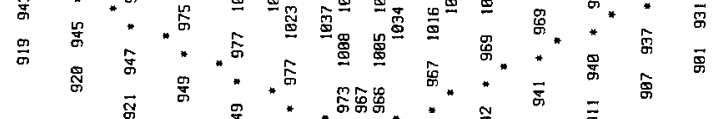


Table B12B-2.16. MFA-2 Sodium Subchannel Temperatures

at Top of Fuel Pin Bundle for BOC 12B-2

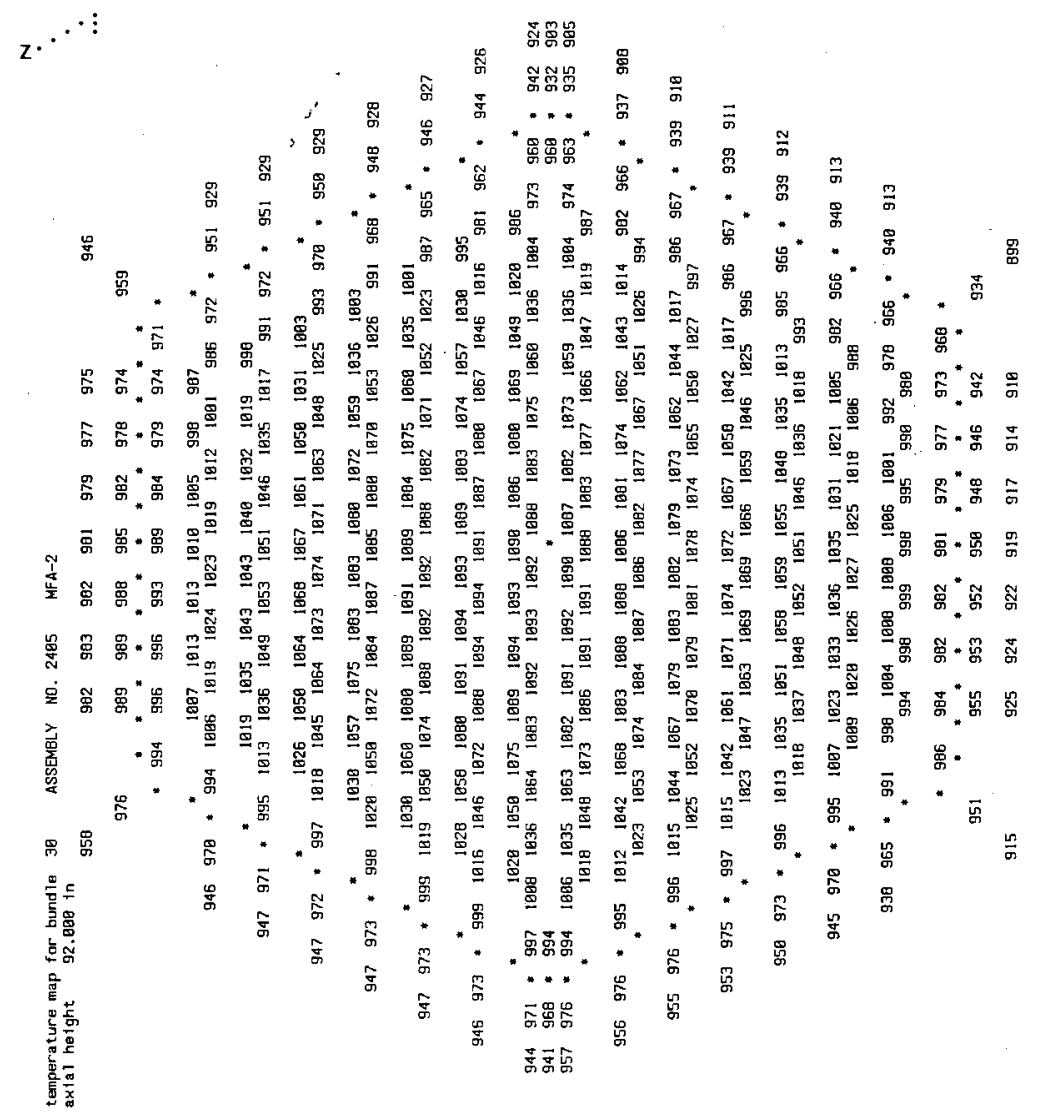


Table E12B-2.1. Fission Power Generated in MFA-2 and Neighboring Assemblies at EOC 12B-2

$\begin{array}{lcccc}\text { CORE } & * * * * * * & \text { POWER IN MEGAWATTS } & * * * * * * \\ \text { POS. } & \text { BELOW CORE } & \text { IN CORE } & \text { ABOVE CORE } & \text { TOTAL PWR } \\ 2405 & 1.152 \mathrm{E}-01 & 3.394 \mathrm{E}+00 & 7.416 \mathrm{E}-02 & 3.584 \mathrm{E}+00 \\ 2304 & 1.434 \mathrm{E}-02 & 4.566 \mathrm{E}+00 & 1.090 \mathrm{E}-02 & 4.591 \mathrm{E}+00 \\ 2404 & 0.000 \mathrm{E}+00 & 5.519 \mathrm{E}+00 & 0.000 \mathrm{E}+00 & 5.519 \mathrm{E}+00 \\ 2406 & 0.000 \mathrm{E}+00 & 0.000 \mathrm{E}+00 & 0.000 \mathrm{E}+00 & 0.000 \mathrm{E}+00 \\ 2506 & 1.286 \mathrm{E}-02 & 3.632 \mathrm{E}+00 & 9.830 \mathrm{E}-03 & 3.654 \mathrm{E}+00 \\ 2507 & 1.487 \mathrm{E}-02 & 3.141 \mathrm{E}+00 & 1.004 \mathrm{E}-02 & 3.166 \mathrm{E}+00 \\ 2508 & 1.397 \mathrm{E}-02 & 3.460 \mathrm{E}+00 & 9.864 \mathrm{E}-03 & 3.483 \mathrm{E}+00\end{array}$

Table E12B-2.2. Assembly Averaged Total and Fast Flux in MFA-2 at EOC $12 \mathrm{~B}-2$

$\begin{array}{cccc} & \text { Core } & \text { Flux } & \left(\mathrm{n} / \mathrm{cm}^{2}-\mathrm{sec}\right) \\ \text { Assembly } & \text { Pos. } & \text { Total } & >0.1 \mathrm{MeV} \\ \text { MFA-2 } & 2405 & 3.439 E+15 & 2.159 \mathrm{E}+15\end{array}$


Table E12B-2.4. Axial Distribution of Total Flux, Fast Flux and Power in MFA-2 at EOC 12B-2

\begin{tabular}{|c|c|c|c|}
\hline $\begin{array}{r}Z(\mathrm{~cm}) \\
-97.78 \\
-92.78 \\
-87.78 \\
-82.78 \\
-77.78 \\
-72.15 \\
-65.90 \\
-60.35 \\
-55.49 \\
-50.62 \\
-47.17 \\
-43.58 \\
-38.45 \\
-33.32 \\
-28.20 \\
-23.07 \\
-17.94 \\
-12.82 \\
-7.69 \\
-2.56 \\
2.56 \\
7.69 \\
12.82 \\
17.94 \\
23.07 \\
28.20 \\
33.32 \\
38.45 \\
43.58 \\
47.17 \\
50.62 \\
55.49 \\
60.35 \\
64.98 \\
69.39 \\
76.46 \\
86.80 \\
98.14 \\
110.00\end{array}$ & $\begin{array}{l}\text { Total Flux } \\
2.7435 \mathrm{E}-02 \\
5.8972 \mathrm{E}-02 \\
9.4633 \mathrm{E}-02 \\
1.3724 \mathrm{E}-01 \\
1.8988 \mathrm{E}-01 \\
2.5038 \mathrm{E}-01 \\
3.1156 \mathrm{E}-01 \\
3.7474 \mathrm{E}-01 \\
4.5532 \mathrm{E}-01 \\
5.4634 \mathrm{E}-01 \\
6.1701 \mathrm{E}-01 \\
6.9064 \mathrm{E}-01 \\
7.9955 \mathrm{E}-01 \\
9.0163 \mathrm{E}-01 \\
9.9123 \mathrm{E}-01 \\
1.0676 \mathrm{E}+00 \\
1.1287 \mathrm{E}+00 \\
1.1736 \mathrm{E}+00 \\
1.2028 \mathrm{E}+00 \\
1.2159 \mathrm{E}+00 \\
1.2126 \mathrm{E}+00 \\
1.1923 \mathrm{E}+00 \\
1.1553 \mathrm{E}+00 \\
1.1019 \mathrm{E}+00 \\
1.0312 \mathrm{E}+00 \\
9.4452 \mathrm{E}-01 \\
8.4443 \mathrm{E}-01 \\
7.3216 \mathrm{E}-01 \\
6.1394 \mathrm{E}-01 \\
5.3513 \mathrm{E}-01 \\
4.6167 \mathrm{E}-01 \\
3.6902 \mathrm{E}-01 \\
2.8591 \mathrm{E}-01 \\
2.3107 \mathrm{E}-01 \\
1.9665 \mathrm{E}-01 \\
1.4750 \mathrm{E}-01 \\
9.5009 \mathrm{E}-02 \\
5.4280 \mathrm{E}-02 \\
2.2985 \mathrm{E}-02\end{array}$ & $\begin{array}{c}\text { Flux }>0.1 \mathrm{MeV} \\
1.4540 \mathrm{E}-02 \\
2.8134 \mathrm{E}-02 \\
4.5080 \mathrm{E}-02 \\
6.7339 \mathrm{E}-02 \\
9.7370 \mathrm{E}-02 \\
1.3641 \mathrm{E}-01 \\
1.8640 \mathrm{E}-01 \\
2.5492 \mathrm{E}-01 \\
3.3988 \mathrm{E}-01 \\
4.4629 \mathrm{E}-01 \\
5.4259 \mathrm{E}-01 \\
6.5032 \mathrm{E}-01 \\
7.8423 \mathrm{E}-01 \\
8.9910 \mathrm{E}-01 \\
9.9397 \mathrm{E}-01 \\
1.0731 \mathrm{E}+00 \\
1.1352 \mathrm{E}+00 \\
1.1796 \mathrm{E}+00 \\
1.2087 \mathrm{E}+00 \\
1.2222 \mathrm{E}+00 \\
1.2196 \mathrm{E}+00 \\
1.2003 \mathrm{E}+00 \\
1.1645 \mathrm{E}+00 \\
1.1129 \mathrm{E}+00 \\
1.0421 \mathrm{E}+00 \\
9.5338 \mathrm{E}-01 \\
8.4913 \mathrm{E}-01 \\
7.2561 \mathrm{E}-01 \\
5.8602 \mathrm{E}-01 \\
4.7962 \mathrm{E}-01 \\
3.8694 \mathrm{E}-01 \\
2.8610 \mathrm{E}-01 \\
2.0517 \mathrm{E}-01 \\
1.5393 \mathrm{E}-01 \\
1.2552 \mathrm{E}-01 \\
8.7626 \mathrm{E}-02 \\
5.1989 \mathrm{E}-02 \\
2.8019 \mathrm{E}-02 \\
1.2342 \mathrm{E}-02 \\
\end{array}$ & $\begin{array}{c}\text { Power } \\
0.0000 \mathrm{E}+00 \\
0.0000 \mathrm{E}+00 \\
0.0000 \mathrm{E}+00 \\
0.0000 \mathrm{E}+00 \\
0.0000 \mathrm{E}+00 \\
0.0000 \mathrm{E}+00 \\
0.0000 \mathrm{E}+00 \\
1.6323 \mathrm{E}-01 \\
1.8205 \mathrm{E}-01 \\
2.0425 \mathrm{E}-01 \\
2.2382 \mathrm{E}-01 \\
7.7500 \mathrm{E}-01 \\
8.5999 \mathrm{E}-01 \\
9.5703 \mathrm{E}-01 \\
9.7004 \mathrm{E}-01 \\
1.0424 \mathrm{E}+00 \\
1.1008 \mathrm{E}+00 \\
1.1024 \mathrm{E}+00 \\
1.1293 \mathrm{E}+00 \\
1.1415 \mathrm{E}+00 \\
1.1499 \mathrm{E}+00 \\
1.1309 \mathrm{E}+00 \\
1.0962 \mathrm{E}+00 \\
1.1049 \mathrm{E}+00 \\
1.0350 \mathrm{E}+00 \\
9.4965 \mathrm{E}-01 \\
9.3177 \mathrm{E}-01 \\
8.1607 \mathrm{E}-01 \\
7.0728 \mathrm{E}-01 \\
1.6172 \mathrm{E}-01 \\
1.3919 \mathrm{E}-01 \\
1.1425 \mathrm{E}-01 \\
9.2964 \mathrm{E}-02 \\
0.0000 \mathrm{E}+00 \\
0.0000 \mathrm{E}+00 \\
0.0000 \mathrm{E}+00 \\
0.0000 \mathrm{E}+00 \\
0.0000 \mathrm{E}+00 \\
0.0000 \mathrm{E}+00\end{array}$ \\
\hline
\end{tabular}


HNF-SD-FF-ANAL-009 Rev. 1

Table E12B-2.6. Fission Power Distribution by Pin in MFA-2 at EOC 12B-2 CORE POSITION 2405

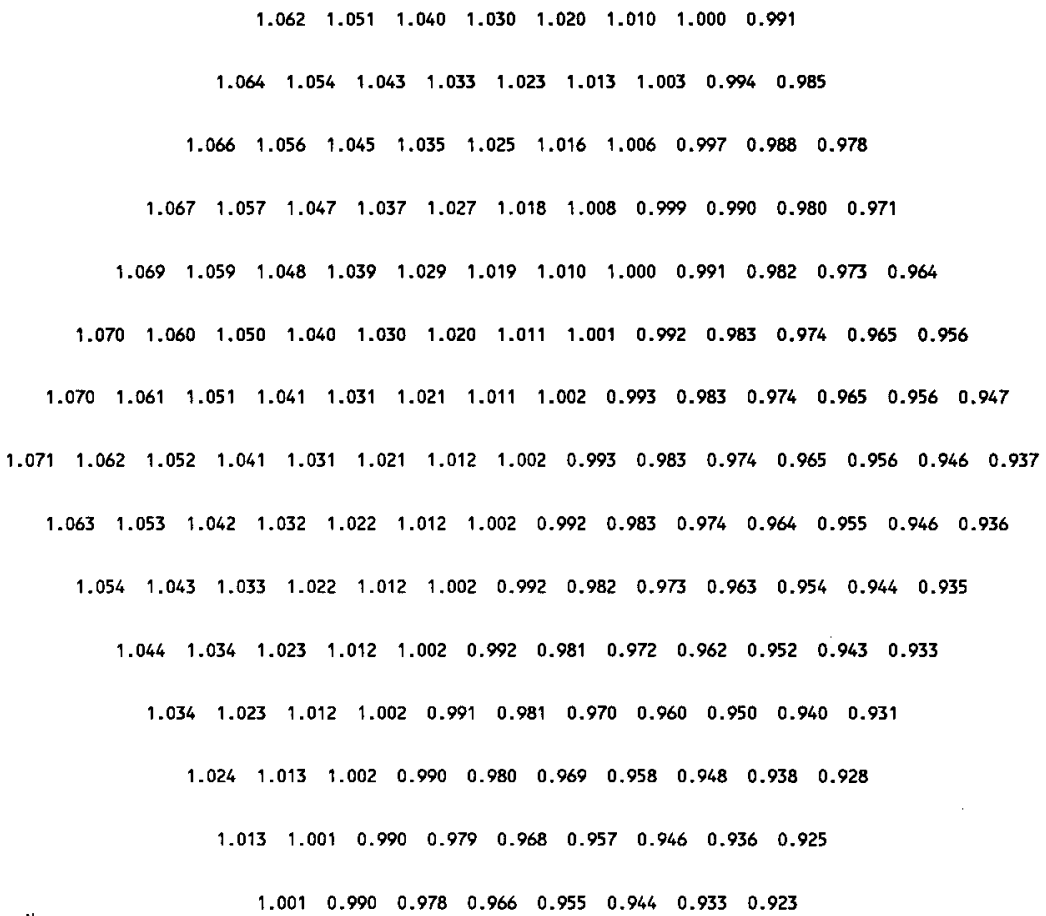




\section{HNF-SD-FF-ANAL-009 Rev. 1}

Table E12B-2.8. Fast Flux Distribution by Pin in MFA-2 at EOC 12B-2 CORE POSITION 2405

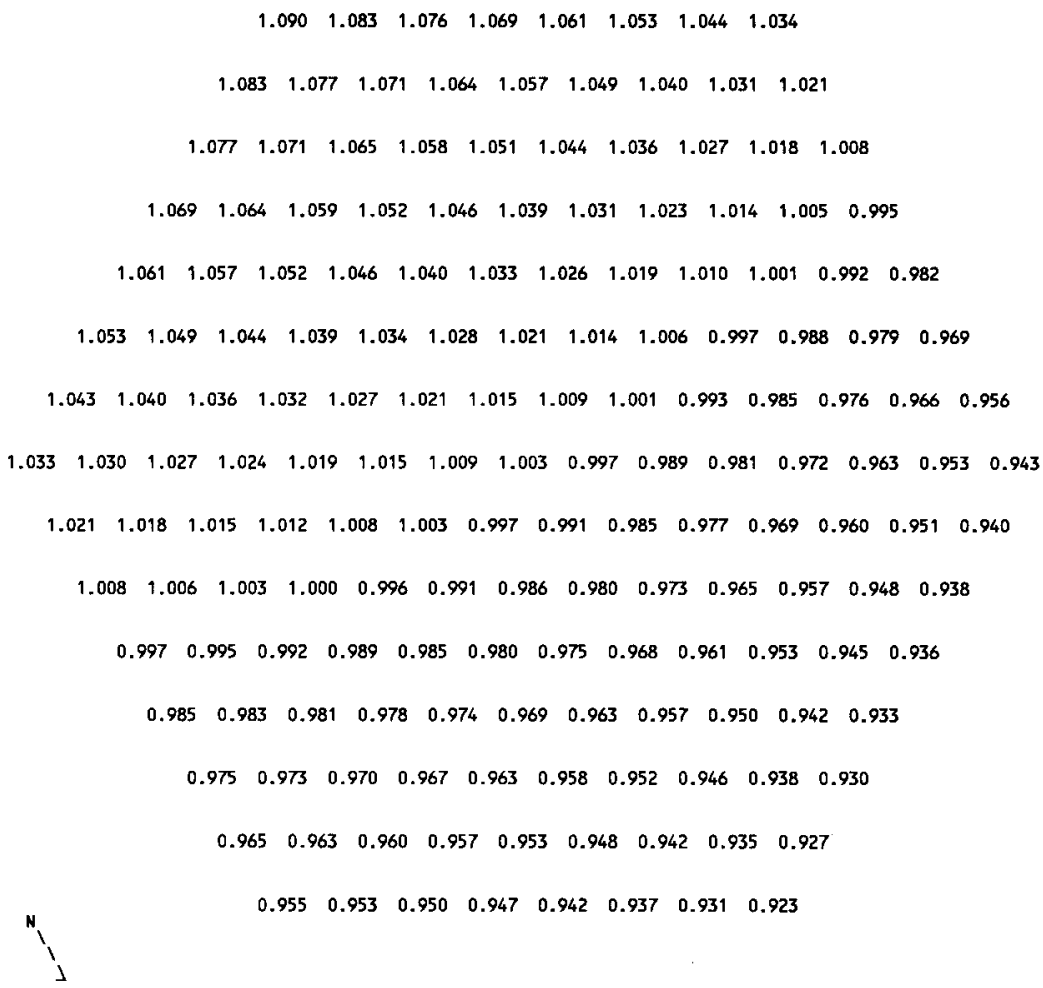


Table E12B-2.9. MFA-2 Duct Wal1

Fast Flux Data at EOC 12B-2

$\begin{array}{cl}\text { Side } & \text { Flux }>0.1 \mathrm{MeV}\left(\mathrm{n} / \mathrm{cm}^{2}-\mathrm{sec}\right) \\ \text { E } & 2.1171 \mathrm{E}+15 \\ \text { SE } & 2.3183 \mathrm{E}+15 \\ \text { SW } & 2.3094 \mathrm{E}+15 \\ \text { W } & 2.1077 \mathrm{E}+15 \\ \text { NW } & 1.9718 \mathrm{E}+15 \\ \text { NE } & 2.0064 \mathrm{E}+15\end{array}$

Table E12B-2.10. Assembly Outlet Temperatures and Flow Rates at EOC 12B-2

$\begin{array}{lcl}\text { CALCULATED } & \\ \text { CORE } & \text { OUTLET TEMP. } & \text { FLOW RATE } \\ \text { POS. } & \text { (DEG F) } & \text { (LB/H) } \\ 2405 & 1025 & 109980 \\ 2304 & 929 & 199130 \\ 2404 & 980 & 195840 \\ 2406 & 840 & 14020 \\ 2506 & 893 & 189360 \\ 2507 & 864 & 189360 \\ 2508 & 879 & 189360\end{array}$

Assembly flows are based on a calculated core pressure drop of 101.2 psi at a total reactor flow rate of $16.74 \mathrm{E}+06 \mathrm{lb} / \mathrm{hr}$. 
temperature map for bundle
axla) height $44.606 \mathrm{in}$

ASSEMBLY NO. 2485 MFA-2

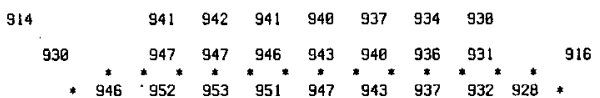

993

$\because$

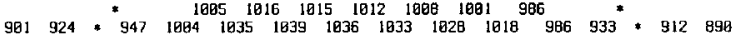

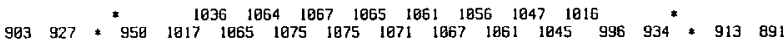

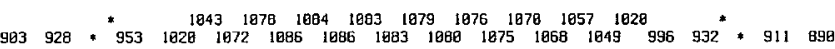

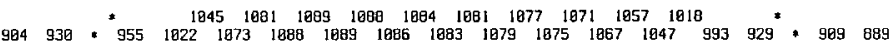

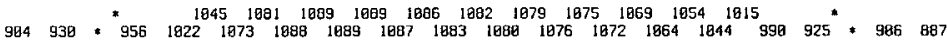

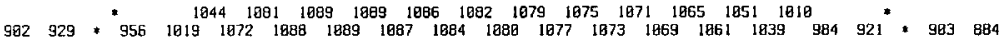

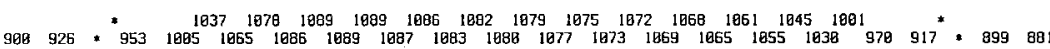

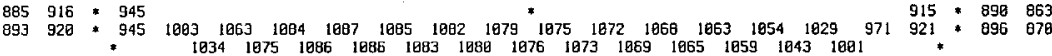

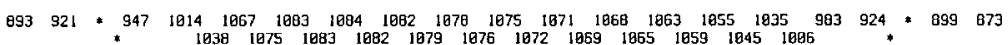

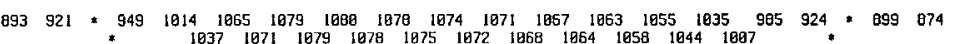

$\begin{array}{lllllllllllllllllllll}092 \quad 921 & * & 949 & 1813 & 1061 & 1075 & 1976 & 1073 & 1070 & 1066 & 1062 & 1054 & 1034 & 984 & 922 & * 999 & 075\end{array}$

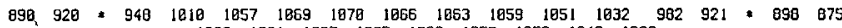

$807 \quad 917 * 946 \quad 1095 \quad 1847 \quad 1056 \quad 1855 \quad 1852 \quad 1048 \quad 1842 \quad 1026 \quad 979 \quad 913 * 897 \quad 874$

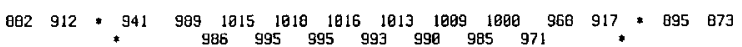

㽣

윰

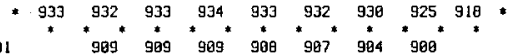

$$
\begin{aligned}
& \begin{array}{lllllllll}
885 & 885 & 884 & 883 & 881 & 878 & 873 & \text { 86 }
\end{array}
\end{aligned}
$$


Table E12B-2.14. MFA-2 Sodium Subchannel Temperatures at Elevation of Upper Axial Blanket for EOC 12B-2

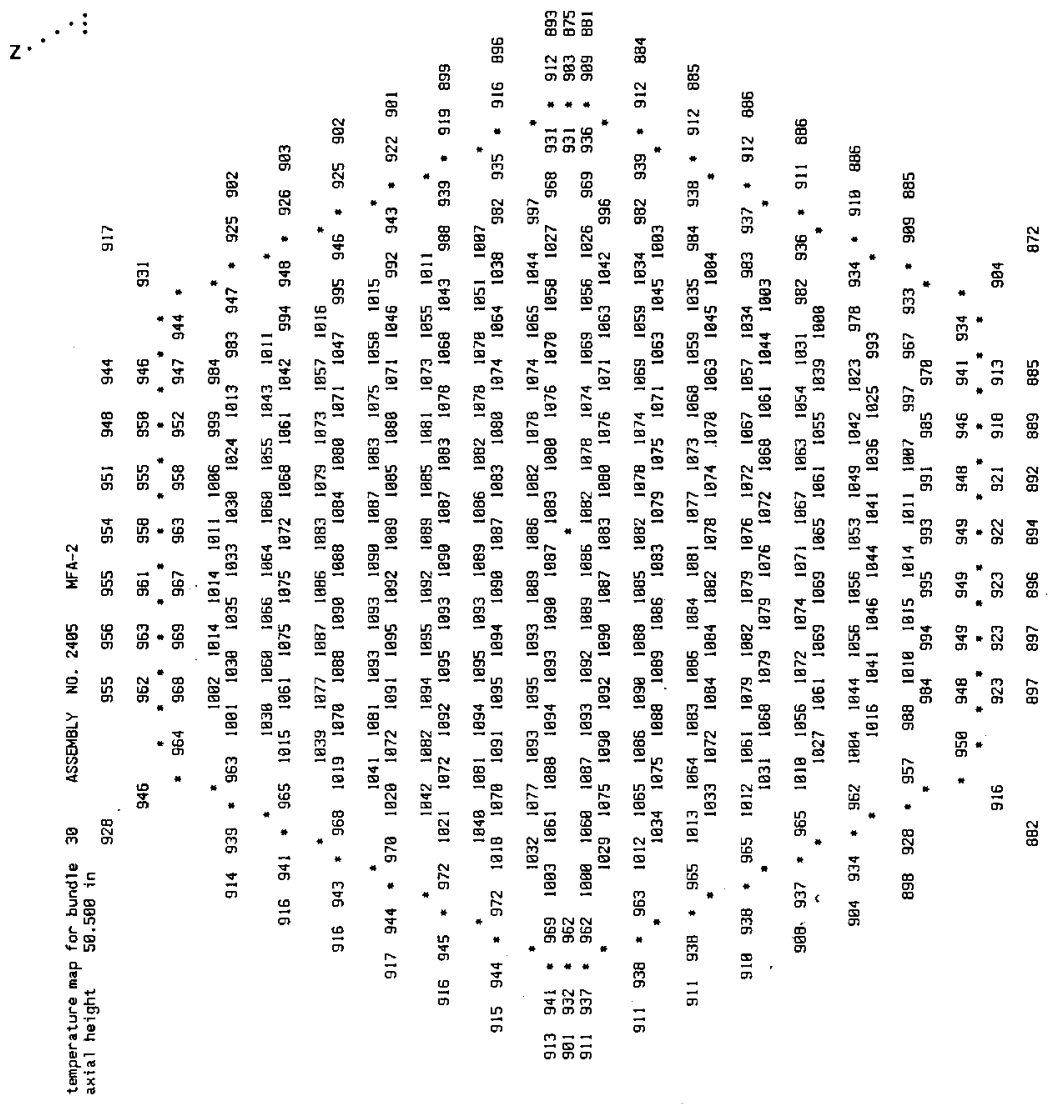


Table E12B-2.16. MFA-2 Sodium Subchannel Temperatures at Top of Fuel Pin Bundle for EOC 12B-2

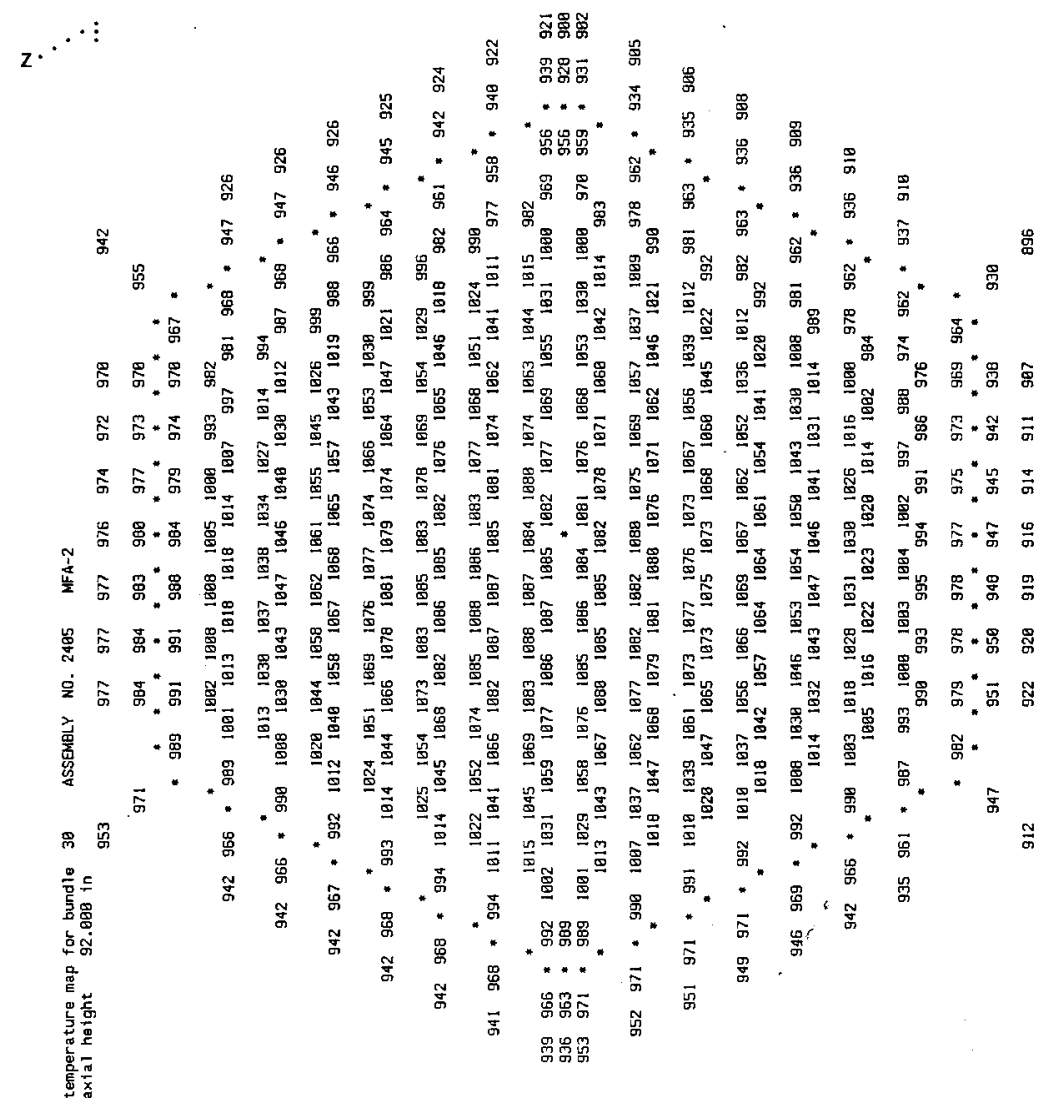


Table El2B-2.18. Composition of MFA-2 at EOC 12B-2

\begin{tabular}{|c|c|c|c|c|c|}
\hline \multirow{3}{*}{$\begin{array}{c}\text { Axial } \\
\text { Level } \\
1\end{array}$} & \multicolumn{2}{|c|}{ Axial Range $(\mathrm{cm})$} & \multirow[b]{2}{*}{ Constituent } & \multicolumn{2}{|c|}{ Atom Density $(\mathrm{a} / \mathrm{b}-\mathrm{cm})$} \\
\hline & $\begin{array}{r}\text { Lower } \\
-128.05\end{array}$ & $\begin{array}{r}\text { Upper } \\
-75.28\end{array}$ & & & End of Cycle \\
\hline & & & $\begin{array}{l}\mathrm{Na}-23 \\
\mathrm{SS}-316\end{array}$ & $\begin{array}{l}5.3150 \mathrm{E}-03 \\
6.4090 \mathrm{E}-02\end{array}$ & $\begin{array}{l}5.3150 \mathrm{E}-03 \\
6.4090 \mathrm{E}-02\end{array}$ \\
\hline 2 & -75.28 & -62.78 & & & \\
\hline & & & $\begin{array}{l}\mathrm{Na}-23 \\
\text { SS }-316\end{array}$ & $\begin{array}{l}1.3177 \mathrm{E}-02 \\
3.4175 \mathrm{E}-02\end{array}$ & $\begin{array}{l}1.3177 \mathrm{E}-02 \\
3.4175 \mathrm{E}-02\end{array}$ \\
\hline 3 & -62.78 & -46.14 & $\begin{array}{l}U-235 \\
U-238 \\
\mathrm{Pu}-239 \\
\mathrm{Pu}-240 \\
\mathrm{FP} \\
0-16 \\
\mathrm{Na}-23 \\
\mathrm{SS}-316\end{array}$ & $\begin{array}{l}1.4143 \mathrm{E}-05 \\
7.0573 \mathrm{E}-03 \\
0.0000 \mathrm{E}+00 \\
0.0000 \mathrm{E}+00 \\
0.0000 \mathrm{E}+00 \\
1.4143 \mathrm{E}-02 \\
9.7416 \mathrm{E}-03 \\
1.9073 \mathrm{E}-02\end{array}$ & $\begin{array}{l}7.7500 \mathrm{E}-06 \\
6.6559 \mathrm{E}-03 \\
2.9345 \mathrm{E}-04 \\
2.5564 \mathrm{E}-05 \\
8.5731 \mathrm{E}-05 \\
1.4143 \mathrm{E}-02 \\
9.7416 \mathrm{E}-03 \\
1.9073 \mathrm{E}-02\end{array}$ \\
\hline 4 & -46.14 & -30.76 & $\begin{array}{l}U-235 \\
U-238 \\
P u-239 \\
P u-240 \\
P u-241 \\
P u-242 \\
A m-241 \\
F P \\
0-16 \\
\mathrm{Na}-23 \\
S S-316\end{array}$ & $\begin{array}{l}1.0160 \mathrm{E}-05 \\
4.4270 \mathrm{E}-03 \\
1.7483 \mathrm{E}-03 \\
2.2290 \mathrm{E}-04 \\
1.9040 \mathrm{E}-05 \\
4.7330 \mathrm{E}-06 \\
7.5700 \mathrm{E}-07 \\
0.0000 \mathrm{E}+00 \\
1.2760 \mathrm{E}-02 \\
9.8100 \mathrm{E}-03 \\
1.8990 \mathrm{E}-02\end{array}$ & $\begin{array}{l}5.9829 \mathrm{E}-06 \\
4.1276 \mathrm{E}-03 \\
1.2365 \mathrm{E}-03 \\
3.1420 \mathrm{E}-04 \\
2.7999 \mathrm{E}-05 \\
5.6321 \mathrm{E}-06 \\
4.3342 \mathrm{E}-06 \\
7.0788 \mathrm{E}-04 \\
1.2760 \mathrm{E}-02 \\
9.8100 \mathrm{E}-03 \\
1.8990 \mathrm{E}-02\end{array}$ \\
\hline 5 & -30.76 & -15.38 & $\begin{array}{l}U-235 \\
U-238 \\
\mathrm{Pu}-239 \\
\mathrm{Pu}-240 \\
\mathrm{Pu}-241 \\
\mathrm{Pu}-242 \\
\mathrm{Am}-241 \\
\mathrm{FP} \\
\mathrm{O}-16 \\
\mathrm{Na}-23 \\
\mathrm{SS}-316\end{array}$ & $\begin{array}{l}1.0160 \mathrm{E}-05 \\
4.4270 \mathrm{E}-03 \\
1.7483 \mathrm{E}-03 \\
2.2290 \mathrm{E}-04 \\
1.9040 \mathrm{E}-05 \\
4.7330 \mathrm{E}-06 \\
7.5700 \mathrm{E}-07 \\
0.0000 \mathrm{E}+00 \\
1.2760 \mathrm{E}-02 \\
9.8100 \mathrm{E}-03 \\
1.8990 \mathrm{E}-02\end{array}$ & $\begin{array}{l}5.2546 \mathrm{E}-06 \\
4.0507 \mathrm{E}-03 \\
1.1309 \mathrm{E}-03 \\
3.1339 \mathrm{E}-04 \\
2.8388 \mathrm{E}-05 \\
5.6784 \mathrm{E}-06 \\
4.1910 \mathrm{E}-06 \\
8.9195 \mathrm{E}-04 \\
1.2760 \mathrm{E}-02 \\
9.8100 \mathrm{E}-03 \\
1.8990 \mathrm{E}-02\end{array}$ \\
\hline 6 & -15.38 & 0.00 & $\begin{array}{l}U-235 \\
U-238 \\
\mathrm{Pu}-239 \\
\mathrm{Pu}-240 \\
\mathrm{Pu}-241 \\
\mathrm{Pu}-242 \\
\mathrm{Am}-241 \\
\mathrm{FP} \\
\mathrm{O}-16 \\
\mathrm{Na}-23 \\
\mathrm{SS}-316\end{array}$ & $\begin{array}{l}1.0160 \mathrm{E}-05 \\
4.4270 \mathrm{E}-03 \\
1.7483 \mathrm{E}-03 \\
2.2290 \mathrm{E}-04 \\
1.9040 \mathrm{E}-05 \\
4.7330 \mathrm{E}-06 \\
7.5700 \mathrm{E}-07 \\
0.0000 \mathrm{E}+00 \\
1.2760 \mathrm{E}-02 \\
9.8100 \mathrm{E}-03 \\
1.8990 \mathrm{E}-02\end{array}$ & $\begin{array}{l}4.8715 \mathrm{E}-06 \\
4.0098 \mathrm{E}-03 \\
1.0803 \mathrm{E}-03 \\
3.1783 \mathrm{E}-04 \\
2.9620 \mathrm{E}-05 \\
5.8324 \mathrm{E}-06 \\
4.1901 \mathrm{E}-06 \\
9.7749 \mathrm{E}-04 \\
1.2760 \mathrm{E}-02 \\
9.8100 \mathrm{E}-03 \\
1.8990 \mathrm{E}-02\end{array}$ \\
\hline & & & & & \\
\hline
\end{tabular}


HNF-SD-FF-ANAL-009 Rev, 1

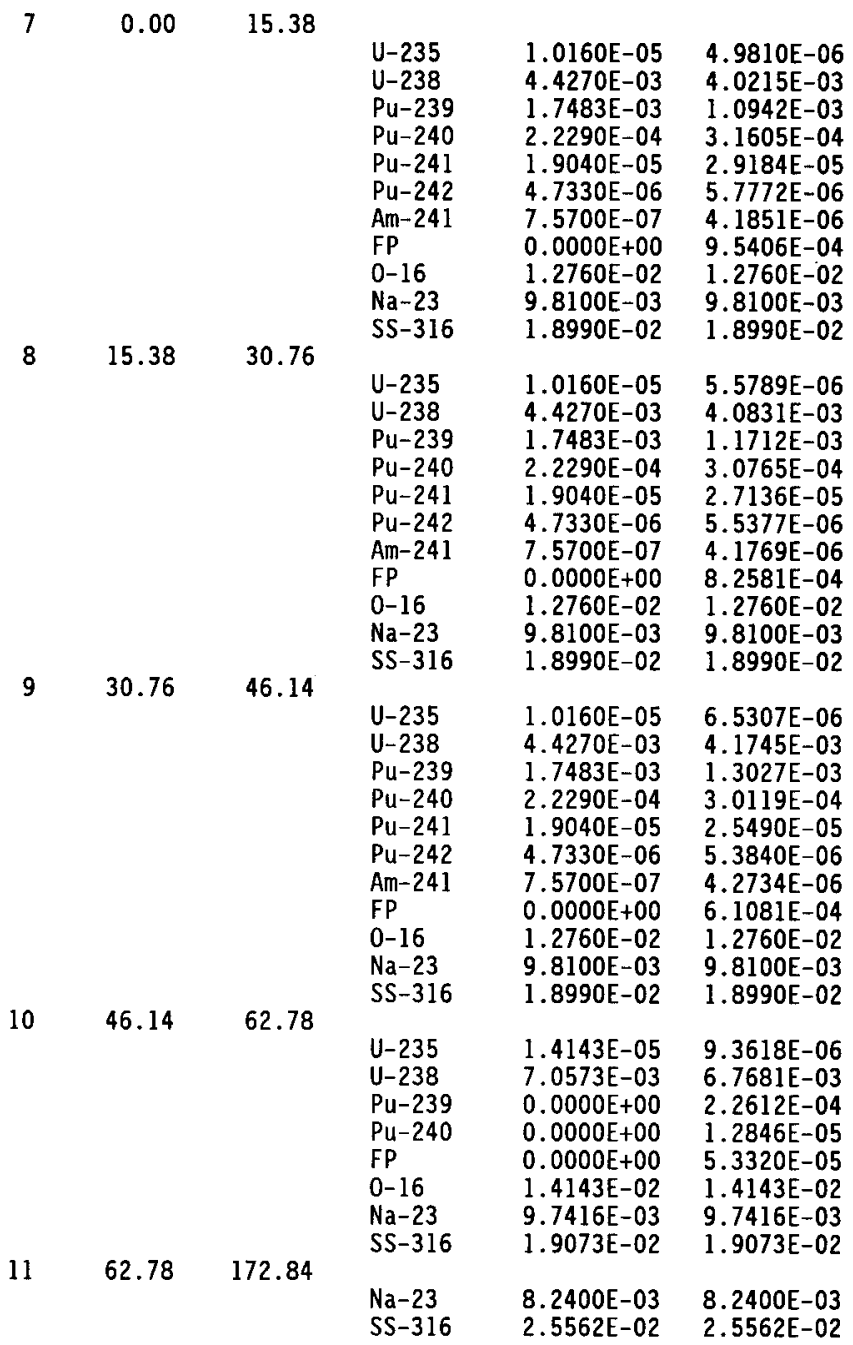


Attachment 1

\section{CHECKLIST FOR PEER REVIEW}

Document Reviewed: If

Scope of Review:

Yes No NA

[] [] []* Previous reviews complete and cover analysis, up to scope of this review,

[X] [ ] [ ] $\quad$ Problem completely defined.

[] []$[x$

$[x[][]$

[D [ ] [ ]

$[x][]$

$[x][]$

Accident scenarios developed in a clear and logical manner.

Necessary assumptions explicitly stated and supported.

Computer codes and data files documented.

Data used in calculations explicitly stated in document.

Data checked for consistency with original source information as

applicable.

[] [] $[\mathrm{x}]$ Mathematical derivations checked including dimensional consistency of results.

$[\mathrm{X}[\mathrm{]}] \mathrm{]}$

[] []$\infty$

Models appropriate and used within range of validity or use outside range of established validity justified.

Hand calculations checked for errors. Spreadsheet results should be treated exactly the same as hand calculations.

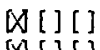

Software input correct and consistent with document reviewed.

$[x][][]$

Software output consistent with input and with results reported in

$[x][][]$

Limits/criteria/guidelines applied to analysis results are appropriate and

$\mathbb{D}[][]$

$[x][]$

Dif [ ] ]

referenced. Limits/criteria/guidelines checked against references.

Safety margins consistent with good engineering practices.

Conclusions consistent with analytical results and applicable limits.

Results and conclusions address all points required in the problem

statement.

[] [ ] $[\mathrm{X}]$

Format consistent with appropriate NRC Regulatory Guide or other standards

[] $\mathbb{N}^{*}$ Review calculations, comments, and/or notes are attached.

$\mathbb{Q}[\mathrm{C}] \mathrm{]}$

Document appróved.

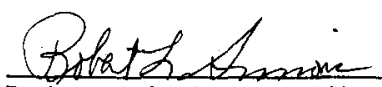

Reviewer (Printed Name and Signature)

\section{$4-9-97$ \\ Date}

* Any calculations, comments, or notes generated as part of this review should be signed, dated and attached to this checklist. Such material should be labeled and recorded in such a manner.. as to be intelligible to a technically qualified third party. 


\begin{tabular}{|c|c|c|c|c|c|}
\hline \multicolumn{6}{|c|}{ DISTRIBUTION SHEET } \\
\hline To & \multirow{2}{*}{\multicolumn{3}{|c|}{$\begin{array}{l}\text { From } \\
\text { Criticality and Shielding }\end{array}$}} & \multicolumn{2}{|c|}{ Page 1 of 1} \\
\hline Distribution & & & & \multicolumn{2}{|c|}{ Date Apri1 9, 1997} \\
\hline \multicolumn{4}{|l|}{ Project Title/Work Order } & \multicolumn{2}{|c|}{ EDT No. } \\
\hline \multicolumn{4}{|c|}{ Irradiation Data for the MFA-1 and MFA-2 Tests in the FFTF } & \multicolumn{2}{|c|}{ ECN No. 605714} \\
\hline Name & MSIN & $\begin{array}{c}\text { Text } \\
\text { With All } \\
\text { Attach. }\end{array}$ & Text Only & $\begin{array}{l}\text { Attach./ } \\
\text { Appendix } \\
\text { Only }\end{array}$ & $\begin{array}{l}\text { EDT/ECN } \\
\text { Only }\end{array}$ \\
\hline Central Files $(1+$ Original $)$ & A3-88 & $x$ & & & \\
\hline E. R. Cramer & HO-34 & & & & $x$ \\
\hline K. D. Dobbin & $\mathrm{H} 0-35$ & $\mathrm{X}$ & & & \\
\hline J. Greenborg & $\mathrm{HO}-35$ & $X$ & & & \\
\hline J. V. Nelson & $H 0-35$ & $x$ & & & \\
\hline R. J. Puigh & HO-31 & $x$ & & & \\
\hline
\end{tabular}

